\title{
Telehealth for Acute and Chronic Care Consultations
}




\section{Comparative Effectiveness Review}

Number 216

\section{Telehealth for Acute and Chronic Care Consultations}

Prepared for:

Agency for Healthcare Research and Quality

U.S. Department of Health and Human Services

5600 Fishers Lane

Rockville, MD 20857

www.ahrq.gov

Contract No. 290-2015-00009-I

Prepared by:

Pacific Northwest Evidence-based Practice Center

Portland, OR

Investigators:

Annette M. Totten, Ph.D.

Ryan N. Hansen, Pharm.D., Ph.D.

Jesse Wagner, M.A.

Lucy Stillman, B.S.

Ilya Ivlev, M.D., Ph.D., M.B.I.

Cynthia Davis-O’Reilly, B.S.

Cara Towle, R.N., M.S.N., M.A.

Jennifer M. Erickson, D.O.

Deniz Erten-Lyons, M.D.

Rongwei Fu, Ph.D.

Jesse Fann, M.D., M.P.H.

Joseph B. Babigumira, M.B.Ch.B., M.S., Ph.D.

Katherine J. Palm-Cruz, M.D.

Marc Avery, M.D.

Marian S. McDonagh, Pharm.D.

AHRQ Publication No. 19-EHC012-EF

April 2019 


\section{Key Messages}

\section{Purpose of Review}

To assess the effectiveness of telehealth consultations and explore supplemental decision analysis.

\section{Key Messages}

- Results vary by setting and condition, with telehealth consultations producing generally either better outcomes or no difference from comparators in settings and clinical indications studied.

o Remote intensive care unit consultations likely reduce mortality.

o Specialty telehealth consultations likely reduce patient time in the emergency department.

o Telehealth consultations in emergency services likely reduce heart attack mortality.

o Remote consultations for outpatient care likely improve access and clinical outcomes.

- More detailed telehealth consultation costs and outcomes data would improve modeling assumptions.

- Future research should employ rigorous methods and standardized outcomes for consistent measurement of telehealth consultation effectiveness. 
This report is based on research conducted by the Pacific Northwest Evidence-based Practice Center (EPC) under contract to the Agency for Healthcare Research and Quality (AHRQ), Rockville, MD (Contract No. 290-2015-00009-I). The findings and conclusions in this document are those of the authors, who are responsible for its contents; the findings and conclusions do not necessarily represent the views of AHRQ. Therefore, no statement in this report should be construed as an official position of AHRQ or of the U.S. Department of Health and Human Services.

\section{None of the investigators have any affiliations or financial involvement that conflicts with the material presented in this report.}

The information in this report is intended to help healthcare decision makers - patients and clinicians, health system leaders, and policymakers, among others-make well-informed decisions and thereby improve the quality of healthcare services. This report is not intended to be a substitute for the application of clinical judgment. Anyone who makes decisions concerning the provision of clinical care should consider this report in the same way as any medical reference and in conjunction with all other pertinent information, i.e., in the context of available resources and circumstances presented by individual patients.

This report is made available to the public under the terms of a licensing agreement between the author and the Agency for Healthcare Research and Quality. This report may be used and reprinted without permission except those copyrighted materials that are clearly noted in the report. Further reproduction of those copyrighted materials is prohibited without the express permission of copyright holders.

AHRQ or U.S. Department of Health and Human Services endorsement of any derivative products that may be developed from this report, such as clinical practice guidelines, other quality enhancement tools, or reimbursement or coverage policies, may not be stated or implied.

This report may periodically be assessed for the currency of conclusions. If an assessment is done, the resulting surveillance report describing the methodology and findings will be found on the Effective Health Care Program website at www.effectivehealthcare.ahrq.gov. Search on the title of the report.

Persons using assistive technology may not be able to fully access information in this report. For assistance contact EffectiveHealthCare@ahrq.hhs.gov.

Suggested citation: Totten AM, Hansen RN, Wagner J, Stillman L, Ivlev I, Davis-O’Reilly C, Towle C, Erickson JM, Erten-Lyons D, Fu R, Fann J, Babigumira JB, Palm-Cruz KJ, Avery M, McDonagh MS. Telehealth for Acute and Chronic Care Consultations. Comparative Effectiveness Review No. 216. (Prepared by Pacific Northwest Evidence-based Practice Center under Contract No. 290-2015-00009-I.) AHRQ Publication No. 19-EHC012-EF. Rockville, MD: Agency for Healthcare Research and Quality; April 2019. Posted final reports are located on the Effective Health Care Program search page.

DOI: https://doi.org/10.23970/AHRQEPCCER216. 
The Agency for Healthcare Research and Quality (AHRQ), through its Evidence-based Practice Centers (EPCs), sponsors the development of systematic reviews to assist public- and private-sector organizations in their efforts to improve the quality of healthcare in the United States. These reviews provide comprehensive, science-based information on common, costly medical conditions, and new healthcare technologies and strategies.

Systematic reviews are the building blocks underlying evidence-based practice; they focus attention on the strength and limits of evidence from research studies about the effectiveness and safety of a clinical intervention. In the context of developing recommendations for practice, systematic reviews can help clarify whether assertions about the value of the intervention are based on strong evidence from clinical studies. For more information about AHRQ EPC systematic reviews, see www.effectivehealthcare.ahrq.gov/reference/purpose.cfm.

AHRQ expects that these systematic reviews will be helpful to health plans, providers, purchasers, government programs, and the healthcare system as a whole. Transparency and stakeholder input are essential to the Effective Health Care Program. Please visit the website (www.effectivehealthcare.ahrq.gov) to see draft research questions and reports or to join an email list to learn about new program products and opportunities for input.

If you have comments on this systematic review, they may be sent by mail to the Task Order Officer named below at: Agency for Healthcare Research and Quality, 5600 Fishers Lane, Rockville, MD 20857, or by email to epc@ahrq.hhs.gov.

Gopal Khanna, M.B.A.

Director

Agency for Healthcare Research and Quality

Stephanie Chang, M.D., M.P.H.

Director

Evidence-based Practice Center Program

Center for Evidence and Practice

Improvement

Agency for Healthcare Research and Quality
Arlene S. Bierman, M.D., M.S.

Director

Center for Evidence and Practice Improvement

Agency for Healthcare Research and Quality

Elise Berliner, Ph.D.

Task Order Officer

Center for Evidence and Practice

Improvement

Agency for Healthcare Research and Quality 


\section{Investigator Affiliations}

Annette M. Totten, Ph.D.

Department of Medical Informatics \&

Clinical Epidemiology

Oregon Health \& Science University

Ryan N. Hansen, Pharm.D., Ph.D.

The CHOICE Institute, School of Pharmacy

University of Washington

Jesse Wagner, M.A.

Department of Medical Informatics \&

Clinical Epidemiology

Oregon Health \& Science University

Lucy Stillman, B.S.

Department of Medical Informatics \&

Clinical Epidemiology

Oregon Health \& Science University

Ilya Ivlev, M.D., Ph.D., M.B.I.

Department of Medical Informatics \&

Clinical Epidemiology

Oregon Health \& Science University

(Dr. Ivlev was supported by National

Library of Medicine Biomedical Informatics

Training Grant \#T15LM007088.)

Cynthia Davis-O’Reilly, B.S.

Department of Medical Informatics \&

Clinical Epidemiology

Oregon Health \& Science University

Cara Towle, R.N., M.S.N., M.A.

Integrated Care Training

Program/Psychiatry Consultation \&

Telepsychiatry

Psychiatry and Behavioral Sciences

University of Washington School of

Medicine
Jennifer M. Erickson, D.O.

Integrated Care Training

Program/Psychiatry Consultation \&

Telepsychiatry

Psychiatry and Behavioral Sciences

University of Washington School of

Medicine

Deniz Erten-Lyons, M.D.

Department of Neurology

Oregon Health \& Science University

Rongwei Fu, Ph.D.

OHSU-PSU School of Public Health

Department of Medical Informatics and

Clinical Epidemiology, School of Medicine

Oregon Health \& Science University

Jesse Fann, M.D., M.P.H.

Division of Population Health, Department

of Psychiatry \& Behavioral Sciences

Departments of Rehabilitation Medicine and

Epidemiology

University of Washington

Psychiatry \& Psychology, Seattle Cancer

Care Alliance

Clinical Research Division, Fred Hutchinson

Cancer Research Center

Joseph B. Babigumira, M.B.Ch.B., M.S., Ph.D.

Department of Global Health, School of

Public Health

University of Washington

Katherine J. Palm-Cruz, M.D.

University of Washington

Marc Avery, M.D.

Health Management Associates

Marian S. McDonagh, Pharm.D.

Department of Medical Informatics \&

Clinical Epidemiology

Oregon Health \& Science University 


\section{Acknowledgments}

The authors gratefully acknowledge the following individuals for their contributions to this project: Elaine Graham, M.L.S., for program guidance and collaborative support; Tracy Dana, M.L.S., for assistance with the literature search; Leah Williams, B.S., for editorial support; Sandra Assasnik, M.A., L.B. Miller, B.A., and Rachel Lockard, B.S., for administrative assistance; Brittany Lazur, M.P.H., for assistance with data abstraction; and our Task Order Officer, Elise Berliner, Ph.D., for support and guidance in developing this report.

\section{Technical Expert Panel}

In designing the study questions and methodology at the outset of this report, the EPC consulted several technical and content experts. Broad expertise and perspectives were sought. Divergent and conflicted opinions are common and perceived as healthy scientific discourse that results in a thoughtful, relevant systematic review. Therefore, in the end, study questions, design, methodologic approaches, and/or conclusions do not necessarily represent the views of individual technical and content experts.

Technical Experts must disclose any financial conflicts of interest greater than $\$ 5,000$ and any other relevant business or professional conflicts of interest. Because of their unique clinical or content expertise, individuals with potential conflicts may be retained. The TOO and the EPC work to balance, manage, or mitigate any potential conflicts of interest identified.

The list of Technical Experts who provided input to this report follows:

Margo Edmunds, Ph.D.*

Vice President, Evidence Generation and

Translation

Academy Health

Washington, DC

Karen Kuntz, Sc.D.

Professor, Division of Health Policy and

Management

School of Public Health, University of

Minnesota

Co-Leader Screening, Prevention, Etiology

and Cancer Survivorship Program

Masonic Cancer Center, University of

Minnesota

Minneapolis, MN
James Marcin, M.D., M.P.H.

Vice Chair, Pediatric Clinical Research

Director, UC Davis Center for Health and

Technology

Chief, Division of Pediatric Critical Care

Medicine

Professor, Department of Pediatrics

UC Davis Children's Hospital

Davis, CA

C. Daniel Mullins, Ph.D.*

Professor, Department of Pharmaceutical

Health Services Research

University of Maryland School of Pharmacy

Baltimore, Maryland

Karen Rheuban, M.D.*

Director, UVA Karen S. Rheuban Center for

Telehealth

Professor of Pediatrics

University of Virginia School of Medicine

Charlottesville, VA 
Sean Tunis, M.D., M.Sc.

President and CEO, Center for Medical

Technology Policy

Baltimore, MD

John D. Whited, M.D., M.H.S.*

Durham Veterans Affairs Health Care

System

Associate Professor of Medicine

Duke University School of Medicine

Durham, NC

*Provided input on Draft Report.
Brian Wood, M.D.

Medical Director, MW AETC ECHO

Telehealth Project

Associate Medical Director, Harborview

Satellite HIV Clinic

Associate Professor of Medicine, Division

of Allergy and Infectious Diseases

University of Washington School of

Medicine

Seattle, WA

\section{Peer Reviewers}

Prior to publication of the final evidence report, EPCs sought input from independent Peer Reviewers without financial conflicts of interest. However, the conclusions and synthesis of the scientific literature presented in this report do not necessarily represent the views of individual reviewers.

Peer Reviewers must disclose any financial conflicts of interest greater than $\$ 5,000$ and any other relevant business or professional conflicts of interest. Because of their unique clinical or content expertise, individuals with potential nonfinancial conflicts may be retained. The TOO and the EPC work to balance, manage, or mitigate any potential nonfinancial conflicts of interest identified.

The list of Peer Reviewers follows:

Larry Davis, M.D.

Professor of Neurology and Neuroscience

University of New Mexico School of

Medicine

Chief, Neurology Service

New Mexico VA Medical Center

Albuquerque, NM

Dan Malone, R.Ph., Ph.D., FAMCP

Professor of Pharmacy Practice and Science

Director, Comparative Effectiveness

Research Group

University of Arizona, College of Pharmacy

Tucson, AZ
Lois Olinger, M.A.

Vice President, Westat

Rockville, MD 


\section{Telehealth for Acute and Chronic Care Consultations}

\section{Structured Abstract}

Objectives. To conduct a systematic review to identify and summarize the available evidence about the effectiveness of telehealth consultations and to explore using decision modeling techniques to supplement the review. Telehealth consultations are defined as the use of telehealth to facilitate collaboration between two or more providers, often involving a specialist, or among clinical team members, across time and/or distance. Consultations may focus on the prevention, assessment, diagnosis, and/or clinical management of acute or chronic conditions.

Data sources. We searched Ovid MEDLINE ${ }^{\circledR}$, the Cochrane Central Register of Controlled Trials (CCRCT), and the Cumulative Index to Nursing and Allied Health Literature (CINAHL ${ }^{\circledR}$ ) to identify studies published from 1996 to May 2018. We also reviewed reference lists of identified studies and systematic reviews, and we solicited published or unpublished studies through an announcement in the Federal Register. Data for the model came both from studies identified via the systematic review and from other sources.

Methods. We included comparative studies that provided data on clinical, cost, or intermediate outcomes associated with the use of any technology to facilitate consultations for inpatient, emergency, or outpatient care. We rated studies for risk of bias and extracted information about the study design, the telehealth interventions, and results. We assessed the strength of evidence and applicability, and then synthesized the findings using quantitative and qualitative methods. An exploratory decision model was developed to assess the potential economic impact of telehealth consultations for traumatic brain injuries in adults.

Results. The search yielded 9,366 potentially relevant citations. Upon review, 8,356 were excluded and the full text of 1,010 articles was pulled for review. Of these, 233 articles met our criteria and were included-54 articles evaluated inpatient consultations; 73, emergency care; and 106, outpatient care.

The overall results varied by setting and clinical topic, but generally the findings are that telehealth improved outcomes or that there was no difference between telehealth and the comparators across the settings and for the clinical indications studied.

Remote intensive care unit (ICU) consultations likely reduce ICU and total hospital mortality with no significant difference in ICU or hospital length of stay; specialty telehealth consultations likely reduce the time patients spend in the emergency department; telehealth for emergency medical services likely reduces mortality for patients with heart attacks; and remote consultations for outpatient care likely improve access and a range of clinical outcomes (moderate strength of evidence in favor of telehealth). Findings with lower confidence are that inpatient telehealth consultations may reduce length of stay and costs; telehealth consultations in emergency care may improve outcomes and reduce costs due to fewer transfers, and also may reduce outpatient visits and costs due to less travel (low strength of evidence in favor of telehealth). Current evidence shows no difference in clinical outcomes with inpatient telehealth specialty consultations, no difference in mortality but also no difference in harms with telestroke consultations, and no difference in satisfaction with outpatient telehealth consultations (low strength of evidence of no difference). Too few studies reported information on potential harms from outpatient telehealth consultations for conclusions to be drawn (insufficient evidence). 
An exploratory cost model underscores the importance of perspective and assumptions in using modeling to extend evidence, and the need for more detailed data on costs and outcomes when telehealth is used for consultations. For example, a model comparing telehealth to transfers and in-person neurosurgical consultations for acute traumatic brain injury identified that the impact of telehealth on costs may depend on multiple factors, including how alternatives are organized (e.g., if the telehealth and in-person options are part of the same healthcare system) and whether the cost of a telehealth versus an in-person consultation differ.

Conclusions. In general, the evidence indicates that telehealth consultations are effective in improving outcomes or providing services, with no difference in outcomes; however, the evidence is stronger for some applications, and less strong or insufficient for others. However, as specific details about the implementation of telehealth consultations and the environment were rarely reported, it is difficult to assess generalizability. Exploring the use of a cost model underscored that the economic impact of telehealth consultations depends on the perspective used in the analysis. The increase in both interest and investment in telehealth suggests the need to develop a research agenda that emphasizes rigor and focuses on standardized outcome comparisons that can inform policy and practice decisions. 


\section{Contents}

Evidence Summary ............................................................................................................................. 1

Introduction ..................................................................................................................................... 1

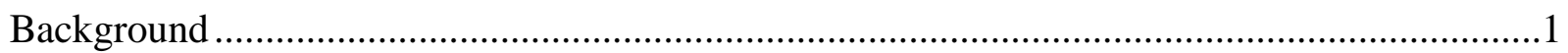

Definitions of Telehealth and Telehealth Consultation for This Project .....................................2

Scope and Questions ............................................................................................................2

Key Questions for the Systematic Review....................................................................... 3

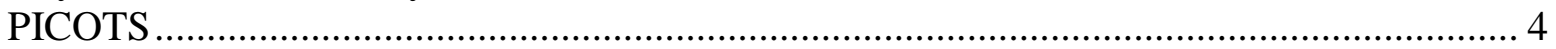

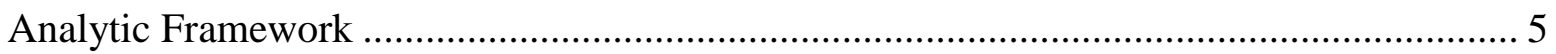

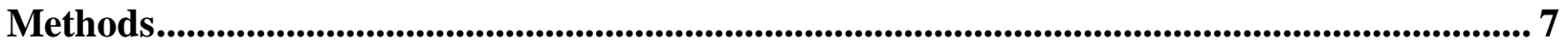

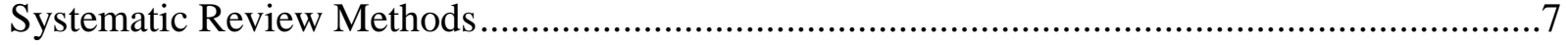

Literature Search Strategy............................................................................................. 7

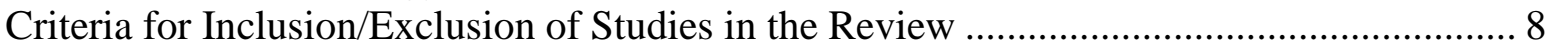

Data Abstraction and Data Management ............................................................................ 8

Assessment of Methodological Risk of Bias of Individual Studies........................................ 8

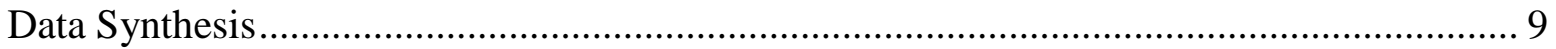

Grading the Strength of Evidence for Major Comparisons and Outcomes ........................... 10

Assessing Applicability ...................................................................................................... 10

Exploratory Cost Model for Telehealth Neurosurgical Consultations .......................................11

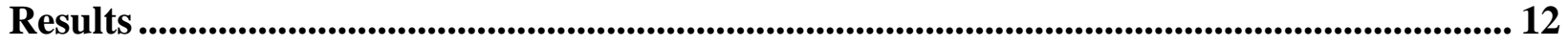

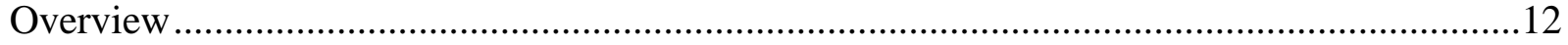

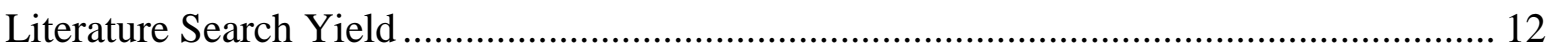

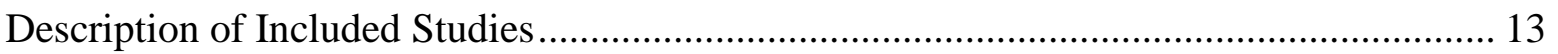

Organization of Results................................................................................................... 17

Systematic Review Results by Patient Setting..........................................................................17

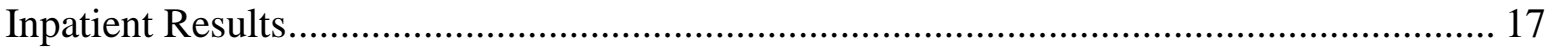

Emergency Care Results ...................................................................................... 52

Outpatient Consultation Results ......................................................................................... 83

Discussion............................................................................................................................................... 127

Key Findings and Strength of Evidence ……………….....................................................127

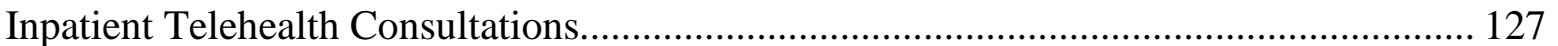

Emergency Care Telehealth Consultations ....................................................................... 129

Outpatient Telehealth Consultations............................................................................. 130

Exploratory Cost Model for Telehealth Neurosurgical Consultations ................................. 132

Findings in Relationship to What Is Already Known ..........................................................132

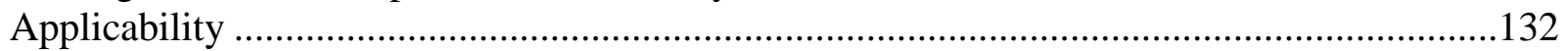

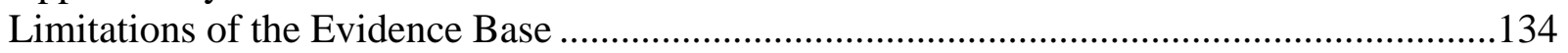

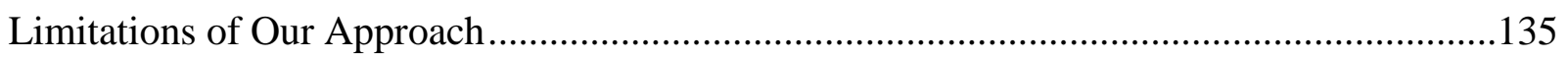

Future Research Needs ................................................................................................136

Implications and Conclusions ..................................................................................................... 139

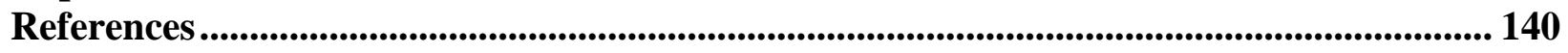

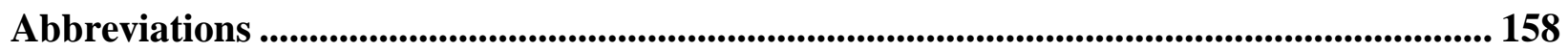

Tables

Table A. Inpatient telehealth consultations: strength of evidence ............................................. ES-6

Table B. Emergency care telehealth consultations: strength of evidence.................................... ES-7

Table C. Outpatient care telehealth consultations: strength of evidence .................................. ES-9 


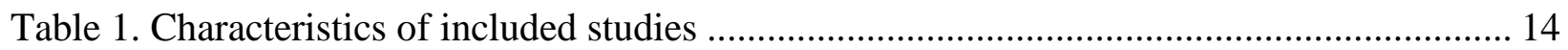

Table 2. Remote intensive care units: summary of evidence...................................................... 18

Table 3. Remote intensive care units: selected outcomes not included in meta-analysis.............. 25

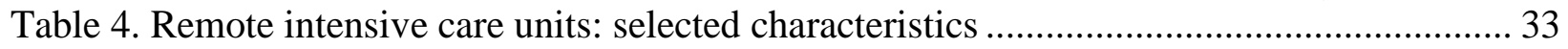

Table 5. Inpatient specialty consultations: summary of evidence .................................................. 36

Table 6. Inpatient specialty consultations: selected outcomes....................................................... 41

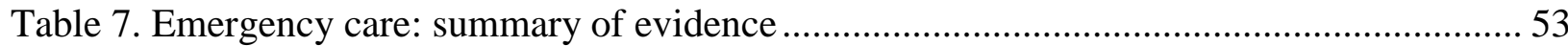

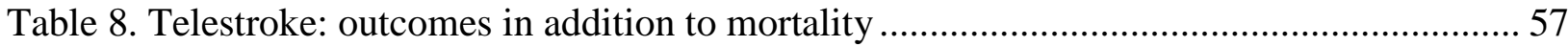

Table 9. Emergency care telehealth specialty consultations: selected outcomes ............................6 66

Table 10. Emergency medical services and urgent care telehealth: selected outcomes ................. 75

Table 11. Outpatient care consultations: summary of evidence …………………………............. 84

Table 12. Characteristics of outpatient consultations and outcomes, percent (counts) ................. 87

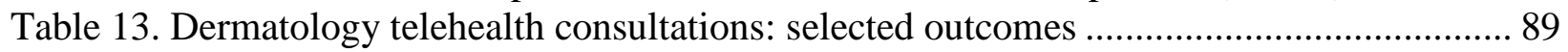

Table 14. Wound care telehealth consultations: selected outcomes ........................................... 96

Table 15. Ophthalmologic telehealth consultations: selected outcomes ..................................... 98

Table 16. Orthopedic telehealth consultations: selected outcomes ............................................. 100

Table 17. Dentistry telehealth consultations: selected outcomes................................................ 102

Table 18. Cancer telehealth consultations: selected outcomes ........................................................ 103

Table 19. Psychiatry telehealth consultations: selected outcomes............................................... 107

Table 20. Infectious disease telehealth consultations: selected outcomes.................................. 109

Table 21. Single specialties using diagnostic technology: selected outcomes ............................. 111

Table 22. Single specialty by type: selected outcomes............................................................... 115

Table 23. Multiple specialty telehealth consultations: selected outcomes ................................... 123

Table 24. Inpatient telehealth consultations: strength of evidence ............................................ 128

Table 25. Emergency care telehealth consultations: strength of evidence ………....................... 129

Table 26. Outpatient care telehealth consultations: strength of evidence.................................... 131

\section{Figures}

Figure 1. Analytic framework for telehealth consultations ........................................................... 6

Figure 2. Literature flow diagram ...................................................................................... 12

Figure 3. Distribution of included articles $(\mathrm{n}=233)$...................................................................... 16

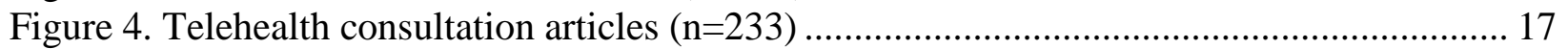

Figure 5. Meta-analysis of intensive care unit mortality ............................................................ 21

Figure 6. Meta-analysis of hospital mortality in intensive care unit studies ................................ 22

Figure 7. Meta-analysis of intensive care unit length of stay ……………………....................... 23

Figure 8. Meta-analysis of hospital length of stay in intensive care unit studies ......................... 24

Figure 9. Impact of telestroke on mortality .................................................................................... 56

\section{Appendixes}

Appendix A. Literature Search Strategies

Appendix B. Inclusion and Exclusion Criteria

Appendix C. Included Studies

Appendix D. Excluded Studies

Appendix E. Telehealth for Consultations Risk of Bias Instructions

Appendix F. Evidence Tables

Appendix G. Risk of Bias

Appendix H. Strength of Evidence 
Appendix I. Exploratory Decision Modeling Methods and Results 


\section{Evidence Summary}

\section{Background}

Telehealth is the use of information and telecommunications technology to provide or support healthcare across time and/or distance. Telehealth's potential benefits are frequently cited, ${ }^{1,2}$ and there is a sizable body of research on telehealth, including systematic reviews and reviews of reviews. ${ }^{3-8}$ Despite this potential, implementation and spread has been slower than expected, ${ }^{9,10}$ though recent trends suggest that use of telehealth may be increasing.

With improvement in technologies, ${ }^{11}$ changes in payment policies, ${ }^{12,13}$ and evolving models for healthcare in general and telehealth in particular, the possibility exists for a rapid acceleration in implementation and wider use of telehealth. However, targeting, supporting, and sustaining increased use of telehealth requires organized and accessible information on the impact of different uses of telehealth. Specifically, synthesis of existing research evidence can help inform decisions about where, in terms of settings and clinical indications, telehealth is likely to improve access, quality, and efficiency. One approach is to assess the evidence about the different roles telehealth can play in healthcare.

Telehealth for consultations allows medical expertise to be available where and when it is needed, minimizing potential time or geographic barriers to care and maximizing the efficient use of scarce resources. Telehealth for consultations has been studied across a range of clinical situations but not previously assessed in a systematic review.

\section{Objective}

The objective of this report is to identify and summarize the available evidence about telehealth consultations. The overarching goal is to maximize the utility of available information by presenting the results in formats that support decision makers at various levels (e.g., regulators, providers, and payers) as they consider policy and practice changes related to telehealth for consultation. To accomplish this we combined a broad systematic review, covering a range of clinical indications, with an exploratory decision model for one selected clinical application. Both systematic reviews and decision analyses have accepted methodologies, but they are not frequently used in tandem. In this sense, this project is experimental as it strives to provide the results of a traditional systematic review of the available research and explore how the addition of decision analysis might be used to increase the utility of evidence for decision makers.

This review focuses on the effectiveness of telehealth for provider-to-provider consultations. Telehealth consultations are defined as the use of telehealth to facilitate collaboration between providers, often involving a specialist consultant, or among clinical team members, across time and/or distance, on the assessment, diagnosis, and/or clinical management of a specific patient or group of patients. While the patient may or may not be involved in the consultation, the consultation is required to be related to a specific patient or group of patients in order to differentiate this activity from training or education (which would not meet our definition of telehealth). Limited information provided by one clinician to another that does not contribute to collaboration (e.g., interpretation of an electroencephalogram, report on an x-ray or scan, or reporting the results of a diagnostic test) is not considered a consultation for this review. 


\section{Systematic Review Key Questions}

The Key Questions for the review were:

1. Are telehealth consultations effective in improving clinical and economic outcomes?

Clinical and economic outcomes may include, but are not limited to, mortality and morbidity, patient-reported outcomes, quality of life, utilization of health services, and cost of services.

2. Are telehealth consultations effective in improving intermediate outcomes?

Intermediate outcomes include both outcomes that precede the ultimate outcomes of interest (e.g., mediators) and secondary outcomes. Intermediate outcomes may include, but are not limited to, access to care, patient and provider satisfaction, behavior, and decisions (e.g., patient completion of treatment, provider antibiotic stewardship); volume of services; and healthcare processes (e.g., time to diagnosis or treatment).

3. Do telehealth consultations result in harms, adverse events, or negative unintended consequences?

4. What are the characteristics of telehealth consultations that have been the subject of comparative studies?

These characteristics may include clinical conditions, characteristics of the providers and patients and their relationships, telehealth modalities, and characteristics of settings, including the type of care and healthcare organization, payment models, as well as geographic and economic characteristics.

5. Do clinical, economic, intermediate, or negative outcomes (i.e., the outcomes in Key Questions 1, 2, and 3) vary across telehealth consultation characteristics (Key Question 4)?

\section{Systematic Review Methods}

The conduct of this systematic review followed the Methods Guide for Effectiveness and Comparative Effectiveness Reviews, ${ }^{14}$ and it is reported according to the PRISMA checklist. The scope, Key Questions, and inclusion criteria of this review were developed in consultation with a group of technical experts. Detailed methods are available in the full report and the posted protocol (https://effectivehealthcare.ahrq.gov/topics/telehealth-acute-chronic/research-protocol/). The protocol was registered with PROSPERO (CRD42017058304).

A research librarian created the search strategy and another research librarian reviewed it before searching Ovid MEDLINE ${ }^{\circledR}$, the Cochrane Central Register of Controlled Trials (CCRCT), and the Cumulative Index to Nursing and Allied Health Literature (CINAHL ${ }^{\circledR}$ ) to identify studies published from 1996 through May 2018. We also reviewed reference lists of identified studies and systematic reviews, and solicited suggestions through an announcement in the Federal Register.

We limited our study inclusion to the use of telehealth for consultations and outcomes that measure clinical and cost effectiveness. Otherwise our criteria were broad, and we included any 
technology and any comparative study, including before-after and retrospective as well as prospective designs, with quantitative outcomes data. Studies could compare telehealth consultations to consultations done in a different mode (e.g., in-person or telephone), no access to specialty care, or usual care which could be an unspecified mix of these options. We excluded descriptive studies, studies assessing only diagnostic concordance, studies where there was no nontelehealth comparison, and modeling studies that used hypothetical data.

Two team members independently reviewed all abstracts and two reviewers independently assessed each full-text article. Disagreements were resolved by discussion among investigators. For included articles, investigators abstracted key characteristics and data about the studies for quantitative and qualitative synthesis. We were able to conduct meta-analyses for some but not all topics and outcomes due to the heterogeneity of outcome measures, study designs, and telehealth interventions. Two investigators independently rated the risk of bias of each study using predefined criteria consistent with the chapter, "Assessing the Risk of Bias of Individual Studies When Comparing Medical Interventions” in the Methods Guide for Effectiveness and Comparative Effectiveness Reviews. ${ }^{14}$ Risk of bias for economic evaluations were assessed using a modified version of the Consensus Health Economic Criteria. ${ }^{15,16}$ Disagreements were resolved by consensus.

Strength of evidence was assessed for each outcome and Key Question as described in the Methods Guide for Effectiveness and Comparative Effectiveness Reviews. ${ }^{14}$ We assigned a strength of evidence grade of high, moderate, low, or insufficient for the body of evidence for each Key Question, based on evaluation of four domains: study limitations, consistency, directness, and precision. High, moderate, and low ratings reflect our confidence in the accuracy and validity of the findings and whether future studies might alter these findings (magnitude or direction). We gave a rating of insufficient when we were unable to draw conclusions due to serious inconsistencies, serious methodological limitations, or lack of evidence. We considered applicability and the strength of evidence when making general assessments across the studies and use qualifiers in key messages and conclusions such as 'likely' for moderate strength of evidence and/or some applicability concerns or 'may' for low strength of evidence and/or significant applicability concerns.

\section{Systematic Review Results}

The literature searches yielded 9,366 potentially relevant citations. Upon review of the article titles and abstracts, 8,356 were excluded and the full text of 1,010 articles were pulled for review. Of these, 233 articles met our inclusion criteria. The most frequent reasons for excluding an article were that the intervention was not a telehealth consultation (ineligible intervention) or that the study did not compare telehealth consultations to usual care or another intervention (ineligible comparison). A list of the included studies is provided in Appendix C of the full report, and the citations for excluded studies are in Appendix D of the full report.

The included studies are diverse in terms of location, technology, and design. The most frequent geographic location for the included studies of telehealth consultations was the United States (110 articles or 47\%); however, more than half of the studies were conducted in other countries. The most common mode or technology used for telehealth consultations was video, which was used in more than half of the studies (55\%). The majority of the studies (66\%) were observational, including prospective cohorts, retrospective cohorts, and before/after studies in which a group of patients from before the implementation of telehealth consultations are compared to a different group of patients after telehealth implementation. In these studies, the 
comparator was often usual care, that is, care without telehealth, and the studies rarely provided more detail (e.g., if consultations were in-person, if care was delivered without consultation, or a mix of both). About one-fifth (19\%) were randomized controlled trials, 12 percent were economic evaluations, and approximately 3 percent were pre-post studies in which outcomes for the same patients were compared pre (without telehealth) and post telehealth consultations. Table 1 in the full report provides more information on the characteristics of the included studies, and detailed information abstracted from each study is provided in Appendix F of the full report.

We categorized the systematic review results into three patient settings: inpatient, emergency department (ED) or emergency medical services (EMS), and outpatient. We chose to organize the systematic review results by patient setting as the settings are likely to have different telehealth technology and requirements as well as differences in payment structures, staffing, and organization of care delivery. The results are summarized by setting in Tables A, B, and C and in the accompanying text.

\section{Inpatient Telehealth Consultations}

\section{Remote Intensive Care Units}

- Clinical outcomes: Intensive care unit (ICU) and hospital mortality are lower with remote ICU (moderate strength of evidence).

- Economic outcomes: Not all studies analyzed costs of remote ICUs or their impact on revenue; those that did used differing methods, and conclusions were inconsistent (insufficient evidence).

- Intermediate outcomes: ICU and hospital length of stay (LOS) are not statistically different with remote ICU (moderate strength of evidence).

- Adverse effects: None of the included studies specifically addressed potential harms or unintended consequences (insufficient evidence).

We identified 21 studies reported in 22 articles evaluating the use of telehealth to provide remote ICU services. Remote ICU services involve off site staff (intensivists, critical care nurses, and sometimes administrative assistants) who monitor ICU patients and provide consultation and management assistance with the care of these patients. Thirteen of these studies used before-after designs comparing outcomes from a period before implementation of remote ICUs to the period after this model of care was in operation in the same hospital or hospitals. The remaining studies include four retrospective and two prospective cohort studies, one cross sectional survey, and one pre-post survey. These studies did not provide details on the nontelehealth care, though it likely included a mix of care by clinicians who are not specialists, less frequent care by specialists, and transfers to other hospitals. We reviewed selected key factors that could help explain the differences in outcomes across studies, including information on the hospitals that were the sites for the studies, the coverage and staffing of the remote ICU interventions, and the time periods in which outcomes were measured. For example, the majority of included studies were conducted in larger teaching hospitals or hospitals affiliated with an academic center; and all of the studies included a physician intensivist, most included nursing, and about half included administrative support. However, none of these factors clearly differentiate between studies reporting a clear benefit from remote ICUs and those reporting no benefit or possible benefits. Furthermore, this limitation means we cannot compare the ICUs included in these studies to all ICUs that might consider employing telehealth based on our data. 


\section{Inpatient Specialist Consultations}

- Clinical outcomes: Mortality or serious morbidity (e.g., cardiac arrest, low birthweight, falls, and disability) improve with telehealth consultations across specialties, but differences are not statistically significant in most studies (low strength of evidence).

- Economic outcomes: Cost savings were realized due to avoided transfers or travel, but telehealth did not save money in all studies (low strength of evidence).

- Intermediate outcomes: The impact of telehealth consultations on intermediate outcomes such as hospital LOS, transfer rate or satisfaction of patients, relatives, or healthcare providers is also positive, but not convincing with differences that are close to significant and estimates that are less precise (low strength of evidence).

- Adverse effects: Only studies of surgery explicitly examined harms, but the study limitations were high based on small sample sizes and high risk of bias (insufficient evidence).

We identified 31 studies (32 articles) that reported the use of telehealth to provide specialty consultations for inpatients. Specialty consultations are provided when the input of a specialist is needed for diagnosis, care planning, or treatment, and a physician with the specialized knowledge is not available at the hospital where the patient is located or at the time when the consultation is needed. The studies of inpatient specialist consultations cover a wide range of clinical indications, ranging from neonatal to geriatric care and from care planning to remote proctoring of surgery. Studies of inpatient consultations were predominately cohort studies, including ten retrospective and 15 prospective cohort designs that compare hospitals with and without telehealth. There were also three before-after studies and three randomized trials that studied inpatient consultations. Most of the cohort studies included multiple sites with the largest prospective study including 3,060 patients in 5 intervention and 5 matched comparison hospitals. Overall, inpatient telehealth consultations are not well-described, making it problematic to relate characteristics of the intervention or environment to effectiveness and to assess the generalizability of the study results to either hospitals that may differ in important ways from those included in the studies or to the use for other specialties not studied. 
Table A. Inpatient telehealth consultations: strength of evidence

\begin{tabular}{|c|c|c|c|c|}
\hline Topic & Outcome (KQ) & $\begin{array}{l}\text { Number } \\
\text { of } \\
\text { Studies } \\
\text { (N) }\end{array}$ & Main Findings & $\begin{array}{l}\text { Strength of } \\
\text { Evidence } \\
\text { (Insufficient, } \\
\text { Low, } \\
\text { Moderate, } \\
\text { High) }\end{array}$ \\
\hline \multirow[t]{6}{*}{$\begin{array}{l}\text { Inpatient } \\
\text { remote ICU }\end{array}$} & $\begin{array}{l}\text { ICU Mortality } \\
(\text { KQ1) }\end{array}$ & 11 & $\begin{array}{l}\text { Lower ICU mortality } \\
\text { RR } 0.69(95 \% \mathrm{Cl} 0.51,0.89)\end{array}$ & Moderate \\
\hline & $\begin{array}{l}\text { Hospital Mortality } \\
(\text { KQ1 })^{\mathrm{a}}\end{array}$ & 12 & $\begin{array}{l}\text { Lower hospital mortality } \\
\text { RR } 0.76(95 \% \mathrm{Cl}, 0.60,0.95)\end{array}$ & Moderate \\
\hline & Cost (KQ1) & 6 & $\begin{array}{l}\text { Unable to summarize across studies: different } \\
\text { methods and inconsistent results. }\end{array}$ & Insufficient \\
\hline & $\begin{array}{l}\text { ICU LOS } \\
(K Q 2)^{a}\end{array}$ & 12 & $\begin{array}{l}\text { No significant difference in ICU LOS } \\
\text { Mean difference (days) }-0.39(95 \% \mathrm{Cl}-0.99,0.15)\end{array}$ & Moderate \\
\hline & $\begin{array}{l}\text { Hospital LOS } \\
(\text { KQ2) }\end{array}$ & 12 & $\begin{array}{l}\text { No significant difference in hospital LOS } \\
\text { Mean difference (days) }-0.14(95 \% \mathrm{Cl}-0.96,0.63)\end{array}$ & Moderate \\
\hline & $\begin{array}{l}\text { Harms } \\
\text { (KQ3) }\end{array}$ & 0 & None reported in identified articles & Insufficient \\
\hline \multirow{5}{*}{$\begin{array}{l}\text { Inpatient } \\
\text { specialty } \\
\text { consultations }\end{array}$} & Mortality (KQ1) & 12 & No significant difference in mortality & Low \\
\hline & $\begin{array}{l}\text { Other clinical } \\
\text { outcomes (KQ1) }\end{array}$ & 6 & $\begin{array}{l}\text { Clinical outcomes better with telehealth but small } \\
\text { differences and most not significantly different }\end{array}$ & Low \\
\hline & Cost (KQ1) & 7 & $\begin{array}{l}\text { Cost savings due to avoiding transfers or travel } \\
\text { when telehealth is used but not in all studies }\end{array}$ & Low \\
\hline & $\begin{array}{l}\text { Intermediate } \\
\text { outcome (KQ2) }\end{array}$ & 27 & $\begin{array}{l}\text { Reductions in LOS and waiting time but all not } \\
\text { significantly different; satisfaction measures good } \\
\text { but not excellent }\end{array}$ & Low \\
\hline & Harms (KQ3) & 3 & $\begin{array}{l}\text { Complications from telehealth in surgery was } \\
\text { compared with standard procedures in small } \\
\text { studies with high risk of bias }\end{array}$ & Insufficient \\
\hline
\end{tabular}

$\mathrm{CI}$ = confidence interval; ICU = intensive care unit; KQ = Key Question; LOS = length of stay; RR = risk ratio

${ }^{\text {a }}$ Based on studies included in the meta-analysis

\section{Emergency Care Telehealth Consultations}

We split emergency care into three subtopics, as follows.

\section{Telestroke}

- Clinical outcomes: The evidence suggests that telestroke does not result in differences in either in-hospital or 3-month mortality (moderate strength of evidence)

- Intermediate outcomes: Changes in thrombolytic therapy (tPA) use and time to treatment with telestroke were not significantly different (low strength of evidence).

- Adverse events: No increased harms, specifically hemorrhage (moderate strength of evidence).

\section{Specialty consultations in emergency departments}

- Clinical outcomes: The impact on clinical outcomes including mortality and functional status is generally positive, though the results are not always statistically significant (low strength of evidence).

- Economic outcomes: Analysis of costs were available only in a few studies, and the results favored savings (low strength of evidence).

- Intermediate outcomes: Teleconsultations have a positive effect on intermediate outcomes such as appropriate triage and transfers and shorter time in the emergency department (moderate strength of evidence).

- Adverse events: No information was available about harms (insufficient evidence). 


\section{EMS and urgent care}

- Clinical outcomes: Telehealth reduces mortality for heart attack patients (moderate strength of evidence).

- Economic outcomes: Reductions in air transfers and referrals contributed to estimates of lower costs (low strength of evidence).

- Intermediate outcomes: Telehealth led to more timely provision of care and a reduction in air transfers and referrals to higher-level care following urgent care (moderate strength of evidence), and these

- Adverse effects: Information on harms was very limited (insufficient evidence).

Telestroke refers to the use of telemedicine to convey information about a patient to a vascular neurologist/stroke specialist for assessment and diagnosis with a focus on time sensitive treatment and transport decisions. The 29 studies that investigated telestroke all compared outcomes from a prior time period or another hospital without telestroke with those with telestroke. All patients were treated, but in the cases without telestroke, patients received care for their stroke but after a delay, which may have limited their treatment options. Ten studies of EMS evaluated an approach similar to telestroke for patients potentially experiencing heart attacks. Fifteen of the 19 studies of specialist consultations in EDs were before-after or cohort studies that did not provide detailed information on the care without telehealth. In the 21 studies of telehealth consultations for EMS or urgent care, in the groups without telehealth, emergency personnel or clinicians made decisions about transfer or treatment without consultant input.

Table B. Emergency care telehealth consultations: strength of evidence

\begin{tabular}{|c|c|c|c|c|}
\hline Topic & $\begin{array}{l}\text { Outcome } \\
\text { (KQ) }\end{array}$ & $\begin{array}{l}\text { Number } \\
\text { of } \\
\text { Studies } \\
\text { (N) }\end{array}$ & Main Findings & $\begin{array}{l}\text { Strength of Evidence } \\
\text { (Insufficient, Low, } \\
\text { Moderate, High) }\end{array}$ \\
\hline \multirow{5}{*}{$\begin{array}{l}\text { Emergency } \\
\text { Care: } \\
\text { Telestroke }\end{array}$} & $\begin{array}{l}\text { Mortality In- } \\
\text { hospital (KQ1) }\end{array}$ & 9 & $\begin{array}{l}\text { RR } 0.89 \\
(95 \% \mathrm{Cl} 0.63,1.43) \text { No difference }\end{array}$ & Moderate \\
\hline & $\begin{array}{l}\text { Mortality 3- } \\
\text { month (KQ1) }\end{array}$ & 7 & $\begin{array}{l}\text { RR } 0.94 \\
(95 \% \mathrm{Cl} 0.82,1.16) \quad \text { No difference }\end{array}$ & Moderate \\
\hline & $\begin{array}{l}\text { tPA } \\
\text { administration } \\
\text { (KQ2) }\end{array}$ & 13 & $\begin{array}{l}\text { Reported tPA use increases; four } \\
\text { significant; majority not statistically } \\
\text { significant or not tested }\end{array}$ & Low \\
\hline & $\begin{array}{l}\text { Time to } \\
\text { Treatment } \\
\text { (KQ2) }\end{array}$ & 23 & $\begin{array}{l}\text { Time to treatment is shorter but not } \\
\text { significant in the majority of studies; a } \\
\text { minority report longer times }\end{array}$ & Low \\
\hline & $\begin{array}{l}\text { Harms (all } \\
\text { Hemorrhage) } \\
\text { (KQ3) }\end{array}$ & 11 & $\begin{array}{l}\text { No difference in hemorrhage, the only } \\
\text { potential harm reported }\end{array}$ & Moderate \\
\hline \multirow[t]{3}{*}{$\begin{array}{l}\text { Emergency } \\
\text { Care: } \\
\text { Specialty } \\
\text { Consultations }\end{array}$} & $\begin{array}{l}\text { Clinical } \\
\text { outcomes } \\
\text { (KQ1) }\end{array}$ & 13 & $\begin{array}{l}\text { Lower mortality reported in most } \\
\text { studies but not statistically significant; } \\
\text { Four studies reporting other clinical } \\
\text { outcomes that were better with } \\
\text { telehealth; one reported significant } \\
\text { differences }\end{array}$ & Low \\
\hline & Cost (KQ1) & 5 & $\begin{array}{l}\text { Lower costs with better or no change } \\
\text { in clinical outcome in most (4) studies; } \\
\text { one study reported higher costs }\end{array}$ & Low \\
\hline & $\begin{array}{l}\text { Intermediate } \\
\text { outcomes } \\
\text { (KQ2) }\end{array}$ & 19 & $\begin{array}{l}\text { Increase in appropriate transfers, } \\
\text { decrease in time to decision and time } \\
\text { in ED with telehealth compared with } \\
\text { standard care }\end{array}$ & Moderate \\
\hline
\end{tabular}




\begin{tabular}{|l|l|c|l|c|}
\hline Topic & $\begin{array}{c}\text { Number } \\
\text { of } \\
\text { Outcome } \\
\text { (KQ) }\end{array}$ & $\begin{array}{c}\text { (N) } \\
\text { (K) }\end{array}$ & Main Findings & $\begin{array}{c}\text { Strength of Evidence } \\
\text { Insufficient, Low, } \\
\text { Moderate, High) }\end{array}$ \\
\hline & Harms (KQ3) & 10 & $\begin{array}{l}\text { No studies reported data on harms } \\
\text { from telehealth }\end{array}$ & Insufficient \\
\hline $\begin{array}{l}\text { Emergency } \\
\text { Care: EMS or } \\
\text { Urgent Care }\end{array}$ & $\begin{array}{l}\text { Clinical } \\
\text { Outcomes } \\
\text { (KQ1) }\end{array}$ & STEMl patients & Moderate \\
\cline { 2 - 5 } & Cost (KQ1) & 5 & $\begin{array}{l}\text { Lower costs due to avoided transfers } \\
\text { or lower staff costs when telehealth is } \\
\text { used }\end{array}$ & Low \\
\cline { 2 - 5 } & $\begin{array}{l}\text { Intermediate } \\
\text { Outcomes } \\
\text { (KQ2) }\end{array}$ & 20 & $\begin{array}{l}\text { Treatment is more timely and fewer air } \\
\text { transfers or referrals to higher level of } \\
\text { care }\end{array}$ & Moderate \\
\cline { 2 - 5 } & Harms (KQ3) & 1 & $\begin{array}{l}\text { One study reported data that could be } \\
\text { interpreted as harms, but not defined } \\
\text { as such by the authors }\end{array}$ & Insufficient \\
\hline
\end{tabular}

CI = confidence interval; ED = emergency department; EMS = emergency medical services; KQ = Key Question; RR = risk ratio; STEMI = ST-elevation myocardial infarction; $\mathrm{tPA}=$ tissue plasminogen activator;

\section{Outpatient Telehealth Consultations}

- Clinical outcomes: Clinical outcomes were reported in just over one-quarter of the studies of telehealth consultations and in 7 of 11 clinical topics. In three topics, there is moderate strength of evidence of the benefits of telehealth (better healing in wound care, higher response to treatment in psychiatry, and improvement in chronic condition outcomes), and in dermatology the findings show no difference in clinical outcomes (low strength of evidence). In three topics (cancer, infectious disease, and multiple specialties) studies were identified, but the results were inconsistent (insufficient evidence).

\section{- Intermediate outcomes}

o Access: Telehealth consultations improved access by reducing wait times and time to treatment and by increasing the number of patients receiving indicated diagnostic tests or treatment (moderate strength of evidence).

o Management and utilization: Telehealth consultations reduced utilization (the number of in-person specialist and hospital visits; number of hospitalizations, and shorter lengths of stay) in most studies. Findings were inconsistent about agreement on diagnosis and management (low strength of evidence).

o Satisfaction: Patients were generally more satisfied with telehealth consultations, particularly when telehealth saved time or expense compared with the alternative. Clinicians tended to be less satisfied with telehealth than in-person consultations, though differences were rarely statistically significant (low strength of evidence).

- Costs: Studies report lower costs and in most cases savings are attributable to reductions in transfers or less transportation. However, the rigor of the measurement, imprecision of estimates and inconsistency in the magnitude of the effects limits confidence in these findings (low strength of evidence).

- Harms: Only two of studies explicitly examined harms, reporting lower rates of complications with telehealth (insufficient evidence).

The 106 included articles evaluating telehealth consultations in the outpatient setting are summarized in Table C. Detailed results split into 11 clinical topics are provided in the full 
report. All of these studies addressed at least one intermediate outcome, and we organized these into three categories: access, management and utilization, and satisfaction.

For the 11 clinical topics, seven reported clinical outcomes (dermatology, wound care, orthopedics, cancer, psychiatry, infectious disease and single specialties). In four of these seven the body of evidence supports better outcomes with telehealth. For 10 topics there were improvements in at least one intermediate outcome. Cost outcomes were identified for nine out of 11 topics, but the conclusions are mixed with lower costs reported across studies for four topics (wound care, orthopedics, cancer, single specialties with diagnostic technology), while for the other five topics the results were inconsistent or cost savings were either minimal or not realized.

Table C. Outpatient care telehealth consultations: strength of evidence

\begin{tabular}{|c|c|c|c|}
\hline Outcome (KQ) & $\begin{array}{l}\text { Number } \\
\text { of } \\
\text { Studies } \\
\text { (N) }\end{array}$ & Main Findings & $\begin{array}{l}\text { Strength of } \\
\text { Evidence } \\
\text { (Insufficient, Low, } \\
\text { Moderate, High) }\end{array}$ \\
\hline $\begin{array}{l}\text { Clinical Outcomes } \\
\text { (KQ1): Dermatology }\end{array}$ & 3 & No significant different in clinical course & Low \\
\hline $\begin{array}{l}\text { Clinical Outcomes } \\
\text { (KQ1): Wound Care }\end{array}$ & 5 & Better healing and fewer amputations & Moderate \\
\hline $\begin{array}{l}\text { Clinical Outcomes } \\
\text { (KQ1): Ophthalmology }\end{array}$ & 0 & No studies reported data on clinical outcomes & Insufficient \\
\hline $\begin{array}{l}\text { Clinical Outcomes } \\
\text { (KQ1): Orthopedics }\end{array}$ & 0 & No studies reported data on clinical outcomes & Insufficient \\
\hline $\begin{array}{l}\text { Clinical Outcomes } \\
\text { (KQ1): Dental }\end{array}$ & 0 & No studies reported data on clinical outcomes & Insufficient \\
\hline $\begin{array}{l}\text { Clinical Outcomes } \\
\text { (KQ1): Cancer }\end{array}$ & 1 & $\begin{array}{l}\text { Rate of serious side effects from chemotherapy } \\
\text { reported in } 1 \text { study. }\end{array}$ & Insufficient \\
\hline $\begin{array}{l}\text { Clinical Outcomes } \\
\text { (KQ1): Psychiatry }\end{array}$ & $\begin{array}{l}3 \text { (in five } \\
\text { articles) }\end{array}$ & Decrease in symptoms and high remission rates & Moderate \\
\hline $\begin{array}{l}\text { Clinical Outcomes } \\
\text { (KQ1): Infectious } \\
\text { Disease }\end{array}$ & 3 & $\begin{array}{l}\text { Inconsistent results for virologic suppression across } \\
\text { studies }\end{array}$ & Insufficient \\
\hline $\begin{array}{l}\text { Clinical Outcomes } \\
\text { (KQ1): ): Single } \\
\text { Conditions with } \\
\text { Diagnostic Technology }\end{array}$ & 0 & No studies reported data on clinical outcomes & Insufficient \\
\hline $\begin{array}{l}\text { Clinical Outcomes } \\
\text { (KQ1): Single } \\
\text { Specialties }\end{array}$ & 6 & $\begin{array}{l}\text { Positive effects on clinical outcomes such as } \\
\text { response to treatment. }\end{array}$ & Moderate \\
\hline $\begin{array}{l}\text { Clinical Outcomes } \\
\text { (KQ1):Multiple } \\
\text { Specialties } \\
\end{array}$ & 4 & $\begin{array}{l}\text { Inconsistent results across studies for unanticipated } \\
\text { or avoidable health services utilization }\end{array}$ & Insufficient \\
\hline Cost (KQ1) & 32 & $\begin{array}{l}\text { Most studies report cost saving with telehealth but } \\
\text { calculations vary and most are dependent on patient } \\
\text { avoided travel and loss of time }\end{array}$ & Low \\
\hline $\begin{array}{l}\text { Intermediate Outcomes: } \\
\text { Access (KQ2) }\end{array}$ & 35 & $\begin{array}{l}\text { Access in terms of time to, or comprehensiveness } \\
\text { of, service is improved with telehealth }\end{array}$ & Moderate \\
\hline $\begin{array}{l}\text { Intermediate Outcomes: } \\
\text { Management and } \\
\text { Utilization (KQ2) }\end{array}$ & 31 & $\begin{array}{l}\text { Mixed results with majority finding some benefit in } \\
\text { terms of avoiding visits and similar diagnosis or } \\
\text { management but a subset of studies report } \\
\text { differences in diagnosis and management with } \\
\text { telehealth compared with standard care }\end{array}$ & Low \\
\hline $\begin{array}{l}\text { Intermediate Outcomes: } \\
\text { Satisfaction (KQ2) }\end{array}$ & 22 & $\begin{array}{l}\text { Satisfaction generally the same; patients higher with } \\
\text { telehealth if time/travel is avoided. Providers the } \\
\text { same or slightly worse for telehealth. }\end{array}$ & Low \\
\hline Harms (KQ3) & 0 & No studies reported data on harms & Insufficient \\
\hline
\end{tabular}

KQ = Key Question 


\section{Exploratory Cost Model for Telehealth Neurosurgical Consultations}

The purpose of exploring decision analysis was to address questions the systematic review alone could not answer. We attempted to construct a model to address the following questions for one selected use:

1. What is the predicted impact on clinical, economic, and intermediate outcomes of telehealth consultations?

2. What is the predicted effect of various proposed payment reforms on clinical, economic, and intermediate outcomes of telehealth consultations?

We selected the use of telehealth for neurosurgical consultations by rural or community hospitals for patients with moderate to severe traumatic brain injury (TBI) for this exploratory model. This topic was selected for two reasons: (1) the systematic review did not identify a body of existing evidence that could adequately inform decisions about this use; and (2) neurosurgery is a specialty that is not widely available in all locations (such as rural areas) where people sustain TBIs, making it the type of use often suggested as appropriate for telehealth. We considered the comparison of (1) immediate transfer after stabilization from the community hospital with no access to neurosurgical consultations to a level I or II trauma center (standard care model) and (2) telehealth consultation to determine if the patient can be managed at the local hospital or should be transferred to a level I or II trauma center (telemedicine model).

The model was built as a decision tree. When data were available in the studies included in the systematic review these were used, but the decision modeling team also undertook targeted searches for published data for specific parameters. Data from the literature were used as input parameters to calculate incremental costs for the two different possibilities from the perspective of the healthcare system. The decision analytic model assumed equivalent patient outcomes (details provided in Appendix I). However, the framework was constructed to allow for future inclusion of differences in patient outcomes based on the Glasgow Outcome Scale (GOS) at 6 months: (1) death, (2) persistent vegetative state, (3) severe disability (lost independence) (4) moderate disability, and (5) good outcome (healthy post-TBI) if and when this evidence becomes available.

The model specification and results of this analysis are included in Appendix I of the full report. Insights from our efforts to model cost outcomes are included in the Discussion summary below with more detail in the Discussion section of the full report.

\section{Discussion}

This review summarizes a large volume of literature and explores the potential for supplementing systematic reviews with decision models. The 233 included articles cover a diversity of clinical uses and settings for telehealth even when the function is focused only on telehealth consultations. The size, diversity, and other characteristics of these studies of telehealth consultations are important to consider when assessing the utility of the evidence base, potential next steps in research, and what overall conclusion can be drawn from this literature.

\section{Applicability}

Our results and synthesis of this large number of studies was organized based on our assessment of the applicability of different subgroups of results. For telehealth consultations we 
found that the setting is often of primary importance, and we analyzed and presented the studies by setting -inpatient, emergency, and outpatient care. We also made some distinctions within settings. For example, for inpatient care we considered the remote ICU studies separately as remote ICU consultation is a very specialized, specific use, but we combined other specialty consultations for inpatient care as they are similar in terms of the function (e.g., to diagnose a condition or to provide direction during a surgery) of the consultation and the types of outcomes. For emergency care we separated telestroke, specialty consults for ED patients, and EMS/urgent care for similar reasons. The issues of applicability for outpatient consultations and our approach were slightly different. We reported the details separately by specialty to allow readers to see the results in these groupings, as people are often interested in a particular specialty. Then we combined the results across specialties in the strength of evidence assessment by outcomes in grouping that we felt were appropriate in terms of findings that are likely applicable across specialties.

\section{Limitations}

There are important limitations to the evidence base on the effectiveness of the use of telehealth for consultations. The most significant is the variation in study designs and the level of rigor of the research methodology. The literature on telehealth consultations consists primarily of studies that are considered weaker designs such as before and after studies without a comparison group and retrospective cohort studies. Very few studies were rated as low risk of bias; most were moderate or high. Importantly, the comparison treatment was poorly described in these studies; such that it was often impossible to know whether usual care referred to in-person care by a consultant, no consultant involvement, or a combination of both. Other limitations are that the outcomes used to evaluate telehealth are inconsistent and the best or most appropriate outcome is not always used when data are limited to what is routinely collected. Also, the studies provide very little information on the context or the environment in which telehealth for consultations was implemented.

There are also limitations to the review process and decision modeling. Searching for telehealth use for a specific function is difficult as the indexing terms in MEDLINE and other citation databases do not exactly match our scope. Also, given the variation in study designs, environments, and outcomes, we completed quantitative synthesis using meta-analysis for some topics, but used qualitative approaches for the majority; we acknowledge that qualitative synthesis is more open to interpretation and judgment.

In exploring the utility of decision models, we modeled the costs of neurological consultation for acute traumatic brain injury, using an analysis that assumes equivalence in patient outcomes. Other assumptions are possible (i.e., that outcomes are better or worse with telehealth), and this model does not help the decision maker consider these possible variations. However, the model was built to allow inclusion of patient outcomes following treatment for cost benefit analyses in the future. When data become available, the impact on mortality or quality adjusted life years could be incorporated into the model and used to inform judgements about the value of additional costs given patient benefits.

\section{Future Research Needs}

While we identified 233 articles that evaluated the effectiveness of telehealth consultations, several questions remain to be addressed in future research. A key priority is the need for 
rigorous, multi-site studies of telehealth consultations in clinical areas and in the types of organizations where the lack of evidence may be a barrier to wider spread implementation.

Future studies are also needed that both expand and standardize outcomes and clarify their objectives. Agreeing on some common metrics across uses of telehealth for consultation would facilitate comparisons across clinical areas and help identify priorities for future expansion of telehealth consultations. Given the wide range of clinical topics, these common metrics may need to be intermediate outcomes, such as measures of access or satisfaction or cost effectiveness. While costs are not the only important outcome, collecting more cost economic data would allow more direct comparisons across clinical topics and both facilitate and inform additional decision analyses, whether these are done for publication of for organizations' internal consideration. At the same time this needs to be balanced with attention to the most important outcomes for a given condition. There are examples, such as telestroke, where the most frequently reported outcome (mortality) may not be the most important, either to patients or in terms of the expected impact of changing care. The assessment of telehealth consultations would also be strengthened by more studies that include contemporary comparison groups, either groups of patients or other organizations, so that the effect of the telehealth consultations could be more successfully isolated from historical changes or the idiosyncrasies of a specific organization. This could involve adding comparison or control sites to pre and post telehealth studies.

The research on telehealth could have more impact if its objectives were clearer. Evaluations of telehealth consultations can consider different perspectives and different levels of implementation and evaluation, but failing to be clear leads to studies with confusing results and lessens the impact of positive results. For example, the work on the decision analyses highlighted the importance of clearly specifying the options being compared, or what is "usual care. In the studies we evaluated for this systematic review, the nontelehealth or "usual care" option consisted of was often not specified and was not always clear what care these patients received.

The decision analysis also highlighted the importance of perspective and the need for better information. Most studies did not clearly state their perspective, though it was often implied that is was a single organization (e.g., a hospital or practice group). This seems unnecessarily limiting, and more studies at higher levels seem warranted. In many ways telehealth consultations could be viewed as a systems-level intervention, more similar to health information exchange and electronic health records, than to a condition-specific treatment.

A major evolution of the research in this area would be to focus on hybrid studies, that is, studies that combine effectiveness and implementation assessments. While the results may be uneven across specific clinical areas, telehealth consultations do generally improve access and clinical outcomes and are likely to improve other outcomes. What is missing is much of the specific information asked for in Key Questions 4 and 5 of this review; that is, what are the characteristics of the context and how do they impact outcomes? A hybrid approach to future research could focus on the information needed to promote successful implementation while still continuing to collect better data demonstrating effectiveness and economic impact.

Reviewing background material for this report and discussing telehealth with the Technical Expert Panel and other experts has convinced us that telehealth consultation are being used, particularly in smaller and rural health systems, and that data are often being collected. However, these organizations and data are not represented in the published literature due to lack of research and analysis capacity. Given the importance to policy and practice issues related to telehealth 
consultations (e.g., payment, scope of work, cross organization, and state licensing), identifying and facilitating the analysis of these data should be a priority and may help strengthen what conclusions can be made about telehealth consultations.

Also during the time period covered in the review and during our work, policies that facilitate telehealth consultation and the number of publications about telehealth increased. However, many of these are descriptive or less rigorous approaches to research. Continuing in this vein will not contribute to the next level of telehealth expansion. Given that more and more resources are being invested in telehealth, it is reasonable to suggest that research evaluating its effectiveness should both increase and improve. The current situation seems to require an organized effort by telehealth advocates, researchers, and policy makers to identify where there are still gaps in the research base and prioritize these in terms of their potential to move the field forward, toward increasing use of telehealth in those settings and instances where it is likely to be beneficial for patients, healthcare providers, health systems or society.

\section{Conclusions}

Although the literature evaluating telehealth consultations is large, it is not possible to make a global, general statement about the clinical and economic effectiveness of telehealth consultations for several reasons. These include the diversity of settings, clinical topics, and outcomes; the limited number of high-quality studies; different approaches to measurement, particularly of costs; and how the perspective may impact the estimation of outcomes. It is possible to conclude it is likely that telehealth is more effective than usual care in several specific situations: Remote ICUs reduce ICU and in-hospital mortality; emergency medical services access to telehealth reduces mortality in patients having heart attacks; remote consultations in emergency care decrease time from presentation to decision, reducing ED time and increasing appropriate transfers and admissions; remote consultations as part of outpatient care improve clinical outcomes in some clinical disciplines and increase access to care in those that have been studied.

For other uses and outcomes the strength of evidence is less definitive. Telehealth consultations may improve inpatient care, emergency stroke care and the management of and satisfaction with outpatient consultations across several specialties. Potential harms or unintended consequences were rarely addressed and future research should address this, if only to confirm they are not significant. Studies of economic outcomes including costs produced mixed results due to major differences in definitions and methods as well as the fact that costs and savings may not accrue to the same organization in an interdependent healthcare system.

Decision models have the potential to build on systematic review results and use evidence in ways that would make it more applicable by tailoring the question, base case, and perspective to the decision maker's situation. But our experience demonstrates that the literature may not be available to provide all the data needed to fully execute a functioning model for all topics of interest. However, decision modeling can provide some insight by quantifying differences in costs across settings and estimating where savings are likely to accrue in the system. While our exploratory assessment was limited to costs, expansion of this approach could allow more targeted identification of scenarios in which telehealth could improve the range of outcomes including clinical outcomes, access, and cost.

Future research about telehealth consultations needs to be more rigorous if it is to inform policy and practice decisions. Specifically, more studies should include multiple sites, collect 
information on the context and environment, and consistently measure a more comprehensive range of economic impacts and costs using standard practices.

\section{References}

1. Castro D, Miller B, Nager A. Unlocking the Potential of Physician-to-Patient Telehealth Services. The Information Technology \& Innovation Foundation; 2014. https://itif.org/publications/2014/05/12/unlo cking-potential-physician-patient-telehealthservices. Accessed Nov 152018.

2. Institute of Medicine. The Role of Telehealth in an Evolving Health Care Environment: Workshop Summary. Washington, DC: The National Academies Press; 2012.

3. Bashshur RL, Shannon GW, Smith BR, et al. The empirical foundations of telemedicine interventions for chronic disease management. Telemed J E Health. 2014;20(9):769-800. doi: 10.1089/tmj.2014.9981. PMID: 24968105.

4. Ekeland AG, Bowes A, Flottorp S. Effectiveness of telemedicine: a systematic review of reviews. Int J Med Inform. 2010;79(11):736-71. doi: 10.1016/j.ijmedinf.2010.08.006. PMID: 20884286.

5. Hersh WR, Wallace JA, Patterson PK, et al. Telemedicine for the Medicare Population. Evidence Report/Technology Assessment No. 24. AHRQ Publication No. 01-E012. Rockville, MD: Agency for Healthcare Research and Quality; 2001.

6. Hersh WR, Hickam DH, Severance SM, et al. Telemedicine for the Medicare Population: Update. Evidence Report/Technology Assessment No. 131. AHRQ Publication No. 06-E007. Rockville, MD: Agency for Healthcare Research and Quality; 2006.

7. Hersh WR, Wallace JA, Patterson PK, et al. Telemedicine for the Medicare Population: Pediatric, Obstetric, and Clinician-Indirect Home Interventions. Evidence Report/Technology Assessment No. 24, supplement. AHRQ publication No. 01E060. Rockville, MD: Agency for Healthcare Research and Quality; 2001.
8. Totten AM, Womack DM, Eden KB, et al. Telehealth: Mapping the Evidence for Patient Outcomes from Systematic Reviews. Technical brief No. 26. AHRQ publication No.16-EHC034-EF. Rockville, MD: Agency for Healthcare Research and Quality; 2016. Available at https://effectivehealthcare.ahrq.gov/sites/def ault/files/pdf/telehealth_technical-brief.pdf.

9. $\quad$ Adler-Milstein J, Kvedar J, Bates DW. Telehealth among US hospitals: several factors, including state reimbursement and licensure policies, influence adoption. Health Aff (Millwood). 2014;33(2):207-15. doi: 10.1377/hlthaff.2013.1054. PMID: 24493762.

10. Broderick A, Lindeman D. Scaling telehealth programs: Lessons from early adopters. The Commonwealth Fund; 2013. https://www.commonwealthfund.org/publica tions/case-study/2013/jan/scaling-telehealthprograms-lessons-early-adopters. Accessed Nov 152018.

11. Beck M. How Telemedicine is Transforming Health Care. The Wall Street Journal; 2016. https://www.wsj.com/articles/howtelemedicine-is-transforming-health-care1466993402. Accessed Nov 152018.

12. Report to the Congress: Medicare and the Health Care Delivery System. Medicare Payment Advisory Commission. Washington, DC: 2016.

13. Report to Congress on Medicaid and CHIP. Medicaid and CHIP Payment and Access Commission. Washington, DC: 2018.

14. Methods Guide for Effectiveness and Comparative Effectiveness Reviews. Agency for Healthcare Research and Quality; 2017.

https://effectivehealthcare.ahrq.gov/topics/ce r-methods-guide/overview. Accessed Nov 212018. 
15. Consensus Health Economic Criteria -

CHEC list. Maastricht University Health Services Research.

https://hsr.mumc.maastrichtuniversity.nl/con

sensus-health-economic-criteria-chec-list.

Accessed Nov 152018.

16. Wijnen B, Van Mastrigt G, Redekop WK, et al. How to prepare a systematic review of economic evaluations for informing evidence-based healthcare decisions: data extraction, risk of bias, and transferability (part 3/3). Expert Rev Pharmacoecon Outcomes Res. 2016 Dec;16(6):723-32. doi: 10.1080/14737167.2016.1246961. PMID: 27762640 . 


\section{Introduction}

\section{Background}

Telehealth is the use of information and telecommunications technology to provide or support healthcare across time and/or distance. It is a tool with the potential to increase access, improve the quality of care, increase patient satisfaction, positively impact patient outcomes, and reduce the cost of care. Telehealth's potential benefits are frequently cited, ${ }^{1,2}$ and there has been a sizable and rapidly growing body of research on telehealth, including systematic reviews and reviews of reviews. ${ }^{3-8}$ Despite this potential, implementation and spread has been slower than many expected ${ }^{9,10}$ though recent trends suggest the speed and scope of spread may be increasing.

With improvement in technologies and changes in payment policies that are accompanying evolving models for healthcare delivery and payment in general and telehealth in particular, the possibility exists for an acceleration in implementation and wider use of telehealth. Faster internet connections, better hardware, and increasing use of technology by the public as well as by professionals are making telehealth use more and more feasible. ${ }^{11}$ Payment policies and delivery models are catching up as demonstrated by recommendations to allow payment for more telehealth services in Medicare ${ }^{12}$ and Medicaid ${ }^{13}$ and plans for large scale expansions such as the Veterans Administration's “Anywhere to Anywhere” program. ${ }^{14}$

However, these changes are necessary but not sufficient to assure the widespread use of telehealth. This is reflected in several recent initiatives that include proposing a framework for telehealth research and policy ${ }^{15}$ and a conceptual framework for developing measures of telehealth use and quality of care. ${ }^{16}$ The research and policy framework developed by a committee of experts sought to identify the broad components of the policy context, how telehealth can be used in practice, and potential outcomes in order to support systematic consideration of telehealth expansion and barriers to implementation. ${ }^{15}$ The conceptual framework for measurement developed by the National Quality Forum (NQF) identified four domains (Access to Care, Financial Impact/Cost, Experience, and Effectiveness) and several subdomains that could be used to define and categorize the potential impact of telehealth. The NQF's report suggests these domains can be used to develop specific measures of the impact of telehealth that can be used across different modes of delivery (e.g., live video, store-and-forward, remote patient monitoring and mobile health) and clinical indications. ${ }^{16}$

As these efforts illustrate, targeting, supporting, and sustaining increased use of telehealth requires organized and accessible information about the impact of telehealth. While a synthesis of existing research evidence can help inform decisions about telehealth implementation and expansion, it is difficult to produce a meaningful and useful synthesis given the broad scope of telehealth and its use in a wide range of clinical setting for several different indications.

One specific way to address this complexity is to assess the evidence about the different roles telehealth can play in healthcare. This project takes this approach and focuses on one specific role: telehealth for consultations. Telehealth for consultations uses technology to allow healthcare providers to involve other providers, often specialists, in the prevention, treatment, and management of acute and chronic conditions. The technology allows medical expertise to be available where and when it is needed, minimizing potential time or geographic barriers to care and maximizing the efficient use of scarce resources. Telehealth for consultations has been studied across a range of clinical situations, including injuries, ${ }^{17}$ burn care, ${ }^{18}$ and infectious disease. ${ }^{19-21}$ Identifying and summarizing the available evidence about the use of telehealth for consultations will help support the best use of this technology across clinical topics in the future. 
The overarching goal of this systematic review is to maximize the utility of available research by presenting the results in formats that support decision makers at various levels (e.g., regulators, providers, and payers) as they consider policy and practice changes related to telehealth for consultation. To accomplish this goal, this project explored combining two evidence synthesis methods: a systematic review of the literature and an extension of the evidence using decision modeling. Both methods have accepted methodologies, but they are not frequently used in tandem. Thus, in this sense, this project is experimental as it strives to both provide the results of a traditional systematic review to identify, organize, and analyze the available research about the use of telehealth for consultations and explore how the addition of decision analysis may be used to increase the utility of evidence for decision makers.

\section{Definitions of Telehealth and Telehealth Consultation for This Project}

Telehealth is defined as the use of information and telecommunications technology in healthcare delivery for a specific patient or group of patients, involving a provider across distance or time to address a diagnosis, health condition, or overarching needs of a patient. The information can be transmitted live, be stored and then forwarded, or be a hybrid of these two possibilities. This definition is similar to that used in the previously published Evidence Map, ${ }^{8}$ although the inclusion and exclusion criteria have been changed to match the scope of this review.

Telehealth consultation is defined as the use of telehealth designed to facilitate collaboration among providers, often involving a specialist consultant, or between clinical team members, across time and/or distance, on the assessment, diagnosis, and/or clinical management of a specific patient or group of patients. While the patient may or may not be involved in the consultation, the consultation is required to be related to a specific patient or group of patients in order to differentiate this activity from training or education (which would not meet our definition of telehealth). Limited information provided by one clinician to another that does not contribute to collaboration (e.g., interpretation of an electroencephalogram [EEG], report on an $\mathrm{x}$-ray or scan, or reporting the results of a diagnostic test) is not considered a consultation for this review.

\section{Scope and Questions}

The Key Questions for the systematic review (SR) are presented below, and the Guiding Questions for the exploratory decision model (DM) are provided later in the report. The Key Questions for the SR were based on questions provided in the scope of work for the Request for Task Order issued by the Effective Health Care program of the Agency of Healthcare Research and Quality. The questions were reviewed, reorganized, and refined by the project team and revised after input from the Technical Expert Panel. There was no formal topic refinement for this project. While the protocol for this review was developed prior to the NQF framework for measurement development, the domains they identified (Access to Care, Financial Impact/Cost, Experience, and Effectiveness) ${ }^{16}$ correspond to the outcomes for Key Question 1 (clinical outcomes-effectiveness; economic outcomes- Financial Impact/Cost) or are included in the outcomes for Key Question 2 (intermediate outcomes include Access to Care and Experience). 
The Guiding Questions for the DM were also included in the scope of work. The topics, specific questions, and scope for the DM were based on the literature triage and initial findings of the SR.

\section{Key Questions for the Systematic Review}

\section{Are telehealth consultations effective in improving clinical and economic outcomes?}

Clinical and economic outcomes may include, but are not limited to, mortality and morbidity, patient-reported outcomes, quality of life, utilization of health services, and cost of services.

\section{Are telehealth consultations effective in improving intermediate} outcomes?

Intermediate outcomes include both outcomes that precede the ultimate outcomes of interest (e.g., mediators) and secondary outcomes. Intermediate outcomes may include, but are not limited to, access to care, patient and provider satisfaction, behavior, and decisions (e.g., patient completion of treatment, provider antibiotic stewardship); volume of services; and healthcare processes (e.g., time to diagnosis or treatment).

8. Do telehealth consultations result in harms, adverse events, or negative unintended consequences?

9. What are the characteristics of telehealth consultations that have been the subject of comparative studies?

These characteristics may include:

a. Clinical conditions addressed. These can include broad categories such as diagnosis and treatment of infectious disease or behavior health as well as specific conditions (e.g., upper respiratory infection, hepatitis C, depression, or addiction) or decisions (e.g., stewardship of antibiotics or antimicrobials, selection of treatments).

b. Characteristics of the providers and patients involved.

c. Relationships among the providers and patients involved, including whether these are new or ongoing relationships.

d. Telehealth modalities and/or methods for sharing patient data and communicating among providers.

e. Whether specifics in (d) meet Medicare's coverage and Health Insurance Portability and Accountability Act (HIPAA) requirements,

f. Settings, including:

- Type of healthcare organization, including the organizational structure (e.g., integrated delivery system, critical access) and the type of care (e.g., longterm care, inpatient, ambulatory care).

- Country.

- Geographic and economic characteristics, such as urban or rural areas, or areas with high versus low socioeconomic resources.

h. Other circumstances (e.g., appropriate transportation, climate).

g. Payment models, requirements, or limits for payment, including: 
- The payer/insurance for the patient (e.g., Medicare, Medicaid, commercial).

- Any parameters for payment (e.g., relative value units) or limits on visits.

- Any eligibility requirements for payment based on patient, provider, setting, or context characteristics.

10. Do clinical, economic, intermediate, or negative outcomes (i.e., the outcomes in Key Questions 1, 2, and 3) vary across telehealth consultation characteristics (Key Question 4)?

\section{PICOTS}

The PICOTS framework is used to define the scope of the review. The population, intervention, comparator, outcomes, timing, and setting (PICOTS) for this review are outlined below.

\section{Populations:}

- Patients of any age, with medical care needs for prevention, treatment, or management of chronic or acute conditions.

- Providers (clinicians or healthcare organizations).

- Payers for healthcare services (public, private, insurers, patients).

\section{Interventions:}

- Telehealth consultations are defined as the use of telehealth designed to facilitate collaboration among providers, often involving a specialist, or between clinical team members, across time and/or distance, on the prevention, assessment, treatment and/or clinical management of a specific patient or group of patients.

- Telehealth consultations can be for any acute or chronic conditions. The literature search focused on both general conditions and specific ones identified as areas of growth and policy interest such as infectious disease, dermatology, and critical care.

- Telehealth consultations can use any technology (e.g., real-time video, store and forward, data transfer).

\section{Comparator:}

- Other locations, patients, or time periods that used any alternative to telehealth for healthcare delivery. The alternatives to telehealth could include consultations conducted in another way (e.g., in-person or telephone), care with no access to specialty services, or usual care, which may or may not be defined and could include: 1) consultations conducted in-person, or 2) care delivered without consultation, or 3) a mix of both.

\section{Outcomes for Each Key Question:}

- Key Question 1: Clinical and economic outcomes

o Clinical outcomes such as patient-reported outcomes, mortality, morbidity, such as function, illness recovery, infection.

o Economic outcomes such as return on investment, cost, volume of visits, and resource use. 
- Key Question 2: Intermediate outcomes

o Access to services

o Patient satisfaction, behavior, and decisions such as completion of treatment, or satisfaction with less travel to access healthcare.

o Provider satisfaction, behavior, and decisions such as choice of treatment or antibiotic stewardship.

o Time to diagnosis, time to treatment, and length of stay.

- Key Question 3: Adverse effects or unintended consequences

o Loss of privacy or breech of data security.

o Misdiagnosis or delayed diagnosis.

o Inappropriate treatment.

0 Increase in resource costs, negative return on investment.

- Key Question 4: Not applicable (this is a descriptive question).

- Key Question 5: Same outcomes as Key Questions 1-3.

\section{Timing:}

- Telehealth consultations can be used at any point in the diagnosis, treatment, or management of a patient.

- Outcome measurement needs to occur after the telehealth consultation.

\section{Setting:}

- The consultation can involve providers and patients in any location. Settings could include inpatient, outpatient, or long-term care, and could be in civilian, Veterans Administration, or military facilities.

\section{Study Designs:}

- Comparative studies, including trials and observational studies.

- Descriptive studies may be used to inform the decision model (DM) as needed but will not be included in the SR.

\section{Analytic Framework}

Figure 1 is the analytic framework, which represents the relationships among the elements of the Key Questions for the systematic review. 
Figure 1. Analytic framework for telehealth consultations

KQ 1

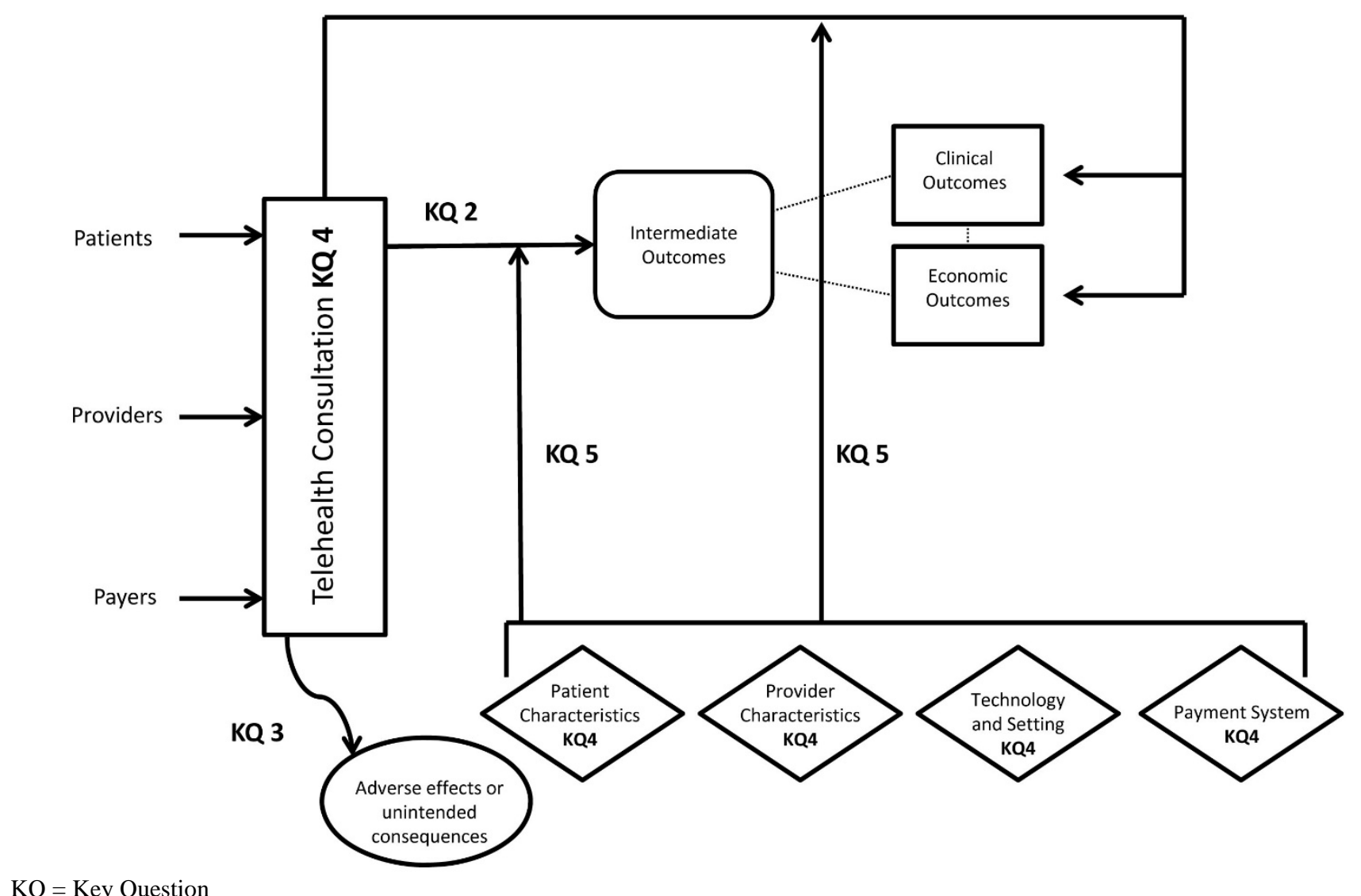

KQ = Key Question 


\section{Methods}

The methods for this systematic review follow the Agency for Healthcare Research and Quality (AHRQ) Methods Guide for Effectiveness and Comparative Effectiveness Reviews (available at http://www.effectivehealthcare.ahrq.gov/methodsguide.cfm) and the PRISMA checklist. ${ }^{22,23}$ The full protocol for the review contains a detailed description of the methods and is available at the Effective Health Care website (http://effectivehealthcare.ahrq.gov/index.cfm). The protocol was registered with PROSPERO (CRD42017058304).

As this project includes a systematic review (SR) and a supplemental decision model (DM), the key elements of the methods are outlined separately.

\section{Systematic Review Methods}

\section{Literature Search Strategy}

The complete search strategies are included in Appendix A.

Publication Date Range: We searched for studies published in a 20-year period, from 1996 through May 2018. This date range captures studies of systems that rely on more current technology. In our evidence tables, we included information on the dates the studies were conducted and the technologies used, as well as the dates of publication.

Literature Databases: Ovid MEDLINE ${ }^{\circledR}$, the Cochrane Central Register of Controlled Trials (CCRCT), and the Cumulative Index to Nursing and Allied Health Literature (CINAHL ${ }^{\circledR}$ ) were searched to capture published literature. The search strategies were developed by a specialist librarian and peer reviewed by a second librarian.

Hand Searching: Reference lists of included articles and selected excluded articles (e.g., systematic and narrative reviews) were reviewed for includable literature.

Supplemental Evidence and Data for Systematic Reviews: The AHRQ Evidence-based Practice Center Scientific Resource Center was asked to notify stakeholders about the opportunity to submit Scientific Information Packets via an announcement in the Federal Register.

Grey Literature: Sources for grey (unpublished) literature included reports produced by government agencies, healthcare provider organizations, or others. With the help of AHRQ we contacted the federal government community of practice on telehealth (FedTel), the American Telemedicine Association, and AcademyHealth to make initial inquiries, and we also followed up on any suggestions made by Technical Expert Panel members.

Process for Selecting Studies: Pre-established criteria were used to determine eligibility for inclusion and exclusion of abstracts in accordance with the Methods Guide for Effectiveness and Comparative Effectiveness Reviews. ${ }^{22}$ To ensure accuracy, all abstracts were independently reviewed by two team members. All citations deemed appropriate for inclusion by at least one of the reviewers were retrieved. Each full-text article was independently reviewed for eligibility by at least two reviewers. We reviewed the full text of any articles suggested by peer reviewers or that arose from the public posting or Supplemental Evidence and Data for Systematic reviews 
processes. Any disagreements about inclusion or exclusion were resolved by discussion and consensus across the investigators.

\section{Criteria for Inclusion/Exclusion of Studies in the Review}

The criteria are based on the Key Questions and are described in detail in Appendix B. Key criteria are described below.

Study Designs: We included comparative studies of any design including trials and cohort studies, as well as pre-post designs (i.e., the comparison can be across time points as well as across different groups). We included economic evaluations that compared two groups and used data derived alongside a primary research study. We reviewed reference lists of existing SRs to identify studies for inclusion. We excluded descriptive studies with no outcomes data or studies that included only outcomes data from one point in time (post only). We also excluded modeling studies that used simulated data, and excluded commentaries, letters, and articles that described telehealth systems or implementations but did not assess impact. We considered whether an excluded article contained information that could be used in the DM even if the study was not included in the SR.

Non-English-Language Studies: We restricted inclusion to English-language articles, but reviewed English-language abstracts of non-English-language articles to identify studies that would otherwise meet inclusion criteria, in order to assess for the likelihood of language bias.

\section{Data Abstraction and Data Management}

The following data were abstracted from studies deemed eligible based on inclusion criteria (Included Studies are listed in Appendix C): study design, year, setting, country, sample size, eligibility criteria, population, and clinical characteristics (e.g., age, sex, race, reason for presentation, diagnosis), intervention characteristics (e.g., duration, training/background of personnel engaged in the consultations), and results relevant to each Key Question as outlined in the PICOTS section in the Introduction. Information relevant for assessing applicability of individual studies included the number of patients randomized/eligible for inclusion in an observational study relative to the number of patients enrolled, and characteristics of the population, telehealth intervention, and administrating personnel. Sources of funding for studies were also recorded when reported. All study data were verified for accuracy and completeness by a second team member. A record of studies excluded at the full-text level with reasons for exclusion is provided in Appendix D.

\section{Assessment of Methodological Risk of Bias of Individual Studies}

We assessed risk of bias for individual controlled trials and observational studies using predefined criteria consistent with the approach recommended in the chapter, Assessing the Risk of Bias of Individual Studies When Comparing Medical Interventions in the Methods Guide for Effectiveness and Comparative Effectiveness Reviews. ${ }^{22}$ Economic evaluations were assessed using a modified version of the Consensus Health Economic Criteria. ${ }^{24,25}$ Our team selected the criteria related specifically to concerns of internal validity and the potential introduction of bias. All studies regardless of design were rated as "low risk of bias," "medium risk of bias," or "high risk of bias." The detailed instructions and criteria used for this evaluation are in Appendix E. 
Studies rated "low risk of bias" are considered to have the least risk of bias, and their results are generally considered valid. "Low risk of bias" studies include clear descriptions of the population, setting, interventions, and comparison groups; a valid method for allocation of patients to treatment; low dropout rates and clear reporting of dropouts; appropriate means for preventing bias; and appropriate measurement of outcomes.

Studies rated "medium risk of bias" are susceptible to some bias, though not enough to invalidate the results. These studies may not meet all the criteria for a rating of low risk of bias, but no flaw is likely to cause major bias. The study may be missing information, making it difficult to assess limitations and potential problems. The "medium risk of bias" category is broad, and studies with this rating will vary in their strengths and weaknesses. The results of some medium risk of bias studies are likely to be valid, while others may be only possibly valid.

Studies rated "high risk of bias" have significant flaws that imply biases of various types that may invalidate the results. They have a serious or "fatal" flaw in design, analysis, or reporting; large amounts of missing information; discrepancies in reporting; or serious problems in the delivery of the intervention. In general, observational studies that do not perform adjustment for potential confounders will be assessed as "high risk of bias." The results of these studies are at least as likely to reflect flaws in the study design as the true difference between the compared interventions. We did not exclude studies rated high risk of bias a priori, but high risk of bias studies are considered to be less reliable than low or medium risk of bias studies when synthesizing the evidence, particularly if there are discrepancies among study results.

Each eligible study was independently reviewed for risk of bias by two team members. Any disagreements were resolved by consensus. If the two reviewers could not arrive at a consensus, the principal investigator or the lead for the decision analysis made a final determination. Team members who were involved in the conduct of a study were not involved in data abstraction or risk of bias assessment for that study.

\section{Data Synthesis}

Based on the data abstraction we constructed comprehensive evidence tables (Appendix F) identifying the study characteristics, results of interest, risk of bias ratings for all included studies, and summary tables included in the text to highlight the main findings. We reviewed and highlighted studies by using a hierarchy-of-evidence approach, where the best evidence is the focus of our synthesis for each Key Question.

Data are presented in summary tables; ranges, descriptive analysis, and interpretation of the results are provided.

We conducted quantitative synthesis (i.e., meta-analysis) for combinations of similar telehealth interventions and outcomes when there were adequate data from included studies. In cases with few studies, lack of data, or when the use of telehealth or outcomes were different, we used qualitative approaches.

Random effects meta-analysis based on the profile likelihood method was conducted to combine the studies, and this method incorporates the uncertainty related to estimating betweenstudy heterogeneity. Statistical heterogeneity was assessed using the standard $\chi^{2}$ test and $I^{2}$ statistic. For binary outcomes (mortality outcomes), we combined risk ratios. If a study reported adjusted risk ratios, we used the adjusted risk ratio instead of the risk ratio calculated based on the raw data. If a study reported adjusted odds ratio, we converted the adjusted odds ratio to an adjusted risk ratio. For continuous outcomes (length of stay), we combined mean differences. For studies where both the intervention and control groups had a pre- and post-period, we used 
the mean difference of differences. We conducted sensitivity analyses by comparing results when outlying studies were included or excluded.

All analyses were conducted using Stata/IC 13.1 (StataCorp LP, College Station, TX).

\section{Grading the Strength of Evidence for Major Comparisons and Outcomes}

The strength of evidence (SOE) for each Key Question was initially assessed by one researcher for each clinical outcome (see PICOTS section in Introduction) by using the approach described in the Methods Guide for Effectiveness and Comparative Effectiveness Reviews. ${ }^{22}$ To ensure consistency and validity of the evaluation, the grades were reviewed by the entire team of investigators for:

- Study limitations (low, medium, or high level of study limitations)

- Consistency (consistent, inconsistent, or unknown/not applicable)

- Directness (direct or indirect)

- Precision (precise or imprecise)

- Reporting bias (suspected or undetected)

The risk of bias for individual studies is provided in Appendix G, while the SOE for each Key Question is in Appendix H. The strength of evidence was assigned an overall grade of high, moderate, low, or insufficient according to a four-level scale by evaluating and weighing the combined results of the above domains:

- High-Very confident that the estimate of effect lies close to the true effect for this outcome. The body of evidence has few or no deficiencies. The findings are stable (i.e., another study would not change the conclusions).

- Moderate-Confident that the estimate of effect lies close to the true effect for this outcome. The body of evidence has some deficiencies. The findings are likely to be stable, but some doubt remains.

- Low-Limited confidence that the estimate of effect lies close to the true effect for this outcome. The body of evidence has major or numerous deficiencies (or both). Additional evidence is needed before concluding either that the findings are stable or that the estimate of effect is close to the true effect.

- Insufficient-No evidence. Investigators are unable to estimate an effect, or have no confidence in the estimate of effect for this outcome. No evidence is available or the body of evidence has unacceptable deficiencies, precluding reaching a conclusion.

\section{Assessing Applicability}

Applicability was considered according to the approach described in the Methods Guide for Effectiveness and Comparative Effectiveness Reviews. ${ }^{22}$ We used the PICOTS framework to consider the applicability of the evidence base for each Key Question, for example, examining the characteristics of the patient populations (e.g., clinical condition), attrition of participants, telehealth intervention (including personnel delivering the intervention) and study setting (e.g., inpatient or outpatient). 
Variability in the studies may limit the ability to generalize the results to other populations and settings. We considered applicability and the strength of evidence when making general assessments across the studies, and we use qualifiers in key messages and conclusions such as 'likely' for moderate strength of evidence and/or some applicability concerns or 'may' for low strength of evidence and/or significant applicability concerns.

\section{Exploratory Cost Model for Telehealth Neurosurgical Consultations}

The purpose of exploring decision analysis was to address questions the SR alone could not answer. We attempted to construct a model to address the following Guiding Questions for one selected use:

3. What is the predicted impact on clinical, economic, and intermediate outcomes of telehealth consultations?

4. What is the predicted effect of various proposed payment reforms on clinical, economic, and intermediate outcomes of telehealth consultations?

We selected the use of telehealth for neurosurgical consultations by rural or community hospitals for patients with moderate to severe traumatic brain injury for this exploratory model. This topic was selected for two reasons: 1) the systematic review did not identify a body of existing evidence that could adequately inform decisions about this use; and 2) neurosurgery is a specialty that is not widely available in all locations (such as rural areas) where people sustain traumatic brain injuries, making it the type of use often suggested as appropriate for telehealth.

The model was built as a decision tree. When data were available in the studies included in the systematic review these were used, but the decision modeling team also conducted targeted searches for published data for specific parameters. This approach is common in decision modeling and allows for the inclusion of data from sources that would not meet the inclusion criteria of the systematic review.

The results of the model are reported as costs, and the incremental difference in costs between the two potential treatment scenarios that produce similar outcomes for similar patients. As current evidence on how or whether patient outcomes differ when the consultation is in person or via telehealth is limited for this particular application, the model was constructed as a "what if analysis" assuming equivalent clinical outcomes, facilitating focus on understanding the drivers of cost differences.

The model specification and results of this analysis are included in Appendix I. Insights from our efforts to model cost outcomes are included in the Discussion. 


\section{Results}

\section{Overview}

\section{Literature Search Yield}

The results of the literature search, triage of abstracts, and the review of full-text articles is summarized in the study flow diagram (Figure 2). Our searches yielded 9,366 potentially relevant citations after reviewing titles and abstracts, 8,356 were excluded and the full text of 1,010 articles were pulled for review. Of these, 233 articles met our inclusion criteria. A list of the included studies is provided in Appendix C.

The most frequent reasons for excluding an article were that the intervention was not a telehealth consultation (ineligible intervention) or that the study did not compare telehealth consultations to usual care or some other intervention (ineligible comparison). The majority of the excluded studies about telehealth consultations were excluded because they provided only descriptive information. The citations for the studies excluded after full-text review and the primary reasons for exclusion are included in Appendix D.

Figure 2. Literature flow diagram

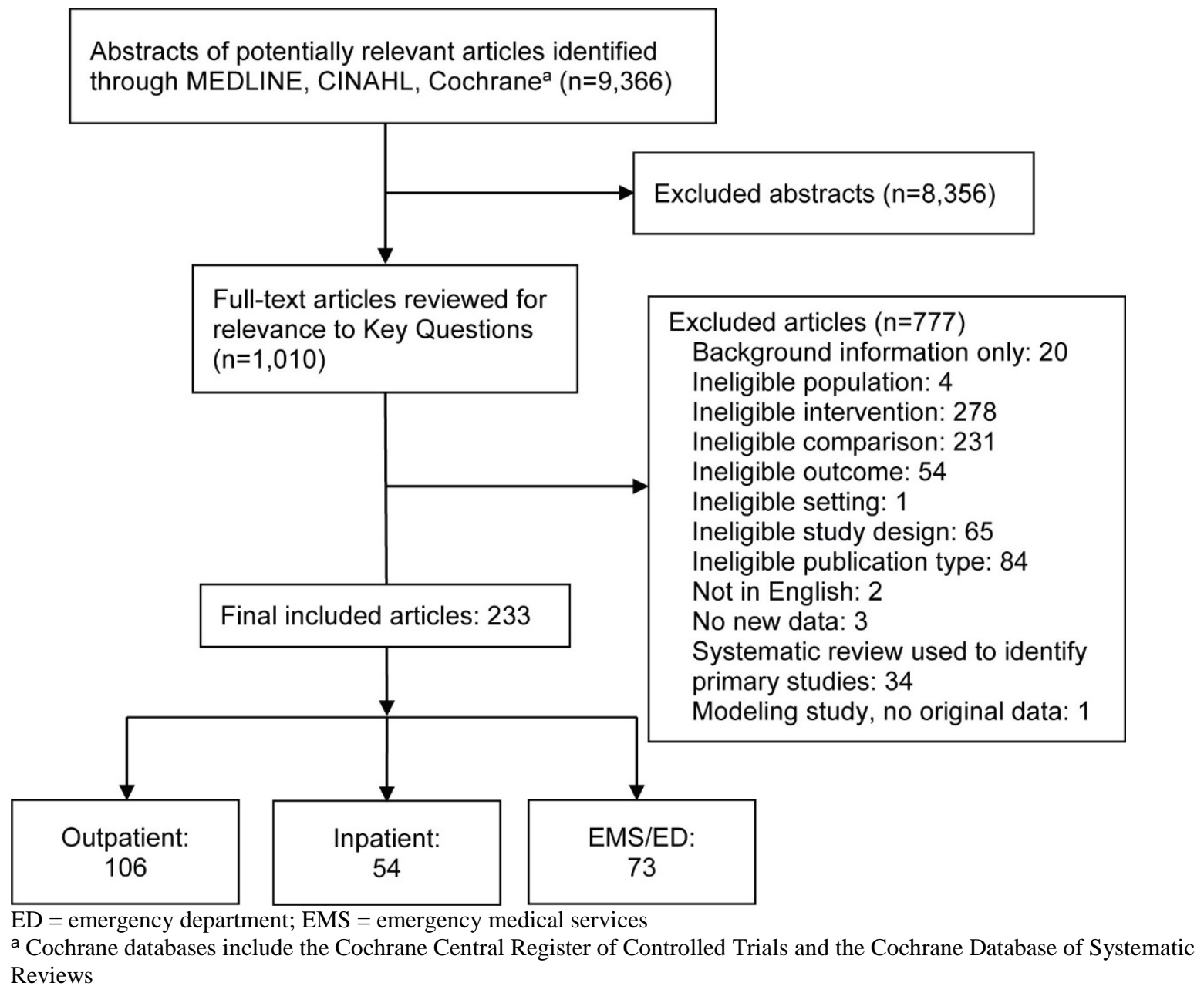




\section{Description of Included Studies}

Table 1 provides information on selected characteristics of the 233 included articles.

The most frequent geographic location for the included studies of telehealth consultations was the United States (110 articles or 47\%); however more than half of the studies were conducted in other countries. Seventy-eight articles (approximately 33\%) were conducted in Europe, 15 in Asia, 19 in Australia or New Zealand, three in South America, four in Canada, and one in Africa (Mali).

While the scope of this review is limited to the use of telehealth for consultations, there was substantial variation in the mode and type/timing of telehealth. The most common mode or technology used for telehealth consultations was video, which was used in just over half of the studies (55\%). Store and forward of images and information was used in almost 16 percent of the studies; 5 percent studied systems that facilitated review of electronic records, 4 percent involved streaming data, and 10 percent used a variety of modalities simultaneously. Ten percent of studies did not provide enough information to categorize the mode or technology. The mode is closely, but not perfectly, related to whether the consultation was synchronous (real time) or asynchronous. In most of the studies the consultations were in real time (72\%), or both real time and asynchronous communications were used (6\%). Twenty percent of studies evaluated consultations that involved asynchronous exchanges.

The study designs and sample sizes also varied. Most of the studies (67\%) were observational, including prospective cohorts, retrospective cohorts, and before/after studies in which a group of patients from before the implementation of telehealth consultations are compared with a different group of patients after telehealth implementation. In these studies, the comparator was often usual care, that is care without telehealth and the studies rarely provided more detail (e.g., if consultations were in-person, if care was delivered without consultation or a mix of both). Nearly one-fifth (19\%) were randomized controlled trials, 12 percent were economic evaluations, and approximately 3 percent were pre-post studies in which outcomes for the same patients were compared prior to and post telehealth consultations. The size of the studies ranged from small (23\% with under 100 subjects) to very large (over 10,000 subjects) with most studies of moderate size (41\% of studies with 101 to 500 patients and 11\% with 501 to 1000 patients). The studies were evaluated for risk of bias (see Methods and Appendix E for details), and 11 percent were rated as low risk of bias, 46 percent as moderate, and 43 percent as high.

The studies also varied in terms of the type of outcomes used in evaluating telehealth. Many studies included more than one outcome. The primary outcomes for this review (Key Question 1) included clinical outcomes (e.g., mortality and morbidity), resource utilization (e.g., length of hospital stay, number of hospitalizations, number of outpatient visits, number of tests), and economic outcomes (e.g., costs of care, costs avoided, and expenditures for telehealth or alternative services). Approximately 40 percent of studies included clinical outcomes, while about a third analyzed economic outcomes. More than 80 percent of the studies included intermediate outcomes, which we defined as patient or provider satisfaction or behavior (e.g., adherence to treatment or frequency in ordering tests). Very few studies ( $<5 \%)$ explicitly considered or reported potential harms.

The identified studies assessed consultations across numerous settings and specialties. Use of telehealth consultations to inform the treatment of patients in hospitals (inpatient setting) included studies of remote intensive care units (ICUs) as well as programs that facilitated consultations for several specific specialties (e.g., neonatal cardiology, pediatrics, geriatrics, 
psychiatry, and surgeries). Included evaluations in emergency care addressed the effectiveness of stroke assessment and specialist consultations with emergency department physicians or with emergency medical personnel. We also included studies of teleconsultations for several types of outpatient care (e.g., dermatology, wound care, ophthalmology, orthopedics, dentistry, cancer, and infectious disease). While the detailed results in the following sections cover a wide range of topics, the topics are limited to those for which comparative studies were identified; not all possible uses of telehealth for consultations are represented. Based on prior work on a telehealth evidence map, ${ }^{8}$ and input from our Technical Expert Panel and other stakeholders there may be topics for which telehealth consultations are used that are not covered in this review. Examples include antibiotic management, pain management, and opioid misuse.

Table 1. Characteristics of included studies

\begin{tabular}{|c|c|c|c|c|}
\hline Characteristic & Categories & $\begin{array}{l}\text { Number } \\
\text { of } \\
\text { Articles }\end{array}$ & $\begin{array}{l}\text { Percentage } \\
\text { of Articles }\end{array}$ & References \\
\hline \multirow{9}{*}{$\begin{array}{l}\text { Geographic } \\
\text { Location }\end{array}$} & United States & 110 & $47.2 \%$ & $26-135$ \\
\hline & Non-UK Europe & 56 & $24 \%$ & $136-191$ \\
\hline & United Kingdom & 22 & $9.4 \%$ & $192-213$ \\
\hline & $\begin{array}{l}\text { Australia or New } \\
\text { Zealand }\end{array}$ & 19 & $8.2 \%$ & $214-232$ \\
\hline & Asia & 15 & $6.4 \%$ & $233-247$ \\
\hline & Canada & 4 & $1.7 \%$ & $248-251$ \\
\hline & South America & 3 & $1.3 \%$ & $252-254$ \\
\hline & NR & 3 & $1.3 \%$ & $255-257$ \\
\hline & Africa & 1 & $0.4 \%$ & 258 \\
\hline \multirow{4}{*}{ Study Design ${ }^{a}$} & $\begin{array}{l}\text { Observational } \\
\text { (Prospective cohort, } \\
\text { retrospective } \\
\text { cohort, before-after) }\end{array}$ & 155 & $66.5 \%$ & $\begin{array}{l}26-33,36-42,44,47,49-51,53,55,60,61,64-68, \\
70-73,75,76,78-80,82-84,86-94,96,97,101,102, \\
104-113,115-117,119-125,131-139,141-145,150, \\
154-156,158,159,161,162,164,165,167,169, \\
171-174,176-179,182,183,185,188-190,193 \\
196-199,203,209,214-216,218,219,222,225- \\
227,229,232,234,236-244,246,248-254,256-258\end{array}$ \\
\hline & $\begin{array}{l}\text { Randomized } \\
\text { controlled trial }\end{array}$ & 44 & $18.9 \%$ & $\begin{array}{l}34,35,46,48,56,58,59,63,69,74,98,100,126, \\
128-130,140,146,149,153,157,163 \\
175,180,186,187,192,194,195,200-202,207,208 \\
210,217,220,224,228,233,235,245,247,255\end{array}$ \\
\hline & $\begin{array}{l}\text { Economic } \\
\text { Evaluation }\end{array}$ & 29 & $12.4 \%$ & $\begin{array}{l}45,52,57,62,77,81,85,95,99,103,118,127,147,151, \\
152,160,166,170,181,184,204-206,211,212,221, \\
223,230,231\end{array}$ \\
\hline & $\begin{array}{l}\text { Pre-post (same } \\
\text { patients) }\end{array}$ & 6 & $2.6 \%$ & $43,54,148,168,191,213$ \\
\hline \multirow[t]{3}{*}{ Risk of Bias ${ }^{a}$} & Low & 27 & $11.4 \%$ & $\begin{array}{l}29,30,47,51,71,80,89,90,94,102,107,137,138,166 \\
172,173,175,178,180,204,207,208,216,218,236 \\
246,255\end{array}$ \\
\hline & Moderate & 109 & $46.2 \%$ & $\begin{array}{l}27,28,36,45,48,56-62,64-68,70,73,76,78,81-88, \\
91,93,95,96,98-100,108,111-113,117,118, \\
120,123,125-127,129-135,139-143,145-148,150, \\
151,155,159,161,162,167-169,171,176 \\
177,179,181,183-185,189,190,192,195,196 \\
203,205,206,210-212,214,217,221,223,224 \\
230-234,238,240,243,247,248,251,253,254\end{array}$ \\
\hline & High & 100 & $42.4 \%$ & $\begin{array}{l}26,29,31-35,37-44,46,49,50,52-55,63,69,72,74,75, \\
77,79,83,92,97,101,103-106,109,110,114-116,119, \\
121,122,124,128,136,144,149,152-154,156-158, \\
160,163-165,170,174,177,182,186-188,191,193, \\
194,197-202,209,213,215,219,220,222,225-229, \\
235,237,239,241,242,244,245,249,250,252, \\
256-258\end{array}$ \\
\hline
\end{tabular}




\begin{tabular}{|c|c|c|c|c|}
\hline Characteristic & Categories & $\begin{array}{l}\text { Number } \\
\text { of } \\
\text { Articles }\end{array}$ & $\begin{array}{l}\text { Percentage } \\
\text { of Articles }\end{array}$ & References \\
\hline \multirow{6}{*}{ Sample Size ${ }^{b}$} & Under 100 & 49 & $22.8 \%$ & $\begin{array}{l}26,32,39,42,43,48,54,69,74,80,81,92,97,100, \\
103-105,108,111,115-117,120,139,140,142, \\
149,157,163,164,184,185,188,190,191,193,203, \\
205,206,217,220,226,228,235,239,241,244,250,257\end{array}$ \\
\hline & $100-500$ & 88 & $40.9 \%$ & $\begin{array}{l}30,34,35,40,45,46,49,51,56-59,64,65,67, \\
68,70,75,76,83-86,88,101,102,112-114,119,121 \\
122,124,126-130,133,135,136,141 \\
143,144,148,150-153,156,159-161,168-171 \\
174,175,178-182,187,189,192,194-196,198-202 \\
204,210,213,216,222-225,229,231-234,237 \\
238,240,243,249,251,253,255,256,258\end{array}$ \\
\hline & $501-1000$ & 24 & $11.2 \%$ & $\begin{array}{l}28,38,41,53,60,66,72,96,98,99,107,125,146,147 \\
158,167,172,209,211,215,218,227,236,242,245- \\
247\end{array}$ \\
\hline & $1001-10,000$ & 36 & $16.7 \%$ & $\begin{array}{l}27,33,36,44,47,50,62,73,79,87,89-91,94,106,109 \\
110,123,132,134,137,138,145,154,155,162,165 \\
166,173,176,177,183,186,207,208,212,214,219 \\
248,254\end{array}$ \\
\hline & $10,001+$ & 9 & $4.2 \%$ & $29,55,61,71,77,78,93,95,131,197$ \\
\hline & NR/unclear & 9 & $4.2 \%$ & $31,37,52,63,82,118,221,230,252$ \\
\hline \multirow{6}{*}{$\begin{array}{l}\text { Mode of } \\
\text { Telehealth }\end{array}$} & Video & 128 & $54.9 \%$ & $\begin{array}{l}26,27,30,32,34,35,39,41-44,46-57,59-63,66,68-70 \\
73,74,76-78,80,81,84-86,88-90,92-96,100,101 \\
104,105,108,111,116-118,120,121,124,133-135 \\
139,141,142,149,151,153,155,156,159-161,163, \\
164,167,169,170,174,178,181,183-185,187,188, \\
194,196-202,205-208,211,212,214,215,217 \\
219-221,223,225-227,229-232,234,235, \\
240,242,245,249,250,252,253,255,256\end{array}$ \\
\hline & $\begin{array}{l}\text { Data store and } \\
\text { forward }\end{array}$ & 37 & $15.9 \%$ & $\begin{array}{l}98,99,113,122,126,128-130,132,146-148 \\
154,157,158,166,175,177,180,190,191 \\
209,213,216,222,228,233,236-239,241,243 \\
244,251,254,258\end{array}$ \\
\hline & $\begin{array}{l}\text { Electronic } \\
\text { chart/record review }\end{array}$ & 11 & $4.7 \%$ & $28,40,45,65,75,79,127,144,171,186,248$ \\
\hline & Mixed modalities & 24 & $10.3 \%$ & $\begin{array}{l}36,58,71,87,103,106,107,110,112,123,131,137,138, \\
140,150,152,168,176,182,193,224,246,247,257\end{array}$ \\
\hline & $\begin{array}{l}\text { Unspecified/ } \\
\text { unclear }\end{array}$ & 24 & $10.3 \%$ & $\begin{array}{l}29,31,33,37,38,64,67,82,83,91,97,102,109,115,119, \\
125,143,145,165,192,195,203,204,210\end{array}$ \\
\hline & Data streaming & 9 & $3.9 \%$ & $72,114,136,162,172,173,179,189,218$ \\
\hline \multirow{4}{*}{ Timing } & Real-time & 168 & $72.1 \%$ & $\begin{array}{l}26,27,29,30,32,34,35,37-39,41-44,46-64,67-74, \\
76-78,80-97,100,101,103-112,114,116, \\
117,120,121,123,124,131,133-137,139,141-143 \\
149-151,153,155,156,159,160,162-164,167-170 \\
172-174,176,178,179,181-185,187,189 \\
193,194,196-201,203,205-208,210,212,214-218 \\
220,221,223,225-227,229-232,234-240,242 \\
246-250,252-257\end{array}$ \\
\hline & Asynchronous & 47 & $20.2 \%$ & $\begin{array}{l}\text { 31,33,40,45,65,75,79,98,99,118,119,122,125-130, } \\
132,144-148,152,154,157,158,166,171, \\
\text { 175,177,180,186,190-192,195,209,213,222, } \\
224,228,233,244,251,258\end{array}$ \\
\hline & Both & 13 & $5.6 \%$ & $\begin{array}{l}28,36,66,113,138,140,161,165,188,202,211,243 \\
245\end{array}$ \\
\hline & NR/unclear & 5 & $2.1 \%$ & $102,115,204,219,241$ \\
\hline
\end{tabular}

NR = not reported; UK = United Kingdom

a Total is more than 233 as some articles analyzed economic and clinical/intermediate outcomes and the risk of bias was different for different outcomes

b Total is number of studies (215) 
As the volume of the literature is large, we divided it according to the patient setting for both additional description and for presenting the results. We used the three categories: inpatient, emergency department or emergency medical services (ED/EMS), and outpatient. Each study included in this systematic review was assigned to one of these three settings. Figure 3 shows the distribution of the included studies across these three categories. Outpatient is largest, including nearly half the studies, with ED/EMS studies constituting nearly a third and inpatient about a quarter of included studies. Within these categories we have also grouped the studies by clinical indication, condition, or specialty. Summary of evidence tables are included at the beginning of each section that provide the number of studies and citation by setting. Figure 4 presents the year of publication for each article by these categories as well.

Figure 3. Distribution of included articles $(n=233)$

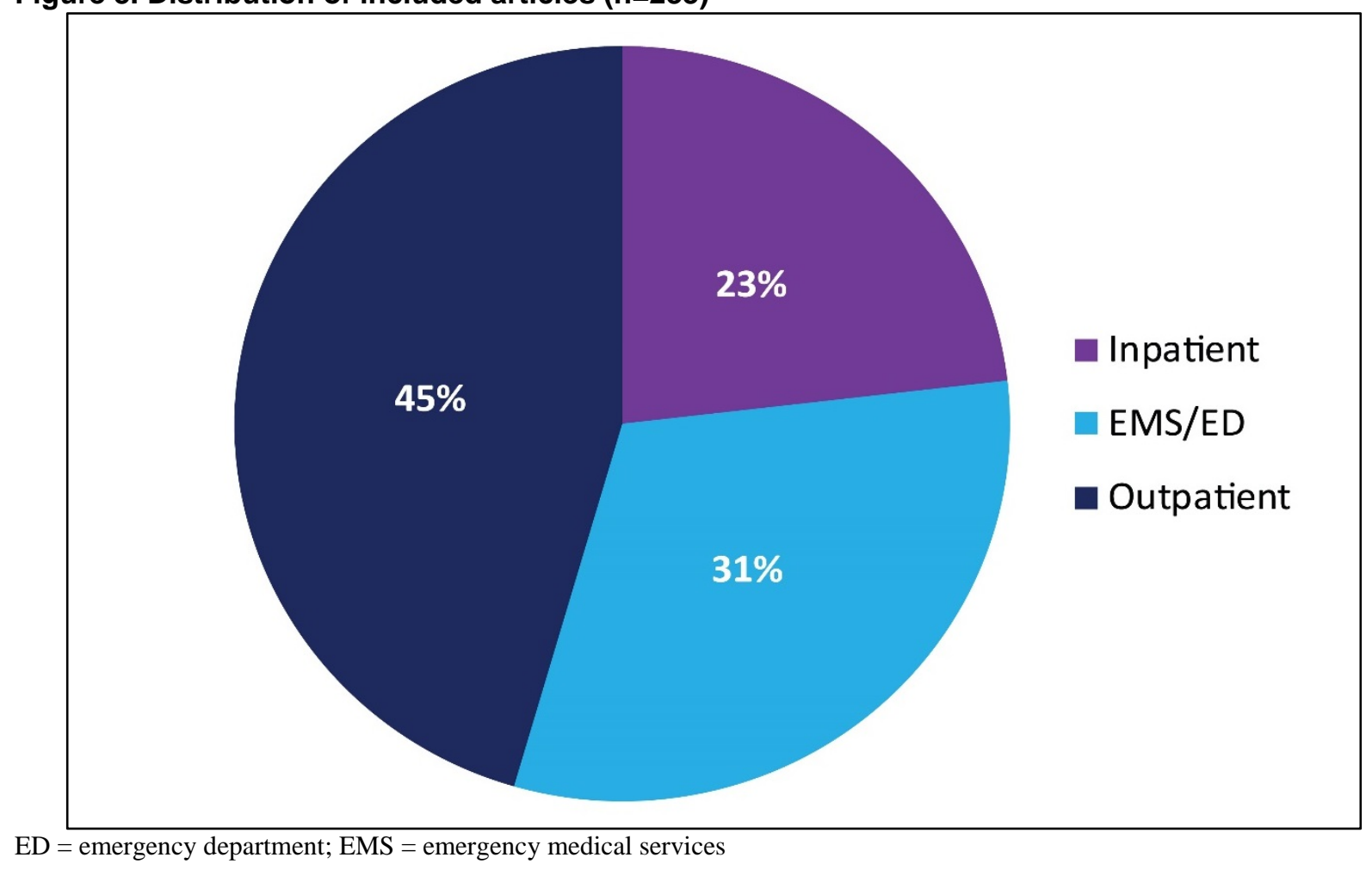


Figure 4. Telehealth consultation articles $(n=233)$

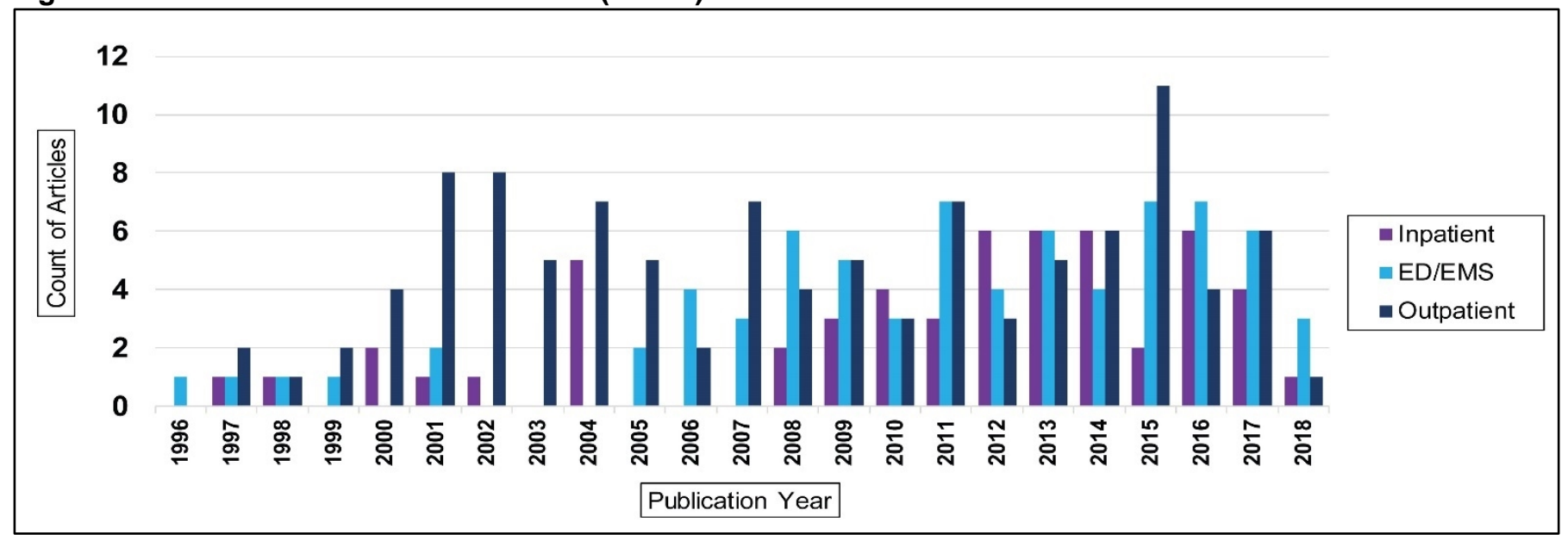

ED = emergency department; EMS = emergency medical services

The tables in Appendixes F (Evidence Tables) and G (Risk of Bias) include the detailed information extracted from each article. Also, the tables later in this Results section provide a summary of study characteristics and outcomes.

\section{Organization of Results}

The results for this review are organized into three sections that correspond to the patient settings (inpatient, ED/EMS, and outpatient). We chose to organize the results by the patient setting as the settings are likely to have different telehealth technology requirements as well as differences in payment structures, staffing, and organization of care delivery. Specifically, what is needed to implement telehealth consultations and the nature of the consultation will likely differ if a patient is in a hospital versus treated out of hospital by EMS, in an ED, or in an outpatient clinic. For example, the logistics and technology needed to facilitate a consultation with a specialist differ for EMS in a moving ambulance where the need is emergent and speed is important, compared with outpatient assessments in physician offices which may require larger networks connecting multiple, stable locations (offices or clinics), or consultations for inpatient treatment that may be more urgent than outpatient and involve linking a smaller number of specialists to allow consultations over distance or at times when a specialist is not on site at the hospital. Each of the three sections describe the literature available to address the five Key Questions. Within each of these sections, the studies are grouped by clinical indications, though we attempted to summarize and draw conclusions across indications where we believe it is appropriate.

\section{Systematic Review Results by Patient Setting}

\section{Inpatient Results}

We divided the research evaluating telehealth for consultations involving inpatient care into two categories: remote intensive care unit (ICU) and specialty consultations. Specialty consultations are further grouped and reported by specific clinical topics. Inpatient care data are shown in Tables 2 through 6 and Figures 5 through 8 . Tables 2 and 5 include the number of articles addressing each topic, a summary assessment of key outcomes across studies, and study 
citations. Figures 5 through 8 and Tables 3, 4, and 6 provide more detail, focusing on key results for each study, and the accompanying text discusses how the studies address the Key Questions for this review. Detailed information we abstracted from each article is provided in Appendix F. The overall rating for risk of bias and the criteria used to assess each article are in Appendix G, and Appendix $\mathrm{H}$ contains the strength of evidence assessment for each topic.

\section{Remote Intensive Care Units}

We identified 21 studies reported in 22 articles evaluating the use of telehealth to provide remote ICU services. Remote ICU (sometimes referred to as tele-ICU) services involve offsite staff (e.g., intensivists, critical care nurses, and sometimes administrative assistants) who monitor ICU patients and provide consultation and management assistance by alerting onsite staff to issues, recommending treatment, and mentoring onsite staff in care delivery. The purpose is to allow hospitals without 24-hour critical care staff to provide high-quality care to critically ill patients and to avoid transferring them to another facility. Remote ICU systems vary but generally include cameras allowing one-way observation of the patient and care provided, mirroring of bedside monitors, and real-time voice communication. Some studies included access to patient record systems while others required special transmission of records from the hospital to the remote ICU staff location.

\section{Remote Intensive Care Units: Key Points}

- Clinical outcomes: ICU and hospital mortality are lower with remote ICU (moderate strength of evidence).

- Economic outcomes: Not all studies analyzed costs of remote ICUs or their impact on revenue; those that did used differing methods, and conclusions were inconsistent (insufficient evidence).

- Intermediate outcomes: ICU and hospital length of stay (LOS) are not statistically different with remote ICU (moderate strength of evidence).

- Adverse effects: None of the included studies specifically addressed potential harms or unintended consequences (insufficient evidence).

Table 2 summarizes the results across the included studies.

Table 2. Remote intensive care units: summary of evidence

\begin{tabular}{|l|l|l|l|l|}
\hline $\begin{array}{l}\text { Number } \\
\text { of } \\
\text { Articles }\end{array}$ & $\begin{array}{l}\text { Clinical } \\
\text { Outcomes }\end{array}$ & $\begin{array}{l}\text { Intermediate } \\
\text { Outcomes }\end{array}$ & Cost & Citations \\
\hline 22 & $\begin{array}{l}* \text { ICU } \\
\text { mortality } \\
\text { lower }\end{array}$ & $\begin{array}{l}\text { * ICU LOS } \\
\text { shorter }\end{array}$ & $\begin{array}{c}\text { ? Cost or } \\
\text { revenue } \\
\text { impact }\end{array}$ & $\begin{array}{l}36,43,44,55,62,71,73,80,81,87,91,94,104,106,107,109,110,123,131,227, \\
240,257\end{array}$ \\
& $\begin{array}{l}\text { * Hospital } \\
\text { Hortality } \\
\text { lower }\end{array}$ & LOS & \\
& $\begin{array}{l}\text { Harms: No } \\
\text { lovidence }\end{array}$ & & \\
\hline
\end{tabular}

ICU = intensive care unit; LOS = length of stay

Key: *superior (telehealth benefit), no difference or inferior (telehealth no benefit), ? inconclusive (inconsistent results) 


\section{Remote Intensive Care Units: Detailed Results}

Figures 5 through 8 and Table 3 includes the results for mortality, LOS, and costs from the included studies of remote ICUs.

All but two studies of remote ICUs were conducted in the United States. The exceptions are a study conducted in India of cardiac intensive care ${ }^{240}$ and a study of remote ICU in Australia and New Zealand. ${ }^{27}$ Thirteen are before-after designs comparing outcomes from a period before implementation of remote ICUs to the period after this model of care was in operation in the same hospital or hospitals. ${ }^{36,55,62,81,87,91,94,107,109,110,123,131,227,257}$ The remaining studies include four retrospective cohorts, ${ }^{44,71,73,240}$ two prospective cohorts, ${ }^{80,106}$ one cross sectional survey, ${ }^{43}$ and one pre-post survey. ${ }^{104}$ The studies did not provide detail on the nontelehealth care, though it likely included a mix of care by nonspecialists, less care by specialists based on availability in person, and transfers to other hospitals if specialists were not available to care for the patients.

\section{Remote Intensive Care Units: Effectiveness in Improving Clinical and Economic Outcomes}

Most of the remote ICU studies included both ICU and in-hospital mortality as primary outcomes. We generated pooled estimates of 12 of these studies that used similar remote ICU interventions across sites and reported adequate data. Figures 5 and 6 contain forest plots for these analyses. If the study did not contain data that could be included in the pooled analysis the results are included in Table 3.

Examining the studies individually reveals that most reported lower mortality in the remote ICUs with a minority reporting no significant difference. The pooled risk ratios and 95\% confidence intervals confirm lower mortality with telehealth. For ICU mortality the risk ratio is 0.69 (95\% confidence interval [CI] 0.51 to 0.89 ), estimating mortality to be 31 percent lower for patients cared for in the remote ICU arms of the 11 included studies in the meta-analysis. Inhospital mortality is also lower: a pooled risk ratio of 0.76 (95\% CI 0.60 to 0.95 ) though the effect is smaller with an estimate of 24 percent lower mortality rates. Hospital mortality rates include patients who die in the ICU and other wards, and the smaller difference may represent patients who survived their ICU stay but died before leaving the hospital.

We conducted sensitivity analyses to assess the stability of the above conclusions. Specifically, we repeated the analyses with and without one study ${ }^{73}$ reporting substantially better odds ratios (that is, lower deaths with remote ICU) after adjustments than the other studies . Omitting this study changes risk ratios (e.g., from 0.69 to 0.72 for ICU mortality), but does not change overall conclusions. Similarly, we repeated analyses stratifying by study design, specially combining only the before-after studies and not including the two studies that used concurrent controls. This changed the risk ratio slightly, but did not alter conclusion.

Six studies evaluated and reported the impact of remote ICU on costs. The studies used very different approaches to assess the economic impact of remote ICUs and the findings were not consistent. Three studies reported benefits: one reported that case volume increased and contributed to higher margins; ${ }^{81}$ another reported that ICU patients contributed to increased revenue because shorter ICU stays allowed more patients to be treated by increasing capacity; ${ }^{36}$ and the third study reported lower ICU total costs (ratio of after to before $0.69, \mathrm{p}=0.031$ ) and attributed this to a decrease in complications after remote ICU implementation. ${ }^{107}$ Other studies reported higher costs ${ }^{62,91}$ or reported very basic estimates. ${ }^{109}$ Given that the evidence is inconsistent and imprecise, we were unable to categorize how remote ICUs affect costs. 


\section{Remote Intensive Care Unit: Effectiveness in Improving Intermediate Outcomes}

Length of stay in the ICU and in the hospital are intermediate outcomes assessed in many studies of remote ICUs. We also pooled results for these outcomes. These analyses combined the mean difference in length of stay (LOS) in days across studies. The data and pooled results based on the 13 studies with usable data are presented in Figures 7 and 8 . The results of studies reporting data that could not be pooled are included in Table 3.

The finding from studies that included ICU LOS were not consistent: some reported shorter LOS and some longer for remote ICU versus usual care. The pooled estimate of the mean difference is -0.39 with a $95 \%$ confidence interval of -0.99 to 0.15 , indicating that the difference is not statistically significantly different from zero. For total in-hospital LOS the results are similar. The pooled difference is -0.14 (95\% CI -0.96 to 0.63 ), which is lower for remote ICU than usual care, but not significantly different. We tested the stability of the results by repeating the analyses including both raw numbers and adjusted estimates when they were provided, by including only the before-after studies, and by excluding studies with the most extreme results. While the magnitude of the difference changed, none of these variations resulted in a difference that was statistically significant. Authors of these studies suggest that patients are surviving longer as reflected in the decline in mortality, but remain in the hospital longer.

Some of these studies assessed other outcomes including nursing staff satisfaction, staff perceptions of teamwork and safety, readmission to the ICU, adherence to guidelines, and transfers to other hospitals. These results are included in Table 3.

\section{Harms, Adverse Events, or Negative Unintended Consequences of Remote ICUs}

None of the studies expressly reported on harms or adverse events. One reported 90-day mortality increased by 6.1 percent in hospitals that adopted remote ICU, but the researchers did not provide comparable data for the control hospitals, making it difficult to assess if this was a harm. ${ }^{71}$ Another study reported that the rate of complications experienced by ICU patients declined with remote ICU. ${ }^{107}$ 
Figure 5. Meta-analysis of intensive care unit mortality

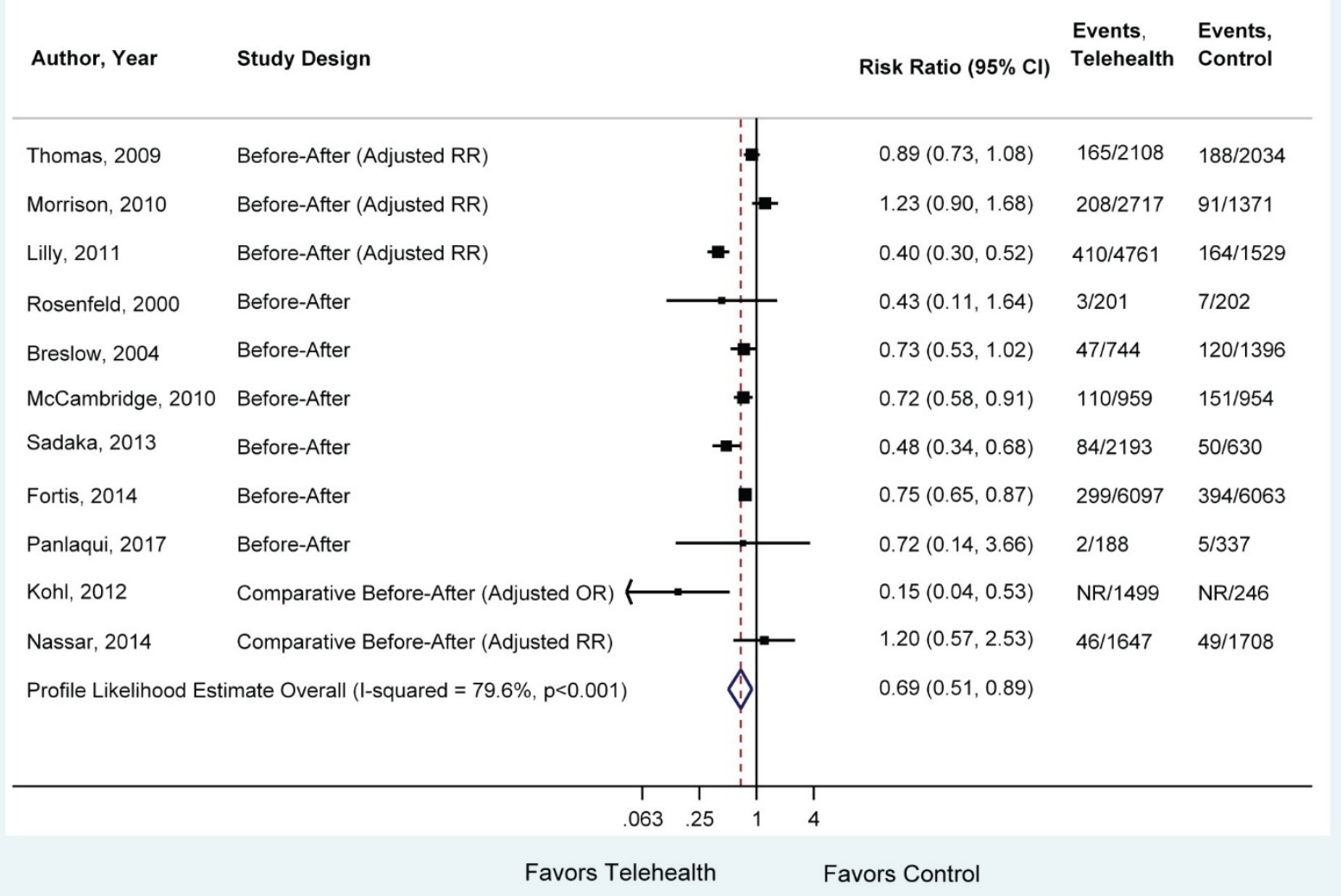

$\mathrm{CI}=$ confidence interval; $\mathrm{NR}=$ not reported; $\mathrm{OR}$ = odds ratio; $\mathrm{RR}=$ risk ratio 
Figure 6. Meta-analysis of hospital mortality in intensive care unit studies

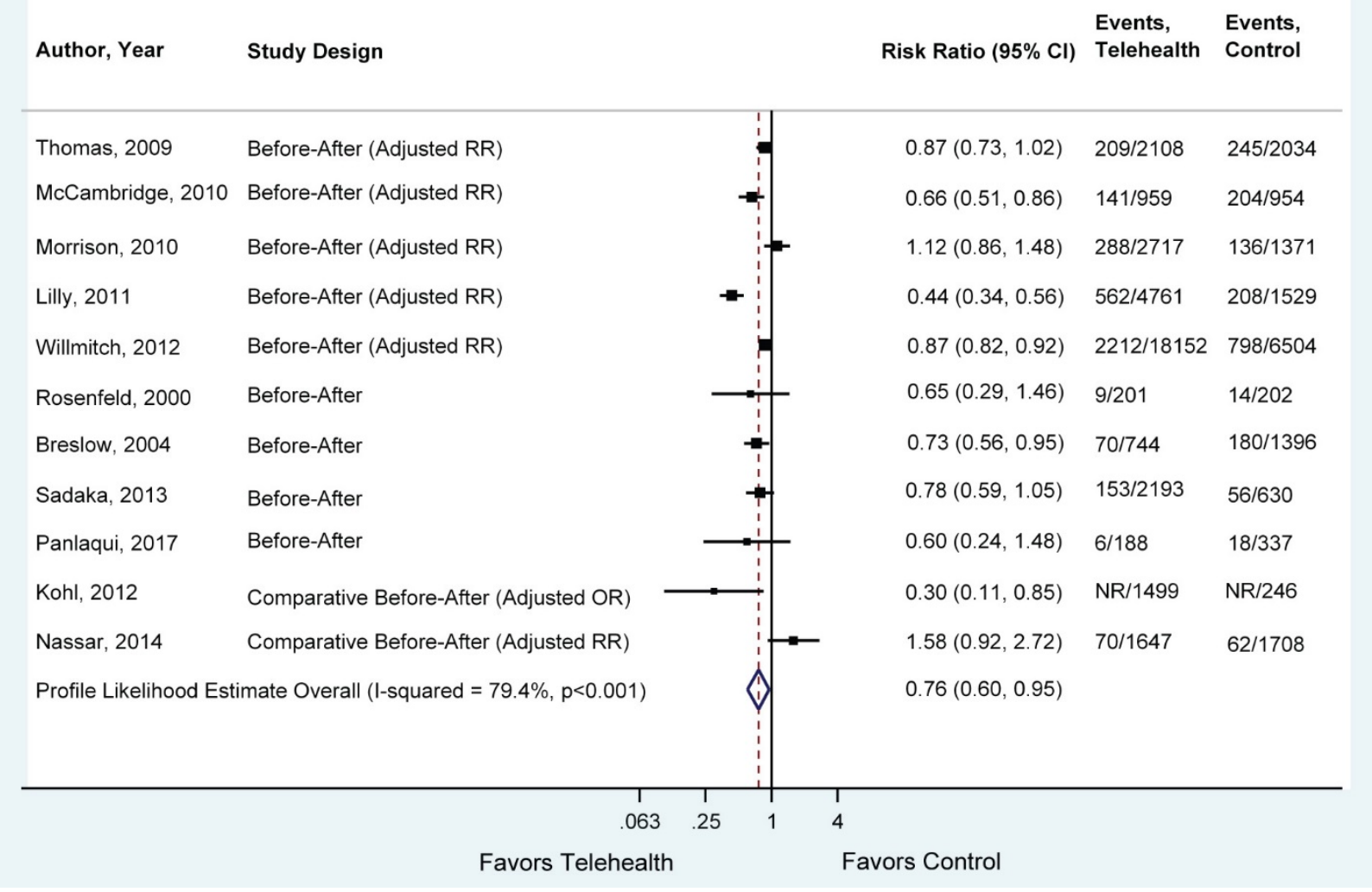

$\mathrm{CI}=$ confidence interval; $\mathrm{NR}=$ not reported; $\mathrm{OR}=$ odds ratio, $\mathrm{RR}=$ risk ratio 
Figure 7. Meta-analysis of intensive care unit length of stay

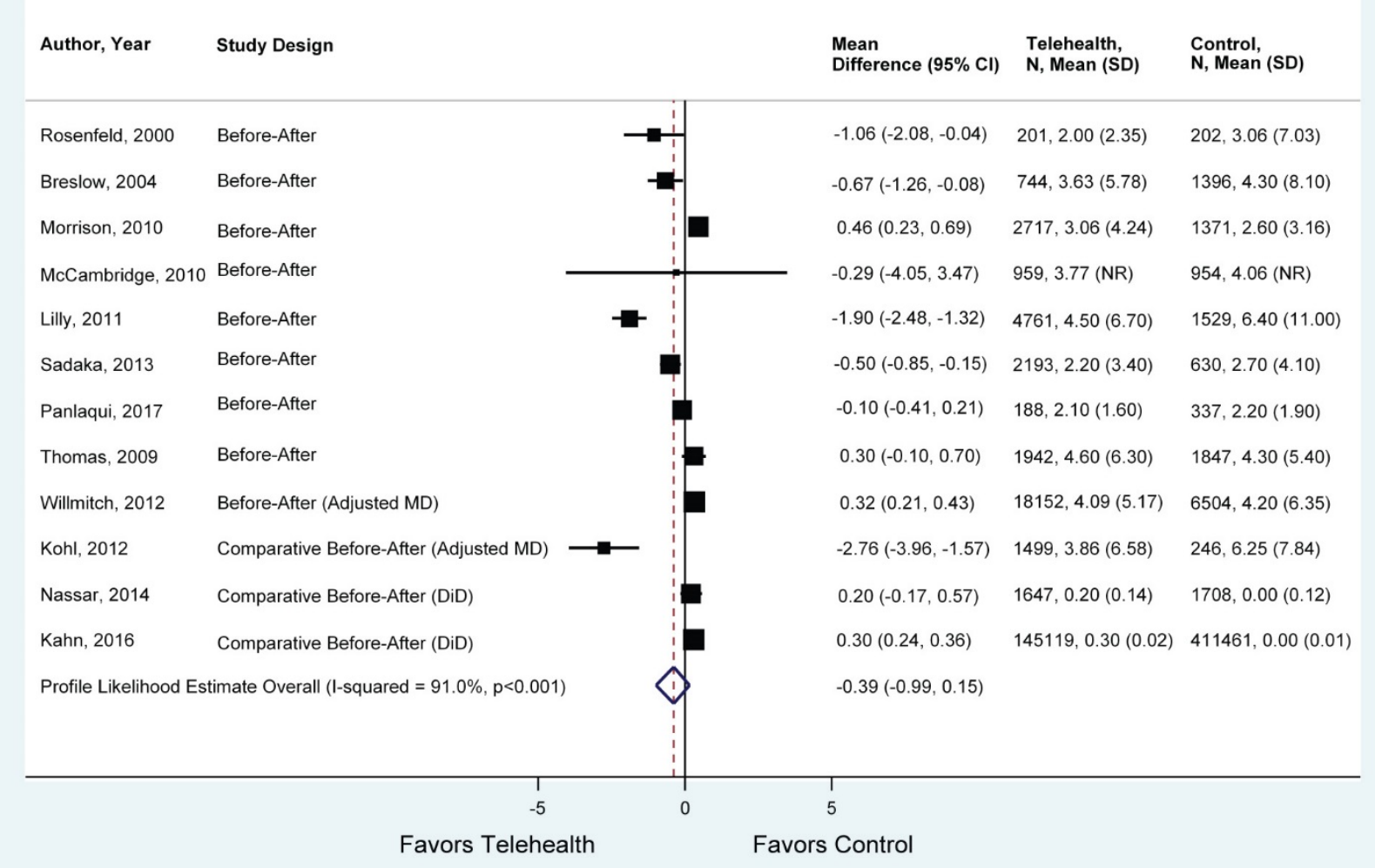

$\mathrm{CI}=$ Confidence Interval; $\mathrm{DiD}$ = difference in difference; $\mathrm{MD}$ = mean difference; $\mathrm{NR}$ = not reported; $\mathrm{SD}$ = standard deviation 
Figure 8. Meta-analysis of hospital length of stay in intensive care unit studies

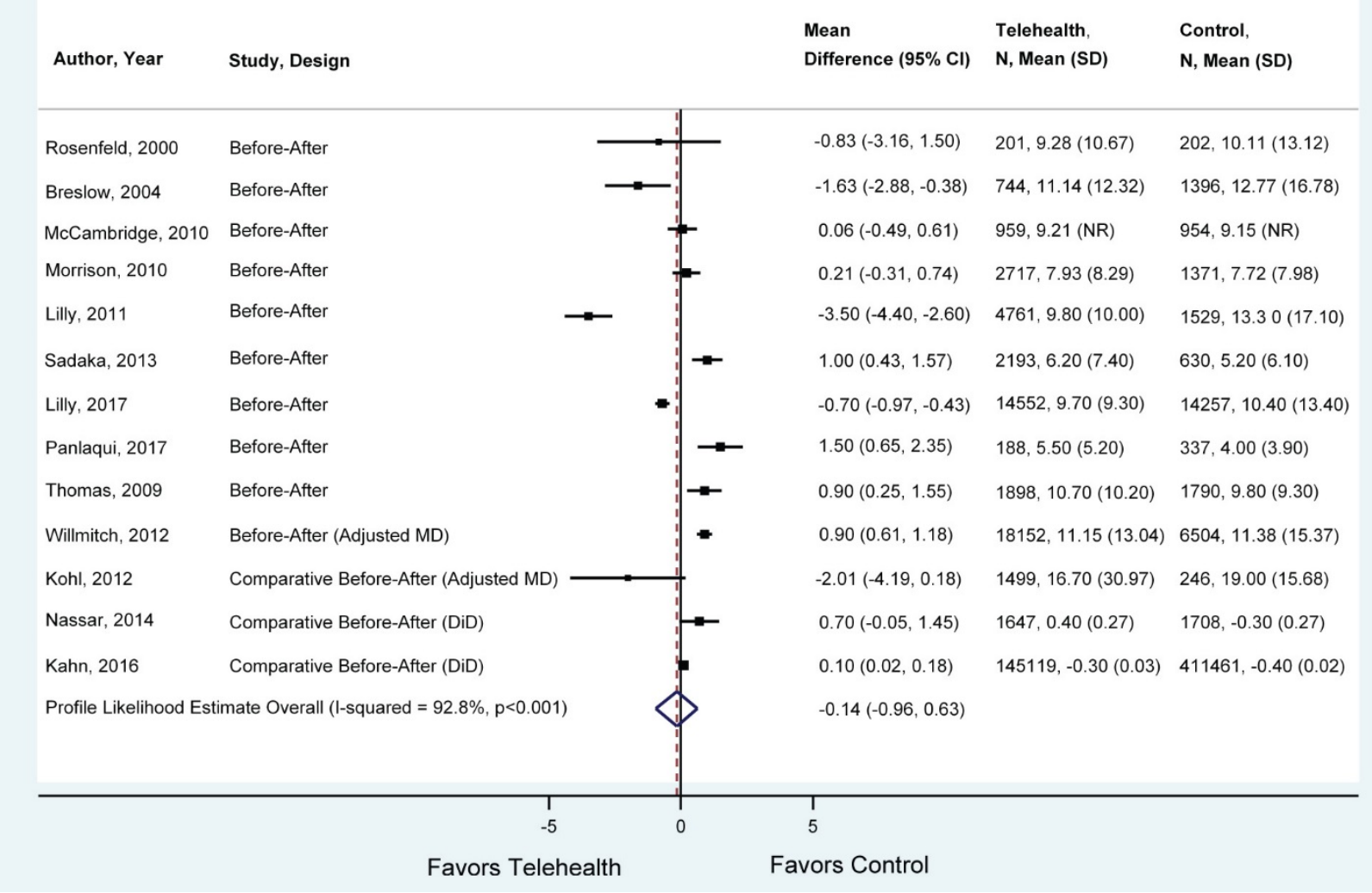

$\mathrm{CI}$ = confidence interval; $\mathrm{DiD}$ = difference in difference; $\mathrm{MD}=$ mean difference; $\mathrm{NR}=$ not reported; $\mathrm{SD}$ = standard deviation 
Table 3. Remote intensive care units: selected outcomes not included in meta-analysis

\begin{tabular}{|c|c|c|c|c|}
\hline $\begin{array}{l}\text { Author, Year } \\
\text { Location } \\
\text { Number of Sites }\end{array}$ & $\begin{array}{l}\text { Study Design } \\
\text { Risk of Bias } \\
\text { Comparison, } n\end{array}$ & Mortality & Intermediate Outcomes & $\begin{array}{l}\text { Costs/Revenue or } \\
\text { Other Resource Use }\end{array}$ \\
\hline $\begin{array}{l}\text { Breslow, } 2004^{36} \\
\text { VA, United States } \\
1 \text { hospital }\end{array}$ & $\begin{array}{l}\text { Before-after } \\
\text { Moderate } \\
\text { A: Before telehealth, } \\
n=1,396 \\
\text { B: After telehealth, } n=744\end{array}$ & $\begin{array}{l}\text { Hospital and ICU mortality in } \\
\text { meta-analysis }\end{array}$ & $\begin{array}{l}\text { Hospital and ICU LOS in meta- } \\
\text { analysis }\end{array}$ & $\begin{array}{l}\text { Revenue, contribution } \\
\text { per month in USD } \\
\text { All patients: } \\
\text { A: } \$ 795,245 \\
\text { B: } \$ 1,319,236 \text { (no } \\
\text { statistical test reported) }\end{array}$ \\
\hline $\begin{array}{l}\text { Chu-Weininger, } 2010^{43} \\
\text { TX, United States } \\
2 \text { community hospitals } \\
1 \text { teaching hospital }\end{array}$ & $\begin{array}{l}\text { Pre-post } \\
\text { High } \\
\text { A: Pre-Telehealth, } n=84 \\
\text { B: Post-Telehealth, } n=71\end{array}$ & NR & $\begin{array}{l}\text { Mean Teamwork Climate score out of } \\
100 \pm \text { SD } \\
\text { A: } 69.7 \pm 25.3 \\
\text { B: } 78.8 \pm 17.2, p=0.009 \\
\text { Mean Safety Climate score } \\
\text { A: } 66.4 \pm 24.6 \\
\text { B: } 73.4 \pm 18.5, p=0.045 \\
\text { Overall hospital Safety Climate score } \\
\text { A: } 69.0 \\
\text { B: } 65.4\end{array}$ & NR \\
\hline $\begin{array}{l}\text { Collins, } 2017^{44} \\
\text { PA, United States } \\
1 \text { surgical ICU } \\
1 \text { virtual ICU } \\
\end{array}$ & $\begin{array}{l}\text { Retrospective cohort } \\
\text { High } \\
\text { A: Surgical ICU, n=6,652 } \\
\text { B: Virtual ICU, } n=1,037\end{array}$ & $\begin{array}{l}\text { ICU mortality, n (\%) } \\
\text { A: } 364(5.5 \%) \\
\text { B: } 3(0.3 \%)\end{array}$ & $\mathrm{NR}$ & NR \\
\hline $\begin{array}{l}\text { Fortis, } 2014^{55} \\
\text { MN, United States } \\
5 \text { ICUs, including } 1 \\
\text { teaching hospital }\end{array}$ & $\begin{array}{l}\text { Retrospective } \\
\text { High } \\
\text { A: No tele-ICU, } n=6,063 \\
\text { B: Tele-ICU, } n=6,097\end{array}$ & ICU mortality in meta-analysis & $\begin{array}{l}\text { Patients readmitted to ICU within } 48 \\
\text { hours } n(\%) \\
\text { A: } 54(0.89 \%) \\
\text { B: } 29(0.49 \%), p=0.0064\end{array}$ & NR \\
\hline
\end{tabular}




\begin{tabular}{|c|c|c|c|c|}
\hline $\begin{array}{l}\text { Author, Year } \\
\text { Location } \\
\text { Number of Sites }\end{array}$ & $\begin{array}{l}\text { Study Design } \\
\text { Risk of Bias } \\
\text { Comparison, } n\end{array}$ & Mortality & Intermediate Outcomes & $\begin{array}{l}\text { Costs/Revenue or } \\
\text { Other Resource Use }\end{array}$ \\
\hline $\begin{array}{l}\text { Franzini, } 2011^{62} \\
\text { Thomas, } 2009^{123} \\
\text { Gulf Coast, United States } \\
5 \text { hospitals }\end{array}$ & $\begin{array}{l}\text { Economic evaluation, } \\
\text { Before-after } \\
\text { Moderate } \\
\text { A: No telehealth, } n=2,034 \\
\text { B: Telehealth, } n=2,108\end{array}$ & $\begin{array}{l}\text { Hospital and ICU mortality in } \\
\text { meta-analysis } \\
\text { Overall: no difference } \\
\text { SAPS II } \leq 50 \text { (less serious): } \\
\text { no difference } \\
\text { SAPS II > } 50 \text { ( } 17 \% \text { of } \\
\text { patients) } \\
\text { ICU: } 40 \% \text { reduction } \\
\text { Hospital: } 37 \% \text { reduction }\end{array}$ & $\begin{array}{l}\text { Hospital and ICU LOS in meta- } \\
\text { analysis } \\
\text { ICU complication rate }(95 \% \text { CI) } \\
\text { A: } 17.9 \%(16.3 \% \text { to } 19.6 \%) \\
\text { B: } 19.2 \%(17.5 \% \text { to } 20.9 \%)\end{array}$ & $\begin{array}{l}\text { Overall ICU cost per } \\
\text { case, in USD } \\
\text { A: } \$ 13,029 \\
\text { B: } \$ 19,324 \\
(48 \% \text { increase) } \\
\text { SAPSII } \leq 50 \text { : significant } \\
\text { increase in cost } \\
\text { (\$6415) with no } \\
\text { significant change in } \\
\text { mortality } \\
\text { SAPS II >50: no } \\
\text { significant increase in } \\
\text { cost (\$2985) with } 11.4 \% \\
\text { significant decrease in } \\
\text { mortality. }\end{array}$ \\
\hline $\begin{array}{l}\text { Gupta, 2014240 } \\
\text { Dehradun, India } \\
1 \mathrm{ICU}\end{array}$ & $\begin{array}{l}\text { Before-after } \\
\text { Moderate } \\
\text { A: Before telehealth, } n=134 \\
\text { B: After telehealth, } n=145\end{array}$ & $\begin{array}{l}\text { 30-Day mortality } \\
\text { A: } 16.4 \% \\
\text { B: } 4.8 \% \\
70 \% \text { reduction, } p=0.001\end{array}$ & $\begin{array}{l}\text { Mean door to needle time, in minutes } \\
\text { A: } \mathbf{1 7 8 . 6 3} \\
\text { B: } \mathbf{2 6 . 2 3 ,} p<0.001 \\
\text { Mean hospital stay } \pm S D \text {, in days } \\
\text { A: } 4.96 \pm 1.18 \\
\text { B: } 4.69 \pm 1.19, p=0.056 \\
\text { Cardiogenic shock, } \% \\
\text { A: } 14.92 \\
\text { B: } 10.35, p=0.248 \\
\text { Ventricular fibrillation/ventricular } \\
\text { tachycardia, } \% \\
\text { A: } 11.94 \\
\text { B: } 8.28, p=0.309 \\
\text { Atrial fibrillation/supra ventricular } \\
\text { tachycardia } \% \\
\text { A: } 14.92 \\
\text { B: } 15.17, p=0.954\end{array}$ & NR \\
\hline
\end{tabular}




\begin{tabular}{|c|c|c|c|c|}
\hline $\begin{array}{l}\text { Author, Year } \\
\text { Location } \\
\text { Number of Sites }\end{array}$ & $\begin{array}{l}\text { Study Design } \\
\text { Risk of Bias } \\
\text { Comparison, } n\end{array}$ & Mortality & Intermediate Outcomes & $\begin{array}{l}\text { Costs/Revenue or } \\
\text { Other Resource Use }\end{array}$ \\
\hline $\begin{array}{l}\text { Kahn, } 2016^{71} \\
\text { United States } \\
389 \text { hospitals without tele- } \\
\text { ICU } \\
132 \text { hospitals with tele-ICU } \\
\text { hospitals }\end{array}$ & $\begin{array}{l}\text { Retrospective cohort } \\
\text { Low } \\
\text { A: No telehealth } \\
\text { Before-telehealth, } \\
\mathrm{n}=419,466 \\
\text { After telehealth, } \mathrm{n}=411,461 \\
\\
\text { B: Telehealth } \\
\text { Before telehealth, } \\
\mathrm{n}=147,517 \\
\text { After telehealth, } \mathrm{n}=145,119\end{array}$ & $\begin{array}{l}\text { 90-Day mortality } \\
\text { [ratio of odds ratios }(95 \% \mathrm{Cl}) \text {, } \\
\text { Group A=Reference] } \\
\text { All hospitals: } 0.96 \text { ( } 0.94 \text { to } \\
\mathbf{0 . 9 8 ) , ~ p < 0 . 0 1} \\
\text { Unadjusted } \\
\text { Before vs. after telehealth } \\
\text { A: } 23.5 \% \text { vs. } 23.07 \%, p<0.01 \\
\text { B: } 24.0 \% \text { vs. } 24.3 \%, p=0.07 \\
\text { Hospitals with telemedicine } \\
12.2 \% \text { significant decrease } \\
81.1 \% \text { no significant change } \\
6.1 \% \text { significant increase }\end{array}$ & $\begin{array}{l}\text { Hospital and ICU LOS in meta- } \\
\text { analysis }\end{array}$ & NR \\
\hline $\begin{array}{l}\text { Kalb, } 2014^{257} \\
\text { NR, authors US-based } \\
11 \text { hospitals }\end{array}$ & $\begin{array}{l}\text { Before-after } \\
\text { High } \\
\text { A: Before Tele-ICU } \\
\text { implementation, } n=N R \\
\text { B}_{1} \text { : After Tele-ICU (2011, } \\
\text { Quarter 3), n=NR } \\
\text { B2: After Tele-ICU (2012, } \\
\text { Quarter 1), n=NR }\end{array}$ & $\begin{array}{l}\text { ICU mortality ratio } \\
\text { A: } 0.34 \\
B_{1}: 0.67, p<0.04 \text { vs. } A \\
B_{2}: 0.65, p<0.03 \text { vs. } A\end{array}$ & $\begin{array}{l}\text { Mean } \% \text { adherence to low tidal } \\
\text { volume-based lung protective } \\
\text { ventilation } \\
\text { A: } 29.5 \% \\
B_{1}: 44.9 \%, p<0.002 \text { vs. A } \\
B_{2}: \mathbf{5 1 . 8} \%, p<0.003 \text { vs. A } \\
\text { Mean ventilator duration } \text { ratio }^{\text {b }} \text {, in } \\
\text { days } \\
\text { A: } 1.08 \\
B_{1}: 0.92, N S \text { vs. A } \\
B_{2}: 0.96, N S \text { vs. A }\end{array}$ & NR \\
\hline $\begin{array}{l}\text { Kohl, } 2012^{73} \\
\text { PA, United States } \\
2 \text { ICUs in the same } \\
\text { hospital: one with } \\
\text { telehealth, one without }\end{array}$ & $\begin{array}{l}\text { Retrospective cohort } \\
\text { Moderate } \\
\mathrm{A}_{1} \text { : No telehealth, prior to } \\
\text { implementation, } \mathrm{n}=220 \\
\mathrm{~A}_{2} \text { : No telehealth, post } \\
\text { telehealth implementation, } \\
\mathrm{n}=285 \\
\mathrm{~B}_{1}: \text { Telehealth, prior to } \\
\text { implementation, } \mathrm{n}=246 \\
\mathrm{~B}_{2}: \text { Telehealth, post } \\
\text { telehealth implementation, } \\
\mathrm{n}=1,499\end{array}$ & $\begin{array}{l}\text { ICU mortality }{ }^{\mathrm{C}}(\mathrm{SEM}) \\
\mathrm{A}_{1}: 0.54(0.06) \\
\mathrm{A}_{2}: 0.42(0.04) \\
\mathrm{B}_{1}: 0.09(0.02) \\
\mathrm{B}_{2}: 0.01(0.003), p=0.003 \\
\text { Mean hospital mortality } \\
(\mathrm{SEM}) \\
\mathrm{A}_{1}: 0.74(0.05) \\
\mathrm{A}_{2}: 0.56(0.04) \\
\mathrm{B}_{1}: 0.13(0.03) \\
\mathrm{B}_{2}: 0.04(0.01), p=0.023\end{array}$ & $\begin{array}{l}\text { Mean ICU LOS }(S E M) \text {, in days } \\
A_{1}: 5.27(\mathbf{0 . 5 2}) \\
A_{2}: 6.09(\mathbf{0 . 4 3}) \\
B_{1}: 6.25(\mathbf{0 . 5 0 )} \\
B_{2}: 3.86(\mathbf{0 . 1 7}), p<0.001 \\
\text { Mean hospital } \text { LOS }^{c}(S E M) \text {, in days } \\
A_{1}: 19.0(1.0) \\
A_{2}: 12.5(1.1) \\
B_{1}: 10.9(0.8) \\
B_{2}: 16.7(0.8), \text { NS }\end{array}$ & NR \\
\hline
\end{tabular}




\begin{tabular}{|c|c|c|c|c|}
\hline $\begin{array}{l}\text { Author, Year } \\
\text { Location } \\
\text { Number of Sites }\end{array}$ & $\begin{array}{l}\text { Study Design } \\
\text { Risk of Bias } \\
\text { Comparison, } n\end{array}$ & Mortality & Intermediate Outcomes & $\begin{array}{l}\text { Costs/Revenue or } \\
\text { Other Resource Use }\end{array}$ \\
\hline $\begin{array}{l}\text { Lilly, } 2011^{80} \\
\text { MA, United States } \\
7 \text { ICUs }\end{array}$ & $\begin{array}{l}\text { Prospective cohort } \\
\text { Low } \\
\text { A: No telehealth, } n=1,529 \\
\text { B: Telehealth, } n=4,761\end{array}$ & $\begin{array}{l}\text { ICU mortality, } n(\%) \\
\text { A: } 164(10.7 \%) \\
\text { B: } 410(8.6 \%), p=0.003 \\
\text { Hospital mortality } \\
\text { A: } 208(13.6) \\
\text { B: } 562(11.8), p=0.005\end{array}$ & $\begin{array}{l}\text { Mean } \pm \text { SD, median (IQR), } \\
\text { ICU LOS, in days } \\
\text { A: } 6.4 \pm 11,2.5(0.2 \text { to } 6.5) \\
\text { B: } 4.5 \pm 6.7,2.4(0.1 \text { to } 4.6), p<0.001 \\
\text { Hospital LOS, in days } \\
\text { A: } 13.3 \pm 17.1,7.9(0.2 \text { to } 15.0) \\
\text { B: } 9.8 \pm 10,6.8(0.2 \text { to } 12.0) \\
\text { p<0.001 }\end{array}$ & NR \\
\hline $\begin{array}{l}\text { Lilly, } 2017^{81} \\
\text { MA, United States } \\
1 \text { academic medical center } \\
\text { ICU }\end{array}$ & $\begin{array}{l}\text { Economic evaluation } \\
\text { Moderate } \\
\text { A: Before telehealth, } \\
n=4,752 \\
B_{1}: \text { After Telehealth, } \\
n=5,735 \\
B_{2}: \text { After Telehealth with } \\
\text { added logistics center, } \\
n=6,581\end{array}$ & NR & $\begin{array}{l}\text { Mean hospital } L O S \pm S D \text {, in days } \\
\text { A vs. } B_{1} \text { included in Meta-Analysis } \\
A: 10.4 \pm 13.4 \\
B_{1}: 9.7 \pm 9.3 \\
B_{2}: 8.8 \pm 8.3 \\
\text { A vs. } B_{2} \mathbf{p}<0.0001 \\
B_{1} \text { vs. } B_{2} \mathbf{p}<0.001\end{array}$ & $\begin{array}{l}\text { Total annual costs, in } \\
\text { USD } \\
\text { A: } \$ 142,766,712 \\
B_{1}: \$ 182,719,738 \\
B_{2}: \$ 200,934,975 \\
\text { Total annual direct } \\
\text { contribution margin } \\
\text { A: } \$ 7,921,584 \\
B_{1}: \$ 37,668,512 \\
B_{2}: \$ 60,586,397\end{array}$ \\
\hline $\begin{array}{l}\text { McCambridge, } 2010^{87} \\
\text { PA, United States } \\
1 \text { hospital }\end{array}$ & $\begin{array}{l}\text { Before-after } \\
\text { Moderate } \\
\text { A: Before telehealth, } n=954 \\
\text { B: After telehealth, } n=959\end{array}$ & $\begin{array}{l}\text { Hospital and ICU mortality in } \\
\text { meta-analysis } \\
\text { Overall } \\
\text { AOR }^{\mathrm{d}}: \mathbf{0 . 6 0 5}, \mathbf{p}=\mathbf{0 . 0 0 2}\end{array}$ & $\begin{array}{l}\text { Hospital and ICU LOS in meta- } \\
\text { analysis } \\
\text { Ventilator use } \\
\text { A: } \mathbf{3 6 . 1 \%} \\
\text { B: } \mathbf{3 1 . 5 \% , p = 0 . 0 4}\end{array}$ & NR \\
\hline $\begin{array}{l}\text { Morrison, } 2010^{91} \\
\text { IL, United States } \\
2 \text { hospitals }\end{array}$ & $\begin{array}{l}\text { Before-after } \\
\text { Moderate } \\
\text { A: Before telehealth, } n=1371 \\
B_{1} \text { : After telehealth, } 1 \text { year } \\
\text { after baseline, } n=1287 \\
B_{2} \text { : After telehealth, } 1 \text { year } \\
\text { after electronic ICU fully } \\
\text { operational, } n=1430\end{array}$ & $\begin{array}{l}\text { Hospital and ICU mortality in } \\
\text { meta-analysis } \\
\text { Total mortality } \\
\text { A: } 9.9 \% \\
B^{1}: 11.1 \% \\
B^{2}: 10.0 \% \\
\text { A vs. B: NS } \\
\text { B vs. } B^{2} \text { : NS }\end{array}$ & $\begin{array}{l}\text { Hospital and ICU LOS in meta- } \\
\text { analysis }\end{array}$ & $\begin{array}{l}\text { Mean } \text { Cost }^{\mathrm{e}} \text {, in USD } \\
\text { A: } \$ 22.43 \\
\mathrm{~B}_{1}: \$ 21.41 \\
\text { B }_{2}: \$ 23.21 \\
\text { A vs. } \mathrm{B}^{1}: \mathrm{NS} \\
\text { B }^{\mathbf{1}} \text { vs. } \mathbf{B}^{\mathbf{2}}: \mathbf{p}=\mathbf{0 . 0 3}\end{array}$ \\
\hline
\end{tabular}




\begin{tabular}{|c|c|c|c|c|}
\hline $\begin{array}{l}\text { Author, Year } \\
\text { Location } \\
\text { Number of Sites }\end{array}$ & $\begin{array}{l}\text { Study Design } \\
\text { Risk of Bias } \\
\text { Comparison, } n\end{array}$ & Mortality & Intermediate Outcomes & $\begin{array}{l}\text { Costs/Revenue or } \\
\text { Other Resource Use }\end{array}$ \\
\hline $\begin{array}{l}\text { Nassar, } 2014^{94} \\
\text { Midwest, United States } \\
7 \text { VA Hospitals }\end{array}$ & $\begin{array}{l}\text { Before-after } \\
\text { Low } \\
A_{1} \text { : Usual care before } \\
\text { telehealth period, } n=1664 \\
A_{2}: \text { Usual care after } \\
\text { telehealth period, } n=1920 \\
B_{1}: \text { Telehealth, before } \\
\text { telehealth period, } n=1708 \\
B_{2}: \text { Telehealth, after } \\
\text { telehealth period } n=1647\end{array}$ & $\begin{array}{l}\text { Hospital and ICU mortality in } \\
\text { meta-analysis }\end{array}$ & $\begin{array}{l}\text { Hospital and ICU LOS in meta- } \\
\text { analysis }\end{array}$ & NR \\
\hline $\begin{array}{l}\text { Panlaqui, } 2017^{227} \\
\text { Australia and New Zealand } \\
1 \text { rural facility } \\
1 \text { regional center }\end{array}$ & $\begin{array}{l}\text { Before- after } \\
\text { High } \\
\text { A: Before telehealth, } n=337 \\
\text { B: After telehealth, } n=188\end{array}$ & $\begin{array}{l}\text { Hospital ICU Mortality: in } \\
\text { meta-analysis } \\
\text { Total mortality, relative risk } \\
(95 \% \mathrm{Cl}) \\
\text { A: } 6.5 \% \\
\text { B: } 4.3 \% \\
\text { RR } 0.98 \text { (0.94 to } 1.02), \\
p=0.28\end{array}$ & $\begin{array}{l}\text { Hospital and ICU LOS: in meta- } \\
\text { analysis } \\
\text { Hospital transfer relative risk ( } 95 \% \\
\mathrm{Cl} \text { ) } \\
\text { A: } \mathbf{3 1 . 8 \%} \\
\text { B: } 22.9 \% \\
\text { RR } 0.88 \text { ( } 0.80 \text { to } 0.98), p=0.03\end{array}$ & NR \\
\hline $\begin{array}{l}\text { Rincon, } 2012^{104} \\
\text { PA, United States } \\
1 \mathrm{ICU}\end{array}$ & $\begin{array}{l}\text { Before-after } \\
\text { High } \\
\text { A: Before telehealth, } n=34 \\
\text { nurses } \\
\text { B: After telehealth, } n=40 \\
\text { nurses }\end{array}$ & NR & $\begin{array}{l}\text { Nurse satisfaction } \\
\text { ICU physicians available } \\
\text { A: } 38 \% \\
\text { B: } 55 \% \text { NS } \\
\text { Adequate physician involvement } \\
\text { A: } 44 \% \\
\text { B: } 65 \%, p=0.007 \\
\text { Opportunity to ask questions } \\
\text { A: } 41 \% \\
\text { B: } 53 \% \text { NS }\end{array}$ & NR \\
\hline
\end{tabular}




\begin{tabular}{|c|c|c|c|c|}
\hline $\begin{array}{l}\text { Author, Year } \\
\text { Location } \\
\text { Number of Sites }\end{array}$ & $\begin{array}{l}\text { Study Design } \\
\text { Risk of Bias } \\
\text { Comparison, } n\end{array}$ & Mortality & Intermediate Outcomes & $\begin{array}{l}\text { Costs/Revenue or } \\
\text { Other Resource Use }\end{array}$ \\
\hline $\begin{array}{l}\text { Romig, } 2012^{106} \\
\text { MD, United States } \\
2 \text { ICU in } 1 \text { hospital }\end{array}$ & $\begin{array}{l}\text { Prospective cohort and } \\
\text { before-after } \\
\text { High } \\
\text { A: No telehealth, } n=612 \\
\text { B: Telehealth, } n=793 ; 403 \\
\text { received telehealth } \\
\text { Nurse survey: } \\
\text { Pre-telehealth, } n=11 \\
\text { Post-telehealth, } n=27\end{array}$ & $\begin{array}{l}\text { ICU mortality before vs. after } \\
\text { telehealth, } \%(\mathrm{n}) \\
\text { A: } 4.9 \%(15 / 305) \text { vs. } 4.6 \% \\
(14 / 307) \\
\text { B: } 1.5 \%(6 / 390) \text { vs. } 3.5 \% \\
(14 / 403)\end{array}$ & $\begin{array}{l}\text { ICU LOS before vs. after telehealth, } \\
\%(n) \text {, in days } \\
\text { A: } 3.9 \text { vs. } 3.8 \\
\text { B: } 5.1 \text { vs. } 4.5 \\
\text { Nurse satisfaction and perceptions of } \\
\text { quality after telehealth, mean } \pm \text { SD } \\
\text { survey score out of } 5 \\
\\
\text { Remote ICU unit } \\
\text { Communications } \\
\text { Pre-telehealth: } 2.99 \pm \mathbf{1 . 1 3} \\
\text { Post-telehealth: } \mathbf{3 . 2 7} \pm \mathbf{1 . 2 7}, \mathrm{p}<0.01 \\
\text { Working conditions } \\
\text { Pre-telehealth: } 3.10 \pm \mathbf{1 . 1 0} \\
\text { Post-telehealth: } \mathbf{3 . 2 3} \pm \mathbf{1 . 1 1}, \mathrm{p}=\mathbf{0 . 0 2} \\
\text { Education } \\
\text { Pre-telehealth: } \mathbf{3 . 5 2} \pm \mathbf{0 . 8 4} \\
\text { Post-telehealth: } \mathbf{3 . 7 6} \pm \mathbf{0 . 7 8 , p} \mathrm{p}<0.03 \\
\text { Control ICU } \\
\text { Significant decline in } 2 \text { scales } \\
\text { Patient care and perceived } \\
\text { effectiveness and education }\end{array}$ & NR \\
\hline
\end{tabular}




\begin{tabular}{|c|c|c|c|c|}
\hline $\begin{array}{l}\text { Author, Year } \\
\text { Location } \\
\text { Number of Sites }\end{array}$ & $\begin{array}{l}\text { Study Design } \\
\text { Risk of Bias } \\
\text { Comparison, } n\end{array}$ & Mortality & Intermediate Outcomes & $\begin{array}{l}\text { Costs/Revenue or } \\
\text { Other Resource Use }\end{array}$ \\
\hline $\begin{array}{l}\text { Rosenfeld, } 2000^{107} \\
\text { MD, United States } \\
1 \text { hospital }\end{array}$ & $\begin{array}{l}\text { Before-after } \\
\text { Low } \\
\text { A } 1 \text { : Before telehealth, }_{\text {baseline } 1, n=225} \\
\text { A } 2 \text { : Before telehealth, }_{\text {baseline } 2, n=202} \\
\text { B: After tele-ICU, } n=201\end{array}$ & $\begin{array}{l}\text { Hospital and ICU mortality in } \\
\text { meta-analysis } \\
\text { Complications } \\
\text { A }_{1}: 15.1 \% \\
A_{2}: 18.8 \% \\
\text { B: } 9.5 \% \text { p }<0.05\end{array}$ & $\begin{array}{l}\text { Hospital and ICU LOS in meta- } \\
\text { analysis }\end{array}$ & $\begin{array}{l}\text { ICU total cost change, } \\
\text { in USD } \\
\mathrm{B}_{\mathrm{V}} \mathrm{A}_{1}: \mathbf{7 5 \%} \\
\text { reduction, } \mathrm{p}=0.002 \\
\mathrm{~B} \text { vs. } \mathrm{A}_{2}: \mathbf{6 9 \%} \\
\text { reduction, } \mathrm{p}=\mathbf{0 . 0 3 1} \\
\text { Hospital total cost } \\
\text { change, in USD } \\
\mathrm{B} \text { vs } \mathrm{A}_{1}: 88 \% \text { reduction, } \\
\mathrm{NS} \\
\mathrm{B} \text { vs. } \mathrm{A}_{2}: 81 \% \text { reduction, } \\
\mathrm{NS} \\
64 \% \text { of difference in } \\
\text { cost between baselines } \\
\text { and intervention were } \\
\text { associated with higher } \\
\text { incidence of } \\
\text { complications during } \\
\text { baseline periods. }\end{array}$ \\
\hline $\begin{array}{l}\text { Ruesch, } 2012^{109} \\
\text { AK, United States } \\
1 \text { hospital }\end{array}$ & $\begin{array}{l}\text { Before-after } \\
\text { High } \\
\text { A: Before telehealth, NR } \\
\text { B: After telehealth, NR }\end{array}$ & $\begin{array}{l}\text { ICU mortality } \\
\text { A: } 17 \\
\text { B: } 24 \\
\text { Hospital mortalityc } \\
\text { A: } 22 \\
\text { B: } 36\end{array}$ & $\begin{array}{l}\text { LOS }^{\mathrm{C}} \text {, in days } \\
\text { ICU } \\
\text { A: } \mathbf{4 . 1} \\
\text { B: } \mathbf{3 . 6 6}, \mathbf{p} \leq \mathbf{0 . 0 5} \\
\text { Hospital } \\
\text { A: } 11.25 \\
\text { B: } 9.48, \mathrm{NS}\end{array}$ & $\begin{array}{l}\text { Actual costs not } \\
\text { reported. Estimated } \\
\text { cost saving based on } \\
\text { changes in LOS were: > } \\
\$ 2.5 \text { million USD, } \\
\text { comparing a calendar } \\
\text { quarter before } \\
\text { implementation with the } \\
\text { last quarter of the } \\
\text { evaluation. }\end{array}$ \\
\hline $\begin{array}{l}\text { Sadaka, } 2013^{110} \\
\text { MO, United States } \\
1 \text { hospital }\end{array}$ & $\begin{array}{l}\text { Before-after } \\
\text { High } \\
\text { A: Before telehealth, } n=630 \\
\text { B: After telehealth, } n=2193\end{array}$ & $\begin{array}{l}\text { Hospital and ICU mortality: in } \\
\text { meta-analysis }\end{array}$ & $\begin{array}{l}\text { Hospital and ICU LOS in meta- } \\
\text { analysis }\end{array}$ & NR \\
\hline
\end{tabular}




\begin{tabular}{|c|c|c|c|c|}
\hline $\begin{array}{l}\text { Author, Year } \\
\text { Location }\end{array}$ & $\begin{array}{l}\text { Study Design } \\
\text { Risk of Bias } \\
\text { Comparison, n }\end{array}$ & Mortality & Intermediate Outcomes & $\begin{array}{l}\text { Costs/Revenue or } \\
\text { Other Resource Use }\end{array}$ \\
\hline $\begin{array}{l}\text { Willmitch, } 2012^{131} \\
\text { FL, United States } \\
5 \text { hospitals }\end{array}$ & $\begin{array}{l}\text { Before-after } \\
\text { Moderate } \\
\text { Comparison: } \\
\text { A: Before telehealth, } \\
n=6,504 \\
B_{1}: \text { After telehealth, } 1 \text { year, } \\
n=6,353 \\
B_{2}: \text { After telehealth, } 2 \text { years, } \\
n=6,018 \\
B_{3}: \text { After telehealth, } 3 \text { years, } \\
n=5,781\end{array}$ & $\begin{array}{l}\text { Hospital mortality in meta- } \\
\text { analysis }\end{array}$ & $\begin{array}{l}\text { Hospital and ICU LOS in meta- } \\
\text { analysis }\end{array}$ & NR \\
\hline
\end{tabular}

AOR = adjusted odds ratio; APACHE IV = Acute Physiology and Chronic Health Evaluation IV; CI = confidence interval; ICU = intensive care unit; IQR = interquartile range; LOS = length of stay; NR = not reported; NS = not significant; SAPS II = Simplified Acute Physiology Score II; SD = standard deviation; SEM = standard error of the mean; USD

= United States Dollars; VA = Veterans Affairs;

${ }^{a}$ Adjusted for APACHE IV status

${ }^{b}$ Number of days of mechanical ventilation/APACHE IV predicted days of mechanical ventilation

c Severity-adjusted

d Adjusted for APACHE IV status and DNR status

e Adjusted total hospital costs divided by 1000

Bold=statistically significant; telehealth superior. Bold and italicized=statistically significant; telehealth inferior. Regular type: not statistically significant. 


\section{Key Characteristics of Remote ICU Studies and Association With Outcomes}

In addition to pooling results, we reviewed selected key factors that could help explain differences in outcomes across studies. Table 4 summarizes information on the hospitals in the studies, the coverage and staffing of the remote ICU interventions, and the time periods when outcomes were measured. However, no studies report significant differences between remote ICUs and in-person critical care in terms of these characteristics.

The majority of studies were conducted in larger teaching hospitals or hospitals affiliated with an academic center. The goal of all studies was to provide critical care for 24 hours, but the amount of remote coverage needed to achieve this goal varied. All of the studies included a physician intensivist, though one added this after 9 months of nursing support alone. ${ }^{109}$ All but one included nursing ${ }^{107}$ and about half included administrative support. Most of the studies collected outcomes data after an implementation period or collected data for multiple periods. The study with the longest followup (3 years) and measurement at multiple periods reported stronger effects in each subsequent year. ${ }^{131}$ One study reporting no overall benefit compared outcomes between sicker and less sick patients and found the remote ICU was associated with a decrease in mortality and a nonsignificant increase in costs for sicker patients and no improvement in mortality and higher costs for less sick patients. ${ }^{62,123}$ This suggests that the benefit may not be uniform across all critical care patients. The largest study examined several characteristics of the subgroup of hospitals that experienced a reduction in 90-day mortality after implementing remote ICUs and found these were more likely to be high volume and located in

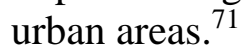

Table 4. Remote intensive care units: selected characteristics

\begin{tabular}{|c|c|c|c|c|}
\hline Study & $\begin{array}{l}\text { Hospital } \\
\text { Number } \\
\text { Characteristics }\end{array}$ & $\begin{array}{l}\text { Remote ICU } \\
\text { Coverage } \\
\text { Staffing }\end{array}$ & $\begin{array}{l}\text { "After" Period } \\
\text { for Outcomes } \\
\text { Measurement }\end{array}$ & $\begin{array}{l}\text { Impact of Remote } \\
\text { ICU } \\
\text { Subgroup } \\
\text { Assessments }\end{array}$ \\
\hline Breslow, $2004^{36}$ & $\begin{array}{l}1 \text { large (650 bed) } \\
\text { tertiary teaching }\end{array}$ & $\begin{array}{l}19 \text { hours (noon -7AM) } \\
\text { - Intensivist, nurse, } \\
\text { administrative assistant } \\
\text { - Attending controlled } \\
\text { level of involvement }\end{array}$ & $\begin{array}{l}\text { Months } 4-10 \text { (6 } \\
\text { months after a 3- } \\
\text { month run in) }\end{array}$ & $\begin{array}{l}\text { Benefit } \\
\text { No subgroups }\end{array}$ \\
\hline Fortis, $2014^{55}$ & $\begin{array}{l}5 \text { hospitals in one } \\
\text { health system }\end{array}$ & $\begin{array}{l}\text { - } 24 \text { hours but different } \\
\text { staff and duties day vs. } \\
\text { night } \\
\text { - Intensivist; nurse } \\
\text { - Full authority }\end{array}$ & $\begin{array}{l}1 \text { year } \\
\text { immediately } \\
\text { following } \\
\text { implementation }\end{array}$ & $\begin{array}{l}\text { Benefit } \\
\text { No subgroups }\end{array}$ \\
\hline $\begin{array}{l}\text { Franzini, } 2011^{62} \\
\text { Thomas, } 2009^{123}\end{array}$ & $\begin{array}{l}\text { 5; } 1 \text { large teaching } \\
\text { hospital; } 2 \text { large } \\
\text { urban hospitals; } 2 \\
\text { small community } \\
\text { hospitals }\end{array}$ & $\begin{array}{l}19 \text { hours (noon -7AM) } \\
\text { weekdays; } 24 \text { hours } \\
\text { weekends } \\
\text { - } 2 \text { Teams of intensivists, } \\
2 \text { nurses, } 1 \\
\text { administrative } \\
\text { technician } \\
\text { - Physicians determined } \\
\text { level }\end{array}$ & $\begin{array}{l}60 \text { to } 120 \text { days } \\
\text { (95 average) } \\
\text { post } \\
\text { implementation } \\
\text { until estimated } \\
\text { sample size } \\
\text { recruited }\end{array}$ & $\begin{array}{l}\text { No benefit } \\
\text { (Overall) } \\
\text { Sicker patients: } \\
\text { decrease in } \\
\text { mortality; increase } \\
\text { in costs }\end{array}$ \\
\hline
\end{tabular}




\begin{tabular}{|c|c|c|c|c|}
\hline Study & $\begin{array}{l}\text { Hospital } \\
\text { Number } \\
\text { Characteristics }\end{array}$ & $\begin{array}{l}\text { Remote ICU } \\
\text { Coverage } \\
\text { Staffing }\end{array}$ & $\begin{array}{l}\text { "After" Period } \\
\text { for Outcomes } \\
\text { Measurement }\end{array}$ & $\begin{array}{l}\text { Impact of Remote } \\
\text { ICU } \\
\text { Subgroup } \\
\text { Assessments }\end{array}$ \\
\hline Kalb, 2014 & $\begin{array}{l}11 \text { moderate-size } \\
\text { community } \\
\text { hospitals, wide } \\
\text { geographic } \\
\text { distribution (details } \\
\text { not reported); all } \\
\text { established } \\
\text { telelCU, phase-in } \\
\text { for ventilator } \\
\text { rounds }\end{array}$ & $\begin{array}{l}\text { - Daily rounds } \\
\text { - Intensivist and critical } \\
\text { care nurse }\end{array}$ & $\begin{array}{l}1.5 \text { years for first } \\
\text { group; } 3 \text { months } \\
\text { for last } \\
\text { (staggered start) }\end{array}$ & $\begin{array}{l}\text { Benefit } \\
\text { No subgroup } \\
\text { comparisons }\end{array}$ \\
\hline Kahn, $2016^{71}$ & $\begin{array}{l}521 \text { hospitals } \\
132 \text { hospitals } \\
\text { adopted telehealth } \\
389 \text { matched } \\
\text { hospitals that did } \\
\text { not }\end{array}$ & - Varied, not reported & 2 years & $\begin{array}{l}\text { Some benefit for } \\
\text { 90-day mortality } \\
\text { (overall) } \\
\text { Large volume and } \\
\text { urban hospitals } \\
\text { more likely to have } \\
\text { significant } \\
\text { reduction in } \\
\text { mortality }\end{array}$ \\
\hline Kohl, $2012^{73}$ & $\begin{array}{l}2,250 \\
1 \text { hospital } \\
2 \text { ICUs one with } \\
\text { and one without } \\
\text { telehealth }\end{array}$ & $\begin{array}{l}\text { - } 24 \text { hours/days all days } \\
\text { - Day time: } 2 \text { critical care } \\
\text { nurses. Evening } 1 \\
\text { intensivist and } 1 \text { nurse } \\
\text { - Rounds every } 1 \text { to } 4 \\
\text { hours as needed }\end{array}$ & $\begin{array}{l}8 \text { to } 20 \text { months } \\
\text { post } \\
\text { implementation }\end{array}$ & $\begin{array}{l}\text { Benefit } \\
\text { No subgroups, } \\
\text { used Apache score } \\
\text { to adjust }\end{array}$ \\
\hline Lilly, $2011^{80}$ & $\begin{array}{l}6,290 \\
2 \text { campuses of an } \\
\text { academic medical } \\
\text { center } \\
7 \text { ICUs }\end{array}$ & $\begin{array}{l}\text { - } 24 \text { hours/day all days } \\
\text { - Intensivist } \\
\text { - Monitoring alarms, } \\
\text { adherence to } \\
\text { guidelines, rounds }\end{array}$ & $\begin{array}{l}2 \text { months after } \\
\text { first site } \\
\text { implementation } \\
\text { to } 1 \text { year } 4 \\
\text { months later } \\
\text { (staggered start } \\
\text { for sites) }\end{array}$ & $\begin{array}{l}\text { Benefit } \\
\text { Compared day and } \\
\text { night admissions, } \\
\text { with more impact } \\
\text { on night } \\
\text { admissions }\end{array}$ \\
\hline Lilly, $2017^{81}$ & $\begin{array}{l}1 \text { academic } \\
\text { medical center on } \\
\text { two campus with } 7 \\
\text { adult ICUs }\end{array}$ & $\begin{array}{l}\text { Off-hours care plan } \\
\text { reviews by intensivist } \\
\text { Electronic early } \\
\text { detection system; } \\
\text { audio-visual link to } \\
\text { patients and nurses }\end{array}$ & $\begin{array}{l}8 \text { years of } \\
\text { followup data } \\
\text { post } \\
\text { implementation }\end{array}$ & $\begin{array}{l}\text { Benefit } \\
\text { Also compared } \\
\text { telehealth with } \\
\text { telehealth } \\
\text { augmented by a } \\
\text { logistic center }\end{array}$ \\
\hline $\begin{array}{l}\text { McCambridge, } \\
2010^{87}\end{array}$ & $\begin{array}{l}1 \text { large }(727 \text { bed }) \\
\text { academic } \\
\text { community } \\
\text { hospital }\end{array}$ & $\begin{array}{l}\text { - } 12 \text { hours (7 PM -7 AM) } \\
\text { - Intensivist and critical } \\
\text { care nurse } \\
\text { - Did admissions and } \\
\text { monitored all patients }\end{array}$ & $\begin{array}{l}10 \text { months after } \\
9 \text {-month } \\
\text { implementation } \\
\text { completed }\end{array}$ & $\begin{array}{l}\text { Benefit } \\
\text { No subgroups }\end{array}$ \\
\hline Morrison, $2010^{91}$ & $\begin{array}{l}2 \text { suburban } \\
\text { community } \\
\text { hospitals; } 650 \text { bed } \\
\text { teaching } 185 \text { bed } \\
\text { not teaching }\end{array}$ & $\begin{array}{l}\text { - Not specified } \\
\text { - Determined by hospital } \\
\text { - Primary provider } \\
\text { controlled level of } \\
\text { involvement }\end{array}$ & $\begin{array}{l}2 \text { 4-month } \\
\text { periods: } 1 \text { year } \\
\text { after baseline } \\
\text { and } 1 \text { year after } \\
\text { implementation }\end{array}$ & $\begin{array}{l}\text { No benefit } \\
\text { Longer followup } \\
\text { and level of } \\
\text { primary provider } \\
\text { involvement did } \\
\text { not change results }\end{array}$ \\
\hline
\end{tabular}




\begin{tabular}{|c|c|c|c|c|}
\hline Study & $\begin{array}{l}\text { Hospital } \\
\text { Number } \\
\text { Characteristics }\end{array}$ & $\begin{array}{l}\text { Remote ICU } \\
\text { Coverage } \\
\text { Staffing }\end{array}$ & $\begin{array}{l}\text { "After" Period } \\
\text { for Outcomes } \\
\text { Measurement }\end{array}$ & $\begin{array}{l}\text { Impact of Remote } \\
\text { ICU } \\
\text { Subgroup } \\
\text { Assessments }\end{array}$ \\
\hline Nassar, $2014^{94}$ & $\begin{array}{l}6,654 \\
7 \text { VA hospitals } \\
8 \text { ICUs } \\
3 \text { academic } \\
\text { medical centers } \\
1 \text { small urban } \\
3 \text { rural hospitals }\end{array}$ & $\begin{array}{l}\text { - } 21 \text { hours a day, all days } \\
\text { - Intensivist and } 2 \text { critical } \\
\text { care nurses } \\
\text { - Alerts, audio and video } \\
\text { monitoring and } \\
\text { communication }\end{array}$ & $\begin{array}{l}6 \text { months after } \\
\text { implementation }\end{array}$ & $\begin{array}{l}\text { Some benefit } \\
\text { Small differences, } \\
\text { not significant in } \\
\text { adjusted analyses } \\
\text { Stratified by risk } \\
\text { and ICU size; no } \\
\text { difference in } \\
\text { results }\end{array}$ \\
\hline Panlaqui, $2017^{227}$ & $\begin{array}{l}525 \\
1 \text { regional center } \\
1 \text { rural facility }\end{array}$ & $\begin{array}{l}\text { - Remote facility initiates } \\
\text { link daily } \\
\text { - Intensivist }\end{array}$ & $\begin{array}{l}1 \text { year after } \\
\text { implementation }\end{array}$ & $\begin{array}{l}\text { No benefit } \\
\text { Adjusted for risk } \\
\text { and age }\end{array}$ \\
\hline Rosenfeld, $2000^{107}$ & $\begin{array}{l}1 \text { community } \\
\text { hospital; academic } \\
\text { affiliated }\end{array}$ & $\begin{array}{l}\text { - } 24 \text { hours } \\
\text { - Intensivists only } \\
\text { (monitoring from home) } \\
\text { - } \text { All monitored }\end{array}$ & $\begin{array}{l}16 \text { weeks during } \\
\text { the study } \\
\text { intervention }\end{array}$ & $\begin{array}{l}\text { Benefit } \\
\text { Benefit attributable } \\
\text { to reduction in } \\
\text { complications. }\end{array}$ \\
\hline Ruesch, $2012^{109}$ & $\begin{array}{l}1 \text { hospital } \\
\text { (Anchorage AK) }\end{array}$ & $\begin{array}{l}\text { - } 24 \text { hour critical care } \\
\text { nurse } \\
\text { - } 10 \text { hours (9 pm to 7am) } \\
\text { intensivist added after } 9 \\
\text { months } \\
\text { - } \text { All monitored }\end{array}$ & $\begin{array}{l}\text { Quarterly for } 1.5 \\
\text { years (6 } \\
\text { quarters) after } \\
\text { implementation }\end{array}$ & $\begin{array}{l}\text { Some benefit } \\
\text { No patient } \\
\text { subgroups }\end{array}$ \\
\hline Sadaka, $2013^{110}$ & $\begin{array}{l}1 \text { community } \\
\text { hospital }\end{array}$ & $\begin{array}{ll}\text { - } & 24 / 7 \\
\text { - } & \text { Intensivists, critical care } \\
& \text { nurses, unit secretaries } \\
\text { - } & \text { Local MD determined } \\
& \text { level of involvement }\end{array}$ & $\begin{array}{l}15 \text { months } \\
\text { starting } \\
\text { immediately with } \\
\text { implementation }\end{array}$ & $\begin{array}{l}\text { Some benefit } \\
\text { AM admission } \\
\text { compared with PM } \\
\text { admission (remote } \\
\text { only): Same as } \\
\text { overall results }\end{array}$ \\
\hline Willmitch, $2012^{131}$ & $\begin{array}{l}5 \text { community } \\
\text { hospitals, mostly } \\
\text { suburban, in one } \\
\text { system }\end{array}$ & $\begin{array}{ll}\text { - } & 24 / 7 \\
\text { - } & \text { Intensivist, critical care } \\
& \text { nurses, unit secretary } \\
\text { - } & \text { Local provider } \\
\text { determined level of } \\
\text { involvement }\end{array}$ & $\begin{array}{l}1,2 \text {, and } 3 \text {, years } \\
\text { after } \\
\text { implementation }\end{array}$ & $\begin{array}{l}\text { Benefit } \\
\text { More benefit seen } \\
\text { in years } 2 \text { and } 3 . \\
\text { Excluding patients } \\
\text { with long stays did } \\
\text { not change } \\
\text { conclusions. }\end{array}$ \\
\hline
\end{tabular}

ICU = Intensive Care Unit; VA = Veterans Affairs

${ }^{a}$ Other studies studied in-hospital and in-ICU mortality rather than 90-day mortality

\section{Inpatient Specialist Consultations}

We identified 32 articles reporting on 31 studies using telehealth to provide specialty consultations for inpatients. Specialty consultations are provided when the input of a specialist is needed for diagnosis, care planning, or treatment and a physician with the specialized knowledge is not available when or where the consultation is needed. Technology for these consultations varies, with some focusing on video interactions that may or may not include the patient, and others consisting of store and forward images, or technology that allows real time collaboration on diagnostic tests or surgery. Generally, specialist consultations are needed to inform decisions, which can be about additional services, such as whether to transfer a patient to a different hospital or whether an in-person followup visit is needed. Consultations may also serve to make or confirm a diagnosis, advise on treatment, or actually provide treatment. 


\section{Inpatient Specialist Consultations: Key Points}

- Clinical outcomes: Mortality or serious morbidity (e.g., cardiac arrest, low birthweight, falls, and disability) improve with telehealth consultations across specialties, but differences are not statistically significant in most studies (low strength of evidence).

- Economic outcomes: Cost savings were realized due to avoided transfers or travel, but telehealth did not save money in all studies (low strength of evidence).

- Intermediate outcomes: The impact of telehealth consultations on intermediate outcomes such as hospital LOS, transfer rate or satisfaction of patients, relatives, or health care providers is also positive, but not convincing with differences that are close to significant and estimates that are less precise (low strength of evidence).

- Adverse effects: Only studies of surgery explicitly examined harms, but study limitations were high based on small sample sizes and high risk of bias (insufficient evidence).

The studies of inpatient specialist consultations cover a wide variety of clinical indications ranging from neonate to geriatric care and from care planning to remote proctoring of surgery. Our ability to draw conclusions is limited to the specialties that have been studied and may or may not be generalizable to other specialties. Table 5 summarizes results across the included studies by clinical specialty and provides an overview of the results. Table 6 provides selected outcomes from each study, and Appendix $\mathrm{H}$ includes the strength of evidence assessment by outcome across these specialties.

Table 5. Inpatient specialty consultations: summary of evidence

\begin{tabular}{|c|c|c|c|c|c|}
\hline $\begin{array}{l}\text { Clinical } \\
\text { Topics } \\
\end{array}$ & $\begin{array}{l}\text { Number } \\
\text { of } \\
\text { Studies }\end{array}$ & Clinical Outcomes & Intermediate Outcomes & Cost & Citations \\
\hline $\begin{array}{l}\text { Neonates } \\
\text { Cardiology }\end{array}$ & 5 & $\begin{array}{l}\sim \text { Death or cardiac } \\
\text { arrest } \\
* \text { Fewer very low } \\
\text { birthweight } \\
\text { deliveries }\end{array}$ & $\begin{array}{l}\text { * LOS, transfers and time to } \\
\text { diagnosis }\end{array}$ & * Net savings & $\begin{array}{l}66,102 \\
103,125 \\
242\end{array}$ \\
\hline $\begin{array}{l}\text { Pediatric } \\
\text { Inpatient }\end{array}$ & $\begin{array}{l}4(5 \\
\text { articles) }\end{array}$ & $\sim$ Hospital mortality & $\begin{array}{l}\text { ? Mixed LOS and satisfaction } \\
\text { * Decrease in } \\
\text { transfers/transport }\end{array}$ & $\begin{array}{l}* \text { Cost saving } \\
\text { and increased } \\
\text { revenue }\end{array}$ & $\begin{array}{l}51,76 \\
84-86\end{array}$ \\
\hline $\begin{array}{l}\text { Pediatric } \\
\text { Sexual Abuse } \\
\text { Assessment }\end{array}$ & 1 & No evidence & $\begin{array}{l}\text { * Improved quality of } \\
\text { assessment }\end{array}$ & No evidence & 88 \\
\hline Geriatrics & 4 & $*$ Fewer falls & $\begin{array}{l}* \text { Decrease time to consult; } \\
\text { increased capacity } \\
* \text { High patient satisfaction } \\
\sim \text { Hospitalizations }\end{array}$ & $\begin{array}{l}\text { * Cost saving } \\
\text { due to avoided } \\
\text { travel }\end{array}$ & $\begin{array}{l}63,221 \\
224,249\end{array}$ \\
\hline Neurology & 3 & ? Mortality & $\begin{array}{l}\text { ? Health service utilization and } \\
\text { transfers }\end{array}$ & No evidence & $\begin{array}{l}165,196, \\
243\end{array}$ \\
\hline $\begin{array}{l}\text { Post stroke } \\
\text { care }\end{array}$ & 1 & $\begin{array}{l}* \text { Lower odds of } \\
\text { poor outcome }\end{array}$ & No evidence & No evidence & 138 \\
\hline Psychiatry & 2 & No evidence & $\begin{array}{l}* \text { Fewer transfers } \\
* \text { Positive rating and willing to } \\
\text { use for next visit }\end{array}$ & $\begin{array}{l}* \text { Lower cost if } \\
\geq 30 \text { cases per } \\
\text { year }\end{array}$ & 164,214 \\
\hline
\end{tabular}




\begin{tabular}{|c|c|c|c|c|c|}
\hline $\begin{array}{l}\text { Clinical } \\
\text { Topics }\end{array}$ & $\begin{array}{l}\text { Number } \\
\text { of } \\
\text { Studies }\end{array}$ & Clinical Outcomes & Intermediate Outcomes & Cost & Citations \\
\hline $\begin{array}{l}\text { Image/Test } \\
\text { Review }\end{array}$ & 5 & $\begin{array}{l}\text { Success rate for } \\
\text { free flap surgery }\end{array}$ & $\begin{array}{l}\text { * More patients sent to day } \\
\text { surgery } \\
\text { * Shorter time to assessment or } \\
\text { response } \\
\sim \text { Transfers, in--person visits } \\
\text { and admissions }\end{array}$ & ? Savings & $\begin{array}{l}26,115 \\
209,237 \\
244\end{array}$ \\
\hline $\begin{array}{l}\text { Mentored } \\
\text { Surgery }\end{array}$ & 3 & $\begin{array}{l}\text { * No complications } \\
\text { or harms }\end{array}$ & $\begin{array}{l}\text { ? Surgery time } \\
* \text { Hospital LOS }\end{array}$ & No evidence & $\begin{array}{l}39,116 \\
149\end{array}$ \\
\hline Critical Care & 2 & * Mortality & $\begin{array}{l}* \text { PCU LOS shorter } \\
<\text { Hospital LOS longer } \\
* \text { Response time }\end{array}$ & $<$ Higher cost & 29,105 \\
\hline $\begin{array}{l}\text { Multiple } \\
\text { Specialties }\end{array}$ & 1 & $\begin{array}{l}\sim \text { No difference in } \\
\text { mortality }\end{array}$ & No evidence & No evidence & 252 \\
\hline
\end{tabular}

LOS = length of stay; PCU = progressive care unit

Key: * superior (telehealth benefit), no difference (no significant difference), < inferior (comparison better than telehealth), ? inconclusive (inconsistent results or insufficient)

\section{Inpatient Specialist Consultations: Detailed Results}

Table 6 includes key results for clinical outcomes (including harms), intermediate outcomes, and costs from the 31 studies about inpatient specialty consultations. Seventeen were conducted in the United States, and the others were conducted in 12 different countries (2 in Australia, 2 in the United Kingdom, and 1 each in Brazil, Finland, Germany, Hong Kong, Israel, Italy, Northern Ireland, Taiwan, Japan, and Spain). Studies of inpatient consultations were predominately cohort studies, including 10 retrospective and 15 prospective cohort designs. There were also three before-after studies and three randomized trials that studied inpatient consultations. Most of the cohort studies included multiple sites with the largest prospective study including 3,060 patients in five intervention and five matched comparison hospitals. ${ }^{138}$ The largest study, a retrospective analysis using data from a large healthcare system included over 16,000 patients. ${ }^{29}$

\section{Inpatient Specialist Consultations: Effectiveness in Improving Clinical and Economic Outcomes}

\section{Mortality}

Twelve studies reported in 13 articles ${ }^{29,76,84-86,102,125,138,165,196,242,243,252}$ about inpatient consultations evaluated mortality outcomes.

Ten studies reported mortality to be the same or not statistically significantly lower. For example, declines in mortality were not significant in one study of telehealth cardiology for neonates implemented in nine hospitals, ${ }^{125}$ in studies of pediatric inpatient consults, ${ }^{7684-86}$ or in a study of a hospital wide multispecialty consult program. ${ }^{252}$ In one pilot study mortality did not differ in a group managed with telehealth compared with a group in which all patients with suspected intracranial bleeds were transferred to a neuro trauma center for in-person neurological care. ${ }^{243}$ Two studies about neurology inpatient teleconsultations reported lower mortality with telehealth and one study of specialized post stroke care reported some benefit from telehealth when the outcome was a composite including death, institutional care or disability: 
- In a comparison of two hospitals, one that used real-time video to secure early teleneurological consults and one that did not, the decline in inpatient mortality was statistically significant (from $10.2 \%$ to $4.9 \%, \mathrm{p}=0.013$ ), but the 3-month mortality rate was not significantly different $(11.7 \% \text { to } 8.6 \%, \mathrm{p}=0.558)^{196}$

- In a study of over 2,000 patients hospitalized with minor head injuries in hospitals without neurosurgery, the adjusted odds of mortality for patients treated in the centers without telehealth compared with those where telehealth was available was not significant (adjusted odds ratio [AOR] 1.25; 95\% CI 0.83 to 1.91), but the odds of death were greater without telehealth when the analysis was limited to patients over 70 years old (AOR 1.14; 95\% CI 1.04 to 1.82$)^{165}$

- The odds of death or institutional care were not significantly different when comparing five hospitals participating in the Telemedical Project for Integrative Stroke Care with five matched control hospitals. When the outcome was redefined as death, institutional care or severe disability, the odds of poor outcome at both 12 months and 3 months were significantly lower in hospitals with telestroke. ${ }^{138}$

\section{Inpatient Specialist Consultations: Other Clinical and Economic Outcomes}

Six studies ${ }^{39,116,149,237,242,249}$ analyzed clinical outcomes other than mortality and reported a significant decline in the rate of very low birthweight deliveries in hospitals without neonatal ICUs after the establishment of telehealth consultations and rounds, ${ }^{242}$ no significant difference in the average number of falls per month in one study of geriatric teleconsultations, ${ }^{249}$ no difference in surgical flap success rates when comparing telehealth and in-person post-operative monitoring, ${ }^{237}$ and no difference in complications in three studies of telehealth mentored surgeries. $^{39,116,149}$

Seven studies reported some assessment of economic outcomes. Studies that found or estimated an economic benefit included an evaluation of remote cardiology assessments for neonates that calculated a net savings of $\$ 13,900$ per infant; ${ }^{103}$ a study of a referral network to a children's hospital that estimated telehealth almost doubled hospital revenue and billing; ${ }^{.0}$ and a study of pediatric intensive care consultations provided to a rural hospital that estimated annual savings of \$300,000 per year for patients and \$279,000 in additional revenue for the rural hospital for patients who were treated with telehealth consultations and not transferred to another location. ${ }^{85}$ An evaluation of a telegeriatrics program calculated lower annual costs with telehealth consultations (73,078 vs. 98,909 Australian dollars), but that savings were only realized when roundtrip travel for an in-person consultation would have exceeded 125 kilometers. ${ }^{221}$ Similarly costs for telehealth consults for inpatient psychiatric care were lower than in-person consultations if a certain number of cases (30 in this study) could be addressed with telehealth per year. ${ }^{164}$ Two studies reported no benefit from telehealth, including one that found no evidence of cost saving for the hospital to offset the capital investment required for a teleconsult service for plastic surgery and burns, ${ }^{209}$ and a study that reported higher mean direct costs (\$12,301without telehealth vs. \$13, 180 with telehealth). ${ }^{29}$

\section{Inpatient Specialist Consultations: Effectiveness in Improving Intermediate Outcomes}

Most of the outcomes reported in the evaluation of inpatient specialty consultations were intermediate outcomes. Twenty-seven studies reported intermediate outcomes such as LOS, rates of transfers to other hospitals, satisfaction, receipt of specific tests or services, and quality/process indicators. 
- Two of the four studies analyzing cardiology consultations and echocardiogram via telehealth reported reductions in LOS, ${ }^{102,125}$ while one study was not large enough to determine if the difference was statistically significant. ${ }^{103}$ One study reported lower, but not significantly different inappropriate transfer rates ${ }^{66}$ while in another study transfers to tertiary care were significantly lower. ${ }^{125}$

- The studies of pediatric inpatient consultations reported no significant difference in LOS, although one study of a single hospital reduced its transfers from 100 to 86 percent $(p=0.04)$ after establishing a pediatric telehealth consult system with a tertiary children's hospital. ${ }^{76}$ Satisfaction with pediatric telehealth consults was generally good, though the mean rating by parents were lower than those of staff. ${ }^{84-86}$ A study evaluating a program designed to increase use of a regional children's hospital increased transfers and LOS. ${ }^{51}$ A study comparing five hospitals with remote access to experts for pediatric sexual abuse evaluations with three hospitals that did not have telehealth found the quality of the assessments was higher with telehealth. ${ }^{88}$

- Geriatric telehealth consultation improved patient performance of self-management, reduced wait time, and increased patient satisfaction. ${ }^{249}$ A video geriatric rounds program provided consultations that were similar in length to in-person visits ${ }^{221}$ while another geriatrics telehealth program decreased the initial consultation time but made no different in overall time spent on the case and did not significantly change triage decisions. ${ }^{224}$

- A hospital with inpatient telehealth neurology consultations had shorter LOS than a comparison hospital but no difference in the number of readmissions or primary care followup visits. ${ }^{196}$ A telehealth consult service allowed some patients with suspected intracranial bleeds to be treated at hospitals without neurosurgical services, reducing the number of transfers but LOS was not different. ${ }^{243}$

- When telehealth was used for consultations, transfers to other hospitals were lower ${ }^{214}$ and the majority of patients, families, and providers who used video telehealth for psychiatric inpatient care planning preferred to have their next conference via video. ${ }^{164}$

- Consults involving image or test data transmission resulted in faster assessment ${ }^{26}$ and response, ${ }^{237}$ though one study reported faster handling by the consultant but no difference in the time it took for the requesting physician to receive a response. ${ }^{115}$

- One randomized trial reported remotely mentored surgeries were shorter than unmentored surgeries (mean of 139 minutes compared to 200 minutes) and that patients had shorter LOS. ${ }^{149}$ Another study that randomized endoscopic surgery to be proctored in-person or remotely found teleproctored surgeries took approximately 4 minutes longer on average; though statistically significant, this difference is unlikely to be clinically meaningful. ${ }^{39} \mathrm{~A}$ third nonrandomized, prospective study reported no difference in the duration of procedures. ${ }^{116}$

\section{Inpatient Specialist Consultations: Harms, Adverse Events, or Negative Unintended Consequences}

The only studies that reported harms were the three of remoted monitored surgery. These studies reported low or no complications, which were not greater when telehealth was compared with in-person supervision. In the study of teleproctored endoscopic sinus surgery, researchers looked for postoperative negative outcomes including cerebral spinal fluid leaks, orbital hematoma, visual disturbance, and need for blood transfusion. These did not occur in any cases in either the teleproctored group or the control group. They also compared the blood loss per 
case and found no significant difference between the groups. ${ }^{39}$ Similarly a study of telementoring for laparoscopic bariatric surgery reported no complications in the telementored group, ${ }^{149}$ and a study of telementoring for robotic surgery reported no intraoperative complications in the telementoring group and one case with complications in the in-person group. ${ }^{116}$

\section{Key Characteristics of Inpatient Specialist Consultation Studies and Correlation With Outcomes}

The studies of inpatient consultations included several specialties; however, the function of the telehealth consultation is essentially the same - to expand access to needed expertise. This expertise is used to assist in the diagnosis, treatment and management of patients. The details of the diagnosis and management decision ranged from deciding whether to transfer a critically ill child to another hospital to treatment plans for stroke patients, homecare clients, or hospitalized psychiatric patients to remote proctored surgery. While some studies mention whether the patient is involved or present in the consultation, others do not. Studies also do not report details of the consulting interaction well. The roles and qualifications of the providers involved are often mentioned, but none of the studies provided extensive details. For example, they do not describe how many different specialists are involved, the nature of the relationships among the different organizations, or the payment model for the specialist consultations.

Overall, inpatient telehealth consultations are not well described, making it problematic to determine how characteristics of the intervention or environment relate to effectiveness. The evidence does not provide insight into how clinical and financial relationships among the organizations and/or the providers should be organized or what may or may not make these not just effective, but also sustainable and replicable. 
Table 6. Inpatient specialty consultations: selected outcomes

\begin{tabular}{|c|c|c|c|c|c|}
\hline Clinical Topic & $\begin{array}{l}\text { Author, Year } \\
\text { Location } \\
\text { Number of Sites } \\
\end{array}$ & $\begin{array}{l}\text { Study Design } \\
\text { Risk of Bias } \\
\text { Comparison, } \mathbf{n} \\
\end{array}$ & $\begin{array}{l}\text { Clinical Outcomes } \\
\text { Harms }\end{array}$ & $\begin{array}{l}\text { Intermediate } \\
\text { Outcomes }\end{array}$ & Economic Outcomes \\
\hline $\begin{array}{l}\text { Neonate } \\
\text { Cardiology }\end{array}$ & $\begin{array}{l}\text { Huang, 20086 } \\
\text { CA, United States } \\
1 \text { community hospital, } 1 \\
\text { university children's } \\
\text { hospital }\end{array}$ & $\begin{array}{l}\text { Before-after } \\
\text { Moderate } \\
\text { A: Before telehealth, } n=280 \\
\text { B: After telehealth, } n=385\end{array}$ & NR & $\begin{array}{l}\text { Echocardiogram upon } \\
\text { admission: } \\
\text { A: } \mathbf{2 7 \%} \\
\text { B: } \mathbf{4 0} \%, \mathbf{p}<\mathbf{0 . 0 0 1} \\
\text { Inappropriate transfers } \\
\text { A: } 7 \\
\text { B: } 2, p=0.06\end{array}$ & NR \\
\hline $\begin{array}{l}\text { Neonate } \\
\text { Cardiology }\end{array}$ & $\begin{array}{l}\text { Kim, } 2013^{242} \\
\text { AR, United States } \\
\\
6 \text { hospitals } \\
3 \text { with, } 3 \text { without } \\
\text { telehealth }\end{array}$ & $\begin{array}{l}\text { Prospective cohort } \\
\text { High } \\
\text { A: No telehealth, site } \\
\text { without NICU, } n=77 \\
\text { B: Telehealth, site without } \\
\text { NICU, } n=181\end{array}$ & $\begin{array}{l}\text { Very low birthweight } \\
\text { A: } \mathbf{1 3 \%} \\
\text { B: } \mathbf{7 \% ,} \mathbf{p}<0.001 \\
\text { Mortality } \\
\text { A: } 13 \% \\
\text { B: } 7 \% \\
\text { Comparison hospitals: no } \\
\text { change; } \\
\text { Statewide mortality } \\
\text { decreased during study } \\
\text { period }\end{array}$ & $\mathrm{NR}$ & NR \\
\hline $\begin{array}{l}\text { Neonate } \\
\text { Cardiology }\end{array}$ & $\begin{array}{l}\text { Rendina, } 1998^{102} \\
\text { NC, United States } \\
2 \text { hospitals }\end{array}$ & $\begin{array}{l}\text { Retrospective cohort } \\
\text { Low } \\
\text { A: Site without telehealth, } \\
n=137 \\
\text { B: Site with telehealth, } \\
n=177\end{array}$ & NR & $\begin{array}{l}\text { Mean LOS in NICU, in } \\
\text { days (\%) } \\
\text { A vs. B: }-12.5 \\
\text { (-17\%), p<0.05 }\end{array}$ & NR \\
\hline $\begin{array}{l}\text { Neonate } \\
\text { Cardiology }\end{array}$ & $\begin{array}{l}\text { Rendina, } 1997^{103} \\
\text { NC, United States } \\
1 \text { university hospital } \\
1 \text { regional medical } \\
\text { center }\end{array}$ & $\begin{array}{l}\text { Economic evaluation } \\
\text { High } \\
\text { A: Usual care, } n=38 \\
\text { B: Telehealth, } n=48\end{array}$ & $\begin{array}{l}\text { Mortality } \\
\text { A: } 1 \\
\text { B: } 1\end{array}$ & $\begin{array}{l}\text { Hospital LOS, in days } \\
\text { A: } 41.2 \\
\text { B: } 35.2 \text {, pooled } \\
\text { variance t-test } p=0.23 \\
\text { Electrocardiographic } \\
\text { interpretation reporting } \\
\text { time } \\
\text { A: } 24 \text { hours } \\
\text { B: } 20 \text { minutes }\end{array}$ & $\begin{array}{l}\text { Net savings, in USD } \\
\$ 13,900 \text { per infant }\end{array}$ \\
\hline
\end{tabular}




\begin{tabular}{|c|c|c|c|c|c|}
\hline Clinical Topic & $\begin{array}{l}\text { Author, Year } \\
\text { Location } \\
\text { Number of Sites }\end{array}$ & $\begin{array}{l}\text { Study Design } \\
\text { Risk of Bias } \\
\text { Comparison, } n\end{array}$ & $\begin{array}{l}\text { Clinical Outcomes } \\
\text { Harms }\end{array}$ & $\begin{array}{l}\text { Intermediate } \\
\text { Outcomes }\end{array}$ & Economic Outcomes \\
\hline $\begin{array}{l}\text { Neonate } \\
\text { Cardiology }\end{array}$ & $\begin{array}{l}\text { Webb, 2013'25 } \\
\text { Multiple locations, } \\
\text { United States } \\
9 \text { sites }\end{array}$ & $\begin{array}{l}\text { Prospective cohort } \\
\text { Moderate } \\
\text { A: Site without telehealth, } \\
n=337 \\
\text { B: Site with telehealth, } \\
n=337\end{array}$ & $\begin{array}{l}\text { Mortality } \\
\text { AOR: } 0.922, \mathrm{NS} \\
\text { Cardiac arrest } \\
\text { AOR: } 0.527, \mathrm{NS}\end{array}$ & $\begin{array}{l}\text { Mean LOS, in days } \\
\text { Total } \\
\text { A: } 1.6 \\
\text { B: } 0.72, p=0.027 \\
\text { ICU } \\
\text { A: } 1.6 \\
\text { B: } 0.65, p=0.027 \\
\text { Mean time to diagnosis, } \\
\text { in minutes } \\
\text { A: } 147 \\
\text { B: } 100, p<0.001 \\
\text { Transport to tertiary } \\
\text { care } \\
\text { A: } 10 \% \\
\text { B: } \mathbf{4} \%, p<0.01\end{array}$ & NR \\
\hline Pediatric Inpatient & $\begin{array}{l}\text { Dharmar, } 2013^{50} \\
\text { CA, USA } \\
1 \text { tertiary children's } \\
\text { hospital } \\
16 \text { remote hospitals }\end{array}$ & $\begin{array}{l}\text { Retrospective cohort } \\
\text { High } \\
\text { A: No telehealth, } n=515 \\
\text { B: Telehealth, } n=1514\end{array}$ & NR & $\begin{array}{l}\text { Mean number of } \\
\text { patients transferred per } \\
\text { year } \\
\text { A: } 143 \\
\text { B: } 285 \\
\text { Mean LOS of } \\
\text { transferred children } \pm \\
\text { SD, in days } \\
\text { A: } 7.7 \pm 14.2 \\
\text { B: } \mathbf{9 . 2} \pm \mathbf{1 5 . 4}, \mathbf{p}<0.05\end{array}$ & $\begin{array}{l}\text { Mean yearly hospital } \\
\text { revenue, in USD } \\
\text { A: } \$ 2.4 \text { million } \\
\text { B: } \$ 4.0 \text { million } \\
\text { Mean yearly } \\
\text { professional billing } \\
\text { revenue, in USD } \\
\text { A: } \$ 313,977 \\
\text { B: } \$ 688,443\end{array}$ \\
\hline
\end{tabular}




\begin{tabular}{|c|c|c|c|c|c|}
\hline Clinical Topic & $\begin{array}{l}\text { Author, Year } \\
\text { Location } \\
\text { Number of Sites }\end{array}$ & $\begin{array}{l}\text { Study Design } \\
\text { Risk of Bias } \\
\text { Comparison, } n\end{array}$ & $\begin{array}{l}\text { Clinical Outcomes } \\
\text { Harms }\end{array}$ & $\begin{array}{l}\text { Intermediate } \\
\text { Outcomes }\end{array}$ & Economic Outcomes \\
\hline Pediatric Inpatient & $\begin{array}{l}\text { Labarbera, } 2013^{76} \\
\text { OR, United States } \\
1 \text { community hospital; } \\
\text { consult from tertiary } \\
\text { children's hospital }\end{array}$ & $\begin{array}{l}\text { Before-after } \\
\text { Moderate } \\
\text { A: Before telehealth, } n=41 \\
\mathrm{~B}_{1} \text { : After telehealth, } \mathrm{n}=56 \\
\mathrm{~B}_{2} \text { : After telehealth and } \\
\text { hospitalist program at } \\
\text { community hospital, } \mathrm{n}=56\end{array}$ & $\begin{array}{l}\text { Mortality } \\
\text { A: } 3 \% \\
B_{1}: 1.8 \% \\
B_{2}: 3.6 \%, \text { NS }\end{array}$ & 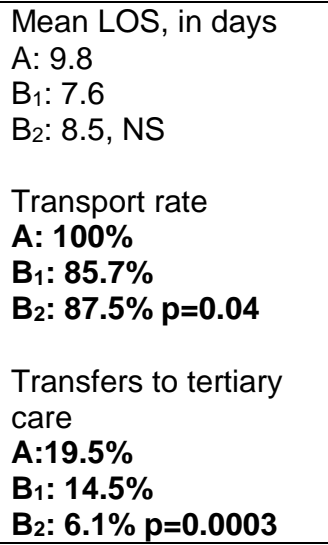 & NR \\
\hline Pediatric Inpatient & $\begin{array}{l}\text { Marcin, 2004a; } 84 \\
\text { Marcin, 2004b } \\
\text { CA, United States } \\
1 \text { hospital }\end{array}$ & $\begin{array}{l}\text { Retrospective cohort, } \\
\text { economic evaluation } \\
\text { Moderate } \\
\mathrm{A}_{1} \text { : No telehealth, } \mathrm{n}=116 \\
\mathrm{~A}_{2}: \text { Patients transferred } \\
\text { from other hospitals, no } \\
\text { telehealth, } \mathrm{n}=86 \\
\mathrm{~B}_{1} \text { : Patients receiving } \\
\text { telehealth, } \mathrm{n}=47 \\
\mathrm{~B}_{2}: \text { All ICU patients during } \\
\text { telehealth period, } \mathrm{n}=180\end{array}$ & $\begin{array}{l}\text { Mortality } \\
\mathrm{A}_{1}: 2.6 \% \\
\mathrm{~A}_{2}: 3.5 \% \\
\mathrm{~B}_{1}: 2.1 \% \\
\mathrm{~B}_{2}: 1.6 \%\end{array}$ & $\begin{array}{l}\text { Mean satisfaction with } \\
\text { telehealth (5-point } \\
\text { scale) } \\
\text { Referring nurse: } \\
4.53 \\
\text { Referring MD: } \\
4.56 \\
\text { Parent or guardian: } \\
4.05\end{array}$ & $\begin{array}{l}\text { Estimated savings, in } \\
\text { USD } \\
\text { Annual cost: } \$ 172,000 \\
\text { Transport: } \\
\$ 300,000 \\
\text { For rural hospital } \\
\text { Estimated revenue } \\
\text { available: } \$ 186,000 \\
\text { Estimated revenue } \\
\text { available due to no } \\
\text { transport: } \\
\$ 279,000\end{array}$ \\
\hline Pediatric Inpatient & $\begin{array}{l}\text { Marcin, 2004c } \\
\text { CA, United States } \\
1 \text { hospital } \\
224\end{array}$ & $\begin{array}{l}\text { Retrospective cohort } \\
\text { Moderate } \\
A_{1}: \text { No telehealth, historical } \\
\text { control } n=127 \\
A_{2} \text { : No telehealth, } n=80 \\
B: \text { Telehealth, } n=17 \\
\text { C: Combination of } A_{2} \text { and } \\
B, n=97\end{array}$ & $\begin{array}{l}\text { Observed/expected } \\
\text { mortality, OR }(95 \% \mathrm{Cl}) \\
\mathrm{A}_{1}: 0.95(0.26 \text { to } 3.48) \\
\mathrm{A}_{2}: 0.44(0.07 \text { to } 1.96) \\
\mathrm{B}: \text { Reference } \\
\mathrm{C}: 0.73(0.06 \text { to } 1.44), \\
\text { NS }\end{array}$ & $\begin{array}{l}\text { ICU LOS, in days } \\
\mathrm{A}_{1}: 3.5 \\
\mathrm{~A}_{2}: 3.4 \\
\mathrm{~B}: 5.9 \\
\mathrm{C}: 3.8, \mathrm{NS} \\
\text { Mean parent } \\
\text { satisfaction with } \\
\text { telehealth: } 3.8 \text { of } 5\end{array}$ & NR \\
\hline
\end{tabular}




\begin{tabular}{|c|c|c|c|c|c|}
\hline Clinical Topic & $\begin{array}{l}\text { Author, Year } \\
\text { Location } \\
\text { Number of Sites }\end{array}$ & $\begin{array}{l}\text { Study Design } \\
\text { Risk of Bias } \\
\text { Comparison, } n\end{array}$ & $\begin{array}{l}\text { Clinical Outcomes } \\
\text { Harms }\end{array}$ & $\begin{array}{l}\text { Intermediate } \\
\text { Outcomes }\end{array}$ & Economic Outcomes \\
\hline $\begin{array}{l}\text { Pediatric Sexual } \\
\text { Assault } \\
\text { Assessment }\end{array}$ & $\begin{array}{l}\text { Miyamoto, } 2014^{88} \\
\text { CA, United States } \\
5 \text { rural telemedicine } \\
\text { hospitals } \\
3 \text { comparison hospitals }\end{array}$ & $\begin{array}{l}\text { Retrospective cohort } \\
\text { Moderate } \\
\text { A: Site without } \\
\text { telemedicine, } n=82 \\
\text { B: Site with telemedicine, } \\
n=101\end{array}$ & NR & $\begin{array}{l}\text { Office of emergency } \\
\text { services child abuse } \\
\text { examination quality } \\
\text { (score range: } 0 \text { to } 5 \text {; } \\
5=\text { good) } \\
\text { Overall assessment } \\
\text { A: } 3.24 \\
\text { B: } 3.88, \text { NS } \\
\text { Total quality score (sum } \\
\text { of } 7 \text { domains) } \\
\text { A: } 29.21 \\
\text { B: } \mathbf{3 1 . 2 0}, p<0.05\end{array}$ & NR \\
\hline Geriatrics & $\begin{array}{l}\text { Chan, et al., } 2001^{249} \\
\text { Hong Kong } \\
1 \text { Nursing home }\end{array}$ & $\begin{array}{l}\text { Before-after } \\
\text { High } \\
\text { A: Before telehealth, } n=N R \\
\text { B: After telehealth, } n=N R \\
\text { Total } n=198\end{array}$ & $\begin{array}{l}\text { Mean monthly falls: } \\
\text { A: } 9.8 \\
\text { B: } 6.8\end{array}$ & $\begin{array}{l}\text { Failed inhaler technique } \\
\text { A: } 93 \% \\
\text { B: } 50 \% \\
\text { Waiting time for consult, } \\
\text { in weeks } \\
\text { A: } 4 \text { to } 13 \\
\text { B: within } 2 \\
\text { Patient satisfaction: } \\
96 \% \text { favorable }\end{array}$ & $\begin{array}{l}11 \% \text { of patients needed } \\
\text { onsite visit at a cost to } \\
\text { nursing home }\end{array}$ \\
\hline Geriatrics & $\begin{array}{l}\text { Grabowski, } 2014^{63} \\
\text { MA, United States } \\
11 \text { nursing homes }\end{array}$ & $\begin{array}{l}\text { RCT } \\
\text { High } \\
\text { Nontelehealth nursing } \\
\text { homes, } n=5 \\
A_{1} \text { : Pre-intervention } \\
A_{2} \text { : Post-intervention } \\
\text { Telehealth nursing homes, } \\
n=6 \\
B_{1}: \text { Pre-intervention } \\
B_{2}: \text { Post-intervention, } n=6\end{array}$ & NR & $\begin{array}{l}\text { Hospitalization per } \\
1,000 \text { nursing home } \\
\text { resident days } \\
A_{1} \text { vs. } A_{2}: 3.8 \text { vs } 3.6, \\
5.3 \% \text { reduction } \\
B_{1} \text { vs. } B_{2}: 3.5 \text { vs. } 3.2, \\
9.7 \% \text { reduction } \\
\\
\text { More engaged } \\
\text { telehealth facilities: } \\
11.3 \% \text { reduction } \\
\text { Less engaged } \\
\text { telehealth facilities: } \\
5.2 \% \text { reduction }\end{array}$ & NR \\
\hline
\end{tabular}




\begin{tabular}{|c|c|c|c|c|c|}
\hline Clinical Topic & $\begin{array}{l}\text { Author, Year } \\
\text { Location } \\
\text { Number of Sites }\end{array}$ & $\begin{array}{l}\text { Study Design } \\
\text { Risk of Bias } \\
\text { Comparison, } n\end{array}$ & $\begin{array}{l}\text { Clinical Outcomes } \\
\text { Harms }\end{array}$ & $\begin{array}{l}\text { Intermediate } \\
\text { Outcomes }\end{array}$ & Economic Outcomes \\
\hline Geriatrics & $\begin{array}{l}\text { Gray, } 2009^{221} \\
\text { Australia } \\
1 \text { hospital } \\
\text { NR }\end{array}$ & $\begin{array}{l}\text { Economic evaluation } \\
\text { Moderate } \\
\text { A: Without telehealth, } n=N R \\
\text { B: With telehealth, } n=12\end{array}$ & $\mathrm{NR}$ & $\begin{array}{l}\text { Mean consultation time, } \\
\text { in minutes }(95 \% \mathrm{CI}) \\
\text { A: } 13.7(11.5 \text { to } 15.9) \\
\text { B: } 15.3(13.6 \text { to } 16.09) \\
\text { Mean consultation time } \\
\text { for new patients ( } 95 \% \\
\text { CI), in minutes } \\
\text { A: } 19.0(15.2 \text { to } 22.8) \\
\text { B: } 19.7(17.0 \text { to } 22.4)\end{array}$ & $\begin{array}{l}\text { Costs per year, in AUD } \\
\text { A: } \$ 90,909 \\
\text { B: } \$ 73,078 \\
\text { In the base-case, cost } \\
\text { savings became } \\
\text { effective when roundtrip } \\
\text { travel is } \geq 125 \mathrm{~km} \\
\text { between locations. }\end{array}$ \\
\hline Geriatrics & $\begin{array}{l}\text { Martin-Khan, } 2016^{224} \\
\text { Australia } \\
3 \text { hospitals } \\
\text { Geriatrician location NR }\end{array}$ & $\begin{array}{l}\mathrm{RCT} \\
\text { Moderate } \\
\text { A: Usual care, } n=81 \\
\text { B: Telehealth, } n=85\end{array}$ & NR & $\begin{array}{l}\text { Mean consultation time } \\
\text { (range) } \pm \text { SD, in } \\
\text { minutes } \\
\text { A: } \mathbf{2 5 . 9 1} \text { (4 to } 77) \pm 9.38 \\
\text { B: } \mathbf{9 . 8 9}(4 \text { to } 35) \pm 5.83 \text {, } \\
\text { p<0.005 } \\
\text { Overall time: no } \\
\text { difference } \\
\text { Triage decisions not } \\
\text { significantly different. }\end{array}$ & NR \\
\hline Neurology & $\begin{array}{l}\text { Craig, 2004196 } \\
\text { North Ireland, UK } \\
2 \text { hospitals }\end{array}$ & $\begin{array}{l}\text { Prospective cohort } \\
\text { Moderate } \\
\text { A: Without telehealth, } \\
n=128 \\
\text { B: With telehealth, } n=164 \\
\text { (not used for all patients) }\end{array}$ & $\begin{array}{l}\text { Inpatient mortality } \\
\text { A: } 10.2 \% \\
\text { B: } 4.9 \% \text { p }=0.013 \\
\text { 3-month mortality } \\
\text { A: } 11.7 \% \\
\text { B: } 8.6 \%, \text { NS }\end{array}$ & $\begin{array}{l}\text { Mean LOS, in days } \\
\text { A: } \mathbf{1 1 . 6} \\
\text { B: } \mathbf{8 . 1}, \mathrm{p}=\mathbf{0 . 0 1 6} \\
\text { HR: } \mathbf{1 . 1 3}, \mathrm{p}=\mathbf{0 . 0 4 5} \\
\\
\text { Hospital readmissions } \\
\text { A: } 16.8 \% \\
\text { B: } 15.0 \%, \mathrm{NS} \\
\text { Mean number of } \\
\text { primary care visits at } 3- \\
\text { months } \\
\text { A: } 2.49 \\
\text { B: } 2.14, \mathrm{NS}\end{array}$ & NR \\
\hline
\end{tabular}




\begin{tabular}{|c|c|c|c|c|c|}
\hline Clinical Topic & $\begin{array}{l}\text { Author, Year } \\
\text { Location } \\
\text { Number of Sites }\end{array}$ & $\begin{array}{l}\text { Study Design } \\
\text { Risk of Bias } \\
\text { Comparison, } n\end{array}$ & $\begin{array}{l}\text { Clinical Outcomes } \\
\text { Harms }\end{array}$ & $\begin{array}{l}\text { Intermediate } \\
\text { Outcomes }\end{array}$ & Economic Outcomes \\
\hline Neurology & $\begin{array}{l}\text { Klein, } 2010^{243} \\
\text { Israel } \\
3 \text { hospitals }\end{array}$ & 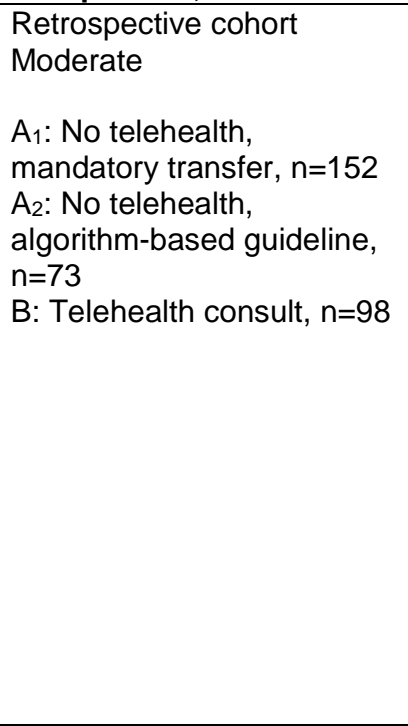 & $\begin{array}{l}\text { Mortality, } \mathrm{n}(\%) \\
\mathrm{A}_{1}: 0(0.0 \%) \\
\mathrm{A}_{2}: 1(1.4 \%) \\
\mathrm{B}: 1(1.0 \%), \mathrm{NS} \\
\text { Need neurosurgery, } \mathrm{n} \\
(\%) \\
\mathrm{A}_{1}: 17(11.2 \%) \\
\mathrm{A}_{2}: 9(12.3 \%) \\
\mathrm{B}: 9(9.2 \%), \mathrm{NS}\end{array}$ & $\begin{array}{l}\text { Transferred, } \mathrm{n}(\%) \\
\mathrm{A}_{1}: 152(100.0 \%) \\
\mathrm{A}_{2}: 54(74.0 \%) \\
\mathrm{B}: 40(40.9 \%) \\
\text { Delayed transfer, } \mathrm{n}(\%) \\
\mathrm{A}_{1}: \mathrm{NA} \\
\mathrm{A}_{2}: 1(1.3 \%) \\
\mathrm{B}: 2(2.0 \%), \mathrm{NS} \\
\text { Length of stay } \\
\mathrm{A}_{1}: 4.19 \\
\mathrm{~A}_{2}: 3.92 \\
\text { B: } 4.48, \mathrm{NS} \\
\text { Need neurological } \\
\text { rehabilitation, } \mathrm{n}(\%) \\
\mathrm{A}_{1}: \mathbf{4}(\mathbf{2 . 6 \% )} \\
\mathrm{A}_{2}: \mathbf{1 5}(\mathbf{2 0 . 8} \%) \\
\mathrm{B}: \mathbf{8}(\mathbf{8 . 2 \% )}, \mathrm{p}<0.001\end{array}$ & NR \\
\hline Neurology & $\begin{array}{l}\text { Migliaretti, } 2013^{165} \\
\text { Italy } \\
\text { Number of hospitals NR }\end{array}$ & $\begin{array}{l}\text { Prospective cohort } \\
\text { High } \\
\text { A: No telehealth, } n=1895 \\
\text { B: Telehealth, } n=462\end{array}$ & $\begin{array}{l}\text { Mortality risk without } \\
\text { telehealth, } \text { AOR }^{\mathrm{b}}(95 \% \\
\mathrm{Cl}) \\
\text { All patients: } 1.25 \text { (0.83 to } \\
\text { 1.91) } \\
\text { People over } 70: 1.14 \\
\text { (1.04 to 1.82) }\end{array}$ & $\mathrm{NR}$ & NR \\
\hline
\end{tabular}




\begin{tabular}{|c|c|c|c|c|c|}
\hline Clinical Topic & $\begin{array}{l}\text { Author, Year } \\
\text { Location } \\
\text { Number of Sites }\end{array}$ & $\begin{array}{l}\text { Study Design } \\
\text { Risk of Bias } \\
\text { Comparison, n }\end{array}$ & $\begin{array}{l}\text { Clinical Outcomes } \\
\text { Harms }\end{array}$ & $\begin{array}{l}\text { Intermediate } \\
\text { Outcomes }\end{array}$ & Economic Outcomes \\
\hline Post Stroke Care & $\begin{array}{l}\text { Audebert, } 2009^{138} \\
\text { Germany } \\
5 \text { intervention hospitals } \\
5 \text { matched comparison } \\
\text { hospitals }\end{array}$ & $\begin{array}{l}\text { Prospective cohort } \\
\text { Low } \\
\text { A: Without telehealth, } \\
n=1938 \\
\text { B: With telehealth, } n=1122\end{array}$ & $\begin{array}{l}\text { Death or institutional } \\
\text { care } \\
12 \text { months } \\
\text { AOR: } 0.89, \mathrm{NS} \\
30 \text { months } \\
\text { AOR: } 0.93 \text {, NS } \\
\text { Poor outcome (death, } \\
\text { institutional care, or } \\
\text { severe disability) } \\
12 \text { months } \\
\text { OR: } \mathbf{0 . 6 5}, \mathrm{p}<0.001 \\
\mathbf{3 0} \text { months } \\
\text { OR: } \mathbf{0 . 8 2 , p = 0 . 0 3 1}\end{array}$ & NR & NR \\
\hline Psychiatry & $\begin{array}{l}\text { Buckley, } 2012^{214} \\
\text { New South Wales, } \\
\text { Australia } \\
19 \text { district hospitals } \\
1 \text { regional hospital }\end{array}$ & $\begin{array}{l}\text { Before-after } \\
\text { Moderate } \\
\text { A: Before telehealth, } \\
n=1153 \\
\text { B: After telehealth, } n=790\end{array}$ & NR & $\begin{array}{l}\text { Patients transferred, \% } \\
(95 \% \mathrm{Cl}) \\
\text { A: } 67 \%(64.0 \text { to } 69.5) \\
B: 60 \%(56.1 \text { to } 63.1), \\
\text { p=0.001 } \\
\text { AOR }: 0.69(0.49 \text { to } \\
0.97)\end{array}$ & NR \\
\hline Psychiatry & $\begin{array}{l}\text { Mielonen, } 2000^{164} \\
\text { Finland } \\
2 \text { remote centers } \\
34 \text { patients }\end{array}$ & $\begin{array}{l}\text { Prospective cohort } \\
\text { High } \\
\text { A: Without telehealth, } n=20 \\
\text { B: With telehealth, } n=14 ; \\
\text { Satisfaction survey, } n=124\end{array}$ & NR & $\begin{array}{l}\text { Staff satisfaction } \\
\text { Video as good (almost } \\
\text { as good) as } \\
\text { conventional meeting: } \\
47 \%(48 \%) \\
\text { Prefer video for next } \\
\text { meeting } \\
\text { Healthcare staff: } 86 \% \\
\text { Patients: } 84 \% \\
\text { Patient relatives: } 92 \%\end{array}$ & $\begin{array}{l}\text { Cost per patient } \\
\text { Videoconference: FIM } \\
2510 \\
\text { Conventional } \\
\text { conference: FIM } 4750 \\
\text { Video is cheaper if } \geq 30 \\
\text { cases per year. } \\
\text { With } 50 \text { cases the } \\
\text { savings would be FIM } \\
117,000\end{array}$ \\
\hline $\begin{array}{l}\text { Test/Image Based } \\
\text { Assessment }\end{array}$ & $\begin{array}{l}\text { Alemi, } 2017^{26} \\
\text { CA, United States } \\
2 \text { hospitals }\end{array}$ & $\begin{array}{l}\text { Prospective cohort } \\
\text { High } \\
\begin{array}{l}\text { A. No telehealth, } n=31 \\
\text { B. Telehealth, } n=29\end{array}\end{array}$ & NR & $\begin{array}{l}\text { Mean time for } \\
\text { assessment (range) } \pm \\
S D, \text { in minutes } \\
\text { A: } \mathbf{3 4}(\mathbf{1 0} \text { to } 60) \pm \mathbf{1 6} \\
\text { B: } \mathbf{1 3}(\mathbf{5} \text { to } \mathbf{3 5}), \pm \mathbf{8}, \\
\text { p<0.001 }\end{array}$ & NR \\
\hline
\end{tabular}




\begin{tabular}{|c|c|c|c|c|c|}
\hline Clinical Topic & $\begin{array}{l}\text { Author, Year } \\
\text { Location } \\
\text { Number of Sites } \\
\end{array}$ & $\begin{array}{l}\text { Study Design } \\
\text { Risk of Bias } \\
\text { Comparison, } n \\
\end{array}$ & $\begin{array}{l}\text { Clinical Outcomes } \\
\text { Harms }\end{array}$ & $\begin{array}{l}\text { Intermediate } \\
\text { Outcomes }\end{array}$ & Economic Outcomes \\
\hline $\begin{array}{l}\text { Test/Image Based } \\
\text { Assessment }\end{array}$ & $\begin{array}{l}\text { Engel, } 2011^{237} \\
\text { Taoyuan, Taiwan } \\
1 \text { hospital }\end{array}$ & $\begin{array}{l}\text { Prospective cohort } \\
\text { High } \\
\text { A: No telehealth, } n=57 \\
\text { B: Telehealth, } n=46\end{array}$ & $\begin{array}{l}\text { Surgery success rate } \\
\text { A: } 95.1 \% \\
\text { B: } 97.8 \%, p=0.4\end{array}$ & $\begin{array}{l}\text { Return to operating } \\
\text { room, } \mathrm{n}(\%) \\
\text { A: } 5(8.8 \%) \\
\text { B: } 4(8.7 \%) \\
\text { Mean response time } \pm \\
\text { SD, in minutes } \\
\text { A: } 180 \pm 104 \\
\text { B: } 8 \pm 3, p=0.01\end{array}$ & NR \\
\hline $\begin{array}{l}\text { Test//mage Based } \\
\text { Assessment }\end{array}$ & $\begin{array}{l}\text { Nagayoshi, } 2016^{244} \\
\text { Kumamoto, Japan } \\
12 \text { rural hospitals } \\
2 \text { centers }\end{array}$ & $\begin{array}{l}\text { Before-after } \\
\text { High } \\
\text { A: Before telehealth, } n=18 \\
\text { B: After telehealth, } n=48\end{array}$ & $\mathrm{NR}$ & $\begin{array}{l}\text { Transfers, } n(\%) \\
\text { A: } \mathbf{1 0}(\mathbf{5 5 \%}) \\
\text { B: } \mathbf{1 0}(\mathbf{2 1 \%}), \mathbf{p}<0.05 \\
\text { Waiting period } \\
\text { A: } 17.2 \text { days } \\
\text { B: } 9.2 \text { days, } p=0.23\end{array}$ & NR \\
\hline $\begin{array}{l}\text { Test/Image Based } \\
\text { Assessment }\end{array}$ & $\begin{array}{l}\text { Sharma, 2016 } \\
\text { PA, United States } \\
2 \text { academic hospitals }\end{array}$ & $\begin{array}{l}\text { Before-after } \\
\text { High } \\
\text { A: Before telehealth, } n=38 \\
\text { B: After telehealth, } n=25\end{array}$ & NR & $\begin{array}{l}\text { Mean handling time, in } \\
\text { minutes }(95 \% \mathrm{Cl}) \\
\text { A: } \mathbf{4 3 . 5}(\mathbf{3 7 . 9} \text { to } \mathbf{4 9 . 0}) \\
\text { B: } \mathbf{2 6 . 9}(\mathbf{1 5 . 4} \text { to } \mathbf{3 8 . 4}) \text {, } \\
\mathbf{p}=\mathbf{0 . 0 0 4} \\
\text { Mean time to response, } \\
\text { in minutes }(95 \% \mathrm{Cl}) \\
\text { A: } 405.7(301.0 \text { to } \\
510.3) \\
\text { B: } 344.7(291.3 \text { to } \\
\text { 398.0), p=0.602 } \\
\text { Teledermatology alone } \\
\text { sufficient: } 10 \text { of } 25 \\
\text { consultations }\end{array}$ & NR \\
\hline
\end{tabular}




\begin{tabular}{|c|c|c|c|c|c|}
\hline Clinical Topic & $\begin{array}{l}\text { Author, Year } \\
\text { Location } \\
\text { Number of Sites }\end{array}$ & $\begin{array}{l}\text { Study Design } \\
\text { Risk of Bias } \\
\text { Comparison, } n \\
\end{array}$ & $\begin{array}{l}\text { Clinical Outcomes } \\
\text { Harms }\end{array}$ & $\begin{array}{l}\text { Intermediate } \\
\text { Outcomes }\end{array}$ & Economic Outcomes \\
\hline $\begin{array}{l}\text { Test/Image Based } \\
\text { Assessment }\end{array}$ & $\begin{array}{l}\text { Wallace, } 2008^{209} \\
\text { United Kingdom } \\
1 \text { hospital providing } \\
\text { consults to >60 sites }\end{array}$ & $\begin{array}{l}\text { Prospective cohort } \\
\text { High } \\
\text { A: No telehealth, } n=607 \\
\text { B: Telehealth, } n=389 ; \\
\text { telehealth used in } 243 \\
\text { referrals }\end{array}$ & NR & 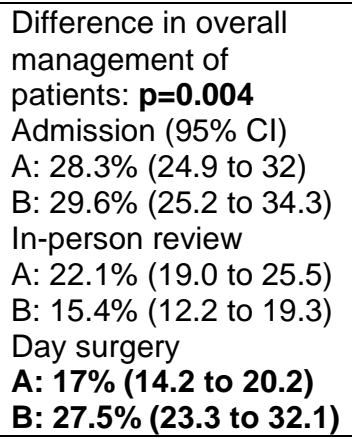 & $\begin{array}{l}\text { No evidence of cost } \\
\text { saving for hospital } \\
\text { (details not reported) } \\
\text { Substantial investment: } \\
£ 70,000\end{array}$ \\
\hline Mentored Surgery & $\begin{array}{l}\text { Burgess, } 2002^{39} \\
\text { HI, United States } \\
1 \text { hospital }\end{array}$ & $\begin{array}{l}\text { Prospective cohort } \\
\text { High } \\
\text { A: No telehealth, } n=42 \\
\text { B: Telehealth, } n=45 \\
\end{array}$ & $\begin{array}{l}\text { No complications or } \\
\text { harms }\end{array}$ & $\begin{array}{l}\text { Mean surgery time, in } \\
\text { minutes: } \\
\text { A: } \mathbf{2 4 . 6 7} \\
B: \mathbf{2 8 . 5 4 , p < 0 . 0 2 7} \\
16 \% \text { increase }\end{array}$ & NR \\
\hline Mentored Surgery & $\begin{array}{l}\text { Fuertes-Guiró, } 2016^{149} \\
\text { Barcelona, Spain } \\
2 \text { community hospitals } \\
1 \text { university hospital }\end{array}$ & $\begin{array}{l}\text { RCT } \\
\text { High } \\
\text { A: No telehealth, } n=16 \\
\text { B: Telementoring, } n=20\end{array}$ & $\begin{array}{l}\text { A: } 3(13 \%) \text { experienced } \\
\text { minor complications }(n=2 \\
\text { bleeding of surgical } \\
\text { wounds; } n=1 \text { urological } \\
\text { infection) }\end{array}$ & $\begin{array}{l}\text { Mean surgery time } \pm \\
S D, \text { in minutes } \\
A: 200 \pm 46 \\
B: 139 \pm 33, p<0.01 \\
\text { Mean hospital stay } \pm \\
\text { SD, in days } \\
\text { A: Mean } 6.7 \pm 0.5 \\
\text { B: Mean } 4.6 \pm 0.5 \\
\text { p }<0.01\end{array}$ & NR \\
\hline Mentor Surgery & $\begin{array}{l}\text { Shin, } 2015^{116} \\
\text { CA, United States } \\
1 \text { academic hospital }\end{array}$ & $\begin{array}{l}\text { Prospective cohort } \\
\text { High } \\
\text { A: No telehealth, } n=29 \\
\text { B: Telementoring, } n=26\end{array}$ & $\begin{array}{l}\text { A: } 1 \text { intraoperative } \\
\text { complication reported } \\
\text { resulting in no } \\
\text { postoperative sequelae } \\
\text { B: } 0 \text { intraoperative } \\
\text { complications } \\
\text { Estimated blood loss } \\
\mathrm{m} / \mathrm{L} \text {, median (range) } \\
\text { A: } 2.5(0 \text { to } 7) \\
\text { B: } 2.5(0 \text { to } 7)\end{array}$ & $\begin{array}{l}\text { Median estimated } \\
\text { duration (range), in } \\
\text { minutes } \\
\text { A: } 15 \text { (5 to } 25) \\
\text { B: } 15 \text { (5 to } 35) \\
\text { Robotic skills } \\
\text { assessment } \\
\text { p>0.05 } \\
\text { Mentors preferred } \\
\text { remote to in-room } \\
\text { p=0.05 }\end{array}$ & NR \\
\hline
\end{tabular}




\begin{tabular}{|c|c|c|c|c|c|}
\hline Clinical Topic & $\begin{array}{l}\text { Author, Year } \\
\text { Location } \\
\text { Number of Sites }\end{array}$ & $\begin{array}{l}\text { Study Design } \\
\text { Risk of Bias } \\
\text { Comparison, } n\end{array}$ & $\begin{array}{l}\text { Clinical Outcomes } \\
\text { Harms }\end{array}$ & $\begin{array}{l}\text { Intermediate } \\
\text { Outcomes }\end{array}$ & Economic Outcomes \\
\hline Critical Care & $\begin{array}{l}\text { Armaignac, } 2018^{29} \\
\text { FL, United States } \\
\text { Large healthcare } \\
\text { system } \\
\text { Number of sites NR }\end{array}$ & $\begin{array}{l}\text { Retrospective cohort } \\
\text { Clinical outcomes: low } \\
\text { Cost outcomes: high } \\
\text { A: Usual care, } n=8,000 \\
\text { B: Telehealth, } n=8,091\end{array}$ & $\begin{array}{l}\text { PCU mortality } \\
\text { A: } 83(1.0 \%) \\
\text { B: } 60(0.7 \%), p=0.048 \\
\text { Hospital mortality } \\
\text { A: } 410(5.2 \%) \\
\text { B: } 342(4.4 \%), p=0.013 \\
\text { Unadjusted HR: } 0.79 \\
\text { (0.68 to } 0.91) \\
\text { Adjusted HRe } 0.56(0.41 \\
\text { to } 0.76)\end{array}$ & $\begin{array}{l}\text { Mean LOS }(95 \% \mathrm{Cl}) \text {, in } \\
\text { days } \\
\text { PCU } \\
\text { A: } 3.2(3.1 \text { to } 3.3) \\
\text { B: } 2.6(2.5 \text { to } 2.7) \\
\text { p }<0.0001 \\
\text { Hospital } \\
\text { A: } 6.8(6.6 \text { to } 6.9) \\
B: 7.3(7.2 \text { to } 7.5) \\
p<0.0001\end{array}$ & $\begin{array}{l}\text { Overall mean direct cost, } \\
\text { in USD } \\
\text { A: } \$ 12,301 \\
B: \$ 13,180 p<0.0001\end{array}$ \\
\hline Critical Care & $\begin{array}{l}\text { Robison, } 2016^{105} \\
\text { DE, United States } \\
1 \text { children's hospital } \\
3 \text { units with telehealth; } 3 \\
\text { without telehealth }\end{array}$ & $\begin{array}{l}\text { Prospective cohort } \\
\text { High } \\
\text { A: Usual care, } n=43 \\
\text { B: Telehealth, } n=48\end{array}$ & NR & $\begin{array}{l}\text { Time to establish } \\
\text { contact, in minutes } \\
\text { A: } \mathbf{3 . 7} \\
\text { B: } \mathbf{2 . 6} \\
\mathbf{p}=\mathbf{0 . 0 1 2} \\
\text { Admitted to PICU } \\
\text { A: } 73 \% \\
\text { B: } 58 \%, p=0.13 \\
\text { Mean number of } \\
\text { Interventions } \\
\text { A: } 1.9 \\
\text { B: } 1.4 \text { NS }\end{array}$ & NR \\
\hline
\end{tabular}




\begin{tabular}{|c|c|c|c|c|c|}
\hline Clinical Topic & $\begin{array}{l}\text { Author, Year } \\
\text { Location } \\
\text { Number of Sites }\end{array}$ & $\begin{array}{l}\text { Study Design } \\
\text { Risk of Bias } \\
\text { Comparison, } n\end{array}$ & $\begin{array}{l}\text { Clinical Outcomes } \\
\text { Harms }\end{array}$ & $\begin{array}{l}\text { Intermediate } \\
\text { Outcomes }\end{array}$ & Economic Outcomes \\
\hline Multiple Specialists & $\begin{array}{l}\text { Steinman, } 20155^{252} \\
\text { Sao Paulo, Brazil } \\
1 \text { spoke hospital } \\
1 \text { hospital providing } \\
\text { teleconsultations }\end{array}$ & $\begin{array}{l}\text { Before-after and } \\
\text { prospective cohort } \\
\text { High } \\
\text { A: } 1 \text { year before telehealth } \\
\text { B: } 1 \text { year after telehealth } \\
n=\text { unclear } \\
\text { Once telehealth established } \\
\text { C: Nontelehealth } \\
\text { consultations } \\
\text { D: Telehealth consultations } \\
n=\text { unclear }\end{array}$ & $\begin{array}{l}\text { AMI mortality } \\
\text { A vs. B: } 17 \% \text { vs. } 14 \% \\
\text { C vs. D: } 14 \% \text { vs. } 8 \% \\
\text { Septic shock mortality } \\
\text { A vs. B: } 66 \% \text { vs. } 68 \% \\
\text { C vs. D: } 71 \% \text { vs } 40 \% \\
\text { Ischemic stroke mortality } \\
\text { A vs. B: } 50 \% \text { vs. } 44 \% \\
\text { C vs. D: } 76 \% \text { vs. } 32 \% \\
\text { Hemorrhagic stroke } \\
\text { mortality } \\
\text { A vs. B: } 23 \% \text { vs. } 28 \% \\
\text { C vs. D: } 37 \% \text { vs. } 16 \% \\
\text { A vs. B comparisons: all } \\
\text { NS } \\
\text { C vs. D comparisons: } \\
\text { p=NR, trending } \\
\text { significant }\end{array}$ & NR & NR \\
\hline
\end{tabular}

AMI = acute myocardial infarction; AOR = adjusted odds ratio; AUD= Australian Dollars; $\mathrm{CI}$ = confidence interval; FIM = Finnish markka; HR = hazard ratio; ICU = intensive care unit; LOS = length of stay; $\mathrm{MD}$ = medical doctor; NA = not applicable; NICU = neonatal intensive care unit; NR = not reported; NS = not significant; OR = odds ratio; PCU = progressive care unit; PICU = pediatric intensive care unit; RCT = randomized control trial; SD = standard deviation; UK = United Kingdom; USD = United States Dollars

${ }^{\text {a }}$ Time periods overlap with other Marcin articles

${ }^{\mathrm{b}}$ Adjusted for sex, age, seriousness of the patient's injury at diagnosis, referral center

c Adjusted for age, sex, clustering in hospitals and repeat visits

${ }^{\mathrm{d}}$ Different values reported in the abstract and text of article

e Scores matched on age, sex, and race, severity of illness, and risk of mortality

Bold=statistically significant; telehealth superior. Bold and italicized=statistically significant; telehealth inferior. Regular type: not statistically significant 


\section{Emergency Care Results}

We identified 73 articles reporting results from 70 studies on the use of telehealth for consultations in emergency care. These are divided below into three functional categories, and are presented and cited in three subsections. The first section summarizes the literature on the use of telehealth for urgent stroke diagnosis and treatment decision. Referred to as telestroke, this has been the most frequently studied application of telehealth for provider-to-provider consultation in emergency care. The second section reports on consultations by various specialists provided as part of care in an emergency room or department. The third section reviews studies of telehealth consultations used by EMS providing out of hospital care or by clinicians providing urgent care for all other topics except stroke.

Table 7 includes the number of articles that addressed each of these subtopics, a summary assessment of key outcomes across the studies, and the citations. Figure 9 and Tables 8, 9, and 10 each provide more detail, focusing on the key results for each study, and the accompanying text discusses selected characteristics of the subset of studies, highlighting any trends or anomalies. Detailed information abstracted from each article is provided in Appendix F. The criteria and overall rating for risk of bias assessment of each study are in Appendix G. The strength of evidence assessment is in Appendix $\mathrm{H}$ and summarizes the evidence by outcome in each of the three categories.

\section{Emergency Care Key Points}

\section{Telestroke:}

- Clinical outcomes: The evidence suggests that telestroke does not result in differences in either in-hospital or 3-month mortality (moderate strength of evidence)

- Intermediate outcomes: Changes in thrombolytic therapy (tissue plasminogen activator [tPA]) use and time to treatment with telestroke were not significantly different (low strength of evidence).

Adverse events: No increased harms, specifically hemorrhage (moderate strength of evidence).

Specialty consultations in emergency departments:

- Clinical outcomes: The impact on clinical outcomes including mortality and functional status is generally positive, though the results are not always statistically significant (low strength of evidence).

- Economic outcomes: Analyses of costs were available only in a few studies, and the results favored savings (low strength of evidence).

- Intermediate outcomes: Teleconsultations have a positive effect on intermediate outcomes such as appropriate triage and transfers and shorter time in the emergency department (moderate strength of evidence).

- Adverse events: No information was available about harms (insufficient evidence).

EMS and urgent care:

- Clinical outcomes: Telehealth reduces morality for heart attack patients (moderate strength of evidence).

- Economic outcomes: Reductions in air transfers and referrals contributed to estimates of lower costs (low strength of evidence). 
- Intermediate outcomes: Telehealth led to more timely provision of care and a reduction in air transfers and referrals to higher-level care following urgent care (moderate strength of evidence), and these

- Adverse effects: Information on harms was very limited (insufficient evidence).

Table 7 provides an overview of the evidence available about the use of telehealth consultations in emergency care.

Table 7. Emergency care: summary of evidence

\begin{tabular}{|c|c|c|c|c|c|}
\hline Topic & $\begin{array}{l}\text { Number } \\
\text { of } \\
\text { Studies }\end{array}$ & $\begin{array}{l}\text { Clinical } \\
\text { Outcomes }\end{array}$ & $\begin{array}{l}\text { Intermediate } \\
\text { Outcomes }\end{array}$ & Cost & Citations \\
\hline Telestroke & 29 & $\begin{array}{l}\sim \text { Mortality } \\
\sim \text { Harms } \\
\sim \text { Function }\end{array}$ & $\begin{array}{l}\text { * Increase in tPA } \\
\text { use } \\
\text { ? No clear effect } \\
\text { on time to } \\
\text { treatment }\end{array}$ & No information & $\begin{array}{l}27,41,48,64,67,68,96,101,120,121 \\
135,137,139,150,155,156,159,161 \\
163,174,176,178,183,193,216,226 \\
236,238,255\end{array}$ \\
\hline $\begin{array}{l}\text { EMS and } \\
\text { Urgent Care }\end{array}$ & $\begin{array}{l}22 \\
\text { (reported } \\
\text { in } 23 \\
\text { articles) }\end{array}$ & $\begin{array}{l}\text { * Reduced } \\
\text { mortality } \\
\text { Harms: } \\
\text { Insufficient } \\
\text { evidence } \\
\end{array}$ & $\begin{array}{l}\text { * Timeliness of } \\
\text { care and } \\
\text { appropriatenes } \\
\text { s of transfers }\end{array}$ & $\begin{array}{l}\text { * Lower costs } \\
\text { related to } \\
\text { fewer } \\
\text { transfers and } \\
\text { lower staff } \\
\text { costs }\end{array}$ & $\begin{array}{l}34,35,77,78,100,113,124,141-143 \\
162,171-173,179,189,197,204 \\
218,225,242,246,254\end{array}$ \\
\hline $\begin{array}{l}\text { ED Specialty } \\
\text { Consultations }\end{array}$ & $\begin{array}{l}19 \\
\text { (reported } \\
\text { in } 21 \\
\text { articles) }\end{array}$ & $\begin{array}{l}\text { * } \text { Lower } \\
\text { mortality, } \\
\text { better } \\
\text { outcomes } \\
\text { Harms: } \\
\text { insufficient } \\
\text { information }\end{array}$ & $\begin{array}{l}\text { * Better transport } \\
\text { triage, shorter } \\
\text { ED time, better } \\
\text { quality of care }\end{array}$ & $\begin{array}{l}\text { } 4 \text { studies } \\
\text { report } \\
\text { savings, but } \\
\text { not always } \\
\text { significant; } 1 \\
\text { increased } \\
\text { costs }\end{array}$ & $\begin{array}{l}47,49,51,53,83,89,90,93,95,97,111, \\
117,133,136,191,235,239,241,245, \\
247,253\end{array}$ \\
\hline
\end{tabular}

ED = emergency department; EMS = emergency medical services; ICU = intensive care unit; LOS = length of stay; tPA = tissue plasminogen activator

Key: * superior (telehealth benefit), no difference, < inferior (comparison better than telehealth), ? inconclusive (inconsistent results)

\section{Detailed Results}

\section{Acute Stroke or Telestroke}

Twenty-nine of the included studies investigated telestroke programs or initiatives. These involve the use of telemedicine to convey information about a patient to a vascular neurologist/stroke specialist for assessment and diagnosis with a focus on determining whether tPA is appropriate. tPA is an effective treatment that can reduce death and disability from acute ischemic stroke when administered within 4.5 hours of the patient developing symptoms. Appropriate use of tPA requires confirming the diagnosis and beginning treatment as soon as possible. Although tPA has been approved for almost two decades and is the standard for initial care, some patients who may benefit from this treatment are not receiving it due to limited access to stroke expertise. Telestroke attempts to solve this access issue by using technology to provide timely consultations for patients at remote locations or at times when vascular neurologists are not physically available. Telestroke programs may involve video and/or audio communications, 
and the transfer of data from an ambulance or ED to the specialist who can then advise on transport or treatment. Usual care is to provide tPA after the in-person assessment if appropriate.

Table 8 and Figure 9 provide selected information and the results from the identified studies of telestroke. Given that there were numerous studies with a similar intervention reporting mortality as the outcome for similar populations, we pooled these results in a meta-analysis represented in Figure 9. Other outcomes from these studies and from studies not providing data on mortality that could be combined in the meta-analysis are provided in Table 8.

\section{Telestroke Effectiveness in Improving Clinical and Economic Outcomes}

Mortality was the most frequently reported clinical outcome in telestroke studies. Given that several studies had small samples sizes and most found no significant difference in mortality, pooled estimates were generated and included 16 studies (Figure 9) to assess whether combining samples would change the overall conclusion. The resulting pooled risk ratios also found no statistically significant benefit and produced the following risk ratios and 95\% confidence intervals: 0.92 (0.62 to 1.34 ) for in-hospital mortality based on nine studies; 0.94 (0.82 to 1.08) for 3-month mortality based on seven studies; and 0.64 (0.35 to 1.16) for the two studies that reported overall mortality.

In Figure 9 the pooled estimates are grouped by in-hospital, 3-month and overall (not defined) mortality to make comparisons across equivalent outcomes. The figure contains the study design, specifies whether the population was all evaluated patients or only those who received tPA, the number of deaths in each arm (with and without telestroke), and the risk ratio for mortality for each study. Two studies calculated adjusted risk ratios and provided raw data. ${ }^{64,67}$ We repeated the meta-analysis with the adjusted values for these studies and confirmed that it would not change the overall conclusion. Another consideration is that within this analysis there are two pairs of studies that may have overlapping populations. Two studies analyzed data from the Telemedical Pilot Project for Integrative Stroke Care (TEMPiS) and report 3-month mortality from different but overlapping timeperiods. ${ }^{137,178}$ Two other studies were both conducted at the University of Pittsburgh Medical Center during overlapping periods and reported inpatient mortality, but they had different authors, funding, and comparisons. ${ }^{27,64}$ Again, we examined the impact of including both or excluding one study in each pair and found that it did not change the conclusion.

Fifteen studies included discharge disposition or a functional measure as a clinical outcome. As several different measures were used, these could not be pooled. Of the 15, only two reported statistically significantly better outcomes with telestroke. ${ }^{137,216}$ The majority (13 studies) reported small differences that did not rise to the level of statistical significance. $27,67,101,135,150,159,161,176,178,193,238,255$

The identified studies of telestroke did not evaluate the costs of telestroke consultations.

\section{Telestroke Effectiveness in Improving Intermediate Outcomes}

The primary intermediate outcomes in these studies are indicators or improved access (rate of providing tPA) and quality of care (timeliness of treatment). These are process measures, not patient results, but increasing both the number of people treated and the timely delivery of treatment is one of the primary goals of most telestroke programs

Thirteen studies reported rates of tPA use with and without telestroke. Of these, four studies reported statistically significant increases. In three studies, the initial rate of tPA use ranged from 0 to 2.8 percent; all three studies increased tPA use with telestroke with rates ranging from 4.3 to 6.8 percent. $^{27,41,137}$ The fourth study reported an initial mean of 34.4 monthly tPA administrations 
across 21 hospitals and increased to a mean of 61.8 administrations with telestroke. ${ }^{96}$ In the rest of the studies the differences were either not significant or statistical tests were not reported.

Timeliness of treatment was included in 23 of 29 studies. Measures included time to treatment (referred to as time to "needle") from either symptom onset or arrival at the hospital (sometimes referred to as "door time”). The results in terms of this outcome are inconsistent. Six studies found telehealth improved timeliness of care by at least one measure, ${ }^{68,96,121,156,161,174}$ three studies found time to treatment was significantly longer with telehealth, ${ }^{135,193,238}$ and the majority of studies reported there was not a statistically significant difference in time to treatment with and without telehealth.

\section{Harms, Adverse Events, or Negative Unintended Consequences}

The primary concern related to harms of telehealth for stroke is that a patient will receive a contraindicated treatment that will lead to negative outcomes or complications. Specifically, tPA given incorrectly can result in intracranial hemorrhage. Eleven studies reported on hemorrhage and all reported either no events or no statistically significant difference in rates comparing telestroke to usual care. ${ }^{27,41,48,67,96,135,159,161,176,193,226}$

\section{Key Characteristics of Telestroke and Association With Outcomes}

The number of sites/hospitals included in each study is listed in Table 8. In all but one study the services are provided by one organization to another; that is, one or two hospitals with stroke expertise (often, referred to as the hub) provide consultations to hospitals that do not have that expertise (the spokes). The one study not following this model ${ }^{238}$ established a telehealth system to allow off site neurologists to provide after-hours coverage within a single organization.

The number of hospitals in these arrangements varies, with some involving only two hospitals (1 hub and 1 spoke) and others involving one or two consulting or hub hospitals and 2 to 25 spoke hospitals. It is possible that in addition to the number of participating hospitals, other aspects of the structure of these relationships, such as how services are paid for or if there are incentives to treat patients in particular locations, could impact the effectiveness of telestroke programs. Unfortunately, there is not enough information available in the published studies to evaluate this.

Information on the number of hospitals involved is one of the only characteristics of the programs other than basic descriptions of the technology (e.g., whether video was one or two way, what test results or images could be transmitted) provided in some but not all of these articles. The studies do not report characteristics of the providers or the environments and information that would allow assessment of whether the patients changed before and after telehealth or were different at the intervention and comparison hospital was not provided. Given that research on emergency care often faces time constraints and challenging environments, less data may be collected, producing less information about the context, and restricting synthesis to the major outcomes and limiting subgroup analysis. 
Figure 9. Impact of telestroke on mortality

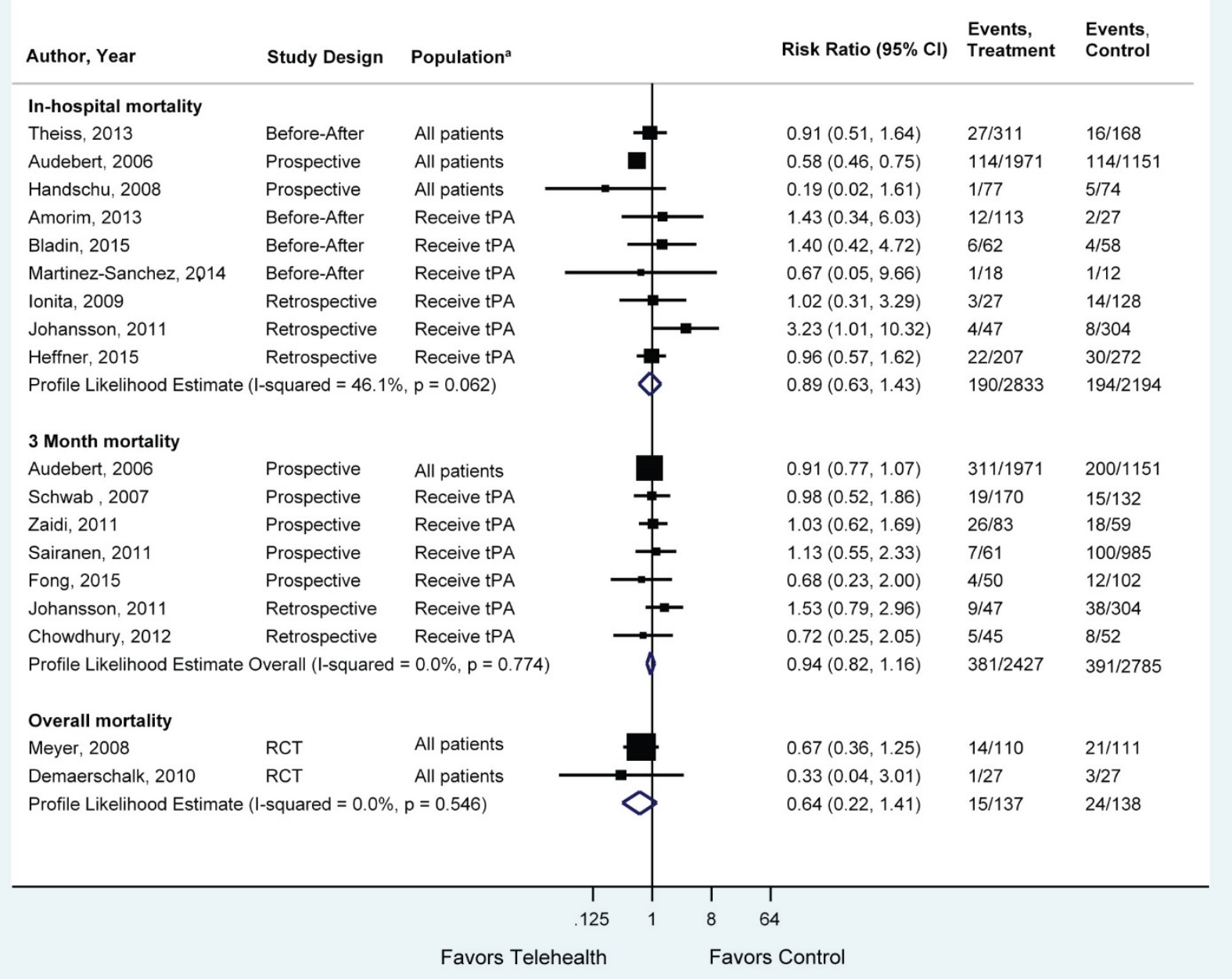

$\mathrm{CI}=$ confidence interval; $\mathrm{RCT}=$ randomized controlled trial; $\mathrm{tPA}=$ tissue plasminogen activator

${ }^{\mathrm{a}}$ Indicates whether original study analyzed all patients or only those who received tPA. 
Table 8. Telestroke: outcomes in addition to mortality

\begin{tabular}{|c|c|c|c|}
\hline $\begin{array}{l}\text { Author, Year } \\
\text { Location } \\
\text { Number of Sites }\end{array}$ & $\begin{array}{l}\text { Study Design } \\
\text { Risk of Bias } \\
\text { Comparison, } n\end{array}$ & $\begin{array}{l}\text { Mortality } \\
\text { Short-Term Outcomes } \\
\text { Long-Term Outcomes }\end{array}$ & $\begin{array}{l}\text { tPA Administration } \\
\text { Time to Treatment } \\
\text { Other Intermediate Outcomes }\end{array}$ \\
\hline $\begin{array}{l}\text { Amorim, } 2013^{27} \\
\text { PA, United States } \\
12 \text { community } \\
\text { hospitals } \\
1 \text { academic } \\
\text { hospital }\end{array}$ & $\begin{array}{l}\text { Before-after } \\
\text { Moderate } \\
\text { A: Before telestroke, } n=919 \\
\text { B: After telestroke, } n=1669\end{array}$ & $\begin{array}{l}\text { Mortality: in meta-analysis } \\
\text { Discharge outcomes } \\
\text { Home: } \\
\text { A: } 33.3 \% \\
\text { B: } 26.5 \% \text {, NS } \\
\text { Rehabilitation: } \\
\text { A: } 33.3 \% \\
\text { B: } 32 \%, \text { NS } \\
\text { Symptomatic Intracerebral } \\
\text { hemorrhage } \\
\text { A: } 3.7 \% \\
\text { B: } 0.9 \%, \text { NS }\end{array}$ & $\begin{array}{l}\text { Overall IV tPA use } \\
\text { A: } \mathbf{2 . 8 \%} \\
\text { B: } \mathbf{6 . 8} \%, \mathbf{p}<\mathbf{0 . 0 0 1} \\
\text { Protocol violations } \\
\text { A: } 0.2 \% \\
\text { B: } 0.3 \%, \text { NS } \\
\text { Onset-to-treatment, in minutes } \\
\text { A: } 129.8 \\
\text { B: } 124.4, \text { NS } \\
\text { Door-to-treatment, in minutes } \\
\text { A: } 74.2 \\
\text { B: } 74.0, \text { NS }\end{array}$ \\
\hline $\begin{array}{l}\text { Audebert, } 2006^{137} \\
\text { Germany } \\
\text { Bavaria } \\
2 \text { academic } \\
\text { hospitals } \\
5 \text { community } \\
\text { intervention } \\
\text { hospitals } \\
5 \text { comparison } \\
\text { hospitals }\end{array}$ & $\begin{array}{l}\text { Prospective cohort } \\
\text { Low } \\
\text { A: Not telestroke, } n=1151 \\
\text { B: Telestroke, } n=1971\end{array}$ & $\begin{array}{l}\text { Mortality: in meta-analysis } \\
\text { Hospital discharge } \\
\text { destination: } \\
\text { Home: } \\
\text { A: } 38 \% \\
\text { B: } 39 \% \\
\text { Dead: } \\
\text { A: } 10 \% \\
\text { B: } 8 \% \\
\text { Rehab unit: } \\
\text { A: } 34 \% \\
\text { B: } 38 \% \\
\text { Nursing home: } \\
\text { A: } 5 \% \\
\text { B: } 3 \% \\
\text { Other hospital: } \\
\text { A: } 13 \% \\
\text { B: } 13 \% \\
\text { p=0.001 } \\
\text { Total \% poor outcomes at } 3 \\
\text { months: } \\
\text { A: } 54 \\
\text { B: } 44, \text { p<0.001 }\end{array}$ & $\begin{array}{l}\text { Thrombolytic treatment } \\
\text { A: } 0 \% \\
\text { B: } 5 \%, p<0.0001 \\
\text { Mean LOS, in days } \\
\text { A: } 11.9 \\
\text { B: } 10.7, p<0.0001\end{array}$ \\
\hline $\begin{array}{l}\text { Bergrath, } 2012^{139} \\
\text { Germany, Aachen } \\
4 \text { ambulances- one } \\
\text { with telehealth } \\
\text { Hospital numbers } \\
\text { NR }\end{array}$ & $\begin{array}{l}\text { Prospective cohort } \\
\text { Moderate } \\
\text { A: Usual care, } n=46 \\
\text { B: Telehealth, } n=18\end{array}$ & NR & $\begin{array}{l}\text { Median EMS alarm to physician arrival time } \\
\text { (IQR), in minutes } \\
\text { A: } \mathbf{7} \text { (4) } \\
\text { B: } \mathbf{5}(\mathbf{2}), p=\mathbf{0 . 0 1 8 2} \\
\text { Median door to brain imaging time (IQR), in } \\
\text { minutes } \\
\text { A: } n=42 ; 57.5 \text { (80) } \\
\text { B: } n=16 ; 59.5(67.5), p=0.6447\end{array}$ \\
\hline
\end{tabular}




\begin{tabular}{|c|c|c|c|}
\hline $\begin{array}{l}\text { Author, Year } \\
\text { Location } \\
\text { Number of Sites }\end{array}$ & $\begin{array}{l}\text { Study Design } \\
\text { Risk of Bias } \\
\text { Comparison, } n\end{array}$ & $\begin{array}{l}\text { Mortality } \\
\text { Short-Term Outcomes } \\
\text { Long-Term Outcomes }\end{array}$ & $\begin{array}{l}\text { tPA Administration } \\
\text { Time to Treatment } \\
\text { Other Intermediate Outcomes }\end{array}$ \\
\hline $\begin{array}{l}\text { Bladin, } 2015^{216} \\
\text { Australia } \\
1 \text { regional } \\
\text { hospital,1 } \\
\text { metropolitan } \\
\text { hospital }\end{array}$ & $\begin{array}{l}\text { Before-after } \\
\text { Low } \\
\text { A: Before telestroke, } n=144 \\
\text { B: After telestroke, } n=138\end{array}$ & $\begin{array}{l}\text { Mortality: in meta-analysis } \\
\text { Discharged home or to } \\
\text { rehabilitation } \\
\text { A: } \mathbf{3 3 \%} \\
\text { B: } \mathbf{8 0 \% , p = 0 . 0 2}\end{array}$ & $\begin{array}{l}\text { tPA use, } n(\%) \\
\text { All strokes <4.5 hours } \\
\text { A: } 10(17 \%) \\
\text { B: } 16(26 \%), N S \\
\text { Ischemic stroke <4.5 hours } \\
\text { A: } 10(19 \%) \\
\text { B: } 16(28 \%), N S \\
\text { Median minutes (IQR) } \\
\text { Door to needle time } \\
\text { A: } 101 \text { (75 to } 153) \\
\text { B: } 85 \text { (72 to } 117), \text { NS } \\
\text { Stroke onset to needle time } \\
\text { A: } 218(180 \text { to } 258) \\
\text { B: } 173:(148 \text { to } 234), N S \\
\text { Median LOS, in days (IQR) } \\
\text { A: } 3 \text { (1 to 6) } \\
\text { B: } 4 \text { (2 to } 6), \text { NS }\end{array}$ \\
\hline $\begin{array}{l}\text { Choi, } 2006^{41} \\
\text { TX, United States } \\
2 \text { community } \\
\text { hospitals one } \\
\text { university hospital }\end{array}$ & $\begin{array}{l}\text { Before-after } \\
\text { High } \\
\text { A: Before telestroke, } n=327 \\
\text { B: After telestroke, } n=328\end{array}$ & $\begin{array}{l}\text { Median pretreatment } \\
\text { NIHSS score } \\
\text { A: NR } \\
\text { B: } 10 \\
\text { Improved by } 4 \text { points on } \\
\text { NIHSS scale } \\
\text { A: NR } \\
\text { B: } 7 \\
\text { Worsened on NIHSS scale } \\
\text { A: NR } \\
\text { B: } 3 \\
\text { Intracerebral hemorrhages } \\
\text { A: NR } \\
\text { B: } 0 \\
\end{array}$ & $\begin{array}{l}\text { tPA use, } n(\%) \\
\text { A: } \mathbf{2}(\mathbf{0 . 8 \% )} \\
\text { B: } \mathbf{1 4}(\mathbf{4 . 3 \% )}, \mathbf{p}<0.001 \\
\text { Median door to needle time (range), in } \\
\text { minutes } \\
\text { A: NR } \\
\text { B: } 85(27 \text { to } 165)\end{array}$ \\
\hline $\begin{array}{l}\text { Chowdhury, } \\
2012^{193} \\
\text { London, United } \\
\text { Kingdom } \\
1 \text { hospital }\end{array}$ & $\begin{array}{l}\text { Retrospective cohort } \\
\text { High } \\
\text { A: Usual care, } n=52 \\
\text { B: Telehealth, } n=45\end{array}$ & $\begin{array}{l}\text { Mortality: in meta-analysis } \\
\text { Favorable outcome } \\
\text { A: } 36.5 \% \\
\text { B: } 42 \%, p=0.9 \\
\text { Symptomatic intracranial } \\
\text { hemorrhage } \\
\text { A: } 7.7 \% \\
\text { B: } 4.4 \%, p=0.7\end{array}$ & $\begin{array}{l}\text { Onset to treatment, median minutes (IQR) } \\
\text { A: } 100 \text { (78 to } 120) \\
\text { B: } 125 \text { (55 to 105) } p=0.001 \\
\text { Admission to treatment, median minutes } \\
\text { (IQR) } \\
\text { A: } 33 \text { (23 to 47) } \\
\text { B: } 61 \text { (43 to 106), } p<0.001\end{array}$ \\
\hline $\begin{array}{l}\text { Demaerschalk, } \\
2010^{48} \\
\text { United States } \\
2 \text { spoke hospitals } \\
1 \text { central hub } \\
\text { hospital }\end{array}$ & $\begin{array}{l}\text { RCT } \\
\text { Moderate } \\
\text { A: Telephone only, } n=27 \\
\text { B: Telehealth, } n=27\end{array}$ & $\begin{array}{l}\text { Mortality: in meta-analysis } \\
\text { Intracerebral hemorrhage } \\
\text { A: } 0 \% \\
\text { B: } 4 \%, p>0.99\end{array}$ & $\begin{array}{l}\text { Onset to decision time } \pm \mathrm{SD} \text {, mean minutes } \\
\text { A: } 164 \pm 28.6 \\
\text { B: } 188.2 \pm 138.2, p=0.07\end{array}$ \\
\hline
\end{tabular}




\begin{tabular}{|c|c|c|c|}
\hline $\begin{array}{l}\text { Author, Year } \\
\text { Location } \\
\text { Number of Sites }\end{array}$ & $\begin{array}{l}\text { Study Design } \\
\text { Risk of Bias } \\
\text { Comparison, } n\end{array}$ & $\begin{array}{l}\text { Mortality } \\
\text { Short-Term Outcomes } \\
\text { Long-Term Outcomes }\end{array}$ & $\begin{array}{l}\text { tPA Administration } \\
\text { Time to Treatment } \\
\text { Other Intermediate Outcomes }\end{array}$ \\
\hline $\begin{array}{l}\text { Dharmasaroja, } \\
2010^{236} \\
\text { Thailand, } \\
\text { Thammasat } \\
1 \text { hub hospital } \\
25 \text { spoke hospitals }\end{array}$ & $\begin{array}{l}\text { Before-after } \\
\text { High } \\
\text { A: Before telehealth, } n=170 \\
\text { B: After telehealth, } n=406\end{array}$ & NR & $\begin{array}{l}\text { tPA use } \\
\text { A: } 8 \% \\
\text { B: } 27 \%\end{array}$ \\
\hline $\begin{array}{l}\text { Fong, et al., } 2015^{238} \\
\text { Hong Kong } \\
1 \text { hospital with } \\
\text { offsite neurologists }\end{array}$ & $\begin{array}{l}\text { Prospective cohort } \\
\text { Moderate } \\
\text { A: No telestroke, } n=102 \\
\text { B: Telestroke, } n=50\end{array}$ & $\begin{array}{l}\text { Mortality: in meta-analysis } \\
\text { Excellent outcome }{ }^{\text {b }} \text { at } 3 \\
\text { months } \\
\text { A: } 43 \% \\
\text { B: } 52 \%, N S \\
\text { In multivariate analyses, } \\
\text { the absence of onsite } \\
\text { neurologists was not } \\
\text { associated with negative } \\
\text { outcomes. }\end{array}$ & $\begin{array}{l}\text { All received tPA } \\
\text { Median minutes (IQR) } \\
\text { Door to needle time } \\
\text { A: } \mathbf{7 1} \text { (60 to } 89) \\
\text { B: } 97 \text { (85 to } 119), p<0.001 \\
\text { Onset to needle time } \\
\text { A: } 133 \text { (109 to } 154) \\
B: 148(134 \text { to } 170), p=0.012\end{array}$ \\
\hline $\begin{array}{l}\text { Handschu, } 2008^{150} \\
\text { Bavaria, Germany } \\
2 \text { stroke centers } \\
2 \text { local hospitals }\end{array}$ & $\begin{array}{l}\text { Prospective cohort } \\
\text { Moderate } \\
\text { A: Telephone consultation, } \\
n=74 \\
\text { B: Telestroke with video, } \\
n=77\end{array}$ & $\begin{array}{l}\text { Mortality: in meta-analysis } \\
\text { Institutional care } 10 \text { days } \\
\text { post-stroke } \\
\text { A: } 5.4 \% \\
\text { B: } 2.6 \%, \text { NS } \\
\text { Admission to stroke ward } \\
\text { A: } 45.9 \% \\
\text { B: } 59.7 \% \text {, NS } \\
\text { Transfer to stroke center } \\
\text { A: } \mathbf{1 4 . 9 \%} \\
\text { B: } \mathbf{9 . 1 \% , p < 0 . 0 5} \\
\text { Diagnosis corrected at } \\
\text { discharge } \\
\text { A: } \mathbf{1 7 . 6 \%} \\
\text { B: } \mathbf{7 . 1 \% , p < 0 . 0 5}\end{array}$ & $\begin{array}{l}\text { Total time for consultation, in minutes } \\
\text { A: } \mathbf{2 7 . 1} \\
\text { B: } \mathbf{4 9 . 8}, \boldsymbol{p}<0.01 \\
\text { LOS, in days } \\
\text { A: } 12.3 \\
\text { B: } 11.4, \text { NS }\end{array}$ \\
\hline $\begin{array}{l}\text { Heffner, } 2015^{64} \\
\text { PA, United States } \\
1 \text { hub hospital } \\
5 \text { spoke hospitals }\end{array}$ & $\begin{array}{l}\text { Retrospective cohort } \\
\text { Moderate } \\
\text { Comparison } \\
A_{1}: \text { Not telestroke, treated } \\
\text { at hub hospital, } n=272 \\
A_{2} \text { : Not telestroke, treated } \\
\text { at spoke then transferred to } \\
\text { hub hospital, } n=73 \\
\text { B: Telestroke, treated at } \\
\text { spoke hospital, } n=134\end{array}$ & Mortality: in meta-analysis & $\begin{array}{l}\text { All received tPA } \\
\text { Door to needle time, in minutes } \\
A_{1}: 71.98 \\
A_{2}: 74.89 \\
B: 76.57 \\
A_{1} \text { vs. } B, N S ; B \text { vs. } A_{2}, N S \\
\text { Onset to needle time, in minutes } \\
A_{1}: 155.6 \\
A_{2}: 133.8 \\
B: 147.57 \\
A_{1} \text { vs. } B, N S ; B \text { vs. } A_{2}, N S \\
\\
\text { LOS, in days }>6 \text { days, OR }(95 \% \mathrm{Cl}) \\
\text { B vs. } A_{1}: 4.696(2.428 \text { to } 9.083) \\
\text { B vs. } A_{1}+A_{2}: 4.280(2.356 \text { to } 7.774) \\
\text { Longer stay in telehealth group }\end{array}$ \\
\hline
\end{tabular}




\begin{tabular}{|c|c|c|c|}
\hline $\begin{array}{l}\text { Author, Year } \\
\text { Location } \\
\text { Number of Sites }\end{array}$ & $\begin{array}{l}\text { Study Design } \\
\text { Risk of Bias } \\
\text { Comparison, } n\end{array}$ & $\begin{array}{l}\text { Mortality } \\
\text { Short-Term Outcomes } \\
\text { Long-Term Outcomes }\end{array}$ & $\begin{array}{l}\text { tPA Administration } \\
\text { Time to Treatment } \\
\text { Other Intermediate Outcomes }\end{array}$ \\
\hline $\begin{array}{l}\text { Hubert, } 2016^{155} \\
\text { Helsinki, Finland } \\
1 \text { central hospital } \\
\text { with telehealth } \\
15 \text { spoke hospitals } \\
2 \text { hub hospitals with } \\
\text { telehealth }\end{array}$ & $\begin{array}{l}\text { Retrospective cohort } \\
\text { Moderate } \\
\text { A: Usual care, } n=912 \\
\text { B: Telehealth, } n=1779\end{array}$ & NR & $\begin{array}{l}\text { Median (IQR); mean } \pm \mathrm{SD} \text {, in minutes } \\
\text { Onset-to-door time } \\
\text { A: } \mathbf{8 8}(\mathbf{6 0} \text { to } 135) ; 105.3 \pm 55.9 \\
\text { B: } 65 \text { (48 to } 101) ; \mathbf{8 0 . 1} \pm \mathbf{4 5 . 3 , p < 0 . 0 0 1} \\
\text { Door-to-needle time } \\
\text { A: } 18 \text { (13 to } 30) ; 25.1 \pm 20.0 \\
\text { B: } 39 \text { (26 to } 56) ; 44.7 \pm 26.7, p<0.001 \\
\text { Onset-to-treatment time } \\
\text { A: } 117 \text { (81 to } 168) ; 130.4 \pm 59.1 \\
\text { B: } 115 \text { (87 to } 155) ; 124.8 \pm 49.4 \\
p=0.452\end{array}$ \\
\hline $\begin{array}{l}\text { Ickenstein, } 2005^{156} \\
\text { Germany } \\
12 \text { community } \\
\text { hospitals } \\
2 \text { specialized stroke } \\
\text { centers }\end{array}$ & $\begin{array}{l}\text { Before-after } \\
\text { High } \\
\text { A: Before telehealth, } n=155 \\
\text { B: After telehealth, } n=164\end{array}$ & NR & $\begin{array}{l}\text { Patients presenting with } 3 \text { hours and } \\
\text { receiving tPA } \\
\text { A: } 10(6 \%) \\
\text { B: } 45(27 \%)\end{array}$ \\
\hline $\begin{array}{l}\text { Ionita, } 2009^{67} \\
\text { NY, United States } \\
1 \text { hub hospital } \\
10 \text { community } \\
\text { hospitals }\end{array}$ & $\begin{array}{l}\text { Retrospective cohort } \\
\text { Moderate } \\
\text { A: Not telestroke, } n=128 \\
\text { B: Telestroke, } n=27\end{array}$ & $\begin{array}{l}\text { Mortality: in meta-analysis } \\
\text { Poor mRS score }{ }^{\mathrm{c}} \text { at } \\
\text { discharge } \\
\text { A: } 61(48 \%) \\
\text { B: } 13(48 \%), \mathrm{NS} \\
\text { Post thrombolytic } \\
\text { intracranial hemorrhage } \\
\text { A: } 26(20 \%) \\
\text { B: } 9(33 \%), \mathrm{NS}\end{array}$ & $\begin{array}{l}\text { Mean time from onset to needle in minutes } \\
\text { A: } 143.9 \\
\text { B: } 130.7, \text { NS }\end{array}$ \\
\hline $\begin{array}{l}\text { Itrat, } 2016^{68} \\
\text { United States } \\
1 \text { Mobile stroke } \\
\text { units w/ telehealth } \\
9 \text { hospitals with } \\
\text { telehealth }\end{array}$ & $\begin{array}{l}\text { Prospective cohort } \\
\text { Moderate } \\
\text { A: Usual care, } n=56 \\
\text { B:Telehealth, } n=100\end{array}$ & NR & $\begin{array}{l}\text { Median minutes (IQR) } \\
\text { Door to computed tomography read } \\
\text { A: } 25 \text { (19 to } 35) \\
\text { B: } 25 \text { (20 to } 29), p=0.59 \\
\text { Door to IV-tPA } \\
\text { A: } 58 \text { (53 to } 68) \\
\text { B: } \mathbf{3 2} \text { (24 to } 47), p<0.001\end{array}$ \\
\hline $\begin{array}{l}\text { Johansson, } 2011^{159} \\
\text { Austria, Salzburg } \\
5 \text { regional hospitals } \\
1 \text { specialized stroke } \\
\text { center }\end{array}$ & $\begin{array}{l}\text { Retrospective cohort } \\
\text { Moderate } \\
\text { A: Usual care, } n=304 \\
\text { B: Telehealth, } n=47\end{array}$ & $\begin{array}{l}\text { Mortality: in meta-analysis } \\
\text { Good functional outcome } \\
\text { at } 3 \text { months } \\
\text { A: } 43 \% \\
\text { B: } 47 \%, p=0.694 \\
\text { In-hospital complications } \\
\text { A: } 22 \% \\
\text { B: } 23 \%, p=0.85 \\
\text { Hemorrhagic bleeding } \\
\text { A: } 7.6 \% \\
\text { B: } 6.4 \%\end{array}$ & $\begin{array}{l}\text { Onset to needle time, mean minutes } \\
\text { A: } 122(n=277) \\
\text { B: } 113(n=42), p=0.263\end{array}$ \\
\hline
\end{tabular}




\begin{tabular}{|c|c|c|c|}
\hline $\begin{array}{l}\text { Author, Year } \\
\text { Location } \\
\text { Number of Sites }\end{array}$ & $\begin{array}{l}\text { Study Design } \\
\text { Risk of Bias } \\
\text { Comparison, } n\end{array}$ & $\begin{array}{l}\text { Mortality } \\
\text { Short-Term Outcomes } \\
\text { Long-Term Outcomes }\end{array}$ & $\begin{array}{l}\text { tPA Administration } \\
\text { Time to Treatment } \\
\text { Other Intermediate Outcomes }\end{array}$ \\
\hline $\begin{array}{l}\text { Martinez-Sanchez, } \\
2014^{161} \\
\text { Madrid, Spain } \\
1 \text { community } \\
\text { hospital } \\
1 \text { stroke center }\end{array}$ & $\begin{array}{l}\text { Before-after } \\
\text { Moderate } \\
\text { A: Before telestroke, } n=259 \\
\text { B: After telestroke, } n=225\end{array}$ & $\begin{array}{l}\text { Mortality: in meta-analysis } \\
\text { Patients treated with tPA, } \\
\text { Favorable outcome, } n(\%) \\
\text { A: } 4(33.3 \%) \\
\text { B: } 10(55.6 \%), p=0.145 \\
\text { Stroke recurrence, } n(\%) \\
\text { A: } 0 \\
B: 1(5.6 \%) \\
\text { Intracranial hemorrhage, } n \\
(\%) \\
\text { A: } 2(16.7) \\
B: 0, p=0.152\end{array}$ & $\begin{array}{l}\text { Received IV rt-PA } \\
\text { A: } 4.7 \% \\
\text { B: } 8 \%, p=0.125 \\
\text { Door to needle time, median minutes (IQR) } \\
\text { A: } 143.5(48) \\
\text { B: } 66(54), p<0.0001\end{array}$ \\
\hline $\begin{array}{l}\text { Mazighi, } 2017^{163} \\
\text { Paris, France } \\
10 \text { community } \\
\text { hospital EDs } \\
1 \text { stroke center }\end{array}$ & $\begin{array}{l}\text { RCT } \\
\text { High } \\
\text { A: Usual care, } n=22 \\
\text { B: Telethrombolysis, } n=25\end{array}$ & NR & $\begin{array}{l}\text { Received IV rt-PA } \\
\text { A: } 4 \\
\text { B: } 21 \\
\text { Delay time from symptom onset to } \\
\text { administration of IV rt-PA, median minutes } \\
\text { (range) } \\
\text { A: } 184 \text { (178 to } 258) \\
\text { B: } 145(110 \text { to } 200) \\
\end{array}$ \\
\hline $\begin{array}{l}\text { Meyer, } 2008^{255} \\
\text { CA, United States } \\
1 \text { hub hospital } \\
4 \text { spoke hospitals } \\
222 \text { (Included in } \\
\text { Demaershalk, } \\
2012 \text { ) }\end{array}$ & $\begin{array}{l}\text { RCT } \\
\text { Low } \\
\text { A: Telephone only, } n=111 \\
\text { B: Telestroke, } n=111\end{array}$ & $\begin{array}{l}\text { Mortality: in meta-analysis } \\
\text { Barthel Index score of } 95 \text { to } \\
100 \text { at } 90 \text { days, } n(\%) \\
\text { A: } 56(54) \\
\text { B: } 45(43) \\
\text { OR: } 0.6, N S \\
\text { mRS score of } 0-1 \text { at } 90 \\
\text { days, } n(\%) \\
\text { A: } 48(47) \\
\text { B: } 36(34) \\
\text { OR: } 0.6, N S\end{array}$ & $\begin{array}{l}\text { tPA use } \\
\text { A: } 23 \% \\
\text { B: } 28 \%, \text { NS } \\
\text { Correct decision } \\
\text { A: } 82 \% \\
\text { B: } 98 \% \\
\text { OR } 10.9, p=0.0009 \\
\text { Onset to needle time, in minutes } \\
\text { A:143 } \\
\text { B:157.2, NS }\end{array}$ \\
\hline $\begin{array}{l}\text { Nagao, } 2012^{226} \\
\text { Melbourne, } \\
\text { Victoria, Australia } \\
1 \text { spoke hospital } \\
1 \text { hub hospital }\end{array}$ & $\begin{array}{l}\text { Before-after } \\
\text { High } \\
\text { A: Before telestroke, } n=30 \\
\text { B: After telestroke, } n=24 \\
\text { (for treatment in place) }\end{array}$ & $\begin{array}{l}\text { Discharge } n(\%) \\
\text { Dead: } \\
\text { A: } 3(13) \\
\text { B: } 3(10) \\
\text { Home: } \\
\text { A: } 14(47) \\
\text { B: } 12 \text { (50) } \\
\text { Other Hospital: } \\
\text { A: } 13(43) \\
\text { B: } 8 \text { (33) p=0.6 } \\
\text { Complications, } n \text { (\%) } \\
\text { Further stroke: } \\
\text { A: } 0 \\
\text { B: } 2 \text { (8.3) } \\
\text { Intracerebral hemorrhage: } \\
\text { A: } 0 \\
\text { B: } 0\end{array}$ & $\begin{array}{l}\text { Received thrombolysis, } n(\%) \\
\text { A: } 0 \\
\text { B: } 8(33 \%)\end{array}$ \\
\hline
\end{tabular}




\begin{tabular}{|c|c|c|c|}
\hline $\begin{array}{l}\text { Author, Year } \\
\text { Location } \\
\text { Number of Sites }\end{array}$ & $\begin{array}{l}\text { Study Design } \\
\text { Risk of Bias } \\
\text { Comparison, } \mathbf{n}\end{array}$ & $\begin{array}{l}\text { Mortality } \\
\text { Short-Term Outcomes } \\
\text { Long-Term Outcomes }\end{array}$ & $\begin{array}{l}\text { tPA Administration } \\
\text { Time to Treatment } \\
\text { Other Intermediate Outcomes }\end{array}$ \\
\hline $\begin{array}{l}\text { Nguyen-Huynh, } \\
2018^{96} \\
\text { Northern California, } \\
\text { United States } \\
21 \text { hospitals } \\
1 \text { stroke center }\end{array}$ & $\begin{array}{l}\text { Before-after } \\
\text { Moderate } \\
\text { A: Before telehealth, } n=310 \\
\text { B: After telehealth, } n=557\end{array}$ & $\begin{array}{l}\text { Symptomatic intracranial } \\
\text { hemorrhage rates } \\
\text { A: } 2.2 \% \\
\text { B: } 3.8 \%, p=0.29\end{array}$ & $\begin{array}{l}\text { Alteplase administration per month, mean } \pm \\
\text { SD } \\
\text { A: } 34.4 \pm 12.4 \\
\text { B: } 61.8 \pm 12.4, p<0.001 \\
\text { Door to needle time, mean minutes } \pm \text { SD } \\
\text { A: } 63.2 \pm 31.2 \\
\text { B: } 41.8 \pm 30.6, p<0.001\end{array}$ \\
\hline $\begin{array}{l}\text { Pedragosa, } 2009^{174} \\
\text { Spain } \\
1 \text { hub hospital } \\
1 \text { community } \\
\text { hospital }\end{array}$ & $\begin{array}{l}\text { Before-after } \\
\text { High } \\
\text { A: Before telestroke, } n=201 \\
\text { B: After telestroke, } n=198\end{array}$ & $\begin{array}{l}\text { Urgent ambulance transfer: } \\
\text { A: } 17 \% \\
\text { B: } 10 \%, p=0.04 \\
\text { Unnecessary transfers to } \\
\text { the stroke center: } \\
\text { A: } \mathbf{5 1 \%} \\
\text { B: } 20 \%, p=0.02 \\
\text { Stroke unit admissions: } \\
\text { A: } 11 \% \\
\text { B: } 8 \%, N S\end{array}$ & $\begin{array}{l}\text { tPA use } \\
\text { A: } 4.5 \% \text { B: } 9.6 \%, N S \\
\text { Onset to needle time, in minutes } \\
\text { A: } \mathbf{2 1 0} \\
\text { B: } \mathbf{1 6 2}, \mathbf{p}=\mathbf{0 . 0 5} \\
\text { tPA in } 0 \text { to } 3 \text { hour window } \\
\text { A: } \mathbf{3 0 \%} \\
\text { B: } \mathbf{6 8 \% ,} \mathbf{p}=\mathbf{0 . 0 4} \\
\text { Specialized neurologist evaluation } \\
\text { A: } \mathbf{1 7 \%} \\
\text { B: } \mathbf{3 8 \% ,} \mathbf{p}<\mathbf{0 . 0 0 1}\end{array}$ \\
\hline $\begin{array}{l}\text { Pervez, } 2010^{101} \\
\text { Boston, MA, United } \\
\text { States } \\
33 \text { hospitals } \\
12 \text { with telehealth } \\
21 \text { without } \\
1 \text { regional stroke } \\
\text { center }\end{array}$ & $\begin{array}{l}\text { Retrospective cohort } \\
\text { High } \\
\text { A: Telephone, } n=181 \\
\text { B: Telehealth (for treatment } \\
\text { in place), } n=115\end{array}$ & $\begin{array}{l}\text { In-hospital mortality } \\
\text { A: } 17.4 \% \\
\text { B: } 14.9 \%, p=0.57 \\
\text { Discharge outcomes } \\
\text { Home: } \\
\text { A: } 30.5 \% \\
\text { B: } 28.6 \%, p=0.74 \\
\text { Inpatient rehabilitation } \\
\text { A: } 55.3 \% \\
\text { B: } 53.3 \%, p=0.67 \\
\text { Ambulatory at discharge } \\
\text { A: } 77.7 \% \\
B: 73.8 \%, p=0.5\end{array}$ & $\begin{array}{l}\text { Median onset to tPA time (IQR), in minutes } \\
\text { A: } 130(102.5 \text { to162.8) } \\
\text { B: } 140(117.3 \text { to165.3), } p=0.06 \\
\text { Mean LOS } \pm S D \text {, in days } \\
\text { A: } 7.6 \pm 6.5 \\
\text { B: } \mathbf{5 . 9} \pm \mathbf{3 . 7}, p<0.001\end{array}$ \\
\hline $\begin{array}{l}\text { Sairanen, } 2011^{176} \\
\text { Helsinki, Finland } \\
5 \text { community } \\
\text { hospitals } \\
1 \text { university hospital }\end{array}$ & $\begin{array}{l}\text { Prospective cohort } \\
\text { Moderate } \\
\text { A: Usual care, } n=985 \\
\text { B: Telehealth, } n=106 \\
61 \text { received thrombolysis }\end{array}$ & $\begin{array}{l}\text { Mortality: in Meta-analysis } \\
\text { Intracerebral bleeding } \\
\text { A: } 9.4 \% \\
\text { B: } 6.7 \%, p=0.427 \\
3 \text { month mRS 0-2 } \\
\text { A: } 58.1 \% \\
\text { B: } 49.1 \%, p=0.214 \\
3 \text { month mRS 0-1 } \\
\text { A: } 36.8 \% \\
\text { B: } 29.4 \%, p=0.289\end{array}$ & NR \\
\hline $\begin{array}{l}\text { Schwab, } 2007^{178} \\
\text { Germany, } \\
\text { Regensburg and } \\
\text { Munich } \\
12 \text { community } \\
\text { hospitals } \\
2 \text { specialized stroke }\end{array}$ & $\begin{array}{l}\text { Prospective cohort } \\
\text { Low } \\
\text { A: Usual care, } n=132 \\
\text { B: Telehealth, } n=170\end{array}$ & $\begin{array}{l}\text { Mortality: in meta-analysis } \\
\text { Good functional outcome at } \\
6 \text { months } \\
\text { A: } 30.9 \% \\
\text { B: } 39.5 \%, p=0.10\end{array}$ & $\begin{array}{l}\text { Onset to treatment time, mean minutes } \\
\text { A: } 143.6 \\
\text { B: } 140.6, p=0.45\end{array}$ \\
\hline
\end{tabular}




\begin{tabular}{|c|c|c|c|}
\hline $\begin{array}{l}\text { Author, Year } \\
\text { Location } \\
\text { Number of Sites }\end{array}$ & $\begin{array}{l}\text { Study Design } \\
\text { Risk of Bias } \\
\text { Comparison, } n\end{array}$ & \begin{tabular}{|l|} 
Mortality \\
Short-Term Outcomes \\
Long-Term Outcomes \\
\end{tabular} & $\begin{array}{l}\text { tPA Administration } \\
\text { Time to Treatment } \\
\text { Other Intermediate Outcomes } \\
\end{array}$ \\
\hline $\begin{array}{l}\text { Switzer, } 2009^{120} \\
\text { GA, United States, } \\
6 \text { rural hospitals } \\
1 \text { hub }\end{array}$ & $\begin{array}{l}\text { Prospective cohort } \\
\text { Moderate } \\
\text { A: ED, } n=26 \\
\text { B: Telehealth, } n=49\end{array}$ & NR & $\begin{array}{l}\text { Onset to treatment time, mean minutes } \pm \text { SD } \\
(95 \% \mathrm{Cl}) \\
\mathrm{A}: 145.88 \pm 46.99(126.9 \text { to } 164.87) \\
\mathrm{B}: 127.57 \pm 36.33 \text { (117.14 to } 138.01) \text {, NS }\end{array}$ \\
\hline $\begin{array}{l}\text { Taqui, } 2017^{121} \\
\text { Cleveland, OH, } \\
\text { United States } \\
1 \text { mobile stroke unit } \\
3 \text { comprehensive } \\
\text { stroke centers } \\
11 \text { primary stroke } \\
\text { centers }\end{array}$ & $\begin{array}{l}\text { Prospective cohort } \\
\text { High } \\
\text { A: Traditional ambulance, } \\
n=53 \\
\text { B: Mobile stroke unit, } \\
n=100\end{array}$ & NR & $\begin{array}{l}\text { Median minutes (IQR) } \\
\text { Alarm-to-thrombolysis time } \\
\text { A: } 94 \text { (78 to } 105) \\
\text { B: } 55.5 \text { (46 to } 65), p<0.0001 \\
\text { Door-to-thrombolysis time } \\
\text { A: } 58 \text { (52 to } 66) \\
\text { B: } 31.5 \text { (24 to } 47), p=0.0012 \\
\text { Symptom-onset-to-thrombolysis time } \\
\text { A: } 122.5(110 \text { to } 176) \\
\text { B: } 97(61 \text { to } 144), p=0.0485 \\
\end{array}$ \\
\hline $\begin{array}{l}\text { Theiss, } 2013^{183} \\
\text { Erfurt, Germany } \\
5 \text { comprehensive } \\
\text { stroke centers } \\
5 \text { Neuro Net } \\
\text { hospitals } \\
5 \text { matched control } \\
\text { hospitals }\end{array}$ & $\begin{array}{l}\text { Before-after } \\
\text { Moderate } \\
\text { A: Control hospitals without } \\
\text { telehealth, } n=168 \\
\text { B: Comprehensive stroke } \\
\text { centers, } n=845 \\
\text { C: Neuro Net hospitals with } \\
\text { stroke telemedicine, } n=311 \\
\end{array}$ & Mortality: in meta-analysis & $\begin{array}{l}\text { Likelihood of receiving tPA } \\
\text { C vs. A, before: OR } 5.7, p=0.07 \\
\text { C vs. A, after: OR 4.5, } p<0.0001 \\
\text { B vs C, before: OR } 3.7, p<0.0001 \\
\text { B vs. C, after: OR } 1.3, p=0.06\end{array}$ \\
\hline $\begin{array}{l}\text { Zaidi, } 2011^{135} \\
\text { Pittsburgh, PA, } \\
\text { United States, } \\
12 \text { spoke hospitals } \\
1 \text { stroke center }\end{array}$ & $\begin{array}{l}\text { Prospective cohort } \\
\text { Moderate } \\
\text { A: Stroke center, } n=59 \\
\text { B: Telestroke (for treatment } \\
\text { in place), } n=83\end{array}$ & $\begin{array}{l}\text { 90-day mortality } \\
\text { A: } 30.4 \% \\
\text { B: } 31.6 \%, p=0.6 \\
\text { Favorable outcome } \\
\text { A: } 37.5 \% \\
\text { B: } 42.1 \%, p=0.7 \\
\text { Asymptomatic intracranial } \\
\text { hemorrhage } \\
\text { A: } 18.6 \% \\
\text { B: } 16.2 \%, p=0.7 \\
\text { Symptomatic intracranial } \\
\text { hemorrhage } \\
\text { A: } 5.1 \% \\
\text { B: } 1.2 \%, p=0.1 \\
\text { mRS } \leq 1 \text { at } 90 \text { days } \\
\text { A: } 22.0 \% \\
\text { B: } 34.9 \% \\
\text { mRS } \leq 2 \text { at } 90 \text { days } \\
\text { A: } 37.5 \% \\
\text { B: } 42.1 \%, p=0.7\end{array}$ & $\begin{array}{l}\text { Mean minutes (SD) } \\
\text { Onset-to-treatment time } \\
\text { A:156.7 (31.6) } \\
\text { B: } 145.5 \text { (42.8), } p=0.09 \\
\text { Arrival-to-treatment time } \\
\text { A: } \mathbf{6 7 . 8} \text { (26.1) } \\
\text { B: } 89.9 \text { (36.3), } p=0.01\end{array}$ \\
\hline
\end{tabular}

$\mathrm{CI}$ = confidence interval; ED = emergency department; EMS = emergency medical services; IQR = interquartile range; IV tPA = intravenous tissue plasminogen activator; IV rtPA =intravenous recombinant tissue plasminogen activator; LOS = length of stay; mRS = modified Rankin Sale; NIHSS = National Institutes of Health Stroke Scale; NR = not reported; NS = not significant; OR = odds ratio; $\mathrm{RCT}$ = randomized controlled trial; $\mathrm{SD}=$ standard deviation; $\mathrm{tPA}$ = tissue plasminogen activator

${ }^{a}$ A moderate or severe ischemic stroke; range 5-23

${ }^{\mathrm{b}}$ An excellent mRS outcome is $0-1$

${ }^{\mathrm{c}}$ A poor mRS score is 4-6

Bold=statistically significant; telehealth superior. Bold and italicized=statistically significant; telehealth inferior. Regular type: not statistically significant 


\section{Emergency Care Telehealth Specialist Consultations}

Twenty-one articles reported on 19 studies of specialist consultations with ED providers. Given the variety of injuries, illnesses and conditions treated in EDs, it is not practical for many EDs to have all potential specialty needs addressed in person, creating the opportunity for telehealth consultants to increase access overall and timeliness. The studies we identified reflected this and included a range of specialties such as trauma, pediatrics, neurology, psychiatry, cardiology, burns, orthopedics and plastic surgery. In ED consultations, like specialty consultations for inpatient care, interactions between providers about specific patients are limited to a single consultation in a short time period. In the case of emergency care, consultations are often executed under time pressure or chaotic conditions.

Table 9 provides general information and the results from these studies. The majority of these studies (13) were conducted in the United States ${ }^{47,49,51,53,83,89,90,93,95,97,111,117,133}$ while two (in three articles) were conducted in Hong Kong ${ }^{239,245,247}$ and one each in Japan, ${ }^{241}$ Italy, ${ }^{191}$ South Korea, ${ }^{235}$ Brazil, ${ }^{253}$ and Turkey. ${ }^{136}$ Several of the studies are small, including less than 100 patients $^{97,111,117,191,235,239,241}$ while at the other extreme, two studies evaluated several thousand patients from multistate ${ }^{95}$ and single state ${ }^{93}$ hospital networks. All of the studies used either video so the specialist could see the patients or transmitted images such as scans or x-rays.

\section{Emergency Care Telehealth Specialist Consultations: Effectiveness in Improving Clinical and Economic Outcomes}

The 13 studies reporting clinical outcomes all reported improvements. Most of these reported lower mortality that was not statistically significantly different. For example, the mortality rate for trauma patients at seven rural EDs declined from 7.8 to 4.8 percent when telehealth consultations provided via video were available but this was not statistically significant. ${ }^{53}$ The exception is a study comparing telephone, teleradiology and video consults for head injuries, which reported that mortality was significantly lower for video consults compared with telephone-only consultations. ${ }^{247}$ While four studies included other clinical outcomes, including function, ${ }^{239,247}$ complications, ${ }^{41,239}$ or diagnosis, ${ }^{136}$ the only significant finding was a lower rate of adverse events during transfer in a small $(n=63)$ study of consultations for brain injury. ${ }^{239}$

Five studies evaluated economic outcomes. Four reported some savings with telehealth, though not always significantly different, while one study reported higher costs. One studies reported a dollar estimate for potential savings per case when transfers were avoided, but did not test this difference. ${ }^{95}$ An evaluation of a statewide network providing psychiatric consultations reported savings in both inpatient charges (significant) and total healthcare charges (not significant) in the 30 days after the ED visit. Having both inpatient and total charges suggests that costs are not just being shifted from one site of care to another, though details about what specific costs were included was not provided. ${ }^{93}$ One study of trauma consultations for seven rural EDs reported a large reduction in hospital costs (from \$7.6 million to \$1.1 million) but did not provide information or details that explain these savings. ${ }^{53} \mathrm{~A}$ study of consultations for sepsis in an ED reported that total hospital costs were lower, but the difference was not significant. ${ }^{83}$ The study reporting higher costs evaluated neurological consults in Hong Kong and found the average cost per patient increased with video consults by approximately 2000 Hong Kong dollars; researchers attributed this to the increased time before a decision was made and the 30 percent failure rate of the video technology used. ${ }^{247}$ 


\section{Emergency Care Telehealth Specialist Consultations: Effectiveness in Improving Intermediate Outcomes}

All of the studies reported at least one intermediate outcome. The most frequently included were rate of transfer and time to treatment or decision. The impact of telehealth consultations appears greater on these intermediate outcomes, and the effect is generally positive. For example, pediatric video consultations compared with phone only or no consultation increased quality of care in one study ${ }^{49}$ and reduced medication error in pediatric emergency care in another; ${ }^{51}$ burn consultations reduced emergency air transports from 100 to 44.3 percent; ${ }^{111}$ psychiatric consults reduced hospital time in the ED, ${ }^{117}$ as well as admissions and increased odds of 30 and 90 day outpatient followup; ${ }^{93}$ and cardiology consultations resulted in faster ${ }^{136}$ and more aggressive $e^{253}$ treatment. The study using video for neurology consultations reported time from referral to decision was longer with both telephone and video consultation when compared with no consultation, but it is unclear if this is a problem or a reflection of more complete assessments. ${ }^{245,247}$

\section{Emergency Care Telehealth Specialist Consultations: Harms, Adverse Events, or Negative Unintended Consequences}

None of the included studies reported on harms or negative unintended consequences of telehealth specialty consultations as part of emergency care.

\section{Key Characteristics of Emergency Care Telehealth Specialist Consultations with Emergency Departments and Overview of Outcomes}

All of the ED telehealth consultations involved visual data. Most were centered on video that allows visual assessment of patients, observation of ED procedures, and audio communication. Only one study included only the transmission of images. ${ }^{191}$ The studies in the United States focused on providing expertise to rural EDs as a means to get specialty assessments to patients in a shorter time than it would take to transfer them to a trauma center where the patient could be assessed by the specialist in person. The non-United States studies appear to have similar goals though increasing access to care in rural areas was not explicitly stated as one of the goals of these studies.

The combination of the similarity in objectives and technology, the wide range of types of specialties and patients, and the lack of detailed information on the environment or specifics of telehealth implementation make identifying subgroups of patients or programs with different outcomes problematic. 
Table 9. Emergency care telehealth specialty consultations: selected outcomes

\begin{tabular}{|c|c|c|c|c|c|}
\hline $\begin{array}{l}\text { Clinical } \\
\text { Topic }\end{array}$ & $\begin{array}{l}\text { Author, Year } \\
\text { Location } \\
\text { Number of Sites }\end{array}$ & $\begin{array}{l}\text { Study Design } \\
\text { Risk of Bias } \\
\text { Comparison, } n\end{array}$ & $\begin{array}{l}\text { Clinical Outcomes } \\
\text { Harms }\end{array}$ & Intermediate Outcomes & Economic \\
\hline ED: General & $\begin{array}{l}\text { Natafgi, } 2017^{95} \\
7 \text { states, US } \\
85 \text { rural hospitals } \\
1 \text { central hub }\end{array}$ & $\begin{array}{l}\text { Economic evaluation } \\
\text { Moderate } \\
\text { A: Tele-ED not } \\
\text { activated, } n=164,291 \\
\text { B: Tele-ED activated, } \\
n=9,048\end{array}$ & $\begin{array}{l}\text { Mortality, n (\%) } \\
\text { A: } 791(0.5 \%) \\
\text { B: } 358(4.0 \%)\end{array}$ & $\begin{array}{l}\text { Transferred, } \mathrm{n}(\%) \\
\text { A: } 1059(0.7 \%) \\
\text { B: } 4224(47.6 \%)\end{array}$ & $\begin{array}{l}\text { Estimated savings } \\
\text { per avoided transfer, } \\
\text { in USD: } \$ 3,823\end{array}$ \\
\hline ED: Trauma & $\begin{array}{l}\text { Duchesne, } 2008^{53} \\
\text { MS, United States } \\
7 \text { rural EDs }\end{array}$ & $\begin{array}{l}\text { Before-after } \\
\text { High } \\
\text { A: Before telehealth, } \\
n=351 \\
B: \text { After telehealth, } \\
n=463 \text { (51 sent to } \\
\text { trauma center) }\end{array}$ & $\begin{array}{l}\text { Mortality, n (\%) } \\
\text { A: } 17(4.8 \%) \\
\text { B: } 4(7.8 \%), \text { NS }\end{array}$ & $\begin{array}{l}\text { Discharge outcomes } \\
\text { Home: } \\
\text { A: } 0 \% \\
\text { B: } 61.3 \% \\
\text { Admitted to community hospital: } \\
\text { A: } 0 \% \\
\text { B: } 13.6 \% \\
\text { Transfer to trauma center: } \\
\text { A: } 100 \% \\
\text { B: } 11 \% \\
\text { LOS at community hospital, in hours, } \\
\text { transfers only } \\
\text { A: } \mathbf{4 7} \\
\text { B: } 1.5, \text { p<0.001 } \\
\text { Mode of transfer } \\
\text { A: } 74.9 \% \text { ground } \\
\text { B: } 70.5 \% \text { ground }\end{array}$ & $\begin{array}{l}\text { Trauma center } \\
\text { costs, in USD for } \\
\text { transfers } \\
\text { A: } \mathbf{\$ 7 , 6 3 2 , 6 2 4} \\
\text { B: } \mathbf{\$ 1 , 1 2 6 , 6 8 3} \\
\text { p<0.001 }\end{array}$ \\
\hline ED: Trauma & $\begin{array}{l}\text { Mohr } 2018^{90} \text { and } \\
2017^{89} \\
\text { ND, USA } \\
36 \text { Critical access } \\
\text { hospitals }\end{array}$ & $\begin{array}{l}\text { Retrospective cohort } \\
\text { Low } \\
\text { A: Usual care, } n=2,371 \\
\text { B: Telehealth, } n=291\end{array}$ & $\begin{array}{l}\text { Mortality } \\
\text { Adjusted OR (95\% Cl) } \\
\text { Telemedicine Use } \\
0.9(0.5 \text { to } 1.4) \text { NS } \\
\text { Telehealth availability } \\
1.2(0.9 \text { to } 1.7) \text { NS }\end{array}$ & $\begin{array}{l}\text { Interhospital transfers } \\
\text { Adjusted OR }(95 \% \mathrm{CI}) \\
\text { Telemedicine Use } \\
1.28(0.94 \text { to } 1.75), \mathrm{NS} \\
\text { Telemedicine Availability } \\
\mathbf{1 . 2}(\mathbf{1 . 1} \text { to } \mathbf{1 . 4 )}, \mathbf{p}<\mathbf{0 . 0 . 0 5}\end{array}$ & NR \\
\hline
\end{tabular}




\begin{tabular}{|c|c|c|c|c|c|}
\hline $\begin{array}{l}\text { Clinical } \\
\text { Topic }\end{array}$ & $\begin{array}{l}\text { Author, Year } \\
\text { Location } \\
\text { Number of Sites }\end{array}$ & $\begin{array}{l}\text { Study Design } \\
\text { Risk of Bias } \\
\text { Comparison, } n\end{array}$ & $\begin{array}{l}\text { Clinical Outcomes } \\
\text { Harms }\end{array}$ & Intermediate Outcomes & Economic \\
\hline $\begin{array}{l}\text { ED: } \\
\text { Pediatrics }\end{array}$ & $\begin{array}{l}\text { Dayal, } 2016^{47} \\
\text { CA, USA } \\
1 \text { Children's } \\
\text { Hospital PICU } \\
75 \text { EDs }\end{array}$ & $\begin{array}{l}\text { Retrospective cohort } \\
\text { Low } \\
\text { A: No telehealth, } \\
n=524 \\
\text { B: Telehealth, } n=582\end{array}$ & $\begin{array}{l}\text { Mortality } n(\%) \\
\text { A: } 23(4.4) \\
\text { B: } 14(2.4), p=0.07 \\
\text { Pediatric Risk Mortality III } \\
\text { score } \\
\text { A: } 4.0 \\
\text { B: } \mathbf{3 . 2}, p<0.05\end{array}$ & $\begin{array}{l}\text { Mean PICU LOS, days } \pm \text { SD } \\
\text { A: } 3.8 \pm 9.4 \\
\text { B: } 3.1 \pm 5.5, p=0.11\end{array}$ & NR \\
\hline $\begin{array}{l}\text { ED: } \\
\text { Pediatrics }\end{array}$ & $\begin{array}{l}\text { Dharmar, 2013 }{ }^{49} \\
\text { CA, United States } \\
5 \text { EDs }\end{array}$ & $\begin{array}{l}\text { Retrospective cohort } \\
\text { Low } \\
\text { Comparison } \\
A_{1}: \text { No telehealth, no } \\
\text { consult, } n=199 \\
\text { A }_{2} \text { : No telehealth, } \\
\text { phone consult, } n=63 \\
\text { B: Telehealth, } n=58\end{array}$ & $\mathrm{NR}$ & 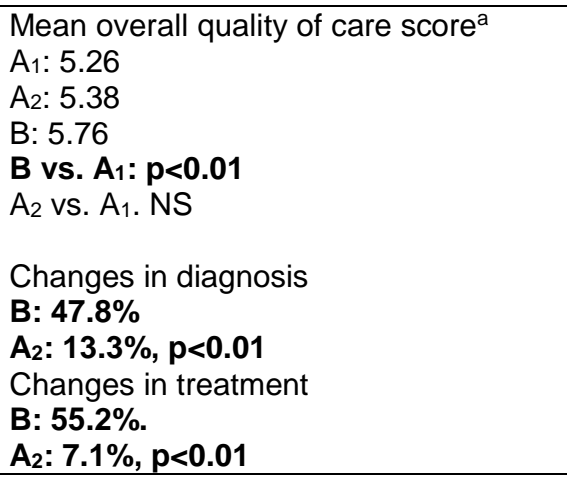 & NR \\
\hline $\begin{array}{l}\text { ED: } \\
\text { Pediatrics }\end{array}$ & $\begin{array}{l}\text { Dharmar, } 2013^{51} \\
\text { CA, United States } \\
8 \text { EDs } \\
1 \text { academic } \\
\text { children's hospital }\end{array}$ & $\begin{array}{l}\text { Retrospective cohort } \\
\text { Low } \\
A_{1} \text { : No telehealth, no } \\
\text { consult, } n=85 \\
A_{2} \text { : No telehealth, } \\
\text { phone consult, } n=76 \\
B: \text { Telehealth, } n=73\end{array}$ & $\begin{array}{l}\text { Mortality, n (\%) } \\
\mathrm{A}_{1}: 2(2.6) \\
\mathrm{A}_{2}: 1(1.2) \\
\mathrm{B}: 3(4.1)\end{array}$ & $\begin{array}{l}\text { Physician-related ED medication errors } \\
(\%) \\
A_{1}: 16(12.5) \\
A_{2}: 18(10.8) \\
B: 5(3.4) \\
\text { B vs. } A_{2}: \mathbf{p}<0.05 \\
\text { B vs. } A_{1}: \mathbf{p}<0.05\end{array}$ & NR \\
\hline
\end{tabular}




\begin{tabular}{|c|c|c|c|c|c|}
\hline $\begin{array}{l}\text { Clinical } \\
\text { Topic }\end{array}$ & $\begin{array}{l}\text { Author, Year } \\
\text { Location } \\
\text { Number of Sites }\end{array}$ & $\begin{array}{l}\text { Study Design } \\
\text { Risk of Bias } \\
\text { Comparison, } n\end{array}$ & $\begin{array}{l}\text { Clinical Outcomes } \\
\text { Harms }\end{array}$ & Intermediate Outcomes & Economic \\
\hline $\begin{array}{l}\text { ED: } \\
\text { Pediatrics }\end{array}$ & $\begin{array}{l}\text { Yang, } 2015^{133} \\
\text { CA, United States } \\
8 \text { rural EDs } \\
1 \text { university hospital }\end{array}$ & $\begin{array}{l}\text { Retrospective cohort } \\
\text { Moderate } \\
\text { A: Telephone, } n=64 \\
\text { B: Telehealth, } n=74\end{array}$ & NR & $\begin{array}{l}\text { Hospital admissions } \\
\text { A: } 87.5 \% \\
\text { B: } 59.5 \%, p<0.05 \\
\text { Observed to expected admission ratio } \\
(95 \% \mathrm{Cl}) \\
\text { Pediatric Risk of Admission II, Overall } \\
\text { A: } 2.58(2.00 \text { to } 3.32) \\
\text { B: } 2.36(1.80 \text { to } 3.10) \\
\text { Revised Pediatric Emergency } \\
\text { Assessment Tool, Overall } \\
\text { A: } 2.57(1.99 \text { to } 3.31) \\
\text { B: } 2.34(1.78 \text { to } 3.07) \\
\end{array}$ & NR \\
\hline $\begin{array}{l}\text { ED: } \\
\text { Neurology }\end{array}$ & $\begin{array}{l}\text { Goh, et al., } 1997^{239} \\
\text { Hong Kong } \\
2 \text { referring hospitals } \\
1 \text { consulting } \\
\text { medical center }\end{array}$ & $\begin{array}{l}\text { Prospective cohort } \\
\text { High } \\
\text { A: No telehealth, } n=28 \\
\text { B: Telehealth, } n=35\end{array}$ & $\begin{array}{l}\text { GOS at } 6 \text { months } \\
\text { Death } \\
\text { A: } 14.3 \% \\
\text { B: } 14.3 \% \\
\text { Vegetative } \\
\text { A: } 7.1 \% \\
\text { B: } 8.6 \% \\
\text { Severe disability } \\
\text { A: } 10.7 \% \\
\text { B: } 2.9 \% \\
\text { Moderate disability } \\
\text { A: } 14.3 \% \\
\text { B: } 14.3 \% \\
\text { Good } \\
\text { A: } 53.6 \% \\
\text { B: } 60 \%, \text { NS } \\
\text { Adverse events during } \\
\text { transfer } \\
\text { A: } \mathbf{3 2 . 1} \% \\
\text { B: } \mathbf{6 . 4 \% , p = 0 . 0 1 7}\end{array}$ & $\begin{array}{l}\text { Therapeutic interventions before transfer } \\
\text { A: } 10.7 \% \\
\text { B: } 32.1 \%, \text { NS } \\
\text { Mean transfer time, in minutes } \\
\text { A: } 80 \\
\text { B: } 72, N S\end{array}$ & $\mathrm{NR}$ \\
\hline
\end{tabular}




\begin{tabular}{|c|c|c|c|c|c|}
\hline $\begin{array}{l}\text { Clinical } \\
\text { Topic }\end{array}$ & $\begin{array}{l}\text { Author, Year } \\
\text { Location } \\
\text { Number of Sites }\end{array}$ & $\begin{array}{l}\text { Study Design } \\
\text { Risk of Bias } \\
\text { Comparison, } n\end{array}$ & $\begin{array}{l}\text { Clinical Outcomes } \\
\text { Harms }\end{array}$ & Intermediate Outcomes & Economic \\
\hline $\begin{array}{l}\text { ED: } \\
\text { Neurology }\end{array}$ & $\begin{array}{l}\text { Wong, 2006 } 247 \\
\text { Poon, 2001245 (pilot } \\
\text { for Wong) } \\
\text { Hong Kong } \\
249 \text { spoke } \\
\text { hospitals } \\
1 \text { hub hospital }\end{array}$ & $\begin{array}{l}\text { RCT } \\
\text { Wong: Moderate } \\
\text { Poon: High } \\
\text { A: No telehealth, } \\
n=235 \\
B_{1}: \text { Telehealth, tele- } \\
\text { radiology, } n=239 \\
B_{2}: \text { Telehealth, video } \\
\text { consult, } n=236\end{array}$ & $\begin{array}{l}\text { Mortality } 6 \text { months after } \\
\text { admission, } n \text { (\%) } \\
\text { A: } 81(35 \%) \\
B_{1}: 59(25 \%) \\
B_{2}: 79(34 \%) \\
B_{1} \text { vs. } A: p=0.025 \\
B_{2} \text { vs. A: } p=0.923 \\
B_{2} \text { vs. } B^{1}: \mathbf{p}=\mathbf{0 . 0 4 3} \\
\text { Favorable outcome after } 6 \\
\text { months, } n(\%) \\
\text { A: } 130(56 \%) \\
B_{1}: 146(47 \%) \\
B_{2}: 124(74 \%) \\
B_{1} \text { vs. A, NS } \\
B_{2} \text { vs. A, NS }\end{array}$ & $\begin{array}{l}\text { Time from referral to decision, in hours } \\
\text { A: } 0.70 \\
B_{1}: 1.01 \\
B_{2}: 1.30 \\
B_{1} \text { vs. } A, N S \\
B_{2} \text { vs. } A: p=0.003 \\
B_{2} \text { vs. } B_{1}, N S \\
\text { Video failure: } 30 \%\end{array}$ & $\begin{array}{l}\text { Average cost per } \\
\text { patient in Hong } \\
\text { Kong dollars (USD) } \\
\text { A: } 14,075(17,237) \\
\text { B: } 14,455(22,906) \\
\text { C: } 16,370(22,587)\end{array}$ \\
\hline ED: Psych & $\begin{array}{l}\text { Narasimhan, } \\
2015^{93} \\
\text { SC, United States } \\
18 \text { hospitals }\end{array}$ & $\begin{array}{l}\text { Prospective cohort } \\
\text { Moderate } \\
\text { A: Site without } \\
\text { telehealth, } n=7,261 \\
\text { B: Site with telehealth, } \\
n=7,261\end{array}$ & NR & $\begin{array}{l}\text { Inpatient admission } \\
\text { OR }: 0.41 p=0.022 \\
\text { LOS in days } \\
\text { OR }:-\mathbf{0 . 4 3 , p = 0 . 0 0 2} \\
\text { 30-day outpatient followup } \\
\text { OR }^{\mathrm{b}}: \mathbf{5 . 4 4 ,} \mathrm{p}<\mathbf{0 . 0 0 1} \\
\\
\text { 90-day outpatient followup } \\
\text { OR: } \mathbf{5 . 6 5}, \mathrm{p}<\mathbf{0 . 0 0 1}\end{array}$ & $\begin{array}{l}\text { Change in charges } \\
30 \text { days after ED } \\
\text { visit, in USD } \\
\text { Inpatient charges: } \\
\mathbf{- 2 , 3 3 8 , p = 0 . 0 4 1} \\
\text { Total charges: } \\
-649, N S\end{array}$ \\
\hline
\end{tabular}




\begin{tabular}{|c|c|c|c|c|c|}
\hline $\begin{array}{l}\text { Clinical } \\
\text { Topic }\end{array}$ & $\begin{array}{l}\text { Author, Year } \\
\text { Location } \\
\text { Number of Sites }\end{array}$ & $\begin{array}{l}\text { Study Design } \\
\text { Risk of Bias } \\
\text { Comparison, } n\end{array}$ & $\begin{array}{l}\text { Clinical Outcomes } \\
\text { Harms }\end{array}$ & Intermediate Outcomes & Economic \\
\hline ED: Psych & $\begin{array}{l}\text { Southard, } 2014^{117} \\
\text { IN, United States } \\
1 \text { rural ED }\end{array}$ & $\begin{array}{l}\text { Before-after } \\
\text { Moderate } \\
\text { A: Before telehealth, } \\
n=24 \\
\text { B: After telehealth, } \\
n=38\end{array}$ & NR & $\begin{array}{l}\text { Mean LOS in ED, in hours } \\
\text { A: } \mathbf{3 1 . 7} \\
\text { B: } \mathbf{1 7 . 0 ,} \mathbf{p}<\mathbf{0 . 0 0 1} \\
\text { Mean order to consult time, in hours } \\
\text { A: } \mathbf{1 6 . 2} \\
\text { B: } \mathbf{5 . 4 , p < 0 . 0 0 1} \\
\text { Door-to-consult time, in hours } \\
\text { A: } \mathbf{2 2 . 7} \\
\text { B: } \mathbf{1 0 . 5}, \mathbf{p}<\mathbf{0 . 0 0 1} \\
\text { Disposition } \\
\text { Inpatient observation } \\
\text { A: } 100 \% \\
\text { B: } 39 \% \\
\text { Home with followup } \\
\text { A: } 0 \% \\
\text { B: } 29 \% \\
\text { Tertiary care center } \\
\text { A: } 0 \% \\
\text { B: } 8 \% \\
\text { Behavioral facility } \\
\text { A: } 0 \\
\text { B: } 24 \%\end{array}$ & NR \\
\hline $\begin{array}{l}\text { Emergency } \\
\text { physician- } \\
\text { Airway } \\
\text { management }\end{array}$ & $\begin{array}{l}\text { Cho, } 2011^{235} \\
\text { Seoul, Korea } \\
2 \text { EDs }\end{array}$ & $\begin{array}{l}\text { RCT } \\
\text { High } \\
\text { A: Usual care, } n=13 \\
\text { B: Telehealth, } n=12\end{array}$ & $\begin{array}{l}\text { Complication rate: } p=0.36 \\
\text { Success rate: } p>0.05\end{array}$ & $\begin{array}{l}\text { Mean intubation time } \pm \text { SD, in seconds } \\
\text { A: } 56 \pm 2 \\
B: 62 \pm 12 \\
p=0.30\end{array}$ & NR \\
\hline $\begin{array}{l}\text { ED: } \\
\text { Cardiology }\end{array}$ & $\begin{array}{l}\text { Astarcioglu, } 2015^{136} \\
\text { Turkey } \\
1 \text { rural hospital }\end{array}$ & $\begin{array}{l}\text { Prospective cohort } \\
\text { High } \\
\text { A: Not telehealth, } n=55 \\
\text { B: Telehealth, } n=53\end{array}$ & $\begin{array}{l}\text { False STEMI: } \\
\text { A: } 8.3 \% \text {. } \\
\text { B: } 0 \%, \text { NS }\end{array}$ & $\begin{array}{l}\text { Mean time in minutes } \\
\text { Door to balloon time, } \\
\text { A: } 130 \\
\text { B: } \mathbf{1 0 9}, \mathbf{p}<0.001 \\
\text { Door to door time } \\
\text { A: } 109 \\
\text { B: } \mathbf{9 1 ,} \mathbf{p}<0.001 \\
\text { Catheterization lab to balloon } \\
\text { A: } 18 \\
\text { B: } 16, \mathrm{NS}\end{array}$ & NR \\
\hline
\end{tabular}




\begin{tabular}{|c|c|c|c|c|c|}
\hline $\begin{array}{l}\text { Clinical } \\
\text { Topic }\end{array}$ & $\begin{array}{l}\text { Author, Year } \\
\text { Location } \\
\text { Number of Sites }\end{array}$ & $\begin{array}{l}\text { Study Design } \\
\text { Risk of Bias } \\
\text { Comparison, } \mathbf{n}\end{array}$ & $\begin{array}{l}\text { Clinical Outcomes } \\
\text { Harms }\end{array}$ & Intermediate Outcomes & Economic \\
\hline $\begin{array}{l}\text { ED: } \\
\text { Cardiology }\end{array}$ & $\begin{array}{l}\text { Macedo, } 2016^{253} \\
\text { Sao Paulo, Brazil } \\
22 \text { EDs } \\
1 \text { reference hospital }\end{array}$ & $\begin{array}{l}\text { Before-after } \\
\text { Moderate } \\
\text { A: Before telehealth, } \\
n=113 \\
\text { B: After telehealth, } \\
n=263\end{array}$ & $\begin{array}{l}\text { In-hospital mortality } \\
\text { A: } 8 \% \\
\text { B: } 3 \% \\
p=0.06\end{array}$ & $\begin{array}{l}\text { Use of pharmacoinvasive strategy } \\
\text { A: } \mathbf{3 8 \%} \\
\text { B: } \mathbf{5 5 . 8 \%} \\
\text { p } \mathbf{0 . 0 0 2}\end{array}$ & NR \\
\hline ED: Burns & $\begin{array}{l}\text { Saffle, } 2009^{111} \\
\text { United States } \\
3 \text { hospitals and } 1 \\
\text { burn center }\end{array}$ & $\begin{array}{l}\text { Before-after } \\
\text { Moderate } \\
\text { A: Before telehealth, } \\
n=28 \\
\text { B: After telehealth, } \\
n=70\end{array}$ & $\begin{array}{l}\text { Mortality, n (\%) } \\
\text { A: } 1 \text { (3.6) } \\
\text { B: } 0\end{array}$ & $\begin{array}{l}\text { Air emergency transport } \\
\text { A: } 100 \% \\
\text { B: } 44.3 \%, p<0.05 \\
\text { Median LOS (IQR), in days } \\
\text { A: } 8(24) \\
\text { B: } 13(23), N S \\
\text { Satisfied with telemedicine visit } \\
\text { Burn center physicians: } 76.9 \% \\
\text { Referring physicians: } 86.4 \% \\
\text { Patients transferred: } 75.9 \% \\
\text { Patients not transferred: } 69.2 \% \\
\text { All respondents: } 78.2 \%\end{array}$ & NR \\
\hline ED: Cancer & $\begin{array}{l}\text { Hashimoto, } 2001^{241} \\
\text { Japan } \\
\text { One district hospital }\end{array}$ & $\begin{array}{l}\text { Before-after } \\
\text { High } \\
\text { A: Before telehealth, } \\
n=17 \\
\text { B: After telehealth, } \\
n=12\end{array}$ & $\begin{array}{l}\text { 1-year survival } \\
\text { A: NR } \\
\text { B: } 72 \% \\
2 \text { year survival } \\
\text { A: NR } \\
\text { B: } 42 \% \\
\text { Mean hospitalization time } \\
\text { A: NR } \\
\text { B: } 2.3 \text { months } \\
\text { Successful ambulation for } \\
\text { patients who were } \\
\text { nonambulant } \\
\text { A: } 25 \% \\
\text { B: } 83 \%, p<0.05\end{array}$ & $\begin{array}{l}\text { Treatment within } 24 \text { hours } \\
\text { A: } 17.6 \% \\
\text { B: } 92 \% \\
\text { Mean onset to radiotherapy time, in days } \\
\text { A: } \mathbf{7 . 1} \\
\text { B: } \mathbf{0 . 8}, \mathbf{p}<0.05\end{array}$ & NR \\
\hline
\end{tabular}




\begin{tabular}{|c|c|c|c|c|c|}
\hline $\begin{array}{l}\text { Clinical } \\
\text { Topic }\end{array}$ & $\begin{array}{l}\text { Author, Year } \\
\text { Location } \\
\text { Number of Sites }\end{array}$ & $\begin{array}{l}\text { Study Design } \\
\text { Risk of Bias } \\
\text { Comparison, } n\end{array}$ & $\begin{array}{l}\text { Clinical Outcomes } \\
\text { Harms }\end{array}$ & Intermediate Outcomes & Economic \\
\hline $\begin{array}{l}\text { ED: Pediatric } \\
\text { Fractures }\end{array}$ & $\begin{array}{l}\text { Zennaro, } 2014^{191} \\
\text { Italy } \\
\text { One hospital }\end{array}$ & $\begin{array}{l}\text { Pre-post } \\
\text { High } \\
\text { A: Pre-telehealth, } n=42 \\
\text { B: Post-telehealth, } \\
n=42\end{array}$ & NR & $\begin{array}{l}\text { In-hospital consultation required } \\
\text { A: } \mathbf{7 6 . 1 \%} \\
\text { B: } \mathbf{3 8 \% , p < 0 . 0 0 1} \\
\text { Immediate activation of other services } \\
\text { A: } 0 \\
\text { B: } \mathbf{3 3 . 3} \%, \mathbf{p}<0.001 \\
\text { Mean time for decision making, in } \\
\text { minutes } \\
\text { A: } \mathbf{5 6 . 2} \\
\text { B: } \mathbf{2 3 . 4 ,} p<0.001\end{array}$ & NR \\
\hline $\begin{array}{l}\text { ED: Plastic } \\
\text { Surgery }\end{array}$ & $\begin{array}{l}\text { Paik, } 2017^{97} \\
\text { NJ, United States } \\
1 \text { university hospital }\end{array}$ & $\begin{array}{l}\text { Retrospective cohort } \\
\text { High } \\
\text { A: Usual care, } n=42 \\
\text { B: Telehealth, } n=42\end{array}$ & NR & $\begin{array}{l}\text { Response time, in minutes } \\
\text { A: } \mathbf{4 8 . 3} \\
\text { B: } \mathbf{8 . 9}, \mathbf{p}<0.001 \\
\text { Overall agreement rate } \\
90.5 \%, n=38\end{array}$ & NR \\
\hline ED: Sepsis & $\begin{array}{l}\text { Machado, } 2018^{83} \\
\text { Columbus, } \mathrm{OH}, \\
\text { USA } \\
1 \text { ED }\end{array}$ & $\begin{array}{l}\text { Retrospective cohort } \\
\text { Clinical: Moderate } \\
\text { Cost: High } \\
\text { A: Usual care, } n=219 \\
\text { B: Telehealth, } n=95\end{array}$ & $\begin{array}{l}\text { Mortality, } n(\%) \\
\text { A: } 49(22.4 \%) \\
\text { B: } 25(26.3 \%), p=0.471\end{array}$ & $\begin{array}{l}\text { Mean time in minutes } \\
\text { To antibiotics } \pm \text { SD } \\
\text { A: } \mathbf{1 6 3 . 4} \pm \mathbf{2 0 4 . 4} \\
\text { B: } \mathbf{1 2 2 . 3} \pm \mathbf{8 3 . 3}, p=0.043 \\
\text { Antibiotics administered within } 3 \text { hours } \\
\text { A: } 71.2 \% \\
\text { B: } 82.1 \%, p=0.097 \\
\text { Mean ED LOS } \pm \text { SD, in days } \\
\text { A: } \mathbf{0 . 1 6} \pm \mathbf{0 . 3 7} \\
\text { B: } \mathbf{0 . 0 8} \pm \mathbf{0 . 2 8}, p=0.036 \\
\text { Mean hospital LOS } \pm \mathrm{SD} \text {, in days } \\
\text { A: } 10 \pm 8.5 \\
\text { B: } 8.6 \pm 5.7, p=0.088 \\
\text { Readmission in } 30 \text { days, } n(\%) \\
\text { A: } 25(11.4 \%) \\
\text { B: } 16(16.8 \%), p=0.204\end{array}$ & $\begin{array}{l}\text { Total hospital costs, } \\
\text { in USD } \\
\text { A: } \$ 24,364 \pm 25,068 \\
\text { B: } \$ 19,713 \pm 16,550 \\
p=0.274\end{array}$ \\
\hline
\end{tabular}




\section{Emergency Medical Services/Urgent Care}

Table 10 contains details and results from 21 studies reported in 23 articles in which telehealth was used to advise EMS personnel or urgent care providers caring for patients outside of the hospital. In these studies, telehealth was used to allow an emergency medicine physician or specialist to contribute to patient assessments and decisions about prehospital treatment and transport. Transport decisions include where the patient should be taken (e.g., the closest hospital, a trauma center, a cardiac center) and what mode of transport should be used (e.g., air, ambulance, personal vehicle).

More than half of these studies (11 of 21) involved transmitting information, frequently including electrocardiograms, about patients experiencing heart attack symptoms. EMS then received assistance with determining if the patient had ST-elevation myocardial infarction (STEMI), directions for preliminary treatment, and advise on whether to transport the patient directly to a location equipped to provide primary percutaneous coronary intervention (PCI). This is important because outcomes are better if patients are treated quickly after symptom onset and diagnosis. For this reason, similar to telestroke, a frequently reported outcome is time to treatment, in this case often referred to as time to balloon, referring to the use of a balloon to open blockages in cases of myocardial infarction (MI).

Among the other nine studies, six studies (reported in 7 articles) addressed the use of telehealth to inform triage and ultimately transport and treatment decisions for a broad range of patients served by EMS, and three (reported in 4 articles) assessed the utility of telehealth in urgent care.

Most studies (7 of 11) of prehospital decisions for STEMI patients were conducted in Europe: five in Italy, ${ }^{143,162,171,172,189}$ one in Germany, ${ }^{141}$ and two in Denmark. ${ }^{173,179}$ Additionally, one each were conducted in Canada, ${ }^{218}$ Brazil, ${ }^{254}$ and the United States. ${ }^{113}$ Similarly, the studies of triage and urgent care are international with most set in the United States, ${ }^{34,35,77,78,100,124}$ and others in South Korea, ${ }^{242}$ Austria, ${ }^{225}$ Taiwan, ${ }^{246}$ Ireland, ${ }^{197}$ and Germany. ${ }^{142}$

\section{Emergency Medical Service/Urgent Care Telehealth: Effectiveness in Improving Clinical and Economic Outcomes}

Ten studies reported clinical outcomes. Nine of these were studies of prehospital cardiac care and compared mortality (some in hospital, some 30-day, one 1-year) for MI patients assessed and treated with and without prehospital telehealth, and in seven of these, ${ }^{162,171-173,189,218,254}$ the mortality rates were significantly lower with telehealth. The studies of more general triage and urgent care did not report clinical outcomes with the exception of one study of pain medications administered by EMS, which reported that complications and nausea and vomiting did not differ. ${ }^{142}$

Five studies included comparisons of costs of staff and equipment or estimates of savings. One study of prehospital cardiac care in Brazil reported the mean cost of admissions was higher for patients treated with telehealth. ${ }^{254}$ Two studies (reported in three articles) of triage and urgent care reported savings due to lower transportation costs. ${ }^{77,78,246}$ One study reported that the telehealth costs were lower than the staff costs of providing the service without telehealth ${ }^{197}$ while another study that examined costs for patients and families as well as the health system reported no significant difference in patient costs and healthy system costs were slightly higher or no different depending on the model used to adjust the costs. ${ }^{204}$ In general, economic outcomes were not reported and the analyses were simple. 


\section{Emergency Medical Service/Urgent Care Telehealth: Effectiveness in Improving Intermediate Outcomes}

All of the included studies reported at least one intermediate outcome. In the studies of prehospital cardiac assessment, nine ${ }^{113,143,162,171-173,179,189,218}$ included a measure of time to treatment (e.g., time to treatment, percent treated within recommended time, total ischemic time), and all of these reported time to treatment was statistically significantly shorter with telehealth. Other outcomes included no difference in guideline adherence ${ }^{141}$ and a statistically significant increase in admissions to tertiary hospitals in a study conducted in Brazil. ${ }^{254}$

The available studies of general triage and treatment conclude that telehealth reduced the number of referrals or transfers. Two of the EMS studies evaluated teleconsultations on decisions about whether to air transport a patient from island locations (Penghu Islands, Taiwan or Palm Island Australia) to a distant hospital and found reductions in air transfers with patients either being treated in place or transferred another way. ${ }^{225,246}$ A study in the United States used telehealth to recommend whether a patient needed to be transported by ambulance or could take personal transportation to a primary care office or ED and found transport by ambulance and time for ambulances to return to service decreased while patient satisfaction did not change. ${ }^{77,78}$

Two urgent care studies included evaluations of minor injury centers where nurse practitioners or nurses provided treatments with telehealth input from physicians in Ireland and the United States. ${ }^{34,35,197}$ These found no significant differences in care needs or patient or clinician assessments.

\section{Emergency Medical Service/Urgent Care Telehealth: Harms, Adverse Events, or Negative Unintended Consequences}

Only one study of pain medications administered by EMS reported clinical outcomes that could also be considered harms or adverse events. A study of pain medication administration by EMS found that complications and nausea and vomiting did not differ. ${ }^{142}$

\section{Key Characteristics of EMS/Urgent Care Telemedicine and Impact of These on Outcomes}

Very little detail was provided in the studies of telehealth for support beyond the type of patients served and brief descriptions of what information was shared. Additionally, different uses of telehealth in this category are evaluated using very different outcomes (e.g., mortality and type of transport). This makes it difficult to identify any subgroups or characteristics that could be used to differentiate successful telehealth interventions within the categories provided (i.e., cardiac care, general triage, and urgent care). Additionally, the categories are fundamentally different, making comparisons across categories inappropriate. 


\begin{tabular}{|c|c|c|c|c|c|}
\hline $\begin{array}{l}\text { Clinical } \\
\text { Topic }\end{array}$ & $\begin{array}{l}\text { Author, Year } \\
\text { Location } \\
\text { Number of Sites }\end{array}$ & $\begin{array}{l}\text { Study Design } \\
\text { Risk of Bias } \\
\text { Comparison, } \mathrm{n}\end{array}$ & $\begin{array}{l}\text { Clinical Outcomes } \\
\text { Harms }\end{array}$ & Intermediate Outcomes & Economic \\
\hline $\begin{array}{l}\text { EMS - } \\
\text { Cardiac }\end{array}$ & $\begin{array}{l}\text { Brokmann, } 2016^{141} \\
\text { Aachen, Germany } \\
5 \text { ambulances } \\
1 \text { telehealth center }\end{array}$ & $\begin{array}{l}\text { Before-after } \\
\text { Moderate } \\
\text { A. Before telehealth, } \\
n=39 \\
\text { B. After telehealth, } \\
n=39\end{array}$ & $\begin{array}{l}\text { NR } \\
\text { No harms detected }\end{array}$ & $\begin{array}{l}\text { Guideline adherence number of } \\
\text { patients } \\
\text { 12-lead ECG } \\
\text { A: } 39 \\
\text { B: } 38, p>0.99 \\
\text { Acetylsalicylic acid } \\
\text { A: } 33 \text {. } \\
\text { B: } 31, p=0.73 \\
\text { Heparin } \\
\text { A: } 33 \\
\text { B: } 34, p>0.99 \\
\text { Morphine } \\
\text { A: } 27 \\
\text { B: } 29, p=0.50 \\
\text { Oxygen } \\
\text { A: } \mathbf{1 8 .} \\
\text { B: } \mathbf{2 9 ,} p=\mathbf{0 . 0 0 7}\end{array}$ & NR \\
\hline $\begin{array}{l}\text { EMS - } \\
\text { Cardiac }\end{array}$ & $\begin{array}{l}\text { Brunetti, } 2014^{143} \\
\text { Apulia, Italy } \\
\text { Meta-analysis: number } \\
\text { of sites NR }\end{array}$ & $\begin{array}{l}\text { Prospective cohort } \\
\text { Moderate } \\
\text { A: Usual care, } n=174 \\
\text { B: Telehealth, } n=123\end{array}$ & NR & $\begin{array}{l}\text { Mean time to balloon } \pm S D \text {, in } \\
\text { minutes } \\
\text { A: } \mathbf{9 4} \pm \mathbf{6 1} \\
\text { B: } \mathbf{4 1} \pm \mathbf{1 7}, \mathbf{p}<\mathbf{0 . 0 0 1}\end{array}$ & NR \\
\hline $\begin{array}{l}\text { EMS - } \\
\text { Cardiac }\end{array}$ & $\begin{array}{l}\text { Chan, } 2012^{218} \\
\text { Canada } \\
\text { Unclear }\end{array}$ & $\begin{array}{l}\text { Retrospective cohort } \\
\text { Low } \\
\text { A: Usual care, } n=427 \\
\text { B: Telehealth, } n=167\end{array}$ & $\begin{array}{l}\text { 30-day mortality } \\
\text { A: } \mathbf{1 3 . 3 \%} \\
\text { B: } \mathbf{5 . 4 \% , p = 0 . 0 0 6} \\
\text { 1-year mortality } \\
\text { A: } 17.5 \% \\
\text { B: } 6.6 \%, p=0.019\end{array}$ & $\begin{array}{l}\text { 90-minute door to balloon time } \\
\text { A: } \mathbf{8 . 7 \%} \\
\text { B: } \mathbf{8 0 . 4 \% , p < 0 . 0 0 1} \\
\text { Post-procedural TIMI flow } \\
\text { grade } 3 \\
\text { A: } \mathbf{9 1 . 4 \% .} \\
\text { B: } \mathbf{9 7 . 6 \% , p = 0 . 0 2}\end{array}$ & NR \\
\hline $\begin{array}{l}\text { EMS - } \\
\text { Cardiac }\end{array}$ & $\begin{array}{l}\text { Marcolino, } 2013^{254} \\
\text { Belo Horizonte, Brazil } \\
18 \text { basic support units } \\
6 \text { advanced support } \\
\text { units }\end{array}$ & $\begin{array}{l}\text { Before-after } \\
\text { Moderate } \\
\text { A: Before telehealth, } \\
n=1242 \\
\text { B: After telehealth, } \\
n=1358\end{array}$ & $\begin{array}{l}\text { AMI in-hospital mortality } \\
\text { A: } 12.3 \% \\
\text { B: } 7.1 \% \\
\text { p<0.001 }\end{array}$ & $\begin{array}{l}\text { AMI hospitalizations, including } \\
\text { ICU stay } \\
\text { A: } 32.4 \% \\
\text { B: } 66.1 \%, p<0.001 \\
\text { Proportion of AMI admitted to } \\
\text { tertiary hospitals } \\
\text { A: } 47.0 \% \\
\text { B: } 69.6 \%, p<0.001\end{array}$ & $\begin{array}{l}\text { Mean cost of } \\
\text { admission } \pm \mathrm{SD} \text {, in } \\
\text { Brazilian reals } \\
\text { A: } 2,480.00 \pm 4054 \\
B: 3,501.00 \pm 3202 \\
\text { p<0.001 }\end{array}$ \\
\hline
\end{tabular}




\begin{tabular}{|c|c|c|c|c|c|}
\hline $\begin{array}{l}\text { Clinical } \\
\text { Topic }\end{array}$ & $\begin{array}{l}\text { Author, Year } \\
\text { Location } \\
\text { Number of Sites }\end{array}$ & $\begin{array}{l}\text { Study Design } \\
\text { Risk of Bias } \\
\text { Comparison, } n \\
\end{array}$ & $\begin{array}{l}\text { Clinical Outcomes } \\
\text { Harms }\end{array}$ & Intermediate Outcomes & Economic \\
\hline $\begin{array}{l}\text { EMS - } \\
\text { Cardiac }\end{array}$ & $\begin{array}{l}\text { Martinoni, } 2011^{162} \\
\text { Italy } \\
\text { Unclear }\end{array}$ & $\begin{array}{l}\text { Retrospective cohort } \\
\text { Moderate } \\
\text { A: Usual care, no } \\
\text { ECG, n=2298 } \\
\text { B: Telehealth, } n=1603\end{array}$ & $\begin{array}{l}\text { 30-day mortality of patients } \\
\text { admitted by EMS } \\
\text { A: } \mathbf{7 . 9 \%} \\
\text { B: } \mathbf{5 . 3 \% , p = 0 . 0 6}\end{array}$ & $\begin{array}{l}\text { First medical contact to balloon, } \\
\text { median minutes (IQR) } \\
\text { A: } 75 \text { (49 to } 112) \\
\text { B: } 50 \text { (30 to78.5), } p<0.001\end{array}$ & NR \\
\hline $\begin{array}{l}\text { EMS - } \\
\text { Cardiac }\end{array}$ & $\begin{array}{l}\text { Ortolani, } 2007^{171} \\
\text { Italy } \\
1 \text { intervention lab }\end{array}$ & $\begin{array}{l}\text { Retrospective cohort } \\
\text { Low } \\
\text { A: Usual care, } n=79 \\
\text { B: Telehealth, } n=42\end{array}$ & $\begin{array}{l}\text { In-hospital cardiac mortality: } \\
\text { A: } 44 \% \\
\text { B: } 21 \% \\
\text { OR: } 0.34, p=0.02 \\
\text { In-hospital all-cause mortality: } \\
\text { A: } 46 \% \text {. } \\
\text { B: } 21 \% \\
\text { OR } 0.32, p=0.01 \\
\text { 1-year survival rate: } \\
\text { A: } 52 \% \text {. } \\
\text { B: } 74 \% \\
\text { OR: } N R, p=0.019\end{array}$ & $\begin{array}{l}\text { Median total ischemic time } \\
\text { (IQR), in minutes } \\
\text { A: } 212(150 \text { to } 366) \\
\text { B: } 142(106 \text { to } 187) \\
\text { Total Ischemic time }<120 \mathrm{~mm} \\
\text { A: } 40(51 \%) . \\
\text { B: } \mathbf{3 2 ( 7 6 \% ) , p = . 0 1}\end{array}$ & NR \\
\hline $\begin{array}{l}\text { EMS - } \\
\text { Cardiac }\end{array}$ & $\begin{array}{l}\text { Ortolani, } 2006^{172} \\
\text { Bologna, Italy } \\
\text { Unclear }\end{array}$ & $\begin{array}{l}\text { Retrospective cohort } \\
\text { Low } \\
A_{1}: \text { Usual care, ED, } \\
n=316 \\
A_{2}: \text { Usual care, local } \\
\text { hospital, } n=176 \\
\text { B: Telehealth, } 166\end{array}$ & $\begin{array}{l}\text { Overall Mortality, } n(\%) \\
\text { A1: } 23(7.3) \\
\text { A2: } 13(7.4) \\
\text { A3: } 8(4.8) \text { NS } \\
\text { In-hospital mortality among } \\
\text { cardiogenic shock subgroup } \\
\text { ( } n=80) \\
\text { A: } 48.1 \%(13 / 27) \\
\text { B: } \mathbf{3 7 . 5 \% ( 9 / 2 4 )} \\
\text { C: } \mathbf{1 3 . 8 \% ( 4 / 2 9 )} \\
\text { p=0.019 }\end{array}$ & $\begin{array}{l}\text { Median treatment delay (IQR), } \\
\text { in minutes } \\
\left.A_{1}: 191 \text { (135 to } 318.7\right) \\
A_{2}: 236(163.7 \text { to } 363.2) \\
B: 146 \text { (108.2 to } 214.5) \\
p=0.001\end{array}$ & NR \\
\hline $\begin{array}{l}\text { EMS - } \\
\text { Cardiac }\end{array}$ & $\begin{array}{l}\text { Pedersen, } 2009^{173} \\
\text { Denmark } \\
\text { Ambulance number NR } \\
1 \text { high volume center }\end{array}$ & $\begin{array}{l}\text { Prospective cohort } \\
\text { Low } \\
\text { A: Usual care, } n=821 \\
\text { B: Telehealth, } n=616\end{array}$ & $\begin{array}{l}\text { All-cause mortality or nonfatal MI } \\
\text { hazard ratio }(95 \% \mathrm{Cl}) \\
\mathbf{0 . 6 7} ;(\mathbf{0 . 4 6} \text { to } \mathbf{0 . 9 7}), \mathbf{p}=\mathbf{0 . 0 3 5}\end{array}$ & $\begin{array}{l}\text { Median door to balloon time } \\
\text { (IQR), in minutes } \\
\text { A: } 103(80 \text { to } 135) \\
\text { B: } 83(67 \text { to } 100) \\
\text { p }<0.001\end{array}$ & NR \\
\hline
\end{tabular}




\begin{tabular}{|c|c|c|c|c|c|}
\hline $\begin{array}{l}\text { Clinical } \\
\text { Topic }\end{array}$ & $\begin{array}{l}\text { Author, Year } \\
\text { Location } \\
\text { Number of Sites } \\
\end{array}$ & $\begin{array}{l}\text { Study Design } \\
\text { Risk of Bias } \\
\text { Comparison, } n \\
\end{array}$ & $\begin{array}{l}\text { Clinical Outcomes } \\
\text { Harms }\end{array}$ & Intermediate Outcomes & Economic \\
\hline $\begin{array}{l}\text { EMS - } \\
\text { Cardiac }\end{array}$ & $\begin{array}{l}\text { Sanchez-Ross, } 2011^{113} \\
\text { NJ, United States } \\
1 \text { university hospital }\end{array}$ & $\begin{array}{l}\text { Prospective cohort } \\
\text { Moderate } \\
\text { A: Not telehealth, } \\
n=50 \\
\text { B: Telehealth, } n=92\end{array}$ & $\begin{array}{l}\text { Mortality } \\
\text { A: } 6 \% \\
\text { B: } 1.1 \%, \text { NS }\end{array}$ & $\begin{array}{l}\text { Median door to balloon time } \\
\text { (IQR), in minutes } \\
\text { A: } 119 \text { ( } 96 \text { to } 178 \text { ) } \\
\text { B: } 63 \text { ( } 42 \text { to } 87), p<0.0004 \\
\text { Median LOS (IQR), in days } \\
\text { A: } 5.5 \text { ( } 3.5 \text { to } 10.5 \text { ) } \\
\text { B: } 3 \text { ( } 2 \text { to } 4), p<0.001\end{array}$ & NR \\
\hline $\begin{array}{l}\text { EMS - } \\
\text { Cardiac }\end{array}$ & $\begin{array}{l}\text { Sejersten, } 2008^{179} \\
\text { Denmark } \\
4 \text { local hospitals } \\
2 \text { coronary centers }\end{array}$ & $\begin{array}{l}\text { Prospective cohort } \\
\text { Moderate } \\
\text { A: Usual care, } n=89 \\
\text { B: Telehealth, } n=168\end{array}$ & $\begin{array}{l}\text { Mortality } \\
\text { A: } 6.9 \% \\
\text { B: } 6.0 \% \\
p=0.67 \\
\text { Arrhythmia } \\
\text { A: } 7 \% \\
\text { B: } 10 \%, p=0.37\end{array}$ & $\begin{array}{l}911 \text { call } \mathrm{PCl} \text {, median minutes } \\
\text { (IQR) } \\
\text { A: } 127 \text { (103 to } 151) \\
\text { B: } 74 \text { (64 to } 94 \text { ), } p<0.001 \\
\text { Door to } \mathrm{PCl} \text {, median minutes } \\
\text { (IQR) } \\
\text { A: } 97 \text { (80 to } 124) \\
\text { B: } 34 \text { (19 to } 46), p<0.001\end{array}$ & NR \\
\hline $\begin{array}{l}\text { EMS - } \\
\text { Cardiac }\end{array}$ & $\begin{array}{l}\text { Zanini, } 2008^{189} \\
\text { Mantova, Italy } \\
6 \text { local hospitals } \\
1 \text { acute care }\end{array}$ & $\begin{array}{l}\text { Retrospective cohort } \\
\text { Moderate } \\
\text { A: Usual care, } n=263 \\
\text { B: Telehealth, } n=136\end{array}$ & $\begin{array}{l}\text { In-hospital Mortality } \\
\text { Total } \\
\text { A: } \mathbf{8 . 7 \%} \\
\text { B: } \mathbf{3} \%, \mathbf{p}=\mathbf{0 . 0 3 9} \\
\text { PCI } \\
\text { A: } 7.2 \% \\
\text { B: } 3.3 \%, p=0.14\end{array}$ & $\begin{array}{l}\text { Onset to balloon time } \pm S D \text {, in } \\
\text { minutes } \\
\text { A: } \mathbf{2 6 2} \pm \mathbf{1 1 2} \\
\text { B: } \mathbf{1 4 8} \pm \mathbf{8 1}, \mathbf{p}<0.001\end{array}$ & NR \\
\hline
\end{tabular}




\begin{tabular}{|c|c|c|c|c|c|}
\hline $\begin{array}{l}\text { Clinical } \\
\text { Topic }\end{array}$ & $\begin{array}{l}\text { Author, Year } \\
\text { Location } \\
\text { Number of Sites }\end{array}$ & $\begin{array}{l}\text { Study Design } \\
\text { Risk of Bias } \\
\text { Comparison, } n\end{array}$ & $\begin{array}{l}\text { Clinical Outcomes } \\
\text { Harms }\end{array}$ & Intermediate Outcomes & Economic \\
\hline $\begin{array}{l}\text { EMS - } \\
\text { Triage and } \\
\text { Transport }\end{array}$ & $\begin{array}{l}\text { Kim, } 2011^{72} \\
\text { Wonju, South Korea } \\
6 \text { ambulances; } 1 \text { hospital }\end{array}$ & $\begin{array}{l}\text { Retrospective cohort } \\
\text { High } \\
\text { A: Not telehealth, } \\
n=750 \\
\text { B: Telehealth, } n=188\end{array}$ & NR & $\begin{array}{l}\text { Mean time in minutes } \\
\text { Arrival to scene } \\
\text { A: } 6.6 \\
\text { B: } 6.6, \mathrm{NS} \\
\text { Treatment time at the scene } \\
\text { A: } \mathbf{6 . 3} \\
\text { B: } \mathbf{4 . 4 ,} \mathbf{p}<0.001 \\
\text { Mean transport time } \\
\text { A: } \mathbf{1 5 . 8} \\
\text { B: } \mathbf{1 9 . 4 ,} \boldsymbol{p}<0.001 \\
\% \text { receiving medical direction } \\
\text { for treatment } \\
\text { A: } \mathbf{0 . 3 \%} \\
\text { B: } \mathbf{8 . 0 \% ,} \mathbf{p}<0.001 \\
\% \text { receiving medical direction } \\
\text { for ambulance diversion } \\
\text { A: } \mathbf{0 . 1 \%} \\
\text { B: } \mathbf{1 4 . 4 \% ,} \mathbf{p}<0.001\end{array}$ & NR \\
\hline $\begin{array}{l}\text { EMS - } \\
\text { Triage and } \\
\text { Transport }\end{array}$ & $\begin{array}{l}\text { Langabeer, 2016;78 } \\
\text { Langabeer, } 2017^{77} \\
\text { TX, United States } \\
1 \text { Fire department }\end{array}$ & $\begin{array}{l}\text { Retrospective cohort } \\
\text { Moderate } \\
\text { Economic evaluation } \\
\text { High } \\
\text { A: Usual care, } n=5570 \\
\text { B: Teleconsult, } \\
n=5570\end{array}$ & $\begin{array}{l}\text { Mortality } \\
\text { A: } 0 \% \\
\text { B: } 0 \%\end{array}$ & $\begin{array}{l}\text { Disposition to ED by } \\
\text { ambulance } \\
\text { A: } 74 \% \\
\text { B: } \mathbf{1 8 \%}, \mathbf{p}<0.001 \\
\text { Patient satisfaction } \\
\text { A: } 87 \% \text {. } \\
\text { B: } 88 \%, p=0.250 \\
\\
\text { Total back in service time, } \\
\text { median minutes (IQR) } \\
\text { A: } 83(20 \text { to } 140) \\
\text { B: } 39(27 \text { to } 90), p<0.001\end{array}$ & $\begin{array}{l}\text { Average unit cost } \\
\text { per patient } \pm \text { SD, in } \\
\text { USD } \\
\text { A: } 270 \pm 77.7 \\
\text { B: } 167 \pm 42.7 \\
\text { p<0.0001 }\end{array}$ \\
\hline
\end{tabular}




\begin{tabular}{|c|c|c|c|c|c|}
\hline $\begin{array}{l}\text { Clinical } \\
\text { Topic }\end{array}$ & $\begin{array}{l}\text { Author, Year } \\
\text { Location } \\
\text { Number of Sites }\end{array}$ & $\begin{array}{l}\text { Study Design } \\
\text { Risk of Bias } \\
\text { Comparison, } n\end{array}$ & $\begin{array}{l}\text { Clinical Outcomes } \\
\text { Harms }\end{array}$ & Intermediate Outcomes & Economic \\
\hline $\begin{array}{l}\text { EMS - } \\
\text { Triage and } \\
\text { Transport }\end{array}$ & $\begin{array}{l}\text { Mathews, } 2008^{225} \\
\text { Australia } \\
1 \text { community }\end{array}$ & $\begin{array}{l}\text { Before-after } \\
\text { High } \\
\text { A: Before telehealth, } \\
n=78 \\
\text { B: After telehealth, } \\
n=113\end{array}$ & NR & 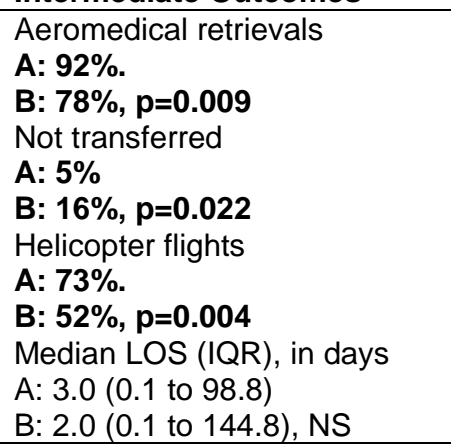 & NR \\
\hline $\begin{array}{l}\text { EMS - } \\
\text { Triage and } \\
\text { Transport }\end{array}$ & $\begin{array}{l}\text { Traub, 2013 }{ }^{124} \\
\text { United States } \\
1 \mathrm{ED}\end{array}$ & $\begin{array}{l}\text { Retrospective cohort } \\
\text { High } \\
\text { A: Usual care, } n=196 \\
\text { B: Telehealth, } n=106 \\
\text { (36 used telehealth) }\end{array}$ & NR & $\begin{array}{l}\text { Mean } \pm \text { SD } \\
\text { Admitted } \\
\text { A: } 64 \pm 32.7 \\
\text { B: } 12 \pm 33.3, p=0.936 \\
\text { LOS } \pm \text { SD }(95 \% \text { Cl), minutes } \\
\text { A: } 258 \pm 172(234 \text { to } 282) \\
\text { B: } 274 \pm 125 \text { (231 to } 316), \\
\text { p=0.525 } \\
\text { Time to physician evaluation } \pm \\
\text { SD (95\% CI), minutes } \\
\text { Full Sample: NS } \\
\text { Subgroup: } n=36 \\
\text { A: } \mathbf{4 2} \pm \mathbf{3 1} \text { ( } \mathbf{3 8} \text { to } 46) \\
\text { B: } \mathbf{1 6} \pm \mathbf{1 5}(\mathbf{1 1} \text { to } \mathbf{2 1 )}, \mathbf{p}<\mathbf{0 . 0 0 1}\end{array}$ & NR \\
\hline $\begin{array}{l}\text { EMS - } \\
\text { Triage and } \\
\text { Transport }\end{array}$ & $\begin{array}{l}\text { Tsai, } 2007^{246} \\
\text { Pengu Island, Taiwan } \\
4 \text { rural EDs } \\
1 \text { rescue command } \\
\text { center }\end{array}$ & $\begin{array}{l}\text { Prospective cohort } \\
\text { Low } \\
\text { A: No telehealth, } \\
n=685 \\
\text { B: Telehealth, } n=137\end{array}$ & NR & $\begin{array}{l}\text { Average flights per month } \\
\text { A: } 19.6 \\
\text { B: } 12.5\end{array}$ & $\begin{array}{l}\text { Annual savings on } \\
\text { emergency air } \\
\text { medical transports, } \\
\text { USD: \$ } \\
448,986\end{array}$ \\
\hline
\end{tabular}




\begin{tabular}{|c|c|c|c|c|c|}
\hline $\begin{array}{l}\text { Clinical } \\
\text { Topic }\end{array}$ & $\begin{array}{l}\text { Author, Year } \\
\text { Location } \\
\text { Number of Sites }\end{array}$ & $\begin{array}{l}\text { Study Design } \\
\text { Risk of Bias } \\
\text { Comparison, } n\end{array}$ & $\begin{array}{l}\text { Clinical Outcomes } \\
\text { Harms }\end{array}$ & Intermediate Outcomes & Economic \\
\hline $\begin{array}{l}\text { EMS - } \\
\text { Triage and } \\
\text { Transport }\end{array}$ & $\begin{array}{l}\text { Patel, } 2015^{100} \\
\text { DE, United States } \\
1 \text { pediatric transport } \\
\text { team } \\
1 \text { command hospital }\end{array}$ & $\begin{array}{l}\text { RCT } \\
\text { Moderate } \\
\text { A: Cell phone, } n=25 \\
\text { B: Telehealth, } n=25\end{array}$ & $\mathrm{NR}$ & $\begin{array}{l}\text { Average call duration, seconds } \\
\text { A: } 186 \\
\text { B: } 139, p=0.055 \\
\text { Medical Command Officer } \\
\text { Survey, } n=12: \\
100 \% \text { found video intuitive } \\
92 \% \text { disposition based on } \\
\text { phone report was difficult } \\
80 \% \text { video provided better } \\
\text { understanding of patient } \\
\text { condition } \\
70 \% \text { video assisted disposition } \\
80 \% \text { believed video should be } \\
\text { used for transport }\end{array}$ & NR \\
\hline $\begin{array}{l}\text { EMS - } \\
\text { Analgesia }\end{array}$ & $\begin{array}{l}\text { Brokmann, 2016 }{ }^{142} \\
\text { Aachen, Germany } \\
5 \text { ambulances } \\
1 \text { telehealth center }\end{array}$ & $\begin{array}{l}\text { Before-after } \\
\text { Moderate } \\
\text { A: Before telehealth, } \\
n=80 \\
\text { B: After telehealth, } \\
n=80\end{array}$ & $\begin{array}{l}\text { Complications } \\
\text { A: } 0 \\
\text { B: } 0 \\
\text { Nausea and vomiting } \\
\text { A: } 11 \% \\
\text { B: } 11 \%\end{array}$ & $\begin{array}{l}\text { Reached adequate prehospital } \\
\text { pain reduction }{ }^{\mathrm{a}}, \\
\text { A: } 31 / 32 \text { patients } \\
\text { B: } 61 / 65 \text { patients, NS } \\
\text { Mean pain reduction during } \\
\text { mission in NRS points } \pm \mathrm{SD} \\
\text { A: } 4.38 \pm \mathbf{2 . 2} \text { points } \\
\text { B: } \mathbf{3 . 7 8} \pm \mathbf{2 . 0} \text { points } \\
\mathbf{p}=\mathbf{0 . 0 1 5 9}\end{array}$ & NR \\
\hline
\end{tabular}




\begin{tabular}{|c|c|c|c|c|c|}
\hline $\begin{array}{l}\text { Clinical } \\
\text { Topic }\end{array}$ & $\begin{array}{l}\text { Author, Year } \\
\text { Location } \\
\text { Number of Sites }\end{array}$ & $\begin{array}{l}\text { Study Design } \\
\text { Risk of Bias } \\
\text { Comparison, } n\end{array}$ & $\begin{array}{l}\text { Clinical Outcomes } \\
\text { Harms }\end{array}$ & Intermediate Outcomes & Economic \\
\hline Urgent Care & $\begin{array}{l}\text { Brennan, 1998;35 } \\
\text { Brennan, } 1999^{34} \\
\text { NJ, United States } \\
2 \text { hospitals } \\
1 \text { central site }\end{array}$ & $\begin{array}{l}\text { RCT } \\
\text { High } \\
\text { A: Usual care, } n=50 \\
\text { B: Telehealth, } n=50\end{array}$ & NR & $\begin{array}{l}\text { Mean throughput time, minutes } \\
\text { A: } 117 \\
\text { B: } 106 \\
72 \text { hour return visit } \\
\text { A: } 0 \% \text {. } \\
\text { B: } 0 \% \text { NS } \\
\text { Need for additional care } \\
\text { A: } 2.4 \% \text {. } \\
\text { B: } 2.3 \% \text { NS } \\
\text { Positive patient-physician } \\
\text { interaction } \\
\text { A: } 100 \% \\
\text { B: } 98 \% \\
\text { Positive patient-nurse } \\
\text { interaction } \\
\text { A: } 98 \% \\
\text { B: } 98 \% \\
\text { Overall patient satisfaction } \\
\text { A: } 95 \% . \\
\text { B: } 98 \% \text { NS }\end{array}$ & NR \\
\hline Urgent Care & $\begin{array}{l}\text { Darkins,1996 } 197 \\
\text { Ireland } \\
1 \text { minor treatment center }\end{array}$ & $\begin{array}{l}\text { Before-after } \\
\text { High } \\
\text { A: Before telehealth, } \\
n=6729 \\
\text { B: After telehealth, } \\
n=9972\end{array}$ & NR & $\begin{array}{l}\text { Referred to ED: } \\
\text { A: } 2.3 \% \\
\text { B: } 1.5 \% \\
\text { Referred to primary care: } \\
\text { A: } 11.9 \% \\
\text { B: } 3.8 \%\end{array}$ & $\begin{array}{l}\text { Direct costs per } \\
\text { year } \\
A: £ 50,000 \text { for } \\
\text { onsite staff } \\
B: £ 7,250 \text { for } \\
\text { equipment }\end{array}$ \\
\hline
\end{tabular}




\begin{tabular}{|c|c|c|c|c|c|}
\hline $\begin{array}{l}\text { Clinical } \\
\text { Topic }\end{array}$ & $\begin{array}{l}\text { Author, Year } \\
\text { Location } \\
\text { Number of Sites }\end{array}$ & $\begin{array}{l}\text { Study Design } \\
\text { Risk of Bias } \\
\text { Comparison, } n\end{array}$ & $\begin{array}{l}\text { Clinical Outcomes } \\
\text { Harms }\end{array}$ & Intermediate Outcomes & Economic \\
\hline Urgent Care & $\begin{array}{l}\text { Noble, } 2005^{204} \\
\text { United Kingdom } \\
\text { Single hospital ED }\end{array}$ & $\begin{array}{l}\text { Economic evaluation } \\
\text { Low } \\
\text { A: Not telehealth, } \\
n=191 \\
\text { B: Telehealth, } n=62\end{array}$ & $\mathrm{NR}$ & $\begin{array}{l}\text { Returned to normal activity in } 7 \\
\text { days }(95 \% \mathrm{Cl}) \\
\text { A: } 47.6 \%(34.9 \% \text { to } 60.6 \%) \\
\text { B: } 47.0 \%(41.0 \% \text { to } 53.2 \%)\end{array}$ & $\begin{array}{l}\text { Mean 7-day cost } \\
\text { difference per } \\
\text { patient }(95 \% \mathrm{Cl}) \\
\text { [95\% bias corrected } \\
\text { CI] } \\
\text { NHS Cost: } \\
£ 39.5(-1.3 \text { to } 80.2) \\
\text { [28.3 to } 73.7] \\
\text { Patient/family cost: } \\
£ 14.3(-26.6 \text { to } \\
55.2)[-11.2 \text { to } 25.9] \\
\text { Total cost: } \\
£ 53.75(-6.97 \text { to } \\
114.46)[24.10 \text { to } \\
101.81]\end{array}$ \\
\hline
\end{tabular}

AMI = acute myocardial infarction; $\mathrm{CI}$ = confidence interval; ECG = electrocardiogram; ED = emergency department; EMS = emergency medical services; GBP = British Pound; ICU = intensive care unit; IQR = interquartile range; LOS = length of stay; NHS = National Health Services; NR = not reported; NS = not significant; NRS = Numerical Rating Scale; $\mathrm{OR}$ = odds ratio; $\mathrm{PCI}$ = percutaneous coronary intervention; $\mathrm{RCT}$ = randomized controlled trial; $\mathrm{SD}$ = standard deviation; $\mathrm{TIMI}=$ thrombolysis in myocardial infarction; USD = United States dollars

Bold=statistically significant; telehealth superior. Bold and italicized=statistically significant; telehealth inferior. Regular type: not statistically significant.

${ }^{a}$ A reduction of the Numerical Rating Scale of $\geq 2$ points or NRS $<5$ at end of mission 


\section{Outpatient Consultation Results}

Collaboration with a specialist as part of outpatient care is what is mostly commonly thought of as a healthcare consultation. We identified 106 articles that evaluated telehealth consultations used to inform diagnosis, treatment, or management of patients receiving care in the outpatient setting. These studies span several specialties and use several different technologies to facilitate consultations. They also vary in the outcomes used to assess effectiveness. To capture and organize this diverse group of studies, we presented the results in three ways. First, we provided an overview of the results summarized by clinical topic in Table 11. Second, the key results are described in text accompanying selected results for each study provided in tables by clinical topic. Third, we looked across clinical topics and summarize how the results for outpatient consultations address the Key Questions for this review.

\section{Organization of Evidence}

The 106 included articles evaluating telehealth consultations in the outpatient setting are summarized in Table 11 below. They are grouped in 11 clinical topics, eight of which are separate specialties wherein we identified three or more articles (i.e., dermatology, wound care, ophthalmology, orthopedics, dentistry, cancer, psychiatry, and infectious diseases). The remaining articles are organized in three additional categories. The last category in the overview table and this section consists of studies of programs designed to facilitate consultations with multiple specialists. These programs connect primary care providers to a hospital or group of specialists rather than one specific specialty. We split the remaining specialties containing one or two included articles into two categories. The first consists of consultations using diagnostic technology as part of the consultation, including echocardiograms, ultrasounds, endoscopies, and Dopplers. In these studies consultations were conducted using real time transmission of images and data, and in some, specialists guided the technician on their use. The other group includes articles about specific specialty consultations that do not use diagnostic technology. Most of these studies evaluate telehealth consultations in the management of chronic conditions including hypertension, diabetes, arthritis and chronic pain.

Given the volume of evidence and range of topics, the key points are followed by the findings across the clinical topics. Then for each of the 11 clinical topics there is a short narrative description of the evidence followed by a table with details from each study.

\section{Outpatient Key Points}

- Clinical outcomes: Clinical outcomes were reported in just over one-quarter of the studies of telehealth consultations and in 7 of 11 clinical topics. In three topics, there is moderate strength of evidence of the benefits of telehealth (better healing in wound care, higher response to treatment in psychiatry, and improvement in chronic condition outcomes), and in dermatology the findings show no difference in clinical outcomes (low strength of evidence). In three topics (cancer, infectious disease, and multiple specialties) studies were identified, but the results were inconsistent (insufficient evidence).

- Intermediate outcomes

o Access: Telehealth consultations improved access by reducing wait times and time to treatment and by increasing the number of patients receiving indicated diagnostic tests or treatment (moderate strength of evidence).

o Management and utilization: Telehealth consultations reduced utilization (the number of in-person specialist and hospital visits; number of hospitalizations, and shorter 
lengths of stay) in most studies. Findings were inconsistent about agreement on diagnosis and management (low strength of evidence).

o Satisfaction: Patients were generally more satisfied with telehealth consultations, particularly when telehealth saved time or expense compared with the alternative. Clinicians tended to be less satisfied with telehealth than in-person consultations, though differences were rarely statistically significant (low strength of evidence).

- Costs: Studies report lower costs and in most cases savings are attributable to reductions in transfers or less transportation. However, the rigor of the measurement, imprecision of estimates and inconsistency in the magnitude of the effects limits confidence in these findings (low strength of evidence).

- Harms: Only two of studies explicitly examined harms, reporting lower rates of complications with telehealth (insufficient evidence).

Table 11. Outpatient care consultations: summary of evidence

\begin{tabular}{|c|c|c|c|c|c|}
\hline $\begin{array}{l}\text { Clinical } \\
\text { Topics }\end{array}$ & $\begin{array}{l}\text { Number of } \\
\text { Studies }\end{array}$ & $\begin{array}{l}\text { Clinical Outcomes and } \\
\text { Harms }\end{array}$ & Intermediate Outcomes & Costs & Citations \\
\hline Ophthalmology & 6 & No evidence & $\begin{array}{l}\text { * Increased screening and } \\
\text { treatment; fewer } \\
\text { surgeon visits; high } \\
\text { satisfaction }\end{array}$ & $\begin{array}{l}\text { Difference } \\
\text { only due to } \\
\text { patient travel } \\
\text { and avoided } \\
\text { transport }\end{array}$ & $\begin{array}{l}122,132,185,188 \\
215,219\end{array}$ \\
\hline Dentistry & 4 & No evidence & $\begin{array}{l}\text { * Reduced time to } \\
\text { treatment }\end{array}$ & $\begin{array}{l}<\text { Outreach } \\
\text { clinics less } \\
\text { expensive } \\
\text { than telehealth } \\
\end{array}$ & $154,167,177,205$ \\
\hline Cancer & $\begin{array}{l}9 \text { in } 10 \\
\text { articles }\end{array}$ & * Fewer side effects & $\begin{array}{l}\text { * Some decrease in time } \\
\text { to treatment; increased } \\
\text { adherence to guidelines } \\
\text { * Satisfaction better or no } \\
\text { different }\end{array}$ & $\begin{array}{l}\text { * Lower costs if } \\
\text { number of } \\
\text { consults is } \\
\text { sufficient }\end{array}$ & $\begin{array}{l}32,52,70,112,181 \\
182,198,200,230 \\
234\end{array}$ \\
\hline Psychiatry & $\begin{array}{l}3 \text { studies } \\
\text { (in } 5 \\
\text { articles) }\end{array}$ & $\begin{array}{l}\text { * Higher response to } \\
\text { treatment; decreased } \\
\text { symptoms }\end{array}$ & * Higher satisfaction & No evidence & $46,56-59$ \\
\hline
\end{tabular}




\begin{tabular}{|c|c|c|c|c|c|}
\hline $\begin{array}{l}\text { Clinical } \\
\text { Topics }\end{array}$ & $\begin{array}{l}\text { Number of } \\
\text { Studies }\end{array}$ & $\begin{array}{l}\text { Clinical Outcomes and } \\
\text { Harms }\end{array}$ & Intermediate Outcomes & Costs & Citations \\
\hline $\begin{array}{l}\text { Single } \\
\text { Specialties }\end{array}$ & 17 & $\begin{array}{l}\text { * Improvements in } \\
\text { chronic condition } \\
\text { outcomes }\end{array}$ & $\begin{array}{l}\text { ? Effects on satisfaction } \\
\text { and management are } \\
\text { unclear }\end{array}$ & $\begin{array}{l}\text { Some limited } \\
\text { impact on } \\
\text { costs }\end{array}$ & $\begin{array}{l}31,42,61,69,74,92 \\
144,145,168,186, \\
194,210,217,220 \\
232,248,250\end{array}$ \\
\hline $\begin{array}{l}\text { Multiple } \\
\text { Specialties }^{a}\end{array}$ & $\begin{array}{l}8 \\
\text { (reported in } \\
10 \text { articles) }\end{array}$ & No evidence & $\begin{array}{l}\text { * Improved management; } \\
\text { higher satisfaction } \\
\text { ? Unclear impact on } \\
\text { emergency department } \\
\text { and hospitalizations } \\
\end{array}$ & $\begin{array}{l}\text { ? Mixed: lower } \\
\text { costs in two } \\
\text { studies; higher } \\
\text { in one large } \\
\text { trial }\end{array}$ & $\begin{array}{l}28,38,60,152,157, \\
184,207,208,212, \\
229\end{array}$ \\
\hline
\end{tabular}

Key: * superior (telehealth benefit), no difference, < inferior (comparison better than telehealth), ? inconclusive (inconsistent results)

a These studies evaluated programs that made consultations available covering different numbers of specialties (i.e., ranging from

4 to 28 ) or any specialty available (disciplines not specified)

\section{Detailed Results}

\section{Results Across Clinical Topics}

\section{Outpatient Telehealth Consultations: Effectiveness in Improving Clinical and Economic Outcomes}

Approximately one-quarter of studies (28) reported clinical outcomes. These were concentrated - though not exclusively limited - to studies in which the consulting relationship was ongoing and involved treating and managing a condition over time as opposed to a single consultation. Most of these (22 of 28) reported a benefit from telehealth. For example, three studies about psychiatric consultations, four about wound care, and three about infectious disease all reported positive outcomes such as reduced symptoms, faster healing, or reduced viral load. Most, but not all of these studies, involved real time, video consultations, and patients were often present. In other studies, specialists reviewed updated records, including images or test results and contacted the treating physician with recommended changes in treatment or requested more information. The four studies of wound care used store and forward approaches to provide images and information, which the specialist reviewed when available and used them to develop a treatment plan that was communicated to the patient and referring clinician at separate time. Three dermatology studies in which the consultation was limited to a single interaction for diagnosis and initial management recommendations reported improvement in patients' conditions or that the clinical course did not differ between telehealth and in-person consultations.

Just under one-third of the studies (32) about outpatient consultations included some assessment of cost or economic impact. These varied from basic estimates of travel costs to detailed assessments of the different sources of fixed and variable costs. However, most are comparatively simple, and while about half (14) of the studies reported some cost savings for teleconsultations, these were mostly limited to avoided travel costs and loss of productivity for patients. In a small number of studies (4), telehealth consultations were not less expensive: for example a study of dental consultations to underserved communities concluded that telehealth consultations were more expensive than outreach visits by dentists, ${ }^{194}$ and a study of a network 
linking primary care to multiple specialists via video found telehealth consultations to be more expensive due to treatment costs and the extra time required to have both the specialist and primary care physician available for real time video consultations. ${ }^{207}$

\section{Outpatient Telehealth Effectiveness in Improving Intermediate Outcomes}

Most studies of outpatient telehealth consultations used intermediate outcomes to assess efficacy. These outcomes included impacts on access to services, health services utilization and the management of patients' conditions, and patient and provider satisfaction. Overall, the results support the use of telehealth consultations, though the amount of evidence varies across the different intermediate outcomes.

Thirty-five studies evaluated whether telehealth consultations improved access to services. We interpreted increased access to include both timelier access to services as well as increased rates of use. These were concentrated in dermatology (6 studies), studies of multiple specialties (6 studies), specialty consultations that included diagnostic technology (3 studies), and ophthalmology (3 studies). The impact on access is related to the type of care. For example, in the dermatology studies, telehealth consultations reduced wait time and time to treatment, and studies of consults with diagnostic technology reported increased numbers of patients receiving indicated tests and in less time with telehealth.

Thirty-one studies reported outcomes related to utilization and management. In some clinical categories, only one study addressed these outcomes, while in other clinical categories, as many as 10 articles studied utilization and management. Not unexpectedly, telehealth consultations reduced the number of in-person specialist and hospital visits; they also were associated with fewer hospitalizations, shorter lengths of stay, and care that is more likely to follow establish guidelines. The one aspect of management for which the findings were less consistent was agreement on diagnosis and management, with some studies reporting a significant difference between telehealth and in-person conclusions or that telehealth was unable to facilitate a diagnosis, though the reasons were not clear (i.e., whether the cause was due to issues with or limitations of the technology or the comfort of the provider in making a diagnosis without a hands-on physical exam).

Twenty-two studies assessed satisfaction with telehealth consultations and generally reported that patients and providers were as satisfied with telehealth consultation as in-person visits. In some cases, patients and families were more satisfied, particularly when the telehealth consultation saved travel and associated time and expense, while providers tended to be slightly less satisfied with telehealth consultations though this difference was not statistically significant.

\section{Outpatient Telehealth Consultations: Harms, Adverse Events, or Negative Unintended Consequences}

Two outpatient studies explicitly addressed harms or unintended consequences in reporting lower rates of complications. ${ }^{30,234}$ One studied complications in cancer treatments, ${ }^{30}$ and the other reported serious adverse events related to hepatitis $C$ treatment. ${ }^{234}$ In part, the overall lack of reporting on harms reflects the relatively short-term followup in most outpatient studies and the focus on intermediate outcomes. Although there are some other findings that are not positive (e.g., a portion, but not the majority of patients reporting they are uncomfortable being videotaped or less than ideal agreement on management), these do not rise to the level of harms. 


\section{Outpatient Telehealth Consultations: Key Characteristics of Studies and Association With Outcomes}

As is evident from the detailed results, the outpatient studies of telehealth consultation include multiple disciplines. For most clinical topics, the studies were conducted in a variety of geographic locations and countries with about 40 percent being conducted in the United States. There are some exceptions, for example, all included studies of telehealth psychiatric consultations were conducted in the United State while all the included dental studies were conducted in European countries. The body of literature also includes studies with different designs and with sample sizes ranging from as small as 11 to several thousand. This variety is interesting; however, there are no patterns evident that associate these general descriptive characteristics with whether telehealth consultations produce a benefit. Additionally, similar to the inpatient and emergency care studies, outpatient studies did not report many details about the environment or context. Notably, they provided very little information on the organizations themselves, any staffing and/or training needed to facilitate telehealth consultations, or payment models for consultations or other care related to the consultation.

There were two characteristics of telehealth consultations that we included in the in-text tables in this section that were not included in the inpatient and emergency care results sections. These are whether consultations occurred in real time or were asynchronous (e.g., store and forward) and when the consultation was a single event or if there were ongoing, continuous interactions between the consultant and referring physician. Table 12 presents percentages of studies with each of these characteristics. More studies were of real time consultations (about two-thirds) rather than asynchronous (about one-third). The distribution between consultations that were one-time and continuing was closer to an even split (56\% and $43 \%$, respectively). We also looked at the percentage of studies with each of these characteristics to determine if they were more or less likely to report that telehealth produced a benefit relative to the comparison group. Fewer studies with real time consultations reported a benefit (44\%) than studies with asynchronous consultations (76\%). This may be because the asynchronous studies more often measured access and time to treatment, and these are consistently better with telehealth. The difference is similar when comparing the percentage of one-time (43\%) and continuing (70\%) consultations that reported results favoring telehealth. The studies of ongoing consultations tended to report clinical outcomes or intermediate outcomes involving the management of chronic conditions (e.g., wound healing, reductions in blood pressures); results that likely required time and repeated input from specialists.

These characteristics are confounded with the clinical topic thereby making it difficult to draw conclusions from this information or generalize further. For example, most of the dermatology studies are asynchronous while all of the studies involving diagnostic technology are real time by definition. It is also likely that other factors that have not been measured may be more strongly associated with benefits. Nevertheless, looking at characteristics across studies and outcomes is an important initial step to increase our understanding of when and how telehealth consultations are most likely to be effective.

Table 12. Characteristics of outpatient consultations and outcomes, percent (counts)

\begin{tabular}{|c|c|c|c|c|}
\hline Characteristic & Real Time & Asynchronous & One Time & Continuing \\
\hline Percent of all outpatient studies ${ }^{a}$ & $63 \%(59+$ of 94$)$ & $36 \%(34$ of 94$)$ & $56 \%(53$ of 94$)$ & $43 \%(40$ of 94$)$ \\
\hline $\begin{array}{l}\text { Percent of studies with the } \\
\text { characteristic reporting a benefit }^{b}\end{array}$ & $44 \%(26$ of 59$)$ & $76 \%(26$ of 34$)$ & $43 \%(23$ of 53$)$ & $70 \%(28$ of 40$)$ \\
\hline
\end{tabular}

a Timing and frequency were both unclear in one study each, + includes studies that used both real time and asynchronous

${ }^{b}$ In any outcome where telehealth was better than the comparator: clinical, intermediate, or cost 


\section{Results for Each Clinical Topic}

In this section, results for each study are presented in tables according to the 11 specialty groups. The accompanying text provides a brief description or highlights key findings.

\section{Dermatology}

Dermatology as a field was an early adopter and has continued to adapt and study telehealth applications. While many studies in the field focus on diagnostic concordance or accuracy, this was not one of our Key Questions for this review, and we included studies researching patient clinical outcomes and intermediate outcomes such as measures of access or health services use. We identified 22 dermatology studies reported in 28 articles, resulting in more studies of telehealth consultations than any other outpatient specialty. In the majority of studies, the consultations were asynchronous: specifically, images and medical history were made available to a dermatologist who reviewed them at a different time, made a diagnosis, and sometimes provided treatment recommendations (16 studies). This process is often referred to as store and forward. A smaller number of studies (5 studies) used video to facilitate real time evaluations and discussion among the dermatologist, referring physician, and patient, while one study employed both store and forward and real time consultations. ${ }^{202}$ In 16 of the 22 studies, the consultation was a one-time interaction about the patient, though physicians may have collaborated on many patients over time. In six studies, the dermatologist was involved in some ongoing care and followup. Store and forward was usually used for consultations involving a single interaction for a given patient, but there were some cases where store and forward consultations were used to initiate a longer relationship. Real time consultations were used for both ongoing and one-time consultations.

Only three dermatology studies evaluated clinical outcomes. In one, more patients recovered (20\%) in the telehealth group than in the group without telehealth $(4.1 \%)$ in the month between their initial visit and the in-person dermatology assessment. ${ }^{146}$ In the telehealth group a consult was used to provide management advice faster, and treatment was started during the time patients waited for an in-person appointment. The other two studies evaluating clinical outcomes compared the clinical course of patients who were evaluated using store and forward dermatology and in face-to-face visits and found no difference in the numbers of patients who improved, had no change, or were worse. ${ }^{98,130}$

Most of the studies evaluated teledermatology in terms of one or more intermediate outcomes (e.g., assessment, satisfaction, and care management) or in terms of costs compared to usual care. Overall, teledermatology improved access by dramatically reducing wait times for visits and time to treatment (e.g., mean wait times for new patients were 9.75 days for teledermatology and 32.9 days for in-person visits, ${ }^{33}$ and time from consultation to operation was 60.6 days for inperson and 26.1 days with telehealth consultations). ${ }^{148}$ The findings for satisfaction and cost were mixed with most studies reporting a benefit (similar satisfaction and lower costs) while the findings related to the impact on management also varied (e.g., reductions in referrals and unnecessary visits: an advantage; but issues with disagreement on diagnosis or inability to make a diagnosis: a disadvantage). The results from each study are presented below in Table 13. 
Table 13. Dermatology telehealth consultations: selected outcomes

\begin{tabular}{|c|c|c|c|}
\hline $\begin{array}{l}\text { Author, Year } \\
\text { Location } \\
\text { Number of Sites }\end{array}$ & $\begin{array}{l}\text { Study Design } \\
\text { Risk of Bias } \\
\text { Timing } \\
\text { Consultation } \\
\text { Frequency } \\
\text { Comparison, } \mathbf{n}\end{array}$ & $\begin{array}{l}\text { Clinical and Intermediate } \\
\text { Outcomes } \\
\text { (Clinical outcomes are } \\
\text { UNDERLINED AND } \\
\text { CAPITALIZED) }\end{array}$ & Cost/Revenue Outcomes \\
\hline $\begin{array}{l}\text { Bezalel, } 2015^{33} \\
\text { FL, United States } \\
1 \text { VA Hospital }\end{array}$ & $\begin{array}{l}\begin{array}{l}\text { Before-after } \\
\text { High }\end{array} \\
\text { Asynchronous } \\
\text { Continuing } \\
\text { A: Before telehealth, } \\
n=1557 \text { new patients } \\
\text { B: After telehealth, } \\
n=1508 \text { new patients } \\
n \text { for established } \\
\text { patients NR }\end{array}$ & $\begin{array}{l}\text { New patient wait time, in days: } \\
\text { A: } 32.9 \\
\text { B: } 9.75, \mathbf{p}<0.001 \\
\text { Established patient wait time, in } \\
\text { days: } \\
\text { A: } 4.14 \\
\text { B: } 1.49, \mathrm{NS}\end{array}$ & NR \\
\hline $\begin{array}{l}\text { Byamba, } 2015^{233} \\
\text { Mongolia } \\
20 \text { rural health } \\
\text { clinics } \\
1 \text { National } \\
\text { Dermatology Center }\end{array}$ & $\begin{array}{l}\text { RCT } \\
\text { Moderate } \\
\text { Asynchronous } \\
\text { One time } \\
\text { A: Not telehealth, } \\
n=229 \\
\text { B: Telehealth, } n=221\end{array}$ & $\begin{array}{l}\text { Hospital referrals, } n(\%) \\
\text { A: } 28(\mathbf{1 2 . 2 \% )} \\
\text { B: } 7(\mathbf{3 . 1 \%}), \mathbf{p}<0.01 \\
\text { Time to receive care, in hours } \\
\text { A: } 322 \\
\text { B: } 53\end{array}$ & $\begin{array}{l}\text { Patients travel expense, in } \\
\text { USD: } \\
\text { A: } \$ 3,174 \\
\text { B: } \$ 320 \\
\text { Total reduction in costs: } \$ 76.36 \\
\text { per patient }\end{array}$ \\
\hline $\begin{array}{l}\text { Carter, } 2017^{40} \\
\text { TX, United States } \\
1 \text { outpatient clinic } \\
1 \text { hospital }\end{array}$ & $\begin{array}{l}\text { Retrospective cohort } \\
\text { High } \\
\text { Asynchronous } \\
\text { One time } \\
\text { A. In-person, n=173 } \\
\text { B. Telehealth, n=79 }\end{array}$ & $\begin{array}{l}\text { Median time to evaluation, in } \\
\text { days } \\
\text { A: } 70.0 \\
\text { B: } 0.5 \\
\text { Median time to treatment, in } \\
\text { days } \\
\text { A: } 73.5 \\
\text { B: } 3.0\end{array}$ & NR \\
\hline $\begin{array}{l}\text { Collins, } 2004^{195} ; \\
\text { Bowns, } 2006^{192} \\
\text { UK } \\
8 \text { General Practices } \\
1 \text { hospital }\end{array}$ & $\begin{array}{l}\text { RCT } \\
\text { Moderate } \\
\text { Asynchronous } \\
\text { Continuing } \\
\text { A: Not telehealth, } \\
n=97 \\
\text { B: Telehealth, } n=111\end{array}$ & $\begin{array}{l}\text { Satisfaction with care received } \\
\text { A: } 90 \% \\
\text { B: } 81 \%, \text { NS } \\
\text { Satisfaction with management } \\
\text { of skin problem: } \\
\text { A: } 87 \% \\
\text { B: } 84 \%, \text { NS }\end{array}$ & NR \\
\hline
\end{tabular}




\begin{tabular}{|c|c|c|c|}
\hline $\begin{array}{l}\text { Author, Year } \\
\text { Location } \\
\text { Number of Sites }\end{array}$ & $\begin{array}{l}\text { Study Design } \\
\text { Risk of Bias } \\
\text { Timing } \\
\text { Consultation } \\
\text { Frequency } \\
\text { Comparison, } \mathbf{n}\end{array}$ & $\begin{array}{l}\text { Clinical and Intermediate } \\
\text { Outcomes } \\
\text { (Clinical outcomes are } \\
\text { UNDERLINED AND } \\
\text { CAPITALIZED) }\end{array}$ & Cost/Revenue Outcomes \\
\hline $\begin{array}{l}\text { Datta, } 2015^{45} \\
\text { NC, United States } \\
3 \text { primary care sites } \\
2 \text { VA Medical } \\
\text { Centers }\end{array}$ & $\begin{array}{l}\text { Economic evaluation } \\
\text { Moderate } \\
\text { Asynchronous } \\
\text { Continuing } \\
\text { A: Usual care, } n=196 \\
\text { B: Telehealth, } n=195\end{array}$ & $\begin{array}{l}\text { Dermatology clinic visits } \\
\text { A: } 303 \\
\text { B: } 214\end{array}$ & $\begin{array}{l}\text { Total mean cost, USD } \\
\text { Societal Perspective } \\
\text { A: } \$ 106,194 \\
\text { B: } \$ 89,523 \\
\text { VA Perspective } \\
\text { A: } \$ 66,145 \\
\text { B: } \$ 59,917 \\
\text { Mean per participant cost } \pm \text { SD } \\
\text { Societal Perspective } \\
\text { A: } \mathbf{5 4 1} \pm \mathbf{4 0 3} \\
\text { B: } \mathbf{4 6 0} \pm \mathbf{4 2 8} \\
\text { Difference (95\% Cl): }-\mathbf{8 2} \text { (-152 } \\
\text { to }-\mathbf{1 2}) \\
\text { VA Perspective } \\
\text { A: } 338 \pm 291 \\
\text { B: } 308 \pm 298 \\
\text { Difference }(95 \% \mathrm{Cl}):-30(-79 \\
\text { to } 20) \text {.NS }\end{array}$ \\
\hline $\begin{array}{l}\text { Eminovic, 2009146; } \\
2010^{147} \\
\text { Netherlands } \\
35 \text { general practices } \\
2 \text { hospitals }\end{array}$ & $\begin{array}{l}\text { RCT; Economic } \\
\text { evaluation } \\
\text { Moderate } \\
\text { Asynchronous } \\
\text { One time } \\
\text { A: Not telehealth, } \\
n=304 \\
\text { B: Telehealth, } n=301\end{array}$ & $\begin{array}{l}\text { RECOVERED AT 1 MONTH } \\
\text { A: } 4.1 \% \\
\text { B: } 20 \% \\
\text { Preventable consultation } \\
\text { A: } \mathbf{1 8 . 3 \% .} \\
\text { B: } \mathbf{3 9 . 0} \% \\
\text { Difference: } \mathbf{2 0 . 7 \%}(\mathbf{9 5 \%} \mathrm{CI} \\
\mathbf{8 . 5 \%} \text { to } \mathbf{3 2 . 9 \% )} \\
\text { General satisfaction } \\
\text { A: } 3.8 \\
\text { B: } 3.8\end{array}$ & $\begin{array}{l}\text { Mean total costs }(95 \% \mathrm{Cl}) \\
\mathrm{A}: € 354.0(228.0 \text { to } 484.0) \\
\mathrm{B}: € 387 \text { (281.0 to } 502.5) \\
\text { Mean out-of-pocket cost, }(95 \% \\
\mathrm{Cl}) \\
\mathrm{A}: € 16.3 \text { (8.1 to } 24.5) \\
\mathrm{B}: € 12.4(5.4 \text { to } 19.6) \\
\text { Mean travel costs } \\
\mathrm{A}: € 15.2 \\
\mathrm{~B}: € 11.5 \\
\text { Mean employer costs, }(95 \% \\
\mathrm{Cl}) \\
\mathrm{A}: € 47.3 \text { (18 to } 83.1) \\
\mathrm{B}: € 46.2(18.4 \text { to } 86.1) \\
\text { Savings when distance is }>75 \\
\mathrm{~km} \text { or when consultation } \\
\text { volume can be increased } \\
>37 \%\end{array}$ \\
\hline $\begin{array}{l}\text { Ferrandiz, } 2007^{148} \\
\text { Seville, Spain } \\
6 \text { primary care } \\
1 \text { University hospital }\end{array}$ & $\begin{array}{l}\text { Prospective cohort } \\
\text { and pre-post } \\
\text { Moderate } \\
\text { Asynchronous } \\
\text { One time } \\
\text { A: Not telehealth, } \\
\text { n=NR } \\
\text { B: Telehealth, n=NR }\end{array}$ & $\begin{array}{l}\text { Mean consultation to operation } \\
\text { wait time }(95 \% \mathrm{Cl}) \text {, in days } \\
\text { A: } \mathbf{6 0 . 6}(\mathbf{5 6 . 2} \text { to } \mathbf{6 4 . 9 )} \\
\text { B: } \mathbf{2 6 . 1}(\mathbf{2 4 . 5} \text { to } 27.7), \mathbf{p}<\mathbf{0 . 0 0 1} \\
\text { Accuracy of telediagnoses } \\
\mathbf{k}=\mathbf{0 . 8 6} \text { (95\% Cl } \mathbf{0 . 8 3} \text { to } \mathbf{0 . 8 9}) \\
\text { Agreement rate between the } \\
\text { surgical technique planned } \\
\text { through teleconsultation and } \\
\text { technique performed: } \mathrm{k}=0.75 \\
\text { (95\% Cl } 0.04 \text { to } 0.79)\end{array}$ & NR \\
\hline
\end{tabular}




\begin{tabular}{|c|c|c|c|}
\hline $\begin{array}{l}\text { Author, Year } \\
\text { Location } \\
\text { Number of Sites }\end{array}$ & $\begin{array}{l}\text { Study Design } \\
\text { Risk of Bias } \\
\text { Timing } \\
\text { Consultation } \\
\text { Frequency } \\
\text { Comparison, } \mathrm{n}\end{array}$ & $\begin{array}{l}\text { Clinical and Intermediate } \\
\text { Outcomes } \\
\text { (Clinical outcomes are } \\
\text { UNDERLINED AND } \\
\text { CAPITALIZED) }\end{array}$ & Cost/Revenue Outcomes \\
\hline $\begin{array}{l}\text { Gilmour, } 1998^{199} \\
\text { UK } \\
3 \text { health centers } \\
3 \text { hospitals }\end{array}$ & $\begin{array}{l}\text { Prospective cohort } \\
\text { High } \\
\text { Real time } \\
\text { One time } \\
\text { A: Not telehealth, } \\
\mathrm{n}=\mathrm{NR} \\
\text { B: Telehealth, } \mathrm{n}=\mathrm{NR}\end{array}$ & $\begin{array}{l}\text { Investigations requested after } \\
\text { consultations: no difference } \\
\text { Followup arrangements: no } \\
\text { difference } \\
\text { Definitive diagnosis made: } \\
\text { A: } 97 \% \\
\text { B: } 60 \%, p=0.002 \\
81 \% \text { management plan correct }\end{array}$ & NR \\
\hline $\begin{array}{l}\text { Hsiao, } 2008^{65} \\
\text { CA, United States } \\
1 \text { VA Medical Center } \\
3 \text { remote primary } \\
\text { care clinics }\end{array}$ & $\begin{array}{l}\text { Retrospective cohort } \\
\text { Moderate } \\
\text { Asynchronous } \\
\text { One time } \\
\text { A: Not telehealth, } \\
\mathrm{n}=77 \\
\text { B: Telehealth, } \mathrm{n}=92\end{array}$ & $\begin{array}{l}\text { Mean days to initial evaluation } \\
\text { A: } \mathbf{4 8} \\
\text { B: } 4, p<0.0001 \\
\text { Mean days to biopsy } \\
\text { A: } 57 \\
\text { B: } \mathbf{3 8}, p=0.034 \\
\text { Mean days to surgery } \\
\text { A: } 125 \\
\text { B: } 104, p=0.006\end{array}$ & NR \\
\hline $\begin{array}{l}\text { Krupinski, } 2004^{75} \\
\text { AZ, United States } \\
1 \text { medical center } \\
1 \text { hospital }\end{array}$ & $\begin{array}{l}\text { Retrospective cohort } \\
\text { High } \\
\text { Asynchronous } \\
\text { One time } \\
\text { A: Not telehealth, } \\
n=50 \\
\text { B: Telehealth, } n=50\end{array}$ & $\begin{array}{l}\text { Recorded notes of action } \\
\text { A: } \mathbf{1 2 \%} \\
\text { B: } \mathbf{4 3 \%} z=\mathbf{3 . 1 4}, \mathbf{p}<0.01 \\
\text { Patients seen again by referring } \\
\text { clinician after referral for same } \\
\text { problem } \\
\text { A: } 10 \% \\
\text { B: } 8 \%, z=0.40, N S\end{array}$ & NR \\
\hline $\begin{array}{l}\text { Lamminen, } 2001^{160} \\
\text { Finland } \\
1 \text { health center } \\
1 \text { University hospital }\end{array}$ & $\begin{array}{l}\text { Economic evaluation } \\
\text { High } \\
\text { Real time } \\
\text { One time } \\
\\
\mathrm{A}_{1} \text { : Ophthalmology, } \\
\mathrm{n}=85 \\
\mathrm{~A}_{2} \text { : Dermatology, } \\
\mathrm{n}=64 \\
\mathrm{~B}_{1} \text { : Tele- } \\
\text { Ophthalmology, } \mathrm{n}=24 \\
\mathrm{~B}_{2} \text { : Tele- } \\
\text { Dermatology, } \mathrm{n}=18 \\
\end{array}$ & NR & $\begin{array}{l}\text { Consultation cost per patient: } \\
\mathrm{A}_{1}: € 126 \\
\mathrm{~A}_{2}: € 143 \\
\mathrm{~B}_{1} \text { and } \mathrm{B}_{2}: € 9,760+37.6 \mathrm{per} \\
\text { patient } \\
\text { Number of teleconsultations to } \\
\text { break even: } 110 \text { in } \\
\text { ophthalmology and } 92 \text { in } \\
\text { dermatology }\end{array}$ \\
\hline $\begin{array}{l}\text { Lim, } 2012^{222} \\
\text { Waikato, New } \\
\text { Zealand } \\
1 \text { community clinic } \\
1 \text { hospital clinic }\end{array}$ & $\begin{array}{l}\text { Prospective cohort } \\
\text { High } \\
\text { Asynchronous } \\
\text { One time } \\
\text { A: Face to face, } \\
n=100 \\
\text { B: Telehealth, } n=200\end{array}$ & $\begin{array}{l}\text { Mean waiting time, in days } \\
\text { A: } 114 \text { days } \\
\text { B: } 39 \text { days } \\
\text { Patient satisfaction survey: } 1 \text { to } \\
5 \text { ( } 5=\text { =xcellent) } \\
\text { Overall experience } \\
\text { A: } 3.8 \\
\text { B: } 4.5\end{array}$ & $\begin{array}{l}\text { Financial cost/patient NZ\$ } \\
\text { A: } \$ 306 \\
\text { B: } \$ 264\end{array}$ \\
\hline
\end{tabular}




\begin{tabular}{|c|c|c|c|}
\hline $\begin{array}{l}\text { Author, Year } \\
\text { Location } \\
\text { Number of Sites }\end{array}$ & $\begin{array}{l}\text { Study Design } \\
\text { Risk of Bias } \\
\text { Timing } \\
\text { Consultation } \\
\text { Frequency } \\
\text { Comparison, } n\end{array}$ & $\begin{array}{l}\text { Clinical and Intermediate } \\
\text { Outcomes } \\
\text { (Clinical outcomes are } \\
\text { UNDERLINED AND } \\
\text { CAPITALIZED) }\end{array}$ & Cost/Revenue Outcomes \\
\hline $\begin{array}{l}\text { Loane, } 2000^{202} \\
\text { Northern Ireland, } \\
\text { UK }^{\mathrm{a}} \\
4 \text { health centers } \\
2 \text { hospitals }\end{array}$ & $\begin{array}{l}\text { RCT } \\
\text { High } \\
\text { Real-time and } \\
\text { asynchronous } \\
\text { One time } \\
\text { A: Usual care, } n=102 \\
B_{1}: \text { Telehealth video, } \\
n=102 \\
B_{2}: \text { Telehealth store } \\
\text { and forward, } n=96 \\
\text { (from original } 102 \\
\text { telehealth video } \\
\text { group) }\end{array}$ & $\begin{array}{l}\text { Required at least one } \\
\text { subsequent hospital } \\
\text { appointment } \\
\text { A: } 45 \% \\
B_{1}: 46 \% \\
B_{2}: 69 \%\end{array}$ & $\mathrm{NR}$ \\
\hline $\begin{array}{l}\text { Loane, } 2001^{223} \\
\text { New Zealand } \\
2 \text { rural health } \\
\text { centers } \\
1 \text { hospital }\end{array}$ & $\begin{array}{l}\text { Economic evaluation } \\
\text { Moderate } \\
\text { Real time } \\
\text { Continuing } \\
\text { A: Not telehealth, } \\
\mathrm{n}=94 \\
\text { B: Telehealth, } \mathrm{n}=109\end{array}$ & $\begin{array}{l}\text { Average consultation time, in } \\
\text { minutes } \\
\text { A: } 21.6 \\
\text { B: } 20.0\end{array}$ & $\begin{array}{l}\text { Total cost of dermatologist's } \\
\text { time spent in performing } \\
\text { consultations, in NZ } \$ \\
\text { A: } \$ 5,724 \\
\text { B: } \$ 6,163 \\
\text { Cost of patient time to attend } \\
\text { consultations, in NZ } \$ \\
\text { A: } \$ 7,838 \\
\text { B: } \$ 1,846 \\
\text { Total travel costs for patients to } \\
\text { attend consultations, in NZ } \$ \\
\text { A: } \$ 16,519 \\
\text { B: } \$ 877 \\
\text { Total societal costs of } \\
\text { consultations, in NZ } \$ \\
\text { A: } \$ 30,081 \\
\text { B: } \$ 34,346\end{array}$ \\
\hline $\begin{array}{l}\text { Loane, } 1999^{201} \\
\text { Northern Ireland, UK } \\
4 \text { health centers } \\
2 \text { hospitals }\end{array}$ & $\begin{array}{l}\text { RCT } \\
\text { High } \\
\text { Real time } \\
\text { One time } \\
\text { A: Not telehealth, } \\
n=103 \\
\text { B: Telehealth, } n=61\end{array}$ & $\begin{array}{l}\text { Mean time, in minutes } \\
\text { Total time, including waiting and } \\
\text { travel } \\
\text { A: } 84.4 \\
\text { B: } 59.3 \\
\text { Wait time } \\
\text { A: } 20 \\
\text { B: } 5.4 \\
\text { Consultation time } \\
\text { A: } 16.8 \\
\text { B: } 22.0 \\
\text { Total travel time } \\
\text { A: } 48.0 \\
\text { B: } 31.6 \\
\text { Mean travel distance, in } \mathrm{km} \\
\text { A: } 25.4 \\
\text { B: } 10.4\end{array}$ & NR \\
\hline
\end{tabular}




\begin{tabular}{|c|c|c|c|}
\hline $\begin{array}{l}\text { Author, Year } \\
\text { Location } \\
\text { Number of Sites }\end{array}$ & $\begin{array}{l}\text { Study Design } \\
\text { Risk of Bias } \\
\text { Timing } \\
\text { Consultation } \\
\text { Frequency } \\
\text { Comparison, } n\end{array}$ & $\begin{array}{l}\text { Clinical and Intermediate } \\
\text { Outcomes } \\
\text { (Clinical outcomes are } \\
\text { UNDERLINED AND } \\
\text { CAPITALIZED) }\end{array}$ & Cost/Revenue Outcomes \\
\hline $\begin{array}{l}\text { Mahendran, } 2005^{213} \\
\text { England, UK }\end{array}$ & $\begin{array}{l}\text { Pre/post } \\
\text { High } \\
\text { Asynchronous } \\
\text { One time } \\
\text { A: Pre-telehealth, } \\
\mathrm{n}=163 \\
\text { B: Post-Telehealth, } \\
\mathrm{n}=163 ; \text { same as } \\
\text { group A }\end{array}$ & $\begin{array}{l}\text { Agreement on management } \\
\text { plan } \\
55 \% \\
\text { Managed appropriately } \\
13 \% \text { required reassurance } \\
\text { only } \\
9 \% \text { recommended further } \\
\text { review } \\
33 \% \text { minor operation } \\
\text { Not adequately managed by } \\
\text { telehealth } \\
15 \% \text { poor image quality } \\
20 \% \text { complex and required in- } \\
\text { person exam and consent } \\
10 \% \text { recommended } \\
\text { unnecessary or wrong surgery }\end{array}$ & NR \\
\hline $\begin{array}{l}\text { Moreno-Ramirez, } \\
2009^{166} \\
\text { Spain } \\
12 \text { Primary Care } \\
\text { Centers } \\
1 \text { hospital }\end{array}$ & $\begin{array}{l}\text { Economic evaluation } \\
\text { Low } \\
\text { Asynchronous } \\
\text { One time } \\
\text { A: Not telehealth, } \\
n=2,009 \\
\text { B: Telehealth, } \\
n=2,009\end{array}$ & $\mathrm{NR}$ & $\begin{array}{l}\text { Unit cost per patient } \\
\text { A: } € \mathbf{1 2 9 . 3 7} \\
\text { B: } \mathbf{€ 7 9 . 7 8 , p = 0 . 0 0 5} \\
\text { Cost ratios A to B } \\
\text { Overall: } 1.62 \\
\text { For benign lesions: } 3.29\end{array}$ \\
\hline $\begin{array}{l}\text { Nordal, } 2001^{169} \\
\text { Norway } \\
1 \text { municipality } \\
1 \text { hospital }\end{array}$ & $\begin{array}{l}\text { Retrospective cohort } \\
\text { Moderate } \\
\text { Real time } \\
\text { One time } \\
\text { A: Not telehealth, } \\
n=N R \\
\text { B: Telehealth, } n=N R\end{array}$ & $\begin{array}{l}\text { Dermatologist satisfaction } \\
22 \% \text { favored face-to-face } \\
14 \% \text { favored telehealth } \\
\\
\text { Patient reports } \\
61 \% \text { no disadvantage to video } \\
18 \% \text { reduced contact with } \\
\text { specialist } \\
7 \% \text { discomfort being recorded } \\
86 \% \text { favored having GP present } \\
\text { for teledermatology }\end{array}$ & NR \\
\hline $\begin{array}{l}\text { Pak, 200798; Pak, } \\
2009^{99} \\
\text { TX, United States }\end{array}$ & $\begin{array}{l}\text { RCT and economic } \\
\text { evaluation } \\
\text { Moderate } \\
\text { Asynchronous } \\
\text { One time } \\
\text { A: Not telehealth, } \\
n=236 \\
\text { B: Telehealth, } n=272\end{array}$ & $\begin{array}{l}\text { CHANGES IN CLINICAL } \\
\text { COURSE } \\
\text { Improved } \\
\text { A: } 65 \% \\
\text { B: } 64 \% \\
\text { No change } \\
\text { A: } 32 \% \\
\text { B: } 33 \% \\
\text { Worse } \\
\text { A: } 3 \% \\
\text { B: } 4 \%, \text { NS }\end{array}$ & $\begin{array}{l}\text { Total costs, in USD } \\
\text { A: } \$ 129,133 \\
\text { B: } \$ 119,402 \\
\text { Direct costs, in USD } \\
\text { A: } \$ 98,365 \\
\text { B: } \$ 103,043 \\
\text { Lost productivity, in USD } \\
\text { A: } \$ 30,768 \\
\text { B: } \$ 16,359 \\
\text { Telehealth is less expensive } \\
\text { when including lost productivity }\end{array}$ \\
\hline
\end{tabular}




\begin{tabular}{|c|c|c|c|}
\hline $\begin{array}{l}\text { Author, Year } \\
\text { Location } \\
\text { Number of Sites }\end{array}$ & $\begin{array}{l}\text { Study Design } \\
\text { Risk of Bias } \\
\text { Timing } \\
\text { Consultation } \\
\text { Frequency } \\
\text { Comparison, } n\end{array}$ & $\begin{array}{l}\text { Clinical and Intermediate } \\
\text { Outcomes } \\
\text { (Clinical outcomes are } \\
\text { UNDERLINED AND } \\
\text { CAPITALIZED) }\end{array}$ & Cost/Revenue Outcomes \\
\hline $\begin{array}{l}\text { Piette, } 2017^{175} \\
\text { Paris, France } \\
39 \text { GPs }\end{array}$ & $\begin{array}{l}\text { RCT } \\
\text { Low } \\
\text { Asynchronous } \\
\text { Continuing } \\
\text { A: Usual care, } n=50 \\
\text { B: Telehealth, } n=53\end{array}$ & $\begin{array}{l}\text { Median delay for specialist } \\
\text { response/treatment initiation } \\
\text { A: } \mathbf{4 0} \text { days } \\
\text { B: } \mathbf{4} \text { days } \\
\text { Adjusted HR: } \mathbf{2 . 5 5}, \mathbf{p}<\mathbf{0 . 0 1 1} \\
\\
\text { Patient global satisfaction, } n(\%) \\
\text { Very satisfied or satisfied } \\
\text { A: } 47(94 \%) \\
\text { B: } 45(84.9 \%) \\
\text { Unsatisfied or very unsatisfied } \\
\text { A: } 3(6 \%) \\
\text { B: } 8(15.1 \%) \\
p=0.99 \\
\text { Patient satisfaction, time to } \\
\text { treatment } \\
\text { Very satisfied or satisfied } \\
\text { A: } 13(26 \%) \\
\text { B: } 38(71.7 \%) \\
\text { Unsatisfied or very unsatisfied } \\
\text { A: } 37(74 \%) \\
\text { B: } 15(28.3 \%) \\
\text { p=0.20 }\end{array}$ & $\mathrm{NR}$ \\
\hline $\begin{array}{l}\text { Whited, } 2002,2003 \text {, } \\
2004^{126-128} \\
\text { NC, United States } \\
2 \text { VA hospitals } \\
3 \text { outpatient clinics }\end{array}$ & $\begin{array}{l}\text { RCT; Economic } \\
\text { evaluation } \\
\text { Moderate (2003 and } \\
\text { 2002) /High (2004) } \\
\text { Asynchronous } \\
\text { One time } \\
\text { A: Not telehealth, } \\
n=140 \\
\text { B: Telehealth, } n=135\end{array}$ & $\begin{array}{l}\text { Mean days } \pm \text { SD (Median) } \\
\text { Time to initial definitive } \\
\text { intervention intention to treat } \\
\text { analysis: } \\
\text { A: } \mathbf{1 1 4 . 3} \pm \mathbf{7 2 . 3}(\mathbf{1 2 7}) \\
\text { B: } \mathbf{7 3 . 8} \pm \mathbf{7 1 . 6}(\mathbf{4 1 )}, \mathbf{p}=\mathbf{0 . 0 0 0 1} \\
\text { Actual clinic visit analysis }{ }^{\mathrm{b}} \text {, } \\
\text { mean days } \pm \text { SD } \\
\text { A: } \mathbf{1 3 5 . 6} \pm \mathbf{9 4 . 3} \\
\text { B: } \mathbf{9 3 . 4} \pm \mathbf{9 6 . 1 ,} \mathbf{p}=\mathbf{0 . 0 0 2 7} \\
\text { Referring clinician overall } \\
\text { satisfaction with consult } \\
\text { Agree: } \\
\text { A: } 23 \% \\
\text { B: } 92 \% \\
\text { Neutral: } \\
\text { A: } 42 \% \\
\text { B: } 5 \% \\
\text { Disagree: } \\
\text { A: } 35 \% \\
\text { B: } 3 \% \\
\text { Patient overall satisfaction with } \\
\text { TH consultation outcome: } 82 \% \\
\text { Preferred TH: } 42 \% \\
\text { Preferred usual care: } 37 \% \\
\text { Neutral, no preference: } 22 \%\end{array}$ & $\begin{array}{l}\text { Total annual cost }{ }^{\mathrm{c}} \text {, in USD } \\
\text { A: } \$ 116,416 \\
\text { B: } \$ 198,016 \\
\text { Per patient } \\
\text { A: } \$ 21.40 \\
\text { B: } \$ 36.40 \\
\text { Incremental cost per patient of } \\
\text { teledermatology }{ }^{\mathrm{c}} \\
+\$ 15.00\end{array}$ \\
\hline
\end{tabular}




\begin{tabular}{|c|c|c|c|}
\hline $\begin{array}{l}\text { Author, Year } \\
\text { Location } \\
\text { Number of Sites }\end{array}$ & $\begin{array}{l}\text { Study Design } \\
\text { Risk of Bias } \\
\text { Timing } \\
\text { Consultation } \\
\text { Frequency } \\
\text { Comparison, } n\end{array}$ & $\begin{array}{l}\text { Clinical and Intermediate } \\
\text { Outcomes } \\
\text { (Clinical outcomes are } \\
\text { UNDERLINED AND } \\
\text { CAPITALIZED) }\end{array}$ & Cost/Revenue Outcomes \\
\hline $\begin{array}{l}\text { Whited, } 2013^{129} ; \\
\text { Whited, } 2013^{130} \\
\text { United States } \\
2 \text { community based } \\
\text { outpatient clinics } \\
1 \text { hospital } \\
2 \text { medical centers }\end{array}$ & $\begin{array}{l}\text { RCT } \\
\text { Moderate } \\
\text { Asynchronous } \\
\text { One time } \\
\text { A: Not telehealth, } \\
n=136 \\
\text { B: Telehealth, } n=125\end{array}$ & $\begin{array}{l}\text { BASELINE TO } 9 \text { MONTHS } \\
\text { Resolved or improved } \\
\text { A: } 72 \% \\
\text { B: } 72 \% \\
\text { Unchanged - not clinically } \\
\text { relevant: } \\
\text { A: } 11 \% \\
\text { B: } 10 \% \\
\text { Unchanged - clinically relevant: } \\
\text { A: } 13 \% \\
\text { B: } 10 \% \\
\text { Worse } \\
\text { A: } 4 \% \\
\text { B: } 8 \% \\
\text { NS }\end{array}$ & $\mathrm{NR}$ \\
\hline $\begin{array}{l}\text { Whited, } 2013^{129} ; \\
\text { Whited, 2013 } \\
\text { (continued) } \\
\text { United States } \\
2 \text { community based } \\
\text { outpatient clinics } \\
1 \text { hospital } \\
2 \text { medical centers }\end{array}$ & $\begin{array}{l}\text { RCT } \\
\text { Moderate } \\
\text { Asynchronous } \\
\text { One time } \\
\text { A: Not telehealth, } \\
n=136 \\
\text { B: Telehealth, } n=125\end{array}$ & $\begin{array}{l}\text { Mean change in quality of life } \\
\text { Skindex- } 16 \text { score }^{\mathrm{d}} \pm \mathrm{SD} \text { : } \\
\text { Composite } \\
\text { Baseline to } 3 \text { months } \\
\text { A: }-5.8 \pm 19.1 \\
\text { B: }-7.8 \pm 21.9, p=0.39 \\
\text { Baseline to } 9 \text { months } \\
\text { A: }-13.2 \pm 21.6 \\
\text { B: }-12.0 \pm 24.5, p=0.66\end{array}$ & NR \\
\hline
\end{tabular}

$\mathrm{CI}=$ confidence interval; EU = euro; GP = general practitioner; HR = hazard ratio; k = kappa; NR = not reported; NS = not significant; NZ = New Zealand dollar; RCT = randomized controlled trial; SD = standard deviation; $\mathrm{TH}=$ telehealth; USD = United States dollars; VA = Veterans Affairs

a Potentially overlapping populations

${ }^{\mathrm{b}}$ Date to clinic visit is used if telehealth patient went to clinic despite no recommended visit

${ }^{c}$ Extrapolated cost data from 275 patients to the total population of patients serviced by dermatology clinic in 2001, $n=5,440$

d Negative indicates improvement

Bold=statistically significant; telehealth superior. Bold and italicized=statistically significant; telehealth inferior. Regular type: not statistically significant.

\section{Wound Care}

Six studies reported on different approaches to telehealth for wound care (Table 14). The studies consisted of small numbers (all <200) of home care, wound clinic, long-term care, and primary care patients. The five studies reporting clinical outcomes used different approaches to telehealth (one real time video ${ }^{256}$ and four image and/or record review ${ }^{180,190,228,251}$ ) but all reported clinical benefit, in terms of better healing or fewer amputations with telehealth expert consultations than with usual care. In all of these studies, consultations continued over the course of the patients' treatment for the wound or ulcer. Costs of telehealth consultations were lower than the cost of in-person consultations, and overall healthcare costs were also lower. 
Table 14. Wound care telehealth consultations: selected outcomes

\begin{tabular}{|c|c|c|c|c|}
\hline $\begin{array}{l}\text { Author, Year } \\
\text { Location } \\
\text { Number of Sites } \\
\text { Number of Patients }\end{array}$ & $\begin{array}{l}\text { Study Design } \\
\text { Risk of Bias } \\
\text { Timing } \\
\text { Consultation } \\
\text { Frequency } \\
\text { Comparison, } \mathbf{n}\end{array}$ & $\begin{array}{l}\text { Clinical Outcomes } \\
\text { Harms }\end{array}$ & $\begin{array}{l}\text { Intermediate } \\
\text { Outcomes }\end{array}$ & $\begin{array}{l}\text { Cost/Revenue } \\
\text { Outcomes }\end{array}$ \\
\hline $\begin{array}{l}\text { Kobza, } 2000^{256} \\
\text { United States } \\
\text { Hospital based home care } \\
\text { agencies number NR }\end{array}$ & $\begin{array}{l}\text { Before-after } \\
\text { High } \\
\text { Real time } \\
\text { Continuing } \\
\text { A: Before } \\
\text { telehealth, } \\
\mathrm{n}=120 \\
\text { B: After } \\
\text { telehealth, } \mathrm{n}=76\end{array}$ & $\begin{array}{l}\text { Rate of healing } \\
\text { Stage II pressure ulcer } \\
\text { A: } 34 \% \\
\text { B: } 83 \% \\
\text { Stage IV pressure } \\
\text { ulcer } \\
\text { A: } 10 \% \\
\text { B: } 38 \% \\
\text { Healing rate for all } \\
\text { wounds improved with } \\
\text { telehealth except } \\
\text { stage III pressure } \\
\text { ulcers } \\
\text { Healing time } \\
\text { decreased in all } \\
\text { categories with } \\
\text { telehealth } \\
\text { Discharge with healed } \\
\text { wounds: } \\
\text { A: } 37 \% \\
\text { B: } 58 \%\end{array}$ & $\begin{array}{l}\text { Mean home visits } \\
\text { per wound patient } \\
\text { A: } 60 \\
\text { B: } 33 \\
\text { Hospitalizations } \\
\text { A: } 18 \% \\
\text { B: } 6 \%\end{array}$ & NR \\
\hline $\begin{array}{l}\text { Santamaria, } 2004^{228} \\
\text { Kimberly, Australia } \\
4 \text { clinics }\end{array}$ & $\begin{array}{l}\text { RCT } \\
\text { High } \\
\text { Asynchronous } \\
\text { Continuing } \\
\text { A: Not } \\
\text { telehealth, } n=43 \\
\text { B: Telehealth, } \\
n=50\end{array}$ & $\begin{array}{l}\text { Healing rate, per week } \\
\text { A: }-4.9 \% \\
\text { B: } 6.8 \%, p=0.012 \\
\text { Amputation } \\
\text { A: } 6 \\
\text { B: } 1 \\
\text { Mortality } \\
\text { A: NR } \\
\text { B: } 2\end{array}$ & NR & $\begin{array}{l}\text { Total } \text { Cost }^{\mathrm{a}} \text {, in } \\
\text { AUD } \\
\text { A: } \$ 862,161 \\
\text { B: } \$ 670,226\end{array}$ \\
\hline $\begin{array}{l}\text { Smith-Strom, } 2018^{180} \\
\text { Norway } \\
3 \text { clinics }\end{array}$ & $\begin{array}{l}\text { RCT } \\
\text { Low } \\
\text { Asynchronous } \\
\text { Continuing } \\
\text { A:Usual care, } \\
n=88 \\
\text { B:Telehealth, } \\
n=94\end{array}$ & $\begin{array}{l}\text { Mortality } \\
\text { A: } 5.7 \% \\
\text { B: } 5.3 \% \\
\text { Mean difference }(95 \% \\
\mathrm{Cl}) \\
-0.4 \% \text { (-6.5 to } 5.7) \\
\text { Amputations } \\
\text { A: } 14.8 \% \\
\text { B: } 6.4 \% \\
\text { Mean difference }(95 \% \\
\text { Cl) } \\
\mathbf{- 8 . 3} \%(-\mathbf{1 6 . 3} \text { to }-\mathbf{0 . 5 )}\end{array}$ & $\begin{array}{l}\text { Ulcers healed } \\
\text { A: } 76.1 \% \\
\text { B: } 79.8 \% \\
\text { Mean time to } \\
\text { healing, in months } \pm \\
\text { SD } \\
\text { A: } 3.8 \pm 3.4 \\
\text { B: } 3.4 \pm 3.2 \\
\text { Mean difference } \\
(95 \% \mathrm{Cl}): \\
-0.43(-1.50 \text { to } 0.65)\end{array}$ & \\
\hline
\end{tabular}




\begin{tabular}{|c|c|c|c|c|}
\hline $\begin{array}{l}\text { Author, Year } \\
\text { Location } \\
\text { Number of Sites } \\
\text { Number of Patients }\end{array}$ & $\begin{array}{l}\text { Study Design } \\
\text { Risk of Bias } \\
\text { Timing } \\
\text { Consultation } \\
\text { Frequency } \\
\text { Comparison, } \mathbf{n}\end{array}$ & $\begin{array}{l}\text { Clinical Outcomes } \\
\text { Harms }\end{array}$ & $\begin{array}{l}\text { Intermediate } \\
\text { Outcomes }\end{array}$ & $\begin{array}{l}\text { Cost/Revenue } \\
\text { Outcomes }\end{array}$ \\
\hline $\begin{array}{l}\text { Specht, } 2001^{118} \\
\text { IA, United States } \\
1 \text { long term care facility }\end{array}$ & $\begin{array}{l}\text { Economic } \\
\text { evaluation } \\
\text { Moderate } \\
\text { Asynchronous } \\
\text { Continuing } \\
\text { A: Not } \\
\text { telehealth, n=NR } \\
\text { B: Telehealth, } \\
n=N R\end{array}$ & NR & $\begin{array}{l}\text { Mean time spent on } \\
\text { appointment } \\
\text { A: } 8.5 \text { hours } \\
\text { B: } 20 \text { minutes }\end{array}$ & $\begin{array}{l}\text { Mean cost } \\
\text { consultation, in } \\
\text { USD } \\
\text { A: } \$ 246.28 \\
\text { B: } \$ 136.16\end{array}$ \\
\hline $\begin{array}{l}\text { Stern, } 2014^{251} \\
\text { Ontario, Canada } \\
12 \text { long term care facilities }\end{array}$ & $\begin{array}{l}\begin{array}{l}\text { Before-after } \\
\text { (stepped wedge) } \\
\text { Moderate }\end{array} \\
\text { Asynchronous } \\
\text { Continuing } \\
\text { Total wounds } \\
\text { analyzed: } 259 \\
\text { among } 137 \text { long- } \\
\text { term care } \\
\text { residents } \\
\text { A: Not } \\
\text { telehealth, } \\
\mathrm{n}=\text { unclear } \\
\text { B: Telehealth, } \\
\mathrm{n}=\text { unclear }\end{array}$ & $\begin{array}{l}\text { Mean rate of healing } \\
1.0058 \text { times slower in } \\
\text { intervention period } \\
\text { ( } 95 \% \mathrm{Cl} 0.985 \text { to } \\
1.027), p=0.6 \\
\\
\text { Time to healing } \\
\text { Intervention HR: } 1.48 \\
(95 \% \mathrm{Cl} 0.79 \text { to } 2.78) \text {, } \\
\mathrm{p}=0.22\end{array}$ & $\begin{array}{l}\text { Visual analogue } \\
\text { scale wound-specific } \\
\text { pain scores } \\
\text { Estimated mean } \\
0.39 \text { units higher } \\
\text { during intervention } \\
\text { period ( } 95 \% \mathrm{Cl}-0.55 \\
\text { to } 1.34), \mathrm{NS} \\
\text { Hospitalizations } \\
\text { Estimated mean rate } \\
1.2 \text { times higher } \\
\text { during intervention } \\
\text { (95\% Cl } 0.62 \text { to } \\
2.36), \mathrm{NS} \\
\text { ED visits } \\
\text { Estimated mean rate } \\
1.3 \text { times larger } \\
\text { during intervention } \\
\text { (95\% Cl } 0.58 \text { to } \\
2.90) \text {, NS }\end{array}$ & $\begin{array}{l}\text { Telehealth } \\
\text { reduced direct } \\
\text { care costs by } \\
\text { CAD } \$ 649 \text { per } \\
\text { resident }^{\mathrm{b}}\end{array}$ \\
\hline $\begin{array}{l}\text { Zarchi, } 2015^{190} \\
\text { Denmark } \\
4 \text { home-care organizations }\end{array}$ & $\begin{array}{l}\text { Prospective } \\
\text { cohort } \\
\text { Moderate } \\
\text { Asynchronous } \\
\text { Continuing } \\
\text { A: Not } \\
\text { telehealth, } n=40 \\
\text { B: Telehealth, } \\
n=50\end{array}$ & $\begin{array}{l}\text { Adjusted telehealth } \\
\text { hazard ratio }(95 \% \mathrm{Cl}) \text {, } \\
\text { p-value } \\
\text { 1-Year wound } \\
\text { healing } \\
2.19(1.15 \text { to } 4.17) \text {, } \\
\text { p=0.017 } \\
\text { Complete wound } \\
\text { healing at 1-year } \\
\text { followup } \\
\text { A: } 45 \% \\
\text { B: } 70 \%\end{array}$ & $\mathrm{NR}$ & NR \\
\hline
\end{tabular}

AUD = Australian dollars; CAD = Canadian dollars; CI = confidence interval; ED = emergency department; NR = not reported; NS = not significant; RCT = randomized controlled trial; SD = standard deviation; USD = United States dollars

a 43 subjects per group were used in the costing analysis to eliminate the effect of the larger group of intervention patients

b 42 participants crossed study phases

Bold=statistically significant; telehealth superior. Bold and italicized=statistically significant; telehealth inferior. Regular type: not statistically significant. 


\section{Ophthalmology}

None of the six ophthalmology studies reported clinical outcomes (Table 15). Two studies reported minimal information on telehealth and nontelehealth costs in ophthalmology with one reporting no difference in the per visit cost and estimating the only savings were from patients avoiding travel, ${ }^{185}$ and the second reported savings due to avoided transfers to a distant hospital for evaluation. ${ }^{215}$ Telehealth did reduce the number of visits to a surgeon in a study of cataract management, ${ }^{188}$ and two other studies reported increases in screening completion rates. ${ }^{122,219}$

Table 15. Ophthalmologic telehealth consultations: selected outcomes

\begin{tabular}{|c|c|c|c|}
\hline $\begin{array}{l}\text { Author, Year } \\
\text { Geographic Location } \\
\text { Number of Sites } \\
\text { Number of Patients }\end{array}$ & $\begin{array}{l}\text { Study Design } \\
\text { Risk of Bias } \\
\text { Timing } \\
\text { Consultation Frequency } \\
\text { Comparison, } n\end{array}$ & Intermediate Outcomes & $\begin{array}{l}\text { Cost/Revenue } \\
\text { Outcomes }\end{array}$ \\
\hline $\begin{array}{l}\text { Blackwell, } 1997^{215} \\
\text { Queensland, Australia } \\
1 \text { remote hospital } \\
1 \text { specialist hospital }\end{array}$ & $\begin{array}{l}\text { Before-after } \\
\text { High } \\
\text { Real time } \\
\text { One-time } \\
\text { A: Before telehealth, } n=315 \\
\text { B: After telehealth, } n=264 ; \\
24 \text { received telehealth }\end{array}$ & $\begin{array}{l}\text { Patients transferred for urgent } \\
\text { care } \\
\text { A: } 17 \\
\text { B: } 4\end{array}$ & $\begin{array}{l}\text { Amount hospital } \\
\text { saved due to } \\
\text { transfers avoided, } \\
\mathrm{NZ} \$ \\
\$ 6,500\end{array}$ \\
\hline $\begin{array}{l}\text { Crossland, } 2016^{219} \\
\text { Rural, regional, urban } \\
\text { Australia } \\
\text { Telehealth } 5 \text { without } \\
\text { ophthalmologic } \\
\text { location NR }\end{array}$ & $\begin{array}{l}\text { Prospective cohort } \\
\text { High } \\
\text { Unclear } \\
\text { Continuing } \\
\text { A: Usual care, } n=577 \\
\text { B: Telehealth, } n=447\end{array}$ & $\begin{array}{l}\text { Screening rates } \\
\text { A: } 22 \% \text { to } 53 \% \\
\text { B: } 100 \% \\
\text { Diagnosed with mild-moderate } \\
\text { diabetic retinopathy } \\
\text { A: } 5 \% \\
\text { B: } 9 \% \\
\text { Appropriate followup } \\
\text { A: } 29 \% \\
\text { B: } 95 \%\end{array}$ & NR \\
\hline $\begin{array}{l}\text { Taylor, } 2007^{122} \\
\text { TN, United States } \\
1 \text { community clinic } \\
1 \text { ophthalmic clinic }\end{array}$ & $\begin{array}{l}\text { Before-after } \\
\text { High } \\
\text { Asynchronous } \\
\text { One-time } \\
\text { A: Before telehealth, } n=294 \\
\text { B: After telehealth, } n=201\end{array}$ & $\begin{array}{l}\text { Screen completion } \\
\text { A: } 31 \% \\
\text { B: } 100 \%\end{array}$ & NR \\
\hline
\end{tabular}




\begin{tabular}{|c|c|c|c|}
\hline $\begin{array}{l}\text { Author, Year } \\
\text { Geographic Location } \\
\text { Number of Sites } \\
\text { Number of Patients }\end{array}$ & $\begin{array}{l}\text { Study Design } \\
\text { Risk of Bias } \\
\text { Timing } \\
\text { Consultation Frequency } \\
\text { Comparison, } n\end{array}$ & Intermediate Outcomes & $\begin{array}{l}\text { Cost/Revenue } \\
\text { Outcomes }\end{array}$ \\
\hline $\begin{array}{l}\text { Tuulonen,1999185 } \\
\text { Oulu, Finland } \\
1 \text { rural healthcare } \\
\text { center } \\
1 \text { University clinic }\end{array}$ & $\begin{array}{l}\text { Before-After } \\
\text { Moderate } \\
\text { Real time } \\
\text { One-time } \\
\text { A: Before telehealth, } n=41 \\
\text { B: After telehealth, } n=29\end{array}$ & $\begin{array}{l}\text { Mean time including travel, in } \\
\text { hours } \\
\text { A: } \mathbf{8 . 5} \\
\text { B: } \mathbf{2 . 0}, \mathbf{p}=\mathbf{0 . 0 0 0 1} \\
\text { Mean time absent from work, } \\
\text { A: } 6.6 \\
\text { B: } 3.3 \text {, test NR } \\
\text { Very satisfied with overall care } \\
\text { A: } 69 \% \\
\text { B: } 86 \%, \mathrm{NS} \\
\text { Selecting TH for next visit } \\
\text { A: } 81 \% \\
\text { B: } 96 \%, \mathrm{NS} \\
\text { Want TH for next visit due to } \\
\text { reduced travel } \\
\text { A: } 97 \% \\
\text { B: } 86 \%, \text { NS }\end{array}$ & $\begin{array}{l}\text { Overall cost of } \\
\text { visits, in USD } \\
\text { A: } \$ 111 \\
\text { B: \$110, no } \\
\text { difference } \\
\text { Reduced travel } \\
\text { saved } \$ 55 \text { per visit } \\
\text { for patients, not } \\
\text { included in overall } \\
\text { cost. }\end{array}$ \\
\hline $\begin{array}{l}\text { Wilson, 2005 } \\
\text { AZ, United States } \\
2 \text { primary care } \\
\text { medical clinics }\end{array}$ & $\begin{array}{l}\text { Before-after } \\
\text { Moderate } \\
\text { Asynchronous } \\
\text { One-time } \\
\text { A: Before telehealth, } \\
n=2,910 \\
\text { B: After telehealth, } n=4,068\end{array}$ & $\begin{array}{l}\text { Retinal exam rate }(95 \% \mathrm{Cl}) \text { for } \\
\text { diabetic patients } \\
\text { A: } \mathbf{5 0 \%}(\mathbf{4 4 \%} \text { to } \mathbf{5 6 \% )} \\
\text { B: } \mathbf{7 5 \%}(\mathbf{7 0 \%} \text { to } \mathbf{8 0} \%), \mathbf{p}<\mathbf{0 . 0 0 0 1} \\
\text { Rate of laser therapy for diabetic } \\
\text { retinopathy } \\
\text { A: } 19.6 \text { per } 1,000 \text { patients with } \\
\text { diabetes } \\
\text { B: } 29.5 \text { per } 1,000 \text { patients with } \\
\text { diabetes } \\
51 \% \text { increase in laser treatment } \\
\text { rate. }\end{array}$ & NR \\
\hline $\begin{array}{l}\text { Zahlmann, } 2002^{188} \\
\text { Germany } \\
5 \text { ophthalmologists }\end{array}$ & $\begin{array}{l}\text { Prospective and } \\
\text { retrospective cohort } \\
\text { High } \\
\text { Both (asynchronous } \\
\text { followed by real time) } \\
\text { One time } \\
\text { A: Not telehealth, } n=20 \\
\text { B: Telehealth, } n=42\end{array}$ & $\begin{array}{l}\text { Mean number of visits } \\
\text { To referring ophthalmologists } \\
\text { A: } 1.85 \\
\text { B: } 2.02, \mathrm{NS} \\
\text { To surgical ophthalmologists } \\
\text { A: } \mathbf{2 . 0 5} \\
\text { B: } \mathbf{1 . 0 7 ,} \mathbf{p}=\mathbf{0 . 0 0 0 1} \\
\text { Mean travel time } \pm \mathrm{SD} \text {, in hours } \\
\text { A: } 2.53 \\
\text { B: } 2.17, \mathrm{NS} \\
\text { Total ophthalmologist } \pm \mathrm{SD} \text {, in } \\
\text { hours } \\
\text { A: } \mathbf{3 . 0 3} \pm \mathbf{0 . 7 3} \\
\text { B: } \mathbf{2 . 0 8} \pm \mathbf{0 . 6 1}, \mathbf{p}=\mathbf{0 . 0 0 0 1} \\
\text { Mean satisfaction with overall } \\
\text { treatment }(10 \text { point scale; } 0=\text { very } \\
\text { positive }) \\
\text { A: } \mathbf{0 . 9 5} \\
\text { B: } \mathbf{0 . 1 4}, \mathbf{p}=\mathbf{0 . 0 1 9}\end{array}$ & NR \\
\hline
\end{tabular}

$\mathrm{CI}=$ confidence interval; NR = not reported; NS = not significant; NZ = New Zealand dollars; $\mathrm{SD}$ = standard deviation; TH = telehealth; USD = United States dollars

Bold=statistically significant; telehealth superior. Bold and italicized=statistically significant; telehealth inferior. Regular type: not statistically significant. 


\section{Orthopedics}

Six articles reported the results of four studies about telehealth in orthopedic consultations (Table 16). One study used consultations and image transmission to assess fractures, ${ }^{158}$ one used telehealth consultations to screen electronic records and recommend treatments for osteoporosis, ${ }^{79}$ and two studies evaluated a range of orthopedic conditions encountered in primary care that would be referred to orthopedic surgeons. ${ }^{151,153}$ None of these studies reported clinical outcomes; they all reported either intermediate or economic outcomes. One of these found that using telehealth to transmit records and x-rays resulted in fewer missed fractures and fewer unnecessary hospital trips. ${ }^{158}$ Availability of orthopedic video consultations with primary care practices resulted in lower costs, successful exams, and management plans that were not significantly different. ${ }^{151,187}$ A US VA project had specialists review records of patients with recent fractures and write recommendations about medications and bone density testing for primary care clinicians. These consults conducted via the electronic record significantly increased adherence to guidelines for recommended treatments. ${ }^{79}$

Table 16. Orthopedic telehealth consultations: selected outcomes

\begin{tabular}{|c|c|c|c|}
\hline $\begin{array}{l}\text { Author, Year } \\
\text { Location } \\
\text { Number of Sites } \\
\text { Number of Patients }\end{array}$ & $\begin{array}{l}\text { Study Design } \\
\text { Risk of Bias } \\
\text { Timing } \\
\text { Consultation } \\
\text { Frequency } \\
\text { Comparison, } \mathbf{n}\end{array}$ & Intermediate Outcomes & Cost/Revenue \\
\hline $\begin{array}{l}\text { Harno, } 2001^{151} \\
\text { Finland } \\
2 \text { hospitals }\end{array}$ & $\begin{array}{l}\text { Economic evaluation } \\
\text { High } \\
\text { Real time } \\
\text { One time } \\
\text { A: Not telehealth, } n=168 \\
\text { B: Telehealth, } n=57\end{array}$ & $\begin{array}{l}\text { Mean time of visit, in minutes: } \\
\text { A: } 12 \\
\text { B: } 13 \\
\text { Diagnosis revised } \\
\text { A: } 16 \% \\
\text { B: } 12 \% \\
\text { Decision to perform surgery } \\
\text { A: } 38 \% \\
\text { B: } 53 \%\end{array}$ & $\begin{array}{l}\text { Total cost per } \\
\text { patient } \\
\text { A: } € 154 \\
\text { B: } € 41 \\
\text { Outpatient is } 45 \% \\
\text { higher } \\
\text { Marginal cost } \\
\text { decreased } 48 \\
\text { Euros for each visit }\end{array}$ \\
\hline $\begin{array}{l}\text { Haukipuro, 2000 } \\
\text { Ohinmaa, 2002 } \\
\text { Vuolio } 2003^{187} \text { (1-year } \\
\text { followup) } \\
\text { Finland } \\
1 \text { outpatient clinic }\end{array}$ & $\begin{array}{l}\text { RCT, Economic } \\
\text { evaluation } \\
\text { High } \\
\text { Real time } \\
\text { One time } \\
\text { A: Not telehealth, } n=69 \\
\text { B: Telehealth, } n=76\end{array}$ & $\begin{array}{l}\text { Mean time of visit including travel, in } \\
\text { hours } \\
\text { A: } 8 \\
\text { B: } 1.5 \\
\text { Mean distance travelled, in km } \\
\text { A: } 170 \\
\text { B: } 8 \\
\text { Success of exam, rated at least } \\
\text { good by practitioner } \\
\text { A: } 99 \% \text { of cases } \\
\text { B: } 80 \% \text { of cases } \\
\text { Management plan for first admission } \\
\text { patients } \\
\text { Operation: } \\
\text { A: } 54 \% \\
\text { B: } 64 \% \\
\text { Followup or further examinations: } \\
\text { A: } 18 \% \\
\text { B: } 18 \% \\
\text { Problem solved at } 1^{\text {st }} \text { visit: } \\
\text { A: } 28 \% \\
\text { B: } 18 \% \text {, NS }\end{array}$ & $\begin{array}{l}\text { Total cost per } \\
\text { patient, including } \\
\text { travel and indirect } \\
\text { costs based on } 100 \\
\text { patients } \\
\text { A: } € 114 \text { Euros } \\
\text { B: €88 Euros } \\
\text { Difference } \\
\text { dependent on } \\
\text { patient travel: } \\
\text { Breakeven point: } \\
80 \text { cases at160 km } \\
200 \text { case at } 80 \mathrm{~km}\end{array}$ \\
\hline
\end{tabular}




\begin{tabular}{|c|c|c|c|}
\hline $\begin{array}{l}\text { Author, Year } \\
\text { Location } \\
\text { Number of Sites } \\
\text { Number of Patients }\end{array}$ & $\begin{array}{l}\text { Study Design } \\
\text { Risk of Bias } \\
\text { Timing } \\
\text { Consultation } \\
\text { Frequency } \\
\text { Comparison, } n\end{array}$ & Intermediate Outcomes & Cost/Revenue \\
\hline $\begin{array}{l}\text { Jacobs, } 2015^{158} \\
\text { Ameland, Netherlands } \\
2 \text { general practices }\end{array}$ & $\begin{array}{l}\text { Before-after } \\
\text { High } \\
\text { Asynchronous } \\
\text { One time } \\
\text { A: Before telehealth, } \\
n=312 \\
\text { B: After telehealth, } n=482\end{array}$ & $\begin{array}{l}\text { Referral to hospital } \\
\text { A: } 26.6 \% \\
\text { B: } 8.1 \% \\
\text { Unnecessary trips to the hospital } \\
\text { A: } 13.1 \% \\
\text { B: } 0.4 \% \\
\text { Missed fractures } \\
\text { A: } 13.6 \% \\
\text { B: } 1.7 \%\end{array}$ & NR \\
\hline $\begin{array}{l}\text { Lee, 201479 } \\
\text { United States } \\
3 \text { VA Medical Centers }\end{array}$ & $\begin{array}{l}\text { Prospective cohort } \\
\text { High } \\
\text { Asynchronous } \\
\text { One time } \\
\text { A: Not telehealth, } n=N R \\
\text { B: Telehealth, } n=N R\end{array}$ & $\begin{array}{l}\text { Difference before and after } \\
\text { introduction of telehealth } \\
\\
\text { Change in treatment rates for } \\
\text { bisphosphonates } \\
\text { A: } 1.8 \% \text { decrease } \\
\text { B: } 2.5 \% \text { increase, } p=0.02 \\
\text { Change in treatment rates for } \\
\text { calcium and/or vitamin D } \\
\text { A: } 1.2 \% \text { decrease } \\
\text { B: } 13.9 \% \text { increase, } p<0.01\end{array}$ & NR \\
\hline
\end{tabular}

NR = not reported; NS = not significant; RCT = randomized controlled trial; VA = Veterans Affairs

Bold=statistically significant; telehealth superior. Bold and italicized=statistically significant; telehealth inferior. Regular type: not statistically significant.

\section{Dental}

Four studies, all conducted in Europe, evaluated the use of telehealth for dental consultations (Table 17). Three focused on specific issues (dental implants, ${ }^{167}$ temporomandibular joint [TMJ] disorders, ${ }^{177}$ and impacted molars ${ }^{154}$ ), and the fourth used video to replace in-person visits for restorative dentistry. ${ }^{205}$ The results were not robust, and no clinical outcomes were reported. The strongest result reported is that telehealth consultations resulted in a significantly shorter time to treatment for TMJ (76.8 days versus 2.3). ${ }^{177}$ The single cost analysis determined that telehealth visits cost less than hospital visits but more than outreach visits (i.e., when dentists go to communities in need of services). ${ }^{205}$ 
Table 17. Dentistry telehealth consultations: selected outcomes

\begin{tabular}{|c|c|c|c|}
\hline $\begin{array}{l}\text { Author, Year } \\
\text { Location } \\
\text { Number of Sites } \\
\text { Number of } \\
\text { Patients }\end{array}$ & $\begin{array}{l}\text { Study Design } \\
\text { Risk of Bias } \\
\text { Timing } \\
\text { Consultation Frequency } \\
\text { Comparison, } n\end{array}$ & Intermediate Outcomes & $\begin{array}{l}\text { Cost/Revenue } \\
\text { Outcomes }\end{array}$ \\
\hline $\begin{array}{l}\text { Herce, } 2011^{154} \\
\text { Seville, Spain } \\
4 \text { primary care } \\
1 \text { university } \\
\text { hospital }\end{array}$ & $\begin{array}{l}\text { Prospective cohort } \\
\text { High } \\
\text { One-time } \\
\text { Store and forward } \\
\text { A: Usual care } n=2,550 \\
\text { B: Telehealth } n=97\end{array}$ & $\begin{array}{l}\text { Time to care outcome measured differently } \\
\text { in A and B } \\
\text { Mean days to intervention }(95 \% \mathrm{Cl}) \\
\text { A: } \mathbf{2 8}(\mathbf{2 4 . 5 1} \text { to } 29.6) \\
\text { Mean days to be added to surgical wait list } \\
\text { B: } \mathbf{3 . 3 3}(\mathbf{2} \text { to } 4.65), \mathbf{p}<\mathbf{0 . 0 0 1} \\
\text { Cancellation rate on day of surgery, }(95 \% \\
\text { Cl) } \\
\text { A: } 8.85 \%(5.62 \text { to } 11.81 \%) \\
\text { B: } 7.8 \%(3.8 \% \text { to } 10.5 \%), p=0.76\end{array}$ & NR \\
\hline $\begin{array}{l}\text { Nickenig,2008 } \\
\text { Germany } \\
1 \text { dental clinic } \\
2 \text { external } \\
\text { experts }\end{array}$ & $\begin{array}{l}\text { Prospective cohort } \\
\text { Moderate } \\
\text { Real time } \\
\text { One time } \\
\text { A: No telehealth, } n=772 \\
\text { B: Telehealth, } n=85\end{array}$ & $\begin{array}{l}\text { Changes in diagnosis } \\
\text { A: } 4 \% \\
\text { B: } 0 \% \\
\text { Change in prosthodontic protocol } \\
\text { A: } 7 \% \\
\text { B: } 3 \% \\
\text { Change in number and position of implants } \\
\text { A: } 19 \% \\
\text { B: } 15 \%\end{array}$ & NR \\
\hline $\begin{array}{l}\text { Salazar- } \\
\text { Fernandez, } \\
2012^{177} \\
\text { Seville, Spain } \\
1 \text { hospital } \\
10 \text { Primary Cares }\end{array}$ & $\begin{array}{l}\text { Prospective cohort } \\
\text { Clinical outcomes: } \\
\text { Moderate } \\
\text { Economic outcomes: High } \\
\text { Asynchronous } \\
\text { Continuing } \\
\text { A: No telehealth, } n=710 \\
\text { B: Telehealth, } n=342\end{array}$ & $\begin{array}{l}\text { Patients referred to maxillofacial surgery } \\
\text { A: } 11.6 \% \\
\text { B: } 10.2 \%, \text { NS } \\
\text { Resolved consultation } \\
\text { A: } 74.5 \% \\
\text { B: } 88 \%, \text { NS } \\
\text { Second consultations } \\
\text { A: } 4.6 \% \\
\text { B: } 0.8 \%, \text { NS } \\
\text { Mean lost working hours } \\
\text { A: } \mathbf{3 2 . 2 4} \\
\text { B: } \mathbf{1 6 . 8 0}, \mathbf{p}=0.01 \\
\text { Mean time to treatment, in days } \\
\text { A: } \mathbf{7 8 . 6} \\
\text { B: } \mathbf{2 . 3}, \mathbf{p}<0.001 \\
\text { Complaints } \\
\text { A: } 0.8 \% \\
\text { B: } 0.3 \%, \text { NS }\end{array}$ & NR \\
\hline $\begin{array}{l}\text { Scuffham, } 2002^{205} \\
\text { UK } \\
2 \text { general dental } \\
\text { practices } \\
1 \text { hospital }\end{array}$ & $\begin{array}{l}\text { Economic evaluation } \\
\text { Moderate } \\
\text { Real time } \\
\text { One time } \\
A_{1} \text { : No telehealth, outreach } \\
\text { visits, } n=N R \\
A_{2} \text { : No telehealth, hospital } \\
\text { visits, } n=N R \\
B: \text { Telehealth, } n=N R\end{array}$ & NR & $\begin{array}{l}\text { Total variable costs } \\
\text { per patient, in GBP } \\
A_{1}: £ 233.86 \\
A_{2}: £ 1,181.52 \\
B: £ 404.10 \\
\text { Total societal costs, } \\
\text { in GBP } \\
A_{1}: £ 403.11 \\
A_{2}: £ 1,181.51 \\
B: £ 582.69\end{array}$ \\
\hline
\end{tabular}

CI = confidence interval; GBP = British pound; NR = not reported; NS = not significant

Bold=statistically significant; telehealth superior. Bold and italicized=statistically significant; telehealth inferior. Regular type: not statistically significant. 


\section{Cancer}

We identified ten articles reporting on nine studies about using telehealth for consultations in cancer care (Table 18). In these studies, telehealth was either used to convene virtual tumor boards and interdisciplinary meetings or to allow oncologists to confer with patients and a local physician to plan for cancer care provision in a remote or rural area. All of these studies used video to communicate, and records and images were shared electronically.

Outcomes in included cancer studies varied. A study of remote oncology guidance for chemotherapy was the only one to measure patient outcomes and reported the rate of serious side effects per patient was lower in the telehealth group. ${ }^{234}$ In the other studies, the effectiveness of cancer teleconsultations was evaluated in terms of care processes, satisfaction, and cost. In one study, using a referral institution and nine other hospitals in the US VA, telehealth provided more comprehensive care and avoided travel, but the time from referral to treatment was not significantly different. ${ }^{112}$ In a cluster RCT of breast cancer planning meetings in Scotland, telemedicine was less expensive, and the ratings of participants were not different except that face-to-face meeting participants felt a consensus was reached more frequently than did telehealth participants. ${ }^{200}$ Likewise, a study conducted in Sweden reported similar ratings of communication by telehealth participants as in face-to-face and in-person tumor boards, similar presentation time, less time traveling and waiting, and overall similar costs because equipment costs balanced out the reduction in travel costs. ${ }^{181,182}$ Another study in Australia reported net savings as the travel avoided exceeded the cost of telehealth equipment. ${ }^{230}$ In two studies the impact of telemedicine on time to treatment is less clear; one reported shorter mean time to initial evaluation (18 vs. 21 days) but longer time from evaluation to surgery ( 28 vs. 48 days $)^{32}$ while the second found no significant difference in the time from visit to surgery. ${ }^{198}$

Table 18. Cancer telehealth consultations: selected outcomes

\begin{tabular}{|c|c|c|c|}
\hline $\begin{array}{l}\text { Author, Year } \\
\text { Location } \\
\text { Number of Sites } \\
\text { Number of } \\
\text { Patients }\end{array}$ & $\begin{array}{l}\text { Study Design } \\
\text { Risk of Bias } \\
\text { Timing } \\
\text { Consultation Frequency } \\
\text { Comparison, } n\end{array}$ & $\begin{array}{l}\text { Clinical Outcomes } \\
\text { Intermediate Outcomes } \\
\text { (Clinical outcomes are UNDERLINED AND } \\
\text { CAPITALIZED) }\end{array}$ & Cost/Revenue Outcomes \\
\hline $\begin{array}{l}\text { Beswick, } 2016^{32} \\
\text { CA, United } \\
\text { States } \\
1 \text { Medical Center } \\
2 \text { remote sites }\end{array}$ & $\begin{array}{l}\text { Prospective cohort } \\
\text { High } \\
\text { Real-time } \\
\text { Continuing } \\
\text { A: Not telehealth, } n=26 \\
\text { B: Telehealth, } n=15\end{array}$ & $\begin{array}{l}\text { Mean time from initial referral to evaluation } \\
\text { (range), in days } \\
\text { A: } 21 \text { (6 to } 61) \\
\text { B: } 18 \text { (6 to } 53) \\
\text { Mean time from evaluation to surgery } \\
\text { (range), in days } \\
\text { A: } 28 \text { (0 to } 55) \\
\text { B: } 48 \text { (11 to } 101) \\
\text { Mean time from referral to surgery (range), } \\
\text { in days } \\
\text { A: } 49 \text { (22 to } 83) \\
\text { B: } 54(17 \text { to } 108)\end{array}$ & NR \\
\hline
\end{tabular}




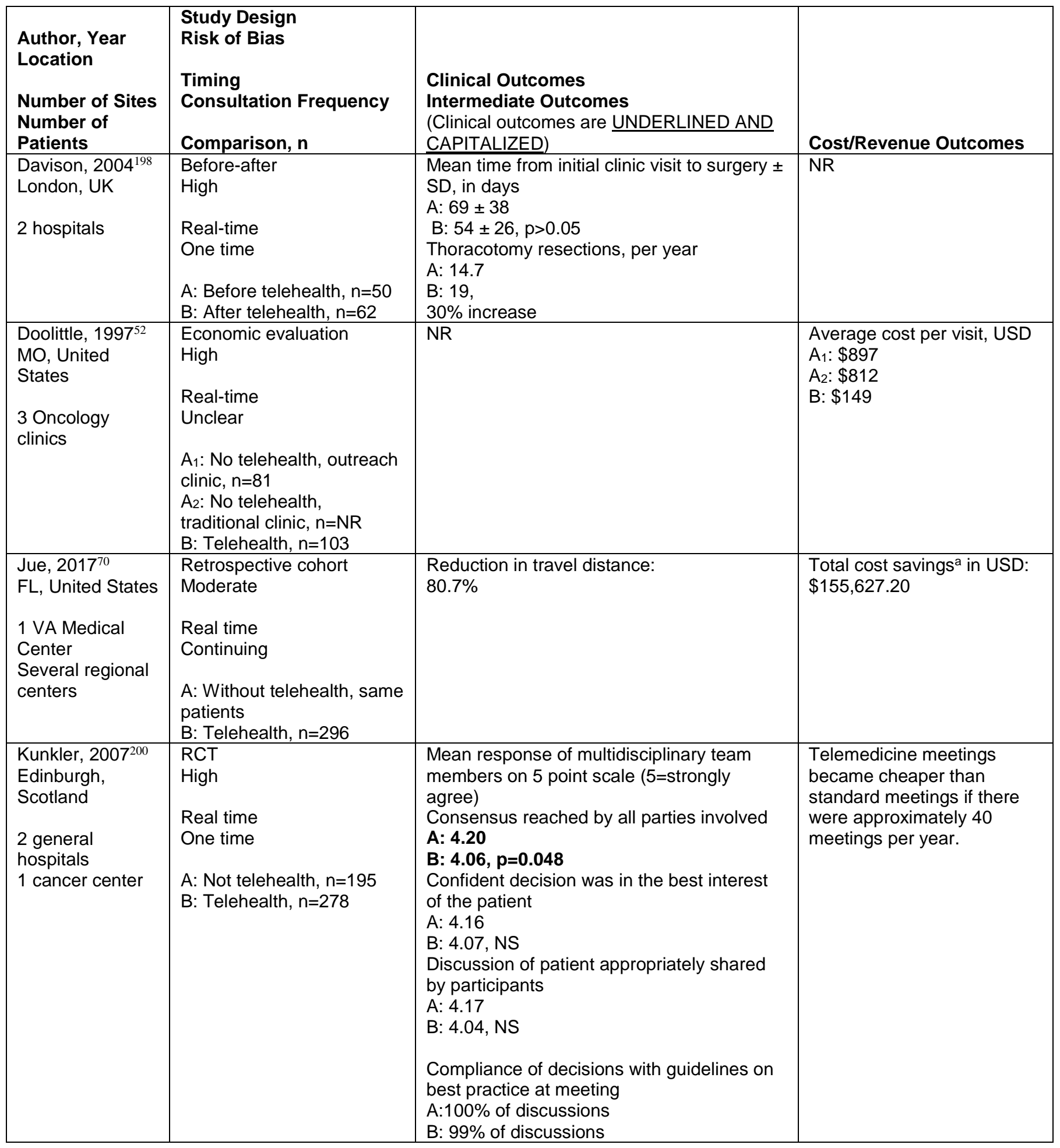




\begin{tabular}{|c|c|c|c|}
\hline $\begin{array}{l}\text { Author, Year } \\
\text { Location } \\
\text { Number of Sites } \\
\text { Number of } \\
\text { Patients }\end{array}$ & $\begin{array}{l}\text { Study Design } \\
\text { Risk of Bias } \\
\text { Timing } \\
\text { Consultation Frequency } \\
\text { Comparison, } \mathbf{n}\end{array}$ & $\begin{array}{l}\text { Clinical Outcomes } \\
\text { Intermediate Outcomes } \\
\text { (Clinical outcomes are UNDERLINED AND } \\
\text { CAPITALIZED) }\end{array}$ & Cost/Revenue Outcomes \\
\hline $\begin{array}{l}\text { Salami, } 2015^{112} \\
\text { United States } \\
1 \text { VA referral } \\
\text { institution } \\
9 \text { VA Medical } \\
\text { Centers }\end{array}$ & $\begin{array}{l}\text { Retrospective cohort } \\
\text { Moderate } \\
\text { Real time } \\
\text { One time } \\
\text { A: Not telehealth, } n=68 \\
\text { B: Telehealth, } n=48\end{array}$ & $\begin{array}{l}\text { Comprehensive clinical evaluation prior to } \\
\text { initiation of treatment } \\
\text { A: } \mathbf{6 4 . 7 \%} \\
\text { B: } 91.7 \%, p=0.001 \\
\text { Guideline driven clinical evaluation prior to } \\
\text { initiation of treatment } \\
\text { A: } 75 \% \\
\text { B: } 100 \%, p<0.001 \\
\text { Assessment of tumor stage } \\
\text { A: } 73.5 \% \\
\text { B: } 91.7 \%, p=0.002 \\
\text { Assessment of transplant eligibility } \\
\text { A: } 85.3 \% \\
\text { B: } 95.8 \%, p=0.006 \\
\text { Median time from referral to evaluation, in } \\
\text { days } \\
\text { A: } 39 \\
\text { B: } 23, p<0.001 \\
\text { Median time from referral to treatment } \\
\text { initiation, in days } \\
\text { A: } 63 \\
B: 55, p=0.152 \\
\text { Median distance travelled by patient to } \\
\text { receive evaluation, in miles } \\
\text { A: } 683 \\
\text { B: } 0, p<0.001\end{array}$ & NR \\
\hline
\end{tabular}




\begin{tabular}{|c|c|c|c|}
\hline $\begin{array}{l}\text { Author, Year } \\
\text { Location } \\
\text { Number of Sites } \\
\text { Number of } \\
\text { Patients } \\
\end{array}$ & $\begin{array}{l}\text { Study Design } \\
\text { Risk of Bias } \\
\text { Timing } \\
\text { Consultation Frequency } \\
\text { Comparison, } n\end{array}$ & $\begin{array}{l}\text { Clinical Outcomes } \\
\text { Intermediate Outcomes } \\
\text { (Clinical outcomes are UNDERLINED AND } \\
\text { CAPITALIZED) }\end{array}$ & Cost/Revenue Outcomes \\
\hline $\begin{array}{l}\text { Stalfors, } 2005^{181} \\
\text { Sweden } \\
1 \text { regional } \\
\text { hospital } \\
2 \text { district general } \\
\text { hospitals }\end{array}$ & $\begin{array}{l}\text { Economic evaluation } \\
\text { Moderate } \\
\text { Real time } \\
\text { One time } \\
\text { A: Not telehealth, } n=50 \\
\text { B: Telehealth, } n=68\end{array}$ & NR & $\begin{array}{l}\text { Combined cost, in SEK } \\
\text { A: } 2,267 \\
\text { B: } 2,036, N S \\
\text { Direct medical, in SEK } \\
\text { A: } 576 \\
\text { B: } 1,550^{\text {b }} \\
\text { Direct nonmedical, in SEK } \\
\text { A: } 886 \\
\text { B: } 176 \\
\text { Indirect nonmedical, in SEK } \\
\text { A: } 805 \\
\text { B: } 310\end{array}$ \\
\hline $\begin{array}{l}\text { Thaker, } 2013^{230} \\
\text { Queensland, } \\
\text { Australia } \\
1 \text { cancer center } \\
6 \text { rural centers }\end{array}$ & $\begin{array}{l}\text { Economic evaluation } \\
\text { Moderate } \\
\text { Real time } \\
\text { Continuing } \\
\text { A: Not telehealth, } n=N R \\
\text { B: Telehealth, } n=147\end{array}$ & NR & $\begin{array}{l}\text { In AUD } \\
\text { Net savings: } \$ 320,118 \\
\text { Total cost of teleconsults: } \\
\$ 442,276 \\
\text { Estimated travel expense } \\
\text { avoided: } \$ 762,394 \\
\text { Travel costs for patients and } \\
\text { escorts: } \$ 658,760 \\
\text { Aeromedical retrievals : } \\
\$ 52,400 \\
\text { Travel for specialists: } \\
\$ 47,634 \\
\text { Accommodation costs for a } \\
\text { proportion of patients: } \\
\$ 3,600 \\
\text { Telehealth costs would need } \\
\text { to increase } 72 \% \text { to negate } \\
\text { savings. }\end{array}$ \\
\hline
\end{tabular}

Abbreviation: AUD = Australian dollars; CI = confidence interval; NR = not reported; NS = not significant; RCT = randomized controlled trial; SD = standard deviation; SEK = Swedish Krona; VA = Veterans Affairs; UK = United Kingdom; USD = United States dollars

${ }^{a}$ Combination of commuting and hotel fees averted

b SEK 1,288 is equipment cost + hypothetical avoided costs reported in article not included

Bold=statistically significant; telehealth superior. Bold and italicized=statistically significant; telehealth inferior. Regular type: not statistically significant

\section{Psychiatry}

Five articles reported the results of two studies of telehealth programs used to provide consultations for the treatment of depression ${ }^{46,57-59}$ and one study of consultations for treatment of posttraumatic stress disorder (PTSD) ${ }^{56}$ in adults (Table 19). Telehealth was used in all of the programs to facilitate a multifaceted comprehensive treatment program. The telehealth versions of these evidence-based treatment programs were designed to expand access to mental healthcare in rural areas or to practices with no mental health services. The studies randomized either practices or patients to the telehealth program or usual care. All three programs reported improvement in clinical outcomes such as decreases in symptoms or higher remission rates of systems after 6 months or a year. Intermediate outcomes, such as medication adherence and satisfaction, were also higher. The one analysis of costs found an expected increase in primary care costs for depression treatment, along with an increase in specialty physical care costs, which 
were attributed to case management referrals for pain management and management of other comorbid chronic conditions. ${ }^{57}$ The same study also found that minority patients responded to treatment at higher rates using telehealth, suggesting that telehealth as part of collaborative care could help ameliorate racial disparities in care. ${ }^{46}$

Table 19. Psychiatry telehealth consultations: selected outcomes

\begin{tabular}{|c|c|c|c|}
\hline $\begin{array}{l}\text { Author, Year } \\
\text { Location } \\
\text { Number of Sites } \\
\text { Number of } \\
\text { Patients }\end{array}$ & $\begin{array}{l}\text { Study Design } \\
\text { Risk of Bias } \\
\text { Timing } \\
\text { Consultation } \\
\text { Frequency } \\
\text { Comparison }\end{array}$ & Clinical Outcomes & Intermediate Outcomes \\
\hline $\begin{array}{l}\text { Fortney, } 2007^{58} ; \\
2011^{57} ; \\
\text { Davis, } 2011^{46} \\
\text { South-central } \\
\text { United States } \\
7 \text { Veterans } \\
\text { Administration } \\
\text { primary care } \\
\text { centers }\end{array}$ & $\begin{array}{l}\text { RCT: Moderate } \\
\text { Economic } \\
\text { evaluation }{ }^{57} \text { : High } \\
\text { Real time and } \\
\text { asynchronous } \\
\text { Continuing } \\
\text { A: Not telehealth, } \\
\mathrm{n}=218 \\
\mathrm{~B}: \text { Telehealth, } \\
\mathrm{n}=177\end{array}$ & 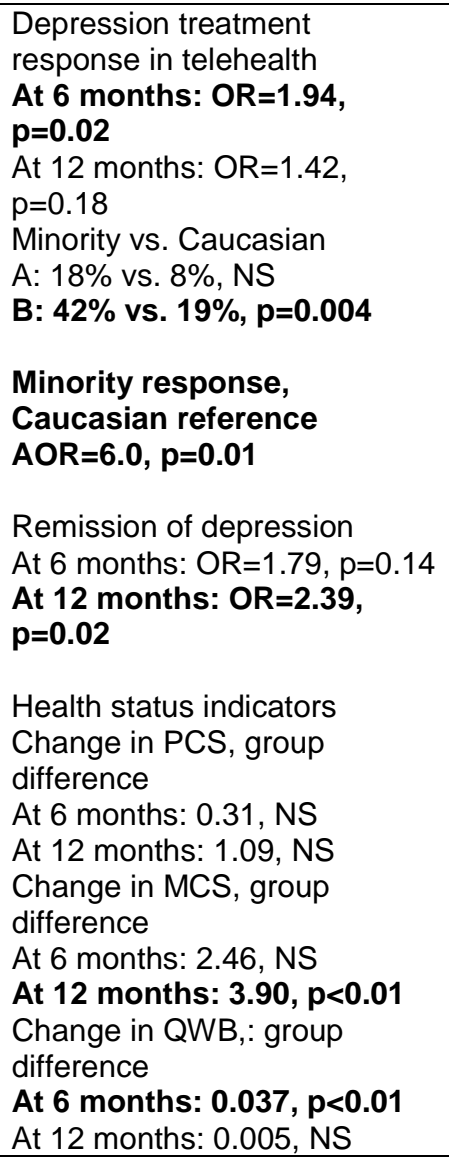 & $\begin{array}{l}\text { Medication adherence at specified } \\
\text { month, OR } \\
6 \text { months: } 2.11, p=0.04 \\
12 \text { months: } 2.72, p<0.01 \\
\text { Treatment satisfaction at specified } \\
\text { month, OR } \\
6 \text { months: } 1.83, p=0.01 \\
12 \text { months: } 1.71, p=0.03 \\
\text { Economic outcomes } \\
\text { Expected increase in primary care: } \\
\text { Encounters: Marginal effect } 0.34 \text {, } \\
p=0.004 \\
\text { Costs: Marginal effect } \$ 61.4, \\
p=0.013 \\
\text { Unexpected increase in specialty } \\
\text { physical healthcare: } \\
\text { Encounters: Marginal effect } 0.42, \\
p=0.001 \\
\text { Costs: Marginal effect } \$ 490.60 \\
p=0.003\end{array}$ \\
\hline $\begin{array}{l}\text { Fortney,201359 } \\
\text { AR, United States } \\
5 \text { Federally } \\
\text { Qualified Health } \\
\text { Centers }\end{array}$ & $\begin{array}{l}\text { RCT } \\
\text { Moderate } \\
\text { Real time } \\
\text { Continuing } \\
\text { A: Not telehealth, } \\
n=185 \\
\text { B: Telehealth, } \\
n=179\end{array}$ & $\begin{array}{l}\text { Response to treatment } \\
\text { AOR: } 7.74, \mathbf{p}<0.0001 \\
\text { Remission } \\
\text { AOR: } 12.69, \mathbf{p}<0.0001 \\
\text { Adherence } \\
\text { AOR: } 1.22, \mathrm{NS} \\
\text { Any specialty mental health } \\
\text { visits } \\
\text { AOR: } 0.56, \mathrm{NS} \\
\text { Primary care visits } \\
\text { AIRR: } 1.16, \mathrm{NS} \\
\text { Depression-related primary } \\
\text { care visits } \\
\text { AIRR: } 0.99, \mathrm{NS}\end{array}$ & $\begin{array}{l}\text { Satisfaction, AOR } \\
\text { At baseline: } 1.08, \mathrm{NS} \\
\text { At } 6 \text { months: } 2.76, \mathrm{p}=0.0012 \\
\text { At } 12 \text { months: } 1.99, \mathrm{p}=0.0313 \\
\text { At } 18 \text { months: } 1.67, \mathrm{NS} \\
\text { Adjusted group difference in } \\
\text { depression severity } \\
\text { Baseline: }-0.04, \mathrm{NS} \\
\text { At } 6 \text { months: }-0.50, \mathrm{p}<0.0001 \\
\text { At } 12 \text { months: }-0.49, \mathrm{p}<0.0001 \\
\text { At } 18 \text { months: }-0.33, \mathrm{p}<0.0001\end{array}$ \\
\hline
\end{tabular}




\begin{tabular}{|c|c|c|c|}
\hline $\begin{array}{l}\text { Author, Year } \\
\text { Location } \\
\text { Number of Sites } \\
\text { Number of } \\
\text { Patients } \\
\end{array}$ & $\begin{array}{l}\text { Study Design } \\
\text { Risk of Bias } \\
\text { Timing } \\
\text { Consultation } \\
\text { Frequency } \\
\text { Comparison }\end{array}$ & Clinical Outcomes & Intermediate Outcomes \\
\hline $\begin{array}{l}\text { Fortney, } 2015^{56} \\
\text { United States } \\
11 \text { Veterans } \\
\text { Administration } \\
\text { outpatient clinics }\end{array}$ & $\begin{array}{l}\text { RCT } \\
\text { Moderate } \\
\text { Real time } \\
\text { Continuing } \\
\text { A: Not telehealth, } \\
n=132 \\
\text { B: Telehealth, } \\
n=133\end{array}$ & 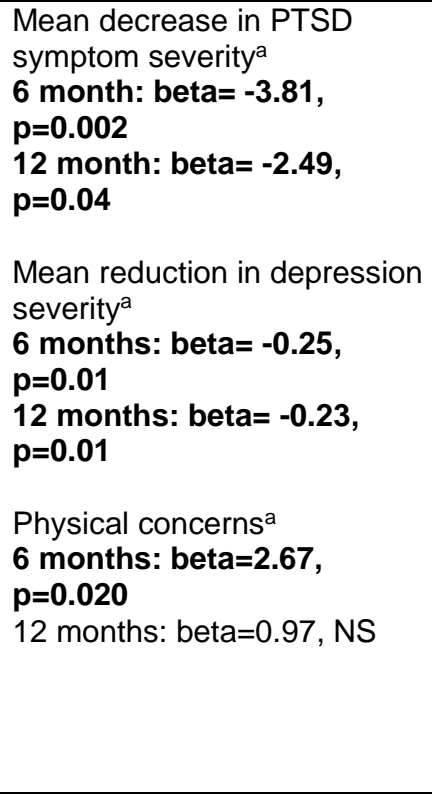 & $\begin{array}{l}\text { Adherence to medication, OR } \\
6 \text { months: } 0.86, N S \\
12 \text { months: } 0.91, N S \\
\text { Any PTSD medication prescriptions, } \\
\text { OR } \\
6 \text { months: } 2.98, N S \\
\text { Prescribed Prazosin prescription, OR } \\
6 \text { months: } 2.43 \text {, NS } \\
\text { Percent attending } \geq 8 \text { psychotherapy } \\
\text { sessions: } \\
\text { A: } 5.3 \% \\
\text { B: } 27.1 \% \\
\text { Percent receiving cognitive } \\
\text { processing therapy: } \\
\text { A: } 12.1 \% \\
\text { B: } 54.9 \% \\
\text { Mean number of cognitive } \\
\text { processing therapy sessions } \\
\text { attended: } \\
\text { A: } \mathbf{0 . 8} \\
\text { B: } \mathbf{4 . 2} \\
\text { RR } \mathbf{9 . 5 1 ,} \text { p }<0.001\end{array}$ \\
\hline
\end{tabular}

AIRR = adjusted incidence rate ratio; AOR = adjusted odds ratio; MCS = Mental Component Summary; NS = not significant; OR = odds ratio; PCS = Physical Component Summary; PTSD = post-traumatic stress disorder; QWB = quality of well-being score; RCT = randomized controlled trial; RR = risk ratio

a PTSD severity measured by Posttraumatic Diagnostic Scale, depression severity measured by Hopkins Symptom Checklist, and physical concerns measured by Physical Component Summary

Bold=statistically significant; telehealth superior. Bold and italicized=statistically significant; telehealth inferior. Regular type: not statistically significant

\section{Infectious Disease}

Four studies addressed the use of telehealth in infectious diseases (Table 20). Two of these studies assessed using telehealth to advise on treatment for patients with hepatitis $\mathrm{C},{ }^{30,108}$ one studied providing subspecialty consultations about patients with HIV in prison, ${ }^{134}$ and one evaluated a system of e-consults for a range of infectious diseases. ${ }^{119}$ Three of the studies used video for real time communication ${ }^{30,108,134}$ while one incorporated the consult into the electronic health record and allowed the specialists to review and respond when they were available. ${ }^{119}$ All four studies were conducted in the United States.

Clinical outcomes focused on viral load or suppression and were not significantly different in the two studies of hepatitis C. ${ }^{30,108}$ The HIV study reported significantly better outcomes after six visits. ${ }^{134}$ An e-consult system or various infectious diseases reduced time to completion of the consultations from a mean of 16.5 days to about one half day (0.6 days). ${ }^{119}$ None of these studies reported economic outcomes. 
Table 20. Infectious disease telehealth consultations: selected outcomes

\begin{tabular}{|c|c|c|c|c|}
\hline $\begin{array}{l}\text { Condition } \\
\text { or } \\
\text { Specialty }\end{array}$ & $\begin{array}{l}\text { Author, Year } \\
\text { Location } \\
\text { Number of Sites } \\
\text { Number of } \\
\text { Patients }\end{array}$ & $\begin{array}{l}\text { Study Design } \\
\text { Risk of Bias } \\
\text { Timing } \\
\text { Consultation } \\
\text { Frequency } \\
\text { Comparison, } \mathbf{n}\end{array}$ & Clinical Outcomes & Intermediate Outcomes \\
\hline $\begin{array}{l}\text { Hepatitis } \\
\text { C }\end{array}$ & $\begin{array}{l}\text { Rossaro, } 2013^{108} \\
\text { CA, United } \\
\text { States } \\
1 \text { physician } \\
5 \text { telemedicine } \\
\text { clinics } \\
1 \text { university clinic }\end{array}$ & $\begin{array}{l}\text { Retrospective } \\
\text { cohort } \\
\text { Moderate } \\
\text { Real time } \\
\text { Continuing } \\
\text { A: No telehealth, } \\
n=40 \\
B: \text { Telehealth, } \\
n=40\end{array}$ & $\begin{array}{l}\text { Sustained virologic response } \\
\text { A: } 43 \% \\
\text { B: } 55 \% \text {, NS }\end{array}$ & $\begin{array}{l}\text { Completion of therapy, } n \\
(\%) \\
\text { A: } \mathbf{2 1}(\mathbf{5 3 \% )} \\
\text { B: } \mathbf{3 1}(\mathbf{7 8 \% )}, \mathbf{p}=\mathbf{0 . 0 3} \\
\text { Mean number of weeks of } \\
\text { therapy } \\
\text { A: } 30.2 \\
\text { B: } 36.7, \mathrm{NS} \\
\text { Mean number of visits } \\
\text { A: } \mathbf{2 . 2} \\
\text { B:19.6, p<0.0001 } \\
\text { Mean number of visits per } \\
\text { week } \\
\text { A: } \mathbf{0 . 0 7} \\
\text { B: } \mathbf{0 . 6 1 ,} \mathbf{p}<0.001 \\
\text { Stopped therapy due to } \\
\text { depression } \\
\text { A: } 2.5 \% \\
\text { B: } 10.0 \% \\
\text { Anti-depressant } \\
\text { medication } \\
\text { A: } 17.5 \% \\
\text { B: } 35.0 \% \\
\text { Reasons for early } \\
\text { termination of therapy: } \\
\text { A: Severe anemia, skin } \\
\text { rash, and weight loss } \\
\text { B: Severe depression, NS }\end{array}$ \\
\hline $\begin{array}{l}\text { Hepatitis } \\
\text { C }\end{array}$ & $\begin{array}{l}\text { Arora, } 2011^{30} \\
\text { NM, United } \\
\text { States } \\
1 \text { university clinic } \\
21 \text { rural clinics }\end{array}$ & $\begin{array}{l}\text { Prospective cohort } \\
\text { Low } \\
\text { Real time } \\
\text { Continuing } \\
\text { A: No telehealth, } \\
n=146 \\
\text { B: Telehealth, } \\
n=261\end{array}$ & $\begin{array}{l}\text { Sustained virologic response } \\
\text { All genotypes } \\
\text { A: } 57.5 \% \\
\text { B: } 58.2 \% \\
\text { Difference in response, } \\
\text { percentage points }(95 \% \mathrm{CI}) \\
\text { All genotypes: } 0.7(-9.2 \text { to } 10.7) \text {, } \\
\text { NS } \\
\text { Genotype } 1: 3.9 \text { (-9.5 to } 17.0) \text {, } \\
\text { NS } \\
\text { Genotype } 2 \text { or } 3:-1.5 \text { (-15.2 to } \\
\text { 13.3), NS } \\
\text { Sustained virologic response in } \\
\text { univariate models } \\
\text { OR: } 1.03, \text { NS } \\
\text { In multivariate models } \\
\text { AOR } 1.10, \text { NS } \\
\text { Serious adverse events } \\
\text { A: } 13.7 \% \\
\text { B: } 6.9 \%, p=0.02\end{array}$ & NR \\
\hline
\end{tabular}




\begin{tabular}{|c|c|c|c|c|}
\hline $\begin{array}{l}\text { Condition } \\
\text { or } \\
\text { Specialty }\end{array}$ & $\begin{array}{l}\text { Author, Year } \\
\text { Location } \\
\text { Number of Sites } \\
\text { Number of } \\
\text { Patients }\end{array}$ & $\begin{array}{l}\text { Study Design } \\
\text { Risk of Bias } \\
\text { Timing } \\
\text { Consultation } \\
\text { Frequency } \\
\text { Comparison, n }\end{array}$ & Clinical Outcomes & Intermediate Outcomes \\
\hline HIV & $\begin{array}{l}\text { Young, 2014 }{ }^{134} \\
\text { IL, United States } \\
1 \text { Correctional } \\
\text { Facility } \\
1 \text { University } \\
\text { Telemedicine } \\
\text { Clinic }\end{array}$ & $\begin{array}{l}\text { Before-after } \\
\text { Moderate } \\
\text { Real time } \\
\text { Continuing } \\
\\
\text { A: Before } \\
\text { telehealth, } n=514 \\
\text { B: After telehealth, } \\
n=687\end{array}$ & $\begin{array}{l}\text { Complete virologic suppression } \\
\text { attained during first } 6 \text { visits } \\
\text { A: } \mathbf{5 9 . 3 \%} \\
\text { B: } \mathbf{9 1 . 1 \%} \\
\text { OR: } \mathbf{7 . 0}(\mathbf{9 5 \%} \text { CI } 5.1 \text { to } 9.8) \text {, } \\
\text { p<0.001 }\end{array}$ & NR \\
\hline Several & $\begin{array}{l}\text { Strymish, } 2017^{119} \\
\text { MA, United } \\
\text { States } \\
5 \text { community- } \\
\text { based outpatient } \\
\text { clinics } \\
3 \text { main campuses }\end{array}$ & $\begin{array}{l}\text { Before-after } \\
\text { High } \\
\text { Asynchronous } \\
\text { One time } \\
\text { A: Before } \\
\text { telehealth, } n=195 \\
\text { B: After telehealth, } \\
n=285\end{array}$ & NR & $\begin{array}{l}\text { Time to completion for e- } \\
\text { consults, mean } \pm \text { SD } \\
\text { A: } 16.5 \text { days } \pm 12.4 \\
\text { B: } 0.6 \text { days } \pm \mathbf{3 . 6}, p<0.05\end{array}$ \\
\hline
\end{tabular}

AOR = adjusted odds ratio; $\mathrm{CI}$ = confidence interval; $\mathrm{HCV}$ = hepatitis $\mathrm{C}$ virus; $\mathrm{NS}$ = not significant; $\mathrm{OR}$ = odds ratio; $\mathrm{SD}$ = standard deviation

Bold=statistically significant; telehealth superior. Bold and italicized=statistically significant; telehealth inferior. Regular type: not statistically significant.

\section{Consultations for Single Conditions Using Diagnostic Technology}

Telehealth was used for consultations for a specific specialty in 10 studies. The consultations involved guiding the use of diagnostic technology and assessing the transmitted information (Table 21). These studies used fetal echocardiograms, ${ }^{114,140,203,211,258}$ ultrasound, ${ }^{37,82}$ endoscopy, ${ }^{206,231}$ and Doppler. ${ }^{54}$ Across these studies, telehealth consultations increased timely access to tests and improved management. Costs were lower, but only due to savings for patients. None of these studies reported patient clinical outcomes or harms. 
Table 21. Single specialties using diagnostic technology: selected outcomes

\begin{tabular}{|c|c|c|c|c|}
\hline $\begin{array}{l}\text { First Author, Year } \\
\text { Location } \\
\text { Specialty and } \\
\text { Technology } \\
\text { Number of Sites }\end{array}$ & $\begin{array}{l}\text { Study Design } \\
\text { Risk of Bias } \\
\text { Timing } \\
\text { Consultation } \\
\text { Frequency } \\
\text { Comparison, } \mathbf{n} \\
\end{array}$ & $\begin{array}{l}\text { Utilization } \\
\text { Outcomes }\end{array}$ & $\begin{array}{l}\text { Other Intermediate } \\
\text { Outcomes }\end{array}$ & $\begin{array}{l}\text { Economic } \\
\text { Outcomes } \\
\end{array}$ \\
\hline $\begin{array}{l}\text { Bagayoko,2014 } \\
\text { Mali } \\
\text { Obstetrics and } \\
\text { fetal } \\
\text { echocardiogram } \\
8 \text { clinics }\end{array}$ & $\begin{array}{l}\text { Prospective cohort } \\
\text { for patient costs } \\
\text { Before-after for } \\
\text { health center costs } \\
\text { High } \\
\text { Real time } \\
\text { (presumed, not } \\
\text { stated) } \\
\text { One time } \\
\text { A: No telehealth, } \\
\text { n=NR } \\
\text { B: Telehealth, n=NR }\end{array}$ & $\begin{array}{l}\text { Attendance rate } \\
\text { increase } \\
\text { A: } 44.9 \% \\
\text { B: } 79.8 \%\end{array}$ & NR & $\begin{array}{l}\text { Mean patient } \\
\text { savings with } \\
\text { telehealth } \\
\text { equivalent to USD } \\
\$ 25\end{array}$ \\
\hline $\begin{array}{l}\text { Boman,2014 } \\
\text { Sweden } \\
\text { Robot assisted } \\
\text { echocardiogram } \\
\text { and cardiology } \\
1 \text { primary } \\
\text { healthcare center, } \\
1 \text { hospital }\end{array}$ & $\begin{array}{l}\text { RCT } \\
\text { Moderate } \\
\text { Real time } \\
\text { echocardiogram; } \\
\text { separate followup } \\
\text { One time } \\
\text { A: No telehealth, } \\
n=19 \\
\text { B: Telehealth, } n=19\end{array}$ & NR & $\begin{array}{l}\text { Median process time (IQR), in } \\
\text { days } \\
\text { A: } \mathbf{1 1 4} \text { ( } \mathbf{7 5} \text { to } 140) \\
\text { B: } 27 \text { (12 to } 60), \mathbf{p}<0.001 \\
\text { Median time from } \\
\text { randomization to } \\
\text { echocardiography (IQR), in } \\
\text { days } \\
\text { A: } \mathbf{8 6} \text { ( } 66 \text { to 117) } \\
\text { B: } 12 \text { (7 to 29), p<0.001 } \\
\text { Median days, (IQR) from } \\
\text { clinical exam to GP signing } \\
\text { off results } \\
\text { A: } 6 \text { (4 to 25) } \\
\text { B: } 5 \text { (0 to 19), NS }\end{array}$ & NR \\
\hline $\begin{array}{l}\text { Britt, } 2006^{37} \\
\text { AR, United States } \\
\text { Obstetric } \\
\text { ultrasound } \\
\text { NR } \\
>90 \text { health unit } \\
\text { sites } \\
>54 \text { hospitals } \\
25 \text { clinical sites }\end{array}$ & $\begin{array}{l}\text { Before-after } \\
\text { High } \\
\text { Real time } \\
\text { One time } \\
\text { A: Before telehealth, } \\
\text { NR } \\
\text { B: After telehealth, } \\
\text { NR }\end{array}$ & $\begin{array}{l}\text { Mean number of } \\
\text { maternal } \\
\text { transports } \\
\text { A: } 278 \\
\text { B: } 237 \\
\text { Mean LOS per } \\
\text { maternal } \\
\text { transport, in days } \\
\text { A: } \mathbf{8 . 0 2} \\
\text { B: } \mathbf{6 . 0 6}, \mathbf{p}=\mathbf{0 . 0 0 3}\end{array}$ & $\begin{array}{l}\text { Mean number of remote } \\
\text { consultations } \\
\text { A: } \mathbf{1 0 8} \\
\text { B: } \mathbf{2 6 9}, p=0.01 \\
\text { Mean number of phone } \\
\text { consultations } \\
\text { A: } \mathbf{5 5} \\
\text { B: } 107, p=0.03 \\
\text { Mean number of phone } \\
\text { consults between doctors } \\
\text { A: } \mathbf{5 5} \\
\text { B: } 107, p=0.03 \\
\text { Mean number of doctors } \\
\text { involved in weekly case } \\
\text { discussions } \\
\text { A: } 4.33 \\
\text { B: } 8.58\end{array}$ & NR \\
\hline
\end{tabular}




\begin{tabular}{|c|c|c|c|c|}
\hline $\begin{array}{l}\text { First Author, Year } \\
\text { Location } \\
\text { Specialty and } \\
\text { Technology } \\
\text { Number of Sites }\end{array}$ & $\begin{array}{l}\text { Study Design } \\
\text { Risk of Bias } \\
\text { Timing } \\
\text { Consultation } \\
\text { Frequency } \\
\text { Comparison, } n\end{array}$ & $\begin{array}{l}\text { Utilization } \\
\text { Outcomes }\end{array}$ & $\begin{array}{l}\text { Other Intermediate } \\
\text { Outcomes }\end{array}$ & $\begin{array}{l}\text { Economic } \\
\text { Outcomes }\end{array}$ \\
\hline $\begin{array}{l}\text { Dowie, } 2007^{211} \\
\text { London, England } \\
\text { Fetal and pediatric } \\
\text { echocardiography } \\
5 \text { hospitals }\end{array}$ & $\begin{array}{l}\text { Economic } \\
\text { evaluation } \\
\text { Moderate } \\
\text { Real time and store } \\
\text { and forward } \\
\text { One time } \\
\text { A: Conventional } \\
\text { referrals } n=387 \\
\text { B: Telehealth } n=117\end{array}$ & & $\begin{array}{l}\text { EuroQOL EQ-5D }(n=37) \\
\text { Mean } \pm \text { SD } \\
\text { A: } 0.72 \pm 0.22 \\
\text { B: } 0.86 \pm 0.14\end{array}$ & $\begin{array}{l}\text { Mean cost of initial } \\
\text { consult } \\
\text { A: } £ 277 \\
\text { B: } £ 411 \\
\text { After 6-month } \\
\text { followup } \\
\text { A: } £ 2,172 \\
\text { B: } £ 3,350, N S\end{array}$ \\
\hline $\begin{array}{l}\text { Endean, } 2001^{54} \\
\text { KY, United States } \\
\text { Vascular surgery } \\
\text { with Doppler probe } \\
1 \text { University } \\
\text { hospital; } 3 \text { clinics }\end{array}$ & $\begin{array}{l}\text { Pre-post } \\
\text { Moderate } \\
\text { Real time } \\
\text { One time } \\
\text { A: Pre-telehealth, } \\
n=32 \\
\text { B: Post-telehealth, } \\
n=32\end{array}$ & NR & $\begin{array}{l}\text { Mean evaluation time, in } \\
\text { minutes } \\
\text { A: } 19.0 \\
\text { B: } 20.6 \\
\text { Overall concordance } n,(\%) \\
29 \text { of } 32(91 \%) \\
\text { Mean physician satisfaction } \\
\text { score with TH consult (7-point } \\
\text { scale, } 7=\text { highest) } \\
5.71 \\
\text { Mean patient satisfaction } \\
\text { score comparing TH to } \\
\text { conventional from }-1 \text { to } 1 \\
\text { (1=better) } \\
0.27\end{array}$ & NR \\
\hline $\begin{array}{l}\text { Long, } 2014^{82} \\
\text { AR, United States } \\
\text { Obstetric } \\
\text { ultrasound } \\
\text { NR } \\
>90 \text { health unit } \\
\text { sites } \\
>54 \text { hospitals } \\
25 \text { clinical sites }\end{array}$ & $\begin{array}{l}\text { Retrospective } \\
\text { Moderate } \\
\text { Real time } \\
\text { One time } \\
\text { A: No telehealth, } \\
n=N R \\
\text { B: Telehealth, } n=N R\end{array}$ & $\begin{array}{l}\text { Pregnancies } \\
\text { receiving } \\
\text { comprehensive } \text { ultrasound }^{\mathrm{a}} \\
\text { A: } \mathbf{9 . 6 \%} \\
\text { B: } \mathbf{1 1 . 3 \%}, \\
\text { p<0.0001 } \\
\text { High-risk } \\
\text { pregnancies } \\
\text { receiving } \\
\text { comprehensive } \\
\text { ultrasounda: } \\
\text { A: } 16.9 \% \\
\text { B: } 19.9 \% \text {, } \\
\text { p<0.001 }\end{array}$ & $\begin{array}{l}\text { High-risk pregnancies with } \\
\text { prenatal care starting each } \\
\text { trimestera }^{\mathrm{a}} \\
\text { First trimester } \\
\text { A: } 74.3 \% \\
\text { B: } 75.0 \% \\
\text { Second trimester: } \\
\text { A: } 21.5 \% \\
\text { B: } 21.1 \% \\
\text { Third trimester: } \\
\text { A: } 4.2 \% \\
\text { B: } 4.0 \%\end{array}$ & NR \\
\hline $\begin{array}{l}\text { McCrossan, } 2012^{203} \\
\text { Northern Ireland, } \\
\text { UK } \\
\text { Fetal } \\
\text { telecardiology } \\
2 \text { hospitals }\end{array}$ & $\begin{array}{l}\text { Prospective cohort } \\
\text { Moderate } \\
\text { Real time } \\
\text { One time } \\
\text { A: No telehealth, } \\
n=N R \\
\text { B: Telehealth, n=NR }\end{array}$ & NR & $\begin{array}{l}\text { Aggregate mean patient } \\
\text { satisfaction, out of } 25 \text { points } \\
\text { A: } 23.2 \\
\text { B: } 23.2 \text {, NS }\end{array}$ & $\begin{array}{l}\text { Mean difference in } \\
\text { days taken off } \\
\text { work } \\
\mathbf{0 . 6 1} \text { days, } \mathbf{p}<\mathbf{0 . 0 1}\end{array}$ \\
\hline
\end{tabular}




\begin{tabular}{|c|c|c|c|c|}
\hline $\begin{array}{l}\text { First Author, Year } \\
\text { Location } \\
\text { Specialty and } \\
\text { Technology } \\
\text { Number of Sites }\end{array}$ & $\begin{array}{l}\text { Study Design } \\
\text { Risk of Bias } \\
\text { Timing } \\
\text { Consultation } \\
\text { Frequency } \\
\text { Comparison, n }\end{array}$ & $\begin{array}{l}\text { Utilization } \\
\text { Outcomes }\end{array}$ & $\begin{array}{l}\text { Other Intermediate } \\
\text { Outcomes }\end{array}$ & $\begin{array}{l}\text { Economic } \\
\text { Outcomes }\end{array}$ \\
\hline $\begin{array}{l}\text { Sharma,2003 }{ }^{114} \\
\text { NY, United States } \\
\text { Fetal } \\
\text { telecardiology } \\
\\
2 \text { hospitals; } 1 \text { with } \\
\text { expertise, } 1 \\
\text { without }\end{array}$ & $\begin{array}{l}\text { Retrospective } \\
\text { cohort } \\
\text { High } \\
\text { Real time } \\
\text { One time } \\
\text { A: No telehealth, } \\
n=195 \\
\text { B: Telehealth, } n=34\end{array}$ & $\begin{array}{l}\text { Mean number of } \\
\text { inadequately } \\
\text { identified } \\
\text { cardiovascular } \\
\text { items, out of } 31 \\
\text { A: } 2.3 \text { items } \\
\text { B: } 2.1 \text { items, NS }\end{array}$ & $\begin{array}{l}\text { Patient satisfaction (5-point } \\
\text { scale, 5=very satisfied) } \\
\text { Comfort during exam } \\
\text { A: } 4.6 \\
\text { B: } 4.3, \text { NS } \\
\text { Amount of information } \\
\text { received during exam } \\
\text { A: } 4.6 \\
\text { B: } 4.3, p=0.05 \\
\text { Doctor's willingness to } \\
\text { answer questions } \\
\text { A: } 4.6 \\
\text { B: } 4.5 \text {, NS } \\
\text { Explanation of exam results } \\
\text { A: } 4.6 \\
\text { B: } 4.4, \text { NS } \\
\text { Overall quality of care and } \\
\text { services } \\
\text { A: } 4.6 \\
\text { B: } 4.5 \text {, NS }\end{array}$ & NR \\
\hline $\begin{array}{l}\text { van der Pol,2010 } 206 \\
\text { Scotland, Shetland } \\
\text { Islands, and } \\
\text { Aberdeen, UK } \\
\text { Cancer } \\
\text { Endoscopy for } \\
\text { airway } \\
2 \text { rural clinics } \\
1 \text { mainland clinic }\end{array}$ & $\begin{array}{l}\text { Economic } \\
\text { evaluation } \\
\text { Moderate } \\
\text { Real time } \\
\text { One time } \\
\text { A: No telehealth, } \\
n=N R \\
\text { B: Telehealth, n=NR }\end{array}$ & NR & NR & $\begin{array}{l}\text { Total per patient } \\
\text { A: } £ 381 \\
\text { B: } £ 353 \\
\text { Per clinic mean } \\
\text { cost } \\
\text { Staff } \\
\text { A: } £ 351 \\
\text { B: } £ 361 \\
\text { Equipment } \\
\text { A: } £ 247 \\
\text { B: } £ 1390 \\
\text { Disposables } \\
\text { A: } £ 32 \\
\text { B: } £ 16 \\
\text { Mean cost per } \\
\text { patient } \\
\text { Staff } \\
\text { A: } £ 18 \\
\text { B: } £ 72 \\
\text { Equipment } \\
\text { A: } £ 12 \\
\text { B: } £ 278 \\
\text { Disposables: } \\
\text { A: } £ 2 \\
\text { B: } £ 3 \\
\text { Travel } \\
\text { A: } £ 349 \\
\text { B: } £ 0\end{array}$ \\
\hline
\end{tabular}




\begin{tabular}{|c|c|c|c|c|}
\hline $\begin{array}{l}\text { First Author, Year } \\
\text { Location } \\
\text { Specialty and } \\
\text { Technology } \\
\text { Number of Sites }\end{array}$ & $\begin{array}{l}\text { Study Design } \\
\text { Risk of Bias } \\
\text { Timing } \\
\text { Consultation } \\
\text { Frequency } \\
\text { Comparison, n }\end{array}$ & $\begin{array}{l}\text { Utilization } \\
\text { Outcomes }\end{array}$ & $\begin{array}{l}\text { Other Intermediate } \\
\text { Outcomes }\end{array}$ & $\begin{array}{l}\text { Economic } \\
\text { Outcomes }\end{array}$ \\
\hline $\begin{array}{l}\text { Xu,2008 } \\
\text { Queensland, } \\
\text { Australia } \\
\text { Pediatric ENT with } \\
\text { endoscopy } \\
1 \text { hospital; several } \\
\text { clinics }\end{array}$ & $\begin{array}{l}\text { Economic } \\
\text { evaluation } \\
\text { Moderate } \\
\text { Real time } \\
\text { Continuing } \\
\text { A: No telehealth, } \\
\mathrm{n}=177 \\
\text { B: Telehealth, } n=88\end{array}$ & NR & $\begin{array}{l}\text { Average number of } \\
\text { consultations per person } \\
\text { A: } 1.5 \\
\text { B: } 1.3\end{array}$ & $\begin{array}{l}\text { Total cost per } \\
\text { consultation, in } \\
\text { AUD } \\
\text { A: } \$ 155 \\
\text { B: } \$ 161 \\
\text { Variable cost per } \\
\text { consultation, in } \\
\text { AUD } \\
\text { A: } \$ 155 \\
\text { B: } \$ 108 \\
\text { Total annual } \\
\text { variable cost, in } \\
\text { AUD } \\
\text { A: } \$ 27,364 \\
\text { B: } \$ 14,160 \\
\text { Difference for } 265 \\
\text { consultations } \\
\text { A vs. B cost- } \\
\text { savings } \\
\text { AUD } \$ 7,621\end{array}$ \\
\hline
\end{tabular}

AUD = Australian dollar; ENT = ear nose and throat; GP = general practitioner; IQR = interquartile range; LOS = length of stay; $\mathrm{NR}$ = not reported; NS = not significant; RCT = randomized control trial; $\mathrm{SD}=$ standard deviation; $\mathrm{TH}=$ telehealth; UAMS = University of Arkansas for Medical Sciences; UK = United Kingdom; USD = United States dollars

${ }^{a}$ Mean percentages: A: 2001-2003; B: 2004-2007

Bold=statistically significant; telehealth superior

\section{Single Specialty Consultations}

An additional 17 studies evaluated the use of teleconsultations to obtain assessments and advice on care from specialists for different conditions (Table 22). Most of these involved consultations designed to assist in managing chronic conditions such as diabetes, ${ }^{69,144,168,210}$ hypertension management, ${ }^{145}$ pain, ${ }^{61}$ and arthritis, ${ }^{250}$ and they did not use diagnostic technology during the consultation. This section also includes specialty consultations in areas such as genetic counseling ${ }^{220,232}$ and urology, ${ }^{42}$ the subject of one or two included studies.

The majority of these consultations were conducted in real time using video to allow the clinicians to interact. The exception is four studies that facilitated asynchronous, ongoing exchanges of information - three between primary care physicians and specialists to facilitate management of hypertension, ${ }^{145}$ diabetes, ${ }^{144}$ and kidney failure ${ }^{186}$ and one in which specialists reviewed sleep studies. ${ }^{31}$

Regardless of the format of telehealth, these studies reported positive effects of telehealth consultations on clinical outcomes (e.g., similar rates of response to treatment or lower mortality rates) and more limited effects on intermediate outcomes (e.g., no difference in satisfaction) and cost savings, which were primarily dependent on patient costs. 
Table 22. Single specialty by type: selected outcomes

\begin{tabular}{|c|c|c|c|c|c|}
\hline $\begin{array}{l}\text { Condition } \\
\text { or Specialty }\end{array}$ & $\begin{array}{l}\text { Author, Year } \\
\text { Location } \\
\text { Number of Sites }\end{array}$ & $\begin{array}{l}\text { Study Design } \\
\text { Risk of Bias } \\
\text { Timing } \\
\text { Consultation } \\
\text { Frequency } \\
\text { Comparison, } \mathrm{n} \\
\end{array}$ & Clinical Outcomes & Intermediate Outcomes & Cost Outcomes \\
\hline Arthritis & $\begin{array}{l}\text { Jong, } 2004^{250} \\
\text { Canada } \\
3 \text { hospitals } \\
6 \text { physicians }\end{array}$ & $\begin{array}{l}\text { Prospective cohort } \\
\text { High } \\
\text { Real time } \\
\text { Continuing } \\
\\
A_{1} \text { : No telehealth, in- } \\
\text { person, } n=2 \\
\text { A}_{2} \text { : No telehealth, } \\
\text { email, } n=4 \\
\text { B: Telehealth, } n=6\end{array}$ & NR & $\begin{array}{l}\text { Physician satisfaction } \\
\text { Higher for video than in-person or } \\
\text { in-person with email, values not } \\
\text { provided }\end{array}$ & $\begin{array}{l}\text { Average cost, in CAD } \\
A_{1}: \$ 975 \text { travel cost } \\
A_{2}: \text { NR } \\
B: \$ 87.50 \text { per half } \\
\text { hour of } \\
\text { videoconference, the } \\
\text { mean length of a } \\
\text { session }\end{array}$ \\
\hline $\begin{array}{l}\text { Blood } \\
\text { Pressure } \\
\text { Control }\end{array}$ & \begin{tabular}{|l|} 
De Luca $2005^{145}$ \\
Naples, Italy \\
1 university clinic \\
23 hospital based clinics \\
60 general practitioners
\end{tabular} & $\begin{array}{l}\text { Prospective cohort } \\
\text { Moderate } \\
\text { Asynchronous } \\
\text { Continuing } \\
\text { A: No Telehealth, } \\
n=1,985 \\
\text { B: Telehealth, } \\
n=1,949\end{array}$ & $\begin{array}{l}\text { Mean reduction in SBP/DBP blood } \\
\text { pressure mmHg } \\
\text { A: 4.1/3.1 } \\
\text { B: } 7.3 / 5.4, p<0.001 \\
\text { Patients with BP }<140 / 90 \mathrm{mmHg} \\
\text { A: } 47 \% \\
\text { B: } 51 \%, \mathbf{p}<0.001 \\
\text { Major cardiovascular events } \\
\text { A: } 4.3 \% \\
\text { B: } 2.9 \%, p<0.02 \\
\text { AOR: } 0.838, p<0.05\end{array}$ & NR & NR \\
\hline
\end{tabular}




\begin{tabular}{|c|c|c|c|c|c|}
\hline $\begin{array}{l}\text { Condition } \\
\text { or Specialty }\end{array}$ & $\begin{array}{l}\text { Author, Year } \\
\text { Location } \\
\text { Number of Sites }\end{array}$ & $\begin{array}{l}\text { Study Design } \\
\text { Risk of Bias } \\
\text { Timing } \\
\text { Consultation } \\
\text { Frequency } \\
\text { Comparison, } n \\
\end{array}$ & Clinical Outcomes & Intermediate Outcomes & Cost Outcomes \\
\hline $\begin{array}{l}\text { Childhood } \\
\text { Obesity }\end{array}$ & $\begin{array}{l}\text { Mulgrew, 201192 } \\
\text { United States, CA } \\
1 \text { weight management } \\
\text { clinic } \\
\text { Remote clinics NR }\end{array}$ & $\begin{array}{l}\text { Prospective cohort } \\
\text { High } \\
\text { Real time } \\
\text { Continuing } \\
\text { A: Face to face, } n=15 \\
\text { B: Telemedicine, } \\
n=10\end{array}$ & NR & $\begin{array}{l}\text { Overall patient satisfaction defined } \\
\text { as a score of } 40 \text { to } 48, \text { mean } \pm \text { SD } \\
\text { A: } 44.5 \pm 3.85 \\
\text { B: } 43.8 \pm 4.83, p=0.42 \\
\text { Satisfaction with consulting } \\
\text { healthcare provider on a } 10 \text { point } \\
\text { scale with } 10 \text { being the best, mean } \\
\pm \text { SD } \\
\text { A: } 9.3 \pm 0.91 \\
\text { B: } 9.4 \pm 1.01\end{array}$ & NR \\
\hline Diabetes & $\begin{array}{l}\text { Basudev, } 2016^{210} \\
\text { London, England } \\
1 \text { virtual clinic } \\
6 \text { general practices }\end{array}$ & $\begin{array}{l}\text { RCT } \\
\text { Moderate } \\
\text { Real time } \\
\text { Continuing } \\
\text { A: Usual care, } n=88 \\
\text { B: Virtual clinic, } n=79\end{array}$ & $\begin{array}{l}\text { Reduction of HbA1c (difference } \pm \\
\text { SD) } \\
\text { A: } 10 \mathrm{mmol} / \mathrm{mol}(-0.8 \pm 1.9 \%) \\
\text { B: } 8 \mathrm{mmol} / \mathrm{mol}(-0.6 \pm 1.7 \%), \\
\mathrm{p}=0.4 \\
\text { Change in SBP } \pm \mathrm{SD} \\
\text { A: Increase: } 2 \pm \mathbf{1 8} \mathbf{m m H g} \\
\text { B: Decrease: } \mathbf{6} \pm 16 \mathrm{mmHg}, \\
\mathrm{p}=\mathbf{0 . 0 0 8} \\
\text { Cholesterol, weight, renal function, } \\
\text { NS }\end{array}$ & NR & NR \\
\hline
\end{tabular}




\begin{tabular}{|c|c|c|c|c|c|}
\hline $\begin{array}{l}\text { Condition } \\
\text { or Specialty }\end{array}$ & $\begin{array}{l}\text { Author, Year } \\
\text { Location } \\
\text { Number of Sites }\end{array}$ & \begin{tabular}{|l|} 
Study Design \\
Risk of Bias \\
Timing \\
Consultation \\
Frequency \\
Comparison, $\mathrm{n}$
\end{tabular} & Clinical Outcomes & Intermediate Outcomes & Cost Outcomes \\
\hline Diabetes & \begin{tabular}{|l|} 
Carallo,2015 \\
Calabria, Italy \\
33 general practitioners
\end{tabular} & $\begin{array}{l}\text { Prospective cohort } \\
\text { High } \\
\text { Asynchronous } \\
\text { Continuing } \\
\text { A: No telehealth, } \\
n=208 \\
\text { B: Telehealth, } n=104\end{array}$ & $\begin{array}{l}\text { Change in HbA1c mmol/mol from } \\
\text { baseline to followup } \\
\text { A: no change } \\
\text { B: }-4, p=0.01 \\
\text { Change in LDL cholesterol } \mathrm{mg} / \mathrm{dL} \\
\text { from baseline to followup: } \\
\text { A: }-\mathbf{9 . 2 ,} p=0.01 \\
\text { B: }-1.4, p=0.001 \\
\text { Change in body mass index } \mathrm{kg} / \mathrm{m}^{2} \\
\text { from baseline to followup: } \\
\text { A: No change } \\
\text { B: -0.03, } p=0.03 \\
\text { No difference between groups in } \\
\text { blood pressures, triglycerides, or } \\
\text { waist size }\end{array}$ & $\begin{array}{l}\text { Mean number of visits } \\
\text { A: } \mathbf{1 . 3} \\
\text { B: } \mathbf{0 . 6}, \mathbf{p}<0.0001 \\
\text { Mean duration of visit, in minutes } \\
\text { A: } 24 \\
\text { B: } 7\end{array}$ & NR \\
\hline Diabetes & \begin{tabular}{|l|} 
lzquierdo, $2009^{69}$ \\
NY, United States \\
25 schools \\
Kindergarden-8 $8^{\text {th }}$ grade
\end{tabular} & $\begin{array}{l}\text { RCT } \\
\text { High } \\
\text { Real time } \\
\text { Continuing } \\
\text { A: No telehealth, } \\
\mathrm{n}=18 \\
\text { B: Telehealth, } \mathrm{n}=23\end{array}$ & $\begin{array}{l}\text { HbA1c value at } 6 \text { months } \\
\text { Values not specified } \\
\text { A: Increase, NS } \\
\text { B: Decrease, } p<0.02 \\
\\
\text { Hospitalizations for diabetic } \\
\text { ketoacidosis } \\
\text { A: } 22.2 \% \\
\text { B: } 4.3 \%\end{array}$ & $\begin{array}{l}\text { Pediatric Quality of Life Diabetes } \\
\text { module: No difference between } \\
\text { groups } \\
\text { Improved emotional function } \\
\text { between months } 6 \text { and } 12 \\
\text { A: Improved, } \boldsymbol{p}<0.04 \\
\text { B: No change } \\
\text { Treatment needed at } 6 \text { vs. } 12 \\
\text { months } \\
\text { A: } 48 \text { vs. } 35 \\
\text { B: } 20 \text { vs. } 9, p=N R \\
\text { Urgent visits } \\
\text { A: No change } \\
\text { B: Significant decrease, } p \text {-value } \\
\text { NR }\end{array}$ & NR \\
\hline
\end{tabular}




\begin{tabular}{|c|c|c|c|c|c|}
\hline $\begin{array}{l}\text { Condition } \\
\text { or Specialty }\end{array}$ & $\begin{array}{l}\text { Author, Year } \\
\text { Location } \\
\text { Number of Sites }\end{array}$ & $\begin{array}{l}\text { Study Design } \\
\text { Risk of Bias } \\
\text { Timing } \\
\text { Consultation } \\
\text { Frequency } \\
\text { Comparison, } n\end{array}$ & Clinical Outcomes & Intermediate Outcomes & Cost Outcomes \\
\hline Diabetes & $\begin{array}{l}\text { Nikkanen,2008 } 168 \\
\text { Oulu Arc Sub region, } \\
\text { Finland } \\
3 \text { health centers }\end{array}$ & $\begin{array}{l}\text { Pre-post } \\
\text { Moderate } \\
\\
\text { Real time } \\
\text { Continuing } \\
\text { A: Pre-telehealth, } \\
n=101 \\
\text { B: Post-telehealth, } \\
n=101\end{array}$ & $\begin{array}{l}\text { Mean HbA1c } \\
\text { A: } 8.0 \% \\
\text { B: } 7.6 \% \\
\text { Difference: }(-0.4), p=0.007 \\
\\
\text { Mean LDL cholesterol, mmol/L } \\
\text { A: } 3.3 \\
\text { B: } 2.7 \\
\text { Difference: }(-0.6), p=0.001 \\
\text { Systolic blood pressure, } \mathrm{mm} \mathrm{Hg} \\
\text { A: } 146 \\
\text { B: } 140 \\
\text { Difference: }(-6), \mathrm{NS} \\
\text { Mean body mass index, } \mathrm{kg} / \mathrm{m}^{2} \\
\text { A: } 30.6 \\
\text { B: } 30.4 \\
\text { Difference: }(-0.2), \mathrm{NS} \\
\\
\text { Subgroup analyses indicate } \\
\text { largest change in HbA1c results in } \\
\text { patients with diabetes mellitus }>10 \\
\text { years and with higher HbA1c at } \\
\text { baseline. }\end{array}$ & NR & NR \\
\hline
\end{tabular}




\begin{tabular}{|c|c|c|c|c|c|}
\hline $\begin{array}{l}\text { Condition } \\
\text { or Specialty }\end{array}$ & $\begin{array}{l}\text { Author, Year } \\
\text { Location } \\
\text { Number of Sites } \\
\end{array}$ & $\begin{array}{l}\text { Study Design } \\
\text { Risk of Bias } \\
\text { Timing } \\
\text { Consultation } \\
\text { Frequency } \\
\text { Comparison, } \mathrm{n} \\
\end{array}$ & Clinical Outcomes & Intermediate Outcomes & Cost Outcomes \\
\hline $\begin{array}{l}\text { Genetic } \\
\text { Counseling }\end{array}$ & \begin{tabular}{|l} 
Zilliacus, $2011^{232}$ \\
New South Wales and \\
Australia \\
\\
3 family cancer clinics \\
4 outreach genetic \\
counseling services
\end{tabular} & $\begin{array}{l}\text { Prospective cohort } \\
\text { Moderate } \\
\text { Real time } \\
\text { One-Time } \\
\text { A: Face to face, } n=89 \\
\text { B: Telehealth, } n=106\end{array}$ & NR & $\begin{array}{l}\text { Mean satisfaction score } \pm \text { SD }(54 \\
\text { point scale; higher= greater } \\
\text { satisfaction) } \\
\text { A: } 40.8 \pm 9.9 \\
\text { B: } 45.6 \pm 8.4, p=0.76 \\
\text { No difference in telegenetics vs. } \\
\text { face-to-face: } \\
\text { Knowledge gained, } p=0.55 \\
\text { Generalized anxiety, } p=0.42 \\
\text { Depression, } p=0.96 \\
\text { Perceived empathy of genetic } \\
\text { clinician, } p=0.13 \\
\text { Perceived empathy of genetic } \\
\text { counselor, } p=0.12 \\
\text { Significant difference in } \\
\text { telegenetics vs. face-to-face: } \\
\text { Meeting patients' expectations, } \\
p=0.009 \\
\text { Promoting perceived personal } \\
\text { control, } p=0.031\end{array}$ & NR \\
\hline $\begin{array}{l}\text { Clinical } \\
\text { Genetics }\end{array}$ & $\begin{array}{l}\text { Gattas, } 2001^{220} \\
\text { Queensland, Australia } \\
1 \text { hospital }\end{array}$ & $\begin{array}{l}\text { RCT } \\
\text { High } \\
\text { Real time } \\
\text { One time } \\
\text { A: No telehealth, } \\
\mathrm{n}=23 \\
\text { B: Telehealth, } \mathrm{n}=44\end{array}$ & NR & $\begin{array}{l}\text { Numerical data not reported } \\
\text { Provider satisfaction A vs. B } \\
\text { Communication: no difference } \\
\text { Ability to maintain eye contact: } \\
\text { slightly lower } \\
\text { Room comfortability: higher } \\
\text { Satisfaction with clinic format: No } \\
\text { difference } \\
\text { Counselor satisfaction: higher } \\
\text { satisfaction with face-to-face } \\
\text { consultations }\end{array}$ & NR \\
\hline
\end{tabular}




\begin{tabular}{|c|c|c|c|c|c|}
\hline $\begin{array}{l}\text { Condition } \\
\text { or Specialty }\end{array}$ & $\begin{array}{l}\text { Author, Year } \\
\text { Location } \\
\text { Number of Sites }\end{array}$ & $\begin{array}{l}\text { Study Design } \\
\text { Risk of Bias } \\
\text { Timing } \\
\text { Consultation } \\
\text { Frequency } \\
\text { Comparison, } n \\
\end{array}$ & Clinical Outcomes & Intermediate Outcomes & Cost Outcomes \\
\hline $\begin{array}{l}\text { Inflammatory } \\
\text { Bowel } \\
\text { Disease }\end{array}$ & $\begin{array}{l}\text { Krier, } 2011^{74} \\
\text { CA, United States } \\
\text { Patient location unclear } \\
1 \text { VA Hospital }\end{array}$ & $\begin{array}{l}\text { RCT } \\
\text { High } \\
\text { Real time } \\
\text { Continuing } \\
\text { A: Usual care, } n=19 \\
\text { B: Telehealth, } n=15\end{array}$ & NR & $\begin{array}{l}\text { Mean duration } \pm S D \text { (range), in } \\
\text { minutes } \\
\text { A: } 59 \pm 10 \text { ( } 26 \text { to } 73) \\
\text { B: } 60 \pm 14 \text { ( } 35 \text { to } 80), p=0.81 \\
\text { Mean wait time } \pm S D \text { (range), in } \\
\text { minutes } \\
\text { A: } 18 \pm 14.5 \text { ( } 5 \text { to } 60) \\
\text { B: } 25 \pm 25 \text { (5 to } 90), p=0.31 \\
\text { Clinic experience, attention to } \\
\text { patient concerns, bedside manner, } \\
\text { and perceived skill level of the } \\
\text { doctor rated excellent by both } \\
\text { groups. }\end{array}$ & NR \\
\hline Nephrology & $\begin{array}{l}\text { Bernstein, } 2010^{248} \\
\text { Manitoba, Canada } \\
1 \text { hospital; } 12 \text { local } \\
\text { centers }\end{array}$ & $\begin{array}{l}\text { Retrospective cohort } \\
\text { Moderate } \\
\text { Real time } \\
\text { Continuing } \\
\\
\text { A: No telehealth, } \\
\mathrm{n}=2,196 \\
\mathrm{~B}_{1}: \text { Telehealth, near } \\
\text { urban center, } \mathrm{n}=285 \\
\mathrm{~B}_{2} \text { : Telehealth, far } \\
\text { from urban center, } \\
\mathrm{n}=182\end{array}$ & $\begin{array}{l}\text { Hazard Ratios } \\
\text { 2- to } 5 \text {-year survival } \\
\text { B }_{1} \text { vs } A: 0.67, p<0.001 \\
\text { B }_{2} \text { vs. } A: 0.72, p<0.05 \\
\\
\text { Diabetic nephropathy } \\
\text { B }_{1} \text { vs } A: 0.63, p<0.001 \\
\text { B }_{2} \text { vs. } A: 0.63, p<0.01\end{array}$ & $\mathrm{NR}$ & NR \\
\hline
\end{tabular}




\begin{tabular}{|c|c|c|c|c|c|}
\hline $\begin{array}{l}\text { Condition } \\
\text { or Specialty }\end{array}$ & $\begin{array}{l}\text { Author, Year } \\
\text { Location } \\
\text { Number of Sites } \\
\end{array}$ & $\begin{array}{l}\text { Study Design } \\
\text { Risk of Bias } \\
\\
\text { Timing } \\
\text { Consultation } \\
\text { Frequency } \\
\text { Comparison, } \mathrm{n} \\
\end{array}$ & Clinical Outcomes & Intermediate Outcomes & Cost Outcomes \\
\hline Nephrology & $\begin{array}{l}\text { Van Gelder, } 2017^{186} \\
\text { Netherlands } \\
7 \text { General practices } \\
\text { Nephrologist location NR }\end{array}$ & $\begin{array}{l}\text { RCT } \\
\text { High } \\
\text { Asynchronous } \\
\text { One time } \\
\\
\text { A: Usual care, } \\
n=1,727 \\
\text { B: Telehealth, } \\
n=1,277 \\
\end{array}$ & NR & $\begin{array}{l}\text { Referral rate } \\
\text { A: } 3.0 \% \\
\text { B: } 2.3 \% \\
\text { OR: } 0.61 \text { ( } 95 \% \mathrm{Cl} 0.31 \text { to } 1.23) \\
\text { Consultation rate } \\
\text { A: } 5.0 \% \\
\text { B: } 6.3 \% \\
\text { OR: } 2.00 \text { ( } 95 \% \mathrm{Cl} 0.75 \text { to } 5.33 \text { ) }\end{array}$ & NR \\
\hline Neurology & $\begin{array}{l}\text { Chua, } 2001^{194} \\
\text { Northern Ireland, UK } \\
\\
1 \text { Regional Neuro Center } \\
2 \text { District Hospitals }\end{array}$ & $\begin{array}{l}\text { RCT } \\
\text { High } \\
\text { Real time } \\
\text { One time } \\
\text { A: Usual care, } n=65 \\
\text { B: Telehealth, } n=76 \\
\end{array}$ & NR & $\begin{array}{l}\text { Number of investigations, } \mathrm{n}(\%) \\
\text { A: } 11(17 \%) \\
\text { B: } 46(61 \%) \\
\\
\text { Reviews after first consultation } \\
\text { A: } 14(22 \%) \\
\text { B: } 22(29 \%)\end{array}$ & $\begin{array}{l}\text { Cost of consultation } \\
\text { A: } £ 49 \\
\text { B: } £ 72\end{array}$ \\
\hline Pain & $\begin{array}{l}\text { Frank, } 2015^{61} \\
\text { United States } \\
47 \text { medical centers } \\
148 \text { community-based } \\
\text { outpatient clinics }\end{array}$ & \begin{tabular}{|l}
$\begin{array}{l}\text { Before-after } \\
\text { Moderate }\end{array}$ \\
Real time \\
Continuing \\
A: No telehealth, \\
$n=299,981$ \\
B: Telehealth, \\
$n=22,454$
\end{tabular} & NR & $\begin{array}{l}\text { HR }(95 \% \mathrm{Cl}) \\
\text { Delivery of out-patient care: } \\
\text { Physical medicine: } 1.10 \text { (1.05 to } \\
\text { 1.14) } \\
\text { Mental health: } 0.99 \text { (0.93 to } 1.05) \\
\text { Substance use disorder: } 0.93 \text { (0.84 } \\
\text { to } 1.03 \text { ) } \\
\text { Specialty pain clinics: } 1.01 \text { (0.94 to } \\
\text { 1.08) } \\
\text { Medication initiation: } \\
\text { Anti-depressant: } 1.09 \text { (1.02 to } \\
\text { 1.15) } \\
\text { Anticonvulsant: } 1.13 \text { (1.06 to } \\
\text { 1.19) } \\
\text { Opioid analgesics: } 1.05 \text { (0.99 to } \\
1.10)\end{array}$ & NR \\
\hline
\end{tabular}




\begin{tabular}{|c|c|c|c|c|c|}
\hline $\begin{array}{l}\text { Condition } \\
\text { or Specialty }\end{array}$ & $\begin{array}{l}\text { Author, Year } \\
\text { Location } \\
\text { Number of Sites }\end{array}$ & $\begin{array}{l}\text { Study Design } \\
\text { Risk of Bias } \\
\text { Timing } \\
\text { Consultation } \\
\text { Frequency } \\
\text { Comparison, } n\end{array}$ & Clinical Outcomes & Intermediate Outcomes & Cost Outcomes \\
\hline Sleep & $\begin{array}{l}\text { Baig, } 2016^{31} \\
\text { WI, United States } \\
1 \text { VA Medical Center } \\
\text { Locations are not clear }\end{array}$ & $\begin{array}{l}\text { Retrospective cohort } \\
\text { High } \\
\text { Asynchronous } \\
\text { One time } \\
\text { A: No telehealth, } \\
n=\text { unclear } \\
\text { B: Telehealth, } \\
n=\text { unclear }\end{array}$ & NR & $\begin{array}{l}\text { Sleep consults per year } \\
\text { A: } 150 \\
\text { B: } 1,851 \\
\text { Number of sleep studies } \\
\text { A: } 282 \\
\text { B: } 833 \\
\text { Wait time for positive airway } \\
\text { pressure prescription } \\
\text { A: }>60 \text { days } \\
\text { B: }<7 \text { days }\end{array}$ & NR \\
\hline $\begin{array}{l}\text { Speech } \\
\text { Pathology }\end{array}$ & $\begin{array}{l}\text { Burns, } 2017^{217} \\
\text { Queensland, Australia } \\
3 \text { Regional Sites } \\
1 \text { Hospital }\end{array}$ & $\begin{array}{l}\text { RCT } \\
\text { Moderate } \\
\text { Real time } \\
\text { Continuing } \\
\text { A: Usual care, } n=39 \\
\text { B: Telehealth, } n=43\end{array}$ & NR & $\begin{array}{l}\text { Mean days from response to } \\
\text { management } \pm \text { SD (range) } \\
\text { A: } 3.2 \pm 3.7 \text { ( } 0 \text { to } 11) \\
B: 3.1 \pm 3.4(0 \text { to } 14), p=0.928 \\
\text { Clinician satisfaction reached } \\
\text { statistical significance for all } \\
\text { parameters assessed in favor of } \\
\text { telehealth. }\end{array}$ & NR \\
\hline Urology & $\begin{array}{l}\text { Chu, } 2015^{42} \\
\text { CA, United States } \\
1 \text { Tertiary care clinic } \\
2 \text { outpatient primary } \\
\text { clinics }\end{array}$ & $\begin{array}{l}\text { Retrospective cohort } \\
\text { High } \\
\text { Real time } \\
\text { One time } \\
\text { A: No telehealth, } \\
n=N R \\
\text { B: Telehealth, } n=N R\end{array}$ & NR & $\begin{array}{l}\text { Estimated time savings } \\
\text { Mean distance: } 277 \text { miles } \\
\text { Mean time: } 290 \text { minutes }\end{array}$ & $\begin{array}{l}\text { Estimated savings, in } \\
\text { USD } \\
\text { Expenses: } \$ 67 \\
\text { Lost opportunity cost } \\
\$ 126 \\
\\
\text { Total patient savings } \\
5 \text { hours } \\
\$ 193 \text { per visit }\end{array}$ \\
\hline
\end{tabular}

CAD = Canadian dollars; CI = confidence interval; DBP = diastolic blood pressure; HbA1c = hemoglobin; HR = hazard ratio; LDL = low density lipoprotein cholesterol; NR = no reported; NS = not significant; OR = odds ratio; RCT = randomized control trial; SBP = systolic blood pressure; SD = standard deviation; UK = United Kingdom; USD = United States dollars

Bold=statistically significant; telehealth superior. Bold and italicized=statistically significant; telehealth inferior. Regular type: not statistically significant. 


\section{Multiple Specialty Consultations}

We identified 10 articles representing eight studies that evaluated telehealth programs facilitating outpatient consultations for multiple specialists (Table 23). Most of these programs created agreements between primary care practices, some within correctional facilities and others in remote locations, with a hospital or medical center that has multiple specialists available. The clinical outcomes are limited to avoiding inpatient, ED, or followup visits, and telehealth consultations resulted in significant reductions in one study ${ }^{28}$ but did not result in significant changes in three other studies that reported these outcomes. ${ }^{60,207,208,212}$ Satisfaction was generally high, but the impact on access was not frequently reported, and most studies did not find differences in management and treatment. The evaluation of costs was mixed, with the largest study reporting higher costs for telehealth. ${ }^{212}$ The higher costs were due to equipment costs and the fact that both the primary care physician and the specialist were present for the telehealth consultation. This added physician time was not offset by cost savings despite a significant reduction in the number of tests and investigations. The range of disciplines and likely range of patient conditions and severity may contribute to the fact that the results across these studies are inconclusive.

Table 23. Multiple specialty telehealth consultations: selected outcomes

\begin{tabular}{|c|c|c|c|c|}
\hline $\begin{array}{l}\text { Author, } \\
\text { Year } \\
\text { Location } \\
\text { Number of } \\
\text { Sites }\end{array}$ & $\begin{array}{l}\text { Study } \\
\text { Design } \\
\text { Risk of Bias } \\
\text { Timing } \\
\text { Consultation } \\
\text { Frequency } \\
\text { Comparison, } \\
\mathbf{n}\end{array}$ & Clinical Outcomes & Intermediate Outcomes & $\begin{array}{l}\text { Economic } \\
\text { Outcomes }\end{array}$ \\
\hline $\begin{array}{l}\text { Angstman, } \\
2009^{28} \\
\text { United } \\
\text { States, MN } \\
\text { Number of } \\
\text { sites } \\
\text { unclear; } 1 \\
\text { medical } \\
\text { organization }\end{array}$ & $\begin{array}{l}\text { Retrospective } \\
\text { cohort } \\
\text { Moderate } \\
\text { Both } \\
\text { One time } \\
\text { A: No } \\
\text { telehealth, } \\
n=500 \\
B: \text { Telehealth, } \\
n=228\end{array}$ & $\begin{array}{l}\text { Unscheduled return } \\
\text { visit within } 2 \text { weeks, } \\
\text { any reason: } \\
\text { A: } \mathbf{2 7 . 6 \%} \\
\text { B: } \mathbf{3 8 . 2 \% , p < 0 . 0 1} \\
\text { OR: } \mathbf{1 . 8 8 , p \leq 0 . 0 1} \\
\text { Unscheduled return } \\
\text { visit within } 2 \text { weeks, } \\
\text { same reason: } \\
\text { A: } 19.6 \% \\
\text { B: } 20.2 \%, \text { NS } \\
\text { OR: } 1.18, N S\end{array}$ & $\mathrm{NR}$ & NR \\
\hline $\begin{array}{l}\text { Brown- } \\
\text { Connolly, } \\
2002^{38} \\
\text { United } \\
\text { States, CA } \\
\text { 34 primary } \\
\text { care and } 4 \\
\text { specialty } \\
\text { sites }\end{array}$ & $\begin{array}{l}\text { Prospective } \\
\text { cohort } \\
\text { High } \\
\text { Real time } \\
\text { Continuing } \\
\text { A: No } \\
\text { telehealth, } \\
n=N R \\
\text { B: Telehealth, } \\
n=N R\end{array}$ & NR & $\begin{array}{l}\text { Distance to specialist } \\
\text { A: } 195 \mathbf{~ k m} \\
\text { B: } 27 \mathbf{~ k m} \\
\text { Difference: } \mathbf{- 1 6 8} \mathbf{k m}, \mathbf{p}<0.05 \\
\text { Travel time, in minutes } \\
\text { A: } 156 \\
\text { B: } 26 \\
\text { Difference: }-130 \text { minutes, NS } \\
\text { Patient response to survey } \\
\text { Telemedicine again: } 90 \% \\
\text { Telemedicine made it easier to get } \\
\text { services: } 91 \% \\
\text { Would get better care in person: } 39 \%\end{array}$ & NR \\
\hline
\end{tabular}




\begin{tabular}{|c|c|c|c|c|}
\hline $\begin{array}{l}\text { Author, } \\
\text { Year } \\
\text { Location } \\
\text { Number of } \\
\text { Sites }\end{array}$ & $\begin{array}{l}\text { Study } \\
\text { Design } \\
\text { Risk of Bias } \\
\text { Timing } \\
\text { Consultation } \\
\text { Frequency } \\
\text { Comparison, } \\
\mathrm{n}\end{array}$ & Clinical Outcomes & Intermediate Outcomes & $\begin{array}{l}\text { Economic } \\
\text { Outcomes }\end{array}$ \\
\hline $\begin{array}{l}\text { Fox, } 2007^{60} \\
\text { United } \\
\text { States, TN } \\
4 \text { adolescent } \\
\text { correctional } \\
\text { facilities }\end{array}$ & $\begin{array}{l}\text { Before-after } \\
\text { Moderate } \\
\text { Real time } \\
\text { Continuous } \\
\text { A: Year } \\
\text { before } \\
\text { telehealth, } \\
n=173 \\
B_{1}: \text { Year after } \\
\text { telehealth, } \\
n=257 \\
B_{2}: 2 \text { years } \\
\text { after } \\
\text { telehealth, } \\
n=276\end{array}$ & 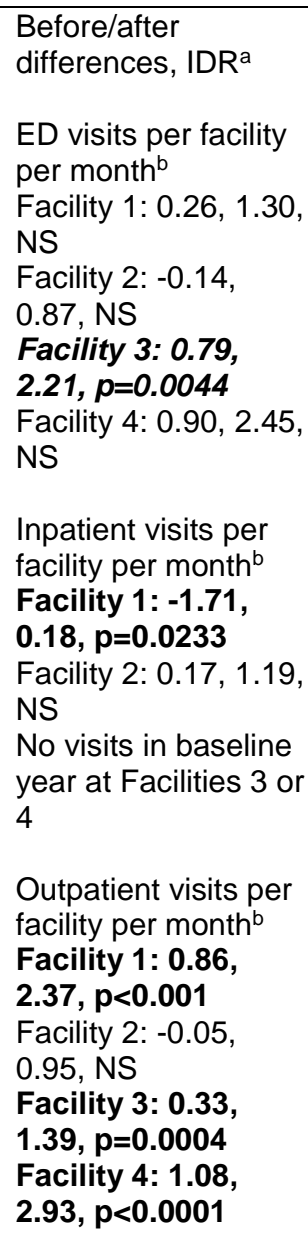 & 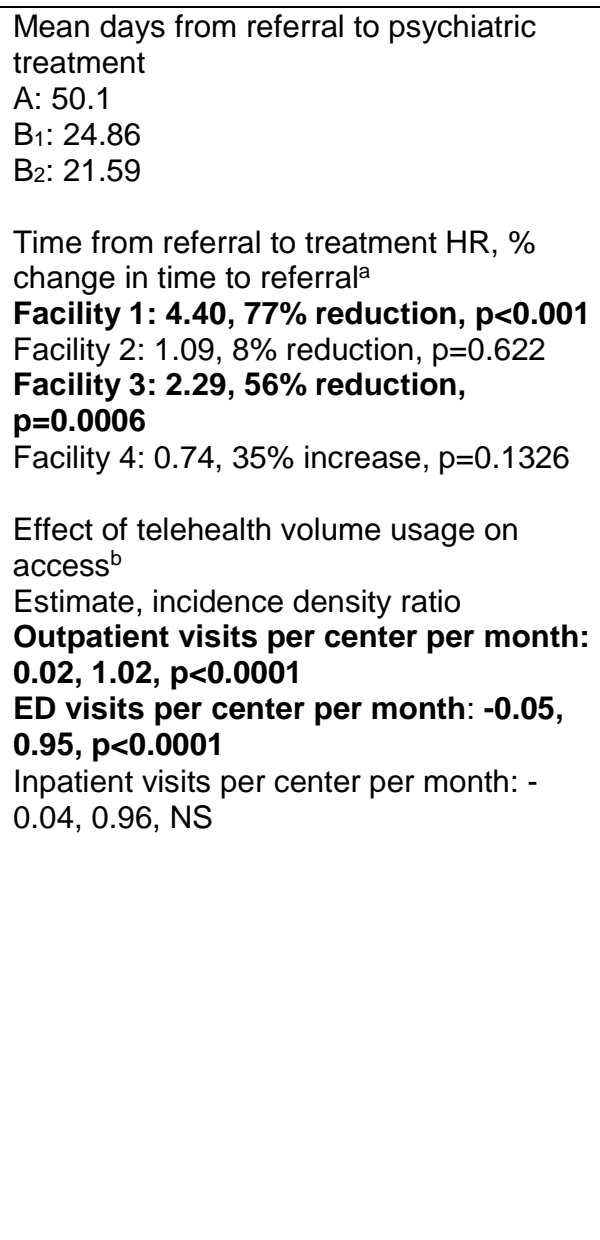 & NR \\
\hline $\begin{array}{l}\text { Harno, } \\
2000^{152} \\
\text { Finland, } \\
\text { Myyrmäki } \\
\text { and Tuusula } \\
2 \text { hospitals, } \\
3 \text { health } \\
\text { centers }\end{array}$ & $\begin{array}{l}\text { Economic } \\
\text { evaluation } \\
\text { Moderate } \\
\text { Asynchronou } \\
\text { s } \\
\text { One time } \\
\text { A: No } \\
\text { telehealth, } \\
n=85 \\
\text { B: Telehealth, } \\
n=207\end{array}$ & $\mathrm{NR}$ & $\begin{array}{l}\text { Patients receiving appointments at } \\
\text { outpatient clinic } \\
\text { A: } 79 \% \\
\text { B: } 43 \% \\
\text { Consultations with diagnosis changes } \\
\text { A: } 25 \% \\
\text { B: } 29 \% \\
\text { Want next appointment via TH } \\
\text { A: } 60 \% \\
\text { B: } 80 \%\end{array}$ & $\begin{array}{l}\text { Variable cost } \\
\text { for outpatient } \\
\text { visits } \\
\text { A: } € 210.81 \\
\text { B: } € 32.06\end{array}$ \\
\hline
\end{tabular}




\begin{tabular}{|c|c|c|c|c|}
\hline $\begin{array}{l}\text { Author, } \\
\text { Year } \\
\text { Location } \\
\text { Number of } \\
\text { Sites }\end{array}$ & $\begin{array}{l}\text { Study } \\
\text { Design } \\
\text { Risk of Bias } \\
\text { Timing } \\
\text { Consultation } \\
\text { Frequency } \\
\text { Comparison, } \\
\mathbf{n}\end{array}$ & Clinical Outcomes & Intermediate Outcomes & $\begin{array}{l}\text { Economic } \\
\text { Outcomes }\end{array}$ \\
\hline $\begin{array}{l}\text { Jaatinen, } \\
2002^{157} \\
\text { Finland, } \\
\text { Satakunta } \\
4 \text { clinics } \\
1 \text { Hospital }\end{array}$ & $\begin{array}{l}\text { RCT } \\
\text { High } \\
\text { Asynchronou } \\
\text { S } \\
\text { Continuous } \\
\text { A: No } \\
\text { telehealth, } \\
n=24 \\
\text { B: Telehealth, } \\
n=54\end{array}$ & NR & $\begin{array}{l}\text { Success relating patient history } \\
\text { Good vs. Moderate vs. Bad } \\
\text { A: } 85 \% \text { vs } 10 \% \text { vs } 5 \% \\
\text { B: } 62 \% \text { vs } 31 \% \text { vs } 8 \% \text {, Ns } \\
\text { Success relating patient physical status } \\
\text { Good vs. Moderate vs. Bad } \\
\text { A: } 90 \% \text { vs } 10 \% \text { vs } 0 \% \\
\text { B: } \mathbf{4 6 \%} \text { vs } 33 \% \text { vs } 21 \%, p=0.01 \\
\text { Success relating overall patient case } \\
\text { Good vs. Moderate vs. Bad } \\
\text { A: } 85 \% \text { vs } 15 \% \text { vs } 13 \% \\
\text { B: } \mathbf{4 8} \% \text { vs } 39 \% \text { vs } 0 \%, p=0.02 \\
\text { Median total time for visit } \\
\text { A: } 3.5 \text { hours } \\
\text { B: } 1.0 \text { hours }\end{array}$ & NR \\
\hline $\begin{array}{l}\text { Smith, } \\
2002^{229} \\
\text { Australia, } \\
\text { Queensland } \\
3 \text { hospitals } \\
\text { (Mackay, } \\
\text { Hervey Bay, } \\
\text { Royal } \\
\text { Children's } \\
\text { Hospital) }\end{array}$ & $\begin{array}{l}\text { Before-after } \\
\text { High } \\
\text { Real time } \\
\text { One time } \\
\text { A: Before } \\
\text { telehealth, } \\
n=N R \\
\text { B: After } \\
\text { telehealth, } \\
n=N R\end{array}$ & $\begin{array}{l}\text { Change in pediatric } \\
\text { admissions from } \\
\text { Mackay region to } \\
\text { Royal Children's } \\
\text { Hospital } \\
\text { A: } 9.7 \text { patients per } \\
\text { month } \\
\text { B: } 6.0 \text { patients per } \\
\text { month } \\
\text { Change in pediatric } \\
\text { admissions From } \\
\text { Hervey Bay region } \\
\text { to Royal Children's } \\
\text { Hospital } \\
\text { A: } 10.0 \text { patients per } \\
\text { month } \\
\text { B: } 12.5 \text { patients per } \\
\text { month }\end{array}$ & $\begin{array}{l}\text { Patient referrals for outpatient } \\
\text { appointments to Brisbane from Mackay } \\
\text { A: } 7.9 \text { patients per month } \\
\text { B: } 5.7 \text { patients per month } \\
\text { Patient referrals for outpatient } \\
\text { appointments to Brisbane from Hervey } \\
\text { Bay } \\
\text { A: } 15.8 \text { patients per month } \\
\text { B: } 15.4 \text { patients per month }\end{array}$ & NR \\
\hline $\begin{array}{l}\text { Tsitlakidis, } \\
2005^{184} \\
\text { Greece, } \\
\text { Lemnos and } \\
\text { Skyros } \\
2 \text { remote } \\
\text { health } \\
\text { centers } \\
1 \text { hospital }\end{array}$ & $\begin{array}{l}\text { Economic } \\
\text { evaluation } \\
\text { Moderate } \\
\text { Real time } \\
\text { One time } \\
\text { A: No } \\
\text { telehealth, } \\
n=N R \\
\text { B: Telehealth, } \\
n=N R\end{array}$ & NR & $\begin{array}{l}\text { Average consultation time, in minutes } \\
\text { A: } 30.0 \\
\text { B: } 5.3 \\
\text { Post-consultation time requirements, in } \\
\text { minutes } \\
\text { A: } 10.0 \\
\text { B: } 2.6\end{array}$ & $\begin{array}{l}\text { Total cost } \\
\text { per patient: } \\
\text { A: } € 270 \\
\text { B: } € 203 \\
\text { Savings } \\
\text { dependent } \\
\text { on distance } \\
\text { travelled and } \\
\text { number of } \\
\text { cases. }\end{array}$ \\
\hline
\end{tabular}




\begin{tabular}{|c|c|c|c|c|}
\hline $\begin{array}{l}\text { Author, } \\
\text { Year } \\
\text { Location } \\
\text { Number of } \\
\text { Sites }\end{array}$ & $\begin{array}{l}\text { Study } \\
\text { Design } \\
\text { Risk of Bias } \\
\text { Timing } \\
\text { Consultation } \\
\text { Frequency } \\
\text { Comparison, } \\
\text { n }\end{array}$ & Clinical Outcomes & Intermediate Outcomes & $\begin{array}{l}\text { Economic } \\
\text { Outcomes }\end{array}$ \\
\hline $\begin{array}{l}\text { Wallace, } \\
2002^{207} \\
\text { Jacklin, } \\
2003^{212} \\
\text { Wallace, } \\
2004^{208} \\
\text { United } \\
\text { Kingdom } \\
2 \text { hospitals } \\
29 \text { practices }\end{array}$ & $\begin{array}{l}\text { RCT } \\
\text { Low } \\
\text { Real time } \\
\text { Continuous } \\
\text { A: No } \\
\text { telehealth, } \\
n=971 \\
\text { B: Telehealth, } \\
n=968\end{array}$ & $\begin{array}{l}\text { Mean difference at } 6 \\
\text { months }(95 \% \mathrm{Cl}) \\
\text { Tests and } \\
\text { investigations: } \\
\mathbf{- 0 . 7 9}(\mathbf{- 1 . 2 1} \text { to }-\mathbf{0 . 3 7}) \\
\text { Emergency visits: } \\
0.002(-0.02 \text { to } 0.03) \\
\text { Inpatient stays: } \\
-0.02 \text { (-0.06 to } 0.01) \\
\text { Day surgery and } \\
\text { inpatient } \\
\text { procedures: } \\
-0.01(-0.04 \text { to } 0.02) \\
\text { Prescriptions } \\
0.57(-0.64 \text { to } 1.78)\end{array}$ & 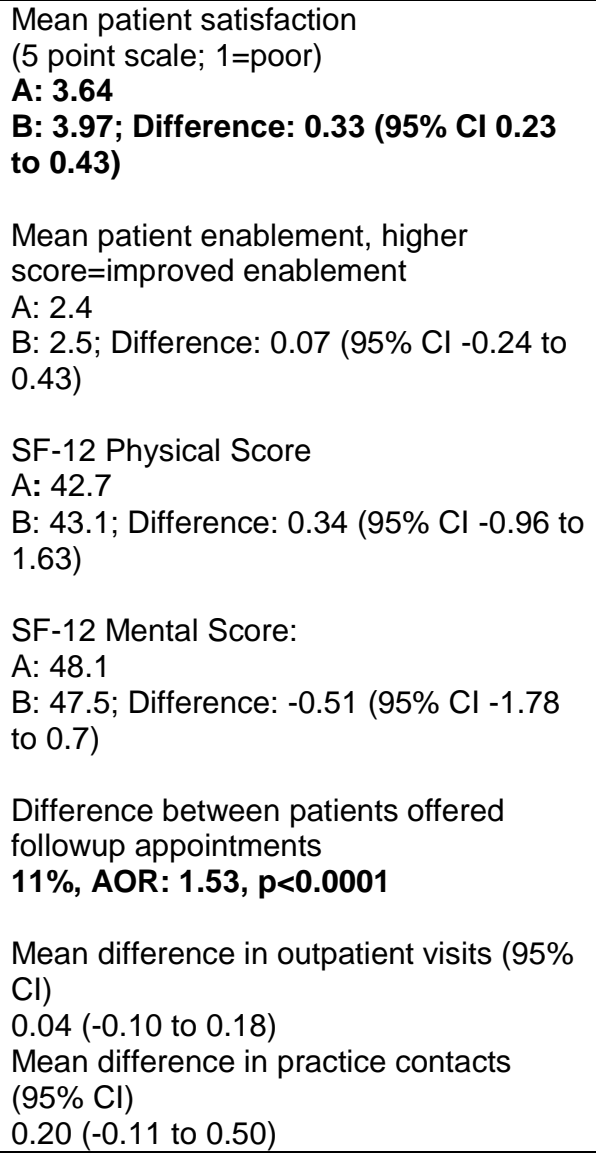 & $\begin{array}{l}\text { Total mean } \\
\text { NHS costs: } \\
\text { A: } £ 625.26 \\
\text { B: } £ 723.98 \\
\text { Difference: } \\
£ 98.72, \\
p=0.03 \\
\text { NHS } \\
\text { adjusted } \\
\text { difference: } \\
£ 93.80 \text { (7.34 } \\
\text { to 180.40) } \\
\text { Total patient } \\
\text { costs: } \\
\text { A: } £ 11.38 \\
\text { B: } £ 3.69 \\
\text { Difference: } \\
£-7.70, \\
\text { p<0.0001 } \\
\text { Costs higher } \\
\text { due to } \\
\text { equipment } \\
\text { and requiring } \\
\text { both GP and } \\
\text { specialist } \\
\text { time. }\end{array}$ \\
\hline
\end{tabular}

AOR = adjusted odds ratio; $\mathrm{CI}$ = confidence interval; $\mathrm{ED}=$ emergency department; $\mathrm{GP}$ = general practitioner; HR = hazard ratio; NHS = National Health Service; NR = not reported; NS = not significant; OR = odds ratio; RCT = randomized control trial; SF12 = Short Form-12; $\mathrm{TH}=$ telehealth

${ }^{a}$ Combined $1+2$ years after telehealth vs. before telehealth

${ }^{\mathrm{b}} \mathrm{N}=144$

Bold=statistically significant; telehealth superior. Bold and italicized=statistically significant; telehealth inferior. Regular type: not statistically significant. 


\section{Discussion}

\section{Key Findings and Strength of Evidence}

These key findings are the result of our comprehensive systematic review and our prototype decision analyses. The systematic review focused on the effectiveness of telehealth consultations in terms of clinical and cost outcomes as well as intermediate outcomes and harms. We organized the results by setting (inpatient, emergency, and outpatient care) and completed the strength of evidence (SOE) assessments by setting as well. Within settings, we further divided the studies into subgroups by clinical focus, which varied across the three settings. Given the wide variety of study designs and outcome measures, we were not able to use meta-analysis and relied on qualitative approaches for summarizing and synthesizing results across studies.

Many of the SOE assessments are low due to a combination of study limitations, inconsistent results, and imprecise estimates of effect. There were a few moderate ratings and no high ratings. Additionally, there were cases in which the SOE was noted as insufficient, reflecting either a lack of studies addressing the specific question or that available evidence did not allow a conclusion to be drawn. In general, harms were not reported, and therefore the evidence is insufficient. The evidence about clinical outcomes and intermediate outcomes is mixed, and more details are provided below. Given our interest in cost modeling for the decision model portion of this project, we paid particular attention to the type of economic outcomes included in the studies, the sources of data, and the rigor of different approaches to assessing costs and utilizations. Overall, the strength of evidence about costs and other economic outcomes is low across the settings due to inconsistencies in both methods and results.

The strongest evidence across groups of studies are moderate SOE ratings for the following combinations of settings and outcomes. For inpatient care, remote intensive care units (ICUs) reduce ICU and hospital mortality while lengths of stay (LOS) are not significantly different. In emergency care, specialty remote consultations increase appropriate transfers and admissions while decreasing the time from presentation to decision and the amount of time spent in an emergency department (intermediate outcomes). When telehealth is used in emergency medical services, mortality is reduced for ST-elevation myocardial infarction (STEMI) patients, and treatment is more timely (clinical and intermediate outcomes). Use of telehealth consultations in outpatient care resulted in improved clinical outcomes for wound care, psychiatry and single chronic disease care, and in increased access to services across specialties (intermediate outcome).

In other combinations of settings and outcomes, the strength of evidence is low or the evidence is insufficient. These are described in the text and tables in the next section with more details provided in the Results sections above.

\section{Inpatient Telehealth Consultations}

To facilitate summarizing and synthesis we split the inpatient studies into remote ICU and specialist consultations for hospitalized patients. Table 24 provides the number of studies reporting each type of outcome, the main findings, and the strength of evidence for these two subgroups.

The results of the identified studies reported provide evidence that remote ICUs decrease mortality in the ICU and during the hospital stay (moderate strength of evidence). ICU and hospital length of stay are slightly shorter (less than one-half day mean difference), but the 
differences are not statistically significant (moderate strength of evidence). A subset of the studies (6) analyzed costs of remote ICUs or their impact on revenue but their methods and conclusions were inconsistent with costs measured in a variety of ways and half reporting savings or increased revenue and half reporting increased costs (insufficient evidence).

The studies of inpatient specialist consultations reported no significant differences in mortality (low strength of evidence), but other clinical outcomes defined as serious morbidity (e.g., cardiac arrest, low birthweight, falls, and disability) improved with telehealth, but these differences were not always statistically significant (low strength of evidence). The impact on intermediate outcomes such as hospital LOS or patient satisfaction is also mostly positive, but with differences that were close to significant and estimates that were less precise (low strength of evidence). Costs were compared in seven studies. Most studies reported savings due to avoided travel or transfers (low strength of evidence). Three studies of remote surgery were the only ones that explicitly examined harms, and while no harms were identified, the studies were small and rated as high risk of bias (insufficient evidence).

Limited information on the characteristics of what is studied (Key Question 4) made it difficult to assess variation in outcomes (Key Question 5), though we did look at hospital characteristics, remote ICU coverage, and the period for outcome measurement for the studies of remote ICUs. While these differed across studies there was no identifiable pattern of association of these characteristics with the results.

Table 24. Inpatient telehealth consultations: strength of evidence

\begin{tabular}{|c|c|c|c|c|}
\hline Topic & Outcome (KQ) & $\begin{array}{l}\text { Number } \\
\text { of } \\
\text { Studies } \\
\text { (N) }\end{array}$ & Main Findings & $\begin{array}{l}\text { Strength of } \\
\text { Evidence } \\
\text { (Insufficient, } \\
\text { Low, } \\
\text { Moderate, } \\
\text { High) } \\
\end{array}$ \\
\hline \multirow[t]{6}{*}{$\begin{array}{l}\text { Inpatient } \\
\text { remote ICU }\end{array}$} & $\begin{array}{l}\text { ICU Mortality } \\
(\text { KQ1 })^{a}\end{array}$ & 11 & $\begin{array}{l}\text { Lower ICU mortality } \\
\text { RR } 0.69(95 \% \mathrm{Cl} 0.51,0.89)\end{array}$ & Moderate \\
\hline & $\begin{array}{l}\text { Hospital Mortality } \\
(\text { KQ1 })^{\mathrm{a}}\end{array}$ & 12 & $\begin{array}{l}\text { Lower hospital mortality } \\
\text { RR } 0.76(95 \% \mathrm{Cl}, 0.60,0.95)\end{array}$ & Moderate \\
\hline & Cost (KQ1) & 6 & $\begin{array}{l}\text { Unable to summarize across studies: different } \\
\text { methods and inconsistent results. }\end{array}$ & Insufficient \\
\hline & $\begin{array}{l}\text { ICU LOS } \\
\left(_{(K Q 2)}{ }^{a}\right.\end{array}$ & 12 & $\begin{array}{l}\text { No significant difference in ICU LOS } \\
\text { Mean difference (days) }-0.39(95 \% \mathrm{Cl}-0.99,0.15)\end{array}$ & Moderate \\
\hline & $\begin{array}{l}\text { Hospital LOS } \\
\text { (KQ2) }^{\mathrm{a}}\end{array}$ & 12 & $\begin{array}{l}\text { No significant difference in hospital LOS } \\
\text { Mean difference (days) }-0.14(95 \% \mathrm{Cl}-0.96,0.63)\end{array}$ & Moderate \\
\hline & $\begin{array}{l}\text { Harms } \\
\text { (KQ3) }\end{array}$ & 0 & None reported in identified articles & Insufficient \\
\hline \multirow{5}{*}{$\begin{array}{l}\text { Inpatient } \\
\text { specialty } \\
\text { consultations }\end{array}$} & Mortality (KQ1) & 12 & No significant difference in mortality & Low \\
\hline & $\begin{array}{l}\text { Other clinical } \\
\text { outcomes (KQ1) }\end{array}$ & 6 & $\begin{array}{l}\text { Clinical outcomes better with telehealth but small } \\
\text { differences and most not significantly different }\end{array}$ & Low \\
\hline & Cost (KQ1) & 7 & $\begin{array}{l}\text { Cost savings due to avoiding transfers or travel } \\
\text { when telehealth is used but not in all studies }\end{array}$ & Low \\
\hline & $\begin{array}{l}\text { Intermediate } \\
\text { outcome (KQ2) }\end{array}$ & 27 & $\begin{array}{l}\text { Reductions in LOS and waiting time but all not } \\
\text { significantly different; satisfaction measures good } \\
\text { but not excellent }\end{array}$ & Low \\
\hline & Harms (KQ3) & 3 & $\begin{array}{l}\text { Complications or harms from telehealth in surgery } \\
\text { was compared with standard procedures in small } \\
\text { studies with high risk of bias }\end{array}$ & Insufficient \\
\hline
\end{tabular}

CI = confidence interval; ICU = intensive care unit; KQ = Key Question; LOS = length of stay; RR = risk ratio

a Based on studies included in the meta-analysis 


\section{Emergency Care Telehealth Consultations}

We divided the emergency care studies into three categories: telestroke, specialist consultations for patients in an emergency department, and emergency medical services (EMS) and urgent care (Table 25).

Across the telestroke studies, there were no significant differences in mortality, either inhospital or at 3 months (moderate strength of evidence). The rates of hemorrhage, the harm most likely to occur if a stroke is treated inappropriately, were also not significantly different with and without telehealth (moderate strength of evidence). Small differences in functional outcomes were not significant; tissue plasminogen activator (tPA) use increased, but the majority of studies reported this change did not rise to the level of statistical significance; and most studies found no significant difference in time to treatment (all low strength of evidence).

The studies of specialty consultations for emergency patients reported lower mortality; however, these differences were not always statistically significant. Similarly, four studies reported other clinical outcomes, with only one, lower complications during transport, achieving statistical significance. Four of five studies reported lower costs (low strength of evidence). Intermediate outcomes focused on the impact on decisions including transfers, hospital admissions and time spent in an emergency department (ED), and there were more consistent finding of benefits from telehealth consultations (moderate strength of evidence). None of the included studies in this category reported harms.

When telehealth is used by EMS to inform decisions on treatment and location of transport for patients with suspected heart attacks, mortality is lower (moderate strength of evidence). We did not identify sufficient evidence to reach a conclusion about harms when telehealth consultations are used in EMS or urgent care (insufficient evidence), though there is some evidence these consultations reduce transfers and referrals (intermediate outcomes; moderate strength of evidence) and costs (low strength of evidence).

Table 25. Emergency care telehealth consultations: strength of evidence

\begin{tabular}{|c|c|c|c|c|}
\hline Topic & $\begin{array}{l}\text { Outcome } \\
\text { (KQ) }\end{array}$ & $\begin{array}{l}\text { Number } \\
\text { of } \\
\text { Studies } \\
\text { (N) }\end{array}$ & Main Findings & $\begin{array}{l}\text { Strength of Evidence } \\
\text { (Insufficient, Low, } \\
\text { Moderate, High) }\end{array}$ \\
\hline \multirow{5}{*}{$\begin{array}{l}\text { Emergency } \\
\text { Care: } \\
\text { Telestroke }\end{array}$} & $\begin{array}{l}\text { In-hospital } \\
\text { mortality (KQ1) }\end{array}$ & 9 & $\begin{array}{l}\text { RR } 0.89 \\
(95 \% \mathrm{Cl} 0.63,1.43) \text { No difference }\end{array}$ & Moderate \\
\hline & $\begin{array}{l}\text { 3-month } \\
\text { mortality (KQ1) }\end{array}$ & 7 & $\begin{array}{l}\text { RR } 0.94 \\
(95 \% \mathrm{Cl} 0.82,1.16) \text { No difference }\end{array}$ & Moderate \\
\hline & $\begin{array}{l}\text { tPA } \\
\text { administration } \\
(\mathrm{KQ} 2)\end{array}$ & 13 & $\begin{array}{l}\text { Reported tPA use increases; four } \\
\text { significant; majority not statistically } \\
\text { significant or not tested }\end{array}$ & Low \\
\hline & $\begin{array}{l}\text { Time to } \\
\text { Treatment } \\
\text { (KQ2) }\end{array}$ & 23 & $\begin{array}{l}\text { Time to treatment is shorter but not } \\
\text { significant in the majority of studies; a } \\
\text { minority report longer times }\end{array}$ & Low \\
\hline & $\begin{array}{l}\text { Harms (all } \\
\text { Hemorrhage) } \\
\text { (KQ3) }\end{array}$ & 11 & $\begin{array}{l}\text { No difference in hemorrhage, the only } \\
\text { potential harm reported }\end{array}$ & Moderate \\
\hline $\begin{array}{l}\text { Emergency } \\
\text { Care: } \\
\text { Specialty } \\
\text { Consultations }\end{array}$ & $\begin{array}{l}\text { Clinical } \\
\text { outcomes } \\
(\mathrm{KQ1})\end{array}$ & 13 & $\begin{array}{l}\text { Lower mortality reported in most } \\
\text { studies but not statistically significant; } \\
\text { Four studies reporting other clinical } \\
\text { outcomes that were better with } \\
\text { telehealth; one reported significant } \\
\text { differences }\end{array}$ & Low \\
\hline
\end{tabular}




\begin{tabular}{|c|c|c|c|c|}
\hline Topic & $\begin{array}{l}\text { Outcome } \\
\text { (KQ) }\end{array}$ & $\begin{array}{l}\text { Number } \\
\text { of } \\
\text { Studies } \\
\text { (N) }\end{array}$ & Main Findings & $\begin{array}{l}\text { Strength of Evidence } \\
\text { (Insufficient, Low, } \\
\text { Moderate, High) }\end{array}$ \\
\hline & Cost (KQ1) & 5 & $\begin{array}{l}\text { Lower costs with better or no change } \\
\text { in clinical outcome in most (4) studies; } \\
\text { one study reported higher costs }\end{array}$ & Low \\
\hline & $\begin{array}{l}\text { Intermediate } \\
\text { outcomes } \\
\text { (KQ2) }\end{array}$ & 19 & $\begin{array}{l}\text { Increase in appropriate transfers, } \\
\text { decrease in time to decision and time } \\
\text { in ED with telehealth compared with } \\
\text { standard care }\end{array}$ & Moderate \\
\hline & Harms (KQ3) & 0 & $\begin{array}{l}\text { No studies reported data on harms } \\
\text { from telehealth }\end{array}$ & Insufficient \\
\hline \multirow[t]{4}{*}{$\begin{array}{l}\text { Emergency } \\
\text { Care: EMS or } \\
\text { Urgent Care }\end{array}$} & $\begin{array}{l}\text { Clinical } \\
\text { Outcomes } \\
\text { (KQ1) }\end{array}$ & 10 & $\begin{array}{l}\text { Telehealth reduced mortality for } \\
\text { STEMI patients }\end{array}$ & Moderate \\
\hline & Cost (KQ1) & 5 & $\begin{array}{l}\text { Lower costs due to avoided transfers } \\
\text { or lower staff costs when telehealth is } \\
\text { used }\end{array}$ & Low \\
\hline & $\begin{array}{l}\text { Intermediate } \\
\text { Outcomes } \\
\text { (KQ2) }\end{array}$ & 20 & $\begin{array}{l}\text { Treatment is more timely and fewer air } \\
\text { transfers or referrals to higher level of } \\
\text { care }\end{array}$ & Moderate \\
\hline & Harms (KQ3) & 1 & $\begin{array}{l}\text { One study reported data that could be } \\
\text { interpreted as harms, but not defined } \\
\text { as such by the authors }\end{array}$ & Insufficient \\
\hline
\end{tabular}

CI = confidence interval; EMS = emergency medical services; KQ = Key Question; RR = risk ratio; STEMI = ST-elevation myocardial infarction; tPA = tissue plasminogen activator

\section{Outpatient Telehealth Consultations}

We grouped the included articles evaluating telehealth consultations in the outpatient setting into 11 clinical topics (Table 26). In eight of the specific specialties we identified, we included three or more articles (i.e., dermatology, wound care, ophthalmology, orthopedics, dentistry, cancer, psychiatry, and infectious disease). The remaining three topics consist of programs designed to facilitate consultations with multiple specialists. The first involves the use of diagnostic technology such as echocardiograms, ultrasounds, endoscopies, and Dopplers. The second group includes articles about specific specialty consultations that do not involve diagnostic technology. These studies evaluated the use of telehealth consultations in the management of chronic conditions including hypertension, diabetes, arthritis and chronic pain. The final category includes studies of telehealth set up to provide consultations across multiple specialties.

Clinical outcomes were improved in several topic areas including wound care, psychiatry, and chronic conditions such as diabetes (moderate strength of evidence). In dermatology, clinical course was found to be similar with and without telehealth (low strength of evidence). For some specialties including ophthalmology, dentistry, cancer, infectious disease, and specialties combined with diagnostic technology, clinical outcomes were either not reported or the results were not sufficient to support a conclusion (insufficient evidence). Only two outpatient studies explicitly addressed harms. Lower costs were reported in most studies that assessed costs, but the methodologies used varied considerably, and most of the positive (cost savings) results hinged on patient savings of travel costs and time rather than cost savings for the health system (low strength of evidence).

Given that all of these studies addressed at least one intermediate outcome, we split the intermediate outcomes into three categories for the SOE assessment: access, management and 
utilization, and satisfaction. Access was improved with telehealth consultations across all specialties. For example, several studies in dermatology reported that time to diagnosis and time to treatment were reduced. Similarly, telehealth consultations using diagnostic technology allowed faster assessment of conditions or more patients to have the comprehensive assessment indicated (e.g., ultrasound for high risk pregnancies) (moderate strength of evidence).

In many cases, telehealth consultations were designed to influence how a condition was managed in terms of what services were utilized (e.g., a hospitalization or travel to a specialist for an in-person exam). Most of the studies reported telehealth consultations had the intended effect of reducing hospital admissions and specialist in-person visits while providing similar diagnoses and management plans, however, a few studies reported differences in diagnosis, planned management, or treatment as these studies assumed the in-person decisions were correct. Because of this inconsistency, the strength of evidence is low.

Satisfaction results differed for patients and family compared with providers, despite being generally positive. Patients appreciated greater access and savings in time, costs, and time off work that traveling for care would require. Clinicians' assessments were more varied, with many rating the telehealth consultations as the same or as good as face-to-face while others reported they were slightly worse (low strength of evidence).

Table 26. Outpatient care telehealth consultations: strength of evidence

\begin{tabular}{|c|c|c|c|}
\hline Outcome (KQ) & $\begin{array}{l}\text { Number } \\
\text { of } \\
\text { Studies } \\
\text { (N) }\end{array}$ & Main Findings & $\begin{array}{l}\text { Strength of } \\
\text { Evidence } \\
\text { (Insufficient, Low, } \\
\text { Moderate, High) }\end{array}$ \\
\hline $\begin{array}{l}\text { Clinical Outcomes } \\
\text { (KQ1): Dermatology }\end{array}$ & 3 & No significant different in clinical course & Low \\
\hline $\begin{array}{l}\text { Clinical Outcomes } \\
\text { (KQ1): Wound Care }\end{array}$ & 5 & Better healing and fewer amputations & Moderate \\
\hline $\begin{array}{l}\text { Clinical Outcomes } \\
\text { (KQ1): Ophthalmology }\end{array}$ & 0 & No studies reported data on clinical outcomes & Insufficient \\
\hline $\begin{array}{l}\text { Clinical Outcomes } \\
\text { (KQ1): Orthopedics }\end{array}$ & 0 & No studies reported data on clinical outcomes & Insufficient \\
\hline $\begin{array}{l}\text { Clinical Outcomes } \\
\text { (KQ1): Dental }\end{array}$ & 0 & No studies reported data on clinical outcomes & Insufficient \\
\hline $\begin{array}{l}\text { Clinical Outcomes } \\
\text { (KQ1): Cancer }\end{array}$ & 1 & $\begin{array}{l}\text { Rate of serious side effects from chemotherapy } \\
\text { reported in } 1 \text { study }\end{array}$ & Insufficient \\
\hline $\begin{array}{l}\text { Clinical Outcomes } \\
\text { (KQ1): Psychiatry }\end{array}$ & $\begin{array}{l}3 \text { (in } 5 \\
\text { articles) }\end{array}$ & Decrease in symptoms and high remission rates & Moderate \\
\hline $\begin{array}{l}\text { Clinical Outcomes } \\
\text { (KQ1): Infectious } \\
\text { Disease }\end{array}$ & 3 & $\begin{array}{l}\text { Inconsistent results for virologic suppression across } \\
\text { studies }\end{array}$ & Insufficient \\
\hline $\begin{array}{l}\text { Clinical Outcomes } \\
\text { (KQ1): ): Single } \\
\text { Conditions with } \\
\text { Diagnostic Technology }\end{array}$ & 0 & No studies reported data on clinical outcomes & Insufficient \\
\hline $\begin{array}{l}\text { Clinical Outcomes } \\
\text { (KQ1): Single } \\
\text { Specialties }\end{array}$ & 6 & $\begin{array}{l}\text { Positive effects on clinical outcomes such as } \\
\text { response to treatment. }\end{array}$ & Moderate \\
\hline $\begin{array}{l}\text { Clinical Outcomes } \\
\text { (KQ1):Multiple } \\
\text { Specialties }\end{array}$ & 4 & $\begin{array}{l}\text { Inconsistent results across studies for unanticipated } \\
\text { or avoidable health services utilization }\end{array}$ & Insufficient \\
\hline Cost (KQ1) & 32 & $\begin{array}{l}\text { Most studies report cost saving with telehealth but } \\
\text { calculations vary and most are dependent on patient } \\
\text { avoided travel and loss of time }\end{array}$ & Low \\
\hline
\end{tabular}




\begin{tabular}{|l|c|l|c|}
\hline Outcome (KQ) & $\begin{array}{c}\text { Number } \\
\text { of } \\
\text { Studies } \\
\text { (N) }\end{array}$ & Main Findings & $\begin{array}{c}\text { Strength of } \\
\text { Evidence } \\
\text { (Insufficient, Low, } \\
\text { Moderate, High) }\end{array}$ \\
\hline $\begin{array}{l}\text { Intermediate Outcomes: } \\
\text { Access (KQ2) }\end{array}$ & 35 & $\begin{array}{l}\text { Access in terms of time to, or comprehensiveness of } \\
\text { service is improved with telehealth }\end{array}$ & Moderate \\
\hline $\begin{array}{l}\text { Intermediate Outcomes: } \\
\text { Management and }\end{array}$ & 31 & $\begin{array}{l}\text { Mixed results with majority finding some benefit in } \\
\text { terms of avoiding visits and similar diagnosis or } \\
\text { management but a subset of studies report } \\
\text { differences in diagnosis and management with } \\
\text { telehealth compared with standard care }\end{array}$ & Low \\
\hline $\begin{array}{l}\text { Intermediate Outcomes: } \\
\text { Satisfaction (KQ2) }\end{array}$ & 22 & $\begin{array}{l}\text { Satisfaction generally the same; patients higher with } \\
\text { telehealth if time/travel is avoided. Providers the } \\
\text { same or slightly worse for telehealth. }\end{array}$ & Low \\
\hline Harms (KQ3) & 2 & $\begin{array}{l}\text { Rates of complications and serious adverse events } \\
\text { reported in two studies }\end{array}$ & Insufficient \\
\hline
\end{tabular}

KQ = Key Question

\section{Exploratory Cost Model for Telehealth Neurosurgical Consultations}

During the systematic review of published studies we identified topics for which decision models and/or economic assessment studies had not been published. After reviewing the possibilities, we selected telehealth consultations in the acute management of patients with traumatic brain injury transported to hospitals not designated level I or II trauma centers. We considered the comparison of (1) immediate transfer after stabilization from the community hospital with no access to neurosurgical consultations to a level I or II trauma center (standard care model) and (2) telehealth consultation to determine if the patient can be managed at the local hospital or should be transferred to a level I or II trauma center (telemedicine model). Data from the literature were used as input parameters to calculate incremental costs for the two different possibilities from the perspective of the healthcare system.

The decision analytic model assumed equivalent patient outcomes (details provided in Appendix I). However, the framework was constructed to allow for future inclusion of differences in patient outcomes based on the Glasgow Outcome Scale (GOS) at 6 months: (1) death, (2) persistent vegetative state, (3) severe disability (lost independence) (4) moderate disability, and (5) good outcome (healthy post-traumatic brain injury) if and when this evidence becomes available.

\section{Findings in Relationship to What Is Already Known}

The literature on telehealth is large and included several systematic reviews of varying size and scope. We did not identify any existing reviews that exactly addressed our Key Questions or matched our requirements and inclusion criteria. We identified reviews that were broad, including telehealth for consultations as well as other functions (e.g., a review on the impact of telemedicine on professional practice and healthcare outcomes ${ }^{259}$ and reviews on single clinical areas (e.g. dentistry ${ }^{260}$ and psychiatry ${ }^{261}$ ). In total, we examined 34 systematic reviews that were related to our topic and used these to identify additional studies for inclusion in this review and to summarize according to our Key Questions.

\section{Applicability}

Our results and synthesis are based on a relatively large number of studies included in this review. While the largest group was conducted in the United States, many were conducted in 
Europe, Asia, Australia, and New Zealand (see Table 1). Table 1 also demonstrates that the included studies represent a range of technologies or modes and both real time and asynchronous consultations. Some details, such as whether the patient was present at the consultation were not reported consistently, but they were reported frequently enough to know that it varied. These and other details about the studies are included in Appendixes F and G.

How we organized and analyzed the included studies was driven by our assessment of the applicability of different subgroups of the results. We analyzed and presented the studies by setting - inpatient, emergency, and outpatient care - because we believe consultations require different infrastructure and serve different purposes in these broad settings. We did not combine across these categories because we do not think the results from one setting are directly applicable to another. For instance, the results of studies about emergency care are not directly applicable to situations where time is not an essential factor and specific expertise is not needed quickly. Similarly, the results of asynchronous dermatology used to assess skin lesions are not as applicable to the use of telehealth to monitor and manage ICU patients as they may be to the use of other specialists for outpatient consultation.

Within settings, we created subgroups based on our assessment of when the results are applicable across conditions and uses. For inpatient care, we kept the remote ICU studies separate, as that is a very specialized, specific use. We combined other specialty consultations for inpatient care as they are similar in terms of the function of the consultation (e.g., to diagnose a condition or to provide direction during a surgery) and the types of outcomes. For example, even though the populations are different, remote neurological consultation or an adult with a traumatic brain injury and a neonate inpatient cardiology consultation are similar in that both are facilitating access to highly specialized expertise in order to make decisions about whether to transport the patient or how the patient should be managed. This similarity may transcend the fact that the populations are very different.

For emergency care we separated telestroke, specialty consults for ED patients, and EMS/urgent care for similar reasons. While time is important in all emergency care, it is the core consideration in telestroke and EMS/urgent care. The use of different specialist consultations in the ED, ranging from pediatrics to psychiatry are for different patients but for similar purposes: to inform the management of patients' presenting conditions, including whether the patient should be admitted, transferred, or discharged home. These patients are often more stable, and the technology necessary to connect consultants to an ED is likely different from that needed to connect consultants to ambulances and first responders.

Our approach and the issues of applicability for outpatient consultations were slightly different. We reported the details and clinical outcomes separately by specialty to allow readers to see the results in these groupings as people are often interested in a particular specialty. Then we combined the results across specialties when assessing costs and intermediate outcomes. We divided intermediate outcomes into three categories as all the studies of outpatient consultations included one or more intermediate outcome and to facilitate considerations of applicability in terms of whether the telehealth consultations were impacting access, management and utilization of health services, or satisfaction.

Applicability is often focused on the populations of patients to whom the results may apply. For this intervention, the setting is of primary importance. The setting, combined with the goal or nature of the intervention (i.e., what the purpose of the telehealth consultation is) and the intended outcome, drive applicability. More nuanced assessments by payment model or 
organizational characteristics would be useful as well but are not possible given the lack of published results.

\section{Limitations of the Evidence Base}

There are important limitations to the evidence base on the effectiveness of the use of telehealth for consultations. The most significant is the variation in study designs and the level of rigor of the research methodology. In our assessment, very few studies were rated as low risk of bias; most were moderate or high. Risk of bias criteria are specific to the study design, which can minimize the fact that some study designs are much more likely to be weak or biased than others. The literature on telehealth consultations consists primarily of studies that would be considered weaker designs such as before and after studies without comparison groups and retrospective cohort studies. In some cases, many of the studies for a specific clinical area would be considered weak designs. For example, most of the studies on remote ICU programs compared outcomes prior to the remote ICU program initiation to a period after implementation. Even though some studies did examine patient characteristics or considered risk adjusted outcomes, it is possible that several other elements of care changed that were not measured or accounted for. In the analyses of costs or other economic outcomes, the designs and approaches also varied and few were rigorous cost analyses. Many estimated costs or savings indirectly, some relying on hypothetical estimates of what would have been spent or saved absent a program. Importantly, the comparison treatment was poorly described in these studies; such that it was often impossible to know what type of care (e.g. in-person care by a consultant versus no consultation) was in the "usual care" groups.

Another limitation is the inconsistency in outcomes used to evaluate effectiveness. Outcomes ranged from mortality to time to diagnosis to avoided appointments. The variation in outcomes across clinical areas makes it difficult to assess the comparative impact of telehealth consultations. Based on the available evidence we are able to conclude that remote ICU reduces mortality and store and forward dermatology reduces time to diagnosis, but it is not possible to say if one is more effective or suggest which may have a larger impact on different types of stakeholders ranging from patients to health systems. Additionally, we found that detailed economic data was rarely provided, and this was a major barrier to decision analyses.

Another concern and potential limitation in this literature is that is it not always clear what the best or most appropriate outcome should be for these studies. Retrospective studies and some prospective studies can be limited by what data are routinely or easily collected. It is possible these are not the most important outcomes for telehealth consultations. For example, telestroke programs report mortality rates, but it is possible that telestroke provides appropriate and timely access to treatment that reduces long-term disability but not necessarily mortality. However, because this requires longer followup, data may not be readily available in existing records. Patient-reported outcomes may be underrepresented for similar reasons. While some studies did include patient satisfaction, these were a minority, and broader, comprehensive measures of patient experience, confidence, or engagement are not common in this literature.

While the range of clinical topics identified was broad, there were clinical topics we expected to find and did not. For example, antibiotic management, pain management, and opioid misuse are not well-represented. It is difficult to determine if these topics are the focus of studies that have not been published yet or if they have not been studied.

Finally, the studies provide very little information on the context or the environment in which telehealth for consultations was implemented. While most, but not all studies, provided at least 
minimal information on the type of technology used (e.g., two way video, mirroring of monitors, still image storage) very little or no information was provided on the details of the workflow, the staffing and other characteristics of the specific practice, department or parent organization. Perhaps most importantly, information was not provided about the type of payment model for the consultation or the followup or ongoing care after the consultation. This is particularly problematic as most studies were in only a single location and few involved multiple sites. Without information about payment models and costs, it is not possible to estimate the economic impact of telehealth as well as the impact on access. The lack of information about the context and environment is at the core of the issues with applicability mentioned above.

\section{Limitations of Our Approach}

There are also limitations to this combined review and decision modeling report that are the result of our processes and decisions. Searching for research about a specific function of telehealth, in this case provider to provider consultation, is difficult as the indexing terms in Medline and other citation databases do not exactly match our scope. We used the MeSH term "Remote Consultation" but as this did not identify several studies known to us, we augmented this with keyword searching and more general MeSH terms. As a result, our search identified citations on this specific function of telehealth but also telehealth more generally. We did not conduct searches using terms for specific clinical areas. Therefore, if the indexing, abstract or title did not include terms related to telehealth and only focused on the clinical topic, we may not have identified the study. We also checked reference lists of included articles, relevant systematic reviews, and reviewed what was submitted in response to our request for information published in the Federal Register, our request for public comments, and peer review. Despite these efforts it is possible that some existing relevant studies are not included.

As the focus of this review is on evidence related to the effectiveness of telehealth consultations, we required that studies include specific types of outcomes (clinical outcomes, costs, and intermediate outcomes including access, satisfaction, and utilization of health services/medical management of the condition). We did not include studies that only reported descriptions of implementation, assessments of technology (e.g., the reliability of transmissions or the quality of video or images), or diagnostic concordance. However, if these types of information were provided in a study along with included outcomes, we did not ignore it. This type of information was not reported consistently in our included studies, so our understanding of these factors and how they relate to included outcomes is limited.

Given the variation in study designs, environments, and outcomes, we used quantitative synthesis (i.e., meta-analysis), for only a small number of situations where the outcomes were mortality or length of stay and the interventions were similar across studies (remote ICU and stroke). For the rest we used a qualitative approach to the strength of evidence framework. We also provided summaries in the text by more specific clinical indications. Qualitative synthesis such as this is more open to interpretation and judgment. We have attempted to be transparent and provide enough detail to allow readers to examine our conclusions, but we acknowledge that there is a significant subjective component to this and that another group of investigators could review the same literature and provide different ratings.

An important limitation to the cost model is the assumption that patient outcomes are equivalent. Should systematic differences or uncertainty exist, then a different model incorporating outcomes would be needed to make valid comparisons of the economic value of the two approaches to care. The model was built to allow inclusion of patient outcomes 
following treatment for cost benefit analyses in the future. While outcomes were assumed to be equivalent in the model included in Appendix I, when more and better data become available, the impact on mortality or function could be used to inform judgements about the value of additional costs given the patient benefits.

\section{Future Research Needs}

While we identified 233 articles that evaluated the effectiveness of telehealth consultations, several questions remain to be addressed in future research. A key priority is the need for rigorous, multi-site studies of telehealth consultations in clinical areas and in the types of organizations where the lack of evidence may be a barrier to wider spread implementation. For example, most of the remote ICU studies were conducted in a single hospital, and the hospitals included urban and academic medical centers, while the suggestion is that rural or underresourced hospitals may benefit most from this type of telehealth. Avoiding transport of critical care patients while still providing technically advanced care could keep patients closer to their families and keep revenue for care in the community. Another example is the use of outpatient teleconsultations involving technology such as echocardiograms, ultrasound, or endoscopy. The studies included in this review that had a remote specialist guiding the use of technology by an appropriate technician located with the patient appear promising. However, not enough studies or sites were included to determine when this might increase access to critical services, improve patient outcomes, and be cost effective.

Future studies are also needed that both expand and standardize outcomes and clarify their objectives. Agreeing on some common metrics across uses of telehealth for consultation would facilitate comparisons across clinical areas and help identify priorities for future expansion of telehealth consultations. Given the wide range of clinical topics, these common metrics may need to be intermediate outcomes, such as measures of access or satisfaction or cost effectiveness. While costs are not the only important outcome, collecting more economic data would allow more direct comparisons across clinical topics and both facilitate and inform additional decision analyses, whether these are done for publication of for organizations' internal consideration. At the same time this needs to be balanced with attention to the most important outcomes for a given condition. As mentioned in limitations of the literature, there are examples, such as telestroke where the most frequently reported outcome (mortality) may not be the most important, either to patients or in terms of the expected impact of changing care. The assessment of telehealth consultations would also be strengthened by more studies that include contemporary comparison groups, either groups of patients or other organizations, so that the effect of the telehealth consultations could be more successfully isolated from historical changes or the idiosyncrasies of a specific organization. This could involve adding comparisons or control sites to before/after telehealth studies.

The research on telehealth could have more impact if its objectives were clearer. Evaluations of telehealth consultations can consider different perspectives and different levels of implementation and evaluation, but failing to be clear leads to studies with confusing results and lessens the impact of positive results. For example, the work on the decision analyses highlighted the importance of clearly specifying the options being compared, or what is "usual care." For a decision analysis, it is important to decide if the alternative to a telehealth consultation is a faceto-face consultation, nonreceipt of a service, or service provided with no consultation. While all may be possible, this shapes the many factors for consideration. In the studies we evaluated for 
this systematic review, what the nontelehealth or "usual care" option consisted of was often not specified, and it was not always clear what care these patients received.

The decision analysis also highlighted the importance of perspective and the need for better information. The assessment of telehealth consultations is different from the perspective of a payer, a health system, a hospital, a practice group, or an individual provider. Most studies did not clearly state their perspective, though it was often implied that is was a single organization (e.g., a hospital or practice group). This seems unnecessarily limiting, and more studies at higher levels seem warranted. In many ways telehealth consultations could be viewed as a systems-level intervention, more similar to health information exchange and electronic health records, than to a condition-specific treatment. While a small subset of studies looked at the use of telehealth consultations across several specialties, they did not look at systems level implementation that would facilitate consultations throughout an organization and spread the cost of the technology, the workflow changes, and any needed training or new skills more broadly across a system. A more definitive test of the hypothesis that telehealth consultations provide better value could come from multi-site trial-based economic evaluations, where patients are randomized to either standard management or a telehealth consultation and cost as well as outcomes data is collected.

A major evolution of the research in this area would be to focus on hybrid studies, that is, studies that combine effectiveness and implementation assessments. While the results may be uneven across specific clinical areas, telehealth consultations do generally improve access and clinical outcomes and are likely to improve other outcomes. What is missing is much of the specific information asked for in Key Questions 4 and 5 of this review; that is, what are the characteristics of the context and how do they impact outcomes? Additionally, having more information on costs could be facilitated by collecting economic data alongside trials or observational studies. This would greatly increase the relevance and completeness of evidence. A hybrid approach to future research could focus on the information needed to promote successful implementation while still continuing to collect better data demonstrating effectiveness and economic impact.

Reviewing background material for this report and discussing telehealth with the Technical Expert Panel and other experts has convinced us that telehealth consultation are being used, particularly in smaller and rural health systems, and that data are often being collected. However, these organizations and data are not represented in the published literature due to lack of research and analysis capacity. Given the importance to policy and practice issues related to telehealth consultations (e.g., payment, scope of work, cross organization and state licensing), identifying and facilitating the analysis of these data should be a priority and may help strengthen what conclusions can be made about telehealth consultations.

Also, during the time period covered in the review and during our work, policies were changed that will likely facilitate telehealth consultation and, perhaps in response, the number of publications about telehealth increased. However, many of these publications are descriptive reports or evaluations using comparatively weak designs. Continuing in this vein, increasing volume without increasing rigor is unlikely to contribute to the next level of telehealth expansion. Given that increasingly more resources are being invested in telehealth, it is reasonable to suggest that research evaluating its effectiveness and impact should improve, employing better designs and better data. Important efforts include defining and delineating potential functions and appropriate outcomes. Clearly defining the function of telehealth in various clinical situation is essential so that future research can avoid combining and comparing across several functions, which may be one reason studies and reviews are often unable to reach 
conclusions or make recommendations. However, comparisons also require common measures, such as those based on the measurement framework produced by the National Quality Forum as a step toward developing common outcome measures for quality assessment, quality improvement, and research. ${ }^{16}$

The current situation seems to require an organized effort by telehealth advocates, researchers, and policymakers. We strove to identify where there are still gaps in the research base for one function of telehealth, but this needs to be done for other functions and then stakeholders need to prioritize identified research gaps in terms of their potential to move the field forward, toward increasing use of telehealth in those settings and instances where it is likely to be beneficial for patients, healthcare providers, health systems, or society. 


\section{Implications and Conclusions}

Although the literature evaluating telehealth consultations is large, it is not possible to make a global, general statement about the clinical and economic effectiveness of telehealth consultations for several reasons. These include the diversity of settings, clinical topics and outcomes; the limited number of high-quality studies; different approaches to measurement, particularly of costs; and how the perspective may impact the estimation of outcomes. It is possible to conclude it is likely that telehealth is more effective than usual care in several specific situations: Remote intensive care units (ICUs) reduce ICU and in-hospital mortality; emergency medical services access to telehealth reduces mortality in patients having heart attacks; remote consultations in emergency care decrease time from presentation to decision, reducing emergency department (ED) time and increasing appropriate transfers and admissions; remote consultations as part of outpatient care improve clinical outcomes in some clinical disciplines and increase access to care in those that have been studied.

For other uses and outcomes the strength of evidence is less definitive. Telehealth consultations may improve inpatient care, emergency stroke care and the management of and satisfaction with outpatient consultations across several specialties. Potential harms or unintended consequences were rarely addressed and future research should address this, if only to confirm they are not significant. Studies of economic outcomes including costs produced mixed results due to major differences in definitions and methods as well as the fact that costs and savings may not accrue to the same organization in an interdependent healthcare system.

Decision models have the potential to build on systematic review results and use evidence in ways that would make it more applicable by tailoring the question, base case, and perspective to the decision maker's situation. But our experience demonstrates that the literature may not be available to provide all the data needed to fully execute a functioning model for all topics of interest. However, decision modeling can provide some insight by quantifying differences in costs across settings and estimating where savings are likely to accrue in the system. While our exploratory assessment was limited to costs, expansion of this approach could allow more targeted identification of scenarios in which telehealth could improve the range of outcomes including clinical outcomes, access, and cost.

Future research about telehealth consultations needs to be more rigorous if it is to inform policy and practice decisions. Specifically, more studies should include multiple sites, collect information on the context and environment, and consistently measure a more comprehensive range of economic impacts and costs using standard practices. 


\section{References}

1. Castro D, Miller B, Nager A. Unlocking the Potential of Physician-to-Patient Telehealth Services. The Information Technology \& Innovation Foundation; 2014. https://itif.org/publications/2014/05/12/unlo cking-potential-physician-patient-telehealthservices. Accessed Nov 152018.

2. Lustig TA. The role of telehealth in an evolving health care environment: workshop summary. Washington, D.C.: The National Academies Press; 2012.

3. Bashshur RL, Shannon GW, Smith BR, et al. The empirical foundations of telemedicine interventions for chronic disease management. Telemed J. E Health. 2014;20(9):769-800. doi: 10.1089/tmj.2014.9981. PMID: 24968105.

4. $\quad$ Ekeland AG, Bowes A, Flottorp S. Effectiveness of telemedicine: a systematic review of reviews. Int. J. Med. Inform. 2010;79(11):736-71. doi: 10.1016/j.ijmedinf.2010.08.006. PMID: 20884286.

5. Hersh WR, Wallace JA, Patterson PK, et al. Telemedicine for the Medicare Population. Evidence Report/Technology Assessment No. 24. AHRQ Publication No. 01-E012. Rockville, MD: Agency for Healthcare Research and Quality; 2001.

6. Hersh WR, Hickam DH, Severance SM, et al. Telemedicine for the Medicare Population: Update. Evidence Report/Technology Assessment No. 131. AHRQ Publication No. 06-E007. Rockville, MD: Agency for Healthcare Research and Quality; 2006.

7. Hersh WR, Wallace JA, Patterson PK, et al. Telemedicine for the Medicare Population: Pediatric, Obstetric, and Clinician-Indirect Home Interventions. Evidence Report/Technology Assessment No. 24, supplement. AHRQ publication No. 01E060. Rockville, MD: Agency for Healthcare Research and Quality; 2001.
8. Totten AM, Womack DM, Eden KB, et al. Telehealth: Mapping the Evidence for Patient Outcomes from Systematic Reviews. Technical brief No. 26. AHRQ publication No.16-EHC034-EF. Rockville, MD: Agency for Healthcare Research and Quality; 2016. Available at https://effectivehealthcare.ahrq.gov/sites/def ault/files/pdf/telehealth_technical-brief.pdf.

9. Adler-Milstein J, Kvedar J, Bates DW. Telehealth among US hospitals: several factors, including state reimbursement and licensure policies, influence adoption. Health Aff. (Millwood). 2014;33(2):207-15. doi: 10.1377/hlthaff.2013.1054. PMID: 24493762.

10. Broderick A, Lindeman D. Scaling telehealth programs: Lessons from early adopters. The Commonwealth Fund; 2013. https://www.commonwealthfund.org/publica tions/case-study/2013/jan/scaling-telehealthprograms-lessons-early-adopters. Accessed Nov 152018.

11. Beck M. How Telemedicine is Transforming Health Care. The Wall Street Journal; 2016. https://www.wsj.com/articles/howtelemedicine-is-transforming-health-care1466993402. Accessed Nov 152018.

12. Report to the Congress: Medicare and the Health Care Delivery System. Medicare Payment Advisory Commission. Washington, DC: 2016.

13. Report to Congress on Medicaid and CHIP. Medicaid and CHIP Payment and Access Commission. Washington, DC: 2018.

14. Wicklund E. VA Finalizes 'Anywhere to Anywhere' Telehealth Program for Vets. mHealthIntelligence.com Xtelligent Media; 2018.

https://mhealthintelligence.com/news/vafinalizes-anywhere-to-anywhere-telehealthprogram-for-vets. Accessed Nov 142018. 
15. Edmunds M, Tuckson R, Lewis J, et al. An Emergent Research and Policy Framework for Telehealth. EGEMS (Washington, DC). 2017;5(2):1303. doi: 10.13063/23279214.1303. PMID: 28459085.

16. National Quality Forum. Creating a Framework to Support Measure Development for Telehealth Department of Health and Human Services. Contract HHSM-500-2012-00009I, Task Order HHSM-500-T0022. Washington, DC: 2017.

17. Hasselberg M, Beer N, Blom L, et al. Image-based medical expert teleconsultation in acute care of injuries. A systematic review of effects on information accuracy, diagnostic validity, clinical outcome, and user satisfaction. PLoS One.

2014;9(6):e98539. doi:

10.1371/journal.pone.0098539. PMID: 24887257.

18. Wallace DL, Hussain A, Khan N, et al. A systematic review of the evidence for telemedicine in burn care: with a UK perspective. Burns. 2012 Jun;38(4):465-80. doi: 10.1016/j.burns.2011.09.024. PMID: 22078804.

19. Assimacopoulos A, Alam R, Arbo M, et al. A brief retrospective review of medical records comparing outcomes for inpatients treated via telehealth versus in-person protocols: is telehealth equally effective as in-person visits for treating neutropenic fever, bacterial pneumonia, and infected bacterial wounds? Telemedicine and eHealth. 2008;14(8):762-8. doi: 10.1089/tmj.2007.0128. PMID: 18954245.

20. Mashru J, Kirlew M, Saginur R, et al. Management of infectious diseases in remote northwestern Ontario with telemedicine videoconference consultations. J. Telemed. Telecare. 2017 Jan;23(1):83-7. doi: 10.1177/1357633X15625136. PMID: 26748393.

21. Parmar P, Mackie D, Varghese S, et al. Use of telemedicine technologies in the management of infectious diseases: a review. Clin. Infect. Dis. 2015;60(7):108494. doi: 10.1093/cid/ciu1143. PMID: 25516192.
22. Methods Guide for Effectiveness and Comparative Effectiveness Reviews. Agency for Healthcare Research and Quality; 2017.

https://effectivehealthcare.ahrq.gov/topics/ce r-methods-guide/overview. Accessed Nov 212018.

23. Moher D, Liberati A, Tetzlaff J, et al. Preferred reporting items for systematic reviews and meta-analyses: the PRISMA statement. Ann. Intern. Med. 2009 Aug 18;151(4):264-9, w64. PMID: 19622511.

24. Consensus Health Economic Criteria CHEC list. Maastricht University Health Services Research. https://hsr.mumc.maastrichtuniversity.nl/con sensus-health-economic-criteria-chec-list. Accessed Nov 152018.

25. Wijnen B, Van Mastrigt G, Redekop WK, et al. How to prepare a systematic review of economic evaluations for informing evidence-based healthcare decisions: data extraction, risk of bias, and transferability (part 3/3). Expert Rev. Pharmacoecon. Outcomes Res. 2016 Dec;16(6):723-32. doi: 10.1080/14737167.2016.1246961. PMID: 27762640.

26. Alemi AS, Seth R, Heaton C, et al. Comparison of video and in-person free flap assessment following head and neck free tissue transfer. Otolaryngol Head Neck Sur. 2017 Jun;156(6):1035-40. doi: 10.1177/0194599816688620. PMID: 28140835.

27. Amorim E, Shih MM, Koehler SA, et al. Impact of telemedicine implementation in thrombolytic use for acute ischemic stroke: the University of Pittsburgh Medical Center telestroke network experience. J. Stroke Cerebrovasc. Dis. 2013 May;22(4):527-31. doi:

10.1016/j.jstrokecerebrovasdis.2013.02.004. PMID: 23489955.

28. Angstman KB, Rohrer JE, Adamson SC, et al. Impact of e-consults on return visits of primary care patients. Health Care Manag (Frederick). 2009 Jul-Sep;28(3):253-7. doi: 10.1097/HCM.0b013e3181b3efa3. PMID: 19668067. 
29. Armaignac DL, Saxena A, Rubens M, et al. Impact of telemedicine on mortality, length of stay, and cost among patients in progressive care units: experience from a large healthcare system*. Crit. Care Med. 2018 04/13;46(5):728-35. doi: 10.1097/CCM.0000000000002994. PMID: 29384782.

30. Arora S, Thornton K, Murata G, et al. Outcomes of treatment for hepatitis $\mathrm{C}$ virus infection by primary care providers. N. Engl. J. Med. 2011 Jun 9;364(23):2199-207. doi: 10.1056/NEJMoa1009370. PMID: 21631316.

31. Baig MM, Antonescu-Turcu A, Ratarasarn K. Impact of Sleep Telemedicine Protocol in Management of Sleep Apnea: A 5-Year VA Experience. Telemed J. E Health. 2016 May;22(5):458-62. doi: 10.1089/tmj.2015.0047. PMID: 26974884.

32. Beswick DM, Vashi A, Song Y, et al. Consultation via telemedicine and access to operative care for patients with head and neck cancer in a Veterans Health Administration population. Head Neck. 2016 Jun;38(6):925-9. doi: 10.1002/hed.24386. PMID: 26899939.

33. Bezalel S, Fabri P, Park HS. Implementation of store-and-forward teledermatology and its associated effect on patient access in a Veterans Affairs dermatology clinic. JAMA Dermatol. 2015 May;151(5):556-7. doi: 10.1001/jamadermatol.2014.5272. PMID: 25671336.

34. Brennan JA, Kealy JA, Gerardi LH, et al. Telemedicine in the emergency department: a randomized controlled trial. J. Telemed. Telecare. 1999;5(1):18-22. doi: 10.1258/1357633991932342. PMID: 10505365.

35. Brennan JA, Kealy JA, Gerardi LH, et al. A randomized controlled trial of telemedicine in an emergency department. J. Telemed. Telecare. 1998;4(1):18-20. doi: 10.1258/1357633981931911. PMID: 9640721.
36. Breslow MJ, Rosenfeld BA, Doerfler M, et al. Effect of a multiple-site intensive care unit telemedicine program on clinical and economic outcomes: an alternative paradigm for intensivist staffing. Crit. Care Med. 2004 Jan;32(1):31-8. doi: 10.1097/01.CCM.0000104204.61296.41. PMID: 14707557.

37. Britt DW, Norton JD, Hubanks AS, et al. A two-period assessment of changes in specialist contact in a high-risk pregnancy telemedical program. Telemed J. E Health. 2006 Feb;12(1):35-41. doi: 10.1089/tmj.2006.12.35. PMID: 16478411.

38. Brown-Connolly NE. Patient satisfaction with telemedical access to specialty services in rural California. J. Telemed. Telecare. 2002;8 Suppl 2:7-10. doi: 10.1177/1357633X020080S204. PMID: 12217115.

39. Burgess LP, Syms MJ, Holtel MR, et al. Telemedicine: teleproctored endoscopic sinus surgery. Laryngoscope. 2002 Feb;112(2):216-9. doi: 10.1097/00005537200202000-00003. PMID: 11889372.

40. Carter ZA, Goldman S, Anderson K, et al. Creation of an internal teledermatology store-and-forward system in an existing electronic health record: a pilot study in a safety-net public health and hospital system. JAMA Dermatol. 2017 Jul 01;153(7):64450. doi: 10.1001/jamadermatol.2017.0204 PMID: 28423156.

41. Choi JY, Porche NA, Albright KC, et al. Using telemedicine to facilitate thrombolytic therapy for patients with acute stroke. Jt Comm J Qual Patient Saf. 2006 Apr;32(4):199-205. PMID: 16649650.

42. Chu S, Boxer R, Madison P, et al. Veterans Affairs telemedicine: bringing urologic care to remote clinics. Urology. 2015 Aug;86(2):255-60. doi: 10.1016/j.urology.2015.04.038. PMID: 26168998.

43. Chu-Weininger MY, Wueste L, Lucke JF, et al. The impact of a tele-ICU on provider attitudes about teamwork and safety climate. Qual Saf Health Care. 2010 Dec;19(6):e39. doi: 10.1136/qshc.2007.024992. PMID: 20427298. 
44. Collins TA, Robertson MP, Sicoutris CP, et al. Telemedicine coverage for post-operative ICU patients. J. Telemed. Telecare. 2017

Feb;23(2):360-4. doi: 10.1177/1357633X16631846. PMID: 27365321.

45. Datta SK, Warshaw EM, Edison KE, et al. Cost and utility analysis of a store-andforward teledermatology referral system: A randomized clinical trial. JAMA Dermatol. 2015;151(12):1323-9. doi: 10.1001/jamadermatol.2015.2362. PMID: 26375589.

46. Davis TD, Deen T, Bryant-Bedell K, et al. Does minority racial-ethnic status moderate outcomes of collaborative care for depression? Psychiatr. Serv. 2011 Nov;62(11):1282-8. doi: 10.1176/appi.ps.62.11.1282. PMID: 22211206.

47. Dayal P, Hojman NM, Kissee JL, et al. Impact of telemedicine on severity of illness and outcomes among children transferred from referring emergency departments to a children's hospital PICU. Pediatr. Crit. Care Med. 2016 Jun;17(6):516-21. doi: 10.1097/PCC.0000000000000761. PMID: 27099972.

48. Demaerschalk BM, Bobrow BJ, Raman R, et al. Stroke team remote evaluation using a digital observation camera in Arizona: the initial mayo clinic experience trial. Stroke. 2010 Jun;41(6):1251-8. doi: 10.1161/STROKEAHA.109.574509. PMID: 20431081.

49. Dharmar M, Romano PS, Kuppermann N, et al. Impact of critical care telemedicine consultations on children in rural emergency departments. Crit. Care Med. 2013

Oct;41(10):2388-95. doi: 10.1097/CCM.0b013e31828e9824. PMID: 23921273.

50. Dharmar M, Sadorra CK, Leigh P, et al. The financial impact of a pediatric telemedicine program: a children's hospital's perspective. Telemed J. E Health. 2013 Jul;19(7):502-8. doi: 10.1089/tmj.2012.0266. PMID: 23837516
51. Dharmar M, Kuppermann N, Romano PS, et al. Telemedicine consultations and medication errors in rural emergency departments. Pediatrics. 2013

Dec;132(6):1090-7. doi: 10.1542/peds.20131374. PMID: 24276844.

52. Doolittle GC, Harmon A, Williams A, et al. A cost analysis of a tele-oncology practice. J. Telemed. Telecare. 1997;3 Suppl 1:20-2. doi: 10.1258/1357633971930850. PMID: 9218371.

53. Duchesne JC, Kyle A, Simmons J, et al. Impact of telemedicine upon rural trauma care. J. Trauma. 2008 Jan;64(1):92-7; discussion 7-8. doi: 10.1097/TA.0b013e31815dd4c4. PMID: 18188104.

54. Endean ED, Mallon LI, Minion DJ, et al. Telemedicine in vascular surgery: does it work? Am. Surg. 2001 Apr;67(4):334-40; discussion 40-1. PMID: 11307999.

55. Fortis S, Weinert C, Bushinski R, et al. A health system-based critical care program with a novel tele-ICU: implementation, cost, and structure details. J. Am. Coll. Surg. 2014 Oct;219(4):676-83. doi: 10.1016/j.jamcollsurg.2014.04.015. PMID: 25154668 .

56. Fortney JC, Pyne JM, Kimbrell TA, et al. Telemedicine-based collaborative care for posttraumatic stress disorder: a randomized clinical trial. JAMA Psychiatry. 2015 Jan;72(1):58-67. doi: 10.1001/jamapsychiatry.2014.1575. PMID: 25409287.

57. Fortney JC, Maciejewski ML, Tripathi SP, et al. A budget impact analysis of telemedicine-based collaborative care for depression. Med. Care. 2011 Sep;49(9):87280. doi: 10.1097/MLR.0b013e31821d2b35. PMID: 21623240.

58. Fortney JC, Pyne JM, Edlund MJ, et al. A randomized trial of telemedicine-based collaborative care for depression. J. Gen. Intern. Med. 2007 Aug;22(8):1086-93. doi: 10.1007/s11606-007-0201-9. PMID: 17492326. 
59. Fortney JC, Pyne JM, Mouden SB, et al. Practice-based versus telemedicine-based collaborative care for depression in rural federally qualified health centers: a pragmatic randomized comparative effectiveness trial. Am. J. Psychiatry. 2013 Apr;170(4):414-25. doi: 10.1176/appi.ajp.2012.12050696. PMID: 23429924.

60. Fox KC, Somes GW, Waters TM. Timeliness and access to healthcare services via telemedicine for adolescents in state correctional facilities. J. Adolesc. Health. 2007 Aug;41(2):161-7. doi:

10.1016/j.jadohealth.2007.05.001. PMID: 17659220.

61. Frank JW, Carey EP, Fagan KM, et al. Evaluation of a telementoring intervention for pain management in the Veterans Health Administration. Pain Med. 2015 Jun;16(6):1090-100. doi: 10.1111/pme.12715. PMID: 25716075.

62. Franzini L, Sail KR, Thomas EJ, et al. Costs and cost-effectiveness of a telemedicine intensive care unit program in 6 intensive care units in a large health care system. $\mathrm{J}$. Crit. Care. 2011 Jun;26(3):329.e1-6. doi: 10.1016/j.jcrc.2010.12.004. PMID: 21376515.

63. Grabowski DC, O'Malley AJ. Use of telemedicine can reduce hospitalizations of nursing home residents and generate savings for medicare. Health Aff. (Millwood). 2014 Feb;33(2):244-50. doi: 10.1377/hlthaff.2013.0922. PMID: 24493767.

64. Heffner DL, Thirumala PD, Pokharna P, et al. Outcomes of spoke-retained telestroke patients versus hub-treated patients after intravenous thrombolysis: telestroke patient outcomes after thrombolysis. Stroke. 2015 Nov;46(11):3161-7. doi: 10.1161/STROKEAHA.115.009980. PMID: 26396027.

65. Hsiao JL, Oh DH. The impact of store-andforward teledermatology on skin cancer diagnosis and treatment. J. Am. Acad. Dermatol. 2008 Aug;59(2):260-7. doi: 10.1016/j.jaad.2008.04.011. PMID: 18485526.
66. Huang T, Moon-Grady AJ, Traugott C, et al. The availability of telecardiology consultations and transfer patterns from a remote neonatal intensive care unit. J. Telemed. Telecare. 2008;14(5):244-8. doi: 10.1258/jtt.2008.080102. PMID: 18632999.

67. Ionita CC, Sharma J, Janicke DM, et al. Acute ischemic stroke and thrombolysis location: comparing telemedicine and stroke center treatment outcomes. Hosp Pract (1995). 2009 Dec;37(1):33-9. doi: 10.3810/hp.2009.12.252. PMID: 20877169.

68. Itrat A, Taqui A, Cerejo R, et al. Telemedicine in prehospital stroke evaluation and thrombolysis: taking stroke treatment to the doorstep. JAMA Neurol. 2016 Feb;73(2):162-8. doi: 10.1001/jamaneurol.2015.3849. PMID: 26641366.

69. Izquierdo R, Morin PC, Bratt K, et al. School-centered telemedicine for children with type 1 diabetes mellitus. J. Pediatr. 2009 Sep;155(3):374-9. doi: 10.1016/j.jpeds.2009.03.014. PMID: 19464030.

70. Jue JS, Spector SA, Spector SA. Telemedicine broadening access to care for complex cases. J. Surg. Res. 2017 Dec;220:164-70. doi: 10.1016/j.jss.2017.06.085. PMID: 29180178.

71. Kahn JM, Le TQ, Barnato AE, et al. ICU telemedicine and critical care mortality: a national effectiveness study. Med. Care. 2016 Mar;54(3):319-25. doi: 10.1097/MLR.0000000000000485. PMID: 26765148.

72. Kim YK, Kim KY, Lee KH, et al. Clinical outcomes on real-time telemetry system in developing emergency medical service system. Telemed J. E Health. 2011 May;17(4):247-53. doi: 10.1089/tmj.2010.0152. PMID: 21480786.

73. Kohl BA, Fortino-Mullen M, Praestgaard A, et al. The effect of ICU telemedicine on mortality and length of stay. J. Telemed. Telecare. 2012;18(5):282-6. doi: 10.1258/jtt.2012.120208. PMID: 22802522. 
74. Krier M, Kaltenbach T, McQuaid K, et al. Potential use of telemedicine to provide outpatient care for inflammatory bowel disease. Am. J. Gastroenterol. 2011 Dec;106(12):2063-7. doi: 10.1038/ajg.2011.329. PMID: 22138934.

75. Krupinski EA, Engstrom M, Barker G, et al. The challenges of following patients and assessing outcomes in teledermatology. J. Telemed. Telecare. 2004;10(1):21-4. doi: 10.1258/135763304322764149. PMID: 15006211.

76. Labarbera JM, Ellenby MS, Bouressa P, et al. The impact of telemedicine intensivist support and a pediatric hospitalist program on a community hospital. Telemed J. E Health. 2013 Oct;19(10):760-6. doi: 10.1089/tmj.2012.0303. PMID: 23937510.

77. Langabeer JR, 2nd, Champagne-Langabeer $\mathrm{T}$, Alqusairi D, et al. Cost-benefit analysis of telehealth in pre-hospital care. J. Telemed. Telecare. 2017 Sep;23(8):747-51. doi: 10.1177/1357633X16680541. PMID: 27913657.

78. Langabeer JR, 2nd, Gonzalez M, Alqusairi $\mathrm{D}$, et al. Telehealth-enabled emergency medical services program reduces ambulance transport to urban emergency departments. West. J. Emerg. Med. 2016 Nov;17(6):713-20. doi: 10.5811/westjem.2016.8.30660. PMID: 27833678.

79. Lee RH, Lyles KW, Pearson M, et al. Osteoporosis screening and treatment among veterans with recent fracture after implementation of an electronic consult service. Calcif. Tissue Int. 2014 Jun;94(6):659-64. doi: 10.1007/s00223-0149849-4. PMID: 24699797.

80. Lilly CM, Cody S, Zhao H, et al. Hospital mortality, length of stay, and preventable complications among critically ill patients before and after tele-ICU reengineering of critical care processes. JAMA. 2011 Jun 01;305(21):2175-83. doi: 10.1001/jama.2011.697. PMID: 21576622.

81. Lilly CM, Motzkus C, Rincon T, et al. ICU Telemedicine Program Financial Outcomes. Chest. 2017 February 01;151(2):286-97. doi: 10.1016/j.chest.2016.11.029. PMID: 27932050.
82. Long MC, Angtuaco T, Lowery C. Ultrasound in telemedicine: its impact in high-risk obstetric health care delivery. Ultrasound Q. 2014 Sep;30(3):167-72. doi: 10.1097/RUQ.0000000000000073. PMID: 25148484.

83. Machado SM, Wilson EH, Elliott JO, et al. Impact of a telemedicine eICU cart on sepsis management in a community hospital emergency department. J. Telemed. Telecare. 2018 Apr;24(3):202-8. doi: 10.1177/1357633X17691862. PMID: 29278979.

84. Marcin JP, Nesbitt TS, Kallas HJ, et al. Use of telemedicine to provide pediatric critical care inpatient consultations to underserved rural Northern California. J. Pediatr. 2004 Mar;144(3):375-80. doi: 10.1016/j.jpeds.2003.12.017. PMID: 15001947.

85. Marcin JP, Nesbitt TS, Struve S, et al. Financial benefits of a pediatric intensive care unit-based telemedicine program to a rural adult intensive care unit: impact of keeping acutely ill and injured children in their local community. Telemed J. E Health. 2004;10 Suppl 2:S-1-5. PMID: 23570207.

86. Marcin JP, Schepps DE, Page KA, et al. The use of telemedicine to provide pediatric critical care consultations to pediatric trauma patients admitted to a remote trauma intensive care unit: a preliminary report. Pediatr. Crit. Care Med. 2004;5(3):251-6. PMID: 15115563.

87. McCambridge M, Jones K, Paxton H, et al. Association of health information technology and teleintensivist coverage with decreased mortality and ventilator use in critically ill patients. Arch. Intern. Med. 2010 Apr 12;170(7):648-53. doi: 10.1001/archinternmed.2010.74. PMID: 20386011.

88. Miyamoto S, Dharmar M, Boyle C, et al. Impact of telemedicine on the quality of forensic sexual abuse examinations in rural communities. Child Abuse Negl. 2014 Sep;38(9):1533-9. doi: 10.1016/j.chiabu.2014.04.015. PMID: 24841062. 
89. Mohr NM, Harland KK, Chrischilles EA, et al. Emergency department telemedicine is used for more severely injured rural trauma patients, but does not decrease transfer: a cohort study. Acad. Emerg. Med.

2017;24(2):177-85. doi:

10.1111/acem.13120. PMID: 28187248.

90. Mohr NM, Vakkalanka JP, Harland KK, et al. Telemedicine use decreases rural emergency department length of stay for transferred North Dakota trauma patients. Telemed J. E Health. 2018 Mar;24(3):194202. doi: 10.1089/tmj.2017.0083. PMID: 28731843.

91. Morrison JL, Cai Q, Davis N, et al. Clinical and economic outcomes of the electronic intensive care unit: results from two community hospitals. Crit. Care Med. 2010 Jan;38(1):2-8. doi: 10.1097/CCM.0b013e3181b78fa8. PMID: 19730249.

92. Mulgrew KW, Shaikh U, Nettiksimmons J. Comparison of parent satisfaction with care for childhood obesity delivered face-to-face and by telemedicine. Telemed J. E Health. 2011 Jun;17(5):383-7. doi: 10.1089/tmj.2010.0153. PMID: 21492028.

93. Narasimhan M, Druss BG, Hockenberry JM, et al. Impact of a telepsychiatry program at emergency departments statewide on the quality, utilization, and costs of mental health services. Psychiatr. Serv. 2015 Nov;66(11):1167-72. doi:

10.1176/appi.ps.201400122. PMID: 26129992.

94. Nassar BS, Vaughan-Sarrazin MS, Jiang L, et al. Impact of an intensive care unit telemedicine program on patient outcomes in an integrated health care system. JAMA Intern Med. 2014 Jul;174(7):1160-7. doi: 10.1001/jamainternmed.2014.1503. PMID: 24819673.

95. Natafgi N, Shane DM, Ullrich F, et al. Using tele-emergency to avoid patient transfers in rural emergency departments: An assessment of costs and benefits. J. Telemed. Telecare. 2017;24(3):193-201. doi: 10.1177/1357633X17696585. PMID: 29278984.
96. Nguyen-Huynh MN, Klingman JG, Avins AL, et al. Novel telestroke program improves thrombolysis for acute stroke across 21 hospitals of an integrated healthcare system. Stroke. 2018 01;49(1):133-9. doi: 10.1161/STROKEAHA.117.018413. PMID: 29247142.

97. Paik AM, Granick MS, Scott S. Plastic surgery telehealth consultation expedites emergency department treatment. J. Telemed. Telecare. 2017 Feb;23(2):321-7. doi: 10.1177/1357633X16639459. PMID: 27056907.

98. Pak H, Triplett CA, Lindquist JH, et al. Store-and-forward teledermatology results in similar clinical outcomes to conventional clinic-based care. J. Telemed. Telecare. 2007;13(1):26-30. doi: 10.1258/135763307779701185. PMID: 17288655.

99. Pak HS, Datta SK, Triplett CA, et al. Cost minimization analysis of a store-andforward teledermatology consult system. Telemed J. E Health. 2009 Mar;15(2):160-5. doi: 10.1089/tmj.2008.0083. PMID: 19292625.

100. Patel S, Hertzog JH, Penfil S, et al. A prospective pilot study of the use of telemedicine during pediatric transport: A high-quality, low-cost alternative to conventional telemedicine systems. Pediatr. Emerg. Care. 2015 Sep;31(9):611-5. doi: 10.1097/PEC.0000000000000544. PMID: 26335229.

101. Pervez MA, Silva G, Masrur S, et al. Remote supervision of IV-tPA for acute ischemic stroke by telemedicine or telephone before transfer to a regional stroke center is feasible and safe. Stroke. 2010 Jan;41(1):e18-24. doi: 10.1161/strokeaha.109.560169. PMID: 19910552.

102. Rendina MC. The effect of telemedicine on neonatal intensive care unit length of stay in very low birthweight infants. Proc. AMIA Symp. 1998:111-5. PMID: 9929192. 
103. Rendina MC, Long WA, deBliek R. Effect size and experimental power analysis in a paediatric cardiology telemedicine system. J. Telemed. Telecare. 1997;3 (Suppl 1):56-7. doi: 10.1258/1357633971930382. PMID: 9218385.

104. Rincon F, Vibbert M, Childs V, et al. Implementation of a model of robotic telepresence (RTP) in the neuro-ICU: effect on critical care nursing team satisfaction. Neurocrit. Care. 2012 Aug;17(1):97-101. doi: 10.1007/s12028-012-9712-2. PMID: 22547040 .

105. Robison J, Slamon NB. A more rapid, rapid response. Pediatr. Crit. Care Med. 2016 Sep;17(9):871-5. doi: 10.1097/PCC.0000000000000855. PMID: 27427880.

106. Romig MC, Latif A, Gill RS, et al. Perceived benefit of a telemedicine consultative service in a highly staffed intensive care unit. J. Crit. Care. 2012 Aug;27(4):426 e9-16. doi: 10.1016/j.jcrc.2011.12.007. PMID: 22421004.

107. Rosenfeld BA, Dorman T, Breslow MJ, et al. Intensive care unit telemedicine: alternate paradigm for providing continuous intensivist care. Crit. Care Med. 2000 Dec;28(12):3925-31. PMID: 11153637.

108. Rossaro L, Torruellas C, Dhaliwal S, et al. Clinical outcomes of hepatitis $\mathrm{C}$ treated with pegylated interferon and ribavirin via telemedicine consultation in Northern California. Dig. Dis. Sci. 2013 Dec;58(12):3620-5. doi: 10.1007/s10620013-2810-y. PMID: 24154637.

109. Ruesch C, Mossakowski J, Forrest J, et al. Using nursing expertise and telemedicine to increase nursing collaboration and improve patient outcomes. Telemed J. E Health. 2012 Oct;18(8):591-5. doi: 10.1089/tmj.2011.0274. PMID: 22957503.

110. Sadaka F, Palagiri A, Trottier S, et al. Telemedicine intervention improves ICU outcomes. Crit. Care Res. Pract. 2013;2013:456389. doi: 10.1155/2013/456389. PMID: 23365729.
111. Saffle JR, Edelman L, Theurer L, et al. Telemedicine evaluation of acute burns is accurate and cost-effective. J. Trauma. 2009 Aug;67(2):358-65. doi: 10.1097/TA.0b013e3181ae9b02. PMID: 19667890.

112. Salami AC, Barden GM, Castillo DL, et al. Establishment of a regional virtual tumor board program to improve the process of care for patients with hepatocellular carcinoma. J. Oncol. Pract. 2015 Jan;11(1):e66-74. doi: 10.1200/JOP.2014.000679. PMID: 25466708.

113. Sanchez-Ross M, Oghlakian G, Maher J, et al. The STAT-MI (ST-Segment Analysis Using Wireless Technology in Acute Myocardial Infarction) trial improves outcomes. JACC Cardiovasc. Interv. 2011 Feb;4(2):222-7. doi: 10.1016/j.jcin.2010.11.007. PMID: 21349462.

114. Sharma S, Parness IA, Kamenir SA, et al. Screening fetal echocardiography by telemedicine: efficacy and community acceptance. J. Am. Soc. Echocardiogr. 2003 Mar;16(3):202-8. doi: 10.1067/mje.2003.46. PMID: 12618726.

115. Sharma P, Kovarik CL, Lipoff JB. Teledermatology as a means to improve access to inpatient dermatology care. J. Telemed. Telecare. 2016 Jul;22(5):304-10. doi: 10.1177/1357633x15603298. PMID: 26377123.

116. Shin DH, Dalag L, Azhar RA, et al. A novel interface for the telementoring of robotic surgery. BJU Int. 2015 Aug;116(2):302-8. doi: 10.1111/bju.12985. PMID: 25381917.

117. Southard EP, Neufeld JD, Laws S. Telemental health evaluations enhance access and efficiency in a critical access hospital emergency department. Telemed J. E Health. 2014 Jul;20(7):664-8. doi: 10.1089/tmj.2013.0257. PMID: 24811858.

118. Specht JK, Wakefield B, Flanagan J. Evaluating the cost of one telehealth application connecting an acute and longterm care setting. J. Gerontol. Nurs. 2001 Jan;27(1):34-9. PMID: 11915095. 
119. Strymish J, Gupte G, Afable MK, et al. Electronic consultations (E-consults): advancing infectious disease care in a large Veterans Affairs healthcare system. Clin. Infect. Dis. 2017 Apr 15;64(8):1123-5. doi: 10.1093/cid/cix058. PMID: 28158475.

120. Switzer JA, Hall C, Gross H, et al. A webbased telestroke system facilitates rapid treatment of acute ischemic stroke patients in rural emergency departments. J. Emerg. Med. 2009;36(1):12-8. doi: 10.1016/j.jemermed.2007.06.041. PMID: 18242925.

121. Taqui A, Cerejo R, Itrat A, et al. Reduction in time to treatment in prehospital telemedicine evaluation and thrombolysis. Neurology. 2017 Apr 04;88(14):1305-12. doi: 10.1212/WNL.0000000000003786. PMID: 28275084.

122. Taylor CR, Merin LM, Salunga AM, et al. Improving diabetic retinopathy screening ratios using telemedicine-based digital retinal imaging technology: the Vine Hill study. Diabetes Care. 2007 Mar;30(3):5748. doi: 10.2337/dc06-1509. PMID: 17327323.

123. Thomas EJ, Lucke JF, Wueste L, et al. Association of telemedicine for remote monitoring of intensive care patients with mortality, complications, and length of stay. JAMA. 2009 Dec 23;302(24):2671-8. doi: 10.1001/jama.2009.1902. PMID: 20040555.

124. Traub SJ, Butler R, Chang YH, et al. Emergency department physician telemedical triage. Telemed J. E Health. 2013 Nov;19(11):841-5. doi: 10.1089/tmj.2013.0026. PMID: 24093916.

125. Webb CL, Waugh CL, Grigsby J, et al. Impact of telemedicine on hospital transport, length of stay, and medical outcomes in infants with suspected heart disease: a multicenter study. J. Am. Soc. Echocardiogr. 2013 Sep;26(9):1090-8. doi: 10.1016/j.echo.2013.05.018. PMID: 23860093.

126. Whited JD, Hall RP, Foy ME, et al. Teledermatology's impact on time to intervention among referrals to a dermatology consult service. Telemed J. E Health. 2002 Fall;8(3):313-21. doi: 10.1089/15305620260353207. PMID: 12419025.
127. Whited JD, Datta S, Hall RP, et al. An economic analysis of a store and forward teledermatology consult system. Telemed J. E Health. 2003 Winter;9(4):351-60. doi: 10.1089/153056203772744671. PMID: 14980092.

128. Whited JD, Hall RP, Foy ME, et al. Patient and clinician satisfaction with a store-andforward teledermatology consult system. Telemed J. E Health. 2004

Winter;10(4):422-31. doi: 10.1089/tmj.2004.10.422. PMID: 15689645.

129. Whited JD, Warshaw EM, Edison KE, et al. Effect of store and forward teledermatology on quality of life: a randomized controlled trial. JAMA Dermatol. 2013

May;149(5):584-91. doi: 10.1001/2013.jamadermatol.380. PMID: 23426111.

130. Whited JD, Warshaw EM, Kapur K, et al. Clinical course outcomes for store and forward teledermatology versus conventional consultation: a randomized trial. J. Telemed. Telecare. 2013 Jun;19(4):197-204. doi: 10.1177/1357633X13487116. PMID: 23666440.

131. Willmitch B, Golembeski S, Kim SS, et al. Clinical outcomes after telemedicine intensive care unit implementation. Crit. Care Med. 2012 Feb;40(2):450-4. doi: 10.1097/CCM.0b013e318232d694. PMID: 22020235.

132. Wilson C, Horton M, Cavallerano J, et al. Addition of primary care-based retinal imaging technology to an existing eye care professional referral program increased the rate of surveillance and treatment of diabetic retinopathy. Diabetes Care. 2005 Feb;28(2):318-22. PMID: 15677786.

133. Yang NH, Dharmar M, Kuppermann N, et al. Appropriateness of disposition following telemedicine consultations in rural emergency departments. Pediatr. Crit. Care Med. 2015 Mar;16(3):e59-64. doi: 10.1097/PCC.0000000000000337. PMID: 25607743. 
134. Young JD, Patel M, Badowski M, et al. Improved virologic suppression with HIV subspecialty care in a large prison system using telemedicine: an observational study with historical controls. Clin. Infect. Dis. 2014 Jul 1;59(1):123-6. doi: 10.1093/cid/ciu222. PMID: 24723283.

135. Zaidi SF, Jumma MA, Urra XN, et al. Telestroke-guided intravenous tissue-type plasminogen activator treatment achieves a similar clinical outcome as thrombolysis at a comprehensive stroke center. Stroke. 2011 Nov;42(11):3291-3. doi: 10.1161/STROKEAHA.111.625046. PMID: 21885843.

136. Astarcioglu MA, Sen T, Kilit C, et al. Timeto-reperfusion in STEMI undergoing interhospital transfer using smartphone and WhatsApp messenger. Am. J. Emerg. Med. 2015 Oct;33(10):1382-4. doi: 10.1016/j.ajem.2015.07.029. PMID: 26299691.

137. Audebert HJ, Schenkel J, Heuschmann PU, et al. Effects of the implementation of a telemedical stroke network: the Telemedic Pilot Project for Integrative Stroke Care (TEMPiS) in Bavaria, Germany. Lancet Neurol. 2006 Sep;5(9):742-8. doi: 10.1016/s1474-4422(06)70527-0. PMID: 16914402.

138. Audebert HJ, Schultes K, Tietz V, et al. Long-term effects of specialized stroke care with telemedicine support in community hospitals on behalf of the Telemedical Project for Integrative Stroke Care (TEMPiS). Stroke. 2009 Mar;40(3):902-8. doi: 10.1161/STROKEAHA.108.529255. PMID: 19023095.

139. Bergrath S, Reich A, Rossaint R, et al. Feasibility of prehospital teleconsultation in acute stroke--a pilot study in clinical routine. PLoS One. 2012;7(5):e36796. doi: 10.1371/journal.pone.0036796. PMID: 22629331.

140. Boman K, Olofsson M, Berggren P, et al. Robot-assisted remote echocardiographic examination and teleconsultation: a randomized comparison of time to diagnosis with standard of care referral approach. JACC Cardiovasc. Imaging. 2014 Aug;7(8):799-803. doi: 10.1016/j.jcmg.2014.05.006. PMID: 25124011.
141. Brokmann JC, Conrad C, Rossaint R, et al. Treatment of acute coronary syndrome by telemedically supported paramedics compared with physician-based treatment: a prospective, interventional, multicenter trial. J. Med. Internet Res. 2016 Dec 01;18(12):e314. doi: 10.2196/jmir.6358. PMID: 27908843.

142. Brokmann JC, Rossaint R, Hirsch F, et al. Analgesia by telemedically supported paramedics compared with physicianadministered analgesia: A prospective, interventional, multicentre trial. Eur. J. Pain. 2016 Aug;20(7):1176-84. doi: 10.1002/ejp.843. PMID: 26914284.

143. Brunetti ND, Di Pietro G, Aquilino A, et al. Pre-hospital electrocardiogram triage with tele-cardiology support is associated with shorter time-to-balloon and higher rates of timely reperfusion even in rural areas: data from the Bari- Barletta/Andria/Trani public emergency medical service 118 registry on primary angioplasty in ST-elevation myocardial infarction. Europ Heart J Acute Cardiovasc Care. 2014 Sep;3(3):204-13. doi: 10.1177/2048872614527009. PMID: 24604713.

144. Carallo C, Scavelli FB, Cipolla M, et al. Management of type 2 diabetes mellitus through telemedicine. PLoS One. 2015;10(5):e0126858. doi: 10.1371/journal.pone.0126858. PMID: 25974092.

145. De Luca N, Izzo R, Iaccarino G, et al. The use of a telematic connection for the followup of hypertensive patients improves the cardiovascular prognosis. J. Hypertens. 2005;23(7):1417-23. PMID: 15942466.

146. Eminovic N, de Keizer NF, Wyatt JC, et al. Teledermatologic consultation and reduction in referrals to dermatologists: a cluster randomized controlled trial. Arch. Dermatol. 2009 May;145(5):558-64. doi: 10.1001/archdermatol.2009.44. PMID: 19451500.

147. Eminovic N, Dijkgraaf MG, Berghout RM, et al. A cost minimisation analysis in teledermatology: model-based approach. BMC Health Serv. Res. 2010;10:251. doi: 10.1186/1472-6963-10-251. PMID: 20738871. 
148. Ferrandiz L, Moreno-Ramirez D, NietoGarcia A, et al. Teledermatology-based presurgical management for nonmelanoma skin cancer: a pilot study. Dermatol. Surg. 2007 Sep;33(9):1092-8. doi: 10.1111/j.15244725.2007.33223.x. PMID: 17760600.

149. Fuertes-Guiro F, Vitali-Erion E, RodriguezFranco A. A program of telementoring in laparoscopic bariatric surgery. Minim. Invasive Ther. Allied Technol. 2016;25(1):8-14. doi: 10.3109/13645706.2015.1083446. PMID: 26360307.

150. Handschu R, Scibor M, Willaczek B, et al. Telemedicine in acute stroke: remote videoexamination compared to simple telephone consultation. J. Neurol. 2008 Nov;255(11):1792-7. doi: 10.1007/s00415008-0066-9. PMID: 19156491.

151. Harno K, Arajarvi E, Paavola T, et al. Clinical effectiveness and cost analysis of patient referral by videoconferencing in orthopaedics. J. Telemed. Telecare. 2001;7(4):219-25. doi: 10.1258/1357633011936435. PMID: 11506757.

152. Harno K, Paavola T, Carlson C, et al. Patient referral by telemedicine: effectiveness and cost analysis of an Intranet system. J. Telemed. Telecare. 2000;6(6):320-9. doi: 10.1258/1357633001935996. PMID: 11265100 .

153. Haukipuro K, Ohinmaa A, Winblad I, et al. The feasibility of telemedicine for orthopaedic outpatient clinics--a randomized controlled trial. J. Telemed. Telecare. 2000;6(4):193-8. doi: 10.1258/1357633001935347. PMID: 11027118

154. Herce J, Lozano R, Salazar CI, et al. Management of impacted third molars based on telemedicine: a pilot study. J. Oral Maxillofac. Surg. 2011 Feb;69(2):471-5. doi: 10.1016/j.joms.2010.09.004. PMID: 21129834.
155. Hubert GJ, Meretoja A, Audebert HJ, et al. Stroke thrombolysis in a centralized and a decentralized system (Helsinki and Telemedical Project for Integrative Stroke Care Network). Stroke. 2016 Dec;47(12):2999-3004. doi: 10.1161/STROKEAHA.116.014258. PMID: 27834751.

156. Ickenstein GW, Horn M, Schenkel J, et al. The use of telemedicine in combination with a new stroke-code-box significantly increases t-PA use in rural communities. Neurocrit. Care. 2005 August 01;3(1):27-32. doi: 10.1385/ncc:3:1:027. PMID: 16159092.

157. Jaatinen PT, Aarnio P, Remes J, et al. Teleconsultation as a replacement for referral to an outpatient clinic. J. Telemed. Telecare. 2002;8(2):102-6. doi: 10.1258/1357633021937550. PMID: 11972945.

158. Jacobs JJ, Jacobs JP, van Sonderen E, et al. Fracture diagnostics, unnecessary travel and treatment: a comparative study before and after the introduction of teleradiology in a remote general practice. BMC Fam. Pract. 2015 May 06;16:53. doi: 10.1186/s12875015-0268-z. PMID: 25943473.

159. Johansson T, Mutzenbach SJ, Ladurner G. Telemedicine in acute stroke care: the TESSA model. J. Telemed. Telecare. 2011;17(5):268-72. doi: 10.1258/jtt.2011.101213. PMID: 21824968.

160. Lamminen H, Lamminen J, Ruohonen K, et al. A cost study of teleconsultation for primary-care ophthalmology and dermatology. J. Telemed. Telecare. 2001;7(3):167-73. doi: 10.1258/1357633011936336. PMID: 11346477.

161. Martinez-Sanchez P, Miralles A, Sanz de Barros R, et al. The effect of telestroke systems among neighboring hospitals: more and better? The Madrid Telestroke Project. J. Neurol. 2014 Sep;261(9):1768-73. doi: 10.1007/s00415-014-7419-3. PMID: 24957298. 
162. Martinoni A, Servi SD, Boschetti E, et al. Importance and limits of pre-hospital electrocardiogram in patients with ST elevation myocardial infarction undergoing percutaneous coronary angioplasty. Eur. J. Cardiovasc. Prev. Rehabil. 2011 June 01;18(3):526-32. doi: 10.1177/1741826710389395. PMID: 21450642.

163. Mazighi M, Meseguer E, Labreuche J, et al. TRUST-tPA trial: Telemedicine for remote collaboration with urgentists for stroke-tPA treatment. J. Telemed. Telecare. 2017 Jan;23(1):174-80. doi: 10.1177/1357633X15615762. PMID: 26656722.

164. Mielonen ML, Ohinmaa A, Moring J, et al. Psychiatric inpatient care planning via telemedicine. J. Telemed. Telecare. 2000;6(3):152-7. doi: 10.1258/1357633001935248. PMID: 10912333.

165. Migliaretti G, Ciaramitaro P, Berchialla P, et al. Teleconsulting for minor head injury: the Piedmont experience. J. Telemed. Telecare. 2013 Jan;19(1):33-5. doi:

10.1177/1357633X12474738. PMID: 23454822.

166. Moreno-Ramirez D, Ferrandiz L, Ruiz-deCasas A, et al. Economic evaluation of a store-and-forward teledermatology system for skin cancer patients. J. Telemed. Telecare. 2009;15(1):40-5. doi: 10.1258/jtt.2008.080901. PMID: 19139219.

167. Nickenig H, Wichmann M, Schlegel A, et al. Use of telemedicine for pre-implant dental assessment -- a comparative study. J. Telemed. Telecare. 2008;14(2):93-7. doi: 10.1258/jtt.2007.070806. PMID: 18348756.

168. Nikkanen T, Timonen M, Ylitalo K, et al. Quality of diabetes care among patients managed by teleconsultation. J. Telemed. Telecare. 2008;14(6):295-9. doi: 10.1258/jtt.2008.080313. PMID: 18776074.

169. Nordal EJ, Moseng D, Kvammen B, et al. A comparative study of teleconsultations versus face-to-face consultations. $\mathrm{J}$. Telemed. Telecare. 2001;7(5):257-65. doi: 10.1258/1357633011936507. PMID: 11571079 .
170. Ohinmaa A, Vuolio S, Haukipuro K, et al. A cost-minimization analysis of orthopaedic consultations using videoconferencing in comparison with conventional consulting. J. Telemed. Telecare. 2002;8(5):283-9. doi: 10.1177/1357633X0200800507. PMID: 12396857.

171. Ortolani P, Marzocchi A, Marrozzini C, et al. Usefulness of prehospital triage in patients with cardiogenic shock complicating ST-elevation myocardial infarction treated with primary percutaneous coronary intervention. Am. J. Cardiol. 2007 Sep 01;100(5):787-92. doi: 10.1016/j.amjcard.2007.03.099. PMID: 17719321.

172. Ortolani P, Marzocchi A, Marrozzini C, et al. Clinical impact of direct referral to primary percutaneous coronary intervention following pre-hospital diagnosis of STelevation myocardial infarction. Eur. Heart J. 2006;27(13):1550-7. doi: 10.1093/eurheartj/ehl006. PMID: 16707549.

173. Pedersen SH, Galatius S, Hansen PR, et al. Field triage reduces treatment delay and improves long-term clinical outcome in patients with acute ST-segment elevation myocardial infarction treated with primary percutaneous coronary intervention. J. Am. Coll. Cardiol. 2009;54(24):2296-302. doi: 10.1016/j.jacc.2009.06.056. PMID: 19958965.

174. Pedragosa A, Alvarez-Sabin J, Molina CA, et al. Impact of a telemedicine system on acute stroke care in a community hospital. J. Telemed. Telecare. 2009;15(5):260-3. doi: 10.1258/jtt.2009.090102. PMID: 19590033.

175. Piette E, Nougairede M, Vuong V, et al. Impact of a store-and-forward teledermatology intervention versus usual care on delay before beginning treatment: A pragmatic cluster-randomized trial in ambulatory care. J. Telemed. Telecare. 2017 Sep;23(8):725-32. doi: 10.1177/1357633X16663328. PMID: 27496852.

176. Sairanen T, Soinila S, Nikkanen M, et al. Two years of Finnish Telestroke: thrombolysis at spokes equal to that at the hub. Neurology. 2011 Mar 29;76(13):114552. doi: 10.1212/WNL.0b013e318212a8d4. PMID: 21368283. 
177. Salazar-Fernandez CI, Herce J, GarciaPalma A, et al. Telemedicine as an effective tool for the management of temporomandibular joint disorders. J. Oral Maxillofac. Surg. 2012;70(2):295-301. doi: 10.1016/j.joms.2011.03.053. PMID: 21803470.

178. Schwab S, Vatankhah B, Kukla C, et al. Long-term outcome after thrombolysis in telemedical stroke care. Neurology. 2007 Aug 28;69(9):898-903. doi: 10.1212/01.wnl.0000269671.08423.14. PMID: 17724293.

179. Sejersten M, Sillesen M, Hansen PR, et al. Effect on treatment delay of prehospital teletransmission of 12-lead electrocardiogram to a cardiologist for immediate triage and direct referral of patients with ST-segment elevation acute myocardial infarction to primary percutaneous coronary intervention. Am. J. Cardiol. 2008;101(7):941-6. doi: 10.1016/j.amjcard.2007.11.038. PMID: 18359312

180. Smith-Strom H, Igland J, Ostbye T, et al. The effect of telemedicine follow-up care on diabetes-related foot ulcers: a clusterrandomized controlled non inferiority trial. Diabetes Care. 2018;41(1):96-103. doi: 10.2337/dc17-1025. PMID: 29187423.

181. Stalfors J, Bjorholt I, Westin T. A cost analysis of participation via personal attendance versus telemedicine at a head and neck oncology multidisciplinary team meeting. J. Telemed. Telecare. 2005;11(4):205-10. doi: 10.1258/1357633054068892. PMID: 16007751.

182. Stalfors J, Holm-Sjogren L, Schwieler A, et al. Satisfaction with telemedicine presentation at a multidisciplinary tumour meeting among patients with head and neck cancer. J. Telemed. Telecare. 2003;9(3):1505. doi: 10.1258/135763303767149951. PMID: 12882214.

183. Theiss S, Gunzel F, Storm A, et al. Using routine data for quality assessment in NeuroNet telestroke care. J. Stroke Cerebrovasc. Dis. 2013 Oct;22(7):984-90. doi: 10.1016/j.jstrokecerebrovasdis.2012.01.012. PMID: 22365711.
184. Tsitlakidis C, Mylonakis J, Niakas D. Economic evaluation of telemedicine for a remotely located population: the case of two Greek islands. Int J Electron Healthc. 2005;1(3):243-60. doi: 10.1504/IJEH.2005.006473. PMID: 18048208.

185. Tuulonen A, Ohinmaa T, Alanko HI, et al. The application of teleophthalmology in examining patients with glaucoma: a pilot study. J. Glaucoma. 1999 Dec;8(6):367-73. PMID: 10604295.

186. van Gelder VA, Scherpbier-de Haan ND, van Berkel S, et al. Web-based consultation between general practitioners and nephrologists: a cluster randomized controlled trial. Fam. Pract. 2017;34(4):4306. doi: 10.1093/fampra/cmw131. PMID: 28158576.

187. Vuolio S, Winblad I, Ohinmaa A, et al. Videoconferencing for orthopaedic outpatients: one-year follow-up. J. Telemed. Telecare. 2003;9(1):8-11. doi: 10.1258/135763303321159620. PMID: 12641886.

188. Zahlmann G, Mertz M, Fabian E, et al. Perioperative cataract OP management by means of teleconsultation. Graefes Arch. Clin. Exp. Ophthalmol. 2002 Jan;240(1):1720. PMID: 11954775.

189. Zanini R, Aroldi M, Bonatti S, et al. Impact of prehospital diagnosis in the management of ST elevation myocardial infarction in the era of primary percutaneous coronary intervention: reduction of treatment delay and mortality. J. Cardiovasc. Med. 2008;9(6):570-5. doi: 10.2459/JCM.0b013e3282f2c9bd. PMID: 18475124.

190. Zarchi K, Haugaard VB, Dufour DN, et al. Expert advice provided through telemedicine improves healing of chronic wounds: prospective cluster controlled study. J. Invest. Dermatol. 2015 Mar;135(3):895-900. doi: 10.1038/jid.2014.441. PMID: 25290685. 
191. Zennaro F, Grosso D, Fascetta R, et al. Teleradiology for remote consultation using iPad improves the use of health system human resources for paediatric fractures: prospective controlled study in a tertiary care hospital in Italy. BMC Health Serv. Res. 2014 Jul 28;14:327. doi: 10.1186/14726963-14-327. PMID: 25070705.

192. Bowns I, Collins K, Walters S, et al. Telemedicine in dermatology: a randomized controlled trial. Health Technol. Assess. 2006;10(43):3-25. PMID: 17049140.

193. Chowdhury M, Birns J, Rudd A, et al. Telemedicine versus face-to-face evaluation in the delivery of thrombolysis for acute ischaemic stroke: a single centre experience. Postgrad. Med. J. 2012 Mar;88(1037):134-7. doi: 10.1136/postgradmedj-2011-130060. PMID: 22267526.

194. Chua R, Craig J, Wootton R, et al. Cost implications of outpatient teleneurology. J. Telemed. Telecare. 2001;7 Suppl 1:62-4. doi: 10.1177/1357633x010070s126. PMID: 11576496.

195. Collins K, Walters S, Bowns I. Patient satisfaction with teledermatology: quantitative and qualitative results from a randomized controlled trial. J. Telemed. Telecare. 2004;10(1):29-33. doi: 10.1258/135763304322764167. PMID: 15006213.

196. Craig J, Chua R, Russell C, et al. A cohort study of early neurological consultation by telemedicine on the care of neurological inpatients. J. Neurol. Neurosurg. Psychiatry. 2004 Jul;75(7):1031-5. PMID: 15201365.

197. Darkins A, Dearden CH, Rocke LG, et al. An evaluation of telemedical support for a minor treatment centre. J. Telemed.

Telecare. 1996;2(2):93-9. doi: 10.1177/1357633X9600200205. PMID: 9375069.

198. Davison AG, Eraut CD, Haque AS, et al. Telemedicine for multidisciplinary lung cancer meetings. J. Telemed. Telecare. 2004;10(3):140-3. doi: 10.1258/135763304323070779. PMID: 15237512 .
199. Gilmour E, Campbell SM, Loane MA, et al. Comparison of teleconsultations and face-toface consultations: preliminary results of a United Kingdom multicentre teledermatology study. Br. J. Dermatol. 1998 Jul;139(1):81-7. PMID: 9764153.

200. Kunkler IH, Prescott RJ, Lee RJ, et al. TELEMAM: a cluster randomised trial to assess the use of telemedicine in multidisciplinary breast cancer decision making. Eur. J. Cancer. 2007 Nov;43(17):2506-14. doi: 10.1016/j.ejca.2007.08.026. PMID: 17962011.

201. Loane MA, Bloomer SE, Corbett R, et al. Patient cost-benefit analysis of teledermatology measured in a randomized control trial. J. Telemed. Telecare. 1999;5 Suppl 1:S1-3. PMID: 10534821.

202. Loane MA, Bloomer SE, Corbett R, et al. A randomized controlled trial to assess the clinical effectiveness of both realtime and store-and-forward teledermatology compared with conventional care. J. Telemed. Telecare. 2000;6 Suppl 1:S1-3. PMID: 10793956.

203. McCrossan BA, Sands AJ, Kileen T, et al. A fetal telecardiology service: patient preference and socio-economic factors. Prenat. Diagn. 2012 Sep;32(9):883-7. doi: 10.1002/pd.3926. PMID: 22718083.

204. Noble SM, Coast J, Benger JR. A costconsequences analysis of minor injuries telemedicine. J. Telemed. Telecare. 2005;11(1):15-9. doi: 10.1177/1357633X0501100104. PMID: 15829038.

205. Scuffham PA, Steed M. An economic evaluation of the Highlands and Islands teledentistry project. J. Telemed. Telecare. 2002;8(3):165-77. doi: 10.1177/1357633X0200800307. PMID: 12097178.

206. van der Pol M, McKenzie L. Costs and benefits of tele-endoscopy clinics in a remote location. J. Telemed. Telecare. 2010;16(2):89-94. doi: 10.1258/jtt.2009.090609. PMID: 20139140. 
207. Wallace P, Haines A, Harrison R, et al. Joint teleconsultations (virtual outreach) versus standard outpatient appointments for patients referred by their general practitioner for a specialist opinion: a randomised trial. Lancet. 2002 Jun 08;359(9322):1961-8. PMID: 12076550.

208. Wallace P, Barber J, Clayton W, et al. Virtual outreach: a randomised controlled trial and economic evaluation of joint teleconferenced medical consultations. Health Technol. Assess. 2004 Dec;8(50):1106, iii-iv. PMID: 15546515.

209. Wallace DL, Jones SM, Milroy C, et al. Telemedicine for acute plastic surgical trauma and burns. J. Plast. Reconstr. Aesthet. Surg. 2008;61(1):31-6. doi: 10.1016/j.bjps.2006.03.045. PMID: 18068653.

210. Basudev N, Crosby-Nwaobi R, Thomas S, et al. A prospective randomized controlled study of a virtual clinic integrating primary and specialist care for patients with Type 2 diabetes mellitus. Diabet. Med. 2016 Jun;33(6):768-76. doi: 10.1111/dme.12985. PMID: 27194175.

211. Dowie R, Mistry H, Young TA, et al. Telemedicine in pediatric and perinatal cardiology: economic evaluation of a service in English hospitals. Int. J. Technol. Assess. Health Care. 2007 Winter;23(1):116-25. doi: 10.1017/s0266462307051653. PMID: 17234025.

212. Jacklin PB, Roberts JA, Wallace P, et al. Virtual outreach: economic evaluation of joint teleconsultations for patients referred by their general practitioner for a specialist opinion. BMJ. 2003 Jul 12;327(7406):84. doi: 10.1136/bmj.327.7406.84. PMID: 12855528.

213. Mahendran R, Goodfield MJ, Sheehan-Dare RA. An evaluation of the role of a store-andforward teledermatology system in skin cancer diagnosis and management. Clin. Exp. Dermatol. 2005 May;30(3):209-14. doi: 10.1111/j.1365-2230.2005.01735.x. PMID: 15807671.
214. Buckley D, Weisser S. Videoconferencing could reduce the number of mental health patients transferred from outlying facilities to a regional mental health unit. Aust. N. Z. J. Public Health. 2012 Oct;36(5):478-82. doi: 10.1111/j.1753-6405.2012.00915.x. PMID: 23025371.

215. Blackwell NA, Kelly GJ, Lenton LM. Telemedicine ophthalmology consultation in remote Queensland. Med. J. Aust. 1997 Dec 1-15;167(11-12):583-6. PMID: 9418795.

216. Bladin CF, Molocijz N, Ermel S, et al. Victorian Stroke Telemedicine Project: implementation of a new model of translational stroke care for Australia. Intern. Med. J. 2015 Sep;45(9):951-6. doi: 10.1111/imj.12822. PMID: 26011155.

217. Burns CL, Ward EC, Hill AJ, et al. Randomized controlled trial of a multisite speech pathology telepractice service providing swallowing and communication intervention to patients with head and neck cancer: Evaluation of service outcomes. Head Neck. 2017 May;39(5):932-9. doi: 10.1002/hed.24706 PMID: 28225567.

218. Chan AW, Kornder J, Elliott H, et al. Improved survival associated with prehospital triage strategy in a large regional ST-segment elevation myocardial infarction program. JACC Cardiovasc. Interv. 2012;5(12):1239-46. doi: 10.1016/j.jcin.2012.07.013. PMID: 23257372.

219. Crossland L, Askew D, Ware R, et al. Diabetic retinopathy screening and monitoring of early stage disease in Australian general practice: tackling preventable blindness within a chronic care model. J Diabetes Res. 2016;2016:1-7. doi: 10.1155/2016/8405395. PMID: 26798655.

220. Gattas MR, MacMillan JC, Meinecke I, et al. Telemedicine and clinical genetics: establishing a successful service. J. Telemed. Telecare. 2001;7 Suppl 2:68-70. doi: 10.1258/1357633011937191. PMID: 11747665.

221. Gray LC, Wright OR, Cutler AJ, et al. Geriatric ward rounds by video conference: a solution for rural hospitals. Med. J. Aust. 2009 Dec 7-21;191(11-12):605-8. PMID: 20028277. 
222. Lim D, Oakley AM, Rademaker M. Better, sooner, more convenient: a successful teledermoscopy service. Australas. J. Dermatol. 2012 Feb;53(1):22-5. doi: 10.1111/j.1440-0960.2011.00836.x. PMID: 22309326.

223. Loane MA, Oakley A, Rademaker M, et al. A cost-minimization analysis of the societal costs of realtime teledermatology compared with conventional care: results from a randomized controlled trial in New Zealand. J. Telemed. Telecare. 2001;7(4):233-8. doi: 10.1258/1357633011936453. PMID: 11506759.

224. Martin-Khan MG, Edwards H, Wootton R, et al. Web-based (online) comprehensive geriatric assessment is more time efficient, and as reliable, as reading patient medical records and conducting traditional in person consultations. J. Telemed. Telecare. 2016 Dec;22(8):478-82. doi: 10.1177/1357633X16674088. PMID: 27799451.

225. Mathews KA, Elcock MS, Furyk JS. The use of telemedicine to aid in assessing patients prior to aeromedical retrieval to a tertiary referral centre. J. Telemed. Telecare. 2008;14(6):309-14. doi: 10.1258/jtt.2008.080417. PMID: 18776077.

226. Nagao KJ, Koschel A, Haines HM, et al. Rural Victorian Telestroke project. Intern. Med. J. 2012 Oct;42(10):1088-95. doi: 10.1111/j.1445-5994.2011.02603.x. PMID: 21981424.

227. Panlaqui OM, Broadfield E, Champion R, et al. Outcomes of telemedicine intervention in a regional intensive care unit: a before and after study. Anaesth. Intensive Care. 2017;45(5):605-10. PMID: 28911290.

228. Santamaria N, Carville K, Ellis I, et al. The effectiveness of digital imaging and remote expert wound consultation on healing rates in chronic lower leg ulcers in the Kimberley region of Western Australia. Primary Intention: The Australian Journal of Wound Management. 2004;12(2):62-70.

229. Smith AC, Williams M, Van der Westhuyzen J, et al. A comparison of telepaediatric activity at two regional hospitals in Queensland. J. Telemed. Telecare. 2002;8 Suppl 3:S3:58-62. PMID: 12661625.
230. Thaker DA, Monypenny R, Olver I, et al. Cost savings from a telemedicine model of care in northern Queensland, Australia. Med. J. Aust. 2013 Sep 16;199(6):414-7. PMID: 24033216.

231. Xu CQ, Smith AC, Scuffham PA, et al. A cost minimisation analysis of a telepaediatric otolaryngology service. BMC Health Serv. Res. 2008 Feb 04;8:30. doi: 10.1186/1472-6963-8-30. PMID: 18241356.

232. Zilliacus EM, Meiser B, Lobb EA, et al. Are videoconferenced consultations as effective as face-to-face consultations for hereditary breast and ovarian cancer genetic counseling? Genet. Med. 2011 Nov;13(11):933-41. doi: 10.1097/GIM.0b013e3182217a19. PMID: 21799430.

233. Byamba K, Syed-Abdul S, Garcia-Romero $\mathrm{M}$, et al. Mobile teledermatology for a prompter and more efficient dermatological care in rural Mongolia. Br. J. Dermatol. 2015 Jul;173(1):265-7. doi: 10.1111/bjd.13607. PMID: 25494968.

234. Chan BA, Larkins SL, Evans R, et al. Do teleoncology models of care enable safe delivery of chemotherapy in rural towns? Med. J. Aust. 2015 Nov 16;203(10):4066.e6. PMID: 26561905.

235. Cho J, Chung HS, Choa M, et al. A pilot study of the Tele-Airway Management System in a hospital emergency department. J. Telemed. Telecare. 2011;17(1):49-53. doi: 10.1258/jtt.2010.100202. PMID: 21097567.

236. Dharmasaroja PA, Muengtaweepongsa S, Kommarkg U. Implementation of telemedicine and stroke network in thrombolytic administration: comparison between walk-in and referred patients. Neurocrit. Care. 2010 Aug;13(1):62-6. doi: 10.1007/s12028-010-9360-3. PMID: 20411354.

237. Engel H, Huang JJ, Tsao CK, et al. Remote real-time monitoring of free flaps via smartphone photography and $3 \mathrm{G}$ wireless Internet: a prospective study evidencing diagnostic accuracy. Microsurgery. 2011 Nov;31(8):589-95. doi: 10.1002/micr.20921. PMID: 22072583. 
238. Fong WC, Ismail M, Lo JW, et al. Telephone and teleradiology-guided thrombolysis can achieve similar outcome as thrombolysis by neurologist on-site. J. Stroke Cerebrovasc. Dis. 2015 Jun;24(6):1223-8. doi: 10.1016/j.jstrokecerebrovasdis.2015.01.022. PMID: 25906936.

239. Goh KY, Tsang KY, Poon WS. Does teleradiology improve inter-hospital management of head-injury? Can. J. Neurol. Sci. 1997 Aug;24(3):235-9. PMID: 9276110.

240. Gupta S, Dewan S, Kaushal A, et al. eICU reduces mortality in STEMI patients in resource-limited areas. Glob Heart. 2014 Dec;9(4):425-7. doi: 10.1016/j.gheart.2014.07.006. PMID: 25592796.

241. Hashimoto S, Shirato H, Kaneko K, et al. Clinical efficacy of telemedicine in emergency radiotherapy for malignant spinal cord compression. J. Digit. Imaging. 2001 Sep;14(3):124-30. PMID: 11720334.

242. Kim EW, Teague-Ross TJ, Greenfield WW, et al. Telemedicine collaboration improves perinatal regionalization and lowers statewide infant mortality. J. Perinatol. 2013 Sep;33(9):725-30. doi: 10.1038/jp.2013.37. PMID: 23579490.

243. Klein Y, Donchik V, Jaffe D, et al. Management of patients with traumatic intracranial injury in hospitals without neurosurgical service. J. Trauma. 2010 Sep;69(3):544-8. doi: 10.1097/TA.0b013e3181c99936. PMID: 20234328.

244. Nagayoshi Y, Oshima S, Ogawa H. Clinical impact of telemedicine network system at rural hospitals without on-site cardiac surgery backup. Telemed J. E Health. 2016 Nov;22(11):960-4. doi: 10.1089/tmj.2015.0225. PMID: 27148833.

245. Poon WS, Leung CH, Lam MK, et al. The comparative impact of video-consultation on neurosurgical health services. Int. J. Med. Inform. 2001 Jul;62(2-3):175-80. PMID: 11470620.
246. Tsai SH, Kraus J, Wu HR, et al. The effectiveness of video-telemedicine for screening of patients requesting emergency air medical transport (EAMT). J. Trauma. 2007 Feb;62(2):504-11. doi: 10.1097/01.ta.0000219285.08974.45. PMID: 17297342.

247. Wong HT, Poon WS, Jacobs P, et al. The comparative impact of video consultation on emergency neurosurgical referrals. Neurosurgery. 2006 Sep;59(3):607-13; discussion -13. doi: 10.1227/01.NEU.0000228926.13395.F9. PMID: 16955042.

248. Bernstein K, Zacharias J, Blanchard JF, et al. Model for equitable care and outcomes for remote full care hemodialysis units. Clin. J. Am. Soc. Nephrol. 2010 Apr;5(4):645-51. doi: 10.2215/CJN.04550709. PMID: 20185604.

249. Chan WM, Woo J, Hui E, et al. The role of telenursing in the provision of geriatric outreach services to residential homes in Hong Kong. J. Telemed. Telecare. 2001;7(1):38-46. doi: 10.1258/1357633011936129. PMID: 11265937.

250. Jong M, Kraishi M. A comparative study on the utility of telehealth in the provision of rheumatology services to rural and northern communities. Int. J. Circumpolar Health. 2004 Dec;63(4):415-21. PMID: 15709316.

251. Stern A, Mitsakakis N, Paulden M, et al. Pressure ulcer multidisciplinary teams via telemedicine: a pragmatic cluster randomized stepped wedge trial in long term care. BMC Health Serv. Res. 2014;14:83. doi: 10.1186/1472-6963-14-83. PMID: 24559218.

252. Steinman M, Morbeck RA, Pires PV, et al. Impact of telemedicine in hospital culture and its consequences on quality of care and safety. Einstein (Sao Paulo). 2015 OctDec;13(4):580-6. doi: 10.1590/S167945082015GS2893. PMID: 26676268.

253. Macedo TA, de Barros ESPG, Simoes SA, et al. Impact of chest pain protocol with access to telemedicine on implementation of pharmacoinvasive strategy in a private hospital network. Telemed J. E Health. 2016 Jul;22(7):549-52. doi: 10.1089/tmj.2015.0178. PMID: 26693879. 
254. Marcolino MS, Brant LC, Araujo JG, et al. Implementation of the myocardial infarction system of care in city of Belo Horizonte, Brazil.[Erratum appears in Arq Bras Cardiol. 2013 Apr;100(4):313]. Arq. Bras. Cardiol. 2013 Apr;100(4):307-14. PMID: 23545995.

255. Meyer BC, Raman R, Hemmen T, et al. Efficacy of site-independent telemedicine in the STRokE DOC trial: a randomised, blinded, prospective study. Lancet Neurol. 2008 Sep;7(9):787-95. doi: 10.1016/S14744422(08)70171-6. PMID: 18676180.

256. Kobza L, Scheurich A. The impact of telemedicine on outcomes of chronic wounds in the home care setting. Ostomy Wound Manage. 2000 Oct;46(10):48-53. PMID: 11889733.

257. Kalb T, Raikhelkar J, Meyer S, et al. A multicenter population-based effectiveness study of teleintensive care unit-directed ventilator rounds demonstrating improved adherence to a protective lung strategy, decreased ventilator duration, and decreased intensive care unit mortality. J. Crit. Care. 2014 Aug;29(4):691.e7-14. doi: 10.1016/j.jcrc.2014.02.017. PMID: 24636928.

258. Bagayoko CO, Traore D, Thevoz L, et al. Medical and economic benefits of telehealth in low- and middle-income countries: results of a study in four district hospitals in Mali. BMC Health Serv. Res. 2014;14 Suppl 1:S9. doi: 10.1186/1472-6963-14-S1-S9. PMID: 25080312.

259. Flodgren G, Rachas A, Farmer AJ, et al. Interactive telemedicine: effects on professional practice and health care outcomes. Cochrane Database of Systematic Reviews. 2015;9:CD002098. doi: http://dx.doi.org/10.1002/14651858.CD0020 98.pub2. PMID: 26343551.

260. Marino R, Ghanim A. Teledentistry: a systematic review of the literature. J. Telemed. Telecare. 2013 Jun;19(4):179-83. doi: 10.1177/1357633X13479704. PMID: 23512650.
261. Hilty DM, Marks SL, Urness D, et al. Clinical and educational telepsychiatry applications: a review. Can. J. Psychiatry. 2004 Jan;49(1):12-23. doi: 10.1177/070674370404900103. PMID: 14763673. 


\section{Abbreviations}

AHRQ

AIRR

AMI

AOR

APACHE

AUD

CAD

CCRCT

CI

CINAHL

DBP

DiD

DM

ED

EEG

ENT

EMS

EPC

FIM

GBP

GOS

GP

$\mathrm{HCV}$

HgbA1C

HR

ICU

IQR

IV tPA

$\mathrm{K}$

KQ

LDL

LOS

MCS

MD

MI

mRS

NA
Agency for Healthcare Research and Quality

adjusted incidence ratio

acute myocardial infarction

adjusted odds ratio

Acute Physiology and Chronic Health Evaluation

Australian dollars

Canadian dollars

Cochrane Central Register of Controlled Trials

confidence interval

Cumulative Index to Nursing and Allied Health Literature

diastolic blood pressure

difference in difference

decision model

emergency department

electroencephalogram

ear, nose, and throat

emergency medical services

Evidence-based Practice Center

Finnish markka

British Pound

Glasgow Outcome Scale

general practitioner

hepatitis $\mathrm{C}$ virus

hemoglobin A1c

hazard ratio

intensive care unit

interquartile range

intravenous tissue plasminogen activator

Kappa

Key Question

low-density lipoprotein cholesterol

length of stay

Mental Component Summary

medical doctor

myocardial infarction

Modified Rankin Sale

not applicable 
NHS

NICU

NIHSS

NQF

NR

NS

OR

PCI

PCS

PCU

PICOTS

PICU

PRISMA

PTSD

QWB

RCT

RR

SAPS

SBP

SD

SEK

SEM

SF-12

SOE

SR

STEMI

TEP

TIMI

TMJ

tPA

UAMS

UK

USD

VA
National Health Services

neonatal intensive care unit

National Institutes of Health Stroke Scale

National Quality Forum

not reported

not significant

odds ratio

percutaneous coronary intervention

Physical Component Summary

progressive care unit

population, intervention, comparator, outcomes, timing, and setting

pediatric intensive care unit

Preferred Reporting Items for Systematic Reviews and Meta-Analyses

posttraumatic stress disorder

quality of well-being score

randomized controlled trial

risk ratio

Simplified Acute Physiology Score

systolic blood pressure

standard deviation

Swedish Krona

standard error of the mean

Short Form-12

strength of evidence

systematic review

ST-elevation myocardial infarction

Technical Expert Panel

thrombolysis in myocardial infarction

temporomandibular joint disorder

tissue plasminogen activator

University of Arkansas for Medical Sciences

United Kingdom

United States Dollars

Veterans Affairs 


\section{Appendix A. Literature Search Strategies}

\section{Search strategies - Telehealth consultation}

\section{Database: Ovid MEDLINE(R) without Revisions 1996 to May Week 42018}

Systematic reviews

1 exp Telemedicine/

2 Mobile Applications/

3 telemedicine journal \& e health.jn.

4 "journal of telemedicine \& telecare".jn.

5 or $/ 1-4$

6 limit 5 to (meta analysis or systematic reviews)

7 meta-analysis.pt.

8 meta-analysis/ or systematic review/ or meta-analysis as topic/ or "meta analysis (topic)"/ or "systematic review (topic)"/ or exp technology assessment, biomedical/

9 ((systematic* adj3 (review* or overview*)) or (methodologic* adj3 (review* or overview*))).ti,ab.

10 ((quantitative adj3 (review* or overview* or synthes*)) or (research adj3 (integrati* or overview*))).ti,ab.

11 ((integrative adj3 (review* or overview*)) or (collaborative adj3 (review* or overview*)) or (pool* adj3 analy*)).ti,ab.

12 (data synthes* or data extraction* or data abstraction*).ti,ab.

13 (handsearch* or hand search*).ti,ab.

14 (mantel haenszel or peto or der simonian or dersimonian or fixed effect* or latin square*).ti,ab.

15 (met analy* or metanaly* or technology assessment* or HTA or HTAs or technology overview* or technology appraisal*).ti,ab.

16 (meta regression* or metaregression*).ti,ab.

17 (meta-analy* or metaanaly* or systematic review* or biomedical technology assessment* or bio-medical technology assessment*).mp,hw.

18 (medline or cochrane or pubmed or medlars or embase or cinahl).ti,ab,hw.

19 (cochrane or (health adj2 technology assessment) or evidence report).jw.

20 (meta-analysis or systematic review).ti,ab.

21 (comparative adj3 (efficacy or effectiveness)).ti,ab.

22 (outcomes research or relative effectiveness).ti,ab.

23 ((indirect or indirect treatment or mixed-treatment) adj comparison*).ti,ab.

24 or/7-23

$25 \quad 5$ and 24

$26 \quad 6$ or 25

27 limit 26 to $\mathrm{yr}=" 2018$ - current"

Randomized controlled trials and controlled observational studies - Broad search strategy

1 exp Telemedicine/

2 Mobile Applications/

3 telemedicine journal \& e health.jn. 
4 "journal of telemedicine \& telecare".jn.

5 or $1-4$

6 limit 5 to (clinical trial, all or comparative study or controlled clinical trial or pragmatic

clinical trial or randomized controlled trial)

75 and (random* or control* or cohort).ti,ab.

86 or 7

9 limit 8 to $y r=" 2018$ - current"

All study designs - Narrow search strategy

1 exp Telemedicine/

2 Mobile Applications/

3 telemedicine journal \& e health.jn.

4 "journal of telemedicine \& telecare".jn.

5 exp Remote Consultation/

6 consult*.mp.

7 (or/1-4) and (5 or 6)

8 limit 7 to $\mathrm{yr}=" 1996$ - 2018"

9 limit 8 to (meta analysis or systematic reviews)

10 meta-analysis.pt.

11 meta-analysis/ or systematic review/ or meta-analysis as topic/ or "meta analysis (topic)"/ or "systematic review (topic)"/ or exp technology assessment, biomedical/

12 ((systematic* adj3 (review* or overview*)) or (methodologic* adj3 (review* or overview*))).ti,ab.

13 ((quantitative adj3 (review* or overview* or synthes*)) or (research adj3 (integrati* or overview*))).ti,ab.

14 ((integrative adj3 (review* or overview*)) or (collaborative adj3 (review* or overview*)) or (pool* adj3 analy*)).ti,ab.

15 (data synthes* or data extraction* or data abstraction*).ti,ab.

16 (handsearch* or hand search*).ti,ab.

17 (mantel haenszel or peto or der simonian or dersimonian or fixed effect* or latin square*).ti,ab.

18 (met analy* or metanaly* or technology assessment* or HTA or HTAs or technology overview* or technology appraisal*).ti,ab.

19 (meta regression* or metaregression*).ti,ab.

20 (meta-analy* or metaanaly* or systematic review* or biomedical technology assessment* or bio-medical technology assessment*).mp,hw.

21 (medline or cochrane or pubmed or medlars or embase or cinahl).ti,ab,hw.

22 (cochrane or (health adj2 technology assessment) or evidence report).jw.

23 (meta-analysis or systematic review).ti,ab.

24 (comparative adj3 (efficacy or effectiveness)).ti,ab.

25 (outcomes research or relative effectiveness).ti,ab.

26 ((indirect or indirect treatment or mixed-treatment) adj comparison*).ti,ab.

27 or $/ 10-26$

$28 \quad 8$ and 27

$29 \quad 9$ or 28

$30 \quad 8$ not 29 
31 limit 30 to (english language and humans)

$32 \quad 7$ and 27

$33 \quad 7$ not 32

34 limit 33 to $\mathrm{yr}=" 2018$ - current"

Database: EBM Reviews - Cochrane Central Register of Controlled Trials May 2018

1 exp Telemedicine/

2 (telemedicine or telehealth or teleconsult*).mp.

31 or 2

43 and (random* or control* or cohort).ti,ab.

5 limit 4 to english language

Database: EBM Reviews - Cochrane Database of Systematic Reviews 2005 to May 31, 2018

1 (telemedicine or telehealth or teleconsult*).mp.

2 limit 1 to new reviews

Database - CINAHL Plus with Full Text

S1 (MM “Telemedicine +")

S2 consult*

S3 S1 AND S2 


\title{
Appendix B. Inclusion and Exclusion Criteria
}

\author{
Full-Text Paper Inclusion/Exclusion Codes: Reasons for full text paper inclusion or exclusion
}

\section{Inclusion}

$1=$ Include in the report

\section{Exclusion}

$\mathbf{2}=$ Background or discussion paper only

$\mathbf{3}=$ Ineligible population (nonhuman, patients without known or suspected acute or chronic conditions)

4 = Ineligible intervention (not a consultation; example: direct patient care, educational/training sessions)

$\mathbf{5}=$ Ineligible comparison or no comparison (descriptive)

$\mathbf{6}$ = Ineligible outcome; does not have an included outcome (e.g., feasibility only, no outcome/just description)

$7=$ Ineligible timing (studies with outcome measures that occur before telehealth consultation)

$\mathbf{8}=$ Ineligible setting (exclude nonhealth care and training/education setting unless there is a consultation)

9 = Ineligible study design (nonsystematic reviews, evaluation of hypothetical uses or needs assessments)

$\mathbf{1 0}=$ Ineligible publication type (opinion, editorial, letter, guideline document not used for background)

$11=$ Wrong years (studies published before 1996)

$12=$ Not in English

$13=$ No new data

$14=$ Systematic review used to identify primary studies

15 = Modeling study, no original data

Table B-1. Inclusion and exclusion criteria

\begin{tabular}{|c|c|c|}
\hline PICOTS & Include & Exclude \\
\hline Populations & $\begin{array}{l}\text { Patients of any age, with medical care } \\
\text { needs for prevention, treatment or } \\
\text { management of chronic or acute } \\
\text { condition. } \\
\text { Providers (clinicians or health care } \\
\text { organizations). Dentistry may be added } \\
\text { only if it fits our criteria for consultation. } \\
\text { Payers for health care services (public, } \\
\text { private, insurers, or patients). }\end{array}$ & $\begin{array}{l}\text { Consultations about people, non-health care } \\
\text { services (e.g., housing or social services). }\end{array}$ \\
\hline Interventions & $\begin{array}{l}\text { Telehealth consultations, defined as the } \\
\text { use of telehealth designed to facilitate } \\
\text { collaboration among providers, often } \\
\text { involving a specialist, or assessment } \\
\text { between clinical team members, across } \\
\text { time and/or distance, on the assessment, } \\
\text { diagnosis, and/or clinical management of } \\
\text { a specific patient or group of patients. } \\
\text { Telehealth consultations can be for any } \\
\text { acute or chronic conditions. The search } \\
\text { will be both general as well as focused on } \\
\text { conditions identified as infectious } \\
\text { disease, dermatology and critical care. } \\
\text { Telehealth consultations can use any } \\
\text { technology (e.g., real-time video, store } \\
\text { and forward). }\end{array}$ & $\begin{array}{l}\text { Provision of direct, ongoing patient care (telehealth } \\
\text { for patient visits) } \\
\text { Strictly educational/training sessions that do not } \\
\text { have a consultation about a specific patient or } \\
\text { patients. } \\
\text { Simple referral for ongoing management with no } \\
\text { collaboration. } \\
\text { Telephone-only consultations do not constitute } \\
\text { telehealth. }\end{array}$ \\
\hline Comparators & $\begin{array}{l}\text { Other locations, patients, or timeperiods } \\
\text { that use in-person consultations or } \\
\text { provide usual care (which could include } \\
\text { no access to specific services). }\end{array}$ & $\begin{array}{l}\text { Comparisons of in-person and telehealth } \\
\text { assessments by the same provider } \\
\text { No comparison/simple reporting of events or } \\
\text { characteristics }\end{array}$ \\
\hline
\end{tabular}




\begin{tabular}{|c|c|c|}
\hline PICOTS & Include & Exclude \\
\hline $\begin{array}{l}\text { Outcomes } \\
\text { Clinical } \\
\text { KQ1 }\end{array}$ & $\begin{array}{l}\text { Clinical outcomes such as mortality, } \\
\text { morbidity, function, recovery and access } \\
\text { to services (e.g., improved medical } \\
\text { condition, access to telepsychiatry } \\
\text { evaluation with psychiatrist in primary } \\
\text { care office) }\end{array}$ & $\begin{array}{l}\text { Descriptive statistics without comparison. } \\
\text { For example, the number of telehealth encounters, } \\
\text { the conditions treated or the actions taken if these } \\
\text { are not compared somehow (across groups, time } \\
\text { periods or raters) }\end{array}$ \\
\hline $\begin{array}{l}\text { Outcomes } \\
\text { Economic } \\
\text { KQ1 }\end{array}$ & $\begin{array}{l}\text { Economic outcomes such as return on } \\
\text { investment, cost, volume of visits, and } \\
\text { resource use }\end{array}$ & \\
\hline $\begin{array}{l}\text { Outcomes } \\
\text { Intermediate } \\
\text { KQ2 }\end{array}$ & $\begin{array}{l}\text { Patient satisfaction, behavior, and } \\
\text { decisions such as completion of } \\
\text { treatment, or satisfaction with less travel } \\
\text { to access health care } \\
\text { Provider satisfaction, behavior, and } \\
\text { decisions such as the choice of treatment } \\
\text { or antibiotic stewardship Improved time to } \\
\text { diagnosis and treatment }\end{array}$ & $\begin{array}{l}\text { Studies looking only at diagnostic concordance or } \\
\text { accuracy or other measures of agreement between } \\
\text { in-person and telehealth consultations }\end{array}$ \\
\hline $\begin{array}{l}\text { Adverse } \\
\text { Effects or } \\
\text { Unintended } \\
\text { Consequences } \\
\text { KQ3 }\end{array}$ & $\begin{array}{l}\text { Loss of privacy or breech of data security } \\
\text { Misdiagnosis or delayed diagnosis } \\
\text { Inappropriate treatment } \\
\text { Increase in resource costs, negative } \\
\text { return on investment }\end{array}$ & \\
\hline Timing & $\begin{array}{l}\text { Telehealth consultations can be used at } \\
\text { any point in the diagnosis, treatment, or } \\
\text { management of patient. } \\
\text { Outcomes measurement needs to occur } \\
\text { after the telehealth consultation }\end{array}$ & $\begin{array}{l}\text { Studies with outcome measures that occur before } \\
\text { the telehealth consultation. }\end{array}$ \\
\hline Setting & $\begin{array}{l}\text { The consultation can involve providers } \\
\text { and patients in any location. These could } \\
\text { include inpatient, outpatient, long-term } \\
\text { care and could be in civilian, VA, or } \\
\text { military facilities. }\end{array}$ & $\begin{array}{l}\text { Not in a training/educational setting (unless there } \\
\text { truly is consultation between providers) } \\
\text { Settings that are not health care related }\end{array}$ \\
\hline $\begin{array}{l}\text { Study Design } \\
\text { and } \\
\text { Publication } \\
\text { Type }\end{array}$ & $\begin{array}{l}\text { Comparative studies, including trials and } \\
\text { observational studies (cohorts or pre/post } \\
\text { historical controls may be considered). } \\
\text { Descriptive studies may be used to inform } \\
\text { the DM as needed but will not be used to } \\
\text { inform the SR. }\end{array}$ & $\begin{array}{l}\text { Nonsystematic reviews, commentaries, or letters. } \\
\text { Evaluations of hypothetical situations (e.g., would } \\
\text { teleconsultation be used if it was available?) } \\
\text { Feasibility assessments (if it was possible to set it } \\
\text { up) } \\
\text { Survey studies are included only if they are about } \\
\text { things that actually happened (no hypothetical } \\
\text { survey studies) }\end{array}$ \\
\hline Years & 1996 to present & 1995 and earlier \\
\hline Language & English & Non-English \\
\hline
\end{tabular}

\section{Key Questions for the Systematic Review}

1. Are telehealth consultations effective in improving clinical and economic outcomes? Clinical and economic outcomes may include, but are not limited to, mortality and morbidity, utilization of health services, cost of services, and access to services.

2. Are telehealth consultations effective in improving intermediate outcomes? Intermediate outcomes include both outcomes that precede the ultimate outcomes of interest and secondary outcomes. These may include, but are not limited to, patient and provider satisfaction, behavior, and decisions; volume of services; and health care process. 
3. Have telehealth consultations resulted in harms, adverse events, or negative unintended consequences?

4. What are the characteristics of telehealth consultations that have been the subject of comparative studies?

The characteristics include:

a. Clinical conditions addressed

b. Characteristics of the providers and patients involved

c. Relationships among the providers and patients involved

d. Telehealth modalities and/or methods for sharing patient data used

e. Whether specifics in (d) meet Medicare's coverage and HIPAA requirements

f. Settings such as type of health care organization, country, or urban or rural area

h. Other circumstances (e.g., appropriate transportation, climate)

g. Payment models or requirements or limits for payment

5. Do clinical, economic, intermediate, or negative outcomes (i.e., the outcomes in KQs 1, 2, and 3) vary across telehealth consultation characteristics (KQ4)?

\section{Outcomes for Each Key Question}

- KQ1: Clinical and economic outcomes

o Clinical outcomes such as mortality, morbidity, function, recovery, infection, and access to services.

o Economic outcomes such as return on investment, cost, volume of visits, and resource use.

- KQ 2: Intermediate outcomes

o Patient satisfaction, behavior, and decisions

o Provider satisfaction, behavior, and decisions

0 Time to diagnosis and time to treatment

- KQ 3: Adverse effects or unintended consequences

o Loss of privacy or breech of data security

o Misdiagnosis or delayed diagnosis

0 Inappropriate treatment

- KQ 4: Not applicable (this is a descriptive question)

- KQ5: Clinical and economic outcomes (see KQ1), intermediate outcomes (see KQ2), and adverse effects or unintended consequences (see KQ3)

\section{Timing}

- Telehealth consultations can be used at any point in the diagnosis, treatment, or management of a patient.

- Outcome measurement needs to occur after the telehealth consultation.

\section{Setting}

- The consultation can involve providers and patients in any location.

\section{Study Designs}

- Comparative studies, including trials and observational studies. 
- Descriptive studies may be used to inform the decision model as needed but will not be included in the systematic review. 


\section{Appendix C. Included Studies}

1. Alemi AS, Seth R, Heaton C, et al. Comparison of video and in-person free flap assessment following head and neck free tissue transfer. Otolaryngol Head Neck Sur. 2017 Jun;156(6):1035-40. doi: 10.1177/0194599816688620. PMID: 28140835.

2. Amorim E, Shih MM, Koehler SA, et al. Impact of telemedicine implementation in thrombolytic use for acute ischemic stroke: the University of Pittsburgh Medical Center telestroke network experience. J. Stroke Cerebrovasc. Dis. 2013 May;22(4):527-31. doi:

10.1016/j.jstrokecerebrovasdis.2013.02.004. PMID: 23489955.

3. Angstman KB, Rohrer JE, Adamson SC, et al. Impact of e-consults on return visits of primary care patients. Health Care Manag (Frederick). 2009 Jul-Sep;28(3):253-7. doi: 10.1097/HCM.0b013e3181b3efa3. PMID: 19668067.

4. Armaignac DL, Saxena A, Rubens M, et al. Impact of telemedicine on mortality, length of stay, and cost among patients in progressive care units: experience from a large healthcare system*. Crit. Care Med. 2018 04/13;46(5):728-35. doi: 10.1097/CCM.0000000000002994. PMID: 29384782.

5. Arora S, Thornton K, Murata G, et al. Outcomes of treatment for hepatitis $\mathrm{C}$ virus infection by primary care providers. N. Engl. J. Med. 2011 Jun 9;364(23):2199-207. doi: 10.1056/NEJMoa1009370. PMID: 21631316.

6. Astarcioglu MA, Sen T, Kilit C, et al. Timeto-reperfusion in STEMI undergoing interhospital transfer using smartphone and WhatsApp messenger. Am. J. Emerg. Med. 2015 Oct;33(10):1382-4. doi: 10.1016/j.ajem.2015.07.029. PMID: 26299691.
7. Audebert HJ, Schenkel J, Heuschmann PU, et al. Effects of the implementation of a telemedical stroke network: the Telemedic Pilot Project for Integrative Stroke Care (TEMPiS) in Bavaria, Germany. Lancet Neurol. 2006 Sep;5(9):742-8. doi: 10.1016/s1474-4422(06)70527-0. PMID: 16914402.

8. Audebert HJ, Schultes K, Tietz V, et al. Long-term effects of specialized stroke care with telemedicine support in community hospitals on behalf of the Telemedical Project for Integrative Stroke Care (TEMPiS). Stroke. 2009 Mar;40(3):902-8. doi: 10.1161/STROKEAHA.108.529255. PMID: 19023095.

9. Bagayoko CO, Traore D, Thevoz L, et al. Medical and economic benefits of telehealth in low- and middle-income countries: results of a study in four district hospitals in Mali. BMC Health Serv. Res. 2014;14 Suppl 1:S9. doi: 10.1186/1472-6963-14-S1-S9. PMID: 25080312.

10. Baig MM, Antonescu-Turcu A, Ratarasarn K. Impact of Sleep Telemedicine Protocol in Management of Sleep Apnea: A 5-Year VA Experience. Telemed J. E Health. 2016 May;22(5):458-62. doi: 10.1089/tmj.2015.0047. PMID: 26974884.

11. Basudev N, Crosby-Nwaobi R, Thomas S, et al. A prospective randomized controlled study of a virtual clinic integrating primary and specialist care for patients with Type 2 diabetes mellitus. Diabet. Med. 2016 Jun;33(6):768-76. doi: 10.1111/dme.12985. PMID: 27194175.

12. Bergrath S, Reich A, Rossaint R, et al. Feasibility of prehospital teleconsultation in acute stroke--a pilot study in clinical routine. PLoS One. 2012;7(5):e36796. doi: 10.1371/journal.pone.0036796. PMID: 22629331.

13. Bernstein K, Zacharias J, Blanchard JF, et al. Model for equitable care and outcomes for remote full care hemodialysis units. Clin. J. Am. Soc. Nephrol. 2010 Apr;5(4):645-51. doi: 10.2215/CJN.04550709. PMID: 20185604. 
14. Beswick DM, Vashi A, Song Y, et al. Consultation via telemedicine and access to operative care for patients with head and neck cancer in a Veterans Health Administration population. Head Neck. 2016 Jun;38(6):925-9. doi: 10.1002/hed.24386. PMID: 26899939.

15. Bezalel S, Fabri P, Park HS. Implementation of store-and-forward teledermatology and its associated effect on patient access in a Veterans Affairs dermatology clinic. JAMA Dermatol. 2015 May;151(5):556-7. doi: 10.1001/jamadermatol.2014.5272. PMID: 25671336 .

16. Blackwell NA, Kelly GJ, Lenton LM. Telemedicine ophthalmology consultation in remote Queensland. Med. J. Aust. 1997 Dec 1-15;167(11-12):583-6. PMID: 9418795.

17. Bladin CF, Molocijz N, Ermel S, et al. Victorian Stroke Telemedicine Project: implementation of a new model of translational stroke care for Australia. Intern. Med. J. 2015 Sep;45(9):951-6. doi: 10.1111/imj.12822. PMID: 26011155.

18. Boman K, Olofsson M, Berggren P, et al. Robot-assisted remote echocardiographic examination and teleconsultation: a randomized comparison of time to diagnosis with standard of care referral approach.

JACC Cardiovasc. Imaging. 2014

Aug;7(8):799-803. doi:

10.1016/j.jcmg.2014.05.006. PMID: 25124011.

19. Bowns I, Collins K, Walters S, et al. Telemedicine in dermatology: a randomized controlled trial. Health Technol. Assess. 2006;10(43):3-25. PMID: 17049140.

20. Brennan JA, Kealy JA, Gerardi LH, et al. A randomized controlled trial of telemedicine in an emergency department. J. Telemed. Telecare. 1998;4(1):18-20. doi: 10.1258/1357633981931911. PMID: 9640721 .

21. Brennan JA, Kealy JA, Gerardi LH, et al. Telemedicine in the emergency department: a randomized controlled trial. J. Telemed. Telecare. 1999;5(1):18-22. doi: 10.1258/1357633991932342. PMID: 10505365.
22. Breslow MJ, Rosenfeld BA, Doerfler M, et al. Effect of a multiple-site intensive care unit telemedicine program on clinical and economic outcomes: an alternative paradigm for intensivist staffing. Crit. Care Med. 2004 Jan;32(1):31-8. doi: 10.1097/01.CCM.0000104204.61296.41. PMID: 14707557.

23. Britt DW, Norton JD, Hubanks AS, et al. A two-period assessment of changes in specialist contact in a high-risk pregnancy telemedical program. Telemed J. E Health. 2006 Feb;12(1):35-41. doi: 10.1089/tmj.2006.12.35. PMID: 16478411.

24. Brokmann JC, Conrad C, Rossaint R, et al. Treatment of acute coronary syndrome by telemedically supported paramedics compared with physician-based treatment: a prospective, interventional, multicenter trial. J. Med. Internet Res. 2016 Dec

01;18(12):e314. doi: 10.2196/jmir.6358. PMID: 27908843.

25. Brokmann JC, Rossaint R, Hirsch F, et al. Analgesia by telemedically supported paramedics compared with physicianadministered analgesia: A prospective, interventional, multicentre trial. Eur. J. Pain. 2016 Aug;20(7):1176-84. doi: 10.1002/ejp.843. PMID: 26914284.

26. Brown-Connolly NE. Patient satisfaction with telemedical access to specialty services in rural California. J. Telemed. Telecare. 2002;8 Suppl 2:7-10. doi: 10.1177/1357633X020080S204. PMID: 12217115.

27. Brunetti ND, Di Pietro G, Aquilino A, et al. Pre-hospital electrocardiogram triage with tele-cardiology support is associated with shorter time-to-balloon and higher rates of timely reperfusion even in rural areas: data from the Bari- Barletta/Andria/Trani public emergency medical service 118 registry on primary angioplasty in ST-elevation myocardial infarction. Europ Heart J Acute Cardiovasc Care. 2014 Sep;3(3):204-13. doi: 10.1177/2048872614527009. PMID: 24604713. 
28. Buckley D, Weisser S. Videoconferencing could reduce the number of mental health patients transferred from outlying facilities to a regional mental health unit. Aust. N. Z. J. Public Health. 2012 Oct;36(5):478-82. doi: 10.1111/j.1753-6405.2012.00915.x. PMID: 23025371.

29. Burgess LP, Syms MJ, Holtel MR, et al. Telemedicine: teleproctored endoscopic sinus surgery. Laryngoscope. 2002 Feb;112(2):216-9. doi: 10.1097/00005537200202000-00003. PMID: 11889372.

30. Burns CL, Ward EC, Hill AJ, et al. Randomized controlled trial of a multisite speech pathology telepractice service providing swallowing and communication intervention to patients with head and neck cancer: Evaluation of service outcomes. Head Neck. 2017 May;39(5):932-9. doi: 10.1002/hed.24706 PMID: 28225567.

31. Byamba K, Syed-Abdul S, Garcia-Romero $\mathrm{M}$, et al. Mobile teledermatology for a prompter and more efficient dermatological care in rural Mongolia. Br. J. Dermatol. 2015 Jul;173(1):265-7. doi:

10.1111/bjd.13607. PMID: 25494968.

32. Carallo C, Scavelli FB, Cipolla M, et al. Management of type 2 diabetes mellitus through telemedicine. PLoS One.

2015;10(5):e0126858. doi:

10.1371/journal.pone.0126858. PMID: 25974092.

33. Carter ZA, Goldman S, Anderson K, et al. Creation of an internal teledermatology store-and-forward system in an existing electronic health record: a pilot study in a safety-net public health and hospital system. JAMA Dermatol. 2017 Jul 01;153(7):64450. doi: 10.1001/jamadermatol.2017.0204 PMID: 28423156.

34. Chan AW, Kornder J, Elliott H, et al. Improved survival associated with prehospital triage strategy in a large regional ST-segment elevation myocardial infarction program. JACC Cardiovasc. Interv. 2012;5(12):1239-46. doi: 10.1016/j.jcin.2012.07.013. PMID: 23257372.
35. Chan BA, Larkins SL, Evans R, et al. Do teleoncology models of care enable safe delivery of chemotherapy in rural towns? Med. J. Aust. 2015 Nov 16;203(10):4066.e6. PMID: 26561905.

36. Chan WM, Woo J, Hui E, et al. The role of telenursing in the provision of geriatric outreach services to residential homes in Hong Kong. J. Telemed. Telecare. 2001;7(1):38-46. doi: 10.1258/1357633011936129. PMID: 11265937.

37. Cho J, Chung HS, Choa M, et al. A pilot study of the Tele-Airway Management System in a hospital emergency department. J. Telemed. Telecare. 2011;17(1):49-53. doi: 10.1258/jtt.2010.100202. PMID: 21097567.

38. Choi JY, Porche NA, Albright KC, et al. Using telemedicine to facilitate thrombolytic therapy for patients with acute stroke. Jt Comm J Qual Patient Saf. 2006 Apr;32(4):199-205. PMID: 16649650.

39. Chowdhury M, Birns J, Rudd A, et al. Telemedicine versus face-to-face evaluation in the delivery of thrombolysis for acute ischaemic stroke: a single centre experience. Postgrad. Med. J. 2012 Mar;88(1037):134-7. doi: 10.1136/postgradmedj-2011-130060.

PMID: 22267526.

40. Chu S, Boxer R, Madison P, et al. Veterans Affairs telemedicine: bringing urologic care to remote clinics. Urology. 2015

Aug;86(2):255-60. doi: 10.1016/j.urology.2015.04.038. PMID: 26168998.

41. Chua R, Craig J, Wootton R, et al. Cost implications of outpatient teleneurology. J. Telemed. Telecare. 2001;7 Suppl 1:62-4. doi: 10.1177/1357633x010070s126. PMID: 11576496.

42. Chu-Weininger MY, Wueste L, Lucke JF, et al. The impact of a tele-ICU on provider attitudes about teamwork and safety climate. Qual Saf Health Care. 2010 Dec;19(6):e39. doi: 10.1136/qshc.2007.024992. PMID: 20427298. 
43. Collins K, Walters S, Bowns I. Patient satisfaction with teledermatology: quantitative and qualitative results from a randomized controlled trial. J. Telemed. Telecare. 2004;10(1):29-33. doi: 10.1258/135763304322764167. PMID: 15006213.

44. Collins TA, Robertson MP, Sicoutris CP, et al. Telemedicine coverage for post-operative ICU patients. J. Telemed. Telecare. 2017

Feb;23(2):360-4. doi: 10.1177/1357633X16631846. PMID: 27365321.

45. Craig J, Chua R, Russell C, et al. A cohort study of early neurological consultation by telemedicine on the care of neurological inpatients. J. Neurol. Neurosurg. Psychiatry. 2004 Jul;75(7):1031-5. PMID: 15201365.

46. Crossland L, Askew D, Ware R, et al. Diabetic retinopathy screening and monitoring of early stage disease in Australian general practice: tackling preventable blindness within a chronic care model. J Diabetes Res. 2016;2016:1-7. doi: 10.1155/2016/8405395. PMID: 26798655.

47. Darkins A, Dearden CH, Rocke LG, et al. An evaluation of telemedical support for a minor treatment centre. J. Telemed. Telecare. 1996;2(2):93-9. doi: 10.1177/1357633X9600200205. PMID: 9375069.

48. Datta SK, Warshaw EM, Edison KE, et al. Cost and utility analysis of a store-andforward teledermatology referral system: A randomized clinical trial. JAMA Dermatol. 2015;151(12):1323-9. doi:

10.1001/jamadermatol.2015.2362. PMID: 26375589.

49. Davis TD, Deen T, Bryant-Bedell K, et al. Does minority racial-ethnic status moderate outcomes of collaborative care for depression? Psychiatr. Serv. 2011 Nov;62(11):1282-8. doi: 10.1176/appi.ps.62.11.1282. PMID: 22211206.

50. Davison AG, Eraut CD, Haque AS, et al. Telemedicine for multidisciplinary lung cancer meetings. J. Telemed. Telecare. 2004;10(3):140-3. doi: 10.1258/135763304323070779. PMID: 15237512 .
51. Dayal P, Hojman NM, Kissee JL, et al. Impact of telemedicine on severity of illness and outcomes among children transferred from referring emergency departments to a children's hospital PICU. Pediatr. Crit. Care Med. 2016 Jun;17(6):516-21. doi: 10.1097/PCC.0000000000000761. PMID: 27099972.

52. De Luca N, Izzo R, Iaccarino G, et al. The use of a telematic connection for the followup of hypertensive patients improves the cardiovascular prognosis. J. Hypertens. 2005;23(7):1417-23. PMID: 15942466.

53. Demaerschalk BM, Bobrow BJ, Raman R, et al. Stroke team remote evaluation using a digital observation camera in Arizona: the initial mayo clinic experience trial. Stroke. 2010 Jun;41(6):1251-8. doi: 10.1161/STROKEAHA.109.574509. PMID: 20431081.

54. Dharmar M, Kuppermann N, Romano PS, et al. Telemedicine consultations and medication errors in rural emergency departments. Pediatrics. 2013

Dec;132(6):1090-7. doi: 10.1542/peds.20131374. PMID: 24276844.

55. Dharmar M, Romano PS, Kuppermann N, et al. Impact of critical care telemedicine consultations on children in rural emergency departments. Crit. Care Med. 2013 Oct;41(10):2388-95. doi: 10.1097/CCM.0b013e31828e9824. PMID: 23921273.

56. Dharmar M, Sadorra CK, Leigh $\mathrm{P}$, et al. The financial impact of a pediatric telemedicine program: a children's hospital's perspective. Telemed J. E Health. 2013 Jul;19(7):502-8. doi: 10.1089/tmj.2012.0266. PMID: 23837516.

57. Dharmasaroja PA, Muengtaweepongsa S, Kommarkg U. Implementation of telemedicine and stroke network in thrombolytic administration: comparison between walk-in and referred patients. Neurocrit. Care. 2010 Aug;13(1):62-6. doi: 10.1007/s12028-010-9360-3. PMID: 20411354.

58. Doolittle GC, Harmon A, Williams A, et al. A cost analysis of a tele-oncology practice. J. Telemed. Telecare. 1997;3 Suppl 1:20-2. doi: 10.1258/1357633971930850. PMID: 9218371. 
59. Dowie R, Mistry H, Young TA, et al. Telemedicine in pediatric and perinatal cardiology: economic evaluation of a service in English hospitals. Int. J. Technol. Assess. Health Care. 2007 Winter;23(1):116-25. doi: 10.1017/s0266462307051653. PMID: 17234025.

60. Duchesne JC, Kyle A, Simmons J, et al. Impact of telemedicine upon rural trauma care. J. Trauma. 2008 Jan;64(1):92-7; discussion 7-8. doi: 10.1097/TA.0b013e31815dd4c4. PMID: 18188104.

61. Eminovic N, de Keizer NF, Wyatt JC, et al. Teledermatologic consultation and reduction in referrals to dermatologists: a cluster randomized controlled trial. Arch. Dermatol. 2009 May;145(5):558-64. doi:

10.1001/archdermatol.2009.44. PMID: 19451500.

62. Eminovic N, Dijkgraaf MG, Berghout RM, et al. A cost minimisation analysis in teledermatology: model-based approach. BMC Health Serv. Res. 2010;10:251. doi: 10.1186/1472-6963-10-251. PMID: 20738871.

63. Endean ED, Mallon LI, Minion DJ, et al. Telemedicine in vascular surgery: does it work? Am. Surg. 2001 Apr;67(4):334-40; discussion 40-1. PMID: 11307999.

64. Engel H, Huang JJ, Tsao CK, et al. Remote real-time monitoring of free flaps via smartphone photography and 3G wireless Internet: a prospective study evidencing diagnostic accuracy. Microsurgery. 2011 Nov;31(8):589-95. doi: 10.1002/micr.20921. PMID: 22072583.

65. Ferrandiz L, Moreno-Ramirez D, NietoGarcia A, et al. Teledermatology-based presurgical management for nonmelanoma skin cancer: a pilot study. Dermatol. Surg. 2007 Sep;33(9):1092-8. doi: 10.1111/j.15244725.2007.33223.x. PMID: 17760600.

66. Fong WC, Ismail M, Lo JW, et al. Telephone and teleradiology-guided thrombolysis can achieve similar outcome as thrombolysis by neurologist on-site. J. Stroke Cerebrovasc. Dis. 2015 Jun;24(6):1223-8. doi: 10.1016/j.jstrokecerebrovasdis.2015.01.022. PMID: 25906936.
67. Fortis S, Weinert C, Bushinski R, et al. A health system-based critical care program with a novel tele-ICU: implementation, cost, and structure details. J. Am. Coll. Surg. 2014 Oct;219(4):676-83. doi: 10.1016/j.jamcollsurg.2014.04.015. PMID: 25154668.

68. Fortney JC, Maciejewski ML, Tripathi SP, et al. A budget impact analysis of telemedicine-based collaborative care for depression. Med. Care. 2011 Sep;49(9):87280. doi: 10.1097/MLR.0b013e31821d2b35. PMID: 21623240.

69. Fortney JC, Pyne JM, Edlund MJ, et al. A randomized trial of telemedicine-based collaborative care for depression. J. Gen. Intern. Med. 2007 Aug;22(8):1086-93. doi: 10.1007/s11606-007-0201-9. PMID: 17492326.

70. Fortney JC, Pyne JM, Kimbrell TA, et al. Telemedicine-based collaborative care for posttraumatic stress disorder: a randomized clinical trial. JAMA Psychiatry. 2015 Jan;72(1):58-67. doi: 10.1001/jamapsychiatry.2014.1575. PMID: 25409287.

71. Fortney JC, Pyne JM, Mouden SB, et al. Practice-based versus telemedicine-based collaborative care for depression in rural federally qualified health centers: a pragmatic randomized comparative effectiveness trial. Am. J. Psychiatry. 2013 Apr;170(4):414-25. doi: 10.1176/appi.ajp.2012.12050696. PMID: 23429924.

72. Fox KC, Somes GW, Waters TM. Timeliness and access to healthcare services via telemedicine for adolescents in state correctional facilities. J. Adolesc. Health. 2007 Aug;41(2):161-7. doi: 10.1016/j.jadohealth.2007.05.001. PMID: 17659220.

73. Frank JW, Carey EP, Fagan KM, et al. Evaluation of a telementoring intervention for pain management in the Veterans Health Administration. Pain Med. 2015 Jun;16(6):1090-100. doi: 10.1111/pme.12715. PMID: 25716075. 
74. Franzini L, Sail KR, Thomas EJ, et al. Costs and cost-effectiveness of a telemedicine intensive care unit program in 6 intensive care units in a large health care system. J. Crit. Care. 2011 Jun;26(3):329.e1-6. doi: 10.1016/j.jcrc.2010.12.004. PMID: 21376515.

75. Fuertes-Guiro F, Vitali-Erion E, RodriguezFranco A. A program of telementoring in laparoscopic bariatric surgery. Minim. Invasive Ther. Allied Technol.

2016;25(1):8-14. doi: 10.3109/13645706.2015.1083446. PMID: 26360307.

76. Gattas MR, MacMillan JC, Meinecke I, et al. Telemedicine and clinical genetics: establishing a successful service. J. Telemed. Telecare. 2001;7 Suppl 2:68-70. doi: 10.1258/1357633011937191. PMID: 11747665.

77. Gilmour E, Campbell SM, Loane MA, et al. Comparison of teleconsultations and face-toface consultations: preliminary results of a United Kingdom multicentre teledermatology study. Br. J. Dermatol. 1998 Jul;139(1):81-7. PMID: 9764153.

78. Goh KY, Tsang KY, Poon WS. Does teleradiology improve inter-hospital management of head-injury? Can. J. Neurol. Sci. 1997 Aug;24(3):235-9. PMID: 9276110.

79. Grabowski DC, O'Malley AJ. Use of telemedicine can reduce hospitalizations of nursing home residents and generate savings for medicare. Health Aff. (Millwood). 2014 Feb;33(2):244-50. doi: 10.1377/hlthaff.2013.0922. PMID: 24493767.

80. Gray LC, Wright OR, Cutler AJ, et al. Geriatric ward rounds by video conference: a solution for rural hospitals. Med. J. Aust. 2009 Dec 7-21;191(11-12):605-8. PMID: 20028277.

81. Gupta S, Dewan S, Kaushal A, et al. eICU reduces mortality in STEMI patients in resource-limited areas. Glob Heart. 2014 Dec;9(4):425-7. doi: 10.1016/j.gheart.2014.07.006. PMID: 25592796.
82. Handschu R, Scibor M, Willaczek B, et al. Telemedicine in acute stroke: remote videoexamination compared to simple telephone consultation. J. Neurol. 2008 Nov;255(11):1792-7. doi: 10.1007/s00415008-0066-9. PMID: 19156491.

83. Harno K, Arajarvi E, Paavola T, et al. Clinical effectiveness and cost analysis of patient referral by videoconferencing in orthopaedics. J. Telemed. Telecare. 2001;7(4):219-25. doi: 10.1258/1357633011936435. PMID: 11506757.

84. Harno K, Paavola T, Carlson C, et al. Patient referral by telemedicine: effectiveness and cost analysis of an Intranet system. J. Telemed. Telecare. 2000;6(6):320-9. doi: 10.1258/1357633001935996. PMID: 11265100 .

85. Hashimoto S, Shirato H, Kaneko K, et al. Clinical efficacy of telemedicine in emergency radiotherapy for malignant spinal cord compression. J. Digit. Imaging. 2001 Sep;14(3):124-30. PMID: 11720334.

86. Haukipuro K, Ohinmaa A, Winblad I, et al. The feasibility of telemedicine for orthopaedic outpatient clinics--a randomized controlled trial. J. Telemed. Telecare. 2000;6(4):193-8. doi: 10.1258/1357633001935347. PMID: 11027118

87. Heffner DL, Thirumala PD, Pokharna P, et al. Outcomes of spoke-retained telestroke patients versus hub-treated patients after intravenous thrombolysis: telestroke patient outcomes after thrombolysis. Stroke. 2015 Nov;46(11):3161-7. doi: 10.1161/STROKEAHA.115.009980. PMID: 26396027.

88. Herce J, Lozano R, Salazar CI, et al. Management of impacted third molars based on telemedicine: a pilot study. J. Oral Maxillofac. Surg. 2011 Feb;69(2):471-5. doi: 10.1016/j.joms.2010.09.004. PMID: 21129834.

89. Hsiao JL, Oh DH. The impact of store-andforward teledermatology on skin cancer diagnosis and treatment. J. Am. Acad. Dermatol. 2008 Aug;59(2):260-7. doi: 10.1016/j.jaad.2008.04.011. PMID: 18485526. 
90. Huang T, Moon-Grady AJ, Traugott C, et al. The availability of telecardiology consultations and transfer patterns from a remote neonatal intensive care unit. J. Telemed. Telecare. 2008;14(5):244-8. doi: 10.1258/jtt.2008.080102. PMID: 18632999.

91. Hubert GJ, Meretoja A, Audebert HJ, et al. Stroke thrombolysis in a centralized and a decentralized system (Helsinki and Telemedical Project for Integrative Stroke Care Network). Stroke. 2016 Dec;47(12):2999-3004. doi: 10.1161/STROKEAHA.116.014258. PMID: 27834751.

92. Ickenstein GW, Horn M, Schenkel J, et al. The use of telemedicine in combination with a new stroke-code-box significantly increases t-PA use in rural communities. Neurocrit. Care. 2005 August 01;3(1):27-32. doi: 10.1385/ncc:3:1:027. PMID: 16159092.

93. Ionita CC, Sharma J, Janicke DM, et al. Acute ischemic stroke and thrombolysis location: comparing telemedicine and stroke center treatment outcomes. Hosp Pract (1995). 2009 Dec;37(1):33-9. doi: 10.3810/hp.2009.12.252. PMID: 20877169.

94. Itrat A, Taqui A, Cerejo R, et al. Telemedicine in prehospital stroke evaluation and thrombolysis: taking stroke treatment to the doorstep. JAMA Neurol. 2016 Feb;73(2):162-8. doi: 10.1001/jamaneurol.2015.3849. PMID: 26641366.

95. Izquierdo R, Morin PC, Bratt K, et al. School-centered telemedicine for children with type 1 diabetes mellitus. J. Pediatr. 2009 Sep;155(3):374-9. doi: 10.1016/j.jpeds.2009.03.014. PMID: 19464030.

96. Jaatinen PT, Aarnio P, Remes J, et al. Teleconsultation as a replacement for referral to an outpatient clinic. J. Telemed. Telecare. 2002;8(2):102-6. doi: 10.1258/1357633021937550. PMID: 11972945.

97. Jacklin PB, Roberts JA, Wallace P, et al. Virtual outreach: economic evaluation of joint teleconsultations for patients referred by their general practitioner for a specialist opinion. BMJ. 2003 Jul 12;327(7406):84. doi: 10.1136/bmj.327.7406.84. PMID: 12855528.
98. Jacobs JJ, Jacobs JP, van Sonderen E, et al. Fracture diagnostics, unnecessary travel and treatment: a comparative study before and after the introduction of teleradiology in a remote general practice. BMC Fam. Pract. 2015 May 06;16:53. doi: 10.1186/s12875015-0268-z. PMID: 25943473.

99. Johansson T, Mutzenbach SJ, Ladurner G. Telemedicine in acute stroke care: the TESSA model. J. Telemed. Telecare. 2011;17(5):268-72. doi: 10.1258/jtt.2011.101213. PMID: 21824968.

100. Jong M, Kraishi M. A comparative study on the utility of telehealth in the provision of rheumatology services to rural and northern communities. Int. J. Circumpolar Health. 2004 Dec;63(4):415-21. PMID: 15709316.

101. Jue JS, Spector SA, Spector SA. Telemedicine broadening access to care for complex cases. J. Surg. Res. 2017

Dec;220:164-70. doi: 10.1016/j.jss.2017.06.085. PMID: 29180178.

102. Kahn JM, Le TQ, Barnato AE, et al. ICU telemedicine and critical care mortality: a national effectiveness study. Med. Care. 2016 Mar;54(3):319-25. doi: 10.1097/MLR.0000000000000485. PMID: 26765148.

103. Kalb T, Raikhelkar J, Meyer S, et al. A multicenter population-based effectiveness study of teleintensive care unit-directed ventilator rounds demonstrating improved adherence to a protective lung strategy, decreased ventilator duration, and decreased intensive care unit mortality. J. Crit. Care. 2014 Aug;29(4):691.e7-14. doi: 10.1016/j.jcrc.2014.02.017. PMID: 24636928.

104. Kim EW, Teague-Ross TJ, Greenfield WW, et al. Telemedicine collaboration improves perinatal regionalization and lowers statewide infant mortality. J. Perinatol. 2013 Sep;33(9):725-30. doi: 10.1038/jp.2013.37. PMID: 23579490.

105. Kim YK, Kim KY, Lee KH, et al. Clinical outcomes on real-time telemetry system in developing emergency medical service system. Telemed J. E Health. 2011 May;17(4):247-53. doi: 10.1089/tmj.2010.0152. PMID: 21480786. 
106. Klein Y, Donchik V, Jaffe D, et al. Management of patients with traumatic intracranial injury in hospitals without neurosurgical service. J. Trauma. 2010 Sep;69(3):544-8. doi: 10.1097/TA.0b013e3181c99936. PMID: 20234328.

107. Kobza L, Scheurich A. The impact of telemedicine on outcomes of chronic wounds in the home care setting. Ostomy Wound Manage. 2000 Oct;46(10):48-53. PMID: 11889733.

108. Kohl BA, Fortino-Mullen M, Praestgaard A, et al. The effect of ICU telemedicine on mortality and length of stay. J. Telemed. Telecare. 2012;18(5):282-6. doi: 10.1258/jtt.2012.120208. PMID: 22802522.

109. Krier M, Kaltenbach T, McQuaid K, et al. Potential use of telemedicine to provide outpatient care for inflammatory bowel disease. Am. J. Gastroenterol. 2011 Dec;106(12):2063-7. doi: 10.1038/ajg.2011.329. PMID: 22138934.

110. Krupinski EA, Engstrom M, Barker G, et al. The challenges of following patients and assessing outcomes in teledermatology. $\mathrm{J}$. Telemed. Telecare. 2004;10(1):21-4. doi: 10.1258/135763304322764149. PMID: 15006211.

111. Kunkler IH, Prescott RJ, Lee RJ, et al. TELEMAM: a cluster randomised trial to assess the use of telemedicine in multidisciplinary breast cancer decision making. Eur. J. Cancer. 2007 Nov;43(17):2506-14. doi: 10.1016/j.ejca.2007.08.026. PMID: 17962011.

112. Labarbera JM, Ellenby MS, Bouressa P, et al. The impact of telemedicine intensivist support and a pediatric hospitalist program on a community hospital. Telemed J. E Health. 2013 Oct;19(10):760-6. doi: 10.1089/tmj.2012.0303. PMID: 23937510.

113. Lamminen $\mathrm{H}$, Lamminen J, Ruohonen $\mathrm{K}$, et al. A cost study of teleconsultation for primary-care ophthalmology and dermatology. J. Telemed. Telecare. 2001;7(3):167-73. doi: 10.1258/1357633011936336. PMID: 11346477.
114. Langabeer JR, 2nd, Champagne-Langabeer $\mathrm{T}$, Alqusairi $\mathrm{D}$, et al. Cost-benefit analysis of telehealth in pre-hospital care. J. Telemed. Telecare. 2017 Sep;23(8):747-51. doi: 10.1177/1357633X16680541. PMID: 27913657.

115. Langabeer JR, 2nd, Gonzalez M, Alqusairi $\mathrm{D}$, et al. Telehealth-enabled emergency medical services program reduces ambulance transport to urban emergency departments. West. J. Emerg. Med. 2016 Nov;17(6):713-20. doi: 10.5811/westjem.2016.8.30660. PMID: 27833678.

116. Lee RH, Lyles KW, Pearson M, et al. Osteoporosis screening and treatment among veterans with recent fracture after implementation of an electronic consult service. Calcif. Tissue Int. 2014 Jun;94(6):659-64. doi: 10.1007/s00223-0149849-4. PMID: 24699797.

117. Lilly CM, Cody S, Zhao H, et al. Hospital mortality, length of stay, and preventable complications among critically ill patients before and after tele-ICU reengineering of critical care processes. JAMA. 2011 Jun 01;305(21):2175-83. doi: 10.1001/jama.2011.697. PMID: 21576622.

118. Lilly CM, Motzkus C, Rincon T, et al. ICU Telemedicine Program Financial Outcomes. Chest. 2017 February 01;151(2):286-97. doi: 10.1016/j.chest.2016.11.029. PMID: 27932050.

119. Lim D, Oakley AM, Rademaker M. Better, sooner, more convenient: a successful teledermoscopy service. Australas. J. Dermatol. 2012 Feb;53(1):22-5. doi: 10.1111/j.1440-0960.2011.00836.x. PMID: 22309326.

120. Loane MA, Bloomer SE, Corbett R, et al. Patient cost-benefit analysis of teledermatology measured in a randomized control trial. J. Telemed. Telecare. 1999;5 Suppl 1:S1-3. PMID: 10534821.

121. Loane MA, Bloomer SE, Corbett R, et al. A randomized controlled trial to assess the clinical effectiveness of both realtime and store-and-forward teledermatology compared with conventional care. J. Telemed. Telecare. 2000;6 Suppl 1:S1-3. PMID: 10793956. 
122. Loane MA, Oakley A, Rademaker M, et al. A cost-minimization analysis of the societal costs of realtime teledermatology compared with conventional care: results from a randomized controlled trial in New Zealand. J. Telemed. Telecare. 2001;7(4):233-8. doi: 10.1258/1357633011936453. PMID: 11506759 .

123. Long MC, Angtuaco T, Lowery C. Ultrasound in telemedicine: its impact in high-risk obstetric health care delivery. Ultrasound Q. 2014 Sep;30(3):167-72. doi: 10.1097/RUQ.0000000000000073. PMID: 25148484.

124. Macedo TA, de Barros ESPG, Simoes SA, et al. Impact of chest pain protocol with access to telemedicine on implementation of pharmacoinvasive strategy in a private hospital network. Telemed J. E Health. 2016 Jul;22(7):549-52. doi: 10.1089/tmj.2015.0178. PMID: 26693879.

125. Machado SM, Wilson EH, Elliott JO, et al. Impact of a telemedicine eICU cart on sepsis management in a community hospital emergency department. J. Telemed. Telecare. 2018 Apr;24(3):202-8. doi: 10.1177/1357633X17691862. PMID: 29278979.

126. Mahendran R, Goodfield MJ, Sheehan-Dare RA. An evaluation of the role of a store-andforward teledermatology system in skin cancer diagnosis and management. Clin. Exp. Dermatol. 2005 May;30(3):209-14. doi: 10.1111/j.1365-2230.2005.01735.x. PMID: 15807671.

127. Marcin JP, Nesbitt TS, Kallas HJ, et al. Use of telemedicine to provide pediatric critical care inpatient consultations to underserved rural Northern California. J. Pediatr. 2004 Mar;144(3):375-80. doi:

10.1016/j.jpeds.2003.12.017. PMID: 15001947.

128. Marcin JP, Nesbitt TS, Struve S, et al. Financial benefits of a pediatric intensive care unit-based telemedicine program to a rural adult intensive care unit: impact of keeping acutely ill and injured children in their local community. Telemed J. E Health. 2004;10 Suppl 2:S-1-5. PMID: 23570207.
129. Marcin JP, Schepps DE, Page KA, et al. The use of telemedicine to provide pediatric critical care consultations to pediatric trauma patients admitted to a remote trauma intensive care unit: a preliminary report. Pediatr. Crit. Care Med. 2004;5(3):251-6. PMID: 15115563.

130. Marcolino MS, Brant LC, Araujo JG, et al. Implementation of the myocardial infarction system of care in city of Belo Horizonte, Brazil.[Erratum appears in Arq Bras Cardiol. 2013 Apr;100(4):313]. Arq. Bras. Cardiol. 2013 Apr;100(4):307-14. PMID: 23545995.

131. Martinez-Sanchez P, Miralles A, Sanz de Barros R, et al. The effect of telestroke systems among neighboring hospitals: more and better? The Madrid Telestroke Project. J. Neurol. 2014 Sep;261(9):1768-73. doi: 10.1007/s00415-014-7419-3. PMID: 24957298.

132. Martin-Khan MG, Edwards H, Wootton R, et al. Web-based (online) comprehensive geriatric assessment is more time efficient, and as reliable, as reading patient medical records and conducting traditional in person consultations. J. Telemed. Telecare. 2016 Dec;22(8):478-82. doi: 10.1177/1357633X16674088. PMID: 27799451.

133. Martinoni A, Servi SD, Boschetti E, et al. Importance and limits of pre-hospital electrocardiogram in patients with ST elevation myocardial infarction undergoing percutaneous coronary angioplasty. Eur. J. Cardiovasc. Prev. Rehabil. 2011 June 01;18(3):526-32. doi: 10.1177/1741826710389395. PMID: 21450642.

134. Mathews KA, Elcock MS, Furyk JS. The use of telemedicine to aid in assessing patients prior to aeromedical retrieval to a tertiary referral centre. J. Telemed. Telecare. 2008;14(6):309-14. doi:

10.1258/jtt.2008.080417. PMID: 18776077.

135. Mazighi M, Meseguer E, Labreuche J, et al. TRUST-tPA trial: Telemedicine for remote collaboration with urgentists for stroke-tPA treatment. J. Telemed. Telecare. 2017 Jan;23(1):174-80. doi: 10.1177/1357633X15615762. PMID: 26656722. 
136. McCambridge M, Jones K, Paxton H, et al. Association of health information technology and teleintensivist coverage with decreased mortality and ventilator use in critically ill patients. Arch. Intern. Med. 2010 Apr 12;170(7):648-53. doi: 10.1001/archinternmed.2010.74. PMID: 20386011.

137. McCrossan BA, Sands AJ, Kileen T, et al. A fetal telecardiology service: patient preference and socio-economic factors. Prenat. Diagn. 2012 Sep;32(9):883-7. doi: 10.1002/pd.3926. PMID: 22718083.

138. Meyer BC, Raman R, Hemmen T, et al. Efficacy of site-independent telemedicine in the STRokE DOC trial: a randomised, blinded, prospective study. Lancet Neurol. 2008 Sep;7(9):787-95. doi: 10.1016/S14744422(08)70171-6. PMID: 18676180.

139. Mielonen ML, Ohinmaa A, Moring J, et al. Psychiatric inpatient care planning via telemedicine. J. Telemed. Telecare. 2000;6(3):152-7. doi: 10.1258/1357633001935248. PMID: 10912333.

140. Migliaretti G, Ciaramitaro P, Berchialla P, et al. Teleconsulting for minor head injury: the Piedmont experience. J. Telemed. Telecare. 2013 Jan;19(1):33-5. doi: 10.1177/1357633X12474738. PMID: 23454822.

141. Miyamoto S, Dharmar M, Boyle C, et al. Impact of telemedicine on the quality of forensic sexual abuse examinations in rural communities. Child Abuse Negl. 2014 Sep;38(9):1533-9. doi: 10.1016/j.chiabu.2014.04.015. PMID: 24841062.

142. Mohr NM, Harland KK, Chrischilles EA, et al. Emergency department telemedicine is used for more severely injured rural trauma patients, but does not decrease transfer: a cohort study. Acad. Emerg. Med. 2017;24(2):177-85. doi: 10.1111/acem.13120. PMID: 28187248.

143. Mohr NM, Vakkalanka JP, Harland KK, et al. Telemedicine use decreases rural emergency department length of stay for transferred North Dakota trauma patients. Telemed J. E Health. 2018 Mar;24(3):194202. doi: 10.1089/tmj.2017.0083. PMID: 28731843.
144. Moreno-Ramirez D, Ferrandiz L, Ruiz-deCasas A, et al. Economic evaluation of a store-and-forward teledermatology system for skin cancer patients. J. Telemed.

Telecare. 2009;15(1):40-5. doi: 10.1258/jtt.2008.080901. PMID: 19139219.

145. Morrison JL, Cai Q, Davis N, et al. Clinical and economic outcomes of the electronic intensive care unit: results from two community hospitals. Crit. Care Med. 2010 Jan;38(1):2-8. doi: 10.1097/CCM.0b013e3181b78fa8. PMID: 19730249.

146. Mulgrew KW, Shaikh U, Nettiksimmons J. Comparison of parent satisfaction with care for childhood obesity delivered face-to-face and by telemedicine. Telemed J. E Health. 2011 Jun;17(5):383-7. doi: 10.1089/tmj.2010.0153. PMID: 21492028.

147. Nagao KJ, Koschel A, Haines HM, et al. Rural Victorian Telestroke project. Intern. Med. J. 2012 Oct;42(10):1088-95. doi: 10.1111/j.1445-5994.2011.02603.x. PMID: 21981424.

148. Nagayoshi Y, Oshima S, Ogawa H. Clinical impact of telemedicine network system at rural hospitals without on-site cardiac surgery backup. Telemed J. E Health. 2016 Nov;22(11):960-4. doi: 10.1089/tmj.2015.0225. PMID: 27148833.

149. Narasimhan M, Druss BG, Hockenberry JM, et al. Impact of a telepsychiatry program at emergency departments statewide on the quality, utilization, and costs of mental health services. Psychiatr. Serv. 2015 Nov;66(11):1167-72. doi: 10.1176/appi.ps.201400122. PMID: 26129992.

150. Nassar BS, Vaughan-Sarrazin MS, Jiang L, et al. Impact of an intensive care unit telemedicine program on patient outcomes in an integrated health care system. JAMA Intern Med. 2014 Jul;174(7):1160-7. doi: 10.1001/jamainternmed.2014.1503. PMID: 24819673.

151. Natafgi N, Shane DM, Ullrich F, et al. Using tele-emergency to avoid patient transfers in rural emergency departments: An assessment of costs and benefits. J. Telemed. Telecare. 2017;24(3):193-201. doi: 10.1177/1357633X17696585. PMID: 29278984. 
152. Nguyen-Huynh MN, Klingman JG, Avins AL, et al. Novel telestroke program improves thrombolysis for acute stroke across 21 hospitals of an integrated healthcare system. Stroke. 2018 01;49(1):133-9. doi: 10.1161/STROKEAHA.117.018413. PMID: 29247142.

153. Nickenig H, Wichmann M, Schlegel A, et al. Use of telemedicine for pre-implant dental assessment -- a comparative study. J. Telemed. Telecare. 2008;14(2):93-7. doi: 10.1258/jtt.2007.070806. PMID: 18348756.

154. Nikkanen T, Timonen M, Ylitalo K, et al. Quality of diabetes care among patients managed by teleconsultation. J. Telemed. Telecare. 2008;14(6):295-9. doi: 10.1258/jtt.2008.080313. PMID: 18776074.

155. Noble SM, Coast J, Benger JR. A costconsequences analysis of minor injuries telemedicine. J. Telemed. Telecare. 2005;11(1):15-9. doi: 10.1177/1357633X0501100104. PMID: 15829038.

156. Nordal EJ, Moseng D, Kvammen B, et al. A comparative study of teleconsultations versus face-to-face consultations. $\mathrm{J}$. Telemed. Telecare. 2001;7(5):257-65. doi: 10.1258/1357633011936507. PMID: 11571079 .

157. Ohinmaa A, Vuolio S, Haukipuro K, et al. A cost-minimization analysis of orthopaedic consultations using videoconferencing in comparison with conventional consulting. J. Telemed. Telecare. 2002;8(5):283-9. doi: 10.1177/1357633X0200800507. PMID: 12396857.

158. Ortolani P, Marzocchi A, Marrozzini C, et al. Usefulness of prehospital triage in patients with cardiogenic shock complicating ST-elevation myocardial infarction treated with primary percutaneous coronary intervention. Am. J. Cardiol. 2007 Sep 01;100(5):787-92. doi: 10.1016/j.amjcard.2007.03.099. PMID: 17719321.
159. Ortolani P, Marzocchi A, Marrozzini C, et al. Clinical impact of direct referral to primary percutaneous coronary intervention following pre-hospital diagnosis of STelevation myocardial infarction. Eur. Heart J. 2006;27(13):1550-7. doi: 10.1093/eurheartj/ehl006. PMID: 16707549.

160. Paik AM, Granick MS, Scott S. Plastic surgery telehealth consultation expedites emergency department treatment. J. Telemed. Telecare. 2017 Feb;23(2):321-7. doi: 10.1177/1357633X16639459. PMID: 27056907.

161. Pak H, Triplett CA, Lindquist JH, et al. Store-and-forward teledermatology results in similar clinical outcomes to conventional clinic-based care. J. Telemed. Telecare. 2007;13(1):26-30. doi: 10.1258/135763307779701185. PMID: 17288655.

162. Pak HS, Datta SK, Triplett CA, et al. Cost minimization analysis of a store-andforward teledermatology consult system. Telemed J. E Health. 2009 Mar;15(2):160-5. doi: 10.1089/tmj.2008.0083. PMID: 19292625.

163. Panlaqui OM, Broadfield E, Champion R, et al. Outcomes of telemedicine intervention in a regional intensive care unit: a before and after study. Anaesth. Intensive Care. 2017;45(5):605-10. PMID: 28911290.

164. Patel S, Hertzog JH, Penfil S, et al. A prospective pilot study of the use of telemedicine during pediatric transport: A high-quality, low-cost alternative to conventional telemedicine systems. Pediatr. Emerg. Care. 2015 Sep;31(9):611-5. doi: 10.1097/PEC.0000000000000544. PMID: 26335229.

165. Pedersen SH, Galatius S, Hansen PR, et al. Field triage reduces treatment delay and improves long-term clinical outcome in patients with acute ST-segment elevation myocardial infarction treated with primary percutaneous coronary intervention. J. Am. Coll. Cardiol. 2009;54(24):2296-302. doi: 10.1016/j.jacc.2009.06.056. PMID: 19958965. 
166. Pedragosa A, Alvarez-Sabin J, Molina CA, et al. Impact of a telemedicine system on acute stroke care in a community hospital. J. Telemed. Telecare. 2009;15(5):260-3. doi: 10.1258/jtt.2009.090102. PMID: 19590033.

167. Pervez MA, Silva G, Masrur S, et al. Remote supervision of IV-tPA for acute ischemic stroke by telemedicine or telephone before transfer to a regional stroke center is feasible and safe. Stroke. 2010 Jan;41(1):e18-24. doi: 10.1161/strokeaha.109.560169. PMID: 19910552.

168. Piette E, Nougairede M, Vuong V, et al. Impact of a store-and-forward teledermatology intervention versus usual care on delay before beginning treatment: A pragmatic cluster-randomized trial in ambulatory care. J. Telemed. Telecare. 2017 Sep;23(8):725-32. doi: 10.1177/1357633X16663328. PMID: 27496852.

169. Poon WS, Leung CH, Lam MK, et al. The comparative impact of video-consultation on neurosurgical health services. Int. J. Med. Inform. 2001 Jul;62(2-3):175-80. PMID: 11470620 .

170. Rendina MC. The effect of telemedicine on neonatal intensive care unit length of stay in very low birthweight infants. Proc. AMIA Symp. 1998:111-5. PMID: 9929192.

171. Rendina MC, Long WA, deBliek R. Effect size and experimental power analysis in a paediatric cardiology telemedicine system. J. Telemed. Telecare. 1997;3 (Suppl 1):56-7. doi: 10.1258/1357633971930382. PMID: 9218385.

172. Rincon F, Vibbert M, Childs V, et al. Implementation of a model of robotic telepresence (RTP) in the neuro-ICU: effect on critical care nursing team satisfaction. Neurocrit. Care. 2012 Aug;17(1):97-101. doi: 10.1007/s12028-012-9712-2. PMID: 22547040 .

173. Robison J, Slamon NB. A more rapid, rapid response. Pediatr. Crit. Care Med. 2016 Sep;17(9):871-5. doi: 10.1097/PCC.0000000000000855. PMID: 27427880.
174. Romig MC, Latif A, Gill RS, et al. Perceived benefit of a telemedicine consultative service in a highly staffed intensive care unit. J. Crit. Care. 2012 Aug;27(4):426 e9-16. doi: 10.1016/j.jcrc.2011.12.007. PMID: 22421004.

175. Rosenfeld BA, Dorman T, Breslow MJ, et al. Intensive care unit telemedicine: alternate paradigm for providing continuous intensivist care. Crit. Care Med. 2000 Dec;28(12):3925-31. PMID: 11153637.

176. Rossaro L, Torruellas C, Dhaliwal S, et al. Clinical outcomes of hepatitis $\mathrm{C}$ treated with pegylated interferon and ribavirin via telemedicine consultation in Northern California. Dig. Dis. Sci. 2013 Dec;58(12):3620-5. doi: 10.1007/s10620013-2810-y. PMID: 24154637.

177. Ruesch C, Mossakowski J, Forrest J, et al. Using nursing expertise and telemedicine to increase nursing collaboration and improve patient outcomes. Telemed J. E Health. 2012 Oct;18(8):591-5. doi: 10.1089/tmj.2011.0274. PMID: 22957503.

178. Sadaka F, Palagiri A, Trottier S, et al. Telemedicine intervention improves ICU outcomes. Crit. Care Res. Pract. 2013;2013:456389. doi: 10.1155/2013/456389. PMID: 23365729.

179. Saffle JR, Edelman L, Theurer L, et al. Telemedicine evaluation of acute burns is accurate and cost-effective. J. Trauma. 2009 Aug;67(2):358-65. doi: 10.1097/TA.0b013e3181ae9b02. PMID: 19667890.

180. Sairanen T, Soinila S, Nikkanen M, et al. Two years of Finnish Telestroke: thrombolysis at spokes equal to that at the hub. Neurology. 2011 Mar 29;76(13):114552. doi: 10.1212/WNL.0b013e318212a8d4. PMID: 21368283.

181. Salami AC, Barden GM, Castillo DL, et al. Establishment of a regional virtual tumor board program to improve the process of care for patients with hepatocellular carcinoma. J. Oncol. Pract. 2015 Jan;11(1):e66-74. doi: 10.1200/JOP.2014.000679. PMID: 25466708. 
182. Salazar-Fernandez CI, Herce J, GarciaPalma A, et al. Telemedicine as an effective tool for the management of temporomandibular joint disorders. J. Oral Maxillofac. Surg. 2012;70(2):295-301. doi: 10.1016/j.joms.2011.03.053. PMID: 21803470.

183. Sanchez-Ross M, Oghlakian G, Maher J, et al. The STAT-MI (ST-Segment Analysis Using Wireless Technology in Acute Myocardial Infarction) trial improves outcomes. JACC Cardiovasc. Interv. 2011 Feb;4(2):222-7. doi: 10.1016/j.jcin.2010.11.007. PMID: 21349462.

184. Santamaria N, Carville K, Ellis I, et al. The effectiveness of digital imaging and remote expert wound consultation on healing rates in chronic lower leg ulcers in the Kimberley region of Western Australia. Primary Intention: The Australian Journal of Wound Management. 2004;12(2):62-70.

185. Schwab S, Vatankhah B, Kukla C, et al. Long-term outcome after thrombolysis in telemedical stroke care. Neurology. 2007 Aug 28;69(9):898-903. doi: 10.1212/01.wnl.0000269671.08423.14. PMID: 17724293.

186. Scuffham PA, Steed M. An economic evaluation of the Highlands and Islands teledentistry project. J. Telemed. Telecare. 2002;8(3):165-77. doi: 10.1177/1357633X0200800307. PMID: 12097178.

187. Sejersten M, Sillesen M, Hansen PR, et al. Effect on treatment delay of prehospital teletransmission of 12-lead electrocardiogram to a cardiologist for immediate triage and direct referral of patients with ST-segment elevation acute myocardial infarction to primary percutaneous coronary intervention. Am. J. Cardiol. 2008;101(7):941-6. doi: 10.1016/j.amjcard.2007.11.038. PMID: 18359312

188. Sharma P, Kovarik CL, Lipoff JB. Teledermatology as a means to improve access to inpatient dermatology care. J. Telemed. Telecare. 2016 Jul;22(5):304-10. doi: 10.1177/1357633x15603298. PMID: 26377123.
189. Sharma S, Parness IA, Kamenir SA, et al. Screening fetal echocardiography by telemedicine: efficacy and community acceptance. J. Am. Soc. Echocardiogr. 2003 Mar;16(3):202-8. doi: 10.1067/mje.2003.46. PMID: 12618726.

190. Shin DH, Dalag L, Azhar RA, et al. A novel interface for the telementoring of robotic surgery. BJU Int. 2015 Aug;116(2):302-8. doi: 10.1111/bju.12985. PMID: 25381917.

191. Smith AC, Williams M, Van der Westhuyzen J, et al. A comparison of telepaediatric activity at two regional hospitals in Queensland. J. Telemed. Telecare. 2002;8 Suppl 3:S3:58-62. PMID: 12661625.

192. Smith-Strom H, Igland J, Ostbye T, et al. The effect of telemedicine follow-up care on diabetes-related foot ulcers: a clusterrandomized controlled non inferiority trial. Diabetes Care. 2018;41(1):96-103. doi: 10.2337/dc17-1025. PMID: 29187423.

193. Southard EP, Neufeld JD, Laws S. Telemental health evaluations enhance access and efficiency in a critical access hospital emergency department. Telemed J. E Health. 2014 Jul;20(7):664-8. doi: 10.1089/tmj.2013.0257. PMID: 24811858.

194. Specht JK, Wakefield B, Flanagan J. Evaluating the cost of one telehealth application connecting an acute and longterm care setting. J. Gerontol. Nurs. 2001 Jan;27(1):34-9. PMID: 11915095.

195. Stalfors J, Bjorholt I, Westin T. A cost analysis of participation via personal attendance versus telemedicine at a head and neck oncology multidisciplinary team meeting. J. Telemed. Telecare. 2005;11(4):205-10. doi: 10.1258/1357633054068892. PMID: 16007751.

196. Stalfors J, Holm-Sjogren L, Schwieler A, et al. Satisfaction with telemedicine presentation at a multidisciplinary tumour meeting among patients with head and neck cancer. J. Telemed. Telecare. 2003;9(3):1505. doi: $10.1258 / 135763303767149951$. PMID: 12882214. 
197. Steinman M, Morbeck RA, Pires PV, et al. Impact of telemedicine in hospital culture and its consequences on quality of care and safety. Einstein (Sao Paulo). 2015 OctDec;13(4):580-6. doi: 10.1590/S167945082015GS2893. PMID: 26676268.

198. Stern A, Mitsakakis N, Paulden M, et al. Pressure ulcer multidisciplinary teams via telemedicine: a pragmatic cluster randomized stepped wedge trial in long term care. BMC Health Serv. Res. 2014;14:83. doi: 10.1186/1472-6963-14-83. PMID: 24559218.

199. Strymish J, Gupte G, Afable MK, et al. Electronic consultations (E-consults): advancing infectious disease care in a large Veterans Affairs healthcare system. Clin. Infect. Dis. 2017 Apr 15;64(8):1123-5. doi: 10.1093/cid/cix058. PMID: 28158475.

200. Switzer JA, Hall C, Gross H, et al. A webbased telestroke system facilitates rapid treatment of acute ischemic stroke patients in rural emergency departments. J. Emerg. Med. 2009;36(1):12-8. doi: 10.1016/j.jemermed.2007.06.041. PMID: 18242925 .

201. Taqui A, Cerejo R, Itrat A, et al. Reduction in time to treatment in prehospital telemedicine evaluation and thrombolysis. Neurology. 2017 Apr 04;88(14):1305-12. doi: 10.1212/WNL.0000000000003786. PMID: 28275084.

202. Taylor CR, Merin LM, Salunga AM, et al. Improving diabetic retinopathy screening ratios using telemedicine-based digital retinal imaging technology: the Vine Hill study. Diabetes Care. 2007 Mar;30(3):5748. doi: 10.2337/dc06-1509. PMID: 17327323.

203. Thaker DA, Monypenny R, Olver I, et al. Cost savings from a telemedicine model of care in northern Queensland, Australia. Med. J. Aust. 2013 Sep 16;199(6):414-7. PMID: 24033216.

204. Theiss S, Gunzel F, Storm A, et al. Using routine data for quality assessment in NeuroNet telestroke care. J. Stroke Cerebrovasc. Dis. 2013 Oct;22(7):984-90. doi: 10.1016/j.jstrokecerebrovasdis.2012.01.012. PMID: 22365711.
205. Thomas EJ, Lucke JF, Wueste L, et al. Association of telemedicine for remote monitoring of intensive care patients with mortality, complications, and length of stay. JAMA. 2009 Dec 23;302(24):2671-8. doi: 10.1001/jama.2009.1902. PMID: 20040555.

206. Traub SJ, Butler R, Chang YH, et al. Emergency department physician telemedical triage. Telemed J. E Health. 2013 Nov;19(11):841-5. doi: 10.1089/tmj.2013.0026. PMID: 24093916.

207. Tsai SH, Kraus J, Wu HR, et al. The effectiveness of video-telemedicine for screening of patients requesting emergency air medical transport (EAMT). J. Trauma. 2007 Feb;62(2):504-11. doi: 10.1097/01.ta.0000219285.08974.45. PMID: 17297342.

208. Tsitlakidis C, Mylonakis J, Niakas D. Economic evaluation of telemedicine for a remotely located population: the case of two Greek islands. Int J Electron Healthc. 2005;1(3):243-60. doi: 10.1504/IJEH.2005.006473. PMID: 18048208.

209. Tuulonen A, Ohinmaa T, Alanko HI, et al. The application of teleophthalmology in examining patients with glaucoma: a pilot study. J. Glaucoma. 1999 Dec;8(6):367-73. PMID: 10604295.

210. van der Pol M, McKenzie L. Costs and benefits of tele-endoscopy clinics in a remote location. J. Telemed. Telecare. 2010;16(2):89-94. doi: 10.1258/jtt.2009.090609. PMID: 20139140.

211. van Gelder VA, Scherpbier-de Haan ND, van Berkel S, et al. Web-based consultation between general practitioners and nephrologists: a cluster randomized controlled trial. Fam. Pract. 2017;34(4):4306. doi: 10.1093/fampra/cmw131. PMID: 28158576.

212. Vuolio S, Winblad I, Ohinmaa A, et al. Videoconferencing for orthopaedic outpatients: one-year follow-up. J. Telemed. Telecare. 2003;9(1):8-11. doi: 10.1258/135763303321159620. PMID: 12641886. 
213. Wallace DL, Jones SM, Milroy C, et al. Telemedicine for acute plastic surgical trauma and burns. J. Plast. Reconstr. Aesthet. Surg. 2008;61(1):31-6. doi: 10.1016/j.bjps.2006.03.045. PMID: 18068653.

214. Wallace P, Barber J, Clayton W, et al. Virtual outreach: a randomised controlled trial and economic evaluation of joint teleconferenced medical consultations. Health Technol. Assess. 2004 Dec;8(50):1106, iii-iv. PMID: 15546515.

215. Wallace P, Haines A, Harrison R, et al. Joint teleconsultations (virtual outreach) versus standard outpatient appointments for patients referred by their general practitioner for a specialist opinion: a randomised trial. Lancet. 2002 Jun 08;359(9322):1961-8. PMID: 12076550.

216. Webb CL, Waugh CL, Grigsby J, et al. Impact of telemedicine on hospital transport, length of stay, and medical outcomes in infants with suspected heart disease: a multicenter study. J. Am. Soc. Echocardiogr. 2013 Sep;26(9):1090-8. doi: 10.1016/j.echo.2013.05.018. PMID: 23860093.

217. Whited JD, Datta S, Hall RP, et al. An economic analysis of a store and forward teledermatology consult system. Telemed J. E Health. 2003 Winter;9(4):351-60. doi: 10.1089/153056203772744671. PMID: 14980092.

218. Whited JD, Hall RP, Foy ME, et al. Teledermatology's impact on time to intervention among referrals to a dermatology consult service. Telemed J. E Health. 2002 Fall;8(3):313-21. doi: 10.1089/15305620260353207. PMID: 12419025.

219. Whited JD, Hall RP, Foy ME, et al. Patient and clinician satisfaction with a store-andforward teledermatology consult system. Telemed J. E Health. 2004 Winter;10(4):422-31. doi: 10.1089/tmj.2004.10.422. PMID: 15689645.
220. Whited JD, Warshaw EM, Edison KE, et al. Effect of store and forward teledermatology on quality of life: a randomized controlled trial. JAMA Dermatol. 2013

May;149(5):584-91. doi: 10.1001/2013.jamadermatol.380. PMID: 23426111.

221. Whited JD, Warshaw EM, Kapur K, et al. Clinical course outcomes for store and forward teledermatology versus conventional consultation: a randomized trial. J. Telemed. Telecare. 2013 Jun;19(4):197-204. doi: 10.1177/1357633X13487116. PMID: 23666440.

222. Willmitch B, Golembeski S, Kim SS, et al. Clinical outcomes after telemedicine intensive care unit implementation. Crit. Care Med. 2012 Feb;40(2):450-4. doi: 10.1097/CCM.0b013e318232d694. PMID: 22020235.

223. Wilson C, Horton M, Cavallerano J, et al. Addition of primary care-based retinal imaging technology to an existing eye care professional referral program increased the rate of surveillance and treatment of diabetic retinopathy. Diabetes Care. 2005 Feb;28(2):318-22. PMID: 15677786.

224. Wong HT, Poon WS, Jacobs P, et al. The comparative impact of video consultation on emergency neurosurgical referrals. Neurosurgery. 2006 Sep;59(3):607-13; discussion -13. doi: 10.1227/01.NEU.0000228926.13395.F9. PMID: 16955042.

225. Xu CQ, Smith AC, Scuffham PA, et al. A cost minimisation analysis of a telepaediatric otolaryngology service. BMC Health Serv. Res. 2008 Feb 04;8:30. doi: 10.1186/1472-6963-8-30. PMID: 18241356.

226. Yang NH, Dharmar M, Kuppermann N, et al. Appropriateness of disposition following telemedicine consultations in rural emergency departments. Pediatr. Crit. Care Med. 2015 Mar;16(3):e59-64. doi: 10.1097/PCC.0000000000000337. PMID: 25607743. 
227. Young JD, Patel M, Badowski M, et al. Improved virologic suppression with HIV subspecialty care in a large prison system using telemedicine: an observational study with historical controls. Clin. Infect. Dis. 2014 Jul 1;59(1):123-6. doi: 10.1093/cid/ciu222. PMID: 24723283.

228. Zahlmann G, Mertz M, Fabian E, et al. Perioperative cataract OP management by means of teleconsultation. Graefes Arch. Clin. Exp. Ophthalmol. 2002 Jan;240(1):1720. PMID: 11954775.

229. Zaidi SF, Jumma MA, Urra XN, et al. Telestroke-guided intravenous tissue-type plasminogen activator treatment achieves a similar clinical outcome as thrombolysis at a comprehensive stroke center. Stroke. 2011 Nov;42(11):3291-3. doi: 10.1161/STROKEAHA.111.625046. PMID: 21885843.

230. Zanini R, Aroldi M, Bonatti S, et al. Impact of prehospital diagnosis in the management of ST elevation myocardial infarction in the era of primary percutaneous coronary intervention: reduction of treatment delay and mortality. J. Cardiovasc. Med. 2008;9(6):570-5. doi: 10.2459/JCM.0b013e3282f2c9bd. PMID: 18475124 .
231. Zarchi K, Haugaard VB, Dufour DN, et al. Expert advice provided through telemedicine improves healing of chronic wounds: prospective cluster controlled study. J. Invest. Dermatol. 2015 Mar;135(3):895-900. doi: 10.1038/jid.2014.441. PMID: 25290685.

232. Zennaro F, Grosso D, Fascetta R, et al. Teleradiology for remote consultation using iPad improves the use of health system human resources for paediatric fractures: prospective controlled study in a tertiary care hospital in Italy. BMC Health Serv. Res. 2014 Jul 28;14:327. doi: 10.1186/14726963-14-327. PMID: 25070705.

233. Zilliacus EM, Meiser B, Lobb EA, et al. Are videoconferenced consultations as effective as face-to-face consultations for hereditary breast and ovarian cancer genetic counseling? Genet. Med. 2011

Nov;13(11):933-41. doi: 10.1097/GIM.0b013e3182217a19. PMID: 21799430. 


\section{Appendix D. Excluded Studies}

1. Army tests battlefield medical systems. Telemed Virtual Real. 1997 Dec;2(12):134. PMID: 10174645. Exclusion reason: Ineligible comparison

2. Abu Dhabi Hospital links to WorldCare Gulf. Telemed Virtual Real. 1997 Sep;2(9):106. PMID: 10170469. Exclusion reason: Ineligible publication type

3. New York doctor goes on the 'Net to save a baby in Argentina. Telemed Virtual Real. 1997 Sep;2(9):105. PMID: 10170467. Exclusion reason: Ineligible study design

4. $\quad$ PhysiTel announces "One World" program for developing nations. Telemed Virtual Real. 1998 Aug;3(8):93. PMID: 10182127. Exclusion reason: Ineligible publication type

5. Industry donations complete "last mile" so tiny hearts can get expert care. Telemed Virtual Real. 1998 Jul;3(7):81. PMID: 10180768. Exclusion reason: Ineligible comparison

6. Italian hospitals connect to Bosnia, Albania. Telemed Virtual Real. 1998 Jun;3(6):70. PMID: 10180761. Exclusion reason: Ineligible comparison

7. Navy uses telemedicine to save sailors, money. Telemed Virtual Real. 1998 Mar;3(3):29. PMID: 10177466. Exclusion reason: Ineligible publication type

8. Report of the Joint Working Group on telemammography/teleradiology and information management. Washington DC, USA. March 15-17, 1999. Acad Radiol. 1999 Nov;6 Suppl 7:S303-24. PMID: 10894305. Exclusion reason: Ineligible publication type

9. Telemedical consultation system: how to choose. Emerg Nurse. 1999;7(6):11-3. Exclusion reason: Ineligible publication type

10. Online second opinion services allow doctors and hospitals to treat patients outside their local markets. Internet Healthc Strateg. 2003 Aug;5(8):7-9. PMID: 12961838.

Exclusion reason: Ineligible publication type
11. Comprehensive care is put to the test in CMS demo. Dis Manag Advis. 2004 Aug;10(8):85-8. PMID: 15473181.

Exclusion reason: Ineligible intervention

12. Web-based consults save money, boost satisfaction: members, docs communicate on nonurgent matters. Case Management Advisor. 2005;16(8):88-90. Exclusion reason: Ineligible study design

13. Mobile health technologies facilitate efficient communication in ophthalmology. Ocular Surgery News. 2013;31(4):1-12. Exclusion reason: Ineligible comparison

14. Telehealth tackles elderly health care. Aust Nurs J. 2013 Jul;21(1):41. PMID: 23936932. Exclusion reason: Ineligible intervention

15. Women linked with maternity services. Midwives. 2014;17(4):8. PMID: 25145078. Exclusion reason: Ineligible intervention

16. National Quality Forum. Creating a Framework to Support Measure Development for Telehealth Department of Health and Human Services. Contract HHSM-500-2012-00009I, Task Order HHSM-500-T0022. Washington, DC: 2017. Exclusion reason: Background information only

17. Aanesen M, Lotherington AT, Olsen F. Smarter elder care? A cost-effectiveness analysis of implementing technology in elder care. Health Informatics J. 2011 Sep;17(3):161-72. doi: 10.1177/1460458211409716. PMID: 21937460. Exclusion reason: Ineligible intervention

18. Aarnio P, Lamminen H, Lepisto J, et al. A prospective study of teleconferencing for orthopaedic consultations. J Telemed Telecare. 1999;5(1):62-6. doi: 10.1258/1357633991932405. PMID: 10505371. Exclusion reason: Ineligible comparison 
19. Aboujaoude E, Salame W. Technology at the service of pediatric mental health: review and assessment. J Pediatr. 2016 Apr;171:20-4. doi: 10.1016/j.jpeds.2015.12.009. PMID: 26743496. Exclusion reason: Ineligible intervention

20. Adams SJ, Burbridge BE, Badea A, et al. Initial experience using a telerobotic ultrasound system for adult abdominal sonography. Can Assoc Radiol J. 2017 Aug;68(3):308-14. doi: 10.1016/j.carj.2016.08.002. PMID: 28159435. Exclusion reason: Ineligible intervention

21. Agarwal S, Day DJ, Sibson L, et al. Thrombolysis delivery by a regional telestroke network--experience from the U.K. National Health Service. J Am Heart Assoc. 2014 Feb 26;3(1):e000408. doi: 10.1161/JAHA.113.000408. PMID: 24572251. Exclusion reason: Ineligible intervention

22. Agarwal S, Tamrat T, Glenton C, et al. Decision-support tools via mobile devices to improve quality of care in primary healthcare settings. Cochrane Database of Syst Rev. 2018(2) PMID: 00075320100000000-11357. Exclusion reason: Ineligible study design

23. Agasthya N, Foo K, Smith T, et al. Use of telemedicine in the pediatric intensive care unit. Crit Care Med. 2018;Conference: 47th society of critical care medicine critical care congress, SCCM. 2018. United states 46(Supplement 1):189. PMID: CN01452195. Exclusion reason: Ineligible publication type

24. Ahmed A, Slosberg E, Prasad P, et al. The successful use of telemedicine in acute variceal hemorrhage. J Clin Gastroenterol. 1999 Sep;29(2):212-3. PMID: 10478893.

Exclusion reason: Ineligible study design

25. Akbik F, Hirsch JA, Chandra RV, et al. Telestroke-the promise and the challenge. Part two-expansion and horizons. J Neurointerv Surg. 2017 Apr;9(4):361-5. doi: 10.1136/neurintsurg-2016-012340. PMID: 26984867. Exclusion reason: Ineligible publication type
26. Akbik F, Hirsch JA, Chandra RV, et al. Telestroke-the promise and the challenge. Part one: growth and current practice. J Neurointerv Surg. 2017 Apr;9(4):357-60. doi: 10.1136/neurintsurg-2016-012291. PMID: 26984868. Exclusion reason: Ineligible publication type

27. Akematsu Y, Nitta S, Morita K, et al. Empirical analysis of the long-term effects of telecare use in Nishi-aizu Town, Fukushima Prefecture, Japan. Technol Health Care. 2013;21(2):173-82. doi: 10.3233/THC-130728. PMID: 23510979. Exclusion reason: Ineligible intervention

28. Akematsu Y, Tsuji M. Does telecare reduce the number of treatment days? Stud Health Technol Inform. 2012;180:507-11. PMID: 22874242. Exclusion reason: Ineligible intervention

29. Akematsu Y, Tsuji M. Measuring the effect of telecare on medical expenditures without bias using the propensity score matching method. Telemed J E Health. 2012 Dec;18(10):743-7. doi: 10.1089/tmj.2012.0019. PMID: 23072633. Exclusion reason: Ineligible intervention

30. Akematsu Y, Tsuji M. Relation between telecare implementation and number of treatment days in a Japanese town. J Telemed Telecare. 2013 Jan;19(1):36-9. doi: 10.1177/1357633X12474743. PMID: 23390215. Exclusion reason: Ineligible intervention

31. $\mathrm{Al}$ Kasab S, Harvey JB, Debenham E, et al. Door to needle time over telestroke- a comprehensive stroke center experience. Telemed J E Health. 2018 Feb;24(2):111-5. doi: 10.1089/tmj.2017.0067. PMID: 28753069. Exclusion reason: Ineligible intervention

32. AlAzab R, Khader Y. Telenephrology application in rural and remote areas of Jordan: benefits and impact on quality of life. Rural Remote Health. 2016 JanMar;16(1):3646. PMID: 26745230. Exclusion reason: Ineligible comparison

33. Allen A, Wheeler T. Telepsychiatry background and activity survey. The development of telepsychiatry. Telemed Today. 1998 Apr-May;6(2):34-7. PMID: 10181178. Exclusion reason: Ineligible comparison 
34. Amarenco P, Nadjar M. Telemedicine for improving emergent management of acute cerebrovascular syndromes. Int J Stroke. 2007;2(1):47-50. doi: 10.1111/j.17474949.2007.00087.x. PMID: 18705988.

Exclusion reason: Ineligible outcome

35. Amoakoh-Coleman M, Borgstein AB, Sondaal SF, et al. Effectiveness of mHealth interventions targeting health care workers to improve pregnancy outcomes in low- and middle-income countries: A systematic review. J Med Internet Res. 2016 Aug 19;18(8):e226. doi: 10.2196/jmir.5533. PMID: 27543152. Exclusion reason: Systematic review used to identify primary studies

36. Andonegui J, Aliseda D, Serrano L, et al. Evaluation of a telemedicine model to follow up patients with exudative agerelated macular degeneration. Retina. 2016 Feb;36(2):279-84. doi: 10.1097/IAE.0000000000000729. PMID: 26383707. Exclusion reason: Ineligible comparison

37. Andrews V. Using telemedicine in clinical decision-making. Practice Nursing. 2014;25(1):42-6. Exclusion reason: Ineligible comparison

38. Anogianakis G, Maglavera S. Medical emergency aid through telematics (MERMAID). Stud Health Technol Inform. 1996;29:255-64. PMID: 10163758.

Exclusion reason: Ineligible comparison

39. Anonymous. Saving sight and lives online. BMJ. 2017 Jan 09;356:j42. doi: 10.1136/bmj.j42. PMID: 28069579. Exclusion reason: Ineligible publication type

40. Arba F, Piccardi B, Baldereschi M, et al. Telemedicine for acute ischaemic stroke. Cochrane Database Syst Rev. 2016(2) PMID: 00075320-100000000-10477. Exclusion reason: Ineligible publication type
41. Armfield NR, Coulthard MG, Slater A, et al. The effectiveness of telemedicine for paediatric retrieval consultations: rationale and study design for a pragmatic multicentre randomised controlled trial. BMC Health Serv Res. 2014 Nov 11;14:546. doi: 10.1186/s12913-014-0546-9. PMID: 25381774. Exclusion reason: Background information only

42. Armstrong AW, Dorer DJ, Lugn NE, et al. Economic evaluation of interactive teledermatology compared with conventional care. Telemed J E Health. 2007 Apr;13(2):91-9. doi: 10.1089/tmj.2006.0035. PMID: 17489695. Exclusion reason: Ineligible intervention

43. Armstrong IJ, Haston WS. Medical decision support for remote general practitioners using telemedicine. J Telemed Telecare. 1997;3(1):27-34. doi: 10.1258/1357633971930166. PMID: 9139758. Exclusion reason: Ineligible comparison

44. Armstrong IJ, Haston WS, Maclean JR. Telepresence for decision support offshore. J Telemed Telecare. 1996;2(3):176-7. PMID: 9375055. Exclusion reason: Ineligible publication type

45. Arora S, Geppert CM, Kalishman S, et al. Academic health center management of chronic diseases through knowledge networks: Project ECHO. Acad Med. 2007 Feb;82(2):154-60. doi: 10.1097/ACM.0b013e31802d8f68. PMID: 17264693. Exclusion reason: Background information only

46. Assis TG, Palhares DM, Alkmim MB, et al. Teledermatology for primary care in remote areas in Brazil. J Telemed Telecare. 2013 Dec;19(8):494-5. doi: 10.1177/1357633X13512059. PMID: 24197397. Exclusion reason: Ineligible comparison

47. Atlas I, Granai CO, Gajewski W, et al. Videoconferencing for gynaecological cancer care: an international tumour board. J Telemed Telecare. 2000;6(4):242-4. doi: 10.1258/1357633001935310. PMID: 11027128. Exclusion reason: Ineligible study design 
48. Audebert H. Telestroke: effective networking. Lancet Neurol. 2006 Mar;5(3):279-82. doi: 10.1016/S14744422(06)70378-7. PMID: 16488384.

Exclusion reason: Ineligible study design

49. Audebert HJ, Kukla C, Clarmann von Claranau S, et al. Telemedicine for safe and extended use of thrombolysis in stroke: the Telemedic Pilot Project for Integrative Stroke Care (TEMPiS) in Bavaria. Stroke. 2005 Feb;36(2):287-91. doi:

10.1161/01.STR.0000153015.57892.66. PMID: 15625294. Exclusion reason: Ineligible comparison

50. Audebert HJ, Kukla C, Vatankhah B, et al. Comparison of tissue plasminogen activator administration management between Telestroke Network hospitals and academic stroke centers: the Telemedical Pilot Project for Integrative Stroke Care in Bavaria/Germany. Stroke. 2006 Jul;37(7):1822-7. doi: 10.1161/01.STR.0000226741.20629.b2. PMID: 16763192. Exclusion reason: Background information only

51. Audebert HJ, Wimmer MLJ, Hahn R, et al. Can telemedicine contribute to fulfill WHO Helsingborg Declaration of Specialized Stroke Care? Cerebrovasc Dis. 2005;20(5):362-9. PMID: 16141717.

Exclusion reason: No new data

52. Ausayakhun S, Skalet AH, Jirawison C, et al. Accuracy and reliability of telemedicine for diagnosis of cytomegalovirus retinitis. Am J Ophthalmol. 2011;152(6):1053-8.e1. PMID: 21861977. Exclusion reason: Ineligible outcome

53. Ayiasi RM, Kolsteren P, Batwala V, et al. Effect of Village Health Team home visits and mobile phone consultations on maternal and newborn care practices in Masindi and Kiryandongo, Uganda: A communityintervention trial. PLoS One. 2016;11(4). doi: 10.1371/journal.pone.0153051. PMID: 27101379. Exclusion reason: Ineligible intervention
54. Azzolini C, Torreggiani A, Eandi C, et al. A teleconsultation network improves the efficacy of anti-VEGF therapy in retinal diseases. J Telemed Telecare. 2013 Dec;19(8):437-42. doi: 10.1177/1357633X13501760. PMID: 24162839. Exclusion reason: Ineligible intervention

55. Baer CA, Williams CM, Vickers L, et al. A pilot study of specialized nursing care for home health patients. J Telemed Telecare. 2004;10(6):342-5. doi: 10.1258/1357633042602099. PMID: 15603632. Exclusion reason: Ineligible outcome

56. Bagot KL, Bladin CF, Vu M, et al. Exploring the benefits of a stroke telemedicine programme: An organisational and societal perspective. J Telemed Telecare. 2016 Dec;22(8):489-94. doi: 10.1177/1357633X16673695. PMID: 27799453. Exclusion reason: Ineligible study design

57. Baker LC, Johnson SJ, Macaulay D, et al. Integrated telehealth and care management program for Medicare beneficiaries with chronic disease linked to savings. Health Aff (Millwood). 2011 Sep;30(9):1689-97. doi: 10.1377/hlthaff.2011.0216. PMID: 21900660. Exclusion reason: Ineligible intervention

58. Baker LC, Macaulay DS, Sorg RA, et al. Effects of care management and telehealth: a longitudinal analysis using medicare data.[Erratum appears in J Am Geriatr Soc. 2013 Nov;61(11):2062]. J Am Geriatr Soc. 2013 Sep;61(9):1560-7. doi: 10.1111/jgs.12407. PMID: 24028359.

Exclusion reason: Ineligible intervention

59. Barbieri JS, Nelson CA, James WD, et al. The reliability of teledermatology to triage inpatient dermatology consultations. JAMA Dermatol. 2014 Apr;150(4):419-24. doi: 10.1001/jamadermatol.2013.9517. PMID: 24522374. Exclusion reason: Ineligible comparison 
60. Barbour PJ, Arroyo J, High S, et al. Telehealth for patients with Parkinson's disease: delivering efficient and sustainable long-term care. Hospital practice (1995) Hospital practice. 2016;44(2):92-7. doi: 10.1080/21548331.2016.1166922. PMID: 26982525. Exclusion reason: Ineligible comparison

61. Barnett ML, Yee Jr HF, Mehrotra A, et al. Los Angeles safety-net program eConsult system was rapidly adopted and decreased wait times to see specialists. Health Aff (Millwood). 2017;76(4):492-9. doi: 10.1377/hlthaff.2016.1283. PMID: 28264951. Exclusion reason: Ineligible comparison

62. Baruffaldi F, Gualdrini G, Toni A. Comparison of asynchronous and realtime teleconsulting for orthopaedic second opinions. J Telemed Telecare. 2002;8(5):297-301. doi: 10.1177/1357633X0200800509. PMID: 12396859. Exclusion reason: Ineligible comparison

63. Bashshur RL, Howell JD, Krupinski EA, et al. The empirical foundations of telemedicine interventions in primary care. Telemed J E Health. 2016 May;22(5):34275. doi: 10.1089/tmj.2016.0045. PMID: 27128779. Exclusion reason: Background information only

64. Bau CT, Chen RC, Huang CY. Construction of a clinical decision support system for undergoing surgery based on domain ontology and rules reasoning. Telemed J E Health. 2014 May;20(5):460-72. doi: 10.1089/tmj.2013.0221. PMID: 24730353. Exclusion reason: Ineligible intervention

65. Bauer MS, Krawczyk L, Tuozzo K, et al. Implementing and sustaining team-based telecare for bipolar disorder: lessons learned from a model-guided, mixed methods analysis. Telemed J E Health. 2018 Jan;24(1):45-53. doi: 10.1089/tmj.2017.0017. PMID: 28665773. Exclusion reason: Ineligible comparison
66. Bekelman DB, Plomondon ME, Carey EP, et al. Primary results of the Patient-Centered Disease Management (PCDM) for Heart Failure Study: A randomized clinical trial. JAMA Internal Medicine. 2015

May;175(5):725-32. doi: 10.1001/jamainternmed.2015.0315. PMID: 25822284. Exclusion reason: Ineligible intervention

67. Bell RC, Yager PH, Clark ME, et al. Telemedicine versus face-to-face evaluations by respiratory therapists of mechanically ventilated neonates and children: A pilot study. Respir Care. 2016 Feb;61(2):149-54. doi: 10.4187/respcare.04080. PMID: 26647456. Exclusion reason: Ineligible outcome

68. Benger JR, Noble SM, Coast J, et al. The safety and effectiveness of minor injuries telemedicine. Emerg Med J. 2004 Jul;21(4):438-45. PMID: 15208226.

Exclusion reason: Ineligible comparison

69. Benson GA, Sidebottom A, Sillah A, et al. Reach and effectiveness of the HeartBeat Connections telemedicine pilot program. J Telemed Telecare. 2018 Apr;24(3):216-23. doi: 10.1177/1357633X17692723. PMID: 29278986. Exclusion reason: Ineligible intervention

70. Bentley CL, Mountain GA, Thompson J, et al. A pilot randomised controlled trial of a Telehealth intervention in patients with chronic obstructive pulmonary disease: challenges of clinician-led data collection. Trials. 2014;15:313. doi: 10.1186/17456215-15-313. PMID: 25100550. Exclusion reason: Ineligible intervention

71. Bilgi M, Erol T, Gullu H, et al. Teleconsultation of coronary angiograms using smartphones and an audio/video conferencing application. Technol Health Care. 2013;21(4):407-14. doi:

10.3233/THC-130733. PMID: 23949176. Exclusion reason: Ineligible intervention

72. Black-Schaffer S, Flotte TJ.

Teledermatopathology. Adv Dermatol. 2001;17:325-38. PMID: 11758122.

Exclusion reason: Ineligible study design

73. Blair R. Psychotherapy online. Health Manag Technol. 2001 Feb;22(2):24-7. PMID: 11225332. Exclusion reason: Ineligible publication type 
74. Bolle SR, Lien AH, Mjaaseth R, et al. Videobased emergency medical interaction. Tidsskr Nor Laegeforen. 2013 Jan 22;133(2):136-8. doi:

10.4045/tidsskr.12.1274. PMID: 23344594.

Exclusion reason: Ineligible comparison

75. Borooah S, Grant B, Blaikie A, et al. Using electronic referral with digital imaging between primary and secondary ophthalmic services: a long term prospective analysis of regional service redesign. Eye (Lond). 2013 Mar;27(3):392-7. doi:

10.1038/eye.2012.278. PMID: 23258310.

Exclusion reason: Ineligible intervention

76. Bott MJ, James B, Collins BT, et al. A prospective clinical trial of telecytopathology for rapid interpretation of specimens obtained during endobronchial ultrasound-fine needle aspiration. Ann Thorac Surg. 2015 Jul;100(1):201-5; discussion 5-6. doi:

10.1016/j.athoracsur.2015.02.090. PMID: 26002445. Exclusion reason: Ineligible intervention

77. Bowen ME, Bosworth HB, Roumie CL. Blood pressure control in a hypertension telemedicine intervention: does distance to primary care matter? J Clin Hypertens. 2013 Oct;15(10):723-30. doi: 10.1111/jch.12172. PMID: 24088280. Exclusion reason: Ineligible intervention

78. Bowry R, Parker S, Rajan SS, et al. Benefits of stroke treatment using a mobile stroke unit compared with standard management: The BEST-MSU study run-in phase. Stroke. 2015 Dec;46(12):3370-4. doi:

10.1161/STROKEAHA.115.011093. PMID: 26508753. Exclusion reason: Ineligible intervention

79. Boxer RJ. Telemedicine: remote cancer care improves communication. Oncology Times. 2017;47(1):1-15. doi:

10.1097/01.COT.0000512175.80464.3f.

Exclusion reason: Ineligible comparison

80. Bradford NK, Armfield NR, Young J, et al. Paediatric palliative care by video consultation at home: a cost minimisation analysis. BMC Health Serv Res. 2014 Jul 28;14:328. doi: 10.1186/1472-6963-14-328. PMID: 25069399. Exclusion reason:

Background information only
81. Bradford NK, Caffery LJ, Smith AC. Telehealth services in rural and remote Australia: a systematic review of models of care and factors influencing success and sustainability. Rural \& Remote Health. 2016 Oct-Dec;16(4):4268. PMID: 27817199.

Exclusion reason: Systematic review used to identify primary studies

82. Branger PJ, van't Hooft A, van der Wouden JC, et al. Shared care for diabetes:

supporting communication between primary and secondary care. Int J Med Inform. 1999 Feb-Mar;53(2-3):133-42. PMID: 10193883. Exclusion reason: Ineligible intervention

83. Brebner JA, Brebner EM, Ruddick-Bracken $\mathrm{H}$. Accident and emergency teleconsultation for primary care--a systematic review of technical feasibility, clinical effectiveness, cost effectiveness and level of local management. J Telemed Telecare. 2006;12 Suppl 1(1):5-8. doi: 10.1258/135763306777978542. PMID: 16884562. Exclusion reason: Systematic review used to identify primary studies

84. Breen P, Murphy K, Browne G, et al. Formative evaluation of a telemedicine model for delivering clinical neurophysiology services part I: utility, technical performance and service provider perspective. BMC Med Inform Decis Mak. 2010 Sep 15;10(1):48. doi: 10.1186/14726947-10-48. PMID: 20843309. Exclusion reason: Ineligible comparison

85. Breen P, Murphy K, Browne G, et al. Formative evaluation of a telemedicine model for delivering clinical neurophysiology services part II: the referring clinician and patient perspective. BMC Med Inform Decis Mak. 2010;10:49. doi: 10.1186/1472-6947-10-49. PMID: 20843310. Exclusion reason: Ineligible intervention

86. Brennan PF. Telehealth: bringing health care to the point of living. Med Care. 1999 Feb;37(2):115-6. PMID: 10024115. Exclusion reason: Ineligible study design

87. Britain CS. Making the connection in rural mental health. Behav Healthc Tomorrow. 1996 Aug;5(4):67-9. PMID: 10159331. Exclusion reason: Ineligible comparison 
88. Britt DW. A qualitative comparative analysis of the conditions affecting early maternal transfer patterns. J Telemed Telecare. 2006;12(8):392-5. doi: 10.1258/135763306779378753. PMID: 17227603. Exclusion reason: Ineligible comparison

89. Brunetti ND, De Gennaro L, Correale M, et al. Pre-hospital electrocardiogram triage with telemedicine near halves time to treatment in STEMI: A meta-analysis and meta-regression analysis of non-randomized studies. Int J Cardiol. 2017 Apr 01;232:5-11. doi: 10.1016/j.ijcard.2017.01.055 PMID: 28089154. Exclusion reason: Systematic review used to identify primary studies

90. Bruns J. Calling for automation. Pediatric call center at a Missouri children's hospital sees lower call times and higher productivity from a fully automated system. Health Manag Technol. 2003 Oct;24(10):22, 5-7. PMID: 14535120. Exclusion reason: Ineligible comparison

91. Bryld LE, Heidenheim M, Dam TN, et al. Teledermatology with an integrated nurseled clinic on the Faroe Islands--7 years' experience. J Eur Acad Dermatol Venereol. 2011 Aug;25(8):987-90. doi:

10.1111/j.1468-3083.2010.03884.x. PMID: 21740465. Exclusion reason: Ineligible outcome

92. Buchman TG, Coopersmith CM, Meissen $\mathrm{HW}$, et al. Innovative interdisciplinary strategies to address the intensivist shortage. Crit Care Med. 2017;45(2):298-304. doi: 10.1097/ccm.0000000000002209. PMID: 28098625. Exclusion reason: Ineligible publication type

93. Burgiss SG, Smith GT, Dimmick SL, et al. Improving telepresence during consultations. Telemed Today. 1998 Aug;6(4):14-5. PMID: 10182370. Exclusion reason: Ineligible comparison

94. Butler LC, Wong A, Whitfill T, et al. The impact of a telepresent team leader on pediatric resuscitation: a randomized controlled trial. Ann Emerg Med. 2016;Conference: american college of emergency physicians, ACEP. 2016 research forum. United states 68(4 Supplement 1):S83-S4. Exclusion reason: Ineligible intervention
95. Byng R, Jones R, Leese M, et al. Exploratory cluster randomised controlled trial of shared care development for longterm mental illness. Br J Gen Pract. 2004;54(501):259-66. PMID: 15113492. Exclusion reason: Ineligible intervention

96. Caffery LJ, Bradford NK, Wickramasinghe SI, et al. Outcomes of using telehealth for the provision of healthcare to Aboriginal and Torres Strait Islander people: a systematic review. Aust N Z J Public Health. 2017 Feb;41(1):48-53. doi: 10.1111/17536405.12600. PMID: 27868300. Exclusion reason: Ineligible outcome

97. Caffery LJ, Farjian M, Smith AC. Telehealth interventions for reducing waiting lists and waiting times for specialist outpatient services: A scoping review. J Telemed Telecare. 2016;42(7):504-12. doi: 10.1177/1357633X16670495. PMID: 27686648. Exclusion reason: Ineligible study design

98. Callahan CW, Malone F, Estroff D, et al. Effectiveness of an Internet-based store-andforward telemedicine system for pediatric subspecialty consultation. Arch Pediatr Adolesc Med. 2005 Apr;159(4):389-93. doi: 10.1001/archpedi.159.4.389. PMID: 15809396. Exclusion reason: Ineligible study design

99. Callahan EJ, Hilty DM, Nesbitt TS. Patient satisfaction with telemedicine consultation in primary care: comparison of ratings of medical and mental health applications. Telemed J. 1998 Winter;4(4):363-9. doi: 10.1089/tmj.1.1998.4.363. PMID: 10220477. Exclusion reason: Ineligible outcome

100. Campana BA, Jarvis-Selinger S, Ho K, et al. Use of telemedicine for an emergency craniotomy in a pediatric trauma. CMAJ. 2004 Aug 31;171(5):444-6. doi: 10.1503/cmaj.1040006. PMID: 15337721. Exclusion reason: Ineligible publication type

101. Campanella N, Morosini P, Sampaolo G, et al. Medical teleconsultation to general practitioners reduces the medical error vulnerability of internal medicine patients. Eur J Intern Med. 2015 Nov;26(9):675-9. doi: 10.1016/j.ejim.2015.08.010. PMID: 26329761. Exclusion reason: Ineligible comparison 
102. Cape J, Whittington C, Bower P. What is the role of consultation-liaison psychiatry in the management of depression in primary care? A systematic review and meta-analysis. Gen Hosp Psychiatry. 2010 May-Jun;32(3):24654. doi: 10.1016/j.genhosppsych.2010.02.003. PMID: 20430227. Exclusion reason: Ineligible intervention

103. Capurro D, Ganzinger M, Perez-Lu J, et al. Effectiveness of eHealth interventions and information needs in palliative care: a systematic literature review. J Med Internet Res. 2014;16(3):e72. doi: 10.2196/jmir.2812. PMID: 24610324. Exclusion reason: Ineligible intervention

104. Caputo MP, Jr. Saving lives with teletrauma video communications. Health Manag Technol. 2005 Sep;26(9):40-1. PMID: 16218278. Exclusion reason: Ineligible comparison

105. Carlisle D. Technology: distant voices, saved lives. Health Serv J. 2013 Mar 14;123(6343):Suppl 6-7. PMID: 23898746. Exclusion reason: Ineligible publication type

106. Cassimatis M, Kavanagh DJ. Effects of type 2 diabetes behavioural telehealth interventions on glycaemic control and adherence: a systematic review. J Telemed Telecare. 2012 Dec;18(8):447-50. doi: 10.1258/jtt.2012.GTH105. PMID: 23209266. Exclusion reason: Ineligible intervention

107. Castela E, Ramalheiro G, Pires A, et al. Five years of teleconsultation: experience of the Cardiology Department of Coimbra Pediatric Hospital. Rev Port Cardiol. 2005 Jun;24(6):835-40. PMID: 16121675.

Exclusion reason: Ineligible comparison

108. Castro A, Larrain A, Fritsch R, et al. [Feasibility of implementing tele-psychiatry in Chile]. Rev Med Chil. 2012 Jun;140(6):789-96. doi: 10.4067/S003498872012000600015. PMID: 23282619. Exclusion reason: Not in English
109. Catic AG, Mattison ML, Bakaev I, et al. ECHO-AGE: an innovative model of geriatric care for long-term care residents with dementia and behavioral issues. J Am Med Dir Assoc. 2014 Dec;15(12):938-42. doi: 10.1016/j.jamda.2014.08.014. PMID: 25306294. Exclusion reason: Ineligible comparison

110. Chalmers A, Arnold H. The implementation and evaluation of a pilot project in telerheumatology [abstract]. J Rheumatol. 2010 Jun;37(6):1322; CRA Meeting Proceedings. Exclusion reason: Ineligible publication type

111. Chan FY, Soong B, Lessing K, et al. Clinical value of real-time tertiary fetal ultrasound consultation by telemedicine: preliminary evaluation. Telemed J. 2000 Summer;6(2):237-42. doi: 10.1089/107830200415171. PMID: 10957736. Exclusion reason: Ineligible comparison

112. Chan FY, Soong B, Watson D, et al. Realtime fetal ultrasound by telemedicine in Queensland. A successful venture? J Telemed Telecare. 2001;7 Suppl 2:7-11. doi: 10.1258/1357633011937290. PMID: 11747644. Exclusion reason: Ineligible comparison

113. Chan HH, Woo J, Chan WM, et al. Teledermatology in Hong Kong: a costeffective method to provide service to the elderly patients living in institutions. Int J Dermatol. 2000 Oct;39(10):774-8. PMID: 11095200. Exclusion reason: Ineligible comparison

114. Chang LW, Kagaayi J, Arem H, et al. Impact of a mHealth intervention for peer health workers on AIDS care in rural Uganda: a mixed methods evaluation of a cluster-randomized trial. AIDS Behav. 2011 Nov;15(8):1776-84. doi: 10.1007/s10461011-9995-x. PMID: 21739286. Exclusion reason: Ineligible intervention

115. Charrier N, Zarca K, Durand-Zaleski I, et al. Efficacy and cost effectiveness of telemedicine for improving access to care in the Paris region: study protocols for eight trials. BMC Health Serv Res. 2016;16:45. doi: 10.1186/s12913-016-1281-1. PMID: 26857558. Exclusion reason: Ineligible publication type 
116. Chavooshi B, Mohammadkhani P, Dolatshahee B. Telemedicine vs. in-person delivery of intensive short-term dynamic psychotherapy for patients with medically unexplained pain: A 12-month randomized, controlled trial. J Telemed Telecare. 2017 Jan;23(1):133-41. doi: 10.1177/1357633X15627382. PMID: 27036877. Exclusion reason: Ineligible intervention

117. Chen HF, Kalish MC, Pagan JA. Telehealth and hospitalizations for Medicare home healthcare patients. Am J Manag Care. 2011 Jun;17(6 Spec No.):e224-30. PMID: 21756016. Exclusion reason: Ineligible intervention

118. Chen J, Jin W, Zhang XX, et al. Telerehabilitation approaches for stroke patients: systematic review and metaanalysis of randomized controlled trials. J Stroke Cerebrovasc Dis. 2015

Dec;24(12):2660-8. doi:

10.1016/j.jstrokecerebrovasdis.2015.09.014. PMID: 26483155. Exclusion reason: Ineligible intervention

119. Chen KC, Yen DH, Chen CD, et al. Effect of emergency department in-hospital teleelectrocardiographic triage and interventional cardiologist activation of the infarct team on door-to-balloon times in STsegment-elevation acute myocardial infarction. Am J Cardiol. 2011 May 15;107(10):1430-5. doi: 10.1016/j.amjcard.2011.01.015. PMID: 21414598. Exclusion reason: Ineligible intervention

120. Chen SY, Chang YH, Hsu HC, et al. Oneyear efficacy and safety of the telehealth system in poorly controlled type 2 diabetic patients receiving insulin therapy. Telemed $\mathrm{J}$ E Health. 2011 Nov;17(9):683-7. doi: 10.1089/tmj.2011.0020 PMID: 21882998.

Exclusion reason: Ineligible intervention

121. Chen TS, Goldyne ME, Mathes EF, et al. Pediatric teledermatology: observations based on 429 consults. J Am Acad Dermatol. 2010 Jan;62(1):61-6. doi: 10.1016/j.jaad.2009.05.039. PMID: 19926163. Exclusion reason: Ineligible outcome
122. Chin TL. Straight from the heart. Health Data Manag. 1998 May;6(5):108-9. PMID: 10179879. Exclusion reason: Ineligible publication type

123. Chipps J, Brysiewicz P, Mars M. Effectiveness and feasibility of telepsychiatry in resource constrained environments? A systematic review of the evidence. Afr J Psychiatry. 2012 Jul;15(4):235-43. doi: 10.4314/ajpsy.v15i4.30. PMID: 22829225. Exclusion reason: Systematic review used to identify primary studies

124. Chittle MD, Rao SK, Jaff MR, et al. Asynchronous vascular consultation via electronic methods: A feasibility pilot. Vasc Med. 2015 Dec;20(6):551-6. doi: 10.1177/1358863X15601734. PMID: 26385414. Exclusion reason: Ineligible comparison

125. Choi Yoo SJ, Nyman JA, Cheville AL, et al. Cost effectiveness of telecare management for pain and depression in patients with cancer: results from a randomized trial. Gen Hosp Psychiatry. 2014 Nov-Dec;36(6):599606. doi:

10.1016/j.genhosppsych.2014.07.004 PMID: 25130518. Exclusion reason: Ineligible intervention

126. Chong CA. Using stroke thrombolysis to describe the role of repetition in learning a cognitive skill. Med Educ. 2016 Feb;50(2):250-8. doi: 10.1111/medu.12936. PMID: 26813003. Exclusion reason: Ineligible comparison

127. Ciccia AH, Roizen N, Garvey M, et al. Identification of neurodevelopmental disabilities in underserved children using telehealth (INvesT): Clinical trial study design. Contemp Clin Trials. 2015 Nov;45(Pt B):226-32. doi: 10.1016/j.cct.2015.10.004. PMID: 26475663. Exclusion reason: Ineligible intervention

128. Cichosz SL, Ehlers LH, Hejlesen O. Health effectiveness and cost-effectiveness of telehealthcare for heart failure: study protocol for a randomized controlled trial. Trials. 2016;17(1). doi: 10.1186/s13063016-1722-5. PMID: 27955682. Exclusion reason: Ineligible study design 
129. Clarke PH. A referrer and patient evaluation of a telepsychiatry consultation-liaison service in South Australia. J Telemed Telecare. 1997;3 Suppl 1:12-4. doi: 10.1258/1357633971930788. PMID: 9218368. Exclusion reason: Ineligible intervention

130. Cloutier A. Distance diagnosis in pediatric cardiology: a model for telemedecine implementation. Telemed Today. 2000 JunJul;8(3):20-1. PMID: 11183301. Exclusion reason: Ineligible comparison

131. Colias M. Virtual access. Despite concerns, more physicians are realizing the value of online medical consultations. Getting reimbursed is another story. Mod Healthc. 2004 Jan 26;34(4):48-9. PMID: 14959560. Exclusion reason: Ineligible intervention

132. Cook J, Mullings C, Vowles R, et al. The use of teledentistry to provide GDPs with advice in orthodontics. Dent Update. 2002 Jun;29(5):249-55. PMID: 12096385.

Exclusion reason: Ineligible comparison

133. Cook PF, Emiliozzi S, Waters C, et al. Effects of telephone counseling on antipsychotic adherence and emergency department utilization. Am J Manag Care. 2008 Dec;14(12):841-6. PMID: 19067501.

Exclusion reason: Ineligible intervention

134. Corcoran H, Hui E, Woo J. The acceptability of telemedicine for podiatric intervention in a residential home for the elderly. J Telemed Telecare. 2003;9(3):1469. doi: 10.1258/135763303767149942. PMID: 12877776. Exclusion reason: Ineligible intervention

135. Court JH, Austin MW. Virtual glaucoma clinics: patient acceptance and quality of patient education compared to standard clinics. Clin Ophthalmol. 2015;9:745-9. doi: 10.2147/opth.s75000. PMID: 25987832.

Exclusion reason: Ineligible intervention

136. Coustasse A, Deslich S, Bailey D, et al. A business case for tele-intensive care units. Perm J. 2014 Fall;18(4):76-84. doi: 10.7812/TPP/14-004. PMID: 25662529. Exclusion reason: Ineligible publication type
137. Craig J, Patterson V, Russell C, et al. Interactive videoconsultation is a feasible method for neurological in-patient assessment. Eur J Neurol. 2000 Nov;7(6):699-702. PMID: 11136358. Exclusion reason: Ineligible comparison

138. Cui Y, Doupe M, Katz A, et al. Economic evaluation of Manitoba Health Lines in the management of congestive heart failure. Healthcare Policy $=$ Politiques de sante. 2013 Nov;9(2):36-50. PMID: 24359716. Exclusion reason: Ineligible intervention

139. Currell R, Urquhart C, Wainwright P, et al. Telemedicine versus face to face patient care: effects on professional practice and health care outcomes. Cochrane Database Syst Rev. 2000(2):CD002098. doi: 10.1002/14651858.CD002098. PMID: 10796678. Exclusion reason: Ineligible study design

140. Cutting S, Conners JJ, Lee VH, et al. Telestroke in an urban setting. Telemed J E Health. 2014 Sep;20(9):855-7. doi: 10.1089/tmj.2013.0348. PMID: 24968197. Exclusion reason: Ineligible comparison

141. Da Silva R, Rados D, Dos Santos E, et al. Teleconsultation support patients with benign prostatic hyperplasia being discharged from specialized care: a randomized noninferiority study. J Urol. 2018;Conference: 2018 Annual Meeting, American Urological Association, AUA 2018. United states 199(4 Supplement 1):e686-e7. Exclusion reason: Ineligible publication type

142. da Silva VD. A remote second-look teleconsultation protocol on breast cytopathology. Pathologica. 1998 Dec;90(6):824-5. PMID: 10221009. Exclusion reason: Ineligible intervention

143. Dadosky A, Overbeck H, Barbetta L, et al. Telemanagement of heart failure patients across the post-acute care continuum. Telemed J E Health. 2018 May;24(5):360-6. doi: 10.1089/tmj.2017.0058. PMID: 28910238. Exclusion reason: Ineligible intervention

144. Dakins DR. Technology opens 'golden window'. Health Data Manag. 2001 Feb;9(2):128-30, 32. PMID: 11272392. Exclusion reason: Ineligible publication type 
145. Dalfrà MG, Nicolucci A, Lapolla A. The effect of telemedicine on outcome and quality of life in pregnant women with diabetes. J Telemed Telecare.

2009;15(5):238-42. doi:

10.1258/jtt.2009.081213. PMID: 19590029.

Exclusion reason: Ineligible intervention

146. Damji KF, Kawaguchi A, Sharafeldin N, et al. Tele-ophthalmology for age related macular degeneration and diabetic retinopathy: a systematic review and meta analysis. Invest Ophthalmol Vis Sci. 2016;Conference:. 2016 annual meeting of the association for research in vision and ophthalmology, ARVO 2016. United states 57(12):5559. Exclusion reason: Systematic review used to identify primary studies

147. Dang S, Gomez-Orozco CA, van Zuilen $\mathrm{MH}$, et al. Providing dementia consultations to veterans using clinical video telehealth: results from a clinical demonstration project. Telemed J E Health. 2018 Mar;24(3):203-9. doi: 10.1089/tmj.2017.0089. PMID: 28686082. Exclusion reason: Ineligible comparison

148. Dario C, Scannapieco G, Scienza R, et al. The neurosurgical telecounseling network in the Veneto Region: 4 years of experience of HEALTH OPTIMUM. Telemed J E Health. 2014 Nov;20(11):1009-14. doi:

10.1089/tmj.2013.0351. PMID: 25272284.

Exclusion reason: Ineligible comparison

149. Daskivich LP, Vasquez C, Martinez C, Jr., et al. Implementation and evaluation of a large-scale teleretinal diabetic retinopathy screening program in the Los Angeles County Department of Health Services. JAMA Intern Med. 2017 May 01;177(5):642-9. doi: 10.1001/jamainternmed.2017.0204. PMID: 28346590. Exclusion reason: Ineligible intervention

150. Daucourt V, Sicotte C, Pelletier-Fleury N, et al. Cost-minimization analysis of a widearea teleradiology network in a French region. Int J Qual Health Care. 2006 Aug;18(4):287-93. doi: 10.1093/intqhc/mzi075. PMID: 16144793. Exclusion reason: Ineligible comparison
151. David CV. Teledermatology: examples of consultative and direct-to-consumer platforms in action. Cutis. 2013 Apr;91(4):E1-4. PMID: 24195085. Exclusion reason: Ineligible publication type

152. Davis P. The application of telehealth to rheumatology. Clin Rheumatol. 2003 Sep;22(3):168-72. doi: 10.1007/s10067-0030747-9. PMID: 14505205. Exclusion reason: Ineligible comparison

153. Dayhoff RE, Kuzmak PM. Providing complete multimedia patient data to consulting radiologists, other specialists, and the referring clinician. J Digit Imaging. 1998 Aug;11(3 Suppl 1):134-6. PMID: 9735451. Exclusion reason: Ineligible comparison

154. de Araujo JS, Regis CT, Gomes RG, et al. Impact of telemedicine in the screening for congenital heart disease in a center from Northeast Brazil. J Trop Pediatr. 2016 12;62(6):471-6. PMID: 27273306.

Exclusion reason: Ineligible comparison

155. Demaerschalk BM, Raman R, Ernstrom K, et al. Efficacy of telemedicine for stroke: pooled analysis of the Stroke Team Remote Evaluation Using a Digital Observation Camera (STRokE DOC) and STRokE DOC Arizona telestroke trials. Telemed J E Health. 2012 Apr;18(3):230-7. doi: 10.1089/tmj.2011.0116 PMID: 22400970.

Exclusion reason: No new data

156. Demaerschalk BM, Vegunta S, Vargas BB, et al. Reliability of real-time video smartphone for assessing National Institutes of Health Stroke Scale scores in acute stroke patients. Stroke. 2012 Dec;43(12):3271-7. doi: 10.1161/STROKEAHA.112.669150. PMID: 23160878. Exclusion reason: Ineligible intervention

157. DeMaio J, Schwartz L, Cooley P, et al. The application of telemedicine technology to a directly observed therapy program for tuberculosis: a pilot project. Clin Infect Dis. 2001 Dec 15;33(12):2082-4. doi: 10.1086/324506. PMID: 11698993. Exclusion reason: Ineligible intervention

158. Demartines N. Telemedicine applications in surgery. Curr Probl Dermatol. 2003;32:94101. PMID: 12471997. Exclusion reason: Ineligible study design 
159. Demiris G, Parker Oliver D, Kruse RL, et al. Telehealth group interactions in the hospice setting: assessing technical quality across platforms. Telemed J E Health. 2013

Apr;19(4):235-40. doi:

10.1089/tmj.2012.0185. PMID: 23506328.

Exclusion reason: Ineligible intervention

160. Demiris G, Speedie SM, Hicks LL.

Assessment of patients' acceptance of and satisfaction with teledermatology. J Med

Syst. 2004 Dec;28(6):575-9. PMID:

15615285. Exclusion reason: Ineligible study design

161. den Hollander D, Mars M. Smart phones make smart referrals: The use of mobile phone technology in burn care - A

retrospective case series. Burns. 2017

Feb;43(1):190-4. doi:

10.1016/j.burns.2016.07.015. PMID:

27575675. Exclusion reason: Ineligible

comparison

162. Dendale P, Keulenaer GD, Troisfontaines P, et al. Effect of a telemonitoring-facilitated collaboration between general practitioner and heart failure clinic on mortality and rehospitalization rates in severe heart failure: the TEMA-HF 1 (TElemonitoring in the MAnagement of Heart Failure) study. Eur J Heart Fail. 2012;14(3):333-40. doi: doi:10.1093/eurjhf/hfr144. Exclusion reason: Ineligible intervention

163. Denis B, Broc G, Gendre I, et al. Tailored telephone counseling to increase adherence of underusers in an organized colorectal cancer screening program with FOBT: A randomized controlled trial.

Gastroenterology. 2012 Digestive Disease

Week 2012, San Diego, CA United States;142(5 SUPPL. 1):S767-S8. Exclusion reason: Ineligible intervention

164. DerGurahian J. Partners: time to plug in. Mod Healthc. 2007 Oct 15;37(41):7. PMID: 17972490. Exclusion reason: Ineligible intervention

165. Di Stefani A, Zalaudek I, Argenziano G, et al. Feasibility of a two-step teledermatologic approach for the management of patients with multiple pigmented skin lesions. Dermatol Surg. 2007 Jun;33(6):686-92. doi: 10.1111/j.1524-4725.2007.33144.x. PMID: 17550445. Exclusion reason: Ineligible intervention
166. Docimo SG, Moore RG, Adams J, et al. Early experience with telerobotic surgery in children. J Telemed Telecare. 1996;2 Suppl 1:48-50. PMID: 9375090. Exclusion reason: Ineligible study design

167. Donnem T, Ervik B, Magnussen K, et al. Bridging the distance: a prospective teleoncology study in Northern Norway. Support Care Cancer. 2012 Sep;20(9):2097103. doi: 10.1007/s00520-011-1319-1. PMID: 22076621. Exclusion reason: Ineligible comparison

168. Doolittle G. A POTS-based tele-hospice project in Missouri. Telemed Today. 1997 Aug;5(4):18-9. PMID: 10174244. Exclusion reason: Ineligible comparison

169. Doolittle GC, Allen A. From acute leukaemia to multiple myeloma: clarification of a diagnosis using teleoncology. J Telemed Telecare. 1996;2(2):119-21. doi: 10.1177/1357633X9600200209. PMID: 9375073. Exclusion reason: Ineligible study design

170. Doty E, Zincone LH, Jr., Balch DC. Telemedicine in the North Carolina prison system. A cost-benefits analysis. Stud Health Technol Inform. 1996;29:239-41. PMID: 10163755. Exclusion reason: Ineligible study design

171. Drummond D, Arnaud C, Guedj R, et al. Google glass for residents dealing with pediatric cardiopulmonary arrest: a randomized, controlled, simulation-based study. Pediatr Crit Care Med.

2017;18(2):120-7. doi: 10.1097/PCC.0000000000000977. PMID: 8165347. Exclusion reason: Ineligible population

172. Ducas RA, Philipp RK, Jassal DS, et al. Cardiac Outcomes Through Digital Evaluation (CODE) STEMI project: prehospital digitally-assisted reperfusion strategies. Can J Cardiol. 2012 JulAug;28(4):423-31. doi: 10.1016/j.cjca.2012.02.005. PMID: 22494815. Exclusion reason: Ineligible intervention 
173. Dullet NW, Geraghty EM, Kaufman T, et al. Impact of a university-based outpatient telemedicine program on time savings, travel costs, and environmental pollutants. Value Health. 2017 Apr;20(4):542-6. doi: 10.1016/j.jval.2017.01.014. PMID: 28407995. Exclusion reason: Ineligible comparison

174. Duran A, Runkle I, Matía P, et al. Family physician and endocrinologist coordination as the basis for diabetes care in clinical practice. BMC Endocr Disord. 2008;8:9-. doi: 10.1186/1472-6823-8-9. PMID: 18671870. Exclusion reason: Ineligible intervention

175. Dutta D, Kendall J, Holmes C, et al. Evaluation of a telephone advice system for remote intravenous thrombolysis in ischemic stroke: data from a United kingdom network. Stroke. 2015 Mar;46(3):867-9. doi: 10.1161/STROKEAHA.114.008190. PMID: 25604248. Exclusion reason: Ineligible intervention

176. Dy P, Morin PC, Weinstock RS. Use of telemedicine to improve glycemic management in a skilled nursing facility: a pilot study. Telemed J E Health. 2013 Aug;19(8):643-5. doi: 10.1089/tmj.2012.0274. PMID: 23758078.

Exclusion reason: Ineligible outcome

177. Eaton LH, Gordon DB, Wyant S, et al. Development and implementation of a telehealth-enhanced intervention for pain and symptom management. Contemp Clin Trials. 2014 Jul;38(2):213-20. doi: 10.1016/j.cct.2014.05.005. PMID: 24846620. Exclusion reason: Ineligible outcome

178. Ebinger M, Fiebach JB, Audebert HJ. Mobile computed tomography: prehospital diagnosis and treatment of stroke. Curr Opin Neurol. 2015 Feb;28(1):4-9. doi: 10.1097/WCO.0000000000000165. PMID: 25490196. Exclusion reason: Ineligible study design

179. Ebinger M, Winter B, Wendt M, et al. Effect of the use of ambulance-based thrombolysis on time to thrombolysis in acute ischemic stroke: a randomized clinical trial. JAMA. 2014 Apr 23-30;311(16):1622-31. doi: 10.1001/jama.2014.2850. PMID: 24756512. Exclusion reason: Ineligible intervention
180. Edison KE, Ward DS, Dyer JA, et al. Diagnosis, diagnostic confidence, and management concordance in live-interactive and store-and-forward teledermatology compared to in-person examination. Telemed J E Health. 2008 Nov;14(9):88995. doi: 10.1089/tmj.2008.0001. PMID: 19035797. Exclusion reason: Ineligible intervention

181. Eedy DJ, Wootton R. Teledermatology: a review. Br J Dermatol. 2001 Apr;144(4):696-707. PMID: 11298526. Exclusion reason: Ineligible study design

182. Egede LE, Acierno R, Knapp RG, et al. Psychotherapy for depression in older veterans via telemedicine: effect on quality of life, satisfaction, treatment credibility, and service delivery perception. J Clin Psychiatry. 2016 Dec;77(12):1704-11. doi: 10.4088/JCP.16m10951. PMID: 27835713. Exclusion reason: Ineligible intervention

183. Egede LE, Gebregziabher M, Walker RJ, et al. Trajectory of cost overtime after psychotherapy for depression in older veterans via telemedicine. J Affect Disord. 2017;207:157-62. doi:

10.1016/j.jad.2016.09.044. PMID:

27721190. Exclusion reason: Ineligible intervention

184. Egede LE, Williams JS, Voronca DC, et al. Randomized controlled trial of technologyassisted case management in low income adults with type 2 diabetes. Diabetes Technol Ther. 2017;19(8):476-82. doi: 10.1089/dia.2017.0006. PMID: 28581821. Exclusion reason: Ineligible intervention

185. Ehlers L, Muskens WM, Jensen LG, et al. National use of thrombolysis with alteplase for acute ischaemic stroke via telemedicine in Denmark: a model of budgetary impact and cost effectiveness. CNS Drugs. 2008;22(1):73-81. PMID: 18072816. Exclusion reason: Ineligible study design

186. Eibl JK, Gauthier G, Pellegrini D, et al. The effectiveness of telemedicine-delivered opioid agonist therapy in a supervised clinical setting. Drug Alcohol Depend. 2017 Jul 01;176:133-8. doi: 10.1016/j.drugalcdep.2017.01.048 PMID: 28535455. Exclusion reason: Ineligible intervention 
187. Eik Filho W, Bonjorno LP, Franco AJ, et al. Evaluation, intervention, and follow-up of patients with diabetes in a primary health care setting in Brazil: the importance of a specialized mobile consultancy. Diabetol Metab Syndr. 2016;8(1):56. doi: 10.1186/s13098-016-0173-1. PMID: 27508006. Exclusion reason: Ineligible intervention

188. Ellis DG, Mayrose J, Jehle DV, et al. A telemedicine model for emergency care in a short-term correctional facility. Telemed J E Health. 2001 Summer;7(2):87-92. doi: 10.1089/153056201750279584. PMID: 11421081. Exclusion reason: Ineligible comparison

189. Estai M, Kanagasingam Y, Tennant M, et al. A systematic review of the research evidence for the benefits of teledentistry. $\mathrm{J}$ Telemed Telecare. 2018 Apr;24(3):147-56. doi: 10.1177/1357633X16689433. PMID: 28118778. Exclusion reason: Systematic review used to identify primary studies

190. Etter M, Feussner H, Siewert JR. Guidelines for teleconsultation in surgery. The German experience. Surg Endosc. 1999 Dec;13(12):1254-5. PMID: 10594281. Exclusion reason: Ineligible study design

191. Evangelista A, Galuppo V, Mendez J, et al. Hand-held cardiac ultrasound screening performed by family doctors with remote expert support interpretation.[Erratum appears in Heart. 2016 May 1;102(9):728; PMID: 27073249]. Heart. 2016 Mar;102(5):376-82. doi: 10.1136/heartjnl2015-308421. PMID: 26802099. Exclusion reason: Ineligible intervention

192. Ezzeddine B, Hymon B, Morneault J, et al. Kettering Medical Center advances cardiac care services. J Cardiovasc Manag. 1999 Jul-Aug;10(4):35-7. PMID: 10557918. Exclusion reason: Ineligible comparison

193. Fahey AJ. Tele-ICU: state of the art care. Ohio Nurses Rev. 2013 Mar-Apr;88(2):6. PMID: 23631101. Exclusion reason: Ineligible comparison

194. Falsone JJ, Moidu K, Sheehan CA, et al. Is telemedicine justifiable? Conn Med. 1998 Jul;62(7):423-7. PMID: 9707797. Exclusion reason: Ineligible comparison
195. Fang JL, Collura CA, Johnson RV, et al. Emergency video telemedicine consultation for newborn resuscitations: the Mayo Clinic experience. Mayo Clin Proc. 2016

Dec;91(12):1735-43. doi: 10.1016/j.mayocp.2016.08.006. PMID: 27887680. Exclusion reason: Ineligible comparison

196. Faruque LI, Wiebe N, Ehteshami-Afshar A, et al. Effect of telemedicine on glycated hemoglobin in diabetes: a systematic review and meta-analysis of randomized trials. CMAJ. 2017 Mar 06;189(9):Ineligible population41-Ineligible outcome4. doi: 10.1503/cmaj.150885. PMID: 27799615.

Exclusion reason: Ineligible intervention

197. Fatehi F, Armfield NR, Dimitrijevic M, et al. Clinical applications of videoconferencing: a scoping review of the literature for the period 2002-2012. J Telemed Telecare. 2014 Oct;20(7):377-83. doi: 10.1177/1357633X14552385. PMID: 25399998. Exclusion reason: Ineligible study design

198. Fatehi F, Gray LC, Russell AW, et al. Validity and reliability of video teleconsultation for the management of diabetes: A randomized controlled trial. Diabetes Technol Ther. 2015;17(18). PMID: 24528569. Exclusion reason: Ineligible intervention

199. Fatehi F, Gray LC, Russell AW, et al. Validity study of video teleconsultation for the management of diabetes: A piot randomized controlled trial. Diabetes Technol Ther. 2015 Oct;17(10):717-25. doi: 10.1089/dia.2014.0416. PMID: 26181716.

Exclusion reason: Ineligible intervention

200. Fefferman N, Strubel N, Prithiani C, et al. Virtual radiology rounds: adding value in the digital era. Pediatr Radiol. 2016;33(9):1645-50. doi: 10.1007/s00247016-3675-y. PMID: 27488506. Exclusion reason: Ineligible comparison

201. Ferrandiz L, Ojeda-Vila T, Corrales A, et al. Internet-based skin cancer screening using clinical images alone or in conjunction with dermoscopic images: a randomized teledermoscopy trial. J Am Acad Dermatol. 2017 Apr;76(4):676-82. doi: 10.1016/j.jaad.2016.10.041. PMID: 28089728. Exclusion reason: Ineligible comparison 
202. Ferrandiz L, Ruiz-de-Casas A, MartinGutierrez FJ, et al. Effect of teledermatology on the prognosis of patients with cutaneous melanoma. Arch Dermatol. 2012

Sep;148(9):1025-8. doi: 10.1001/archdermatol.2012.778. PMID: 22986852. Exclusion reason: Ineligible intervention

203. Fields BG, Behari PP, McCloskey S, et al. Remote ambulatory management of Veterans with obstructive sleep apnea. Sleep. 2016;39(3):501-9. PMID: CN01142074. Exclusion reason: Ineligible intervention

204. Fierson WM, Capone A, Jr. Telemedicine for evaluation of retinopathy of prematurity. Pediatrics. 2015 Jan;135(1):e238-54. doi: 10.1542/peds.2014-0978. PMID: 25548330. Exclusion reason: Ineligible intervention

205. Fijalkowski N, Zheng LL, Henderson MT, et al. Stanford University Network for Diagnosis of Retinopathy of Prematurity (SUNDROP): four-years of screening with telemedicine. Curr Eye Res. 2013

Feb;38(2):283-91. doi: 10.3109/02713683.2012.754902. PMID: 23330739. Exclusion reason: Ineligible intervention

206. Fijalkowski N, Zheng LL, Henderson MT, et al. Stanford University Network for Diagnosis of Retinopathy of Prematurity (SUNDROP): five years of screening with telemedicine. Ophthalmic Surg Lasers Imaging. 2014 Mar-Apr;45(2):106-13. doi: 10.3928/23258160-20140122-01. PMID: 24444469. Exclusion reason: Ineligible intervention

207. Filip M, Linzer P, Samal F, et al. Medical consultations and the sharing of medical images involving spinal injury over mobile phone networks. Am J Emerg Med. 2012 Jul;30(6):961-5. doi:

10.1016/j.ajem.2011.05.007. PMID: 21795006. Exclusion reason: Ineligible outcome

208. Filipi JM, Khairat S. Tracking and visualizing headache trends on a mobile or desktop website. Stud Health Technol Inform. 2013;192:1199. PMID: 23920973. Exclusion reason: Ineligible intervention
209. Finnane A, Dallest K, Janda M, et al. Teledermatology for the diagnosis and management of skin cancer: A systematic review. JAMA Dermatology. 2017 Mar 01;153(3):319-27. doi: 10.1001/jamadermatol.2016.4361. PMID: 27926766. Exclusion reason: Systematic review used to identify primary studies

210. Finnane AR, Siller G, Soyer HP. Teledermatologists' management of emergency skin conditions. Med J Aust. 2015 Oct 05;203(7):286. PMID: 26424060. Exclusion reason: Ineligible comparison

211. Fitzpatrick D, Grabarz D, Wang L, et al. How effective is a virtual consultation process in facilitating multidisciplinary decision-making for malignant epidural spinal cord compression? Int J Radiat Oncol Biol Phys. 2012 Oct 01;84(2):e167-72. doi: 10.1016/j.ijrobp.2012.03.057. PMID: 22682804. Exclusion reason: Ineligible outcome

212. Fleischman A, Hourigan SE, Lyon HN, et al. Creating an integrated care model for childhood obesity: a randomized pilot study utilizing telehealth in a community primary care setting. Clin Obes. 2016 Dec;6(6):3808. doi: 10.1111/cob.12166. PMID: 27863024. Exclusion reason: Ineligible intervention

213. Flodgren, Gerd, Rachas, et al. Interactive telemedicine: effects on professional practice and health care outcomes [Systematic Review]. Cochrane Database Syst Rev. 2015(9) PMID: 26343551.

Exclusion reason: Systematic review used to identify primary studies

214. Fogliardi R, Frumento E, Rincon D, et al. Telecardiology: results and perspectives of an operative experience. J Telemed Telecare. 2000;6 Suppl 1:S162-4. PMID: 10794007. Exclusion reason: Ineligible comparison

215. Fonseca AS, Goldenberg DC, Stocchero GF, et al. Validation of videoconference with smartphones in telemedicine facial trauma care: analysis of concordance to on-site evaluation. Ann Plast Surg. 2016 Oct;77(4):433-7. doi: 10.1097/SAP.0000000000000623. PMID: 26418788. Exclusion reason: Ineligible outcome 
216. Fonyad L, Krenacs T, Nagy P, et al. Validation of diagnostic accuracy using digital slides in routine histopathology. Diagn Pathol. 2012;7:35. doi: 10.1186/17461596-7-35. PMID: 22463804. Exclusion reason: Ineligible intervention

217. Ford JA, Pereira A. Does teledermatology reduces secondary care referrals and is it acceptable to patients and doctors?: a service evaluation. J Eval Clin Pract. 2015 Aug;21(4):710-6. doi: 10.1111/jep.12373. PMID: 25903046. Exclusion reason: Ineligible intervention

218. Ford SC, Ajani ZA, Chen Q, et al. Comparison of standard emergency room care with tele-stroke evaluation in acute intracerebral hemorrhage management. Stroke. Conference: American Heart Association/American Stroke Association. 2016;47(no pagination). Exclusion reason: Ineligible publication type

219. Forni A, Skehan N, Hartman CA, et al. Evaluation of the impact of a tele-ICU pharmacist on the management of sedation in critically ill mechanically ventilated patients. Ann Pharmacother. 2010 Mar;44(3):432-8. doi: 10.1345/aph.1M576. PMID: 20164471. Exclusion reason: Ineligible intervention

220. Fortney JC, Pyne JM, Turner EE, et al. Telepsychiatry integration of mental health services into rural primary care settings. Int Rev Psychiatry. 2015;27(6):525-39. doi: 10.3109/09540261.2015.1085838. PMID: 26634618. Exclusion reason: Background information only

221. Fraizer C. Online consultations: new entry portals. J Med Pract Manage. 2004 JulAug;20(1):16-7. PMID: 15500016.

Exclusion reason: Ineligible comparison

222. Franklin CL, Cuccurullo LA, Walton JL, et al. Face to face but not in the same place: a pilot study of prolonged exposure therapy. J Trauma Dissociation. 2017;18(1):116-30. PMID: 27348462 Exclusion reason: Ineligible intervention
223. Friedman RH, Kazis LE, Jette A, et al. A telecommunications system for monitoring and counseling patients with hypertension. Impact on medication adherence and blood pressure control. Am J Hypertens. 1996 Apr;9(4 Pt 1):285-92. PMID: 8722429. Exclusion reason: Ineligible intervention

224. Fruhauf J, Hofman-Wellenhof R, Kovarik C, et al. Mobile teledermatology in subSaharan Africa: a useful tool in supporting health workers in low-resource centres. Acta Derm Venereol. 2013 Jan;93(1):122-3. doi: 10.2340/00015555-1404. PMID: 22735496. Exclusion reason: Ineligible comparison

225. Fuertes-Guiro F, Girabent-Farres M. Opportunity cost of the dermatologist's consulting time in the economic evaluation of teledermatology. J Telemed Telecare. 2017 Aug;23(7):657-64. doi: 10.1177/1357633X16660876. PMID: 27450572. Exclusion reason: Systematic review used to identify primary studies

226. Furukawa M, Furukawa MK, Mizojiri G, et al. Telemedicine in laryngology. Telemed J. 1998 Winter;4(4):329-33. doi: 10.1089/tmj.1.1998.4.329. PMID: 10220473. Exclusion reason: Ineligible intervention

227. Gackowski A, Czekierda L, Chrustowicz A, et al. Development, implementation, and multicenter clinical validation of the TeleDICOM--advanced, interactive teleconsultation system. J Digit Imaging. 2011 Jun;24(3):541-51. doi: 10.1007/s10278-010-9303-8. PMID: 20495992. Exclusion reason: Ineligible study design

228. Gagnon L. Telemedicine offers effective screening option for AMD. Ocular Surgery News. 2007;25(12):18. Exclusion reason: Ineligible publication type

229. Gagnon MP, Ngangue P, Payne-Gagnon J, et al. m-Health adoption by healthcare professionals: a systematic review. J Am Med Inform Assoc. 2016 Jan;23(1):212-20. doi: 10.1093/jamia/ocv052. PMID: 26078410. Exclusion reason: Ineligible intervention 
230. Galvez JA, Rehman MA. Telemedicine in anesthesia: an update. Curr Opin Anaesthesiol. 2011 Aug;24(4):459-62. doi: 10.1097/ACO.0b013e328348717b. PMID: 21659874. Exclusion reason: Ineligible publication type

231. Gamble JE, Savage GT, Icenogle ML. Value-chain analysis of a rural health program: toward understanding the cost benefit of telemedicine applications. Hosp Top. 2004 Winter;82(1):10-7. PMID: 15490956. Exclusion reason: Ineligible publication type

232. Gardiner S, Hartzell TL. Telemedicine and plastic surgery: a review of its applications, limitations and legal pitfalls. J Plast Reconstr Aesthet Surg. 2012 Mar;65(3):e4753. doi: 10.1016/j.bjps.2011.11.048. PMID: 22178033. Exclusion reason: Ineligible study design

233. Gattu R, Teshome G, Lichenstein R. Telemedicine applications for the pediatric emergency medicine: a review of the current literature. Pediatr Emerg Care. 2016

Feb;32(2):123-30. doi: 10.1097/PEC.0000000000000712. PMID: 26835573. Exclusion reason: Background information only

234. Gellis ZD, Kenaley BL, Ten Have T. Integrated telehealth care for chronic illness and depression in geriatric home care patients: the Integrated Telehealth Education and Activation of Mood (I-TEAM) study. J Am Geriatr Soc. 2014 May;62(5):889-95. doi: 10.1111/jgs.12776. PMID: 24655228. Exclusion reason: Ineligible intervention

235. Gerlach UA, Vrakas G, Holdaway L, et al. Skype clinics after intestinal transplantation - follow-up beyond post codes. Clin Transplant. 2016 Jul;30(7):760-6. doi: 10.1111/ctr.12745. PMID: 27140671. Exclusion reason: Ineligible intervention

236. Gifford AJ, Colebatch AJ, Litkouhi S, et al. Remote frozen section examination of breast sentinel lymph nodes by telepathology. ANZ J Surg. 2012 Nov;82(11):803-8. doi: 10.1111/j.1445-2197.2012.06191.x. PMID: 22924988. Exclusion reason: Ineligible intervention
237. Gillespie G. A view from afar. Vanderbilt University uses a telemedicine program to help diagnose a devastating eye disease before it's too late. Health Data Manag. 2003 Jun;11(6):92, 4. PMID: 12825447.

Exclusion reason: Ineligible comparison

238. Gillespie SM, Shah MN, Wasserman EB, et al. Reducing emergency department utilization through engagement in telemedicine by senior living communities. Telemed J E Health. 2016 Jun;22(6):489-96. doi: 10.1089/tmj.2015.0152. PMID: 26741194. Exclusion reason: Ineligible intervention

239. Glaessl A, Coras B, Popal H, et al. A survey among dermatologists in practice about teledermatology. Curr Probl Dermatol. 2003;32:172-5. PMID: 12472008.

Exclusion reason: Ineligible comparison

240. Glinkowski W, Michonski J, Glinkowska B, et al. Telediagnostic 3D school screening of back curvatures and posture using structured light method - pilot study. Stud Health Technol Inform. 2012;176:291-4. PMID: 22744511. Exclusion reason: Ineligible outcome

241. Glueckauf RL. Telehealth and chronic disabilities: New frontier for research and development. Rehabil Psychol. 2002;47(1):3-7. Exclusion reason: Ineligible study design

242. Goldman RD, Wei JJ, Cheyne J, et al. Impact of follow-up calls from the pediatric emergency department on return visits within 72 hours: a randomized controlled trial. Pediatr Emerg Care. 2014

Sep;30(9):613-6. doi: 10.1097/PEC.0000000000000207. PMID: 25162691. Exclusion reason: Ineligible intervention

243. Goldmeier D, Ivens D. Distant healing. Int J STD AIDS. 2000 Mar;11(3):203. PMID: 10726950. Exclusion reason: Ineligible publication type

244. Goldschmidt RH, Willard CL, Liljestrand P. Rural human immunodeficiency virus care. Arch Fam Med. 1998 Jan-Feb;7(1):14. PMID: 9443690. Exclusion reason: Ineligible intervention 
245. Goldstein D, Fanberg H. Digital doctoring 2002. Manag Care Interface. 2001 Jul;14(7):32-4. PMID: 11481813. Exclusion reason: Ineligible publication type

246. Gonzalez F, Iglesias R, Gomez-Ulla F, et al. Telemedicine reduces referral of diabetic patients to ophthalmologists. J Telemed Telecare. 2003;9(5):307-8. doi: 10.1258/135763303769211373. PMID: 14599339. Exclusion reason: Ineligible comparison

247. Gordon AS, Adamson WC, DeVries AR. Virtual visits for acute, nonurgent care: a claims analysis of episode-level utilization. J Med Internet Res. 2017 Feb 17;19(2):e35. doi: 10.2196/jmir.6783. PMID: 28213342. Exclusion reason: Ineligible intervention

248. Govender SM, Mars M. The use of telehealth services to facilitate audiological management for children: A scoping review and content analysis. J Telemed Telecare. 2017 Apr;23(3):392-401. doi: 10.1177/1357633X16645728. PMID: 27130158. Exclusion reason: Ineligible outcome

249. Granlund H. Aspects of quality: face-to-face versus teleconsulting. Curr Probl Dermatol. 2003;32:158-66. PMID: 12472006.

Exclusion reason: Ineligible study design

250. Granlund H, Thoden CJ, Carlson C, et al. Realtime teleconsultations versus face-toface consultations in dermatology: immediate and six-month outcome. J Telemed Telecare. 2003;9(4):204-9. doi: 10.1258/135763303322225526. PMID: 12952690. Exclusion reason: Ineligible intervention

251. Grant B, Morgan GJ, McCrossan BA, et al. Remote diagnosis of congenital heart disease: the impact of telemedicine. Arch Dis Child. 2010 Apr;95(4):276-80. doi: 10.1136/adc.2008.146456. PMID: 19948507. Exclusion reason: Ineligible outcome

252. Gray L, Wright O, Armfield NR, et al. Geriatric ward rounds conducted by videoconference. J Telemed Telecare. 2007;13:S3:103-S3. PMID: 20028277.

Exclusion reason: Ineligible comparison
253. Gray LC, Fatehi F, Martin-Khan M, et al. Telemedicine for specialist geriatric care in small rural hospitals: preliminary data. J Am Geriatr Soc. 2016 Jun;64(6):1347-51. doi: 10.1111/jgs.14139. PMID: 27321617. Exclusion reason: Ineligible comparison

254. Gray W, O'Brien D, Taleb F, et al. Benefits and pitfalls of telemedicine in neurosurgery. J Telemed Telecare. 1997;3(2):108-10. doi: 10.1258/1357633971930959. PMID: 9206282. Exclusion reason: Ineligible study design

255. Greiner A, Mess WH, Schmidli J, et al. Cyber medicine enables remote neuromonitoring during aortic surgery. J Vasc Surg. 2012 May;55(5):1227-32; discussion 32-3. doi: 10.1016/j.jvs.2011.11.121. PMID: 22341581. Exclusion reason: Ineligible outcome

256. Grossman D, Grindlay K. Safety of medical abortion provided through telemedicine compared with in person. Obstet Gynecol. 2017 Oct;130(4):778-82. doi: 10.1097/AOG.0000000000002212. PMID: 28885427. Exclusion reason: Ineligible intervention

257. Groves RH, Jr., Holcomb BW, Jr., Smith ML. Intensive care telemedicine: evaluating a model for proactive remote monitoring and intervention in the critical care setting. Stud Health Technol Inform. 2008;131:131-46. PMID: 18305328. Exclusion reason: Ineligible study design

258. Guedon-Moreau L, Lacroix D, Sadoul N, et al. Costs of remote monitoring vs. ambulatory follow-ups of implanted cardioverter defibrillators in the randomized ECOST study. Europace. 2014

Aug;16(8):1181-8. doi: 10.1093/europace/euu012. PMID: 24614572. Exclusion reason: Ineligible intervention

259. Guilfoyle C, Wootton R, Hassall S, et al. User satisfaction with allied health services delivered to residential facilities via videoconferencing. J Telemed Telecare. 2003;9 Suppl 1:S52-4. doi: 10.1258/135763303322196349. PMID: 12952723. Exclusion reason: Ineligible intervention 
260. Gupta A, Cavallerano J, Sun JK, et al. Evidence for telemedicine for diabetic retinal disease. Semin Ophthalmol.

2017;32(1):22-8. doi:

10.1080/08820538.2016.1228403. PMID:

27748634. Exclusion reason: Ineligible

outcome

261. Gupta N, Chawla N, Gupta D, et al. Community triage of otology patients using a store-and-forward telemedicine device: A feasibility study. Ear Nose Throat J. 2017;96(7):246-9. PMID: 28719707.

Exclusion reason: Ineligible comparison

262. Gupta SC, Sinha SK, Dagar AB. Evaluation of the effectiveness of diagnostic \& management decision by teleophthalmology using indigenous equipment in comparison with in-clinic assessment of patients. Indian J Med Res. 2013 Oct;138(4):531-5. PMID: 24434260. Exclusion reason: Ineligible outcome

263. Gurwin J, Tomlinson LA, Quinn GE, et al. A tiered approach to retinopathy of prematurity screening (tarp) using aweight gain predictive model and a telemedicine system. JAMA Ophthalmol. 2017;135(2):131-6. doi: 10.1001/jamaophthalmol.2016.5203. PMID: 28056115. Exclusion reason: Ineligible outcome

264. Guss B, Mishkin D, Sharma R. Using telemedicine to address crowding in the ED. Hosp Case Manag. 2016;22(8):173-5. PMID: 30133205. Exclusion reason: Ineligible publication type

265. Guttmann-Bauman I, Kono J, Lin AL, et al. Use of telehealth videoconferencing in pediatric type 1 diabetes in oregon. Telemed J E Health. 2018 Jan;24(1):86-8. doi: 10.1089/tmj.2017.0072. PMID: 28654350.

Exclusion reason: Ineligible intervention

266. Hagland M. IT and point-of-care decision support. Health Manag Technol. 1998 Oct;19(11):10-2, 4, 69-70. PMID: 10185156. Exclusion reason: Ineligible intervention
267. Hailey D, Roine R, Ohinmaa A. Systematic review of evidence for the benefits of telemedicine. J Telemed Telecare. 2002;8 Suppl 1:1-30. doi: 10.1258/1357633021937604. PMID: 12020415. Exclusion reason: Systematic review used to identify primary studies

268. Hall-Barrow J, Hall RW, Burke BL, Jr. Telemedicine and neonatal regionalization of care - ensuring that the right baby gets to the right nursery. Pediatr Ann. 2009 Oct;38(10):557-61. doi: 10.3928/0090448120090918-02. PMID: 19968193. Exclusion reason: Ineligible publication type

269. Halterman JS, Fagnano M, Tajon RS, et al. Effect of the school-based telemedicine enhanced asthma management (SB-TEAM) program on asthma morbidity: a randomized clinical trial. AMA pediatr. 2018;172(3): e174938. doi: 10.1001/jamapediatrics.2017.4938. PMID: 29309483. Exclusion reason: Ineligible intervention

270. Hamann D, Mortensen WS, Hamann CR, et al. Experiences in adoption of teledermatology in Mohs micrographic surgery: using smartglasses for intraoperative consultation and defect triage. Surg Innov. 2014 Dec;21(6):653-4. doi: 10.1177/1553350614552735. PMID: 25389145. Exclusion reason: Ineligible intervention

271. Hamilton A. On the virtual couch. Time. 1999 May 24;153(20):71. PMID: 10620914. Exclusion reason: Ineligible intervention

272. Hammack GG. Telemedicine in corrections. Curr Probl Dermatol. 2003;32:148-52. PMID: 12472004. Exclusion reason: Ineligible study design

273. Hammett L, Harvath TA, Flaherty-Robb M, et al. Remote wound care consultation for nursing homes: using a web-based assessment and care planning tool. J Gerontol Nurs. 2007 Nov;33(11):27-35; quiz 6-7. PMID: 18019116. Exclusion reason: Ineligible comparison 
274. Hanna GM, Fishman I, Edwards DA, et al. Development and patient satisfaction of a new telemedicine service for pain management at Massachusetts General Hospital to the island of Martha's Vineyard. Pain Med. 2016 Sep;17(9):1658-63. doi: 10.1093/pm/pnw069. PMID: 27121891.

Exclusion reason: Ineligible comparison

275. Hark LA, Katz LJ, Myers JS, et al. Philadelphia telemedicine glaucoma detection and follow-up study: methods and screening results. Am J Ophthalmol. 2017 Sep;181:114-24. doi: 10.1016/j.ajo.2017.06.024. PMID: 28673747. Exclusion reason: Ineligible intervention

276. Harley J, McLaren P, Blackwood G, et al. The use of videoconferencing to enhance tertiary mental health service provision to the island of Jersey. J Telemed Telecare. 2002;8 Suppl 2:36-8. doi:

10.1177/1357633X020080S216. PMID: 12217127. Exclusion reason: Ineligible intervention

277. Harrison M. Systems begin to reap the rewards of globally integrated healthcare. Mod Healthc. 2016 Feb 22;46(8):25. PMID: 27079048. Exclusion reason: Ineligible publication type

278. Hassan R, Siregar JA, NA ARM. The implementation of teleneurosurgery in the management of referrals to a neurosurgical department in hospital sultanah amninah johor bahru. Malays J Med Sci. 2014 Mar;21(2):54-62. PMID: 24876808.

Exclusion reason: Ineligible intervention

279. Hassol A. Surprises from the rural telemedicine survey. Telemed Today. 1996 Nov-Dec;4(6):5, 41. PMID: 10165151. Exclusion reason: Ineligible comparison

280. Havenga E, Swanepoel W, le Roux T, et al. Tele-intervention for children with hearing loss: A comparative pilot study. J Telemed Telecare. 2017 Jan;23(1):116-25. doi: 10.1177/1357633X15617886. PMID: 26670208. Exclusion reason: Ineligible intervention
281. Heautot JF, Gibaud B, Catroux B, et al. Influence of the teleradiology technology (N-ISDN and ATM) on the inter-hospital management of neurosurgical patients. Med Inform Internet Med. 1999 AprJun;24(2):121-34. PMID: 10399710. Exclusion reason: Ineligible comparison

282. Heidgerken AD, Adkins J, Storch EA, et al. Telehealth intervention for adolescents with type 1 diabetes. J Pediatr. 2006 May;148(5):707-8. doi: 10.1016/j.jpeds.2006.01.001. PMID: 16737898. Exclusion reason: Ineligible publication type

283. Herbert MS, Afari N, Liu L, et al. Telehealth versus in-person acceptance and commitment therapy for chronic pain: a randomized noninferiority trial. J Pain. 2017;18(2):200-11. doi: 10.1016/j.jpain.2016.10.014. PMID: 27838498. Exclusion reason: Ineligible intervention

284. Hex N, Tuggey J, Wright D, et al. Telemedicine in care homes in Airedale, Wharfedale and Craven. Clin Gov. 2015;20(3):146-54. doi: doi:10.1108/CGIJ07-2015-0022. Exclusion reason: Ineligible intervention

285. High WA, Houston MS, Calobrisi SD, et al. Assessment of the accuracy of low-cost store-and-forward teledermatology consultation. J Am Acad Dermatol. 2000 May;42(5 Pt 1):776-83. PMID: 10775853. Exclusion reason: Ineligible intervention

286. Hilt RJ, Romaire MA, McDonell MG, et al. The Partnership Access Line: evaluating a child psychiatry consult program in Washington State. JAMA Pediatr. 2013 Feb;167(2):162-8. doi: 10.1001/2013.jamapediatrics.47. PMID: 23247331. Exclusion reason: Ineligible intervention

287. Hilty DM, Cobb HC, Neufeld JD, et al. Telepsychiatry reduces geographic physician disparity in rural settings, but is it financially feasible because of reimbursement? Psychiatr Clin North Am. 2008;31(1):85-94. doi: 10.1016/j.psc.2007.11.010. PMID: 18295040. Exclusion reason: Ineligible outcome 
288. Hilty DM, Marks SL, Urness D, et al. Clinical and educational telepsychiatry applications: a review. Can J Psychiatry. 2004 Jan;49(1):12-23. doi: 10.1177/070674370404900103. PMID: 14763673. Exclusion reason: Ineligible study design

289. Hilty DM, Yellowlees PM, Nesbitt TS. Evolution of telepsychiatry to rural sites: changes over time in types of referral and in primary care providers' knowledge, skills and satisfaction. Gen Hosp Psychiatry. 2006 Sep-Oct;28(5):367-73. doi: 10.1016/j.genhosppsych.2006.05.009. PMID: 16950370. Exclusion reason: Ineligible comparison

290. Hobson EV, Baird WO, Bradbury M, et al. Telehealth in motor neurone disease. A mixed methods, randomised controlled, pilot study of the use of the TiM telehealth system to deliver highly specialised care in motor neurone disease, at a distance. 27th international symposium on ALS/MND. Ireland. Conference start. 2016;17:54-5. Exclusion reason: Ineligible publication type

291. Hobson EV, Baird WO, Cooper CL, et al. Using technology to improve access to specialist care in amyotrophic lateral sclerosis: A systematic review. Amyotrophic Lateral sclerosis \& Frontotemporal Degeneration. 2016 Jul-Aug;17(5-6):313-24. doi: 10.3109/21678421.2016.1165255. PMID: 27027466. Exclusion reason: Systematic review used to identify primary studies

292. Hofmeyer J, Leider JP, Satorius J, et al. Implementation of telemedicine consultation to assess unplanned transfers in rural longterm care facilities, 2012-2015: a pilot study. J Am Med Dir Assoc. 2016;177(5):1006-10. doi: 10.1016/j.jamda.2016.06.014. PMID: 27477614. Exclusion reason: Ineligible intervention

293. Host BK, Turner AW, Muir J. Real-time teleophthalmology video consultation: an analysis of patient satisfaction in rural Western Australia. Clin Exp Optom. 2018 Jan;101(1):129-34. doi: 10.1111/cxo.12535. PMID: 28436157. Exclusion reason: Ineligible comparison
294. Houtchens BA, Clemmer TP, Holloway HC, et al. Telemedicine and international disaster response: medical consultation to Armenia and Russia via a Telemedicine Spacebridge. Prehosp Disaster Med. 1993 JanMar;8(1):57-66. PMID: 11536928. Exclusion reason: Ineligible comparison

295. Howell D. It is unclear whether specialist palliative care teleconsultation leads to an improvement in patient symptom scores. Evid Based Nurs. 2018 01;21(1):1. doi: 10.1136/eb-2017-102842. PMID: 29223969. Exclusion reason: Ineligible publication type

296. Howell G, Ardilles T, Bonham AJ. Implementation of a remote intensive care unit monitoring system correlates with improvements in patient outcomes. Chest. 2008;134(4, Supplement 2):58S. doi: 10.1378/chest.134.4_MeetingAbstracts.s580 03. Exclusion reason: Ineligible publication type

297. Hsieh CH, Jeng SF, Chen CY, et al. Teleconsultation with the mobile cameraphone in remote evaluation of replantation potential. J Trauma. 2005 Jun;58(6):120812. PMID: 15995472. Exclusion reason: Ineligible comparison

298. Hsu M-H, Chu T-B, Yen J-C, et al. Development and implementation of a national telehealth project for long-term care: A preliminary study. Comput Methods Programs Biomed. 2010 2010/03/01/;97(3):286-92. doi: 10.1016/j.cmpb.2009.12.008. PMID: 20092907. Exclusion reason: Ineligible intervention

299. Huang VW, Reich KM, Fedorak RN. Distance management of inflammatory bowel disease: systematic review and metaanalysis. World J Gastroenterol. 2014 Jan 21;20(3):829-42. doi: 10.3748/wjg.v20.i3.829. PMID: 24574756. Exclusion reason: Ineligible intervention

300. Hudson TJ, Fortney JC, Pyne JM, et al. Reduction of patient-reported antidepressant side effects, by type of collaborative care. Psychiatr Serv. 2015 Mar 1;66(3):272-8. doi: 10.1176/appi.ps.201300570. PMID: 25727115. Exclusion reason: Ineligible intervention 
301. Hui E, Woo J. Telehealth for older patients: the Hong Kong experience. J Telemed Telecare. 2002;8 Suppl 3:S3:39-41. PMID: 12661617. Exclusion reason: Ineligible intervention

302. Hui E, Woo J, Hjelm M, et al. Telemedicine: a pilot study in nursing home residents. Gerontology. 2001 Mar-Apr;47(2):82-7. doi: 52778. PMID: 11287732. Exclusion reason: Ineligible intervention

303. Hwang JS, Lappan CM, Sperling LC, et al. Utilization of telemedicine in the U.S. military in a deployed setting. Mil Med. 2014 Nov;179(11):1347-53. doi: 10.7205/MILMED-D-14-00115. PMID: 25373065. Exclusion reason: Background information only

304. Ignatius E, Perala S, Makela K. Use of videoconferencing for consultation in dental prosthetics and oral rehabilitation. $\mathrm{J}$ Telemed Telecare. 2010;16(8):467-70. doi: 10.1258/jtt.2010.100303. PMID: 21030487. Exclusion reason: Ineligible comparison

305. Irving M, Stewart R, Spallek H, et al. Using teledentistry in clinical practice as an enabler to improve access to clinical care: A qualitative systematic review. J Telemed Telecare. 2018 Apr;24(3):129-46. doi: 10.1177/1357633X16686776. PMID: 28092220. Exclusion reason: Systematic review used to identify primary studies

306. Ishani A, Christopher J, Palmer D, et al. Telehealth by an interprofessional team in patients with CKD: a randomized controlled trial. Am J Kidney Dis. 2016;68(1):41-9. doi: 10.1053/j.ajkd.2016.01.018. PMID: 26947216. Exclusion reason: Ineligible intervention

307. Izquierdo R, Meyer S, Starren J, et al. Detection and remediation of medically urgent situations using telemedicine case management for older patients with diabetes mellitus. Ther Clin Risk Manag. 2007;3(3):485-9. PMID: 18488079.

Exclusion reason: Ineligible comparison
308. J.P. vdH, N.F. dK, J.D. B, et al. Teledermatology applied following patient selection by general practitioners in daily practice improves efficiency and quality of care at lower cost. Br J Dermatol.

2011;165(5):1058-65. doi: doi:10.1111/j.1365-2133.2011.10509.x. PMID: 21729026. Exclusion reason: Ineligible comparison

309. Janardhanan L, Leow YH, Chio MT, et al. Experience with the implementation of a web-based teledermatology system in a nursing home in Singapore. J Telemed Telecare. 2008;14(8):404-9. doi: 10.1258/jtt.2008.080105. PMID: 19047449. Exclusion reason: Ineligible comparison

310. Jani PD, Forbes L, Choudhury A, et al. Evaluation of diabetic retinal screening and factors for ophthalmology referral in a telemedicine network. JAMA Ophthalmol. 2017 Jul 01;135(7):706-14. doi: 10.1001/jamaophthalmol.2017.1150. PMID: 28520833. Exclusion reason: Ineligible intervention

311. Jeganathan VS, Hall HN, Sanders R. Electronic referrals and digital imaging systems in ophthalmology: a global perspective. Asia Pac J Ophthalmol (Phila). 2017 Jan-Feb;6(1):3-7. doi: 10.22608/APO.2016110. PMID: 28161930. Exclusion reason: Ineligible comparison

312. Jerant AF, Azari R, Nesbitt TS. Reducing the cost of frequent hospital admissions for congestive heart failure: a randomized trial of a home telecare intervention. Med Care. 2001 Nov;39(11):1234-45. PMID: 11606877. Exclusion reason: Ineligible intervention

313. Jian G, Mao J, Jiang J. Telediagnosis of 168 cases of renal disease. J Telemed Telecare. 2002;8(6):360-1. doi: 10.1258/135763302320939275. PMID: 12537926. Exclusion reason: Ineligible comparison

314. Jin AJ, Martin D, Maberley D, et al. Evaluation of a mobile diabetes care telemedicine clinic serving Aboriginal communities in northern British Columbia, Canada. Int J Circumpolar Health. 2004 2004/09/01;63(sup2):124-8. doi: 10.3402/ijch.v63i0.17871. PMID: 15736635. Exclusion reason: Ineligible intervention 
315. Jodar-Sanchez F, Ortega F, Parra C, et al. Cost-utility analysis of a telehealth programme for patients with severe chronic obstructive pulmonary disease treated with long-term oxygen therapy. J Telemed Telecare. 2014 Sep;20(6):307-16. doi: 10.1177/1357633X14544421. PMID: 25052387. Exclusion reason: Ineligible intervention

316. Johnson A. Digital doctors: telemedicine improves medical care in remote areas, but faces some policy obstacles. State Legis. 2006 Jun;32(6):30-2. PMID: 16791976.

Exclusion reason: Ineligible publication type

317. Johnston B, Yellowlees P. Telepsychiatry consultations in primary care coordinated by virtual care navigators. Psychiatr Serv. 2016 Jan;67(1):142. doi: 10.1176/appi.ps.660905. PMID: 26725496. Exclusion reason: Ineligible publication type

318. Jones BN, 3rd, Colenda CC. Telemedicine and geriatric psychiatry. Psychiatr Serv. 1997 Jun;48(6):783-5. doi: 10.1176/ps.48.6.783. PMID: 9175185. Exclusion reason: Ineligible comparison

319. Jones NC, Nazarian RM, Duncan LM, et al. Interinstitutional whole slide imaging teleconsultation service development: assessment using internal training and clinical consultation cases. Arch Pathol Lab Med. 2015 May;139(5):627-35. doi: 10.5858/arpa.2014-0133-OA. PMID: 25415180. Exclusion reason: Ineligible intervention

320. Joubert J, Reid C, Barton D, et al. Integrated care improves risk factor modification one year after stroke: initial results of the ICARUSS model. J Neurol Neurosurg Psychiatry. 2008doi:

10.1136/jnnp.2008.148122. Exclusion reason: Ineligible intervention

321. Jury SC, Kornberg AJ. Integrating telehealth in to 'business as usual': Is it really possible? J Telemed Telecare. 2016 Dec;22(8):499503. doi: 10.1177/1357633X16675802. PMID: 27799455. Exclusion reason: Ineligible comparison
322. Kagan J, Levy J. Improve outcomes and pay-for-performance rewards by facilitating joint decision making by physicians and patients to seek specialty consultation. Stud Health Technol Inform. 2017;244:84-. doi: 10.3233/978-1-61499-824-2-84. Exclusion reason: Ineligible publication type

323. Kalankesh LR, Pourasghar F, Nicholson L, et al. Effect of telehealth interventions on hospitalization indicators: A systematic review. Perspect Health Inf Manag. 2016;13(Fall):1h. PMID: 27843425. Exclusion reason: Ineligible intervention

324. Kaliyadan F, Amin TT, Kuruvilla J, et al. Mobile teledermatology--patient satisfaction, diagnostic and management concordance, and factors affecting patient refusal to participate in Saudi Arabia. J Telemed Telecare. 2013 Sep;19(6):315-9. doi: 10.1177/1357633X13501778. PMID: 24163295. Exclusion reason: Ineligible comparison

325. Kaliyadan F, Venkitakrishnan S.

Teledermatology: clinical case profiles and practical issues. Indian J Dermatol Venereol Leprol. 2009 Jan-Feb;75(1):32-5. PMID: 19172028. Exclusion reason: Ineligible comparison

326. Kane-Gill SL, Niznik JD, Kellum JA, et al. Use of telemedicine to enhance pharmacist services in the nursing facility. Consult Pharm. 2017 Feb 01;32(2):93-8. doi: 10.4140/TCP.n.2017.93. PMID: 28569660. Exclusion reason: Systematic review used to identify primary studies

327. Kanthraj GR, Srinivas CR. Store and forward teledermatology. Indian J Dermatol Venereol Leprol. 2007 Jan-Feb;73(1):5-12. PMID: 17314439. Exclusion reason: Ineligible comparison

328. Kassam F, Amin S, Sogbesan E, et al. The use of teleglaucoma at the University of Alberta. J Telemed Telecare. 2012 Oct;18(7):367-73. doi: 10.1258/jtt.2012.120313. PMID: 22977196. Exclusion reason: Ineligible comparison

329. Kassar K, Roe C, Desimone M. Use of telemedicine for management of diabetes in correctional facilities. Telemed J E Health. 2017 Jan;23(1):55-9. doi:

10.1089/tmj.2016.0036. PMID: 27223479.

Exclusion reason: Ineligible intervention 
330. Kawaguchi A, Sharafeldin N, Sundaram A, et al. Tele-ophthalmology for age-related macular degeneration and diabetic retinopathy screening: A systematic review and meta-analysis. Telemed J E Health. 2017 Aug 7doi: 10.1089/tmj.2017.0100. PMID: 28783458. Exclusion reason: Ineligible outcome

331. Kawaguchi A, Sharafeldin N, Sundaram A, et al. Tele-Ophthalmology for age-related macular degeneration and diabetic retinopathy screening: A systematic review and meta-analysis. Telemed J E Health. 2018 Apr;24(4):301-8. doi: 10.1089/tmj.2017.0100. PMID: 28783458. Exclusion reason: Systematic review used to identify primary studies

332. Kazley AS, McLeod AC, Wager KA. Telemedicine in an international context: definition, use, and future. Advances in Health Care Management. 2012;12:143-69. PMID: 22894049. Exclusion reason: Ineligible outcome

333. Keaton L, Steiner V, Masterson M, et al. An E-rehabilitation team helps caregivers deal with stroke. J Allied Health. 2004;2(4):17pp. Exclusion reason: Ineligible comparison

334. Kedar I, Ternullo JL, Weinrib CE, et al. Internet based consultations to transfer knowledge for patients requiring specialised care: retrospective case review. BMJ. 2003 Mar 29;326(7391):696-9. doi: 10.1136/bmj.326.7391.696. PMID: 12663408. Exclusion reason: Ineligible comparison

335. Keogh K, Clark P, Valery PC, et al. Use of telehealth to treat and manage chronic viral hepatitis in regional Queensland. J Telemed Telecare. 2016 Dec;22(8):459-64. doi: 10.1177/1357633X16673794. PMID: 27799448. Exclusion reason: Ineligible comparison

336. Kew KM, Cates CJ. Remote versus face-toface check-ups for asthma. Cochrane Database of Syst Rev. 2016(4). doi: 10.1002/14651858.CD011715.pub2. PMID: 27087257. PMID: 00075320-10000000010099. Exclusion reason: Ineligible intervention
337. Khan K, Shuaib A, Whittaker T, et al. Telestroke in Northern Alberta: a two year experience with remote hospitals. Can J Neurol Sci. 2010 Nov;37(6):808-13. PMID: 21059543. Exclusion reason: Ineligible comparison

338. Khoja S, Scott R, Husyin N, et al. Impact of simple conventional and Telehealth solutions on improving mental health in Afghanistan. J Telemed Telecare. 2016 Dec;22(8):495-8. doi: 10.1177/1357633X16674631. PMID: 27799454. Exclusion reason: Ineligible intervention

339. Khouri AS, Szirth BC, Salti HI, et al. DICOM transmission of simultaneous stereoscopic images of the optic nerve in patients with glaucoma. J Telemed Telecare. 2007;13(7):337-40. doi: 10.1258/135763307782215389. PMID: 17958934. Exclusion reason: Ineligible intervention

340. Kieffer JA, Drew PG. How the Internet could wring $\$ 7.3$ billion out of professional fees. Diagn Imaging (San Franc). 2000 Jul;22(7):33-5. PMID: 11010305. Exclusion reason: Ineligible comparison

341. Kim YN, Shin DG, Park S, et al. Randomized clinical trial to assess the effectiveness of remote patient monitoring and physician care in reducing office blood pressure. Hypertens Res. 2015 Jul;38(7):491-7. doi: 10.1038/hr.2015.32 PMID: 25787041. Exclusion reason: Ineligible intervention

342. Kincade K. Telemedicine supports primarycare delivery at outlying VA centers. Telemed Telehealth Netw. 1998 Apr;4(2):8, 10-1. PMID: 10181485. Exclusion reason: Ineligible comparison

343. King AB, Wolfe GS. Evaluation of a diabetes specialist-guided primary care diabetes treatment program. J Am Acad Nurse Pract. 2009 Jan;21(1):24-30. doi: 10.1111/j.1745-7599.2008.00370.x. PMID: 19125892. Exclusion reason: Ineligible intervention 
344. Kingue S, Angandji P, Menanga AP, et al. Efficiency of an intervention package for arterial hypertension comprising telemanagement in a Cameroonian rural setting: The TELEMED-CAM study. Pan Afr Med J. 2013;15:153. doi: 10.11604/pamj.2013.15.153.2655. PMID: 24396559. Exclusion reason: Ineligible intervention

345. Kinney AY, Steffen LE, Brumbach BH, et al. Randomized noninferiority trial of telephone delivery of BRCA1/2 genetic counseling compared with in-person counseling: 1-year follow-up. J Clin Oncol. 2016 Aug 20;34(24):2914-24. doi: 10.1200/JCO.2015.65.9557. PMID: 27325848. Exclusion reason: Ineligible intervention

346. Kinsella A. Disabled populations \& telerehabilitation--new approaches. Caring. 1999 Aug;18(8):20-2, 4, 6-7. PMID: 10557968. Exclusion reason: Ineligible intervention

347. Kirkizlar E, Serban N, Sisson JA, et al. Evaluation of telemedicine for screening of diabetic retinopathy in the Veterans Health Administration. Ophthalmology. 2013 Dec;120(12):2604-10. doi: 10.1016/j.ophtha.2013.06.029. PMID: 24084501. Exclusion reason: Ineligible intervention

348. Kirkpatrick AW, McKee I, McKee JL, et al. Remote just-in-time telementored trauma ultrasound: A double-factorial randomized controlled trial examining fluid detection and remote knobology control through an ultrasound graphic user interface display. Am J Surg. 2016;211(5):894-902. doi: 10.1016/j.amjsurg.2016.01.018. PMID: 27020901. Exclusion reason: Ineligible intervention

349. Kit PL, Wong SS, D'Rozario V, et al. Exploratory findings on novice group counselors' initial co-facilitating experiences in in-class support groups with adjunct online support groups. The Journal for Specialists in Group Work. 2014;39(4):316-44. doi: 10.1080/01933922.2014.954737. Exclusion reason: Ineligible intervention
350. Kitamura C, Zurawel-Balaura L, Wong RK. How effective is video consultation in clinical oncology? A systematic review. Current Oncology. 2010;17(3):17-27. PMID: 20567623. Exclusion reason: Ineligible intervention

351. Klaz I, Wohl Y, Nathansohn N, et al. Teledermatology: quality assessment by user satisfaction and clinical efficiency. Isr Med Assoc J. 2005 Aug;7(8):487-90. PMID: 16106771. Exclusion reason: Ineligible comparison

352. Klersy C, De Silvestri A, Gabutti G, et al. Economic impact of remote patient monitoring: an integrated economic model derived from a meta-analysis of randomized controlled trials in heart failure. Eur J Heart Fail. 2011 Apr;13(4):450-9. doi: 10.1093/eurjhf/hfq232. PMID: 21193439. Exclusion reason: Ineligible intervention

353. Knol A, van den Akker TW, Damstra RJ, et al. Teledermatology reduces the number of patient referrals to a dermatologist. J Telemed Telecare. 2006;12(2):75-8. doi: 10.1258/135763306776084365. PMID: 16539753. Exclusion reason: Ineligible comparison

354. Knox L, Rahman RJ, Beedie C. Quality of life in patients receiving telemedicine enhanced chronic heart failure disease management: A meta-analysis. J Telemed Telecare. 2017 Aug;23(7):639-49. doi: 10.1177/1357633X16660418. PMID: 27450573. Exclusion reason: Ineligible intervention

355. Kofos D, Pitetti R, Orr R, et al. Telemedicine in pediatric transport: a feasibility study. Pediatrics. 1998 Nov;102(5):Ineligible comparison8. PMID: 9794988. Exclusion reason: Ineligible intervention

356. Kohl B, Gutsche J, Kim P, et al. Effect of telemedicineon mortality and length of stay in a University hospital [abstract]. Crit Care Med. 2007;35:A111. Exclusion reason: Ineligible publication type

357. Kohri T, Sakamaki T, Hasegawa T, et al. Prospective multicenter case-control study of telemedicine for home medical care. Stud Health Technol Inform. 2013;192:963. PMID: 23920737. Exclusion reason: Ineligible intervention 
358. Kon AA, Marcin JP. Using telemedicine to improve communication during paediatric resuscitations. J Telemed Telecare.

2005;11(5):261-4. doi:

10.1258/1357633054471920. PMID:

16035970. Exclusion reason: Ineligible study design

359. Kopycka-Kedzierawski DT, Billings RJ. Comparative effectiveness study to assess two examination modalities used to detect dental caries in preschool urban children. Telemed J E Health. 2013 Nov;19(11):83440. doi: 10.1089/tmj.2013.0012. PMID: 24053114. Exclusion reason: Ineligible intervention

360. Kovacikova L, Zahorec M, Skrak P, et al. Transatlantic medical consultation and second opinion in pediatric cardiology has benefit past patient care: A case study in videoconferencing. Congenit Heart Dis. 2017 Jul;12(4):491-6. doi:

10.1111/chd.12480. PMID: 28523862.

Exclusion reason: Ineligible comparison

361. Kraco K. Long-distance dermatology. Minn Med. 2004 Mar;87(3):11. PMID: 15080284. Exclusion reason: Ineligible comparison

362. Kreutzer J, Akutsu H, Fahlbusch R, et al. Teleradiology in neurosurgery: experience in 1024 cases. $\mathrm{J}$ Telemed Telecare. 2008;14(2):67-70. doi: 10.1258/jtt.2007.060605. PMID: 18348750.

Exclusion reason: Ineligible comparison

363. Krishna MT, Knibb RC, Huissoon AP. Is there a role for telemedicine in adult allergy services? Clin Exp Allergy. 2016 May;46(5):668-77. doi: 10.1111/cea.12701. PMID: 26742680. Exclusion reason: Ineligible study design

364. Kulcsar Z, Albert D, Ercolano E, et al. Telerheumatology: a technology appropriate for virtually all. Semin Arthritis Rheum. 2016 Dec;46(3):380-5. doi: 10.1016/j.semarthrit.2016.05.013. PMID: 27395561. Exclusion reason: Ineligible comparison

365. Kumar G, Falk DM, Bonello RS, et al. The costs of critical care telemedicine programs: a systematic review and analysis. Chest. 2013 Jan;143(1):19-29. doi: 10.1378/chest.11-3031. PMID: 22797291.

Exclusion reason: Systematic review used to identify primary studies
366. Kumar KR, Yogesan K, Constable IJ. Teleophthalmology in India. Is it here to stay? Indian J Ophthalmol. 2003 Dec;51(4):295-6. PMID: 14750615. Exclusion reason: Ineligible study design

367. Kumar N, Busarla SVP, Sayed S, et al. Telecytology in East Africa: a feasibility study of forty cases using a static imaging system. J Telemed Telecare. 2012;18(1):712. doi: 10.1258/jtt.2011.110308. PMID: 22052967. Exclusion reason: Ineligible comparison

368. Kumari Rani P, Raman R, Manikandan M, et al. Patient satisfaction with teleophthalmology versus ophthalmologistbased screening in diabetic retinopathy. $\mathrm{J}$ Telemed Telecare. 2006;12(3):159-60. doi: 10.1258/135763306776738639. PMID: 16638238. Exclusion reason: Ineligible intervention

369. Kurji K, Kiage D, Rudnisky CJ, et al. Improving diabetic retinopathy screening in Africa: patient satisfaction with teleophthalmology versus ophthalmologistbased screening. Middle East Afr J Ophthalmol. 2013 Jan-Mar;20(1):56-60. doi: 10.4103/0974-9233.106388. PMID: 23580853. Exclusion reason: Ineligible outcome

370. Kurzynski MW. Telemedicine for the family doctor. Curr Probl Dermatol. 2003;32:83-6. PMID: 12471995. Exclusion reason: Ineligible comparison

371. Kwak MJ, Kim JM, Shin IH, et al. Real-time medical control using a wireless audio-video transmission device in a pre-hospital emergency service in Korea. J Telemed Telecare. 2009;15(8):404-8. doi: 10.1258/jtt.2009.090504. PMID: 19948707. Exclusion reason: Ineligible comparison

372. Kyle E, Aitken P, Elcock M, et al. Use of telehealth for patients referred to a retrieval service: timing, destination, mode of transport, escort level and patient care. J Telemed Telecare. 2012 Apr;18(3):147-50. doi: 10.1258/jtt.2012.SFT106. PMID: 22362826. Exclusion reason: Ineligible comparison 
373. Laghari FJ, Hammer MD. Telestroke imaging: A review. J Neuroimaging. 2017 Jan;27(1):16-22. doi: 10.1111/jon.12402. PMID: 27805298. Exclusion reason: Ineligible study design

374. Lama T, Karmacharya B, Chandler C, et al. Telephone management of severe wasp stings in rural Nepal: a case report. $\mathrm{J}$ Telemed Telecare. 2011;17(2):105-8. doi: 10.1258/jtt.2010.100606. PMID: 21139015. Exclusion reason: Ineligible study design

375. Lambrecht CJ. Telemedicine in trauma care: description of 100 trauma teleconsults. Telemed J. 1997 Winter;3(4):265-8. doi: 10.1089/tmj.1.1997.3.265. PMID: 10176007. Exclusion reason: Ineligible comparison

376. Lambrecht CJ. Telemedicine in trauma care. Telemed Today. 1998 Feb;6(1):25. PMID: 10178359. Exclusion reason: Ineligible comparison

377. Lamel SA, Haldeman KM, Ely H, et al. Application of mobile teledermatology for skin cancer screening. J Am Acad Dermatol. 2012 Oct;67(4):576-81. doi: 10.1016/j.jaad.2011.11.957. PMID: 22243769. Exclusion reason: Ineligible intervention

378. Lamminen H, Nevalainen J, Alho A, et al. Experimental telemedicine in orthopaedics. J Telemed Telecare. 1996;2(3):170-3. doi: 10.1258/1357633961930013. PMID: 9375053. Exclusion reason: Ineligible comparison

379. Lamminen H, Tuomi ML, Lamminen J, et al. A feasibility study of realtime teledermatology in Finland. J Telemed Telecare. 2000;6(2):102-7. doi: 10.1258/1357633001935121. PMID: 10824378. Exclusion reason: Ineligible comparison

380. LaMonte MP, Bahouth MN, Hu P, et al. Telemedicine for acute stroke: triumphs and pitfalls. Stroke. 2003 Mar;34(3):725-8. doi: 10.1161/01.STR.0000056945.36583.37. PMID: 12624298. Exclusion reason: Ineligible comparison
381. Lasierra N, Alesanco A, Gilaberte Y, et al. Lessons learned after a three-year store and forward teledermatology experience using internet: Strengths and limitations. Int J Med Inform. 2012;81(5):332-43. doi: 10.1016/j.ijmedinf.2012.02.008. PMID: 22425394. Exclusion reason: Ineligible comparison

382. Latifi R, Gunn JK, Bakiu E, et al. Access to specialized care through telemedicine in limited-resource country: initial 1,065 teleconsultations in Albania. Telemed J E Health. 2016 Dec;22(12):1024-31. PMID: 27219617. Exclusion reason: Ineligible comparison

383. Latifi R, Olldashi F, Dogjani A, et al. Telemedicine for neurotrauma in Albania: Initial results from case series of 146 patients. World Neurosurg. 2018 Apr;112:e747-e53. doi: 10.1016/j.wneu.2018.01.146. PMID: 29410169. Exclusion reason: Ineligible comparison

384. Lawton S, Timmons S. Stakeholders' experience of teledermatology in a nurse-led community clinic: a case study. Health Informatics J. 2005;11(2):111-22. Exclusion reason: Ineligible comparison

385. Lazzarini PA, Clark D, Mann RD, et al. Does the use of store-and-forward telehealth systems improve outcomes for clinicians managing diabetic foot ulcers? A pilot study. J Foot Ankle Res. 2011 05/20;4(Suppl 1):P31-P. doi: 10.1186/17571146-4-S1-P31. Exclusion reason: Ineligible comparison

386. Leavitt ER, Kessler S, Pun S, et al. Teledermatology as a tool to improve access to care for medically underserved populations: A retrospective descriptive study. J Am Acad Dermatol. 2016 Dec;75(6):1259-61. doi: 10.1016/j.jaad.2016.07.043. PMID: 27846951. Exclusion reason: Ineligible publication type

387. Lee ACW, Parmanto B, Saptono A, et al. The VISYTER telerehabilitation system for globalizing physical therapy consultation: issues and challenges for telehealth implementation. J Phys Ther Educ. 2012 Winter2012;26(1):90-6. Exclusion reason: Ineligible comparison 
388. Lee RH, Pearson M, Lyles KW, et al. Geographic scope and accessibility of a centralized, electronic consult program for patients with recent fracture. Rural Remote Health. 2016 Jan-Mar;16(1):3440. PMID: 26745338. Exclusion reason: Ineligible intervention

389. Leggett P, Graham L, Steele K, et al. Telerheumatology--diagnostic accuracy and acceptability to patient, specialist, and general practitioner. Br J Gen Pract. 2001 Sep;51(470):746-8. PMID: 11593837. Exclusion reason: Ineligible comparison

390. Leichter SB, Bowman K, Adkins RA, et al. Impact of remote management of diabetes via computer: the 360 study--a proof-ofconcept randomized trial. Diabetes Technol Ther. 2013 May;15(5):434-8. doi: 10.1089/dia.2012.0323. PMID: 23537419. Exclusion reason: Ineligible intervention

391. Leigh H, Cruz H, Mallios R. Telepsychiatry appointments in a continuing care setting: kept, cancelled and no-shows. J Telemed Telecare. 2009;15(6):286-9. doi: 10.1258/jtt.2009.090305. PMID: 19720765. Exclusion reason: Ineligible intervention

392. Leroi I, Woolham J, Gathercole R, et al. Does telecare prolong community living in dementia? A study protocol for a pragmatic, randomised controlled trial. Trials 2013;14:349. doi: 10.1186/1745-6215-14349. PMID: 24152600. Exclusion reason: Ineligible intervention

393. Levin K, Madsen JR, Petersen I, et al. Telemedicine diabetes consultations are cost-effective, and effects on essential diabetes treatment parameters are similar to conventional treatment: 7-year results from the Svendborg Telemedicine Diabetes Project. J Diabetes Sci Technol. 2013 May 01;7(3):587-95. doi:

10.1177/193229681300700302. PMID: 23759390. Exclusion reason: Ineligible intervention

394. Levin YS, Warshaw EM. Teledermatology: a review of reliability and accuracy of diagnosis and management. Dermatol Clin. 2009 Apr;27(2):163-76, vii. doi: 10.1016/j.det.2008.11.012. PMID: 19254660. Exclusion reason: Ineligible study design
395. Lewis D, Morris S, Uzun O, et al. Paediatric cardiac telemedicine--transmitting echocardiography images in Wales. $\mathrm{J}$ Telemed Telecare. 2010;16(4):198-200. doi: 10.1258/jtt.2010.004010. PMID: 20511574. Exclusion reason: Ineligible comparison

396. Lewis J, Ray P, Liaw ST. Recent worldwide developments in eHealth and mHealth to more effectively manage cancer and other chronic diseases - A systematic review. Yearb Med Inform. 2016 Nov 10(1):93-108. doi: 10.15265/IY-2016-020. PMID: 27830236. Exclusion reason: Systematic review used to identify primary studies

397. Lewis K, Gilmour E, Harrison PV, et al. Digital teledermatology for skin tumours: a preliminary assessment using a receiver operating characteristics (ROC) analysis. J Telemed Telecare. 1999;5 Suppl 1:S57-8. PMID: 10534843. Exclusion reason: Ineligible outcome

398. Li B, Powell AM, Hooper PL, et al. Prospective evaluation of teleophthalmology in screening and recurrence monitoring of neovascular age-related macular degeneration: a randomized clinical trial. JAMA Ophthalmol. 2015 Mar;133(3):27682. doi: 10.1001/jamaophthalmol.2014.5014. PMID: 25473945. Exclusion reason: Ineligible intervention

399. Li Z, Wu C, Olayiwola JN, et al. Telemedicine-based digital retinal imaging vs standard ophthalmologic evaluation for the assessment of diabetic retinopathy. Conn Med. 2012 Feb;76(2):85-90. PMID: 22670358. Exclusion reason: Ineligible outcome

400. Liddy C, Drosinis P, Deri Armstrong C, et al. What are the cost savings associated with providing access to specialist care through the Champlain BASE eConsult service? A costing evaluation. BMJ Open. 2016 Jun 23;6(6):e010920. doi: 10.1136/bmjopen2015-010920. PMID: 27338880. Exclusion reason: Ineligible comparison

401. Liddy C, Drosinis P, Keely E. Electronic consultation systems: worldwide prevalence and their impact on patient care-a systematic review. Fam Pract. 2016 Jun;33(3):274-85. doi: 10.1093/fampra/cmw024. PMID: 27075028. Exclusion reason: Systematic review used to identify primary studies 
402. Liddy C, Rowan MS, Afkham A, et al. Building access to specialist care through econsultation. Open Med. 2013;7(1):e1-8. PMID: 23687533. Exclusion reason: Ineligible comparison

403. Liddy C, Smyth C, Poulin PA, et al. Improving access to chronic pain services through eConsultation: A cross-sectional study of the champlain BASE eConsult service. Pain Med. 2016 Apr 03;17(6):104957. doi: 10.1093/pm/pnw038. PMID: 27040667. Exclusion reason: Ineligible comparison

404. Lilly CM, McLaughlin JM, Zhao H, et al. A multicenter study of ICU telemedicine reengineering of adult critical care. Chest. 2014 Mar 1;145(3):500-7. doi:

10.1378/chest.13-1973. PMID: 24306581.

Exclusion reason: Ineligible intervention

405. Lim AC, Egerton IB, See A, et al. Accuracy and reliability of store-and-forward teledermatology: preliminary results from the St George Teledermatology Project. Australas J Dermatol. 2001 Nov;42(4):24751. PMID: 11903155. Exclusion reason: Ineligible intervention

406. Limido A, Mare C, Giani S, et al. [PROVA E TRASPORTA Project: results of teletransmission of the electrocardiogram from community hospitals and emergency service ambulances in the management of STelevation acute coronary syndromes]. G Ital Cardiol (Rome). 2006 Jul;7(7):498-504. PMID: 16977789. Exclusion reason: Ineligible intervention

407. Lin JC. Applying telecommunication technology to health-care delivery. IEEE Eng Med Biol Mag. 1999 Jul-Aug;18(4):2831. PMID: 10429899. Exclusion reason: Ineligible comparison

408. Lin KH, Chen CH, Chen YY, et al. Bidirectional and multi-user telerehabilitation system: clinical effect on balance, functional activity, and satisfaction in patients with chronic stroke living in long-term care facilities. Sensors. 2014;14(7):12451-66. doi: 10.3390/s140712451. PMID: 25019632.

Exclusion reason: Ineligible intervention
409. Linton O. Subspecialization consulting. Acad Radiol. 2004 May;11(5):602-3. doi: 10.1016/j.acra.2004.01.008. PMID:

15147627. Exclusion reason: Ineligible publication type

410. Liou JK, Soon MS, Chen CH, et al. Shared care combined with telecare improves glycemic control of diabetic patients in a rural underserved community. Telemed J E Health. 2014 Feb;20(2):175-8. doi: 10.1089/tmj.2013.0037. PMID: 24320193. Exclusion reason: Ineligible intervention

411. Lipana LS, Bindal D, Nettiksimmons J, et al. Telemedicine and face-to-face care for pediatric obesity. Telemed J E Health. 2013 Oct;19(10):806-8. doi:

10.1089/tmj.2012.0292. PMID: 23980937. Exclusion reason: Ineligible intervention

412. Liu SX, Lee MC, Atakhorrami M, et al. Economic assessment of home-based COPD management programs. COPD. 2013 Dec;10(6):640-9. doi: 10.3109/15412555.2013.813447. PMID: 23848542. Exclusion reason: Ineligible intervention

413. Liu W, Saxon DR, McNair B, et al. Endocrinology telehealth consultation improved glycemic control similar to faceto-face visits in veterans. J Diabetes Sci Technol. 2016 Sep;10(5):1079-86. doi: 10.1177/1932296816648343. PMID: 27170633. Exclusion reason: Ineligible intervention

414. Livingstone J, Solomon J. An assessment of the cost-effectiveness, safety of referral and patient satisfaction of a general practice teledermatology service. London J Prim Care (Abingdon). 2015;7(2):31-5. PMID: 26217401. Exclusion reason: Ineligible comparison

415. Lloyd AR, Clegg J, Lange J, et al. Safety and effectiveness of a nurse-led outreach program for assessment and treatment of chronic hepatitis $C$ in the custodial setting. Clin Infect Dis. 2013 Apr;56(8):1078-84. doi: 10.1093/cid/cis1202. PMID: 23362288. Exclusion reason: Ineligible comparison 
416. Loane MA, Bloomer SE, Corbett R, et al. A comparison of real-time and store-andforward teledermatology: a cost-benefit study. Br J Dermatol. 2000

Dec;143(6):1241-7. PMID: 11122028.

Exclusion reason: Ineligible comparison

417. Loane MA, Corbett R, Bloomer SE, et al. Diagnostic accuracy and clinical management by realtime teledermatology. Results from the Northern Ireland arms of the UK Multicentre Teledermatology Trial. J Telemed Telecare. 1998;4(2):95-100. doi: 10.1258/1357633981932028. PMID: 9744165. Exclusion reason: Ineligible comparison

418. Loane MA, Gore HE, Bloomer SE, et al. Preliminary results from the Northern Ireland arms of the UK Multicentre Teledermatology Trial: is clinical management by realtime teledermatology possible? J Telemed Telecare. 1998;4 Suppl 1:3-5. doi: 10.1258/1357633981931425. PMID: 9640716. Exclusion reason: Ineligible comparison

419. Loh PK, Donaldson M, Flicker L, et al. Development of a telemedicine protocol for the diagnosis of Alzheimer's disease. $\mathrm{J}$ Telemed Telecare. 2007;13(2):90-4. doi: 10.1258/135763307780096159. PMID: 17359573. Exclusion reason: Ineligible intervention

420. Longan R. Telemedicine in prison. Stud Health Technol Inform. 1998;50:48-52. PMID: 10180585. Exclusion reason: Ineligible comparison

421. Lopez-Torres J, Rabanales J, Simarro MJ, et al. Effectiveness of a telemedicine programme for patients with metabolic syndrome. Technol Health Care. 2015;23(2):161-9. doi: 10.3233/THC140888. PMID: 25515052. Exclusion reason: Ineligible intervention

422. Lorton L, Legler JD. A telemedicine trial. J AHIMA. 1996 Apr;67(4):40-2. PMID: 10155779. Exclusion reason: Ineligible study design
423. Lowitt MH, Kessler, II, Kauffman CL, et al. Teledermatology and in-person examinations: a comparison of patient and physician perceptions and diagnostic agreement. Arch Dermatol. 1998 Apr;134(4):471-6. PMID: 9554300. Exclusion reason: Ineligible intervention

424. Luchsinger JA, Palmas W, Teresi JA, et al. Improved diabetes control in the elderly delays global cognitive decline. J Nutr Health Aging. 2011 Jun;15(6):445-9. PMID: 21623465. Exclusion reason: Ineligible intervention

425. Ludwick DA, Lortie C, Doucette J, et al. Evaluation of a telehealth clinic as a means to facilitate dermatologic consultation: pilot project to assess the efficiency and experience of teledermatology used in a primary care network. J Cutan Med Surg. 2010 Jan-Feb;14(1):7-12. PMID: 20128984. Exclusion reason: Ineligible comparison

426. Ludzik J, Witkowski AM, RotermanKonieczna I. New telemedicine techniques in dermatology - evaluation with reflectance confocal microscopy via cloud-based platform. Folia Med Cracov. 2016;56(3):219. PMID: 28275268. Exclusion reason: Ineligible outcome

427. Lumb PD. Technology application: disruptive, empowering, controlling? J Crit Care. 2013 Jun;28(3):221-2. doi: 10.1016/j.jcrc.2013.03.016. PMID: 23623035. Exclusion reason: Ineligible publication type

428. Lundell S, Holmner A, Rehn B, et al. Telehealthcare in COPD: a systematic review and meta-analysis on physical outcomes and dyspnea. Respir Med. 2015 Jan;109(1):11-26. doi: 10.1016/j.rmed.2014.10.008. PMID: 25464906. Exclusion reason: Ineligible intervention

429. Lupoli J, Articola C, Stufflebearn L. Technology makes virtual checkups reality. Caring. 2006 Jul;25(7):6-8. PMID: 16915945. Exclusion reason: Ineligible intervention 
430. Lynch M, Tachakra S, Dawood M, et al. Using telemedicine for remote trauma medicine. What the public thinks. Emerg Nurse. 2000 May;8(2):8-9. doi: 10.7748/en2000.05.8.2.8.c1317. PMID: 11935544. Exclusion reason: Ineligible study design

431. Mackintosh N, Terblanche M, Maharaj R, et al. Telemedicine with clinical decision support for critical care: a systematic review. Systematic Reviews. 2016 Oct 18;5(1):176. PMID: 27756376. Exclusion reason: Systematic review used to identify primary studies

432. MacLeod KJ, Marcin JP, Boyle C, et al. Using telemedicine to improve the care delivered to sexually abused children in rural, underserved hospitals. Pediatrics. 2009 Jan;123(1):223-8. doi: 10.1542/peds.2007-1921. PMID: 19117886. Exclusion reason: Ineligible comparison

433. Magann EF, McKelvey SS, Hitt WC, et al. The use of telemedicine in obstetrics: a review of the literature. Obstet Gynecol Surv. 2011 Mar;66(3):170-8. doi: 10.1097/OGX.0b013e3182219902. PMID: 21689487. Exclusion reason: Ineligible study design

434. Mair F, McClusky C, Wilsgaard T, et al. The added value of video for consultations in telemedicine for minor injuries work. $\mathrm{J}$ Telemed Telecare. 2011;17(8):427-31. doi: 10.1258/jtt.2011.110318. PMID: 22036927. Exclusion reason: Ineligible comparison

435. Makai P, Perry M, Robben SH, et al. Evaluation of an eHealth intervention in chronic care for frail older people: why adherence is the first target. J Med Internet Res. 2014;16(6):e156. doi:

10.2196/jmir.3057. PMID: 24966146.

Exclusion reason: Ineligible intervention

436. Mallett R. Teledermatology in practice: the Peterborough experience. British Journal of Healthcare Computing \& Information Management. 2000;17(5):14-7. Exclusion reason: Ineligible intervention
437. Malone F, Callahan CW, Chan DS, et al. Caring for children with asthma through teleconsultation: "ECHO-Pac, The Electronic Children's Hospital of the Pacific". Telemed J E Health. 2004 Summer;10(2):138-46. doi: 10.1089/tmj.2004.10.138. PMID: 15319043. Exclusion reason: Ineligible comparison

438. Mamlin BW, Tierney WM. The promise of information and communication technology in healthcare: extracting value from the chaos. Am J Med Sci. 2016 Jan;351(1):5968. doi: 10.1016/j.amjms.2015.10.015. PMID: 26802759. Exclusion reason: Ineligible study design

439. Mammas CS, Geropoulos S, Saatsakis G, et al. Telepathology as a method to optimize quality in organ transplantation: a feasibility and reliability study of the virtual benching of liver graft. Stud Health Technol Inform. 2013;190:276-8. doi: 10.3233/978-1-61499276-9-276. PMID: 23823447. Exclusion reason: Ineligible intervention

440. Mammas CS, Kavantzas N, Geropoulos S, et al. Telepathology as a method to optimize quality in organ transplantation: a feasibility and reliability study of the virtual benching of renal graft. Stud Health Technol Inform. 2013;190:270-2. doi: 10.3233/978-1-61499276-9-270. PMID: 23823445. Exclusion reason: Ineligible intervention

441. Mammas CS, Lazaris A, Geropoulos S, et al. Telemedicine systems in organ transplantation: a feasibility and reliability study of the integrated teleradiological and tele-pathological evaluation of liver graft. Stud Health Technol Inform. 2013;190:2857. doi: 10.3233/978-1-61499-276-9-285. PMID: 23823450. Exclusion reason: Ineligible intervention

442. Mammas CS, Lazaris A, Geropoulos S, et al. Telepathology as a method to optimize quality in organ transplantation: a feasibility and reliability study of the virtual benching of pancreas graft. Stud Health Technol Inform. 2013;190:273-5. doi: 10.3233/9781-61499-276-9-273. PMID: 23823446.

Exclusion reason: Ineligible intervention 
443. Mani S, Joseph LH, Sharma S. Feasibility of telemedicine or telephone-based family intervention for rural paediatric obesity: Cluster randomized control trial. J Telemed Telecare. 2016 06;22(4):264-5. doi: 10.1177/1357633X15601524. PMID: 26362563. Exclusion reason: Ineligible publication type

444. Mann T, Colven R. A picture is worth more than a thousand words: enhancement of a pre-exam telephone consultation in dermatology with digital images. Acad Med. 2002 Jul;77(7):742-3. PMID: 12114161.

Exclusion reason: Ineligible comparison

445. Marcelo A, Fatmi Z, Firaza PN, et al. An online method for diagnosis of difficult TB cases for developing countries. Stud Health Technol Inform. 2011;164:168-73. PMID: 21335706. Exclusion reason: Ineligible outcome

446. Marchell R, Locatis C, Burges G, et al. Comparing high definition live interactive and store-and-forward consultations to inperson examinations. Telemed J E Health. 2017 Mar;23(3):213-8. doi: 10.1089/tmj.2016.0093. PMID: 27705083. Exclusion reason: Ineligible outcome

447. Marchell R, Locatis C, Burgess G, et al. Patient and provider satisfaction with teledermatology. Telemed J E Health. 2017 Aug;23(8):684-90. doi: 10.1089/tmj.2016.0192. PMID: 28375822. Exclusion reason: Ineligible intervention

448. Marcolino MS, Pereira Afonso dos Santos J, Santos Neves D, et al. Teleconsultations to provide support for primary care practitioners and improve quality of care-the experience of a large scale telehealth service in Brazil. Stud Health Technol Inform. 2015;216:987. PMID: 26262289.

Exclusion reason: Background information only

449. Marcolino MS, Santos TMM, Stefanelli FC, et al. Cardiovascular emergencies in primary care: an observational retrospective study of a large-scale telecardiology service. Sao Paulo Med J. 2017 Sep-Oct;135(5):481-7. doi: 10.1590/1516-3180.2017.0090110617. PMID: 29116311. Exclusion reason: Ineligible outcome
450. Marconi GP, Chang T, Pham PK, et al. Traditional nurse triage vs physician telepresence in a pediatric ED. Am J Emerg Med. 2014 Apr;32(4):325-9. doi: 10.1016/j.ajem.2013.12.032. PMID: 24445223. Exclusion reason: Ineligible intervention

451. Margolis A, Young H, Lis J, et al. A telepharmacy intervention to improve inhaler adherence in veterans with chronic obstructive pulmonary disease. Am J Health Syst Pharm. 2013 Nov 1;70(21):1875-6. doi: 10.2146/ajhp120241. PMID: 24128957.

Exclusion reason: Ineligible intervention

452. Marino R, Ghanim A. Teledentistry: a systematic review of the literature. $\mathrm{J}$ Telemed Telecare. 2013 Jun;19(4):179-83. doi: 10.1177/1357633X13479704. PMID: 23512650. Exclusion reason: Systematic review used to identify primary studies

453. Marshall M, Shah R, Stokes-Lampard H. Online consulting in general practice: making the move from disruptive innovation to mainstream service. BMJ. 201803 26;360:k1195. doi: 10.1136/bmj.k1195. PMID: 29581174. Exclusion reason: Ineligible publication type

454. Martinelli T, Bosson JL, Bressollette L, et al. Robot-based tele-echography: clinical evaluation of the TER system in abdominal aortic exploration. J Ultrasound Med. 2007 Nov;26(11):1611-6. PMID: 17957055. Exclusion reason: Ineligible intervention

455. Martinez A, Villarroel V, Seoane J, et al. A study of a rural telemedicine system in the Amazon region of Peru. J Telemed Telecare. 2004;10(4):219-25. doi: 10.1258/1357633041424412. PMID: 15273032. Exclusion reason: Ineligible intervention

456. Martin-Khan M, Flicker L, Wootton R, et al. The diagnostic accuracy of telegeriatrics for the diagnosis of dementia via video conferencing. J Am Med Dir Assoc. 2012 Jun;13(5):487 e19-24. doi: 10.1016/j.jamda.2012.03.004. PMID: 22572552. Exclusion reason: Ineligible intervention 
457. Martin-Khan M, Handyside J, Salih S, et al. A pilot telegeriatrics service in rural Australia. J Telemed Telecare. 2007;13:S3:104-5. Exclusion reason: Ineligible comparison

458. Martin-Khan M, Varghese P, Wootton R, et al. Physical examination and diagnosis of dementia for video consultation. J Am Geriatr Soc. 2008 May;56(5):947-9. doi: 10.1111/j.1532-5415.2008.01658.x. PMID: 18454756. Exclusion reason: Ineligible intervention

459. Martin-Khan M, Verghese P, Wootton R, et al. Successes and failures in assessing cognitive function in older adults using video consultation. J Telemed Telecare. 2007;13:S3:60-2. Exclusion reason: Ineligible intervention

460. Martin-Khan M, Wootton R, Whited J, et al. A systematic review of studies concerning observer agreement during medical specialist diagnosis using videoconferencing. J Telemed Telecare. 2011;17(7):350-7. doi: 10.1258/jtt.2011.101113. PMID: 21983223. Exclusion reason: Ineligible outcome

461. Martin-Khan MG, Edwards H, Wootton R, et al. Reliability of an online geriatric assessment procedure using the interRAI acute care assessment system. J Am Geriatr Soc. 2017 Sep;65(9):2029-36. doi: 10.1111/jgs.14895. PMID: 28832897. Exclusion reason: Ineligible intervention

462. Mashru J, Kirlew M, Saginur R, et al. Management of infectious diseases in remote northwestern Ontario with telemedicine videoconference consultations. J Telemed Telecare. 2017 Jan;23(1):83-7. doi: 10.1177/1357633X15625136. PMID: 26748393. Exclusion reason: Background information only

463. Masi C, Hamlish T, Davis A, et al. Using an established telehealth model to train urban primary care providers on hypertension management. J Clin Hypertens. 2012 Jan;14(1):45-50. doi: 10.1111/j.17517176.2011.00559.x. PMID: 22235823. Exclusion reason: Ineligible intervention
464. Mastrogiannis DS, Igwe E, Homko CJ. The role of telemedicine in the management of the pregnancy complicated by diabetes. Curr Diab Rep. 2013 Feb;13(1):1-5. doi: 10.1007/s11892-012-0352-x. PMID: 23242646. Exclusion reason: Ineligible intervention

465. McConnochie KM, Conners GP, Brayer AF, et al. Effectiveness of telemedicine in replacing in-person evaluation for acute childhood illness in office settings. Telemed J E Health. 2006 Jun;12(3):308-16. doi: 10.1089/tmj.2006.12.308. PMID: 16796498. Exclusion reason: Ineligible outcome

466. McConnochie KM, Conners GP, Brayer AF, et al. Differences in diagnosis and treatment using telemedicine versus in-person evaluation of acute illness. Ambul Pediatr. 2006 Jul-Aug;6(4):187-95; discussion 96-7. doi: 10.1016/j.ambp.2006.03.002. PMID: 16843248. Exclusion reason: Ineligible intervention

467. McConnochie KM, Wood NE, Alarie C, et al. Care offered by an information-rich pediatric acute illness connected care model. Telemed J E Health. 2016 Jun;22(6):465-72. doi: 10.1089/tmj.2015.0161. PMID: 26701609. Exclusion reason: Ineligible comparison

468. McConnochie KM, Wood NE, Kitzman HJ, et al. Telemedicine reduces absence resulting from illness in urban child care: evaluation of an innovation. Pediatrics. 2005 May;115(5):1273-82. doi: 10.1542/peds.2004-0335. PMID: 15867035. Exclusion reason: Ineligible intervention

469. McCue MJ, Mazmanian PE, Hampton CL, et al. Cost-minimization analysis: A followup study of a telemedicine program. Telemed J. 1998 Winter;4(4):323-7. doi: 10.1089/tmj.1.1998.4.323. PMID: 10220472. Exclusion reason: Ineligible intervention

470. McDougall JA, Ferucci ED, Glover J, et al. Telerheumatology: A systematic review. Arthritis Care Res (Hoboken). 2017 Oct;69(10):1546-57. doi: 10.1002/acr.23153. PMID: 27863164. Exclusion reason: Systematic review used to identify primary studies 
471. McFarland LV, Raugi GJ, Taylor LL, et al. Implementation of an education and skills programme in a teledermatology project for rural veterans. J Telemed Telecare. 2012

Mar;18(2):66-71. doi:

10.1258/jtt.2011.110518. PMID: 22198956.

Exclusion reason: Background information only

472. McGill AF, North JB. Teleconference fracture clinics: a trial for rural hospitals. ANZ J Surg. 2012 Jan-Feb;82(1-2):2-3. doi: 10.1111/j.1445-2197.2011.05952.x. PMID: 22507484. Exclusion reason: Ineligible comparison

473. McGill M, Constantino M, Yue DK. Integrating telemedicine into a national diabetes footcare network. Pract Diabetes Int. 2000;17(7):235-8. Exclusion reason: Ineligible comparison

474. McGowan J, Hogg W, Zhong J, et al. A cost-consequences analysis of a primary care librarian question and answering service. PLoS One. 2012;7(3):e33837. doi: 10.1371/journal.pone.0033837. PMID: 22442727. Exclusion reason: Ineligible intervention

475. McGrail KM, Ahuja MA, Leaver CA. Virtual visits and patient-centered care: results of a patient survey and observational study. J Med Internet Res. 2017 May 26;19(5):e177. doi: 10.2196/jmir.7374. PMID: 28550006. Exclusion reason: Ineligible intervention

476. McKoy KC, DiGregorio S, Stira L. Asynchronous teledermatology in an urban primary care practice. Telemed J E Health. 2004;10 Suppl 2:S-70-80. PMID: 23570217. Exclusion reason: Ineligible comparison

477. McLaren PM, Blunden J, Lipsedge ML, et al. Telepsychiatry in an inner-city community psychiatric service. J Telemed Telecare. 1996;2(1):57-9. doi: 10.1258/1357633961929178. PMID: 9375043. Exclusion reason: Ineligible study design

478. McLaren SW. Teledentistry: An alternative method to in-office consultations. Quintessence Int. 2017;31(8):179-80. doi: 10.3290/j.qi.a37796. PMID: 28232960. Exclusion reason: Ineligible publication type
479. McLaughlin S, Tobin RJ, Leonard S, et al. The role of digital photography and electronic referral in the triage of patients with suspected skin cancer. Br J Dermatol. 2006 Jan;154(1):188-90. doi: 10.1111/j.1365-2133.2005.07009.x. PMID: 16403120. Exclusion reason: Ineligible comparison

480. McManus J, Salinas J, Morton M, et al. Teleconsultation program for deployed soldiers and healthcare professionals in remote and austere environments. Prehosp Disaster Med. 2008 May-Jun;23(3):210-6; discussion 7. PMID: 18702266. Exclusion reason: Ineligible comparison

481. McNelis J, Schwall GJ, Collins JF. Robotic remote presence technology in the surgical intensive care unit. J Trauma Acute Care Surg. 2012 Feb;72(2):527-30. doi: 10.1097/TA.0b013e31822f7d3b. PMID: 22327990. Exclusion reason: Ineligible intervention

482. McWilliams T, Hendricks J, Twigg D, et al. Telehealth for paediatric burn patients in rural areas: a retrospective audit of activity and cost savings. Burns (03054179). 2016;22(8):1487-93. doi: 10.1016/j.burns.2016.03.001. PMID: 27575678. Exclusion reason: Ineligible comparison

483. Meade B, Barnett P. Emergency care in a remote area using interactive video technology: a study in prehospital telemedicine. J Telemed Telecare. 2002;8(2):115-7. doi: 10.1258/1357633021937587. PMID: 11972948. Exclusion reason: E8

484. Meade DM. Cut to the chase! How can you get ED docs to OK what you need? Emerg Med Serv. 1996 Aug;25(8):47-52. PMID: 10159378. Exclusion reason: Ineligible intervention

485. Medeiros de Bustos E, Berthier E, Chavot D, et al. Evaluation of a French regional telemedicine network dedicated to neurological emergencies: A 14-year study. Telemed J E Health. 2018 Feb;24(2):155-60. doi: 10.1089/tmj.2017.0035. PMID: 29346039. Exclusion reason: Ineligible comparison 
486. Mehrotra A, Paone S, Martich GD, et al. A comparison of care at e-visits and physician office visits for sinusitis and urinary tract infection. JAMA Intern Med. 2013 Jan 14;173(1):72-4. doi: 10.1001/2013.jamainternmed.305. PMID: 23403816. Exclusion reason: Ineligible intervention

487. Mekhjian H, Warisse J, Gailiun M, et al. An Ohio telemedicine system for prison inmates: a case report. Telemed J. 1996 Spring;2(1):17-24. doi: 10.1089/tmj.1.1996.2.17. PMID: 10165346. Exclusion reason: Ineligible comparison

488. Menchetti M, Sighinolfi C, Di Michele V, et al. Effectiveness of collaborative care for depression in Italy. A randomized controlled trial. Gen Hosp Psychiatry. 2013 2013/11/01/;35(6):579-86. doi: 10.1016/j.genhosppsych.2013.07.009. PMID: 23969143. Exclusion reason: Ineligible intervention

489. Mendez I, Jong M, Keays-White D, et al. The use of remote presence for health care delivery in a northern Inuit community: a feasibility study. Int J Circumpolar Health. 2013;72doi: 10.3402/ijch.v72i0.21112. PMID: 23984292. Exclusion reason: Ineligible comparison

490. Mendu ML, McMahon GM, Licurse A, et al. Electronic consultations in nephrology: pilot implementation and evaluation. Am J Kidney Dis. 2016 Nov;68(5):821-3. doi: 10.1053/j.ajkd.2016.05.029. PMID: 27503182. Exclusion reason: Ineligible comparison

491. Menelli S. Save time and effort--and make more money--with electronic communication. Midwifery Today Int Midwife. 2007 Summer(82):22. PMID: 17725120. Exclusion reason: Ineligible outcome

492. Metting EI, Riemersma RA, Kocks JH, et al. Feasibility and effectiveness of an asthma/COPD service for primary care: a cross-sectional baseline description and longitudinal results. NPJ Prim Care Respir Med. 2015;25:14101. doi: 10.1038/npjpcrm.2014.101. PMID: 25569634. Exclusion reason: Ineligible comparison
493. Mettner J. Long distance service. Minn Med. 2004 Sep;87(9):24-7, 56-7. PMID: 15495870. Exclusion reason: Ineligible publication type

494. Mettner J. The doctor is in another town: telepsychiatry brings care to people in rural Minnesota. Minn Med. 2013 Oct;96(10):225. PMID: 24494373. Exclusion reason: Ineligible intervention

495. Meulepas MA, Jacobs JE, Smeenk FWJM, et al. Effect of an integrated primary care model on the management of middle-aged and old patients with obstructive lung diseases. Scand J Prim Health Care. 2007 10/15/received;25(3):186-92. doi: 10.1080/02813430701573943. PMID: 17846938. Exclusion reason: Ineligible intervention

496. Miladinovic M, Mladenovic D, Mihailovic $\mathrm{B}$, et al. Evaluation of telemedicine in the management of dentogenous infections. Vojnosanit Pregl. 2013 Jun;70(6):569-75. PMID: 23885523. Exclusion reason: Ineligible comparison

497. Mireskandari M, Kayser G, Hufnagl P, et al. Teleconsultation in diagnostic pathology: experience from Iran and Germany with the use of two European telepathology servers. J Telemed Telecare. 2004;10(2):99-103. doi: 10.1258/135763304773391549. PMID: 15068646. Exclusion reason: Ineligible intervention

498. Modai I, Jabarin M, Kurs R, et al. Cost effectiveness, safety, and satisfaction with video telepsychiatry versus face-to-face care in ambulatory settings. Telemed J E Health. 2006 Oct;12(5):515-20. doi: 10.1089/tmj.2006.12.515. PMID: 17042703. Exclusion reason: Ineligible intervention

499. Mofid M, Nesbitt T, Knuttel R. The other side of teledermatology: patient preferences. J Telemed Telecare. 2007;13(5):246-50. doi: 10.1258/135763307781458967. PMID: 17697512. Exclusion reason: Ineligible intervention

500. Mooney E, Hood AF, Lampros J, et al. Comparative diagnostic accuracy in virtual dermatopathology. Skin Res Technol. 2011 May;17(2):251-5. doi: 10.1111/j.16000846.2010.00493.x. PMID: 21251087. Exclusion reason: Ineligible intervention 
501. Moreno-Ramirez D, Ferrandiz L, Bernal AP, et al. Teledermatology as a filtering system in pigmented lesion clinics. J Telemed Telecare. 2005;11(6):298-303. doi: 10.1258/1357633054893364. PMID: 16168166. Exclusion reason: Ineligible intervention

502. Moreno-Ramirez D, Ferrandiz L, Galdeano $\mathrm{R}$, et al. Teledermatoscopy as a triage system for pigmented lesions: a pilot study. Clin Exp Dermatol. 2006 Jan;31(1):13-8. doi: 10.1111/j.1365-2230.2005.02000.x. PMID: 16309470. Exclusion reason: Ineligible intervention

503. Moreno-Ramírez D, Raya-Maldonado J, Morales-Conde $\mathrm{M}$, et al. Increasing frequency of seborrheic keratosis diagnoses as a favorable consequence of teledermatology-based skin cancer screening: A cross-sectional study of 34,553 patients. Am J Clin Dermatol. 2017;18(5):681-5. doi: 10.1007/s40257-0170283-z. PMID: 28397109. Exclusion reason: Ineligible comparison

504. Morgan AE, Lappan CM, Fraser SL, et al. Infectious disease teleconsultative support of deployed healthcare providers. Mil Med. 2009 Oct;174(10):1055-60. PMID: 19891217. Exclusion reason: Ineligible comparison

505. Morrell K, Hyers M, Stuchiner T, et al. Implementation of a tele-swallow safety study. Stroke. Conference: American Heart Association/American Stroke Association. 2016;47(no pagination). Exclusion reason: Ineligible study design

506. Morris J, Campbell-Richards D, Wherton J, et al. Webcam consultations for diabetes: findings from four years of experience in Newham. Practical Diabetes. 2017;22(7):4550. Exclusion reason: Ineligible intervention

507. Mort A, Eadie L, Regan L, et al. Combining transcranial ultrasound with intelligent communication methods to enhance the remote assessment and management of stroke patients: Framework for a technology demonstrator. Health Informatics J. 2016 Sep;22(3):691-701. doi: 10.1177/1460458215580353. PMID: 25975807. Exclusion reason: Ineligible comparison
508. Morton CA, Downie F, Auld S, et al. Community photo-triage for skin cancer referrals: an aid to service delivery. Clin Exp Dermatol. 2011 Apr;36(3):248-54. doi: 10.1111/j.1365-2230.2010.03960.x. PMID: 21070338. Exclusion reason: Ineligible intervention

509. Moskowitz A, Chan YF, Bruns J, et al. Emergency physician and stroke specialist beliefs and expectations regarding telestroke. Stroke. 2010 Apr;41(4):805-9. doi: 10.1161/STROKEAHA.109.574137. PMID: 20167910. Exclusion reason: Ineligible comparison

510. Mounessa JS, Chapman S, Braunberger T, et al. A systematic review of satisfaction with teledermatology. J Telemed Telecare. 2018 May;24(4):263-70. doi: 10.1177/1357633X17696587. PMID: 28350281. Exclusion reason: Systematic review used to identify primary studies

511. Mourin Moral FJ, Anadon MJ, de-Marcos L, et al. Clicker system improvement with a web technology system. Med Educ. 2015 Nov;49(11):1161-2. doi: 10.1111/medu.12874. PMID: 26494095. Exclusion reason: Ineligible comparison

512. Moya M, Valdez J, Yonas H, et al. The impact of a telehealth web-based solution on neurosurgery triage and consultation. Telemed J E Health. 2010 Nov;16(9):945-9. doi: 10.1089/tmj.2010.0044. PMID: 21034238. Exclusion reason: Ineligible comparison

513. Mrak G, Paladino J, Dzubur A, et al. Telemedicine in neurosurgery: teleradiology connections in the Republic of Croatia. J Telemed Telecare. 2009;15(3):142-4. doi: 10.1258/jtt.2009.003012. PMID: 19364899. Exclusion reason: Ineligible outcome

514. Mueller KI, Alstadhaug KB, Bekkelund SI. Telemedicine in diagnosis and management of non-acute headaches: An open-labelled noninferiority randomised controlled study. Eur J Neurol. 2016;23(785) PMID: 27301460. Exclusion reason: Ineligible intervention 
515. Mueller KJ, Potter AJ, MacKinney AC, et al. Lessons from tele-emergency: improving care quality and health outcomes by expanding support for rural care systems. Health Aff (Millwood). 2014 Feb;33(2):22834. doi: 10.1377/hlthaff.2013.1016. PMID: 24493765. Exclusion reason: Background information only

516. Muir J, Xu C, Paul S, et al. Incorporating teledermatology into emergency medicine. Emerg Med Australas. 2011 Oct;23(5):5628. doi: 10.1111/j.1742-6723.2011.01443.x. PMID: 21995470. Exclusion reason: Ineligible intervention

517. Muir KW, Grubber J, Mruthyunjaya P, et al. Progression of diabetic retinopathy in the hypertension intervention nurse telemedicine study. JAMA Ophthalmol. 2013 Jul;131(7):957-8. doi: 10.1001/jamaophthalmol.2013.81. PMID: 23702951. Exclusion reason: Ineligible intervention

518. Mulholland HC, Casey F, Brown D, et al. Application of a low cost telemedicine link to the diagnosis of neonatal congenital heart defects by remote consultation. Heart. 1999 Aug;82(2):217-21. PMID: 10409539.

Exclusion reason: Ineligible comparison

519. Muller KI, Alstadhaug KB, Bekkelund SI. A randomized trial of telemedicine efficacy and safety for nonacute headaches. Neurology. 2017 Jul 11;89(2):153-62. doi: 10.1212/WNL.0000000000004085. PMID: 28615434. Exclusion reason: Ineligible intervention

520. Muller KI, Alstadhaug KB, Bekkelund SI. Telemedicine in the management of nonacute headaches: a prospective, openlabelled non-inferiority, randomised clinical trial. Cephalalgia. 2017;37(9):855-63. doi: 10.1177/0333102416654885. PMID: 27301460. Exclusion reason: Ineligible intervention

521. Muller KI, Alstadhaug KB, Bekkelund SI. Headache patients' satisfaction with telemedicine: a 12-month follow-up randomized non-inferiority trial. Eur J Neurol. 2017. doi: 10.1111/ene.13294. PMID: 28432757. Exclusion reason: Ineligible intervention
522. Muller-Barna P, Hubert GJ, Boy S, et al. TeleStroke units serving as a model of care in rural areas: 10-year experience of the TeleMedical project for integrative stroke care. Stroke. 2014 Sep;45(9):2739-44. doi: 10.1161/STROKEAHA.114.006141. PMID: 25147327. Exclusion reason: Ineligible comparison

523. Myers K, Vander Stoep A, Zhou C, et al. Effectiveness of a telehealth service delivery model for treating attention-

deficit/hyperactivity disorder: a communitybased randomized controlled trial. J Am Acad Child Adolesc Psychiatry. 2015 Apr;54(4):263-74. doi: 10.1016/j.jaac.2015.01.009. PMID: 25791143. Exclusion reason: Ineligible comparison

524. Narasimha S, Madathil KC, Agnisarman S, et al. Designing telemedicine systems for geriatric patients: A review of the usability studies. Telemed J E Health. 2017

Jun;23(6):459-72. doi: 10.1089/tmj.2016.0178. PMID: 27875667. Exclusion reason: Ineligible study design

525. Nazareth S, Kontorinis N, Muwanwella N, et al. Successful treatment of patients with hepatitis $\mathrm{C}$ in rural and remote Western Australia via telehealth. J Telemed Telecare. 2013 Feb;19(2):101-6. doi:

10.1258/jtt.2012.120612. PMID: 23528788. Exclusion reason: Ineligible intervention

526. Nhu T. Telemedicine identifies need for ROP referrals. Ocular Surgery News. 2015;33(3):16-. Exclusion reason: Ineligible publication type

527. Nijssen-Jordan C, Jennett P, Johnston R. Failure of a paediatric teleconsultation project in a Canadian urban environment. J Telemed Telecare. 2001;7 Suppl 2:16. doi: 10.1258/1357633011937326. PMID: 11747647. Exclusion reason: Ineligible comparison

528. Nilsen LL, Moen A. Teleconsultation collaborative work and opportunities for learning across organizational boundaries. $\mathrm{J}$ Telemed Telecare. 2008;14(7):377-80. doi: 10.1258/jtt.2008.007012. PMID: 18852321. Exclusion reason: Ineligible comparison 
529. Norman V, French R, Hassan E, et al. Effect of a telemedicine facilitated program on intensive careunit length of stay (LOS) and financial performance [abstract]. Crit Care Med. 2009;37:A3. Exclusion reason: Ineligible publication type

530. Nymark LS, Davies P, Shabestari O, et al. Analysis of the impact of the Birmingham OwnHealth program on secondary care utilization and cost: a retrospective cohort study. Telemed J E Health. 2013

Dec;19(12):949-55. doi: 10.1089/tmj.2013.0011. PMID: 23909885.

Exclusion reason: Ineligible intervention

531. Ochs K. Communication in rural health care. Kans Nurse. 1995 Aug;70(7):4. PMID: 7494390. Exclusion reason: Ineligible comparison

532. Odnoletkova I, Goderis G, Nobels F, et al. Optimizing diabetes control in people with type 2 diabetes through nurse-led telecoaching. Diabet Med. 2016 Jun;33(6):777-85. doi: 10.1111/dme.13092. PMID: 26872105. Exclusion reason: Ineligible intervention

533. O'Donovan J, Bersin A. Controlling Ebola through mHealth strategies. Lancet Glob Health. 2015 Jan;3(1):e22. doi: 10.1016/S2214-109X(14)70357-2. PMID: 25539965. Exclusion reason: Ineligible publication type

534. Ogasawara K, Abe T. WTP (Willingness To Pay) for tele-health consultation service in Hokkaido, Japan. Stud Health Technol Inform. 2013;192:1026-. doi: 10.3233/9781-61499-289-9-1026. PMID: 23920800.

Exclusion reason: Ineligible intervention

535. O'Hanley P. Triage through technology: New Brunswick Tele-Care service reduces ER visits. Hosp Q. 1997 Winter;1(2):46-7. PMID: 10345291. Exclusion reason: Ineligible comparison

536. Ohashi K, Sakamoto N, Watanabe M, et al. Development of a telediagnosis endoscopy system over secure internet. Methods Inf Med. 2008;47(2):157-66. PMID: 18338087. Exclusion reason: Ineligible intervention
537. Okita AL, Molina Tinoco LJ, Patatas OH, et al. Use of smartphones in telemedicine: comparative study between standard and teledermatological evaluation of highcomplex care hospital inpatients. Telemed J E Health. 2016 Sep;22(9):755-60. doi: 10.1089/tmj.2015.0086. PMID: 26959500. Exclusion reason: Ineligible outcome

538. Okoroh EM, Kroelinger CD, Smith AM, et al. US and territory telemedicine policies: identifying gaps in perinatal care. Am J Obstet Gynecol. 2016;19(1):772.e1-.e6. doi: 10.1016/j.ajog.2016.08.020. PMID: 27565048. Exclusion reason: Background information only

539. Oliveira EN, Cainelli J, Pinto ME, et al. Integrated system to automatize information collecting for the primary health care at home. Stud Health Technol Inform. 2013;192:442-6. PMID: 23920593.

Exclusion reason: Ineligible intervention

540. Oliveira TC, Branquinho MJ, Goncalves L. State of the art in telemedicine - concepts, management, monitoring and evaluation of the telemedicine programme in Alentejo (Portugal). Stud Health Technol Inform. 2012;179:29-37. PMID: 22925783.

Exclusion reason: Ineligible outcome

541. Oxley T, Rogers S. Reducing the tyranny of distance, bringing specialist expertise to rural EDs: Virtual trauma and critical care unit. Australas Emerg Nurs J.

2009;12(4):165-6. doi: 10.1016/j.aenj.2009.08.049. Exclusion reason: Ineligible publication type

542. Padrick MM, Smith SNC, McMurry TL, et al. NIH stroke scale assessment via iPadbased mobile telestroke during ambulance transport is feasible-pilot data from the improving treatment with rapid evaluation of acute stroke via mobile telemedecine (iTREAT) study. Stroke. 2015;46(11). Exclusion reason: Ineligible outcome

543. Paiva T, Coelho H, Almeida A, et al. Neuroteleconsultation for general practitioners. Stud Health Technol Inform. 2000;78:329-41. PMID: 11151604.

Exclusion reason: Ineligible comparison 
544. Palmas W, Shea S, Starren J, et al. Medicare payments, healthcare service use, and telemedicine implementation costs in a randomized trial comparing telemedicine case management with usual care in medically underserved participants with diabetes mellitus (IDEATel). J Am Med Inform Assoc. 2010 Mar-Apr;17(2):196202. doi: 10.1136/jamia.2009.002592. PMID: 20190064. Exclusion reason: Ineligible intervention

545. Palmer MJ, Barnard S, Perel P, et al. Mobile phone-based interventions for improving adherence to medication prescribed for the primary prevention of cardiovascular disease in adults. Cochrane Database Syst Rev. 2017(5) doi:

10.1002/14651858.CD012675.pub2. PMID: 29932455. Exclusion reason: Ineligible study design

546. Papai G, Racz I, Czuriga D, et al. Transtelephonic electrocardiography in the management of patients with acute coronary syndrome. J Electrocardiol. 2014 MayJun;47(3):294-9. doi: 10.1016/j.jelectrocard.2014.02.007. PMID: 24636796. Exclusion reason: Ineligible intervention

547. Pappas Y, Seale C. The physical examination in telecardiology and televascular consultations: a study using conversation analysis. Patient Educ Couns. 2010 Oct;81(1):113-8. doi: 10.1016/j.pec.2010.01.005. PMID: 20144523. Exclusion reason: Ineligible comparison

548. Parham GP, Mwanahamuntu MH, Pfaendler KS, et al. eC3--a modern telecommunications matrix for cervical cancer prevention in Zambia. J Low Genit Tract Dis. 2010 Jul;14(3):167-73. doi: 10.1097/LGT.0b013e3181cd6d5e. PMID: 20592550. Exclusion reason: Ineligible comparison

549. Parra MW, Castillo RC, Rodas EB, et al. International trauma teleconference: evaluating trauma care and facilitating quality improvement. Telemed J E Health. 2013 Sep;19(9):699-703. doi: 10.1089/tmj.2012.0254. PMID: 23841490. Exclusion reason: Ineligible comparison
550. Pastora-Bernal JM, Martin-Valero R, BaronLopez FJ, et al. Evidence of benefit of telerehabitation after orthopedic surgery: a systematic review. J Med Internet Res. 2017 Apr 28;19(4):e142. doi: 10.2196/jmir.6836. PMID: 28455277. Exclusion reason: Ineligible intervention

551. Patterson BJ, Kaboli PJ, Tubbs T, et al. Rural access to clinical pharmacy services. J Am Pharm Assoc (2003). 2014 SepOct;54(5):518-25. doi: 10.1331/JAPhA.2014.13248. PMID: 25216881. Exclusion reason: Ineligible intervention

552. Patterson V, Conneally P. Intercontinental telemedicine for acute neurology. J Telemed Telecare. 2005;11(6):320-2. doi: 10.1258/1357633054893300. PMID: 16168170. Exclusion reason: Ineligible study design

553. Patterson V, Craig J, Pang KA, et al. Successful management of unexplained coma by telemedicine. J Telemed Telecare. 1999;5(2):134-6. doi: 10.1258/1357633991933341. PMID: 10628026. Exclusion reason: Ineligible study design

554. Patterson V, Samant S, Singh MB, et al. Diagnosis of epileptic seizures by community health workers using a mobile app: A comparison with physicians and a neurologist. Seizure. 2018 Feb;55:4-8. doi: 10.1016/j.seizure.2017.12.006. PMID: 29291457. Exclusion reason: Ineligible outcome

555. Paynter M. Evaluating use of telemedicine within a minor injury unit. Nurs Times. 2008;104(42):30-1. PMID: 18980079. Exclusion reason: Ineligible publication type

556. Pena NV, Torres M, Cardona JA, et al. Impact of telemedicine assessment on glycemic variability in children with type 1 diabetes mellitus. Diabetes Technol Ther. 2013 Feb;15(2):136-42. doi: 10.1089/dia.2012.0243. PMID: 23289433. Exclusion reason: Ineligible intervention 
557. Perednia DA, Gaines JA, Butruille TW. Comparison of the clinical informativeness of photographs and digital imaging media with multiple-choice receiver operating characteristic analysis. Arch Dermatol. 1995 Mar;131(3):292-7. PMID: 7887658. Exclusion reason: Ineligible intervention

558. Peterson A. Improving type 1 diabetes management with mobile tools: a systematic review. J Diabetes Sci Technol. 2014 Jul;8(4):859-64. doi: 10.1177/1932296814529885. PMID: 24876414. Exclusion reason: Systematic review used to identify primary studies

559. Phabphal K, Hirunpatch S. The effectiveness of low-cost teleconsultation for emergency head computer tomography in patients with suspected stroke. J Telemed Telecare. 2008;14(8):439-42. doi: 10.1258/jtt.2008.080603. PMID: 19047455. Exclusion reason: Ineligible intervention

560. Phillips CM, Burke WA, Allen MH, et al. Reliability of telemedicine in evaluating skin tumors. Telemed J. 1998 Spring;4(1):59. doi: 10.1089/tmj.1.1998.4.5. PMID: 9599075. Exclusion reason: Ineligible intervention

561. Piccolo D, Smolle J, Argenziano G, et al. Teledermoscopy--results of a multicentre study on 43 pigmented skin lesions. J Telemed Telecare. 2000;6(3):132-7. doi: 10.1258/1357633001935202. PMID: 10912329. Exclusion reason: Ineligible intervention

562. Piccolo D, Soyer HP, Chimenti S, et al. Diagnosis and categorization of acral melanocytic lesions using teledermoscopy. J Telemed Telecare. 2004;10(6):346-50. doi: 10.1258/1357633042602017. PMID: 15603633. Exclusion reason: Ineligible intervention

563. Pizarro AM, Gregorio JP, Lapao LV. The effect of pharmaceutical services-based eHealth intervention on chronic patient health outcomes. Stud Health Technol Inform. 2015;210:464-6. PMID: 25991189. Exclusion reason: Ineligible intervention
564. Pressman AR, Kinoshita L, Kirk S, et al. A novel telemonitoring device for improving diabetes control: protocol and results from a randomized clinical trial. Telemed J E Health. 2014 Feb;20(2):109-14. doi: 10.1089/tmj.2013.0157. PMID: 24404816. Exclusion reason: Ineligible intervention

565. Purcell R, McInnes S, Halcomb EJ. Telemonitoring can assist in managing cardiovascular disease in primary care: a systematic review of systematic reviews. BMC Fam Pract. 2014;15:43. doi: 10.1186/1471-2296-15-43. PMID: 24606887. Exclusion reason: Ineligible intervention

566. Pyne JM, Fortney JC, Mouden S, et al. Costeffectiveness of on-site versus off-site collaborative care for depression in rural FQHCs. Psychiatr Serv. 2015 May 1;66(5):491-9. doi: 10.1176/appi.ps.201400186. PMID: 25686811. Exclusion reason: Ineligible intervention

567. Raaber N, Duvald I, Riddervold I, et al. Geographic information system data from ambulances applied in the emergency department: effects on patient reception. Scand J Trauma Resusc Emerg Med. 2016 Mar 31;24:39. doi: 10.1186/s13049-0160232-5. PMID: 27029399. Exclusion reason: Ineligible intervention

568. Ramnath VR, Ho L, Maggio LA, et al. Centralized monitoring and virtual consultant models of tele-ICU care: a systematic review. Telemed J E Health. 2014 Oct;20(10):936-61. doi: 10.1089/tmj.2013.0352. PMID: 25226571. Exclusion reason: Systematic review used to identify primary studies

569. Ramnath VR, Khazeni N. Centralized monitoring and virtual consultant models of tele-ICU care: a side-by-side review. Telemed J E Health. 2014 Oct;20(10):96271. doi: 10.1089/tmj.2014.0024. PMID: 25225795. Exclusion reason: Background information only

570. Rand ER, Lappan CM, Lee JC, et al. Paging the worldwide cardiology consultant: the Army Knowledge Online Telemedicine Consultation Program in cardiology. Mil Med. 2009 Nov;174(11):1144-8. PMID: 19960820. Exclusion reason: Ineligible comparison 
571. Rappaport ES, Reynolds HN, Baucom S, et al. Telehealth support of managed care for a correctional system: The open architecture telehealth model. Telemed J E Health. 2018 Jan;24(1):54-60. doi:

10.1089/tmj.2016.0275. PMID: 28682706. Exclusion reason: Ineligible comparison

572. Rasekaba TM, Furler J, Blackberry I, et al. Telemedicine interventions for gestational diabetes mellitus: A systematic review and meta-analysis. Diabetes Res Clin Pract. 2015 Oct;110(1):1-9. doi: 10.1016/j.diabres.2015.07.007. PMID: 26264410. Exclusion reason: Ineligible intervention

573. Razavi H, Copeland SP, Turner AW. Increasing the impact of teleophthalmology in Australia: Analysis of structural and economic drivers in a state service. Aust $\mathrm{J}$ Rural Health. 2017 Feb;25(1):45-52. doi: 10.1111/ajr.12277. PMID: 26781722.

Exclusion reason: Ineligible comparison

574. Reid DS, Weaver LE, Sargeant JM, et al. Telemedicine in Nova Scotia: report of a pilot study. Telemed J. 1998 Fall;4(3):24958. doi: 10.1089/tmj.1.1998.4.249. PMID: 9831749. Exclusion reason: Ineligible comparison

575. Reponen J, Ilkko E, Jyrkinen L, et al. Initial experience with a wireless personal digital assistant as a teleradiology terminal for reporting emergency computerized tomography scans. J Telemed Telecare. 2000;6(1):45-9. doi:

10.1258/1357633001933943. PMID: 10824391. Exclusion reason: Ineligible outcome

576. Rimal D, Huang Fu JH, Gillett D. Our experience in using telehealth for paediatric plastic surgery in Western Australia. ANZ J Surg. 2017 Apr;87(4):277-81. doi: 10.1111/ans.13925. PMID: 28219124. Exclusion reason: Ineligible outcome

577. Rincon TA, Manos EL, Pierce JD. Telehealth intensive care unit nurse surveillance of sepsis. Comput Inform Nurs. 2017 Sep;35(9):459-64. doi: 10.1097/CIN.0000000000000355. PMID: 28445172. Exclusion reason: Ineligible outcome
578. Ritter LA, Robinette TR, Cofano J. Evaluation of a statewide telemedicine program. Calif J Health Promot. 2010;8(1):1-9. Exclusion reason: Ineligible comparison

579. Rixon L, Hirani SP, Cartwright M, et al. A RCT of telehealth for COPD patient's quality of life: the whole system demonstrator evaluation. Clin Respir J. 2017;11(4):459-69. doi: 10.1111/crj.12359. PMID: 26260325. Exclusion reason: Ineligible intervention

580. Roberts S, Spain B, Hicks C, et al. Telemedicine in the Northern Territory: an assessment of patient perceptions in the preoperative anaesthetic clinic. Aust J Rural Health. 2015 Jun;23(3):136-41. doi: 10.1111/ajr.12140. PMID: 25615954. Exclusion reason: Ineligible comparison

581. Robinson A. Video-conferencing: underused by rural general practitioners. Aust Health Rev. 2002;25(6):131-5. PMID: 12536872. Exclusion reason: Ineligible comparison

582. Robinson JD, Turner JW, Wood KS. Patient perceptions of acute care telemedicine: a pilot investigation. Health Commun. 2015;30(12):1269-76. doi: 10.1080/10410236.2014.936335. PMID: 25668582. Exclusion reason: Ineligible comparison

583. Rocca MA, Kudryk VL, Pajak JC, et al. The evolution of a teledentistry system within the Department of Defense. Proc AMIA Symp. 1999;Annual Symposium.:921-4. PMID: 10566495. Exclusion reason: Ineligible comparison

584. Rockhill CM, Tse YJ, Fesinmeyer MD, et al. Telepsychiatrists' medication treatment strategies in the children's attentiondeficit/hyperactivity disorder telemental health treatment study. J Child Adolesc Psychopharmacol. 2016 Oct;26(8):662-71. PMID: 26258927. Exclusion reason: No new data

585. Rodas EB, Latifi R, Cone S, et al. Telesurgical presence and consultation for open surgery. Arch Surg. 2002 Dec;137(12):1360-3; discussion 3. PMID: 12470100. Exclusion reason: Ineligible intervention 
586. Rodriguez Villa S, Alonso Alvarez C, de Dios Del Valle R, et al. Five-year experience of tele-ophthalmology for diabetic retinopathy screening in a rural population. Arch Soc Esp Oftalmol. 2016 Sep;91(9):426-30. doi: 10.1016/j.oftal.2016.01.023. PMID: 26900042. Exclusion reason: Ineligible comparison

587. Romero G, Sanchez P, Garcia M, et al. Randomized controlled trial comparing store-and-forward teledermatology alone and in combination with web-camera videoconferencing. Clin Exp Dermatol. 2010 Apr;35(3):311-7. doi: 10.1111/j.13652230.2009.03503.x. PMID: 19874323.

Exclusion reason: Ineligible comparison

588. Ronis SD, McConnochie KM, Wang H, et al. Urban telemedicine enables equity in access to acute illness care. Telemed J E Health. 2017 Feb;23(2):105-12. doi: 10.1089/tmj.2016.0098. PMID: 27383822. Exclusion reason: Ineligible intervention

589. Roots A, Bhalla A, Birns J. Telemedicine for stroke: a systematic review. J Neurosci Nurs. 2011;7(2):481-9. Exclusion reason: Systematic review used to identify primary studies

590. Rosen D, McCall JD, Primack BA. Telehealth protocol to prevent readmission among high-risk patients with congestive heart failure. Am J Med. 2017 Nov;130(11):1326-30. doi: 10.1016/j.amjmed.2017.07.007. PMID: 28756266. Exclusion reason: Ineligible intervention

591. Rosina R, Starling J, Nunn K, et al. Telenursing: clinical nurse consultancy for rural paediatric nurses. J Telemed Telecare. 2002;8 Suppl 3:S3:48-9. PMID: 12661621. Exclusion reason: Ineligible comparison

592. Ross J, Stevenson F, Lau R, et al. Factors that influence the implementation of ehealth: a systematic review of systematic reviews (an update). Implementation Science. 201610 26;11(1):146. PMID: 27782832. Exclusion reason: Systematic review used to identify primary studies
593. Ross MD, Twombly IA, Bruyns C, et al. Telecommunications for health care over distance: the Virtual Collaborative Clinic. Stud Health Technol Inform. 2000;70:28691. PMID: 10977558. Exclusion reason: Ineligible comparison

594. Rothenberg SS, Yoder S, Kay S, et al. Initial experience with surgical telementoring in pediatric laparoscopic surgery using remote presence technology. J Laparoendosc Adv Surg Tech A. 2009;19:S219-22. doi: 10.1089/lap.2008.0133. PMID: 18976120.

Exclusion reason: Ineligible comparison

595. Rottger J, Irving AM, Broere J, et al. Use of telecommunications in a rural emergency. Brain surgery by fax. J Telemed Telecare. 1997;3(1):59-60. doi: 10.1258/1357633971930210. PMID: 9139763. Exclusion reason: Ineligible study design

596. Rouleau G, Gagnon MP, Cote J, et al. Impact of information and communication technologies on nursing care: results of an overview of systematic reviews. J Med Internet Res. 2017 Apr 25;19(4):e122. doi: 10.2196/jmir.6686. PMID: 28442454.

Exclusion reason: Ineligible study design

597. Rubin MN, Wellik KE, Channer DD, et al. A systematic review of telestroke. Postgrad Med. 2013 Jan;125(1):45-50. doi: 10.3810/pgm.2013.01.2623. PMID: 23391670. Exclusion reason: Systematic review used to identify primary studies

598. Saifu HN, Asch SM, Goetz MB, et al. Evaluation of human immunodeficiency virus and hepatitis $C$ telemedicine clinics. Am J Manag Care. 2012 Apr;18(4):207-12. PMID: 22554009. Exclusion reason: Ineligible intervention

599. Saleem Y, Taylor MH, Khalifa N. Forensic telepsychiatry in the United Kingdom. Behav Sci Law. 2008;26(3):333-44. doi: 10.1002/bsl.810. PMID: 18548516. Exclusion reason: Ineligible study design

600. Saleh N, Abdel Hay R, Hegazy R, et al. Can teledermatology be a useful diagnostic tool in dermatology practice in remote areas? An Egyptian experience with 600 patients. J Telemed Telecare. 2017 Feb;23(2):233-8. doi: 10.1177/1357633X16633944. PMID: 26940796. Exclusion reason: Ineligible outcome 
601. Samson PC, Gupta NK, Vira MA, et al. The use of videoconferencing for monitoring inpatient post-operative urologic patients. J Urol. 2018;AUA 2018(4 Supplement 1):e1093-e4. Exclusion reason: Ineligible publication type

602. Samuels A. International telepsychiatry: a link between New Zealand and Australia. Aust N Z J Psychiatry. 1999 Apr;33(2):2846. PMID: 10336233. Exclusion reason: Ineligible publication type

603. Sanders KA, Figiel C, Kiely JM, et al. Expanding access to intravenous tissue-type plasminogen activator treatment with a practice-based telestroke system. J Stroke Cerebrovasc Dis. 2013 Nov;22(8):e546-8. doi:

10.1016/j.jstrokecerebrovasdis.2013.06.013. PMID: 23834847. Exclusion reason: Background information only

604. Saurman E, Perkins D, Roberts R, et al. Responding to mental health emergencies: implementation of an innovative telehealth service in rural and remote New South Wales, Australia. J Emerg Nurs. 2011 Sep;37(5):453-9. doi: 10.1016/j.jen.2010.11.005. PMID: 21889653. Exclusion reason: Ineligible intervention

605. Savard L, Borstad A, Tkachuck J, et al. Telerehabilitation consultations for clients with neurologic diagnoses: cases from rural Minnesota and American Samoa. NeuroRehabilitation. 2003;18(2):93-102. PMID: 12867672. Exclusion reason: Ineligible comparison

606. Sawyer MA, Lim RB, Wong SY, et al. Telementored laparoscopic cholecystectomy: a pilot study. Stud Health Technol Inform. 2000;70:302-8. PMID: 10977561. Exclusion reason: Ineligible intervention

607. Saysell E, Routley C. Telemedicine in community-based palliative care: evaluation of a videolink teleconference project. Int J Palliat Nurs. 2003 Nov;9(11):489-95. doi: 10.12968/ijpn.2003.9.11.11874. PMID: 14676726. Exclusion reason: Ineligible comparison
608. Scalvini S, Rivadossi F, Comini L, et al. Telemedicine: the role of specialist second opinion for GPs in the care of hypertensive patients. Blood Press. 2011 Jun;20(3):15865. doi: 10.3109/08037051.2010.542646. PMID: 21241165. Exclusion reason: Ineligible outcome

609. Schenkel J, Reitmeir P, Von Reden S, et al. [Cost analysis of telemedical treatment of stroke]. Gesundheitswesen. 2013 Jul;75(7):405-12. doi: 10.1055/s-00321321779 PMID: 22864846. Exclusion reason: Not in English

610. Schermerhorn SM, Auchincloss PJ, Kraft K, et al. Patella fracture in US servicemember in an austere location. J Spec Oper Med. 2018 2018;18(1):142-4. PMID: 29533450. Exclusion reason: Ineligible publication type

611. Scherpbier-de Haan ND, van Gelder VA, Van Weel C, et al. Initial implementation of a web-based consultation process for patients with chronic kidney disease. Ann Fam Med. 2013 Mar-Apr;11(2):151-6. doi: 10.1370/afm.1494. PMID: 23508602.

Exclusion reason: Ineligible comparison

612. Schnieder P. Telemedicine's legal \& financial battlefields. Healthc Inform. 1996 Oct;13(10):50-2, 4, 6. PMID: 10162035. Exclusion reason: Ineligible comparison

613. Schopp L. Telehealth and traumatic brain injury: creative community-based care. Telemed Today. 2000 Feb;8(1):4-6, 33. PMID: 10848027. Exclusion reason: Ineligible publication type

614. Schroeder SD. Quality focus: South Dakota Health IT summit. S D Med. 2013

Dec;66(12):516. PMID: 24527552. Exclusion reason: Ineligible publication type

615. Schwamm LH, Holloway RG, Amarenco P, et al. A review of the evidence for the use of telemedicine within stroke systems of care: a scientific statement from the American Heart Association/American Stroke Association. Stroke. 2009 Jul;40(7):261634. doi: 10.1161/strokeaha.109.192360. PMID: 19423852. Exclusion reason: Ineligible study design 
616. Schwarz F. Peer consultants: Missing link in the treatment of chronic pain. Can Fam Physician. 2015 Oct;61(10):837-8, 44-5. PMID: 26472783. Exclusion reason: Ineligible comparison

617. Scott WW, Jr., Rosenbaum JE, Ackerman SJ, et al. Subtle orthopedic fractures: teleradiology workstation versus film interpretation. Radiology. 1993

Jun;187(3):811-5. doi:

10.1148/radiology.187.3.8497636. PMID: 8497636. Exclusion reason: Ineligible intervention

618. Scuffham P. Systematic review of cost effectiveness in telemedicine. Quality of cost effectiveness studies in systematic reviews is problematic. BMJ. 2002 Sep 14;325(7364):598; author reply PMID: 12228145. Exclusion reason: Systematic review used to identify primary studies

619. Seckeler MD, Gordon BM, Williams DA, et al. Use of smart technology for remote consultation in the pediatric cardiac catheterization laboratory. Congenit Heart Dis. 2015 Nov-Dec;10(6):Background information only88-94. doi: 10.1111/chd.12308. PMID: 26559927.

Exclusion reason: Ineligible comparison

620. Seemann R, Guevara G, Undt G, et al. Clinical evaluation of tele-endoscopy using UMTS cellphones. Surg Endosc. 2010;24(11):2855-9. doi: 10.1007/s00464010-1066-2. PMID: 20440517. Exclusion reason: Ineligible comparison

621. Selsky D. A tale of two clinics: How virtual labor services revolutionized two CIED clinics. EP Lab Digest. 2014;14(9):34-7. Exclusion reason: Ineligible publication type

622. Selvam A. Using telemedicine to get faster, more accurate treatment for stroke patients. Mod Healthc. 2013 Nov 25;43(47):26. PMID: 24416872. Exclusion reason: Ineligible publication type
623. Senel E, Sabancilar E, Mansuroglu C, et al. A preliminary study of the contribution of telemicroscopy to the diagnosis and management of skin tumours in teledermatology. J Telemed Telecare. 2014 Jun;20(4):178-83. doi: 10.1177/1357633x14533885. PMID: 24786415. Exclusion reason: Ineligible intervention

624. Serghis D. Time for telehealth? Aust Nurs J. 1999 Jul;7(1):16-9. PMID: 10568912.

Exclusion reason: Ineligible comparison

625. Setterberg SR, Busseri MA, Fleissner RM, et al. Remote assessment of the use of seclusion and restraint with paediatric psychiatric patients. J Telemed Telecare. 2003;9(3):176-9. doi: 10.1258/135763303767150003. PMID: 12877781. Exclusion reason: Ineligible outcome

626. Shahpori R, Kushniruk A, Hebert M, et al. Tele-ICU - a Canadian review. Stud Health Technol Inform. 2011;164:420-4. PMID: 21335747. Exclusion reason: Ineligible study design

627. Shaikh Q, Kamal AK. Can telemedicine improve stroke outcomes? JPMA J Pak Med Assoc. 2012 Dec;62(12):1352-3. PMID: 23866493. Exclusion reason: Ineligible publication type

628. Shanit D, Cheng A, Greenbaum RA. Telecardiology: supporting the decisionmaking process in general practice. $\mathrm{J}$ Telemed Telecare. 1996;2(1):7-13. doi: 10.1258/1357633961929105. PMID: 9375036. Exclusion reason: Ineligible comparison

629. Sharp NJ. A teleconsultation briefing. Another example of "ya gotta be there". CIN Plus. 1999 Jan 01;2(1):3. PMID: 10890846. Exclusion reason: Ineligible publication type

630. Shaw EC, Hanby AM, Wheeler K, et al. Observer agreement comparing the use of virtual slides with glass slides in the pathology review component of the POSH breast cancer cohort study. J Clin Pathol. 2012 May;65(5):403-8. doi: 10.1136/jclinpath-2011-200369. PMID: 22447915. Exclusion reason: Ineligible intervention 
631. Shi L, Wu H, Dong J, et al. Telemedicine for detecting diabetic retinopathy: a systematic review and meta-analysis. $\mathrm{Br} \mathrm{J}$ Ophthalmol. 2015 Jun;99(6):823-31. doi: 10.1136/bjophthalmol-2014-305631. PMID: 25563767. Exclusion reason: Ineligible outcome

632. Shiels WE, 2nd. Pediatric teleradiology outsourcing: downside considerations. Pediatr Radiol. 2010;40(8):1349-52. doi: 10.1007/s00247-010-1666-y. PMID: 20523984. Exclusion reason: Ineligible study design

633. Shipherd JC, Kauth MR, Matza A. Nationwide interdisciplinary E-consultation on transgender are in the Veterans Health Administration. Telemed J E Health. 2016 Dec;22(12):1008-12. PMID: 27159795.

Exclusion reason: Ineligible comparison

634. Shore JH, Brooks E, Savin DM, et al. An economic evaluation of telehealth data collection with rural populations. Psychiatr Serv. 2007 Jun;58(6):830-5. doi: 10.1176/ps.2007.58.6.830. PMID: 17535944. Exclusion reason: Ineligible intervention

635. Sibson L. The use of telemedicine technology to support in pre-hospital patient care. Journal of Paramedic Practice. 2014;6(7):344-53. Exclusion reason: Ineligible study design

636. Silva PS, Horton MB, Clary D, et al. Identification of diabetic retinopathy and ungradable image rate with ultrawide field imaging in a national teleophthalmology program. Ophthalmology. 2016 Jun;123(6):1360-7. doi: 10.1016/j.ophtha.2016.01.043. PMID: 26949120. Exclusion reason: Ineligible intervention

637. Silverman ME. Long distance diagnosis. J Med Assoc Ga. 2000 Nov;89(4):18. PMID: 11143678. Exclusion reason: Ineligible publication type

638. Siwicki B. Providing the proof. Health Data Manag. 1997 Sep;5(9):108-10. PMID: 10169993. Exclusion reason: Ineligible publication type
639. Slipy SM. Telemedicine and interconnection services reduce costs at several facilities. Prison and health system partner with Ameritech. Health Manag Technol. 1995 Jul;16(8):52, 5. PMID: 10143565.

Exclusion reason: Ineligible publication type

640. Smith AC. Telepaediatrics. J Telemed Telecare. 2007;13(4):163-6. doi: 10.1258/135763307780908021. PMID: 17565770. Exclusion reason: Ineligible comparison

641. Smith AC, Kairl JA, Kimble R. Post-acute care for a paediatric burns patient in regional Queensland. J Telemed Telecare.

2002;8(5):302-4. doi: 10.1177/1357633X0200800510. PMID: 12396860. Exclusion reason: Ineligible study design

642. Smith AC, Williams M, Justo R. The multidisciplinary management of a paediatric cardiac emergency. J Telemed Telecare. 2002;8(2):112-4. doi: 10.1258/1357633021937578. PMID: 11972947. Exclusion reason: Ineligible study design

643. Smith HA. Telepsychiatry. Psychiatr Serv. 1998 Nov;49(11):1494-5. PMID: 9826259. Exclusion reason: Ineligible publication type

644. Smith SM, Cousins G, Clyne B, et al. Shared care across the interface between primary and specialty care in management of long term conditions. Cochrane Database Syst Rev. 2017(2). doi: 10.1002/14651858.CD004910.pub3. PMID: 28230899. Exclusion reason: Systematic review used to identify primary studies

645. Smith-Strom H, Iversen MM, Graue M, et al. An integrated wound-care pathway, supported by telemedicine, and competent wound management-Essential in follow-up care of adults with diabetic foot ulcers. Int $\mathrm{J}$ Med Inform. 2016;94:59-66. doi: 10.1016/j.ijmedinf.2016.06.020. PMID: 27573312. Exclusion reason: Ineligible study design

646. Solenski N, Southerland A, Shephard T, et al. The EQUITe telestroke study. Stroke. 2016;47(no pagination). Exclusion reason: Ineligible publication type 
647. Solomons NM, Lamb AE, Lucas FL, et al. Examination of the patient-focused impact of cancer telegenetics among a rural population: Comparison with traditional inperson services. Telemed J E Health. 2018 Feb;24(2):130-8. doi: 10.1089/tmj.2017.0073. PMID: 28737998. Exclusion reason: Ineligible intervention

648. Soriano Marcolino M, Minelli Figueira R, Pereira Afonso Dos Santos J, et al. The experience of a sustainable large scale Brazilian telehealth network. Telemed J E Health. 2016 Nov;22(11):899-908. PMID: 27167901. Exclusion reason: Ineligible comparison

649. Southern S, Ramakrishnan V. The digital second opinion. Br J Plast Surg. 1998 Dec;51(8):649-50. PMID: 10209475. Exclusion reason: Ineligible publication type

650. Speyer R, Denman D, Wilkes-Gillan S, et al. Effects of telehealth by allied health professionals and nurses in rural and remote areas: A systematic review and metaanalysis. J Rehabil Med. 2018 Feb 28;50(3):225-35. doi: 10.2340/165019772297. PMID: 29257195. Exclusion reason: Ineligible intervention

651. Sreelatha OK, Ramesh SV. Teleophthalmology: improving patient outcomes? Clin Ophthalmol. 2016;10:28595. doi: 10.2147/opth.s80487. PMID: 26929592. Exclusion reason: Ineligible study design

652. Stamford P, Bickford T, Hsiao H, et al. The significance of telemedicine in a rural emergency department. IEEE Eng Med Biol Mag. 1999 Jul-Aug;18(4):45-52. PMID: 10429901. Exclusion reason: Ineligible comparison

653. Staubach P, Muhler AK, Peveling-Oberhag A, et al. Telemedicine in children with dermatological diseases-a prospective randomized study in the department of dermatologiy university medical center Mainz/Germany. Pediatr Dermatol. 2017;ESPD. 2017(Supplement 2):S49-S50. Exclusion reason: Ineligible publication type
654. Stern J, Heneghan C, Sclafani AP, et al. Telemedicine applications in otolaryngology. J Telemed Telecare. 1998;4 Suppl 1:74-5. doi: 10.1258/1357633981931551. PMID: 9640745. Exclusion reason: Ineligible outcome

655. Stevanovic A, Beckers SK, Czaplik M, et al. Telemedical support for prehospital Emergency Medical Service (TEMS trial): study protocol for a randomized controlled trial. Trials. 2017;18(1). doi: 10.1186/s13063-017-1781-2. PMID: 28126019. Exclusion reason: Ineligible publication type

656. Stillerova T, Liddle J, Gustafsson L, et al. Could everyday technology improve access to assessments? A pilot study on the feasibility of screening cognition in people with Parkinson's disease using the Montreal Cognitive Assessment via Internet videoconferencing. Aust Occup Ther J. 2016;63(6):373-80. doi: 10.1111/14401630.12288. PMID: 27059159. Exclusion reason: Ineligible intervention

657. Sudan R, Salter M, Lynch T, et al. Bariatric surgery using a network and teleconferencing to serve remote patients in the Veterans Administration Health Care System: feasibility and results. Am J Surg. 2011;202(1):71-6. doi: 10.1016/j.amjsurg.2010.06.016. PMID: 21601825. Exclusion reason: Ineligible comparison

658. Suhonen R. Teledermatology trial in Finland. J Telemed Telecare. 1997;3(1):612. PMID: 9139764. Exclusion reason: Ineligible publication type

659. Sullivan DH, Chapman M, Mullen PE. Videoconferencing and forensic mental health in Australia. Behav Sci Law. 2008;26(3):323-31. doi: 10.1002/bsl.815. PMID: 18548513. Exclusion reason: Ineligible comparison

660. Swartz D. Remote access: the last mile is getting shorter. Telemed Today. 1999 Dec;7(6):27-30. PMID: 10848018.

Exclusion reason: Ineligible publication type 
661. Switzer JA, Demaerschalk BM, Xie J, et al. Cost-effectiveness of hub-and-spoke telestroke networks for the management of acute ischemic stroke from the hospitals' perspectives. Circ Cardiovasc Qual Outcomes. 2013 Jan 01;6(1):18-26. doi: 10.1161/CIRCOUTCOMES.112.967125. PMID: 23212458. Exclusion reason: Ineligible study design

662. Switzer JA, Singh R, Mathiassen L, et al. Telestroke: variations in intravenous thrombolysis by spoke hospitals. J Stroke Cerebrovasc Dis. 2015 Apr;24(4):739-44. doi:

10.1016/j.jstrokecerebrovasdis.2014.09.028. PMID: 25649506. Exclusion reason: Background information only

663. T. H, F. LJ, E. HS, et al. A randomized controlled trial of shared care versus routine care for patients receiving oral anticoagulant therapy. J Intern Med. 2002;252(4):322-31. doi: doi:10.1046/j.1365-2796.2002.01039.x. PMID: 12366605. Exclusion reason: Ineligible intervention

664. Tachakra S, Dawood M, Wiley C, et al. Telemedical consultation system. Emerg Nurse. 1999 Oct;7(6):11-3. PMID: 10614311. Exclusion reason: Ineligible comparison

665. Tachakra S, Mullett ST, Freij R, et al. Confidentiality and ethics in telemedicine. J Telemed Telecare. 1996;2 Suppl 1:68-71. PMID: 9375097. Exclusion reason: Ineligible comparison

666. Tachakra S, Rajani R. Social presence in telemedicine. J Telemed Telecare. 2002;8(4):226-30. doi: 10.1258/135763302320272202. PMID: 12217106. Exclusion reason: Ineligible outcome

667. Tachakra S, Stinson A. Suitable cases for teleconsultation by ENPs. Emerg Nurse. 2000 Dec-2001 Jan;8(8):9-11. PMID: 11935735. Exclusion reason: Ineligible comparison

668. Tachakra S, Uko Uche C, Stinson A. Four years' experience of telemedicine support of a minor accident and treatment service. $\mathrm{J}$ Telemed Telecare. 2002;8 Suppl 2:87-9. doi: 10.1177/1357633X020080S240. PMID: 12217151. Exclusion reason: Ineligible comparison
669. Tachakra S, Wiley C, Dawood M, et al. Evaluation of telemedical support to a freestanding minor accident and treatment service. J Telemed Telecare. 1998;4(3):1405. doi: 10.1258/1357633981932118. PMID: 10321041. Exclusion reason: Ineligible comparison

670. Tagliaferri S, Ippolito A, Cuccaro P, et al. Governance of innovation and appropriateness of hospitalization for highrisk pregnancy: the TOCOMAT system. Telemed J E Health. 2013 Jul;19(7):542-8. doi: 10.1089/tmj.2012.0205. PMID: 23146064. Exclusion reason: Ineligible intervention

671. Taleb AC, Bohm GM, Avila M, et al. The efficacy of telemedicine for ophthalmology triage by a general practitioner. J Telemed Telecare. 2005;11 Suppl 1:83-5. doi: 10.1258/1357633054461958. PMID: 16036006. Exclusion reason: Ineligible intervention

672. Tan E, Yung A, Jameson M, et al. Successful triage of patients referred to a skin lesion clinic using teledermoscopy (IMAGE IT trial). Br J Dermatol. 2010 Apr;162(4):803-11. doi: 10.1111/j.13652133.2010.09673.x. PMID: 20222920. Exclusion reason: Ineligible outcome

673. Tan IJ, Dobson LP, Bartnik S, et al. Realtime teleophthalmology versus face-to-face consultation: A systematic review. J Telemed Telecare. 2017 Aug;23(7):629-38. doi: 10.1177/1357633X16660640. PMID: 27444188. Exclusion reason: Ineligible outcome

674. Tan LF, Mason N, Gonzaga WJ. Virtual visits for upper respiratory tract infections in adults associated with positive outcome in a Cox Model. Telemed J E Health. 2017 Mar;23(3):200-4. doi: 10.1089/tmj.2016.0018. PMID: 27351543. Exclusion reason: Ineligible intervention

675. Taylor G. Mhealth and video conferencing: better care in rural North Carolina. Behav Healthc. 2013 Sep-Oct;33(5):47-8. PMID: 24298707. Exclusion reason: Ineligible publication type 
676. Taylor P. An assessment of the potential effect of a teledermatology system. $\mathrm{J}$ Telemed Telecare. 2000;6 Suppl 1:S74-6. PMID: 10793979. Exclusion reason: Ineligible comparison

677. Taylor P, Kennedy C, Murdoch I, et al. Assessment of benefit in tele-ophthalmology using a consensus panel. J Telemed Telecare. 2003;9(3):140-5. doi: 10.1258/135763303767149933. PMID: 12877775. Exclusion reason: Ineligible comparison

678. Terkelsen CJ, Lassen JF, Nørgaard BL, et al. Reduction of treatment delay in patients with ST-elevation myocardial infarction: impact of pre-hospital diagnosis and direct referral to primary percutanous coronary intervention. Eur Heart J. 2005;26(8):770-7. doi: 10.1093/eurheartj/ehi100. PMID: 15684279. Exclusion reason: Ineligible comparison

679. Thapa A, Kc B, Shakya B. Cost effective use of free-to-use apps in neurosurgery (FAN) in developing countries: from clinical decision making to educational courses, strengthening health care delivery. World Neurosurg. 2016 Nov;95:270-5. doi: 10.1016/j.wneu.2016.08.001. PMID: 27535637. Exclusion reason: Ineligible comparison

680. Thijssing L, van der Heijden JP, Chavannes $\mathrm{NH}$, et al. Telepulmonology: effect on quality and efficiency of care. Respir Med. 2014 Feb;108(2):314-8. doi: 10.1016/j.rmed.2013.10.017. PMID: 24210893. Exclusion reason: Ineligible comparison

681. Thijssing L, van der Heijden JP, Chavannes $\mathrm{NH}$, et al. Telepulmonology in the Netherlands: effect on quality and efficiency of care. Stud Health Technol Inform. 2013;192:1087. PMID: 23920861.

Exclusion reason: Ineligible comparison

682. Thomas SM, Jeyaraman MM, Hodge WG, et al. The effectiveness of teleglaucoma versus in-patient examination for glaucoma screening: a systematic review and metaanalysis. PLoS One. 2014;9(12):e113779. doi: 10.1371/journal.pone.0113779. PMID: 25479593. Exclusion reason: Systematic review used to identify primary studies
683. Timonen O. The teleconsultation in general practice. A randomized, controlled study of a remote consultation experiment using a videoconferencing system. Int $\mathrm{J}$

Circumpolar Health. 2004 Sep;63(3):28990. PMID: 15526933. Exclusion reason: Ineligible intervention

684. Torabi E, Froehle CM, Lindsell CJ, et al. Monte Carlo simulation modeling of a regional stroke team's use of telemedicine. Acad Emerg Med. 2016 Jan;23(1):55-62. doi: 10.1111/acem.12839. PMID: 26720746. Exclusion reason: Ineligible population

685. Torous J, Keshavan M, Gutheil T. Promise and perils of digital psychiatry. Asian J Psychiatr. 2014 Aug;10:120-2. doi: 10.1016/j.ajp.2014.06.006. PMID: 25042968. Exclusion reason: Ineligible publication type

686. Treter S, Perrier N, Sosa JA, et al. Telementoring: a multi-institutional experience with the introduction of a novel surgical approach for adrenalectomy. Ann Surg Oncol. 2013 Aug;20(8):2754-8. doi: 10.1245/s10434-013-2894-9. PMID: 23512076. Exclusion reason: Ineligible comparison

687. Tsai HH, Pong YP, Liang CC, et al. Teleconsultation by using the mobile camera phone for remote management of the extremity wound: a pilot study. Ann Plast Surg. 2004 Dec;53(6):584-7. PMID: 15602257. Exclusion reason: Ineligible comparison

688. Turnbull J, Pope C, Martin D, et al. Do telephones overcome geographical barriers to general practice out-of-hours services? Mixed-methods study of parents with young children. J Health Serv Res Policy. 2010 Jan;15(1):21-7. doi: 10.1258/jhsrp.2009.009023. PMID: 19843639. Exclusion reason: Ineligible intervention

689. Uchino K, Massaro L, Jovin TG, et al. Protocol adherence and safety of intravenous thrombolysis after telephone consultation with a stroke center. J Stroke Cerebrovasc Dis. 2010 Nov-Dec;19(6):41723. doi: 10.1016/j.jstrokecerebrovasdis.2009.07.013. PMID: 21051004. Exclusion reason: Ineligible intervention 
690. Unützer J, Katon W, Callahan CM, et al. Collaborative care management of late-life depression in the primary care setting: A randomized controlled trial. JAMA. 2002;288(22):2836-45. doi: 10.1001/jama.288.22.2836. PMID: 12472325. Exclusion reason: Ineligible intervention

691. Urquhart AC, Antoniotti NM, Berg RL. Telemedicine--an efficient and costeffective approach in parathyroid surgery. Laryngoscope. 2011 Jul;121(7):1422-5. doi: 10.1002/lary.21812. PMID: 21647908. Exclusion reason: Ineligible intervention

692. Valenzuela Espinoza A, Devos S, Van Hooff R, et al. Cost-utility of in-ambulance telemedicine for stroke patients. Value Health. 2016; ISPOR 2016(7):A699. doi: 10.2196/mhealth.8288. PMID: 29175808. Exclusion reason: Ineligible publication type

693. Valenzuela Espinoza A, Van Hooff RJ, De Smedt A, et al. Development and Pilot Testing of 24/7 In-Ambulance Telemedicine for Acute Stroke: Prehospital Stroke Study at the Universitair Ziekenhuis BrusselProject. Cerebrovasc Dis. 2016;42(1-2):1522. doi: $10.1159 / 000444175$. PMID: 26950076. Exclusion reason: Ineligible comparison

694. Vallejos QM, Quandt SA, Feldman SR, et al. Teledermatology consultations provide specialty care for farmworkers in rural clinics. J Rural Health. 2009 Spring2009;25(2):198-202. doi: 10.1111/j.1748-0361.2009.00218.x. PMID: 19785587. Exclusion reason: Ineligible comparison

695. van den Akker TW, Reker CH, Knol A, et al. Teledermatology as a tool for communication between general practitioners and dermatologists. J Telemed Telecare. 2001;7(4):193-8. doi: 10.1258/1357633011936390. PMID: 11506753. Exclusion reason: Ineligible comparison

696. van den Berg N, Schumann M, Kraft K, et al. Telemedicine and telecare for older patients--a systematic review. Maturitas. 2012 Oct;73(2):94-114. doi: 10.1016/j.maturitas.2012.06.010. PMID: 22809497. Exclusion reason: Ineligible intervention
697. Van Den Wijngaart LS, Roukema J, Boehmer ALM, et al. A virtual asthma clinic for children: fewer routine outpatient visits, same asthma control. Eur Respir J. 2017;50(4). doi: 10.1183/13993003.004712017. PMID: 28982775. Exclusion reason: Ineligible intervention

698. van der Heijden JP, Thijssing L, Witkamp L, et al. Accuracy and reliability of teledermatoscopy with images taken by general practitioners during everyday practice. J Telemed Telecare. 2013 Sep;19(6):320-5. doi: 10.1177/1357633X13503437. PMID: 24163296. Exclusion reason: Ineligible outcome

699. van Hezewijk M, van den Akker ME, van de Velde CJ, et al. Costs of different follow-up strategies in early breast cancer: a review of the literature. Breast. 2012 Dec;21(6):693700. doi: 10.1016/j.breast.2012.09.009. PMID: 23084960. Exclusion reason: Ineligible intervention

700. Van Hooff RJ, Cambron M, Van Dyck R, et al. Prehospital unassisted assessment of stroke severity using telemedicine: a feasibility study. Stroke. 2013 Oct;44(10):2907-9. doi: 10.1161/STROKEAHA.113.002079. PMID: 23920013. Exclusion reason: Ineligible population

701. Van Oeveren L, Donner J, Fantegrossi A, et al. Telemedicine-assisted intubation in rural emergency departments: a national emergency airway registry study. Ann Emerg Med. 2016;Conference: American college of emergency physicians, ACEP. 2016 research forum. United States 68(4 Supplement 1):S24-S5. doi: 10.1089/tmj.2016.0140. PMID: 27673565. Exclusion reason: Ineligible comparison

702. Van Oeveren L, Donner J, Fantegrossi A, et al. Telemedicine-assisted intubation in rural emergency departments: a National Emergency Airway Registry Study. Telemed J E Health. 2017 Apr;23(4):290-7. doi: 10.1089/tmj.2016.0140. PMID: 27673565. Exclusion reason: Ineligible comparison 
703. Veras M, Kairy D, Rogante M, et al. Outcome measures in tele-rehabilitation and virtual reality for stroke survivors: protocol for a scoping review. Glob J Health Sci. 2016 Jan;8(1):79-82. doi:

10.5539/gjhs.v8n1p79 PMID: 26234991.

Exclusion reason: Ineligible study design

704. Verberk WJ, Kessels AG, Thien T. Telecare is a valuable tool for hypertension

management, a systematic review and metaanalysis. Blood Press Monit. 2011

Jun;16(3):149-55. doi:

10.1097/MBP.0b013e328346e092. PMID: 21527847. Exclusion reason: Ineligible intervention

705. Verhoeven F, Tanja-Dijkstra K, Nijland N, et al. Asynchronous and synchronous teleconsultation for diabetes care: a systematic literature review. J Diabetes Sci Technol. 2010 May;4(3):666-84. PMID: 20513335. Exclusion reason: Systematic review used to identify primary studies

706. Verma M, Raman R, Mohan RE. Application of tele-ophthalmology in remote diagnosis and management of adnexal and orbital diseases. Indian J Ophthalmol. 2009;57(5):381-4. doi: 10.4103/03014738.55078. PMID: 19700877. Exclusion reason: Ineligible comparison

707. Vinekar A, Gilbert C, Dogra M, et al. The KIDROP model of combining strategies for providing retinopathy of prematurity screening in underserved areas in India using wide-field imaging, tele-medicine, non-physician graders and smart phone reporting. Indian J Ophthalmol. 2014 Jan;62(1):41-9. doi: 10.4103/03014738.126178. PMID: 24492500. Exclusion reason: Ineligible outcome

708. Vitacca M, Paneroni M, Grossetti F, et al. Is there any additional effect of tele-assistance on long-term care programmes in hypercapnic COPD patients? A retrospective study. COPD. 2016 Oct;13(5):576-82. doi: 10.3109/15412555.2016.1147542. PMID: 27018995. Exclusion reason: Ineligible intervention

709. Vladzymyrskyy AV. Our experience with telemedicine in traumatology and orthopedics. Ulus Travma Acil Cerrahi Derg. 2004 Jul;10(3):189-91. PMID: 15286891. Exclusion reason: Ineligible comparison
710. Voelkel N, Hubert ND, Backhaus R, et al. Thrombolysis in postoperative stroke. Stroke. 2017 Nov;48(11):3034-9. doi: 10.1161/STROKEAHA.117.017957. PMID: 28939674. Exclusion reason: Ineligible comparison

711. Volpe T, Boydell KM, Pignatiello A. Mental health services for Nunavut children and youth: evaluating a telepsychiatry pilot project. Rural Remote Health. 2014;14(2):2673. PMID: 24831454.

Exclusion reason: Ineligible intervention

712. von Wangenheim A, de Souza Nobre LF, Tognoli H, et al. User satisfaction with asynchronous telemedicine: a study of users of Santa Catarina's system of telemedicine and telehealth. Telemed J E Health. 2012 Jun;18(5):339-46. doi: 10.1089/tmj.2011.0197. PMID: 22703378. Exclusion reason: Ineligible outcome

713. Vowden K, Vowden P. A pilot study on the potential of remote support to enhance wound care for nursing-home patients. J Wound Care. 2013 Sep;22(9):481-8. PMID: 24005782. Exclusion reason: Ineligible outcome

714. Vrecar I, Hristovski D, Peterlin B. Telegenetics: an update on availability and use of telemedicine in clinical genetics service. J Med Syst. 2017 Feb;41(2):21. doi: 10.1007/s10916-016-0666-3. PMID: 27987158. Exclusion reason: Ineligible study design

715. Vuononvirta T, Timonen M, KeinanenKiukaanniemi S, et al. The attitudes of multiprofessional teams to telehealth adoption in northern Finland health centres. J Telemed Telecare. 2009;15(6):290-6. doi: 10.1258/jtt.2009.090108. PMID: 19720766. Exclusion reason: Background information only

716. Vyas KS, Hambrick HR, Shakir A, et al. A systematic review of the use of telemedicine in plastic and reconstructive surgery and dermatology. Ann Plast Surg. 2017 Jun;78(6):736-68. doi: 10.1097/SAP.0000000000001044. PMID: 28328635. Exclusion reason: Systematic review used to identify primary studies 
717. Wade VA, Eliott JA, Hiller JE. A qualitative study of ethical, medico-legal and clinical governance matters in Australian telehealth services. J Telemed Telecare. 2012

Mar;18(2):109-14. doi: 10.1258/jtt.2011.110808. PMID: 22267306. Exclusion reason: Ineligible comparison

718. Wakefield B, Flanagan J, Specht JK. Telehealth: an opportunity for gerontological nursing practice. J Gerontol Nurs. 2001 Jan;27(1):10-4. PMID: 11915091. Exclusion reason: Ineligible publication type

719. Wakefield BJ. Telehealth. J Gerontol Nurs. 2001 Jan;27(1):9. PMID: 11915101.

Exclusion reason: Ineligible publication type

720. Wakefield BJ, Buresh KA, Flanagan JR, et al. Interactive video specialty consultations in long-term care. J Am Geriatr Soc. 2004 May;52(5):789-93. doi: 10.1111/j.15325415.2004.52220.x. PMID: 15086663. Exclusion reason: Ineligible comparison

721. Wakefield BJ, Holman JE, Ray A, et al. Effectiveness of home telehealth in comorbid diabetes and hypertension: a randomized, controlled trial. Telemed J E Health. 2011 May;17(4):254-61. doi: 10.1089/tmj.2010.0176. PMID: 21476945. Exclusion reason: Ineligible intervention

722. Wakefield BJ, Holman JE, Ray A, et al. Outcomes of a home telehealth intervention for patients with diabetes and hypertension. Telemed J E Health. 2012 Oct;18(8):575-9. doi: 10.1089/tmj.2011.0237. PMID: 22873700. Exclusion reason: Ineligible intervention

723. Wallace DL, Hussain A, Khan N, et al. A systematic review of the evidence for telemedicine in burn care: with a UK perspective. Burns. 2012 Jun;38(4):465-80. doi: 10.1016/j.burns.2011.09.024. PMID: 22078804. Exclusion reason: Systematic review used to identify primary studies

724. Wallace DL, Smith RW, Pickford MA. A cohort study of acute plastic surgery trauma and burn referrals using telemedicine. J Telemed Telecare. 2007;13(6):282-7. doi: 10.1258/135763307781644933. PMID: 17785024. Exclusion reason: Ineligible intervention
725. Walter S, Kostopoulos P, Haass A, et al. Diagnosis and treatment of patients with stroke in a mobile stroke unit versus in hospital: a randomised controlled trial. Lancet Neurol. 2012 May;11(5):397-404. doi: 10.1016/S1474-4422(12)70057-1. PMID: 22497929. Exclusion reason: Ineligible intervention

726. Wang TT, Li JM, Zhu CR, et al. Assessment of utilization and cost-effectiveness of telemedicine program in western tegions of China: a 12-year study of 249 hospitals across 112 cities. Telemed J E Health. 2016 Nov;22(11):909-20. PMID: 27314300. Exclusion reason: Ineligible comparison

727. Waran V, Selladurai BM, Bahuri NF, et al. Teleconferencing using multimedia messaging service (MMS) for long-range consultation of patients with neurosurgical problems in an acute situation. J Trauma. 2008;64(2):362-5. doi: 10.1097/TA.0b013e318070cc88. PMID: 18301199. Exclusion reason: Ineligible comparison

728. Warshaw EM, Lederle FA, Grill JP, et al. Accuracy of teledermatology for pigmented neoplasms. J Am Acad Dermatol. 2009 Nov;61(5):753-65. doi: 10.1016/j.jaad.2009.04.032. PMID: 19679375. Exclusion reason: Ineligible comparison

729. Warshaw EM, Lederle FA, Grill JP, et al. Accuracy of teledermatology for nonpigmented neoplasms. J Am Acad Dermatol. 2009 Apr;60(4):579-88. doi: 10.1016/j.jaad.2008.11.892. PMID: 19217689. Exclusion reason: Ineligible comparison

730. Wasowski A, Skarzynski H, Lorens A, et al. The telefitting method used in the national network of teleaudiology: assessment of quality and cost effectiveness. J Hear Sci. 2012;2(2):81-5. Exclusion reason: Ineligible comparison

731. Watanabe SM, Fairchild A, Pituskin E, et al. Improving access to specialist multidisciplinary palliative care consultation for rural cancer patients by videoconferencing: report of a pilot project. Support Care Cancer. 2013 Apr;21(4):12017. doi: 10.1007/s00520-012-1649-7. PMID: 23161339. Exclusion reason: Ineligible comparison 
732. Watzke B, Heddaeus D, Steinmann M, et al. Effectiveness and cost-effectiveness of a guideline-based stepped care model for patients with depression: study protocol of a cluster-randomized controlled trial in routine care. BMC Psychiatry. 2014;14:230. doi: 10.1186/s12888-014-0230-y. PMID: 25182269. Exclusion reason: Ineligible intervention

733. Wechsler LR, Tsao JW, Levine SR, et al. Teleneurology applications: report of the telemedicine work group of the American Academy of Neurology. Neurology. 2013 Feb 12;80(7):670-6. doi: 10.1212/WNL.0b013e3182823361. PMID: 23400317. Exclusion reason: Background information only

734. Weinerman B, den Duyf J, Hughes A, et al. Can subspecialty cancer consultations be delivered to communities using modern technology?--A pilot study. Telemed J E Health. 2005 Oct;11(5):608-15. doi: 10.1089/tmj.2005.11.608. PMID: 16250826. Exclusion reason: Ineligible publication type

735. Weinlich M, Nieuwkamp N, Stueben U, et al. Telemedical assistance for in-flight emergencies on intercontinental commercial aircraft. J Telemed Telecare. 2009;15(8):409-13. doi: 10.1258/jtt.2009.090501. PMID: 19948708. Exclusion reason: Ineligible comparison

736. Weiss M, Klier F, Fischer B, et al. Telemedicine project RATEMA--radiation accident telecommunication medical assistance system. J Telemed Telecare. 1996;2 Suppl 1:9-12. PMID: 9375078. Exclusion reason: Ineligible comparison

737. Werner A, Anderson LE. Rural telepsychiatry is economically unsupportable: the Concorde crashes in a cornfield. Psychiatr Serv. 1998 Oct;49(10):1287-90. doi: 10.1176/ps.49.10.1287. PMID: 9779895. Exclusion reason: Background information only

738. Wheeler T. Telemedicine and special needs children. Telemed Today. 1998 Aug;6(4):16-20. PMID: 10182371. Exclusion reason: Ineligible comparison
739. Wheeler T. Urban kids receive telemedical care at school. Telemed Today. 1998 AprMay;6(2):6. PMID: 10181180. Exclusion reason: Ineligible publication type

740. Whited JD. Teledermatology. Current status and future directions. Am J Clin Dermatol. 2001;2(2):59-64. PMID: 11705304.

Exclusion reason: Ineligible publication type

741. Whited JD, Datta SK, Aiello LM, et al. A modeled economic analysis of a digital teleophthalmology system as used by three federal health care agencies for detecting proliferative diabetic retinopathy. Telemed J E Health. 2005 Dec;11(6):641-51. doi: 10.1089/tmj.2005.11.641. PMID: 16430383. Exclusion reason: Ineligible intervention

742. Wierwille JL, Pukay-Martin ND, Chard $\mathrm{KM}$, et al. Effectiveness of PTSD telehealth treatment in a VA clinical sample. Psychol Serv. 2016 Nov;13(4):373-9. PMID: 27657798. Exclusion reason: Ineligible intervention

743. Wilcox ME, Adhikari NK. The effect of telemedicine in critically ill patients: systematic review and meta-analysis. Crit Care. 2012 Jul 18;16(4):R127. doi: 10.1186/cc11429. PMID: 22809335.

Exclusion reason: Ineligible intervention

744. Wilkinson JR, Korom-Djakovic D, Spindler $\mathrm{M}$, et al. Patient and provider perception of video telehealth in Parkinson's disease (PD). Mov Disord. 2015;30(14). Exclusion reason: Ineligible comparison

745. Wilkinson JR, Spindler M, Wood SM, et al. High patient satisfaction with telehealth in Parkinson disease: a randomized controlled study. Neurol Clin Pract. 2016;6(3):241-51. doi: 10.1212/CPJ.0000000000000252. PMID: 27347441. Exclusion reason: Ineligible intervention

746. Williams BH. The AFIP center for telemedicine application--pathology for the twenty-first century. Telemed Virtual Real. 1998 Jun;3(6):64-5. PMID: 10180759.

Exclusion reason: Ineligible publication type 
747. Williams PA, Giles M. Viability of healthcare service delivery alternatives for the Australian mining sector. Stud Health Technol Inform. 2012;182:170-9. PMID: 23138092. Exclusion reason: Ineligible study design

748. Wilson M, Mazowita G, Ignaszewski A, et al. Family physician access to specialist advice by telephone: Reduction in unnecessary specialist consultations and emergency department visits. Can Fam Physician. 2016 Nov;62(11):e668-e76. PMID: 28661886. Exclusion reason: Ineligible intervention

749. Winchester JF. Telecommunications and the dialysis patient. Am J Kidney Dis. 1999 Jul;34(1):XXXVI-XXXVIII. doi: 10.1053/AJKD034xxxvi. PMID: 10401040. Exclusion reason: Ineligible publication type

750. Wood EW, Strauss RA, Janus C, et al. Telemedicine consultations in oral and maxillofacial surgery: a follow-up study. $\mathrm{J}$ Oral Maxillofac Surg. 2016 Feb;74(2):2628. doi: 10.1016/j.joms.2015.09.026. PMID: 26501427. Exclusion reason: Ineligible comparison

751. Woodward MA, Bavinger JC, Amin S, et al. Telemedicine for ophthalmic consultation services: use of a portable device and layering information for graders. J Telemed Telecare. 2017 Feb;23(2):365-70. doi: 10.1177/1357633X16634544. PMID: 26936864. Exclusion reason: Ineligible intervention

752. Wootton R. Twenty years of telemedicine in chronic disease management--an evidence synthesis. J Telemed Telecare. 2012 Jun;18(4):211-20. doi: 10.1258/jtt.2012.120219. PMID: 22674020. Exclusion reason: Ineligible study design

753. Wootton R, Geissbuhler A, Jethwani K, et al. Long-running telemedicine networks delivering humanitarian services: experience, performance and scientific output. Bull World Health Organ. 2012 May 1;90(5):341-7D. doi: 10.2471/BLT.11.099143. PMID: 22589567. Exclusion reason: Ineligible intervention
754. Wootton R, Vladzymyrskyy A, Zolfo M, et al. Experience with low-cost telemedicine in three different settings. Recommendations based on a proposed framework for network performance evaluation. Glob Health Action. 2011;4doi: 10.3402/gha.v4i0.7214 PMID: 22162965. Exclusion reason: Ineligible study design

755. Wu PH, Chang SC, Huang WT, et al. Effectiveness of nurse case management model compared with usual care in lymphoma patients. Cancer Nurs. 2015; International Conference on Cancer Nursing(var.pagings) 38 (4 SUPPL. 1):S55. Exclusion reason: Ineligible intervention

756. Wu TC, Parker SA, Jagolino AL, et al. Can telemedicine replace an on-board vascular neurologist in deciding about tissue plasminogen activator treatment? A prespecified substudy of the BEST-MSU study. American Heart Association/American Stroke Association. 2017;48(no pagination). Exclusion reason: Ineligible publication type

757. Yager P, Dapul H, Murphy S, et al. Comparison of face-to-face versus telemedicine patient assessment in a pediatric intensive care unit. Crit Care Med. 2013;40(12 SUPPL. 1):58. Exclusion reason: Ineligible intervention

758. Yager PH, Cummings BM, Whalen MJ, et al. Nighttime telecommunication between remote staff intensivists and bedside personnel in a pediatric intensive care unit: a retrospective study. Crit Care Med. 2012 Sep;40(9):2700-3. doi: 10.1097/CCM.0b013e3182591dab. PMID: 22732287. Exclusion reason: Ineligible comparison

759. Yang CW, Wang HC, Chiang WC, et al. Impact of adding video communication to dispatch instructions on the quality of rescue breathing in simulated cardiac arrests--a randomized controlled study. Resuscitation. 2008 Sep;78(3):327-32. doi: 10.1016/j.resuscitation.2008.03.232. PMID: 18583016. Exclusion reason: Ineligible intervention 
760. Yang CW, Wang HC, Chiang WC, et al. Interactive video instruction improves the quality of dispatcher-assisted chest compression-only cardiopulmonary resuscitation in simulated cardiac arrests. Crit Care Med. 2009 Feb;37(2):490-5. doi: 10.1097/CCM.0b013e31819573a5. PMID: 19114904. Exclusion reason: Ineligible intervention

761. Yang NH, Dharmar M, Yoo BK, et al. Economic evaluation of pediatric telemedicine consultations to rural emergency departments. Med Decis Making. 2015 Aug;35(6):773-83. doi: 10.1177/0272989X15584916. PMID: 25952744. Exclusion reason: Modeling study, no original data

762. Yogesan K, Constable IJ, Barry CJ, et al. Evaluation of a portable fundus camera for use in the teleophthalmologic diagnosis of glaucoma. J Glaucoma. 1999;8(5):297-301. PMID: 10529928. Exclusion reason: Ineligible outcome

763. Yoo SK, Kim SH, Jung SM, et al. A multispecialist teleconsultation system using multiple unicasting. Telemed J E Health. 2007 Oct;13(5):565-71. doi: 10.1089/tmj.2007.0006. PMID: 17999618. Exclusion reason: Ineligible comparison

764. You JS, Park S, Chung SP, et al. Usefulness of a mobile phone with video telephony in identifying the correct landmark for performing needle thoracocentesis. Emerg Med J. 2009 Mar;26(3):177-9. doi: 10.1136/emj.2008.060541. PMID: 19234005. Exclusion reason: Ineligible study design

765. Young LB, Chan PS, Cram P. Staff acceptance of tele-ICU coverage: a systematic review. Chest. 2011 Feb;139(2):279-88. doi: 10.1378/chest.101795. PMID: 21051386. Exclusion reason: Systematic review used to identify primary studies

766. Young LB, Chan PS, Lu X, et al. Impact of telemedicine intensive care unit coverage on patient outcomes: a systematic review and meta-analysis. Arch Intern Med. 2011 Mar 28;171(6):498-506. doi: 10.1001/archinternmed.2011.61. PMID: 21444842. Exclusion reason: Ineligible intervention
767. Yuan ZW, Liu ZR, Wei D, et al. Mobile stroke: an experience of intravenous thrombolysis guided by teleconsultation based on Google Glass. CNS Neurosci Ther. 2015 Jul;21(7):607-9. doi:

10.1111/cns.12413. PMID: 26096048.

Exclusion reason: Ineligible population

768. Zanaboni P, Scalvini S, Bernocchi P, et al. Teleconsultation service to improve healthcare in rural areas: acceptance, organizational impact and appropriateness. BMC Health Serv Res. 2009 Dec 18;9:238. doi: 10.1186/1472-6963-9-238. PMID: 20021651. Exclusion reason: Ineligible comparison

769. Zanaty M, Chalouhi N, Starke RM, et al. Epidemiology of a large telestroke cohort in the Delaware valley. Clin Neurol Neurosurg. 2014 Oct;125:143-7. doi: 10.1016/j.clineuro.2014.06.006. PMID: 25128655. Exclusion reason: Ineligible comparison

770. Zanol KE, Anderson P. Telemedicine beyond the technology. Mo Med. 1999 Mar;96(3):88-91. PMID: 10202434. Exclusion reason: Ineligible publication type

771. Zeineh J. AST and telepathology benefit labs. MLO Med Lab Obs. 2004 Jun;36(6):20, 2-3. PMID: 15242178.

Exclusion reason: Ineligible publication type

772. Zelickson BD. Teledermatology in the nursing home. Curr Probl Dermatol. 2003;32:167-71. PMID: 12472007. Exclusion reason: Ineligible comparison

773. Zelickson BD, Homan L. Teledermatology in the nursing home. Arch Dermatol. 1997 Feb;133(2):171-4. PMID: 9041829.

Exclusion reason: Ineligible comparison

774. Zembowicz A, Ahmad A, Lyle SR. A comprehensive analysis of a web-based dermatopathology second opinion consultation practice. Arch Pathol Lab Med. 2011 Mar;135(3):379-83. doi: 10.1043/2010-0187-OA.1. PMID: 21366464. Exclusion reason: Ineligible comparison 
775. Zheng W, Nickasch M, Lander L, et al.

Treatment outcome comparison between telepsychiatry and face-to-face

buprenorphine medication-assisted treatment

for opioid use disorder: a 2-year

retrospective data analysis. J Addict Med.

2017;11(2):138-44. doi:

10.1097/ADM.0000000000000287. PMID:

28107210. Exclusion reason: Ineligible

intervention

776. Ziemba SJ, Bradley NS, Landry LA, et al.

Posttraumatic stress disorder treatment for

Operation Enduring Freedom/Operation

Iraqi Freedom combat veterans through a

civilian community-based telemedicine

network. Telemed J E Health. 2014

May;20(5):446-50. doi:

10.1089/tmj.2013.0312. PMID: 24617961.

Exclusion reason: Ineligible intervention

777. Zincone LH, Jr., Doty E, Balch DC.

Financial analysis of telemedicine in a prison system. Telemed J. 1997

Winter;3(4):247-55. doi:

10.1089/tmj.1.1997.3.247. PMID:

10176005. Exclusion reason: Ineligible intervention 


\section{Appendix E. Telehealth for Consultations Risk of Bias Instructions}

\section{Step 1: Determine Study Design}

Table E-1. Description of study design categorization

\begin{tabular}{|c|c|}
\hline Category & Details \\
\hline Randomized Controlled Trial & $\begin{array}{l}\text { Key characteristic: People or groups (hospitals/clinics) are assigned by } \\
\text { the researchers and assignment is random, not based on } \\
\text { assessment/need or other non-random criteria. } \\
\text { Maybe called RCT, Randomized Trial, Randomized Study } \\
\text { - } \quad \text { Randomization is about Random Assignment to different } \\
\text { treatments. Random Sampling is different and does NOT } \\
\text { make something an RCT. }\end{array}$ \\
\hline Observational Cohort & $\begin{array}{l}\text { Key characteristics: } \\
\text { 1. The study compares different people-usually some treated } \\
\text { with telehealth and some without. } \\
\text { 2. How patients are treated. This is observed as it happens, it is } \\
\text { not controlled by the study, i.e., the researchers do not } \\
\text { determine who receives the intervention } \\
\text { We are including } 3 \text { types of studies in this category: } \\
\text { Prospective cohorts: Patients are identified and followed into the future } \\
\text { to obtain data } \\
\text { Retrospective cohort: Patient are identified, but all the data already } \\
\text { exists and the researchers look back into the past to collect it } \\
\text { Before-after cohorts: A group of patients from before an } \\
\text { intervention/system change are compared to a different group of } \\
\text { patients after a change. These may be called Pre Post by the authors, } \\
\text { but we are distinguishing studies that compare different groups of } \\
\text { patients from those that compare the same patients at different times. }\end{array}$ \\
\hline Pre-Post & $\begin{array}{l}\text { The same patients are compared at different points in time or under } \\
\text { different conditions. Patients serve as their own controls. } \\
\text { This includes studies where the telehealth consultation is done twice on } \\
\text { the same patient, once using telehealth and once face-to-face. }\end{array}$ \\
\hline Economic evaluations & $\begin{array}{l}\text { Key characteristics: } \\
\text { 1. The study assesses the economic impact of one intervention } \\
\text { compared to another or to usual care. } \\
\text { 2. The study uses economic or cost data from primary research. } \\
\text { This is to differentiate between studies that use hypothetical } \\
\text { data or data that was derived from other published sources. } \\
\text { Studies that do not use full economic analysis methods, but assess a } \\
\text { certain cost as a direct outcome (e.g., patient costs incurred in } \\
\text { telehealth versus not telehealth), can be assessed using the } \\
\text { appropriate criteria related to its study design (i.e., RCT, cohort, pre- } \\
\text { post) }\end{array}$ \\
\hline
\end{tabular}

RCT = randomized controlled trial 


\section{Step 2: Assess Criteria for the Study Design}

\section{Responses are:}

Yes: indicating the criteria is met, bias is reduced

No: indicating the criteria is not met; bias may be introduced

Unclear: article has some mention, but not clear what was done; bias may be present

NR: indicating the article did not report on the criteria; bias may be present

NA: not applicable

Table E-2. Criteria for randomized controlled trials

\begin{tabular}{|c|c|}
\hline Criteria & Details \\
\hline $\begin{array}{l}\text { Was randomization sequence } \\
\text { generated adequately? }\end{array}$ & $\begin{array}{l}\text { Adequate: computer generated, random number table, coin flip } \\
\text { Key: truly random, not a characteristic of the patient or the situation } \\
\text { Inadequate examples: day of week, patient's birthday, last two digits of medical } \\
\text { record number (may be based on something) }\end{array}$ \\
\hline $\begin{array}{l}\text { Was allocation concealment } \\
\text { adequate? }\end{array}$ & $\begin{array}{l}\text { Adequate: assignment to groups is not evident in advance to the person } \\
\text { recruiting and assigning the patient. Examples: centralized call in to another } \\
\text { location; computer generated; opaque sealed envelopes (maybe) } \\
\text { Inadequate examples: An open list, envelopes }\end{array}$ \\
\hline $\begin{array}{l}\text { Were eligibility criteria } \\
\text { specified? }\end{array}$ & $\begin{array}{l}\text { Specified: list of key characteristics stated and applied } \\
\text { Not specified: information not provided about who would be included or } \\
\text { excluded }\end{array}$ \\
\hline $\begin{array}{l}\text { Were groups similar at baseline } \\
\text { or did the analysis control for } \\
\text { any important baseline } \\
\text { differences? }\end{array}$ & $\begin{array}{l}\text { Look for a Table } 1 \text { that compares key characteristics. } \\
\text { Adequate: Most differences not statistically significantly different; small } \\
\text { differences between groups, OR if there is a difference despite randomization } \\
\text { that seems important, they control for that characteristic in analysis. }\end{array}$ \\
\hline $\begin{array}{l}\text { Were participants analyzed in } \\
\text { the groups they were originally } \\
\text { assigned to? }\end{array}$ & $\begin{array}{l}\text { This may be called "intention to treat." } \\
\text { This means people stay in the group to which they were assigned for analysis } \\
\text { even if they get the other treatment. }\end{array}$ \\
\hline $\begin{array}{l}\text { Was attrition low and/or } \\
\text { adherence high? }\end{array}$ & $\begin{array}{l}\text { We want YES to be positive. We want most people to remain in the study. A cut } \\
\text { off often used is losing fewer than } 20 \% \text { of the people. } \\
\text { Another question to consider is, do people complete the intervention as } \\
\text { intended? }\end{array}$ \\
\hline $\begin{array}{l}\text { Were outcome assessors } \\
\text { and/or data analyst blinded? Or } \\
\text { are primary outcome measures } \\
\text { unlikely to be biased? }\end{array}$ & $\begin{array}{l}\text { For Yes they should say the person measuring the outcome or analyzing the } \\
\text { data does not know which groups patients or organizations are in. } \\
\text { This may be No for many telehealth studies. } \\
\text { Outcomes unlikely to be biased include death, hospitalization and service } \\
\text { utilization. Outcomes that might be biased might be functional assessment, } \\
\text { quality of life, and satisfaction. }\end{array}$ \\
\hline $\begin{array}{l}\text { Were reliable measures of } \\
\text { outcomes and confounders } \\
\text { used and implemented } \\
\text { consistently across all study } \\
\text { participants/groups? }\end{array}$ & $\begin{array}{l}\text { This is about using measures that provide consistent information and in most } \\
\text { cases have been tested or evaluated. }\end{array}$ \\
\hline $\begin{array}{l}\text { Were the outcomes } \\
\text { prespecified and were all the } \\
\text { prespecified outcomes } \\
\text { reported? }\end{array}$ & $\begin{array}{l}\text { This may be hard to tell from a journal article. Ideally they should specify their } \\
\text { outcomes in methods and then give data for all that were mentioned. }\end{array}$ \\
\hline
\end{tabular}


Table E-3. Criteria for cohort (must compare different patients - includes different patients before and after telehealth)

\begin{tabular}{|c|c|}
\hline Criteria & Details \\
\hline Study Design & $\begin{array}{l}\text { Specify: (see definitions above) } \\
\text { Retrospective Cohort } \\
\text { Prospective Cohort } \\
\text { Before After }\end{array}$ \\
\hline $\begin{array}{l}\text { Were eligibility or selection } \\
\text { criteria for the study population } \\
\text { pre-specified and clearly } \\
\text { described? }\end{array}$ & For Yes: It should be clear who is included in the study and who is excluded \\
\hline $\begin{array}{l}\text { Did the study attempt to enroll a } \\
\text { random sample or consecutive } \\
\text { or all patients meeting inclusion } \\
\text { criteria in a defined period? }\end{array}$ & $\begin{array}{l}\text { For Yes: Articles should say "all" or consecutive and be specific. } \\
\text { For No: Anything that suggests the study picked a subgroup based on a criteria } \\
\text { that might influence outcomes. }\end{array}$ \\
\hline $\begin{array}{l}\text { Were groups similar or did the } \\
\text { design and analyses account } \\
\text { for important potential } \\
\text { confounding and modifying } \\
\text { variables appropriately? }\end{array}$ & $\begin{array}{l}\text { For Yes: The study should specify what confounders or modifiers are important } \\
\text { and how they are addressed. You should be convinced that how confounders } \\
\text { were addressed was done well. } \\
\text { Confounding is when a variable is related to both the intervention and the } \\
\text { outcome. Example: health status if sicker patients use telehealth and healthier } \\
\text { use in-person visits and your outcome is hospitalizations. } \\
\text { In Before After studies one thing to consider is whether there was a major } \\
\text { change or something different likely to affect the outcome in the different time } \\
\text { periods and whether the researchers try to address this. } \\
\text { Modifying variables: When the effect of the intervention is different for different } \\
\text { groups. Example: Older patients are less satisfied with telehealth than younger } \\
\text { people. Modifiers may be adjusted for or the results may be presented by } \\
\text { subgroups (subgroups is an acceptable approach). }\end{array}$ \\
\hline $\begin{array}{l}\text { Were valid and reliable } \\
\text { measures used and were they } \\
\text { adequately ascertained? } \\
\text { (inclusion/exclusion, } \\
\text { confounding, outcomes) }\end{array}$ & $\begin{array}{l}\text { This is about using measures that provide consistent information and in most } \\
\text { cases have been tested or evaluated. } \\
\text { It is also important to consider how the outcomes were ascertained. That is, } \\
\text { does the study describe how they got the outcomes they were studying? For } \\
\text { example, does the study tell you how they determined diagnosis outcomes or } \\
\text { how they got the data for costs? }\end{array}$ \\
\hline $\begin{array}{l}\text { Were outcome assessors } \\
\text { and/or data analysts blinded to } \\
\text { treatment or are the outcomes } \\
\text { objective and unlikely to be } \\
\text { biased? }\end{array}$ & $\begin{array}{l}\text { For Yes: Studies should state that the person measuring the outcome or } \\
\text { analyzing the data does not know which groups patients or organizations are in. } \\
\text { This may be NO for many telehealth studies. } \\
\text { Outcomes unlikely to be biased include death, hospitalization and service } \\
\text { utilization. Outcomes that might be biased include functional assessment, } \\
\text { quality of life, and satisfaction. }\end{array}$ \\
\hline $\begin{array}{l}\text { Was there NO or only small } \\
\text { amounts of missing data? Or if } \\
\text { there was missing data, was it } \\
\text { handled appropriately? }\end{array}$ & $\begin{array}{l}\text { Missing data is about specific variables missing, NOT about patients dropping } \\
\text { out (that is the next criteria). } \\
\text { NA is an option if the study is retrospective. If data is missing, they may } \\
\text { describe analytic methods for addressing this. If they are complex, make a note } \\
\text { and ask. In general, dropping cases or carrying the last value forward are not } \\
\text { acceptable. Acceptable approaches might be using "bootstrapping," near } \\
\text { neighbor, or matching; that is, some methods attempt to estimate the missing } \\
\text { data and are clear about their assumptions. }\end{array}$ \\
\hline $\begin{array}{l}\text { Is there NO important } \\
\text { differential loss to followup or } \\
\text { overall low loss to followup or } \\
\text { attrition? }\end{array}$ & $\begin{array}{l}\text { No: If a large number of people are lost to followup or if a smaller number are } \\
\text { lost but there are more in one group than another or result in the groups being } \\
\text { different (for example, all the men drop out of one group). } \\
\text { NA is an option here for Retrospective Cohorts. NA is not negative for } \\
\text { retrospective studies. }\end{array}$ \\
\hline $\begin{array}{l}\text { Were outcomes prespecified } \\
\text { and were prespecified } \\
\text { outcomes reported? }\end{array}$ & $\begin{array}{l}\text { This may be hard to tell from a journal article. Ideally they should specify their } \\
\text { outcomes in methods and then give data for the all that were mentioned. }\end{array}$ \\
\hline
\end{tabular}


Table E-4. Criteria for pre-post (same patients, different time periods)

\begin{tabular}{|c|c|}
\hline Criteria & Details \\
\hline Study Design & Confirm Pre Post (same people; different time periods) \\
\hline $\begin{array}{l}\text { Were eligibility or selection } \\
\text { criteria for the study population } \\
\text { prespecified and clearly } \\
\text { described? }\end{array}$ & $\begin{array}{l}\text { For Yes: It should be clear who is included in the study and who is excluded it } \\
\text { should be clear who is in and who is out. }\end{array}$ \\
\hline $\begin{array}{l}\text { Did the study attempt to enroll } \\
\text { a random sample or } \\
\text { consecutive or all patients } \\
\text { meeting inclusion criteria in the } \\
\text { Pre Period? }\end{array}$ & $\begin{array}{l}\text { For Yes: Articles should say "all" or consecutive and be specific. } \\
\text { For No: Anything that suggests the study picked a subgroup based on a criteria } \\
\text { that might influence outcomes. }\end{array}$ \\
\hline $\begin{array}{l}\text { Did the design and analyses } \\
\text { account for important potential } \\
\text { confounding and modifying } \\
\text { variables appropriately? }\end{array}$ & $\begin{array}{l}\text { For Yes: The study should specify what confounders are important and how they } \\
\text { are addressed. You should be convinced that how they were addressed was } \\
\text { done well. For these studies the confounders are what might be different in the } \\
\text { Pre and Post time periods. } \\
\text { In Pre Post studies one thing to consider is whether there was a major change } \\
\text { or something different likely to affect the outcome in the different time periods } \\
\text { and did the researchers try to address this. }\end{array}$ \\
\hline $\begin{array}{l}\text { Were valid and reliable } \\
\text { measures used and were they } \\
\text { adequately ascertained? } \\
\text { (inclusion/exclusion, } \\
\text { confounding, outcomes) }\end{array}$ & $\begin{array}{l}\text { This is about using measures that provide consistent information and in most } \\
\text { cases have been tested or evaluated. } \\
\text { It is also important to consider how the outcomes were ascertained. That is, } \\
\text { does the study describe how they got the outcomes they were studying? For } \\
\text { example, does the study tell you how they determined diagnosis outcomes or } \\
\text { how they got the data for costs? }\end{array}$ \\
\hline $\begin{array}{l}\text { Were outcome assessors } \\
\text { and/or data analysts blinded to } \\
\text { treatment or are the outcomes } \\
\text { objective and unlikely to be } \\
\text { biased? }\end{array}$ & $\begin{array}{l}\text { For Yes: Studies should state that the person measuring the outcome or } \\
\text { analyzing the data does not know which groups patients or organizations are in. } \\
\text { This may be NO for many telehealth studies. } \\
\text { Outcomes unlikely to be biased include death, hospitalization and service } \\
\text { utilization. Outcomes that might be biased include functional assessment, quality } \\
\text { of life, and satisfaction. }\end{array}$ \\
\hline $\begin{array}{l}\text { Was there NO or only small } \\
\text { amounts of missing data? Or if } \\
\text { there was missing data, was it } \\
\text { handled appropriately? }\end{array}$ & $\begin{array}{l}\text { Missing data is about specific variables missing, NOT about patients dropping } \\
\text { out (that is the next criteria). } \\
\text { If data is missing, they may describe analytic methods for addressing this. If they } \\
\text { are complex, make a note and ask. In general, dropping cases or carrying the } \\
\text { last value forward are not acceptable. Acceptable approaches might be using } \\
\text { "bootstrapping," near neighbor, or matching; that is, some methods attempt to } \\
\text { estimate the missing data and are clear about their assumptions. }\end{array}$ \\
\hline $\begin{array}{l}\text { Is there low loss to followup or } \\
\text { attrition? }\end{array}$ & $\begin{array}{l}\text { No: If a large number of people are lost from baseline to end point-usually less } \\
\text { than } 20 \% \text {. You may need to look at the numbers included at the two points in } \\
\text { time if the article does not tell you this. }\end{array}$ \\
\hline $\begin{array}{l}\text { Were outcomes prespecified } \\
\text { and were prespecified } \\
\text { outcomes reported? }\end{array}$ & $\begin{array}{l}\text { This may be hard to tell from a journal article. Ideally they should specify their } \\
\text { outcomes in methods and then give data for the all that were mentioned. }\end{array}$ \\
\hline
\end{tabular}

Criteria and details for economic evaluations adapted from Consensus Health Economic Criteria - CHEC List. ${ }^{1,2}$ 
Table E-5. Criteria for economic evaluations

\begin{tabular}{|c|c|}
\hline Criteria & Details \\
\hline Study Design & Confirm Economic Evaluation \\
\hline $\begin{array}{l}\text { Are competing alternatives } \\
\text { clearly described? }\end{array}$ & $\begin{array}{l}\text { A detailed description should be given of the competing interventions. This } \\
\text { should encompass a clear and specific statement of the primary objective of } \\
\text { each alternative, as well as relevant factors, such as intensity, duration, and } \\
\text { frequency. }\end{array}$ \\
\hline $\begin{array}{l}\text { Is the economic study design } \\
\text { appropriate to the stated } \\
\text { objective? }\end{array}$ & $\begin{array}{l}\text { An appropriate economic study design is a full economic evaluation (comparison } \\
\text { of costs and effects of two or more interventions) based on primary research } \\
\text { (cohort, case-control, randomised controlled trial). }\end{array}$ \\
\hline $\begin{array}{l}\text { Are all important and relevant } \\
\text { costs for each alternative } \\
\text { identified? }\end{array}$ & $\begin{array}{l}\text { The costs should be measured appropriately in physical units. The instrument by } \\
\text { which the costs are measured should be valid and clearly stated (e.g., interview, } \\
\text { questionnaire, cost-diary). }\end{array}$ \\
\hline $\begin{array}{l}\text { Are all costs measured } \\
\text { appropriately in physical units? }\end{array}$ & $\begin{array}{l}\text { The sources of valuation should be clearly stated for each cost price of every } \\
\text { volume parameter and their reference year. The main cost should be calculated } \\
\text { based on depleted sources, no tariffs should be used. }\end{array}$ \\
\hline $\begin{array}{l}\text { Are costs valued } \\
\text { appropriately? }\end{array}$ & $\begin{array}{l}\text { A full identification of all important and relevant outcomes should be given in } \\
\text { relation to the perspective and the research question. }\end{array}$ \\
\hline $\begin{array}{l}\text { Are all important and relevant } \\
\text { outcomes for each alternative } \\
\text { identified? }\end{array}$ & $\begin{array}{l}\text { The outcome measurement should result from the outcome identification and } \\
\text { this should be straightforward (e.g., if mortality is a main outcome measure this } \\
\text { should be taken into account in the analysis). The instrument by which the } \\
\text { outcomes are measured should be valid and clearly stated. }\end{array}$ \\
\hline $\begin{array}{l}\text { Are all outcomes measured } \\
\text { appropriately? }\end{array}$ & $\begin{array}{l}\text { The method of outcome valuation should be clearly stated. Examples of } \\
\text { valuation methods are Discrete Choice Experiments (e.g., Conjoint analysis, } \\
\text { Contingent valuation), direct utility assessment (e.g., VAS, TTO, SG), Indirect } \\
\text { utility assessment (e.g., HUI, EQ-5D, QWB), Person trade off, etc. }\end{array}$ \\
\hline $\begin{array}{l}\text { Are outcomes valued } \\
\text { appropriately? }\end{array}$ & $\begin{array}{l}\text { An incremental analysis should examine the additional costs from one } \\
\text { intervention over another, compared to the additional outcomes that it delivers. } \\
\text { The incremental costs-effectiveness ratio is obtained by dividing the costs } \\
\text { differences by the outcome differences for the alternatives. }\end{array}$ \\
\hline $\begin{array}{l}\text { Is an incremental analysis of } \\
\text { costs and outcomes of } \\
\text { alternatives performed? }\end{array}$ & $\begin{array}{l}\text { Discounting is done appropriately if all costs and outcomes are converted to one } \\
\text { single year, based on a motivated discount rate. }\end{array}$ \\
\hline $\begin{array}{l}\text { Are all future costs and } \\
\text { outcomes discounted } \\
\text { appropriately? }\end{array}$ & $\begin{array}{l}\text { All variables in the analysis are potential candidates for the sensitivity analysis. } \\
\text { Only variables that are certain or which have a minimal impact on the study } \\
\text { results (based on the preliminary analysis) can be excluded from the sensitivity } \\
\text { analysis. Furthermore, a justification should be given over the range of the } \\
\text { variables used in the sensitivity analysis. }\end{array}$ \\
\hline $\begin{array}{l}\text { Are all important variables, } \\
\text { whose values are uncertain, } \\
\text { appropriately subjected to } \\
\text { sensitivity analysis? }\end{array}$ & $\begin{array}{l}\text { This can be done by being explicit about the viewpoint of analysis and by } \\
\text { indicating how particular costs and outcomes vary by location, setting, patient } \\
\text { population, care provider, etc. }\end{array}$ \\
\hline
\end{tabular}

\section{Step 3: Overall Rating}

Key points

- All criteria are not created equal. Just adding up the Yes/No counts is not the goal.

- Considerations vary by study design

o RCTs: if the randomization is not done well, a study cannot be low risk of bias as the benefits of randomization may be void. 
o Observational and Pre Post: It is important that confounding and selection are addressed in the design and analysis because the groups are not created through randomization. 


\section{Appendix E References}

1. Wijnen B, Van Mastrigt G, Redekop WK, et al. How to prepare a systematic review of economic evaluations for informing evidence-based healthcare decisions: data extraction, risk of bias, and transferability (part 3/3). Expert Rev. Pharmacoecon. Outcomes Res. 2016 Dec;16(6):723-32. doi: 10.1080/14737167.2016.1246961. PMID: 27762640 .
2. Consensus Health Economic Criteria CHEC list. Maastricht University Health Services Research.

https://hsr.mumc.maastrichtuniversity.nl/con sensus-health-economic-criteria-chec-list. Accessed Nov 152018. 


\section{Appendix F. Evidence Tables}

Table F-1. Inpatient evidence table: study characteristics

\begin{tabular}{|c|c|c|c|c|}
\hline $\begin{array}{l}\text { Author, Year } \\
\text { (See Appendix C } \\
\text { for full citation) }\end{array}$ & $\begin{array}{l}\text { Geographic } \\
\text { Location of Studies }\end{array}$ & Study Period & Study Design & Comparison and Intervention \\
\hline Alemi, 2017 & $\begin{array}{l}\text { San Francisco, } \\
\text { California } \\
\text { USA } \\
\end{array}$ & September 2015 to May 2016 & Prospective Cohort & $\begin{array}{l}\text { A: In person } \\
\text { B: Remote assessment }\end{array}$ \\
\hline Armaignac, 2018 & $\begin{array}{l}\text { Coral Gables, Florida } \\
\text { USA }\end{array}$ & December 2011 to August 2016 & Retrospective Cohort & $\begin{array}{l}\text { A: Usual care } \\
\text { B: Telehealth }\end{array}$ \\
\hline Audebert, 2009 & Germany & July 2003 to March 2005 & Prospective Cohort & $\begin{array}{l}\text { A: Hospitals not part of TEMPIS: without stroke } \\
\text { telehealth consultation availability and no stroke ward } \\
\text { B: Hospitals with a stroke telemedicine consultation } \\
\text { availability and a stroke ward set up with a } \\
\text { multidisciplinary team educated earlier by stroke MD } \\
\text { and RNs. }\end{array}$ \\
\hline Breslow, 2004 & \begin{tabular}{|l|} 
Virginia \\
USA
\end{tabular} & \begin{tabular}{|l|} 
A: July 1999 to June 2000 \\
B: Jan 1,2000 to June 2001 \\
\end{tabular} & Before-After & $\begin{array}{l}\text { A: Before telehealth } \\
\text { B: After telehealth }\end{array}$ \\
\hline Buckley, 2012 & $\begin{array}{l}\text { Murrumbidgee Local } \\
\text { Health District } \\
\text { New South Wales }\end{array}$ & January 2002 to December 2010 & Before-After & $\begin{array}{l}\text { A: Before telehealth } \\
\text { B: After telehealth }\end{array}$ \\
\hline $\begin{array}{l}\text { Burgess, et al., } \\
2002\end{array}$ & $\begin{array}{l}\text { Honolulu, Hawaii } \\
\text { USA }\end{array}$ & NR & Prospective Cohort & $\begin{array}{l}\text { A: Conventional sinus surgery } \\
\text { B: Teleproctored sinus surgery }\end{array}$ \\
\hline Chan, et al., 2001 & Hong Kong & August 1998 to July 1999 & Before-After & $\begin{array}{l}\text { A: Before telehealth } \\
\text { B: After telehealth }\end{array}$ \\
\hline $\begin{array}{l}\text { Chu-Weininger, } \\
2010\end{array}$ & $\begin{array}{l}\text { Houston, Texas } \\
\text { USA }\end{array}$ & June 2005 to October 2005 & Pre/Post & $\begin{array}{l}\text { A: Pre-telehealth } \\
\text { B: Post-telehealth }\end{array}$ \\
\hline Collins, 2017 & $\begin{array}{l}\text { Pennsylvania, } \\
\text { Philadelphia } \\
\text { USA }\end{array}$ & January 2008 to July 2011 & Retrospective Cohort & $\begin{array}{l}\text { A: Surgical intensive care unit } \\
\text { B: Virtual intensive care unit }\end{array}$ \\
\hline Craig, 2004 & United Kingdom & January 1999 to June 1999 & Prospective Cohort & $\begin{array}{l}\text { A: Hospital without a teleneurology consultation } \\
\text { B: Hospital with a teleneurology consultation }\end{array}$ \\
\hline Dharmar, 2013 & $\begin{array}{l}\text { Northern California } \\
\text { USA }\end{array}$ & July 2003 to December 2010 & Retrospective Cohort & $\begin{array}{l}\text { A: No telehealth } \\
\text { B: Telehealth }\end{array}$ \\
\hline Engel, 2011 & $\begin{array}{l}\text { Taoyuan, Taiwan } \\
1 \text { hospital } \\
\end{array}$ & May and June 2009 & Prospective Cohort & $\begin{array}{l}\text { A: In person } \\
\text { B: Telehealth } \\
\end{array}$ \\
\hline Fortis, 2014 & $\begin{array}{l}\text { Minneapolis, } \\
\text { Minnesota } \\
\text { USA } \\
\end{array}$ & 2011 and 2012 & Retrospective Cohort & $\begin{array}{l}\text { A: No tele-ICU } \\
\text { B: Tele-ICU }\end{array}$ \\
\hline $\begin{array}{l}\text { Franzini, } 2011 \\
\text { same patients as } \\
\text { Thomas, } 2009 \\
\end{array}$ & $\begin{array}{l}\text { Gulf Coast region } \\
\text { USA }\end{array}$ & $\begin{array}{l}\text { A: January } 2003 \text { to August } 2005 \\
\text { B: July } 2004 \text { to July } 2006\end{array}$ & $\begin{array}{l}\text { Economic Evaluation, } \\
\text { Before-After }\end{array}$ & $\begin{array}{l}\text { A: Before tele-ICU } \\
\text { B: After tele-ICU }\end{array}$ \\
\hline
\end{tabular}




\begin{tabular}{|c|c|c|c|c|}
\hline $\begin{array}{l}\text { Author, Year } \\
\text { (See Appendix C } \\
\text { for full citation) }\end{array}$ & $\begin{array}{l}\text { Geographic } \\
\text { Location of Studies }\end{array}$ & Study Period & Study Design & Comparison and Intervention \\
\hline Fuertes-Guiró, 2016 & $\begin{array}{l}\text { Barcelona, Spain } \\
2 \text { community } \\
\text { hospitals } \\
1 \text { University hospitals }\end{array}$ & March 2013 and March 2014 & RCT & $\begin{array}{l}\text { A: No mentor } \\
\text { B: Telementoring }\end{array}$ \\
\hline Grabowski, 2014 & $\begin{array}{l}\text { Massachusetts } \\
\text { USA }\end{array}$ & October 2009 to September 2011 & RCT & $\begin{array}{l}\text { A1: Nursing homes without telehealth, pre-intervention } \\
\text { A2: Nursing homes without telehealth, post- } \\
\text { intervention } \\
\text { B1: Nursing homes with telehealth, pre-intervention } \\
\text { B2: Nursing homes with telehealth, post-intervention }\end{array}$ \\
\hline Gray, 2009 & Australia & January 2007 to September 2008 & Economic Evaluation & \begin{tabular}{|l|} 
A: In-person visits \\
B: Telehealth videoconferencing \\
\end{tabular} \\
\hline Gupta, 2014 & $\begin{array}{l}\text { Dehradun } \\
\text { India }\end{array}$ & $\begin{array}{l}\text { Before: April } 2012 \text { to March } 2013 \\
\text { After: April } 2013 \text { to March } 2014 \\
\end{array}$ & Before-After & $\begin{array}{l}\text { A: Before telehealth } \\
\text { B: After telehealth }\end{array}$ \\
\hline Huang, 2008 & $\begin{array}{l}\text { California } \\
\text { USA } \\
\end{array}$ & 2001 to 2006 & Before-After & $\begin{array}{l}\text { A: NICU echocardiograms before telehealth } \\
\text { B: NICU echocardiograms after telehealth }\end{array}$ \\
\hline Kahn, 2016 & USA & \begin{tabular}{|l|}
2001 to 2010 \\
(4 years of data in each hospital) \\
\end{tabular} & Retrospective Cohort & $\begin{array}{l}\text { A: Hospitals without ICU telehealth } \\
\text { B: Hospitals with ICU telehealth }\end{array}$ \\
\hline Kalb, 2014 & NR & January 2010 to March 2012 & Before-After & $\begin{array}{l}\text { A: Before tele-ICU implementation } \\
\text { B: After tele-ICU (2011, Quarter 3) } \\
\text { C: After tele-ICU (2012, Quarter 1) }\end{array}$ \\
\hline Kim, 2013 & $\begin{array}{l}\text { Arkansas } \\
\text { USA }\end{array}$ & March 2009 to March 2010 & Prospective Cohort & $\begin{array}{l}\text { The following hospitals were compared before and } \\
\text { after implementation of TH: } \\
\text { 1. Non-TH hospital, non-NICU } \\
\text { 2. Non-TH hospital, with NICU } \\
\text { 3. TH-hospital - non-NICU } \\
\text { 4. TH-hospital, with NICU } \\
\text { 5. University hospital } \\
\text { 6. Statewide infant mortality } \\
\text { Outcomes comparison: } \\
\text { A: Nontelehealth hospital, without NICU } \\
\text { B: Telehealth hospital, without NICU }\end{array}$ \\
\hline Klein, 2010 & Israel & $\begin{array}{l}\text { January 1, } 2005 \text { to December 31, } \\
2006\end{array}$ & Retrospective Cohort & $\begin{array}{l}\text { A1: Transfer all patients with traumatic ICB to trauma } \\
\text { center with neurosurgical service } \\
\text { A2: Transfer decisions made by clinical algorithm } \\
\text { B: Transfer decisions made by telehealth }\end{array}$ \\
\hline Kohl, 2012 & $\begin{array}{l}\text { Philadelphia, } \\
\text { Pennsylvania } \\
\text { USA }\end{array}$ & April 2003 to June 2006 & Retrospective Cohort & $\begin{array}{l}\text { A1: No telehealth, prior to implementation } \\
\text { A2: No telehealth, post telehealth implementation } \\
\text { B1: Telehealth, prior to implementation } \\
\text { B2: Telehealth, post telehealth implementation }\end{array}$ \\
\hline
\end{tabular}




\begin{tabular}{|c|c|c|c|c|}
\hline $\begin{array}{l}\text { Author, Year } \\
\text { (See Appendix C } \\
\text { for full citation) }\end{array}$ & $\begin{array}{l}\text { Geographic } \\
\text { Location of Studies }\end{array}$ & Study Period & Study Design & Comparison and Intervention \\
\hline Labarbera, 2013 & $\begin{array}{l}\text { Oregon } \\
\text { USA }\end{array}$ & January 2006 to October 2009 & Before-After & $\begin{array}{l}\text { A: Before implementation of telehealth (consults only } \\
\text { via telephone) } \\
\text { B: After implementation of telehealth (consults mostly } \\
\text { via videoconference, some by telephone), but before } \\
\text { pediatric hospitalist program in community hospital } \\
\text { C: After telehealth and after pediatric hospitalist } \\
\text { program at community hospital }\end{array}$ \\
\hline Lilly, 2011 & $\begin{array}{l}\text { Massachusetts } \\
\text { USA }\end{array}$ & April 2005 to September 2007 & Prospective Cohort & $\begin{array}{l}\text { A: Pre-telehealth } \\
\text { B: Telehealth }\end{array}$ \\
\hline Lilly, 2017 & $\begin{array}{l}\text { Massachusetts } \\
\text { USA }\end{array}$ & January 2004 to May 2013 & Economic Evaluation & $\begin{array}{l}\text { A: Pre-telehealth } \\
\text { B: Post-telehealth } \\
\text { C: Post-telehealth with added Logistics Center }\end{array}$ \\
\hline Marcin, 2004a & $\begin{array}{l}\text { USA } \\
\text { California }\end{array}$ & April 2000 to April 2002 & Retrospective Cohort & $\begin{array}{l}\text { A1: Pre-telehealth control group } \\
\text { A2: Patients transferred, control group } \\
\text { B1: Patients receiving telehealth } \\
\text { B2: All ICU patients during telehealth period }\end{array}$ \\
\hline Marcin, 2004b & $\begin{array}{l}\text { USA } \\
\text { California }\end{array}$ & April 2000 to April 2002 & Retrospective Cohort & $\begin{array}{l}\text { A: Patients who received telemedicine consultations } \\
\text { B: Patients who were in ICU who did not receive } \\
\text { telemedicine consultations }\end{array}$ \\
\hline Marcin, 2004c & $\begin{array}{l}\text { Redding, California } \\
\text { USA }\end{array}$ & February 1998 to September 2000 & Retrospective Cohort & $\begin{array}{l}\text { A1: Patients cared for in ICU prior to telemedicine } \\
\text { A2: Patients who did not have telehealth consult after } \\
\text { telehealth program initiated } \\
\text { B: Patients who had telehealth consult } \\
\text { C: All trauma patients admitted to adult ICU during } \\
\text { telehealth program regardless of if they had telehealth } \\
\text { consult }\end{array}$ \\
\hline Martin-Khan, 2016 & Australia & Unclear & RCT & $\begin{array}{l}\text { A: Usual care } \\
\text { B: Telehealth }\end{array}$ \\
\hline $\begin{array}{l}\text { McCambridge, } \\
2010\end{array}$ & USA & $\begin{array}{l}\text { A: September } 2002 \text { to December } \\
2003 \\
\text { B: January } 2004 \text { to September } \\
2004\end{array}$ & Before-After & $\begin{array}{l}\text { A: Before implementation of health information } \\
\text { technology with remote intensivist coverage (HITB- } \\
\text { RIC) } \\
\text { B: After implementation of HITB-RIC }\end{array}$ \\
\hline Mielonen, 2000 & Finland & November 1997 to 1998 & Prospective Cohort & $\begin{array}{l}\text { A: Conventional consults } \\
\text { B: Telehealth consults }\end{array}$ \\
\hline Migliaretti, 2013 & Italy & 2009 & Prospective Cohort & $\begin{array}{l}\text { A: Patients admitted to the same hospital in } 2009 \text { who } \\
\text { did not receive a neurosurgery teleconsultation } \\
\text { B: Patients admitted in } 2009 \text { for whom a neurosurgery } \\
\text { teleconsultation was obtained }\end{array}$ \\
\hline Miyamoto, 2014 & $\begin{array}{l}\text { California } \\
\text { USA }\end{array}$ & January 2004 to December 2009 & Retrospective Cohort & $\begin{array}{l}\text { A: Comparison site } \\
\text { B: telehealth site (hospital access to telehealth } \\
\text { consultations during forensic examinations) }\end{array}$ \\
\hline
\end{tabular}




\begin{tabular}{|c|c|c|c|c|}
\hline $\begin{array}{l}\text { Author, Year } \\
\text { (See Appendix C } \\
\text { for full citation) }\end{array}$ & $\begin{array}{l}\text { Geographic } \\
\text { Location of Studies }\end{array}$ & Study Period & Study Design & Comparison and Intervention \\
\hline $\begin{array}{l}\text { Morrison, et al., } \\
2010\end{array}$ & $\begin{array}{l}\text { Chicago, Illinois } \\
\text { USA }\end{array}$ & December, 2002 to October 2004 & Before-After & $\begin{array}{l}\text { A: Baseline (before telehealth; } 12 / 1 / 2002 \text { to } 3 / 1 / 2003) \\
\text { B: elCU Wave } 1(12 / 1 / 2003 \text { to } 3 / 1 / 2004) \\
\text { C: elCU Wave } 2(7 / 1 / 2004 \text { to } 10 / 31 / 2004)\end{array}$ \\
\hline Nagayoshi, 2016 & Kumamoto, Japan & January 2010 to December 2014 & Before-After & $\begin{array}{l}\text { A: Before telehealth } \\
\text { B: After telehealth }\end{array}$ \\
\hline Nassar, 2014 & $\begin{array}{l}\text { Upper Midwest } \\
\text { USA } \\
7 \text { VA Hospitals }\end{array}$ & 2011 to 2012 & Before-After & $\begin{array}{l}\text { Usual care } \\
\text { A1: Usual care before telehealth period } \\
\text { A2: Usual care after telehealth period } \\
\text { Telehealth } \\
\text { B1: Telehealth, before telehealth period } \\
\text { B2: Telehealth, after telehealth period }\end{array}$ \\
\hline Panlaqui, 2017 & $\begin{array}{l}\text { Australia and New } \\
\text { Zealand }\end{array}$ & 2010 to 2015 & Before-After & $\begin{array}{l}\text { A: Before telehealth } \\
\text { B: After telehealth }\end{array}$ \\
\hline Rendina, 1997 & $\begin{array}{l}\text { Chapel Hill, North } \\
\text { Carolina } \\
\text { USA }\end{array}$ & 1994 and 1995 & Economic Evaluation & $\begin{array}{l}\text { A: Usual care } \\
\text { B: Telehealth }\end{array}$ \\
\hline Rendina, 1998 & USA, North Carolina & 3 years, 1994 to 1996 & Retrospective Cohort & $\begin{array}{l}\text { A: Hospital without telehealth } \\
\text { B: Hospital with telehealth }\end{array}$ \\
\hline Rincon, 2012 & $\begin{array}{l}\text { Philadelphia, } \\
\text { Pennsylvania } \\
\text { USA }\end{array}$ & January to February 2011 & Before-After & $\begin{array}{l}\text { A: Before telehealth } \\
\text { B: After telehealth }\end{array}$ \\
\hline Robison, 2016 & $\begin{array}{l}\text { Wilmington, Delaware } \\
\text { USA }\end{array}$ & February 2014 to October 2014 & Prospective Cohort & $\begin{array}{l}\text { A: Usual care } \\
\text { B: Telehealth }\end{array}$ \\
\hline Romig, 2012 & $\begin{array}{l}\text { Baltimore, Maryland } \\
\text { USA }\end{array}$ & January 2010 to April 2010 & \begin{tabular}{|l|} 
Before-After, \\
Prospective Cohort
\end{tabular} & $\begin{array}{l}\text { A: No telehealth, } n=612 \\
\text { B: Telehealth, } n=793 ; 403 \text { received telehealth } \\
\text { Nurse survey: } \\
\text { Before telehealth, } n=11 \\
\text { After telehealth, } n=27\end{array}$ \\
\hline Rosenfeld, 2000 & $\begin{array}{l}\text { Baltimore, Maryland } \\
\text { USA }\end{array}$ & $\begin{array}{l}\text { September } 1997 \text { to December } \\
1997\end{array}$ & $\begin{array}{l}\text { Before-After (two time } \\
\text { periods before } \\
\text { telehealth) }\end{array}$ & $\begin{array}{l}\text { A: Surgical ICU without onsite ICU physician staff } \\
\text { directly responsible for patient care. Two baseline } \\
\text { periods to control for seasonal variations: } \\
\text { A.1: } 9 / 1 / 96-12 / 18 / 96 \text { and } \\
\text { A-2: } 2 / 1 / 97-5 / 18 / 97 \\
\text { B Intervention: } 9 / 1 / 97 \text { to } 12 / 18 / 97 \text { during which one of } \\
4 \text { intensivists provided round-the-clock monitoring of } \\
\text { ICU patients from their homes }\end{array}$ \\
\hline Ruesch, 2012 & $\begin{array}{l}\text { Alaska } \\
\text { USA }\end{array}$ & $\begin{array}{l}\text { A: NR } \\
\text { B: January } 2009 \text { to December } \\
2009\end{array}$ & Before-After & $\begin{array}{l}\text { A: Before telemedicine } \\
\text { B: Telehealth with nurse support } \\
\text { C: Telehealth with nurse support and physician } \\
\text { support }\end{array}$ \\
\hline
\end{tabular}




\begin{tabular}{|c|c|c|c|c|}
\hline $\begin{array}{l}\text { Author, Year } \\
\text { (See Appendix C } \\
\text { for full citation) }\end{array}$ & $\begin{array}{l}\text { Geographic } \\
\text { Location of Studies }\end{array}$ & Study Period & Study Design & Comparison and Intervention \\
\hline Sadaka, 2013 & \begin{tabular}{|l|} 
Missouri \\
USA
\end{tabular} & July 2009 to March 2011 & Before-After & $\begin{array}{l}\text { A: Before telelCU } \\
\text { B: After telelCU }\end{array}$ \\
\hline Sharma, 2016 & $\begin{array}{l}\text { Philadelphia, } \\
\text { Pennsylvania } \\
\text { USA }\end{array}$ & March 2014 to July 2014 & Before-After & $\begin{array}{l}\text { A: Before telehealth } \\
\text { B: After telehealth }\end{array}$ \\
\hline Shin, 2015 & $\begin{array}{l}\text { Los Angeles, } \\
\text { California } \\
\text { USA } \\
\end{array}$ & October 2013 to May 2014 & Prospective Cohort & $\begin{array}{l}\text { A: In-room mentoring } \\
\text { B: Tele-mentoring }\end{array}$ \\
\hline Steinman, 2015 & $\begin{array}{l}\text { Sao Paulo } \\
\text { Brazil }\end{array}$ & May 2012 to May 2013 & $\begin{array}{l}\text { Before-After, } \\
\text { Prospective Cohort }\end{array}$ & $\begin{array}{l}\text { A: } 1 \text { year before telehealth } \\
\text { B: } 1 \text { year after telehealth } \\
\text { Once telehealth established } \\
\text { C: Nontelehealth consultations } \\
\text { D: Telehealth consultations } \\
\text { n=unclear }\end{array}$ \\
\hline $\begin{array}{l}\text { Thomas, } 2009 \\
\text { same patients as } \\
\text { Franzini, } 2011 \\
\end{array}$ & USA & $\begin{array}{l}\text { A: January } 2003 \text { to August } 2005 \\
\text { B: July } 2004 \text { to July } 2006\end{array}$ & $\begin{array}{l}\text { Economic Evaluation, } \\
\text { Before-After }\end{array}$ & $\begin{array}{l}\text { A: Before remote monitoring of ICU patients } \\
\text { B: After remote monitoring of ICU patients }\end{array}$ \\
\hline Wallace, 2008 & United Kingdom & March 2003 to May 2003 & Prospective Cohort & $\begin{array}{l}\text { A: Site without telehealth } \\
\text { B: Site with telehealth }\end{array}$ \\
\hline Webb, 2013 & $\begin{array}{l}9 \text { sites: Ann Arbor MI, } \\
\text { Chicago IL, Denver } \\
\text { CO, Wash DC, } \\
\text { Winston-Salem NC, } \\
\text { Detroit MI, Pittsburg } \\
\text { PA, Charlottesville } \\
\text { VA, Houston TX, } \\
\text { Portland OR } \\
\text { USA } \\
\end{array}$ & July 1999 to Dec 2001 & Prospective Cohort & $\begin{array}{l}\text { A: Babies born at hospital without access to telehealth } \\
\text { B: Babies born at hospital with access to telehealth }\end{array}$ \\
\hline Willmitch, 2012 & $\begin{array}{l}\text { Florida } \\
\text { USA }\end{array}$ & December 2004 to July 2007 & Before-After & $\begin{array}{l}\text { A: Before telehealth } \\
\text { B: } 1 \text { year after telehealth } \\
\text { C: } 2 \text { years after telehealth } \\
\text { D: } 3 \text { years after telehealth }\end{array}$ \\
\hline
\end{tabular}

eICU = electronic intensive care unit; ICU = intensive care unit; NICU = neonatal intensive care unit; NR = not reported; RCT = randomized control trial; TEMPIS = Telemedic Pilot Project for Integrative Stroke Care; $\mathrm{TH}=$ telehealth

See Appendix C. Included Studies for full citations 
Table F-2. Inpatient evidence table: patient characteristics

\begin{tabular}{|c|c|c|c|c|c|c|c|c|}
\hline $\begin{array}{l}\text { Author, Year } \\
\text { (See Appendix C } \\
\text { for full citation) }\end{array}$ & Number Analyzeda & Population & $\begin{array}{l}\text { Referring } \\
\text { Providers } \\
\text { Specialtyl } \\
\text { Type }\end{array}$ & $\begin{array}{l}\text { Consulting Provider } \\
\text { Specialty/Type }\end{array}$ & $\begin{array}{l}\text { Patient } \\
\text { Present? }\end{array}$ & $\begin{array}{l}\text { Patient } \\
\text { Setting }\end{array}$ & $\begin{array}{l}\text { Modalities of } \\
\text { Telehealth } \\
\text { Consultation } \\
\text { Intervention }\end{array}$ & Timing \\
\hline Alemi, 2017 & $\begin{array}{l}60 \\
\text { A: } 31 \\
B: 29 \\
\end{array}$ & Adults & ICU nurse & On-call resident & Yes & Inpatient & Video & Real-time \\
\hline Armaignac, 2018 & $\begin{array}{l}16,091 \\
A: 8,000 \\
B: 8,091\end{array}$ & Adults & $\begin{array}{l}\text { Physicians and } \\
\text { nurses }\end{array}$ & $\begin{array}{l}\text { Physicians and } \\
\text { nurses }\end{array}$ & Yes & Inpatient & NR & Real-time \\
\hline Audebert, 2009 & $\begin{array}{l}3,060 \\
\text { A: } 1,938 \\
\text { B: } 1,122\end{array}$ & Any patients with stroke & $\begin{array}{l}\text { Local } \\
\text { physicians at } \\
\text { the hospital } \\
\text { that are } \\
\text { involved in } \\
\text { caring for } \\
\text { stroke patients }\end{array}$ & Neurology & Yes & Inpatient & $\begin{array}{l}\text { Videoconference } \\
\text { and review of } \\
\text { CT/MRI brain } \\
\text { images }\end{array}$ & Mixed \\
\hline Breslow, 2004 & $\begin{array}{l}2,140 \\
\text { A: } 1,396 \\
\text { B: } 744\end{array}$ & Not specified & $\begin{array}{l}\text { ICU attending } \\
\text { physician }\end{array}$ & Intensivist & Unclear & $\begin{array}{l}\text { Inpatient } \\
\text { (ICU) }\end{array}$ & $\begin{array}{l}\text { Mixed } \\
\text { (videoconference, } \\
\text { records, bedside } \\
\text { monitoring) } \\
\end{array}$ & Mixed \\
\hline Buckley, 2012 & $\begin{array}{l}1,943 \\
\text { A: } 1,153 \\
\text { B: } 790\end{array}$ & Adults & $\begin{array}{l}\text { Community } \\
\text { mental health } \\
\text { clinicians }\end{array}$ & $\begin{array}{l}\text { Psychiatrist or senior } \\
\text { mental health } \\
\text { professionals }\end{array}$ & Yes & Inpatient & videoconference & Real-time \\
\hline $\begin{array}{l}\text { Burgess, et al., } \\
2002\end{array}$ & $\begin{array}{l}87 \\
\text { A: } 42 \text { patients (83 } \\
\text { sinus sides) } \\
\text { B: } 45 \text { patients (83 } \\
\text { sinus sides) } \\
\end{array}$ & NR & $\begin{array}{l}\text { Otolaryngology } \\
\text { residents }\end{array}$ & Attending surgeon & Yes & Inpatient & Videoconference & Real-time \\
\hline Chan, et al., 2001 & $\begin{array}{l}198 \\
\text { A: NR } \\
\text { B: NR }\end{array}$ & Adults in nursing facility & Skilled nursing & Geriatrics & Yes & \begin{tabular}{|l|} 
Inpatient \\
(residential \\
nursing \\
home)
\end{tabular} & Videoconference & Real-time \\
\hline $\begin{array}{l}\text { Chu-Weininger, } \\
2010\end{array}$ & $\begin{array}{l}84, \text { same patients } \\
\text { A: } 84 \\
\text { B: } 71 \\
\end{array}$ & Nurses and doctors & ICU nurse & Intensivist & Yes & Inpatient & Videoconference & Real-time \\
\hline Collins, 2017 & $\begin{array}{l}7,689 \\
\text { A: } 6,652 \\
\text { B: } 1,037 \\
\end{array}$ & Adult & $\begin{array}{l}\text { Nurse } \\
\text { practitioner }\end{array}$ & Intensivist & Yes & Inpatient & Videoconference & Real-time \\
\hline Craig, 2004 & $\begin{array}{l}292 \\
\text { A: } 128 \\
B: 164\end{array}$ & $\geq 12$ & $\begin{array}{l}\text { Junior general } \\
\text { physician }\end{array}$ & Neurologist & Yes & Inpatient & Videoconference & Real-time \\
\hline
\end{tabular}




\begin{tabular}{|c|c|c|c|c|c|c|c|c|}
\hline $\begin{array}{l}\text { Author, Year } \\
\text { (See Appendix C } \\
\text { for full citation) }\end{array}$ & Number Analyzed ${ }^{a}$ & Population & $\begin{array}{l}\text { Referring } \\
\text { Providers } \\
\text { Specialtyl } \\
\text { Type }\end{array}$ & $\begin{array}{l}\text { Consulting Provider } \\
\text { Specialty/Type }\end{array}$ & $\begin{array}{l}\text { Patient } \\
\text { Present? }\end{array}$ & $\begin{array}{l}\text { Patient } \\
\text { Setting }\end{array}$ & $\begin{array}{l}\text { Modalities of } \\
\text { Telehealth } \\
\text { Consultation } \\
\text { Intervention } \\
\end{array}$ & Timing \\
\hline Dharmar, 2013 & $\begin{array}{l}2,029 \\
\text { A: } 515 \\
B: 1,514 \\
\end{array}$ & Pediatric & ED physician & Pediatric critical care & Yes & Inpatient & Video & Real-time \\
\hline Engel, 2011 & $\begin{array}{l}103 \text { flaps } \\
\text { A: } 57 \\
\text { B: } 46\end{array}$ & Age range (13 to 83 years) & $\begin{array}{l}\text { Nurses and } \\
\text { house officers }\end{array}$ & Plastic surgeon & Yes & Inpatient & $\begin{array}{l}\text { Remote } \\
\text { smartphone } \\
\text { photographic } \\
\text { assessments }\end{array}$ & Real-time \\
\hline Fortis, 2014 & $\begin{array}{l}12,160 \\
\text { A: } 6,063 \\
B: 6,097 \\
\end{array}$ & ICU patients & $\begin{array}{l}\text { Nurse } \\
\text { hospitalist }\end{array}$ & Intensivist & Yes & Inpatient & Videoconference & Real-time \\
\hline $\begin{array}{l}\text { Franzini, } 2011 \\
\text { same patients as } \\
\text { Thomas, } 2009\end{array}$ & $\begin{array}{l}3,970 \\
\text { A: } 2,034 \\
\text { B: } 2,108\end{array}$ & NR & $\begin{array}{l}\mathrm{ICU} \\
\text { intensivists }\end{array}$ & ICU intensivists & Yes & Inpatient & Videoconference & Real-time \\
\hline Fuertes-Guiró, 2016 & \begin{tabular}{|l|}
36 \\
A: 16 \\
B: 20 \\
2 conversions in \\
nonmentored group \\
A \\
\end{tabular} & Rural & Surgeon & Mentoring surgeon & Yes & Inpatient & Videoconference & Real-time \\
\hline Grabowski, 2014 & $\begin{array}{l}\text { A1 and A2: same } 5 \\
\text { nursing homes } \\
\text { B1 and B2: same } 6 \\
\text { nursing homes }\end{array}$ & Adults & Nurse & On-call physician & Yes & $\begin{array}{l}\text { Nursing } \\
\text { Home }\end{array}$ & Videoconference & Real-time \\
\hline Gray, 2009 & $\begin{array}{l}\text { NR } \\
\text { A: NR } \\
\text { B: } 12 \\
\end{array}$ & Rural geriatric patients & $\begin{array}{l}\text { Junior house } \\
\text { doctor and } \\
\text { nurses } \\
\end{array}$ & Geriatrician & Yes & Inpatient & $\begin{array}{l}\text { Videoconference } \\
\text { (pathology results } \\
\text { viewed online) }\end{array}$ & Real-time \\
\hline Gupta, 2014 & $\begin{array}{l}279 \\
\text { A: } 13 \\
\text { B: } 145 \\
\end{array}$ & Adult & ICU Physician & Cardiologist & Yes & Inpatient & Audiovisual & Real-time \\
\hline Huang, 2008 & $\begin{array}{l}665 \\
\text { A. } 280 \\
\text { B. } 385 \\
\end{array}$ & NICU patients & Neonatologist & Cardiologist & $\begin{array}{l}\text { Yes, } \\
\text { occasionally }\end{array}$ & Inpatient & $\begin{array}{l}\text { Videoconference, } \\
\text { telephone for } \\
\text { recommendations }\end{array}$ & Mixed \\
\hline
\end{tabular}




\begin{tabular}{|c|c|c|c|c|c|c|c|c|}
\hline $\begin{array}{l}\text { Author, Year } \\
\text { (See Appendix C } \\
\text { for full citation) }\end{array}$ & Number Analyzed ${ }^{a}$ & Population & $\begin{array}{l}\text { Referring } \\
\text { Providers } \\
\text { Specialtyl } \\
\text { Type } \\
\end{array}$ & $\begin{array}{l}\text { Consulting Provider } \\
\text { Specialty/Type }\end{array}$ & \begin{tabular}{|l} 
Patient \\
Present?
\end{tabular} & \begin{tabular}{|l} 
Patient \\
Setting
\end{tabular} & $\begin{array}{l}\text { Modalities of } \\
\text { Telehealth } \\
\text { Consultation } \\
\text { Intervention } \\
\end{array}$ & Timing \\
\hline Kahn, 2016 & \begin{tabular}{|l|}
521 sites \\
$1,123,563$ patients \\
\\
A: No telehealth \\
Before-telehealth, \\
$n=419,466$ \\
After telehealth, \\
$n=411,461$ \\
\\
B: Telehealth \\
Before telehealth, \\
$n=147,517$ \\
After telehealth, \\
$n=145,119$
\end{tabular} & $\begin{array}{l}\text { Medicare fee-for-service } \\
\text { beneficiaries over } 65 \\
\text { years old }\end{array}$ & ICU provider & $\begin{array}{l}\text { Support nurses and } \\
\text { physicians }\end{array}$ & NR & $\begin{array}{l}\text { Inpatient, } \\
\text { ICU }\end{array}$ & $\begin{array}{l}\text { Videoconference, } \\
\text { data streaming, } \\
\text { alarms, and EHR }\end{array}$ & Real-time \\
\hline Kalb, 2014 & $\begin{array}{l}11 \text { Hospitals } \\
\text { A: NR } \\
\text { B: NR } \\
\text { C: NR } \\
\end{array}$ & $\begin{array}{l}\text { Moderate-sized } \\
\text { community hospital ICUs }\end{array}$ & ICU nurses & ICU physicians & Yes & ICU & $\begin{array}{l}\text { Clinical } \\
\text { monitoring, } \\
\text { patient data, and } \\
\text { videoconference } \\
\end{array}$ & Real-time \\
\hline Kim, 2013 & \begin{tabular}{|l|}
767 \\
A. Non-TH - non- \\
NICU - pre: 90 \\
B. Non-TH- non- \\
NICU- post: 91 \\
C. Non-TH-NICU - \\
pre - 102 \\
D. Non-TH-NICU- \\
post - 111 \\
E. TH hospital non- \\
NICU - pre: 50 \\
F. TH hospital non- \\
NICU - post: 27 \\
G. TH hospital with \\
NICU - pre: 25 \\
H. TH hospital with \\
NICU - post: 33 \\
I. University pre-116 \\
J. University post - \\
122
\end{tabular} & VLBW neonates & Physicians & $\begin{array}{l}\text { Obstetric faculty at } \\
\text { university hospital, } \\
\text { Neonatology faculty at } \\
\text { university hospital }\end{array}$ & $\begin{array}{l}\text { Yes, for } \\
\text { some }\end{array}$ & Inpatient & Videoconference & Real-time \\
\hline
\end{tabular}




\begin{tabular}{|c|c|c|c|c|c|c|c|c|}
\hline $\begin{array}{l}\text { Author, Year } \\
\text { (See Appendix C } \\
\text { for full citation) }\end{array}$ & Number Analyzed ${ }^{a}$ & Population & $\begin{array}{l}\text { Referring } \\
\text { Providers } \\
\text { Specialtyl } \\
\text { Type } \\
\end{array}$ & $\begin{array}{l}\text { Consulting Provider } \\
\text { Specialty/Type }\end{array}$ & $\begin{array}{l}\text { Patient } \\
\text { Present? }\end{array}$ & $\begin{array}{l}\text { Patient } \\
\text { Setting } \\
\end{array}$ & $\begin{array}{l}\text { Modalities of } \\
\text { Telehealth } \\
\text { Consultation } \\
\text { Intervention } \\
\end{array}$ & Timing \\
\hline Klein, 2010 & $\begin{array}{l}323 \\
\text { A1: } 152 \\
\text { A2: } 73 \\
\text { B: } 98 \\
\end{array}$ & General & Trauma & Neurosurgery & Unclear & EMS/ED & $\begin{array}{l}\text { Telephone, test } \\
\text { results }\end{array}$ & Mixed \\
\hline Kohl, 2012 & $\begin{array}{l}2,250 \\
\text { A1: } 220 \\
\text { A2: } 285 \\
\text { B1: } 246 \\
\text { B2: } 1,499 \\
\end{array}$ & NR & Nurse & $\begin{array}{l}\text { Nurses and } \\
\text { intensivists }\end{array}$ & Yes & $\begin{array}{l}\text { Inpatient } \\
\text { ICU }\end{array}$ & Videoconference & Real-time \\
\hline Labarbera, 2013 & $\begin{array}{l}153 \\
\text { A. } 41 \\
\text { B. } 56 \\
\text { C. } 56 \\
\end{array}$ & Pediatrics $(<18)$ & Physician & Pediatric intensivist & $\begin{array}{l}\text { Unclear if } \\
\text { for all video- } \\
\text { conference } \\
\text { consults } \\
\end{array}$ & EMS/ED & $\begin{array}{l}\text { Videoconference } \\
\text { or telephone }\end{array}$ & Real-time \\
\hline Lilly, 2011 & $\begin{array}{l}6,290 \\
\text { A: } 1,529 \\
B: 4,761 \\
\end{array}$ & Adults & Nurse & Intensivists & Yes & $\begin{array}{l}\text { Inpatient } \\
\text { ICU }\end{array}$ & Videoconference & Real-time \\
\hline Lilly, 2017 & $\begin{array}{l}17,068 \\
\text { A: } 4,752 \\
\text { B: } 5,735 \\
\text { C: } 6,581 \\
\end{array}$ & Adults & Nurse & Intensivists & Yes & $\begin{array}{l}\text { Inpatient } \\
\text { ICU }\end{array}$ & Videoconference & Real-time \\
\hline Marcin, 2004a & $\begin{array}{l}429 \\
\text { A1: } 116 \\
\text { A2: } 86 \\
\text { B1: } 47 \\
\text { B2: } 180 \\
\end{array}$ & Pediatrics & $\begin{array}{l}\text { Attending } \\
\text { physician }\end{array}$ & Pediatric intensivist & Yes & Inpatient & Videoconference & Real-time \\
\hline Marcin, 2004b & $\begin{array}{l}137 \\
\text { A: } 47 \\
\text { B: } 90\end{array}$ & Pediatrics & Physician & Pediatric intensivist & Yes & Inpatient & Videoconference & Real-time \\
\hline Marcin, 2004c & $\begin{array}{l}321 \\
\text { A1: } 127 \\
\text { A2: } 80 \\
\text { B: } 17 \\
\text { C: } 97\end{array}$ & Pediatrics $(<16)$ & $\begin{array}{l}\text { Community } \\
\text { hospital } \\
\text { physicians }\end{array}$ & Pediatric intensivist & $\begin{array}{l}\text { Yes } \\
\text { (unclear if } \\
\text { for every } \\
\text { consult) }\end{array}$ & Inpatient & Videoconference & Real-time \\
\hline Martin-Khan, 2016 & $\begin{array}{l}166 \\
A: 81 \\
B: 85\end{array}$ & Adult & RN & Geriatricians & No & Inpatient & Web-based & Asynchronous \\
\hline
\end{tabular}




\begin{tabular}{|c|c|c|c|c|c|c|c|c|}
\hline $\begin{array}{l}\text { Author, Year } \\
\text { (See Appendix C } \\
\text { for full citation) }\end{array}$ & Number Analyzed ${ }^{a}$ & Population & $\begin{array}{l}\text { Referring } \\
\text { Providers } \\
\text { Specialtyl } \\
\text { Type }\end{array}$ & $\begin{array}{l}\text { Consulting Provider } \\
\text { Specialty/Type }\end{array}$ & $\begin{array}{l}\text { Patient } \\
\text { Present? }\end{array}$ & $\begin{array}{l}\text { Patient } \\
\text { Setting }\end{array}$ & $\begin{array}{l}\text { Modalities of } \\
\text { Telehealth } \\
\text { Consultation } \\
\text { Intervention }\end{array}$ & Timing \\
\hline $\begin{array}{l}\text { McCambridge, } \\
2010\end{array}$ & $\begin{array}{l}1,913 \\
\text { A: } 954 \\
B: 959\end{array}$ & Adults & Critical care & $\begin{array}{l}\text { Critical care, } \\
\text { intensivists }\end{array}$ & Yes & $\begin{array}{l}\text { Inpatient } \\
\text { (ICU) }\end{array}$ & $\begin{array}{l}\text { Electronic } \\
\text { records, digitally } \\
\text { generated alerts, } \\
\text { computer } \\
\text { assisted order } \\
\text { entry, radiological } \\
\text { information, two- } \\
\text { way audio, one- } \\
\text { way video system }\end{array}$ & Real-time \\
\hline Mielonen, 2000 & $\begin{array}{l}34 \\
\text { A: } 20 \\
\text { B: } 14 \\
\text { Satisfaction survey, } \\
n=124 \\
\end{array}$ & Psychiatric inpatients & NR & $\begin{array}{l}\text { Health care staff at } \\
\text { department of } \\
\text { psychiatry }\end{array}$ & Yes & Inpatient & Videoconference & Real-time \\
\hline Migliaretti, 2013 & $\begin{array}{l}2,357 \\
\text { A: } 1,895 \\
\text { B: } 462\end{array}$ & $\begin{array}{l}\text { Diagnosis of head injury } \\
\text { (194 females and } 268 \\
\text { males) with a diagnosis of } \\
\text { head injury. The majority } \\
\text { of the patients were over } \\
70 \text { years old ( } 67 \% \text { over } 70 \\
\text { years) }\end{array}$ & $\begin{array}{l}\text { Not clearl } \\
\text { admitting } \\
\text { physicians/ } \\
\text { non neuro- } \\
\text { surgeons }\end{array}$ & Neurosurgery & No & Inpatient & $\begin{array}{l}\text { Mixed-telephone } \\
\text { and transmitting } \\
\text { CT images via } \\
\text { telemedicine } \\
\text { system }\end{array}$ & Mixed \\
\hline Miyamoto, 2014 & $\begin{array}{l}183 \\
\text { A: } 82 \\
B: 101\end{array}$ & Children, rural & NR & $\begin{array}{l}\text { Advanced practice } \\
\text { nurse practitioners } \\
\text { (certified pediatric and } \\
\text { adult sexual assault } \\
\text { nurse examiners) }\end{array}$ & No & Outpatient & $\begin{array}{l}\text { Commercially } \\
\text { available } \\
\text { telemedicine unit } \\
\text { with an integrated } \\
\text { colposcope and } \\
\text { high definition } \\
\text { digital video } \\
\text { camera; } \\
\text { videoconference }\end{array}$ & Real-time \\
\hline $\begin{array}{l}\text { Morrison, et al., } \\
2010\end{array}$ & \begin{tabular}{|l|}
4,088 \\
A: 1,371 \\
B: 1,287 \\
C: 1,430 \\
\end{tabular} & Adults & $\begin{array}{l}\text { Physicians and } \\
\text { surgeons }\end{array}$ & \begin{tabular}{|l} 
Intensivists and \\
critical care nurses
\end{tabular} & Unclear & $\begin{array}{l}\text { Inpatient } \\
\text { (ICU) }\end{array}$ & NR & Real-time \\
\hline Nagayoshi, 2016 & $\begin{array}{l}66 \\
\text { A: } 18 \\
\text { B: } 48 \\
\end{array}$ & Adult & Cardiologist & $\begin{array}{l}\text { Cardiologist with on- } \\
\text { site cardiac surgery }\end{array}$ & No & Inpatient & Central server & Asynchronous \\
\hline
\end{tabular}




\begin{tabular}{|c|c|c|c|c|c|c|c|c|}
\hline $\begin{array}{l}\text { Author, Year } \\
\text { (See Appendix C } \\
\text { for full citation) }\end{array}$ & Number Analyzed a & Population & $\begin{array}{l}\text { Referring } \\
\text { Providers } \\
\text { Specialtyl } \\
\text { Type } \\
\end{array}$ & $\begin{array}{l}\text { Consulting Provider } \\
\text { Specialty/Type }\end{array}$ & $\begin{array}{l}\text { Patient } \\
\text { Present? }\end{array}$ & $\begin{array}{l}\text { Patient } \\
\text { Setting } \\
\end{array}$ & $\begin{array}{l}\text { Modalities of } \\
\text { Telehealth } \\
\text { Consultation } \\
\text { Intervention }\end{array}$ & Timing \\
\hline Nassar, 2014 & $\begin{array}{l}6,654 \\
\text { A: } 3,584 \\
\text { A1: } 1,664 \\
\text { A2: } 1,920 \\
\text { B: } 3,355 \\
\text { B1: } 1,708 \\
\text { B2: } 1,647\end{array}$ & Adults & ICU staff & Intensivist & Yes & Inpatient & Videoconference & Real-time \\
\hline Panlaqui, 2017 & $\begin{array}{l}525 \\
\text { A: } 337 \\
\text { B: } 188\end{array}$ & Adult & $\begin{array}{l}\text { General } \\
\text { practitioner } \\
\text { and nurses }\end{array}$ & Intensivist & Yes & Inpatient & Videoconference & Real-time \\
\hline Rendina, 1997 & $\begin{array}{l}86 \\
\text { A: } 38 \\
\text { B: } 48 \\
\end{array}$ & Pediatric & NR & Cardiologist & Yes & Inpatient & $\begin{array}{l}\text { Videoconference } \\
\text { and digital } \\
\text { images }\end{array}$ & Real-time \\
\hline Rendina, 1998 & $\begin{array}{l}314 \\
\text { A: } 137 \\
\text { B: } 177 \text { (75 born } \\
\text { before telehealth } \\
\text { system available) }\end{array}$ & Neonates & Not specified & Cardiology & Unclear & $\begin{array}{l}\text { Inpatient, } \\
\text { ICU }\end{array}$ & Not specified & Not specified \\
\hline Rincon, 2012 & $\begin{array}{l}74 \\
\text { A: } 34 \text { Nurses } \\
\text { B: } 40 \text { Nurses }\end{array}$ & Nurses & ICU nurse & ICU specialist & Yes & Inpatient & Videoconference & Real-time \\
\hline Robison, 2016 & $\begin{array}{l}91 \\
\text { A: } 43 \\
B: 48\end{array}$ & Pediatric & ICU nurse & Critical care intensivist & Yes & Inpatient & Videoconference & Real-time \\
\hline Romig, 2012 & $\begin{array}{l}1405 \\
\text { A: } 612 \\
\text { B: } 793 ; 403 \\
\text { received telehealth } \\
\text { Nurse survey: } \\
\text { Pre-telehealth, } \\
\text { n=11 } \\
\text { Post-telehealth, } \\
\text { n=27 }\end{array}$ & ICU & ICU specialist & ICU intensivist & Unclear & $\mathrm{ICU}$ & $\begin{array}{l}\text { Videoconference } \\
\text { and physiologic } \\
\text { monitoring }\end{array}$ & Real-time \\
\hline
\end{tabular}




\begin{tabular}{|c|c|c|c|c|c|c|c|c|}
\hline $\begin{array}{l}\text { Author, Year } \\
\text { (See Appendix C } \\
\text { for full citation) }\end{array}$ & Number Analyzed ${ }^{a}$ & Population & $\begin{array}{l}\text { Referring } \\
\text { Providers } \\
\text { Specialtyl } \\
\text { Type } \\
\end{array}$ & $\begin{array}{l}\text { Consulting Provider } \\
\text { Specialty/Type }\end{array}$ & $\begin{array}{l}\text { Patient } \\
\text { Present? }\end{array}$ & $\begin{array}{l}\text { Patient } \\
\text { Setting }\end{array}$ & $\begin{array}{l}\text { Modalities of } \\
\text { Telehealth } \\
\text { Consultation } \\
\text { Intervention } \\
\end{array}$ & Timing \\
\hline Rosenfeld, 2000 & $\begin{array}{l}628 \\
\text { A1: } 225 \\
\text { A2: } 202 \\
\text { B: } 201\end{array}$ & $\begin{array}{l}\text { All patients in ICU during } \\
\text { study period (exclusion } \\
\text { criteria }<16 \text { years old, }<4- \\
\text { hour stay in ICU, transfer } \\
\text { to another hospital ICU, } \\
\text { missing APACHE III data) }\end{array}$ & $\begin{array}{l}\text { ICU attending } \\
\text { and house } \\
\text { staff } \\
\text { physicians; } \\
\text { also some } \\
\text { interaction with } \\
\text { nursing staff. }\end{array}$ & ICU intensivist & Yes & $\begin{array}{l}\text { Inpatient } \\
\text { (ICU) }\end{array}$ & $\begin{array}{l}\text { Videoconference, } \\
\text { bedside } \\
\text { monitoring as } \\
\text { well as lab data } \\
\text { (via telephone } \\
\text { access system), } \\
\text { ECGs, radiology, } \\
\text { consultant notes, } \\
\text { and bedside data } \\
\text { flowsheets were } \\
\text { scanned and } \\
\text { transmitted daily. }\end{array}$ & Real-time \\
\hline Ruesch, 2012 & $\begin{array}{l}\text { 1,308 } \\
\text { A: NR } \\
\text { B: NR } \\
\text { C: NR }\end{array}$ & NR & Nurse & $\begin{array}{l}\text { Intensivist, critical } \\
\text { care }\end{array}$ & Unclear & $\begin{array}{l}\text { Inpatient } \\
\text { (ICU) }\end{array}$ & $\begin{array}{l}\text { Digitally } \\
\text { generated alerts, } \\
\text { presumably } \\
\text { others because } \\
\text { collaboration is } \\
\text { discussed but not } \\
\text { specified }\end{array}$ & Real-time \\
\hline Sadaka, 2013 & $\begin{array}{l}2,823 \\
\text { A: } 630 \\
\text { B: } 2,193\end{array}$ & Adults & Intensivist & Intensivist & Yes & $\begin{array}{l}\text { Inpatient } \\
\text { (ICU) }\end{array}$ & $\begin{array}{l}\text { Digitally } \\
\text { generated alerts, } \\
\text { videoconference, } \\
\text { still images }\end{array}$ & Real-time \\
\hline Sharma, 2016 & $\begin{array}{l}63 \\
\text { A: } 38 \\
\text { B: } 25\end{array}$ & NR & Resident & $\begin{array}{l}\text { Attending } \\
\text { dermatologist }\end{array}$ & Unclear & Inpatient & $\begin{array}{l}\text { Smartphone and } \\
\text { internet-based } \\
\text { app }\end{array}$ & Unclear \\
\hline Shin, 2015 & $\begin{array}{l}55 \\
\text { A: } 29 \\
\text { B: } 26\end{array}$ & NR & $\begin{array}{l}\text { Robotic } \\
\text { surgery } \\
\text { trainees }\end{array}$ & $\begin{array}{l}\text { Robotic fellows or } \\
\text { attending physicians } \\
\text { from the } \\
\text { Urology Department } \\
\text { who had completed at } \\
\text { least } 150 \text { console } \\
\text { cases as primary } \\
\text { surgeon }\end{array}$ & Yes & Inpatient & $\begin{array}{l}\text { Videoconference } \\
\text { and telestrate }\end{array}$ & Real-time \\
\hline
\end{tabular}




\begin{tabular}{|c|c|c|c|c|c|c|c|c|}
\hline $\begin{array}{l}\text { Author, Year } \\
\text { (See Appendix C } \\
\text { for full citation) }\end{array}$ & Number Analyzed ${ }^{a}$ & Population & $\begin{array}{l}\text { Referring } \\
\text { Providers } \\
\text { Specialtyl } \\
\text { Type } \\
\end{array}$ & $\begin{array}{l}\text { Consulting Provider } \\
\text { Specialty/Type }\end{array}$ & $\begin{array}{l}\text { Patient } \\
\text { Present? }\end{array}$ & $\begin{array}{l}\text { Patient } \\
\text { Setting }\end{array}$ & \begin{tabular}{|l|} 
Modalities of \\
Telehealth \\
Consultation \\
Intervention \\
\end{tabular} & Timing \\
\hline Steinman, 2015 & Unclear & $\begin{array}{l}\text { Patient diagnosis with: } \\
\text { stroke, sepsis, acute MI, } \\
\text { trauma, cardiac arrest, } \\
\text { acute hepatic failure, } \\
\text { diabetic ketoacidosis, } \\
\text { heart failure, brain tumor, } \\
\text { pulmonary thrombo- } \\
\text { embolism, cardiac } \\
\text { arrhythmia, acute } \\
\text { mesenteric ischemia, } \\
\text { hemorrhagic shock, } \\
\text { exogenous intoxication. }\end{array}$ & ED and ICU & ED and ICU & Yes & ED or ICU & $\begin{array}{l}\text { Videoconference } \\
\text { and PACS }\end{array}$ & Real-time \\
\hline $\begin{array}{l}\text { Thomas, } 2009 \\
\text { same patients as } \\
\text { Franzini, } 2011\end{array}$ & $\begin{array}{l}4,142 \\
\text { A: } 2,034 \\
B: 2,108\end{array}$ & NR & NR & Intensivist & Yes & $\begin{array}{l}\text { Inpatient } \\
\text { (ICU) }\end{array}$ & $\begin{array}{l}\text { Videoconference, } \\
\text { digitally } \\
\text { generated alerts, } \\
\text { fax }\end{array}$ & Real-time \\
\hline Wallace, 2008 & $\begin{array}{l}996 \\
\text { A: } 607 \\
\text { B: } 389 \text { TH } \\
\text { available; } 243 \text { used } \\
\text { TH }\end{array}$ & $\begin{array}{l}\text { Various adults and } \\
\text { children }\end{array}$ & NR & $\begin{array}{l}\text { Plastic surgery trauma } \\
\text { and burn specialists }\end{array}$ & No & $\begin{array}{l}\text { Referring } \\
\text { hospitals } \\
\text { or minor } \\
\text { injury units }\end{array}$ & $\begin{array}{l}\text { Store and forward } \\
\text { images }\end{array}$ & Asynchronous \\
\hline Webb, 2013 & $\begin{array}{l}674 \\
\text { Matched pairs } \\
\text { A: } 337 \\
\text { B: } 337\end{array}$ & $\begin{array}{l}\text { Infants aged }<6 \text { weeks } \\
\text { with mild or no heat } \\
\text { disease }\end{array}$ & OB & Neonatology & Yes & Inpatient & $\begin{array}{l}\text { Mixed: } \\
\text { echocardiology } \\
\text { studies and } \\
\text { telephone } \\
\text { consultations } \\
\text { between } \\
\text { providers }\end{array}$ & Asynchronous \\
\hline Willmitch, 2012 & $\begin{array}{l}24,656 \\
\text { A. } 6,504 \\
\text { B. } 6,353 \\
\text { C. } 6,018 \\
\text { D. } 5,781 \\
\end{array}$ & Adults & Critical care & $\begin{array}{l}\text { Intensivist, critical } \\
\text { care }\end{array}$ & Yes & $\begin{array}{l}\text { Inpatient } \\
\text { (ICU) }\end{array}$ & $\begin{array}{l}\text { Digitally } \\
\text { generated alerts, } \\
\text { two way voice, } \\
\text { one way video }\end{array}$ & Real-time \\
\hline
\end{tabular}

CT = computed tomography; ED = emergency department; EHR = electronic health record; EMS = emergency medical services; ICU = intensive care unit; NICU = neonatal intensive care unit; NR = not reported; PACS = picture archiving and communication system; $\mathrm{TH}$ = telehealth; VLBW = very low birth weight

${ }^{a}$ For definitions of interventions and comparisons (e.g., A vs. B), see Table F-1

See Appendix C. Included Studies for full citations 
Table F-3. Inpatient evidence table: results

\begin{tabular}{|c|c|c|c|}
\hline $\begin{array}{l}\text { Author, Year } \\
\text { (See Appendix C } \\
\text { for full citation) }\end{array}$ & $\begin{array}{l}\text { Results: } \\
\text { KQ1: Clinical and Economic Outcomes }\end{array}$ & $\begin{array}{l}\text { Results: } \\
\text { KQ2: Intermediate Outcomes }\end{array}$ & $\begin{array}{l}\text { Results: } \\
\text { KQ3: Adverse Effects } \\
\text { or Unintended } \\
\text { Consequences }\end{array}$ \\
\hline Alemi, 2017 & NR & $\begin{array}{l}\text { Time for assessment (range) (SD), in } \\
\text { minutes } \\
\text { A: } 34 \text { (10 to } 60),(16) \\
B: 13 \text { (5 to } 35),(8) \\
p<0.001\end{array}$ & None \\
\hline Armaignac, 2018 & $\begin{array}{l}\text { PCU mortality, } n(\%) \\
\text { A: } 83(1 \%) \\
\text { B: } 60(0.7 \%) \\
p=0.048 \\
\text { Hospital mortality, } n(\%) \\
\text { A: } 410(5.2 \%) \\
\text { B: } 342(4.4 \%) \\
p=0.013 \\
\text { Unadjusted HR: } 0.79(0.68 \text { to } 0.91) \\
\text { Adjusted HR: } 0.56(0.41 \text { to } 0.76) \\
\\
\text { Overall mean direct cost, in USD } \\
\text { A: } \$ 12,301 \\
\text { B: } \$ 13,180, p<0.0001\end{array}$ & $\begin{array}{l}\text { Mean LOS }(95 \% \mathrm{Cl}) \text {, in days } \\
\text { PCU } \\
\text { A: } 3.2(3.1 \text { to } 3.3) \\
\text { B: } 2.6(2.5 \text { to } 2.7) \\
\text { p<0.0001 } \\
\text { Hospital } \\
\text { A: } 6.8(6.6 \text { to } 6.9) \\
\text { B: } 7.3(7.2 \text { to } 7.5) \\
p<0.0001\end{array}$ & NR \\
\hline
\end{tabular}




\begin{tabular}{|c|c|c|c|}
\hline $\begin{array}{l}\text { Author, Year } \\
\text { (See Appendix C } \\
\text { for full citation) }\end{array}$ & $\begin{array}{l}\text { Results: } \\
\text { KQ1: Clinical and Economic Outcomes }\end{array}$ & $\begin{array}{l}\text { Results: } \\
\text { KQ2: Intermediate Outcomes }\end{array}$ & $\begin{array}{l}\text { Results: } \\
\text { KQ3: Adverse Effects } \\
\text { or Unintended } \\
\text { Consequences } \\
\end{array}$ \\
\hline Audebert, 2009 & $\begin{array}{l}\text { Mortality: The cumulative survival rates after } 30 \text { months were } 68.0 \% \\
\text { in the intervention group and } 65.5 \% \text { in the control group (log rank } \\
\text { test: } p=0.271) \text {. } \\
\text { Reduced death or institutional care, AOR }(95 \% \mathrm{Cl}) \text {, p-value } \\
12 \text { months: } \\
0.89 \text { ( } 0.75 \text { to } 1.07), p=0.223 \\
30 \text { months: } \\
0.93 \text { ( } 0.78 \text { to } 1.1), p=0.400 \\
\text { Poor outcome (death, institutional care, severe disability), AOR (95\% } \\
\text { Cl), p-value } \\
12 \text { months: } \\
0.65 \text { ( } 0.54 \text { to } 0.78), p<0.001 \\
30 \text { months: } \\
0.82 \text { ( } 0.68 \text { to } 0.98), p=0.031 \\
\text { Combined mortality and institutional care: The statistically significant } \\
\text { difference in univariate analysis at } 12 \text { months in favor of the TEMPiS } \\
\text { group did not remain significant after correction for possible } \\
\text { confounders. There was only a trend in favor of the intervention } \\
\text { group at } 30 \text { months without significance in multivariable analysis. } \\
\text { Followup rates (combined death, institutional care, or severe } \\
\text { disability) } \\
6 \text { months: } \\
\text { A: } 96.0 \% \\
\text { B: } 96.8 \% \\
30 \text { months: } \\
\text { A: } 95.6 \% \\
\text { B: } 95.7 \% \\
\text { The outcome death and dependency was significantly less frequent } \\
\text { in the intervention group at both followup times }\end{array}$ & \begin{tabular}{|l|l} 
NR \\
\end{tabular} & NR \\
\hline
\end{tabular}




\begin{tabular}{|c|c|c|c|}
\hline $\begin{array}{l}\text { Author, Year } \\
\text { (See Appendix C } \\
\text { for full citation) }\end{array}$ & $\begin{array}{l}\text { Results: } \\
\text { KQ1: Clinical and Economic Outcomes }\end{array}$ & $\begin{array}{l}\text { Results: } \\
\text { KQ2: Intermediate Outcomes }\end{array}$ & $\begin{array}{l}\text { Results: } \\
\text { KQ3: Adverse Effects } \\
\text { or Unintended } \\
\text { Consequences }\end{array}$ \\
\hline Breslow, 2004 & $\begin{array}{l}\text { Mortality, p-value; relative risk }(95 \% \mathrm{Cl}) \\
\text { Aggregate ICU mortality: } \\
\text { A: } 8.6 \% \\
\text { B: } 6.3 \%, p<0.05 ; 0.73 \text { ( } 0.53 \text { to } 1.02) \\
\text { Aggregate hospital mortality: } \\
\text { A: } 12.9 \% \\
\text { B: } 9.4 \%, p<0.05 ; 0.73 \text { ( } 0.55 \text { to } 0.95) \\
\text { MICU ICU mortality: } \\
\text { A: } 13.9 \% \\
\text { B: } 9.5 \%, p<0.05 ; 0.68 \text { ( } 0.46 \text { to } 0.98) \\
\text { MICU hospital mortality: } \\
\text { A: } 19.8 \% \\
\text { B: } 13.9 \%, p<0.05 ; 0.71 \text { (0.52 to } 0.95) \\
\text { SICU ICU mortality: } \\
\text { A: } 4.2 \% \\
\text { B: } 3.4 \%, N S ; 0.81(0.43 \text { to } 1.55) \\
\text { SICU hospital mortality: } \\
\text { A: } 7.2 \% \\
\text { B: } 5.5 \%, N S ; 0.75(0.46 \text { to } 1.23) \\
\text { Mean LOS, in days }(95 \% \text { CI) } \\
\text { Aggregate ICU: } \\
\text { A: } 4.3(3.93 \text { to } 4.78) \\
\text { B: } 3.63(3.21 \text { to } 4.04), p<0.05 \\
\text { Aggregate hospital ICU: } \\
\text { A: } 12.77(11.89 \text { to } 13.65) \\
\text { B: } 11.4(10.26 \text { to } 12.03), N S \\
\text { MICU ICU: } \\
\text { A: } 5.62 \text { (4.88 to } 6.35) \\
\text { B: } 4.84 \text { (4.16 to } 5.51), p<0.05 \\
\text { MICU hospital: } \\
\text { A: } 14.93 \text { (13.4 to } 16.45) \\
\text { B: } 13.61(12.11 \text { to } 15.17), N S \\
\text { SICU ICU: } \\
\text { A: } 3.30 \text { (2.83 to } 3.78) \\
\text { B: } 2.49 \text { (2.2 to } 2.97), p<0.05 \\
\text { SICU hospital: } \\
\text { A: } 11.00 \text { (10.01 to } 11.98) \\
\text { B: } 8.83 \text { (7.89 to } 9.76), p<0.05\end{array}$ & NR & NR \\
\hline
\end{tabular}




\begin{tabular}{|c|c|c|c|}
\hline $\begin{array}{l}\text { Author, Year } \\
\text { (See Appendix C } \\
\text { for full citation) }\end{array}$ & $\begin{array}{l}\text { Results: } \\
\text { KQ1: Clinical and Economic Outcomes }\end{array}$ & $\begin{array}{l}\text { Results: } \\
\text { KQ2: Intermediate Outcomes }\end{array}$ & $\begin{array}{l}\text { Results: } \\
\text { KQ3: Adverse Effects } \\
\text { or Unintended } \\
\text { Consequences }\end{array}$ \\
\hline $\begin{array}{l}\text { Breslow, } 2004 \\
\text { (continued) }\end{array}$ & $\begin{array}{l}\text { Percent patients with LOS } \geq 7 \text { days, median ICU LOS (IQR) } \\
\text { All patients: } \\
\text { A: } 13.9 \%, 14.0(10.0 \text { to } 25.8) \\
\text { B: } 11.6 \%, 14.1(9.4 \text { to } 21.0), \mathrm{NS} \\
\text { MICU patients: } \\
\text { A: } 19.3 \%, 14.0(10.0 \text { to } 19.5) \\
\text { B: } 17.5 \%, 13.9(9.3 \text { to } 19.5), \mathrm{NS} \\
\text { siCU patients: } \\
\text { A: } 9.4 \%, 14.5(10.0 \text { to } 25.3) \\
\text { B: } 5.9 \%, 15.0(10.3 \text { to } 23.7), p<0.05 \\
\\
\text { Revenue, contribution per month, in USD } \\
\text { All patients: } \\
\text { A: } \$ 795,245 \\
\text { B: } \$ 1,319,236 \\
\text { MICU: } \\
\text { A: } 334,273 \\
\text { B: } \$ 505,669 \\
\text { SICU: } \\
\text { A: } \$ 483,221 \\
\text { B: } \$ 862,591\end{array}$ & (continued) & (continued) \\
\hline Buckley, 2012 & $\begin{array}{l}\text { Patients transferred }(95 \% \mathrm{Cl}) \\
\text { A: } 66.8 \%(64.0 \text { to } 69.5) \\
\text { B: } 59.6 \%(56.1 \text { to } 63.1)\left(\mathrm{X}^{2}=10.42, \mathrm{p}=0.001\right) \text {. } \\
\text { After adjusting for age, sex, clustering in hospitals and repeat visits } \\
\text { the odds of transfer were } 0.69(95 \% \mathrm{Cl} 0.49 \text { to } 0.97) \text { of previous. }\end{array}$ & NR & NR \\
\hline $\begin{array}{l}\text { Burgess, et al., } \\
2002\end{array}$ & NR & $\begin{array}{l}\text { Average time to complete operative } \\
\text { case, in minutes } \\
\text { A: } 24.67 \\
\text { B: } 28.54, p<0.027\end{array}$ & $\begin{array}{l}\text { No cases of } \\
\text { complications/harms }\end{array}$ \\
\hline Chan, et al., 2001 & $89 \%$ of visits accomplished via telemedicine; $11 \%$ needed onsite visit & $\begin{array}{l}\text { Failed inhaler technique } \\
\text { A: } 93 \% \\
\text { B: } 50 \% \\
\\
\text { Waiting time for consult, in weeks } \\
\text { A: } 4 \text { to } 13 \\
\text { B: } \geq 2 \\
\text { Patient satisfaction: } \\
96 \% \text { favorable }\end{array}$ & $\begin{array}{l}\text { Mean monthly falls } \\
\text { A: } 9.8 \\
\text { B: } 6.8, \text { NS }\end{array}$ \\
\hline
\end{tabular}




\begin{tabular}{|c|c|c|c|}
\hline $\begin{array}{l}\text { Author, Year } \\
\text { (See Appendix C } \\
\text { for full citation) }\end{array}$ & $\begin{array}{l}\text { Results: } \\
\text { KQ1: Clinical and Economic Outcomes }\end{array}$ & $\begin{array}{l}\text { Results: } \\
\text { KQ2: Intermediate Outcomes } \\
\end{array}$ & $\begin{array}{l}\text { Results: } \\
\text { KQ3: Adverse Effects } \\
\text { or Unintended } \\
\text { Consequences } \\
\end{array}$ \\
\hline $\begin{array}{l}\text { Chu-Weininger, } \\
2010\end{array}$ & NR & $\begin{array}{l}\text { Mean teamwork climate score (SD) } \\
\text { A: } 69.7(25.3) \\
\text { B: } 78.8(17.2) \\
p=0.009 \\
\text { Mean safety climate score (SD) } \\
\text { A: } 66.4(24.6) \\
\text { B: } 73.4(18.5) \\
\text { p=0.045 } \\
\text { Overall hospital safety climate score } \\
\text { A: } 69.0 \\
\text { B: } 65.4\end{array}$ & NR \\
\hline Collins, 2017 & NR & $\begin{array}{l}\text { ICU mortality, n (\%) } \\
\text { A: } 364(5.5 \%) \\
\text { B: } 3(0.3 \%)\end{array}$ & NR \\
\hline Craig, 2004 & $\begin{array}{l}\text { Inpatient mortality } \\
\text { A: } 10.2 \% \\
\text { B: } 4.9 \%, p=0.013 \\
\text { 3-month mortality } \\
\text { A: } 11.7 \% \\
\text { B: } 8.6 \%, p=0.558 \\
\text { Mean LOS (SD), in days } \\
\text { A: } 11.6(22.3) \\
\text { B: } 8.1(16.9), p=0.016 \\
\text { HR } 1.13(95 \% \mathrm{Cl} 1.003 \text { to } 1.282), p=0.045\end{array}$ & $\begin{array}{l}\text { No differences in measures comparing } \\
\text { quality of care, the use of inpatient } \\
\text { hospital resources, and medical services } \\
\text { in the followup period between } \mathrm{TCH} \text { and } \\
\text { Erne patients. } \\
\text { Hospital readmissions } \\
\text { A: } 16.8 \% \\
\text { B: } 15.0 \%, p=0.862 \\
\text { Mean number primary care visits at 3- } \\
\text { months } \\
\text { A: } 2.49 \\
\text { B: } 2.14, p=0.519\end{array}$ & NR \\
\hline Dharmar, 2013 & $\begin{array}{l}\text { Mean hospital revenue per year, in USD } \\
\text { A: } \$ 2.4 \text { million } \\
\text { B: } \$ 4.0 \text { million } \\
\text { Mean professional billing revenue per year, in USD } \\
\text { A: } \$ 313,977 \\
\text { B: } \$ 688,443\end{array}$ & $\begin{array}{l}\text { Mean number of patients transferred per } \\
\text { year } \\
\text { A: } 143 \\
\text { B: } 285 \\
\text { Mean LOS of transferred children (SD), } \\
\text { in days } \\
\text { A: } 7.7(14.2) \\
\text { B: } 9.2(15.4) \\
\text { p }<0.05\end{array}$ & NR \\
\hline
\end{tabular}




\begin{tabular}{|c|c|c|c|}
\hline $\begin{array}{l}\text { Author, Year } \\
\text { (See Appendix C } \\
\text { for full citation) }\end{array}$ & $\begin{array}{l}\text { Results: } \\
\text { KQ1: Clinical and Economic Outcomes }\end{array}$ & $\begin{array}{l}\text { Results: } \\
\text { KQ2: Intermediate Outcomes }\end{array}$ & $\begin{array}{l}\text { Results: } \\
\text { KQ3: Adverse Effects } \\
\text { or Unintended } \\
\text { Consequences }\end{array}$ \\
\hline Engel, 2011 & $\begin{array}{l}\text { Surgery success rate } \\
\text { A: } 95.1 \% \\
\text { B: } 97.8 \%, p=0.4\end{array}$ & $\begin{array}{l}\text { Return to operating room } \\
\text { A: } 5(8.8 \%) \\
\text { B: } 4(8.7 \%) \\
\text { Mean response time } \pm S D \text {, in minutes } \\
\text { A: } 180 \pm 104 \\
B: 8 \pm 3, p=0.01\end{array}$ & NR \\
\hline Fortis, 2014 & $\begin{array}{l}\text { Mortality (unadjusted) } \\
\text { A: } 6.5 \% \\
\text { B: } 4.9 \% p<0.0002\end{array}$ & $\begin{array}{l}\text { Patients readmitted to ICU (\%) } \\
\text { A: } 54(0.89 \%) \\
\text { B: } 29(0.49 \%), p=0.0064\end{array}$ & NR \\
\hline $\begin{array}{l}\text { Franzini, } 2011 \\
\text { same patients as } \\
\text { Thomas, } 2009\end{array}$ & $\begin{array}{l}\text { Overall: no difference } \\
\text { SAPS II } \leq 50 \text { (less serious): no difference } \\
\text { SAPS II > } 50 \text { (17\% of patients) } \\
\text { ICU: } 40 \% \text { reduction } \\
\text { Hospital: } 37 \% \text { reduction } \\
\\
\text { Cost } \% \text { increase), in USD } \\
\text { Average daily cost } \\
\text { A: } \$ 2,851 \\
\text { B: } \$ 3,653 \text { (28\% increase) } \\
\text { Overall ICU cost per case: } \\
\text { A: } \$ 13,029 \\
\text { B: } \$ 19,324 \\
\text { Difference: } \$ 6295 \text { (48\% increase) } \\
\text { Average cost per patient: } \\
\text { A: } \$ 20,231 \\
\text { B: } \$ 25,846 \\
\text { Difference: } \$ 5615 \text { (28\% increase) } \\
\text { SAPSII } \leq 50: \text { increase cost by } \$ 6415 \text { with no change in mortality } \\
\text { SAPS II > } 50: \text { increase cost } \$ 2985 \text { with } 11.4 \% \text { decrease in mortality }\end{array}$ & NR & NR \\
\hline Fuertes-Guiró, 2016 & $\begin{array}{l}\text { Mean length of surgery (SD), in minutes } \\
\text { A: } 200(46) \\
\text { B: } 139(33), p<0.01 \\
\text { Mean hospital stay (SD), in days } \\
\text { A: } 6.7(0.5) \\
\text { B: } 4.6(0.5), p<0.01\end{array}$ & NR & $\begin{array}{l}\text { Three patients (12.5\%) } \\
\text { who underwent surgery } \\
\text { without telementoring } \\
\text { suffered from minor } \\
\text { complications (bleeding } \\
\text { of surgical wounds, two } \\
\text { cases, and urological } \\
\text { infection, one case) }\end{array}$ \\
\hline
\end{tabular}




\begin{tabular}{|c|c|c|c|}
\hline $\begin{array}{l}\text { Author, Year } \\
\text { (See Appendix C } \\
\text { for full citation) }\end{array}$ & $\begin{array}{l}\text { Results: } \\
\text { KQ1: Clinical and Economic Outcomes }\end{array}$ & $\begin{array}{l}\text { Results: } \\
\text { KQ2: Intermediate Outcomes }\end{array}$ & $\begin{array}{l}\text { Results: } \\
\text { KQ3: Adverse Effects } \\
\text { or Unintended } \\
\text { Consequences } \\
\end{array}$ \\
\hline Grabowski, 2014 & Only estimated cost savings & $\begin{array}{l}\text { Hospitalization rate per } 1,000 \text { nursing } \\
\text { home resident days } \\
\text { A1: } 3.78 \\
\text { A2: } 3.58 \\
\text { B1: } 3.50 \\
\text { B2: } 3.16 \\
\text { Reduction in hospitalizations } \\
\text { A1 and A2: } 5.3 \% \\
\text { B1 and B2: } 9.7 \% \\
\text { Telehealth only } \\
\text { More engaged: } 11.3 \% \\
\text { Less engaged: } 5.2 \%\end{array}$ & NR \\
\hline Gray, 2009 & $\begin{array}{l}\text { Costs per year, in AUD (including salary, office space, travel time, } \\
\text { DSL costs, travel costs, and set up costs of } \$ 30,000 \text { amortized over } 3 \\
\text { years with depreciation rate of } 25 \% \text { ) } \\
\text { A: } \$ 73,078 \\
\text { B: } \$ 90,909 \\
\text { In the base-case, cost savings became effective when roundtrip } \\
\text { travel time is } \geq 125 \mathrm{~km} \text { between locations. }\end{array}$ & $\begin{array}{l}\text { Mean consultation time }(95 \% \mathrm{Cl}) \text {, in } \\
\text { minutes } \\
\text { A: } 13.7(11.5 \text { to } 15.9) \\
\text { B: } 15.3(13.6 \text { to } 16.09) \\
\text { Mean consultation time for new patients } \\
(95 \% \mathrm{Cl}) \text {, in minutes } \\
\text { A: } 19.0(15.2 \text { to } 22.8) \\
\text { B: } 19.7(17.0 \text { to } 22.4)\end{array}$ & NR \\
\hline Gupta, 2014 & $\begin{array}{l}\text { 30-day mortality } \\
\text { A: } 16.4 \% \\
\text { B: } 4.8 \% \\
70 \% \text { reduction } \\
p=0.001\end{array}$ & $\begin{array}{l}\text { Mean door to needle, in minutes } \\
\text { A: } 178.63 \\
B: 26.23 \\
p<0.001 \\
\text { Mean hospital stay } \pm S D \text {, in days } \\
\text { A: } 4.96 \pm 1.18 \\
B: 4.69 \pm 1.19 \\
p=0.056\end{array}$ & $\begin{array}{l}\text { Cardiogenic shock, } n(\%) \\
\text { A: } 20(14.92) \\
\text { B: } 15(10.35), p=0.248 \\
\text { Ventricular } \\
\text { fibrillation/ventricular } \\
\text { tachycardia (\%) } \\
\text { A: } 16(11.94) \\
\text { B: } 12(8.28), p=0.309 \\
\text { Atrial fibrillation/supra } \\
\text { ventricular tachycardia } \\
(\%) \\
\text { A: } 20(14.92) \\
B: 22(15.17), p=0.954\end{array}$ \\
\hline Huang, 2008 & $\begin{array}{l}\text { Increase in percentage of normal first echocardiograms } \\
\text { A: } 31 \% \\
\text { B: } 37 \% ; p=0.03 \\
\text { Echocardiogram use increased from } 27 \% \text { of admissions prior to } \\
\text { telehealth to } 40 \% \text { of admissions after telehealth }(p<0.001)\end{array}$ & $\begin{array}{l}\text { Inappropriate transfers } \\
\text { A: } 7 \\
\text { B: } 2, p=0.06 \\
\text { No difference in overall rate of transfer }\end{array}$ & NR \\
\hline
\end{tabular}




\begin{tabular}{|c|c|c|c|}
\hline $\begin{array}{l}\text { Author, Year } \\
\text { (See Appendix C } \\
\text { for full citation) }\end{array}$ & $\begin{array}{l}\text { Results: } \\
\text { KQ1: Clinical and Economic Outcomes }\end{array}$ & $\begin{array}{l}\text { Results: } \\
\text { KQ2: Intermediate Outcomes }\end{array}$ & $\begin{array}{l}\text { Results: } \\
\text { KQ3: Adverse Effects } \\
\text { or Unintended } \\
\text { Consequences }\end{array}$ \\
\hline Kahn, 2016 & $\begin{array}{l}\text { 90-day mortality } \\
\text { [ratio of odds ratios exponentiated from difference-in-differences } \\
\text { (95\% CI), p-value; Group A= Reference] } \\
\text { All hospitals: } 0.96 \text { ( } 0.94 \text { to } 0.98), p<0.01 \\
\text { Rural hospitals: } 1.06 \text { ( } 0.99 \text { to } 1.13), p=0.09 \\
\text { Nonteaching hospitals: } 0.97(0.95 \text { to } 1.01), p=0.11 \\
\text { Small hospitals ( }<100 \text { beds): } 0.97 \text { (0.92 to } 1.03), p=0.36 \\
\\
\text { Unadjusted mortality } \\
\text { Pre period vs. post period } \\
\text { A: } 23.5 \% \text { vs. } 23.07 \%, p<0.01 \\
\text { B: } 24.0 \% \text { vs. } 24.3 \%, p=0.07 \\
\text { Characteristics of before/after } 90-\text { day mortality in telemedicine } \\
\text { hospitals: Hospitals that significantly increased mortality ( } n=9) \text { vs. } \\
\text { Hospitals with no mortality differences ( } n=107) \text { vs. hospitals that } \\
\text { significantly reduced mortality ( } n=16) \\
\text { Mean \# hospital beds (SD): } 195 \text { (143) vs. } 183 \text { (142) vs. } 265 \text { (261), } \\
p=0.17 \\
\text { Academic status } \\
\text { Nonteaching: } 66.7 \% \text { vs. } 63.6 \% \text { vs. } 50.0 \% \\
\text { Small teaching: } 22.2 \% \text { vs. } 22.4 \% \text { vs. } 31.3 \% \\
\text { Large teaching: } 11.1 \% \text { vs. } 14.0 \% \text { vs. } 18.8 \% p=0.85 \\
\text { Mean number of ICU admissions (SD): } 1037 \text { (1027) vs. } 738 \text { (640) vs. } \\
1484 \text { (1598), } p<0.01\end{array}$ & $\begin{array}{l}\text { ICU mean LOS (SD), in days } \\
\text { A: } \\
\text { Pre-telehealth: } 4.8(6.6) \\
\text { Post-telehealth: } 4.8(6.4) \\
\text { B: } \\
\text { Pre-telehealth: } 4.7(6.6) \\
\text { Post-telehealth: } 5.0(6.6) \\
\text { Pre-period: TH vs. non TH p=0.04 } \\
\text { TH pre-period vs. TH post-period p<0.01 } \\
\text { Hospital mean LOS (SD), in days } \\
\text { A: } \\
\text { Pre-telehealth: } 8.2(9.1) \\
\text { Post-telehealth: } 7.8(8.7) \\
\text { B: } \\
\text { Pre-telehealth: } 8.6(9.3) \\
\text { Post-telehealth: } 8.3(8.9) \\
\text { Pre-period: TH vs. non-TH p<0.01 } \\
\text { TH pre-period vs. TH post-period } p<0.01\end{array}$ & NR \\
\hline Kalb, 2014 & $\begin{array}{l}\text { ICU mortality ratio (APACHE IV-adjusted) } \\
\text { A: } 0.34 \\
\text { B: ( } 3 \text { months post implementation): } 0.67, p<0.04 \text { vs. A } \\
\text { C: } 0.65, p<0.03 \text { vs. A }\end{array}$ & $\begin{array}{l}\text { Mean \% adherence to low tidal volume- } \\
\text { based lung protective ventilation (SD) } \\
\text { A: } 29.5(18.2) \\
\text { B: } 44.9(15.7), p<0.002 \text { vs. A } \\
\text { C: } 51.8(22.7), p<0.003 \text { vs. A } \\
\text { Mean ventilator duration ratio (SD), in } \\
\text { days (number of days of mechanical } \\
\text { ventilation/APACHE IV predicted days of } \\
\text { mechanical ventilation) } \\
\text { A: } 1.08(0.34) \\
\text { B: } 0.92(0.28), p=0.09 \text { vs. A } \\
\text { C: } 0.96(0.24), p=0.37 \text { vs. A }\end{array}$ & NR \\
\hline
\end{tabular}




\begin{tabular}{|c|c|c|c|}
\hline $\begin{array}{l}\text { Author, Year } \\
\text { (See Appendix C } \\
\text { for full citation) }\end{array}$ & $\begin{array}{l}\text { Results: } \\
\text { KQ1: Clinical and Economic Outcomes }\end{array}$ & $\begin{array}{l}\text { Results: } \\
\text { KQ2: Intermediate Outcomes }\end{array}$ & $\begin{array}{l}\text { Results: } \\
\text { KQ3: Adverse Effects } \\
\text { or Unintended } \\
\text { Consequences } \\
\end{array}$ \\
\hline Kim, 2013 & $\begin{array}{l}\text { Very low birthweight deliveries, } \mathrm{n}(\%) \\
\text { A: } 50 ; 13 \% \\
\text { B: } 27 ; 7 \% \\
\mathrm{p}=0.0099 \\
\text { There were no changes in other hospitals } \\
\\
\text { Mortality } \\
\text { A: } 13 \% \\
\text { B: } 6.7 \% \\
\text { Statewide infant mortality decreased during study period } \\
\text { No change in bronchopulmonary dysplasia or necrotizing enterocolitis }\end{array}$ & NR & $\begin{array}{l}\text { TH-hospitals non-NICU } \\
\text { had incidence of IVH } \\
\text { slightly increase }(p=0.03)\end{array}$ \\
\hline Klein, 2010 & $\begin{array}{l}\text { Mortality, } \mathrm{n}(\%) \\
\text { A1: } 0(0) \\
\text { A2: } 1(1.4) \\
\text { B: } 1(1.0) \\
p=0.391 \\
\text { Need for neurosurgery, } \mathrm{n}(\%) \\
\text { A1: } 17(11.2) \\
\text { A2: } 9(12.3) \\
\text { B: } 9(9.2) \\
p=0.793\end{array}$ & \begin{tabular}{|l} 
Transferred, $\mathrm{n}(\%)$ \\
A1: $152(100)$ \\
A2: $54(74)$ \\
B: $40(40.9)$ \\
Delayed transfer, $\mathrm{n}(\%)$ \\
A1: NA \\
A2: $1(1.3)$ \\
B: $2(2.04)$ \\
$p=0.234$ \\
Length of stay, $\mathrm{n}(\mathrm{SD})$ \\
A1: $4.19(6.0)$ \\
A2: $3.92(3.5)$ \\
B: $4.48(5.1)$ \\
$\mathrm{p}=0.787$ \\
Need for neurological rehabilitation, $\mathrm{n}$ \\
$(\%)$ \\
A: $4(2.6)$ \\
$\mathrm{C}: 15(20.8)$ \\
B: $8(8.2)$ \\
$\mathrm{p}<0.001$
\end{tabular} & NR \\
\hline
\end{tabular}




\begin{tabular}{|c|c|c|c|}
\hline $\begin{array}{l}\text { Author, Year } \\
\text { (See Appendix C } \\
\text { for full citation) }\end{array}$ & $\begin{array}{l}\text { Results: } \\
\text { KQ1: Clinical and Economic Outcomes }\end{array}$ & $\begin{array}{l}\text { Results: } \\
\text { KQ2: Intermediate Outcomes }\end{array}$ & $\begin{array}{l}\text { Results: } \\
\text { KQ3: Adverse Effects } \\
\text { or Unintended } \\
\text { Consequences } \\
\end{array}$ \\
\hline Kohl, 2012 & $\begin{array}{l}\text { Mean severity adjusted ICU mortality (SEM) } \\
\text { A1: } 0.54(0.06) \\
\text { A2: } 0.42(0.04) \\
\text { B1: } 0.09(0.02) \\
\text { B2: } 0.01(0.003) \\
p=0.003 \\
\text { Mean severity adjusted hospital mortality (SEM) } \\
\text { A1: } 0.74(0.05) \\
\text { A2: } 0.56(0.04) \\
\text { B1: } 0.13(0.03) \\
\text { B2: } 0.04(0.01) \\
p=0.023\end{array}$ & $\begin{array}{l}\text { Mean severity adjusted ICU LOS (SEM), } \\
\text { in days } \\
\text { A1: } 5.27(0.52) \\
\text { A2: } 6.09(0.43) \\
\text { B1: } 6.25(0.50) \\
\text { B2: } 3.86(0.17) \\
\text { p<0.001 } \\
\text { Mean severity adjusted hospital LOS } \\
\text { (SEM), in days } \\
\text { A1: } 19.0(1.0) \\
\text { A2: } 12.5(1.1) \\
\text { B1: } 10.9(0.8) \\
\text { B2: } 16.7(0.8) \\
\text { NS }\end{array}$ & NR \\
\hline Labarbera, 2013 & \begin{tabular}{|l} 
Mortality \\
A: $3 \%$ \\
B: $1.8 \%$ \\
C: $3.6 \%, p=0.38$ \\
Mean LOS (SD), in days \\
A: $9.8(11.9)$ \\
B: $7.6(5.8)$ \\
C: $8.5(7.8), p=0.47$
\end{tabular} & $\begin{array}{l}\text { Transport rate: } \\
\text { A: } 100 \% \\
\text { B: } 85.7 \% \\
\text { C: } 87.5 \% p=0.04 \\
\text { Lower for both telemedicine cohorts } \\
\text { Transfers to tertiary care } \\
\text { A: } 19.5 \% \\
\text { B1: } 14.5 \% \\
\text { B2: } 6.1 \% p=0.0003 \\
\\
\text { Cohorts } 2 \text { and } 3 \text { re-analyzed based on if } \\
\text { they received telephone or telemedicine } \\
\text { consult - of those with TH consult } 72.7 \% \\
\text { were transferred with } 7.5 \% \text { diverting to } \\
\text { tertiary ward and of those with telephone } \\
\text { consult } 100 \% \text { were transferred and } \\
12.3 \% \text { diverting to tertiary ward } \\
\text { (p<0.001) }\end{array}$ & NR \\
\hline
\end{tabular}




\begin{tabular}{|c|c|c|c|}
\hline $\begin{array}{l}\text { Author, Year } \\
\text { (See Appendix C } \\
\text { for full citation) }\end{array}$ & $\begin{array}{l}\text { Results: } \\
\text { KQ1: Clinical and Economic Outcomes }\end{array}$ & $\begin{array}{l}\text { Results: } \\
\text { KQ2: Intermediate Outcomes }\end{array}$ & $\begin{array}{l}\text { Results: } \\
\text { KQ3: Adverse Effects } \\
\text { or Unintended } \\
\text { Consequences }\end{array}$ \\
\hline Lilly, 2011 & $\begin{array}{l}\text { ICU mortality, } n(\%) \\
\text { A: } 164(10.7 \%) \\
\text { B: } 410(8.6 \%) \\
p=0.003 \\
\text { Hospital mortality, n (\%) } \\
\text { A: } 208(13.6) \\
\text { B: } 562(11.8) \\
p=0.005\end{array}$ & $\begin{array}{l}\text { Mean ICU LOS (SD), and median (IQR), } \\
\text { in days } \\
\text { A: } 6.4(11), 2.5(0.2 \text { to } 6.5) \\
B: 4.5(6.7), 2.4(0.1 \text { to } 4.6) \\
p<0.001 \\
\text { Mean hospital LOS, and median (IQR), } \\
\text { in days } \\
\text { A: } 13.3(17.1) 7.9(0.2 \text { to } 15.0) \\
B: 9.8(10) 6.8(0.2 \text { to } 12.0) \\
p<0.001\end{array}$ & $\begin{array}{l}\text { Complications n/total } \\
\text { patients eligible (\%) } \\
\text { Ventilator associated } \\
\text { pneumonia } \\
\text { A: } 76 / 584(13 \%) \\
\text { B: } 32 / 1949(1.6 \%) \\
\text { OR }(95 \% \mathrm{Cl}): 0.15(0.09 \\
\text { to } 0.23), \mathrm{p}<0.001 \\
\text { Catheter-related } \\
\text { bloodstream infection } \\
\text { A: } 19 / 1529(1) \\
\text { B: } 29 / 4761(0.6) \\
\text { OR }(95 \% \mathrm{Cl}): 0.50(0.27 \\
\text { to } 0.93), \mathrm{p}=0.005 \\
\text { Acute kidney injury } \\
\text { A: } 174 / 1452(12) \\
\text { B: } 540 / 4565(12) \\
\text { OR }(95 \% \mathrm{Cl}): 1.00(0.71 \\
\text { to } 1.69), \mathrm{p}=0.38\end{array}$ \\
\hline Lilly, 2017 & $\begin{array}{l}\text { Total annual costs, in USD } \\
\text { A: } \$ 142,766,712 \\
\text { B: } \$ 182,719,738 \\
\text { C: } \$ 200,934,975 \\
\text { Total annual direct contribution margin } \\
\text { A: } \$ 7,921,584 \\
\text { B: } \$ 37,668,512 \\
\text { C: } \$ 60,586,397\end{array}$ & $\begin{array}{l}\text { Mean hospital LOS (SD), in days } \\
\text { A: } 10.4(13.4) \\
\text { B: } 9.7(9.3) \\
\text { C: } 8.8(8.3) \\
\text { A and C } p<0.0001 \\
\text { B and C } p<0.001\end{array}$ & $\begin{array}{l}\text { Complications (specifics } \\
\text { NR) } \\
\text { A: } 30.6 \% \\
\text { B: } 55.5 \% \\
\text { C: } 62.8 \% \\
\text { B vs. A: } p<0.0001 \\
\text { C vs. A: } p<0.0001 \\
\text { C vs. B: } p<0.0001\end{array}$ \\
\hline Marcin, 2004a & $\begin{array}{l}\text { Patients receiving telemedicine consult were sicker (higher PRISM } \\
\text { score) than those not receiving consult or historic controls } \\
\text { Mortality observed/predicted } \\
\text { A1: } 2.6 \% / 7.1 \% \\
\text { A2: } 3.5 \% / 5.1 \% \\
\text { B1: } 2.1 \% / 8.9 \% \\
\text { B2: } 1.6 \% / 4.6 \%\end{array}$ & $\begin{array}{l}\text { Overall satisfaction with telehealth, mean } \\
\text { (SD) } \\
\text { 5-point scale, 5=extremely satisfied } \\
\text { Referring nurse respiratory therapist: } \\
4.53(0.15) \\
\text { Referring MD: } 4.56(0.09) \\
\text { Parent or guardian: } 4.05(0.19)\end{array}$ & NA \\
\hline
\end{tabular}




\begin{tabular}{|c|c|c|c|}
\hline $\begin{array}{l}\text { Author, Year } \\
\text { (See Appendix C } \\
\text { for full citation) }\end{array}$ & $\begin{array}{l}\text { Results: } \\
\text { KO1: Clinical and Economic Outcomes }\end{array}$ & $\begin{array}{l}\text { Results: } \\
\text { KO2: Intermediate Outcomes }\end{array}$ & $\begin{array}{l}\text { Results: } \\
\text { KQ3: Adverse Effects } \\
\text { or Unintended } \\
\text { Consequences }\end{array}$ \\
\hline Marcin, 2004b & $\begin{array}{l}\text { Annual cost savings, in USD } \\
\text { To patients receiving TH consult: } \$ 172,000 \\
\text { To patients not transferred due to TH consults: } \$ 300,000 \\
\\
\text { Revenue generated, in USD } \\
\text { From patients receiving TH consult: } \$ 186,000 \\
\text { From patients not transported after TH consult: } \$ 279,000\end{array}$ & NA & NA \\
\hline Marcin, 2004c & $\begin{array}{l}\text { Feasibility: } \\
\text { Telemedicine is feasible in this setting. Patients who received } \\
\text { consults were younger and more severely injured (severity =18.3 vs. } \\
14.7, p=0.07) \\
\text { Mean ICU LOS (SD), p-value } \\
\text { A1: } 3.5(6.2) \\
\text { A2: } 3.4(5.8) \\
\text { B: } 5.9(8.1) \\
\text { C: } 3.8(6.3), p=0.31 \\
\text { Observed/expected mortality odds ratio }(95 \% \mathrm{Cl}) \\
\text { A1: } 0.95(0.26 \text { to } 3.48) \\
\text { A2: } 0.44(0.07 \text { to } 1.96) \\
\text { B: } \text { Reference } \\
\text { C: } 0.73(0.06 \text { to } 1.44) \text { NS }\end{array}$ & $\begin{array}{l}\text { Parents were satisfied with } \\
\text { communication, quality of medical care } \\
\text { and overall telemedicine. Providers were } \\
\text { satisfied with quality and ease of use of } \\
\text { equipment and overall telemedicine. } \\
\text { Mean parent satisfaction: } \\
3.8 \text { on a 5-point scale }\end{array}$ & NA \\
\hline Martin-Khan, 2016 & NR & $\begin{array}{l}\text { Total average consultation time (range); } \\
\text { SD, in minutes } \\
\text { A: } 25.91 \text { (4 to } 77) ; 9.38 \\
\text { B: } 9.89 \text { (4 to } 35) ; 5.83 \\
\text { p<0.005 } \\
\text { Triage decisions not significantly } \\
\text { different }\end{array}$ & NR \\
\hline
\end{tabular}




\begin{tabular}{|c|c|c|c|}
\hline $\begin{array}{l}\text { Author, Year } \\
\text { (See Appendix C } \\
\text { for full citation) }\end{array}$ & $\begin{array}{l}\text { Results: } \\
\text { KQ1: Clinical and Economic Outcomes } \\
\end{array}$ & $\begin{array}{l}\text { Results: } \\
\text { KQ2: Intermediate Outcomes }\end{array}$ & $\begin{array}{l}\text { Results: } \\
\text { KQ3: Adverse Effects } \\
\text { or Unintended } \\
\text { Consequences } \\
\end{array}$ \\
\hline $\begin{array}{l}\text { McCambridge, } \\
2010\end{array}$ & $\begin{array}{l}\text { Mortality } \\
\text { Hospital mortality, } \mathrm{n}(\%) \\
\text { A: } 204(21.4 \%) \\
\text { B: } 141(14.7 \%), p<0.001 \\
\text { ICU mortality } \\
\text { A: } 15.8 \% \\
\text { B: } 11.5 \%, p=0.006 \\
\text { Standardized mortality ratio } \\
\text { A: } 1.075 \\
\text { B: } 0.758 \\
\text { Mortality logistical regression (controlling for APACHE IV and DNR } \\
\text { status): } \\
\text { OR } 0.605 \text { (SE } 0.159, p=0.002)\end{array}$ & $\begin{array}{l}\text { Ventilator use } \\
\text { A: } 36.1 \% \\
\text { B: } 31.5 \%, p=0.04 \\
\text { Hospital LOS, in days } \\
\text { A: } 9.2 \\
\text { B: } 9.2, p=0.83 \\
\text { ICU LOS, in days } \\
\text { A: } 4.1 \\
\text { B: } 3.8, p=0.88\end{array}$ & NR \\
\hline Mielonen, 2000 & $\begin{array}{l}\text { Significant cost reduction associated with care planning conferences } \\
\text { via Videoconference vs. conventional in person consults. } \\
\text { Cost per patient were } \\
\text { FIM 2,510 videoconferences } \\
\text { FIM 4,750 conventional } \\
\text { Video is cheaper if there are } 30 \text { cases per year } \\
\text { With } 50 \text { cases the savings would be FIM } 117,000\end{array}$ & $\begin{array}{l}\text { Satisfaction with quality of } \\
\text { communication } 90 \% \text { of respondents } \\
\text { were satisfied with quality of } \\
\text { communication of videoconferencing } \\
\text { Staff satisfaction: } \\
47 \% \text { video as good as conventional } \\
\text { meeting } \\
48 \% \text { video almost as good as } \\
\text { conventional } \\
\text { Preference for next meeting to be video: } \\
86 \% \text { of health care staff } \\
84 \% \text { patients } \\
92 \% \text { relatives of patients }\end{array}$ & NR \\
\hline Migliaretti, 2013 & $\begin{array}{l}\text { Stratified analysis possible showing modest benefit in mortality risk } \\
\text { on those over } 70 \text { years old with consultation with a neurosurgeon. } \\
\text { Mortality }(95 \% \mathrm{Cl}) \\
\text { OR } 1.32(1.08 \text { to } 1.74) \\
\text { AOR } 1.25 \text { (0.83 to } 1.91) \text { NS } \\
\text { People over } 70: \\
\text { AOR } 1.14 \text { ( } 1.04 \text { to } 1.82) \\
\end{array}$ & $\begin{array}{l}\text { Duration of consultations: } \\
\text { Within } 22 \text { mins: } 50 \% \\
\text { More than } 60 \text { mins: } 10 \%\end{array}$ & NR \\
\hline
\end{tabular}




\begin{tabular}{|c|c|c|c|}
\hline $\begin{array}{l}\text { Author, Year } \\
\text { (See Appendix C } \\
\text { for full citation) }\end{array}$ & $\begin{array}{l}\text { Results: } \\
\text { KQ1: Clinical and Economic Outcomes }\end{array}$ & $\begin{array}{l}\text { Results: } \\
\text { KQ2: Intermediate Outcomes }\end{array}$ & $\begin{array}{l}\text { Results: } \\
\text { KQ3: Adverse Effects } \\
\text { or Unintended } \\
\text { Consequences } \\
\end{array}$ \\
\hline Miyamoto, 2014 & NR & $\begin{array}{l}\text { Mean quality scores out of } 5 \text { with } 5 \text { being } \\
\text { "excellent," for OES child abuse } \\
\text { examination forms ( } 95 \% \text { Cl): } \\
\text { General information and authorization: } \\
\text { A: } 4.98 \text { ( }(4.93 \text { to } 5.02) \\
\text { B: } 4.96 \text { ( } 4.90 \text { to } 5.02) \\
\text { Consent: } \\
\text { A: } 4.46 \text { ( } 4.16 \text { to } 4.76) \\
\text { B: } 4.94 \text { ( } 4.85 \text { to } 5.03), p<0.05 \\
\text { Patient history: } \\
\text { A: } 4.72 \text { ( } 4.51 \text { to } 4.92) \\
\text { B: } 4.43 \text { (4.17 to } 4.70) \\
\text { General examination: } \\
\text { A: } 4.38 \text { (4.10 to } 4.66) \\
\text { B: } 4.76 \text { ( } 4.62 \text { to } 4.89), p<0.05 \\
\text { Genital/perianal examination: } \\
\text { A: } 3.28 \text { (2.95 to } 3.61) \\
\text { B: } 4.08 \text { (3.85 to } 4.32), p<0.05 \\
\text { Examination findings: } \\
\text { A: } 3.20 \text { (2.87 to } 3.52) \\
\text { B: } 3.77 \text { ( }(3.50 \text { to } 4.04), p<0.05 \\
\text { Overall assessment: } \\
\text { A: } 3.24 \text { ( } 2.96 \text { to } 3.53) \\
\text { B: } 3.88 \text { (3.63 to } 4.13), p<0.05 \\
\text { Total quality score: } \\
\text { A: } 29.21 \text { (28.22 to } 30.20) \\
\text { B: } 31.20 \text { (30.39 to } 32.02), p<0.05\end{array}$ & NR \\
\hline
\end{tabular}




\begin{tabular}{|c|c|c|c|}
\hline $\begin{array}{l}\text { Author, Year } \\
\text { (See Appendix C } \\
\text { for full citation) }\end{array}$ & $\begin{array}{l}\text { Results: } \\
\text { KQ1: Clinical and Economic Outcomes }\end{array}$ & $\begin{array}{l}\text { Results: } \\
\text { KQ2: Intermediate Outcomes }\end{array}$ & $\begin{array}{l}\text { Results: } \\
\text { KQ3: Adverse Effects } \\
\text { or Unintended } \\
\text { Consequences } \\
\end{array}$ \\
\hline $\begin{array}{l}\text { Miyamoto, } 2014 \\
\text { (continued) }\end{array}$ & (continued) & $\begin{array}{l}\text { Mean completeness and accuracy } \\
\text { scores for the forensic examination, out } \\
\text { of } 5 \text { with } 5 \text { being "excellent" }(95 \% \mathrm{Cl}) \\
\text { Photo/video quality: } \\
\text { A: } 2.57 \text { ( } 2.35 \text { to } 3.80) \\
\text { B: } 3.86 \text { (3.66 to } 4.06), p<0.05 \\
\text { Complete/thorough exam: } \\
\text { A: } 3.83 \text { (3.64 to } 4.02) \\
\text { B: } 4.49(4.35 \text { to } 4.63), p<0.05 \\
\text { Diagnostic accuracy: } \\
\text { A: } 3.68 \text { (3.46 to } 3.91) \\
\text { B: } 4.14 \text { (3.94 to } 4.34), p<0.05 \\
\text { Total completeness and accuracy score: } \\
\text { A: } 11.88 \text { (11.13 to } 12.63) \\
\text { B: } 14.52(13.84 \text { to } 15.20), p<0.05\end{array}$ & (continued) \\
\hline
\end{tabular}




\begin{tabular}{|c|c|c|c|}
\hline $\begin{array}{l}\text { Author, Year } \\
\text { (See Appendix C } \\
\text { for full citation) }\end{array}$ & $\begin{array}{l}\text { Results: } \\
\text { KQ1: Clinical and Economic Outcomes }\end{array}$ & $\begin{array}{l}\text { Results: } \\
\text { KQ2: Intermediate Outcomes }\end{array}$ & $\begin{array}{l}\text { Results: } \\
\text { KQ3: Adverse Effects } \\
\text { or Unintended } \\
\text { Consequences } \\
\end{array}$ \\
\hline $\begin{array}{l}\text { Morrison, et al., } \\
2010\end{array}$ & 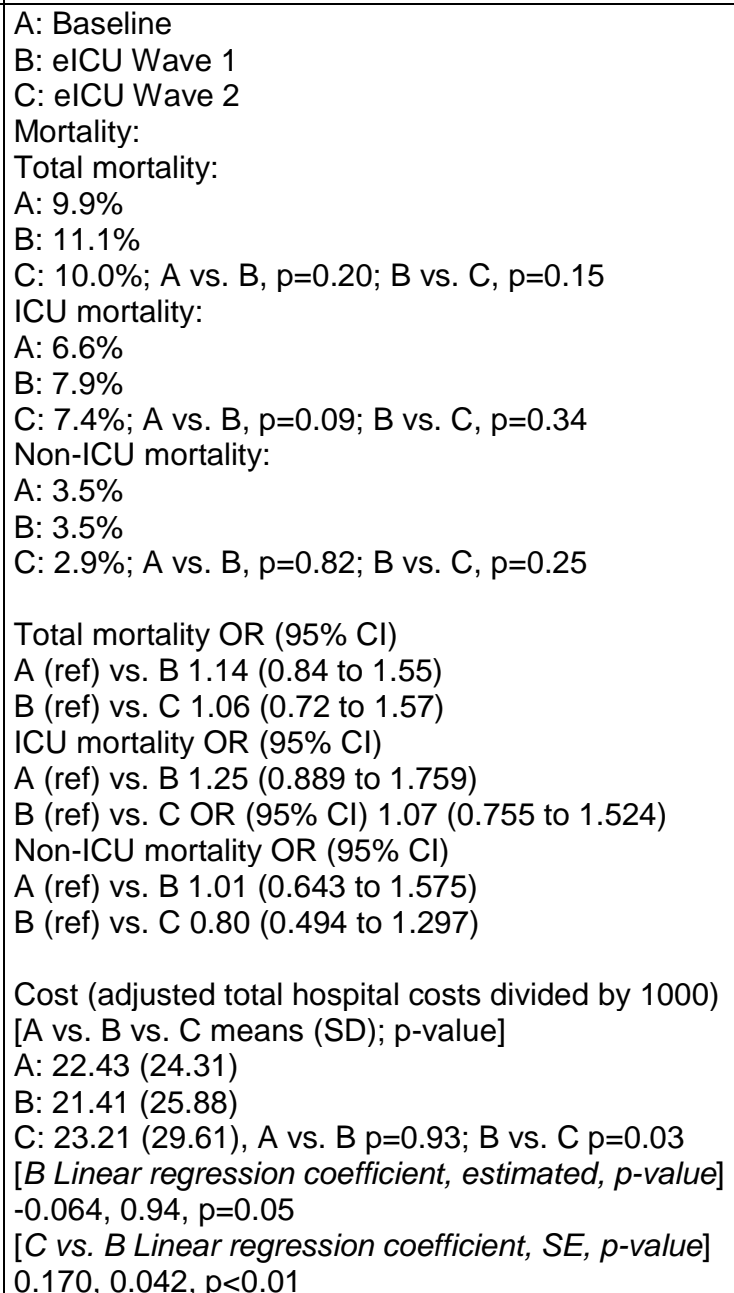 & $\begin{array}{l}\text { Mean hospital LOS (SD) } \\
\text { A: } 7.72(7.98) \\
\text { B: } 7.98(7.94) \\
\text { C: } 7.89 \text { (8.60) } \\
\text { A vs. B p=0.48; B vs. C } p=0.56 \\
\text { Mean ICU LOS (SD) } \\
\text { A: } 2.60 \text { (3.16) } \\
\text { B: } 2.92(3.94) \\
\text { C: } 3.18(4.49) \\
\text { A vs. B p=0.15; B vs. C } p=0.09 \\
\text { [B Linear regression coefficient, } \\
\text { estimated, p-value] } \\
\text { Hospital LOS: } 0.004,1.00, p=0.91 \\
\text { ICU LOS: O.051, } 1.05, p=0.11 \\
\text { [C vs. B Linear regression coefficient, } \\
\text { SE, p-value] } \\
\text { Hospital LOS: } 0.115,0.046, p=0.01 \\
\text { ICU LOS: } 0.078,0.035, p=0.03\end{array}$ & NA \\
\hline
\end{tabular}




\begin{tabular}{|c|c|c|c|}
\hline $\begin{array}{l}\text { Author, Year } \\
\text { (See Appendix C } \\
\text { for full citation) }\end{array}$ & $\begin{array}{l}\text { Results: } \\
\text { KQ1: Clinical and Economic Outcomes }\end{array}$ & $\begin{array}{l}\text { Results: } \\
\text { KQ2: Intermediate Outcomes }\end{array}$ & $\begin{array}{l}\text { Results: } \\
\text { KQ3: Adverse Effects } \\
\text { or Unintended } \\
\text { Consequences } \\
\end{array}$ \\
\hline Nagayoshi, 2016 & NR & $\begin{array}{l}\text { Patient transfer } \\
\text { A: } 10(55 \%) \\
\text { B: } 10(21 \%) \\
p<0.05 \\
\text { Waiting period, in days } \\
\text { A: } 17.2 \\
\text { B: } 9.2 \\
p=0.23\end{array}$ & NR \\
\hline Nassar, 2014 & $\begin{array}{l}\text { Mortality risk adjusted odds ratio }(95 \% \mathrm{Cl}) \text {, after telehealth vs. before } \\
\text { telehealth } \\
\text { ICU } \\
\text { A: } 0.88 \text { (0.52 to } 1.49), p=0.65 \\
\text { B: } 1.07(0.60 \text { to } 1.90), p=0.82 \\
\text { Hospital } \\
\text { A: } 0.82(0.57 \text { to } 1.19), p=0.30 \\
\text { B: } 1.33(0.86 \text { to } 2.07), p=0.20 \\
30 \text {-day } \\
\text { A: } 0.79(0.62 \text { to } 1.01), p=0.06 \\
\text { B: } 1.10 \text { (0.82 to } 1.47), p=0.52 \\
\text { Unadjusted mortality, } n \\
\text { ICU } \\
\text { A: } B \\
\text { Before telehealth }=67 \\
\text { After telehealth }=65 \\
\text { B: } \\
\text { Before telehealth }=49 \\
\text { After telehealth }=46 \\
\text { Hospital } \\
\text { A: } \\
\text { Before telehealth }=115 \\
\text { After telehealth }=111 \\
\text { B: } \\
\text { Before telehealth }=62 \\
\text { After telehealth }=70\end{array}$ & $\begin{array}{l}\text { Risk adjusted odds ratio relative LOS } \\
(95 \% \text { Cl), after telehealth vs. before } \\
\text { telehealth } \\
\text { ICU } \\
\text { A: } 1.00(0.93 \text { to } 1.08) p=0.99 \\
\text { B: } 1.02(0.92 \text { to } 1.14) p=0.68 \\
\text { Hospital } \\
\text { A: } 0.93(0.86 \text { to } 1) p=0.05 \\
\text { B: } 1.03(0.96 \text { to } 1.11) p=0.43 \\
\text { Mean LOS (SD), in days } \\
\text { ICU } \\
\text { A: } \\
\text { Before telehealth=2.9 }(3.7) \\
\text { After telehealth }=2.9(3.4) \\
\text { B: } \\
\text { Before telehealth=2.6 }(3.6) \\
\text { After telehealth=2.8 }(4.7) \\
\text { p-value: } \\
\text { Telehealth before vs. after }=0.15 \\
\text { Control before vs. after }=0.72 \\
\text { After period, telehealth vs. control }=0.18 \\
\text { Hospital } \\
\text { A: } \\
\text { Before telehealth=6.8 }(7.9) \\
\text { After telehealth=6.5 }(8.2) \\
\text { B: } \\
\text { Before telehealth=6.9 }(8.5) \\
\text { After telehealth=7.3 }(6.9) \\
\text { p-value: } \\
\text { Telehealth before vs. after }=0.18 \\
\text { Control before vs. after }=0.35 \\
\text { After period, telehealth vs. control }=0.11\end{array}$ & NR \\
\hline
\end{tabular}




\begin{tabular}{|c|c|c|c|}
\hline $\begin{array}{l}\text { Author, Year } \\
\text { (See Appendix C } \\
\text { for full citation) }\end{array}$ & $\begin{array}{l}\text { Results: } \\
\text { KQ1: Clinical and Economic Outcomes }\end{array}$ & $\begin{array}{l}\text { Results: } \\
\text { KQ2: Intermediate Outcomes }\end{array}$ & $\begin{array}{l}\text { Results: } \\
\text { KQ3: Adverse Effects } \\
\text { or Unintended } \\
\text { Consequences } \\
\end{array}$ \\
\hline Panlaqui, 2017 & $\begin{array}{l}\text { Total mortality } \\
\text { A: } 6.5 \% \\
\text { B: } 4.3 \% \\
\text { RR } 0.98 \text { (0.94 to } 1.02), p=0.28 \\
\text { ICU } \\
\text { A: } 1.6 \% \\
\text { B: } 1.1 \% \\
\text { RR } 1 \text { (0.98 to } 1.03), p=0.67 \\
\text { Hospital } \\
\text { A: } 5.4 \% \\
\text { B: } 3.2 \% \\
\text { RR } 1.02(0.99 \text { to } 1.06), p=0.25\end{array}$ & $\begin{array}{l}\text { Hospital transfer, relative risk }(95 \% \mathrm{Cl}) \\
\text { A: } 31.8 \% \\
\text { B: } 22.9 \% \\
0.88(0.80 \text { to } 0.98) \\
p=0.03 \\
\text { Mean hospital LOS in days (SD) } \\
\text { A: } 4.0(3.9) \\
\text { B: } 5.5(5.2), p<0.0002 \\
\text { Mean ICU LOS in days (SD) } \\
\text { A: } 2.2(1.9) \\
\text { B: } 2.1(1.6), p=0.6\end{array}$ & NR \\
\hline Rendina, 1997 & $\begin{array}{l}\text { Mortality } \\
\text { A: } 1 \\
\text { B: } 1 \\
\text { Net savings in USD: } \\
\$ 13,900 \text { per infant }\end{array}$ & $\begin{array}{l}\text { Hospital LOS, in days } \\
\text { A: } 41.2 \\
\text { B: } 35.2 \\
\text { Pooled variance t-test } p=0.23 \\
\\
\text { Reporting time } \\
\text { A: } 24 \text { hours } \\
\text { B: } 20 \text { minutes }\end{array}$ & NR \\
\hline Rendina, 1998 & $\begin{array}{l}\text { LOS in NICU } \\
\text { A vs. B; regression sign, } p \text {-value } \\
\text { Telemedicine: }-, p=0.001 \\
\text { Birth weight } x \text { telemedicine: }+, p=0.0009 \\
\text { Low birthweight neonates }(<960 \text { grams) -, } p=0.05 \\
\text { Higher birthweight neonates }(960 \text { grams): NS } \\
\text { LOS reduction with telemedicine: } 12.5 \text { days }(17 \%), p<0.05\end{array}$ & NR & NR \\
\hline
\end{tabular}




\begin{tabular}{|c|c|c|c|}
\hline $\begin{array}{l}\text { Author, Year } \\
\text { (See Appendix C } \\
\text { for full citation) }\end{array}$ & $\begin{array}{l}\text { Results: } \\
\text { KQ1: Clinical and Economic Outcomes }\end{array}$ & $\begin{array}{l}\text { Results: } \\
\text { KQ2: Intermediate Outcomes }\end{array}$ & $\begin{array}{l}\text { Results: } \\
\text { KQ3: Adverse Effects } \\
\text { or Unintended } \\
\text { Consequences } \\
\end{array}$ \\
\hline Rincon, 2012 & NR & $\begin{array}{l}\text { Nurse satisfaction } \\
\text { ICU physicians available } \\
\text { A: } 38 \% \\
\text { B: } 55 \% \\
\text { p=NS } \\
\text { Adequate physician involvement } \\
\text { A: } 44 \% \\
\text { B: } 65 \% \\
p=0.007 \\
\text { Opportunity to ask questions } \\
\text { A: } 41 \% \\
\text { B: } 53 \% \\
\text { p=NS } \\
\end{array}$ & NR \\
\hline Robison, 2016 & NR & $\begin{array}{l}\text { Face time interface versus arrival at the } \\
\text { bedside, in minutes } \\
\text { A: } 3.7 \\
\text { B: } 2.6 \\
p=0.012 \\
\text { Admitted to PICU } \\
\text { A: } 73 \% \\
\text { B: } 58 \%, p=0.13 \\
\text { Interventions } \\
\text { A: } 1.9 \\
\text { B: } 1.4, p=\text { not significant }\end{array}$ & NR \\
\hline
\end{tabular}




\begin{tabular}{|c|c|c|c|}
\hline $\begin{array}{l}\text { Author, Year } \\
\text { (See Appendix C } \\
\text { for full citation) }\end{array}$ & $\begin{array}{l}\text { Results: } \\
\text { KQ1: Clinical and Economic Outcomes }\end{array}$ & $\begin{array}{l}\text { Results: } \\
\text { KQ2: Intermediate Outcomes }\end{array}$ & $\begin{array}{l}\text { Results: } \\
\text { KQ3: Adverse Effects } \\
\text { or Unintended } \\
\text { Consequences } \\
\end{array}$ \\
\hline Romig, 2012 & $\begin{array}{l}\text { ICU Mortality rate before vs. after telehealth, \% (n) } \\
\text { A: } 4.9 \%(15 / 305) \text { vs. } 4.6 \%(14 / 307) \\
\text { B: } 1.5 \%(6 / 30) \text { vs. } 3.5 \%(14 / 403) \\
\text { ICU LOS before vs. after telehealth, } \%(n) \text {, in days } \\
\text { A: } 3.9 \text { vs. } 3.8 \\
\text { B: } 5.1 \text { vs. } 4.5\end{array}$ & $\begin{array}{l}\text { Nurse satisfaction and perceptions of } \\
\text { quality after telehealth, mean survey } \\
\text { score out of } 5 \text { (SD) } \\
\\
\text { Remote ICU unit } \\
\text { Communications } \\
\text { Pre-telehealth: } 2.99(1.13) \\
\text { Post-telehealth: } 3.27(1.27), p<0.01 \\
\text { Psychological working conditions and } \\
\text { burnout } \\
\text { Pre-telehealth: } 3.10(1.10) \\
\text { Post-telehealth: } 3.23(1.11), p=0.02 \\
\text { Education } \\
\text { Pre-telehealth: } 3.52(0.84) \\
\text { Post-telehealth: } 3.76(0.78), p<0.03 \\
\text { Control ICU } \\
\text { Significant decline in } 2 \text { scales } \\
\text { Patient care and perceived effectiveness } \\
\text { and education }\end{array}$ & NA \\
\hline
\end{tabular}




\begin{tabular}{|c|c|c|c|}
\hline $\begin{array}{l}\text { Author, Year } \\
\text { (See Appendix C } \\
\text { for full citation) }\end{array}$ & $\begin{array}{l}\text { Results: } \\
\text { KQ1: Clinical and Economic Outcomes }\end{array}$ & $\begin{array}{l}\text { Results: } \\
\text { KQ2: Intermediate Outcomes }\end{array}$ & $\begin{array}{l}\text { Results: } \\
\text { KQ3: Adverse Effects } \\
\text { or Unintended } \\
\text { Consequences }\end{array}$ \\
\hline Rosenfeld, 2000 & $\begin{array}{l}\text { Mortality } \\
\text { ICU: } \\
\text { A1: } 9.8 \% \\
\text { A2: } 3.5 \% \\
\text { B: } 1.5 \%, p<0.05 \\
\text { Hospital: } \\
\text { A1: } 11.6 \% \\
\text { A2: } 6.93 \% \\
\text { B: } 4.48 \%, p<0.05 \\
\text { Cost reduction, in USD } \\
\text { ICU: costs reduced by 25\% (from A1) and 31\% (from A2), including } \\
\text { lower routine, radiology and therapy costs. } \\
\text { ICU total cost change } \\
\text { B vs. A1: } 0.75 \text { ( } p=0.002) \\
\text { B vs. A2: } 0.69 \text { ( } p=0.031) \\
\text { Hospital total costs } \\
\text { B vs. A1: } 0.88 \text { ( } p=0.15) \\
\text { B vs. A2: } 0.81 \text { ( } p=0.12) \\
\text { Hospital: costs were } 12 \% \text { (than A1) and } 19 \% \text { (than A2) lower but not } \\
\text { stat significant. ICU-based costs, as percent of total hospital costs, } \\
\text { were lower during B, by } 62 \% \text { (A1) and } 53 \% \text { (A2). }\end{array}$ & $\begin{array}{l}\text { Mean LOS }(95 \% \mathrm{Cl}) \\
\text { ICU } \\
\text { A1: } 2.71(2.14 \text { to } 3.03) \\
\text { A2: } 3.06(1.95 \text { to } 3.89) \\
\text { B: } 2.0(1.66 \text { to } 2.3), p<0.01 \\
\text { Hospital } \\
\text { A1: } 9.18(8.04 \text { to } 10.44) \\
\text { A2 } 10.11(8.32 \text { to } 11.94) \\
\text { B: } 9.28(7.87 \text { to } 10.82) \\
\text { ICU: LOS shorter in B by } 26 \% \text { (A1) and } \\
35 \% \text { (A2); and } 26 \% \text { lower than predicted, } \\
\text { whereas A1 and A2 did not differ from } \\
\text { predicted. } \\
\text { Hospital: No differences in LOS. } \\
\end{array}$ & $\begin{array}{l}\text { Complications } \\
\text { A1: } 15.1 \% \\
\text { A2: } 18.8 \% \\
\text { B: } 9.5 \% \\
\text { p<0.05 } \\
\text { Costs related to } \\
\text { complications: } 64 \% \text { of } \\
\text { difference in cost } \\
\text { between baselines and } \\
\text { intervention were } \\
\text { associated with higher } \\
\text { incidence of } \\
\text { complications during } \\
\text { baseline periods; and } \\
\text { regardless of study } \\
\text { period, hospital costs of } \\
\text { patients with } \\
\text { complications was } 3 x \\
\text { costs of patients without } \\
\text { complications. }\end{array}$ \\
\hline
\end{tabular}




\begin{tabular}{|c|c|c|c|}
\hline $\begin{array}{l}\text { Author, Year } \\
\text { (See Appendix C } \\
\text { for full citation) }\end{array}$ & $\begin{array}{l}\text { Results: } \\
\text { KQ1: Clinical and Economic Outcomes }\end{array}$ & $\begin{array}{l}\text { Results: } \\
\text { KQ2: Intermediate Outcomes }\end{array}$ & $\begin{array}{l}\text { Results: } \\
\text { KQ3: Adverse Effects } \\
\text { or Unintended } \\
\text { Consequences }\end{array}$ \\
\hline Ruesch, 2012 & $\begin{array}{l}\text { Hospital LOS (actual; predicted), in days } \\
\text { A: } 11.25 ; 9.75 \\
\text { B: } 9.48 ; 10.9 \text { (statistical significance NR) } \\
\text { ICU LOS (actual; predicted), in days } \\
\text { A: } 4.1 \text { days actual; } 2.87 \text { predicted } \\
\text { B: } 3.66 \text { actual; } 3.89 \text { predicted days (statistical significance NR) } \\
\text { Severity-adjusted mortality (actual; predicted) } \\
\text { Hospital: } \\
\text { A: } 22 ; 25.5 \\
\text { B: } 36 ; 57.7 \text { predicted indicating the saving of } 22 \text { lives (statistical } \\
\text { significance NR) } \\
\text { ICU: } \\
\text { A: } 17 ; 17.6 \\
\text { B: } 24.0 ; 39.4 \\
\text { (statistical significance NR) } \\
\text { Actual costs not reported. Estimated cost saving based on changes } \\
\text { in LOS were over } \$ 2.5 \text { million USD, comparing a calendar quarter } \\
\text { preimplementation and the last quarter of the evaluation }\end{array}$ & $\begin{array}{l}\text { Ventilator-associated pneumonia } \\
\text { compliance: } 6 \% \text { increase from } 87.2 \% \text { vs. } \\
93.3 \%, p=0.02 \\
\text { Peptic ulcer disease bundle compliance: } \\
0.5 \%, \text { NS } \\
\text { Deep vein thrombosis compliance } \\
\text { bundle: } 1 \%, \text { NS } \\
\text { Frequency of hypoglycemia: } \\
\text { A: } 2.8 \% \\
\text { B: } 1.3 \% \\
\text { Missed SCIP opportunities on } \\
\text { postoperative day } 2 \text { for cardiac surgery: } \\
\text { A: } 69 \% \\
\text { B: } 43 \% \\
\text { Nurse documentation of patient restraint } \\
\text { compliance: } \\
\text { A: } 74 \% \\
\text { B: } 100 \%\end{array}$ & NR \\
\hline Sadaka, 2013 & $\begin{array}{l}\text { ICU mortality } \\
\text { A: } 7.9 \% \\
\text { B: } 3.8 \% \\
\text { OR } 0.46,95 \% \mathrm{Cl}(0.32 \text { to } 0.66) \\
p=0.0001 \\
\text { Hospital mortality } \\
\text { A: } 8.8 \% \\
\text { B: } 6.9 \% \\
\text { OR } 0.76,95 \% \mathrm{Cl}(0.55 \text { to } 1.0) \\
p=0.1\end{array}$ & $\begin{array}{l}\text { ICU LOS (SD), in days } \\
\text { A: } 2.7(4.1) \\
\text { B: } 2.2(3.4) \\
\text { HR } 1.16,95 \% \mathrm{Cl}(1.00 \text { to } 1.40) \\
\text { p=0.01 } \\
\text { Hospital LOS (SD), in days } \\
\text { A: } 5.2(6.1) \\
\text { B: } 6.2(7.4) \\
\text { HR } 1.30,95 \% \mathrm{Cl}(1.25 \text { to } 1.35) \\
\text { p=0.00 }\end{array}$ & NR \\
\hline
\end{tabular}




\begin{tabular}{|c|c|c|c|}
\hline $\begin{array}{l}\text { Author, Year } \\
\text { (See Appendix C } \\
\text { for full citation) }\end{array}$ & $\begin{array}{l}\text { Results: } \\
\text { KQ1: Clinical and Economic Outcomes }\end{array}$ & $\begin{array}{l}\text { Results: } \\
\text { KQ2: Intermediate Outcomes }\end{array}$ & $\begin{array}{l}\text { Results: } \\
\text { KQ3: Adverse Effects } \\
\text { or Unintended } \\
\text { Consequences } \\
\end{array}$ \\
\hline Sharma, 2016 & NR & $\begin{array}{l}\text { Mean handling time }(95 \% \mathrm{Cl}) \text {, in minutes } \\
\text { A: } 43.5(37.9 \text { to } 49.0) \\
\text { B: } 26.9(15.4 \text { to } 38.4) \\
\text { p=0.004 } \\
\text { Time to response } \\
\text { A: } 405.7(301.0 \text { to } 510.3) \\
\text { B: } 344.7 \text { (291.3 to } 398.0) \\
\text { p=0.602 } \\
\text { Teledermatology alone sufficiently } \\
\text { answered consultations in } 10 \text { of } 25 \text { study } \\
\text { consultations. }\end{array}$ & NR \\
\hline Shin, 2015 & $\begin{array}{l}\text { Estimated blood loss } \mathrm{m} / \mathrm{L} \text {, median (range) } \\
\text { A: } 2.5(0 \text { to } 7) \\
\text { B: } 2.5(0 \text { to } 7)\end{array}$ & $\begin{array}{l}\text { Median (range) } \\
\text { Estimated duration, in minutes } \\
\text { A: } 15.0 \text { (5 to } 25) \\
\text { B: } 15.0 \text { (5 to } 35) \\
\text { Robotic skills assessment } \\
p>0.05 \\
\text { Mentors preferred remote to in room } \\
p=0.05\end{array}$ & $\begin{array}{l}\text { There was one } \\
\text { intraoperative } \\
\text { complication reported. } \\
\text { During an in-room } \\
\text { mentored robotic partial } \\
\text { nephrectomy, a colon } \\
\text { serosal injury occurred } \\
\text { from bipolar energy of a } \\
\text { fenestrated bipolar } \\
\text { forceps. This was } \\
\text { immediately recognized } \\
\text { by the mentor and over- } \\
\text { sutured, resulting in no } \\
\text { postoperative sequelae. } \\
\text { No intraoperative } \\
\text { complications were noted } \\
\text { in remote mentored } \\
\text { cases. }\end{array}$ \\
\hline
\end{tabular}




\begin{tabular}{|c|c|c|c|}
\hline $\begin{array}{l}\text { Author, Year } \\
\text { (See Appendix C } \\
\text { for full citation) }\end{array}$ & $\begin{array}{l}\text { Results: } \\
\text { KQ1: Clinical and Economic Outcomes }\end{array}$ & $\begin{array}{l}\text { Results: } \\
\text { KQ2: Intermediate Outcomes }\end{array}$ & $\begin{array}{l}\text { Results: } \\
\text { KQ3: Adverse Effects } \\
\text { or Unintended } \\
\text { Consequences } \\
\end{array}$ \\
\hline Steinman, 2015 & $\begin{array}{l}\text { Mortality by condition } \\
\text { AMl } \\
\text { A vs. B: } 17 \% \text { vs. } 14 \% \\
\text { C vs. D: } 14.4 \% \text { vs. } 7.6 \% \\
\text { Septic shock } \\
\text { A vs. B: } 65.7 \% \text { vs. } 67.9 \% \\
\text { C vs. D: } 70.9 \% \text { vs. } 40.4 \% \\
\text { Ischemic stroke } \\
\text { A vs. B: } 50 \% \text { vs. } 43.8 \% \\
\text { C vs. D: } 75.6 \% \text { vs. } 32.1 \% \\
\text { Hemorrhagic stroke } \\
\text { A vs. B: } 23.4 \% \text { vs. } 27.8 \% \\
\text { C vs. D: } 36.9 \% \text { vs. } 15.6 \% \\
\text { A vs. B comparisons are all NS } \\
\text { C vs. D comparisons trend toward significant but p=NR }\end{array}$ & $\begin{array}{l}\text { Change in treatment plan for } 18(7.1 \%) \\
\text { patients the telehealth consult influenced } \\
\text { making a definite diagnosis, for } 239 \\
(92.9 \%) \text { the telehealth consult } \\
\text { contributed to clinical management. }\end{array}$ & NR \\
\hline $\begin{array}{l}\text { Thomas, } 2009 \\
\text { same patients as } \\
\text { Franzini, } 2011\end{array}$ & $\begin{array}{l}\text { Mortality } \\
\text { ICU mortality }(95 \% \mathrm{Cl}) \\
\text { A: } 9.2 \%(8.0 \text { to } 10.5) \\
\text { B: } 7.8 \%(6.7 \text { to } 9.0) \\
\text { A to B decrease: } 1.4 \% \\
(-0.3 \% \text { to } 3.2 \%) \mathrm{p}=0.12 \\
\text { RR } 0.88 ;(0.71 \text { to } 1.08) \\
\\
\text { Hospital mortality }(95 \% \mathrm{Cl}) \\
\text { A: } 12.0 \%(10.6 \% \text { to } 13.5 \%) \\
\text { B: } 9.9 \%(8.6 \% \text { to } 11.2 \%) \\
\text { A to B decrease, } 2.1 \% ;(0.2 \% \text { to } 4.1 \%) ; p=0.03 \\
\text { RR: } 0.85 ;(95 \% \mathrm{Cl} 0.71 \text { to } 1.03)\end{array}$ & $\begin{array}{l}\text { ICU LOS }(95 \% \mathrm{Cl}) \text {, in days } \\
\text { A: } 4.3(4.0 \text { to } 4.5) \\
\text { B: } 4.6(4.3 \text { to } 4.9) \\
\text { Hospital LOS } \\
\text { A: } 9.8(9.4 \text { to } 10.2) \\
\text { B: } 10.7(10.2 \text { to } 11.1)\end{array}$ & $\begin{array}{l}\text { ICU complication rates } \\
\text { ( } 95 \% \text { CI) } \\
\text { A: } 17.9 \%(16.3 \text { to } 19.6) \\
\text { B: } 19.2 \%(17.5 \text { to } 20.9) \\
\text { The CAIC-reduced model } \\
\text { revealed that the rate of } \\
\text { complications was } \\
\text { associated with SAPS II } \\
\text { score }(p=0.001) \text { but not } \\
\text { with tele-ICU } \\
\text { implementation ( } p=0.15) .\end{array}$ \\
\hline
\end{tabular}




\begin{tabular}{|c|c|c|c|}
\hline $\begin{array}{l}\text { Author, Year } \\
\text { (See Appendix C } \\
\text { for full citation) }\end{array}$ & $\begin{array}{l}\text { Results: } \\
\text { KQ1: Clinical and Economic Outcomes }\end{array}$ & $\begin{array}{l}\text { Results: } \\
\text { KQ2: Intermediate Outcomes }\end{array}$ & $\begin{array}{l}\text { Results: } \\
\text { KQ3: Adverse Effects } \\
\text { or Unintended } \\
\text { Consequences }\end{array}$ \\
\hline Wallace, 2008 & $\begin{array}{l}\text { No evidence of cost saving for hospital (details not reported) } \\
\text { Capital outlay was significant (£70K) }\end{array}$ & $\begin{array}{l}\text { Difference in overall management of } \\
\text { referrals } \\
p=0.004 \\
\text { Admission }(95 \% \mathrm{Cl}) \\
\text { A: } 28.3 \%(24.9 \text { to } 32) \\
\text { B: } 29.6 \%(25.2 \text { to } 34.3) \\
\\
\text { In person review }(95 \% \mathrm{Cl}) \\
\text { A: } 22.1 \%(19.0 \text { to } 25.5) \\
\text { B: } 15.4 \%(12.2 \text { to } 19.3) \\
\\
\text { Day surgery }(95 \% \mathrm{Cl}) \\
\text { A: } 17 \%(14.2 \text { to } 20.2) \\
\text { B: } 27.5 \%(23.3 \text { to } 32.1) \\
\\
\text { Inappropriate referral }(95 \% \mathrm{Cl}) \\
\text { A: } 0.5 \%(0.2 \text { to } 1.4) \\
0.3 \%(0.0 \text { to } 1.4)\end{array}$ & NR \\
\hline Webb, 2013 & $\begin{array}{l}\text { Mortality, morbidity: control patients significantly more likely to } \\
\text { receive inotropic support and indomethacin. } \\
\text { Adjusted OR }(95 \% \mathrm{Cl}) \\
\text { Death } \\
0.922(0.389 \text { to } 2.136) \\
\text { Cardiac arrest } \\
0.527(0.184 \text { to } 1.505) \\
\\
\text { Mean LOS, in days } \\
\text { Total } \\
\text { A: } 1.6(6.4) \\
\text { B: } 0.72(4.1) p=0.027 \\
\text { ICU } \\
\text { A: } 1.6(6.2) \\
\text { B: } 0.65(4.0) p=0.027 \\
\text { no differences in LOS in pediatric wards and well nursery. } \\
\text { Transport to tertiary care: } \\
\text { A: } 10 \% \\
\text { B: } 4 \% \\
\text { p }<0.01 \\
\text { AOR } 0.435 \text { ( } 95 \% \text { CI } 0.229 \text { to } 0.827)\end{array}$ & $\begin{array}{l}\text { Time to diagnosis: } \\
\text { Mean (SD), in minutes } \\
\text { A: } 147(60) \\
\text { B: } 100(67) p<0.001 \\
\text { *telemedicine patients were located } \\
\text { significantly farther from tertiary } \\
\text { hospitals. }\end{array}$ & $\begin{array}{l}\text { No significant differences } \\
\text { in death, cardiac arrest, } \\
\text { and intraventricular } \\
\text { hemorrhage or in use of } \\
\text { prostaglandin infusion, } \\
\text { mechanical ventilation, or } \\
\text { extracorporeal } \\
\text { membrane oxygenation. }\end{array}$ \\
\hline
\end{tabular}




\begin{tabular}{|c|c|c|c|}
\hline $\begin{array}{l}\text { Author, Year } \\
\text { (See Appendix C } \\
\text { for full citation) }\end{array}$ & $\begin{array}{l}\text { Results: } \\
\text { KQ1: Clinical and Economic Outcomes }{ }^{\mathrm{a}} \\
\end{array}$ & $\begin{array}{l}\text { Results: } \\
\text { KQ2: Intermediate Outcomes } \\
\end{array}$ & $\begin{array}{l}\text { Results: } \\
\text { KQ3: Adverse Effects } \\
\text { or Unintended } \\
\text { Consequences } \\
\end{array}$ \\
\hline Willmitch, 2012 & $\begin{array}{l}\text { Hospital mortality } \\
1 \text { year post } \\
\text { RR } 0.92(95 \% \mathrm{Cl} 0.82 \text { to } 1.03) \mathrm{p}=0.142 \\
2 \text { years post } \\
\text { RR } 0.88(95 \% \mathrm{Cl} 0.78 \text { to } 0.98) \mathrm{p}=0.025 \\
3 \text { years post: } \\
\text { RR } 0.77(0.69-0.87) \mathrm{p}<0.001 \\
\text { Severity-adjusted } \\
\text { Before/After } \\
\text { Mean LOS, in days }(95 \% \mathrm{Cl}) \\
\text { Hospital } \\
\text { A: } 11.86(11.55 \text { to } 12.21) \\
\text { D: } 10.16(9.80 \text { to } 10.53), p<0.001 \\
\text { ICU } \\
\text { A: } 4.35(4.22 \text { to } 4.49) \\
\text { D: } 3.80(3.65 \text { to } 3.94) p<0.001\end{array}$ & NR & NR \\
\hline
\end{tabular}

AOR = adjusted odds ratio; APACHE IV = Acute Physiology and Chronic Health Evaluation; AUD = Australian dollars; CAIC = consistent Akaike information criterion model; $\mathrm{CI}$ = confidence interval; DNR = do not resuscitate; FIM = Finnish markka; HR = hazard ratio; ICU = intensive care unit; IQR = interquartile range; IVH = intraventricular hemorrhage; LOS = length of stay; MICU = medical intensive care unit; NICU = neonatal intensive care unit; NR = not reported; NS = not significant; OES = Office of Emergency Services; PCU = progressive care unit; PICU = pediatric intensive care unit; RR = relative risk; SAPS II = Simplified Acute Physiology Score II; SCIP = Surgical Care Improvement Project; SD = standard deviation; SEM = standard error mean; SICU = surgical intensive care unit; TCH = Tyrone County Hospital; TEMPiS = Telemedic Pilot Project for Integrative Stroke Care; TH = telehealth; USD = United States dollars

${ }^{a}$ For definitions of interventions and comparisons (e.g., A vs. B), see Table F-1

See Appendix C. Included Studies for full citations 
Table F-4. Emergency medical services/emergency department evidence table: study characteristics

\begin{tabular}{|c|c|c|c|c|}
\hline $\begin{array}{l}\text { Author, Year } \\
\text { (See Appendix C for full } \\
\text { citation) }\end{array}$ & $\begin{array}{l}\text { Geographic Location } \\
\text { of Studies }\end{array}$ & Study Period & Study Design & Comparison and Intervention \\
\hline Amorim, 2013 & $\begin{array}{l}\text { Pennsylvania } \\
\text { USA }\end{array}$ & $\begin{array}{l}\text { March } 2005 \text { to December } \\
2008\end{array}$ & Before-After & $\begin{array}{l}\text { A: Before telestroke implementation } \\
\text { B: After telestroke implementation }\end{array}$ \\
\hline Astarcioglu, 2015 & Turkey & $\begin{array}{l}\text { January } 2015 \text { to May } \\
2015\end{array}$ & Prospective Cohort & $\begin{array}{l}\text { A: Concurrently treated STEMI patients who were } \\
\text { not triaged } \\
\text { B: Smartphone triaged STEMI patients in the ER }\end{array}$ \\
\hline Audebert, 2006 & $\begin{array}{l}\text { Bavaria } \\
\text { Germany }\end{array}$ & July 2003 to March 2005 & Prospective Cohort & $\begin{array}{l}\text { A: } 5 \text { hospitals without specialized stroke care } \\
\text { B: } 5 \text { hospitals without specialized stroke care with } \\
\text { telehealth stroke support }\end{array}$ \\
\hline Bergrath, 2012 & $\begin{array}{l}\text { Aachen } \\
\text { Germany }\end{array}$ & $\begin{array}{l}\text { May } 2010 \text { to September } \\
2010\end{array}$ & Prospective Cohort & $\begin{array}{l}\text { A: Usual care } \\
\text { B: Telehealth }\end{array}$ \\
\hline Bladin, 2015 & Australia & $\begin{array}{l}\text { A: January to December } \\
2010 \\
\text { B: October } 2011 \text { to } \\
\text { October } 2012 \\
\end{array}$ & Before-After & $\begin{array}{l}\text { A: Before telestroke protocol initiation } \\
\text { B: After telestroke initiation }\end{array}$ \\
\hline Brennan, 1998 and 1999 & $\begin{array}{l}\text { Northwest New Jersey } \\
\text { USA }\end{array}$ & NR & RCT & \begin{tabular}{|l|} 
A: Usual care \\
B: Telehealth
\end{tabular} \\
\hline Brokmann, 2016b & $\begin{array}{l}\text { Aachen } \\
\text { Germany }\end{array}$ & August 2012 to July 2013 & Before-After & $\begin{array}{l}\text { A: Before telehealth } \\
\text { B: After telehealth }\end{array}$ \\
\hline Brokmann, 2016 & $\begin{array}{l}\text { Aachen } \\
\text { Germany }\end{array}$ & $\begin{array}{l}\text { Before: April } 2011 \text { to } \\
\text { March } 2012 \\
\text { After: August } 2012 \text { to } \\
\text { August } 2013\end{array}$ & Before-After & $\begin{array}{l}\text { A: Before telehealth } \\
\text { B: After telehealth }\end{array}$ \\
\hline Brunetti, 2014 & \begin{tabular}{|l|}
$\begin{array}{l}\text { Apulia } \\
\text { Italy }\end{array}$ \\
\end{tabular} & \begin{tabular}{|l|} 
October 2012 to April \\
2013 \\
\end{tabular} & Prospective Cohort & $\begin{array}{l}\text { A: Usual care } \\
\text { B: Telehealth } \\
\end{array}$ \\
\hline Chan, 2012 & $\begin{array}{l}\text { British Columbia } \\
\text { Canada }\end{array}$ & April 2009 to March 2011 & Retrospective Cohort & $\begin{array}{l}\text { A Usual care } \\
\text { B: Telehealth } \\
\end{array}$ \\
\hline Cho, 2011 & \begin{tabular}{|l|} 
Seoul \\
Korea \\
\end{tabular} & \begin{tabular}{|l|} 
March 2008 to February \\
2009 \\
\end{tabular} & RCT & \begin{tabular}{|l|} 
A: Usual care \\
B: Tele-airway \\
\end{tabular} \\
\hline Choi, 2006 & $\begin{array}{l}\text { Texas } \\
\text { USA }\end{array}$ & $\begin{array}{l}\text { A: January } 2003 \text { to March } \\
2004 \\
\text { B: April } 2004 \text { to May } 2005\end{array}$ & Before-After & $\begin{array}{l}\text { A: Patients treated with tPA without telestroke } \\
\text { B: Patients treated with tPA with telestroke }\end{array}$ \\
\hline Chowdhury, 2012 & $\begin{array}{l}\text { London } \\
\text { United Kingdom }\end{array}$ & July 2007 to Dec 2009 & Retrospective Cohort & $\begin{array}{l}\text { A: Usual care } \\
\text { B: Telehealth }\end{array}$ \\
\hline Darkins, 1996 & Ireland & $\begin{array}{l}\text { A: September } 1993 \text { to } \\
\text { September } 1994 \\
\text { B: October } 1994 \text { to } \\
\text { October } 1995\end{array}$ & Before-After & $\begin{array}{l}\text { A: Before telehealth } \\
\text { B: After telehealth }\end{array}$ \\
\hline Dayal, 2016 & $\begin{array}{l}\text { Sacramento, California } \\
\text { USA }\end{array}$ & 2010 to 2014 & Retrospective Cohort & $\begin{array}{l}\text { A: Usual care } \\
\text { B: Telehealth }\end{array}$ \\
\hline
\end{tabular}




\begin{tabular}{|c|c|c|c|c|}
\hline $\begin{array}{l}\text { Author, Year } \\
\text { (See Appendix C for full } \\
\text { citation) }\end{array}$ & $\begin{array}{l}\text { Geographic Location } \\
\text { of Studies }\end{array}$ & Study Period & Study Design & Comparison and Intervention \\
\hline Demaerschalk, 2010 & USA & $\begin{array}{l}\text { December } 2007 \text { to } \\
\text { October } 2008\end{array}$ & $\mathrm{RCT}$ & $\begin{array}{l}\text { A: Telephone only } \\
\text { B: Telehealth }\end{array}$ \\
\hline Dharmar, 2013 & $\begin{array}{l}\text { Rural California } \\
\text { USA }\end{array}$ & $\begin{array}{l}\text { 4-year period } \\
\text { (2 years before tele and } 2 \\
\text { years after) } \\
\text { Dates not specified }\end{array}$ & Retrospective Cohort & $\begin{array}{l}\text { A: No consultation completed } \\
\text { B: Emergency consults completed by teleconsult } \\
\text { C: Emergency consults completed by telephone }\end{array}$ \\
\hline Dharmar, 2013 & $\begin{array}{l}\text { California } \\
\text { USA }\end{array}$ & $\begin{array}{l}\text { January } 2003 \text { to } \\
\text { December } 2009\end{array}$ & Retrospective Cohort & $\begin{array}{l}\text { A1: No telehealth, no consult } \\
\text { A2: No telehealth, phone consult } \\
\text { B: Telehealth } \\
\end{array}$ \\
\hline Dharmasaroja, 2010 & $\begin{array}{l}\text { Thammasat } \\
\text { Thailand }\end{array}$ & $\begin{array}{l}\text { A: June } 2007 \text { to February } \\
2008 \\
\text { B: March } 2008 \text { to March } \\
2009\end{array}$ & Before-After & $\begin{array}{l}\text { A: Before implementation of telestroke network } \\
\text { B: After implementation of telestroke network }\end{array}$ \\
\hline Duchesne, 2008 & USA & $\begin{array}{l}\text { January } 2000 \text { to January } \\
2005\end{array}$ & Before-After & $\begin{array}{l}\text { A: Before trauma telehealth implementation } \\
\text { B: After trauma telehealth implementation }\end{array}$ \\
\hline Fong, 2015 & Hong Kong & \begin{tabular}{|l|} 
January 2009 to \\
December 2012 \\
\end{tabular} & Prospective Cohort & $\begin{array}{l}\text { A: Neurologist on site } \\
\text { B: Telestroke, no neurologist on site }\end{array}$ \\
\hline Goh, 1997 & Hong Kong & March 1995 to May 1996 & Prospective Cohort & $\begin{array}{l}\text { A: Usual care } \\
\text { B: Teleradiology }\end{array}$ \\
\hline Handschu, 2008 & $\begin{array}{l}\text { Bavaria } \\
\text { Germany }\end{array}$ & $\begin{array}{l}1 \text { year } \\
\text { Dates not specified }\end{array}$ & Prospective Cohort & $\begin{array}{l}\text { A: Usual care (telephone consult) } \\
\text { B: Telestroke with video }\end{array}$ \\
\hline Hashimoto, 2001 & Japan & 1994 to 1999 & Before-After & $\begin{array}{l}\text { A: Before telehealth } \\
\text { B: After telehealth } \\
\end{array}$ \\
\hline Heffner, 2015 & $\begin{array}{l}\text { Pennsylvania } \\
\text { USA }\end{array}$ & 2006 to 2014 & Retrospective Cohort & $\begin{array}{l}\text { A: Patients treated at regional stroke center } \\
\text { B: Patients treated at hospital served by telehealth } \\
\text { and remained at the local hospital } \\
\text { C: Patients treated at hospital served by telehealth } \\
\text { and transferred to regional stroke center }\end{array}$ \\
\hline Hubert, 2016 & $\begin{array}{l}\text { Helsinki } \\
\text { Finland } \\
\end{array}$ & \begin{tabular}{|l|} 
January 2011 to \\
December 2013 \\
\end{tabular} & Retrospective Cohort & $\begin{array}{l}\text { A: Usual care } \\
\text { B: Telehealth }\end{array}$ \\
\hline Ickenstein, 2005 & Germany & \begin{tabular}{|l|} 
December 2001 to \\
December 2003
\end{tabular} & Before-After & $\begin{array}{l}\text { A: Before telehealth } \\
\text { B: After telehealth }\end{array}$ \\
\hline Ionita, 2009 & $\begin{array}{l}\text { New York } \\
\text { USA }\end{array}$ & \begin{tabular}{|l|} 
January 2006 to \\
December 2008
\end{tabular} & Retrospective Cohort & $\begin{array}{l}\text { A: Patients treated with thrombolysis at a regional } \\
\text { medical center } \\
\text { B: Patients treated with thrombolysis at community } \\
\text { hospitals via telemedicine }\end{array}$ \\
\hline Itrat, 2016 & USA & $\begin{array}{l}\text { July } 2014 \text { to November } \\
2014\end{array}$ & Prospective Cohort & $\begin{array}{l}\text { A: Usual care } \\
\text { B:Telehealth }\end{array}$ \\
\hline Johansson, 2011 & $\begin{array}{l}\text { Salzburg } \\
\text { Austria }\end{array}$ & 2006 to 2009 & Retrospective Cohort & $\begin{array}{l}\text { A: Usual care } \\
\text { B: Telehealth }\end{array}$ \\
\hline
\end{tabular}




\begin{tabular}{|c|c|c|c|c|}
\hline $\begin{array}{l}\text { Author, Year } \\
\text { (See Appendix C for full } \\
\text { citation) }\end{array}$ & $\begin{array}{l}\text { Geographic Location } \\
\text { of Studies }\end{array}$ & Study Period & Study Design & Comparison and Intervention \\
\hline Kim, 2011 & $\begin{array}{l}\text { Wonju } \\
\text { South Korea }\end{array}$ & June 2007 to March 2008 & Retrospective Cohort & $\begin{array}{l}\text { A: Patients without telemetry system } \\
\text { B: Patients with telemetry system }\end{array}$ \\
\hline Langabeer, 2016 and 2017 & $\begin{array}{l}\text { Houston, Texas } \\
\text { USA }\end{array}$ & $\begin{array}{l}\text { January to December } \\
2015\end{array}$ & $\begin{array}{l}\text { Retrospective Cohort; } \\
\text { Economic Evaluation }\end{array}$ & $\begin{array}{l}\text { A: Usual care } \\
\text { B: Teleconsult }\end{array}$ \\
\hline Macedo, 2016 & \begin{tabular}{|l|} 
San Paulo \\
Brazil \\
\end{tabular} & 2011 to 2014 & Before-After & $\begin{array}{l}\text { A: Before telehealth } \\
\text { B: After telehealth }\end{array}$ \\
\hline Machado, 2018 & $\begin{array}{l}\text { Columbus, Ohio } \\
\text { USA }\end{array}$ & $\begin{array}{l}\text { July } 2010 \text { and February } \\
2013\end{array}$ & Retrospective Cohort & $\begin{array}{l}\text { A: Usual care } \\
\text { B: Telehealth }\end{array}$ \\
\hline Marcolino, 2013 & $\begin{array}{l}\text { Belo Horizonte } \\
\text { Brazil }\end{array}$ & 2009 to 2011 & Before-After & $\begin{array}{l}\text { A: Before telehealth } \\
\text { B: After telehealth }\end{array}$ \\
\hline Martinez-Sanchez, 2014 & $\begin{array}{l}\text { Madrid } \\
\text { Spain }\end{array}$ & $\begin{array}{l}\text { Pre: March } 2008 \text { to } \\
\text { February } 2010 \\
\text { Post: March } 2010 \text { to } \\
\text { February } 2013\end{array}$ & Before-After & $\begin{array}{l}\text { A: Before telestroke } \\
\text { B: After telestroke }\end{array}$ \\
\hline Martinoni, 2011 & Italy & 18 month period & Retrospective Cohort & $\begin{array}{l}\text { A: Usual care } \\
\text { B: Telehealth }\end{array}$ \\
\hline Mathews, 2008 & Australia & $\begin{array}{l}\text { September } 2006 \text { to } \\
\text { March } 2007\end{array}$ & Before-After & $\begin{array}{l}\text { A: All patients referral for rural transfer for 6-month } \\
\text { period before tele-link } \\
\text { B: All patients referral for rural transfer for 6-month } \\
\text { period after tele-link who received a teleconsult. } \\
\text { C: All patients referral for rural transfer for 6-month } \\
\text { period after tele-link who did not receive a } \\
\text { teleconsult }\end{array}$ \\
\hline Mazighi, 2017 & \begin{tabular}{|l|} 
Paris \\
France \\
\end{tabular} & April 2006 to March 2010 & RCT & $\begin{array}{l}\text { A: Usual care } \\
\text { B: Telethrombolysis }\end{array}$ \\
\hline Meyer, 2008 & NR & $\begin{array}{l}\text { January } 2004 \text { to August } \\
2007\end{array}$ & RCT & $\begin{array}{l}\text { A: Telephone only consultation } \\
\text { B: Telemedicine }\end{array}$ \\
\hline $\begin{array}{l}\text { Mohr, } 2018 \\
\text { (same patients as Mohr } \\
\text { 2017) }\end{array}$ & $\begin{array}{l}\text { North Dakota } \\
\text { USA }\end{array}$ & 2008 to 2014 & Retrospective Cohort & $\begin{array}{l}\text { A: Usual care } \\
\text { B: Telehealth }\end{array}$ \\
\hline $\begin{array}{l}\text { Mohr, 2017(same patients as } \\
\text { Mohr 2018) }\end{array}$ & $\begin{array}{l}\text { North Dakota } \\
\text { USA }\end{array}$ & 2008 to 2014 & Retrospective Cohort & $\begin{array}{l}\text { A: Usual care } \\
\text { B: Telehealth }\end{array}$ \\
\hline Nagao, 2012 & $\begin{array}{l}\text { Melbourne, Victoria } \\
\text { Australia }\end{array}$ & $\begin{array}{l}\text { Telestroke: October } 2009 \\
\text { to September } 2010 \text { and } \\
\text { control group: October } \\
2008 \text { to September } 2009\end{array}$ & Before-After & $\begin{array}{l}\text { A: Before telestroke } \\
\text { B: After telestroke }\end{array}$ \\
\hline Narasimhan, 2015 & USA, South Carolina & March 2009 to June 2013 & Prospective Cohort & $\begin{array}{l}\text { A: Patients with the same mental health conditions } \\
\text { and demographic characteristics but were treated in } \\
\text { hospitals that did not have telepsychiatry. } \\
\text { B: Telepsychiatry recipients }\end{array}$ \\
\hline
\end{tabular}




\begin{tabular}{|c|c|c|c|c|}
\hline $\begin{array}{l}\text { Author, Year } \\
\text { (See Appendix C for full } \\
\text { citation) }\end{array}$ & $\begin{array}{l}\text { Geographic Location } \\
\text { of Studies }\end{array}$ & Study Period & Study Design & Comparison and Intervention \\
\hline Natafgi, 2017 & $\begin{array}{l}7 \text { states } \\
85 \text { rural hospitals } \\
\text { Hub located in South } \\
\text { Dakota } \\
\text { USA }\end{array}$ & $\begin{array}{l}\text { October } 2009 \text { to February } \\
2014\end{array}$ & Economic Evaluation & $\begin{array}{l}\text { A: Tele-ED not activated } \\
\text { B: Tele-ED activated }\end{array}$ \\
\hline Nguyen-Huynh, 2018 & \begin{tabular}{|l|} 
Northern CA \\
USA
\end{tabular} & 2015 to 2016 & Before-After & $\begin{array}{l}\text { A: Before telehealth } \\
\text { B: After telehealth }\end{array}$ \\
\hline Noble, 2005 & $\begin{array}{l}\text { Single hospital ED } \\
\text { United Kingdom }\end{array}$ & NR & Economic Evaluation & $\begin{array}{l}\text { A: Hospitals without teleconsultations } \\
\text { B: Hospitals with teleconsultations }\end{array}$ \\
\hline Ortolani 2006 & $\begin{array}{l}\text { Bologna } \\
\text { Italy }\end{array}$ & $\begin{array}{l}\text { January } 2003 \text { to } \\
\text { December } 2004\end{array}$ & Retrospective Cohort & $\begin{array}{l}\text { A: ED } \\
\text { B: Local hospital } \\
\text { C: Telehealth }\end{array}$ \\
\hline Ortolani, 2007 & Italy & $\begin{array}{l}\text { January } 2003 \text { to } \\
\text { December } 2005\end{array}$ & Retrospective Cohort & $\begin{array}{l}\text { A: Conventional hospital-based triage } \\
\text { B: Pre-hospital telemedicine ambulance triage }\end{array}$ \\
\hline Paik, 2017 & $\begin{array}{l}\text { Newark, New Jersey } \\
\text { USA }\end{array}$ & $\begin{array}{l}\text { May 1, } 2013 \text { to May 31, } \\
2013\end{array}$ & Retrospective Cohort & $\begin{array}{l}\text { A: Usual care } \\
\text { B: Telehealth }\end{array}$ \\
\hline Patel, 2015 & $\begin{array}{l}\text { Wilmington, Delaware } \\
\text { USA }\end{array}$ & \begin{tabular}{|l|} 
July 2012 to September \\
2012 \\
\end{tabular} & RCT & $\begin{array}{l}\text { A: Cell phone } \\
\text { B: Telehealth }\end{array}$ \\
\hline Pedersen, 2009 & Denmark & \begin{tabular}{|l|} 
January 2005 to July \\
2008
\end{tabular} & Prospective Cohort & $\begin{array}{l}\text { A: Usual care } \\
\text { B: Telehealth }\end{array}$ \\
\hline Pedragosa, 2009 & Spain & \begin{tabular}{|l|} 
January 2006 to \\
December 2007
\end{tabular} & Before-After & $\begin{array}{l}\text { A: Before telemedicine } \\
\text { B: After telemedicine }\end{array}$ \\
\hline Pervez, 2010 & $\begin{array}{l}\text { Boston, Massachusetts } \\
\text { USA }\end{array}$ & $\begin{array}{l}\text { January } 2003 \text { to March } \\
2008\end{array}$ & Retrospective Cohort & $\begin{array}{l}\text { A: Telephone } \\
\text { B: Telehealth (for treatment in place) }\end{array}$ \\
\hline Poon, 2001 & Hong Kong & $\begin{array}{l}\text { October } 1998 \text { to July } \\
1999\end{array}$ & RCT & $\begin{array}{l}\text { A: Telephone consultation } \\
\text { B: Teleradiology and telephone consultation } \\
\text { C. Video-consultation }\end{array}$ \\
\hline Saffle, 2009 & USA & 2003 to August 2007 & Before-After & $\begin{array}{l}\text { A: Before telemedicine for acute burn } \\
\text { implementation } \\
\text { B: After telemedicine for acute burn implementation }\end{array}$ \\
\hline Sairanen, 2011 & \begin{tabular}{|l|} 
Helsinki \\
Finland \\
\end{tabular} & 2007 to 2009 & Prospective Cohort & $\begin{array}{l}\text { A: Usual care } \\
\text { B: Telehealth }\end{array}$ \\
\hline Sanchez-Ross, 2011 & \begin{tabular}{|l|} 
New Jersey \\
USA
\end{tabular} & \begin{tabular}{|l|} 
June 2006 to February \\
2009
\end{tabular} & Prospective Cohort & $\begin{array}{l}\text { A: Usual care } \\
\text { B: STAT-MI Network }\end{array}$ \\
\hline Schwab, 2007 & $\begin{array}{l}\text { Regensburg and } \\
\text { Munich } \\
\text { Germany }\end{array}$ & $\begin{array}{l}\text { February } 2003 \text { to } \\
\text { November } 2004\end{array}$ & Prospective Cohort & $\begin{array}{l}\text { A: Usual care } \\
\text { B: Telehealth }\end{array}$ \\
\hline Sejersten, 2008 & Denmark & \begin{tabular}{|l|} 
October 2003 to October \\
2005
\end{tabular} & Prospective Cohort & $\begin{array}{l}\text { A: Usual care } \\
\text { B: Telehealth }\end{array}$ \\
\hline Southard, 2014 & $\begin{array}{l}\text { Indiana } \\
\text { USA }\end{array}$ & $\begin{array}{l}\text { January } 2009 \text { to January } \\
2010\end{array}$ & Before-After & $\begin{array}{l}\text { A: Before telemedicine } \\
\text { B: After telemedicine }\end{array}$ \\
\hline
\end{tabular}




\begin{tabular}{|c|c|c|c|c|}
\hline $\begin{array}{l}\text { Author, Year } \\
\text { (See Appendix C for full } \\
\text { citation) }\end{array}$ & $\begin{array}{l}\text { Geographic Location } \\
\text { of Studies }\end{array}$ & Study Period & Study Design & Comparison and Intervention \\
\hline Switzer, 2009 & $\begin{array}{l}\text { Augusta, Georgia } \\
\text { USA }\end{array}$ & \begin{tabular}{|l|} 
February 2003 to March \\
2006
\end{tabular} & Prospective Cohort & $\begin{array}{l}\text { A: Emergency department } \\
\text { B: Telehealth }\end{array}$ \\
\hline Taqui, 2017 & $\begin{array}{l}\text { Cleveland, Ohio } \\
\text { USA }\end{array}$ & $\begin{array}{l}\text { July } 2014 \text { to November } \\
2014\end{array}$ & Prospective Cohort & $\begin{array}{l}\text { A: Traditional ambulance } \\
\text { B: Mobile stroke unit }\end{array}$ \\
\hline Theiss, 2013 & $\begin{array}{l}\text { Erfurt } \\
\text { Germany }\end{array}$ & 2006 to 2009 & Before-After & $\begin{array}{l}\text { A: Control hospitals without telemedicine } \\
\text { B: Comprehensive stroke centers } \\
\text { C: Neuro Net hospitals with stroke telemedicine }\end{array}$ \\
\hline Traub, 2013 & $\begin{array}{l}\text { Unclear } \\
\text { USA }\end{array}$ & April to June 2012 & Retrospective Cohort & $\begin{array}{l}\text { A: Usual care } \\
\text { B: Telehealth } \\
\end{array}$ \\
\hline Tsai, 2007 & Taiwan & $\begin{array}{l}\text { November } 1999 \text { to } \\
\text { August } 2003\end{array}$ & Prospective Cohort & $\begin{array}{l}\text { A: Emergency air medical transport system before } \\
\text { implementing preflight screening and } \\
\text { teleconsultation } \\
\text { B: Emergency air medical transport system after } \\
\text { implementing preflight screening and } \\
\text { teleconsultation }\end{array}$ \\
\hline Wong, 2006 & Hong Kong & $\begin{array}{l}\text { October } 1998 \text { to } \\
\text { September } 2001\end{array}$ & RCT & $\begin{array}{l}\text { A: Telephone only consultation (standard of care) } \\
\text { B: Telephone + teleradiology (sharing of images) } \\
\text { C: Videoconference }\end{array}$ \\
\hline Yang, 2015 & $\begin{array}{l}\text { Northern, California } \\
\text { USA }\end{array}$ & $\begin{array}{l}\text { January } 2003 \text { and May } \\
2012\end{array}$ & Retrospective Cohort & $\begin{array}{l}\text { A: Telephone } \\
\text { B: Telehealth }\end{array}$ \\
\hline Zaidi, 2011 & $\begin{array}{l}\text { Pittsburgh, } \\
\text { Pennsylvania } \\
\text { USA }\end{array}$ & July 2008 to July 2009 & Prospective Cohort & $\begin{array}{l}\text { A: Stroke center } \\
\text { B: Telestroke }\end{array}$ \\
\hline Zanini, 2008 & $\begin{array}{l}\text { Mantova } \\
\text { Italy }\end{array}$ & June 2003 to June 2005 & Retrospective Cohort & $\begin{array}{l}\text { A: Usual care } \\
\text { B: Telehealth }\end{array}$ \\
\hline Zennaro, 2014 & Italy & $\begin{array}{l}\text { September } 2013 \text { to } \\
\text { October } 2013\end{array}$ & Pre/Post & $\begin{array}{l}\text { A: Pediatric patients with fractures receiving } \\
\text { telephone consultation from orthopedic surgeon on } \\
\text { call } \\
\text { B: Same pediatric patients with fractures receiving } \\
\text { telephone consultation from orthopedic surgeon on } \\
\text { call this time including an image of the x-ray sent to } \\
\text { the consulting orthopedic surgeon via an iPad }\end{array}$ \\
\hline
\end{tabular}

elevation myocardial infarction

See Appendix C. Included Studies for full citations 
Table F-5. Emergency medical services/emergency department evidence table: patient characteristics

\begin{tabular}{|c|c|c|c|c|c|c|c|c|}
\hline $\begin{array}{l}\text { Author, Year } \\
\text { (See Appendix C } \\
\text { for full citation) }\end{array}$ & $\begin{array}{l}\text { Number } \\
\text { Analyzed }^{\mathrm{a}}\end{array}$ & Population & $\begin{array}{l}\text { Referring } \\
\text { Providers } \\
\text { Specialty/Type }\end{array}$ & $\begin{array}{l}\text { Consulting } \\
\text { Provider } \\
\text { Specialty/Type }\end{array}$ & $\begin{array}{l}\text { Patient } \\
\text { Present? }\end{array}$ & $\begin{array}{l}\text { Patient } \\
\text { Setting }\end{array}$ & $\begin{array}{l}\text { Modalities of } \\
\text { Telehealth } \\
\text { Consultation } \\
\text { Intervention } \\
\end{array}$ & Timing \\
\hline Amorim, 2013 & \begin{tabular}{|l|}
2,588 \\
A: 919 \\
B: 1,669 \\
\end{tabular} & Rural patients & ED physician & Neurologists & Yes & ED/EMS & Videoconference & Real-time \\
\hline Astarcioglu, 2015 & $\begin{array}{l}108 \\
A: 55 \\
B: 53\end{array}$ & $\begin{array}{l}\text { Adults, } \\
\text { average age } \\
62.2 \text { in Group } \\
\text { A and } 64.6 \text { in } \\
\text { Group B, } \\
\text { majority } \\
\text { male, with } \\
\text { expected } \\
\text { comorbidities } \\
\text { for STEMl } \\
\text { patients }\end{array}$ & ED & $\begin{array}{l}\text { Interventional } \\
\text { cardiology }\end{array}$ & No & ED/EMS & WhatsApp & Real-time \\
\hline Audebert, 2006 & $\begin{array}{l}3,122 \\
\text { A: } 1,151 \\
\text { B: } 1,971 \\
\end{array}$ & Adults & $\begin{array}{l}\text { Multiple (non- } \\
\text { neurology) }\end{array}$ & Neurology & Yes & ED/EMS & $\begin{array}{l}\text { Videoconference, } \\
\text { radiological data } \\
\text { transfer }\end{array}$ & Real-time \\
\hline Bergrath, 2012 & $\begin{array}{l}64 \\
\text { A: } 46 \\
\text { B: } 18 \\
\end{array}$ & NR & EMS physician & $\begin{array}{l}\text { Hospital-based } \\
\text { EMS physician }\end{array}$ & Yes & ED/EMS & Videoconference & Real-time \\
\hline Bladin, 2015 & $\begin{array}{l}282 \\
\text { A: } 144 \\
B: 138\end{array}$ & Adult & $\begin{array}{l}\text { ED physicians } \\
\text { (with medical } \\
\text { registrars and } \\
\text { nursing staff) }\end{array}$ & $\begin{array}{l}\text { Neurologists/stroke } \\
\text { specialist }\end{array}$ & $\begin{array}{l}\text { Unclear - patient } \\
\text { was present in } \\
\text { the ED, but did } \\
\text { not seem like the } \\
\text { consulting } \\
\text { specialist saw } \\
\text { the patient } \\
\text { directly }\end{array}$ & ED/EMS & $\begin{array}{l}\text { Telephone and } \\
\text { radiology images; } \\
\text { video camera } \\
\text { unclear }\end{array}$ & Real-time \\
\hline $\begin{array}{l}\text { Brennan, } 1998 \\
\text { and } 1999\end{array}$ & $\begin{array}{l}100 \\
\text { A: } 50 \\
\text { B: } 50 \\
\end{array}$ & NR & Nurse & $\begin{array}{l}\text { Emergency } \\
\text { physician }\end{array}$ & Yes & ED/EMS & Videoconference & Real-time \\
\hline $\begin{array}{l}\text { Brokmann, } \\
2016 b\end{array}$ & $\begin{array}{l}160 \\
\text { A: } 80 \\
\text { B: } 80 \\
\text { Complete } \\
\text { Numerical } \\
\text { Rating Scale } \\
\text { A: } 32 / 80 \\
\text { B: } 65 / 80\end{array}$ & $\begin{array}{l}\text { Pediatric and } \\
\text { adults }\end{array}$ & Paramedic & EMS physician & Yes & ED/EMS & Video & Real-time \\
\hline
\end{tabular}




\begin{tabular}{|c|c|c|c|c|c|c|c|c|}
\hline $\begin{array}{l}\text { Author, Year } \\
\text { (See Appendix C } \\
\text { for full citation) }\end{array}$ & $\begin{array}{l}\text { Number } \\
\text { Analyzed }^{\mathrm{a}}\end{array}$ & Population & $\begin{array}{l}\text { Referring } \\
\text { Providers } \\
\text { Specialty/Type } \\
\end{array}$ & $\begin{array}{l}\text { Consulting } \\
\text { Provider } \\
\text { Specialty/Type }\end{array}$ & $\begin{array}{l}\text { Patient } \\
\text { Present? }\end{array}$ & $\begin{array}{l}\text { Patient } \\
\text { Setting } \\
\end{array}$ & $\begin{array}{l}\text { Modalities of } \\
\text { Telehealth } \\
\text { Consultation } \\
\text { Intervention } \\
\end{array}$ & Timing \\
\hline Brokmann, 2016 & \begin{tabular}{|l|}
78 \\
A: 39 \\
B: 39 \\
\end{tabular} & Adults & Paramedic & EMS physician & Yes & ED/EMS & Video & Real-time \\
\hline Brunetti, 2014 & $\begin{array}{l}297 \\
\text { A: } 174 \\
\text { B: } 123\end{array}$ & Adults & EMS & Cardiologist & Yes & ED/EMS & $\begin{array}{l}\text { Smart phone and } \\
\text { electronic EKG }\end{array}$ & Real-time \\
\hline Chan, 2012 & $\begin{array}{l}594 \\
\text { A: } 427 \\
\text { B: } 167\end{array}$ & Adults & Paramedic & ED physician & Yes & ED/EMS & $\begin{array}{l}\text { ECG transmitted } \\
\text { electronically }\end{array}$ & Real-time \\
\hline Cho, 2011 & \begin{tabular}{|l|}
25 \\
A: 13 \\
B: 12 \\
\end{tabular} & Adults & $\begin{array}{l}\text { Emergency } \\
\text { residents }\end{array}$ & $\begin{array}{l}\text { Emergency } \\
\text { physician }\end{array}$ & Yes & ED/EMS & Videoconference & Real-time \\
\hline Choi, 2006 & $\begin{array}{l}655 \\
\text { A: } 327 \\
B: 328 \\
\end{array}$ & Stroke & $\begin{array}{l}\text { Academic } \\
\text { medical center }\end{array}$ & Neurology & Yes & ED/EMS & Videoconference & Real-time \\
\hline $\begin{array}{l}\text { Chowdhury, } \\
2012\end{array}$ & $\begin{array}{l}97 \\
\text { A: } 52 \\
\text { B: } 45\end{array}$ & Adults & $\begin{array}{l}\text { ED doctors and } \\
\text { senior stroke } \\
\text { nurse }\end{array}$ & Stroke specialist & Yes & ED/EMS & \begin{tabular}{|l|} 
Videoconference \\
plus S\&F scans
\end{tabular} & Real-time \\
\hline Darkins, 1996 & $\begin{array}{l}16,700 \\
\text { A: } 6,729 \\
\text { B: } 9,972,51 \\
\text { seen using TH }\end{array}$ & Emergency & $\begin{array}{l}\text { Academic } \\
\text { medical center }\end{array}$ & $\begin{array}{l}\text { Emergency- } \\
\text { orthopedic }\end{array}$ & Yes & ED/EMS & Videoconference & Real-time \\
\hline Dayal, 2016 & $\begin{array}{l}1,106 \\
\text { A: } 524 \\
\text { B: } 582 \\
\end{array}$ & Pediatric & ED physician & $\begin{array}{l}\text { Pediatric critical } \\
\text { care physician }\end{array}$ & Yes & ED/EMS & Videoconference & Real-time \\
\hline $\begin{array}{l}\text { Demaerschalk, } \\
2010\end{array}$ & \begin{tabular}{|l|}
54 \\
A: 27 \\
B: 27 \\
\end{tabular} & Adults & ED physician & Stroke neurologist & Yes & ED/EMS & Videoconference & Real-time \\
\hline Dharmar, 2013 & $\begin{array}{l}320 \\
\text { A: } 199 \\
\text { B: } 58 \\
\text { C: } 63 \\
\end{array}$ & $\begin{array}{l}\text { Pediatric } \\
\text { emergencies }\end{array}$ & $\begin{array}{l}\text { Academic } \\
\text { medical center }\end{array}$ & $\begin{array}{l}\text { Pediatric critical } \\
\text { care }\end{array}$ & Yes & ED/EMS & $\begin{array}{l}\text { Videoconference or } \\
\text { telephone }\end{array}$ & Real-time \\
\hline Dharmar, 2013 & \begin{tabular}{|l|}
234 \\
A1: 85 \\
A2: 76 \\
B: 73 \\
\end{tabular} & Children & ED physician & $\begin{array}{l}\text { Pediatric Critical } \\
\text { Care }\end{array}$ & Yes & ED/EMS & Videoconference & Real-time \\
\hline $\begin{array}{l}\text { Dharmasaroja, } \\
2010\end{array}$ & $\begin{array}{l}576 \\
\text { A: } 170 \\
B: 406\end{array}$ & Adults & $\begin{array}{l}\text { Neurology } \\
\text { residents }\end{array}$ & Neurology & Yes & ED/EMS & $\begin{array}{l}\text { Telephone, transfer } \\
\text { of radiological data }\end{array}$ & Real-time \\
\hline
\end{tabular}




\begin{tabular}{|c|c|c|c|c|c|c|c|c|}
\hline $\begin{array}{l}\text { Author, Year } \\
\text { (See Appendix C } \\
\text { for full citation) }\end{array}$ & \begin{tabular}{|l} 
Number \\
Analyzed $^{\mathrm{a}}$
\end{tabular} & Population & $\begin{array}{l}\text { Referring } \\
\text { Providers } \\
\text { Specialty/Type } \\
\end{array}$ & $\begin{array}{l}\text { Consulting } \\
\text { Provider } \\
\text { Specialty/Type }\end{array}$ & $\begin{array}{l}\text { Patient } \\
\text { Present? }\end{array}$ & $\begin{array}{l}\text { Patient } \\
\text { Setting }\end{array}$ & \begin{tabular}{|l|} 
Modalities of \\
Telehealth \\
Consultation \\
Intervention \\
\end{tabular} & Timing \\
\hline Duchesne, 2008 & $\begin{array}{l}814 \\
\text { A: } 351 \\
\text { B: } 463 \text { (51 sent } \\
\text { to trauma } \\
\text { center) } \\
\end{array}$ & Rural patients & $\begin{array}{l}\text { Nurse } \\
\text { practitioner }\end{array}$ & Physician & Yes & ED/EMS & Videoconference & Real-time \\
\hline Fong, 2015 & $\begin{array}{l}152 \\
\text { A: } 102 \\
\text { B: } 50 \\
\end{array}$ & Not specified & Internist & Neurologist & Yes & ED/EMS & $\begin{array}{l}\text { Telephone, } \\
\text { teleradiology }\end{array}$ & Real-time \\
\hline Goh, 1997 & $\begin{array}{l}63 \\
\text { A: } 28 \\
\text { B: } 35 \\
\end{array}$ & Not specified & Not specified & Neurologist & Unclear & ED/EMS & $\begin{array}{l}\text { Telephone, } \\
\text { teleradiology }\end{array}$ & Real-time \\
\hline Handschu, 2008 & $\begin{array}{l}151 \\
\text { A: } 74 \\
B: 77\end{array}$ & Not specified & Not specified & Neurologist & Yes & ED/EMS & $\begin{array}{l}\text { Videoconference, } \\
\text { data transfer }\end{array}$ & Real-time \\
\hline Hashimoto, 2001 & $\begin{array}{l}29 \\
\text { A: } 17 \\
\text { B: } 12\end{array}$ & Not specified & Not specified & Radiation-oncology & Yes & ED/EMS & $\begin{array}{l}\text { Digital images of } \\
\text { any scans }\end{array}$ & Unclear \\
\hline Heffner, 2015 & $\begin{array}{l}479 \\
\text { A: } 272 \\
\text { B: } 134 \\
\text { C: } 73 \\
\end{array}$ & Not specified & Neurologist & Neurologist & Yes & ED/EMS & Not specified & Real-time \\
\hline Hubert, 2016 & $\begin{array}{l}2,691 \\
\text { A: } 912 \\
\text { B: } 1,779 \\
\end{array}$ & Adult & ED physician & Neurologist & Yes & ED/EMS & Videoconference & Real-time \\
\hline Ickenstein, 2005 & $\begin{array}{l}319 \\
\text { A: } 155 \\
B: 164\end{array}$ & Adults & ED physician & Neurologist & Yes & ED/EMS & Videoconference & Real-time \\
\hline Ionita, 2009 & $\begin{array}{l}155 \\
\text { A: } 128 \\
\text { B: } 27 \\
\end{array}$ & Not specified & Not specified & Neurology & Yes & ED/EMS & Not specified & Real-time \\
\hline Itrat, 2016 & $\begin{array}{l}156 \\
\text { A: } 56 \\
\text { B: } 100\end{array}$ & Adult & EMS & $\begin{array}{l}\text { Vascular } \\
\text { neurologist }\end{array}$ & Yes & ED/EMS & Videoconference & Real-time \\
\hline Johansson, 2011 & $\begin{array}{l}351 \\
\text { A: } 304 \\
B: 47\end{array}$ & Adults & ED physician & Stroke neurologist & Yes & ED/EMS & Videoconference & Real-time \\
\hline
\end{tabular}




\begin{tabular}{|c|c|c|c|c|c|c|c|c|}
\hline $\begin{array}{l}\text { Author, Year } \\
\text { (See Appendix C } \\
\text { for full citation) }\end{array}$ & $\begin{array}{l}\text { Number } \\
\text { Analyzed }^{a}\end{array}$ & Population & $\begin{array}{l}\text { Referring } \\
\text { Providers } \\
\text { SpecialtylType }\end{array}$ & $\begin{array}{l}\text { Consulting } \\
\text { Provider } \\
\text { Specialty/Type }\end{array}$ & $\begin{array}{l}\text { Patient } \\
\text { Present? }\end{array}$ & $\begin{array}{l}\text { Patient } \\
\text { Setting }\end{array}$ & $\begin{array}{l}\text { Modalities of } \\
\text { Telehealth } \\
\text { Consultation } \\
\text { Intervention }\end{array}$ & Timing \\
\hline Kim, 2011 & $\begin{array}{l}938 \\
\text { A: } 750 \\
B: 188\end{array}$ & $\begin{array}{l}\text { Suburban } \\
\text { and urban } \\
\text { emergency } \\
\text { patients seen } \\
\text { by EMS } \\
\text { system }\end{array}$ & EMT & $\begin{array}{l}\text { Public health } \\
\text { doctors }\end{array}$ & Yes & ED/EMS & $\begin{array}{l}\text { Code division } \\
\text { multiple access for } \\
\text { real-time } \\
\text { information } \\
\text { exchange, patient } \\
\text { data monitoring via } \\
\text { online method, and } \\
\text { cellular phone }\end{array}$ & Real-time \\
\hline $\begin{array}{l}\text { Langabeer, } 2016 \\
\text { and } 2017\end{array}$ & $\begin{array}{l}11,140 \\
A: 5,570 \\
B: 5,570\end{array}$ & Adults & Paramedic & EMS physician & Yes & ED/EMS & Video & Real-time \\
\hline Macedo, 2016 & $\begin{array}{l}376 \\
\text { A: } 113 \\
\text { B: } 263 \\
\end{array}$ & Adult & ED physician & Cardiologist & Yes & ED/EMS & Videoconference & Real-time \\
\hline Machado, 2018 & $\begin{array}{l}314 \\
\text { A: } 219 \\
\text { B: } 95 \\
\end{array}$ & Adults & ED clinicians & Intensivist & Yes & ED/EMS & NR & Real-time \\
\hline Marcolino, 2013 & $\begin{array}{l}2,600 \\
A: 1,242 \\
B: 1,358\end{array}$ & Adults & Hospitalist & Cardiac specialist & Yes & ED/EMS & $\begin{array}{l}\text { Telephone and } \\
\text { electronic EKG }\end{array}$ & Real-time \\
\hline $\begin{array}{l}\text { Martinez- } \\
\text { Sanchez, } 2014\end{array}$ & $\begin{array}{l}484 \\
\text { A: } 259 \\
\text { B: } 225 \\
\end{array}$ & Adults & ED physician & Neurologist & Yes & ED/EMS & Videoconference & Mixed \\
\hline Martinoni, 2011 & $\begin{array}{l}3,901 \\
\text { A: } 2,298 \\
\text { B: } 1,603 \\
\end{array}$ & Adults & EMS & Cardiologist & Yes & ED/EMS & ECG transmission & Real-time \\
\hline Mathews, 2008 & $\begin{array}{l}191 \\
\text { A: } 78 \\
B: 113\end{array}$ & $\begin{array}{l}\text { Rural } \\
\text { emergency }\end{array}$ & Urban hospital & Multiple & Unclear & ED/EMS & Videoconference & Real-time \\
\hline Mazighi, 2017 & $\begin{array}{l}47 \\
\text { A: } 22 \\
\text { B: } 25\end{array}$ & Adults & ER physician & Stroke neurologist & Yes & ED/EMS & Video & Real-time \\
\hline Meyer, 2008 & $\begin{array}{l}222 \\
A: 111 \\
B: 111\end{array}$ & $\begin{array}{l}\text { Adults at } \\
\text { least } 18 \\
\text { years old }\end{array}$ & $\begin{array}{l}\text { Emergency } \\
\text { nurse } \\
\text { practitioners }\end{array}$ & NR & Unclear & ED/EMS & Videoconference & Real-time \\
\hline $\begin{array}{l}\text { Mohr, } 2018 \\
\text { (same patients } \\
\text { as Mohr 2017) }\end{array}$ & $\begin{array}{l}2,662 \\
\text { A: } 2,371 \\
\text { B: } 291 \\
\end{array}$ & Adults & $\begin{array}{l}\text { Rural ER } \\
\text { physician }\end{array}$ & ED physician & Yes & ED/EMS & Video & Real-time \\
\hline
\end{tabular}




\begin{tabular}{|c|c|c|c|c|c|c|c|c|}
\hline $\begin{array}{l}\text { Author, Year } \\
\text { (See Appendix C } \\
\text { for full citation) }\end{array}$ & $\begin{array}{l}\text { Number } \\
\text { Analyzed }^{\mathrm{a}}\end{array}$ & Population & $\begin{array}{l}\text { Referring } \\
\text { Providers } \\
\text { Specialty/Type } \\
\end{array}$ & $\begin{array}{l}\text { Consulting } \\
\text { Provider } \\
\text { Specialty/Type }\end{array}$ & $\begin{array}{l}\text { Patient } \\
\text { Present? }\end{array}$ & $\begin{array}{l}\text { Patient } \\
\text { Setting } \\
\end{array}$ & $\begin{array}{l}\text { Modalities of } \\
\text { Telehealth } \\
\text { Consultation } \\
\text { Intervention } \\
\end{array}$ & Timing \\
\hline $\begin{array}{l}\text { Mohr, } 2017 \\
\text { (same patients } \\
\text { as Mohr 2018) }\end{array}$ & \begin{tabular}{|l|}
2,662 \\
A: 2,371 \\
B: 291 \\
\end{tabular} & Adults & $\begin{array}{l}\text { Rural ER } \\
\text { physician }\end{array}$ & $\begin{array}{l}\text { ER physician and } \\
\text { ED nurse }\end{array}$ & Yes & ED/EMS & Video & Real-time \\
\hline Nagao, 2012 & $\begin{array}{l}54 \\
\text { A: } 30 \\
B: 24\end{array}$ & Adults & $\begin{array}{l}\text { Emergency } \\
\text { physician }\end{array}$ & Neurologist & Yes & ED/EMS & Videoconference & Real-time \\
\hline $\begin{array}{l}\text { Narasimhan, } \\
2015\end{array}$ & $\begin{array}{l}14,522 \\
A: 7,261 \\
B: 7,261\end{array}$ & $\begin{array}{l}\text { All patients } \\
\text { seen in ED } \\
\text { for psychiatry } \\
\text { issues }\end{array}$ & ED doctors & $\begin{array}{l}\text { Psychiatrists with } \\
\text { at least one year } \\
\text { experience in } \\
\text { emergency } \\
\text { psychiatry }\end{array}$ & Yes & ED/EMS & Videoconference & Real-time \\
\hline Natafgi, 2017 & \begin{tabular}{|l|}
173,339 \\
A: 164,291 \\
B: 9,048 \\
\end{tabular} & Not specified & $\begin{array}{l}\text { Rural ER } \\
\text { physician }\end{array}$ & $\begin{array}{l}\text { Emergency } \\
\text { medicine physician }\end{array}$ & Yes & ED/EMS & Videoconference & Real-time \\
\hline $\begin{array}{l}\text { Nguyen-Huynh, } \\
2018\end{array}$ & $\begin{array}{l}\text { A: } 310 \\
\text { B: } 557 \\
\end{array}$ & Adult & ED physician & Neurologist & Yes & ED/EMS & Videoconference & Real-time \\
\hline Noble, 2005 & $\begin{array}{l}253 \\
\text { A: } 191 \\
B: 62\end{array}$ & Not specified & Nurse & ED & Yes & ED/EMS & $\begin{array}{l}\text { Unclear; pictures or } \\
\text { video }\end{array}$ & Unclear \\
\hline Ortolani, 2006 & \begin{tabular}{|l|}
658 \\
A: 316 \\
B: 176 \\
C: 166 \\
\end{tabular} & Adults & EMS physician & Cardiologist & Yes & ED/EMS & ECG transmission & Real-time \\
\hline Ortolani, 2007 & $\begin{array}{l}121 \\
\text { A: } 79 \\
B: 42\end{array}$ & $\begin{array}{l}\text { Patients with } \\
\text { STEMI with } \\
\text { cardiogenic } \\
\text { shock }\end{array}$ & $\begin{array}{l}\text { Ambulance } \\
\text { personnel (1 } \\
\text { physician, } 2 \\
\text { paramedics) }\end{array}$ & Cardiologists & No & ED/EMS & $\begin{array}{l}\text { Records (EKG } \\
\text { trace sent) }\end{array}$ & Asynchronous \\
\hline Paik, 2017 & $\begin{array}{l}84 \\
\text { A: } 42 \\
\text { B: } 42 \\
\end{array}$ & NR & ED clinicians & $\begin{array}{l}\text { Plastic surgeon } \\
\text { and orthopedics }\end{array}$ & Yes & ED/EMS & IPad app & Real-time \\
\hline Patel, 2015 & \begin{tabular}{|l|}
50 \\
A: 25 \\
B: 25 \\
\end{tabular} & Pediatric & $\begin{array}{l}\text { Pediatric } \\
\text { transport team }\end{array}$ & $\begin{array}{l}\text { Medical command } \\
\text { officer }\end{array}$ & Yes & ED/EMS & Videoconference & Real-time \\
\hline Pedersen, 2009 & $\begin{array}{l}1,437 \\
\text { A: } 821 \\
\text { B: } 616\end{array}$ & Adults & EMS & Cardiologist & Yes & ED/EMS & $\begin{array}{l}\text { ECG } \\
\text { transmissionmobile } \\
\text { phone with fax }\end{array}$ & Real-time \\
\hline Pedragosa, 2009 & $\begin{array}{l}399 \\
\text { A: } 201 \\
\text { B: } 198\end{array}$ & Rural patients & ED physician & Neurologist & Yes & ED/EMS & Videoconference & Real-time \\
\hline
\end{tabular}




\begin{tabular}{|c|c|c|c|c|c|c|c|c|}
\hline $\begin{array}{l}\text { Author, Year } \\
\text { (See Appendix C } \\
\text { for full citation) }\end{array}$ & $\begin{array}{l}\text { Number } \\
\text { Analyzed }^{a}\end{array}$ & Population & $\begin{array}{l}\text { Referring } \\
\text { Providers } \\
\text { Specialty/Type }\end{array}$ & $\begin{array}{l}\text { Consulting } \\
\text { Provider } \\
\text { Specialty/Type }\end{array}$ & $\begin{array}{l}\text { Patient } \\
\text { Present? }\end{array}$ & $\begin{array}{l}\text { Patient } \\
\text { Setting }\end{array}$ & $\begin{array}{l}\text { Modalities of } \\
\text { Telehealth } \\
\text { Consultation } \\
\text { Intervention } \\
\end{array}$ & Timing \\
\hline Pervez, 2010 & \begin{tabular}{|l|}
296 \\
A: 181 \\
B: 115 \\
\end{tabular} & Adults & $\begin{array}{l}\text { Emergency } \\
\text { physician }\end{array}$ & Neurologist & Yes & ED/EMS & Video & Real-time \\
\hline Poon, 2001 & $\begin{array}{l}327 \\
\text { A: NR } \\
\text { B: NR }\end{array}$ & $\begin{array}{l}\text { Neurosurgical } \\
\text { patients }\end{array}$ & Physician & Neurosurgeon & $\begin{array}{l}\text { Yes for } \\
\text { videoconference, } \\
\text { unclear for } \\
\text { others }\end{array}$ & ED/EMS & Videoconference & Mixed \\
\hline Saffle, 2009 & $\begin{array}{l}98 \\
\text { A: } 28 \\
\text { B: } 70 \\
\end{array}$ & General & Physician & Physician & Yes & ED/EMS & Videoconference & Real-time \\
\hline Sairanen, 2011 & $\begin{array}{l}1,091 \\
\text { A: } 985 \\
\text { B: } 106,61 \\
\text { received } \\
\text { thrombolysis }\end{array}$ & Adults & $\begin{array}{l}\text { Emergency } \\
\text { physician }\end{array}$ & Neurologist & Yes & ED/EMS & $\begin{array}{l}\text { Videoconference } \\
\text { and review of } \\
\text { images, if provided }\end{array}$ & Real-time \\
\hline $\begin{array}{l}\text { Sanchez-Ross, } \\
2011\end{array}$ & $\begin{array}{l}142 \\
\text { A: } 50 \\
\text { B: } 92 \\
\end{array}$ & \begin{tabular}{|l} 
Patients \\
confirmed to \\
have STEMI \\
\end{tabular} & ED physician & Cardiology & Yes & ED/EMS & $\begin{array}{l}\text { Telephone, test } \\
\text { results }\end{array}$ & Mixed \\
\hline Schwab, 2007 & $\begin{array}{l}302 \\
\text { A: } 132 \\
\text { B: } 170 \\
\end{array}$ & Adult & ED physician & Neurologist & Yes & ED/EMS & Videoconference & Real-time \\
\hline Sejersten, 2008 & $\begin{array}{l}257 \\
\text { A: } 89 \\
B: 168 \\
\end{array}$ & Adults & EMT & Cardiologist & Yes & ED/EMS & $\begin{array}{l}\text { ECG to mobile } \\
\text { phone }\end{array}$ & Real-time \\
\hline Southard, 2014 & $\begin{array}{l}62 \\
\text { A: } 24 \\
B: 38\end{array}$ & Rural patients & ED physician & $\begin{array}{l}\text { Mental health } \\
\text { specialists: } \\
\text { social workers, } \\
\text { licensed mental } \\
\text { health counselors, } \\
\text { and counseling } \\
\text { psychologists }\end{array}$ & Unclear & ED/EMS & Videoconference & Real-time \\
\hline Switzer, 2009 & \begin{tabular}{|l|}
75 \\
A: 26 \\
B: 49 \\
\end{tabular} & Adult & ED physician & Neurologist & Yes & ED/EMS & Videoconference & Real-time \\
\hline Taqui, 2017 & \begin{tabular}{|l|}
153 \\
$A: 53$ \\
$B: 100$ \\
\end{tabular} & Adults & $\begin{array}{l}\text { EMT mobile } \\
\text { stroke unit }\end{array}$ & $\begin{array}{l}\text { Vascular } \\
\text { neurologist }\end{array}$ & Yes & ED/EMS & Videoconference & Real-time \\
\hline
\end{tabular}




\begin{tabular}{|c|c|c|c|c|c|c|c|c|}
\hline $\begin{array}{l}\text { Author, Year } \\
\text { (See Appendix C } \\
\text { for full citation) }\end{array}$ & $\begin{array}{l}\text { Number } \\
\text { Analyzed }^{\mathrm{a}}\end{array}$ & Population & $\begin{array}{l}\text { Referring } \\
\text { Providers } \\
\text { Specialty/Type } \\
\end{array}$ & $\begin{array}{l}\text { Consulting } \\
\text { Provider } \\
\text { Specialty/Type }\end{array}$ & $\begin{array}{l}\text { Patient } \\
\text { Present? }\end{array}$ & $\begin{array}{l}\text { Patient } \\
\text { Setting } \\
\end{array}$ & \begin{tabular}{|l|} 
Modalities of \\
Telehealth \\
Consultation \\
Intervention \\
\end{tabular} & Timing \\
\hline Theiss, 2013 & $\begin{array}{l}1,324 \\
\text { A: } 168 \\
\text { B: } 845 \\
\text { C: } 311 \\
\end{array}$ & Adults & $\begin{array}{l}\text { Emergency } \\
\text { physician }\end{array}$ & Neurologist & Yes & ED/EMS & Videoconference & Real-time \\
\hline Traub, 2013 & $\begin{array}{l}302 \\
\text { A: } 196 \\
\text { B: } 106,36 \\
\text { used telehealth } \\
\end{array}$ & & Nurse & $\begin{array}{l}\text { Emergency } \\
\text { physician }\end{array}$ & Yes & ED/EMS & Videoconference & Real-time \\
\hline Tsai, 2007 & \begin{tabular}{|l|}
822 \\
A: 685 \\
B: 137 \\
\end{tabular} & General & Physician & Physician & Unclear & ED/EMS & $\begin{array}{l}\text { Videoconference, } \\
\text { medical record } \\
\text { review }\end{array}$ & Real-time \\
\hline Wong, 2006 & $\begin{array}{l}710 \\
\text { A: } 235 \\
\text { B: } 239 \\
\text { C: } 236\end{array}$ & $\begin{array}{l}\text { Consecutive } \\
\text { patients from } \\
\text { the district } \\
\text { general } \\
\text { hospital } \\
\text { requiring } \\
\text { emergency } \\
\text { neurosurgical } \\
\text { consultation } \\
\text { (mean age } \\
58,60 \% \\
\text { male) }\end{array}$ & Not specified & Neurosurgeon & Yes & ED/EMS & $\begin{array}{l}\text { Telephone + } \\
\text { teleradiology or } \\
\text { videoconference }\end{array}$ & Real-time \\
\hline Yang, 2015 & $\begin{array}{l}138 \\
\text { A: } 64 \\
\text { B: } 74 \\
\end{array}$ & Pediatric & ED physician & $\begin{array}{l}\text { Pediatric critical } \\
\text { care physician }\end{array}$ & Yes & ED/EMS & Videoconference & Real-time \\
\hline Zaidi, 2011 & $\begin{array}{l}142 \\
A: 59 \\
B: 83\end{array}$ & Adult & NR & Neurologist & Yes & ED/EMS & Videoconference & Real-time \\
\hline Zanini, 2008 & $\begin{array}{l}399 \\
\text { A: } 263 \\
\text { B: } 136\end{array}$ & Adults & Ambulance staff & Cardiologist & Yes & ED/EMS & $\begin{array}{l}\text { ECG transmission } \\
\text { Phone }\end{array}$ & Real-time \\
\hline Zennaro, 2014 & $\begin{array}{l}42 \\
\text { A: } 42 \\
\text { B: } 42 \text { (same } \\
\text { patients as A) }\end{array}$ & $\begin{array}{l}\text { Pediatric } \\
\text { patients } \\
\text { presenting } \\
\text { with fractures }\end{array}$ & Pediatrician & $\begin{array}{l}\text { Orthopedic } \\
\text { surgeon }\end{array}$ & No & ED/EMS & $\begin{array}{l}\text { Telephone +/- } \\
\text { sending images via } \\
\text { iPad }\end{array}$ & Asynchronous \\
\hline
\end{tabular}

ECG = electrocardiogram; ED = emergency department; EKG = electrocardiogram; EMS = emergency medical services; EMT = emergency medical technician; NR = not reported; S\&F = store and forward; STEMI = ST-elevation myocardial infarction; $\mathrm{TH}=$ telehealth

${ }^{a}$ For definitions of interventions and comparisons (e.g., A vs. B), see Table F-4

See Appendix C. Included Studies for full citations 
Table F-6. Emergency medical services/emergency department evidence table: results

\begin{tabular}{|c|c|c|c|}
\hline $\begin{array}{l}\text { Author, Year } \\
\text { (See Appendix C } \\
\text { for full citation) }\end{array}$ & $\begin{array}{l}\text { Results: } \\
\text { KQ1: Clinical and Economic Outcomes }\end{array}$ & $\begin{array}{l}\text { Results: } \\
\text { KQ2: Intermediate Outcomes }{ }^{a}\end{array}$ & $\begin{array}{l}\text { Results: } \\
\text { KQ3: Adverse Effects or } \\
\text { Unintended } \\
\text { Consequences }^{\mathrm{a}} \\
\end{array}$ \\
\hline Amorim, 2013 & $\begin{array}{l}\text { Overall IV tPA use } \\
\text { A: } 2.8 \% \\
\text { B: } 6.8 \%, p<0.001 \\
\text { For patients receiving IV tPA ( } \mathrm{n}=27 \text { vs. 113) } \\
\text { [mean (SD), p-value] } \\
\text { Onset to treatment minutes: } \\
\text { A: } 129.8(34) \\
\text { B: } 124.4(34), p=0.49 \\
\text { Door to treatment minutes: } \\
\text { A: } 74.2(32.1) \\
\text { B: } 74.0(29.1), p=0.98 \\
\text { Symptomatic intracerebral hemorrhage: } \\
\text { A: } 3.7 \% \\
\text { B: } 0.9 \%, p=0.34 \\
\text { In-hospital mortality: } \\
\text { A: } 7.4 \% \\
\text { B: } 10.9 \%, p=0.59 \\
\text { Discharge outcomes: } \\
\text { Home: } \\
\text { A: } 33.3 \% \\
\text { B: } 26.5 \%, p=0.48 \\
\text { Rehabilitation: } \\
\text { A: } 33.3 \% \\
\text { B: } 32 \%, p=0.95\end{array}$ & NR & NR \\
\hline Astarcioglu, 2015 & $\begin{array}{l}\text { Door to balloon time reduction of } 21 \text { minutes (95\% Cl } 9.1 \text { to } \\
32.3) \\
\text { Door to door time reduction of } 18 \text { minutes }(95 \% \mathrm{Cl} 11.45 \text { to } \\
32.95) \text {, the majority of the door to balloon estimate. } \\
\text { Cath lab to balloon time was not different, } 2 \text { minutes } \\
\text { (95\% Cl }-2.97 \text { to } 8.17) \\
\text { False STEMI did not occur in Group B, but did in } 8.3 \% \text { of } \\
\text { Group A (NS) }\end{array}$ & NR & NR \\
\hline
\end{tabular}




\begin{tabular}{|c|c|c|c|}
\hline $\begin{array}{l}\text { Author, Year } \\
\text { (See Appendix C } \\
\text { for full citation) }\end{array}$ & $\begin{array}{l}\text { Results: } \\
\text { KQ1: Clinical and Economic Outcomes }\end{array}$ & $\begin{array}{l}\text { Results: } \\
\text { KQ2: Intermediate Outcomes }\end{array}$ & $\begin{array}{l}\text { Results: } \\
\text { KQ3: Adverse Effects or } \\
\text { Unintended } \\
\text { Consequences }^{\mathrm{a}}\end{array}$ \\
\hline Audebert, 2006 & $\begin{array}{l}\text { Mean LOS (SD), in days } \\
\text { A: } 11.9(6) \\
\text { B: } 10.7(6) \text { p<0.0001 } \\
\text { Hospital discharge destination } p=0.001 \\
\text { Home: } \\
\text { A: } 38 \% \\
\text { B: } 39 \% \\
\text { Rehab unit: } \\
\text { A: } 34 \% \\
\text { B: } 38 \% \\
\text { NH: } \\
\text { A: } 5 \% \\
\text { B: } 3 \% \\
\text { Other hospital: } \\
\text { A: } 13 \% \\
\text { B: } 13 \% \\
\text { Dead: } \\
\text { A: } 10 \% \\
\text { B: } 8 \% \\
\text { 3-month outcomes } p<0.0001 \\
\text { Dead: } \\
\text { A: } 19 \% \\
\text { B: } 17 \% \\
\text { Institution: } \\
\text { A: } 14 \% \\
\text { B: } 13 \% \\
\text { Home, severe disability: } \\
\text { A: } 21 \% \\
\text { B: } 14 \% \\
\text { Home without severe disability: } \\
\text { A: } 46 \% \\
\text { B: } 56 \%\end{array}$ & $\begin{array}{l}\text { Diagnostic procedures } \\
\text { All p<0.001 } \\
\text { Rapid brain imaging: } \\
\text { A: } 32 \% \\
\text { B: } 74 \% \\
\text { Carotid artery sonography: } \\
\text { A: } 62 \% \\
\text { B: } 83 \% \\
\text { Standardized test for dysphagia } \\
\text { A: } 48 \% \\
\text { B: } 73 \% \\
\text { Treatments all } p<0.0001 \\
\text { Thrombolytic treatment: } \\
\text { A: } 0 \% \\
\text { B: } 5 \% \\
\text { Physiotherapy: } \\
\text { A: } 49 \% \\
\text { B: } 85 \% \\
\text { Speech therapy: } \\
\text { A: } 10 \% \\
\text { B: } 82 \% \\
\text { OT: } \\
\text { A: } 7 \% \\
\text { B: } 74 \%\end{array}$ & NR \\
\hline Bergrath, 2012 & NR & $\begin{array}{l}\text { Median EMS alarm to physician arrival (IQR), in } \\
\text { minutes } \\
\text { A: } 7 \text { (4) } \\
\text { B: } 5 \text { (2), } p=0.0182 \\
\text { Median door to brain imaging (IQR), in minutes } \\
\text { A: } n=42 ; 57 \text { (80) } \\
\text { B: } n=16 ; 59.5(67.5), p=0.6447\end{array}$ & NR \\
\hline
\end{tabular}




\begin{tabular}{|c|c|c|c|}
\hline $\begin{array}{l}\text { Author, Year } \\
\text { (See Appendix C } \\
\text { for full citation) }\end{array}$ & $\begin{array}{l}\text { Results: } \\
\text { KQ1: Clinical and Economic Outcomes }\end{array}$ & $\begin{array}{l}\text { Results: } \\
\text { KQ2: Intermediate Outcomes }\end{array}$ & $\begin{array}{l}\text { Results: } \\
\text { KQ3: Adverse Effects or } \\
\text { Unintended } \\
\text { Consequences }^{\mathrm{a}}\end{array}$ \\
\hline Bladin, 2015 & $\begin{array}{l}\text { Mortality } \\
\text { A: } 7 \% \\
\text { B: } 10 \%, p=0.58 \\
\text { Median LOS, in days (IQR) } \\
\text { A: } 3(1 \text { to } 6) \\
\text { B: } 4 \text { (2 to } 6), p=0.34 \\
\text { Discharged from hospital } \\
\text { A: } 93 \% \\
\text { B: } 90 \% \text { tPA use (within } 4.5 \text { hours of onset window) } \\
\text { All strokes: } \\
\text { A: } 10(17 \%) \\
\text { B: } 16(26 \%), p=0.26 \\
\text { Ischemic strokes: } \\
\text { A: } 10(19 \%) \\
\text { B: } 16(28 \%), p=0.28\end{array}$ & $\begin{array}{l}\text { Median door to medical contact, in minutes } \\
\text { A: } 13 \\
\text { B: } 13 \text {, ( } p=0.94) \\
\text { Median door to CT scan, in minutes } \\
\text { A: } 63 \\
\text { B: } 34 \text { ( } p=0.006) \\
\text { Median door to VST consult, in minutes } \\
\text { A: no data } \\
\text { B: } 68 \\
\text { Median minutes for patients receiving tPA: } \\
\text { Door to CT scan: } \\
\text { A: } 15 \\
\text { B: } 20 \text { ( } p=0.63 \text { ) } \\
\text { Door to needle time: } \\
\text { A: } 101 \\
\text { B: } 85 \text { ( } p=0.32) \\
\text { Stroke onset to needle: } \\
\text { A: } 218 \\
\text { B: } 173 \text { ( } p=0.11) \\
\text { Door to VST consultation: } \\
\text { A: No data } \\
\text { B: } 50 \\
\text { Discharge destination } \\
\text { Home: } \\
\text { A: } 14(24 \%) \\
\text { B: } 21(34 \%) \\
\text { Nursing home: } \\
\text { A: } 2(3 \%) \\
\text { B: } 3(5 \%) \\
\text { Rehab: } \\
\text { A: } 22(38 \%) \\
\text { B: } 19(31 \%)\end{array}$ & NR \\
\hline
\end{tabular}




\begin{tabular}{|c|c|c|c|}
\hline $\begin{array}{l}\text { Author, Year } \\
\text { (See Appendix C } \\
\text { for full citation) }\end{array}$ & $\begin{array}{l}\text { Results: } \\
\text { KQ1: Clinical and Economic Outcomes }\end{array}$ & $\begin{array}{l}\text { Results: } \\
\text { KQ2: Intermediate Outcomes } \\
\end{array}$ & $\begin{array}{l}\text { Results: } \\
\text { KQ3: Adverse Effects or } \\
\text { Unintended } \\
\text { Consequences }^{\mathrm{a}} \\
\end{array}$ \\
\hline $\begin{array}{l}\text { Brennan, } 1998 \\
\text { and } 1999\end{array}$ & NR & $\begin{array}{l}\text { Average throughput time, in minutes } \\
\text { A: } 117 \\
\text { B: } 10672 \\
\text { Hour return visit } \\
\text { A: } 0 \% \\
\text { B: } 0 \% \\
\text { Need for additional care } \\
\text { A: } 2.4 \% \\
\text { B: } 2.3 \%, p=0.99 \\
\text { Positive patient-physician interaction } \\
\text { A: } 100 \% \\
\text { B: } 98 \%, p=0.32 \\
\text { Positive patient-nurse interaction } \\
\text { A: } 98 \% \\
\text { B: } 98 \%, p=0.97 \\
\text { Overall patient satisfaction } \\
\text { A: } 95 \% \\
\text { B: } 98 \%, p=0.54\end{array}$ & NR \\
\hline $\begin{array}{l}\text { Brokmann, } \\
\text { 2016b }\end{array}$ & NR & $\begin{array}{l}\text { Adequate initial pain reduction Numerical Rating } \\
\text { Scale (NRS) } \\
\text { A: A: } 31 / 32 \\
\text { B: } 61 / 65 \\
\text { NRS reduction during mission } \\
\text { A: } 4.38 \pm 2.2 \text { points } \\
\text { B: } 3.78 \pm 2.0 \\
p=0.0159\end{array}$ & $\begin{array}{l}\text { Complications } \\
\text { A: } 0 \\
\text { B: } 0 \\
\text { Nausea and vomiting } \\
\text { A: } 11 \% \\
\text { B: } 11 \%\end{array}$ \\
\hline
\end{tabular}




\begin{tabular}{|c|c|c|c|}
\hline $\begin{array}{l}\text { Author, Year } \\
\text { (See Appendix C } \\
\text { for full citation) }\end{array}$ & $\begin{array}{l}\text { Results: } \\
\text { KQ1: Clinical and Economic Outcomes } \\
\end{array}$ & $\begin{array}{l}\text { Results: } \\
\text { KQ2: Intermediate Outcomes }{ }^{a}\end{array}$ & $\begin{array}{l}\text { Results: } \\
\text { KQ3: Adverse Effects or } \\
\text { Unintended } \\
\text { Consequences }^{\mathrm{a}}\end{array}$ \\
\hline Brokmann, 2016 & NR & $\begin{array}{l}\text { Correct handling12-lead ECG } \\
\text { A: } 39 \\
\text { B: } 38, p>0.99 \\
\text { Acetylsalicylic acid } \\
\text { A: } 33 \\
\text { B: } 31, p=0.73 \\
\text { Heparin } \\
\text { A: } 33 \\
\text { B: } 34, p>0.99 \\
\text { Morphine } \\
\text { A: } 27 \\
\text { B: } 29, p=0.50 \\
\text { Oxygen } \\
\text { A: } 18 \\
\text { B: } 29, p=0.007 \\
\end{array}$ & None detected \\
\hline Brunetti, 2014 & NR & $\begin{array}{l}\text { Mean time to balloon } \pm S D \text {, in minutes } \\
\text { A: } 94 \pm 61 \\
\text { B: } 41 \pm 17, p<0.001\end{array}$ & NR \\
\hline Chan, 2012 & $\begin{array}{l}\text { 30-day mortality } \\
\text { A: } 13.3 \% \\
\text { B: } 5.4 \% \\
p=0.006\end{array}$ & $\begin{array}{l}\text { 90-minute door to balloon } \\
\text { A: } 8.7 \% \\
\text { B: } 80.4 \%, p<0.001 \\
\text { Post-procedural thrombolysis in myocardial infarction } \\
\text { flow grade } 3 \text { after the emergency procedure } \\
\text { A: } 91.4 \% \\
\text { B: } 97.6 \%, p=0.02 \\
\end{array}$ & NR \\
\hline Cho, 2011 & NR & $\begin{array}{l}\text { Mean intubation times (SD), in seconds } \\
\text { A: } 56(2) \\
\text { B: } 62(12) p=0.30 \\
\text { Complication rate } p=0.36 \\
\text { Success rate } p>0.05\end{array}$ & $\begin{array}{l}\text { Complication rate was not } \\
\text { significantly different } \\
p=0.36\end{array}$ \\
\hline Choi, 2006 & $\begin{array}{l}\text { O Received tPA } \\
\text { A: } 0.81 \% \\
\text { B: } 4.3 \% \\
p<0.001 \\
\text { No intracerebral hemorrhages }\end{array}$ & $\begin{array}{l}\text { Median door to needle: } 85 \text { minutes (range } 27 \text { to 165) } \\
\text { Received tPA } n=14 \\
\text { ( } 24 \text { hours posttreatment) } \\
7 \text { improved by } 4 \text { points on NIHSS; } 3 \text { worsened }\end{array}$ & $\begin{array}{l}1 \text { patient required phone } \\
\text { call consultation due to } \\
\text { equipment failure. No } \\
\text { patients developed } \\
\text { hemorrhages occurred. }\end{array}$ \\
\hline
\end{tabular}




\begin{tabular}{|c|c|c|c|}
\hline $\begin{array}{l}\text { Author, Year } \\
\text { (See Appendix C } \\
\text { for full citation) }\end{array}$ & $\begin{array}{l}\text { Results: } \\
\text { KQ1: Clinical and Economic Outcomes }\end{array}$ & $\begin{array}{l}\text { Results: } \\
\text { KQ2: Intermediate Outcomes }\end{array}$ & $\begin{array}{l}\text { Results: } \\
\text { KQ3: Adverse Effects or } \\
\text { Unintended } \\
\text { Consequences }^{\mathrm{a}}\end{array}$ \\
\hline $\begin{array}{l}\text { Chowdhury, } \\
2012\end{array}$ & $\begin{array}{l}\text { 3-month mortality } \\
\text { A: } 15.5 \% \\
\text { B: } 11 \%, p=0.6 \\
\text { Favorable outcome } \\
\text { A: } 36.5 \% \\
\text { B: } 42 \%, p=0.9\end{array}$ & $\begin{array}{l}\text { Median onset to treatment (IQR), in minutes } \\
\text { A: } 100 \text { (78 to } 120) \\
\text { B: } 125 \text { (55 to } 105), p=0.001 \\
\text { Median admission to treatment, in minutes } \\
\text { A: } 33 \text { (23 to } 47) \\
\text { B: } 61 \text { (43 to } 106), p<0.001\end{array}$ & $\begin{array}{l}\text { Symptomatic intracranial } \\
\text { hemorrhage } \\
\text { A: } 7.7 \% \\
\text { B: } 4.4 \% \\
p=0.7\end{array}$ \\
\hline Darkins, 1996 & $\begin{array}{l}\text { Difference in cost, in GBP } \\
\text { A: } £ 50,000 \text { for onsite staff } \\
B: £ 7,250 \text { for equipment }\end{array}$ & $\begin{array}{l}\text { ED referrals: } \\
\text { A: } 2.3 \% \\
\text { B: } 1.5 \% \\
\text { Primary care referrals: } \\
\text { A: } 11.9 \% \\
\text { B: } 3.8 \%\end{array}$ & NR \\
\hline Dayal, 2016 & $\begin{array}{l}\text { Mortality } n(\%) \\
\text { A: } 23(4.4) \\
\text { B: } 14(2.4) \\
p=0.07\end{array}$ & $\begin{array}{l}\text { Mean PICU LOS (SD), in days } \\
\text { A: } 3.8(9.4) \\
B: 3.1(5.5) \\
p=0.11\end{array}$ & NR \\
\hline $\begin{array}{l}\text { Demaerschalk, } \\
2010\end{array}$ & $\begin{array}{l}\text { Mortality } \\
\text { A: } 11 \% \\
\text { B: } 4 \%\end{array}$ & $\begin{array}{l}\text { Mean onset to decision time } \pm \text { SD, in minutes } \\
A: 164 \pm 28.6 \\
B: 188.2 \pm 138.2 \\
p=0.07\end{array}$ & $\begin{array}{l}\text { Intracerebral hemorrhage } \\
\text { A: } 0 \% \\
\text { B: } 4 \% \\
p>0.99 \\
\end{array}$ \\
\hline Dharmar, 2013 & NR & $\begin{array}{l}\text { Assessment of quality of care ( } 7 \text { point rating) } \\
\text { Unadjusted, overall } \\
\text { Mean }(95 \% \mathrm{Cl}) \\
\text { A: } 5.20(5.07 \text { to } 5.34) \\
\text { B: } 5.60(5.42 \text { to } 5.79) \\
\text { C: } 5.37(5.16 \text { to } 5.59) \\
\text { A to } B: p<0.05 \\
\text { Adjusted meandifference }(95 \% \mathrm{Cl}) \\
\text { B to A: } 0.50(0.17 \text { to } 0.84), p=0.05 \\
\text { Linear regression of overall quality: } \\
\text { A: } N A \text { (reference) } \\
\text { B: } n=58 ; \beta=0.50(95 \% \mathrm{Cl} 0.17 \text { to } 0.84] \\
\text { C: } n=63 ; \beta=0.12(95 \% \mathrm{Cl}-0.14 \text { to } 0.39) \\
\text { Referring ED physicians changed diagnosis: } \\
\text { B: } 47.8 \% \\
\text { C: } 13.3 \% ; p<0.01 \\
\text { Changed in therapeutic interventions: } \\
\text { B: } 55.2 \% \\
\text { C: } 7.1 \% ; p<0.01\end{array}$ & $\mathrm{NR}$ \\
\hline
\end{tabular}




\begin{tabular}{|c|c|c|c|}
\hline $\begin{array}{l}\text { Author, Year } \\
\text { (See Appendix C } \\
\text { for full citation) }\end{array}$ & $\begin{array}{l}\text { Results: } \\
\text { KQ1: Clinical and Economic Outcomes }\end{array}$ & $\begin{array}{l}\text { Results: } \\
\text { KQ2: Intermediate Outcomes }\end{array}$ & $\begin{array}{l}\text { Results: } \\
\text { KQ3: Adverse Effects or } \\
\text { Unintended } \\
\text { Consequences }^{\mathrm{a}} \\
\end{array}$ \\
\hline Dharmar, 2013 & $\begin{array}{l}\text { Mortality } \\
\text { n (\%) } \\
\text { A1: } 2(2.6) \\
\text { A2: } 1(1.2) \\
\text { B: } 3(4.1)\end{array}$ & $\begin{array}{l}\text { Physician-related ED medication errors, } \mathrm{n}(\%) \\
\text { A1: } 16(12.5) \\
\text { A2: } 18(10.8) \\
\text { B: } 5(3.4) \\
\text { B vs. A2: } p<0.05 \\
\text { B vs. A1: } p<0.05\end{array}$ & NR \\
\hline $\begin{array}{l}\text { Dharmasaroja, } \\
2010\end{array}$ & Unclear & $\begin{array}{l}\text { Receipt of tPA } \\
\text { A: } 8 \% \\
\text { B: } 27 \%\end{array}$ & $\begin{array}{l}\text { Protocol violations } 5 \text { of } \\
124 \text { patients after } \\
\text { telehealth, no data } \\
\text { provided from before } \\
\text { period }\end{array}$ \\
\hline Duchesne, 2008 & $\begin{array}{l}\text { Mortality, } n(\%): \\
\text { A: } 17(4.8) \\
\text { B: } 4(7.8) \\
\text { Hospital costs: } \\
\text { A: } \$ 7,632,624 \\
\text { B: } \$ 1,126,683 \\
\text { p<0.001 } \\
\text { LOS at local community hospital, in hours } \\
\text { A: } 47 \\
\text { B: } 1.5, p<0.001\end{array}$ & $\begin{array}{l}\text { Discharge outcomes } \\
\text { Home: } \\
\text { A: NRB: } 61.3 \% \\
\text { Admitted to local community hospital: } \\
\text { A: NRB: } 13.6 \% \\
\text { Transfer to trauma center: } \\
\text { A: } 100 \% \\
\text { B: } 11 \% \\
\text { Mode of transfer } \\
\text { A: } 74.9 \% \text { ground } \\
\text { B: } 70.5 \% \text { ground } \\
\end{array}$ & NA \\
\hline Fong, 2015 & $\begin{array}{l}\text { Excellent outcome mRS } 0-1 \\
\text { (at } 3 \text { months) } \\
\text { A: } 43 \% \\
\text { B: } 52 \% p=0.30 \\
\text { In multivariate analyses, the absence of onsite neurologists } \\
\text { was not associated with negative outcomes. } \\
\text { Mortality } \\
\text { A: } 11.9 \% \\
\text { B: } 8.3 \%, p=0.58\end{array}$ & $\begin{array}{l}\text { Median minutes (IQR) } \\
\text { Onset to door } \\
\text { A: } 54 \text { (43 to } 70) \\
\text { B: } 54 \text { (43 to } 70), p=0.15 \\
\text { Door to } C T \\
\text { A: } 26 \text { ( } 20 \text { to } 38) \\
\text { B: } 30 \text { (25 to } 43), p=0.29 \\
\text { CT to needle } \\
\text { A: } 41 \text { (32 to } 57) \\
\text { B: } 67 \text { (50 to } 82), p<0.001 \\
\text { Door to needle } \\
\text { A: } 71 \text { (60 to } 89) \\
\text { B: } 97 \text { (85 to } 19), p<0.001 \\
\text { Onset to needle } \\
\text { A: } 133 \text { (109 to } 154) \\
\text { B: } 148 \text { (134 to } 170), p=0.012 \\
\end{array}$ & $\begin{array}{l}\text { Symptomatic intracranial } \\
\text { hemorrhage rate } \\
\text { A: } 4.9 \% \\
\text { B: } 4.0 \%, p=1.0\end{array}$ \\
\hline
\end{tabular}




\begin{tabular}{|c|c|c|c|}
\hline $\begin{array}{l}\text { Author, Year } \\
\text { (See Appendix C } \\
\text { for full citation) }\end{array}$ & $\begin{array}{l}\text { Results: } \\
\text { KQ1: Clinical and Economic Outcomes }\end{array}$ & $\begin{array}{l}\text { Results: } \\
\text { KQ2: Intermediate Outcomes }\end{array}$ & $\begin{array}{l}\text { Results: } \\
\text { KQ3: Adverse Effects or } \\
\text { Unintended } \\
\text { Consequences }^{\mathrm{a}} \\
\end{array}$ \\
\hline Goh, 1997 & $\begin{array}{l}\text { Glasgow Outcome Scale } \\
\text { Death: } \\
\text { A: } 14.3 \% \\
\text { B: } 14.3 \% \\
\text { Vegetative: } \\
\text { A: } 7.1 \% \\
\text { B: } 8.6 \% \\
\text { Severe disability: } \\
\text { A: } 10.7 \% \\
\text { B: } 2.9 \% \\
\text { Moderate disability: } \\
\text { A: } 14.3 \% \\
\text { B: } 14.3 \% \\
\text { Good: } \\
\text { A: } 53.6 \% \\
\text { B: } 60 \%\end{array}$ & $\begin{array}{l}\text { Treatments } \\
\text { Overall therapeutic interventions prior to transfer: } \\
\text { A: } 10.7 \% \\
\text { B: } 32.1 \%, p=0.062 \\
\text { Individual interventions: } \\
\text { Endotracheal tube: } \\
\text { A: } 7.1 \% \\
\text { B: } 9.6 \% \\
\text { Mannitol: } \\
\text { A: } 3.6 \% \\
\text { B: } 12.9 \% \\
\text { Other: } \\
\text { A: } 0 \\
\text { B: } 9.6 \%\end{array}$ & $\begin{array}{l}\text { Overall adverse events: } \\
\text { A: } 32.1 \% \\
\text { B: } 6.4 \%, p=0.017 \\
\text { Individual adverse events } \\
\text { Hypoxia: } \\
\text { A: } 3.6 \% \\
\text { B: } 0.0 \% \\
\text { Hypotension: } \\
\text { A: } 3.6 \% \\
\text { B: } 3.2 \% \\
\text { Neurologic deterioration: } \\
\text { A: } 17.7 \% \\
\text { B: } 0.0 \% \\
\text { Convulsions: } \\
\text { A: } 3.6 \% \\
\text { B: } 0.0 \% \\
\text { Missed injuries: } \\
\text { A: } 3.6 \% \\
\text { B: } 3.2 \%\end{array}$ \\
\hline Handschu, 2008 & $\begin{array}{l}\text { Mortality } 10 \text { days post-stroke } \\
\text { A: } 6.8 \% \\
\text { B: } 1.3 \%, p<0.05 \\
\text { Institutional care } 10 \text { days post-stroke } \\
\text { A: } 5.4 \% \\
\text { B: } 2.6 \%, \text { NS } \\
\text { Diagnosis corrected at discharge: } \\
\text { A: } 17.6 \% \\
\text { B: } 7.1 \%, p<0.05 \\
\text { Length of stay, in days: } \\
\text { A: } 12.3 \\
\text { B: } 11.4, N S \\
\text { Admission to stroke ward: } \\
\text { A: } 45.9 \% \\
\text { B: } 59.7 \%, \text { NS } \\
\text { Transfer to stroke center: } \\
\text { A: } 14.9 \% \\
\text { B: } 9.1 \%, p<0.05 \\
\text { Total time for consultation, in minutes: } \\
\text { A: } 27.1 \\
\text { B: } 49.8, p<0.01\end{array}$ & NR & NR \\
\hline
\end{tabular}




\begin{tabular}{|c|c|c|c|}
\hline $\begin{array}{l}\text { Author, Year } \\
\text { (See Appendix C } \\
\text { for full citation) }\end{array}$ & $\begin{array}{l}\text { Results: } \\
\text { KO1: Clinical and Economic Outcomes }\end{array}$ & $\begin{array}{l}\text { Results: } \\
\text { KO2: Intermediate Outcomes }\end{array}$ & $\begin{array}{l}\text { Results: } \\
\text { KQ3: Adverse Effects or } \\
\text { Unintended } \\
\text { Consequences }\end{array}$ \\
\hline Hashimoto, 2001 & $\begin{array}{l}\text { 1-year survival: } \\
\text { A: NR } \\
\text { B: } 72 \% \\
\text { 2-year survival: } \\
\text { A: NR } \\
\text { B: } 42 \% \\
\text { Mean hospitalization time (range), in months: } \\
\text { A: NR } \\
\text { B: } 2.3 \text { (1 to } 3 \text { ) }\end{array}$ & $\begin{array}{l}\text { Treatment within } 24 \text { hours } \\
\text { A: } 17.6 \% \\
\text { B: } 92 \% \\
\text { Successful ambulation for patients who were } \\
\text { nonambulant } \\
\text { A: } n=8,25 \% \\
B: n=6,83 \% \text { ( } p<0.05) \\
\text { Mean onset to radiotherapy time (SD), in days } \\
\text { A: } 7.1(7.9) \\
\text { B: } 0.8(0.4), p<0.05\end{array}$ & NR \\
\hline Heffner, 2015 & $\begin{array}{l}\text { Multivariate analysis OR }(95 \% \mathrm{Cl}) \\
\text { In-hospital mortality } \\
\text { B vs. A: } 11.046(2.785 \text { to } 43.810) \\
\text { B+C vs. A: } 6.835(2.157 \text { to } 21.659) \\
\text { LOS, in days }>6 \text { days OR }(95 \% \mathrm{Cl}) \\
\text { B vs. A: } 4.696(2.428 \text { to } 9.083) \\
\text { B+ C vs. A: } 4.280(2.356 \text { to } 7.774) \\
\\
\text { B vs. A, OR }(95 \% \text { Cl) } \\
\text { Modified Rankin Scale score of } 6: 1.542 \text { (0.635 to } 3.746) \\
\text { Pneumonia } 0.560(0.163 \text { to } 1.929) \\
\text { UTI } 1.486(0.652 \text { to } 3.390) \\
\text { MI/A fib } 0.741(0.259 \text { to } 2.123) \\
\text { GI bleed } 0.702(0.132 \text { to } 3.720) \\
\text { Falls } 0.982(0.116 \text { to } 8.301) \\
\text { Intubated } 0.043(0.007 \text { to } 0.256) \\
\text { Seizure } 0.821(0.142 \text { to } 4.752)\end{array}$ & $\begin{array}{l}\text { Door to needle, in minutes (SD) } \\
\text { A: } 71.98(30.2) \\
\text { B: } 76.57(23.5) \\
\text { A to } B p=0.151 \\
\text { C: } 74.89(28.3) \\
B \text { to } C p=0.683 \\
\text { Onset to needle, in minutes (SD) } \\
\text { A: } 155.6(43.3) \\
\text { B: } 147.57(40.1) \\
\text { A to } B p=0.072 \\
\text { C: } 133.8(42.5) \\
B \text { to } C p=0.028\end{array}$ & NR \\
\hline Hubert, 2016 & NR & $\begin{array}{l}\text { Median (IQR); mean } \pm \text { SD, in minutes } \\
\text { Onset to door time } \\
\text { A: } 88 \text { (60 to } 135) ; 105.3 \pm 55.9 \\
\text { B: } 65 \text { (48 to } 101) ; 80.1 \pm 45.3 \\
\text { p<0.001 } \\
\text { Door to needle time } \\
\text { A: } 18 \text { (13 to } 30) ; 25.1 \pm 20.0 \\
\text { B: } 39 \text { (26 to } 56) ; 44.7 \pm 26.7 \\
\text { p<0.001 } \\
\text { Onset-to-treatment time } \\
\text { A: } 117 \text { (81 to } 168) ; 130.4 \pm 59.1 \\
\text { B: } 115 \text { (87 to } 155) ; 124.8 \pm 49.4 \\
\text { p=0.452 }\end{array}$ & NR \\
\hline
\end{tabular}




\begin{tabular}{|c|c|c|c|}
\hline $\begin{array}{l}\text { Author, Year } \\
\text { (See Appendix C } \\
\text { for full citation) }\end{array}$ & $\begin{array}{l}\text { Results: } \\
\text { KQ1: Clinical and Economic Outcomes }\end{array}$ & $\begin{array}{l}\text { Results: } \\
\text { KQ2: Intermediate Outcomes }\end{array}$ & $\begin{array}{l}\text { Results: } \\
\text { KQ3: Adverse Effects or } \\
\text { Unintended } \\
\text { Consequences }^{\mathrm{a}}\end{array}$ \\
\hline Ickenstein, 2005 & NR & $\begin{array}{l}\text { Patients presenting with } 3 \text { hours and receiving tPA } \\
\text { A: } 10(6 \%) \\
\text { B: } 45(27 \%)\end{array}$ & NR \\
\hline Ionita, 2009 & $\begin{array}{l}\text { Discharge Modified Rankin scale }>3 \\
\text { A: } 61(48 \%) \\
\text { B: } 13(48 \%) p<0.96 \\
\text { Post-thrombolytic intracranial hemorrhage } \\
\text { A: } 26(20 \%) \\
\text { B: } 9(33 \%) p<0.15 \\
\text { Inpatient mortality } \\
\text { A: } 14(11 \%) \\
\text { B: } 3(11 \%) p<0.98 \\
\text { Analysis of variables predictive of mortality or intracranial } \\
\text { hemorrhage } \\
\text { A vs. B =NS }\end{array}$ & $\begin{array}{l}\text { Mean time from onset to needle (SD), in minutes } \\
\text { A: } 143.9(29.5) \\
\text { B: } 130.7(42.1), p<0.13\end{array}$ & NR \\
\hline Itrat, 2016 & NR & $\begin{array}{l}\text { Median door to CT read (IQR), in minutes } \\
\text { A: } 25 \text { (19 to } 35) \\
\text { B: } 25 \text { (20 to } 29) \\
p=0.59 \\
\text { Median door to IV-tPA (IQR), in minutes } \\
\text { A: } 58 \text { (53 to } 68) \\
B: 32 \text { (24 to } 47) \\
p<0.001\end{array}$ & NR \\
\hline Johansson, 2011 & $\begin{array}{l}\text { Good functional outcome at } 3 \text { months } \\
\text { (mRS, dichotomized analysis } 0 \text { to } 1 \text { ) } \\
\text { A: } 43 \% \\
\text { B: } 47 \% \\
p=0.694 \\
\text { Overall mortality at } 3 \text { months } \\
\text { A: } 13 \% \\
\text { B: } 19 \% \\
p=0.248\end{array}$ & $\begin{array}{l}\text { Mean onset to needle, in minutes } \\
\text { A: } 122(n=277) \\
B: 113(n=42) \\
p=0.263\end{array}$ & $\begin{array}{l}\text { Hemorrhagic bleeding } \\
\text { A: } 7.6 \% \\
\text { B: } 6.4 \%\end{array}$ \\
\hline
\end{tabular}




\begin{tabular}{|c|c|c|c|}
\hline $\begin{array}{l}\text { Author, Year } \\
\text { (See Appendix C } \\
\text { for full citation) }\end{array}$ & $\begin{array}{l}\text { Results: } \\
\text { KQ1: Clinical and Economic Outcomes }\end{array}$ & $\begin{array}{l}\text { Results: } \\
\text { KQ2: Intermediate Outcomes } \\
\end{array}$ & $\begin{array}{l}\text { Results: } \\
\text { KQ3: Adverse Effects or } \\
\text { Unintended } \\
\text { Consequences }^{\mathrm{a}} \\
\end{array}$ \\
\hline Kim, 2011 & NR & $\begin{array}{l}\text { Mean time to the scene, in minutes (SD) } \\
\text { A: } 6.6(4.7) \\
\text { B: } 6.6(5.3), p=0.944 \\
\text { Mean treatment time at the scene, in minutes (SD) } \\
\text { A: } 6.3(5.9) \\
\text { B: } 4.4(3.5), p<0.001 \\
\text { Mean transport time, in minutes (SD) } \\
\text { A: } 15.8(9.4) \\
\text { B: } 19.4(9.9), p<0.001 \\
\% \text { receiving medical direction for treatment } \\
\text { A: } 0.3 \\
\text { B: } 8.0, p<0.001 \\
\% \text { receiving medical direction for ambulance } \\
\text { diversion } \\
\text { A: } 0.1 \% \\
\text { B: } 14.4 \%, p<0.001\end{array}$ & NR \\
\hline $\begin{array}{l}\text { Langabeer, } 2016 \\
\text { and } 2017\end{array}$ & $\begin{array}{l}\text { Mortality } \\
\text { A: } 0 \% \\
\text { B: } 0 \% \\
\text { Average unit cost per patient (SD), in USD } \\
\text { A: } \$ 270(77.7) \\
\text { B: } \$ 167(42.7), p<0.0001\end{array}$ & $\begin{array}{l}\text { Ambulance utilization disposition to ED by } \\
\text { ambulance } \\
\text { A: } 74 \% \\
\text { B: } 18 \%, p<0.001 \\
\text { Patient satisfaction } \\
\text { A: } 87 \% \\
\text { B: } 88 \%, p=0.250 \\
\text { Unit productivity } \\
\text { Median total back in service time, in minutes (IQR) } \\
\text { A: } 83(20 \text { to } 140) \\
\text { B:39 (27 to } 90), p<0.001\end{array}$ & NR \\
\hline Macedo, 2016 & $\begin{array}{l}\text { Mortality }(\%) \\
\text { A: } 8 \\
\text { B: } 3 \\
p=0.06\end{array}$ & $\begin{array}{l}\text { Use of pharmacoinvasive strategy } \\
\text { A: } 38 \% \\
B: 55.8 \% \\
p=0.002\end{array}$ & NR \\
\hline
\end{tabular}




\begin{tabular}{|c|c|c|c|}
\hline $\begin{array}{l}\text { Author, Year } \\
\text { (See Appendix C } \\
\text { for full citation) }\end{array}$ & $\begin{array}{l}\text { Results: } \\
\text { KQ1: Clinical and Economic Outcomes }\end{array}$ & $\begin{array}{l}\text { Results: } \\
\text { KQ2: Intermediate Outcomes }\end{array}$ & $\begin{array}{l}\text { Results: } \\
\text { KQ3: Adverse Effects or } \\
\text { Unintended } \\
\text { Consequences }^{\mathrm{a}}\end{array}$ \\
\hline Machado, 2018 & $\begin{array}{l}\text { Mortality \%(n) } \\
\text { A: } 22.4 \%(49) \\
B: 26.3 \%(25) \\
p=0.471 \\
\text { Total hospital costs, in USD } \\
\text { A: } \$ 24,364 \pm 25,068 \\
B: \$ 19,713 \pm 16,550 \\
p=0.274\end{array}$ & $\begin{array}{l}\text { Mean time to antibiotics } \pm \text { SD, in minutes } \\
\text { A: } 163.4 \pm 204.4 \\
\text { B: } 122.3 \pm 83.3 \\
p=0.043 \\
\text { Antibiotics administered within } 3 \text { hours } \\
\text { A: } 71.2 \% \\
\text { B: } 82.1 \% \\
p=0.097 \\
\text { LOS } \pm \text { SD, in days } \\
\text { A: } 10 . \pm 8.5 \\
\text { B: } 8.6 \pm 5.7 \\
p=0.088 \\
\text { Readmission in } 30 \text { days, } \%(n) \\
\text { A: } 11.4(25) \\
\text { B: } 16.8(16) \\
p=0.204\end{array}$ & NR \\
\hline Marcolino, 2013 & $\begin{array}{l}\text { In-hospital mortality } \\
\text { A: } 12.3 \% \\
\text { B: } 7.1 \% \\
p<0.001 \\
\text { The mean cost of admission, in Brazilian reals } \\
\text { A: } \$ 2,480.00 \\
\text { B: } \$ 3,501.00 \\
p<0.001\end{array}$ & $\begin{array}{l}\text { Admissions including ICU stay } \\
\text { A: } 32.4 \% \\
\text { B: } 66.1 \% \\
\text { p<0.001 } \\
\text { Patients admitted to tertiary hospitals } \\
\text { A: } 47.0 \% \\
\text { B: } 69.6 \% \\
\text { p<0.001 }\end{array}$ & NR \\
\hline $\begin{array}{l}\text { Martinez- } \\
\text { Sanchez, } 2014\end{array}$ & $\begin{array}{l}\text { In-hospital mortality } \\
\text { A: } 1(16.7 \%) \\
\text { B: } 1(10 \%) \\
p=1.0 \\
\text { Patients treated with tPA, } \\
\text { Favorable outcome, } n(\%) \\
\text { A: } 4(33.3) \\
\text { B: } 10(55.6), p=0.145\end{array}$ & $\begin{array}{l}\text { Received IV rtPA } \\
\text { A: } 4.7 \% \\
\text { B: } 8 \%, p=0.125 \\
\text { Median door to needle (IQR), in minutes } \\
\text { A: } 143.5(48) \\
\text { B: } 66(54), p<0.0001\end{array}$ & $\begin{array}{l}\text { Stroke recurrence, } n(\%) \\
\text { A: } 0 \\
B: 1(5.6 \%) \\
\text { Intracranial hemorrhage, } \\
n(\%) \\
\text { A: } 2(16.7) \\
B: 0, p=0.152\end{array}$ \\
\hline Martinoni 2011 & $\begin{array}{l}\text { 30-day mortality of patients admitted by EMS } \\
\text { A: } 7.9 \% \\
\text { B: } 5.3 \%, p=0.06\end{array}$ & $\begin{array}{l}\text { First medical contact to balloon, median minutes } \\
\text { (IQR) } \\
\text { A: } 75 \text { (49 to } 112) \\
\text { B: } 50 \text { ( } 30 \text { to } 78.5), p<0.001\end{array}$ & NR \\
\hline
\end{tabular}




\begin{tabular}{|c|c|c|c|}
\hline $\begin{array}{l}\text { Author, Year } \\
\text { (See Appendix C } \\
\text { for full citation) }\end{array}$ & $\begin{array}{l}\text { Results: } \\
\text { KQ1: Clinical and Economic Outcomes }\end{array}$ & $\begin{array}{l}\text { Results: } \\
\text { KQ2: Intermediate Outcomes }\end{array}$ & $\begin{array}{l}\text { Results: } \\
\text { KQ3: Adverse Effects or } \\
\text { Unintended } \\
\text { Consequences }^{\mathrm{a}}\end{array}$ \\
\hline Mathews, 2008 & $\begin{array}{l}\text { Median LOS (range), in days } \\
\text { A: } 3.0(0.1 \text { to } 98.8) \\
\text { B: } 2.0(0.1 \text { to } 144.8), p=0.31\end{array}$ & $\begin{array}{l}\text { Referring doctors felt better able to manage } 59 \% \text { of } \\
\text { cases. } 98 \% \text { felt the equipment was easy to use. } \\
\text { Aeromedical retrievals } \\
\text { A: } 92 \% \\
\text { B: } 78 \%, p=0.009 \\
\text { Not transferred } \\
\text { A: } 5 \% \\
\text { B: } 16 \%, p=0.022 \\
\text { Helicopter flights } \\
\text { A: } 73 \% \\
\text { B: } 52 \%, p=0.004\end{array}$ & $\begin{array}{l}9 \% \text { of cases were felt to } \\
\text { not be better managed }\end{array}$ \\
\hline Mazighi, 2017 & NR & $\begin{array}{l}\text { Received IV rtPA } \\
\text { A: } 4 \\
\text { B: } 21 \\
\text { Median onset to IV rtPA (range), in minutes } \\
\text { A: } 184 \text { (178 to } 258) \\
\text { B: } 145 \text { (110 to } 200)\end{array}$ & NR \\
\hline
\end{tabular}




\begin{tabular}{|c|c|c|c|}
\hline $\begin{array}{l}\text { Author, Year } \\
\text { (See Appendix C } \\
\text { for full citation) }\end{array}$ & $\begin{array}{l}\text { Results: } \\
\text { KQ1: Clinical and Economic Outcomes }\end{array}$ & $\begin{array}{l}\text { Results: } \\
\text { KQ2: Intermediate Outcomes }{ }^{a}\end{array}$ & $\begin{array}{l}\text { Results: } \\
\text { KQ3: Adverse Effects or } \\
\text { Unintended } \\
\text { Consequences }^{\mathrm{a}} \\
\end{array}$ \\
\hline Meyer, 2008 & $\begin{array}{l}\text { Barthel Index score or } 95 \text { to } 100 \text { at } 90 \text { days: } \\
\text { A: } 54 \% \\
\text { B: } 43 \% \\
\text { OR: } 0.6 \text { ( } 95 \% \mathrm{Cl} 0.4 \text { to } 1.1), p=0.13 \\
\text { Modified Rankin scale score of } 0 \text { to } 1 \text { at } 90 \text { days: } \\
\text { A: } 47 \% \\
\text { B: } 34 \% \\
\text { OR: } 0.6(95 \% \mathrm{Cl} 0.3 \text { to } 1.1), p=0.09 \\
\text { Overall mortality: } 13 \% \text { vs. } 19 \% ; 1.6(0.8-3.4), p=0.27 \\
\text { tPA use: } \\
\text { A: } 23 \% \\
\text { B: } 28 \%, p=0.42\end{array}$ & $\begin{array}{l}\text { Mean times (SD), in minutes } \\
\text { Onset to decision: } \\
\text { A: } 230.6(222.4) \\
\text { B: } 258.0(229.9), p=0.07 \\
\text { Onset to thrombolysis ( } n=30 \text { vs. } 55) \text { : } \\
\text { A: } 143.0(33.1) \\
\text { B: } 157.2(37.7), p=0.14 \\
\text { Times from door/call: } \\
\text { Door to decision ( } n=69 \text { vs. } 77) \text { : } \\
\text { A: } 95.5 \text { (64.1) } \\
\text { B: } 99.8(43.5), p=0.20 \\
\text { Call to decision: } \\
\text { A: } 55.2(33.9) \\
\text { B: } 64.7(29.1), p=0.03 \\
\text { Times from consent: } \\
\text { Consent to decision: } \\
\text { A: } 22.9(23.6) \\
\text { B: } 32.0(17.3), p=0.0001 \\
\text { Consent to thrombolysis ( } n=24 \text { vs. } 30) \text { : } \\
\text { A: } 44.8(21.4) \\
\text { B: } 51.2(17.8), p=0.16 \\
\text { Decision to thrombolysis ( } n=24 \text { vs. } 30) \text { : } \\
\text { A: } 15.6(8.5) \\
\text { B: } 10.0 \text { (9.8), } p=0.02 \\
\% \text { correct decision; OR }(95 \% \text { Cl) } \\
\text { Level } 2 b(S D A C): \\
\text { A: } 82 \% \\
\text { B: } 98 \% ; 10.9(2.7 \text { to } 44.6) ; p=0.0009\end{array}$ & NR \\
\hline $\begin{array}{l}\text { Mohr, } 2018 \\
\text { (same patients } \\
\text { as Mohr 2017) }\end{array}$ & $\begin{array}{l}\text { Mortality, adjusted OR }(95 \% \mathrm{Cl}) \\
\text { Telehealth used: } 0.9(0.5 \text { to } 1.4) \\
\text { Telehealth available: } 1.2(0.9 \text { to } 1.7)\end{array}$ & $\begin{array}{l}\text { ED LOS, mean adjusted difference }(95 \% \mathrm{Cl}) \\
\text { Telehealth used: }-12.2(-27.6 \text { to } 3.2) \\
\text { Telehealth available: } 15.4(9.8 \text { to } 21.0) \\
\text { Interhospital transfer, adjusted OR }(95 \% \mathrm{Cl}) \\
\text { Telehealth used: } 1.2(0.8 \text { to } 1.8) \\
\text { Telehealth available: } 1.2(1.1 \text { to } 1.4) \\
\text { CT scans, adjusted OR (95\% Cl) } \\
\text { Telehealth used: } 0.9(0.7 \text { to } 1.2) \\
\text { Telehealth available: } 1.6(1.3 \text { to } 1.9) \\
\text { X-Rays, adjusted OR }(95 \% \mathrm{Cl}) \\
\text { Telehealth used: } 1.1(0.8 \text { to } 1.4) \\
\text { Telehealth available: } 1.3(1.1 \text { to } 1.5)\end{array}$ & NR \\
\hline
\end{tabular}




\begin{tabular}{|c|c|c|c|}
\hline $\begin{array}{l}\text { Author, Year } \\
\text { (See Appendix C } \\
\text { for full citation) }\end{array}$ & $\begin{array}{l}\text { Results: } \\
\text { KQ1: Clinical and Economic Outcomes }\end{array}$ & $\begin{array}{l}\text { Results: } \\
\text { KQ2: Intermediate Outcomes }\end{array}$ & $\begin{array}{l}\text { Results: } \\
\text { KQ3: Adverse Effects or } \\
\text { Unintended } \\
\text { Consequences }^{\mathrm{a}}\end{array}$ \\
\hline $\begin{array}{l}\text { Mohr, } 2017 \\
\text { (same patients } \\
\text { as Mohr 2018) }\end{array}$ & $\begin{array}{l}\text { Mortality, } \mathrm{n}(\%) \\
\text { A: } 16(0.6 \%) \\
\text { B: } 8(2.7 \%)\end{array}$ & $\begin{array}{l}\text { Discharged home, } \mathrm{n}(\%) \\
\text { A: } 923(36.8 \%) \\
\text { B: } 66(22.2 \%) \\
\text { Admitted, } \mathrm{n}(\%) \\
\text { A: } 395(15.7 \%) \\
\text { B: } 43(14.5 \%) \\
\text { Inter-hospital transfer, } \mathrm{n}(\%) \\
\text { A: } 1127(44.9 \%) \\
\text { B: } 170(57.2 \%) \\
\text { Univariate analysis, inter-hospital transfer } \\
\text { A: } 8.5 \% \\
\text { B: } 13.1 \%,(95 \% \mathrm{Cl} 2.3 \% \text { to } 6.9 \%) \\
\text { Multivariable analysis, inter-hospital transfer } \\
\text { AOR: } 1.28,(95 \% \mathrm{Cl} 0.94 \text { to } 1.75)\end{array}$ & NR \\
\hline Nagao, 2012 & $\begin{array}{l}\text { Mortality, } n(\%) \\
\text { A: } 3(13 \%) \\
\text { B: } 3(10 \%), p=0.6(D F 3) \\
\text { Discharged home, } n(\%) \\
\text { A: } 14(47 \%) \\
\text { B: } 12(50 \%) \\
\text { Other hospital, } n(\%) \\
\text { A: } 13(43 \%) \\
\text { B: } 8(33 \%)\end{array}$ & $\begin{array}{l}\text { Thrombolysed, } \mathrm{n}(\%) \\
\text { A: } 0 \\
\text { B: } 8(33 \%) \\
\text { Intracerebral hemorrhage } \\
\text { A: } 0 \\
\text { B: } 0\end{array}$ & $\begin{array}{l}\text { Complications } \\
\text { Further stroke, } n(\%) \\
\text { A: } 0 \\
\text { B: } 2(8.3 \%)\end{array}$ \\
\hline $\begin{array}{l}\text { Narasimhan, } \\
2015\end{array}$ & $\begin{array}{l}\text { Adjusted utilization outcomes OR }(95 \% \mathrm{Cl}), \mathrm{p} \text {-value } \\
\text { Inpatient admission } \\
0.41(0.19 \text { to }-0.88), p=0.022 \\
\text { LOS, in days } \\
-0.43(-0.17 \text { to }-0.14), p=0.002 \\
\text { Change in inpatient charges, } 30 \text { days post ED visit: } \\
-2,338(-4,582 \text { to }-94), p=0.041 \\
\text { Change in total health care charges, } 30 \text { days post ED visit: } \\
-649(-3,221 \text { to } 1,902), p=0.614\end{array}$ & $\begin{array}{l}\text { Adjusted utilization outcomes OR }(95 \% \mathrm{Cl}), \mathrm{p} \text {-value } \\
30 \text {-day outpatient followup } \\
5.44 \text { ( } 4.40 \text { to } 6.72), p<0.001 \\
90 \text { day outpatient followup } \\
5.65 \text { (4.60 to } 6.93), p<0.001\end{array}$ & NR \\
\hline Natafgi, 2017 & $\begin{array}{l}\text { Mortality, } \mathrm{n}(\%) \\
\text { A: } 791(0.5 \%) \\
\text { B: } 358(4.0 \%) \\
\text { Transferred, } \mathrm{n}(\%) \\
\text { A: } 1059(0.7 \%) \\
\text { B: } 4224(47.6 \%) \\
\text { Estimated savings per avoided transfer, in USD: } \$ 3,823\end{array}$ & NR & NR \\
\hline
\end{tabular}




\begin{tabular}{|c|c|c|c|}
\hline $\begin{array}{l}\text { Author, Year } \\
\text { (See Appendix C } \\
\text { for full citation) }\end{array}$ & $\begin{array}{l}\text { Results: } \\
\text { KQ1: Clinical and Economic Outcomes } \\
\end{array}$ & $\begin{array}{l}\text { Results: } \\
\text { KQ2: Intermediate Outcomes } \\
\end{array}$ & $\begin{array}{l}\text { Results: } \\
\text { KQ3: Adverse Effects or } \\
\text { Unintended } \\
\text { Consequences }^{\mathrm{a}} \\
\end{array}$ \\
\hline $\begin{array}{l}\text { Nguyen-Huynh, } \\
2018\end{array}$ & NR & $\begin{array}{l}\text { Alteplase administration per month, mean } \pm S D \\
\text { A: } 34.4 \pm 12.4 \\
\text { B: } 61.8 \pm 12.4, p<0.001 \\
\text { Door to needle time, mean minutes } \pm S D \\
\text { A: } 63.2 \pm 31.2 \\
\text { B: } 41.8 \pm 30.6, p<0.001\end{array}$ & $\begin{array}{l}\text { Symptomatic intracranial } \\
\text { hemorrhage rates } \\
\text { A: } 2.2 \% \\
\text { B: } 3.8 \%, p=0.29\end{array}$ \\
\hline Noble, 2005 & $\begin{array}{l}\text { Returned to normal activity in } 7 \text { days, }(95 \% \mathrm{Cl}) \\
\text { A: } 47.6 \%(34.9 \text { to } 60.6) \\
\text { B: } 47.0 \%(41.0 \text { to } 53.2) \\
\text { Mean } 7 \text {-day cost difference per patient in GPB }(95 \% \mathrm{Cl}) \text {, } \\
\text { [95\% bias corrected } \mathrm{Cl}] \\
\text { NHS cost: } £ 39.47(-1.28 \text { to } 80.21),[28.31 \text { to } 73.67] \\
\text { Patient/family cost: } £ 14.28 \text { (-26.59 to } 55.15),[-11.18 \text { to } \\
25.85] \\
\text { Total cost: } £ 53.75 \text { (-6.97 to } 114.46),[24.10 \text { to } 101.81]\end{array}$ & $\begin{array}{l}\text { Required a change in treatment }(95 \% \mathrm{Cl}) \\
\text { A: } 6.3 \%(1.8 \text { to } 15.5) \\
\text { B: } 9.6 \%(6.4 \text { to } 13.8)\end{array}$ & NR \\
\hline Ortolani, 2006 & $\begin{array}{l}\text { Overall mortality, } n(\%) \\
\text { A: } 23(7.3) \\
\text { B: } 13(7.4) \\
\text { C: } 8(4.8) \\
p=0.537 \\
\text { In-hospital mortality among cardiogenic shock subgroup } \\
(n=80) \\
\text { A: } 48.1 \%(13 / 27) \\
\text { B: } 37.5 \%(9 / 24) \\
\text { C: } 13.8 \%(4 / 29) \\
p=0.019\end{array}$ & $\begin{array}{l}\text { Median treatment delay (IQR), in minutes } \\
\text { A: } 191 \text { (135 to } 318.7) \\
\text { B: } 236(163.7 \text { to } 363.2) \\
\text { C: } 146(108.2 \text { to } 214.5), p=0.001\end{array}$ & $\begin{array}{l}\text { Nothing designated } \\
\text { specifically as } \\
\text { complications, however } \\
\text { not sure if some of the } \\
\text { results reported in the } \\
\text { table are considered } \\
\text { complications. }\end{array}$ \\
\hline
\end{tabular}




\begin{tabular}{|c|c|c|c|}
\hline $\begin{array}{l}\text { Author, Year } \\
\text { (See Appendix C } \\
\text { for full citation) }\end{array}$ & $\begin{array}{l}\text { Results: } \\
\text { KQ1: Clinical and Economic Outcomes }\end{array}$ & $\begin{array}{l}\text { Results: } \\
\text { KQ2: Intermediate Outcomes }\end{array}$ & $\begin{array}{l}\text { Results: } \\
\text { KQ3: Adverse Effects or } \\
\text { Unintended } \\
\text { Consequences }^{\mathrm{a}} \\
\end{array}$ \\
\hline Ortolani, 2007 & $\begin{array}{l}\text { Mortality } \\
\text { In-hospital cardiac mortality: } \\
\text { A: } 44 \% \\
\text { B: } 21 \% \\
\text { OR: } 0.34 \text { ( } 95 \% \mathrm{Cl} 0.14 \text { to } 0.81), p=0.02 \\
\text { In-hospital all-cause mortality: } \\
\text { A: } 46 \% \\
\text { B: } 21 \% \\
\text { OR: } 0.32(95 \% \mathrm{Cl} 0.14 \text { to } 0.77), p=0.01 \\
1-y e a r \text { survival rate: } \\
\text { A: } 52 \% \\
\text { B: } 74 \% \\
\text { OR: NR, } p=0.019 \\
\text { Median total ischemic time, in minutes } \\
\text { A: } 212(150 \text { to } 366) \\
\text { B: } 142(106 \text { to } 187)\end{array}$ & NR & NR \\
\hline Paik, 2017 & NR & $\begin{array}{l}\text { Response time, in minutes } \\
\text { A: } 48.3 \\
\text { B: } 8.9 \\
p<0.001 \\
\text { Overall agreement, } n(\%): 38(90.5 \%) \\
\end{array}$ & NR \\
\hline Patel, 2015 & ( & $\begin{array}{l}\text { Average call duration, in seconds } \\
\text { A: } 186 \\
\text { B: } 139, p=0.055 \\
\text { Medical Command Officer Survey, } n=12 \\
100 \% \text { found video intuitive } \\
92 \% \text { disposition based on phone report was difficult } \\
80 \% \text { video provided better understanding of patient } \\
\text { condition } \\
70 \% \text { video assisted disposition } \\
80 \% \text { believed video should be used for transport } \\
\end{array}$ & NR \\
\hline Pedersen 2009 & $\begin{array}{l}\text { All-cause mortality or nonfatal MI hazard ratio }(95 \% \mathrm{Cl}) \\
0.67 ;(0.46 \text { to } 0.97), p=0.035\end{array}$ & $\begin{array}{l}\text { Median door to balloon time (IQR), in minutes } \\
\text { A: } 103 \text { (80 to } 135) \\
\text { B: } 83(67 \text { to } 100) \\
\text { p }<0.001\end{array}$ & NR \\
\hline
\end{tabular}




\begin{tabular}{|c|c|c|c|}
\hline $\begin{array}{l}\text { Author, Year } \\
\text { (See Appendix C } \\
\text { for full citation) }\end{array}$ & $\begin{array}{l}\text { Results: } \\
\text { KQ1: Clinical and Economic Outcomes }\end{array}$ & $\begin{array}{l}\text { Results: } \\
\text { KQ2: Intermediate Outcomes }\end{array}$ & $\begin{array}{l}\text { Results: } \\
\text { KQ3: Adverse Effects or } \\
\text { Unintended } \\
\text { Consequences }^{\mathrm{a}}\end{array}$ \\
\hline Pedragosa, 2009 & $\begin{array}{l}\text { Urgent ambulance transfer: } \\
\text { A: } 17 \% \\
\text { B: } 10 \%, p=0.04 \\
\text { Specialized neurologist evaluation: } \\
\text { A: } 17 \% \\
\text { B: } 38 \%, p<0.001 \\
\text { Unnecessary transfers to the stroke center: } \\
\text { A: } 51 \% \\
\text { B: } 20 \%, p=0.02 \\
\text { Stroke unit admissions: } \\
\text { A: } 11 \% \\
\text { B: } 8 \%, p=0.34 \\
\text { tPA use: } \\
\text { A: } 4.5 \% \\
\text { B: } 9.6 \%, p=0.07 \\
\text { tPA in } 0 \text { to } 3 \text { hour window } \\
\text { A: } 30 \% \text { in } 2006 \\
\text { B: } 68 \% \text { in } 2007, p=0.04 \\
\end{array}$ & $\begin{array}{l}\text { Onset to needle, in minutes } \\
\text { A: } 210 \\
B: 162, p=0.05\end{array}$ & NR \\
\hline Pervez 2010 & $\begin{array}{l}\text { In-hospital mortality } \\
\text { A: } 17.4 \% \\
\text { B: } 14.9 \%, p=0.57 \\
\text { Discharge outcomes } \\
\text { Home } \\
\text { A: } 30.5 \% \\
\text { B: } 28.6 \% \\
p=0.74 \\
\text { Inpatient rehabilitation } \\
\text { A: } 55.3 \% \\
\text { B: } 53.3 \% \\
p=0.67 \\
\text { Ambulatory at discharge } \\
\text { A: } 77.7 \% \\
\text { B: } 73.8 \% \\
p=0.5\end{array}$ & $\begin{array}{l}\text { Mean LOS } \pm S D \text {, in days } \\
\text { A: } 7.6 \pm 6.5 \\
\text { B: } 5.9 \pm 3.7 \\
\text { p<0.001 } \\
\text { Median onset to tPA time (IQR), in minutes } \\
\text { A: } 130(102.5,162.8) \\
\text { B: } 140(117.3,165.3) \\
\text { p }=0.06\end{array}$ & $\begin{array}{l}\text { Symptomatic ICH }<36 \\
\text { hours } \\
\text { A: } 5.2 \% \\
\text { B: } 3.9 \% \\
p=0.58 \\
\text { Systemic hemorrhage } \\
<36 \text { hours } \\
\text { A: } 2.6 \% \\
\text { B: } 0.6 \% \\
p=0.14\end{array}$ \\
\hline
\end{tabular}




\begin{tabular}{|c|c|c|c|}
\hline $\begin{array}{l}\text { Author, Year } \\
\text { (See Appendix C } \\
\text { for full citation) }\end{array}$ & $\begin{array}{l}\text { Results: } \\
\text { KQ1: Clinical and Economic Outcomes }\end{array}$ & $\begin{array}{l}\text { Results: } \\
\text { KQ2: Intermediate Outcomes }\end{array}$ & $\begin{array}{l}\text { Results: } \\
\text { KQ3: Adverse Effects or } \\
\text { Unintended } \\
\text { Consequences }^{\mathrm{a}} \\
\end{array}$ \\
\hline Poon, 2001 & $\begin{array}{l}\text { Favorable outcome } \\
\text { A: } 38.2 \% \\
\text { B: } 30.8 \% \\
\text { C: } 43.5 \% \\
\text { (no statistical test reported) }\end{array}$ & $\begin{array}{l}\text { Time between referral and decision to transfer, in } \\
\text { minutes } \\
\text { A: } 1.8 \\
\text { B: } 49.2 \\
\text { C: } 65.4 \\
\text { Actual time spent in consultation, in minutes } \\
\text { A: } 1.8 \\
\text { B: } 13.2 \\
\text { C: } 10.2 \\
\text { Time between referral and image received, in } \\
\text { minutes } \\
\text { A: NA } \\
\text { B: } 36 \\
\text { C: } 55\end{array}$ & NR \\
\hline Saffle, 2009 & $\begin{array}{l}\text { Patients requiring air transportation } \\
\text { A: } 100 \% \\
\text { B: } 44.3 \% \\
p<0.05 \\
\text { Mortality, } n(\%) \\
\text { A: } 1(3.6 \%) \\
\text { B: } 0\end{array}$ & $\begin{array}{l}\text { Received emergency air transport } \\
\text { A: } 100 \% \\
\text { B: } 44.3 \% \\
\text { p } 0.05 \\
\text { Larger burn size } \\
\text { A: } 6.5 \% \\
\text { B: } 9.0 \% \\
\text { p=NS } \\
\text { Median LOS (IQR), in days } \\
\text { A: } 8(24) \\
\text { B: } 13(23) \\
\text { p=NS } \\
\text { Referring physicians felt that telemedicine changed } \\
\text { their decision to transport ("strongly agree" or } \\
\text { "agree"), in almost half of cases } \\
\text { Satisfied with telemedicine visit ( } \% \text { strongly agree) } \\
\text { Burn center physicians: } 76.9 \% \\
\text { Referring physicians: } 86.4 \% \\
\text { Patients transferred: } 75.9 \% \\
\text { Patients non transferred: } 69.2 \% \\
\text { All respondents: } 78.2 \%\end{array}$ & NR \\
\hline
\end{tabular}




\begin{tabular}{|c|c|c|c|}
\hline $\begin{array}{l}\text { Author, Year } \\
\text { (See Appendix C } \\
\text { for full citation) }\end{array}$ & $\begin{array}{l}\text { Results: } \\
\text { KQ1: Clinical and Economic Outcomes }\end{array}$ & $\begin{array}{l}\text { Results: } \\
\text { KQ2: Intermediate Outcomes }\end{array}$ & $\begin{array}{l}\text { Results: } \\
\text { KQ3: Adverse Effects or } \\
\text { Unintended } \\
\text { Consequences }^{\mathrm{a}}\end{array}$ \\
\hline Sairanen, 2011 & $\begin{array}{l}\text { Mortality } 3 \text { months, } \mathrm{n}(\%) \\
\text { A: } 93 / 985(10.2 \%) \\
\text { B: } 7 / 61(11.5 \%) \\
\text { mRS 0-2 (3 months), n (\%) } \\
\text { A: 556/985 (58.1\%) } \\
\text { B: } 28 / 57(49.1 \%) \\
\text { mRS 0-1 (3 months), n (\%) } \\
\text { A: 352/957 (36.8\%) } \\
\text { B: } 17 / 57(29.4 \%) \\
\end{array}$ & NR & $\begin{array}{l}\text { Intracranial bleeding, } n \\
(\%) \\
\text { A: } 93 / 985(9.4 \%) \\
\text { B: } 4 / 60(6.7 \%)\end{array}$ \\
\hline $\begin{array}{l}\text { Sanchez-Ross, } \\
2011\end{array}$ & $\begin{array}{l}\text { A: } n=43 \\
\text { B: } n=72 \\
\text { Median length of stay (IQR), in days } \\
\text { A: } 5.5 \text { ( } 3 \text { to } 10.5) \\
\text { B: } 3 \text { (2 to } 4), U=378, p<0.001 \\
\text { Mortality } \\
\text { A: } 6 \% \\
\text { B: } 1.1 \%, p=0.125 \\
\text { Peak troponin, ng/ml } \\
\text { A: } 87.6(38.4 \text { to } 227) \\
\text { B: } 39.5(11 \text { to } 120.5), U=889.5, p=0.005 \\
\text { CPK-MB, ng/ml } \\
\text { A: } 290.3(102.4 \text { to } 484) \\
\text { B: } 126.1(37.2 \text { to } 280.5), U=883, p=0.001 \\
\text { Post-Infarction LVEF } \\
\text { A: } 35 \%(25 \% \text { to } 52 \%) \\
\text { B: } 50 \%(35 \% \text { to } 55 \%), U=1075, p=0.004\end{array}$ & $\begin{array}{l}\text { Median door to balloon time (IQR), in minutes } \\
\text { A: } n=43 ; 119 \text { (96 to } 178) \\
\text { B: } n=72 ; 63 \text { (42 to } 87), U=779.5, p<0.00004\end{array}$ & NR \\
\hline Schwab , 2007 & $\begin{array}{l}3 \text { month mortality } \\
\text { A: } 11.5 \% \\
\text { B: } 11.2 \% \\
6 \text { month mortality } \\
\text { A: } 13 \% \\
\text { B: } 14.2 \%, p=0.45 \\
\text { Good functional outcome at } 6 \text { months } \\
\text { A: } 30.9 \% \\
\text { B: } 39.5 \%, p=0.10\end{array}$ & $\begin{array}{l}\text { Mean onset to treatment time, in minutes } \pm \text { SD }(95 \% \\
\text { Cl) } \\
\text { A: } 145.88 \pm 46.99(126.9 \text { to } 164.87) \\
\text { B: } 127.57 \pm 36.33(117.14 \text { to } 138.01) \\
p=0.45\end{array}$ & NR \\
\hline
\end{tabular}




\begin{tabular}{|c|c|c|c|}
\hline $\begin{array}{l}\text { Author, Year } \\
\text { (See Appendix C } \\
\text { for full citation) }\end{array}$ & $\begin{array}{l}\text { Results: } \\
\text { KQ1: Clinical and Economic Outcomes }\end{array}$ & $\begin{array}{l}\text { Results: } \\
\text { KQ2: Intermediate Outcomes }\end{array}$ & $\begin{array}{l}\text { Results: } \\
\text { KQ3: Adverse Effects or } \\
\text { Unintended } \\
\text { Consequences }^{\mathrm{a}}\end{array}$ \\
\hline Sejersten, 2008 & $\begin{array}{l}\text { Mortality } \\
\text { A: } 6.9 \% \\
\text { B: } 6.0 \% \\
p=0.67\end{array}$ & $\begin{array}{l}\text { Time to percutaneous coronary intervention }(\mathrm{PCl}) \text {, in } \\
\text { minutes } \\
\mathrm{A}: 127 \\
\mathrm{~B}: 74 \\
\mathrm{p}<0.001 \\
\text { Door to } \mathrm{PCl} \text {, in minutes } \\
\mathrm{A}: 97 \\
\mathrm{~B}: 34 \\
\mathrm{p}<0.001\end{array}$ & $\begin{array}{l}\text { Arrhythmia } \\
\text { A: } 6(7 \%) \\
\text { B: } 17(10 \%)\end{array}$ \\
\hline Southard, 2014 & $\begin{array}{l}\text { Mean LOS, in hours (range), (SD), median } \\
\text { A: } 31.7,(12.6 \text { to } 65.9),(14.1), 26.3 \\
B: 17.0,(3.0 \text { to } 69.5),(18.0), 8.2 \\
p<0.001\end{array}$ & $\begin{array}{l}\text { Mean order to consult time, in hours (range), (SD), } \\
\text { median } \\
\text { A: } 16.2,(0.5 \text { to } 52.9),(13.2), 14.2 \\
\text { B: } 5.4,(0.02 \text { to } 25.1),(6.4), 2.6 \\
p<0.001 \\
\text { Mean door to consult time, in hours (range), (SD), } \\
\text { median } \\
\text { A: } 22.7,(6.3 \text { to } 56.6),(12.6), 19.6 \\
\text { B: } 10.5,(1.7 \text { to } 50.9),(10.2), 5.9 ; p<0.001\end{array}$ & NR \\
\hline Switzer, 2009 & NR & $\begin{array}{l}\text { Onset to treatment time (SD), }(95 \% \mathrm{Cl}) \text {, in minutes } \\
\text { A: } 145.88 \text { (46.99), (126.9 to } 164.87) \\
\text { B: } 127.57(36.33),(117.14 \text { to } 138.01)\end{array}$ & NR \\
\hline Taqui, 2017 & NR & $\begin{array}{l}\text { Median alarm to CT scan completion times, in } \\
\text { minutes } \\
\text { A: } 56 \\
B: 33 \\
p<0.0001 \\
\text { Median alarm to thrombolysis times, in minutes } \\
\text { A: } 94 \\
B: 55.5 \\
p<0.0001 \\
\text { Median door to thrombolysis times, in minutes } \\
\text { A: } 58 \\
B: 31.5 \\
p=0.0012 \\
\text { Symptom onset to thrombolysis times, in minutes } \\
\text { A: } 122.5 \\
B: 97 \\
p=0.0485\end{array}$ & NR \\
\hline
\end{tabular}




\begin{tabular}{|c|c|c|c|}
\hline $\begin{array}{l}\text { Author, Year } \\
\text { (See Appendix C } \\
\text { for full citation) }\end{array}$ & $\begin{array}{l}\text { Results: } \\
\text { KQ1: Clinical and Economic Outcomes }\end{array}$ & $\begin{array}{l}\text { Results: } \\
\text { KQ2: Intermediate Outcomes }\end{array}$ & $\begin{array}{l}\text { Results: } \\
\text { KQ3: Adverse Effects or } \\
\text { Unintended } \\
\text { Consequences }^{\mathrm{a}}\end{array}$ \\
\hline Theiss, 2013 & $\begin{array}{l}\text { Mortality all stroke patients } \\
\text { Before } \\
\text { A: } 12.4 \% \\
\text { B: } 9.6 \% \\
\text { C: } 10.4 \% \\
\text { After } \\
\text { A: } 9.6 \% \\
\text { B: } 8.8 \% \\
\text { C: } 8.7 \% \\
\end{array}$ & $\begin{array}{l}\text { Likelihood of receiving tPA } \\
\text { C vs A, before: OR 5.7, } p=0.07 \\
\text { C vs A, after: OR 4.5, } p<0.0001 \\
\text { B vs C, before: OR 3.7, } p<0.0001 \\
\text { B vs C, after: OR 1.3, } p=0.06\end{array}$ & NR \\
\hline Traub, 2013 & NR & $\begin{array}{l}\text { Admitted, mean (SD) } \\
\text { A: } 64(32.65) \\
\text { B: } 12(33.33) \\
p=0.9363 \\
\text { Mean LOS (SD), }(95 \% \mathrm{Cl}) \text {, in minutes } \\
\text { A: } 258.08(171.71),(233.89 \text { to } 282.27) \\
\text { B: } 273.58(125.35),(231.17 \text { to } 316.00) \\
p=0.5246 \\
\\
\text { Mean time to physician evaluation (SD), (95\% Cl), in } \\
\text { minutes } \\
\text { A: } 42.14 \text { (30.59), (37.80 to } 46.48) \\
\text { B: } 15.94(15.22),(10.80 \text { to } 21.09) \\
p<0.0001\end{array}$ & NR \\
\hline Tsai, 2007 & $\begin{array}{l}\text { Total annual savings on emergency air medical transports, } \\
\text { in USD } \\
\$ 448,986\end{array}$ & $\begin{array}{l}\text { Average flights per month } \\
\text { A: } 19.6 \\
\text { B: } 12.5 \\
\text { Reduction of } 36.3 \% \text { (no statistical test reported) }\end{array}$ & NR \\
\hline
\end{tabular}




\begin{tabular}{|c|c|c|c|}
\hline $\begin{array}{l}\text { Author, Year } \\
\text { (See Appendix C } \\
\text { for full citation) }\end{array}$ & $\begin{array}{l}\text { Results: } \\
\text { KQ1: Clinical and Economic Outcomes }\end{array}$ & $\begin{array}{l}\text { Results: } \\
\text { KQ2: Intermediate Outcomes }\end{array}$ & $\begin{array}{l}\text { Results: } \\
\text { KQ3: Adverse Effects or } \\
\text { Unintended } \\
\text { Consequences }^{a}\end{array}$ \\
\hline Wong, 2006 & $\begin{array}{l}\text { Favorable outcome at } 6 \text { months } \\
\text { A: } 130, n=232 \\
\text { B: } 146, n=311 \\
\text { C: } 124, n=167 \\
\text { Total mortality at } 6 \text { months, } n(\%) \\
\text { A: } 81(35 \%) \\
\text { B: } 59(25 \%) \\
\text { C: } 79(34 \%) \\
\text { B vs. A: } p=0.025 \\
\text { C vs. A: } p=0.923 \\
\text { C vs. } B: p=0.043 \\
\text { Average cost per patient, in Hong Kong dollars } \\
\text { A: } 14,075 \\
\text { B: } 14,455 \\
\text { C: } 16,370\end{array}$ & $\begin{array}{l}\text { Diagnostic accuracy: } \\
\text { A: } 63.8 \% \\
\text { B: } 89.1 \% \\
\text { C: } 87.7 \% \\
\text { B vs. A: } p<0.0005 \\
\text { C vs. A: } p<0.0005 \\
\text { Time from referral to decision, in hours (SD) } \\
\text { A: } 0.70(1.9) \\
\text { B: } 1.0(1.8) \\
\text { C: } 1.30(2.5) \\
\text { C vs. A } p=0.003\end{array}$ & $\begin{array}{l}30 \% \text { of failure rate in the } \\
\text { videoconferencing arm } \\
\text { due to lack of a dedicated } \\
\text { team of medical escort } \\
\text { staff and the fixed site VC } \\
\text { at the Accident and } \\
\text { Emergency Department }\end{array}$ \\
\hline Yang, 2015 & NR & $\begin{array}{l}\text { Hospital admissions } \\
\text { A: } 87.5 \% \\
\text { B: } 59.5 \%, p<0.05 \\
\text { Observed/expected admission ratio }(95 \% \mathrm{Cl}) \\
\text { Pediatric Risk of Admission II, overall } \\
\text { A: } 2.58 \text { (2.00 to } 3.32) \\
\text { B: } 2.36(1.80 \text { to } 3.10) \\
\text { Revised Pediatric Emergency Assessment Tool, } \\
\text { overall } \\
\text { A: } 2.57(1.99 \text { to } 3.31) \\
\text { B: } 2.34(1.78 \text { to } 3.07)\end{array}$ & NR \\
\hline Zaidi, 2011 & $\begin{array}{l}90 \text { day mortality } \\
\text { A: } 30.4 \% \\
\text { B: } 31.6 \% \\
p=0.6 \\
\text { mRS } \leq 1 \text { at } 90 \text { days } \\
\text { A: } 22.0 \% \\
\text { B: } 34.9 \% \\
\text { mRS } \leq 2 \text { at } 90 \text { days } \\
\text { A: } 37.5 \% \\
\text { B: } 42.1 \% \\
p=0.7\end{array}$ & $\begin{array}{l}\text { Mean onset to treatment time (SD), in minutes } \\
\text { A: } 156.7(31.6) \\
\text { B: } 145.5(42.8), p=0.09 \\
\text { Mean arrival to treatment time (SD), in minutes } \\
\text { A: } 67.8(26.1) \\
\text { B: } 89.9(36.3), p=0.01 \\
\text { Favorable outcome } \\
\text { A: } 37.5 \% \\
\text { B: } 42.1 \% \\
p=0.7\end{array}$ & $\begin{array}{l}\text { Asymptomatic intracranial } \\
\text { hemorrhage } \\
\text { A: } 18.6 \% \\
\text { B: } 16.2 \% \\
p=0.7 \\
\text { Symptomatic intracranial } \\
\text { hemorrhage } \\
\text { A: } 5.1 \% \\
\text { B: } 1.2 \% \\
p=0.1\end{array}$ \\
\hline
\end{tabular}




\begin{tabular}{|l|l|l|l|}
\hline $\begin{array}{l}\text { Author, Year } \\
\text { (See Appendix C } \\
\text { for full citation) }\end{array}$ & $\begin{array}{l}\text { Results: } \\
\text { KQ1: Clinical and Economic Outcomes }\end{array}$ & $\begin{array}{l}\text { Results: } \\
\text { KQ3: Adverse Effects or } \\
\text { Unintended } \\
\text { Consequences }^{\text {a }}\end{array}$ \\
\hline Zanini, 2008 & $\begin{array}{l}\text { Total in-hospital mortality } \\
\text { A: } 23 / 263(8.7 \%)\end{array}$ & $\begin{array}{l}\text { NR } \\
\text { KQ2: Intermediate Outcomes }\end{array}$ \\
& $\begin{array}{l}\text { B: } 4 / 136(3 \%) \\
\text { p=0.039 }\end{array}$ & $\begin{array}{l}\text { Onset to balloon (SD), in minutes } \\
\text { A: } 262(112) \\
\text { B: } 148(81) \\
\text { p<0.001 }\end{array}$ \\
\hline Zennaro, 2014 & $\begin{array}{l}\text { In-hospital consultation required } \\
\text { A: } 76.1 \%\end{array}$ & $\begin{array}{l}\text { Mean time for decisionmaking (SD), in minutes } \\
\text { A: } 56.2(16.1) \\
\text { B: } 23.4(21.8), p<0.001\end{array}$ \\
& B: $38 \%$ & NR \\
& Immediate activation of services & Diagnostic confidence \\
& A: $0 \%$ & A: $9.92(0.31)$ \\
B: $33.3 \%$ & B: $9.91(0.32), p=0.88$ \\
\hline
\end{tabular}

CI = confidence interval; CPK-MB = creatine kinase-muscle/brain; CT = computed tomography; ECG = electrocardiogram; ED = emergency department; EMS = emergency medical services; GBP = Great British Pound; GI = gastrointestinal; ICH = intracranial hemorrhage; ICU = intensive care unit; IQR = interquartile range; IV = intravenous; LOS = length of stay; LVEF = left ventricular ejection fraction; MI/A fib = myocardial infarction/ atrial fibrillation; mRS = modified Rankin Scale; NA = not applicable; NR = not reported; NRS = numerical rating scale; NS = not significant; NHS = National Health Service; OR = odds ratio; OT = occupational therapy; PCI = percutaneous coronary intervention; PICU = pediatric intensive care unit; SD = standard deviation; SDAC = STRokE DOC adjudicating committee; STEMI = ST-elevation myocardial infarction; tPA = tissue plasminogen activator; USD = United States dollars; UTI = urinary tract infection; VC = video consultation; VST = Virtual Studio Technology

${ }^{\text {a }}$ For definitions of interventions and comparisons (e.g., A vs. B), see Table F-4

See Appendix C. Included Studies for full citations

Table F-7. Outpatient evidence table: study characteristics

\begin{tabular}{|c|c|c|c|c|}
\hline $\begin{array}{l}\text { Author, Year } \\
\text { (See Appendix C for full } \\
\text { citation) }\end{array}$ & $\begin{array}{l}\text { Geographic Location } \\
\text { of Studies }\end{array}$ & Study Period & Study Design & $\begin{array}{l}\text { Comparison and } \\
\text { Intervention }\end{array}$ \\
\hline Angstman, 2009 & $\begin{array}{l}\text { Minnesota } \\
\text { USA }\end{array}$ & November 2005 to April 2008 & Retrospective Cohort & $\begin{array}{l}\text { A: Usual care, seen by } \\
\text { specialist in face-to-face } \\
\text { consult } \\
\text { B: Virtual consult } \\
\end{array}$ \\
\hline Arora, 2011 & $\begin{array}{l}\text { New Mexico } \\
\text { USA }\end{array}$ & NR & Prospective Cohort & $\begin{array}{l}\text { A: University HCV clinic } \\
\text { (control) } \\
\text { B: ECHO site }\end{array}$ \\
\hline Bagayoko, 2014 & Mali & March 2012 to March 2013 & $\begin{array}{l}\text { Prospective Cohort for patient costs; } \\
\text { Before-After for health center costs }\end{array}$ & $\begin{array}{l}\text { A: Control sites } \\
\text { B: Telehealth sites }\end{array}$ \\
\hline Baig, 2016 & $\begin{array}{l}\text { Milwaukee, Wisconsin } \\
\text { USA }\end{array}$ & 2008 to 2012 & Retrospective Cohort & $\begin{array}{l}\text { A: Usual care } \\
\text { B: Telehealth }\end{array}$ \\
\hline Basudev, 2016 & $\begin{array}{l}\text { London } \\
\text { England }\end{array}$ & 12-month period & RCT & $\begin{array}{l}\text { A: Usual care } \\
\text { B: Virtual clinic }\end{array}$ \\
\hline
\end{tabular}




\begin{tabular}{|c|c|c|c|c|}
\hline $\begin{array}{l}\text { Author, Year } \\
\text { (See Appendix C for full } \\
\text { citation) }\end{array}$ & $\begin{array}{l}\text { Geographic Location } \\
\text { of Studies }\end{array}$ & Study Period & Study Design & $\begin{array}{l}\text { Comparison and } \\
\text { Intervention }\end{array}$ \\
\hline Bernstein, 2010 & $\begin{array}{l}\text { Manitoba } \\
\text { Canada }\end{array}$ & January 1990 to October 2005 & Retrospective Cohort & $\begin{array}{l}\text { A: No telehealth } \\
\text { B1: Local community care } \\
\text { (telehealth) near urban center } \\
\text { B2: Local community care } \\
\text { (telehealth) far from urban } \\
\text { center }\end{array}$ \\
\hline Beswick, 2016 & $\begin{array}{l}\text { California } \\
\text { USA }\end{array}$ & August 2013 to March 2015 & Retrospective Cohort & $\begin{array}{l}\text { A: Usual care } \\
\text { B: Telehealth }\end{array}$ \\
\hline Bezalel, 2015 & $\begin{array}{l}\text { Tampa, Florida } \\
\text { USA }\end{array}$ & \begin{tabular}{|l|} 
A: January to May 2012 \\
B: January to May 2013
\end{tabular} & Before-After & $\begin{array}{l}\text { A: Store and forward } \\
\text { teledermatology not heavily } \\
\text { used } \\
\text { B: Store and forward } \\
\text { teledermatology fully } \\
\text { implemented }\end{array}$ \\
\hline Blackwell, 1997 & $\begin{array}{l}\text { Queensland } \\
\text { Australia } \\
\end{array}$ & December 1995 to February 1997 & Before-After & $\begin{array}{l}\text { A: Before telehealth } \\
\text { B: After telehealth }\end{array}$ \\
\hline Boman, 2014 & Sweden & 2010 to 2013 & RCT & $\begin{array}{l}\text { A: Standard of care } \\
\text { consultation } \\
\text { B: Remote consulting and } \\
\text { imaging }\end{array}$ \\
\hline Britt, 2006 & $\begin{array}{l}\text { Little Rock, Arkansas } \\
\text { USA }\end{array}$ & \begin{tabular}{|l|} 
A: December 2002 to May 2003 \\
B: December 2003 to May 2004
\end{tabular} & Before-After & $\begin{array}{l}\text { A: Before ANGELS program } \\
\text { (some limited telehealth } \\
\text { available) } \\
\text { B: After ANGELS program } \\
\text { (more comprehensive } \\
\text { telehealth services) }\end{array}$ \\
\hline Brown-Connolly, 2002 & $\begin{array}{l}\text { California } \\
\text { USA }\end{array}$ & September 1999 to April 2001 & Prospective Cohort & $\begin{array}{l}\text { A: Face-to-face } \\
\text { B: Telehealth } \\
\text { Comparison is same patients } \\
\text { submitting travel information }\end{array}$ \\
\hline Burns, 2017 & $\begin{array}{l}\text { Queensland } \\
\text { Australia } \\
\end{array}$ & July 2013 to October 2015 & RCT & $\begin{array}{l}\text { A: Usual care } \\
\text { B: Telehealth }\end{array}$ \\
\hline Byamba, 2015 & Mongolia & September 2013 to January 2014 & RCT & $\begin{array}{l}\text { A: GPs with access to tele- } \\
\text { consultations; } \\
\text { B: GPs without tele- } \\
\text { consultations (patients were } \\
\text { Referred to dermatologists). }\end{array}$ \\
\hline Carallo, 2015 & $\begin{array}{l}\text { Calabria } \\
\text { Italy }\end{array}$ & 18 months beginning in 2011 & Prospective Cohort & $\begin{array}{l}\text { A: Usual care } \\
\text { B: Quarterly visits to primary } \\
\text { care doctor, phone support } \\
\text { by diabetes specialist }\end{array}$ \\
\hline
\end{tabular}




\begin{tabular}{|c|c|c|c|c|}
\hline $\begin{array}{l}\text { Author, Year } \\
\text { (See Appendix C for full } \\
\text { citation) }\end{array}$ & $\begin{array}{l}\text { Geographic Location } \\
\text { of Studies }\end{array}$ & Study Period & Study Design & $\begin{array}{l}\text { Comparison and } \\
\text { Intervention }\end{array}$ \\
\hline Carter, 2017 & $\begin{array}{l}\text { Dallas, Texas } \\
\text { USA }\end{array}$ & \begin{tabular}{|l|} 
Before May to December 2013 \\
After May to December 2014
\end{tabular} & Retrospective Cohort & $\begin{array}{l}\text { A: In-person } \\
\text { B: Remote assessment }\end{array}$ \\
\hline Chan, 2015 & $\begin{array}{l}\text { Queensland } \\
\text { Australia }\end{array}$ & May 2007 and April 2012 & Retrospective Cohort & $\begin{array}{l}\text { A: Usual care } \\
\text { B: Teleoncology }\end{array}$ \\
\hline Chu, 2015 & $\begin{array}{l}\text { Los Angeles, California } \\
\text { USA }\end{array}$ & September 2013 to March 2014 & Retrospective Cohort & $\begin{array}{l}\text { A: No telehealth } \\
\text { B: Telehealth } \\
\end{array}$ \\
\hline Chua, 2001 & \begin{tabular}{|l|} 
Northern Ireland \\
\end{tabular} & NR & RCT & $\begin{array}{l}\text { A: Usual care } \\
\text { B: Telehealth }\end{array}$ \\
\hline $\begin{array}{l}\text { Collins, 2004; Bowns, } \\
2006\end{array}$ & United Kingdom & NR & RCT & $\begin{array}{l}\text { A: Traditional outpatient } \\
\text { consultation } \\
\text { B: Asynchronous } \\
\text { teleconsultation }\end{array}$ \\
\hline Crossland, 2016 & $\begin{array}{l}\text { Rural, regional, urban } \\
\text { Australia }\end{array}$ & February 2011 to February 2014 & Prospective Cohort & \begin{tabular}{|l|} 
A: Usual care \\
B: Telehealth \\
\end{tabular} \\
\hline Datta, 2015 & $\begin{array}{l}\text { Durham, North Carolina } \\
\text { USA }\end{array}$ & December 2008 to March 2011 & Economic Evaluation & $\begin{array}{l}\text { A: Usual care } \\
\text { B: Telehealth }\end{array}$ \\
\hline Davis, 2011 & USA & 12 months & RCT & $\begin{array}{l}\text { A: Usual care } \\
\text { B: Collaborative care via } \\
\text { telehealth }\end{array}$ \\
\hline Davison, 2004 & \begin{tabular}{|l|} 
London \\
United Kingdom
\end{tabular} & November 2000 to November 2001 & Before-After & \begin{tabular}{|l|} 
A: Before telehealth \\
B: After telehealth \\
\end{tabular} \\
\hline De Luca, 2005 & Italy & June 1999 to December 1999 & Prospective Cohort & $\begin{array}{l}\text { A: Face-to-face } \\
\text { B: Telehealth }\end{array}$ \\
\hline Doolittle, 1997 & $\begin{array}{l}\text { Kansas City, Missouri } \\
\text { USA }\end{array}$ & May 1995 to April 1996 & Economic Evaluation & $\begin{array}{l}\text { A: Fly in outreach clinic } \\
\text { B: Traditional Clinic } \\
\text { C: Telehealth }\end{array}$ \\
\hline Dowie, 2007 & \begin{tabular}{|l|} 
London \\
England \\
\end{tabular} & $\begin{array}{l}15 \text { month period } \\
\text { Specific dates NR }\end{array}$ & Economic Evaluation & $\begin{array}{l}\text { A: Conventional referrals } \\
\text { B: Telehealth }\end{array}$ \\
\hline Eminovic, 2009, 2010 & Netherlands & February 2004 to January 2006 & RCT; Economic Evaluation & \begin{tabular}{|l|} 
A: Usual practice \\
B: Teleconsultation \\
\end{tabular} \\
\hline Endean, 2001 & $\begin{array}{l}\text { Kentucky } \\
\text { USA }\end{array}$ & NR & Pre/Post & $\begin{array}{l}\text { A: Onsite vascular evaluation } \\
\text { B: Telehealth vascular } \\
\text { evaluation }\end{array}$ \\
\hline Ferrandiz, 2007 & \begin{tabular}{|l|} 
Seville \\
Spain \\
\end{tabular} & March 2005 to February 2006 & $\begin{array}{l}\text { Prospective Cohort (pre/post for } \\
\text { clinical accuracy) }\end{array}$ & \begin{tabular}{|l|} 
A: Conventional system \\
B: Teleconsultation \\
\end{tabular} \\
\hline Fortney, 2013 & $\begin{array}{l}\text { Arkansas } \\
\text { USA }\end{array}$ & 18 months between 2007 and 2009 & RCT & $\begin{array}{l}\text { A: Practice based } \\
\text { collaborative care } \\
\text { B: Telehealth based } \\
\text { collaborative care } \\
\end{array}$ \\
\hline
\end{tabular}




\begin{tabular}{|c|c|c|c|c|}
\hline $\begin{array}{l}\text { Author, Year } \\
\text { (See Appendix C for full } \\
\text { citation) }\end{array}$ & $\begin{array}{l}\text { Geographic Location } \\
\text { of Studies }\end{array}$ & Study Period & Study Design & $\begin{array}{l}\text { Comparison and } \\
\text { Intervention }\end{array}$ \\
\hline Fortney, 2007 & $\begin{array}{l}\text { South-Central } \\
\text { USA }\end{array}$ & 12 months & RCT & $\begin{array}{l}\text { A: Usual care } \\
\text { B: Collaborative care via } \\
\text { telehealth }\end{array}$ \\
\hline Fortney, 2011 & USA & April 2003 to September 2004 & RCT & $\begin{array}{l}\text { A: Usual care } \\
\text { B: Telehealth }\end{array}$ \\
\hline Fortney, 2015 & USA & November 2009 to September 2011 & RCT & $\begin{array}{l}\text { Telehealth outreach including } \\
\text { medications and CPT }\end{array}$ \\
\hline Fox, 2007 & $\begin{array}{l}\text { Tennessee } \\
\text { USA }\end{array}$ & $\begin{array}{l}3 \text { years } \\
\text { Dates not specified }\end{array}$ & Before-After & $\begin{array}{l}\text { A: Year before telehealth } \\
\text { program implemented } \\
\text { B: One year after telehealth } \\
\text { program implemented } \\
\text { C: Two years after telehealth } \\
\text { program implemented }\end{array}$ \\
\hline Frank, 2015 & USA & July 2011 to December 2013 & Before-After & $\begin{array}{l}\text { A: Patient panels of primary } \\
\text { care providers with pain that } \\
\text { never presented at SCAN- } \\
\text { ECHO } \\
\text { B: Patient panels of primary } \\
\text { care providers with pain that } \\
\text { have presented at the SCAN- } \\
\text { ECHO meetings }\end{array}$ \\
\hline Gattas, 2001 & \begin{tabular}{|l} 
Queensland \\
Australia \\
\end{tabular} & NR & RCT & $\begin{array}{l}\text { A: Face-to-face } \\
\text { B: Teleconsultations } \\
\end{array}$ \\
\hline Gilmour, 1998 & United Kingdom & 1995 to 1996 & Prospective Cohort & \begin{tabular}{|l|} 
A: Face-to-face \\
B: Telehealth \\
\end{tabular} \\
\hline Harno, 2000 & Finland & 1998 & Economic Evaluation & $\begin{array}{l}\text { A: Hyvinkaa Hospital (no } \\
\text { telehealth) } \\
\text { B: Peijas Hospital (telehealth) }\end{array}$ \\
\hline Harno, 2001 & Finland & 1998 & Economic Evaluation & $\begin{array}{l}\text { A: Outpatient clinic } \\
\text { B: Videoconference }\end{array}$ \\
\hline $\begin{array}{l}\text { Haukipuro same patients } \\
\text { as Ohinmaa and Vuolio, } \\
2000\end{array}$ & Finland & 1998 & RCT; Economic Evaluation & $\begin{array}{l}\text { A: Conventional hospital } \\
\text { outpatient clinic } \\
\text { B: Videoconferencing }\end{array}$ \\
\hline Herce, 2011 & $\begin{array}{l}\text { Seville } \\
\text { Spain }\end{array}$ & January and December 2009 & Prospective Cohort & \begin{tabular}{|l|} 
A: Usual care \\
B: Telehealth \\
\end{tabular} \\
\hline Hsiao, 2008 & $\begin{array}{l}\text { San Francisco, } \\
\text { California } \\
\text { USA }\end{array}$ & January 2003 to July 2007 & Retrospective Cohort & $\begin{array}{l}\text { A: Face-to-face dermatology } \\
\text { consultation } \\
\text { B: Teledermatology } \\
\text { consultation }\end{array}$ \\
\hline
\end{tabular}




\begin{tabular}{|c|c|c|c|c|}
\hline $\begin{array}{l}\text { Author, Year } \\
\text { (See Appendix C for full } \\
\text { citation) }\end{array}$ & $\begin{array}{l}\text { Geographic Location } \\
\text { of Studies }\end{array}$ & Study Period & Study Design & $\begin{array}{l}\text { Comparison and } \\
\text { Intervention }\end{array}$ \\
\hline Izquierdo, 2009 & $\begin{array}{l}\text { Onondaga County, } \\
\text { New York } \\
\text { USA }\end{array}$ & NR & RCT & $\begin{array}{l}\text { A: Usual care: in-person visits } \\
\text { to diabetes center every three } \\
\text { months } \\
\text { B: Telehealth: monthly visit }\end{array}$ \\
\hline Jaatinen, 2002 & $\begin{array}{l}\text { Satakunta } \\
\text { Finland }\end{array}$ & 5 months (from December 1999) & RCT & $\begin{array}{l}\text { A: Control group } \\
\text { (conventional referral letter } \\
\text { sent to hospital outpatient } \\
\text { clinic or telephone call) } \\
\text { B: Telehealth group } \\
\text { (teleconsultation form or } \\
\text { telereferral form and web- } \\
\text { based telehealth system) }\end{array}$ \\
\hline $\begin{array}{l}\text { Jacklin, } 2003 \\
\text { Same study as Wallace } \\
2002,2004\end{array}$ & England & 1999 to NR & RCT & $\begin{array}{l}\text { A: Face-to-face consultation } \\
\text { B: Teleconsult (joint with } \\
\text { referring general practitioner } \\
\text { also participating with patient) }\end{array}$ \\
\hline Jacobs, 2015 & $\begin{array}{l}\text { Ameland } \\
\text { Netherlands }\end{array}$ & 2006 and 2009 & Before-After & $\begin{array}{l}\text { A: Before teleradiology } \\
\text { B: After teleradiology }\end{array}$ \\
\hline Jong, 2004 & Canada & NR & Prospective Cohort & $\begin{array}{l}\text { A: Physicians visiting clinic } \\
\text { B: Physicians using email } \\
\text { C: Physicians } \\
\text { videoconferencing }\end{array}$ \\
\hline Jue, 2017 & $\begin{array}{l}\text { Florida } \\
\text { USA }\end{array}$ & July 2012 and June 2014 & Prospective Cohort & $\begin{array}{l}\text { A: Usual care, travel to Miami } \\
\text { facility } \\
\text { B: Telehealth }\end{array}$ \\
\hline Kobza, 2000 & NR & December 1998 to February 2000 & Before-After & $\begin{array}{l}\text { A: Usual care } \\
\text { B: Telehealth }\end{array}$ \\
\hline Krier, 2011 & $\begin{array}{l}\text { Palo Alto, California } \\
\text { USA }\end{array}$ & NR & RCT & \begin{tabular}{|l|} 
A: Usual care \\
B: Telehealth \\
\end{tabular} \\
\hline Krupinski, 2004 & $\begin{array}{l}\text { Tucson, Arizona } \\
\text { USA }\end{array}$ & 1999 to 2002 & Retrospective Cohort & $\begin{array}{l}\text { A: Cases that were referred } \\
\text { to dermatology and seen in } \\
\text { person } \\
\text { B: Teledermatology referrals }\end{array}$ \\
\hline Kunkler, 2007 & $\begin{array}{l}\text { Edinburgh, Scotland } \\
\text { United Kindom }\end{array}$ & March 2004 to April 2005 & RCT & $\begin{array}{l}\text { A: In-person meetings } \\
\text { B: Telehealth linkage to } \\
\text { breast specialists in a cancer } \\
\text { center }\end{array}$ \\
\hline
\end{tabular}




\begin{tabular}{|c|c|c|c|c|}
\hline $\begin{array}{l}\text { Author, Year } \\
\text { (See Appendix C for full } \\
\text { citation) }\end{array}$ & $\begin{array}{l}\text { Geographic Location } \\
\text { of Studies }\end{array}$ & Study Period & Study Design & $\begin{array}{l}\text { Comparison and } \\
\text { Intervention }\end{array}$ \\
\hline Lamminen, 2001 & Finland & September 1996 to May 1997 & Economic Evaluation & $\begin{array}{l}\text { A 1: Conventional } \\
\text { consultations - } \\
\text { Ophthalmology } \\
\text { A 2: Conventional - } \\
\text { Dermatology } \\
\text { B 1: Teleophthalmology } \\
\text { B 2: Teledermatology }\end{array}$ \\
\hline Lee, 2014 & USA & April 2011 to 2013 & Prospective Cohort & $\begin{array}{l}\text { A: } 6 \text { months before E-consult } \\
\text { program started } \\
\text { B: } 6 \text { months beginning one } \\
\text { year after E-consult program } \\
\text { started }\end{array}$ \\
\hline Lim, 2012 & $\begin{array}{l}\text { Waikato } \\
\text { New Zealand } \\
\end{array}$ & 8 month period & Prospective Cohort & $\begin{array}{l}\text { A: Face-to-face } \\
\text { B: Telehealth } \\
\end{array}$ \\
\hline Loane, 2000 & Northern Ireland & 12 month period Dates NR & RCT & $\begin{array}{l}\text { A: Usual care } \\
\text { B: Telehealth video } \\
\text { C: Telehealth store and } \\
\text { forward }\end{array}$ \\
\hline Loane, 1999 & $\begin{array}{l}\text { Northern Ireland } \\
\text { United Kingdom }\end{array}$ & $\begin{array}{l}9 \text { months } \\
\text { Dates not specified }\end{array}$ & RCT & $\begin{array}{l}\text { A: Traditional hospital } \\
\text { consultation } \\
\text { B: Teledermatology } \\
\text { consultation }\end{array}$ \\
\hline Loane, 2001 & New Zealand & $\begin{array}{l}10 \text { months } \\
\text { Dates not specified }\end{array}$ & Economic Evaluation & $\begin{array}{l}\text { A: Traditional hospital } \\
\text { appointment } \\
\text { B: Teledermatology } \\
\text { appointment } \\
\end{array}$ \\
\hline Long, 2014 & $\begin{array}{l}\text { Arkansas } \\
\text { USA }\end{array}$ & 2001 to 2007 & Retrospective Cohort & $\begin{array}{l}\text { A: Before telehealth service } \\
\text { available } \\
\text { B. After telehealth service } \\
\text { available (numbers not } \\
\text { reported for which } \\
\text { ultrasounds were via } \\
\text { telehealth vs conventional) }\end{array}$ \\
\hline Mahendran, 2005 & England & $\begin{array}{l}\text { 18-month period, dates not } \\
\text { specified }\end{array}$ & Pre/Post & $\begin{array}{l}\text { A: Face-to-face consultation } \\
\text { with dermatologist } \\
\text { B: Store and forward, image } \\
\text { consultation with } \\
\text { dermatologist }\end{array}$ \\
\hline
\end{tabular}




\begin{tabular}{|c|c|c|c|c|}
\hline $\begin{array}{l}\text { Author, Year } \\
\text { (See Appendix C for full } \\
\text { citation) }\end{array}$ & $\begin{array}{l}\text { Geographic Location } \\
\text { of Studies }\end{array}$ & Study Period & Study Design & $\begin{array}{l}\text { Comparison and } \\
\text { Intervention }\end{array}$ \\
\hline McCrossan, 2012 & $\begin{array}{l}\text { Northern Ireland } \\
\text { United Kingdom }\end{array}$ & $\begin{array}{l}20 \text { months } \\
\text { Dates not specified }\end{array}$ & Prospective Cohort & $\begin{array}{l}\text { A: Hospital without remote } \\
\text { fetal echocardiograms } \\
\text { B: Hospital with tele fetal } \\
\text { echocardiograms }\end{array}$ \\
\hline Moreno-Ramirez, 2009 & Spain & 2004 to 2005 & Economic Evaluation & $\begin{array}{l}\text { A: Conventional care } \\
\text { B: Teledermatology } \\
\end{array}$ \\
\hline Mulgrew, 2011 & $\begin{array}{l}\text { California } \\
\text { USA }\end{array}$ & October 2008 to December 2009 & Prospective Cohort & $\begin{array}{l}\text { A. Face-to-face } \\
\text { B: Telehealth }\end{array}$ \\
\hline Nickenig, 2008 & Germany & July 2003 to July 2005 & Prospective Cohort & $\begin{array}{l}\text { A: Conventional care } \\
\text { B: Telehealth }\end{array}$ \\
\hline Nikkanen, 2008 & $\begin{array}{l}\text { Oulu Arc Sub region } \\
\text { Finland }\end{array}$ & 10 to 14 months, 2005 & Pre/Post & $\begin{array}{l}\text { A: First visit to telehealth } \\
\text { supported system } \\
\text { B: Follow-up visit to } \\
\text { telehealth supported system }\end{array}$ \\
\hline Nordal, 2001 & Norway & 1994-1995 & Retrospective Cohort & $\begin{array}{l}\text { A1: Patients with a GP were } \\
\text { seen by one dermatologist } \\
\text { over the video-link. } \\
\text { A2: Patients with a GP were } \\
\text { seen by one dermatologist } \\
\text { face-to-face. }\end{array}$ \\
\hline $\begin{array}{l}\text { Ohinmaa } \\
\text { Same patients as Vuolio } \\
\text { and Haukipuro, } 2002\end{array}$ & Finland & NR & RCT; Economic Evaluation & $\begin{array}{l}\text { A: Conventional outpatient } \\
\text { visits } \\
\text { B: Video-conferencing }\end{array}$ \\
\hline Pak, 2009; Pak, 2007 & $\begin{array}{l}\text { Texas } \\
\text { USA }\end{array}$ & NR & RCT; Economic Evaluation & $\begin{array}{l}\text { A: Usual care } \\
\text { B: Teledermatology } \\
\text { consultation }\end{array}$ \\
\hline Piette, 2017 & \begin{tabular}{|l|} 
Paris \\
France \\
\end{tabular} & February and June 2014 & RCT & \begin{tabular}{|l|} 
A: Usual care \\
B: Telehealth \\
\end{tabular} \\
\hline Rossaro, 2013 & $\begin{array}{l}\text { California } \\
\text { USA }\end{array}$ & 2006 to 2010 & Retrospective Cohort & $\begin{array}{l}\text { A: HCV treatment at the } \\
\text { University of California Davis } \\
\text { Hepatology Clinic } \\
\text { B: HCV treatment at different } \\
\text { telehealth sites }\end{array}$ \\
\hline Salami, 2015 & USA & 2009 to 2013 & Retrospective Cohort & $\begin{array}{l}\text { A: Patients evaluated through } \\
\text { standard interfacility } \\
\text { consultation. } \\
\text { B: Patients evaluated through } \\
\text { the Virtual Tumor Board }\end{array}$ \\
\hline Salazar-Fernandez, 2012 & $\begin{array}{l}\text { Seville } \\
\text { Spain }\end{array}$ & January 2008 to February 2010 & Prospective Cohort & $\begin{array}{l}\text { A: Conventional systems } \\
\text { B: Store and Forward }\end{array}$ \\
\hline
\end{tabular}




\begin{tabular}{|c|c|c|c|c|}
\hline $\begin{array}{l}\text { Author, Year } \\
\text { (See Appendix C for full } \\
\text { citation) }\end{array}$ & $\begin{array}{l}\text { Geographic Location } \\
\text { of Studies }\end{array}$ & Study Period & Study Design & $\begin{array}{l}\text { Comparison and } \\
\text { Intervention }\end{array}$ \\
\hline Santamaria, 2004 & $\begin{array}{l}\text { Kimberly } \\
\text { Australia }\end{array}$ & October 2002 to October 2003 & $\mathrm{RCT}$ & $\begin{array}{l}\text { A: Standard wound care } \\
\text { B: Telehealth }\end{array}$ \\
\hline Scuffham, 2002 & United Kingdom & June 2000 to May 2001 & Economic Evaluation & $\begin{array}{l}\text { A: Outreach visits } \\
\text { B: Face-to-face at hospital } \\
\text { C: Telehealth }\end{array}$ \\
\hline Sharma, 2003 & $\begin{array}{l}\text { New York } \\
\text { USA }\end{array}$ & May 1998 to August 1998 & Retrospective Cohort & $\begin{array}{l}\text { A: Pregnant women } \\
\text { undergoing in-person fetal } \\
\text { echocardiograms who were } \\
\text { referred from outlying primary } \\
\text { care center. } \\
\text { B. Pregnant women } \\
\text { undergoing fetal } \\
\text { echocardiograms via } \\
\text { telehealth at their local } \\
\text { hospital. }\end{array}$ \\
\hline Smith, 2002 & $\begin{array}{l}\text { Queensland } \\
\text { Australia } \\
\end{array}$ & November 2000 to January 2002 & Before-After & $\begin{array}{l}\text { A: Before pediatric telehealth } \\
\text { B: After pediatric telehealth }\end{array}$ \\
\hline Smith-Strom, 2018 & Norway & 2012 to 2016 & RCT & \begin{tabular}{|l|} 
A: Usual care \\
B: Telehealth \\
\end{tabular} \\
\hline Specht, 2001 & $\begin{array}{l}\text { Iowa } \\
\text { USA }\end{array}$ & NR & Economic Evaluation & $\begin{array}{l}\text { A: In-person visit at an acute } \\
\text { care facility for a wound care } \\
\text { specialist visit } \\
\text { B: Telehealth consultation } \\
\text { with a wound care specialist } \\
\text { at an acute care facility. } \\
\end{array}$ \\
\hline Stalfors, 2003 & Sweden & $\begin{array}{l}\text { September } 1998 \text { to September } \\
1999\end{array}$ & Prospective Cohort & $\begin{array}{l}\text { A: Face-to-face patient } \\
\text { presentation at tumor } \\
\text { meeting } \\
\text { B: Telehealth patient } \\
\text { presentation at tumor } \\
\text { meeting }\end{array}$ \\
\hline Stalfors, 2005 & Sweden & $\begin{array}{l}\text { September } 1998 \text { to } \\
\text { September } 1999\end{array}$ & Economic Evaluation & $\begin{array}{l}\text { A: Face-to-face consultation } \\
\text { B: Telehealth }\end{array}$ \\
\hline Stern, 2014 & $\begin{array}{l}\text { Ontario } \\
\text { Canada }\end{array}$ & 17 months & Before-After (stepped wedge) & $\begin{array}{l}\text { A: Not telehealth } \\
\text { B: Enhanced multidisciplinary } \\
\text { team supported by telehealth }\end{array}$ \\
\hline Strymish, 2017 & $\begin{array}{l}\text { Boston, Massachusetts } \\
\text { USA }\end{array}$ & 2014 & Before-After & $\begin{array}{l}\text { A: Face-to-face } \\
\text { B: Telehealth }\end{array}$ \\
\hline Taylor, 2007 & $\begin{array}{l}\text { Nashville, Tennessee } \\
\text { USA }\end{array}$ & September 2003to August 2004 & Before-After & $\begin{array}{l}\text { A: Referral for exam } \\
\text { B: Digital screen }\end{array}$ \\
\hline
\end{tabular}




\begin{tabular}{|c|c|c|c|c|}
\hline $\begin{array}{l}\text { Author, Year } \\
\text { (See Appendix C for full } \\
\text { citation) }\end{array}$ & $\begin{array}{l}\text { Geographic Location } \\
\text { of Studies }\end{array}$ & Study Period & Study Design & $\begin{array}{l}\text { Comparison and } \\
\text { Intervention }\end{array}$ \\
\hline Thaker, 2013 & $\begin{array}{l}\text { Queensland } \\
\text { Australia }\end{array}$ & $\begin{array}{l}\text { March } 12007 \text { to November } 30 \\
2011\end{array}$ & Economic Evaluation & $\begin{array}{l}\text { A: Model generated usual } \\
\text { care costs } \\
\text { B: Cost of patients managed } \\
\text { by telehealth system }\end{array}$ \\
\hline Tsitlakidis, 2005 & $\begin{array}{l}\text { Lemnos and Skyros } \\
\text { Greece }\end{array}$ & October 2002 to October 2003 & Economic Evaluation & $\begin{array}{l}\text { A: Hospital referrals (to } \\
\text { Athens) } \\
\text { B: Telehealth }\end{array}$ \\
\hline Tuulonen, 1999 & \begin{tabular}{|l|} 
Oulu \\
Finland
\end{tabular} & February 1998 to May 1998 & Before-After & $\begin{array}{l}\text { A: Conventional outpatient } \\
\text { visit } \\
\text { B: Telehealth }\end{array}$ \\
\hline van der Pol, 2010 & $\begin{array}{l}\text { Scotland, Shetland } \\
\text { Islands and Aberdeen } \\
\text { United Kingdom }\end{array}$ & 2007 & Economic Evaluation & $\begin{array}{l}\text { A: Mainland endoscopy clinic } \\
\text { B: Tele-endoscopy clinic }\end{array}$ \\
\hline Van Gelder, 2017 & Netherlands & March 2001 to June 2012 & RCT & $\begin{array}{l}\text { A: Usual care } \\
\text { B: Telehealth }\end{array}$ \\
\hline $\begin{array}{l}\text { Vuolio } \\
\text { Same patients as } \\
\text { Ohinmaa and Hakipuro, } \\
2003\end{array}$ & Finland & February 1997 to June 1998 & RCT; Economic Evaluation & $\begin{array}{l}\text { A: Outpatient clinic } \\
\text { B: Videoconference }\end{array}$ \\
\hline Wallace, 2002 & United Kingdom & 1999 to NR & RCT & $\begin{array}{l}\text { A: Standard outpatient } \\
\text { specialist visit } \\
\text { B: Virtual visit between } \\
\text { patient, general practitioner, } \\
\text { and specialist }\end{array}$ \\
\hline Wallace, 2004 & United Kingdom & NR & RCT & $\begin{array}{l}\text { A: Usual care } \\
\text { B: Virtual outreach group }\end{array}$ \\
\hline Whited, 2002 & $\begin{array}{l}\text { Durham, North Carolina } \\
\text { USA }\end{array}$ & NR & RCT; Economic Evaluation & $\begin{array}{l}\text { A: Usual care (text-based } \\
\text { electronic consult request) } \\
\text { B: Teledermatology } \\
\text { consultation (digital images } \\
\text { and a standardized history, in } \\
\text { addition to the text-based } \\
\text { electronic consult) }\end{array}$ \\
\hline Whited, 2003 & $\begin{array}{l}\text { Durham, North Carolina } \\
\text { USA }\end{array}$ & 2001 & RCT; Economic Evaluation & $\begin{array}{l}\text { A: Usual care } \\
\text { B: Telehealth }\end{array}$ \\
\hline
\end{tabular}




\begin{tabular}{|c|c|c|c|c|}
\hline $\begin{array}{l}\text { Author, Year } \\
\text { (See Appendix C for full } \\
\text { citation) }\end{array}$ & $\begin{array}{l}\text { Geographic Location } \\
\text { of Studies }\end{array}$ & Study Period & Study Design & $\begin{array}{l}\text { Comparison and } \\
\text { Intervention }\end{array}$ \\
\hline Whited, 2004 & $\begin{array}{l}\text { Durham, North Carolina } \\
\text { USA }\end{array}$ & NR & RCT; Economic Evaluation & $\begin{array}{l}\text { A: Usual care (text-based } \\
\text { electronic consult) } \\
\text { B: Teledermatology } \\
\text { consultation (digital images } \\
\text { and a standardized history, in } \\
\text { addition to the text-based } \\
\text { electronic consult) }\end{array}$ \\
\hline Whited, 2013a, 2013b & USA & 2008 to 2011 & RCT & $\begin{array}{l}\text { A: Conventional care } \\
\text { B: Teledermatology }\end{array}$ \\
\hline Wilson, 2005 & $\begin{array}{l}\text { Phoenix, Arizona } \\
\text { USA }\end{array}$ & 1999 to 2003 & Retrospective Cohort & $\begin{array}{l}\text { A: Before telehealth } \\
\text { B: After telehealth }\end{array}$ \\
\hline $\mathrm{Xu}, 2008$ & $\begin{array}{l}\text { Queensland } \\
\text { Australia }\end{array}$ & November 2000 to January 2002 & Economic Evaluation & $\begin{array}{l}\text { A: Conventional outpatient } \\
\text { ENT consultation } \\
\text { B: Tele-ENT service }\end{array}$ \\
\hline Young, 2014 & $\begin{array}{l}\text { Chicago, Illinois } \\
\text { USA }\end{array}$ & July 2009 to June 2012 & Before-After & $\begin{array}{l}\text { A: Before telehealth } \\
\text { B: After telehealth }\end{array}$ \\
\hline Zahlmann, 2002 & Germany & NR & $\begin{array}{l}\text { Prospective and Retrospective } \\
\text { Cohort }\end{array}$ & $\begin{array}{l}\text { A: Normal treatment } \\
\text { B: Teleconsultation }\end{array}$ \\
\hline Zarchi, 2015 & Denmark & February 2011 to September 2012 & Prospective Cohort & $\begin{array}{l}\text { A: Wound care without } \\
\text { telehealth } \\
\text { B: Wound care with } \\
\text { telehealth }\end{array}$ \\
\hline Zilliacus, 2011 & $\begin{array}{l}\text { New South Wales and } \\
\text { Australia }\end{array}$ & December 2007 to December 2009 & Prospective Cohort & $\begin{array}{l}\text { A: Face-to-face } \\
\text { B: Telehealth }\end{array}$ \\
\hline
\end{tabular}

ANGELS = Antenatal and Neonatal Guidelines, Education and Learning System; CPT = cognitive processing therapy; ECHO = Extension for Community Healthcare Outcomes; $\mathrm{ENT}=$ ear, nose, throat specialist (otorhinolaryngologist); GP = general practitioner; HCV = hepatitis C virus; NR = not reported; RCT = randomized control trial; SCAN-ECHO = Specialty Care Access Network-Extension for Community Healthcare Outcomes

See Appendix C. Included Studies for full citations

Table F-8. Outpatient evidence table: patient characteristics

\begin{tabular}{|c|c|c|c|c|c|c|c|c|}
\hline $\begin{array}{l}\text { Author, Year } \\
\text { (See Appendix C } \\
\text { for full citation) }\end{array}$ & $\begin{array}{l}\text { Number } \\
\text { Analyzed }^{a}\end{array}$ & Population & $\begin{array}{l}\text { Referring } \\
\text { Providers } \\
\text { Specialty/Type } \\
\end{array}$ & $\begin{array}{l}\text { Consulting Provider } \\
\text { Specialty/Type }\end{array}$ & \begin{tabular}{|l} 
Patient \\
Present?
\end{tabular} & $\begin{array}{l}\text { Patient } \\
\text { Setting } \\
\end{array}$ & $\begin{array}{l}\text { Modalities of } \\
\text { Telehealth } \\
\text { Consultation } \\
\text { Intervention } \\
\end{array}$ & Timing \\
\hline Angstman, 2009 & \begin{tabular}{|l|}
728 \\
A: 500 \\
B: 228 \\
\end{tabular} & Adults & $\begin{array}{l}\text { General } \\
\text { practitioners }\end{array}$ & Not specified & $\begin{array}{l}\text { A: Yes } \\
\text { B: No }\end{array}$ & Outpatient & $\begin{array}{l}\text { Electronic health } \\
\text { documentation, test } \\
\text { results }\end{array}$ & Mixed \\
\hline Arora, 2011 & \begin{tabular}{l|}
407 \\
A: 146 \\
B: 261 \\
\end{tabular} & $\begin{array}{l}\text { Adults between 18- } \\
65 \text { with HCV }\end{array}$ & Primary care & $\begin{array}{l}\text { Hepatology, infectious } \\
\text { diseases, psychiatry, and } \\
\text { pharmacology }\end{array}$ & No & Outpatient & Videoconference & Real-time \\
\hline
\end{tabular}




\begin{tabular}{|c|c|c|c|c|c|c|c|c|}
\hline $\begin{array}{l}\text { Author, Year } \\
\text { (See Appendix C } \\
\text { for full citation) }\end{array}$ & $\begin{array}{l}\text { Number } \\
\text { Analyzed }^{a}\end{array}$ & Population & $\begin{array}{l}\text { Referring } \\
\text { Providers } \\
\text { Specialty/Type } \\
\end{array}$ & $\begin{array}{l}\text { Consulting Provider } \\
\text { Specialty/Type }\end{array}$ & \begin{tabular}{|l} 
Patient \\
Present?
\end{tabular} & $\begin{array}{l}\text { Patient } \\
\text { Setting } \\
\end{array}$ & \begin{tabular}{|l|} 
Modalities of \\
Telehealth \\
Consultation \\
Intervention \\
\end{tabular} & Timing \\
\hline Bagayoko, 2014 & $\begin{array}{l}211 \\
\text { A: NR } \\
\text { B: NR }\end{array}$ & Rural & NR & Obstetrics and cardiology & Yes & Outpatient & $\begin{array}{l}\text { EKG and obstetric } \\
\text { ultrasounds transmitted } \\
\text { via laptops with low } \\
\text { bandwidth internet } \\
\text { connection } \\
\end{array}$ & Asynchronous \\
\hline Baig, 2016 & $\begin{array}{l}\text { Unclear } \\
\text { A: Unclear } \\
\text { B: Unclear } \\
\end{array}$ & Adults & $\begin{array}{l}\text { Primary care } \\
\text { physician }\end{array}$ & Sleep specialist & No & Outpatient & E-Consult & Asynchronous \\
\hline Basudev, 2016 & \begin{tabular}{|l|}
167 \\
A: 88 \\
B: 79 \\
\end{tabular} & Adult & Primary care & Diabetes specialist & No & Outpatient & NR & Real-time \\
\hline Bernstein, 2010 & $\begin{array}{l}2,663 \\
\text { A: } 2,196 \\
\text { B: } 285 \\
\text { C: } 182\end{array}$ & $\begin{array}{l}\text { People age } 17 \text { to } \\
70+, \text { mean age } \\
59.1 \text { years. } 56 \% \\
\text { male. } 65 \% \\
\text { Caucasian, } 28 \% \\
\text { aboriginal. 50\% } \\
\text { diabetic } \\
\text { nephropathy, } \\
\text { though higher rates } \\
\text { of DN in local } \\
\text { communities. 5- } \\
\text { year mortality } 56 \% \\
\end{array}$ & Primary care & Nephrology team & No & Outpatient & $\begin{array}{l}\text { Record/flowsheet } \\
\text { review }\end{array}$ & Real-time \\
\hline Beswick, 2016 & $\begin{array}{l}41 \\
\text { A: } 26 \\
\text { B: } 15 \\
\end{array}$ & Adults & $\begin{array}{l}\text { Nurse and speech } \\
\text { pathologist }\end{array}$ & Head and neck surgeon & Yes & Outpatient & $\begin{array}{l}\text { Audiovisual } \\
\text { teleconference }\end{array}$ & Real-time \\
\hline Bezalel, 2015 & $\begin{array}{l}3,065 \\
\text { A: } 1,557 \text { new } \\
\text { patients } \\
\text { B: } 1,508 \text { new } \\
\text { patients } \\
n \text { for } \\
\text { established } \\
\text { patients NR }\end{array}$ & $\begin{array}{l}\text { Adults (VA } \\
\text { patients) }\end{array}$ & NR & Dermatologist & No & Outpatient & Unclear/NR & Asynchronous \\
\hline Blackwell, 1997 & $\begin{array}{l}579 \\
\text { A:315 } \\
\text { B: } 264 ; 24 \\
\text { received } \\
\text { telehealth } \\
\end{array}$ & NR & $\begin{array}{l}\text { Emergency } \\
\text { department director }\end{array}$ & Ophthalmology specialist & Yes & ED & Videoconference & Real-time \\
\hline
\end{tabular}




\begin{tabular}{|c|c|c|c|c|c|c|c|c|}
\hline $\begin{array}{l}\text { Author, Year } \\
\text { (See Appendix C } \\
\text { for full citation) }\end{array}$ & $\begin{array}{l}\text { Number } \\
\text { Analyzed }^{a}\end{array}$ & Population & $\begin{array}{l}\text { Referring } \\
\text { Providers } \\
\text { Specialty/Type } \\
\end{array}$ & $\begin{array}{l}\text { Consulting Provider } \\
\text { Specialty/Type }\end{array}$ & \begin{tabular}{|l} 
Patient \\
Present?
\end{tabular} & $\begin{array}{l}\text { Patient } \\
\text { Setting } \\
\end{array}$ & \begin{tabular}{|l} 
Modalities of \\
Telehealth \\
Consultation \\
Intervention \\
\end{tabular} & Timing \\
\hline Boman, 2014 & $\begin{array}{l}38 \\
\text { A: } 19 \\
\text { B: } 19\end{array}$ & Adults & General practitioner & Cardiologist & Yes & Outpatient & $\begin{array}{l}\begin{array}{l}\text { Store and forward } \\
\text { (history, ECG) and } \\
\text { "bidirectional } \\
\text { communication" (likely } \\
\text { videoconference) }\end{array} \\
\end{array}$ & Mixed \\
\hline Britt, 2006 & NR & $\begin{array}{l}\text { Rural; Women with } \\
\text { high-risk } \\
\text { pregnancies }\end{array}$ & NR & $\begin{array}{l}\text { Maternal-fetal medicine } \\
\text { specialists and associated } \\
\text { residents }\end{array}$ & Unclear & \begin{tabular}{|l|} 
Inpatient for \\
transports \\
and hospital \\
days \\
Outpatient \\
for \\
consultations \\
\end{tabular} & $\begin{array}{l}\text { NR - videoconference, } \\
\text { presumably }\end{array}$ & Real-time \\
\hline $\begin{array}{l}\text { Brown-Connolly, } \\
2002\end{array}$ & $\begin{array}{l}741 \\
\text { A: NR } \\
\text { B: NR } \\
\end{array}$ & $\begin{array}{l}\text { Adults and } \\
\text { Pediatrics }\end{array}$ & Primary care & Various & Yes & Outpatient & NR & Real-time \\
\hline Burns, 2017 & \begin{tabular}{|l|}
82 \\
A: 39 \\
B: 43 \\
\end{tabular} & Adult & $\begin{array}{l}\text { Regional speech } \\
\text { pathologist }\end{array}$ & Hospital speech pathologist & Yes & Outpatient & Videoconference & Real-time \\
\hline Byamba, 2015 & $\begin{array}{l}450 \\
\text { A: } 229 \\
\text { B: } 221 \\
\end{array}$ & Rural patients & General practitioner & Dermatologist & No & Outpatient & $\begin{array}{l}\text { Clinical notes and } \\
\text { pictures }\end{array}$ & Asynchronous \\
\hline Carallo, 2015 & $\begin{array}{l}312 \\
\text { A: } 208 \\
B: 104\end{array}$ & $\begin{array}{l}\text { Adults with type II } \\
\text { diabetes, without } \\
\text { major } \\
\text { cardiovascular } \\
\text { disease } \\
\end{array}$ & Primary care & Diabetes & Unclear & Outpatient & Electronic health record & Asynchronous \\
\hline Carter, 2017 & $\begin{array}{l}252 \\
\text { A: } 173 \\
\text { B: } 79 \\
\end{array}$ & Adults & $\begin{array}{l}\text { Primary care } \\
\text { physician }\end{array}$ & Dermatology & Yes & Outpatient & Electronic health record & Asynchronous \\
\hline Chan, 2015 & $\begin{array}{l}206 \\
\text { A: } 117 \\
\text { B: } 89 \\
\end{array}$ & rural & General physician & Oncologist & Yes & Outpatient & Videoconference & Real-time \\
\hline Chu, 2015 & $\begin{array}{l}97 \\
\text { A: NR } \\
\text { B: NR } \\
\end{array}$ & Veterans & Primary care & Urology & Yes & Outpatient & Videoconference & Real-time \\
\hline Chua, 2001 & $\begin{array}{l}141 \\
\text { A: } 65 \\
\text { B: } 76 \\
\end{array}$ & Adult & $\begin{array}{l}\text { Primary care } \\
\text { physician }\end{array}$ & Neurologist & Yes & Outpatient & Videoconference & Real-time \\
\hline
\end{tabular}




\begin{tabular}{|c|c|c|c|c|c|c|c|c|}
\hline $\begin{array}{l}\text { Author, Year } \\
\text { (See Appendix C } \\
\text { for full citation) }\end{array}$ & $\begin{array}{l}\text { Number } \\
\text { Analyzed }^{a}\end{array}$ & Population & $\begin{array}{l}\text { Referring } \\
\text { Providers } \\
\text { Specialty/Type } \\
\end{array}$ & $\begin{array}{l}\text { Consulting Provider } \\
\text { Specialty/Type }\end{array}$ & $\begin{array}{l}\text { Patient } \\
\text { Present? }\end{array}$ & $\begin{array}{l}\text { Patient } \\
\text { Setting } \\
\end{array}$ & $\begin{array}{l}\text { Modalities of } \\
\text { Telehealth } \\
\text { Consultation } \\
\text { Intervention } \\
\end{array}$ & Timing \\
\hline $\begin{array}{l}\text { Collins, 2004; } \\
\text { Bowns, } 2006\end{array}$ & $\begin{array}{l}208 \\
\text { A: } 97 \\
\text { B: } 111\end{array}$ & $\begin{array}{l}\text { Adults } 16 \text { or older, } \\
\text { with no diagnoses } \\
\text { of dermatological or } \\
\text { mental health } \\
\text { problems }\end{array}$ & General practitioner & Dermatology & No & Outpatient & Not specified & Asynchronous \\
\hline Crossland, 2016 & $\begin{array}{l}1,024 \\
\text { A: } 577 \\
\text { B: } 447 \\
\end{array}$ & Adults & $\begin{array}{l}\text { General } \\
\text { practitioners }\end{array}$ & Ophthalmologists & Unclear & Outpatient & $\begin{array}{l}\text { Teleconferences } \\
\text { and/or email }\end{array}$ & Unclear \\
\hline Datta, 2015 & $\begin{array}{l}391 \\
\text { A: } 196 \\
\text { B: } 195 \\
\end{array}$ & Adults & $\begin{array}{l}\text { Primary care } \\
\text { physician }\end{array}$ & Dermatologist & & Outpatient & Records and images & Asynchronous \\
\hline Davis, 2011 & $\begin{array}{l}360 \\
\text { A: } 200 \\
B: 160 \\
\end{array}$ & $\begin{array}{l}\text { Veterans (adults) } \\
75 \% \text { white; }>80 \% \\
\text { male }\end{array}$ & Not specified & $\begin{array}{l}\text { Nurse, pharmacist, } \\
\text { psychiatrist }\end{array}$ & No & Outpatient & Videoconference & Real-time \\
\hline Davison, 2004 & $\begin{array}{l}112 \\
\text { A: } 50 \\
\text { B: } 62\end{array}$ & Adults & $\begin{array}{l}\text { Chest medicine } \\
\text { lung cancer clinical } \\
\text { nurse }\end{array}$ & $\begin{array}{l}\text { Thoracic surgeon and } \\
\text { radiologist }\end{array}$ & No & Outpatient & Video & Real-time \\
\hline De Luca, 2005 & $\begin{array}{l}3,934 \\
\text { A: } 1,985 \\
\text { B: } 1,949 \\
\end{array}$ & Adults & Primary care & Cardiologist & Yes & Outpatient & $\begin{array}{l}\text { Internet based digital } \\
\text { network }\end{array}$ & Asynchronous \\
\hline Doolittle, 1997 & $\begin{array}{l}\text { NR } \\
\text { A: } 81 \\
\text { B: NR } \\
\text { C: } 103 \\
\end{array}$ & NR & NR & Oncologist & Yes & Outpatient & Videoconference & Real-time \\
\hline Dowie, 2007 & $\begin{array}{l}504 \\
\text { A: } 387 \\
\text { B: } 117 \\
\end{array}$ & $\begin{array}{l}\text { Pregnant women } \\
\text { and pediatric }\end{array}$ & Unclear & Cardiologist & Yes and no & Outpatient & Videoconference & Mixed \\
\hline $\begin{array}{l}\text { Eminovic, 2009, } \\
2010\end{array}$ & $\begin{array}{l}605 \\
\text { A: } 304 \\
\text { B: } 301 \\
\end{array}$ & $\begin{array}{l}\text { Pediatric and adult } \\
\text { patients }\end{array}$ & General practitioner & Dermatology & Yes & Outpatient & $\begin{array}{l}\text { Digital camera and } \\
\text { website }\end{array}$ & Asynchronous \\
\hline Endean, 2001 & $\begin{array}{l}32 \\
\text { A: } 32 \\
\text { B: } 32\end{array}$ & Adults & NR & Vascular specialist & Yes & Outpatient & Videoconference & Real-time \\
\hline Ferrandiz, 2007 & $\begin{array}{l}134 \\
\text { A: NR } \\
\text { B: NR }\end{array}$ & Adults & Primary care & Dermatologist & Unclear & Outpatient & $\begin{array}{l}\text { Digital pictures inserted } \\
\text { into Word document } \\
\text { containing other clinical } \\
\text { information. Document } \\
\text { sent via intranet to } \\
\text { email account of skin } \\
\text { cancer clinic. } \\
\end{array}$ & Asynchronous \\
\hline
\end{tabular}




\begin{tabular}{|c|c|c|c|c|c|c|c|c|}
\hline $\begin{array}{l}\text { Author, Year } \\
\text { (See Appendix C } \\
\text { for full citation) }\end{array}$ & $\begin{array}{l}\text { Number } \\
\text { Analyzed }^{a}\end{array}$ & Population & $\begin{array}{l}\text { Referring } \\
\text { Providers } \\
\text { SpecialtylType } \\
\end{array}$ & $\begin{array}{l}\text { Consulting Provider } \\
\text { Specialty/Type }\end{array}$ & \begin{tabular}{|l} 
Patient \\
Present?
\end{tabular} & $\begin{array}{l}\text { Patient } \\
\text { Setting }\end{array}$ & $\begin{array}{l}\text { Modalities of } \\
\text { Telehealth } \\
\text { Consultation } \\
\text { Intervention }\end{array}$ & Timing \\
\hline Fortney, 2013 & $\begin{array}{l}364 \\
\text { A: } 185 \\
\text { B: } 179 \\
\end{array}$ & $\begin{array}{l}\text { Adults, majority low } \\
\text { income, rural }\end{array}$ & Primary care & $\begin{array}{l}\text { Pharmacy, psychology, } \\
\text { psychiatry, care } \\
\text { management }\end{array}$ & Sometimes & Outpatient & $\begin{array}{l}\text { Telephone, } \\
\text { videoconference }\end{array}$ & Real-time \\
\hline Fortney, 2007 & $\begin{array}{l}395 \\
\text { A: } 218 \\
\text { B: } 177\end{array}$ & $\begin{array}{l}\text { Adults, majority } \\
\text { older white men }\end{array}$ & Primary care & Psychiatry & Yes & Outpatient & $\begin{array}{l}\text { Phone, medical } \\
\text { records, video }\end{array}$ & Real-time \\
\hline Fortney, 2011 & $\begin{array}{l}395 \\
\text { A: } 218 \\
\text { B: } 177\end{array}$ & Adults & $\begin{array}{l}\text { Primary care } \\
\text { provider }\end{array}$ & Psychiatrists & Yes & Outpatient & $\begin{array}{l}\text { Mixed (interactive } \\
\text { video, telephone) }\end{array}$ & Real-time \\
\hline Fortney, 2015 & $\begin{array}{l}265 \\
\text { A: } 132 \\
\text { B: } 133\end{array}$ & $\begin{array}{l}\text { Veterans, mostly } \\
\text { male }\end{array}$ & $\begin{array}{l}\text { PTSD providers in } \\
\text { CBOCs were } \\
\text { recruited as were } \\
\text { patients with PTSD } \\
\text { diagnoses }\end{array}$ & $\begin{array}{l}\text { Telepsychiatrists, } \\
\text { telephone nurse care } \\
\text { manager }\end{array}$ & Yes & Outpatient & $\begin{array}{l}\text { Telephone for care } \\
\text { managers and } \\
\text { pharmacist. } \\
\text { Psychotherapy and } \\
\text { psychiatric consultation } \\
\text { via videoconference. } \\
\text { Care managers also } \\
\text { used web based } \\
\text { decision support } \\
\text { system. }\end{array}$ & Real-time \\
\hline Fox, 2007 & $\begin{array}{l}706 \\
\text { A: } 173 \\
\text { B: } 257 \\
\text { C: } 276 \\
\end{array}$ & $\begin{array}{l}\text { Adolescents in } \\
\text { state correctional } \\
\text { facilities }\end{array}$ & General nurse & Multiple & Yes & $\begin{array}{l}\text { Other } \\
\text { (correctional } \\
\text { facility) }\end{array}$ & Videoconference & Real-time \\
\hline Frank, 2015 & $\begin{array}{l}322,059 \\
\text { A: } 299,981 \\
\text { B: } 22,454\end{array}$ & $\begin{array}{l}\text { Adult VA patients } \\
\text { with pain that have } \\
\text { an established } \\
\text { primary care } \\
\text { provider at the VA }\end{array}$ & $\begin{array}{l}\text { Primary care } \\
\text { provider }\end{array}$ & Pain specialist & No & Outpatient & Videoconference & Real-time \\
\hline Gattas, 2001 & $\begin{array}{l}62 \\
\text { A: } 23 \text { (8 } \\
\text { providers, } 8 \\
\text { counselors, } 5 \\
\text { patients) } \\
\text { B: } 44 \text { (16 } \\
\text { providers, } 16 \\
\text { counselors, } 12 \\
\text { patients) }\end{array}$ & NR & Genetic counselor & Genetics & Yes & Outpatient & Videoconference & Real-time \\
\hline Gilmour, 1998 & $\begin{array}{l}126 \\
\text { A: NR } \\
B: N R\end{array}$ & $\begin{array}{l}\text { Pediatrics and } \\
\text { adults }\end{array}$ & General practitioner & Dermatologist & Yes & Outpatient & Videoconference & Real-time \\
\hline
\end{tabular}




\begin{tabular}{|c|c|c|c|c|c|c|c|c|}
\hline $\begin{array}{l}\text { Author, Year } \\
\text { (See Appendix C } \\
\text { for full citation) }\end{array}$ & $\begin{array}{l}\text { Number } \\
\text { Analyzed }^{\mathrm{a}}\end{array}$ & Population & $\begin{array}{l}\text { Referring } \\
\text { Providers } \\
\text { Specialty/Type }\end{array}$ & $\begin{array}{l}\text { Consulting Provider } \\
\text { Specialty/Type }\end{array}$ & $\begin{array}{l}\text { Patient } \\
\text { Present? }\end{array}$ & $\begin{array}{l}\text { Patient } \\
\text { Setting }\end{array}$ & $\begin{array}{l}\text { Modalities of } \\
\text { Telehealth } \\
\text { Consultation } \\
\text { Intervention }\end{array}$ & Timing \\
\hline Harno, 2000 & $\begin{array}{l}292 \\
\text { A: } 85 \\
\text { B: } 207\end{array}$ & Adults & Primary care & $\begin{array}{l}\text { Various hospital outpatient } \\
\text { specialties }\end{array}$ & No & Outpatient & $\begin{array}{l}\text { Electronic referral } \\
\text { system; referrals } \\
\text { transferred by intranet } \\
\text { to electronic mail boxes } \\
\text { in the hospital } \\
\text { information system; } \\
\text { video-networking in } \\
\text { separate study. }\end{array}$ & Asynchronous \\
\hline Harno, 2001 & $\begin{array}{l}225 \\
\text { A: } 168 \\
B: 57 \\
\end{array}$ & Adults & Unclear & Orthopedics & Yes & Outpatient & $\begin{array}{l}\text { Email } \\
\text { Videoconference }\end{array}$ & Real-time \\
\hline $\begin{array}{l}\text { Haukipuro same } \\
\text { pts as Ohinmaa } \\
\text { and Vuolio, } 2000\end{array}$ & $\begin{array}{l}145 \\
\text { A: } 69 \\
\text { B: } 76\end{array}$ & Adult & General practitioner & Orthopedics & Yes & Outpatient & Videoconference & Real-time \\
\hline Herce, 2011 & $\begin{array}{l}2,647 \\
\text { A: } 2,550 \\
B: 97\end{array}$ & Adult & $\begin{array}{l}\text { Primary care } \\
\text { dentist }\end{array}$ & Oral surgeon & No & Outpatient & Digital images & Asynchronous \\
\hline Hsiao, 2008 & $\begin{array}{l}169 \\
\text { A: } 77 \\
\text { B: } 92\end{array}$ & $\begin{array}{l}\text { Veterans seen in } \\
\text { the San Francisco } \\
\text { VA or outlying VA } \\
\text { clinics }\end{array}$ & $\begin{array}{l}\text { Primary care } \\
\text { provider }\end{array}$ & Dermatology & No & Outpatient & $\begin{array}{l}\text { Electronic consult } \\
\text { through a shared } \\
\text { medical record system. }\end{array}$ & Asynchronous \\
\hline Izquierdo, 2009 & $\begin{array}{l}41 \\
\text { A: } 18 \\
\text { B: } 23 \\
\end{array}$ & $\begin{array}{l}\text { Kindergarten } \\
\text { through eighth } \\
\text { grade }\end{array}$ & School nurse & Diabetes nurse practitioner & Yes & $\begin{array}{l}\text { Outpatient } \\
\text { (School) }\end{array}$ & Videoconference & Real-time \\
\hline Jaatinen, 2002 & $\begin{array}{l}78 \\
\text { A: } 24 \\
\text { B: } 54\end{array}$ & Adults & $\begin{array}{l}\text { General } \\
\text { practitioners }\end{array}$ & $\begin{array}{l}\text { Internists, surgeons, } \\
\text { geriatricians }\end{array}$ & Unclear & Outpatient & Web-based system & Asynchronous \\
\hline $\begin{array}{l}\text { Jacklin, } 2003 \\
\text { Same study as } \\
\text { Wallace 2002, } \\
2004\end{array}$ & $\begin{array}{l}1,939 \\
\text { A: } 971 \\
\text { B: } 968\end{array}$ & $\begin{array}{l}\text { Not clearly defined } \\
\text { in this paper, refer } \\
\text { to another previous } \\
\text { paper, essentially } \\
\text { any patient that met } \\
\text { inclusion criteria } \\
\text { not defined in this } \\
\text { paper, that needed } \\
\text { a consultation }\end{array}$ & General practitioner & $\begin{array}{l}\text { Not specified, seems like } \\
\text { any consultation }\end{array}$ & Yes & Outpatient & Videoconference & Real-time \\
\hline
\end{tabular}




\begin{tabular}{|c|c|c|c|c|c|c|c|c|}
\hline $\begin{array}{l}\text { Author, Year } \\
\text { (See Appendix C } \\
\text { for full citation) }\end{array}$ & $\begin{array}{l}\text { Number } \\
\text { Analyzed }^{a}\end{array}$ & Population & $\begin{array}{l}\text { Referring } \\
\text { Providers } \\
\text { Specialty/Type } \\
\end{array}$ & $\begin{array}{l}\text { Consulting Provider } \\
\text { Specialty/Type }\end{array}$ & \begin{tabular}{|l} 
Patient \\
Present?
\end{tabular} & $\begin{array}{l}\text { Patient } \\
\text { Setting } \\
\end{array}$ & \begin{tabular}{|l|} 
Modalities of \\
Telehealth \\
Consultation \\
Intervention \\
\end{tabular} & Timing \\
\hline Jacobs, 2015 & $\begin{array}{l}794 \\
\text { A: } 312 \\
\text { B: } 482\end{array}$ & $\begin{array}{l}\text { All patients who } \\
\text { visited GP with } \\
\text { trauma related to } \\
\text { the musculoskeletal } \\
\text { system }\end{array}$ & General practitioner & Radiologist and surgeon & No & $\begin{array}{l}\text { ED/EMS: } \\
\text { Urgent visit } \\
\text { to GP office } \\
\text { acting as ED }\end{array}$ & $\begin{array}{l}\text { Data transfer: digital x- } \\
\text { rays }\end{array}$ & Asynchronous \\
\hline Jong, 2004 & $\begin{array}{l}12 \\
\text { A: } 2 \\
\text { B: } 4 \\
\text { C: } 6 \\
\text { For cost } \\
\text { outcomes, } \\
\text { patient sample } \\
\text { size NR } \\
\end{array}$ & NR & Primary care & Rheumatology & Not always & Outpatient & Videoconference & Real-time \\
\hline Jue, 2017 & \begin{tabular}{|l}
296 \\
A: Same \\
patients \\
B: 296 \\
\end{tabular} & Adults & Nurse practitioner & Surgical oncologist & Yes & Outpatient & Videoconference & Real-time \\
\hline Kobza, 2000 & $\begin{array}{l}196 \\
\text { A: } 120 \\
\text { B: } 76 \\
\end{array}$ & Adults & Home health nurse & Wound care specialist & Yes & Home & Videoconference & Real-time \\
\hline Krier, 2011 & \begin{tabular}{|l|}
34 \\
A: 19 \\
B: 15 \\
\end{tabular} & Adults & $\begin{array}{l}\text { Gastroenterology } \\
\text { fellows }\end{array}$ & $\begin{array}{l}\text { Gastroenterology and } \\
\text { endoscopy specialist }\end{array}$ & Yes & Outpatient & Videoconference & Real-time \\
\hline Krupinski, 2004 & $\begin{array}{l}100 \\
A: 50 \\
B: 50\end{array}$ & $\begin{array}{l}\text { Rural patients } \\
\text { referred for } \\
\text { dermatology } \\
\text { consult }\end{array}$ & $\begin{array}{l}\text { Primary care } \\
\text { provider }\end{array}$ & Dermatology & No & Outpatient & Records and images & Asynchronous \\
\hline Kunkler, 2007 & $\begin{array}{l}473 \\
\text { A: } 195 \\
\text { B: } 278\end{array}$ & Predominantly rural & NR & $\begin{array}{l}\text { Consultant breast } \\
\text { surgeons, medical and } \\
\text { clinical oncologists, } \\
\text { radiologists, pathologists, } \\
\text { and breast care nurses } \\
\end{array}$ & No & Outpatient & $\begin{array}{l}\text { Viewing of } \\
\text { mammograms, CT } \\
\text { scans and pathology } \\
\text { as well as } \\
\text { videoconferencing } \\
\end{array}$ & Real-time \\
\hline Lamminen, 2001 & $\begin{array}{l}191 \\
A: 85 \\
B: 64 \\
C: 24 \\
D: 18\end{array}$ & NR & General practitioner & $\begin{array}{l}\text { Ophthalmology and } \\
\text { dermatology }\end{array}$ & Yes & Outpatient & Videoconference & Real-time \\
\hline
\end{tabular}




\begin{tabular}{|c|c|c|c|c|c|c|c|c|}
\hline $\begin{array}{l}\text { Author, Year } \\
\text { (See Appendix C } \\
\text { for full citation) }\end{array}$ & $\begin{array}{l}\text { Number } \\
\text { Analyzed }^{\mathrm{a}}\end{array}$ & Population & $\begin{array}{l}\text { Referring } \\
\text { Providers } \\
\text { SpecialtylType }\end{array}$ & $\begin{array}{l}\text { Consulting Provider } \\
\text { Specialty/Type }\end{array}$ & $\begin{array}{l}\text { Patient } \\
\text { Present? }\end{array}$ & $\begin{array}{l}\text { Patient } \\
\text { Setting }\end{array}$ & $\begin{array}{l}\text { Modalities of } \\
\text { Telehealth } \\
\text { Consultation } \\
\text { Intervention }\end{array}$ & Timing \\
\hline Lee, 2014 & $\begin{array}{l}3,081 \\
\text { A: NR } \\
\text { B: NR }\end{array}$ & Adults & Primary care & Osteology & No & Outpatient & $\begin{array}{l}\text { Laboratory data, } \\
\text { clinical risk factors, } \\
\text { prior treatment, } \\
\text { electronic notes } \\
\end{array}$ & Asynchronous \\
\hline Lim, 2012 & $\begin{array}{l}300 \\
\text { A: } 100 \\
B: 200\end{array}$ & Pediatric/adults & General practitioner & Dermatologist & No & Outpatient & Secure server & Asynchronous \\
\hline Loane, 2000 & $\begin{array}{l}204 \\
\text { A: } 102 \\
\text { B: } 102 \text { Video } \\
\text { C: } 96 \text { (96 of } \\
\text { the } 102 \text { were } \\
\text { also store and } \\
\text { forward) }\end{array}$ & Pediatric and adults & $\begin{array}{l}\text { Primary care } \\
\text { physician }\end{array}$ & Dermatologist & Yes & Outpatient & Video & Mixed \\
\hline Loane, 1999 & $\begin{array}{l}164 \\
\text { A: } 103 \\
B: 61\end{array}$ & $\begin{array}{l}\text { Adults and children } \\
\text { (range: } 3 \text { months to } \\
84 \text { years; mean } \\
\text { age } 42 \text { years) } \\
\end{array}$ & $\begin{array}{l}\text { General } \\
\text { practitioners }\end{array}$ & Dermatologist & Yes & Outpatient & $\begin{array}{l}\text { Low-cost } \\
\text { videoconferencing } \\
\text { units (VC7000, British } \\
\text { Telecom) } \\
\end{array}$ & Real-time \\
\hline Loane, 2001 & $\begin{array}{l}203 \\
\text { A: } 94 \\
\text { B: } 109\end{array}$ & $\begin{array}{l}\text { Adults and children } \\
\text { (range: } 3 \text { months to } \\
84 \text { years; mean } \\
\text { age } 42 \text { years) }\end{array}$ & $\begin{array}{l}\text { General } \\
\text { practitioners }\end{array}$ & Dermatologist & Yes & Outpatient & $\begin{array}{l}\text { Low-cost personal } \\
\text { computers with } \\
\text { videoconferencing; } \\
\text { video cameras to } \\
\text { transmit close-up } \\
\text { images of skin lesions. }\end{array}$ & Real-time \\
\hline Long, 2014 & $\begin{array}{l}\text { NR } \\
\text { A: NR } \\
\text { B: NR }\end{array}$ & $\begin{array}{l}\text { Maternal Medicaid } \\
\text { population }\end{array}$ & $\begin{array}{l}\text { Community } \\
\text { providers }\end{array}$ & $\begin{array}{l}\text { Maternal fetal medicine } \\
\text { specialists (maternal fetal } \\
\text { medicine nurses staff } 24 / 7 \\
\text { call center) }\end{array}$ & $\begin{array}{l}\text { Yes for } \\
\text { some } \\
\text { ultrasounds, } \\
\text { but not } \\
\text { clear on } \\
\text { how many. }\end{array}$ & Outpatient & $\begin{array}{l}\text { Six-fold telehealth } \\
\text { model - includes } \\
\text { education and training } \\
\text { for providers, 24/7 call } \\
\text { center for support, } \\
\text { telehealth network, } \\
\text { clinical research, case } \\
\text { management and } \\
\text { evidence based } \\
\text { guidelines and } \\
\text { protocols. }\end{array}$ & Real-time \\
\hline
\end{tabular}




\begin{tabular}{|c|c|c|c|c|c|c|c|c|}
\hline $\begin{array}{l}\text { Author, Year } \\
\text { (See Appendix C } \\
\text { for full citation) }\end{array}$ & $\begin{array}{l}\text { Number } \\
\text { Analyzed }^{\mathrm{a}}\end{array}$ & Population & $\begin{array}{l}\text { Referring } \\
\text { Providers } \\
\text { Specialty/Type } \\
\end{array}$ & $\begin{array}{l}\text { Consulting Provider } \\
\text { Specialty/Type }\end{array}$ & $\begin{array}{l}\text { Patient } \\
\text { Present? }\end{array}$ & $\begin{array}{l}\text { Patient } \\
\text { Setting } \\
\end{array}$ & $\begin{array}{l}\text { Modalities of } \\
\text { Telehealth } \\
\text { Consultation } \\
\text { Intervention } \\
\end{array}$ & Timing \\
\hline Mahendran, 2005 & $\begin{array}{l}163 \\
\text { A: } 163 \\
\text { B: } 163 ; \text { same } \\
\text { as group A }\end{array}$ & $\begin{array}{l}\text { Any patient with } \\
\text { suspicious skin } \\
\text { lesion presenting to } \\
\text { their GP during a } \\
\text { specified } 18 \\
\text { months period. }\end{array}$ & General practitioner & Dermatology & No & Outpatient & $\begin{array}{l}\text { Store and forward: } \\
\text { records }\end{array}$ & Asynchronous \\
\hline McCrossan, 2012 & $\begin{array}{l}66 \\
\text { A: NR } \\
\text { B: NR }\end{array}$ & \begin{tabular}{|l|} 
Pregnant women, \\
31.3 years (range = \\
17.1 to 40.8 years) \\
Neonates, 23 \\
weeks (range $=21$ \\
to 26 weeks) \\
\end{tabular} & NR & Fetal cardiologist & Yes & Outpatient & Mixed & Real-time \\
\hline $\begin{array}{l}\text { Moreno-Ramirez, } \\
2009\end{array}$ & $\begin{array}{l}4,018 \\
\text { A: } 2,009 \\
\text { B: } 2,009 \\
\end{array}$ & \begin{tabular}{|l|}
$N R$ \\
\end{tabular} & General practitioner & Dermatologist & Yes & Outpatient & $\begin{array}{l}\text { Digital Camera and } \\
\text { Intranet }\end{array}$ & Asynchronous \\
\hline Mulgrew, 2011 & $\begin{array}{l}25 \\
\text { A: } 15 \\
\text { B: } 10\end{array}$ & Pediatric & $\begin{array}{l}\text { Primary care } \\
\text { physician }\end{array}$ & $\begin{array}{l}\text { Pediatrician specializing in } \\
\text { weight management }\end{array}$ & Yes & Outpatient & Videoconference & Real-time \\
\hline Nickenig, 2008 & $\begin{array}{l}857 \\
\text { A: } 772 \\
\text { B: } 85 \\
\end{array}$ & NR & Dentist & Implant Specialist & No & Outpatient & Videoconference & Real-time \\
\hline Nikkanen, 2008 & $\begin{array}{l}101 \\
\text { A: } 101 \\
\text { B: } 101 \text { (same } \\
\text { as A) }\end{array}$ & Adults & Diabetes & Diabetes & & Outpatient & $\begin{array}{l}\text { Videoconference, } \\
\text { electronic records, } \\
\text { electronic stethoscope }\end{array}$ & Real-time \\
\hline Nordal, 2001 & $\begin{array}{l}121 \\
\text { A1: NR } \\
\text { A2: NR }\end{array}$ & $\begin{array}{l}\text { Adult men and } \\
\text { women (aged 17- } \\
82 \text { years) }\end{array}$ & $\begin{array}{l}\text { University Hospital } \\
\text { of Troms } \varnothing\end{array}$ & Dermatologist & Yes & Outpatient & Videoconference & Real-time \\
\hline $\begin{array}{l}\text { Ohinmaa } \\
\text { Same patients as } \\
\text { Vuolio and } \\
\text { Haukipuro, } 2002 \\
\end{array}$ & $\begin{array}{l}145 \\
\text { A: } 69 \\
B: 76\end{array}$ & Adults & General practitioner & Orthopedics & Yes & Outpatient & Videoconference & Real-time \\
\hline $\begin{array}{l}\text { Pak, 2009; Pak, } \\
2007\end{array}$ & $\begin{array}{l}508 \\
\text { A: } 236 \\
B: 272\end{array}$ & $\begin{array}{l}\text { Adult ( } \geq 18 \text { years } \\
\text { old) who were } \\
\text { referred from the } \\
\text { Department of } \\
\text { Defense } \\
\end{array}$ & Clinician & Dermatologist & No & Outpatient & $\begin{array}{l}\text { Store and forward: } \\
\text { records }\end{array}$ & Asynchronous \\
\hline Piette, 2017 & $\begin{array}{l}103 \\
\text { A: } 50 \\
B: 53\end{array}$ & Adults & General practitioner & Dermatology & No & Outpatient & Email & Asynchronous \\
\hline
\end{tabular}




\begin{tabular}{|c|c|c|c|c|c|c|c|c|}
\hline $\begin{array}{l}\text { Author, Year } \\
\text { (See Appendix C } \\
\text { for full citation) }\end{array}$ & $\begin{array}{l}\text { Number } \\
\text { Analyzed }^{a}\end{array}$ & Population & $\begin{array}{l}\text { Referring } \\
\text { Providers } \\
\text { Specialty/Type } \\
\end{array}$ & $\begin{array}{l}\text { Consulting Provider } \\
\text { SpecialtylType }\end{array}$ & $\begin{array}{l}\text { Patient } \\
\text { Present? }\end{array}$ & $\begin{array}{l}\text { Patient } \\
\text { Setting }\end{array}$ & $\begin{array}{l}\text { Modalities of } \\
\text { Telehealth } \\
\text { Consultation } \\
\text { Intervention } \\
\end{array}$ & Timing \\
\hline Rossaro, 2013 & $\begin{array}{l}80 \\
\text { A: } 40 \\
\text { B: } 40 \\
\end{array}$ & Adults & Primary care & Hepatologist & No & Outpatient & Videoconference & Real-time \\
\hline Salami, 2015 & $\begin{array}{l}116 \\
\text { A: } 68 \\
\text { B: } 48\end{array}$ & Adult men & $\begin{array}{l}\text { Oncology, } \\
\text { gastroenterology, } \\
\text { hepatology, } \\
\text { surgery, other }\end{array}$ & \begin{tabular}{|l|} 
Radiology, \\
gastroenterology, \\
hepatology, \\
transplantation, surgical \\
oncology, medical \\
oncology, radiology
\end{tabular} & $\begin{array}{l}\text { Not } \\
\text { specified }\end{array}$ & Outpatient & $\begin{array}{l}\text { VA electronic medical } \\
\text { record, audiovisual } \\
\text { teleconferencing }\end{array}$ & Real-time \\
\hline $\begin{array}{l}\text { Salazar- } \\
\text { Fernandez, } 2012\end{array}$ & $\begin{array}{l}1,052 \\
\text { A: } 710 \\
\text { B: } 342 \\
\end{array}$ & Adults & Dentist & Oral and maxillofacial & Yes & Outpatient & Store and forward & Asynchronous \\
\hline Santamaria, 2004 & $\begin{array}{l}\text { A : } 43 \\
\text { B: } 50\end{array}$ & Adults & Wound care nurses & Wound care consultant & No & Outpatient & Imaging system & Asynchronous \\
\hline Scuffham, 2002 & $\begin{array}{l}25 \\
\text { A: NR } \\
\text { B: NR } \\
\text { C: NR }\end{array}$ & Adults & General dentist & Specialist dentist & Yes & Outpatient & Videoconference & Real-time \\
\hline Sharma, 2003 & $\begin{array}{l}229 \\
\text { A: } 195 \\
\text { B: } 34\end{array}$ & Adults aged 25-89 & NR & $\begin{array}{l}\text { Staff echocardiographer, } \\
\text { pediatric cardiology fellow }\end{array}$ & Yes & $\begin{array}{l}\text { Primary care } \\
\text { center }\end{array}$ & $\begin{array}{l}\text { Live screening of } \\
\text { echocardiogram } \\
\text { transmitted via data } \\
\text { transmission }\end{array}$ & Real-time \\
\hline Smith, 2002 & $\begin{array}{l}387 \\
\text { A: NR } \\
\text { B: NR }\end{array}$ & Children & Pediatricians & $\begin{array}{l}\text { Physicians in subspecialty } \\
\text { fields including burns, } \\
\text { cardiology, dermatology, } \\
\text { diabetes, endocrinology, } \\
\text { neurology, nephrology, } \\
\text { oncology, orthopedics and } \\
\text { respiratory medicine }\end{array}$ & Unclear & Outpatient & $\begin{array}{l}\text { Videoconference, } \\
\text { email, fax, telephone }\end{array}$ & Real-time \\
\hline Smith-Strom, 2018 & \begin{tabular}{|l|}
182 \\
A: 88 \\
B: 94 \\
\end{tabular} & Adult & Community nurses & Specialist nurse & Yes & Outpatient & $\begin{array}{l}\text { Web-based record and } \\
\text { mobile phone }\end{array}$ & Asynchronous \\
\hline Specht, 2001 & $\begin{array}{l}\mathrm{NR} \\
\text { A: NR } \\
\text { B: NR }\end{array}$ & $\begin{array}{l}\text { Adults in a long } \\
\text { term care facility. }\end{array}$ & $\begin{array}{l}\text { Primary nurse } \\
\text { and/or skin care } \\
\text { nurse at the LTC }\end{array}$ & $\begin{array}{l}\text { Chronic wound nurse } \\
\text { expert }\end{array}$ & Yes & $\begin{array}{l}\text { Long term } \\
\text { care facility }\end{array}$ & $\begin{array}{l}\text { Video using Teledoc } \\
5000\end{array}$ & Asynchronous \\
\hline
\end{tabular}




\begin{tabular}{|c|c|c|c|c|c|c|c|c|}
\hline $\begin{array}{l}\text { Author, Year } \\
\text { (See Appendix C } \\
\text { for full citation) }\end{array}$ & $\begin{array}{l}\text { Number } \\
\text { Analyzed }^{\mathrm{a}}\end{array}$ & Population & $\begin{array}{l}\text { Referring } \\
\text { Providers } \\
\text { Specialty/Type }\end{array}$ & $\begin{array}{l}\text { Consulting Provider } \\
\text { Specialty/Type }\end{array}$ & $\begin{array}{l}\text { Patient } \\
\text { Present? }\end{array}$ & $\begin{array}{l}\text { Patient } \\
\text { Setting }\end{array}$ & $\begin{array}{l}\text { Modalities of } \\
\text { Telehealth } \\
\text { Consultation } \\
\text { Intervention }\end{array}$ & Timing \\
\hline Stalfors, 2003 & \begin{tabular}{|l}
104 \\
(completed \\
patient \\
satisfaction \\
survey) \\
A: $46(39)$ \\
B: $58(45)$ \\
\end{tabular} & Not specified & $\begin{array}{l}\text { Ear nose and } \\
\text { throat surgeon }\end{array}$ & Multiple specialties & Yes & Outpatient & $\begin{array}{l}\text { Videoconference, still } \\
\text { images }\end{array}$ & Real-time \\
\hline Stalfors, 2005 & $\begin{array}{l}118 \\
\text { A: } 50 \\
\text { B: } 68\end{array}$ & $\begin{array}{l}\text { Patients with head } \\
\text { and neck cancers } \\
\text { that are discussed } \\
\text { in the } \\
\text { multidisciplinary } \\
\text { team meeting }\end{array}$ & NR & Multidisciplinary team & Yes & Outpatient & Videoconference & Real-time \\
\hline Stern, 2014 & $\begin{array}{l}\text { Total wounds } \\
\text { analyzed: } 259 \\
\text { among } 137 \\
\text { LTC residents }\end{array}$ & $\begin{array}{l}\text { Long term care } \\
\text { facilities with }>100 \\
\text { beds in Toronto } \\
\text { Central and Central } \\
\text { LHINs with } \\
\text { pressure ulcer } \\
\text { prevalence }>5.5 \%\end{array}$ & Unclear & $\begin{array}{l}\text { Skin and wound care, } \\
\text { plastic surgeon, } \\
\text { occupational therapist, } \\
\text { chiropodist }\end{array}$ & Unclear & $\begin{array}{l}\text { Long term } \\
\text { care facility }\end{array}$ & Email, telephone, data & Asynchronous \\
\hline Strymish, 2017 & $\begin{array}{l}480 \\
\text { A: } 195 \\
\text { B: } 285 \\
\end{array}$ & Adults & NR & Specialist & No & Unclear & e-consult & Asynchronous \\
\hline Taylor, 2007 & $\begin{array}{l}495 \\
\text { A: } 294 \\
\text { B: } 201 \\
\end{array}$ & Adults & $\begin{array}{l}\text { Primary care } \\
\text { physician }\end{array}$ & Expert technical graders & No & Outpatient & Digital images & Asynchronous \\
\hline Thaker, 2013 & \begin{tabular}{|l|}
$N R$ \\
A: NR \\
B: 147
\end{tabular} & Not specified & Not specified & Oncologist & $\begin{array}{l}\text { Yes, local } \\
\text { physician } \\
\text { not always } \\
\text { present }\end{array}$ & Outpatient & Videoconference & Real-time \\
\hline Tsitlakidis, 2005 & $\begin{array}{l}38 \\
\text { A: NR } \\
\text { B: NR }\end{array}$ & $\begin{array}{l}\text { Children and adults } \\
\text { (majority of patients } \\
\text { were between } 20 \\
\text { and } 30 \text { years of } \\
\text { age) }\end{array}$ & $\begin{array}{l}\text { Officers with } \\
\text { medical } \\
\text { background, } \\
\text { conscripts with } \\
\text { medical } \\
\text { background (not } \\
\text { yet specialized), } \\
\text { flight surgeons, } \\
\text { general medical } \\
\text { staff }\end{array}$ & NR & Yes & Outpatient & $\begin{array}{l}\text { PC-based ISDN } \\
\text { videoconferencing }\end{array}$ & Real-time \\
\hline
\end{tabular}




\begin{tabular}{|c|c|c|c|c|c|c|c|c|}
\hline $\begin{array}{l}\text { Author, Year } \\
\text { (See Appendix C } \\
\text { for full citation) }\end{array}$ & $\begin{array}{l}\text { Number } \\
\text { Analyzed }^{\mathrm{a}}\end{array}$ & Population & $\begin{array}{l}\text { Referring } \\
\text { Providers } \\
\text { SpecialtylType }\end{array}$ & $\begin{array}{l}\text { Consulting Provider } \\
\text { Specialty/Type }\end{array}$ & $\begin{array}{l}\text { Patient } \\
\text { Present? }\end{array}$ & $\begin{array}{l}\text { Patient } \\
\text { Setting }\end{array}$ & $\begin{array}{l}\text { Modalities of } \\
\text { Telehealth } \\
\text { Consultation } \\
\text { Intervention }\end{array}$ & Timing \\
\hline Tuulonen, 1999 & $\begin{array}{l}70 \\
\text { A: } 41 \\
\text { B: } 29 \\
\end{array}$ & Adults & General practitioner & Ophthalmology & Yes & Outpatient & Videoconference & Real-time \\
\hline van der Pol, 2010 & $\begin{array}{l}90 \\
\text { A: NR } \\
\text { B: NR }\end{array}$ & Adults & $\begin{array}{l}\text { Anesthesiologist or } \\
\text { surgeon }\end{array}$ & Endoscopy specialist & Yes & Outpatient & Videoconference & Real-time \\
\hline Van Gelder, 2017 & $\begin{array}{l}3,004 \\
\text { A: } 1,727 \\
\text { B: } 1,277 \\
\end{array}$ & Adult & General practitioner & Nephrologist & No & Outpatient & Electronic health record & Asynchronous \\
\hline $\begin{array}{l}\text { Vuolio } \\
\text { Same patients as } \\
\text { Ohinmaa and } \\
\text { Hakipuro, } 2003\end{array}$ & $\begin{array}{l}145 \\
\text { A: } 69 \\
B: 76\end{array}$ & Adults & General practitioner & Orthopedics & Yes & Outpatient & Videoconference & Real-time \\
\hline Wallace, 2002 & $\begin{array}{l}1,939 \\
\text { A: } 971 \\
\text { B: } 968 \\
155 \text { post- } \\
\text { randomization } \\
\text { exclusions } \\
\end{array}$ & $\begin{array}{l}\text { Adults \& Pediatrics } \\
\text { in London (urban) } \\
\text { and Shrewsbury } \\
\text { (rural) }\end{array}$ & General practitioner & $\begin{array}{l}\text { Multiple: orthopedics, } \\
\text { urology, ENT, } \\
\text { gastroenterology and other } \\
\text { medical specialists }\end{array}$ & Present & $\begin{array}{l}\text { Outpatient } \\
\text { (Primary } \\
\text { Care) }\end{array}$ & PC-based video & Real-time \\
\hline Wallace, 2004 & $\begin{array}{l}1,939 \\
\text { A: } 971 \\
\text { B: } 968 \\
\end{array}$ & Not specified & General practice & Multiple specialties & Yes & Outpatient & Videoconference & Real-time \\
\hline Whited, 2002 & $\begin{array}{l}275 \\
\text { A: } 140 \\
\text { B: } 135 \\
\end{array}$ & Adults & $\begin{array}{l}\text { General medicine, } \\
\text { internal medicine, } \\
\text { women's health }\end{array}$ & Dermatology & Unclear & Outpatient & $\begin{array}{l}\text { Mixed (images, } \\
\text { standardized history, } \\
\text { standardized consult) } \\
\end{array}$ & Asynchronous \\
\hline Whited, 2003 & $\begin{array}{l}275 \\
\text { A: } 140 \\
B: 135\end{array}$ & Adults & $\begin{array}{l}\text { Primary care } \\
\text { physician }\end{array}$ & Dermatologist & No & Outpatient & Digital records & Asynchronous \\
\hline Whited, 2004 & $\begin{array}{l}\text { Clinicians: } 91 \\
\text { A: } 53 \\
\text { B: } 38 \\
\text { Patients: } 275 \\
\text { A: } 140 \\
\text { B: } 135 \\
\end{array}$ & Adults & Primary care & Dermatology & Yes & Outpatient & $\begin{array}{l}\text { Mixed (images, } \\
\text { standardized history, } \\
\text { standardized consult) }\end{array}$ & Asynchronous \\
\hline
\end{tabular}




\begin{tabular}{|c|c|c|c|c|c|c|c|c|}
\hline $\begin{array}{l}\text { Author, Year } \\
\text { (See Appendix C } \\
\text { for full citation) }\end{array}$ & $\begin{array}{l}\text { Number } \\
\text { Analyzed }^{\mathrm{a}} \\
\end{array}$ & Population & $\begin{array}{l}\text { Referring } \\
\text { Providers } \\
\text { Specialty/Type } \\
\end{array}$ & $\begin{array}{l}\text { Consulting Provider } \\
\text { Specialty/Type }\end{array}$ & \begin{tabular}{|l} 
Patient \\
Present?
\end{tabular} & $\begin{array}{l}\text { Patient } \\
\text { Setting }\end{array}$ & \begin{tabular}{|l|} 
Modalities of \\
Telehealth \\
Consultation \\
Intervention \\
\end{tabular} & Timing \\
\hline $\begin{array}{l}\text { Whited, 2013a, } \\
\text { 2013b }\end{array}$ & $\begin{array}{l}\text { Randomized: } \\
391 \\
\text { A: } 196 \\
\text { B: } 195 \\
\text { Analyzed: } 261 \\
\text { A: } 136 \\
\text { B: } 125 \\
\end{array}$ & Adults, $97.5 \%$ men & Primary care & Dermatology & No & Outpatient & $\begin{array}{l}\text { Store and forward } \\
\text { electronic medical } \\
\text { record, digital images, } \\
\text { text }\end{array}$ & Asynchronous \\
\hline Wilson, 2005 & $\begin{array}{l}6,978 \\
\text { A: } 2,910 \\
\text { B: } 4,068 \\
\end{array}$ & Adults & $\begin{array}{l}\text { Primary care } \\
\text { physician }\end{array}$ & Image reading Center & No & Outpatient & Digital images & Asynchronous \\
\hline Xu, 2008 & $\begin{array}{l}265 \\
\text { A: } 177 \\
B: 88 \\
\end{array}$ & Pediatric & $\begin{array}{l}\text { Pediatricians and } \\
\text { other physicians }\end{array}$ & ENT specialists & No & Outpatient & Videoconference & Real-time \\
\hline Young, 2014 & $\begin{array}{l}1,201 \\
\text { A: } 514 \\
\text { B: } 687 \\
\end{array}$ & Adult inmates & Correctional nurse & $\begin{array}{l}\text { Infectious disease } \\
\text { physician }\end{array}$ & Yes & Prison & Videoconference & Real-time \\
\hline Zahlmann, 2002 & $\begin{array}{l}62 \\
\text { A: } 20 \\
B: 42 \\
\end{array}$ & Adults & General practitioner & Ophthalmology & Yes & Outpatient & Videoconference & Mixed \\
\hline Zarchi, 2015 & $\begin{array}{l}90 \\
\text { A: } 40 \\
\text { B: } 50 \\
\end{array}$ & $\begin{array}{l}\text { Chronic wound } \\
\text { patients in home } \\
\text { care }\end{array}$ & Home-care nurses & $\begin{array}{l}\text { Hospital-based wound- } \\
\text { expert teams }\end{array}$ & NR & Outpatient & Store and forward & Asynchronous \\
\hline Zilliacus, 2011 & \begin{tabular}{|l|}
195 \\
A: 89 \\
B: 106 \\
Completed \\
questionnaires
\end{tabular} & Adult women & Genetic counselor & Genetic specialist & Yes & Outpatient & Videoconference & Real-time \\
\hline
\end{tabular}

CBOC = community-based outpatient clinics; CT = computed tomography; DN = diabetic nephropathy; E-consult = electronic consultations; ECG = electrocardiogram; ED =

emergency department; EKG = electrocardiogram; EMS = emergency medical services; ENT = ear, nose, throat specialist (otorhinolaryngologist); GP = general practitioner; HCV

= hepatitis C virus; LHIN = Local Health Integration Networks; LTC = long-term care; NR = not reported; PTSD = post-traumatic stress disorder; VA = Veteran's Affairs

${ }^{a}$ For definitions of interventions and comparisons (e.g., A vs. B), see Table F-7

See Appendix C. Included Studies for full citations 
Table F-9. Outpatient evidence table: results

\begin{tabular}{|c|c|c|c|}
\hline $\begin{array}{l}\text { Author, Year } \\
\text { (See Appendix C } \\
\text { for full citation) }\end{array}$ & $\begin{array}{l}\text { Results: } \\
\text { KQ1: Clinical and Economic Outcomes }\end{array}$ & $\begin{array}{l}\text { Results: } \\
\text { KQ2: Intermediate Outcomes }\end{array}$ & $\begin{array}{l}\text { Results: } \\
\text { KQ3: Adverse Effects or } \\
\text { Unintended Consequences }\end{array}$ \\
\hline Angstman, 2009 & $\begin{array}{l}\text { Unscheduled return visit to primary care within } 2 \\
\text { weeks, any reason } \\
n \text { (percent), p-value } \\
\text { A: } 138(27.6) \\
\text { B: } 87(38.2), p<0.01 \\
\text { OR }(95 \% \text { Cl) } \\
1.88(1.33 \text { to } 2.66), p \leq 0.01 \\
\text { No return visit within } 2 \text { weeks, any reason: } \\
\text { A: } 362(72.4) \\
\text { B: } 141(61.8), p<0.01 \\
\text { Unscheduled return visit to primary care within } 2 \\
\text { weeks, same reason } \\
\text { A: } 98(19.6) \\
\text { B: } 46(20.2), p=0.86 \\
\text { OR }(95 \% C l) \\
1.18(0.79 \text { to } 1.76), p=0.43 \\
\text { No return visit within } 2 \text { weeks for the same reason: } \\
\text { A: } 402(80.4) \\
\text { B: } 182(79.8), p=0.86\end{array}$ & NR & NR \\
\hline
\end{tabular}




\begin{tabular}{|c|c|c|c|}
\hline $\begin{array}{l}\text { Author, Year } \\
\text { (See Appendix C } \\
\text { for full citation) }\end{array}$ & $\begin{array}{l}\text { Results: } \\
\text { KQ1: Clinical and Economic Outcomes }\end{array}$ & $\begin{array}{l}\text { Results: } \\
\text { KQ2: Intermediate Outcomes }\end{array}$ & $\begin{array}{l}\text { Results: } \\
\text { KQ3: Adverse Effects or } \\
\text { Unintended Consequences }\end{array}$ \\
\hline Arora, 2011 & 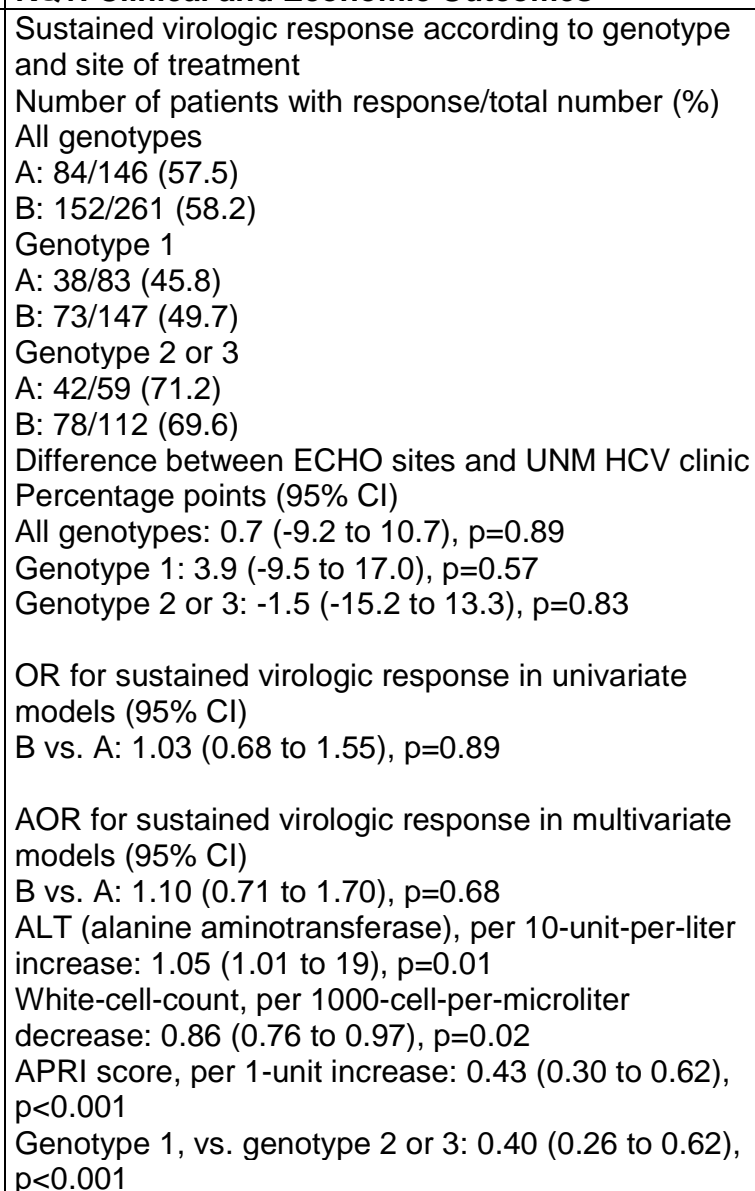 & NR & NR \\
\hline
\end{tabular}




\begin{tabular}{|c|c|c|c|}
\hline $\begin{array}{l}\text { Author, Year } \\
\text { (See Appendix C } \\
\text { for full citation) }\end{array}$ & $\begin{array}{l}\text { Results: } \\
\text { KQ1: Clinical and Economic Outcomes }\end{array}$ & $\begin{array}{l}\text { Results: } \\
\text { KQ2: Intermediate Outcomes }\end{array}$ & $\begin{array}{l}\text { Results: } \\
\text { KQ3: Adverse Effects or } \\
\text { Unintended Consequences }\end{array}$ \\
\hline Bagayoko, 2014 & $\begin{array}{l}\text { Patient savings, in USD } \\
\text { Mean: } \$ 25 \\
\text { Maximum: } \$ 70 \\
\text { (in a country with a per capita GDP of } \$ 1,300 \text { USD per } \\
\text { year) } \\
\text { Volume: } \\
\text { General trend toward more consultations at both } \\
\text { project and related sites but greater rate of attendance } \\
\text { at telehealth sites. } \\
\text { Rate of attendance } \\
\text { A: } 44.9 \% \\
\text { B: } 79.8 \% \\
\text { Telehealth tools accounted for an increase in } \\
\text { attendance at the following project centers: } 35 \% \text { in } \\
\text { Dioila District Hospital, } 10 \% \text { in Bankass, and } 8 \% \text { in } \\
\text { Kolokani and Djenne. }\end{array}$ & $\begin{array}{l}\text { Diagnostic concordance, whether the initial } \\
\text { diagnosis made by the physician changed after } \\
\text { tests or answer from expert; out of } 103 \text { total } \\
\text { cases: } \\
\text { Little changed }=6 \text { cases } \\
\text { Not changed at all= } 26 \text { cases } \\
\text { Changed completely, a lot, or moderately }=73 \\
\text { cases } \\
\text { Whether tests or input from remote expert had } \\
\text { modified treatment (of } 211 \text { total cases): } \\
\text { No change }=3 \text { cases } \\
\text { Little change }=5 \text { cases } \\
\text { Moderate or complete change }=203 \text { cases }\end{array}$ & NR \\
\hline Baig, 2016 & $\mathrm{NR}$ & $\begin{array}{l}\text { Sleep consults per year } \\
\text { A: } 150 \\
B: 1,851 \\
\text { Number of sleep studies } \\
\text { A: } 282 \\
B: 833 \\
\text { Wait time for positive airway pressure } \\
\text { prescription, in days } \\
A: \geqq 60 \\
B: \leqq 7\end{array}$ & NR \\
\hline Basudev, 2016 & NR & $\begin{array}{l}\text { Glycemic control } \mathrm{HbA} 1 \mathrm{c}, \text { in } \mathrm{mmol} / \mathrm{mol} \\
\text { (difference } \pm \text { SD) } \\
\text { A: } 10(0.8 \pm 1.9 \%) \\
\text { B: } 8(0.6 \pm 1.7 \%), p=0.4 \\
\text { Mean reduction in systolic blood pressure } \pm \\
\text { SD, in } \mathrm{mmHg} \\
\text { A: } 2 \pm 18 \\
\text { B: } 6 \pm 16, p=0.008\end{array}$ & NR \\
\hline
\end{tabular}




\begin{tabular}{|c|c|c|c|}
\hline $\begin{array}{l}\text { Author, Year } \\
\text { (See Appendix C } \\
\text { for full citation) }\end{array}$ & $\begin{array}{l}\text { Results: } \\
\text { KQ1: Clinical and Economic Outcomes }\end{array}$ & $\begin{array}{l}\text { Results: } \\
\text { KQ2: Intermediate Outcomes }\end{array}$ & $\begin{array}{l}\text { Results: } \\
\text { KQ3: Adverse Effects or } \\
\text { Unintended Consequences }\end{array}$ \\
\hline Bernstein, 2010 & $\begin{array}{l}\text { 2- to } 5 \text {-year survival, HR } \\
\text { B1 vs } A: 0.67, p<0.001 \\
\text { B2 vs. A: } 0.72, p<0.05 \\
\text { Diabetic nephropathy, HR } \\
\text { B1 vs A: } 0.63, p<0.001 \\
\text { B2 vs. A: } 0.63, p<0.01 \\
\text { Other diagnoses not or marginally significant }\end{array}$ & NR & NR \\
\hline Beswick, 2016 & NR & $\begin{array}{l}\text { Mean time from initial referral to evaluation } \\
\text { (range), in days } \\
\text { A: } 21 \text { (6 to } 61) \\
\text { B: } 18 \text { (6 to } 53) \\
\text { Mean time from evaluation to surgery (range), } \\
\text { in days } \\
\text { A: } 28 \text { ( } 0 \text { to } 55) \\
\text { B } 48 \text { (11 to } 101) \\
\text { Mean time from referral to surgery (range), in } \\
\text { days } \\
\text { A: } 49 \text { (22 to } 83) \\
\text { B: } 54(17 \text { to } 108) \\
\end{array}$ & NR \\
\hline Bezalel, 2015 & NR & $\begin{array}{l}\text { New patient wait times, in days } \\
\text { A: } 32.9 \\
\text { B: } 9.75, p<0.001 \\
\text { Established patient wait times, in days } \\
\text { A: } 4.14 \\
\text { B: } 1.49, p=0.37 \\
\text { Correlation between quantity of telehealth } \\
\text { consultations completed and percentage of } \\
\text { new patients seen at main dermatology clinic } \\
\text { within } 30 \text { days: } R^{2}=0.88, p<0.05 \\
\text { Correlation between quantity of telehealth } \\
\text { consultations completed and new patient wait } \\
\text { times: } R^{2}=0.95, p<0.001 \\
\text { Correlation between quantity of telehealth } \\
\text { consultations completed and established } \\
\text { patient wait times: } R^{2}=0.10, p=0.36 \\
\end{array}$ & NR \\
\hline Blackwell, 1997 & Savings due to transfer, over 3 months: $\$ 6,500$ & $\begin{array}{l}\text { Patients transferred for urgent care } \\
\text { A: } 17 \\
\text { B: } 4\end{array}$ & $\begin{array}{l}\text { No adverse outcomes were } \\
\text { identified }\end{array}$ \\
\hline
\end{tabular}




\begin{tabular}{|c|c|c|c|}
\hline $\begin{array}{l}\text { Author, Year } \\
\text { (See Appendix C } \\
\text { for full citation) }\end{array}$ & $\begin{array}{l}\text { Results: } \\
\text { KQ1: Clinical and Economic Outcomes }\end{array}$ & $\begin{array}{l}\text { Results: } \\
\text { KQ2: Intermediate Outcomes }\end{array}$ & $\begin{array}{l}\text { Results: } \\
\text { KQ3: Adverse Effects or } \\
\text { Unintended Consequences }\end{array}$ \\
\hline Boman, 2014 & NR & $\begin{array}{l}\text { Median total process time, in days } \\
\text { A: } 114 \\
\text { B: } 27, p<0.001 \\
\text { Median time from randomization to attaining a } \\
\text { specialist consultation, in days } \\
\text { A: } 86 \\
\text { B: } 12, p<0.001 \\
\text { Clinical examination to GP signs off the results, } \\
\text { in days } \\
\text { A: } 6 \\
\text { B: } 5, p=0.35 \\
\text { Favorable response to telehealth and found } \\
\text { comparable to standard care: } \\
89 \% \text { (17/19) of patients } \\
\text { Satisfied with information provided in remote } \\
\text { consultation: } \\
100 \% \text { of patients } \\
\text { Felt they had received faster care compared to } \\
\text { standard care and felt that telehealth was } \\
\text { superior: } 95 \%\end{array}$ & NR \\
\hline
\end{tabular}




\begin{tabular}{|c|c|c|c|}
\hline $\begin{array}{l}\text { Author, Year } \\
\text { (See Appendix C } \\
\text { for full citation) }\end{array}$ & $\begin{array}{l}\text { Results: } \\
\text { KQ1: Clinical and Economic Outcomes }\end{array}$ & $\begin{array}{l}\text { Results: } \\
\text { KQ2: Intermediate Outcomes }\end{array}$ & \begin{tabular}{|l|} 
Results: \\
KQ3: Adverse Effects or \\
Unintended Consequences
\end{tabular} \\
\hline Britt, 2006 & $\begin{array}{l}\text { Mean number of maternal transports to UAMS } \\
\text { A: } 278 \\
\text { B: } 237 \\
\\
\text { Mean LOS per maternal transport (SD), in days } \\
\text { A: } 8.02(10.38) \\
\text { B: } 6.06(7.32), p=0.003\end{array}$ & $\begin{array}{l}\text { Mean number of remote consultations (phone } \\
\text { and fax system existed prior to program) } \\
\text { A: } 108 \\
\text { B: } 269, p=0.01 \\
\text { Mean number of phone consultations: } \\
\text { A: } 55 \\
\text { B: } 107, p=0.03 \\
\text { Mean number of phone consults between } \\
\text { doctors: } \\
\text { A: } 55 \\
\text { B: } 107, p=0.03 \\
\text { Total doctors involved in weekly case } \\
\text { discussions: } \\
\text { A: } 33 \\
\text { B: } 98, p=N R \\
\text { Mean number of doctors involved in weekly } \\
\text { case discussions (SD) } \\
\text { A: } 4.33 \text { (1.5) } \\
\text { B: } 8.58 \text { (4.17) } \\
\text { ifferent doctors participating in } \\
\text { teleconsultations: } \\
\text { A: } 22 \\
\text { B: } 36, N S \\
\text { Different counties participating in } \\
\text { teleconsultations: } \\
\text { A: } 12 \\
\text { B: } 28, p=0.04\end{array}$ & \\
\hline $\begin{array}{l}\text { Brown-Connolly, } \\
2002\end{array}$ & NR & $\begin{array}{l}\text { Distance to specialist, in } \mathrm{km} \\
\text { A: } 195 \\
\text { B: } 27 \\
\text { Difference: }-168 \mathrm{~km}, \mathrm{p}<0.05 \\
\text { Travel time, in minutes } \\
\text { A: } 156 \\
\text { B: } 26 \\
\text { Difference: }-130 \text { minutes, NS } \\
90 \% \text { would use telehealth again } \\
91 \% \text { telehealth made it easier to get services } \\
39 \% \text { would get better care in person }\end{array}$ & NR \\
\hline
\end{tabular}

\section{F-102}




\begin{tabular}{|c|c|c|c|}
\hline $\begin{array}{l}\text { Author, Year } \\
\text { (See Appendix C } \\
\text { for full citation) }\end{array}$ & $\begin{array}{l}\text { Results: } \\
\text { KQ1: Clinical and Economic Outcomes }\end{array}$ & $\begin{array}{l}\text { Results: } \\
\text { KQ2: Intermediate Outcomes }\end{array}$ & $\begin{array}{l}\text { Results: } \\
\text { KQ3: Adverse Effects or } \\
\text { Unintended Consequences }\end{array}$ \\
\hline Burns, 2017 & NR & $\begin{array}{l}\text { Mean response to management (SD, range), in } \\
\text { days } \\
\text { A: } 3.20(3.74,0 \text { to } 11) \\
\text { B: } 3.12(3.44,0 \text { to } 14) \\
\text { p=0.928 } \\
\text { Clinician satisfaction reached statistical } \\
\text { significance for all parameters assessed in } \\
\text { favor of telehealth }\end{array}$ & NR \\
\hline Byamba, 2015 & $\begin{array}{l}\text { Patient travel expense, in USD }(n) \\
\text { A: } \$ 3,174(28) \\
\text { B: } \$ 320(7) \\
\text { Reduction in costs } \$ 76.36 \text { per patient } \\
\text { Variety of diagnosis: } \\
\text { A: } 4 \text { diagnoses } \\
\text { B: } 12 \text { diagnoses, } p=0.03 \\
\text { Hospital referrals, } n(\%) \\
\text { A: } 28(12.2 \%) \\
\text { B: } 7(3.1 \%) \\
p<0.01\end{array}$ & $\begin{array}{l}\text { Time to receive care, in hours } \\
\text { A: } 322 \\
\text { B: } 53\end{array}$ & NR \\
\hline Carallo, 2015 & $\begin{array}{l}\text { Changes in diabetic profile: } \\
\text { HbA1c mmol/mol from baseline to followup } \pm \text { SD } \\
\text { A: No change } \\
\text { B: } 58 \pm 6 \text { decreased to } 54 \pm 8 p=0.01 \\
\text { LDL cholesterol } \mathrm{mg} / \mathrm{dL} \text { from baseline to followup } \pm \text { SD } \\
\text { A: } 107.5 \pm 40.6 \text { to } 98.3 \pm 37.7, p=0.01 \\
\text { B: } 101.7 \pm 36.9 \text { to } 90.3 \pm 34.4, p=0.001 \\
\text { BMl } \mathrm{kg} / \mathrm{m}^{2} \text { from baseline to followup } \pm S D \\
\text { A: No change } \\
\text { B: } 31.0 \pm 4.8 \text { to } 30.5 \pm 4.6, p=0.03 \\
\text { No difference between groups: } \\
\text { Blood pressures } \\
\text { Triglycerides } \\
\text { Waist size }\end{array}$ & $\begin{array}{l}\text { Access to specialist } \\
\text { Mean number of visits } \pm \text { SD } \\
\text { A: } 1.3 \pm 1.5 \\
\text { B: } 0.6 \pm 0.9, p<0.0001 \\
\text { Mean duration of visit } \pm S D \text {, in minutes } \\
\text { A: } 24 \pm 11 \\
\text { B: } 7 \pm 3\end{array}$ & NR \\
\hline
\end{tabular}




\begin{tabular}{|c|c|c|c|}
\hline $\begin{array}{l}\text { Author, Year } \\
\text { (See Appendix C } \\
\text { for full citation) }\end{array}$ & $\begin{array}{l}\text { Results: } \\
\text { KQ1: Clinical and Economic Outcomes }\end{array}$ & $\begin{array}{l}\text { Results: } \\
\text { KQ2: Intermediate Outcomes }\end{array}$ & $\begin{array}{l}\text { Results: } \\
\text { KQ3: Adverse Effects or } \\
\text { Unintended Consequences }\end{array}$ \\
\hline Carter, 2017 & NR & $\begin{array}{l}\text { Median time to evaluation, in days } \\
\text { A: } 70.0 \\
\text { B: } 0.5 \\
\text { Median time to treatment, in days } \\
\text { A: } 73.5 \\
\text { B: } 3.0\end{array}$ & NR \\
\hline Chan, 2015 & $\begin{array}{l}\text { No toxicity related deaths in either group. } \\
\text { Inpatient hospital admissions } \\
\text { A: } 35.3 \% \\
\text { B: } 28 \%\end{array}$ & $\mathrm{NR}$ & $\begin{array}{l}\text { Rate of serious side effects } \\
\text { A: } 9.5 \% \\
\text { B: } 4.4 \% \\
\text { Side effects include: } \\
\text { Neutropenia } \\
\text { A: } 18 \% \\
\text { B: } 29 \% \\
\text { Nausea and vomiting } \\
\text { A: } 1.7 \% \\
\text { B: } 0 \\
\text { Diarrhea } \\
\text { A: } 6.9 \% \\
\text { B: } 1.1 \% \\
\text { Neuropathy } \\
\text { A: } 1.7 \% \\
\text { B: } 3.3 \% \\
\text { Fatigue } \\
\text { A: } 4.3 \% \\
\text { B: } 0 \\
\text { Other } \\
\text { A: } 26 \% \\
\text { B: } 16 \% \\
\text { Hospital admissions } \\
\text { A: } 43 \% \\
\text { B: } 36 \% \\
\end{array}$ \\
\hline Chu, 2015 & $\begin{array}{l}\text { Estimated savings, in USD } \\
\text { Expenses: } \$ 67 \\
\text { Lost opportunity cost: } \$ 126 \\
\\
\text { Total patient savings } \\
5 \text { hours } \\
\$ 193 \text { per visit } \\
\end{array}$ & \begin{tabular}{|l} 
Estimated savings \\
Mean distance: 277 miles \\
Mean time: 290 minutes
\end{tabular} & NR \\
\hline Chua, 2001 & $\begin{array}{l}\text { Cost of consultation } \\
\text { A: } £ 49 \\
\text { B: } £ 72\end{array}$ & $\begin{array}{l}\text { Reviews after first consultation } \\
\text { A: } 14(22 \%) \\
\text { B: } 22(29 \%)\end{array}$ & NR \\
\hline
\end{tabular}

\section{F-104}




\begin{tabular}{|c|c|c|c|}
\hline $\begin{array}{l}\text { Author, Year } \\
\text { (See Appendix C } \\
\text { for full citation) }\end{array}$ & $\begin{array}{l}\text { Results: } \\
\text { KQ1: Clinical and Economic Outcomes }\end{array}$ & $\begin{array}{l}\text { Results: } \\
\text { KQ2: Intermediate Outcomes }\end{array}$ & $\begin{array}{l}\text { Results: } \\
\text { KQ3: Adverse Effects or } \\
\text { Unintended Consequences }\end{array}$ \\
\hline $\begin{array}{l}\text { Collins, 2004; } \\
\text { Bowns, } 2006\end{array}$ & NR & $\begin{array}{l}\text { Response rate }(n) \\
\text { A: } 70 \%(68) \\
\text { B: } 72 \%(80) \\
\text { Satisfaction } \\
\text { Overall high and no significant difference } \\
\text { Satisfaction with care you received: } \\
\text { A: } 90 \% \\
\text { B: } 81 \%, p=0.16 \\
\text { Satisfaction with way skin problem was } \\
\text { managed: } \\
\text { A: } 87 \% \\
\text { B: } 84 \%, p=0.59\end{array}$ & NR \\
\hline Crossland, 2016 & NR & $\begin{array}{l}\text { Screening rates: } \\
\text { A: } 22 \text { to } 53 \% \\
\text { B: } 100 \% \\
\text { Diagnosed with mild-moderate diabetic } \\
\text { retinopathy } \\
\text { A: } 5 \% \\
\text { B: } 9 \% \\
\text { Appropriate followup recorded } \\
\text { A: } 29 \% \\
\text { B: } 95 \%\end{array}$ & NR \\
\hline Datta, 2015 & $\begin{array}{l}\text { Total mean cost, in USD } \\
\text { Societal perspective } \\
\text { A: } \$ 106,194 \\
\text { B: } \$ 89,523 \\
\text { VA perspective } \\
\text { A: } \$ 66,145 \\
\text { B: } \$ 59,917 \\
\text { Mean cost per participant (SD) } \\
\text { Societal perspective } \\
\text { A: } 541(403) \\
\text { B: } 460(428) \\
\text { Difference (95\% CI): }-82(-152 \text { to }-12) \text {, statistically } \\
\text { significant } \\
\text { VA perspective } \\
\text { A: } 338(291) \\
\text { B: } 308(298) \\
\text { Difference (95\% Cl): }-30(-79 \text { to } 20), \text { NS }\end{array}$ & $\begin{array}{l}\text { Dermatology clinic visits } \\
\text { A: } 303 \\
\text { B: } 214 \\
\text { Mean change in utility score, baseline to month } \\
9(S D) \\
\text { A: } 0.02(0.18) \\
\text { B: } 0.03(0.19) \\
p=0.50\end{array}$ & NR \\
\hline
\end{tabular}

\section{F-105}




\begin{tabular}{|c|c|c|c|}
\hline $\begin{array}{l}\text { Author, Year } \\
\text { (See Appendix C } \\
\text { for full citation) }\end{array}$ & $\begin{array}{l}\text { Results: } \\
\text { KQ1: Clinical and Economic Outcomes }\end{array}$ & $\begin{array}{l}\text { Results: } \\
\text { KQ2: Intermediate Outcomes }\end{array}$ & \begin{tabular}{|l|} 
Results: \\
KQ3: Adverse Effects or \\
Unintended Consequences \\
\end{tabular} \\
\hline Davis, 2011 & 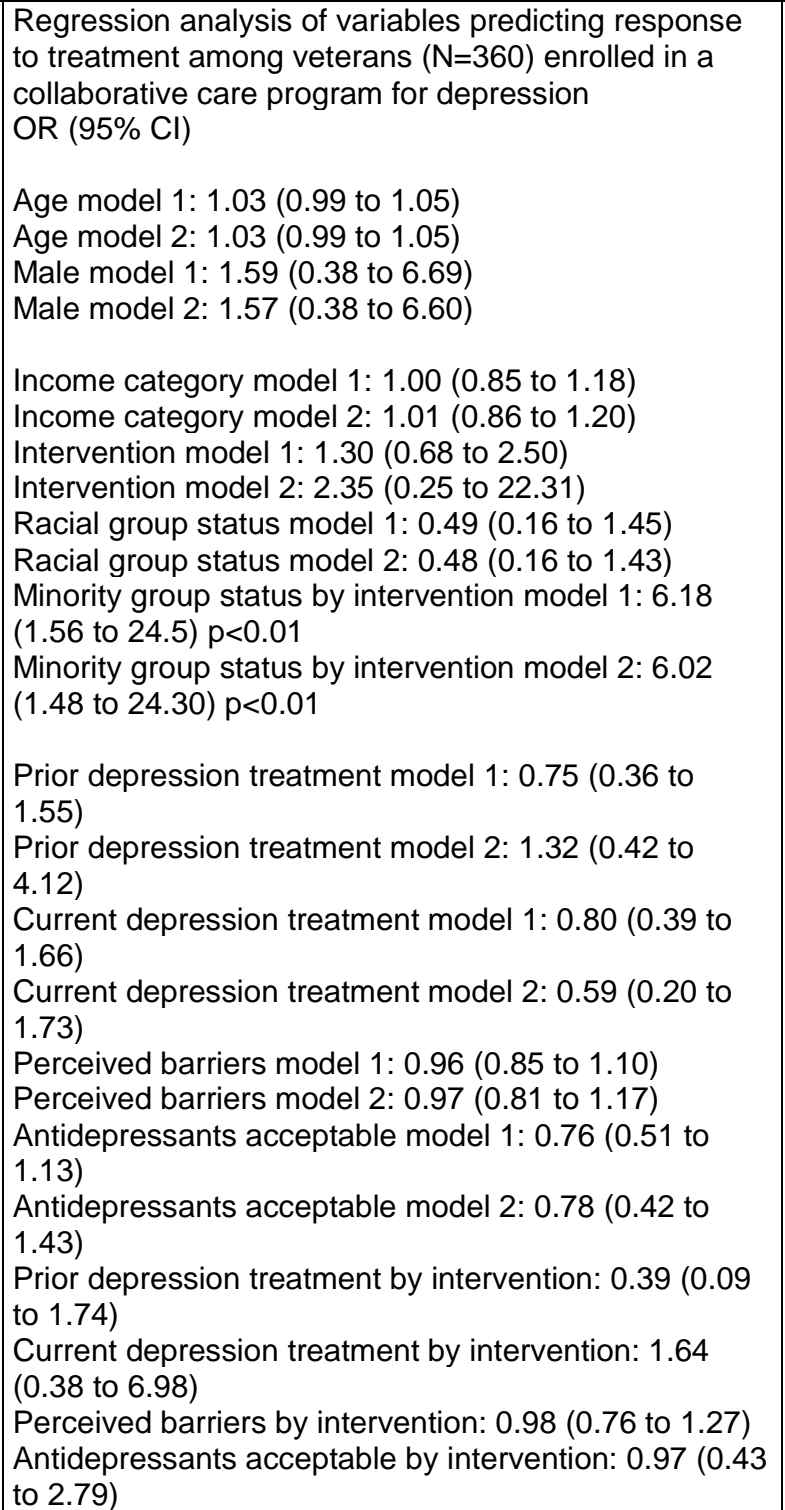 & NR & NR \\
\hline
\end{tabular}




\begin{tabular}{|c|c|c|c|}
\hline $\begin{array}{l}\text { Author, Year } \\
\text { (See Appendix C } \\
\text { for full citation) }\end{array}$ & $\begin{array}{l}\text { Results: } \\
\text { KQ1: Clinical and Economic Outcomes }\end{array}$ & $\begin{array}{l}\text { Results: } \\
\text { KQ2: Intermediate Outcomes }\end{array}$ & $\begin{array}{l}\text { Results: } \\
\text { KQ3: Adverse Effects or } \\
\text { Unintended Consequences }\end{array}$ \\
\hline Davison, 2004 & NR & $\begin{array}{l}\text { Mean time from first seen in clinic to surgery } \\
\text { (SD), in days } \\
\text { A: } 69(38) \\
\text { B: } 54(26) \\
\text { p>0.05 } \\
\text { Thoracotomy resections, per year } \\
\text { A: } 14.7 \\
\text { B: } 19 \\
\text { Telehealth meetings associated with a } 30 \% \\
\text { increase in resection rate }\end{array}$ & NR \\
\hline De Luca, 2005 & $\begin{array}{l}\text { Reduction in blood pressure (SBP/DBP) } \\
\text { A: } 4.1 \pm 0.4 / 3.1 \\
\text { B: } 7.3 \pm 0.4 / 5.4 \\
\pm 0.26 \mathrm{mmHg}, \mathrm{p}<0.001 \\
\text { Percentage of patients with } \mathrm{BP}<140 / 90 \mathrm{mmHg} \\
\text { A: } 47 \% \\
\text { B: } 51 \%, p<0.001 \\
\text { Major cardiovascular events (MACE) } \\
\text { A: } 4.3 \% \\
\text { B: } 2.9 \%, p<0.02 \\
\text { Adjusted OR } \\
\text { 0.838, (95\% CI } 0.73 \text { to } 0.964, p<0.05) \\
\text { TVCR score } \\
\text { A: (baseline) } 3.5 \pm 0.03 \\
\text { A: (followup) } 3.4 \pm 0.03, \mathrm{NS} \\
\text { B: (baseline) } 3.5 \pm 0.02 \\
\text { B: (followup) } 3.2 \pm 0 \mathrm{p}<0.01\end{array}$ & NR & NR \\
\hline Doolittle, 1997 & $\begin{array}{l}\text { Average cost per visit, in USD } \\
\text { A: } \$ 897 \\
\text { B: } \$ 149 \\
\text { C: } \$ 812\end{array}$ & NR & NR \\
\hline Dowie, 2007 & $\begin{array}{l}\text { Mean cost of the initial consult } \\
\text { A: } £ 277 \\
\text { B: } £ 411 \\
\text { After } 6 \text { month followup } \\
\text { A: } £ 2,172 \\
\text { B: } £ 3,350 \\
\text { Nonsignificant }\end{array}$ & $\begin{array}{l}\text { EuroQOL EQ-5D } \\
\text { Mean (SD), n } \\
\text { A: } 0.72(0.22), 11 \\
\text { B: } 0.86(0.14), 26\end{array}$ & NR \\
\hline
\end{tabular}




\begin{tabular}{|c|c|c|c|}
\hline $\begin{array}{l}\text { Author, Year } \\
\text { (See Appendix C } \\
\text { for full citation) }\end{array}$ & $\begin{array}{l}\text { Results: } \\
\text { KQ1: Clinical and Economic Outcomes }\end{array}$ & $\begin{array}{l}\text { Results: } \\
\text { KQ2: Intermediate Outcomes }\end{array}$ & $\begin{array}{l}\text { Results: } \\
\text { KQ3: Adverse Effects or } \\
\text { Unintended Consequences }\end{array}$ \\
\hline $\begin{array}{l}\text { Eminovic, 2009, } \\
2010\end{array}$ & $\begin{array}{l}\text { Patient recovery at } 1 \text { month } \\
\text { A: } 4.1 \% \\
\text { B: } 20 \% \\
\text { Preventable consultation } \\
\text { A: } 18.3 \% \\
\text { B: } 39.0 \% \\
\text { Difference }(95 \% \mathrm{Cl}): 20.7 \%(8.5 \% \text { to } 32.9 \%)\end{array}$ & $\begin{array}{l}\text { No significant differences in patient satisfaction } \\
\text { were found between groups. }\end{array}$ & NR \\
\hline Endean, 2001 & NR & $\begin{array}{l}\text { Mean evaluation time, in minutes } \\
\text { A: } 19.0 \\
\text { B: } 20.6 \\
\text { Overall concordance, } n \text { (\%) } \\
29 \text { of } 32(91 \%) \\
\text { Mean physician satisfaction score with TH } \\
\text { consult ( } 7 \text { point scale, } 7=\text { highest) } \\
5.71 \\
\text { Mean patient satisfaction score comparing TH } \\
\text { to conventional from }-1 \text { to } 1 \text { (1=better) } \\
0.27\end{array}$ & NR \\
\hline Ferrandiz, 2007 & NR & $\begin{array}{l}\text { Mean consultation to operation wait time }(95 \% \\
\mathrm{Cl}) \text {, in days } \\
\mathrm{A}: 60.57 \text { (56.20 to } 64.93) \\
\mathrm{B}: 26.10 \text { ( } 24.51 \text { to } 27.70), \mathrm{p}<0.001 \\
\text { Accuracy of telediagnoses: } \mathrm{k}=0.86(95 \% \mathrm{Cl} \\
0.83 \text { to } 0.89) \\
\text { Agreement rate between surgical technique } \\
\text { planned through teleconsultation and } \\
\text { technique performed: } \mathrm{k}=0.75(95 \% \mathrm{Cl} 0.04 \text { to } \\
0.79)\end{array}$ & NR \\
\hline
\end{tabular}

\section{F-108}




\begin{tabular}{|c|c|c|c|}
\hline $\begin{array}{l}\text { Author, Year } \\
\text { (See Appendix C } \\
\text { for full citation) }\end{array}$ & $\begin{array}{l}\text { Results: } \\
\text { KQ1: Clinical and Economic Outcomes }\end{array}$ & $\begin{array}{l}\text { Results: } \\
\text { KQ2: Intermediate Outcomes }\end{array}$ & $\begin{array}{l}\text { Results: } \\
\text { KQ3: Adverse Effects or } \\
\text { Unintended Consequences }\end{array}$ \\
\hline Fortney, 2013 & $\begin{array}{l}\text { AOR }(95 \% \mathrm{Cl}), \mathrm{p} \text {-value } \\
7.74(3.94 \text { to } 15.20), \mathrm{p}<0.0001 \\
\text { Remission } \\
12.69(4.81 \text { to } 33.46), p<0.0001 \\
\text { Adherence } \\
1.22(0.38 \text { to } 3.89), p=0.737 \\
\text { Any specialty mental health visits } \\
0.56 \text { (0.24 to } 1.82), p=0.4253 \\
\\
\text { Adjusted incidence rate ratio }(95 \% \mathrm{Cl}), \mathrm{p} \text {-value } \\
\text { Primary care visits } \\
1.16(0.98 \text { to } 1.36), p=0.0807 \\
\text { Depression-related primary care visits } \\
0.99(0.72 \text { to } 1.37) p=0.9579\end{array}$ & $\begin{array}{l}\text { AOR }(95 \% \mathrm{Cl}), \mathrm{p} \text {-value } \\
\text { Satisfaction } \\
\text { Baseline } \\
1.08 \text { (0.64 to } 1.83), \mathrm{p}=0.7654 \\
6 \text { months } \\
2.76 \text { ( } 1.50 \text { to } 5.01), \mathrm{p}=0.0012 \\
12 \text { months } \\
1.99 \text { (1.06 to } 3,71), \mathrm{p}=0.0313 \\
18 \text { months } \\
1.67 \text { (0.89 to } 3.13), \mathrm{p}=0.107 \\
\\
\text { Depression severity } \\
\text { Adjusted group difference }(95 \% \mathrm{Cl}) \\
\text { Baseline: }-0.04(-0.18 \text { to } 0.10), p=0.5935 \\
6 \text { months: }-0.50(-0.65 \text { to }-0.35), p<0.0001 \\
12 \text { months: }-0.49(-0.65 \text { to }-0,33), p<0.0001 \\
18 \text { months: }-0.33(-0.49 \text { to }-0.18), p<0.0001\end{array}$ & NR \\
\hline
\end{tabular}




\begin{tabular}{|c|c|c|c|}
\hline $\begin{array}{l}\text { Author, Year } \\
\text { (See Appendix C } \\
\text { for full citation) }\end{array}$ & $\begin{array}{l}\text { Results: } \\
\text { KQ1: Clinical and Economic Outcomes }\end{array}$ & $\begin{array}{l}\text { Results: } \\
\text { KQ2: Intermediate Outcomes }\end{array}$ & \begin{tabular}{|l|} 
Results: \\
KQ3: Adverse Effects or \\
Unintended Consequences \\
\end{tabular} \\
\hline Fortney, 2007 & 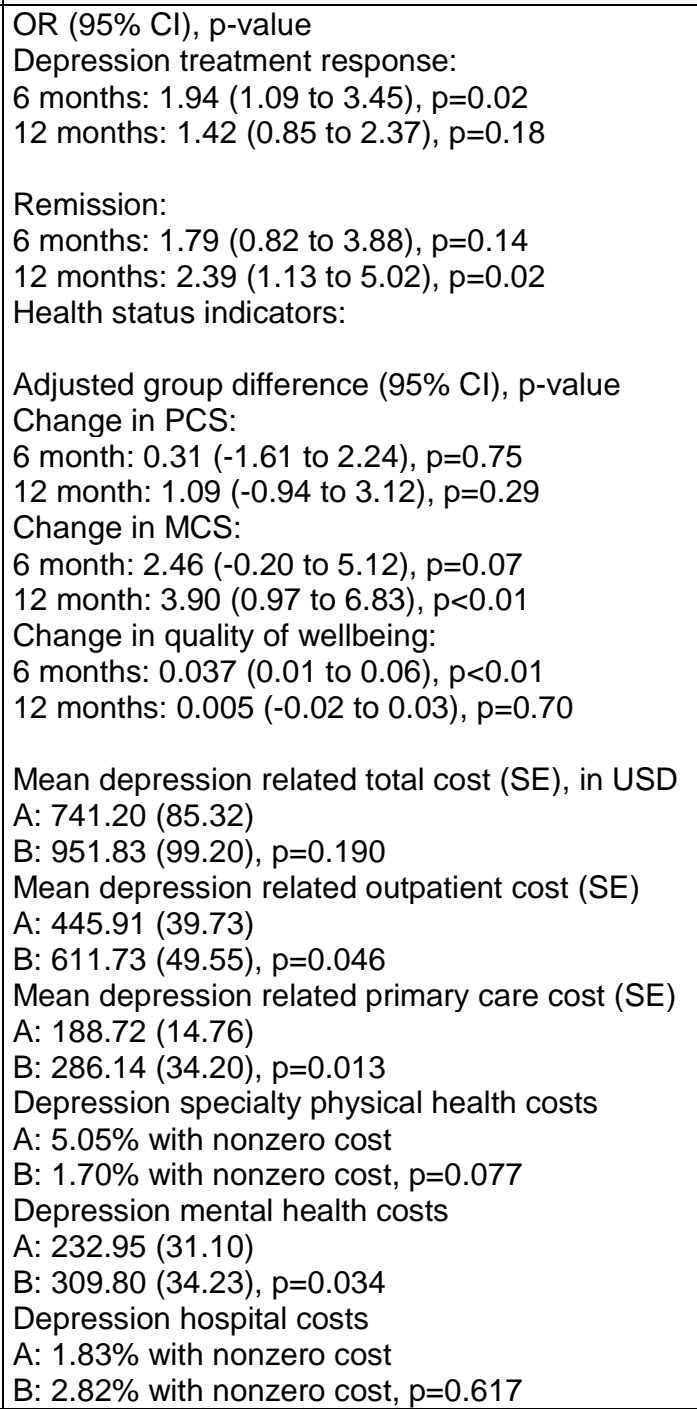 & $\begin{array}{l}\text { OR }(95 \% \mathrm{Cl}), \mathrm{p} \text {-value } \\
\text { Medication adherence: } \\
6 \text { months: } 2.11 \text { ( } 1.02 \text { to } 4.36), \mathrm{p}=0.04 \\
12 \text { months: } 2.72 \text { ( } 1.36 \text { to } 5.44), \mathrm{p}<0.01 \\
\text { Treatment satisfaction: } \\
6 \text { months: } 1.83 \text { (1.14 to } 2.93), \mathrm{p}=0.01 \\
12 \text { months: } 1.71 \text { (1.06 to } 2.77), \mathrm{p}=0.03\end{array}$ & NR \\
\hline
\end{tabular}




\begin{tabular}{|c|c|c|c|}
\hline $\begin{array}{l}\text { Author, Year } \\
\text { (See Appendix C } \\
\text { for full citation) }\end{array}$ & $\begin{array}{l}\text { Results: } \\
\text { KQ1: Clinical and Economic Outcomes }\end{array}$ & $\begin{array}{l}\text { Results: } \\
\text { KQ2: Intermediate Outcomes }\end{array}$ & $\begin{array}{l}\text { Results: } \\
\text { KQ3: Adverse Effects or } \\
\text { Unintended Consequences }\end{array}$ \\
\hline Fortney, 2011 & $\begin{array}{l}\text { Mean total outpatient and inpatient costs (SE), in USD } \\
\text { A: } 5384.76(540.79) \\
B: 7469.42(1366.66) \\
p=0.141\end{array}$ & $\begin{array}{l}\text { Mean outpatient encounters (SE) } \\
\text { A: } 18.84(1.08) \\
B: 22.21(1.26) \\
p=0.008\end{array}$ & NR \\
\hline Fortney, 2015 & 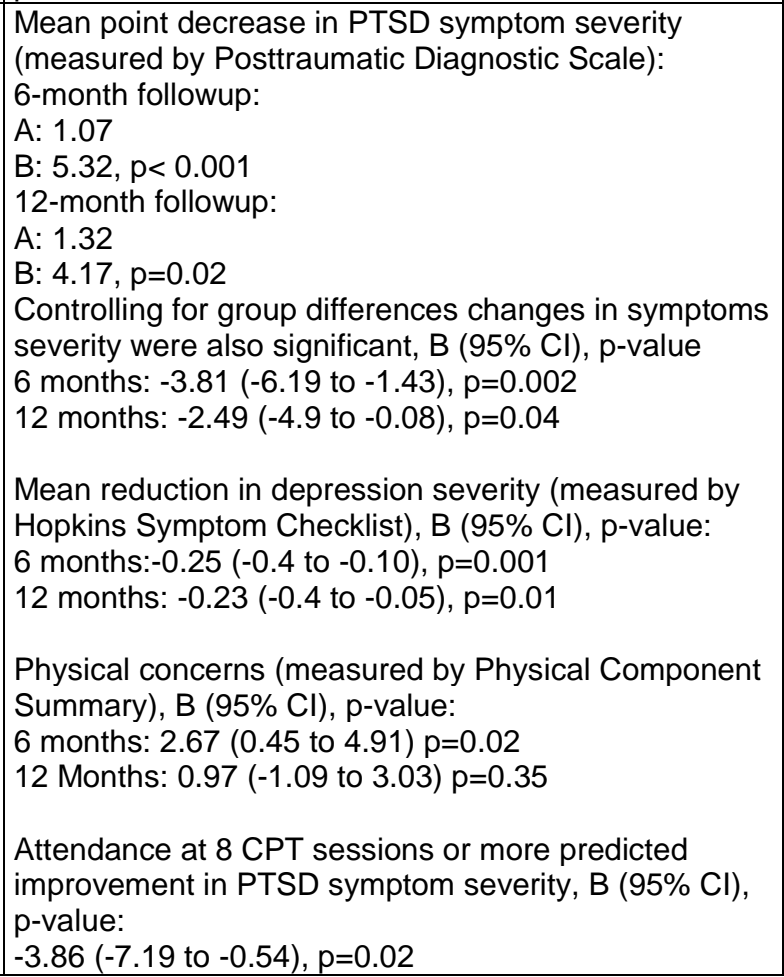 & $\begin{array}{l}\text { AOR }(95 \% \mathrm{Cl}), \mathrm{p} \text {-value } \\
\text { Medication } \\
\text { PTSD medication prescriptions in first } 6 \\
\text { months: } \\
\text { Telehealth: } 2.98 \text { (1.03 to } 8.68), p=0.45 \\
\text { Prazosin prescriptions: } \\
6 \text { months: } 2.43 \text { (1.14 to } 5.20), p=0.2 \\
12 \text { months: } 3.58 \text { (1.71 to } 7.48), p<0.001 \\
\text { Adherence to medication: } \\
\text { OR (95\% Cl), p-value } \\
6 \text { months: } 0.86 \text { (0.46 to } 1.62), p=0.64 \\
12 \text { months: } 0.91 \text { (0.47 to } 1.78), p=0.79 \\
\text { Cognitive processing therapy (CPT) } \\
\text { Percent receiving some cognitive processing } \\
\text { therapy: } \\
\text { A: } 12.1 \% \\
\text { B: } 54.9 \% \\
\text { Percent attending at least } 8 \text { psychotherapy } \\
\text { sessions: } \\
\text { A: } 5.3 \% \\
\text { B: } 27.1 \% \\
\text { Mean number of CPT sessions attended: } \\
\text { A: } 0.8 \\
\text { B: } 4.2 \\
\text { Risk ratio: } 9.51 \text { (95\% Cl } 4.58 \text { to } 19.77 \text { ), } \\
p<0.001\end{array}$ & NR \\
\hline
\end{tabular}




\begin{tabular}{|c|c|c|c|}
\hline $\begin{array}{l}\text { Author, Year } \\
\text { (See Appendix C } \\
\text { for full citation) }\end{array}$ & $\begin{array}{l}\text { Results: } \\
\text { KQ1: Clinical and Economic Outcomes }\end{array}$ & $\begin{array}{l}\text { Results: } \\
\text { KQ2: Intermediate Outcomes }\end{array}$ & $\begin{array}{l}\text { Results: } \\
\text { KQ3: Adverse Effects or } \\
\text { Unintended Consequences }\end{array}$ \\
\hline Fox, 2007 & $\begin{array}{l}\text { Health care utilization: } \\
\text { [Estimate, } p \text {-value, incidence density ratio (IDR): A vs } \\
\text { aggregate } 2 \text { years after telehealth] } \\
\text { Outpatient visits per center per month ( } n=144 ; 4 \\
\text { facilities) } \\
\text { Facility } 1: 0.86, p<0.001,2.37 \\
\text { Facility } 2:-0.05, p=0.6998,0.95 \\
\text { Facility } 3: 0.33, p=0.0004,1.39 \\
\text { Facility } 4: 1.08, p<0.0001,2.93 \\
\text { ED visits per center per month ( } n=144 ; 4 \text { facilities) } \\
\text { Facility } 1: 0.26, p=0.2977,1.30 \\
\text { Facility } 2:-0.14, p=0.4203,0.87 \\
\text { Facility } 3: 0.79, p=0.0044,2.21 \\
\text { Facility } 4: 0.90, p=0.1543,2.45 \\
\text { Inpatient visits per center per month ( } n=144 ; 4 \\
\text { facilities) } \\
\text { Facility } 1:-1.71, p=0.0233,0.18 \\
\text { Facility } 2: 0.17, p=0.8689,1.19 \\
\text { No visits in baseline year at facilities } 3 \text { or } 4 \\
\text { Effect of telehealth volume usage on access }(n=144) \\
\text { [Estimate, } p \text {-value, incidence density ratio (IDR)] } \\
\text { Outpatient visits per center per month: } 0.02, p<0.0001 \text {, } \\
1.0204 \\
\text { ED visits per center per month: }-0.05, p<0.0001, \\
0.9524 \\
\text { Inpatient visits per center per month: }-0.04, p=0.1954 \text {, } \\
0.9615\end{array}$ & $\begin{array}{l}\text { Timeliness of care rates: } \\
\text { Mean time from referral to psychiatric } \\
\text { treatment, in days } \\
\text { A: } 50.1 \\
\text { B: } 24.86 \\
\text { C: } 21.59 \\
\text { Time from referral to treatment by facility ( } n=4 \\
\text { facilities): [hazard ratio, \% change in time to } \\
\text { referral, p-value: A vs Aggregate } 2 \text { years after } \\
\text { telehealth] } \\
\text { Facility } 1: 4.40,77.27 \% \text { reduction, } p<0.001 \\
\text { Facility } 2: 1.09,8.26 \% \text { reduction, } p=0.622 \\
\text { Facility } 3: 2.29,56.33 \% \text { reduction, } p=0.0006 \\
\text { Facility } 4: 0.74,35.14 \% \text { increase, } p=0.1326\end{array}$ & NR \\
\hline
\end{tabular}




\begin{tabular}{|c|c|c|c|}
\hline $\begin{array}{l}\text { Author, Year } \\
\text { (See Appendix C } \\
\text { for full citation) }\end{array}$ & $\begin{array}{l}\text { Results: } \\
\text { KQ1: Clinical and Economic Outcomes }\end{array}$ & $\begin{array}{l}\text { Results: } \\
\text { KQ2: Intermediate Outcomes }\end{array}$ & $\begin{array}{l}\text { Results: } \\
\text { KQ3: Adverse Effects or } \\
\text { Unintended Consequences }\end{array}$ \\
\hline Frank, 2015 & NR & $\begin{array}{l}\text { HR }(95 \% \mathrm{Cl}) \\
\text { Delivery of out-patient care: } \\
\text { Physical medicine } \\
1.10(1.05 \text { to } 1.14) \\
\text { Mental health } \\
0.99(0.93 \text { to } 1.05) \\
\text { Substance use disorder } \\
0.93 \text { (0.84 to } 1.03) \\
\text { Specialty pain clinics } \\
1.01(0.94 \text { to } 1.08) \\
\text { Medication initiation } \\
\text { Anti-depressant } \\
1.09(1.02 \text { to } 1.15) \\
\text { Anticonvulsant } \\
1.13(1.06 \text { to } 1.19) \\
\text { Opioid analgesics } \\
1.05(0.99 \text { to } 1.10)\end{array}$ & NR \\
\hline Gattas, 2001 & NR & $\begin{array}{l}\text { Patient satisfaction: } \\
\text { Communication } \\
\text { Ability to maintain eye contact } \\
\text { Comfort level of room } \\
\text { Satisfaction with clinic format } \\
\text { NS } \\
\text { No numerical data available } \\
\text { Provider satisfaction: } \\
\text { Communication: no difference } \\
\text { Ability to maintain eye contact: slightly lower } \\
\text { Room comfortability: higher } \\
\text { Satisfaction with clinic format: no difference } \\
\text { Counselor satisfaction: } \\
\text { Counselors reported higher satisfaction with } \\
\text { face-to-face consultations }\end{array}$ & NA \\
\hline
\end{tabular}




\begin{tabular}{|c|c|c|c|}
\hline $\begin{array}{l}\text { Author, Year } \\
\text { (See Appendix C } \\
\text { for full citation) }\end{array}$ & $\begin{array}{l}\text { Results: } \\
\text { KQ1: Clinical and Economic Outcomes }\end{array}$ & $\begin{array}{l}\text { Results: } \\
\text { KQ2: Intermediate Outcomes }\end{array}$ & $\begin{array}{l}\text { Results: } \\
\text { KQ3: Adverse Effects or } \\
\text { Unintended Consequences } \\
\end{array}$ \\
\hline Gilmour, 1998 & $\begin{array}{l}\text { Diagnosis } \\
\text { Agreement } 59 \% \\
\text { Telehealth unable } 11 \% \\
\text { Telehealth missed } 6 \% \\
\text { Telehealth wrong } 4 \% \\
\text { Diagnosis made } \\
\text { A: } 97 \% \\
\text { B: } 60 \%, p=0.002 \\
81 \% \text { management plan correct }\end{array}$ & NR & NR \\
\hline Harno, 2000 & $\begin{array}{l}\text { Variable cost for outpatient visits, in euros: } \\
\text { A: } € 210.81 \\
\text { B: } € 32.06\end{array}$ & $\begin{array}{l}\text { Volume of referrals, referrals/inhabitants } \\
\text { A: } 3.8 / 1000 \\
\text { B: } 7.5 / 1000 \\
\text { Proportion of patients receiving appointments } \\
\text { at outpatient clinic: } \\
\text { A: } 79 \% \\
\text { B: } 43 \% \\
\text { Proportion of referrals from GP for on-line } \\
\text { medical advice responded to by } \\
\text { teleconsultation: } \\
\text { A: NR } \\
\text { B: } 78 \% \\
\text { Patients referred by GP for outpatient visit } \\
\text { receiving teleconsultation: } \\
\text { A: NR } \\
\text { B: } 32 \% \\
\text { Proportion of referrals to Peijas resulting in } \\
\text { teleconsultation (by GPs' estimates of urgency } \\
\text { of referral) } \\
\text { Most urgent (needing treatment within one } \\
\text { week): } \\
\text { A: NR } \\
\text { B: } 10 \% \\
\text { Least urgent (needing treatment }>30 \text { days): } \\
\text { A: NR } \\
\text { B: } 50 \%\end{array}$ & NR \\
\hline
\end{tabular}

\section{F-114}




\begin{tabular}{|c|c|c|c|}
\hline $\begin{array}{l}\text { Author, Year } \\
\text { (See Appendix C } \\
\text { for full citation) }\end{array}$ & $\begin{array}{l}\text { Results: } \\
\text { KQ1: Clinical and Economic Outcomes }\end{array}$ & $\begin{array}{l}\text { Results: } \\
\text { KQ2: Intermediate Outcomes }\end{array}$ & $\begin{array}{l}\text { Results: } \\
\text { KQ3: Adverse Effects or } \\
\text { Unintended Consequences }\end{array}$ \\
\hline $\begin{array}{l}\text { Harno, } 2000 \\
\text { (continued) }\end{array}$ & (continued) & $\begin{array}{l}\text { Feasibility of telehealth system rated by } \\
\text { specialists at Peijas Hospital (used telehealth): } \\
\text { Excellent or good: } 67 \% \text { of the cases studied } \\
\text { Bad or very bad: } 10 \% \text { of cases studied } \\
\text { Feasibility of telehealth system rated by } \\
\text { specialists in Hyvinkaa Hospital (did not use } \\
\text { telehealth): } \\
\text { Excellent or good: } 19 \% \text { of cases studied } \\
\text { Bad or very bad: } 72 \% \text { of cases studied } \\
\text { Diagnostic effectiveness- proportion of } \\
\text { consultations with revisions to diagnoses } \\
\text { A: } 25 \% \\
\text { B: } 29 \% \\
\text { Patient satisfaction- wanted next appointed via } \\
\text { telehealth: } \\
\text { A: } 60 \% \\
\text { B: } 80 \%\end{array}$ & (continued) \\
\hline Harno, 2001 & $\begin{array}{l}\text { Total cost per patient, in euros } \\
\text { A: €154.44 } \\
\text { B: €41.22 } \\
\text { Outpatient } 45 \% \text { higher } \\
\text { Marginal cost decreased } € 48 \text { for each visit }\end{array}$ & $\begin{array}{l}\text { Mean time, in minutes } \\
\text { A: } 12 \\
\text { B: } 13 \\
\text { Diagnosis revised } \\
\text { A: } 16 \% \\
\text { B: } 12 \% \\
\text { Decision to perform surgery } \\
\text { A: } 38 \% \\
\text { B: } 53 \%\end{array}$ & NR \\
\hline
\end{tabular}

\section{F-115}




\begin{tabular}{|c|c|c|c|}
\hline $\begin{array}{l}\text { Author, Year } \\
\text { (See Appendix C } \\
\text { for full citation) }\end{array}$ & $\begin{array}{l}\text { Results: } \\
\text { KQ1: Clinical and Economic Outcomes }\end{array}$ & $\begin{array}{l}\text { Results: } \\
\text { KQ2: Intermediate Outcomes }\end{array}$ & $\begin{array}{l}\text { Results: } \\
\text { KQ3: Adverse Effects or } \\
\text { Unintended Consequences }\end{array}$ \\
\hline $\begin{array}{l}\text { Haukipuro same } \\
\text { pts as Ohinmaa } \\
\text { and Vuolio, } 2000\end{array}$ & NR & $\begin{array}{l}\text { Average total time of visit, including travel, in } \\
\text { hours } \\
\text { A: } 8 \\
\text { B: } 1.5 \\
\text { Average distance travelled, in } \mathrm{km} \\
\text { A: } 170 \\
\text { B: } 8 \\
\text { Success of exam, rated at least good by } \\
\text { practitioner } \\
\text { A: } 99 \% \text { of cases } \\
\text { B: } 80 \% \text { of cases }\end{array}$ & NR \\
\hline Herce, 2011 & NR & $\begin{array}{l}\text { Mean time to surgical wait list }(95 \% \mathrm{Cl}) \text {, in } \\
\text { days } \\
\text { A: } 28 \text { (24.51 to } 29.6) \\
\text { B: } 3.33 \text { (2 to } 4.65), p<0.001 \\
\text { On the day surgery cancellation rate } \\
\text { A: } 8.85 \%(5.62 \text { to } 11.81 \%), p<0.005 \\
\text { B: } 7.8 \%(3.8 \% \text { to } 10.5 \%), p=0.76\end{array}$ & NR \\
\hline Hsiao, 2008 & $\begin{array}{l}\text { Mean days (SD, } 95 \% \mathrm{Cl}) \\
\text { To initial evaluation: } \\
\text { A: } 48(38,40 \text { to } 57) \\
\text { B: } 4(5,3 \text { to } 5), p<0.0001 \\
\text { To biopsy: } \\
\text { A: } 57(52,45 \text { to } 68) \\
\text { B: } 38(41,30 \text { to } 47), p=0.034 \\
\text { To surgery: } \\
\text { A: } 125(63,111 \text { to } 140) \\
\text { B: } 104(67,90 \text { to } 118), p=0.006 \\
13 \text { encounters with teledermatology, that may have } \\
\text { created a bias in the initial bias, were removed from } \\
\text { analysis: } \\
\text { Teledermatology still associated with shorter intervals } \\
\text { to initial evaluation ( } p=0.0001) \\
\text { Teledermatology no longer statistically significant for } \\
\text { the time intervals to biopsy ( } p=0.054 \text { ) or to surgery } \\
\text { ( } p=0.053 \text { ) }\end{array}$ & $\mathrm{NR}$ & NR \\
\hline
\end{tabular}




\begin{tabular}{|c|c|c|c|}
\hline $\begin{array}{l}\text { Author, Year } \\
\text { (See Appendix C } \\
\text { for full citation) }\end{array}$ & $\begin{array}{l}\text { Results: } \\
\text { KQ1: Clinical and Economic Outcomes }\end{array}$ & $\begin{array}{l}\text { Results: } \\
\text { KQ2: Intermediate Outcomes }\end{array}$ & $\begin{array}{l}\text { Results: } \\
\text { KQ3: Adverse Effects or } \\
\text { Unintended Consequences }\end{array}$ \\
\hline Izquierdo, 2009 & $\begin{array}{l}\text { HbA1c values at } 6 \text { months } \\
\text { A: Increase (not significant) } \\
\text { B: Decrease ( } p<0.02) \\
\text { Outcomes at } 3 \text { months } \\
\text { Urgent encounters } \\
\text { A: } 59 \\
\text { B: } 63 \\
\text { Urgent calls } \\
\text { A: } 20 \\
\text { B: } 14 \\
\text { Treatment needed } \\
\text { A: } 52 \\
\text { B: } 50 \\
\text { Outcomes at } 6 \text { months } \\
\text { Urgent encounters } \\
\text { A: } 49 \\
\text { B: } 25 \\
\text { Urgent calls } \\
\text { A: } 14 \\
\text { B: } 2 \\
\text { Treatment needed } \\
\text { A: } 48 \\
\text { B: } 20 \\
\text { Outcomes at } 9 \text { months } \\
\text { Urgent encounters } \\
\text { A: } 47 \\
\text { B: } 27 \\
\text { Urgent calls } \\
\text { A: } 27 \\
\text { B: } 6 \\
\text { Treatment needed } \\
\text { A: } 43 \\
\text { B: } 23\end{array}$ & $\begin{array}{l}\text { Effect of telehealth on Pediatric Quality of Life } \\
\text { Modules } \\
\text { Diabetes dimension: no difference between } \\
\text { groups } \\
\text { Treatment } 1 \text { dimension, after } 6 \text { months } \\
\text { A: NS } \\
\text { B: } p \leq 0.04 \\
\text { Treatment } 2 \text { dimension, before summer break: } \\
\text { A: NS } \\
\text { B: } p<0.02 \\
\text { Treatment } 2 \text { dimension, after } 6 \text { months: } \\
\text { A: Significant improvement } \\
\text { B: Stayed at } 6 \text { month levels } \\
\text { Worry dimension: unaffected } \\
\text { Effect of telehealth on Pediatric Quality of Life } \\
\text { Generic Score Scales } \\
\text { Improved physical functioning: } \\
\text { A: NS } \\
\text { B: NS } \\
\text { Improved emotional functioning, baseline to } 6 \\
\text { months: } \\
\text { A: NS } \\
\text { B: } p<0.01 \\
\text { Improved emotional functioning, baseline to } 6 \\
\text { to } 12 \text { months } \\
\text { A: } p<0.04 \\
\text { B: NS } \\
\text { School functioning } \\
\text { A: NS } \\
\text { B: NS } \\
\text { Social functioning } \\
\text { A: NS } \\
\text { B: NS }\end{array}$ & NR \\
\hline
\end{tabular}




\begin{tabular}{|c|c|c|c|}
\hline $\begin{array}{l}\text { Author, Year } \\
\text { (See Appendix C } \\
\text { for full citation) }\end{array}$ & $\begin{array}{l}\text { Results: } \\
\text { KQ1: Clinical and Economic Outcomes }\end{array}$ & $\begin{array}{l}\text { Results: } \\
\text { KQ2: Intermediate Outcomes }\end{array}$ & $\begin{array}{l}\text { Results: } \\
\text { KQ3: Adverse Effects or } \\
\text { Unintended Consequences }\end{array}$ \\
\hline $\begin{array}{l}\text { Izquierdo, } 2009 \\
\text { (Cont.) }\end{array}$ & $\begin{array}{l}\text { Outcomes at } 12 \text { months } \\
\text { Urgent encounters } \\
\text { A: } 35 \\
\text { B: } 10 \\
\text { Urgent calls } \\
\text { A: } 23 \\
\text { B: } 2 \\
\text { Treatment needed } \\
\text { A: } 30 \\
\text { B: } 9 \\
\text { Hospitalizations for diabetic ketoacidosis } \\
\text { A: } 4 \\
\text { B: } 1\end{array}$ & $\begin{array}{l}\text { Students and parents TH satisfaction survey } \\
\text { results ( } 0=\text { not comfortable at all, } 5=\text { very } \\
\text { comfortable), mean score } \pm \mathrm{SD} \\
\text { Felt comfortable with form of communication: } \\
4.68 \pm 0.89 \\
\text { Encounter was convenient: } 4.41 \pm 0.91 \\
\text { Lack of physical contact is acceptable: } 4.54 \pm \\
0.86 \\
\text { Concerns about privacy of personal medical } \\
\text { information: } 0.73 \pm 1.39 \\
\\
\text { Overall, satisfied with TH services: } 4.590 .96 \\
\text { Willing to use TH services again: } 91 \%\end{array}$ & (Cont.) \\
\hline Jaatinen, 2002 & NR & $\begin{array}{l}\text { Success of telehealth in relating patient history: } \\
\text { good vs moderate vs bad } \\
\text { A: } 85 \% \text { vs } 10 \% \text { vs } 5 \% \\
\text { B: } 62 \% \text { vs } 31 \% \text { vs } 8 \% \\
p=0.23 \\
\text { Success of telehealth in relating physical } \\
\text { status: good vs moderate vs bad } \\
\text { A: } 90 \% \text { vs } 10 \% \text { vs } 0 \% \\
\text { B: } 46 \% \text { vs } 33 \% \text { vs } 21 \% \\
p=0.01 \\
\text { Success of telehealth in relating overall patient } \\
\text { case: good vs moderate vs bad } \\
\text { A: } 85 \% \text { vs } 15 \% \text { vs } 0 \% \\
\text { B: } 48 \% \text { vs } 39 \% \text { vs } 13 \% \\
p=0.02 \\
\text { Median total time for visit, in hours } \\
\text { A: } 3.5 \\
\text { B (teleconsultation): } 1.0 \\
\text { B (telereferral): } 1.0\end{array}$ & NR \\
\hline
\end{tabular}




\begin{tabular}{|c|c|c|c|}
\hline $\begin{array}{l}\text { Author, Year } \\
\text { (See Appendix C } \\
\text { for full citation) }\end{array}$ & $\begin{array}{l}\text { Results: } \\
\text { KQ1: Clinical and Economic Outcomes }\end{array}$ & $\begin{array}{l}\text { Results: } \\
\text { KQ2: Intermediate Outcomes }\end{array}$ & $\begin{array}{l}\text { Results: } \\
\text { KQ3: Adverse Effects or } \\
\text { Unintended Consequences }\end{array}$ \\
\hline $\begin{array}{l}\text { Jacklin, } 2003 \\
\text { Same study as } \\
\text { Wallace } 2002 \\
2004\end{array}$ & $\begin{array}{l}\text { Estimated mean cost of index consultation, in pounds } \\
\text { A: } £ 32 \\
\text { B: } £ 164 \\
\text { Mean difference: } £ 132 \\
\text { Total patient costs, in pounds } \\
\text { A: } £ 11.38 \text { (33.85) } \\
\text { B: } £ 3.69 \text { (16.89) } \\
\text { Difference: } £-7.70 \text { (95\% Cl -10.35 to -5.05), p<0.0001 } \\
\text { Mean total NHS costs (SD), in pounds } \\
\text { A: } £ 625.26 \text { (1199.77) } \\
\text { B: } £ 723.98 \text { (832.07) } \\
\text { Difference: } £ 98.72 \text { (95\% Cl } 9.98 \text { to } 187.46), p=0.03 \\
\text { NHS adjusted difference, in pounds } \\
£ 93.80 \text { (7.34 to } 180.40) \\
\text { Mean cost per patient among specialties: } \\
\text { Urology: mean cost were lower in B but NS } \\
\text { all other specialties, mean cost per patient was } \\
\text { significantly higher in B } \\
\text { Cost of prescriptions: } \\
\text { Not significantly different } \\
\text { No differences between the two groups in terms of } \\
\text { tests, investigations and other health services in two } \\
\text { arms of the trial. } \\
\text { Cost } \\
\text { Less travel time, less time off work and slightly lower } \\
\text { cost in patients on the virtual consultation arm. }\end{array}$ & $\begin{array}{l}\text { Followup: } \\
\text { A significantly greater proportion of patients in } \\
\text { the virtual outreach group were offered a } \\
\text { followup appointment }\end{array}$ & NR \\
\hline Jacobs, 2015 & $\begin{array}{l}\text { Referral to hospital } \\
\text { A: } 26.6 \% \\
\text { B: } 8.1 \% \\
\text { Unnecessary trips to the hospital } \\
\text { A: } 13.1 \% \\
\text { B: } 0.4 \%\end{array}$ & NR & $\begin{array}{l}\text { Missed fractures } \\
\text { A: } 13.6 \% \\
\text { B: } 1.7 \%\end{array}$ \\
\hline
\end{tabular}




\begin{tabular}{|c|c|c|c|}
\hline $\begin{array}{l}\text { Author, Year } \\
\text { (See Appendix C } \\
\text { for full citation) }\end{array}$ & $\begin{array}{l}\text { Results: } \\
\text { KQ1: Clinical and Economic Outcomes }\end{array}$ & $\begin{array}{l}\text { Results: } \\
\text { KQ2: Intermediate Outcomes }\end{array}$ & $\begin{array}{l}\text { Results: } \\
\text { KQ3: Adverse Effects or } \\
\text { Unintended Consequences }\end{array}$ \\
\hline Jong, 2004 & $\begin{array}{l}\text { Physician satisfaction } \\
\text { Accessibility to rheumatologist: } \\
\text { A: All rated satisfactory } \\
\text { B: All rated satisfactory } \\
\text { C: All rated very satisfactory } \\
\text { Usefulness of method of consultation: } \\
\text { A: No rating provided } \\
\text { B: No rating provided } \\
\text { C: No rating provided } \\
\text { Impact on management: } \\
\text { A: No rating provided; did not improve management } \\
\text { B: No rating provided; improved management } \\
\text { C: No rating provided; improved management }\end{array}$ & $\begin{array}{l}\text { Costs, in dollars } \\
\text { A: } \$ 975 \text { for flight to on-site } \\
\text { B: } \$ 87.50 \text { for } 1 / 2 \text { hour of teleconsultation }\end{array}$ & NR \\
\hline Jue, 2017 & $\begin{array}{l}\text { Total cost savings for travel and hotel based on } \\
\text { distance from patient home to the Miami VA Facility } \\
\$ 155,627.20\end{array}$ & $\begin{array}{l}\text { Reduction in travel distance } \\
\text { B: } 80.7 \%\end{array}$ & NR \\
\hline Kobza, 2000 & $\begin{array}{l}\text { Stage II pressure ulcer healing rate } \\
\text { A: } 34 \% \\
\text { B: } 83 \% \\
\text { Stage IV pressure ulcer healing rate } \\
\text { A: } 10 \% \\
\text { B: } 38 \% \\
\text { Healing rate for all wounds improved with telehealth, } \\
\text { except for stage III pressure ulcers } \\
\text { Healing time decreased in all categories with } \\
\text { telehealth } \\
\text { Discharge with healed wounds } \\
\text { A: } 37 \% \\
\text { B: } 58 \%\end{array}$ & $\begin{array}{l}\text { Mean number of home visits } \\
\text { A: } 60 \\
\text { B: } 33 \\
\text { Hospitalizations } \\
\text { A: } 18 \% \\
\text { B: } 6 \%\end{array}$ & NR \\
\hline
\end{tabular}




\begin{tabular}{|c|c|c|c|}
\hline $\begin{array}{l}\text { Author, Year } \\
\text { (See Appendix C } \\
\text { for full citation) }\end{array}$ & $\begin{array}{l}\text { Results: } \\
\text { KQ1: Clinical and Economic Outcomes }\end{array}$ & $\begin{array}{l}\text { Results: } \\
\text { KQ2: Intermediate Outcomes }\end{array}$ & $\begin{array}{l}\text { Results: } \\
\text { KQ3: Adverse Effects or } \\
\text { Unintended Consequences }\end{array}$ \\
\hline Krier, 2011 & NR & $\begin{array}{l}\text { Mean duration, in minutes (SD, range) } \\
\text { A: } 59(10,26 \text { to } 73) \\
B: 60(14,35 \text { to } 80) \\
p=0.81 \\
\text { Mean wait time, in minutes (SD, range) } \\
\text { A: } 18(14.5,5 \text { to } 60) \\
B: 25(25,5 \text { to } 90) \\
p=0.31 \\
\text { The two groups similarly rated as excellent } \\
\text { their clinic experience and the major clinical } \\
\text { satisfaction indices of attention to patient } \\
\text { concerns, bedside manner, and perceived skill } \\
\text { level of the doctor. }\end{array}$ & NR \\
\hline Krupinski, 2004 & $\begin{array}{l}\text { In-person group had less evidence in the records } \\
\text { about actions taken as a result of the consultation. } \\
\text { Recorded notes on action taken: } \\
\text { A: } 12 \% \\
\text { B: } 43 \% \\
\text { Z }=3.14, p<0.01 \\
\text { Patients seen again: } \\
\text { A: } 10 \% \\
\text { B: } 8 \% \\
\text { Z }=0.40, p>0.05 \\
\end{array}$ & NR & NR \\
\hline Kunkler, 2007 & $\begin{array}{l}\text { Compliance of decisions with guidelines on best } \\
\text { practice at meeting } \\
\text { A: } 116 / 116(100 \%) \\
\text { B: } 136 / 137(99 \%) \\
\text { Telehealth meetings cheaper than standard meetings: } \\
\text { approximately } 40 \text { meetings per year. }\end{array}$ & $\begin{array}{l}\text { Mean (SE) response of MDT members on a } 5 \\
\text { point scale with } 5 \text { being strongly agree, to the } \\
\text { following } 3 \text { statements: } \\
\text { Consensus was reached by all parties involved } \\
\text { A: } 4.20(0.067) \\
\text { B: } 4.06(0.058), p=0.048 \\
\text { Confident decision was in the best interests of } \\
\text { the patient } \\
\text { A: } 4.16(0.064) \\
\text { B: } 4.07(0.056), p=0.12 \\
\text { Discussion of patient was appropriately shared } \\
\text { by participants } \\
\text { A: } 4.17(0.079) \\
\text { B: } 4.04(0.066), p=0.12\end{array}$ & NR \\
\hline
\end{tabular}




\begin{tabular}{|l|l|l|l|}
\hline $\begin{array}{l}\text { Author, Year } \\
\text { (See Appendix C } \\
\text { for full citation) }\end{array}$ & $\begin{array}{l}\text { Results: } \\
\text { KQ1: Clinical and Economic Outcomes }\end{array}$ & $\begin{array}{l}\text { Results: } \\
\text { KQ3: Adverse Effects or } \\
\text { KQ2: Intermediate Outcomes }\end{array}$ \\
\hline Uamminen, 2001 & $\begin{array}{l}\text { Cost of consultation per patient, in Euros } \\
\text { A1: } € 126\end{array}$ & NR \\
A2: €143 & $\begin{array}{l}\text { NR } \\
\text { There were cost savings in relation to } \\
\text { teleconsultations when the annual numbers of patients } \\
\text { were more than 110 in ophthalmology and 92 in } \\
\text { dermatology. }\end{array}$ & \\
\hline
\end{tabular}




\begin{tabular}{|c|c|c|c|}
\hline $\begin{array}{l}\text { Author, Year } \\
\text { (See Appendix C } \\
\text { for full citation) }\end{array}$ & $\begin{array}{l}\text { Results: } \\
\text { KQ1: Clinical and Economic Outcomes }\end{array}$ & $\begin{array}{l}\text { Results: } \\
\text { KQ2: Intermediate Outcomes }\end{array}$ & $\begin{array}{l}\text { Results: } \\
\text { KQ3: Adverse Effects or } \\
\text { Unintended Consequences }\end{array}$ \\
\hline Lee, 2014 & 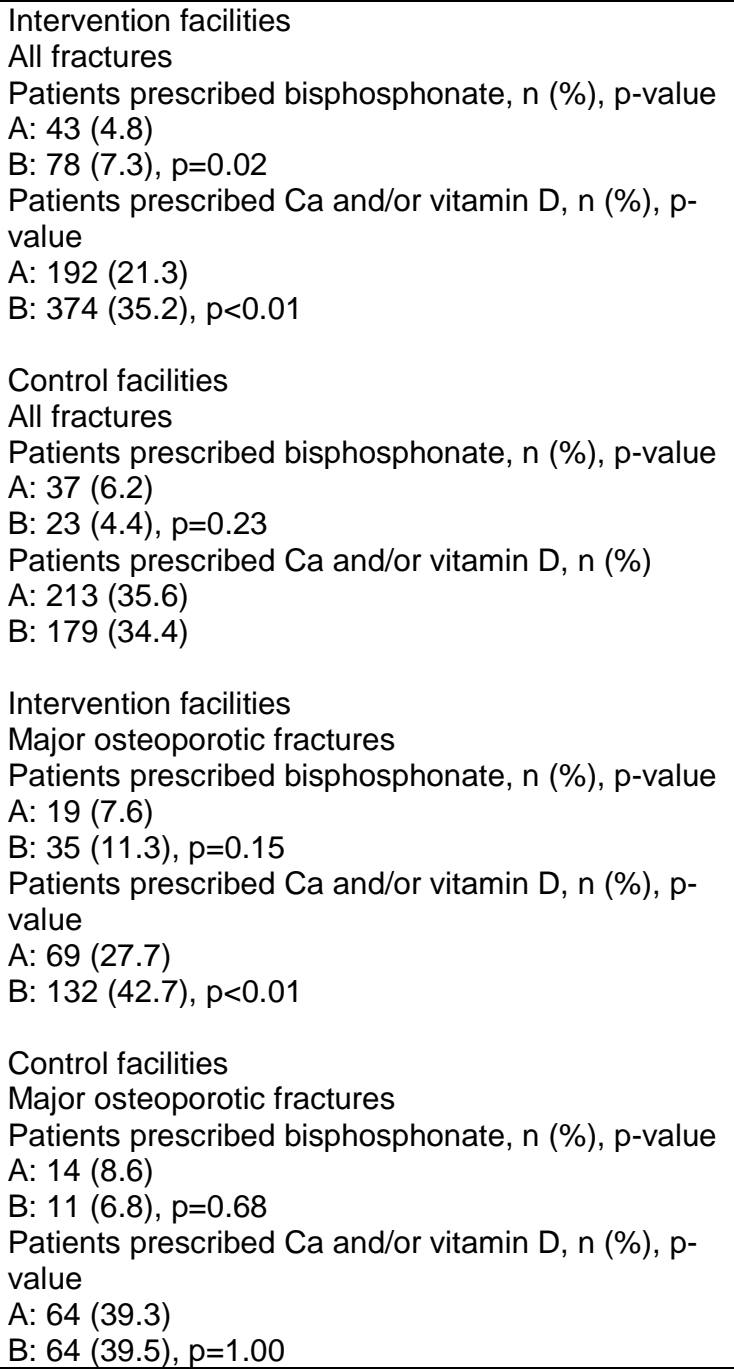 & NR & NR \\
\hline
\end{tabular}




\begin{tabular}{|c|c|c|c|}
\hline $\begin{array}{l}\text { Author, Year } \\
\text { (See Appendix C } \\
\text { for full citation) }\end{array}$ & $\begin{array}{l}\text { Results: } \\
\text { KQ1: Clinical and Economic Outcomes }\end{array}$ & $\begin{array}{l}\text { Results: } \\
\text { KQ2: Intermediate Outcomes }\end{array}$ & $\begin{array}{l}\text { Results: } \\
\text { KQ3: Adverse Effects or } \\
\text { Unintended Consequences }\end{array}$ \\
\hline Lim, 2012 & NR & $\begin{array}{l}\text { Mean waiting time, in days } \\
\text { A: } 114 \\
\text { B: } 39 \\
\text { Patient satisfaction survey: } 1 \text { (poor) to } 5 \\
\text { (excellent) } \\
\text { Overall experience } \\
\text { A: } 3.8 \\
\text { B: } 4.5 \\
\text { Financial cost per patient, in NZ\$ } \\
\text { A: } \$ 306.48 \\
\text { B: } \$ 264.48\end{array}$ & NR \\
\hline Loane, 2000 & NR & $\begin{array}{l}\text { Required at least one subsequent hospital } \\
\text { appointment } \\
\text { A: } 45 \% \\
\text { B: } 46 \% \\
\text { C: } 69 \%\end{array}$ & NR \\
\hline Loane, 1999 & NR & $\begin{array}{l}\text { Mean wait time to see doctor, in minutes } \\
\text { A: } 20 \\
\text { B: } 5.4 \\
\text { Mean consultation time with doctor, in minutes } \\
\text { A: } 16.8 \\
\text { B: } 22.0 \\
\text { Mean total travel time, in minutes } \\
\text { A: } 48.0 \\
\text { B: } 31.6 \\
\text { Mean total time involved in attending } \\
\text { appointment, including waiting, consultation } \\
\text { and travel, in minutes } \\
\text { A: } 84.4 \\
\text { B: } 59.3 \\
\text { Mean total distance involved in attending } \\
\text { appointments, in km } \\
\text { A: } 25.4 \\
\text { B: } 10.4\end{array}$ & NR \\
\hline
\end{tabular}

\section{F-124}




\begin{tabular}{|c|c|c|c|}
\hline $\begin{array}{l}\text { Author, Year } \\
\text { (See Appendix C } \\
\text { for full citation) }\end{array}$ & $\begin{array}{l}\text { Results: } \\
\text { KQ1: Clinical and Economic Outcomes }\end{array}$ & $\begin{array}{l}\text { Results: } \\
\text { KQ2: Intermediate Outcomes }\end{array}$ & $\begin{array}{l}\text { Results: } \\
\text { KQ3: Adverse Effects or } \\
\text { Unintended Consequences }\end{array}$ \\
\hline Loane, 2001 & $\begin{array}{l}\text { Total cost of dermatologist's time spent in performing } \\
\text { consultations, in NZ\$ } \\
\text { A: } \$ 5724.00 \\
\text { B: } \$ 6162.80 \\
\text { Cost of patient time to attend consultations, in NZ\$ } \\
\text { A: } \$ 7838.17 \\
\text { B: } \$ 1845.54 \\
\text { Total travel costs for patients to attend consultations, } \\
\text { in NZ\$ } \\
\text { A: } \$ 16,519.15 \\
\text { B: } \$ 876.64 \\
\text { Total societal costs of consultations, in NZ\$ } \\
\text { A: } \$ 30,081.33 \\
\text { B: } \$ 34,345.55\end{array}$ & $\begin{array}{l}\text { Mean consultation time, in minutes } \\
\text { A: } 21.60 \\
\text { B: } 20.04\end{array}$ & NR \\
\hline Long, 2014 & $\begin{array}{l}\% \text { of pregnancies receiving comprehensive } \\
\text { ultrasound: } \\
\text { A: } 9.6 \% \\
\text { B: } 11.3 \%, p<0.0001 \\
\% \text { of high-risk pregnancies receiving comprehensive } \\
\text { ultrasound: } \\
\text { A: } 16.9 \% \\
\text { B: } 19.9 \%, p<0.001\end{array}$ & $\begin{array}{l}\text { \% of high-risk pregnancies with prenatal care } \\
\text { starting each trimester: } \\
\text { First trimester } \\
\text { A: } 74.3 \% \\
\text { B: } 75.0 \% \\
\text { Second trimester } \\
\text { A: } 21.5 \% \\
\text { B: } 21.1 \% \\
\text { Third trimester } \\
\text { A: } 4.2 \% \\
\text { B: } 4.0 \% \\
\text { Statistical significance NR }\end{array}$ & NR \\
\hline
\end{tabular}

\section{F-125}




\begin{tabular}{|c|c|c|c|}
\hline $\begin{array}{l}\text { Author, Year } \\
\text { (See Appendix C } \\
\text { for full citation) }\end{array}$ & $\begin{array}{l}\text { Results: } \\
\text { KQ1: Clinical and Economic Outcomes }\end{array}$ & $\begin{array}{l}\text { Results: } \\
\text { KQ2: Intermediate Outcomes }\end{array}$ & $\begin{array}{l}\text { Results: } \\
\text { KQ3: Adverse Effects or } \\
\text { Unintended Consequences }\end{array}$ \\
\hline Mahendran, 2005 & NR & $\begin{array}{l}\text { Telehealth diagnosis (face-to-face is gold } \\
\text { standard): } \\
48 \% \text { agree } \\
15 \% \text { uncertain but actual diagnosis mentioned } \\
20 \% \text { incorrect or could not be made } \\
15 \% \text { image not adequate } \\
\\
\text { Agreement on management plan for same } \\
\text { patients evaluated } \\
55 \% \\
\text { Managed appropriately } \\
13 \% \text { required reassurance only } \\
9 \% \text { recommended further review } \\
33 \% \text { minor operation } \\
\text { Not adequately managed by telehealth } \\
15 \% \text { poor image quality } \\
20 \% \text { complex and required in-person exam } \\
\text { and consent } \\
10 \% \text { telehealth recommended surgery not } \\
\text { needed or wrong surgery }\end{array}$ & $\begin{array}{l}\text { Diagnostic and referral issues: } \\
\text { In } 20 \% \text { of cases the diagnosis was } \\
\text { either incorrect or could not be } \\
\text { made. } \\
45 \% \text { of teledermatology referrals } \\
\text { were not adequately managed by } \\
\text { the consultants. } \\
10 \% \text { of all cases would have been } \\
\text { inappropriately booked directly for } \\
\text { surgery if teledermatology was } \\
\text { used. }\end{array}$ \\
\hline McCrossan, 2012 & $\begin{array}{l}\text { Mean difference in days taken off work }(95 \% \mathrm{Cl}), \mathrm{p} \text { - } \\
\text { value: } \\
0.61 \text { ( } 0.39 \text { to } 0.82), p<0.01\end{array}$ & $\begin{array}{l}\text { Mean aggregate patient satisfaction (SD) } \\
\text { A: } 23.2 / 25(2.2) \\
B: 23.2 / 25(2.0) \\
p=0.92\end{array}$ & NR \\
\hline $\begin{array}{l}\text { Moreno-Ramirez, } \\
2009\end{array}$ & $\begin{array}{l}\text { Unit cost per patient, in Euros } \\
\text { A: } € 129.37 \\
\text { B: } € 79.78 \\
p=0.005 \\
\text { For benign lesions conventional care was } 3.29 \text { times } \\
\text { more expensive }\end{array}$ & NR & NR \\
\hline Mulgrew, 2011 & $\mathrm{NR}$ & $\begin{array}{l}\text { Mean overall patient satisfaction defined as a } \\
\text { score of } 5 \text { or } 6 \text { (almost always, or always) (SD) } \\
\text { A: } 44.5(3.85) \\
\text { B: } 43.8(4.83) \\
\text { p=0.42 } \\
\text { Satisfaction with consulting health care } \\
\text { provider } \\
\text { A: } 9.3(0.91) \\
\text { B: } 9.4(1.01)\end{array}$ & NR \\
\hline
\end{tabular}

\section{F-126}




\begin{tabular}{|c|c|c|c|}
\hline $\begin{array}{l}\text { Author, Year } \\
\text { (See Appendix C } \\
\text { for full citation) }\end{array}$ & $\begin{array}{l}\text { Results: } \\
\text { KQ1: Clinical and Economic Outcomes }\end{array}$ & $\begin{array}{l}\text { Results: } \\
\text { KQ2: Intermediate Outcomes }\end{array}$ & $\begin{array}{l}\text { Results: } \\
\text { KQ3: Adverse Effects or } \\
\text { Unintended Consequences }\end{array}$ \\
\hline Nickenig, 2008 & NR & $\begin{array}{l}\text { Concept changes in diagnosis, } n(\%) \\
\text { A: } 36(4 \%) \\
\text { B: } 0(0 \%) \\
\text { Prosthodontic protocol, } n(\%) \\
\text { A: } 67(7 \%) \\
\text { B: } 3(3 \%) \\
\text { Number and position of implants, } n(\%) \\
\text { A: } 148(19 \%) \\
\text { B: } 13(15 \%)\end{array}$ & NR \\
\hline Nikkanen, 2008 & $\begin{array}{l}\text { Mean HbA1c (SD) } \\
\text { A: } 8.0 \%(1.9) \\
\text { B: } 7.6 \%(1.5) \\
\text { Difference: }-0.4 ; p=0.007 \\
\text { LDL cholesterol (mmol/L) } \\
\text { A: } 3.3(0.9) \\
\text { B: } 2.7(0.8) \\
\text { Difference: }-0.6 ; p=0.001 \\
\text { Systolic blood pressure (in } \mathrm{mmHg}) \\
\text { A: } 146(22) \\
\text { B: } 140(16) \\
\text { Difference: }-6 ; p=0.004 \\
\text { Body mass index, in } \mathrm{kg} / \mathrm{m} 2 \\
\text { A: } 30.6(6.3) \\
\text { B: } 30.46(6.7) \\
\text { Difference: }-0.2 ; p=0.58 \\
\text { Subgroup analyses indicate largest change in HbA1c } \\
\text { results in patients with DM }>10 \text { years and with higher } \\
\text { HbA1c at baseline. }\end{array}$ & NR & NR \\
\hline
\end{tabular}




\begin{tabular}{|c|c|c|c|}
\hline $\begin{array}{l}\text { Author, Year } \\
\text { (See Appendix C } \\
\text { for full citation) }\end{array}$ & $\begin{array}{l}\text { Results: } \\
\text { KQ1: Clinical and Economic Outcomes }\end{array}$ & $\begin{array}{l}\text { Results: } \\
\text { KQ2: Intermediate Outcomes }\end{array}$ & $\begin{array}{l}\text { Results: } \\
\text { KQ3: Adverse Effects or } \\
\text { Unintended Consequences }\end{array}$ \\
\hline Nordal, 2001 & NR & $\begin{array}{l}\text { Diagnostic agreement: } \\
72 \% \text { complete concordance } \\
14 \% \text { had partial concordance } \\
13 \% \text { were discordant } \\
\text { Provider mean score of confidence in } \\
\text { diagnoses: } \\
\text { Face-to-face: } 2.7 \\
\text { Telecommunication: } 2.4 \\
\text { p>0.05 } \\
\text { Provider preferences: } \\
22 \% \text { favored face-to-face } \\
14 \% \text { of ratings favored telehealth } \\
\text { Time spent for a consultation: } \\
\text { Face-to-face: } 10.1 \text { minutes } \\
\text { Telecommunication: } 9.5 \text { minutes } \\
\text { Patient reports: } \\
61 \% \text { no disadvantage to video } \\
18 \% \text { reduced contact with specialist } \\
7 \% \text { discomfort being recorded. } \\
86 \% \text { favored having GP present for } \\
\text { teledermatology } \\
\text { patient satisfaction: } \\
\text { Face-to-face: } 8 \% \\
\text { Telecommunications: } 26 \% \\
\text { p=0.0006 }\end{array}$ & NR \\
\hline
\end{tabular}




\begin{tabular}{|c|c|c|c|}
\hline $\begin{array}{l}\text { Author, Year } \\
\text { (See Appendix C } \\
\text { for full citation) }\end{array}$ & $\begin{array}{l}\text { Results: } \\
\text { KQ1: Clinical and Economic Outcomes }\end{array}$ & $\begin{array}{l}\text { Results: } \\
\text { KQ2: Intermediate Outcomes }\end{array}$ & $\begin{array}{l}\text { Results: } \\
\text { KQ3: Adverse Effects or } \\
\text { Unintended Consequences }\end{array}$ \\
\hline $\begin{array}{l}\text { Ohinmaa } \\
\text { Same patients as } \\
\text { Vuolio and } \\
\text { Haukipuro, } 2002\end{array}$ & $\begin{array}{l}\text { Mean time spent by patient, in hours } \\
\text { A: } 8 \\
\text { B: } 1.5 \\
\text { Mean distance travelled, in } \mathrm{km} \\
\text { A: } 170 \\
\text { B: } 8 \\
\text { Cost: } \\
\text { Total cost, including travel and indirect costs based on } \\
100 \text { patients, per patient in euros } \\
\text { A: } € 114.0 \\
\text { B: €87.8 } \\
\text { Difference dependent on patient travel: } \\
\text { Breakeven point: } \\
80 \text { cases if } 160 \mathrm{~km} \\
200 \text { case if } 80 \mathrm{~km} \\
\text { Total cost savings from use of teleconsultation } € 2,620\end{array}$ & NR & NR \\
\hline $\begin{array}{l}\text { Pak, 2009; Pak, } \\
2007\end{array}$ & $\begin{array}{l}\text { Changes in clinical course } \\
\text { Improved } \\
\text { A: } 65 \% \\
\text { B: } 64 \% \\
\text { No change } \\
\text { A: } 32 \% \\
\text { B: } 33 \% \\
\text { Worse } \\
\text { A: } 3 \% \\
\text { B: } 4 \% \text {, NS } \\
\text { Total average cost per patient, in USD } \\
\text { A: } \$ 129,133(\$ 372) \\
\text { B: } \$ 119,402(\$ 340) \\
\text { Direct costs, in USD } \\
\text { A: } \$ 98,365 \\
\text { B: } \$ 103,043 \\
\text { Lost productivity, in USD } \\
\text { A: } \$ 30,768 \\
\text { B: } \$ 16,359\end{array}$ & NR & NR \\
\hline
\end{tabular}




\begin{tabular}{|c|c|c|c|}
\hline $\begin{array}{l}\text { Author, Year } \\
\text { (See Appendix C } \\
\text { for full citation) }\end{array}$ & $\begin{array}{l}\text { Results: } \\
\text { KQ1: Clinical and Economic Outcomes }\end{array}$ & $\begin{array}{l}\text { Results: } \\
\text { KQ2: Intermediate Outcomes }\end{array}$ & $\begin{array}{l}\text { Results: } \\
\text { KQ3: Adverse Effects or } \\
\text { Unintended Consequences }\end{array}$ \\
\hline Piette, 2017 & NR & $\begin{array}{l}\text { Median delay between the initial GP's } \\
\text { consultation and the reply allowing for } \\
\text { treatment to begin, in days } \\
\text { A: } 40 \\
\text { B: } 4 \\
\text { Adjusted hazard ratio= } 2.55 ; p<0.011 \\
\text { Patients' global satisfaction } \\
\text { Very satisfied or satisfied } \\
\text { A: } 47(94 \%) \\
\text { B: } 45(84.9 \%) \\
\text { p=0.99 } \\
\text { Unsatisfied or very unsatisfied } \\
\text { A: } 3(6 \%) \\
\text { B: } 8(15.1 \%) \\
\text { Patients' satisfaction about the delay before } \\
\text { care } \\
\text { Very satisfied or satisfied } \\
\text { A: } 13(26 \%) \\
\text { B: } 38(71.7 \%) \\
\text { p=0.20 } \\
\text { Unsatisfied or very unsatisfied } \\
\text { A: } 37(74 \%) \\
\text { B: } 15(28.3 \%)\end{array}$ & NR \\
\hline
\end{tabular}




\begin{tabular}{|c|c|c|c|}
\hline $\begin{array}{l}\text { Author, Year } \\
\text { (See Appendix C } \\
\text { for full citation) }\end{array}$ & $\begin{array}{l}\text { Results: } \\
\text { KQ1: Clinical and Economic Outcomes }\end{array}$ & $\begin{array}{l}\text { Results: } \\
\text { KQ2: Intermediate Outcomes }\end{array}$ & $\begin{array}{l}\text { Results: } \\
\text { KQ3: Adverse Effects or } \\
\text { Unintended Consequences }\end{array}$ \\
\hline Rossaro, 2013 & $\begin{array}{l}\text { Sustained virological response } \\
\text { A: } 16(43 \%) \\
\text { B: } 21(55 \%), p=0.36\end{array}$ & $\begin{array}{l}\text { Completion of therapy, } n(\%) \\
\text { A: } 21(53 \%) \\
\text { B: } 31(78 \%), p=0.03 \\
\text { Mean number of weeks of therapy } \\
\text { A: } 30.2 \\
\text { B: } 36.7, p=0.07 \\
\text { Mean number of visits } \\
\text { A: } 2.2 \\
\text { B: } 19.6<0.0001 \\
\text { Mean number of visits per week of therapy } \\
\text { A: } 0.07 \\
\text { B: } 0.61, p<0.001 \\
\text { Stopped therapy due to depression } \\
\text { A: } 1 \\
\text { B: } 4 \\
\text { Anti-depressant medication } \\
\text { A: } 7 \\
\text { B: } 14 \\
\text { Reasons for early termination of therapy: } \\
\text { A: Severe anemia, skin rash, and weight loss } \\
\text { B: Severe depression, NS }\end{array}$ & $\begin{array}{l}\text { Side effects were similar for both } \\
\text { groups - not significant } \\
\text { Neutropenia } \\
\text { GI side effects } \\
\text { Fatigue } \\
\text { Depression } \\
\text { Weight loss } \\
\text { Insomnia } \\
\text { Skin rash } \\
\text { Anemia } \\
\text { A: } 53 \% \\
\text { B: } 25 \% \\
\text { p=0.02 } \\
\text { Patients discontinued therapy due } \\
\text { to depression } \\
\text { A: } 1 \\
\text { B: } 4 \\
\text { On anti-depressant medication } \\
\text { A: } 7 \\
\text { B: } 14\end{array}$ \\
\hline
\end{tabular}




\begin{tabular}{|c|c|c|c|}
\hline $\begin{array}{l}\text { Author, Year } \\
\text { (See Appendix C } \\
\text { for full citation) }\end{array}$ & $\begin{array}{l}\text { Results: } \\
\text { KQ1: Clinical and Economic Outcomes }\end{array}$ & $\begin{array}{l}\text { Results: } \\
\text { KQ2: Intermediate Outcomes }\end{array}$ & $\begin{array}{l}\text { Results: } \\
\text { KQ3: Adverse Effects or } \\
\text { Unintended Consequences }\end{array}$ \\
\hline Salami, 2015 & NR & $\begin{array}{l}\text { Comprehensive clinical evaluation prior to } \\
\text { initiation of treatment } n \text { (\%) } \\
\text { A: } 44(64.7 \%) \\
\text { B: } 44(91.7 \%), p=0.001 \\
\text { Guideline-driven clinical evaluation prior to } \\
\text { initiation of treatment } n(\%) \\
\text { A: } 51(75.0 \%) \\
\text { B: } 48(100.0 \%), p<0.001 \\
\text { Assessment of tumor stage } n(\%) \\
\text { A: } 50(73.5 \%) \\
\text { B: } 44(91.7 \%), p=0.002 \\
\text { Assessment of transplant eligibility } n \text { (\%) } \\
\text { A: } 58(85.3) \\
\text { B: } 46(95.8), p=0.006 \\
\text { Median time from referral to evaluation (range), } \\
\text { in days } \\
\text { A: } 39(11 \text { to } 387) \\
\text { B: } 23(8 \text { to } 97), p<0.001 \\
\text { Median time from referral to treatment initiation } \\
\text { (range), in days } \\
\text { median } \\
\text { A: } 63(27 \text { to } 231) \\
\text { B: } 55(27 \text { to } 180), p=0.152 \\
\text { Median miles travelled by patient to receive } \\
\text { evaluation (range) } \\
\text { A: } 683 \text { (0 to } 3327) \\
\text { B: } 0 \text { (0 to 0), } p<0.001\end{array}$ & NR \\
\hline
\end{tabular}




\begin{tabular}{|c|c|c|c|}
\hline $\begin{array}{l}\text { Author, Year } \\
\text { (See Appendix C } \\
\text { for full citation) }\end{array}$ & $\begin{array}{l}\text { Results: } \\
\text { KQ1: Clinical and Economic Outcomes }\end{array}$ & $\begin{array}{l}\text { Results: } \\
\text { KQ2: Intermediate Outcomes }\end{array}$ & $\begin{array}{l}\text { Results: } \\
\text { KQ3: Adverse Effects or } \\
\text { Unintended Consequences }\end{array}$ \\
\hline $\begin{array}{l}\text { Salazar- } \\
\text { Fernandez, } 2012\end{array}$ & $\begin{array}{l}\text { Mean cost of lost working hours per patient }(95 \% \mathrm{Cl}) \\
\text { A: } 32.24(31.6 \text { to } 32.8) \\
\text { B: } 16.8(16.2 \text { to } 17.3), p=0.01\end{array}$ & $\begin{array}{l}\text { Patients referred to maxillofacial surgery } \\
\text { A: } 83(11.6 \%) \\
\text { B: } 35(10.2 \%), p=0.28 \\
\text { Resolved consultation } \\
\text { A: } 529(74.5 \%) \\
\text { B: } 304(88 \%), p=0.07 \\
\text { Second consultations } \\
\text { A: } 29(4.6 \%) \\
\text { B: } 3(0.8 \%), p=0.07 \\
\text { Mean lost working hours } \\
\text { A: } 32.24 \\
\text { B: } 16.8, p=0.01 \\
\text { Mean time until treatment }(95 \% \mathrm{Cl}) \text {, in days } \\
\text { A: } 78.6(77.0 \text { to } 80) \\
\text { B: } 2.3(2.2 \text { to } 2.4), p=0 \\
\text { Number of complaints } \\
\text { A: } 6(0.8 \%) \\
\text { B: } 1(0.3 \%), p=0.1 \\
\text { Mean lost working hours }(95 \% \mathrm{Cl}) \\
\text { A: } 32.24(31.6 \text { to } 32.8) \\
\text { B: } 16.8(16.2 \text { to } 17.3), p=0.01\end{array}$ & NR \\
\hline Santamaria, 2004 & $\begin{array}{l}\text { Average healing rate, per week } \\
\text { A: }-4.9 \% \\
\text { B: } 6.8 \%, p=0.012 \\
\text { Amputations } \\
\text { A: } 6 \\
\text { B: } 1 \\
\text { Mortality } \\
\text { A: NR } \\
\text { B: } 2 \\
\text { Total cost, in AUD } \\
\text { A: } \$ 862,161 \\
\text { B: } \$ 670,226 ; n=43\end{array}$ & NR & NR \\
\hline
\end{tabular}

\section{F-133}




\begin{tabular}{|c|c|c|c|}
\hline $\begin{array}{l}\text { Author, Year } \\
\text { (See Appendix C } \\
\text { for full citation) }\end{array}$ & $\begin{array}{l}\text { Results: } \\
\text { KQ1: Clinical and Economic Outcomes }\end{array}$ & $\begin{array}{l}\text { Results: } \\
\text { KQ2: Intermediate Outcomes }\end{array}$ & $\begin{array}{l}\text { Results: } \\
\text { KQ3: Adverse Effects or } \\
\text { Unintended Consequences }\end{array}$ \\
\hline Scuffham, 2002 & $\begin{array}{l}\text { Total variable costs per patient, in pounds } \\
\text { A: } £ 233.86 \\
\text { B: } £ 1181.52 \\
\text { C: } £ 404.10 \\
\text { Total societal costs, in pounds } \\
\text { A: } £ 403.11 \\
\text { B: } £ 1181.51 \\
\text { C: } £ 582.69 \\
\text { Outreach visits are least expensive }\end{array}$ & NR & NR \\
\hline Sharma, 2003 & $\begin{array}{l}\text { Mean inadequately identified cardiovascular items (out } \\
\text { of } 31 \text { during pilot) } \\
\text { A: } 2.3 \\
\text { B: } 2.1 \\
\text { p }=0.2\end{array}$ & $\begin{array}{l}\text { Mean patient satisfaction score, (5-point scale, } \\
\text { 5=very satisfied), p-value } \\
\text { Comfort during exam: } \\
\text { A: } 4.6 \\
\text { B: } 4.3, N S \\
\text { Amount of information received during exam: } \\
\text { A: } 4.6 \\
\text { B: } 4.3, p=0.05 \\
\text { Willingness of doctor to answer questions: } \\
\text { A: } 4.6 \\
\text { B: } 4.5, N S \\
\text { Explanation of results of exam: } \\
\text { A: } 4.6 \\
\text { B: } 4.4, N S \\
\text { Care and concern of doctor: } \\
\text { A: } 4.6 \\
\text { B: } 4.4, N S \\
\text { Translator's ability to speak language: } \\
\text { A: } 4.5 \\
\text { B: } 4.6, N S \\
\text { Overall quality of care and services: } \\
\text { A: } 4.6 \\
\text { B: } 4.5, N S\end{array}$ & NR \\
\hline
\end{tabular}




\begin{tabular}{|c|c|c|c|}
\hline $\begin{array}{l}\text { Author, Year } \\
\text { (See Appendix C } \\
\text { for full citation) }\end{array}$ & $\begin{array}{l}\text { Results: } \\
\text { KQ1: Clinical and Economic Outcomes }\end{array}$ & $\begin{array}{l}\text { Results: } \\
\text { KQ2: Intermediate Outcomes }\end{array}$ & $\begin{array}{l}\text { Results: } \\
\text { KQ3: Adverse Effects or } \\
\text { Unintended Consequences }\end{array}$ \\
\hline Smith, 2002 & NR & $\begin{array}{l}\text { Change in pediatric admissions from Mackay } \\
\text { region to Royal Children's Hospital, in patients } \\
\text { per month } \\
\text { A: } 9.7 \\
\text { B: } 6.0 \\
\text { Change in pediatric admissions From Hervey } \\
\text { Bay region to Royal Children's Hospital, in } \\
\text { patients per month } \\
\text { A: } 10.0 \\
\text { B: } 12.5 \\
\text { Patient referrals for outpatient appointments to } \\
\text { Brisbane from Mackay, in patients per month } \\
\text { A: } 7.9 \\
\text { B: } 5.7 \\
\text { Patient referrals for outpatient appointments to } \\
\text { Brisbane from Hervey Bay, in patients per } \\
\text { month } \\
\text { A: } 15.8 \\
\text { B: } 15.4 \\
\text { Pediatric outpatient department visits in } \\
\text { Mackay } \\
\text { A: } 78 \\
\text { B: } 134 \\
\text { No change in outpatient department visits in } \\
\text { Hervey Bay }\end{array}$ & NR \\
\hline \begin{tabular}{l|} 
Smith-Strom, 2018 \\
\\
\end{tabular} & $\begin{array}{l}\text { Mortality, } \mathrm{n}(\%) \\
\text { A: } 5(5.7 \%) \\
\text { B: } 5(5.3 \%) \\
\text { Mean difference }(95 \% \mathrm{Cl}):-0.4 \%(-6.5 \text { to } 5.7) \\
\text { Amputation, } \mathrm{n}(\%) \\
\text { A: } 13(14.8 \% \\
\text { B: } 6(6.4 \%) \\
\text { Mean difference }(95 \% \mathrm{Cl}):-8.3 \%(-16.3 \text { to }-0.5)\end{array}$ & $\begin{array}{l}\text { Wounds healed, } n(\%) \\
\text { A: } 67(76.1 \%) \\
\text { B: } 75(79.8 \%) \\
\text { Mean healing time, in months } \\
\text { A: } 3.8 \\
\text { B: } 3.4 \\
\text { Mean difference }(95 \% \mathrm{Cl}):-0.43(-1.50 \text { to } 0.65)\end{array}$ & NR \\
\hline
\end{tabular}

\section{F-135}




\begin{tabular}{|l|l|l|l|}
\hline $\begin{array}{l}\text { Author, Year } \begin{array}{l}\text { (See Appendix C } \\
\text { for full citation) }\end{array} \\
\text { Specht, 2001 }\end{array}$ & $\begin{array}{l}\text { Results: } \\
\text { KQ1: Clinical and Economic Outcomes }\end{array}$ & $\begin{array}{l}\text { Results: } \\
\text { KQ2: Intermediate Outcomes }\end{array}$ & $\begin{array}{l}\text { KQ3: Adverse Effects or } \\
\text { Unintended Consequences }\end{array}$ \\
\hline & A: \$246.28 of chronic wound consultation, in USD & NR & \\
B: \$136.16 & & \\
& Cost avoidance & & \\
& Consultant: \$36.66/consultation & & \\
& Patient transportation cost avoided: \$191.28 & & \\
& Average time spent on appointment & & \\
& A: 8.5 hours, including travel time & & \\
B: 20 minutes & & \\
\hline
\end{tabular}




\begin{tabular}{|c|c|c|c|}
\hline $\begin{array}{l}\text { Author, Year } \\
\text { (See Appendix C } \\
\text { for full citation) }\end{array}$ & $\begin{array}{l}\text { Results: } \\
\text { KQ1: Clinical and Economic Outcomes }\end{array}$ & $\begin{array}{l}\text { Results: } \\
\text { KQ2: Intermediate Outcomes }\end{array}$ & \begin{tabular}{|l|} 
Results: \\
KQ3: Adverse Effects or \\
Unintended Consequences
\end{tabular} \\
\hline Stalfors, 2003 & NR & $\begin{array}{l}\text { Mean time spent including travel and waiting } \\
\text { time } \pm 95 \% \mathrm{Cl} \text {, in hours } \\
\text { A: } 8.9 \pm 0.8 \\
\text { B: } 3.4 \pm 0.5 \\
\text { Mean presentation time } \pm 95 \% \mathrm{Cl} \text {, in minutes } \\
\text { A: } 14.2 \pm 1.4 \\
\text { B: } 13.3 \pm 2.03 \\
\text { Information received during conference rated } \\
\text { as very good } \\
\text { A: } 69 \% \\
\text { B: } 44 \%, p<0.05 \\
\text { Information received during conference rated } \\
\text { as good } \\
\text { A: } 26 \% \\
\text { B: } 44 \%, p<0.05 \\
\text { Information received during conference rated } \\
\text { as insufficient } \\
\text { A: } 0 \% \\
\text { B: } 4 \% \\
\text { Information received during conference rated } \\
\text { as bad } \\
\text { A: } 0 \% \\
\text { B: } 0 \% \\
\text { Satisfaction with information about future } \\
\text { treatment rated as very good } \\
\text { A: } 67 \% \\
\text { B: } 56 \% \\
\text { Satisfaction with information about future } \\
\text { treatment rated as good } \\
\text { A: } 21 \% \\
\text { B: } 38 \% \\
\text { Satisfaction with information about future } \\
\text { treatment rated as insufficient } \\
\text { A: } 0 \% \\
\text { B: } 2 \%\end{array}$ & NR \\
\hline
\end{tabular}

\section{F-137}




\begin{tabular}{|c|c|c|c|}
\hline $\begin{array}{l}\text { Author, Year } \\
\text { (See Appendix C } \\
\text { for full citation) }\end{array}$ & $\begin{array}{l}\text { Results: } \\
\text { KQ1: Clinical and Economic Outcomes }\end{array}$ & $\begin{array}{l}\text { Results: } \\
\text { KQ2: Intermediate Outcomes }\end{array}$ & $\begin{array}{l}\text { Results: } \\
\text { KQ3: Adverse Effects or } \\
\text { Unintended Consequences } \\
\end{array}$ \\
\hline $\begin{array}{l}\text { Stalfors, } 2003 \\
\text { Continued }\end{array}$ & (see above) & $\begin{array}{l}\text { Satisfaction with information about future } \\
\text { treatment rated as bad: } \\
\text { A: } 0 \% \\
\text { B: } 0 \% \\
\text { No answer: } \\
\text { A: } 13 \% \\
\text { B: } 4 \% \\
\text { Answering "totally agree" to statement (n) } \\
\text { I felt calm and secure during the presentation: } \\
\text { A: } 31 \\
\text { B: } 36 \\
\text { I did not feel worse than at any other } \\
\text { investigation: } \\
\text { A: } 19 \\
\text { B: } 25 \\
\text { It felt good to know my doctor had this kind of } \\
\text { support from specialists: } \\
\text { A: } 31 \\
\text { B: } 38 \\
\text { It is as though they listened to me and that I } \\
\text { was a participant: } \\
\text { A: } 20 \\
\text { B: } 30 \\
\text { It felt as though everybody received all the } \\
\text { important information: } \\
\text { A: } 18 \\
\text { B: } 31 \\
\text { It felt good to have my doctor by my side: } \\
\text { A: } 9 \\
\text { B: } 38, p<0.05 \\
\text { Answering "not at all" to statement (n) } \\
\text { It felt as if everybody was talking about me, but } \\
\text { not to me: } \\
\text { A: } 15 \\
\text { B: } 31 \text {, p<0.001 }\end{array}$ & (see above) \\
\hline
\end{tabular}

\section{F-138}




\begin{tabular}{|c|c|c|c|}
\hline $\begin{array}{l}\text { Author, Year } \\
\text { (See Appendix C } \\
\text { for full citation) }\end{array}$ & $\begin{array}{l}\text { Results: } \\
\text { KQ1: Clinical and Economic Outcomes }\end{array}$ & $\begin{array}{l}\text { Results: } \\
\text { KQ2: Intermediate Outcomes }\end{array}$ & $\begin{array}{l}\text { Results: } \\
\text { KQ3: Adverse Effects or } \\
\text { Unintended Consequences }\end{array}$ \\
\hline Stalfors, 2005 & $\begin{array}{l}\text { Combined cost, in SEK } \\
\text { A: } 2,267 \\
\text { B: } 2,036, p>0.2 \\
\text { Direct medical cost, in SEK } \\
\text { A: } 576 ; 429 / \text { patient for district general hospital } \\
\text { physician } \\
\text { B: } 1,550 ; 1,288 / \text { patient is the equipment cost } \\
\text { Direct nonmedical cost, in SEK } \\
\text { A: } 886 \\
\text { B: } 176 \\
\text { Indirect nonmedical cost, in SEK } \\
\text { A: } 805 \\
\text { B: } 310\end{array}$ & NR & NR \\
\hline Stern, 2014 & $\begin{array}{l}\text { Average rate of healing } 1.0058 \text { times slower in } \\
\text { intervention period }(95 \% \mathrm{Cl} 0.985 \text { to } 1.027, \mathrm{p}=0.60) \\
\\
\text { Time to healing, } \mathrm{HR}(95 \% \mathrm{Cl}) \\
\text { Intervention: } 1.48(0.79 \text { to } 2.78), \mathrm{p}=0.22 \\
\\
\text { Estimated probability of healing }(95 \% \mathrm{Cl}) \text { : } \\
\text { A: } 35.0 \%(22.4 \text { to } 45.6) \\
\mathrm{B}: 53.4 \%(41.4 \text { to } 62.9) \\
\text { Estimated incidence rate of intervention: } 1.12 \text { times } \\
\text { larger in intervention ( } 95 \% \mathrm{Cl} 0.74 \text { to } 1.68, \mathrm{p}=0.59) \\
\text { Estimated mean hospitalization rate: } 1.2 \text { times larger } \\
\text { during intervention ( } 95 \% \mathrm{Cl} 0.62 \text { to } 2.36, \mathrm{p}=0.59) \\
\text { Estimated mean ED visit rate: } 1.3 \text { times larger during } \\
\text { intervention ( } 95 \% \mathrm{Cl} 0.58 \text { to } 2.90, \mathrm{p}=0.52) \\
\text { Direct care costs: } \\
\text { Intervention estimated to reduce direct care costs by } \\
\$ 649 \text { per resident }\end{array}$ & $\begin{array}{l}\text { Estimated mean VAS wound-specific pain } \\
\text { scores: } 0.39 \text { units higher during intervention } \\
\text { period ( } 95 \% \mathrm{Cl}-0.55 \text { to } 1.34, p=1.34 \text { ) }\end{array}$ & NR \\
\hline Strymish, 2017 & NR & $\begin{array}{l}\text { Time to completion for e-consults averaged } \\
\text { (SD), in days } \\
\text { A: } 16.5(12.4) \\
\text { B: } 0.6(3.6), p<0.05\end{array}$ & NR \\
\hline
\end{tabular}

\section{F-139}




\begin{tabular}{|c|c|c|c|}
\hline $\begin{array}{l}\text { Author, Year } \\
\text { (See Appendix C } \\
\text { for full citation) }\end{array}$ & $\begin{array}{l}\text { Results: } \\
\text { KQ1: Clinical and Economic Outcomes }\end{array}$ & $\begin{array}{l}\text { Results: } \\
\text { KQ2: Intermediate Outcomes }\end{array}$ & $\begin{array}{l}\text { Results: } \\
\text { KQ3: Adverse Effects or } \\
\text { Unintended Consequences }\end{array}$ \\
\hline Taylor, 2007 & NR & $\begin{array}{l}\text { Screen completion } \\
\text { A: } 31 \% \\
\text { B: } 100 \% \\
\end{array}$ & NR \\
\hline Thaker, 2013 & $\begin{array}{l}\text { Net savings, in AUD: } \$ 320,118 \\
\text { Total cost of teleconsults, in AUD: } \$ 442,276 \\
\text { Estimated travel expense avoided, in AUD: } \$ 762,394 \\
\text { Travel costs for patients and escorts, in AUD: } \\
\$ 658,760 \\
\text { Aeromedical retrievals, in AUD: } \$ 52,400 \\
\text { Travel for specialists, in AUD: } \$ 47,634 \\
\text { Accommodation costs for a proportion of patients, in } \\
\text { AUD: } \$ 3600\end{array}$ & NR & NR \\
\hline Tsitlakidis, 2005 & $\begin{array}{l}\text { Total cost per patient, in euros } \\
\text { A: } 270.061 € \\
\text { B: } 203.578 € \\
\text { Savings dependent on distance travelled and number } \\
\text { of cases }\end{array}$ & $\begin{array}{l}\text { Average consultation time, in minutes } \\
\text { A: } 30.0 \\
\text { B: } 5.3 \\
\text { Post-consultation time requirements, in } \\
\text { minutes } \\
\text { A: } 10.0 \\
\text { B: } 2.6 \\
\end{array}$ & NR \\
\hline Tuulonen, 1999 & $\begin{array}{l}\text { Cost of visits per case, in USD } \\
\text { No difference } \\
\text { A: } \$ 111 \\
\text { B: } \$ 110 \\
\text { Decreased travel saved } \$ 55 \text { per visit for telehealth } \\
\text { patients, not included in overall cost. }\end{array}$ & $\begin{array}{l}\text { Total mean (SD) time spent for visit including } \\
\text { travel, in hours } \\
\text { A: } 8.5(2.4) \\
\text { B: } 2.0(1.0), p=0.0001 \\
\text { Mean time absent from work, in hours } \\
\text { A: } 6.6 \\
\text { B: } 3.3 \\
\% \text { Very satisfied with overall care } \\
\text { A: } 69 \% \\
\text { B: } 86 \% \\
\% \text { Selected telehealth for next visit } \\
\text { A: } 81 \% \\
\text { B: } 96 \% \\
\text { Reduction in travel as reason for wanting } \\
\text { telehealth for next visit } \\
\text { A: } 97 \% \\
\text { B: } 86 \%\end{array}$ & NR \\
\hline
\end{tabular}




\begin{tabular}{|c|c|c|c|}
\hline $\begin{array}{l}\text { Author, Year } \\
\text { (See Appendix C } \\
\text { for full citation) }\end{array}$ & $\begin{array}{l}\text { Results: } \\
\text { KQ1: Clinical and Economic Outcomes }\end{array}$ & $\begin{array}{l}\text { Results: } \\
\text { KQ2: Intermediate Outcomes }\end{array}$ & \begin{tabular}{|l|} 
Results: \\
KQ3: Adverse Effects or \\
Unintended Consequences \\
\end{tabular} \\
\hline van der Pol, 2010 & $\begin{array}{l}\text { Total cost per patient, in pounds } \\
\text { A: } £ 380.52 \\
\text { B: } £ 353.43 \\
\text { Average cost per clinic, in pounds } \\
\text { Staff: } \\
\text { A: } £ 350.52 \\
\text { B: } £ 360.54 \\
\text { Equipment: } \\
\text { A: } £ 247.34 \\
\text { B: } £ 1390.42 \\
\text { Disposables: } \\
\text { A: } £ 32.40 \\
\text { B: } £ 16.20 \\
\text { Average cost per patient, in pounds } \\
\text { Staff: } \\
\text { A: } £ 17.73 \\
\text { B: } £ 72.11 \\
\text { Equipment: } \\
\text { A: } £ 12.37 \\
\text { B: } £ 278.08 \\
\text { Disposables: } \\
\text { A: } £ 1.62 \\
\text { B: } £ 3.24 \\
\text { Travel } \\
\text { A: } £ 349 \\
\text { B: } 0\end{array}$ & NR & NR \\
\hline Van Gelder, 2017 & NR & $\begin{array}{l}\text { Referral rate } \\
\text { A: } 3.0 \% \\
\text { B: } 2.3 \% \\
\text { OR } 0.61 \text { ( } 95 \% \mathrm{Cl} 0.31 \text { to } 1.23) \\
\text { Consultation rate } \\
\text { A: } 5.0 \% \\
\text { B: } 6.3 \% \\
\text { OR } 2.00(95 \% \mathrm{Cl} 0.75 \text { to } 5.33)\end{array}$ & NR \\
\hline
\end{tabular}




\begin{tabular}{|c|c|c|c|}
\hline $\begin{array}{l}\text { Author, Year } \\
\text { (See Appendix C } \\
\text { for full citation) }\end{array}$ & $\begin{array}{l}\text { Results: } \\
\text { KQ1: Clinical and Economic Outcomes }\end{array}$ & $\begin{array}{l}\text { Results: } \\
\text { KQ2: Intermediate Outcomes }\end{array}$ & $\begin{array}{l}\text { Results: } \\
\text { KQ3: Adverse Effects or } \\
\text { Unintended Consequences }\end{array}$ \\
\hline $\begin{array}{l}\text { Vuolio } \\
\text { Same patients as } \\
\text { Ohinmaa and } \\
\text { Hakipuro, } 2003\end{array}$ & NR & $\begin{array}{l}\text { Management plan } \\
\text { Operation } \\
\text { A: } 54 \% \\
\text { B: } 64 \% \\
\text { Follow-up } \\
\text { A: } 18 \% \\
\text { B: } 18 \% \\
\text { Problem solved at first visit } \\
\text { A: } 28 \% \\
\text { B: } 18 \% \\
\end{array}$ & NR \\
\hline Wallace, 2002 & 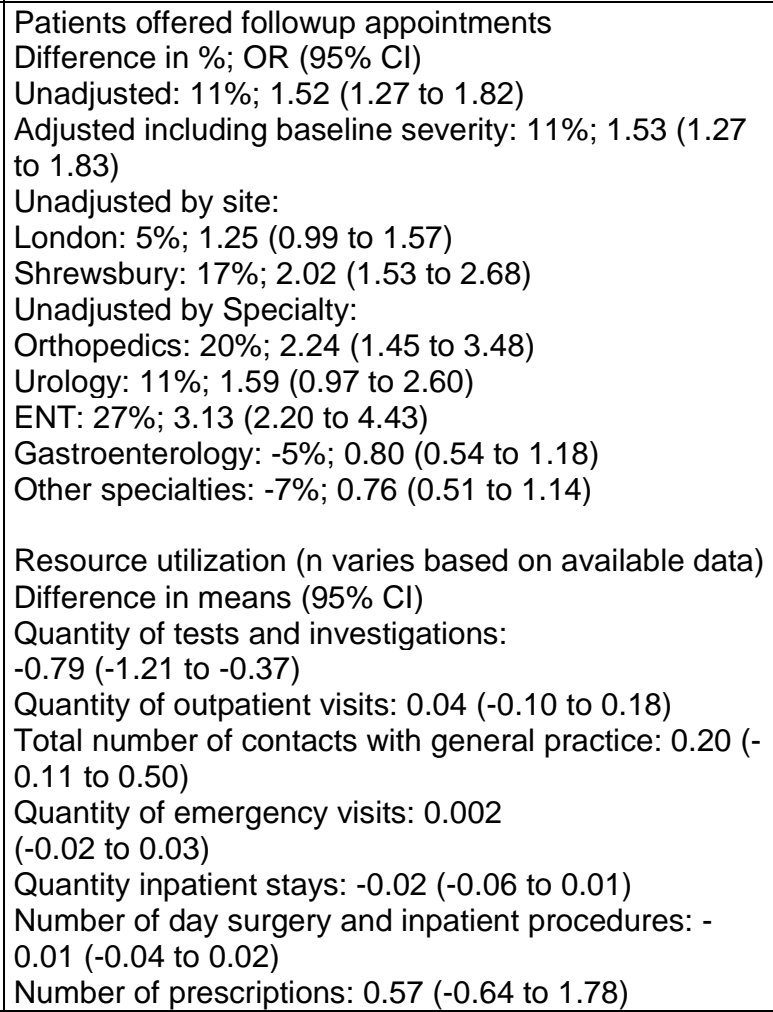 & $\begin{array}{l}\text { Mean patient satisfaction (5 point scale; } \\
\text { 1=poor) } \\
\text { A: } 3.64(1.06) \\
\text { B: } 3.97(0.99) \\
\text { Difference: } 0.33 \text { (0.23 to } 0.43) \\
\text { Mean patient enablement (higher scores } \\
\text { indicate improved enablement) (SD) } \\
\text { A: } 2.4(3.1) \\
\text { B: } 2.5(3.2) \\
\text { Difference: } 0.07(-0.24 \text { to } 0.43)\end{array}$ & NR \\
\hline
\end{tabular}




\begin{tabular}{|c|c|c|c|}
\hline $\begin{array}{l}\text { Author, Year } \\
\text { (See Appendix C } \\
\text { for full citation) }\end{array}$ & $\begin{array}{l}\text { Results: } \\
\text { KQ1: Clinical and Economic Outcomes }\end{array}$ & $\begin{array}{l}\text { Results: } \\
\text { KQ2: Intermediate Outcomes }\end{array}$ & $\begin{array}{l}\text { Results: } \\
\text { KQ3: Adverse Effects or } \\
\text { Unintended Consequences }\end{array}$ \\
\hline Wallace, 2004 & $\begin{array}{l}\text { SF-12 } \\
\text { Physical score } \\
\text { A: } 42.7(12.2) \\
\text { B: } 43.1(12.0) ; p=0.61 \\
\text { Mental score } \\
\text { A: } 48.1 \text { (11.9) } \\
\text { B: } 47.5(11.8) ; p=0.43 \\
\text { Costs, GBP } \\
\text { NHS (health system) } \\
\text { Difference (B-A) } \\
90.80 \text { (2.07-179.54) } \\
\text { Patient } \\
-7.70(-10.35 \text { to }-5.05) \\
\end{array}$ & $\begin{array}{l}\text { Offer of followup appointment } \\
\text { A: } 41 \% \\
\text { B: } 52 \% \\
\text { AOR }(95 \% \mathrm{Cl}) \\
1.53(1.27 \text { to } 1.83), p<0.001 \\
\text { Mean patient satisfaction (1=poor; } 5=\text { excellent) } \\
\text { (SD) } \\
\text { A: } 3.64(1.06) \\
\text { B: } 3.97(0.99)\end{array}$ & NR \\
\hline Whited, 2002 & NR & $\begin{array}{l}\text { Time to initial definitive intervention, in days } \\
\text { ITT analysis mean (SD), median: } \\
\text { A: } 114.3(72.3), 127.0 \\
\text { B: } 73.8,(71.6), 41.0 ; p=0.0001 \\
\\
\text { Actual clinic visit analysis mean (SD), median: } \\
\text { A: } 135.6(94.3), 137.5 \\
\text { B: } 93.4(96.1), 50.0 ; p=0.0027\end{array}$ & NR \\
\hline Whited, 2003 & $\begin{array}{l}\text { Extrapolated cost data from } 275 \text { patients to the total } \\
\text { population of patients serviced by dermatology clinic in } \\
2001, n=5440 \\
\text { Total annual cost, in USD } \\
\text { A: } \$ 116,416 \\
\text { B: } \$ 198,016 \\
\text { Per patient } \\
\text { A: } \$ 21.40 \\
\text { B: } \$ 36.40 \\
\text { Incremental cost per patient of teledermatology: } \\
+\$ 15.00\end{array}$ & $\begin{array}{l}\text { Median time to initial definitive intervention, in } \\
\text { days } \\
\text { A: } 137.5 \\
\text { B: } 50 \\
p=0.0027\end{array}$ & NR \\
\hline
\end{tabular}




\begin{tabular}{|c|c|c|c|}
\hline $\begin{array}{l}\text { Author, Year } \\
\text { (See Appendix C } \\
\text { for full citation) }\end{array}$ & $\begin{array}{l}\text { Results: } \\
\text { KQ1: Clinical and Economic Outcomes }\end{array}$ & $\begin{array}{l}\text { Results: } \\
\text { KQ2: Intermediate Outcomes }\end{array}$ & $\begin{array}{l}\text { Results: } \\
\text { KQ3: Adverse Effects or } \\
\text { Unintended Consequences }\end{array}$ \\
\hline Whited, 2004 & NR & $\begin{array}{l}\text { Clinicians survey } \\
\text { Satisfaction with consult process: } \\
\text { Agree: } \\
\text { A: } 23 \% \\
\text { B: } 92 \% \\
\text { Neutral: } \\
\text { A: } 42 \% \\
\text { B: } 5 \% \\
\text { Disagree: } \\
\text { A: } 35 \% \\
\text { B: } 3 \% \\
\text { Patients survey } \\
\text { - Patients largely satisfied } \\
\text { - Largest source of dissatisfaction, } \\
\text { Usual care: Wait time for appointment } \\
\text { - Teledermatology: some dissatisfied with time } \\
\text { it took to learn their results } \\
\text { - Teledermatology patients were generally } \\
\text { satisfied with the outcome of consultations and } \\
\text { were confident that dermatologists could help } \\
\text { them by viewing digital images }\end{array}$ & NR \\
\hline
\end{tabular}




\begin{tabular}{|c|c|c|c|}
\hline $\begin{array}{l}\text { Author, Year } \\
\text { (See Appendix C } \\
\text { for full citation) }\end{array}$ & $\begin{array}{l}\text { Results: } \\
\text { KQ1: Clinical and Economic Outcomes }\end{array}$ & $\begin{array}{l}\text { Results: } \\
\text { KQ2: Intermediate Outcomes }\end{array}$ & $\begin{array}{l}\text { Results: } \\
\text { KQ3: Adverse Effects or } \\
\text { Unintended Consequences }\end{array}$ \\
\hline $\begin{array}{l}\text { Whited, 2013a, } \\
\text { 2013b }\end{array}$ & 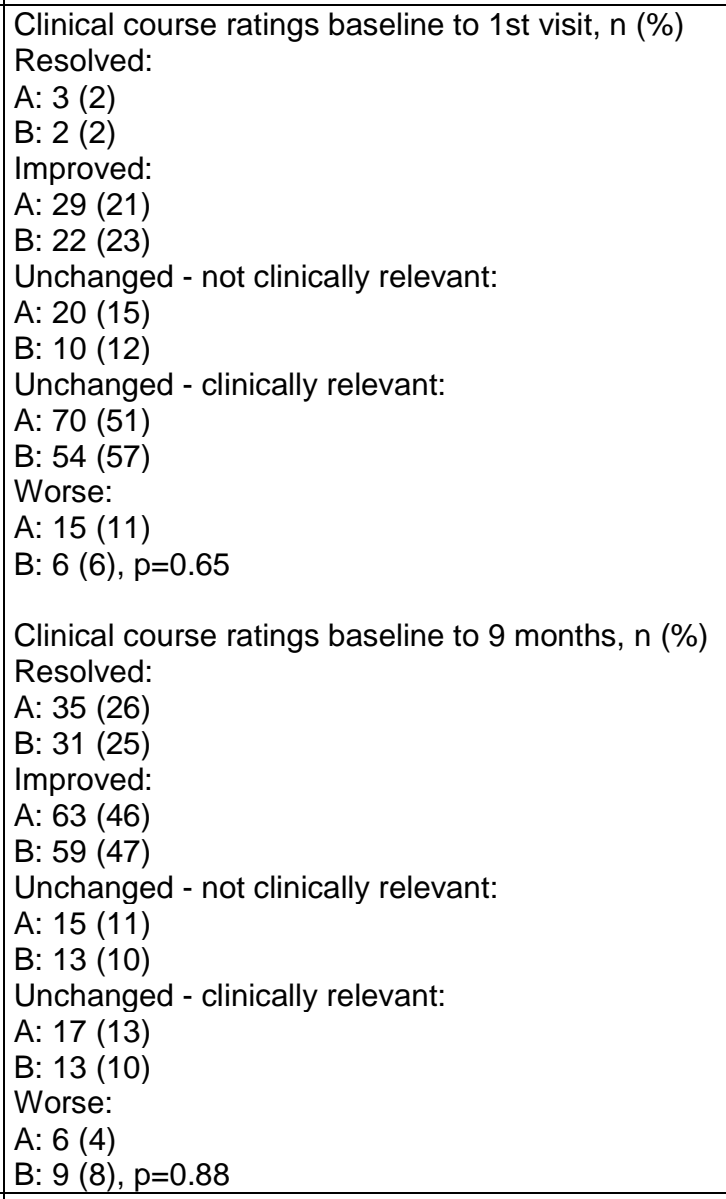 & $\begin{array}{l}\text { Quality of life change in skindex-16 baseline to } \\
3 \text { months mean } \\
\text { Symptoms } \\
\text { A: }-8.0(22.9) \\
\text { B: }-8.7(29.8), p=0.81 \\
\text { Emotions } \\
\text { A: - } 8.9(25.3) \\
\text { B: }-11.6(27.2) \\
\text { Functioning } \\
\text { A: }-0.05(20.9) \\
\text { B: }-3.2(20.2), p=0.22 \\
\text { Composite } \\
\text { A: }-5.8(19.1) \\
\text { B: }-7.8(21.9), p=0.39 \\
\text { Baseline to } 9 \text { months } \\
\text { Symptoms } \\
\text { A: }-14.4(28.2) \\
\text { B: }-10.3(30.6), p=0.22 \\
\text { Emotions } \\
\text { A: }-18.1(25.1) \\
\text { B: }-19.7(30.7), p=0.61 \\
\text { Functioning } \\
\text { A: -6.9 (22.3) } \\
\text { B: -6.0, } p=0.73 \\
\text { Composite } \\
\text { A: }-13.2(21.6) \\
\text { B: }-12.0(24.5), p=0.66\end{array}$ & NR \\
\hline Wilson, 2005 & NR & $\begin{array}{l}\text { Retinal exams rate }(95 \% \mathrm{Cl}) \text { for diabetic } \\
\text { patients } \\
\text { A: } 50 \%(44 \% \text { to } 56 \%) \\
\text { B: } 75 \%(70 \% \text { to } 80 \%), p<0.0001 \\
\text { Rate of laser therapy for diabetic retinopathy } \\
\text { A: } 19.6 \text { per } 1,000 \text { patients with diabetes } \\
\text { B: } 29.5 \text { per } 1,000 \text { patients with diabetes } \\
51 \% \text { increase in laser treatment rate } \\
\end{array}$ & NR \\
\hline
\end{tabular}

\section{F-145}




\begin{tabular}{|c|c|c|c|}
\hline $\begin{array}{l}\text { Author, Year } \\
\text { (See Appendix C } \\
\text { for full citation) }\end{array}$ & $\begin{array}{l}\text { Results: } \\
\text { KQ1: Clinical and Economic Outcomes }\end{array}$ & $\begin{array}{l}\text { Results: } \\
\text { KQ2: Intermediate Outcomes }\end{array}$ & $\begin{array}{l}\text { Results: } \\
\text { KQ3: Adverse Effects or } \\
\text { Unintended Consequences }\end{array}$ \\
\hline $\mathrm{Xu}, 2008$ & $\begin{array}{l}\text { Mean number of consultations per person: } \\
\text { A: } 1.5 \\
\text { B: } 1.3 \\
\text { Total cost per consultation, in dollars } \\
\text { A: } \$ 155 \\
\text { B: } \$ 161 \\
\text { Variable cost per consultation, in dollars } \\
\text { A: } \$ 155 \\
\text { B: } \$ 108 \\
\text { Total annual variable cost, in dollars } \\
\text { A: } \$ 27,364 \\
\text { B: } \$ 14,160 \\
\text { Difference between conducting } 265 \text { consultations } \\
\text { A vs. B cost-savings: } \$ 7,621\end{array}$ & NR & NR \\
\hline Young, 2014 & NR & $\begin{array}{l}\text { Complete virologic suppression during the first } \\
6 \text { visits was significantly greater in the } \\
\text { telehealth group } \\
\text { A: } 59.3 \% \\
\text { B: } 91.1 \% \\
\text { OR } 7.0 \text { (95\% CI } 5.1 \text { to } 9.8) ; p<0.001\end{array}$ & NR \\
\hline
\end{tabular}




\begin{tabular}{|c|c|c|c|}
\hline $\begin{array}{l}\text { Author, Year } \\
\text { (See Appendix C } \\
\text { for full citation) }\end{array}$ & $\begin{array}{l}\text { Results: } \\
\text { KQ1: Clinical and Economic Outcomes }\end{array}$ & $\begin{array}{l}\text { Results: } \\
\text { KQ2: Intermediate Outcomes }\end{array}$ & $\begin{array}{l}\text { Results: } \\
\text { KQ3: Adverse Effects or } \\
\text { Unintended Consequences }\end{array}$ \\
\hline Zahlmann, 2002 & NR & 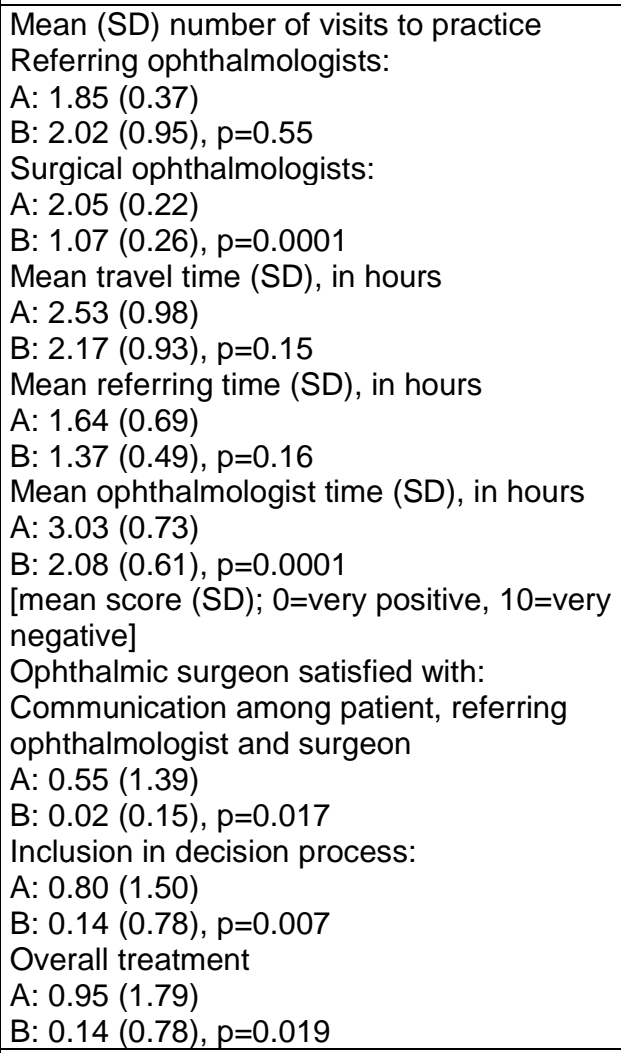 & NR \\
\hline Zarchi, 2015 & $\begin{array}{l}\text { Complete wound healing at 1-year followup } \\
\text { A: } 45 \% \\
\text { B: } 70 \% \\
\text { Complete wound healing, adjusted hazard ratio }(95 \% \\
\text { CI), p-value } \\
\text { A: Reference } \\
\text { B: } 2.19 \text { (1.15 to } 4.17), p=0.017\end{array}$ & NR & NR \\
\hline
\end{tabular}




\begin{tabular}{|c|c|c|c|}
\hline $\begin{array}{l}\text { Author, Year } \\
\text { (See Appendix C } \\
\text { for full citation) }\end{array}$ & $\begin{array}{l}\text { Results: } \\
\text { KQ1: Clinical and Economic Outcomes }\end{array}$ & $\begin{array}{l}\text { Results: } \\
\text { KQ2: Intermediate Outcomes }\end{array}$ & $\begin{array}{l}\text { Results: } \\
\text { KQ3: Adverse Effects or } \\
\text { Unintended Consequences }\end{array}$ \\
\hline Zilliacus, 2011 & NR & $\begin{array}{l}\text { Generalized satisfaction with genetic } \\
\text { counseling; } n, \text { mean (SD) } \\
\text { A: } 84,40.8(9.9) \\
B: 87,45.6(8.4) ; p=0.76 \\
\text { No significant differences were found between } \\
\text { telegenetics and face-to-face genetic } \\
\text { counseling in terms of knowledge gained } \\
p=0.55 \\
\text { Cancer-specific anxiety } \\
p=0.13 \\
\text { Generalized anxiety } \\
p=0.42 \\
\text { Depression } \\
p=0.96 \\
\text { Perceived empathy of the genetic clinician } \\
p=0.13 \\
\text { Perceived empathy of the genetic counselor } \\
p=0.12 \\
\text { Telegenetics performed significantly better } \\
\text { than face-to-face counseling in meeting } \\
\text { patients' expectations } \\
p=0.009 \\
\text { Promoting perceived personal control } \\
p=0.031\end{array}$ & NR \\
\hline
\end{tabular}

AOR = adjusted odds ratio; APRI = aspartate aminotransferase:platelet ratio index; AUD = Australian dollar; BMI = body mass index; BP = blood pressure; CI = confidence interval; CPT = cognitive processing therapy; DBP = diastolic blood pressure; DM = diabetes mellitus; ECHO = Extension for Community Healthcare Outcomes; ED = emergency department; EuroQOL EQ- 5D = standardized instrument that measures health-related quality of life; GBP = Great British Pound; GDP = gross domestic product; GP = general practitioner; GI = gastrointestinal ; HbA1c = hemoglobin A1c; HCV = hepatitis C virus; HR = hazard ratio; IDR = incident density ratio; ITT = intention-to-treat; LDL = lowdensity lipoproteins; LOS = length of stay; MACE = major cardiovascular events; MCS = mental component score; MDT = multidisciplinary team; NHS = National Health Service; NR = not reported; NS = not significant; NZ\$ = New Zealand dollars; OR = odds ratio; PCS = physical component score; PTSD = post-traumatic stress disorder; SBP = systolic blood pressure; SD = standard deviation; SE = standard error; SEK = Swedish krona; SF-12 = Short Form Health Survey 12; TH = telehealth; TVCR; UAMS = University of Arkansas for Medical Sciences; UNM = University of New Mexico; USD = United States dollars; VA = Veteran's Affairs; VAS = visual analog scale

${ }^{a}$ For definitions of interventions and comparisons (e.g., A vs. B), see Table F-7

See Appendix C. Included Studies for full citations 


\section{Appendix G. Risk of Bias}

Table G-1. Risk of bias: cohort studies, part 1

\begin{tabular}{|c|c|c|c|c|c|c|}
\hline $\begin{array}{l}\text { Author, Year } \\
\text { (See Appendix C for } \\
\text { full citation) }\end{array}$ & Study Design & $\begin{array}{l}\text { Eligibility } \\
\text { prespecified? }\end{array}$ & $\begin{array}{l}\text { Random sample or } \\
\text { consecutivelall } \\
\text { patients meeting } \\
\text { inclusion criteria? }\end{array}$ & $\begin{array}{l}\text { Groups similar or } \\
\text { design and } \\
\text { analyses account } \\
\text { for important } \\
\text { potential } \\
\text { confounding and } \\
\text { modifying variables } \\
\text { appropriately? }\end{array}$ & $\begin{array}{l}\text { Valid and reliable } \\
\text { measures used and } \\
\text { adequately } \\
\text { ascertained? }\end{array}$ & $\begin{array}{l}\text { Outcome assessors } \\
\text { and/or data } \\
\text { analysts blinded or } \\
\text { are primary } \\
\text { outcomes unlikely } \\
\text { to be biased? }\end{array}$ \\
\hline Alemi, 2016 & Prospective Cohort & No & Yes & Unclear & No & No \\
\hline Amorim, 2013 & Before-After & Yes & Yes & No & Yes & NR \\
\hline Angstman, 2009 & Retrospective Cohort & Yes & Yes & Unclear & Yes & Yes \\
\hline Armaignac, 2018 & Retrospective Cohort & $\begin{array}{l}\text { Clinical outcomes: } \\
\text { Yes } \\
\text { Cost: Yes } \\
\end{array}$ & $\begin{array}{l}\text { Clinical outcomes: } \\
\text { No } \\
\text { Cost: Yes } \\
\end{array}$ & $\begin{array}{l}\text { Clinical outcomes: } \\
\text { Yes } \\
\text { Cost: Unclear } \\
\end{array}$ & $\begin{array}{l}\text { Clinical outcomes: } \\
\text { Yes } \\
\text { Cost: Unclear } \\
\end{array}$ & $\begin{array}{l}\text { Clinical outcomes: } \\
\text { Yes } \\
\text { Cost: Unclear } \\
\end{array}$ \\
\hline Arora, 2011 & Prospective Cohort & Yes & Yes & Yes & Yes & Yes \\
\hline Astarcioglu, 2015 & Prospective Cohort & No & Unclear & Yes & Yes & Unclear \\
\hline Audebert, 2006 & Prospective Cohort & Yes & Yes & Yes & Yes & No \\
\hline Audebert, 2009 & Prospective Cohort & Yes & Yes & Yes & Yes & Yes \\
\hline Bagayoko, 2014 & $\begin{array}{l}\text { Prospective Cohort } \\
\text { for patient costs } \\
\text { Before-After for } \\
\text { health center costs } \\
\end{array}$ & Yes & Yes & NR & Unclear & NR \\
\hline Baig, 2016 & Retrospective Cohort & No & NR & NR & Yes & Yes \\
\hline Bergrath, 2012 & Prospective Cohort & Yes & Yes & Unclear & Yes & Yes \\
\hline Bernstein, 2010 & Retrospective Cohort & Yes & Yes & Unclear & Yes & Yes \\
\hline Beswick, 2016 & Prospective Cohort & Yes & NR & No & No & Yes \\
\hline Bezalel, 2015 & Before-After & No & Unclear & NR & Unclear & Unclear \\
\hline Blackwell, 1997 & Before-After & Yes & Yes & NR & No & No \\
\hline Bladin, 2015 & Before-After & Yes & Yes & Yes & Yes & Yes \\
\hline Breslow, 2004 & Before-After & Yes & Yes & Unclear & Yes & Yes \\
\hline Britt, 2006 & Before-After & No & Yes & Unclear & Unclear & Unclear \\
\hline Brokmann, 2016 & Before-After & Yes & Yes & Unclear & Yes & No \\
\hline Brokmann, 2016b & Before-After & Yes & Yes & Yes & No & No \\
\hline $\begin{array}{l}\text { Brown-Connolly, } \\
2002\end{array}$ & Prospective Cohort & No & Unclear & Unclear & Unclear & No \\
\hline Brunetti, 2014 & Prospective Cohort & Yes & Yes & No & Yes & Yes \\
\hline Buckley, 2012 & Before-After & Yes & Yes & Unclear & Yes & Yes \\
\hline Burgess, 2002 & Prospective Cohort & Yes & Unclear & Unclear & No & No \\
\hline Carallo, 2015 & Prospective Cohort & No & No & Yes & Unclear & Yes \\
\hline
\end{tabular}




\begin{tabular}{|c|c|c|c|c|c|c|}
\hline $\begin{array}{l}\text { Author, Year } \\
\text { (See Appendix C for } \\
\text { full citation) }\end{array}$ & Study Design & $\begin{array}{l}\text { Eligibility } \\
\text { prespecified? }\end{array}$ & $\begin{array}{l}\text { Random sample or } \\
\text { consecutivelall } \\
\text { patients meeting } \\
\text { inclusion criteria? }\end{array}$ & $\begin{array}{l}\text { Groups similar or } \\
\text { design and } \\
\text { analyses account } \\
\text { for important } \\
\text { potential } \\
\text { confounding and } \\
\text { modifying variables } \\
\text { appropriately? }\end{array}$ & $\begin{array}{l}\text { Valid and reliable } \\
\text { measures used and } \\
\text { adequately } \\
\text { ascertained? }\end{array}$ & $\begin{array}{l}\text { Outcome assessors } \\
\text { and/or data } \\
\text { analysts blinded or } \\
\text { are primary } \\
\text { outcomes unlikely } \\
\text { to be biased? }\end{array}$ \\
\hline Carter, 2017 & Retrospective Cohort & Yes & No & No & Yes & No \\
\hline Chan, 2001 & Before-After & Yes & Yes & Unclear & Unclear & No \\
\hline Chan, 2012 & Prospective Cohort & Yes & Yes & Yes & Yes & Yes \\
\hline Chan, 2015 & Retrospective Cohort & Yes & Yes & No & Yes & Yes \\
\hline Choi, 2006 & Before-After & No & Unclear & No & Unclear & No \\
\hline Chowdhury, 2012 & Retrospective Cohort & Yes & Yes & No & Unclear & Yes \\
\hline Chu, 2015 & Retrospective Cohort & No & NR & Unclear & No & No \\
\hline Collins, 2017 & Retrospective Cohort & Yes & Yes & No & No & Yes \\
\hline Craig, 2004 & Prospective Cohort & Yes & Yes & No & Yes & No \\
\hline Crossland, 2016 & Prospective Cohort & Yes & Yes & Yes & No & NR \\
\hline Darkins, 1996 & Before-After & No & Yes & Unclear & Unclear & No \\
\hline Davison, 2004 & Before-After & No & Yes & NR & No & Yes \\
\hline Dayal, 2016 & Retrospective Cohort & Yes & Yes & Yes & Yes & Yes \\
\hline De Luca, 2005 & Prospective Cohort & Yes & Yes & Yes & Yes & No \\
\hline Dharmaaroja, 2010 & Before-After & Yes & Yes & No & Unclear & Unclear \\
\hline Dharmar, 2013 & Retrospective Cohort & Yes & Yes & No & Unclear & Yes \\
\hline Dharmar, 2013 & Retrospective Cohort & Yes & Yes & Yes & Yes & Yes \\
\hline Dharmar, 2013 & Retrospective Cohort & Yes & Yes & Yes & Yes & No \\
\hline Duchesne, 2008 & Before-After & No & Yes & No & Unclear & Unclear \\
\hline Engel, 2011 & Prospective Cohort & No & Yes & NR & Yes & Yes \\
\hline Ferrandiz, 2007 & $\begin{array}{l}\text { Prospective Cohort } \\
\text { and Pre-Post }\end{array}$ & Yes & Unclear & Unclear & Yes & Yes \\
\hline Fong, 2015 & Prospective Cohort & Yes & Unclear & No & Unclear & NR \\
\hline Fortis, 2014 & Retrospective Cohort & No & Unclear & No & Yes & Yes \\
\hline Fox, 2007 & Before-After & Yes & Unclear & Unclear & Yes & Yes \\
\hline Frank, 2015 & Before-After & Yes & Yes & Unclear & Yes & No \\
\hline Gilmour, 1998 & Prospective Cohort & No & Unclear & Unclear & Yes & No \\
\hline Goh, 1997 & Prospective Cohort & No & Yes & Unclear & Yes & Yes \\
\hline Gupta, 2014 & Before-After & Yes & Unclear & Yes & Yes & Yes \\
\hline Handschu, 2008 & Prospective Cohort & Yes & Yes & Unclear & Unclear & No \\
\hline Hashimoto, 2001 & Before-After & Unclear & Yes & No & Unclear & Unclear \\
\hline Heffner, 2015 & Retrospective Cohort & Yes & Yes & Yes & Unclear & No \\
\hline Herce, 2011 & Prospective Cohort & No & Yes & Unclear & No & Yes \\
\hline Hsiao, 2008 & Retrospective Cohort & Yes & Yes & Unclear & Yes & Yes \\
\hline Huang, 2008 & Before-After & Yes & Yes & Unclear & Yes & Yes \\
\hline
\end{tabular}




\begin{tabular}{|c|c|c|c|c|c|c|}
\hline $\begin{array}{l}\text { Author, Year } \\
\text { (See Appendix C for } \\
\text { full citation) }\end{array}$ & Study Design & $\begin{array}{l}\text { Eligibility } \\
\text { prespecified? }\end{array}$ & $\begin{array}{l}\text { Random sample or } \\
\text { consecutivelall } \\
\text { patients meeting } \\
\text { inclusion criteria? }\end{array}$ & $\begin{array}{l}\text { Groups similar or } \\
\text { design and } \\
\text { analyses account } \\
\text { for important } \\
\text { potential } \\
\text { confounding and } \\
\text { modifying variables } \\
\text { appropriately? }\end{array}$ & $\begin{array}{l}\text { Valid and reliable } \\
\text { measures used and } \\
\text { adequately } \\
\text { ascertained? }\end{array}$ & $\begin{array}{l}\text { Outcome assessors } \\
\text { and/or data } \\
\text { analysts blinded or } \\
\text { are primary } \\
\text { outcomes unlikely } \\
\text { to be biased? }\end{array}$ \\
\hline Hubert, 2016 & Retrospective Cohort & Yes & Yes & No & Yes & Yes \\
\hline Ickenstein, 2005 & Before-After & Yes & Yes & NR & \begin{tabular}{|l|} 
Yes \\
\end{tabular} & No \\
\hline Ionita, 2009 & Retrospective Cohort & Yes & Yes & Yes & Unclear & Yes \\
\hline Itrat, 2016 & Prospective Cohort & Yes & No & Yes & Yes & Yes \\
\hline Jacobs, 2015 & Before-After & Yes & Yes & NR & Unclear & No \\
\hline Johansson, 2011 & Retrospective Cohort & Yes & Yes & Unclear & Yes & Yes \\
\hline Jong, 2004 & Prospective Cohort & No & Unclear & Unclear & No & No \\
\hline Jue, 2017 & Retrospective Cohort & Yes & Yes & NR & Yes & Yes \\
\hline Kahn, 2016 & Retrospective Cohort & Yes & \begin{tabular}{|l|} 
Yes \\
\end{tabular} & Unclear & Yes & Yes \\
\hline Kalb, 2014 & Before-After & No & Unclear & Unclear & Yes & Yes \\
\hline Kim, 2013 & Prospective Cohort & Yes & Unclear & Unclear & Yes & Yes \\
\hline Kim, 2011 & Retrospective Cohort & Yes & Unclear & No & No & No \\
\hline Klein, 2010 & Retrospective Cohort & Yes & Yes & No & Yes & Yes \\
\hline Kobza, 2000 & Before-After & Yes & Unclear & NR & Yes & No \\
\hline Kohl, 2012 & Retrospective Cohort & Yes & Yes & No & Yes & Yes \\
\hline Krupinski, 2004 & Retrospective Cohort & Yes & No & NR & Yes & Yes \\
\hline LaBarbera, 2013 & Before-After & Yes & Yes & Yes & Yes & No \\
\hline Langabeer, 2016 & $\begin{array}{l}\text { Retrospective Cohort } \\
\text { (Case Control) }\end{array}$ & Yes & Yes & Yes & Yes & No \\
\hline Lee, 2014 & Prospective Cohort & Yes & Yes & Unclear & Yes & Yes \\
\hline Lilly, 2011 & Prospective Cohort & Yes & Yes & Yes & Yes & Yes \\
\hline Lim, 2012 & Prospective Cohort & Yes & No & No & Unclear & No \\
\hline Long, 2014 & Retrospective Cohort & Yes & Yes & Unclear & Yes & No \\
\hline Macedo, 2016 & Before-After & Yes & Unclear & Yes & Yes & Yes \\
\hline Machado, 2018 & Retrospective Cohort & $\begin{array}{l}\text { Clinical outcomes: } \\
\text { Unclear } \\
\text { Cost: No } \\
\end{array}$ & $\begin{array}{l}\text { Clinical outcomes: } \\
\text { Yes } \\
\text { Cost: Yes } \\
\end{array}$ & $\begin{array}{l}\text { Clinical outcomes: } \\
\text { Yes } \\
\text { Cost: Unclear } \\
\end{array}$ & $\begin{array}{l}\text { Clinical outcomes: } \\
\text { Yes } \\
\text { Costs: Unclear }\end{array}$ & $\begin{array}{l}\text { Clinical outcomes: } \\
\text { Yes } \\
\text { Costs: Unclear }\end{array}$ \\
\hline Marcin, 2004a & Retrospective Cohort & Yes & Yes & No & Yes & Unclear \\
\hline Marcin, 2004c & Retrospective Cohort & Yes & Yes & No & Yes & Yes \\
\hline Marcolino, 2013 & Before-After & Unclear & Yes & No & Yes & Yes \\
\hline $\begin{array}{l}\text { Martinez-Sanchez, } \\
2014\end{array}$ & Before-After & Yes & Unclear & Yes & Yes & Yes \\
\hline Martinoni, 2011 & Retrospective Cohort & Yes & Yes & Yes & Yes & Yes \\
\hline Mathews, 2008 & Before-After & Yes & No & Unclear & Unclear & No \\
\hline McCambridge, 2010 & Before-After & Yes & Yes & Yes & Unclear & NR \\
\hline
\end{tabular}




\begin{tabular}{|c|c|c|c|c|c|c|}
\hline $\begin{array}{l}\text { Author, Year } \\
\text { (See Appendix C for } \\
\text { full citation) }\end{array}$ & Study Design & $\begin{array}{l}\text { Eligibility } \\
\text { prespecified? }\end{array}$ & $\begin{array}{l}\text { Random sample or } \\
\text { consecutivelall } \\
\text { patients meeting } \\
\text { inclusion criteria? }\end{array}$ & $\begin{array}{l}\text { Groups similar or } \\
\text { design and } \\
\text { analyses account } \\
\text { for important } \\
\text { potential } \\
\text { confounding and } \\
\text { modifying variables } \\
\text { appropriately? }\end{array}$ & $\begin{array}{l}\text { Valid and reliable } \\
\text { measures used and } \\
\text { adequately } \\
\text { ascertained? }\end{array}$ & $\begin{array}{l}\text { Outcome assessors } \\
\text { and/or data } \\
\text { analysts blinded or } \\
\text { are primary } \\
\text { outcomes unlikely } \\
\text { to be biased? }\end{array}$ \\
\hline McCrossan, 2012 & Prospective Cohort & Yes & Yes & Yes & No & No \\
\hline Mielonen, 2000 & Prospective Cohort & Yes & Unclear & Unclear & Unclear & No \\
\hline Migliaretti, 2013 & Prospective Cohort & Yes & Yes & NR & Unclear & No \\
\hline Miyamoto, 2014 & Retrospective Cohort & Yes & Yes & No & Unclear & Unclear \\
\hline $\begin{array}{l}\text { Mohr, } 2017 \\
\text { Mohr, } 2018 \\
\end{array}$ & Retrospective Cohort & Yes & Yes & Yes & Yes & Yes \\
\hline Morrison, 2010 & Before-After & Yes & Yes & Yes & Unclear & NR \\
\hline Mulgrew, 2011 & Prospective Cohort & Yes & Unclear & No & Yes & No \\
\hline Nagao, 2012 & Before-After & Yes & Unclear & No & No & Yes \\
\hline Nagayoshi, 2016 & Before-After & No & Unclear & NR & Unclear & Yes \\
\hline Narasimhan, 2015 & $\begin{array}{l}\text { Prospective Cohort } \\
\text { with historical } \\
\text { controls }\end{array}$ & Yes & NR & Yes & Yes & Yes \\
\hline Nassar, 2014 & Before-After & Yes & Yes & Yes & Yes & Yes \\
\hline Nguyen-Huynh, 2018 & Before-After & No & Unclear & Yes & Yes & Yes \\
\hline Nickenig, 2008 & Prospective Cohort & Yes & Yes & Unclear & Yes & No \\
\hline Nordal, 2001 & Retrospective Cohort & Yes & Yes & Yes & Unclear & Unclear \\
\hline Ortolani, 2007 & Retrospective Cohort & Yes & Yes & No & Yes & Unclear \\
\hline Ortolani, 2006 & Retrospective Cohort & Yes & Yes & Yes & Yes & Yes \\
\hline Paik, 2017 & Retrospective Cohort & Yes & Yes & Yes & Unclear & No \\
\hline Panlaqui, 2017 & Before-After & Yes & NR & Yes & Yes & Yes \\
\hline Pedersen, 2009 & Prospective Cohort & Yes & Yes & Yes & Yes & Yes \\
\hline Pedragosa, 2009 & Before-After & Yes & Yes & Unclear & Yes & No \\
\hline Pervez, 2010 & Retrospective Cohort & Yes & Yes & No & No & Yes \\
\hline Rendina, 1998 & Retrospective Cohort & Yes & Yes & Yes & Yes & Yes \\
\hline Rincon, 2012 & Before-After & No & Unclear & NR & No & No \\
\hline Robison, 2016 & Prospective Cohort & \begin{tabular}{|l|} 
No \\
\end{tabular} & Yes & NR & Unclear & No \\
\hline Romig, 2012 & $\begin{array}{l}\text { Before-After and } \\
\text { Prospective Cohort }\end{array}$ & Unclear & Unclear & Unclear & Yes & NR \\
\hline Rosenfeld, 2000 & Before-After & Yes & Yes & Yes & Yes & Unclear \\
\hline Rossaro, 2013 & Retrospective Cohort & Yes & Yes & Unclear & Yes & Unclear \\
\hline Ruesch, 2012 & Before-After & Unclear & unclear & Unclear & Yes & NR \\
\hline Sadaka, 2013 & Before-After & Unclear & Unclear & Yes & Unclear & No \\
\hline Saffle, 2009 & Before-After & Yes & Yes & No & Unclear & Unclear \\
\hline Sairanen, 2011 & Prospective Cohort & Yes & Yes & No & Yes & Yes \\
\hline
\end{tabular}




\begin{tabular}{|c|c|c|c|c|c|c|}
\hline $\begin{array}{l}\text { Author, Year } \\
\text { (See Appendix C for } \\
\text { full citation) }\end{array}$ & Study Design & $\begin{array}{l}\text { Eligibility } \\
\text { prespecified? }\end{array}$ & $\begin{array}{l}\text { Random sample or } \\
\text { consecutivelall } \\
\text { patients meeting } \\
\text { inclusion criteria? }\end{array}$ & $\begin{array}{l}\text { Groups similar or } \\
\text { design and } \\
\text { analyses account } \\
\text { for important } \\
\text { potential } \\
\text { confounding and } \\
\text { modifying variables } \\
\text { appropriately? }\end{array}$ & $\begin{array}{l}\text { Valid and reliable } \\
\text { measures used and } \\
\text { adequately } \\
\text { ascertained? }\end{array}$ & $\begin{array}{l}\text { Outcome assessors } \\
\text { and/or data } \\
\text { analysts blinded or } \\
\text { are primary } \\
\text { outcomes unlikely } \\
\text { to be biased? }\end{array}$ \\
\hline Salami, 2015 & Retrospective Cohort & Yes & Unclear & No & Yes & Yes \\
\hline $\begin{array}{l}\text { Salazar-Fernandez, } \\
2012\end{array}$ & Prospective Cohort & $\begin{array}{l}\text { Clinical: Yes } \\
\text { Economic: Yes }\end{array}$ & $\begin{array}{l}\text { Clinical: Yes } \\
\text { Economic: Yes }\end{array}$ & \begin{tabular}{|l|} 
Clinical: Yes \\
Economic: Yes \\
\end{tabular} & \begin{tabular}{|l} 
Clinical: Yes \\
Economic: NR \\
\end{tabular} & $\begin{array}{l}\text { Clinical: No } \\
\text { Economic: Unclear }\end{array}$ \\
\hline Sanchez-Ross, 2011 & Prospective Cohort & Yes & Yes & Unclear & Yes & Yes \\
\hline Schwab, 2007 & Prospective Cohort & Yes & Yes & Yes & Yes & No \\
\hline Sejersten, 2008 & Prospective Cohort & Yes & Yes & No & Yes & Yes \\
\hline Sharma, 2003 & Prospective Cohort & Yes & Yes & NR & No & No \\
\hline Sharma, 2016 & Before-After & No & Unclear & Unclear & Yes & Yes \\
\hline Shin, 2015 & Prospective Cohort & No & No & NR & Yes & No \\
\hline Smith, 2002 & Before-After & Yes & Unclear & NR & No & No \\
\hline Southard, 2014 & Before-After & Yes & Yes & Unclear & Yes & Yes \\
\hline Stalfors, 2003 & Prospective Cohort & No & Yes & Yes & Unclear & No \\
\hline Steinman, 2015 & $\begin{array}{l}\text { Before-After and } \\
\text { Prospective Cohort }\end{array}$ & No & Unclear & NR & Unclear & No \\
\hline Stern, 2014 & Before-After & Yes & Yes & Yes & Yes & Yes \\
\hline Strymish, 2017 & Before-After & Yes & Yes & No & Yes & Yes \\
\hline Switzer, 2009 & Prospective Cohort & Yes & Yes & NR & Yes & Yes \\
\hline Taqui, 2017 & Prospective Cohort & Yes & Unclear & No & Yes & Yes \\
\hline Taylor, 2007 & Before-After & Yes & Yes & NR & Yes & Yes \\
\hline Theiss, 2013 & Before-After & Yes & Unclear & No & Yes & Yes \\
\hline Thomas, 2009 & Before-After & Unclear & Yes & Yes & Yes & No \\
\hline Traub, 2013 & Retrospective Cohort & Yes & No & Yes & No & No \\
\hline Tsai, 2007 & Prospective Cohort & Yes & Yes & No & Yes & Yes \\
\hline Tuulonen, 1999 & Before-After & Yes & Yes & NR & \begin{tabular}{|l|} 
Yes \\
\end{tabular} & No \\
\hline Wallace, 2008 & Prospective Cohort & No & Yes & NR & Unclear & No \\
\hline Webb, 2013 & Prospective Cohort & Yes & Yes & Yes & Unclear & Yes \\
\hline Willmitch, 2012 & Before-After & No & Unclear & Yes & Yes & No \\
\hline Wilson, 2005 & Before-After & Yes & \begin{tabular}{|l|} 
Yes \\
\end{tabular} & Unclear & Yes & Yes \\
\hline Yang, 2015 & Retrospective Cohort & Yes & Yes & Yes & Yes & Yes \\
\hline Young, 2014 & Before-After & Yes & Yes & Yes & Yes & Yes \\
\hline Zahlmann, 2002 & $\begin{array}{l}\text { Prospective and } \\
\text { Retrospective Cohort }\end{array}$ & Yes & Unclear & NR & No & No \\
\hline Zaidi, 2011 & Prospective Cohort & Yes & Yes & Yes & Yes & No \\
\hline Zanini, 2008 & Retrospective Cohort & Yes & Yes & Yes & Yes & Yes \\
\hline Zarchi, 2015 & Prospective Cohort & Yes & NR & Yes & Yes & No \\
\hline
\end{tabular}




\begin{tabular}{|c|c|c|c|c|c|c|}
\hline $\begin{array}{l}\text { Author, Year } \\
\text { (See Appendix C for } \\
\text { full citation) }\end{array}$ & Study Design & $\begin{array}{l}\text { Eligibility } \\
\text { prespecified? }\end{array}$ & $\begin{array}{l}\text { Random sample or } \\
\text { consecutivelall } \\
\text { patients meeting } \\
\text { inclusion criteria? }\end{array}$ & $\begin{array}{l}\text { Groups similar or } \\
\text { design and } \\
\text { analyses account } \\
\text { for important } \\
\text { potential } \\
\text { confounding and } \\
\text { modifying variables } \\
\text { appropriately? }\end{array}$ & $\begin{array}{l}\text { Valid and reliable } \\
\text { measures used and } \\
\text { adequately } \\
\text { ascertained? }\end{array}$ & $\begin{array}{l}\text { Outcome assessors } \\
\text { and/or data } \\
\text { analysts blinded or } \\
\text { are primary } \\
\text { outcomes unlikely } \\
\text { to be biased? }\end{array}$ \\
\hline Zilliacus, 2011 & Prospective Cohort & Yes & Unclear & Yes & Yes & Yes \\
\hline
\end{tabular}

See Appendix C. Included Studies for full citations

Table G-2. Risk of bias: cohort studies, part 2

\begin{tabular}{|c|c|c|c|c|c|}
\hline $\begin{array}{l}\text { Author, Year } \\
\text { (See Appendix C } \\
\text { for full citation) }\end{array}$ & $\begin{array}{l}\text { No or only small } \\
\text { amounts of missing } \\
\text { data? If missing data, } \\
\text { was it handled } \\
\text { appropriately? }\end{array}$ & $\begin{array}{l}\text { Nollow loss } \\
\text { to followup } \\
\text { or attrition? }\end{array}$ & $\begin{array}{l}\text { Outcomes } \\
\text { prespecified and } \\
\text { reported? }\end{array}$ & Funding Source & $\begin{array}{l}\text { Risk of Bias } \\
\text { Rating }\end{array}$ \\
\hline Alemi, 2016 & Yes & Yes & Yes & None & High \\
\hline Amorim, 2013 & Yes & Yes & Yes & Supported in part by CLIQS0000235 & Moderate \\
\hline Angstman, 2009 & Yes & NA & Yes & Mayo Clinic Departmental funds & Moderate \\
\hline Armaignac, 2018 & $\begin{array}{l}\text { Clinical outcomes: Yes } \\
\text { Cost: Unclear }\end{array}$ & NA & Yes & No conflicts reported & $\begin{array}{l}\text { Clinical } \\
\text { outcomes: Low } \\
\text { Cost: High } \\
\end{array}$ \\
\hline Arora, 2011 & Yes & Yes & Yes & $\begin{array}{l}\text { AHRQ; Robert Wood Johnson foundation; New Mexico } \\
\text { Department of Health; New Mexico State Legislature }\end{array}$ & Low \\
\hline Astarcioglu, 2015 & NR & Yes & Yes & $\mathrm{NR}$ & High \\
\hline Audebert, 2006 & Yes & Yes & Yes & $\begin{array}{l}\text { Bavarian health insurance companies; Bavarian State } \\
\text { Ministry for Employment and Social Order, Family and } \\
\text { Women; German Stroke Foundation (Stiftung Deutsche } \\
\text { Schlaganfall-Hilfe) }\end{array}$ & Low \\
\hline Audebert, 2009 & Yes & Yes & Yes & German Federal Ministry of Research (BMBF) & Low \\
\hline Bagayoko, 2014 & Unclear & NA & Yes & $\begin{array}{l}\text { Global Health Research Initiative - partnership of } \\
\text { International Development Research Centre and } \\
\text { Foreign Affairs Trade and Development Canada }\end{array}$ & High \\
\hline Baig, 2016 & Yes & NA & Yes & NR & High \\
\hline Bergrath, 2012 & Yes & Yes & No & $\begin{array}{l}\text { German Federal Ministry of Economics and } \\
\text { Technology; Philips Healthcare; P3 Communications }\end{array}$ & Moderate \\
\hline Bernstein, 2010 & Yes & NA & Yes & No conflicts reported & Moderate \\
\hline Beswick, 2016 & Yes & Unclear & Yes & NR & High \\
\hline Bezalel, 2015 & NA & NA & Yes & James A. Haley Veterans' Hospital & High \\
\hline Blackwell, 1997 & Unclear & Unclear & Yes & $\begin{array}{l}\text { Optical Prescription Spectacle Makers Industries } \\
\text { Limited }\end{array}$ & High \\
\hline
\end{tabular}




\begin{tabular}{|c|c|c|c|c|c|}
\hline $\begin{array}{l}\text { Author, Year } \\
\text { (See Appendix C } \\
\text { for full citation) }\end{array}$ & $\begin{array}{l}\text { No or only small } \\
\text { amounts of missing } \\
\text { data? If missing data, } \\
\text { was it handled } \\
\text { appropriately? }\end{array}$ & $\begin{array}{l}\text { No/low loss } \\
\text { to followup } \\
\text { or attrition? }\end{array}$ & $\begin{array}{l}\text { Outcomes } \\
\text { prespecified and } \\
\text { reported? }\end{array}$ & Funding Source & $\begin{array}{l}\text { Risk of Bias } \\
\text { Rating }\end{array}$ \\
\hline Bladin, 2015 & Yes & Yes & Yes & Boehringer Ingelheim & Low \\
\hline Breslow, 2004 & Yes & Yes & Yes & VISICU & Moderate \\
\hline Britt, 2006 & NR & NA & Yes & NR & High \\
\hline Brokmann, 2016 & NR & Yes & Yes & $\begin{array}{l}\text { European Union and the Ministry of Innovation, Science } \\
\text { and Research of North Rhine Westphalia; Philips } \\
\text { Healthcare; P3 communications }\end{array}$ & Moderate \\
\hline Brokmann, 2016b & No & Yes & Yes & $\begin{array}{l}\text { European Union and the Ministry of } \\
\text { Innovation, Science and Research of North Rhine } \\
\text { Westphalia, German }\end{array}$ & Moderate \\
\hline $\begin{array}{l}\text { Brown-Connolly, } \\
2002\end{array}$ & No & No & No & Blue Cross of California State Sponsored Programs & High \\
\hline Brunetti, 2014 & NR & NR & Yes & None & Moderate \\
\hline Buckley, 2012 & NR & NA & Yes & None & Moderate \\
\hline Burgess, 2002 & NR & Yes & Yes & $\begin{array}{l}\text { Department of Defense, Pacific E-health Innovation } \\
\text { Center, Pacific Regional Program Office }\end{array}$ & High \\
\hline Carallo, 2015 & NR & NR & No & No conflicts reported & High \\
\hline Carter, 2017 & NR & Yes & Yes & NR & High \\
\hline Chan, 2001 & NR & NR & Yes & SK Yee Memorial Fund & High \\
\hline Chan, 2012 & NR & NR & Yes & Authors report no relationship with industry & Low \\
\hline Chan, 2015 & Yes & NA & Yes & NR & Moderate \\
\hline Choi, 2006 & Unclear & Unclear & No & Department of Defense Grant & High \\
\hline Chowdhury, 2012 & NR & NA & Yes & NR, no competing interests & High \\
\hline Chu, 2015 & NR & NA & Yes & No conflicts reported & High \\
\hline Collins, 2017 & No & NA & No & None & High \\
\hline Craig, 2004 & Yes & Yes & Yes & $\begin{array}{l}\text { Neurology Research Fund; Research and Development } \\
\text { Office, Department of Health and Social Services for } \\
\text { Northern Ireland }\end{array}$ & Moderate \\
\hline Crossland, 2016 & No & Yes & No & $\begin{array}{l}\text { National Health and Medical Research Council } \\
\text { Partnership Project Grant }\end{array}$ & High \\
\hline Darkins, 1996 & Yes & NR & Yes & NR & High \\
\hline Davison, 2004 & NR & Yes & No & $\begin{array}{l}\text { Eastern Region Information Technology; Dean Allen } \\
\text { and Barry Allen }\end{array}$ & High \\
\hline Dayal, 2016 & NR & NA & Yes & No conflicts reported & Low \\
\hline De Luca, 2005 & NR & NR & Yes & NR & Moderate \\
\hline Dharmaaroja, 2010 & NR & NR & Yes & Thammasat University & High \\
\hline Dharmar, 2013 & NR & NA & Yes & No conflicts reported & High \\
\hline Dharmar, 2013 & Unclear & NA & Yes & NR & Low \\
\hline
\end{tabular}




\begin{tabular}{|c|c|c|c|c|c|}
\hline $\begin{array}{l}\text { Author, Year } \\
\text { (See Appendix C } \\
\text { for full citation) }\end{array}$ & $\begin{array}{l}\text { No or only small } \\
\text { amounts of missing } \\
\text { data? If missing data, } \\
\text { was it handled } \\
\text { appropriately? }\end{array}$ & $\begin{array}{l}\text { No/low loss } \\
\text { to followup } \\
\text { or attrition? }\end{array}$ & $\begin{array}{l}\text { Outcomes } \\
\text { prespecified and } \\
\text { reported? }\end{array}$ & Funding Source & $\begin{array}{l}\text { Risk of Bias } \\
\text { Rating }\end{array}$ \\
\hline Dharmar, 2013 & Unclear & NA & Yes & $\begin{array}{l}\text { AHRQ, Emergency Medical Services for Children; } \\
\text { Office for the Advancement of Telehealth; William } \\
\text { Randolph Hearst Foundation }\end{array}$ & Low \\
\hline Duchesne, 2008 & Unclear & NA & No & $\begin{array}{l}\text { Bower Foundation; Mississippi State Department of } \\
\text { Health }\end{array}$ & High \\
\hline Engel, 2011 & NR & NA & Yes & NR & High \\
\hline Ferrandiz, 2007 & Yes & Yes & Yes & Insitutos Carlos III & Moderate \\
\hline Fong, 2015 & Yes & Yes & Yes & None & Moderate \\
\hline Fortis, 2014 & NR & NA & Yes & No conflicts reported & High \\
\hline Fox, 2007 & NR & Yes & Yes & NR & Moderate \\
\hline Frank, 2015 & NR & NR & Yes & VHA & Moderate \\
\hline Gilmour, 1998 & Yes & Yes & Yes & NHS R\&D Programme & High \\
\hline Goh, 1997 & NR & NR & Yes & Not reported & High \\
\hline Gupta, 2014 & NR & NA & Yes & No conflicts reported & Moderate \\
\hline Handschu, 2008 & Yes & Yes & Yes & $\begin{array}{l}\text { Bavarian State Ministry of Labor and Social Welfare, } \\
\text { Family and Women }\end{array}$ & Moderate \\
\hline Hashimoto, 2001 & NR & NA & Yes & NR & High \\
\hline Heffner, 2015 & NR & NA & Yes & $\begin{array}{l}\text { University of Pittsburgh School of Medicine's Dean's } \\
\text { Summer Research Program }\end{array}$ & Moderate \\
\hline Herce, 2011 & NR & Unclear & Yes & NR & High \\
\hline Hsiao, 2008 & Yes & Yes & Yes & $\begin{array}{l}\text { University of California at San Francisco Dean's } \\
\text { Research Fellowship }\end{array}$ & Moderate \\
\hline Huang, 2008 & NR & NA & Yes & Children's Miracle Network & Moderate \\
\hline Hubert, 2016 & Yes & NA & Yes & $\begin{array}{l}\text { Conflicts and acknowledgement provided, NR actual } \\
\text { funding }\end{array}$ & Moderate \\
\hline Ickenstein, 2005 & NR & NA & Yes & $\begin{array}{l}\text { Bavarian State Ministry for Employment and Social } \\
\text { Order, Family and Women; German Stroke Foundation; } \\
\text { Bavarian health insurance companies }\end{array}$ & High \\
\hline Ionita, 2009 & NR & NA & Yes & NR & Moderate \\
\hline Itrat, 2016 & Yes & Yes & Yes & $\begin{array}{l}\text { The Cleveland Clinic; Milton and Tamar Maltz Family } \\
\text { Foundation }\end{array}$ & Moderate \\
\hline Jacobs, 2015 & NR & NA & No & The Friesland Insurance Company & High \\
\hline Johansson, 2011 & No & NA & Yes & NR & Moderate \\
\hline Jong, 2004 & NR & NR & Unclear & No conflicts reported & High \\
\hline Jue, 2017 & Yes & NA & Yes & None & Moderate \\
\hline Kahn, 2016 & Yes & NA & Yes & National Institutes of Health & Low \\
\hline Kalb, 2014 & NR & \begin{tabular}{|l|} 
NA \\
\end{tabular} & Yes & No extramural support & High \\
\hline Kim, 2013 & Unclear & Unclear & Yes & Centers for Medicare and Medicaid Service; ANGELS & High \\
\hline
\end{tabular}




\begin{tabular}{|c|c|c|c|c|c|}
\hline $\begin{array}{l}\text { Author, Year } \\
\text { (See Appendix C } \\
\text { for full citation) }\end{array}$ & $\begin{array}{l}\text { No or only small } \\
\text { amounts of missing } \\
\text { data? If missing data, } \\
\text { was it handled } \\
\text { appropriately? }\end{array}$ & $\begin{array}{l}\text { No/low loss } \\
\text { to followup } \\
\text { or attrition? }\end{array}$ & $\begin{array}{l}\text { Outcomes } \\
\text { prespecified and } \\
\text { reported? }\end{array}$ & Funding Source & $\begin{array}{l}\text { Risk of Bias } \\
\text { Rating }\end{array}$ \\
\hline Kim, 2011 & Yes & NA & No & Ministry of Health and Welfare (Korea) & High \\
\hline Klein, 2010 & NR & NA & Yes & NR & Moderate \\
\hline Kobza, 2000 & NR & NR & Yes & NR & High \\
\hline Kohl, 2012 & No & NA & Yes & NR & Moderate \\
\hline Krupinski, 2004 & No & No & Yes & NR & High \\
\hline LaBarbera, 2013 & NR & NA & Unclear & $\begin{array}{l}\text { Jackson Foundation; Friends of Doernbecher; Charlotte } \\
\text { Coleman Frey Fellowship }\end{array}$ & Moderate \\
\hline Langabeer, 2016 & Yes & NA & Yes & NR no conflicts & Moderate \\
\hline Lee, 2014 & NR & NR & Yes & $\begin{array}{l}\text { VISN } 6 \text { Specialty Care Office; Duke Claude A. Pepper } \\
\text { Center }\end{array}$ & high \\
\hline Lilly, 2011 & Yes & Yes & Yes & University of Massachusetts & Low \\
\hline Lim, 2012 & Yes & Yes & No & Waikato Health Board & High \\
\hline Long, 2014 & NR & NA & Yes & $\begin{array}{l}\text { Federal and Arkansas Medicaid; } 2010 \text { American } \\
\text { Recovery and Reinvestment Act grant for the Arkansas } \\
\text { Healthcare, Higher Education, Public Safety, and } \\
\text { Research Integrated Broadband Initiative }\end{array}$ & Moderate \\
\hline Macedo, 2016 & Yes & NA & Yes & Amil Life Sciences & Moderate \\
\hline Machado, 2018 & $\begin{array}{l}\text { Clinical outcomes: Unclear } \\
\text { Costs: Unclear }\end{array}$ & NA & Yes & Ohio Health Research Institute & $\begin{array}{l}\text { Clinical } \\
\text { outcomes: } \\
\text { Moderate } \\
\text { Cost: High }\end{array}$ \\
\hline Marcin, 2004a & NR & NA & Yes & $\begin{array}{l}\text { California Telehealth and Telemedicine Foundation; } \\
\text { Mercy Foundation North }\end{array}$ & Moderate \\
\hline Marcin, 2004c & NR & NA & Yes & $\begin{array}{l}\text { The California Telehealth and Telemedicine Foundation; } \\
\text { Mercy Foundation North }\end{array}$ & Moderate \\
\hline Marcolino, 2013 & NR & NA & Yes & No external funding & Moderate \\
\hline $\begin{array}{l}\text { Martinez-Sanchez, } \\
2014\end{array}$ & NR & NR & Yes & $\begin{array}{l}\text { Cohesion Funds of the Spanish National Health } \\
\text { System; Mutua Madrilena Foundation; Spanish Ministry } \\
\text { of Science and Innovation }\end{array}$ & Moderate \\
\hline Martinoni, 2011 & NR & Yes & Yes & Eli-Lilly Italy & Moderate \\
\hline Mathews, 2008 & No & NR & Yes & NR & High \\
\hline $\begin{array}{l}\text { McCambridge, } \\
2010\end{array}$ & Yes & Yes & Yes & Dorothy Rider Pool Health Care Trust & Moderate \\
\hline McCrossan, 2012 & Yes & Yes & Yes & Royal Belfast Hospital for Sick Children & Moderate \\
\hline Mielonen, 2000 & NR & NR & Yes & Finnish Office for Health Technology Assessment & High \\
\hline Migliaretti, 2013 & NR & NR & Yes & NR & High \\
\hline Miyamoto, 2014 & No & NA & Yes & $\begin{array}{l}\text { Maternal and Child Health Bureau of HRSA; William } \\
\text { Randolph Hearst Foundation }\end{array}$ & Moderate \\
\hline
\end{tabular}




\begin{tabular}{|c|c|c|c|c|c|}
\hline $\begin{array}{l}\text { Author, Year } \\
\text { (See Appendix C } \\
\text { for full citation) }\end{array}$ & $\begin{array}{l}\text { No or only small } \\
\text { amounts of missing } \\
\text { data? If missing data, } \\
\text { was it handled } \\
\text { appropriately? } \\
\end{array}$ & $\begin{array}{l}\text { No/low loss } \\
\text { to followup } \\
\text { or attrition? }\end{array}$ & $\begin{array}{l}\text { Outcomes } \\
\text { prespecified and } \\
\text { reported? }\end{array}$ & Funding Source & $\begin{array}{l}\text { Risk of Bias } \\
\text { Rating } \\
\end{array}$ \\
\hline $\begin{array}{l}\text { Mohr, } 2017 \\
\text { Mohr, } 2018 \\
\end{array}$ & No & NA & Yes & Rural Telehealth Research Center & Low \\
\hline Morrison, 2010 & Yes & Yes & Yes & Washington Square Health Foundation, & Moderate \\
\hline Mulgrew, 2011 & NA & NA & Yes & AHRQ & High \\
\hline Nagao, 2012 & No & NA & Yes & $\begin{array}{l}\text { Telematics Course Development Fund; Institute for } \\
\text { Broadband Enabled Society Seed Funding } \\
\end{array}$ & High \\
\hline Nagayoshi, 2016 & NR & NA & Yes & No conflicts reported & High \\
\hline Narasimhan, 2015 & No & NR & Yes & National Institutes of Health; Duke Endowment & Moderate \\
\hline Nassar, 2014 & NR & Yes & Yes & $\begin{array}{l}\text { National Institute of Arthritis and Musculoskeletal and } \\
\text { Skin Disease; US Department of Veterans Affairs }\end{array}$ & Low \\
\hline $\begin{array}{l}\text { Nguyen-Huynh, } \\
2018\end{array}$ & NR & Yes & No & Permanente Medical Group & Moderate \\
\hline Nickenig, 2008 & Yes & Yes & Yes & Institute of Aerospace Medicine - Germany & Moderate \\
\hline Nordal, 2001 & Yes & Yes & Yes & Norwegian Ministry of Social Welfare and Health & Moderate \\
\hline Ortolani 2007 & NA & NA & Yes & Fanti Melloni Foundation & Moderate \\
\hline Ortolani, 2006 & NR & NA & Yes & Fanti Melloni Foundation & Low \\
\hline Paik, 2017 & NR & NA & Yes & None & High \\
\hline Panlaqui, 2017 & NR & NR & Yes & NR & High \\
\hline Pedersen, 2009 & NR & Yes & Yes & $\begin{array}{l}\text { The Danish Heart Foundation; The Murermester Laurits } \\
\text { Peter Christensen and Wife Kirsten Sigrid Christensen } \\
\text { Foundation }\end{array}$ & Low \\
\hline Pedragosa, 2009 & NR & Unclear & No & $\begin{array}{l}\text { Instituto de Salud Carlos III: Evaluación de Tecnologías } \\
\text { Sanitarias Salud }\end{array}$ & High \\
\hline Pervez, 2010 & No & NA & Yes & $\begin{array}{l}\text { AHRQ; NIH; American Heart Association-Bugher } \\
\text { Foundation; Harvard NeuroDiscovery Center; Deane } \\
\text { Institute for Integrative Research in Atrial Fibrillation and } \\
\text { Stroke; Esther U Sharp Fund; Conway Fellowship Fund; } \\
\text { Lakeside Fund; Levitt Fund }\end{array}$ & High \\
\hline Rendina, 1998 & No & NA & Yes & NR & Low \\
\hline Rincon, 2012 & NR & None & Yes & No conflicts reported & High \\
\hline Robison, 2016 & NR & NR & Yes & Nemours Fund for Children's Health & High \\
\hline Romig, 2012 & No & No & Yes & $\begin{array}{l}\text { The Johns Hopkins Hospital and the Johns Hopkins } \\
\text { Department of Anesthesiology and Critical Care } \\
\text { Medicine. }\end{array}$ & High \\
\hline Rosenfeld, 2000 & Yes & Yes & Yes & NR & Low \\
\hline Rossaro, 2013 & No & NA & Yes & $\begin{array}{l}\text { National Center for Advancing Translational Sciences; } \\
\text { National Institutes of Health }\end{array}$ & Moderate \\
\hline Ruesch, 2012 & Unclear & Unclear & Unclear & NR & High \\
\hline
\end{tabular}




\begin{tabular}{|c|c|c|c|c|c|}
\hline $\begin{array}{l}\text { Author, Year } \\
\text { (See Appendix C } \\
\text { for full citation) }\end{array}$ & $\begin{array}{l}\text { No or only small } \\
\text { amounts of missing } \\
\text { data? If missing data, } \\
\text { was it handled } \\
\text { appropriately? }\end{array}$ & $\begin{array}{l}\text { Nollow loss } \\
\text { to followup } \\
\text { or attrition? }\end{array}$ & $\begin{array}{l}\text { Outcomes } \\
\text { prespecified and } \\
\text { reported? }\end{array}$ & Funding Source & $\begin{array}{l}\text { Risk of Bias } \\
\text { Rating }\end{array}$ \\
\hline Sadaka, 2013 & Unclear & Yes & Yes & NR & High \\
\hline Saffle, 2009 & Yes & Yes & Yes & U.S. Department of Commerce & Moderate \\
\hline Sairanen, 2011 & No & Yes & Yes & State Provincial Office of Southern Finland & Moderate \\
\hline Salami, 2015 & Yes & NA & Yes & $\begin{array}{l}\text { Office of Rural Health - Veterans Integrated Service } \\
\text { Network } 16 \text { Clinical Systems Program, Telehealth, and } \\
\text { Rural Access Program }\end{array}$ & Moderate \\
\hline $\begin{array}{l}\text { Salazar-Fernandez, } \\
2012\end{array}$ & $\begin{array}{l}\text { Clinical: Yes } \\
\text { Economic: NR }\end{array}$ & $\begin{array}{l}\text { Clinical: Yes } \\
\text { Economic: } \\
\text { Yes }\end{array}$ & $\begin{array}{l}\text { Clinical: Yes } \\
\text { Economic: Yes }\end{array}$ & NR & $\begin{array}{l}\text { Clinical: } \\
\text { Moderate } \\
\text { Economic: } \\
\text { High } \\
\end{array}$ \\
\hline $\begin{array}{l}\text { Sanchez-Ross, } \\
2011\end{array}$ & NR & NR & Yes & No conflicts reported & Moderate \\
\hline Schwab, 2007 & Yes & Yes & Yes & $\begin{array}{l}\text { Bavarian State Ministry for Employment and Social } \\
\text { Order, Family and Women; German Stroke Foundation }\end{array}$ & Low \\
\hline Sejersten, 2008 & Yes & Yes & Yes & $\begin{array}{l}\text { Carl og Katy Kajsings Legat; Klestrup og hustru } \\
\text { Henriette Klestrups Mendel }\end{array}$ & Moderate \\
\hline Sharma, 2003 & NR & Unclear & Yes & The Fan Fox and Leslie R. Samuels Foundation Inc. & High \\
\hline Sharma, 2016 & NR & NR & Yes & Penn Medicine Center for Health Care Innovation & High \\
\hline Shin, 2015 & Yes & NA & Yes & Industry conflicts declared & High \\
\hline Smith, 2002 & NR & Unclear & No & Commonwealth Department of Health and Aged Care & High \\
\hline Southard, 2014 & NR & NA & Yes & Indiana State Office of Rural health & Moderate \\
\hline Stalfors, 2003 & Unclear & Yes & Yes & $\begin{array}{l}\text { Kunskap och Kompetens Foundation; Assar } \\
\text { Gabrielsson Foundation; Jubilee Clinic Research } \\
\text { Foundation; Goteborg Medical Society; ACTA } \\
\text { Foundation }\end{array}$ & High \\
\hline Steinman, 2015 & NR & NR & No & Brazilian Ministry of Health & High \\
\hline Stern, 2014 & No & No & Yes & $\begin{array}{l}\text { Canadian Patient Safety Institute; Central Community } \\
\text { Care Access Center; Ministry of Health and Long Term } \\
\text { Care }\end{array}$ & Moderate \\
\hline Strymish, 2017 & NR & NA & No & No conflicts reported & High \\
\hline Switzer, 2009 & Yes & Yes & Yes & NR & Moderate \\
\hline Taqui, 2017 & No & NA & Yes & $\begin{array}{l}\text { Cleveland Clinic; Milton and Tamar Maltz Family } \\
\text { Foundation }\end{array}$ & High \\
\hline Taylor, 2007 & Unclear & NA & No & $\begin{array}{l}\text { HCA Foundation; Nashville Memorial Foundation; } \\
\text { Research to Prevent Blindness }\end{array}$ & High \\
\hline Theiss, 2013 & NR & Unclear & Yes & German Ministry of Education and Research & Moderate \\
\hline Thomas, 2009 & Yes & NR & Yes & $\begin{array}{l}\text { Agency for Healthcare Research and Quality; National } \\
\text { Center for Research Resources; National Institutes of } \\
\text { Health }\end{array}$ & Moderate \\
\hline
\end{tabular}

G-11 


\begin{tabular}{|c|c|c|c|c|c|}
\hline $\begin{array}{l}\text { Author, Year } \\
\text { (See Appendix C } \\
\text { for full citation) }\end{array}$ & $\begin{array}{l}\text { No or only small } \\
\text { amounts of missing } \\
\text { data? If missing data, } \\
\text { was it handled } \\
\text { appropriately? }\end{array}$ & $\begin{array}{l}\text { No/low loss } \\
\text { to followup } \\
\text { or attrition? }\end{array}$ & $\begin{array}{l}\text { Outcomes } \\
\text { prespecified and } \\
\text { reported? }\end{array}$ & Funding Source & $\begin{array}{l}\text { Risk of Bias } \\
\text { Rating } \\
\end{array}$ \\
\hline Traub, 2013 & $\mathrm{NR}$ & NA & Yes & No conflicts reported & High \\
\hline Tsai, 2007 & Yes & Yes & Yes & Department of Health, Taiwan & Low \\
\hline Tuulonen, 1999 & Unclear & Yes & Yes & NR & Moderate \\
\hline Wallace, 2008 & NR & NR & Yes & No conflicts reported & High \\
\hline Webb, 2013 & Unclear & Yes & Yes & $\begin{array}{l}\text { American Society of Echocardiography and the Seabury } \\
\text { Foundation }\end{array}$ & Moderate \\
\hline Willmitch, 2012 & Yes & Yes & Yes & NR & Moderate \\
\hline Wilson, 2005 & Yes & Unclear & Yes & NR & Moderate \\
\hline Yang, 2015 & No & NA & Yes & $\begin{array}{l}\text { AHRQ, HRSA Office for the Advancement of } \\
\text { Telehealth; California Healthcare Foundation; William } \\
\text { Randolph Hearst Foundations }\end{array}$ & Moderate \\
\hline Young, 2014 & NR & NR & Yes & NIDA grant & Moderate \\
\hline Zahlmann, 2002 & NR & NR & No & NR & High \\
\hline Zaidi, 2011 & NR & Yes & Yes & NR & Moderate \\
\hline Zanini, 2008 & NR & NA & Yes & NR & Moderate \\
\hline Zarchi, 2015 & Yes & Yes & Yes & NR & Moderate \\
\hline Zilliacus, 2011 & Yes & No & Yes & $\begin{array}{l}\text { NHMRC Career Development Award and by Strategic } \\
\text { Research Partnership Grant from the New South Wales } \\
\text { Cancer Council }\end{array}$ & Moderate \\
\hline
\end{tabular}

NA = not applicable; $\mathrm{NR}=$ not reported

See Appendix C. Included Studies for full citations

Table G-3. Risk of bias: randomized controlled trials, part 1

\begin{tabular}{|c|c|c|c|c|c|c|}
\hline $\begin{array}{l}\text { Author, Year } \\
\text { (See Appendix C for } \\
\text { full citation) }\end{array}$ & $\begin{array}{l}\text { Randomization } \\
\text { adequate? }\end{array}$ & $\begin{array}{l}\text { Allocation } \\
\text { concealment } \\
\text { adequate? }\end{array}$ & $\begin{array}{l}\text { Eligibility criteria } \\
\text { specified? }\end{array}$ & $\begin{array}{l}\text { Groups similar or } \\
\text { controlled for important } \\
\text { baseline differences? }\end{array}$ & $\begin{array}{l}\text { Participants analyzed in } \\
\text { groups originally } \\
\text { assigned? }\end{array}$ & $\begin{array}{l}\text { Attrition low } \\
\text { and/or adherence } \\
\text { high? }\end{array}$ \\
\hline Basudev, 2016 & \begin{tabular}{|l|} 
Yes \\
\end{tabular} & Yes & Yes & Yes & NR & No \\
\hline Boman, 2014 & Unclear & Unclear & Yes & Yes & Yes & Yes \\
\hline $\begin{array}{l}\text { Brennan, } 1998 \\
\text { Brennan, } 1999\end{array}$ & NR & Yes & Yes & Yes & NR & Yes \\
\hline Burns, 2017 & Yes & Yes & Yes & Unclear & NR & Yes \\
\hline Byamba, 2015 & Yes & Unclear & Unclear & Unclear & Yes & Unclear \\
\hline Cho, 2011 & Yes & No & Yes & No & NR & Yes \\
\hline Chua, 2001 & No & NR & Yes & Yes & NR & No \\
\hline $\begin{array}{l}\text { Collins, } 2004 \\
\text { Bowns, } 2006 \\
\end{array}$ & Yes & Yes & Yes & Unclear & Yes & No \\
\hline Davis, 2011 & NR & NR & Yes & Yes & Yes & Yes \\
\hline Demaerschalk, 2010 & Yes & Yes & Yes & Yes & NR & No \\
\hline
\end{tabular}




\begin{tabular}{|c|c|c|c|c|c|c|}
\hline $\begin{array}{l}\text { Author, Year } \\
\text { (See Appendix C for } \\
\text { full citation) }\end{array}$ & $\begin{array}{l}\text { Randomization } \\
\text { adequate? }\end{array}$ & $\begin{array}{l}\text { Allocation } \\
\text { concealment } \\
\text { adequate? } \\
\end{array}$ & $\begin{array}{l}\text { Eligibility criteria } \\
\text { specified? }\end{array}$ & $\begin{array}{l}\text { Groups similar or } \\
\text { controlled for important } \\
\text { baseline differences? }\end{array}$ & $\begin{array}{l}\text { Participants analyzed in } \\
\text { groups originally } \\
\text { assigned? }\end{array}$ & $\begin{array}{l}\text { Attrition low } \\
\text { and/or adherence } \\
\text { high? }\end{array}$ \\
\hline Eminovic, 2009 & Yes & Yes & Yes & Unclear & Yes & Yes \\
\hline Fortney, 2007 & NR & NR & Yes & Yes & Yes & No \\
\hline Fortney, 2013 & Yes & No & Yes & Yes & Yes & No \\
\hline Fortney, 2015 & Yes & No & Yes & Yes & Yes & No \\
\hline Fuertes-Guiro, 2016 & NR & NR & No & Yes & NR & Yes \\
\hline Gattas, 2001 & NR & NR & No & NR & NR & No \\
\hline Grabowski, 2014 & \begin{tabular}{|l|} 
NR \\
\end{tabular} & NR & Yes & Yes & Yes & Yes \\
\hline Haukipuro, 2000 & Unclear & NR & Yes & Yes & NR & Yes \\
\hline Izquierdo, 2009 & \begin{tabular}{|l|} 
NR \\
\end{tabular} & No & Yes & Unclear & NR & Unclear \\
\hline Jaatinen, 2002 & No & Unclear & Yes & NR & NR & No \\
\hline Krier, 2011 & No & No & Yes & No & No & Yes \\
\hline Kunkler, 2007 & NR & NR & Yes & Yes & Yes & No \\
\hline Loane, 1999 & Yes & No & Yes & Unclear/NR & Unclear & Unclear \\
\hline Loane, 2000 & Yes & Yes & Yes & \begin{tabular}{|l|} 
NR \\
\end{tabular} & \begin{tabular}{|l|} 
NR \\
\end{tabular} & Yes \\
\hline Martin-Khan, 2016 & Yes & Yes & Yes & Unclear & NR & Yes \\
\hline Mazighi, 2017 & Yes & Yes & Yes & No & Yes & No \\
\hline Meyer, 2008 & Yes & Yes & Yes & Yes & Yes & Yes \\
\hline Pak, 2007 & Unclear & No & Yes & Yes & Yes & No \\
\hline Patel, 2015 & NR & Yes & Yes & Yes & NR & Yes \\
\hline Piette, 2017 & Yes & Yes & Yes & Yes & Yes & Yes \\
\hline Poon, 2001 & NR & NR & Yes & NR & Yes & Yes \\
\hline Santamaria, 2004 & NR & NR & Yes & No & NR & NR \\
\hline Smith-Strom, 2018 & Yes & Yes & Yes & Yes & Yes & Yes \\
\hline van Gelder, 2017 & Unclear & Yes & Yes & Yes & No & Yes \\
\hline Vuolio, 2002 & Unclear & No & Yes & Yes & NR & Yes \\
\hline $\begin{array}{l}\text { Wallace, } 2002 \\
\text { Jacklin, } 2003\end{array}$ & Yes & No & Yes & Yes & Yes & Yes \\
\hline Wallace, 2004 & Yes & Yes & Yes & Yes & Yes & Yes \\
\hline Whited, 2002 & Unclear & Yes & Unclear & Yes & Yes & Yes \\
\hline Whited, 2004 & Unclear & Yes & Yes & Unclear & NR & Yes \\
\hline $\begin{array}{l}\text { Whited, } 2013 \\
\text { Whited,2013b }\end{array}$ & Yes & Yes & Yes & Yes & Yes & Yes \\
\hline Wong, 2006 & NR & Yes & Yes & Yes & Yes & No \\
\hline
\end{tabular}

NR = not reported

See Appendix C. Included Studies for full citations 
Table G-4. Risk of bias: randomized controlled trials, part 2

\begin{tabular}{|c|c|c|c|c|c|}
\hline $\begin{array}{l}\text { Author, Year } \\
\text { (See Appendix } \\
\text { C for full } \\
\text { citation) }\end{array}$ & $\begin{array}{l}\text { Outcome assessors } \\
\text { and/or data analysts } \\
\text { blinded or are primary } \\
\text { outcomes unlikely to be } \\
\text { biased? }\end{array}$ & $\begin{array}{l}\text { Outcome and } \\
\text { confounder measures } \\
\text { reliable and implemented } \\
\text { consistently across } \\
\text { groups? }\end{array}$ & $\begin{array}{l}\text { Outcomes } \\
\text { prespecified } \\
\text { and reported? }\end{array}$ & Funding Source & $\begin{array}{l}\text { Risk of } \\
\text { Bias } \\
\text { Rating } \\
\end{array}$ \\
\hline Basudev, 2016 & Yes & Yes & Yes & Diabetes UK; Royal College of General Practitioners & Moderate \\
\hline Boman, 2014 & No & Unclear & Yes & GE Healthcare and Philips Healthcare & Moderate \\
\hline $\begin{array}{l}\text { Brennan, } 1998 \\
\text { Brennan, } 1999\end{array}$ & No & Unclear & Yes & $\begin{array}{l}\text { Emergency Medical Associates; VTEL Corporation; } \\
\text { Andris Tek, Inc.; Northwest Covenant Medical } \\
\text { Center }\end{array}$ & High \\
\hline Burns, 2017 & No & Yes & Yes & $\begin{array}{l}\text { Asset Strategic Plan - Central Integrated Regional } \\
\text { Cancer Services; E0I-3, Statewide Telehealth } \\
\text { Services; Royal Brisbane And Women's Hospital; } \\
\text { Australian Centre for Health Services Innovation }\end{array}$ & Moderate \\
\hline Byamba, 2015 & No & Yes & Yes & $\begin{array}{l}\text { National Science Council Project; Ministry of Health } \\
\text { and Welfare, Taiwan; Taipei Medical University }\end{array}$ & Moderate \\
\hline Cho, 2011 & Unclear & No & Yes & $\begin{array}{l}\text { Yonsei University College of Medicine; Ministry of } \\
\text { Knowledge Economy; Korea Institute for } \\
\text { Advancement in Technology }\end{array}$ & High \\
\hline Chua, 2001 & Yes & No & Yes & $\begin{array}{l}\text { Research and Development Office of the Northern } \\
\text { Ireland Department of Health and Social Services }\end{array}$ & High \\
\hline $\begin{array}{l}\text { Collins, } 2004 \\
\text { Bowns, } 2006 \\
\end{array}$ & Yes & Yes & Yes & $\begin{array}{l}\text { UK NHS R\&D Health Technology Assessment } \\
\text { Programme }\end{array}$ & Moderate \\
\hline Davis, 2011 & No & Unclear & Yes & Veterans Affairs & High \\
\hline $\begin{array}{l}\text { Demaerschalk, } \\
2010\end{array}$ & Yes & Yes & Yes & Arizona Department of Health Services; Mayo Clinic & Moderate \\
\hline Eminovic, 2009 & No & No & Yes & $\begin{array}{l}\text { Dutch Ministry of Economic Affairs; Dutch } \\
\text { Organization for Health Research and Development; } \\
\text { KSYOS Health Management Research }\end{array}$ & Moderate \\
\hline Fortney, 2007 & Yes & Yes & Yes & $\begin{array}{l}\text { Veterans Affairs; Veterans Affairs HSR\&D; VA South } \\
\text { Central Mental Illness Research Education and } \\
\text { Clinical Center }\end{array}$ & Moderate \\
\hline Fortney, 2013 & Yes & Yes & Yes & National Institute of Mental Health & Moderate \\
\hline Fortney, 2015 & Yes & Yes & Yes & $\begin{array}{l}\text { Veterans Affairs; Veterans Affairs HSR\&D; VA South } \\
\text { Central Mental Illness Research Education and } \\
\text { Clinical Center }\end{array}$ & Moderate \\
\hline $\begin{array}{l}\text { Fuertes-Guiro, } \\
2016\end{array}$ & Yes & Unclear & Yes & No conflicts reported & High \\
\hline Gattas, 2001 & No & No & No & NR & High Risk \\
\hline $\begin{array}{l}\text { Grabowski, } \\
2014\end{array}$ & Yes & No & Yes & The Commonwealth Fund & High \\
\hline Haukipuro, 2000 & No & Unclear & No & Finnish Office for Health Technology Assessment & High \\
\hline
\end{tabular}




\begin{tabular}{|c|c|c|c|c|c|}
\hline $\begin{array}{l}\text { Author, Year } \\
\text { (See Appendix } \\
\text { C for full } \\
\text { citation) }\end{array}$ & $\begin{array}{l}\text { Outcome assessors } \\
\text { andlor data analysts } \\
\text { blinded or are primary } \\
\text { outcomes unlikely to be } \\
\text { biased? }\end{array}$ & $\begin{array}{l}\text { Outcome and } \\
\text { confounder measures } \\
\text { reliable and implemented } \\
\text { consistently across } \\
\text { groups? }\end{array}$ & $\begin{array}{l}\text { Outcomes } \\
\text { prespecified } \\
\text { and reported? }\end{array}$ & Funding Source & $\begin{array}{l}\text { Risk of } \\
\text { Bias } \\
\text { Rating }\end{array}$ \\
\hline Izquierdo, 2009 & Yes & Yes & Yes & $\begin{array}{l}\text { DHHS, NY State Dept. of Health; Children's Miracle } \\
\text { Network }\end{array}$ & High \\
\hline Jaatinen, 2002 & No & No & Yes & $\begin{array}{l}\text { Foundation of High Technology in Satakunta; } \\
\text { Satakunta Hospital District }\end{array}$ & High \\
\hline Krier, 2011 & Yes & Yes & Yes & None & High \\
\hline Kunkler, 2007 & No & Unclear & Yes & $\begin{array}{l}\text { Department of Health Research and Development; } \\
\text { New Opportunities Fund } \\
\end{array}$ & High \\
\hline Loane, 1999 & Unclear & Unclear & Yes & NHS R\&D Programme & High \\
\hline Loane, 2000 & No & Yes & Yes & $\begin{array}{l}\text { NHS R\&D Programme; Southern Health and Social } \\
\text { Services Board; Glaxo and Steifel }\end{array}$ & High \\
\hline $\begin{array}{l}\text { Martin-Khan, } \\
2016\end{array}$ & Yes & No & Yes & $\begin{array}{l}\text { National Health and Medical Research Council } \\
\text { (Australia) }\end{array}$ & Moderate \\
\hline Mazighi, 2017 & Yes & Yes & Yes & $\begin{array}{l}\text { French Ministry of Health; SOS-Attaque Cerebral } \\
\text { Association; Department a la Recherche Clinique et } \\
\text { au Developpement de l'Assistance publique } \\
\text { Hopitaux de Paris with the Unite de Recherche } \\
\text { Clinique Paris Nord }\end{array}$ & High \\
\hline Meyer, 2008 & Yes & Yes & Yes & $\begin{array}{l}\text { National Institutes of Neurological Disorders and } \\
\text { Stroke }\end{array}$ & Low \\
\hline Pak, 2007 & Yes & Yes & Yes & $\begin{array}{l}\text { VA Telemedicine and Advanced Technology } \\
\text { Research Center }\end{array}$ & Moderate \\
\hline Patel, 2015 & No & Unclear & Yes & Nemours Fund for Children's Health & Moderate \\
\hline Piette, 2017 & No & Yes & Yes & $\begin{array}{l}\text { Pole de Sante Universitaire Gennevilliers Villeneuve } \\
\text { La Garenne }\end{array}$ & Low \\
\hline Poon, 2001 & No & No & Yes & NR & High \\
\hline $\begin{array}{l}\text { Santamaria, } \\
2004\end{array}$ & No & Yes & Yes & Western Australian Department of Health & High \\
\hline $\begin{array}{l}\text { Smith-Strom, } \\
2018\end{array}$ & Yes & Yes & Yes & $\begin{array}{l}\text { Norwegian Directorate of Health and Innovation, } \\
\text { Western Norway Regional Health Authority; } \\
\text { Norwegian Diabetes Association; Western Norway } \\
\text { University of Applied Sciences; Norwegian Research } \\
\text { Council }\end{array}$ & Low \\
\hline $\begin{array}{l}\text { van Gelder, } \\
2017\end{array}$ & No & Yes & Yes & Dutch Kidney Foundation; Amgen & High \\
\hline Vuolio, 2002 & No & Yes & Yes & Finnish Office of Health Technology & High \\
\hline $\begin{array}{l}\text { Wallace, } 2002 \\
\text { Jacklin, } 2003\end{array}$ & Unclear & Yes & Yes & $\begin{array}{l}\text { National Health Service Research and Development } \\
\text { Health Technology Assessment programme; British } \\
\text { Telecom; Merck Foundation }\end{array}$ & Low \\
\hline Wallace, 2004 & Yes & Yes & Yes & NHS R\&D HTA Programme & Low \\
\hline
\end{tabular}

G-15 


\begin{tabular}{|l|l|l|l|l|}
\hline $\begin{array}{l}\text { Author, Year } \\
\begin{array}{l}\text { See Appendix } \\
\text { C for full } \\
\text { citation) }\end{array}\end{array}$ & $\begin{array}{l}\text { Outcome assessors } \\
\text { and/or data analysts } \\
\text { blinded or are primary } \\
\text { outcomes unlikely to be } \\
\text { biased? }\end{array}$ & $\begin{array}{l}\text { Outcome and } \\
\text { confounder measures } \\
\text { reliable and implemented } \\
\text { consistently across } \\
\text { groups? }\end{array}$ & $\begin{array}{l}\text { Outcomes } \\
\text { prespecified } \\
\text { and reported? }\end{array}$ & Funding Source \\
\hline Whited, 2002 & Yes & Yes & $\begin{array}{l}\text { Yes } \\
\text { Service; VA Health Services Research and } \\
\text { Development Service Research Career } \\
\text { Development Award }\end{array}$ \\
\hline Whited, 2004 & No & $\begin{array}{l}\text { Bias } \\
\text { Rating }\end{array}$ \\
\hline $\begin{array}{l}\text { Whited, 2013 } \\
\text { Whited,2013b }\end{array}$ & No & No Health Services Research and Development & $\begin{array}{l}\text { Moderate } \\
\text { Development Service Research Career } \\
\text { Development Award }\end{array}$ \\
\hline Wong, 2006 & Unclear & High & Veterans Affairs HSR\&D \\
\hline
\end{tabular}

$\mathrm{NR}=$ not reported

See Appendix C. Included Studies for full citations

\section{Table G-5. Risk of bias: pre-post studies}

\begin{tabular}{|c|c|c|c|c|c|c|c|c|c|c|c|}
\hline $\begin{array}{l}\text { Author, } \\
\text { Year } \\
\text { (See } \\
\text { Appendix C } \\
\text { for full } \\
\text { citation) }\end{array}$ & $\begin{array}{l}\text { Study } \\
\text { Design }\end{array}$ & \begin{tabular}{|l|} 
\\
Eligibility \\
prespecified
\end{tabular} & $\begin{array}{l}\text { Random } \\
\text { sample or } \\
\text { consecutivel } \\
\text { all patients } \\
\text { meeting } \\
\text { inclusion } \\
\text { criteria? }\end{array}$ & \begin{tabular}{|l|} 
Design and \\
analyses \\
account for \\
important \\
potential \\
confounding \\
and modifying \\
variables \\
appropriately? \\
\end{tabular} & $\begin{array}{l}\text { Valid and } \\
\text { reliable } \\
\text { measures } \\
\text { used and } \\
\text { adequately } \\
\text { ascertained? }\end{array}$ & $\begin{array}{l}\text { Outcome } \\
\text { assessors } \\
\text { andlor data } \\
\text { analysts } \\
\text { blinded or } \\
\text { are primary } \\
\text { outcomes } \\
\text { unlikely to } \\
\text { be biased? }\end{array}$ & $\begin{array}{l}\text { No or only } \\
\text { small amounts } \\
\text { of missing } \\
\text { data? } \\
\text { If missing } \\
\text { data, was it } \\
\text { handled } \\
\text { appropriately? }\end{array}$ & \begin{tabular}{|l|} 
\\
No/Low \\
loss to \\
followup \\
or \\
attrition? \\
\end{tabular} & $\begin{array}{l}\text { Outcomes } \\
\text { pre- } \\
\text { specified } \\
\text { and } \\
\text { reported? }\end{array}$ & $\begin{array}{l}\text { Funding } \\
\text { Source }\end{array}$ & $\begin{array}{l}\text { Risk of } \\
\text { Bias } \\
\text { Rating }\end{array}$ \\
\hline $\begin{array}{l}\text { Chu- } \\
\text { Weininger, } \\
2010\end{array}$ & \begin{tabular}{l|} 
Pre- \\
Post
\end{tabular} & Yes & Yes & Unclear & Yes & No & Yes & No & Yes & AHRQ; NIH & High \\
\hline $\begin{array}{l}\text { Endean, } \\
2001\end{array}$ & $\begin{array}{l}\text { Pre- } \\
\text { Post }\end{array}$ & No & Yes & No & NR & No & NR & Yes & Yes & $\begin{array}{l}\text { Health } \\
\text { Resources and } \\
\text { Services } \\
\text { Administration } \\
\text { (HRSA) grant } \\
\text { from the Office } \\
\text { for the } \\
\text { Advancement } \\
\text { for Telehealth }\end{array}$ & High \\
\hline
\end{tabular}




\begin{tabular}{|c|c|c|c|c|c|c|c|c|c|c|c|}
\hline $\begin{array}{l}\text { Author, } \\
\text { Year } \\
\text { (See } \\
\text { Appendix C } \\
\text { for full } \\
\text { citation) }\end{array}$ & $\begin{array}{l}\text { Study } \\
\text { Design }\end{array}$ & $\begin{array}{l}\text { Eligibility } \\
\text { prespecified }\end{array}$ & $\begin{array}{l}\text { Random } \\
\text { sample or } \\
\text { consecutivel } \\
\text { all patients } \\
\text { meeting } \\
\text { inclusion } \\
\text { criteria? }\end{array}$ & $\begin{array}{l}\text { Design and } \\
\text { analyses } \\
\text { account for } \\
\text { important } \\
\text { potential } \\
\text { confounding } \\
\text { and modifying } \\
\text { variables } \\
\text { appropriately? }\end{array}$ & $\begin{array}{l}\text { Valid and } \\
\text { reliable } \\
\text { measures } \\
\text { used and } \\
\text { adequately } \\
\text { ascertained? }\end{array}$ & \begin{tabular}{|l} 
Outcome \\
assessors \\
andlor data \\
analysts \\
blinded or \\
are primary \\
outcomes \\
unlikely to \\
be biased?
\end{tabular} & $\begin{array}{l}\text { No or only } \\
\text { small amounts } \\
\text { of missing } \\
\text { data? } \\
\text { If missing } \\
\text { data, was it } \\
\text { handled } \\
\text { appropriately? }\end{array}$ & $\begin{array}{l}\text { No/Low } \\
\text { loss to } \\
\text { followup } \\
\text { or } \\
\text { attrition? }\end{array}$ & $\begin{array}{l}\text { Outcomes } \\
\text { pre- } \\
\text { specified } \\
\text { and } \\
\text { reported? }\end{array}$ & $\begin{array}{l}\text { Funding } \\
\text { Source }\end{array}$ & $\begin{array}{l}\text { Risk of } \\
\text { Bias } \\
\text { Rating }\end{array}$ \\
\hline $\begin{array}{l}\text { Nikkanen, } \\
2008\end{array}$ & \begin{tabular}{|l|} 
Pre- \\
Post
\end{tabular} & No & Yes & No & Yes & Yes & Yes & Yes & Yes & NR & $\begin{array}{l}\text { Modera } \\
\text { te }\end{array}$ \\
\hline $\begin{array}{l}\text { Mahendran, } \\
2005\end{array}$ & $\begin{array}{l}\text { Pre- } \\
\text { Post }\end{array}$ & Yes & Yes & No & Yes & No & Yes & Yes & Yes & NR & High \\
\hline $\begin{array}{l}\text { Zennaro, } \\
2014\end{array}$ & $\begin{array}{l}\text { Pre- } \\
\text { Post }\end{array}$ & Yes & No & Unclear & No & Unclear & NR & Yes & Yes & $\begin{array}{l}\text { No conflicts } \\
\text { reported }\end{array}$ & High \\
\hline
\end{tabular}

NR = not reported

See Appendix C. Included Studies for full citations

Table G-6. Risk of bias: economic assessments, part 1

\begin{tabular}{|c|c|c|c|c|c|c|c|c|}
\hline $\begin{array}{l}\text { Author, Year } \\
\text { (See } \\
\text { Appendix C } \\
\text { for full } \\
\text { citation) }\end{array}$ & $\begin{array}{l}\text { Are competing } \\
\text { alternatives } \\
\text { clearly } \\
\text { described? }\end{array}$ & $\begin{array}{l}\text { Is the economic } \\
\text { study design } \\
\text { appropriate to } \\
\text { the stated } \\
\text { objective? }\end{array}$ & $\begin{array}{l}\text { Are all important } \\
\text { and relevant costs } \\
\text { for each } \\
\text { alternative } \\
\text { identified? }\end{array}$ & $\begin{array}{l}\text { Are all costs } \\
\text { measured } \\
\text { appropriately } \\
\text { in physical } \\
\text { units? }\end{array}$ & $\begin{array}{l}\text { Are costs } \\
\text { valued } \\
\text { appropriately? }\end{array}$ & $\begin{array}{l}\text { Are all } \\
\text { important and } \\
\text { relevant } \\
\text { outcomes for } \\
\text { each } \\
\text { alternative } \\
\text { identified? }\end{array}$ & $\begin{array}{l}\text { Are all } \\
\text { outcomes } \\
\text { measured } \\
\text { appropriately? }\end{array}$ & $\begin{array}{l}\text { Are outcomes } \\
\text { valued } \\
\text { appropriately? }\end{array}$ \\
\hline Datta, 2015 & Yes & Yes & Yes & Yes & Yes & Yes & Yes & Yes \\
\hline $\begin{array}{l}\text { Doolittle, } \\
1997\end{array}$ & Yes & Yes & Unclear & Unclear & Unclear & NR & NR & NR \\
\hline Dowie, 2007 & Yes & Yes & Yes & Yes & Yes & Yes & Unclear & Unclear \\
\hline $\begin{array}{l}\text { Eminovic, } \\
2010\end{array}$ & Yes & Yes & Yes & Yes & Yes & NR & NR & NR \\
\hline Fortney, 2011 & Yes & Yes & Unclear & Unclear & Unclear & Unclear & Yes & Unclear \\
\hline $\begin{array}{l}\text { Franzini, } \\
2011\end{array}$ & Yes & Yes & Yes & Yes & Yes & No & Yes & NR \\
\hline Gray, 2009 & Yes & Yes & No & Yes & Yes & NR & NR & NR \\
\hline Harno, 2000 & Yes & Yes & Unclear & Yes & Yes & NR & NR & NR \\
\hline Harno, 2001 & Yes & Unclear & Unclear & No & Yes & NR & NR & NR \\
\hline Jacklin, 2003 & Yes & \begin{tabular}{|l|} 
Yes \\
\end{tabular} & Yes & No & Yes & Yes & No & Yes \\
\hline $\begin{array}{l}\text { Lamminen, } \\
2001\end{array}$ & Yes & Unclear & No & Yes & Yes & Unclear & Yes & Yes \\
\hline $\begin{array}{l}\text { Langabeer, } \\
2017\end{array}$ & Yes & Yes & Yes & Yes & Yes & No & Yes & Yes \\
\hline
\end{tabular}




\begin{tabular}{|c|c|c|c|c|c|c|c|c|}
\hline $\begin{array}{l}\text { Author, Year } \\
\text { (See } \\
\text { Appendix C } \\
\text { for full } \\
\text { citation) }\end{array}$ & $\begin{array}{l}\text { Are competing } \\
\text { alternatives } \\
\text { clearly } \\
\text { described? }\end{array}$ & $\begin{array}{l}\text { Is the economic } \\
\text { study design } \\
\text { appropriate to } \\
\text { the stated } \\
\text { objective? }\end{array}$ & $\begin{array}{l}\text { Are all important } \\
\text { and relevant costs } \\
\text { for each } \\
\text { alternative } \\
\text { identified? }\end{array}$ & $\begin{array}{l}\text { Are all costs } \\
\text { measured } \\
\text { appropriately } \\
\text { in physical } \\
\text { units? }\end{array}$ & $\begin{array}{l}\text { Are costs } \\
\text { valued } \\
\text { appropriately? }\end{array}$ & $\begin{array}{l}\text { Are all } \\
\text { important and } \\
\text { relevant } \\
\text { outcomes for } \\
\text { each } \\
\text { alternative } \\
\text { identified? } \\
\end{array}$ & $\begin{array}{l}\text { Are all } \\
\text { outcomes } \\
\text { measured } \\
\text { appropriately? }\end{array}$ & $\begin{array}{l}\text { Are outcomes } \\
\text { valued } \\
\text { appropriately? }\end{array}$ \\
\hline Lilly, 2017 & Yes & Yes & Unclear & Yes & Unclear & Unclear & Yes & Yes \\
\hline Loane, 2001 & Yes & Yes & Unclear & Yes & Yes & Yes & Yes & Yes \\
\hline Marcin, 2004 & Yes & Yes & Unclear & Yes & Yes & Yes & Yes & Yes \\
\hline $\begin{array}{l}\text { Moreno- } \\
\text { Ramirez, } \\
2009\end{array}$ & Yes & Yes & Unclear & Yes & Yes & Yes & Yes & Yes \\
\hline Natafgi, 2007 & Yes & Yes & Unclear & Yes & NR & No & Yes & NR \\
\hline Noble, 2005 & Yes & Yes & Yes & Yes & Yes & Yes & Yes & \begin{tabular}{|l|} 
Yes \\
\end{tabular} \\
\hline $\begin{array}{l}\text { Ohinmaa, } \\
2002\end{array}$ & Yes & Yes & Unclear & Yes & No & No & No & Unclear \\
\hline Pak, 2009 & Yes & Yes & Yes & Yes & Yes & NR & NR & NR \\
\hline $\begin{array}{l}\text { Rendina, } \\
1997\end{array}$ & Yes & Unclear & No & Yes & Unclear & No & Unclear & Unclear \\
\hline $\begin{array}{l}\text { Scuffham, } \\
2002\end{array}$ & Yes & No & Yes & Yes & Yes & Yes & Yes & Yes \\
\hline Specht, 2001 & Yes & Yes & Yes & Yes & Yes & NR & NR & NR \\
\hline Stalfors, 2005 & Yes & Yes & Yes & Yes & Yes & NR & NR & NR \\
\hline Thaker, 2013 & Yes & Yes & Yes & Yes & No & NR & NR & NR \\
\hline $\begin{array}{l}\text { Tsitlakidis, } \\
2005\end{array}$ & Yes & No & Yes & Yes & Yes & NR & NR & NR \\
\hline $\begin{array}{l}\text { van der Pol, } \\
2010\end{array}$ & Yes & Yes & Yes & NR & Yes & Yes & Yes & Yes \\
\hline Whited, 2003 & Yes & Yes & Yes & Yes & Yes & No & Yes & Yes \\
\hline $\mathrm{Xu}, 2008$ & Yes & No & Yes & Yes & Yes & NR & NR & NR \\
\hline
\end{tabular}

$\mathrm{NR}=$ not reported

See Appendix C. Included Studies for full citations

Table G-7. Risk of bias: economic assessments, part 2

\begin{tabular}{|c|c|c|c|c|c|}
\hline $\begin{array}{l}\text { Author, Year } \\
\text { (See Appendix } \\
\text { C for full } \\
\text { citation) }\end{array}$ & $\begin{array}{l}\text { Is an incremental } \\
\text { analysis of costs } \\
\text { and outcomes of } \\
\text { alternatives } \\
\text { performed? }\end{array}$ & $\begin{array}{l}\text { Are all future } \\
\text { costs and } \\
\text { outcomes } \\
\text { discounted } \\
\text { appropriately? }\end{array}$ & $\begin{array}{l}\text { Are all important } \\
\text { variables, whose values } \\
\text { are uncertain, } \\
\text { appropriately subjected } \\
\text { to sensitivity analysis? }\end{array}$ & Funding Source & \begin{tabular}{|l} 
Risk of \\
Bias \\
Rating \\
\end{tabular} \\
\hline Datta, 2015 & Unclear & NA & No & $\begin{array}{l}\text { Department of Veterans Affairs Office of Research and } \\
\text { Development, Health Services Research and Development }\end{array}$ & Moderate \\
\hline Doolittle, 1997 & No & NR & No & $\mathrm{NIH}$ & High \\
\hline
\end{tabular}




\begin{tabular}{|c|c|c|c|c|c|}
\hline $\begin{array}{l}\text { Author, Year } \\
\text { (See Appendix } \\
\text { C for full } \\
\text { citation) }\end{array}$ & $\begin{array}{l}\text { Is an incremental } \\
\text { analysis of costs } \\
\text { and outcomes of } \\
\text { alternatives } \\
\text { performed? }\end{array}$ & $\begin{array}{l}\text { Are all future } \\
\text { costs and } \\
\text { outcomes } \\
\text { discounted } \\
\text { appropriately? }\end{array}$ & $\begin{array}{l}\text { Are all important } \\
\text { variables, whose values } \\
\text { are uncertain, } \\
\text { appropriately subjected } \\
\text { to sensitivity analysis? }\end{array}$ & Funding Source & $\begin{array}{l}\text { Risk of } \\
\text { Bias } \\
\text { Rating }\end{array}$ \\
\hline Dowie, 2007 & No & NR & Yes & $\begin{array}{l}\text { Department of Health and the Charitable Funds Committee of } \\
\text { the Royal Brompton; Harefield NHS Trust }\end{array}$ & Moderate \\
\hline $\begin{array}{l}\text { Eminovic, } \\
2010\end{array}$ & Yes & NR & Yes & $\begin{array}{l}\text { ZonMw (Dutch Organization for Health Research and } \\
\text { Development), through the Health Care Efficiency Research } \\
\text { programme }\end{array}$ & Moderate \\
\hline Fortney, 2011 & Yes & NA & No & $\begin{array}{l}\text { VA HSR\&D Center for Mental Health and Outcomes Research, } \\
\text { VA HSR\&D Research Career Awards, VA HSR\&D Postdoctoral } \\
\text { Fellowship Program, and the VA South Central Mental Illness } \\
\text { Research Education and Clinical Center }\end{array}$ & Moderate \\
\hline Franzini, 2011 & Yes & NR & No & Agency for Healthcare Research and Quality; NIH/NCRR & Moderate \\
\hline Gray, 2009 & No & NR & No & The Princess Alexandra Hospital Private Practice Fund & Moderate \\
\hline Harno, 2000 & Yes & NR & No & $\begin{array}{l}\text { The Finnish Office for Health Care and Technology Assessment } \\
\text { (FinOHTA); European Union Social Fund }\end{array}$ & Moderate \\
\hline Harno, 2001 & No & NR & No & $\begin{array}{l}\text { The Finnish Office for Health Care and Technology Assessment } \\
\text { (FinOHTA); European Union Social Fund }\end{array}$ & High \\
\hline Jacklin, 2003 & No & NR & Unclear & NHS; BT and the MSD Foundation & Moderate \\
\hline $\begin{array}{l}\text { Lamminen, } \\
2001\end{array}$ & No & NA & No & Finnish Cultural Foundation & High \\
\hline $\begin{array}{l}\text { Langabeer, } \\
2017\end{array}$ & Yes & NR & No & Internal & High \\
\hline Lilly, 2017 & Yes & NA & No & None & Moderate \\
\hline Loane, 2001 & Yes & NA & Yes & NR & Moderate \\
\hline Marcin, 2004 & Yes & NA & No & California Telehealth and Telemedicine Center & Moderate \\
\hline $\begin{array}{l}\text { Moreno- } \\
\text { Ramirez, } 2009\end{array}$ & Yes & NR & Yes & Instituto Carlos III & Low \\
\hline Natafgi, 2007 & Yes & NA & Yes & $\begin{array}{l}\text { Leona M. and Harry B. Helmsley Charitable Trust; Federal Office } \\
\text { of Rural Health Policy in the Health Resources \& Services } \\
\text { Administration (HRSA) to the Rural Telehealth Research \& } \\
\text { Journal of Telemedicine and Telecare Center; Avera Health }\end{array}$ & Moderate \\
\hline Noble, 2005 & No & NA & Yes & Unclear & Low \\
\hline $\begin{array}{l}\text { Ohinmaa, } \\
2002\end{array}$ & Yes & Unclear & Yes & Finnish Office for Health Technology Assessment (FinOHTA) & High \\
\hline Pak, 2009 & Yes & NR & No & $\begin{array}{l}\text { U.S. Army's Telemedicine and Advanced Technology Research } \\
\text { Center }\end{array}$ & Moderate \\
\hline Rendina, 1997 & Yes & NA & No & G. Wiliam Henry and the US NLM & High \\
\hline $\begin{array}{l}\text { Scuffham, } \\
2002\end{array}$ & Yes & Yes & Yes & $\begin{array}{l}\text { National Health Service Research and Development Programme } \\
\text { in Primary Dental Care and the Scottish Council for } \\
\text { Postgraduate Medical and Dental }\end{array}$ & Moderate \\
\hline Specht, 2001 & No & NR & No & VA HSRD & Moderate \\
\hline
\end{tabular}




\begin{tabular}{|c|c|c|c|c|c|}
\hline $\begin{array}{l}\text { Author, Year } \\
\text { (See Appendix } \\
\text { C for full } \\
\text { citation) }\end{array}$ & $\begin{array}{l}\text { Is an incremental } \\
\text { analysis of costs } \\
\text { and outcomes of } \\
\text { alternatives } \\
\text { performed? }\end{array}$ & $\begin{array}{l}\text { Are all future } \\
\text { costs and } \\
\text { outcomes } \\
\text { discounted } \\
\text { appropriately? }\end{array}$ & $\begin{array}{l}\text { Are all important } \\
\text { variables, whose values } \\
\text { are uncertain, } \\
\text { appropriately subjected } \\
\text { to sensitivity analysis? }\end{array}$ & Funding Source & $\begin{array}{l}\text { Risk of } \\
\text { Bias } \\
\text { Rating }\end{array}$ \\
\hline Stalfors, 2005 & No & NR & No & $\begin{array}{l}\text { Kunskap och Kompetens Foundation, the Assar Gabrielsson } \\
\text { Foundation; Jubilee Clinic Research Foundation; Gothenburg } \\
\text { Medical Society; ACTA Foundation }\end{array}$ & Moderate \\
\hline Thaker, 2013 & No & No & No & $\mathrm{NR}$ & Moderate \\
\hline $\begin{array}{l}\text { Tsitlakidis, } \\
2005\end{array}$ & Yes & NR & No & NR & Moderate \\
\hline $\begin{array}{l}\text { van der Pol, } \\
2010\end{array}$ & Yes & NR & No & Scottish Centre for Telehealth & Moderate \\
\hline Whited, 2003 & Yes & NR & Yes & VA Health Services Research and Development Service & Moderate \\
\hline $\mathrm{Xu}, 2008$ & Yes & NR & No & $\begin{array}{l}\text { Commonwealth Department of Health and Ageing (Medical } \\
\text { Specialist Outreach and Assistance Programme); Royal } \\
\text { Children's Hospital Foundation; Queensland Health }\end{array}$ & Moderate \\
\hline
\end{tabular}

$\mathrm{NR}$ = not reported; NA = not applicable

See Appendix C. Included Studies for full citations 


\section{Appendix H. Strength of Evidence}

Table H-1. Strength of evidence

\begin{tabular}{|c|c|c|c|c|c|c|c|c|}
\hline Topic & Outcome (KQ) & $\begin{array}{l}\text { Number } \\
\text { of } \\
\text { Studies } \\
\text { (N) }\end{array}$ & $\begin{array}{c}\text { Study } \\
\text { Limitations } \\
\text { (Low, } \\
\text { Medium, } \\
\text { High) }\end{array}$ & $\begin{array}{l}\text { Directness } \\
\text { (Direct, } \\
\text { Indirect) }\end{array}$ & $\begin{array}{l}\text { Consistency } \\
\text { Consistent, } \\
\text { Inconsistent, } \\
\text { Unknown }\end{array}$ & $\begin{array}{l}\text { Precision } \\
\text { (Precise, } \\
\text { Imprecise) }\end{array}$ & Main Findings & $\begin{array}{l}\text { Strength of } \\
\text { Evidence } \\
\text { Grade } \\
\text { (Insufficient, } \\
\text { Low, } \\
\text { Moderate, } \\
\text { High) }\end{array}$ \\
\hline \multirow[t]{6}{*}{$\begin{array}{l}\text { Inpatient } \\
\text { remote ICU }\end{array}$} & ICU Mortality (KQ1)a & 11 & Medium & Direct & Consistent & Precise & $\begin{array}{l}\text { RR } 0.69(95 \% \mathrm{Cl} 0.51,0.89) \text { Lower } \\
\text { ICU mortality with telehealth }\end{array}$ & Moderate \\
\hline & $\begin{array}{l}\text { Hospital Mortality } \\
\text { (KQ1) }^{\mathrm{a}}\end{array}$ & 12 & Medium & Direct & Consistent & Precise & $\begin{array}{l}\text { RR } 0.76(95 \% \mathrm{Cl}, 0.60,0.95) \text { Lower } \\
\text { hospital mortality with telehealth }\end{array}$ & Moderate \\
\hline & $\begin{array}{l}\text { ICU LOS } \\
(\text { KQ2) }\end{array}$ & 12 & Medium & Direct & Consistent & Precise & $\begin{array}{l}\text { Mean difference } \\
-0.39(95 \% \mathrm{Cl}-0.99,0.15) \text { No } \\
\text { significant difference in ICU LOS }\end{array}$ & Moderate \\
\hline & $\begin{array}{l}\text { Hospital LOS } \\
(\text { KQ2) }\end{array}$ & 12 & Medium & Direct & Consistent & Precise & $\begin{array}{l}\text { Mean difference } \\
-0.14(95 \% \mathrm{Cl}-0.96,0.63) \text { No } \\
\text { significant difference in hospital } \\
\text { length of stay }\end{array}$ & Moderate \\
\hline & \begin{tabular}{|l|}
$\begin{array}{l}\text { Harms } \\
\text { (KQ3) }\end{array}$ \\
\end{tabular} & 0 & NA & NA & NA & NA & $\begin{array}{l}\text { None expressly reported in } \\
\text { identified articles }\end{array}$ & Insufficient \\
\hline & Cost (KQ1) & 6 & Medium & Indirect & Inconsistent & Imprecise & $\begin{array}{l}\text { Unable to summarize across } \\
\text { studies: different methods and } \\
\text { inconsistent results. }\end{array}$ & Insufficient \\
\hline $\begin{array}{l}\text { Inpatient } \\
\text { specialty } \\
\text { consultations }\end{array}$ & Mortality (KQ1) & 12 & Medium & Direct & Consistent & Imprecise & No significant difference in mortality & Low \\
\hline \multirow[t]{4}{*}{$\begin{array}{l}\text { Inpatient } \\
\text { specialty } \\
\text { consultations }\end{array}$} & $\begin{array}{l}\text { Other clinical } \\
\text { outcomes (KQ1) }\end{array}$ & 6 & Medium & Direct & Consistent & Imprecise & $\begin{array}{l}\text { Clinical outcomes better with } \\
\text { telehealth, but not significantly } \\
\text { different }\end{array}$ & Low \\
\hline & \begin{tabular}{|l} 
Intermediate \\
outcome (KQ2)
\end{tabular} & 27 & Medium & Direct & Consistent & Imprecise & $\begin{array}{l}\text { Reductions in LOS and wait time but } \\
\text { all not significantly different; } \\
\text { satisfaction measures good but not } \\
\text { excellent }\end{array}$ & Low \\
\hline & Harms (KQ3) & 3 & High & Direct & Consistent & Imprecise & $\begin{array}{l}\text { Complications only reported in small } \\
\text { studies of teleproctored surgery with } \\
\text { high risk of bias }\end{array}$ & Insufficient \\
\hline & Cost (KQ1) & 7 & High & Direct & Inconsistent & Imprecise & $\begin{array}{l}\text { Cost savings due to avoiding } \\
\text { transfers or travel, but not in all } \\
\text { studies }\end{array}$ & Low \\
\hline
\end{tabular}




\begin{tabular}{|c|c|c|c|c|c|c|c|c|}
\hline Topic & Outcome (KQ) & $\begin{array}{l}\text { Number } \\
\text { of } \\
\text { Studies } \\
\text { (N) }\end{array}$ & $\begin{array}{l}\text { Study } \\
\text { Limitations } \\
\text { (Low, } \\
\text { Medium, } \\
\text { High) }\end{array}$ & $\begin{array}{l}\text { Directness } \\
\text { (Direct, } \\
\text { Indirect) }\end{array}$ & $\begin{array}{l}\text { Consistency } \\
\text { Consistent, } \\
\text { Inconsistent, } \\
\text { Unknown }\end{array}$ & $\begin{array}{l}\text { Precision } \\
\text { (Precise, } \\
\text { Imprecise) }\end{array}$ & Main Findings & $\begin{array}{l}\text { Strength of } \\
\text { Evidence } \\
\text { Grade } \\
\text { (Insufficient, } \\
\text { Low, } \\
\text { Moderate, } \\
\text { High) }\end{array}$ \\
\hline \multirow{6}{*}{$\begin{array}{l}\text { Emergency } \\
\text { Care: } \\
\text { Telestroke }\end{array}$} & $\begin{array}{l}\begin{array}{l}\text { Mortality In-hospital } \\
\text { (KQ1) }^{\mathrm{a}}\end{array} \\
\end{array}$ & 9 & Medium & Direct & Consistent & Precise & $\begin{array}{l}\text { RR } 0.89 \\
(95 \% \mathrm{Cl} 0.63,1.43) \text { No difference }\end{array}$ & Moderate \\
\hline & $\begin{array}{l}\text { Mortality } 3 \text { month } \\
\text { (KQ1) }^{\mathrm{a}}\end{array}$ & 7 & Medium & Direct & Consistent & Precise & $\begin{array}{l}\text { RR } 0.94 \\
(95 \% \mathrm{Cl} 0.82,1.16) \text { No difference }\end{array}$ & Moderate \\
\hline & Function (KQ1) & 15 & Medium & Mixed & Consistent & Imprecise & $\begin{array}{l}\text { Small differences in most studies } \\
\text { were not statistically significant }\end{array}$ & Low \\
\hline & $\begin{array}{l}\text { tPA administration } \\
\text { (KQ2) }\end{array}$ & 13 & Medium & Direct & Consistent & Imprecise & $\begin{array}{l}\text { Reported tPA use increases; four } \\
\text { significant the rest not significant or } \\
\text { not tested }\end{array}$ & Low \\
\hline & $\begin{array}{l}\text { Time to Treatment } \\
\text { (KQ2) }\end{array}$ & 23 & Medium & Direct & Inconsistent & Imprecise & $\begin{array}{l}\text { Time to treatment is not significantly } \\
\text { shorter in the majority of studies, } \\
\text { with a minority reported shorter or } \\
\text { longer times }\end{array}$ & Low \\
\hline & \begin{tabular}{|l} 
Harms (all \\
Hemorrhage) (KQ3)
\end{tabular} & 11 & High & Direct & Consistent & Imprecise & No difference in harms & Moderate \\
\hline \multirow[t]{4}{*}{$\begin{array}{l}\text { Emergency } \\
\text { Care: specialty } \\
\text { consultations }\end{array}$} & $\begin{array}{l}\text { Clinical outcome } \\
\text { (KQ1) }\end{array}$ & 13 & Medium & Direct & Consistent & Imprecise & $\begin{array}{l}\text { Lower, but not statistically } \\
\text { significant; mortality reported in } \\
\text { most studies. Four studies report } \\
\text { better clinical outcomes but only one } \\
\text { difference was statistically } \\
\text { significant }\end{array}$ & Low \\
\hline & $\begin{array}{l}\text { Intermediate } \\
\text { outcome (KQ2) }\end{array}$ & 19 & Medium & Direct & Consistent & Precise & $\begin{array}{l}\text { Increase in appropriate transfers } \\
\text { and care, decrease in time to } \\
\text { decision and time in ED }\end{array}$ & Moderate \\
\hline & Harms (KQ3) & 0 & NA & NA & NA & NA & $\begin{array}{l}\text { No studies reported data on harms } \\
\text { from telehealth }\end{array}$ & Insufficient \\
\hline & Cost (KQ1) & 5 & Medium & Direct & Inconsistent & Imprecise & $\begin{array}{l}\text { Four studies report lower costs with } \\
\text { better or no change in clinical } \\
\text { outcomes; one reported higher } \\
\text { costs }\end{array}$ & Low \\
\hline \multirow{2}{*}{$\begin{array}{l}\text { Emergency } \\
\text { Care: EMS or } \\
\text { Urgent Care }\end{array}$} & $\begin{array}{l}\text { Clinical Outcomes } \\
\text { (KQ1) }\end{array}$ & 10 & Medium & Direct & Consistent & Precise & $\begin{array}{l}\text { Telehealth reduced mortality for } \\
\text { STEMI patients }\end{array}$ & Moderate \\
\hline & $\begin{array}{l}\text { Intermediate } \\
\text { Outcomes (KQ2) }\end{array}$ & 20 & Medium & Direct & Consistent & Imprecise & $\begin{array}{l}\text { Treatment is more timely and fewer } \\
\text { air transfers or referrals to higher } \\
\text { level of care }\end{array}$ & Moderate \\
\hline
\end{tabular}




\begin{tabular}{|c|c|c|c|c|c|c|c|c|}
\hline \multirow[t]{3}{*}{ Topic } & Outcome (KQ) & $\begin{array}{l}\text { Number } \\
\text { of } \\
\text { Studies } \\
\text { (N) }\end{array}$ & $\begin{array}{c}\text { Study } \\
\text { Limitations } \\
\text { (Low, } \\
\text { Medium, } \\
\text { High) } \\
\end{array}$ & $\begin{array}{l}\text { Directness } \\
\text { (Direct, } \\
\text { Indirect) } \\
\end{array}$ & $\begin{array}{l}\text { Consistency } \\
\text { Consistent, } \\
\text { Inconsistent, } \\
\text { Unknown }\end{array}$ & $\begin{array}{l}\text { Precision } \\
\text { (Precise, } \\
\text { Imprecise) }\end{array}$ & Main Findings & $\begin{array}{l}\text { Strength of } \\
\text { Evidence } \\
\text { Grade } \\
\text { (Insufficient, } \\
\text { Low, } \\
\text { Moderate, } \\
\text { High) } \\
\end{array}$ \\
\hline & Cost (KQ1) & 5 & Medium & Indirect & Consistent & Imprecise & $\begin{array}{l}\text { Lower costs due to avoided } \\
\text { transfers or lower staff costs in most } \\
\text { studies }\end{array}$ & Low \\
\hline & Harms (KQ3) & 1 & Medium & Direct & NA & Imprecise & $\begin{array}{l}\text { One study reported data that could } \\
\text { be interpreted as harms, but not } \\
\text { defined as such by the authors }\end{array}$ & Insufficient \\
\hline \multirow[t]{11}{*}{ Outpatient } & $\begin{array}{l}\text { Clinical Outcomes } \\
\text { (KQ1): Dermatology }\end{array}$ & 3 & Medium & Direct & Consistent & imprecise & $\begin{array}{l}\text { No significant different in clinical } \\
\text { course }\end{array}$ & Low \\
\hline & $\begin{array}{l}\text { Clinical Outcomes } \\
\text { (KQ1): Wound Care }\end{array}$ & 5 & Medium & Direct & Consistent & Precise & $\begin{array}{l}\text { Better healing and fewer } \\
\text { amputations }\end{array}$ & Moderate \\
\hline & $\begin{array}{l}\text { Clinical Outcomes } \\
\text { (KQ1): } \\
\text { Ophthalmology }\end{array}$ & 0 & NA & NA & NA & NA & $\begin{array}{l}\text { No studies reported data on clinical } \\
\text { outcomes }\end{array}$ & Insufficient \\
\hline & $\begin{array}{l}\text { Clinical Outcomes } \\
\text { (KQ1): Orthopedics }\end{array}$ & 0 & NA & NA & NA & NA & $\begin{array}{l}\text { No studies reported data on clinical } \\
\text { outcomes }\end{array}$ & Insufficient \\
\hline & $\begin{array}{l}\text { Clinical Outcomes } \\
\text { (KQ1): Dental }\end{array}$ & 0 & NA & NA & NA & NA & $\begin{array}{l}\text { No studies reported data on clinical } \\
\text { outcomes }\end{array}$ & Insufficient \\
\hline & $\begin{array}{l}\text { Clinical Outcomes } \\
\text { (KQ1): Cancer }\end{array}$ & 1 & Medium & Direct & NA & Imprecise & $\begin{array}{l}\text { Rate of serious side effects from } \\
\text { chemotherapy reported in } 1 \text { study }\end{array}$ & Insufficient \\
\hline & $\begin{array}{l}\text { Clinical Outcomes } \\
\text { (KQ1): Psychiatry }\end{array}$ & $\begin{array}{l}3 \text { (in five } \\
\text { articles) }\end{array}$ & Medium & Direct & Consistent & Precise & $\begin{array}{l}\text { Decrease in symptoms and high } \\
\text { remission rates }\end{array}$ & Moderate \\
\hline & $\begin{array}{l}\text { Clinical Outcomes } \\
\text { (KQ1): Infectious } \\
\text { Disease }\end{array}$ & 3 & Medium & Direct & Inconsistent & Imprecise & $\begin{array}{l}\text { Inconsistent results for virologic } \\
\text { suppression across studies }\end{array}$ & Insufficient \\
\hline & $\begin{array}{l}\text { Clinical Outcomes } \\
\text { (KQ1): ): Single } \\
\text { Conditions with Dx } \\
\text { Technology }\end{array}$ & 0 & NA & NA & NA & NA & $\begin{array}{l}\text { No studies reported data on clinical } \\
\text { outcomes }\end{array}$ & Insufficient \\
\hline & $\begin{array}{l}\text { Clinical Outcomes } \\
\text { (KQ1): Single } \\
\text { Specialties }\end{array}$ & 6 & Medium & Direct & Consistent & Precise & $\begin{array}{l}\text { Positive effects on clinical outcomes } \\
\text { including response to treatment }\end{array}$ & Moderate \\
\hline & $\begin{array}{l}\text { Clinical Outcomes } \\
\text { (KQ1):Multiple } \\
\text { Specialties } \\
\end{array}$ & 4 & Medium & Indirect & Inconsistent & Imprecise & $\begin{array}{l}\text { Inconsistent results across studies } \\
\text { for unanticipated or avoidable health } \\
\text { services utilization }\end{array}$ & Insufficient \\
\hline
\end{tabular}




\begin{tabular}{|c|c|c|c|c|c|c|c|c|}
\hline \multirow[t]{6}{*}{ Topic } & Outcome (KQ) & $\begin{array}{l}\text { Number } \\
\text { of } \\
\text { Studies } \\
\text { (N) }\end{array}$ & $\begin{array}{l}\text { Study } \\
\text { Limitations } \\
\text { (Low, } \\
\text { Medium, } \\
\text { High) }\end{array}$ & $\begin{array}{l}\text { Directness } \\
\text { (Direct, } \\
\text { Indirect) }\end{array}$ & $\begin{array}{l}\text { Consistency } \\
\text { Consistent, } \\
\text { Inconsistent, } \\
\text { Unknown }\end{array}$ & $\begin{array}{l}\text { Precision } \\
\text { (Precise, } \\
\text { Imprecise) }\end{array}$ & Main Findings & $\begin{array}{l}\text { Strength of } \\
\text { Evidence } \\
\text { Grade } \\
\text { (Insufficient, } \\
\text { Low, } \\
\text { Moderate, } \\
\text { High) }\end{array}$ \\
\hline & $\begin{array}{l}\text { Intermediate } \\
\text { Outcomes: Access } \\
\text { (KQ2) }\end{array}$ & 35 & Medium & Direct & Consistent & Precise & $\begin{array}{l}\text { Access in terms of time to or } \\
\text { comprehensiveness of service is } \\
\text { improved }\end{array}$ & Moderate \\
\hline & $\begin{array}{l}\text { Intermediate } \\
\text { Outcomes : } \\
\text { Management and } \\
\text { Utilization (KQ2) }\end{array}$ & 31 & Medium & Indirect & Inconsistent & Imprecise & $\begin{array}{l}\text { Mixed results with majority finding } \\
\text { some benefit in terms of avoiding } \\
\text { visits and similar diagnosis or } \\
\text { management but a subset of studies } \\
\text { report differences in diagnosis and } \\
\text { management. }\end{array}$ & Low \\
\hline & $\begin{array}{l}\text { Intermediate } \\
\text { Outcomes: } \\
\text { Satisfaction (KQ2) }\end{array}$ & 22 & $\begin{array}{l}\text { Medium to } \\
\text { High }\end{array}$ & Direct & $\begin{array}{l}\text { Inconsistent } \\
\text { (across } \\
\text { respondent types; } \\
\text { consistent within } \\
\text { types) } \\
\end{array}$ & Imprecise & $\begin{array}{l}\text { Satisfaction generally the same; } \\
\text { patients higher if time/travel is } \\
\text { avoided. Providers the same or } \\
\text { slightly worse for telehealth }\end{array}$ & Low \\
\hline & Cost (KQ1) & 32 & $\begin{array}{l}\text { Medium to } \\
\text { High }\end{array}$ & Direct & Consistent & Imprecise & $\begin{array}{l}\text { Most but not all studies report cost } \\
\text { saving, but calculations vary and } \\
\text { most are dependent on patient } \\
\text { avoided travel and loss of time }\end{array}$ & Low \\
\hline & Harms (KQ3) & 2 & Medium & Indirect & Consistent & Imprecise & $\begin{array}{l}\text { Two studies reported on different } \\
\text { complications and serious adverse } \\
\text { events related to treatments for } \\
\text { different conditions }\end{array}$ & Insufficient \\
\hline
\end{tabular}

ED = emergency department, EMS = emergency medical services, ICU = intensive care unit, KQ = key question, LOS = length of stay, NA = not applicable, tPA = tissue plasminogen activator

${ }^{a}$ Based on studies included in meta-analysis 


\section{Appendix I. Exploratory Decision Modeling Methods and Results}

\section{Decision Model Topic Selection}

Unlike a traditional decision analysis, where the question of interest is typically prespecified, the novel purpose of this decision analysis was to address questions the systematic review (SR) alone cannot answer; the scope was dependent on the findings of the SR. Specifically, we used a decision model to estimate the potential economic impact of telehealth consultations for a selected clinical condition. In order to determine the clinical condition that was ultimately modeled, the studies included in the SR were categorized into one of three clinical practice settings: outpatient, inpatient, and emergency department or emergency medical services. These three settings were chosen as they were believed to be distinct in terms of the kind of consultations that may occur, represent different payment methodologies, and may require different technology and infrastructure. After the included studies were classified by practice setting, two investigators independently evaluated the studies included in the SR to identify a relevant topic, then sought feedback from authors of the SR and the project's technical expert panel.

The clinical areas within each practice setting were considered as potential topics for decision models (DMs) based on multiple criteria. Clinical areas with substantial published decision analysis or economic evaluation papers were excluded so that possible areas were limited to those with novel scientific questions. Next, those clinical specialties within practice settings with less than two published analyses that included both cost and outcome endpoints were excluded on the basis that they would lack adequate published information to inform a credible decision analysis. Additionally, studies that were classified as 'multiple' clinical areas or that included a broad mix of clinical indications, as was the case in many of the emergency department articles, were excluded as their scope was deemed too broad for our exploration of modeling approaches. The remaining clinical areas within each practice setting were considered by the two investigators for adequacy and scientific merit of potential decision analytic modeling.

Areas excluded given the identification of prior economic evaluations:

- Outpatient: dermatology, orthopedics

- Inpatient: intensive care units (ICU)

- Emergency department: psychiatry, radiology

Areas excluded due to our assessment of lack of publications/data to inform decision analysis:

- Outpatient: chronic pain, cardiology, dentistry, endoscopy, genetic counseling, hepatology, nephrology, oncology, pathology, rheumatology, urology

- Inpatient: burns/trauma, OB/GYN, psychology, wound care

- Emergency department: burns, cardiology

Final selection of two candidate areas for the decision analyses was made by consensus between the two members of the decision analysis team and then presented to the entire study team for consideration. These topics were then presented to the Technical Expert Panel for 
specific input on the modeling frameworks using an online survey, and ultimately neurosurgery consultations for patients with traumatic brain injury was selected.

\section{Cost Model for Telehealth Neurosurgical Consultations}

\section{Model Context}

Understanding the impact telehealth could have on cost is a major factor when considering policy changes to promote telehealth or when deciding to invest in implementing a telehealth system. In order to test the idea that a model could address questions the SR could not, we created a decision model designed to help health decision makers understand how telehealth consultations may impact health care costs for a given situation. We selected the use of telehealth for neurosurgical consultations by rural or community hospitals for patients with moderate to severe traumatic brain injury (TBI) for this model. This topic was selected for two reasons: 1) the systematic review did not identify a body of existing evidence that could adequately inform decisions about this use; and 2) neurosurgery is a specialty that is not widely available in all locations (such as rural areas) where people sustain TBIs.

The SR identified one randomized controlled trial (RCT) and three observational studies that assessed the impact of telehealth, but for three different patient populations in three different countries. A study in North Ireland found that patients with moderate TBI who were treated with telehealth had lower inpatient mortality but equivalent 3-month mortality as patients treated without telehealth. ${ }^{3}$ Another study in North Ireland, the only RCT in this group, reported higher rates of laboratory tests and imaging in the telehealth group. ${ }^{4}$ This study was the only to report economic outcomes and found telehealth consultations to cost on average approximately $47 \%$ more than in-person consultations. A study conducted in Italy reported no difference in mortality rates after telehealth was made available for patients hospitalized with minor TBI, ${ }^{5}$ and a study in Israel identified no significant difference in mortality when patients with neurological symptoms (not necessarily TBI) were either transferred to a center with specialist care or managed with a telehealth consult. ${ }^{6}$

Traumatic brain injury (TBI) represents a significant source of mortality and serious disability globally. ${ }^{7,8}$ Patients with TBI pose a challenge to the health care system in terms of resource demands for diagnosis and treatment planning for several reasons. Assessing a TBI patient and determining the severity of injury and appropriate treatment is complex. Much of the damage from TBI may not be from the primary injury, but from complications that develop in the hours and days following injury — these consequences are referred to as secondary injuries. ${ }^{9}$ For example, brain swelling can lead to increased intracranial pressure and more cell damage. Whether or not these secondary injuries occur, how quickly they can be identified, and their severity all vary. This may make it difficult to accurately identify the extent of the injury at the time of initial assessment. Additionally, the current tool frequently used in TBI assessment, the Glasgow Coma Scale (GCS), can be difficult to administer, particularly out-of-hospital, and requires both training and experience for it to be used reliably. ${ }^{10}$ While the severity of injury for some patients is likely to be obvious, warranting direct transport to a trauma center (e.g., those who have a low GCS score indicating coma or those with poly trauma), for a subgroup of patients with TBI, the severity of injury may not be readily apparent, and they may be transported to a hospital that is not a trauma center and does not have neurosurgical and neurotrauma expertise. 
Patients in level I and II trauma centers are managed by neurosurgeons or neurointensivists. However, TBI patients are often transported or present to lower level centers/community hospitals given the uncertainties inherent in assessment and progression of TBI outlined above. These hospitals often have limited numbers of neurosurgeons or may lack these specialists altogether. Prior studies suggest that patients with mild TBI can be safely managed without neurosurgical consultation, even in the presence of abnormal CT scans. ${ }^{11}$ However, in patients who may have moderate to severe TBI, care providers without specialty training and limited experience may be more reluctant to bypass neurosurgical consultation given the uncertainty, the potential severity, the potential need for neurosurgical intervention (NSI), and for fear of missing a feature of TBI that could lead to adverse consequences. Standard care at many of these hospitals is to stabilize the patient and transfer to a trauma center where this expertise is available.

There are several problems with transferring all suspected serious TBI patients and indications that this is not an efficient use of resources. A significant portion of patients transferred to trauma centers are discharged after a short stay or without undergoing surgery or treatment only available at the trauma center. This "over-transferring” blocks beds from patients who need trauma center care, reducing access. Also, transferring injured patients by air, sea, or land is costly, maybe harmful to the patient, and may place a burden on family who must travel to where their family member is treated. It is also important to acknowledge that many treatments for TBI are not benign and a transferred patient could be subjected to care with risks that may not outweigh the benefits.

An alternative to transferring all patients with a TBI to a trauma center is to use telehealth for neurosurgical consultation. This approach has the potential to allow providers at hospitals without this expertise to consult neurosurgeons to assess whether patients need to be transported. This could increase efficiency by allowing neurosurgeons to provide consultative services without travel, by avoiding transport and higher levels of care when they are not needed, by making them more available when they are, and provide both patients and onsite treating clinicians with peace of mind.

To supplement the systematic review and provide additional information for this specific use case, we developed and report a cost model of this type of telehealth consultation service for patients with moderate-severe TBI who are not transported to a high-level trauma center. Given that the telehealth intervention occurs in a short time period following injury and that the most severe TBI patients are likely to immediately transfer regardless of the availability of telehealth, we held patient outcomes constant (by assuming they would be equivalent). Although we found limited direct evidence, we believe this assumption is plausible based on indirect evidence from studies by Gale and colleagues on the impact of transfer distance and delay on outcomes in TBI. ${ }^{12}$ This study found that emergency personnel in the field were triaging and transporting more severe cases directly to higher-level trauma centers, and that neither time nor distance to the trauma center independently contributed to mortality. These findings were incorporated into the model by assuming that most severe patients will result in immediate transport regardless of the availability of telehealth and will not be affected by the existence of the two options (telehealth and standard care) and holding the outcomes of patients the same in the two branches of the model.

Because there is not sufficient direct evidence on how or whether patient outcomes differ when the consultation is in person or via telehealth, we did a "what if analysis" assuming equivalent clinical outcomes, focusing on understanding the drivers of cost differences. This is a 
critical assumption that must be considered when interpreting the model findings as the estimates of economic impact are specific to this particular segment of the TBI population and are limited to considering cost differences, without incorporating potential variations in clinical outcomes.

\section{Analytic Approach}

The objective of the model was to explore the cost implications of using telehealth as an alternative to transferring all TBI patients from a community or rural hospital to a trauma center. The model assesses costs from the perspective of a health care system that must decide how to allocate the scarce resources of neurotrauma expertise and trauma center beds. This scenario could be evaluated at different levels or perspectives and including different types of costs. Approaches could focus on the patient's family's travel costs, the telehealth return on investment for an individual hospital, the impact on a health system or region that includes a mix of hospital types, or the cost to society of using limited resources inefficiently.

We chose to create a model from the perspective of a health care system because health systems organize care for patients across multiple settings and, in this way, are similar to some current organizational and payment demonstrations that cross settings. Also, health care systems are a type of entity likely to consider investing in telehealth. The decision for a health system, that is, the alternatives explored in the model, are: Alternative 1-transfer all patients to a trauma center as soon as possible, or Alternative 2-invest in telehealth to allow remote neurosurgical consultation. With a telehealth consultation, experts view scans, monitors, and the patient and interact with the treating physicians and nurses. The consulting specialists can contribute to the initial assessment and recommend transfer or advise on management in place if the patient is not transferred.

Data to parameterize the model were obtained from the published literature. The results of the model were costs and incremental costs.

The basics of the model are outlined below:

Model structure: Decision tree

Audience: Health care system decisionmakers/administrators; people considering whether to invest in telehealth for an integrated health care system

Perspective: Health care system

Target patient population: New head injuries initially transported to a community or rural hospital (not a level I or II trauma center)

Intervention: Telehealth consultations between the treating medical team at the community hospital and a neurosurgeon or neurointensivist at a trauma center about whether to transfer the patient to a trauma center or manage the patient in place with ongoing consultation.

Comparator: In-person assessment and management at the trauma center after transferTime horizon: 30 daysOutcomes of interest: Cost to deliver care, provider time, patient time, patient travel time 


\section{Model Structure}

The decision analytic model was formulated as a decision tree (Figure I-1) using TreeAge Pro 2017. The base case patient in the model was an adult in whom moderate to severe TBI occurs in a rural area and did not result in a direct, emergent transfer to a level I or II trauma center, rather the patient was transported to a community or local hospital.

Patients with moderate to severe TBI who were not initially transported to a level I or II trauma center were assumed to either die early from their injury, either at the scene or during transport, or survive early mortality to be evaluated and treated. In our base case analysis, we assumed that early mortality rates between the two groups were equivalent, ${ }^{12}$ though mortality is a possible patient outcome later, and patient outcomes were allowed to vary later in the trajectory. This is a critical assumption and may not directly apply to all TBI patients.

Our analysis was designed to identify the components of costs for telehealth with local patient management and usual care (immediate transfer) in this situation so that a health system considering telehealth could better understand what drives the costs of these two options.

With telemedicine, some patients who would have been transferred are managed locally. We initially attempted to model both differences in costs and outcomes for these patients; however, we did not identify sufficient evidence to support whether the outcomes of these patients would be better or worse if they were transferred. Additionally, in many cases telehealth is implemented (and advocated for) based on the assumption of equivalence. Technology, image quality, and the accuracy of telehealth diagnoses have improved, and the studies included in the systematic review do not report harms due to inaccurate telehealth diagnoses. One potential cause of difference for outcomes of TBI, delay to time for treatment, has not been found to impact patient mortality for patients in well-organized trauma systems, though the comparisons made in this study are different from those evaluated in our model. ${ }^{12}$

Given no direct evidence of difference in clinical outcomes, we set outcomes to be equivalent to isolate the effect of telemedicine on costs. Because of the lack of direct evidence, there is uncertainty about which approach has better outcomes. Based on consultation with experts and the limited evidence available, we claim that our assumption of no difference is plausible, but acknowledge that other assumptions would be plausible as well.

Following initial survival of their injury, patients were modeled to undergo NSI depending on need, assessed through in-person consultation or teleconsultation. Transfer to a level I or II trauma center in the telemedicine model was contingent upon need for NSI following teleconsultation (i.e., patients who did not need NSI were managed at the rural or community facility). NSI was assumed to include one or more of the following procedures: ${ }^{13}$ placement of an intraparenchymal intracranial pressure (ICP) monitor, placement of an external ventricular drain, craniotomy, angiographic study, brain targeted therapy (osmotic therapy, hyperventilation, or sedation to lower ICP), placement of a lumbar drain, or diagnosis of a missed spinal injury.

In order to allow for future evaluation of patient outcomes, the modeling framework was constructed to include optional tracking of patient status, however this feature is not included in the currently reported results. Patient outcomes were categorized based on the Glasgow Outcome Scale (GOS) at 6 months: (1) death, (2) persistent vegetative state, (3) severe disability (loss of independence) (4) moderate disability, and (5) good outcome (return to pre TBI level of function or independent function). These outcomes were assumed to be equivalent for patients in the standard and telemedicine models who were not triaged for immediate transport by personnel in the field. ${ }^{12}$ Patients that survived their initial injury and did not undergo a NSI were assumed to have a good outcome. 
Figure I-1. Neurosurgical telehealth consultations decision-analytic model (decision tree)

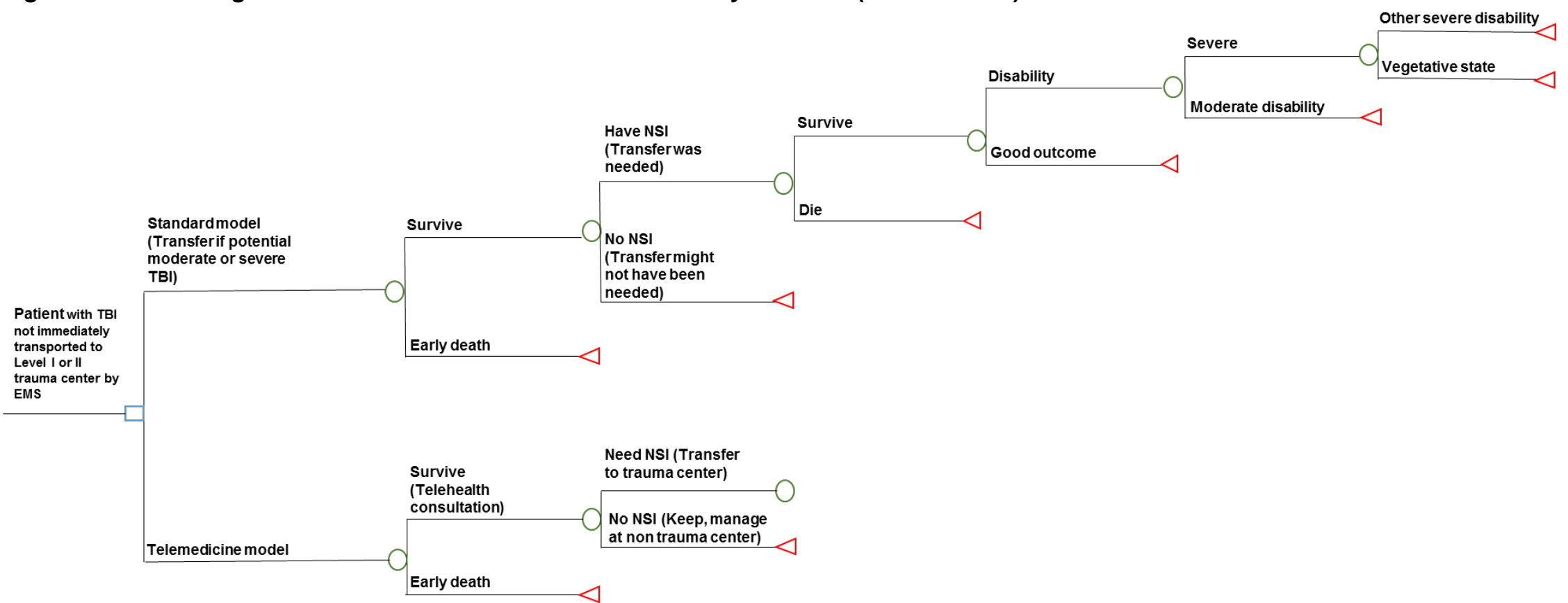

EMS = emergency medical services; NSI = neurosurgical intervention; TBI = traumatic brain injury Squares represent choice nodes; circles represent chance nodes; triangles represent end nodes 


\section{Probabilities}

The probabilities of possible events are summarized in Table I-1. Data to inform the individual node probabilities were currently unavailable from the published literature, thus the probabilities in each comparator were assumed to be equal. Though we note that a current project at the University of New Mexico funded through the Center for Medicare \& Medicaid Innovation (CMMI) is collecting these data.

Table I-1. Parameters used in the neurosurgical decision-analytic model

\begin{tabular}{|c|c|c|c|c|}
\hline Parameter & Baseline & Low & High & Reference \\
\hline \multicolumn{5}{|l|}{ Probabilities } \\
\hline Early Death & 0.09 & 0.07 & 0.11 & 14 \\
\hline Neurosurgical Intervention (NSI) & 0.10 & 0.08 & 0.12 & 14 \\
\hline Death after NSI & 0.10 & 0.08 & 0.12 & 14 \\
\hline Disability after TBI & 0.34 & 0.29 & 0.44 & 12 \\
\hline $\begin{array}{l}\text { Severe Disability (conditional, vs. } \\
\text { moderate) }\end{array}$ & 0.50 & 0.41 & 0.61 & 12 \\
\hline $\begin{array}{l}\text { Vegetative State (conditional, vs. other } \\
\text { severe disability) }\end{array}$ & 0.20 & 0.13 & 0.36 & 12 \\
\hline \multicolumn{5}{|l|}{ Costs } \\
\hline In-person neurosurgical consultation & $\$ 103.00$ & $\$ 52$ & $\$ 155$ & $15^{\star}$ \\
\hline Neurosurgical teleconsultation & $\$ 100.85$ & $\$ 50$ & $\$ 151$ & $15^{\star}$ \\
\hline \multicolumn{5}{|l|}{ Ambulance transportation } \\
\hline Land-based & $\$ 450.07$ & $\$ 225$ & $\$ 675$ & 16 \\
\hline Air/Sea ambulance & $\$ 10,105.34$ & $\$ 5,053$ & $\$ 15,158$ & 16 \\
\hline Percent land-based transfers & 0.75 & 0.60 & 0.90 & \\
\hline Neurosurgery & $\$ 25,376$ & $\$ 12,688$ & $\$ 38,064$ & $17^{\star \star \star}$ \\
\hline ICU care & $\$ 11,913$ & $\$ 5,957$ & $\$ 17,870$ & $17^{\star \star \star \star}$ \\
\hline Hospitalization (nontrauma center) & $\$ 5,002$ & $\$ 2,501$ & $\$ 7,503$ & $17^{\star \star \star \star \star \star}$ \\
\hline Hospitalization (trauma center, no NSI) & $\$ 7,682$ & $\$ 3,841$ & $\$ 11,523$ & $17^{\star \star \star \star \star}$ \\
\hline Hospitalization (trauma center with NSI) & $\$ 11,913$ & $\$ 5,957$ & $\$ 17,870$ & $17^{\star \star \star \star}$ \\
\hline Disability (moderate) & $\$ 85,000$ & $\$ 42,500$ & $\$ 127,500$ & 18, Assumption \\
\hline Disability (severe) & $\$ 1,000,000$ & $\$ 500,000$ & $\$ 1,500,000$ & 18, Assumption \\
\hline Vegetative state & $\$ 3,000,000$ & $\$ 1,500,000$ & $\$ 4,500,000$ & 18, Assumption \\
\hline $\begin{array}{l}\text { NSI = neurosurgical intervention; TBI = traumat } \\
\text { *CPT code 99221 } \\
* \text { **CPT code G0425 } \\
* * * \text { DRG } 025 \\
* * * * \text { DRG 082, Traumatic stupor and coma, com } \\
* * * * * \text { DRG 083, Traumatic stupor and coma, co } \\
* * * * * * \text { DRG 084, Traumatic stupor and coma, C }\end{array}$ & $\begin{array}{l}\text { hour with ma } \\
1 \text { hour with } \mathrm{c} \\
>1 \text { hour witho }\end{array}$ & $\begin{array}{l}\text { complications } \\
\text { lications } \\
\text { omplications }\end{array}$ & & \\
\hline
\end{tabular}

\section{Costs}

We estimated costs from the health care system perspective. Cost estimates were obtained from a variety of sources including the literature and primary analyses (Table I-1).

For ambulance transfers from local hospitals to trauma centers, we abstracted mean unit costs of air and land ambulance transportation from the Marketscan ${ }^{\circledR}$ databases ${ }^{16}$ from 2015 and assumed those mean costs of initial transportation were equivalent between the two scenarios and thus excluded them from the model. For the telemedicine model, we assumed that when transportation occurred, 75 percent occurred by road (as opposed to air or sea transport).

We also included costs of initial in-person consultation following TBI, telemedicine consultation following TBI, intensive care unit (ICU) costs, and hospitalization costs. 
Hospitalization costs were categorized by the site of service using diagnosis related group classifications of complications within the same class of traumatic stupor and coma. While diagnostic related groups (DRGs) are the basis for payments, not actual costs, they are constructed to represent costs. Trauma centers generally receive higher reimbursements through modifiers, and the different levels are used as proxies as follows: trauma center admissions requiring NSI were assumed to be at the cost of admissions with major complications. Trauma center admissions without NSI were assumed at the cost of admissions with complications as a proxy for higher cost admissions at trauma centers relative to community (nontrauma) hospitals. Nontrauma center admissions were assumed at the no complication DRG.

We assumed that patients who were subject to early mortality incurred ICU costs but no additional hospitalization costs. We used a discount rate of 3 percent, as recommended by the $2^{\text {nd }}$ Panel on Cost-Effectiveness in Healthcare and Medicine. ${ }^{19}$

\section{Base Case Analysis}

We estimated the incremental costs between the two decision options.

\section{Sensitivity Analyses}

We performed univariate (one-way) sensitivity analyses to assess the robustness of the model results. Each input parameter was varied independently as part of this sensitivity analyses to the minimum and maximum of its $95 \%$ confidence interval (CI) (or $+/-20 \%$ when the CI was not available), holding all other input parameters at their base case value. The resulting incremental cost values for each input's minimum and maximum were stored and plotted in tornado diagrams of descending order of influence on the overall model results.

\section{Base Case Analysis Results}

We present the results of the base-case analysis in Table I-2. Compared with the standard model, the telemedicine model results in cost savings of \$1,937 per patient.

Table I-2. Modeled costs and incremental costs comparing the telemedicine model to the standard model

\begin{tabular}{|l|l|l|}
\hline & Standard Model & Telemedicine Model \\
\hline Mean cost & $\$ 42,377$ & $\$ 40,440$ \\
\hline Incremental cost difference & & $\$ 1,937$ \\
\hline
\end{tabular}

\section{Univariate Sensitivity Analysis}

We present the results of the univariate sensitivity analysis as a tornado diagram for the impact on incremental cost (Figure I-2) comparing the telemedicine model to the standard model. The width of the bars represents the potential range of the estimate given the potential variation in each variable with the other variables held constant. As indicated by their order (highest impact on top), assuming equal patient outcomes, the incremental cost of telehealth compared with the standard model was most sensitive to the cost of hospitalizations without NSI in a trauma center. Continuing down the diagram the next most influential features are the cost of hospitalizations in nontrauma centers, and the cost of air ambulance transportation. The lower positions in the diagram and the narrower bars show that the cost of the consultation, whether in person or via telehealth, make a much smaller contribution to the variance in the cost estimate. 
Figure I-2. Tornado diagram of one-way sensitivity analysis showing impact of different variables on the incremental cost comparing the telemedicine and standard models

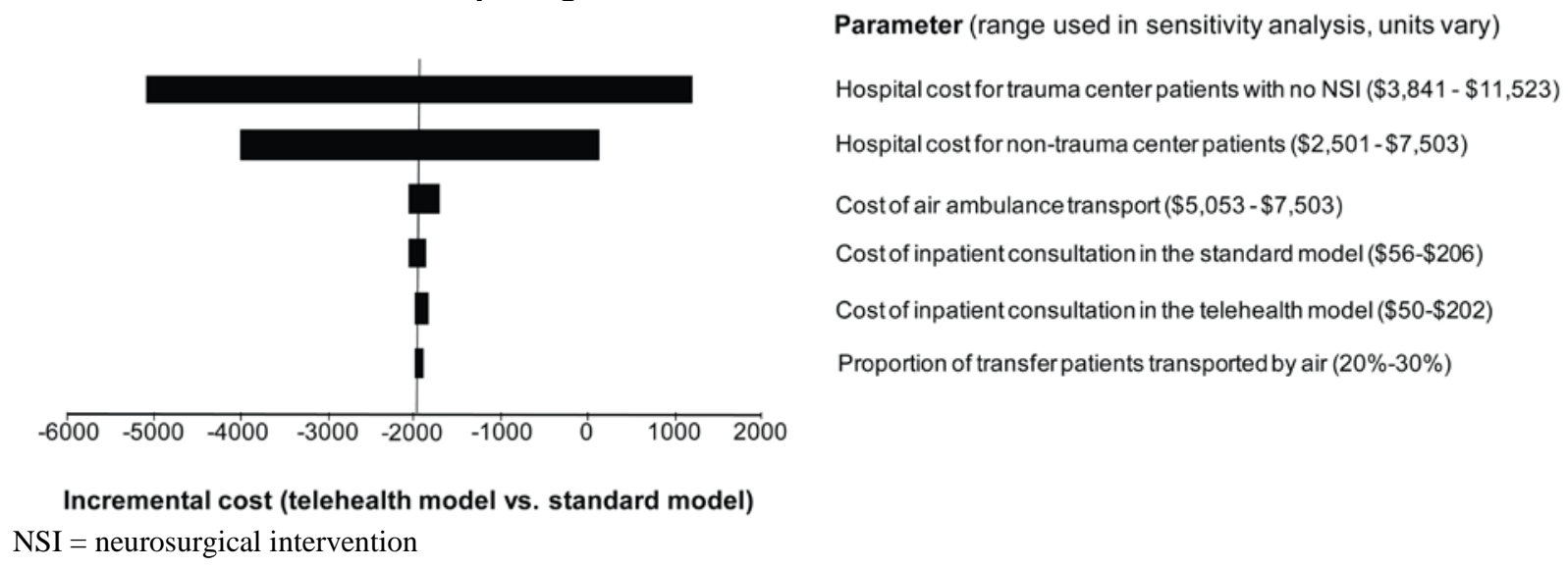

\section{Model Summary}

This analysis suggests that, assuming equivalent patient outcomes, for TBI patients not initially transported to a trauma center, the telemedicine model results in a decrease in health care system costs compared with standard management involving immediate transfer from the community hospital to a trauma center of any patients with potentially moderate or severe TBI. The analyses highlights that savings are driven by differences in the cost of hospitalizations at trauma centers and non-trauma hospitals (DRG reimbursements are used as a proxy for these differences as they are assumed to be relatively related to costs within the health system). This estimated decrease is realized by health systems through lower costs for managing a TBI patient in community hospitals relative to managing patients transferred to a trauma center where the patient did not require NSI. However, it is important to remember that this analysis assumes patient outcomes are equivalent for patients who are managed using a telehealth consultation and patients transferred to a trauma center for in-person assessment and management (standard care); an assumption for which the evidence is not robust.

Sensitivity analyses indicate that several model cost input parameters contribute to the overall uncertainty in the model results, and that the cost of the patient's hospitalization in different types of hospitals (with different costs) is the most important factor and more important than the cost of the telehealth consultation. Ultimately, assuming equivalent patient outcomes, the relative difference in hospital admission costs between community hospitals and trauma centers drives our findings. This means that cost savings are realized if telehealth allows a patient to remain and be treated in the lower cost hospital. But the magnitude of the savings is dependent on how much the costs differ for the specific hospital options for each patient.

\section{Limitations of Cost Model}

We based our selection of topics for the decision analysis on information available midway through the review as we wanted to create the products in parallel and report the results together. Our topic selection may have been different had we completed the review first or if we had established a priori data requirements for the decision analysis and structured the review to provide these. Though we used the SR to identify a topic for the DM, the modeling process allowed us to better understand some of the limitations in the existing literature, particularly in how studies report and analyze costs. In an iterative fashion, while constructing the DM, we 
revisited the included articles in the SR to better determine how investigators defined, reported and measured costs related to telehealth consultations. Ultimately, we found considerable variation in methods for acquiring and reporting cost data, making it difficult to assess cost outcomes across studies.

There are also several limitations to the decision modeling process that are important to consider. As with all models, the results are highly dependent on the analytic framework, assumptions, and available data to inform the calculations. An important assumption in this neurosurgical cost minimization model was equivalence in patient outcomes between the two approaches to patient management. Making this assumption allows consideration and evaluation of health care delivery costs in different settings given identical outcomes. This was done as rigorous data on outcome differences were not available.

Other models could be constructed that incorporate clinical outcomes as well as costs. These would provide more information and would allow the consideration of the balance of costs and outcomes. For example, if mortality or functional outcomes are worse for telehealth, then savings from telehealth could be outweighed by loss of life or disability. Alternatively, if outcomes are the same, consideration of the use of telehealth could focus exclusively on costs. It is also possible that telehealth could produce better outcomes if in-person consultations result in unneeded transfers and care or if transfers for in-person care negatively impact the patient's family by requiring them to travel further in order to be with the patient.

If systematic differences or uncertainty exist, then the cost modeling framework would be less relevant and a different model incorporating outcomes would be needed to make valid comparisons of the economic value of the two approaches to care. The model was built to allow inclusion of patient outcomes following treatment for cost benefit analyses in the future. When more and better data become available, the impact on mortality or quality adjusted life years (QALYs) could be used to inform judgements about the value of additional costs or savings given the patient benefits.

Most of the input parameters for the model were taken from the literature - informed by our systematic review, but some were also assumptions based on clinician and economist input. Initial transportation costs also represent an important cost component, which we assumed to be equivalent between the standard and telemedicine models as a conservative assumption. More granular data on the distances of patient transportation between the two scenarios could be important. Costs and potential outcomes could be affected by the differences in time and distance when all patients in the standard model are being transported to a trauma center versus a closer community hospital.

A more definitive test of the hypothesis that telehealth consultations provide better value for money could come from a trial-based economic evaluation, where patients are randomized to either standard management or a telehealth consultation. Given the limitations of the data available, we chose conservative values when multiple options were available, thereby underestimating overall effects.

\section{Key Findings and Implications}

The base case analysis found that, given the assumptions above, compared with the standard model of no telehealth consultation and transfer of patients with suspected moderate or severe TBI to a trauma center, the telemedicine model results in an incremental cost savings of \$1,937 per patient from the perspective of the health care system and payers. Sensitivity analyses indicate that telehealth may be cost-saving to the health care system and payers, but these 
savings are dependent on the exact costs and relative differences in costs for the different types of hospitalizations (i.e., the costs of the Level I or II trauma center for a patient not requiring a neurosurgical intervention (NSI) and the costs for a patient not transferred and cared for at the local hospital).

Specifically, the sensitivity analyses identified that the parameter that had the highest influence on the model results and that could change the direction of conclusion (e.g. cause the telehealth model to be more expensive) was if the cost of treating patients who did not require NSI in a trauma center reached the top of its range. In addition, when community hospital costs for NSI patients approached the upper end of its range, closing the difference in cost between community hospital and trauma center admissions, the telemedicine model became more costly. Furthermore, the assumption of equivalence in outcomes is fundamental to the relevance of these findings. In a scenario where mortality outcomes are not equivalent, any difference in costs could be easily outweighed by incremental differences in life years gained or lost.

These findings may be most relevant to alternative payment and service delivery models, such as accountable care organizations, and value based insurance designs, which have the ability to allocate patients to different settings, or from the perspective of payers/insurers who are responsible for reimbursement across several types of hospitals. Telehealth consultations appear to increase efficiency for a multi-hospital health system or payers, in that telehealth can be used to decide to treat patients in a lower cost setting (in this case in the local hospital), rather than transferring a patient who does not need NSI to a higher cost setting (e.g. a tertiary care center). However, if telehealth is only evaluated from the perspective of a single hospital, the conclusion might differ. For example, a community hospital may see an increase in revenue from patients who are retained in-house rather than transferred with telehealth but would not see the savings a health system would from avoiding a more expensive hospitalization. From these two different perspectives, the return on an investment in telehealth would differ because the amount of savings or change in revenue are likely different. Modeling could be expanded to compare these perspectives and identify when telehealth does result in savings.

It is also important to consider that the differences in costs may also vary across regions, depending on what services are available and how the health care system is organized. For example, some regions have diverse systems with independent community hospitals while others are covered by larger systems consisting of both community and tertiary care centers under the same umbrella organization. According to the 2016 Snapshot of U.S. Health Systems from the Comparative Health System Performance Initiative, these larger systems represent almost 43\% of hospitals in the U.S. ${ }^{20}$ with at least 18 hospitals per system. For these larger systems, the lower overall cost of care when telehealth is used to support treatment of patients who do not need NSI in lower cost hospitals represents a real opportunity for both cost and staff efficiency.

Furthermore, the reimbursement structure or payment model matters substantially in both the cost estimates and the incentives. For example, in an accountable care model, a large health system could reduce overall costs of care by shifting patients to the lower cost setting while retaining the same per person payment/reimbursement. However, under fee-for-service contracts, the hospitals may be reimbursed based on the location of care (regardless of what was needed) and the insurer/payer may or may not realize a difference in costs, depending on the reimbursement scheme and DRG modifiers. Thus, if the reimbursement in the community hospital were the same as the trauma center for a patient who did not undergo NSI, then there would be no cost difference. 


\section{Appendix I References}

1. Wijnen B, Van Mastrigt G, Redekop WK, et al. How to prepare a systematic review of economic evaluations for informing evidence-based healthcare decisions: data extraction, risk of bias, and transferability (part 3/3). Expert Rev. Pharmacoecon. Outcomes Res. 2016 Dec;16(6):723-32. doi: 10.1080/14737167.2016.1246961. PMID: 27762640.

2. Consensus Health Economic Criteria CHEC list. Maastricht University Health Services Research. https://hsr.mumc.maastrichtuniversity.nl/con sensus-health-economic-criteria-chec-list. Accessed Nov 152018.

3. Craig J, Chua R, Russell C, et al. A cohort study of early neurological consultation by telemedicine on the care of neurological inpatients. J. Neurol. Neurosurg. Psychiatry. 2004 Jul;75(7):1031-5. PMID: 15201365.

4. Chua R, Craig J, Wootton R, et al. Cost implications of outpatient teleneurology. J. Telemed. Telecare. 2001;7 Suppl 1:62-4. doi: 10.1177/1357633x010070s126. PMID: 11576496.

5. Migliaretti G, Ciaramitaro P, Berchialla P, et al. Teleconsulting for minor head injury: the Piedmont experience. J. Telemed. Telecare. 2013 Jan;19(1):33-5. doi: 10.1177/1357633X12474738. PMID: 23454822.

6. Klein Y, Donchik V, Jaffe D, et al. Management of patients with traumatic intracranial injury in hospitals without neurosurgical service. J. Trauma. 2010 Sep;69(3):544-8. doi: 10.1097/TA.0b013e3181c99936. PMID: 20234328.

7. Rubiano AM, Carney N, Chesnut R, et al. Global neurotrauma research challenges and opportunities. Nature. 2015 Nov 19;527(7578):S193-7. doi:

10.1038/nature16035. PMID: 26580327.
8. Report to Congress on Traumatic Brain Injury in the United States: Epidemiology and Rehabilitation. Centers for Disease Control and Prevention. National Center for Injury Prevention and Control; Division of Unintentional Injury Prevention. Atlanta, GA: 2015. Available at

https://www.cdc.gov/traumaticbraininjury/p df/tbi_report to_congress_epi_and_rehaba.pdf.

9. Prins M, Greco T, Alexander D, et al. The pathophysiology of traumatic brain injury at a glance. Dis. Model. Mech. 2013

Nov;6(6):1307-15. doi: 10.1242/dmm.011585. PMID: 24046353.

10. Chou R, Totten AM, Pappas M, et al. Glasgow Coma Scale for Field Triage of Trauma: A Systematic Review. Comparative Effectiveness Reviews, No. 182. AHRQ Publication No. 16(17)-EHC041-EF. Rockville, MD: Agency for Healthcare Research and Quality; 2017. Available at https://www.ncbi.nlm.nih.gov/books/NBK4 10016/ PMID: 28125195.

11. Huynh T, Jacobs DG, Dix S, et al. Utility of neurosurgical consultation for mild traumatic brain injury. Am. Surg. 2006 Dec;72(12):1162-5; discussion6-7. PMID: 17216813.

12. Gale SC, Peters J, Hansen A, et al. Impact of transfer distance and time on rural brain injury outcomes. Brain Inj. 2016;30(4):43740. PMID: 26963433.

13. Wu C, Orringer DA, Lau D, et al. Cumulative incidence and predictors of neurosurgical interventions following nonsevere traumatic brain injury with mildly abnormal head imaging findings. J Trauma Acute Care Surg. 2012 Nov;73(5):1247-53. doi: 10.1097/TA.0b013e318265d24e. PMID: 23064607.

14. Kosty J, Macyszyn L, Lai K, et al. Relating quality of life to Glasgow outcome scale health states. J. Neurotrauma. 2012;29(7):1322-7.

15. Centers for Medicare \& Medicaid Services. Physician Fee Schedule Search. 2017. https://www.cms.gov/apps/physician-feeschedule/search/search-criteria.aspx. Accessed August 12018. 
16. Truven Health MarketScan ${ }^{\circledR}$ Research Databases. 2015.

17. Defense Health Agency.

TRICARE/CHAMPUS Adjusted

Standardized Amounts (ASAs) \& DRG

Weights Summary for FY 2016. 2015.

https://health.mil/Military-Health-

Topics/Business-Support/Rates-andReimbursement/Diagnosis-Related-GroupRates/TRICARE-CHAMPUS-ASA-andDRG-Weights-Summary/Fiscal-Year-2016. Accessed August 12018.

18. Edmonds C. The steep cost of brain injury recovery: celebrities and 'concussion' get attention, but more help is needed. 2015. https://www.nextavenue.org/the-steep-costof-brain-injury-recovery/. Accessed August 12018.
19. Sanders GD, Neumann PJ, Basu A, et al. Recommendations for conduct, methodological practices, and reporting of cost-effectiveness analyses: second panel on cost-cffectiveness in health and medicine.

JAMA. 2016 Sep 13;316(10):1093-103. doi: 10.1001/jama.2016.12195. PMID: 27623463.

20. Snapshot of U.S. Health Systems, 2016. Agency for Healthcare Research and Quality; 2017.

https://www.ahrq.gov/sites/default/files/wysi wyg/snapshot-of-us-health-systems2016v2.pdf Accessed August 12018. 